\title{
5TH WORLD CONGRESS of Paediatric Cardiology and Cardiac Surgery
}

1126

Significance of Exercise Induced Electrocardiograms in long term after Total Cavo Pulmonary Connection Sachiko Kido, Japan

Toshikatsu Tanaka, Department of Cardiology, Hyogo Children's

Hospital, Japan

Hideki Fujita, Department of Cardiology, Hyogo Children's Hospital, Japan

Hirofumi Saiki, Department of Cardiology, Hyogo Children's Hospital, Japan

Kenta Tominaga, Department of Cardiology, Hyogo Children's

Hospital, Japan

Background: The modifications for Fontan procedure aimed at decreasing the incidence of arrhythmias, however a significant percentage of patients of modified Fontan operation have evidence of sinus nodal dysfunction or other arrhythmias, which can be the causes of sudden death or hospitalization in their long term outcome.

Objects: Under ambulatory monitoring many patients are less active than in usual life, so to evaluate the exact risk in daily life and during exercise for patients after total cavo pulmonary connection (TCPC) especially in long term outcome, we investigated their exercise electrocadiography.

Methods: We reviewed standard electrocardiogram (ECG) and exercise ECG (Double Master or Treadmill) of 34 consecutive patients over 15 years old who underwent TCPC procedures between 1988 and 1997 and followed over 10 years.

Results: 2 patients have the history of supraventricular tachycardia. Dysrhythmias at rest are found in 7 patients (20.5\%); junctional rhythm in 4 , atrioventricular block (I) in 1, significant irregular sinus rhythm in 1 and bradycardia (35bpm) in 1. Otherwise in ECG on exercise, 21 patients (62\%) have dysrhythmias; apparent PAC (>couplet, plural P) in 7, PVC (bigeminy 2, multifocal 1, symptom 1) in 6 , atrioventricular block (at max work load) in 2, irregular sinus rhythm (bigeminy like) in 2 and junctional rhythm + SAblock + PAC/PVC in 4. Conclusion: With exercise electrocardiography, cardiac dysrhysmias/arrhythmias after TCPC become tangible, and the prevalence of arrhythmia on exercise in long term outcome is rather high.

\author{
1127 \\ Surgical Treatment of Arrhythmias in Adults with \\ Congenital Heart Disease \\ Halkawt A Nuri, IRCCS Policlinico San Donato, Milan, Italy, Italy \\ Alessandro Giamberti, IRCCS Policlinico San Donato, Milan, Italy, \\ Italy \\ Jaspal S Dua, IRCCS Policlinico San Donato, Milan, Italy, Italy \\ Massimo Chessa, IRCCS Policlinico San Donato, Milan, Italy, Italy \\ Sara Foresti, IRCCS Policlinico San Donato, Milan, Italy, Italy \\ Angelo Micheletti, IRCCS Policlinico San Donato, Milan, Italy, Italy \\ Mario Carminati, IRCCS Policlinico San Donato, Milan, Italy, Italy \\ Alessandro Frigiola, IRCCS Policlinico San Donato, Milan, Italy, Italy
}

Objectives: Supraventricular and ventricular arrhythmias are a major cause of morbidity and mortality in adult patients with congenital heart disease (CHD). Intraoperative ablation offers an alternative to the complex surgical Cox-Maze procedure for these patients. We present the results of our preliminary experience with intraoperative monopolar irrigated radiofrequency ablation (IRA) in adults with CHD undergoing elective cardiac surgery.

Methods: Since September 2002, 58 adults with a mean age of 39 years with CHD underwent IRA during cardiac surgery. We performed 34 right-sided Maze procedures, 14 Cox-Maze III procedures and 10 right ventricular ablations. In addition, we implanted a pace-maker in 14 patients.

Results: Two patients $(2 / 58 ; 3.4 \%)$ died of causes not related to the intraoperative ablation. Over an average follow-up period of 40 months the remaining 56 patients are alive in NYHA class I or II. All patients were discharged on oral antiarrhythmic treatment for 3-months. All patients underwent ambulatory 24-hrs Holter monitoring 3 and 6-months after the ablation procedure and 9 underwent programmed ventricular tachycardia stimulation 6months post-procedure.

Forty-nine patients are still in spontaneous sinus rhythm, 3 are in sinus rhythm on chronic oral antiarrhythmic treatment for recurrence of atrial fibrillation, 3 are in stable atrial fibrillation and 1 has pacemaker rhythm. There were no complications from the IRA.

Conclusions: Intraoperative IRA is a safe and effective procedure to control arrhythmic problems in adults with CHD. This 
procedure should be taken into consideration when transcatheter ablation fails or when elective cardiac surgery is planned.

\section{8}

Diastolic Ventricular Interaction in Patients after Atrial Switch for Transposition of the Great Arteries

Pak-cheong Chow, Department of Paediatric Cardiology, Queen Mary Hospital, Univeristy of Hong Kong, Hong Kong

Xue-cun Liang, Department of Paediatric Cardiology, Queen Mary

Hospital, University of Hong Kong, Hong Kong

Yiu-fai Cheung, Department of Paediatric Cardiology, Queen Mary

Hospital, University of Hong Kong, Hong Kong

Objective: We tested the hypothesis that systemic right ventricular (RV) diastolic dysfunction and diastolic ventricular interaction occur after atrial switch operation for transposition of the great arteries (TGA).

Methods: Twenty-nine patients aged $20.8 \pm 4.1$ years post-atrial switch operation and 27 healthy controls were studied. Twodimensional longitudinal systolic strain and systolic (SRs), early diastolic (SRe), and late diastolic (SRa) strain rates of both ventricles were determined using tissue speckle echocardiography. Early diastolic trans-atrioventricular velocity (E) and myocardial early diastolic myocardial velocity (e) at ventricular free wall-annular junction were measured. Geometry of the morphologic left ventricle was quantified by the diastolic eccentricity index (EI).

Results: In both systemic and subpulmonic ventricles, SRe and SRa were significantly lower and trans-atrioventricular E/e ratios higher in patients than controls (all $\mathrm{p}<0.001$ ). Significant leftward bulging of septum in patients was suggested by the greater left ventricular (LV) EI $(\mathrm{p}<0.001)$. As a group, LV EI correlated negatively with LV SRe $(\mathrm{r}=-0.62, \mathrm{p}<0.001)$ and LV SRa $(r=-0.51, p<0.001)$, and positively with mitral E/e ratio $(r=0.33, p=0.02)$. In patients, $R V$ SRe correlated with LV SRe $(r=0.49, p=0.008)$, and RV SRa correlated with LV $\mathrm{SRa}(\mathrm{r}=0.46, \mathrm{p}=0.01)$. Furthermore, in patients, the systemic RV SRe correlated with RV SRs $(r=0.52, p=0.004)$ and LV systolic strain $(\mathrm{r}=0.41, \mathrm{p}=0.03)$.

Conclusion: Systemic RV diastolic dysfunction occurs after atrial switch operation for TGA and correlates with subpulmonic ventricular diastolic dysfunction. The observed diastolic ventricular interaction is probably mediated through alteration of septal geometry.

\section{8}

Where's the thin red line between benefit and non-benefit left-to-right shunt closure

Supaporn Roymanee, Prince of Songkla University, Had Yai, Songkla, Thailand

Saranwan Phetphisal, Prince of Songkla University, Had Yai, Songkla, Thailand

Nakharin Tonklang, Prince of Songkla University, Had Yai, Songkla, Thailand

Background: In severe pulmonary arterial hypertension (PAH) associated with left-to-right shunts or impending Eisenmenger physiology patients, there were not only no consensus criteria to predict reversibility of PAH but also unclear benefit outcomes post shunt closure.

Method: We reviewed hemodynamic parameters in three patients with severe PAH associated left-to-right shunt who underwent uneventful transcatheter closure and intermediate post procedure showed decrement of $\mathrm{PAH}$, also with improvement in theirs exercise performance and quality of life.

Result: All patients have equalization of pulmonary artery pressure (PAP) and systemic artery pressure (AoP) combined with high pulmonary vascular resistance $(\mathrm{Rp})$. Pre and post $100 \%$ oxygen administration data were collected, results as show in Table.

Post oxygen administration, parameters showed common features as follows: 1) $\mathrm{Rp}$ decreased $>25 \%$, 2) Rp:Rs decreased $>25 \%$, 3) Qp:Qs $>2$, 4) The ratio of systolic or mean between PAP and AoP decreased $>20 \%$, 5) Lower diastolic blood pressure of PAP as compare with AoP at least $20 \mathrm{mmHg}, 6$ ) Aortic saturation $(\mathrm{SaO} 2)$ was $>95 \%$. We propose that beside trial occlusion which require more equipments, time consumption and costly, patients who have at least 4 from 6 criteria will achieve costless, safe and benefit transcatheter closure.

Conclusion: We should focus on hemodynamic data changing after oxygen administration, to predict reversibility of PAH and beneficial effect from left-to-right shunt closure.

\begin{tabular}{|c|c|c|c|c|c|c|c|c|}
\hline Lesion & $\begin{array}{c}\text { Age } \\
\text { (year) }\end{array}$ & Condition & $\begin{array}{c}\mathrm{SaO} 2 \\
(\%)\end{array}$ & $\begin{array}{c}\text { PAP } \\
(\mathrm{S} / \mathrm{D} / \mathrm{M})\end{array}$ & $\begin{array}{c}\text { AoP } \\
(\mathrm{S} / \mathrm{D} / \mathrm{M})\end{array}$ & Qp:Qs & Rp:Rs & $\underset{\left(\mathrm{WU} / \mathrm{m}^{2}\right)}{\mathrm{Rp}_{\mathrm{p}}}$ \\
\hline \multirow{2}{*}{$\begin{array}{l}\text { 1. ASD } \\
23 \mathrm{~mm}\end{array}$} & 56 & - room air & 94 & $101 / 29 / 53$ & $101 / 69 / 78$ & 1.2 & 0.55 & 20.8 \\
\hline & & $-100 \%$ O2 & 99 & $101 / 28 / 49$ & $123 / 77 / 95$ & 2.3 & 0.23 & 11.9 \\
\hline \multirow[t]{2}{*}{$\begin{array}{l}\text { 2. PDA } \\
24 \mathrm{~mm}\end{array}$} & 38 & - room air & 95 & $92 / 50 / 67$ & $90 / 51 / 68$ & 1.5 & 0.67 & 9.2 \\
\hline & 1 & $-100 \%$ O2 & 99 & $85 / 69 / 54$ & $\begin{array}{l}85 / 69 / 54 \\
109 / 55 / 78\end{array}$ & 2.8 & 0.30 & 2.6 \\
\hline $\begin{array}{l}\text { 3. PDA } \\
10 \mathrm{~mm}\end{array}$ & & $-100 \% \mathrm{O} 2$ & 100 & $94 / 46 / 67$ & $157 / 58 / 78$ & 2.8 & 0.23 & 3.3 \\
\hline
\end{tabular}

\section{9}

Mortality and serious morbidity associated with Fontan revision surgery

Zaheer Ahmad, Congenital Cardiac Centre, Southampton University Hospital NHS Trust, United Kingdom

Gruschen Veldtman, Congenital Cardiac Centre, Southampton University Hospital NHS Trust, United Kingdom

Hunaid Vohra, Congenital Cardiac Centre, Southampton University Hospital NHS Trust, United Kingdom

Ransika De Silva, Congenital Cardiac Centre, Southampton University Hospital NHS Trust, United Kingdom

Saket Badle, Congenital Cardiac Centre, Southampton University Hospital NHS Trust, United Kingdom

Robert Whistance, Congenital Cardiac Centre, Southampton University Hospital NHS Trust, United Kingdom

Anthony P Salmon, Congenital Cardiac Centre, Southampton University Hospital NHS Trust, United Kingdom

Marcus Haw, Congenital Cardiac Centre, Southampton University Hospital NHS Trust, United Kingdom

Objective: Fontan revision surgery though effective in relieving late arrhythmia burden associated with a failing Fontan circulation, has important mortality risk. Peri-operative serious morbidity, however, is poorly described. We sought to define mortality $[<30$ days, in hospital and late mortality] and serious morbidity [renal replacement therapy (RRT), prolonged ICU stay $>10$ days] and a composite endpoint (CE) of death, ECMO, RRT, prolonged ICU stays and ventilation $>3$ days, in this group.

Methods: Hospital records were retrospectively reviewed for baseline and peri-operative characteristics, as well as outcomes as defined by the endpoints above. 
Results: 40 patients (19 female, mean age $24 \pm 9$ years, range: 9-48 years) had Fontan revision surgery during 1997 through 2008, for arrhythmia in 29 patients, pathway obstruction in 2 patients, arrhythmia and pathway obstruction in 5 patients and effort intolerance in 3. Mean bypass time was $237 \pm 93$ mins. The 30-day mortality was $7 \%(\mathrm{n}=3)$ and in-hospital mortality $12 \%(\mathrm{n}=5)$. Five-year actuarial survival was $76 \pm 7 \%$ and $65 \pm 11 \%$ at 10 years. Serious morbidity included: RRT ( $\mathrm{n}=7,17.5 \% ; 5$ died), prolonged ICU stay $(\mathrm{n}=8,20 \% ; 4$ died), ECMO $(n=1)$ and CE $(n=13,32 \%, 5$ died $)$. Ventricular dysfunction was an independent predictor of RRT and prolonged ICU stay $(\mathrm{p}<0.01)$, and the composite end point. The use of circulatory arrest was associated with prolonged ICU stay and prolonged ventilation $(\mathrm{p}<0.05)$.

Conclusions: Fontan revision surgery though having a relatively low 30-day mortality, is commonly associated with serious morbidity. Specialized and dedicated peri-operative care is paramount in achieving good outcomes. Risk stratification remains challenging in this group.

\section{0}

Influence of Interventional Cardiology on the Management of Adults with Congenital Heart Disease Massimo Chessa, IRCCS Policlinico San Donato, Milan, Italy, Italy Jaspal S Dua, IRCCS Policlinico San Donato, Milan, Italy, Italy Diana Negura, IRCCS Policlinico San Donato, Milan, Italy, Italy Luciane Piazza, IRCCS Policlinico San Donato, Milan, Italy, Italy Angelo Micheletti, IRCCS Policlinico San Donato, Milan, Italy, Italy Gianfranco Butera, IRCCS Policlinico San Donato, Milan, Italy, Italy Alessandro Giamberti, IRCCS Policlinico San Donato, Milan, Italy, Italy

Mario Carminati, IRCCS Policlinico San Donato, Milan, Italy, Italy

Objective: Transcatheter interventional strategy for management of adults with congenital heart disease (ACHD) is increasingly the preferred invasive treatment option. Recently, several techniques have been developed towards this. We aim to assess the impact of these procedures on the management of ACHD.

Methods: Retrospective study of ACHD patients (age $\geqslant 18 \mathrm{yrs}$ ), who underwent cardiac catheterisation (CC) from January 2000 to December 2008. Data collected included diagnosis, type of procedure and complications.

Results: 1784 (54\% women) ACHD patients underwent CC (mean age $42 \pm 14.5$ years). $19 \%$ had diagnostic and the rest, interventional procedures.

The majority were ASD and PFO closures (82\%) with a major complication rate of $<2.5 \%$ (ASDs). The other procedures in descending frequency were: aortic coarctation stenting, VSD closure, PDA embolization, pulmonary valvuloplasty, pulmonary artery stenting and others. There was only 1 death. Over the last 9 -years, the number of patients undergoing surgery was constant ( $\sim 80$ /year) but the numbers of those undergoing interventional CC increased steadily from 100/yr in 2000 to >225 in 2008.

Conclusion: The evolving use of interventional catheterisation in ACHD has major implications on healthcare organizations for providing optimal care, especially for patients with complex lesions. Increasingly, ACHD with simpler lesions are being managed by interventional catheterisation, hence the percentage of complex cases for surgery is increasing. Hopefully, new developments like transcutaneous valves \& hybrid procedures will help to lessen this surgical load.

In conclusion, we believe that the interventional treatment option for ACHD is safe, successful and will have a wide-ranging impact on patients and healthcare agencies.
2047

Adults With Congenital Heart Disease - Chennai Experience $K M$ Cherian, Frontier Lifeline \& the Dr K.M. Cherian Heart Foundation, India

India has a population of 1.2 Billion, second to China. Diagnosis and treatment for congenital heart disease has become prevalent only during the last 20 years. In this presentation, we report our experience with a series of 711 patients above the age of 18 years with congenital heart disease seen in a single institution between 2004 and 2008. We have reported earlier, a series of 1142 adults with congenital heart disease at the international society of Adult congenital heart disease meeting held in 1998 at Houston. The above series has not been quoted in this paper at all since the Institution has changed and the follow up has not been sufficient. Both the earlier and the present series are a single surgeon's experience from two institutions. There are other institutions in Chennai performing congenital heart surgery. The combined experience of all the institution in Chennai will be more extensive. In the present study, Patients are assessed using a questionnaire to obtain information on their Present age, Education, Employment status, marital status, if married, how many children, whether children are healthy/having congenital malformations, whether on continued medical treatment, Quality of life, frequency of follow-up, current financial status including whether independent or in service. More than $70 \%$ of patients were followed-up so far and the follow-up of remaining patients will be completed and the results will be presented.

\author{
2063 \\ Long-term Result of Anatomical Repair for Discordant \\ Atrioventricular Connections: Comparison of Anatomical \\ and Physiological Repair \\ Takeshi Konuma, Tokyo Women's Medical University Divisions of \\ Cardiovascular Surgery, Japan \\ Yasuharu Imai, Tokyo Women's Medical University Divisions of \\ Cardiovascular Surgery, Japan \\ Kohta Agematsu, Japan \\ Kiyotaka Yano, Japan \\ Masaki Sasoh, Department of Cardiovascular Surgery, Heart Institute of \\ Japan, Tokyo Women's Medical University, Japan \\ Toshio Nakanishi, Tokyo Women's Medical University, Pediatric \\ Cardiology, Japan \\ Hiromi Kurosawa, Tokyo Women's Medical University, Cardiovascular \\ Surgery, Japan
}

Background: Anatomical repair (Double-Switch operation) is expected to have improved long term result by using morphological left ventricle and mitral valve in the systemic circulation compared to conventional repair for discordant atrioventricular connections, but longer-term impact remains unknown. In this study we reviewed the late result of various type procedures and analyzed the risk factor, mortality and reintervention.

Methods: Since 1972, a total of 207 patients with discordant atrioventricular connections (1-TGA) underwent surgical repair and we excluded single ventricle patients in this study. Conventional repair was 55 patients (including 31 conventional Rastelli patients) (group I), Fontan type procedure were 43 patients (group II), Double-Switch operation were 90 patients (group III). Furthermore in group III, we compared 72 patients who had Rastelli procedure (group A) and 18 patients underwent arterial switch (group B).

Results: The Kaplan-Meier estimated survival rates at 20 years was $70 \%$ in group I, $86 \%$ in group II, and $79 \%$ in group III 
$(p=0.26)$. Freedom from reintervention was $49 \%$ in group I, $52 \%$ in group II, and $43 \%$ in group III $(p=0.03)$. In DoubleSwitch operation, survival ratio was $78 \%$ in group A, $81 \%$ in group $B(p=0.84)$, and Freedom from reintervention was $37 \%$ in group $A, 74 \%$ in group $B(p=0.05)$.

Conclusion: There were no significant differences between groups in survival ratio, but tricuspid regurgitation was risk factor for conventional repair. In Double-Switch procedure, combination of Rastelli and atrial switch procedure was risk factor for reintervention, but mortality was same to the group of arterial and atrial switch.

\section{4}

\section{Morphologic variation in Hypoplastic Left Heart Syndrome}

Douglas A Wall, The Prince Charles Hospital/Birmingham Childrens Hospital, Australia

Oliver Stumper, Birmingham Childrens Hospital, United Kingdom Zdenka Reinhardt, Birmingham Childrens Hospital, United Kingdom Timothy Jones, Birmingham Childrens Hospital, United Kingdom David J Barron, Birmingham Childrens Hospital, United Kingdom William J Brawn, Birmingham Childrens Hospital, United Kingdom

Background: To date conflicting studies exist regarding differing morphological variants of hypoplastic left heart syndrome and their impact on mortality. Although Aortic Atresia (AA) is identified by some as a risk factor for mortality, recent data suggests that mitral valve patency is important within this context.

Methods: 222 consecutive patients who underwent the Norwood procedure from May 2002 to May 2007 are included in this study. All patients in the study had systemic right ventricles and pulmonary blood flow provided by a right ventricle-to-pulmonary artery conduit at stage 1 palliation. The study population included only patients with Hypoplastic Left Heart Syndrome (HLHS) and were divided into three groups: aortic atresia-mitral atresia (AA$\mathrm{MA}$ ), aortic stenosis-mitral stenosis (AS-MS), and aortic atresiamitral stenosis (AA-MS). Statistical analysis was conducted using a 2 tailed Fisher exact test and differences were considered statistically significant when $\mathrm{p}<0.05$.

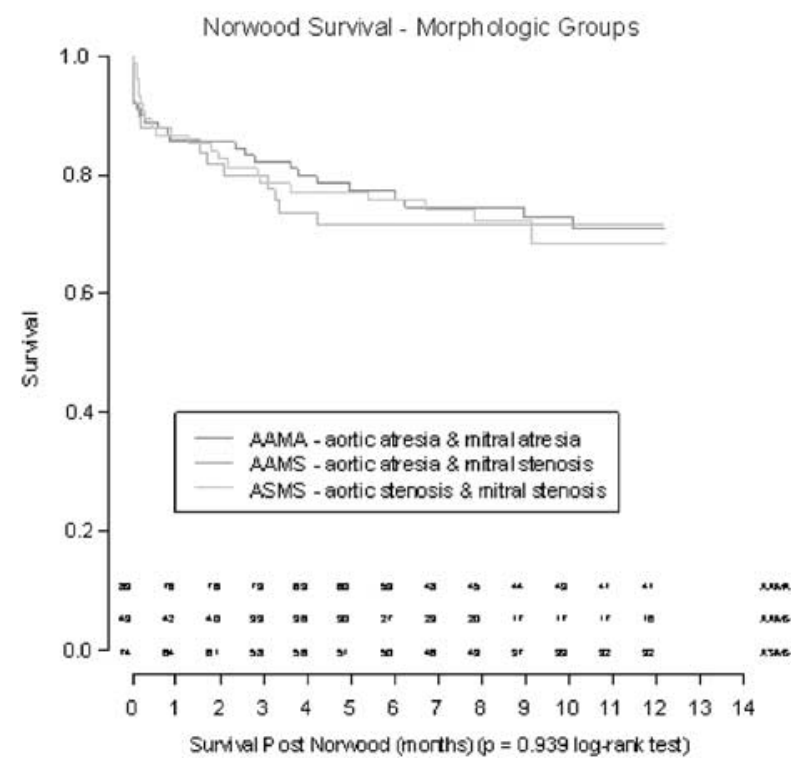

Results: Of the 222 patients who underwent the Norwood procedure, 212 had a diagnosis of hypoplastic left heart syndrome. Of these 89 had AA-MA, 74 had AS-MS, and 49 had AA-MS. A Kaplan-Meier survival curve is presented below showing no significant survival differences between morphologic groups $(p=0.93)$. Using 30 day mortality, no statistical differences could be demonstrated between either of the morphologic groups.

Conclusions: The current surgical management of Hypoplastic Left Heart Syndrome at Birmingham Childrens Hospital confers equivalent survival advantage to the different morphologic groups seen in this condition.

\section{0}

Impairment in brain maturation on magnetic resonance imaging is common in infants undergoing surgery for congenital heart disease

Julia K Gunn, Murdoch Childrens Research Institute, Melbourne, Australia

Rod Hunt, The Royal Children's Hopsital, Australia

Lee Coleman, The Royal Children's Hopsital, Australia

John Beca, Starship Children's Hospital, Auckland, New Zealand

Terrie Inder, Washington University, St Louis, United States

Lara Shekerdemian, The Royal Children's Hopsital, Melbourne,

Australia

Objective: To identify brain injury and maturation impairment in young infants undergoing cardiac surgery.

Methods: 74 full-term infants undergoing surgery for CHD were enrolled in a prospective study of brain injury. Brain MRI was undertaken prior to surgery (MRI 1), 1 week following surgery (MRI 2) and at 2 months (MRI 3). T1, T2 and DWI were obtained with $1 \mathrm{~mm}$ slices in axial and coronal planes. Two blinded assessors scored images for white matter injury (WMI), grey matter injury (GMI), maturation (cortical folding and myelination) and CSF spaces. $\mathrm{Chi}^{2}$ analysis was used to compare groups.

Results: Median age at surgery was 7 days (IQR 3-15). Surgical procedures were: Norwood-type palliation (24), systemicpulmonary artery shunts (13), arterial switch operations (20), common arterial trunk repairs (5), aortic arch repairs (10) and PA banding (2). Cardiopulmonary bypass was required in 63 (85\%) patients. The age at MRI scan, and associated abnormalities are given in the Table. Maturation impairment was present in 70 $(38 \%)$ of MRI scans. This was unrelated to gestational age, but was associated with early WMI, and subsequent increased CSF spaces $(\mathrm{p}<0.05)$. It was more common in cardiac anomalies requiring either a shunt procedure or truncus repair $(\mathrm{p}=0.007)$. Punctate white matter lesions were common on MRI 2. These were not related to either surgical procedure or the use of bypass. Conclusions: WMI is common in young infants undergoing cardiac surgery. Impairment of maturation is also common, and is related to cardiac anomaly. This may predict subsequent brain injury and development.

\begin{tabular}{|c|c|c|c|c|}
\hline MRI abnormality & MRI 1 (69) & MRI 2 (59) & MRI 3 (54) & $\begin{array}{l}\text { Total patients } \\
(74)\end{array}$ \\
\hline $\begin{array}{l}\text { Median age of scan } \\
\text { (IQR) }\end{array}$ & $\begin{array}{c}4(3-15) \\
\text { days }\end{array}$ & $\begin{array}{c}18(14-25) \\
\text { days }\end{array}$ & $\begin{array}{c}61\left(\begin{array}{c}(42-74) \\
\text { days }\end{array}\right.\end{array}$ & \\
\hline WMI & $9(13 \%)$ & $25(42 \%)$ & $9(17 \%)$ & $41(55 \%)$ \\
\hline GMI & $5(7 \%)$ & $3(5 \%)$ & $5(9 \%)$ & $7(9 \%)$ \\
\hline CSF space & $3(4 \%)$ & $20(34 \%)$ & $22(41 \%)$ & $31(42 \%)$ \\
\hline Maturation delay & $31(45 \%)$ & $29(49 \%)$ & $10(13 \%)$ & $40(54 \%)$ \\
\hline
\end{tabular}


2101

Analysis of early outcomes of neonatal congenital heart surgery in EACTS Congenital Database. Is body weight below $2,5 \mathrm{~kg}$ still the risk factor for hospital mortality? Andrzej Kansy, Cardiothoracic Department, Childrens' Health

Memoral Hospital Warsaw, Poland

Zdzislaw Tobota, Poland

Przemyslaw Maruszewski, Poland

Bohdan Maruszewski, Cardiothoracic Department, Childrens' Health Memoral Hospital Warsaw

Objective: Low body weight has been confirmed as risk factor for early death after neonatal congenital heart surgery. We have studied the data on 12271 neonatal CHS procedures of the EACTS Congenital Database, 1999-2008 after excluding PDA procedures.

Methods: Patients were divided into two groups with body weight $2,5 \mathrm{~kg}$ as discriminator and into two surgical eras until and after 2004. 1885 procedures were performed in neonates below $2,5 \mathrm{~kg}$ b.w. 6385 procedures were performed before 2005 and 5886 since. Age, body weight below $2,5 \mathrm{~kg}$, technical difficulty, Aristotle Basic Score, CPB time, AoX time, univentricular physiology and DHCA were studied as potential risk factors for hospital mortality and for onset of complications.

Results: Hospital mortality was $11,6 \%, 16,15 \%$ below $2,5 \mathrm{~kg}$ and $9,14 \%$ above $(p<0,001)$. Mortality decreased between two surgical eras from $13,16 \%$ to $9,96 \%(p<0,0001)$, but only in neonates with body weight above $2,5 \mathrm{~kg}(12,5 \%$ v. $9 \%, \mathrm{p}<0,0001)$. Multivariate analysis showed that in recent era technical difficulty, ABS, AoX time and DHCA were neutralized as risk factors for hospital mortality. Body weight below $2,5 \mathrm{~kg}$, CPB time and univentricular physiology remain significant determinants of early death. Body weight below 2,5 kg was neutralized as a risk factor for onset of complications after 2004. Recently technical difficulty, $\mathrm{ABS}, \mathrm{CPB}$ time, univentricular physiology and DHCA remain significant predictors of onset of complications.

Conclusion: Body weight below 2,5 kg, univentricular physiology and $\mathrm{CPB}$ time continue to be risk factors for hospital death in neonatal cardiac surgery.

\section{2}

The Effect of Granulocyte Colony-Stimulating Factor on Expression of Selected Regulatory Proteins Responsible for Cell Injury or Survival in the Brain of Newborn Piglets Following Cardiopulmonary Bypass and Circulatory Arrest Peter Pastuszko, Rady Children's Hospital of San Diego, San Diego, $C A$, United States

Afsaneh Pirzadeh, Department of Anesthesiology \& Critical Care, The Children's Hospital of Philadelphia, Philadelphia, PA, United States Erin Reade, Department of Anesthesiology \& Critical Care, The Children's Hospital of Philadelphia, Philadelphia, PA, United States Joanna Kubin, Department of Biochemistry \& Biophysics, The University of Pennsylvania School of Medicine, Philadelphia, PA, United States

Alberto Mendoza, Department of Biochemistry \& Biophysics, The University of Pennsylvania School of Medicine, Philadelphia, PA, United States

Gregory J Schears, Department of Anesthesiology \& Critical Care, Mayo Clinic, Rochester, MN, United States

William J Greeley, Department of Anesthesiology \& Critical Care, The Children's Hospital of Philadelphia, Philadelphia, PA, United States Anna Pastuszko, Department of Biochemistry \& Biophysics, The University of Pennsylvania School of Medicine, Philadelphia, PA, United States
Objectives: Granulocyte colony-stimulating factor (G-CSF) has anti-inflammatory and anti-apoptotic properties. We investigated the effect of G-CSF on regulatory proteins involved in initiation (Bax) or inhibition (Jak-2 and Bcl-2) of apoptosis in the brain of newborn piglets following cardiopulmonary bypass and circulatory arrest.

Methods: Anesthetized piglets were placed on $\mathrm{CPB}$, cooled to $18^{\circ} \mathrm{C}$, subjected to $30 \mathrm{~min}$ of DHCA and $1 \mathrm{hr}$ of LF at $20 \mathrm{ml} / \mathrm{kg}$ / min, rewarmed to $37^{\circ} \mathrm{C}$ and monitored for $6 \mathrm{hrs}$. G-CSF was injected $2 \mathrm{hr}$ prior to bypass at $17 \mathrm{mg} / \mathrm{kg}$ (G-CSF-17) or $34 \mathrm{mg} /$ $\mathrm{kg}$ (G-CSF-34). Protein expression is presented as \% of no-GCSF values (means $(6) \pm \mathrm{SE}$ ).

Results: In striatum, Bcl-2 was higher by $73 \pm 4 \%(\mathrm{p}<0.05)$ and $119 \pm 8 \%(p<0.01)$ in G-CSF-17 and G-CSF-34 groups, respectively. In GCSF-34 group Bax was lower by $19 \pm 3 \%$ $(\mathrm{p}<0.05)$ and Jak-2 was higher by $72 \pm 10 \%(\mathrm{p}<0.05) . \mathrm{Bcl}-2 /$ Bax ratio was 0.77, 1.16 and 1.99 in no-G-CSF, G-CSF-17, and GCSF-34 groups, respectively. Bcl-2/Bax increase suggests decreased susceptibility to apoptosis. In hippocampus, Bcl-2 was higher by $35 \pm 6 \%(\mathrm{p}<0.05)$ and $137 \pm 11 \%(\mathrm{p}<0.01)$ in the G-CSF-17 and G-CSF-34 groups, respectively. Bcl-2/Bax ratio was $0.68,0.89$ and 1.56 in no-G-CSF, G-CSF-17 and GCSF-34 groups, respectively. Jak-2 increased $24 \pm 7 \%(\mathrm{p}<0.05)$ in G-CSF-17 and $76 \pm 4 \%(\mathrm{p}<0.01)$ in G-CSF-34 groups. In frontal cortex, $\mathrm{Bcl}-2$ in the G-CSF-34 group increased $61 \pm 7 \%$ $(\mathrm{p}<0.05)$. Bcl-2/Bax ratio was $0.66,0.64$ and 1.0 in no-G-CSF, G-CSF-17 and G-CSF-34 groups, respectively.

Conclusions: In the piglet model of circulatory arrest with low flow bypass, G-CSF provides significant protection, particularly in striatum and hippocampus, from apoptotic neuronal injury.

\section{3}

Homograft Performance in 84 Pediatric Ross Patients Results from the German-Dutch Ross Registry

Jürgen Hörer, Department of Cardiovascular Surgery, German Heart Center Munich at the Technical University, Munich, Germany, Germany

Julie Cleuziou, Department of Cardiovascular Surgery, German Heart Center Munich at the Technical University, Munich, Germany, Germany

Thorsten Hanke, Department of Cardiac Surgery, University Clinic Schleswig-Holstein, Campus Luebeck, Luebeck, Germany, Germany Johanna J M Takkenberg, Department of Cardiothoracic Surgery, Erasmus University Medical Center, Rotterdam, the Netherlands, The Netherlands

Ulrich Stierle, Department of Cardiac Surgery, University Clinic Schleswig-Holstein, Campus Luebeck, Luebeck, Germany, Germany Hans H Sievers, Department of Cardiac Surgery, University Clinic Schleswig-Holstein, Campus Luebeck, Luebeck, Germany, Germany Rüdiger Lange, Department of Cardiac Surgery, University Clinic Schleswig-Holstein, Campus Luebeck, Luebeck, Germany, Germany

Background: The Ross operation may be the ideal aortic valve replacement in pediatric patients. However, reoperations for replacement of the homograft $(\mathrm{HG})$ in the pulmonary position are inevitable. This study was conducted to determine influencing factors for the development of homograft stenosis (HS) and regurgitation (HR) in an international pediatric Ross operated cohort.

Methods: Prospectively collected data of 84 children $<16$ years of age at the time of the Ross operation $(62 \mathrm{~m}, 22 \mathrm{f}$, mean age $9.4 \pm 5.0$ years), were analyzed using hierarchical multilevel modeling. Mean follow-up was $6.5 \pm 4.2$ years. 
Results: Median HG diameter z-value was 0.2 (range -2.2 to +7.3). Mean HG pressure gradient at implantation was $5.1 \mathrm{mmHg}$ with a significant annual increase of $4.1 \mathrm{mmHg} /$ year $(p<0.001)$. Higher donor age was significantly associated with lower mean pressure gradient at implantation $(p=0.037)$. Larger $\mathrm{z}$-value had no significant influence on the annual increase of $\mathrm{HG}$ $(p=0.87)$. Mean HR at implantation was 0.9 without significant annual increase $(0.02$ grade/year, $p=0.35)$. Higher recipient age $(p=0.002)$, and higher donor age $(p<0.0001)$ were significantly associated with lower mean HR at implantation. However, larger $\mathrm{z}$-value $(\mathrm{p}=0.014)$ was significantly associated with a higher annual increase of $\mathrm{HR}$

Conclusion: Relevant HR is rare in the midterm in children following the Ross Operation. However, there is significant annual increase in HG pressure gradient which is not influenced by the use of larger grafts upon initial operation. In contrast, oversizing of the HG may lead to a higher annual increase of HR.

\section{4 \\ Experience of Anatomical Repair for Ebstein's Anomaly in 100 patients \\ Qingyu Wu, First Hospital of Tsinghua Universty, China Guangyu Pan, First Hospital of Tsinghua Universty, China Lianyi Wang, First Hospital of Tsinghua Universty, China Xingpeng Chen, First Hospital of Tsinghua Universty, China Hongyin Li, First Hospital of Tsinghua Universty, China}

Object: To report our experience of anatomical repair for Ebstein's anomaly.

Methods: Between December 1997 and October 2008, 100 (male 39 , female 61) consecutive patients aged 9 months to 63 years were operated for Ebstein's anomaly with anatomical repair technique, among them 7 had previous tricuspid repair surgery in other centers. Tricuspid incompetence was moderate in 26 patients and severe in 74 . The main surgical technique includes excision of atrialized right ventricle, detach and repair the leaflet, transposition of the leaflet and anulus plication of tricuspid valve. In some patients the septal leaflet was severely hypoplastic or absent a new leaflet was created using autologous pericardium.

Results: All patients survived and recovered uneventfully. No patient died or had re-operation. Postoperative echocardiography showed that tricuspid incompetence disappeared in 87 and was mild in 13. Right ventricular function was good. Patients have been doing well during a mean follow-up of 48 months ( 2 months to 10 years). The latest follow up echocardiography showed that tricuspid incompetence disappeared in 63 patients, mild in 24, moderate in 10 and severe in 3 .

Conclusions: The novel anatomical repair technique for the correction of Ebstein's anomaly was successfully applied in 100 consecutive patients with satisfactory early and midterm results. In most patients with Ebstein's anomaly tricuspid valve replacement can be avoided.

\section{7}

Electrical seizures are common during the peri-operative period in young infants undergoing surgery for congenital heart disease

Julia K Gunn, Murdoch Childrens Research Institute, Melbourne, Australia

Rod Hunt, The Royal Children's Hopsital, Australia

John Beca, Starship Children's Hospital, Auckland, New Zealand

Terrie Inder, Washinton University, St Louis, United States

Lara Shekerdemian, The Royal Children's Hopsital, Melbourne, Australia
Objective: To determine the incidence of peri-operative seizures in young infants undergoing cardiopulmonary bypass (CPB). Methods: 61 full-term infants undergoing surgery for CHD were enrolled in a prospective study of brain injury. Two-channel amplitude integrated EEG (aEEG) monitoring was undertaken prior to and during induction of anaesthesia, throughout surgery and for up to 72 hours post-operatively, with a further recording one week later. A blinded assessor reviewed the aEEG recordings for seizure activity on the background and raw traces. $\mathrm{Chi}^{2}$ analysis was used to compare groups.

Results: Median age at surgery was 6 days (IQR 2-55). Surgical procedures were as follows: Norwood/ Damus operations (24), systemic-to-pulmonary artery shunt (6), arterial switch operations (18), common arterial trunk repairs (5), complex aortic arch repairs (6) and miscellaneous (2). 22 (35\%) infants had perioperative electrical seizures. Only 1 had a clinical correlate. They were pre-operative in $2(4 \%)$, intra-operative in $11(20 \%)$ and during the post-operative phase in 13 (22\%). Intra-operative seizures occurred under anaesthetic prior to CPB (3), during CPB (6), during rewarming (5) and following CPB (3). The aEEG background activity was less likely to normalise within 24 hours in those infants who experienced seizures $(p=0.02)$. Severely abnormal background aEEG activity in the first 6 hours after $\mathrm{CPB}$ was strongly associated with post-operative seizures $(\mathrm{p}<0.0001)$.

Conclusions: Sub-clinical electrical seizures are common in infants undergoing $\mathrm{CPB}$, both during and after surgery. Further follow up of the survivors is essential to determine the long-term significance of these findings.

\section{8}

Acceptable Survival for Children with Single Ventricle Physiology after Mechanical Support

Kimberly L Gandy, Children's Hospital of Wisconsin, United States Nancy S Ghanayem, Children's Hospital of Wisconsin, United States Julie A Deavers, Children's Hospital of Wisconsin, United States Mike E Mitchell, Childen's Hospital of Wisconsin, United States Shun-Hwa Li, Children's Research Institute, United States Jeanne Braby, Children's Hospital of Wisconsin, United States Simpson M Pippa, Children's Hospital of Wisconsin, United States James S Tweddell, Children's Hospital of Wisconsin, United States

Background: Mechanical circulatory support (MCS) may be necessary in patients with single ventricle anatomy (SVA) due to intractable low cardiac output, shunt occlusion, and cardiac arrest. Methods: We evaluated our experience from March 1989 to January 2008 of patients with SVA $(n=530)$ on MCS. Our institutional cardiac database and the ELSO databases were queried.

Results: Sixty-one (12\%) patients required MCS. Three patients were supported with ventricular assist devices other than ECMO. Two patients $(3.3 \%)$ required ECMO support prior to surgical staging; 39/61 (64\%) patients were placed on ECMO support after stage 1 palliation; 10/61 (16\%) after bidirectional Glenn; and 10/61 (16\%) after Fontan completion. Target flows were directed by venous oximetry and/or near infrared spectroscopy. Thirty-five (57\%) were placed on mechanical support emergently for cardiac arrest (ECPR). Twenty-six (34\%) were placed on MCS electively or for failure to wean from cardiopulmonary bypass. Seven patients required two ECMO runs; one patient required three ECMO runs. Twenty of 61 patients (33\%) are alive at a follow-up of 9.9 to 175 months. Among single ventricle patients undergoing ECPR, 13/35 (35\%) survived long-term compared to $7 / 26(27 \%)$ without an arrest $(p=0.43)$. One of 
eight patients (12.5\%) requiring multiple ECMO runs survived to discharge after transplantation.

Conclusions: Single ventricle patients can have acceptable survival after MCS in the postoperative period. The need for ECPR in patients receiving MCS does not affect survival. ECMO and other forms of ventricular support should be aggressively offered to single ventricle patients when necessary.

\section{1}

Arterial intima-media thickness, endothelial function and apolipoproteins in adolescents frequently exposed to tobacco smoke

Katariina H Kallio, Research Centre of Applied and Preventive Cardiovascular Medicine, University of Turku, Finland, Finland Eero V Jokinen, Department of Pediatrics, University of Helsinki, Finland, Finland

Olli G Simell, Department of Pediatrics, University of Turku, Finland, Finland

Olli T Raitakari, Department of Clinical Physiology, University of Turku, Finland, Finland

Objective: To examine the associations between frequent exposure to tobacco smoke, arterial changes related to early atherosclerosis and serum lipid profile in adolescents.

Methods: Healthy 13-year-old adolescents from the atherosclerosis prevention trial (STRIP) were studied. Maximum carotid (cIMT) and aortic intima-media thickness (aIMT), and brachial artery flow-mediated dilation (FMD) were measured in 494 adolescents using high resolution ultrasound. Serum lipid, lipoprotein, and apolipoprotein (Apo) values were determined using standard methods. Exposure to tobacco smoke was measured annually between ages 8 and 13 years using serum cotinine concentrations, analyzed with gas chromatography. To define longitudinal exposure, cotinine values of children having serum cotinine measured 2 to 6 times during follow-up were averaged and divided into tertiles (exposure groups): low $(\mathrm{n}=160)$, intermediate $(\mathrm{n}=171)$, and high $(\mathrm{n}=163)$.

Results: Adolescents with higher longitudinal exposure to tobacco smoke had increased cIMT (exposure groups $[$ mean $\pm \mathrm{SD}$ ]: low $0.502 \pm 0.079 \mathrm{~mm}$, intermediate $0.525 \pm$ $0.070 \mathrm{~mm}$, high $0.535 \pm 0.066 \mathrm{~mm} ; \mathrm{p}<0.001)$, and increased aIMT (exposure groups: low $0.527 \pm 0.113 \mathrm{~mm}$, intermediate $0.563 \pm 0.139 \mathrm{~mm}$, high $0.567 \pm 0.126 \mathrm{~mm} ; \mathrm{p}=0.004)$. The FMD decreased when cotinine level increased (exposure groups: low $10.43 \pm 4.34 \%$, intermediate $9.78 \pm 4.38 \%$, high $8.82 \pm 4.14 \% ; \mathrm{p}=0.001)$. These associations were unchanged after adjusting for traditional atherosclerosis risk factors. Moreover, ApoB $(p=0.004), A p o B / A p o A-I$ ratio $(p=0.013)$, and triglycerides $(p=0.037)$ increased with increase in cotinine level. Conclusions: Frequent exposure to tobacco smoke is independently associated with arterial changes of preclinical atherosclerosis and increased ApoB levels among healthy adolescents.

\section{2}

Determining Vascular Risk Profiles for Early Atherosclerosis in Paediatric Rheumatic Disease Timothy J Bradley, The Hospital for Sick Children, Canada Pascal N Tyrrell, The Hospital for Sick Children, Canada Lawrence Ng, The Hospital for Sick Children, Canada Cameron Slorach, The Hospital for Sick Children, Canada Joseph Beyene, The Hospital for Sick Children, Canada Rayfel Schneider, The Hospital for Sick Children, Canada
Brian M Feldman, The Hospital for Sick Children, Canada Earl D Silverman, The Hospital for Sick Children

Objectives: Increased rates of atherosclerotic disease in adults with Systemic Lupus Erythematosus (SLE) and Rheumatoid Arthritis have not been explained by exposure to traditional cardiovascular risk factors alone, prompting investigation into the role of chronic inflammation. The aims of this study were to determine and compare the prevalence of early vascular markers of atherosclerosis in children with SLE, Systemic Juvenile Inflammatory Arthritis (SJIA) and Juvenile Dermatomyositis (JDM). Methods: Vascular indices, including carotid intima-media thickness (CIMT), flow-mediated dilatation (FMD) and pulse wave velocity (PWV), were compared within groups to normal population data and between groups using parametric methods. Results: Of the $101 \mathrm{SLE}$ (age 15.2 \pm 2.6 years, $\mathrm{M}: \mathrm{F}=18: 83$ ), 16 SJIA (age 13.4 \pm 2.6 years, $\mathrm{M}: \mathrm{F}=6: 10$ ) and 24 JDM (age $13.4 \pm 2.6$ years, $\mathrm{M}: \mathrm{F}=14: 10)$, SLE were older and more predominantly female. Mean CIMT were lower in SLE and SJIA compared to controls (both $\mathrm{P}<0.001$ ), but no different between groups (SLE $0.40 \pm 0.05$ vs. SJIA $0.38 \pm 0.06$ vs. JDM $0.42 \pm 0.07 \mathrm{~mm})$. Mean FMD response was lower in SLE compared to controls $(\mathrm{P}<0.001)$, but no different between groups (SLE $8.2 \pm 4.3$ vs. SJIA $8.0 \pm 3.4$ vs. JDM $8.2 \pm 3.5 \%$ ). Mean PWV were higher in SLE and JDM compared to controls (both $\mathrm{P}<0.01$ ), but not significantly different between the groups (SLE $4.6 \pm 1.5$ vs. SJIA $4.5 \pm 2.3$ vs. JDM $4.0 \pm 0.8 \mathrm{~m} / \mathrm{s}$ ). Conclusions: Risk profiles for early vascular markers of atherosclerosis differ according to the underlying paediatric rheumatic disease process. Disease activity, therapy-related and diseasespecific inflammatory factors are likely playing a role in modifying these cardiovascular risk profiles and warrant further investigation.

\section{5}

Population-based echocardiographic screening for Rheumatic Heart Disease in high-risk New Zealand children

Rachel H Webb, Starship Children's Hospital \& the University of Auckland, New Zealand

Adrian A Trenholme, School of Population Child and Youth Health, University of Auckland, New Zealand

Diana Lennon, KidzFirst Children's Hospital, Counties Manukau District Health Board, New Zealand

Ross W Nicholson, KidzFirst Children's Hospital, Counties Manukau District Health Board, New Zealand

Tom L Gentles, Department of Paediatric and Congenital Cardiac Services, Starship Children's Hospital, New Zealand

John Stirling, Department of Paediatric and Congenital Cardiac Services, Starship Children's Hospital, New Zealand

Clare O'Donnell, Department of Paediatric and Congenital Cardiac

Services, Starship Children's Hospital, New Zealand

Nigel J Wilson, Department of Paediatric and Congenital Cardiac Services, Starship Children's Hospital, New Zealand

Objective: There is a high incidence of Acute Rheumatic Fever (ARF) in young Pacific and Maori New Zealanders. Echocardiography has been shown to detect greater prevalence of rheumatic heart disease (RHD) than cardiac auscultation. We aimed to determine the prevalence of RHD in children from an urban population at high risk for ARF.

Methods: Portable echocardiography was conducted in $7 / 29$ randomly selected South Auckland schools on 1142 predominantly Maori and Pacific students aged 10-13 years between 
August 2007-August 2008. Vivid E (GE) machines were used. Mitral and aortic valves were assessed using parasternal and apical views. Findings were classified as definite, probable or possible RHD according to modified WHO criteria (table). Students with abnormal screening echocardiograms were referred for hospital cardiology review and detailed echocardiography. Hospital echocardiograms were blind-reported by 2 or 3 cardiologists.

Results: 93 (8.1\%) had abnormalities suggestive of RHD and $20(1.8 \%)$ had incidental congenital heart defects (CHD) The prevalence of definite and probable RHD was per 20.1/ 1000 (95\% CI 18-53), the majority with mild disease. Physiologic regurgitation was diagnosed in 27 students after hospital assessment (false positive screening rate $2.4 \%$ ).

Conclusions: We found high rates of undetected RHD in this population. Echocardiographic screening programmes for RHD combined with secondary prophylaxis have the potential to prevent severe RHD in high-risk populations. RHD screening has resource implications: $10 \%$ of the study cohort required careful cardiology evaluation for accurate diagnosis of both RHD and CHD and differentiation from physiologic regurgitation.

\begin{tabular}{|c|c|c|c|c|c|c|}
\hline RHD & Murmur & Doppler & Morphology & $\mathbf{N}$ & Prev/1000 & $95 \% \mathrm{CI}$ \\
\hline Definite & + & + & + & 6 & 5.3 & $(2-11)$ \\
\hline Probable & - & + & + & 17 & 14.9 & $(9-24)$ \\
\hline Possible & - & + & - & 25 & 21.9 & $(14-32)$ \\
\hline Possible & - & - & + & 8 & 7.0 & $(3-14)$ \\
\hline Total & & & & 56 & 49.0 & $(37-63)$ \\
\hline
\end{tabular}

\section{6}

Rheumatic Fever Prophylactic Programme: analysis of the pattern of presentation and evolution after the control of recurrences

Cleonice CC Mota, Division of Paediatric and Foetal Cardiology/ Department of Paediatrics/Hospital das Clínicas-Federal University of Minas Gerais, Brazil

Zilda MA Meira, Division of Paediatric and Foetal Cardiology/ Department of Paediatrics/Hospital das Clínicas-Federal University of Minas Gerais, Brazil

Fátima DM Rocha, Division of Paediatric and Foetal Cardiology/ Department of Paediatrics/Hospital das Clínicas-Federal University of Minas Gerais, Brazil

Objective: To compare the profile of clinical presentation and evolution in control patients before and after the implementation of the Prophylactic Programme.

\begin{tabular}{llll}
\hline $\begin{array}{l}\text { Clinical/epidemio data } \\
\text { Pattern of severity }\end{array}$ & $\begin{array}{l}\text { Group 1 } \\
(\mathbf{n}=248)\end{array}$ & $\begin{array}{l}\text { Group 2 } \\
(\mathbf{n}=454)\end{array}$ & $\mathrm{p}$ \\
\hline $\begin{array}{l}\text { Age at first attack: mean/median } \\
\quad \text { years) }\end{array}$ & $8.8 \pm 5.5 / 8.8$ & $9.4 \pm 3.1 / 9.0$ & $\mathrm{NS}$ \\
$\begin{array}{l}\text { Large/small joints(\%) } \\
\text { MR + AR + MS/other }\end{array}$ & $86.7 / 13.3$ & $84.5 / 15.5$ & $\mathrm{NS}$ \\
$\quad 94.2 / 5.8$ & $95.6 / 4.4$ & $\mathrm{NS}$ \\
$\begin{array}{l}\text { associations(\%) } \\
\text { Recurrences (\%) }\end{array}$ & 22.4 & 7.4 & $<0.001$ \\
Severe/mild carditis(\%) & $49.8 / 15.7$ & $30.1 / 30.6$ & $<0.001$ \\
$\begin{array}{l}\text { Hospital admissions(\% } \\
\text { Surgeries(\%) }\end{array}$ & 45.4 & 9.1 & $<0.001$ \\
Deaths(\%) & 13.3 & 1.5 & $<0.001$ \\
\hline
\end{tabular}

Methods: Comparative study involving 702 children and adolescents with rheumatic fever, covering the periods 07/77-07/88 (Group I) and 08/88-02/00 (Group II). The following variables were analysed: age at first episode, affected joints, classification of valvar lesion, recurrences, degree of carditis, besides frequency of stenotic valvar lesions, surgeries, hospital admissions and deaths.

Results: The age ranged from 2.7 to 19.9 years. Carditis $(69,7 \%)$ and arthritis $(70,4 \%)$ were seen most frequently. There was no difference regarding gender, except for chorea $(p=0,001)$. The comparative analysis of the clinical/epidemiological data and pattern of severity are showed in Table 1 .

Conclusion: After the implementation of the programme, the clinical presentation remained unchanged, however, there were significant differences in the pattern of severity, especially in the frequency of hospital admissions, surgeries and deaths, due to the healthcare team's action to control recurrences.

\section{7}

TEE guided minimally invasive perventricular device occlusion of ventricular septal defects without cardiopulmonary bypass: multi-center results and experience Quansheng Xing, Children's Heart Center, Qingdao Children's Hospital, China

Qi An, Department of Cardiac Surgery, West China Hospital, Sichuan University, China

Xiaozhoub Wang, Department of Cardiac Surgery, Shanghai Chest

Hospital, China

Zewei Zhang, Department of Cardiac Surgery, Zejiang University

Children's Hospital, China

Qin Wu, Children's Heart Center, Qingdao Children's Hospital, China

Silin Pan, Children's Heart Center, Qingdao Children's Hospital, China

Objective: Introduce the transesophageal echocardiography (TEE) guided minimally invasive perventricular device occlusion of ventricular septal defects (VSDs) without cardiopulmonary bypass (CPB) and report the results and experience.

Methods: A newly invented delivery system(China Patent No:2007200273818)was used to perform minimal invasive occlusion of VSDs in a series of 254 cases (from Mar 2007 to Sep 2008). For those with perimembranous(191 cases) or muscular(17) VSDs, a 3-5 cm inferior sternotomy was made, but intracristal or supracristal VSDs(46), the left third intercostals space parasternally. Under TEE guidance, through the chosen $\mathrm{RV}$ free wall point, the delivery system with occlusion device of appropriate size was introduced into RV cavity, defect and then left ventricular (LV) cavity. Then following the LV disc, waist and the RV disc were deployed in $L V$, septum and RV respectively. After complete TEE assessing for absence of residual shunting, obstruction, valve interference and arrhythmia, the device was released.

Results: The procedure was successful in 238 cases. It took us less than 30 minutes $(5-28 \mathrm{~min})$ to implant the device. All the patients were discharged 48-96 hours after the operation. During fellow-up period, there was no incidence of residual shunt, noticeable aortic or tricuspid insufficiency and arrhythmia. The other 16 cases(6.3\%) were converted to successful traditional surgical closure with $\mathrm{CPB}$ for the reason of inaccurate assess of intracardiac anomolies.

Conclusion: This minimally invasive procedure for occlusion of VSDs in the operating room appears to be excellent with encouraging outcomes. Cardiopulmonary bypass, severe surgical trauma and prolonged hospital stay are all avoided.

\section{8}

An optimal surgical technique of congenital aortic arch disease

Qingyu Wu, First Hospital of Tsinghua University, China

Xingpeng Chen, First Hospital of Tsinghua University, China 
Jicheng Xi, First Hospital of Tsinghua University, China Rui Zhu, First Hospital of Tsinghua University, China

Objective: To report our experience on treating congenital aortic arch disease using autologous pulmonary artery patch are reported here. Method: From Apr. 1993 to Oct. 2008, 23 patients with congenital aortic arch disease received one-stage complete correction using autologous pulmonary artery patch, including 15 male and 8 female aged from 6 weeks to 6.5 years old $(10.6 \pm 25.3$ months) There were 5 CoA, 5 IAA and 13 hypoplasia of aortic arch. Concomitant anomalies included VSD in 16, ASD in 6 , aortic stenosis in 1, LVOT obstruction in 3 and dextracardia in 1 . There were 7 patients with moderate and 16 with severe pulmonary hypertension. The operation was performed with DHCA, CPB and selective cerebral perfusion. The anterior wall of main pulmonary artery was excised to reconstruct the aortic arch. Then the main pulmonary artery was reconstructed by direct suture (18) or autologous pericardium plasty(5). Other cardiac anomalies were corrected concomitantly. Results: All patients had uneventful surgery. There was 1 inhospital death $(5.9 \%)$, no brain injury. Postoperative echocardiography and CT study showed intracardiac anomalies corrected without residual stenosis. 22 patients have been doing well during a follow up period of 2 months to 16 years. No aortic or subaortic stenosis found.

Conclusion: One-stage complete correction can be achieved with good result in congenital aortic disease associated with other cardiac anomalies. With the benefit of growth potential and less tension, autologous pulmonary artery tissue is an optimal choice in aortic arch reconstruction.

\section{9}

\section{Right ventricle-to-pulmonary artery shunt in Norwood procedure for children with hypoplastic left heart syndrome -200 cases results}

Katarzyna Januszewska, Klinikum Grosshadern, Ludwig Maximilians University, Munich, Germany

Reiner Kozlik-Feldman, Department of Pediatric Cardiology, Klinikum Grosshadern, Ludwig Maximilians University, Munich, Germany Zbigniew Kordon, Department of Pediatric Cardiology, Jagiellonian University, Polish-American Children's Hospital, Cracow, Poland Marcus Loeff, Department of Pediatric Cardiology, Klinikum Grosshadern, Ludwig Maximilians University, Munich, Germany Sabine Greil, Department of Pediatric Cardiology, Klinikum Grosshadern, Ludwig Maximilians University, Munich, Germany Julia Birnbaum, Department of Pediatric Cardiology, Klinikum Grosshadern, Ludwig Maximilians University, Munich, Germany Bruno Reichart, Department of Cardiac Surgery, Klinikum Grosshadern, Ludwig Maximilians University, Munich, Germany Edward Malec, Department of Cardiac Surgery, Klinikum Grosshadern, Ludwig Maximilians University, Munich, Germany

Objective: The reintroduction of the right ventricle-to-pulmonary artery shunt (RV-PA) caused the significant improvement of the surgical results in management of the hypoplastic left heart syndrome (HLHS) in many centers. The aim of this study was to present the multistage surgical treatment long term results of children with HLHS-type defects after RV-PA application in the first stage palliation.

Methods: Between 2001 and 2008 a consecutive series of 200 children with hypoplastic left heart syndrome type of single ventricle underwent Norwood procedure (NP) with application of RV-PA. Hemodynamic, angiographic, echocardiographic, electrocardiographic and clinical perioperative and long term data were retrospectively analyzed.
Results: The mean follow-up was $4.49+/-1.7$ years and was complete in $92.5 \%$ cases. To date, 153 children (mean age $6.12+/$ -1.67 months) underwent second stage procedure: 126 patients hemi-Fontan operation, 25 children - unilateral or bilateral bidirectional Glenn anastomosis and two - double ventricle correction. The last stage palliation - the Fontan operation (FO) underwent 75 children in the mean age of $29.54+/-7.1$ months. The early and late mortality after each stage procedure was as follows: stage I $-11 \%$ and $5.6 \%$, stage II $-3.9 \%$ and $4.8 \%$ and stage III $-0 \%$ and $0 \%$, respectively. The most often postoperative complications were: after NP - infection (18.0\%), after stage II chylothorax (5.2\%) and after FO - effusions (73.3\%).

Conclusions: The Norwood operation with RV-PA is a good preliminary stage in reconstructive surgical treatment for children with hypoplastic left heart syndrome type of single ventricle.

\section{0}

Hard choices for high-risk patients with Critical Left Ventricular Outflow Obstruction: Contemporary comparison of hybrid versus surgical strategies Christian Pizarro, Alfred I duPont Hospital for Children, United States Jacek Kolcz, Alfred I duPont Hospital for Children, United States Christopher D Derby, Alfred I duPont Hospital for Children, United States

Dore Klenk, Alfred I duPont Hospital for Children, United States Gina Baffa, Alfred I duPont Hospital for Children, United States Wolfgang A Radtke, Alfred I duPont Hospital for Children, United States

Surgical management of high-risk newborns with critical left ventricular outflow obstruction (LVOTO) requires complex procedures associated with significant morbidity and mortality. We sought to compare the outcomes of the hybrid versus surgical strategies (Norwood, Ross-Konno) for the management of critical LVOTO in a contemporary non-randomized cohort of neonates considered high-risk candidates.

Retrospective review of all patients undergoing management of critical LVOTO between January 2001 and December 2008. High-risk conditions included prematurity, low birth weight, associated genetic, associated cardiac and non-cardiac pathology. Analysis was performed based on intention to treat. End-points for outcome were early and 6-month mortality.

The cohort included 57 patients (22 hybrid and 35 surgical [31 Norwood, 4 biventricular repair]. The cohort had a median age of 4 (1-62) days, mean weight of $2.7 \pm 0.5 \mathrm{kgs}$ and Aristotle comprehensive score of $18.6 \pm 2.9$ Analysis of the entire cohort identified low birth weight $(\mathrm{p}=.0007)$ prematurity $(\mathrm{p}=.004)$ and organ dysfunction $(\mathrm{p}=.04)$ as risk factors for early death. Six-month mortality was associated with need for reintervention $(p=.017)$ in the surgical group, and aortic atresia $(p=.03)$ in the hybrid group. Logistic regression identified low birth weight $(\mathrm{p}=.05$ OR $5.6[0.9-34.6])$, organ dysfunction $(\mathrm{p}=.05 \mathrm{OR}$ $4.7[0.9-22.5])$, and anatomic diagnosis ( $p=.03$ OR 0.06[.005-.93]) as predictors of mortality for the entire cohort. There was no difference for early and mid-term mortality.

Although the hybrid approach reduces the initial surgical insult, important interstage mortality and ongoing morbidity result in similar survival than surgical strategy. Patient-related factors have a greater impact on outcome that the surgical strategy.

\section{1}

Recoarctation After the Norwood Stage 1 Procedure Douglas A Wall, The Prince Charles Hospital/Birmingham Childrens Hospital, Australia 
John Stickley, Birmingham Childrens Hospital, United Kingdom Oliver Stumper, Birmingham Childrens Hospital, United Kingdom Zdenka Reinhardt, Birmingham Childrens Hospital, United Kingdom Sara Meli, Birmingham Childrens Hospital, United Kingdom Timothy Jones, Birmingham Childrens Hospital, United Kingdom David J Barron, Birmingham Childrens Hospital, United Kingdom William J Brawn, Birmingham Childrens Hospital, United Kingdom

Background: The purpose of this study was to indentify incidence, functional significance and treatment modalities in aortic arch recoarctation after the Norwood procedure.

Methods: 222 consecutive patients who underwent the Norwood procedure from May 2002 to May 2007 were reviewed retrospectively. All patients in the study had systemic right ventricles, pulmonary blood flow provided by a right ventricleto-pulmonary artery conduit and an initial arch intervention (patch) constructed using pulmonary homograft. Freedom from intervention prior to Stage 2 or death were primary outcome measures.

Results: Recoarctation was observed in 30 of 222 patients (15.3\%). Of these 30 patients only $9(30 \%)$ had an initial diagnosis of coarctation at stage 1 Norwood palliation. Mortality in the coarctation cohort was 10\% (3 patients). Freedom from aortic arch reintervention prior to stage 2 was $84.7 \%$. No patients required surgery for recoarctation either before or at 2 nd stage palliation. All interventions for recoarctation involved balloon angioplasty with a median balloon diameter of $7 \mathrm{~mm}$ (range of 4-8 mm) inflated $2(1-3)$ times. Gradients reduced from $20 \mathrm{mmHg}(5-55)$ to $8 \mathrm{mmHg}(0-20)$. Right ventricular systolic function was normal at hospital discharge in $87 \%$ of patients $(26$ patients) with $97 \%$ of patients having no more than mild tricuspid regurgitation (29 patients). The functional significance of recoarctation was that 10 patients had moderate tricuspid regurgitation at catheter intervention and right ventricular systolic function was normal in only 16 patients $(53 \%)$.

Conclusions: Recoarctation after Stage 1 Norwood palliation remains a significant problem and has deleterious effects for the systemic right ventricle.

\section{2}

Reconstruction of the Heart and Pericardium with Decellularized Porcine Extracellular Matrix Scaffold: Human Applications in Congenital Heart Disease Frank G Scholl, Joe DiMaggio Children's Hospital, United States Richard Perryman, Joe DiMaggio Children's Hospital, United States Kak C Chan, Joe DiMaggio Children's Hospital, United States Lilliam Valdes-Cruz, Joe DiMaggio Children's Hospital, United States Mark M Boucek, Joe DiMaggio Children's Hospital, United States

The ideal material for repair of congenitally malformed hearts would encourage regeneration of native tissue with growth potential. Decellularized porcine small intestinal submucosa extracellular matrix (SIS-ECM) promotes tissue remodeling and regeneration in animal models and non-cardiac human applications. This is a retrospective review using SIS-ECM for reconstruction of congenital heart defects.

From June 2007 to September 2008 we performed 19 operations using intracardiac (IC) and extracardiac (EC) SIS-ECM patches. Age ranged from 8 days to 7 years. EC implantations included: pericardial in 9 , pulmonary artery in 3 , superior venacava in 2 and aortoplasty in 1. IC implantation included: ASD in 3, RVOT in 3 , pulmonary valve monocusp creation in 2, valve leaflet augmentation in 2 and repair of unroofed coronary sinus in 1. Follow up was complete.
There were 2 deaths, unrelated to the SIS-ECM. Mean follow up was 7.27 months. There were no complications related to the SIS-ECM. The monocusp valves appeared competent and not stenotic. One patient who underwent tricuspid anterior leaflet augmentation with SIS-ECM, required TV replacement four months later for severe regurgitation following a catheter based procedure. Analysis showed resorption of the SIS-ECM, regeneration of the valve leaflet with organized collagen and reendothelialization. Figure one is an electronmicrograph showing pre-collagen production and organized deposition of collagen.

SIS-ECM is safe for repair of congenital heart defects. It can be used in valve reconstruction and allows for tissue regeneration of the leaflet. The potential for growth is unknown, longer term follow-up will assess this important factor.

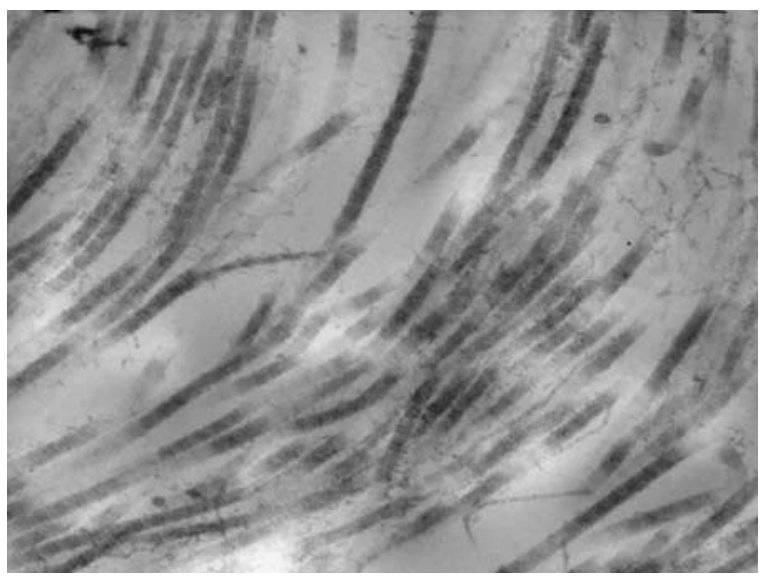

3023

Differential effects of the permanent epicardial pacing site on left ventricular synchrony and function in the young: Implications for lead placement

Jan Janousek, Heart Center Leipzig, Germany

Roman A Gebauer, Department of Pediatric Cardiology, University of Leipzig, Heart Center, Leipzig, Germany, Germany

Viktor Tomek, Kardiocentrum and Cardiovascular Research Center, University Hospital Motol, Prague, Czech Republic, Czech Republic Petr Kubus, Kardiocentrum and Cardiovascular Research Center, University Hospital Motol, Prague, Czech Republic, Czech Republic Vit Razek, Department of Pediatric Cardiology, University of Leipzig, Heart Center, Leipzig, Germany, Germany

Roman Gebauer, Kardiocentrum and Cardiovascular Research Center, University Hospital Motol, Prague, Czech Republic, Czech Republic Tomas Matejka, Kardiocentrum and Cardiovascular Research Center, University Hospital Motol, Prague, Czech Republic, Czech Republic Martin Kostelka, Department of Cardiac Surgery, University of Leipzig, Heart Center, Leipzig, Germany, Germany

Objective: Analysis of LV synchrony and function with respect to the epicardial pacing site in the young.

Methods: LV synchrony (M-mode, speckle tracking) and function were evaluated in 32 young patients with complete non-surgical $(\mathrm{N}=15)$ or surgical $(\mathrm{N}=17)$ atrioventricular block (structural heart disease in 21/32) and permanent epicardial pacing from LV apex $(\mathrm{N}=19)$, RV apex $(\mathrm{N}=7)$ and $\mathrm{RV}$ free wall $(\mathrm{N}=6)$ (median age at implantation/duration of pacing: 5.5/22.8, 6.0/ 57.4 and $0.8 / 66.3$ months, respectively).

Results: LV synchrony and function differed significantly between the LV apical, RV apical and RV free wall pacing sites being worst in the latter: septal to posterior wall motion delay $($ SPWMD $)=$ median 0,69 and $136 \mathrm{~ms}(\mathrm{P}<0.001)$, septal to 
lateral mechanical delay $(\mathrm{SLMD})=54 \pm 29, \quad 73 \pm 24$ and $129 \pm 70 \mathrm{~ms} \quad(\mathrm{P}=0.001)$, apical to basal mechanical delay $(\mathrm{ABMD})=96 \pm 37,106 \pm 50$ and $79 \pm 18 \mathrm{~ms}$ (P NS) and LV ejection fraction $(\mathrm{EF})=57 \pm 9, \quad 49 \pm 12$ and $33 \pm 10 \%$ $(\mathrm{P}<0.001)$, respectively. LV EF correlated negatively with SPWMD (R2 $=0.454, \mathrm{P}<0.001)$ and SLMD $(\mathrm{R} 2=0.320$, $\mathrm{P}<0.001)$ but not with ABMD. LV apical paced patients showed no correlation between duration of pacing and LV EF. Pacing site $(\mathrm{P}=0.014)$ and SPWMD $(\mathrm{P}=0.044)$, but not the duration of pacing, block etiology or presence of structural heart disease were significant multivariable predictors of LV EF.

Conclusions: LV apical pacing preserves septal to lateral mechanical LV synchrony and systolic function and should be the preferred epicardial pacing site in young patients with systemic LV. (Supported by Research Project No 00064203 of University Hospital Motol, Prague, Czech Republic).

\section{4}

A two year prospective population-based molecular autopsy evaluation of post-mortem negative sudden death in 0-40 year olds

Jonathan R Skinner, Starship Childrens Hospital, Auckland, New Zealand

Jackie Crawford, Starship Childrens Hospital, Auckland, New Zealand Carey-Anne Eddy, University of Auckland, New Zealand

Katherine Neas, Central and Southern Regional Clinical Genetic Service, New Zealand

Simon Stables, National Forensic Service, Lab Plus, Auckland, New Zealand

Lloyd Denmark, Lab Plus, Auckland, New Zealand

Donald Love, Lab Plus, Auckland, New Zealand

Andrew Shelling, University of Auckland, New Zealand

Background: Referral-based genetic studies of sudden unexplained death suggest about $30 \%$ of $1-40$ year old deaths (SUDY) and 0-10\% of infant deaths(SIDS) are due to long QT syndrome.

Aims: To ascertain the value of post mortem long QT genetic analysis in a prospective population-based study of SIDS and SUDY. Methods: Coroners and pathologists in New Zealand (population 4.2 million) agreed a national policy of molecular autopsy in SIDS/SUDY. Over 2 years long QT (LQT) genes 1, 2, 3, 5, 6, and 7 were sequenced.

Results: Autopsy DNA was originally stored from 69 SIDS and 71 SUDY, In 59, other tests revealed a diagnosis (eg. bacterial infection 9, cardiomyopathy 7, myocarditis 5). Of the others, 47 LQT genetic tests are completed. 42 (89\%) were uninformative. 3 of 32 SIDS (9\%) had the unclassified variant R1193Q in the SCN5A gene (of questionable pathogenicity).

Two of seven (29\%) 1-18 yr olds had LQT gene variants. A $17 \mathrm{yr}$ old girl (nocturnal death) had variant R67 H in minK (LQT5), previously seen in a LQTS family. A $10 \mathrm{yr}$ old boy (death following light activity) had the novel variant T96R in KCNQ1 (LQT1), which displayed abnormal ion channel electrophysiology (loss-of-function and positively shifted voltage dependence of activation). None of 8 19-40 year olds had variants.

Conclusions: Early results show that molecular autopsy for LQTS in post mortem-negative sudden death is of value in 1-18 year olds. Larger numbers are still required in those $<1$ year and over 18 years of age.

\section{5}

Are Findings Of Dual Atrioventricular Nodal Physiology in Children Predictive Of Atrioventricular Nodal Reentrant Tachycardia?
Anthony C McCanta, University of Colorado, United States Kathryn K Collins, University of Colorado, United States Michael S Schaffer, University of Colorado, United States

Objective: This study retrospectively reviews the charts of children with dual atrioventricular (AV) nodal physiology after ablation of an accessory pathway (AP), but whose AV node was not modified. The purpose of this study is to identify the risk of developing atrioventricular nodal reentrant tachycardia (AVNRT) in children with dual AV nodal physiology.

Methods: This is a single center retrospective chart review of patients who underwent intracardiac electrophysiology study (EPS) at The Children's Hospital, Denver from 1993 to 2008. Patients with AP mediated supraventricular tachycardia (SVT) who underwent ablation of the AP and in whom postablation EPS demonstrated dual AV nodal physiology were identified from the electrophysiology database. Last reported follow-up was recorded with the primary outcome being development of SVT. All patients with recurrent SVT underwent repeat EPS.

Results: Of the 77 patients included in the study, follow-up was obtained for a median duration of 1 year 2 months $(1 \mathrm{~m}-12$ y 3 $\mathrm{m})$. Median age at follow up was 15 years 0 months (6 y 5 m-21 y $3 \mathrm{~m}$ ). Recurrent SVT occurred in 9 of the 77 patients $(11.6 \%)$. Recurrent AP mediated tachycardia was found in 7 (9.1\%). Of the 7 patients with recurrent AP mediated tachycardia, nonsustained AVNRT was induced in 1 patient (1.3\%). AVNRT was induced in 2 patients $(2.6 \%)$ whose initial accessory pathway ablation was successful. Altogether, AVNRT was found in 3 patients (3.8\%) with dual AV nodal physiology.

Conclusions: This study supports the hypothesis that dual AV nodal physiology does not predict AVNRT.

\section{6 \\ Preservation of LV synchrony: Consideration in Pediatric pacing \\ Mohd Shafullah Serdari, National Heart Institute, Malaysia \\ Yazmin Yusoff, National Heart Institute, Malaysia \\ Hanif Osman, National Heart Institute, Malaysia \\ Hasri Samion, National Heart Institute, Malaysia \\ Sivakumar Sivalingam, National Heart Institute, Malaysia \\ Kiew Kong Pau, National Heart Institute, Malaysia \\ Chee Chin Hiew, National Heart Institute, Malaysia}

Objective: The objective of the study is to evaluate the intraventricular (LV) synchrony pattern in children with permanent pacemaker.

Methods: LV synchrony was evaluated in 40 children with complete heart block and life-time pacemaker dependency. Their median age was 7.2 years (range 0.1-18.0) and all has had a minimum of 1 year post pacemaker implantation follow-up. Nineteen had epicardial LV apical pacing, 13 had RV apical (8 epicardial and 5 endocardial) pacing and the remaining 8 had endocardial Septal pacing. The lead location was confirmed by $\mathrm{X}$-ray and ECG imaging. Echocardiography and velocity vector imaging (VVI) were performed (Sequoia, Siemens Medical Solutions, Mountain View, CA) to evaluate the LV synchrony in all patients. Ten normal children were also evaluated as control. Results: LV apical paced is superior to RV septal and RV apical paced children in term of LV synchrony. The mean intraventricular mechanical delay by short axis of LV apical, RV septal and $\mathrm{RV}$ apical pacing were $43+/-10 \mathrm{msec}$ vs $47+/-13 \mathrm{msec}$ vs $47+/-14$ msec. Whilst the mean Septal to posterior wall 
motion delay were: $37+/-10 \mathrm{msec}$ vs $43+/-8 \mathrm{msec}$ vs $48+/$ $-20 \mathrm{msec}$. The intraventricular mechanical delay by short axis and septal to posterior wall in control group were $30+/$ $-10 \mathrm{msec}$ and $29+/-10 \mathrm{msec}$ respectively.

Conclusions: Epicardial LV apical pacing is superior to endocardial $\mathrm{RV}$ septal and RV apical pacing in terms of preservation of left ventricular synchrony.
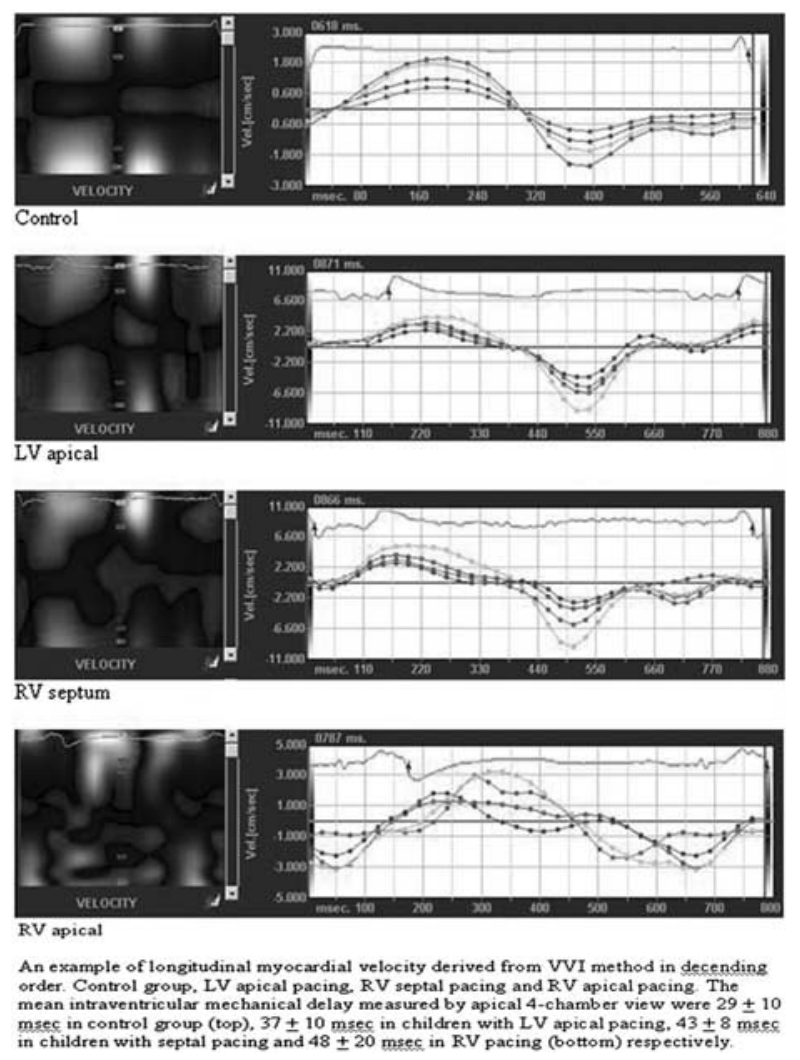

3027

Effect Of Changes In Ventilation On Arterial Carbon Dioxide Levels And Cerebral And Somatic Tissue Oxygenation In Infants Following Cardiopulmonary Bypass And Cardiac Surgery

Marino S Festa, Children's Hospital at Westmead, Australia

Krista Mos, Erasmus MC, The Netherlands

Stephanie Fijten, Erasmus MC, The Netherlands

Andrew Cole, Children's Hospital at Westmead, Australia

David Winlaw, Children's Hospital at Westmead, Australia

Objective: To study the change in cerebral and somatic tissue oxygenation ( $\mathrm{rSO} 2 \mathrm{i})$ in response to alterations in minute volume ventilation and arterial carbon dioxide (PaCO2) concentration in infants following $\mathrm{CPB}$ and cardiac surgery.

Method: Prospective randomised cross-over study in a tertiary cardiac centre, approved by the Local Research and Ethics Committee. Exclusion criteria included age $>12$ months, univentricular repair, need for extracorporal support and clinical evidence of seizures, focal neurology or raised intracranial pressure.

Intervention: $50 \%$ increase/decrease in minute ventilation to alter $\mathrm{PaCO} 2$ levels within normal range at 1 hour and 18 -hours post PICU admission.
Main outcome measurements: Cerebral and Somatic rSO2i (NIRS). Secondary outcome measurements: Haemodynamic status (CI, SVRI, SVI, HR) and amplitude integrated electroencephalography (aEEG) monitoring.

Results: Eleven infants undergoing biventricular repair of congenital heart lesions on CPB were studied. Mean age 3 months (range 1-8). Periods of hyper- and hypoventilation resulted in changed $\mathrm{PaCO} 2$ level (median $5.7 \mathrm{mmHg}$ (range 0.6-17.0)) from baseline, but did not cause significant changes in overall CI or SVRI. Changes in $\mathrm{PaCO} 2$ were associated with significant and linear changes in cerebral but not somatic rSO2i in the 2 nd hour and 18 th hour after PICU admission. Blinded waveform analysis of aEEG recordings is awaited.

Conclusion: Post-operative changes in minute ventilation and arterial $\mathrm{PaCO} 2$ result in changes in oxygenation in cerebral but not somatic tissues. Post-operative hyperventilation and low arterial $\mathrm{PaCO} 2$ is a potential cause of decreased cerebral tissue oxygenation in babies following CPB and biventricular repair.

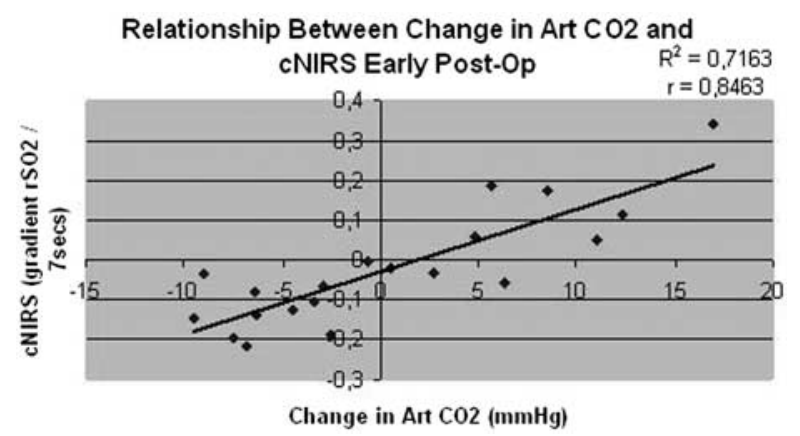

3028

Brain MRI Injury Before and After Neonatal Cardiac Surgery With High-Flow Bypass and Maximized Oxygen Delivery Strategy

Dean B Andropoulos, Texas Children's Hospital/Baylor College of Medicine, United States

Jill V Hunter, Texas Children's Hospital/Baylor College of Medicine, United States

David P Nelson, Texas Children's Hospital/Baylor College of Medicine, United States

Stephen A Stayer, Texas Children's Hospital/Baylor College of Medicine, United States

Ann R Stark, Texas Children's Hospital/Baylor College of Medicine, United States

Emmitt D McKenzie, Texas Children's Hospital/Baylor College of Medicine, United States

Jeffrey S Heinle, Texas Children's Hospital/Baylor College of Medicine, United States

Charles D Fraser, Texas Children's Hospital/Baylor College of Medicine, United States

Introduction: New intraparenchymal brain injury on MRI, consisting of white matter injury (WMI), infarction, or intraparenchymal hemorrhage, is observed in $36-73 \%$ of neonates after cardiac surgery with CPB. (1-3) We performed brain MRI before and after neonatal cardiac surgery, using a high flow CPB protocol, with the hypothesis that incidence of brain injury would be reduced compared to previous studies, and to establish a baseline incidence for neuroprotection studies.

Methods: CPB protocol included $150 \mathrm{ml} / \mathrm{kg} / \mathrm{min}$ flows, $\mathrm{pH}$ stat management, hematocrit $>30 \%$, and high flow antegrade cerebral perfusion. (4) Regional brain oxygen saturation (rSO2) 
$<50 \%$ was treated by protocol. Brain MRI was performed preoperatively, 7 days postoperatively, and at age 3-6 months. Results: 24 of 67 patients (36\%) had new postoperative WMI, infarction, or hemorrhage, and 15\% had new WMI. (Figure 1). Associations with new postoperative injury included preoperative brain injury $(p<0.001)$, and low brain maturity score $(p=0.05)$. 45 patients had a $3 \mathrm{rd}$ scan, with $27 \%$ incidence of new lesions; $58 \%$ of previous lesions partially or completely resolved.

Discussion: Despite evidence-based strategy to maximize brain oxygen delivery, and a reduction in incidence of new injury and WMI compared to previous reports, there is a significant incidence of both pre- and postoperative MRI abnormality in this population. Some lesions resolve in the first 6 months after surgery. Novel perioperative neuroprotective strategies are needed to assess whether long term improvement in neurodevelopmental outcomes can be achieved.

References

1. Stroke 2007;38:736; 2. J Thorac Cardiovasc Surg 2006;131:190; 3. Circulation 2002;106[suppl I] I-109; 4. Pediatr Card Surg Annu. 2008:61.
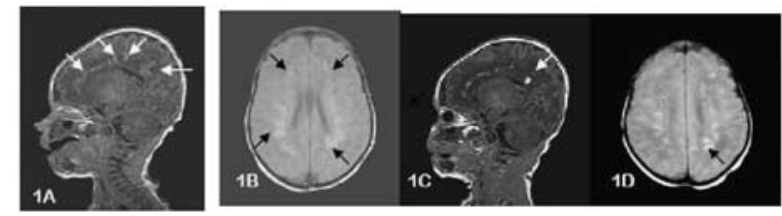

\section{9}

Effect of Ischemic Postconditioning in Infant with Tetralogy of Fallot

Wanjun Luo, Xiangya Hospital, China

Bei Li, XIANGYA HOSPITAL, China

Ri Chen, XIANGYA HOSPITAL, China

Rimao Huang, XIANGYA HOSPITAL, China

Objective: The postoperative course in children with cyanotic congenital heart defect is generally more complicated than that of acyanotic patients. The ischemic postconditioning provide protection from myocardial injury. We conducted a randomized trial to evaluate the effect of postconditioning in children undergoing repair of tetralogy of Fallot.

Methods: Sixty and six children with tetralogy of Fallot were randomly assigned to ischemic postconditioning group ( $\mathrm{n}=34$, aged $2.4 \pm 1.0 \mathrm{yrs})$ or control group $(\mathrm{n}=32$, aged $2.4 \pm 1.2 \mathrm{yrs})$. The ischemic postconditioning was performed by intermittent aortic clamping after reperfusion. The morbidity, mortality, ventilation time, length of ICU stay, inotropic score, release of troponin I and lactate were assayed.

Results: The two groups were comparable. There were one death in postconditioned group and one in control. The cardiopulmonary complications was reduced in postconditioned patients (8.8.\%, 3/34) compared with control $(37.5 \%, 12 / 32, \mathrm{P}<0.05)$. The troponin I was significantly lower $(\mathrm{P}<0.05)$ with reduced inotrope score $(5.5 \pm 3.3$ vs $10.1 \pm 4.9, \mathrm{P}<0.01)$ and lactate release $(\mathrm{P}<0.05)$ in postconditioned patients. The ventilation time was significantly reduced in postconditioned patients compared with control $(14 \pm 13 \mathrm{~h}$ vs $31 \pm 33 \mathrm{~h}, \mathrm{p}<0.05)$. There was a significant decreased in the ICU stay in the postconditioned patients $(42 \pm 25 \mathrm{~h}$ vs $71 \pm 49 \mathrm{~h}, \mathrm{P}<0.05)$.

Conclusions: The study suggests that ischemic postconditioning may provide clinical benefits with respect to the cardiopulmonary morbidity, ventilation time, ICU stay, requirement of inotrope in children undergoing repair for tetralogy of Fallot.
3030

Differential Neuronal Vulnerability varies according to Mechanism of CPB Insult

Nobuyuki Ishibashi, Children's National Medical Center, Washington, DC, United States

Yusuke Iwata, Children's National Medical Center, Washington, DC, United States

Toru Okamura, Children's National Medical Center, Washington, DC, United States

David Zurakowski, Children's Hospital Boston, Boston, MA, United States

Hart GW Lidov, Children's Hospital Boston, Boston, MA,

United States

Richard A Jonas, Children's National Medical Center, Washington,

DC, United States

Objective: We used a piglet model with different mechanisms of bypass-related brain injury to study (1) neuronal vulnerability, (2) relation between cerebral microcirculation and regional neuronal damage and (3) accuracy of intra-operative monitoring in predicting damage.

Methods: Eighty piglets assigned to bypass groups with different ischemic insults: ultra-low flow at $25 \mathrm{oC}$ (control), circulatory arrest at $25 \mathrm{oC}$ (hypo-oxygen supply stress), and ultra-low flow at 34oC (high-oxygen consumption stress). Near-infrared spectroscopy (NIRS) was used to measure tissue-oxygenation index (TOI) and oxyhemoglobin ( $\mathrm{HbO} 2)$ nadir time. Functional capillary density (FCD) and leukocyte activation in cortex was assessed by intravital micrsocopy. Histological neuronal damage scores was the primary outcome. Secondary measures included functional outcome.

Results: Significant neuronal damage was observed in cortex, hippocampus, caudate nucleus, and Purkinje cells. Histological score correlated with impaired FCD and leukocyte activation. TOI and $\mathrm{HbO} 2$ nadir time predicted degree of injury. Histological damage in cortex and Purkinje cells was not different between bypass states, while the caudate nucleus was vulnerable to hypoxic supply stress and the hippocampus was vulnerable to high-oxygen consumption stress. Neurological recovery was lower under hypo-oxygen supply stress than highconsumption stress. Neurological recovery was significantly correlated with neuronal damage in cortex, Purkinje cells, and caudate nucleus but did not correlate with damage in the hippocampus.

Conclusion: Vulnerability of neurons depends on the mechanism of the bypass-related ischemic insult. NIRS can predict injury. Injury to hippocampal neurons which are critical to memory may not be apparent in assessing acute neurological recovery but is important for long-term brain function.

3031

Different organ injury mechanisms following cardiopulmonary bypass and circulatory arrest in a paediatric model

Jonathan McGuinness, Our Lady's Children's Hospital, Crumlin, Dublin 12, Ireland., Ireland

Niamh M Keenan, Department of Surgical Research, Royal College of Surgeons in Ireland, Dublin, Ireland., Ireland

Darren McLoughlin, Department of Surgical Research, Royal College of Surgeons in Ireland, Dublin, Ireland., Ireland

John S Byrne, Department of Surgical Research, Royal College of Surgeons in Ireland, Dublin, Ireland., Ireland Colin Canavan, Department of Surgical Research, Royal College of Surgeons in Ireland, Dublin, Ireland., Ireland 
David Bouchier-Hayes, Department of Surgical Research, Royal College of Surgeons in Ireland, Dublin, Ireland., Ireland

J Mark Redmond, Our Lady's Children's Hospital, Crumlin, Dublin 12, Ireland., Ireland

Introduction: Multiple organ dysfunction(MOD) following cardiopulmonary bypass(CPB) and circulatory arrest for repair of complex congenital cardiac defects causes major morbidity. The exact mechanism and timing of injury is unclear.

Methods: Using a juvenile porcine $\mathrm{CPB}$ and circulatory arrest model, we monitored the development of MOD in the first $24 \mathrm{hrs}$ post-operatively. Five experiments were completed at $8 \mathrm{hrs}$, and 5 at $24 \mathrm{hrs}$. All results are reported as mean $+/-$ standard error of mean, all $\mathrm{p}<0.05$ on ANOVA.

Results: A low cardiac output state developed in all cases at 10-12 hrs. Cardiac injury manifested as a troponin peak at $6 \mathrm{hrs}$ (baseline: $0.224+/-0.1 \mathrm{ng} / \mathrm{ml} ; 6 \mathrm{hrs}: 44.36+/-8.67 \mathrm{ng} / \mathrm{ml}$ ), and diastolic dysfunction at $4-10$ hrs. Pulmonary injury was demonstrated by a reduction in dynamic lung compliance at $10 \mathrm{hrs}$ (baseline: $10.12+/-0.124 \mathrm{ml} / \mathrm{cmH} 2 \mathrm{O} ; \quad 10 \mathrm{hrs}: 5.7+/-0.29 \mathrm{ml} /$ $\mathrm{cmH} 2 \mathrm{O}$ ), followed by a drop in $\mathrm{PO} 2$ at $12 \mathrm{hrs}$ (baseline:49.54 +/ $-3.02 \mathrm{kPa} ; 12 \mathrm{hrs}: 35.72+/-7.19 \mathrm{kPa}$ ), and a reduction in the $\mathrm{PO} 2 / \mathrm{FiO} 2$ ratio into ARDS range. These changes were preceded by peaks of IL-6 at 4hrs (baseline: $0+/-0 \mathrm{pg} / \mathrm{ml}$; $4 \mathrm{hrs}: 314.07+/$ $-93.47 \mathrm{pg} / \mathrm{ml}$ ), and WCC at $6 \mathrm{hrs}$ (baseline: $17.07+/-4 \times 10^{9} /$ L; 6 hrs: $25.25+/-7.13 \times 10^{9} / \mathrm{L}$ ); with marked pulmonary neutrophil infiltration and oedema at 8 hrs.

Renal injury presented as progressive tubular structural damage from 2-12 hrs (urinary $\mathrm{N}$-acetyl-glucosaminidase: $2 \mathrm{hrs:}+/-0.27 \mathrm{U} / \mathrm{l} ; \quad 12 \mathrm{hrs}: 2+/-0.31 \mathrm{U} / \mathrm{l})$, and subsequent impairment of tubular function at 4 hrs with some recovery at 24 hrs (fractional excretion of urinary sodium: baseline: $0.76+$ / $-0.22 ; 4$ hrs: $0.32+/-0.11 ; 16$ hrs: $0.04+/-0.01 ; 24$ hrs: $0.2+/-0.1)$. Throughout this period, renal NIRS readings were $>65 \%$.

Conclusions: Post-operative pulmonary dysfunction appears related to the systemic inflammatory response, while renal medullary injury begins before its onset and begins to resolve despite a low cardiac output state, suggesting an intra-operative ischemic insult.

\section{2}

Non-invasive Cardiac Output Measurement Using Physioflow ${ }^{\circledR}$ in Paediatric Patients- a comparison with MRI Katherine L Taylor, Department of Anesthesia, Hospital for Sick Children, Toronto, Canada

Lars Grosse-Wortmann, Departments of Cardiology and Radiology, Labatt Family Heart Center,Hospital for Sick Children, Toronto, Canada

Cedric Manlhiot, Cardiovascular Clinical Research Unit, Labatt Family Heart Centre, Hospital for Sick Children, Toronto, Canada

Helen Holtby, Hospital for Sick Children, Toronto, Canada

Objectives: The study was designed to evaluate the reliability and accuracy of cardiac output (CO) measurement using electrical bioimpedance cardiography, (Physioflow ${ }^{\mathrm{R}}$ ) (COPF), in paediatric patients with and without cardiac disease undergoing MRI. Methods: After Institutional Ethics Committee approval and informed consent, patients undergoing cardiac MRI were recruited. $\mathrm{CO}$ was calculated by flow measurement. Immediately before or after the MRI, 6 Physioflow ${ }^{\circledR}$ (PF) ECG electrodes were applied for continuous real time measurements every 15 seconds over 10 minutes (COPF). The level of patient sedation was constant for both $\mathrm{CO}$ measurements. $\mathrm{CO}$ was averaged and compared with the $\mathrm{CO}$ measurements using BlandAltman analysis.

Results: Preliminary results of 16 patients ( 8 males $/ 8$ females) are presented. Median age (range) was 2.8 years $(0.2-8.0$ years) and median (range) BSA $0.55 \mathrm{~m}(0.16-1.00 \mathrm{~m}) .44 \%$ of patients had intracardiac shunts, $25 \%$ had severe physiological abnormalities. Median number of PF measurements per patient was 53 (21-377). A total of 959/1599 PF measurements were valid (60\%). The Physioflow ${ }^{\circledR}$ was unable to calculate CI in $8 \%$ of data entries and identified 33\% entries as artifact. A further 14\% data entries were deemed implausible by investigators, $33 \%$ of these entries were not detected as such by PF. Mean $\mathrm{CI}=3.1 \pm 1.1$, mean $\mathrm{CI}=3.0 \pm 0.5$, mean bias $(\mathrm{PF}-\mathrm{MRI})=-0.20 \mathrm{p}=0.40$, limits of agreement $(95 \%)=-3.40-+1.60$. One third of patients had measurements within $20 \%$ of each other, $87 \%$ of patients within $40 \%$. COMRI affected agreement $(\mathrm{r}=+0.86, \mathrm{p}<0.001)$.

Impact: Preliminary data demonstrate that CI is not accurate when compared to CI. Recruitment of patients with more homogenous cardiac physiology continues.

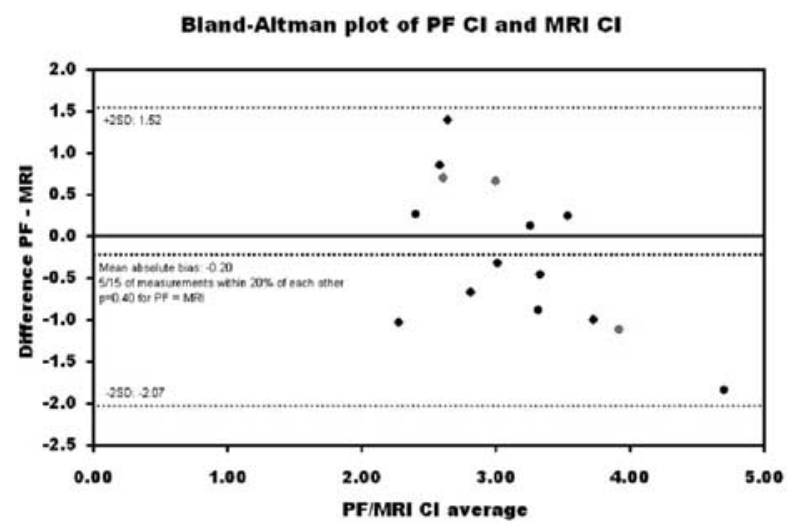

3045

Exercise induces significant biventricular intraventricular mechanical dyssynchrony in children with TOF

Lucy Roche, The Hospital for Sick Children, Toronto, Canada Lars Grosse-Wortmann, The Hospital for Sick Children, Toronto, Canada

Paul F Kantor, The Hospital for Sick Children, Toronto, Canada Andrew N Redington, The Hopsital for Sick Children, Toronto, Canada

Mark K Friedberg, The Hosiptal for Sick Children, Toronto, Canada

Objective: To investigate the effect of exercise on right (RV) and left (LV) intraventricular dyssynchrony and their relation to ventricular function and exercise capacity in children with repaired tetralogy of Fallot (TOF).

Methods: We prospectively studied 29 children with TOF with echocardiography at rest and at peak exercise, MRI and metabolic exercise testing. Dyssynchrony results were compared with healthy controls at rest $(\mathrm{n}=42)$ and peak bicycle ergometry $(\mathrm{n}=25)$

LV dyssynchrony index (LVDI) was assessed by tissue Doppler imaging (TDI) of apical 4-, 3- and 2-chamber views and the SD of time to peak $\mathrm{S}$ velocity in $12 \mathrm{LV}$ segments calculated.

RV dyssynchrony index (RVDI) was assessed by TDI of apical 4-chamber and RVOT views and the SD of time to peak S velocity in $8 \mathrm{RV}$ segments calculated.

All indices were corrected for heart rate.

Results: Controls and patients with TOF were of similar age and had similar dyssynchrony indices at rest (table). There were 
marked differences in their response to exercise (figure). In contrast to controls, exercise caused increasing LVDI and RVDI in children with TOF.

Although the exercise capacity of those with TOF was impaired, (mean peak $\mathrm{VO} 2=63 \%$ predicted) this was unassociated with the degree of either LVDI or RVDI on exertion (LVDI $\mathrm{r}=0.04 \mathrm{p}=\mathrm{NS}$, RVDI $\mathrm{r}=0.23 \mathrm{p}=\mathrm{NS}$ ). LVDI and RVDI were not related to $\operatorname{LVEF}(r=0.08 \mathrm{p}=\mathrm{NS})$ or RVEF $(\mathrm{r}=0.29 \mathrm{p}=\mathrm{NS})$.

Conclusion: Exercise induces significant LV and RV intraventricular mechanical dyssynchrony in children with TOF although this alone does not account for their impaired exercise capacity.

\begin{tabular}{|c|c|c|c|}
\hline & Controls & Children with TOF & $\mathrm{p}$ value \\
\hline Age at study (years) & 12.4 & 12.2 & 0.82 \\
\hline $\begin{array}{l}\text { Mean resting LV dyssynchrony } \\
\text { index (HR corrected) }\end{array}$ & 33.9 & 38.6 & 0.16 \\
\hline $\begin{array}{l}\text { Mean resting RV dyssynchrony } \\
\text { index (HR corrected) }\end{array}$ & 43.6 & 37.7 & 0.03 \\
\hline $\begin{array}{l}\text { Mean exercise LV dyssynchrony } \\
\text { index (HR corrected) }\end{array}$ & 33.1 & 49.6 & 0.002 \\
\hline $\begin{array}{l}\text { Mean exercise RV dyssynchrony } \\
\text { index (HR corrected) }\end{array}$ & 26.1 & 61.1 & $<0.0001$ \\
\hline
\end{tabular}

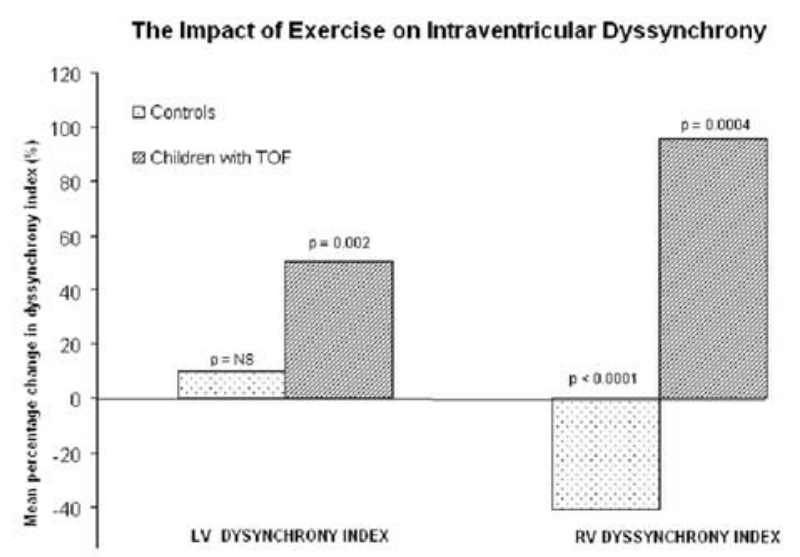

NB:p value is that associated with change from rest to exercise

3046

Pulse oximetry screening (POS) in newborns can reduce the diagnostic gap in critical congenital heart disease (cCHD) -2 years experience from a prospective multicenter trial

Frank T Riede, Heart Center, University of Leipzig, Germany Cornelia Worner, Heart Center, University of Leipzig, Germany Ingo Dähnert, Heart Center, University of Leipzig, Germany Martin Kostelka, Heart Center, University of Leipzig, Germany Peter Schneider, Heart Center, University of Leipzig, Germany

Introduction: POS has been proposed as an effective, non invasive, inexpensive tool allowing earlier diagnosis of cCHD.

Aim of the study: Test the hypothesis that POS can reduce the diagnostic gap in CCHD in daily clinical routine in the setting of tertiary, secondary and primary care centers.

Methods: Prospective multicenter trial in Saxony, Germany. POS was performed in healthy term newborns at the age of 24-72 hours. An oxygen saturation $(\mathrm{SpO} 2)$ of $\geqslant 96 \%$ on lower extremities was defined as normal. If a $\mathrm{SpO} 2$ of $\leqslant 95 \%$ was measured and confirmed after 1 hour, complete clinical examination and echocardiography were performed.
Results: From July 2006-June 2008, 42691 newborns from 34 institutions have been included in the study. 70 children were excluded due to prenatal diagnosis $(n=52)$ or clinical signs of cCHD $(\mathrm{n}=18)$ before POS. 741 newborns not receive POS, mainly due to early discharge after birth $(n=673,91 \%)$. In 41950 newborns POS was performed. POS was true positive in 14 (TAPVD $\mathrm{n}=5$, TGA(-VSD) $\mathrm{n}=4$, PA-VSD $\mathrm{n}=2$, HLHS, Taussig-Bing-Syndrome, PTA-IAA $n=1$ each), false positive in 33 (healthy $n=12, \operatorname{PPHN} n=15$, Sepsis 6), true negative in 41899 and false negative in 4 (ISTA(-VSD) $n=3$, TGA-VSD $n=1$ ) children. Sensitivity, specificity, positive and negative predictive value were $77,78 \%, 99,92 \%, 29,79 \%$ and $99,99 \%$, respectively. Discussion: POS can reduce the postnatal diagnostic gap in cCHD and also detect PPHN or Sepsis. False positive results leading to unnecessary evaluation of healthy newborns are rare. POS should be proposed as a routine procedure in postnatal care.

\section{7}

Serial NT-proBNP in Acute Decompensated Pediatric Heart Failure

Paul F Kantor, Hospital for Sick Children, Toronto, Canada, Canada Derek TH Wong, Hospital for Sick Children, Toronto, Canada,

Canada

Kristen George, Hospital for Sick Children, Toronto, Canada, Canada Judith Wilson, Canada

Background: N-terminal pro-B-type natriuretic peptide (NTproBNP) is elevated in pediatric patients with congestive heart failure (CHF). In adults, it is predictive of outcome. However, the role of NT-proBNP in pediatrics is uncertain. We present the largest pediatric study following patients with acute $\mathrm{CHF}$ with serial NT-proBNP.

Methods: Patients admitted for acute CHF between May 2005 and July 2007, were enrolled. Patients less than 3 months of age or those requiring cardiovascular surgery were excluded. Demographic and clinical data were recorded. NT-proBNP levels were obtained within 24 hours of admission and serially thereafter.

Results: 24 patients were enrolled - 10 required mechanical support. NT-proBNP levels were 100-1000 times above normal and peaked at day $2-3$ of admission, decreasing over time irrespective of whether mechanical support was needed or not. NT-proBNP decreased more rapidly in patients who did not require mechanical support. Of those patients on mechanical support, NT-proBNP declined faster after being placed on mechanical support. At discharge, NT-proBNP levels were significantly decreased compared to admission levels in all patients but were still significantly above the normal reference ranges.

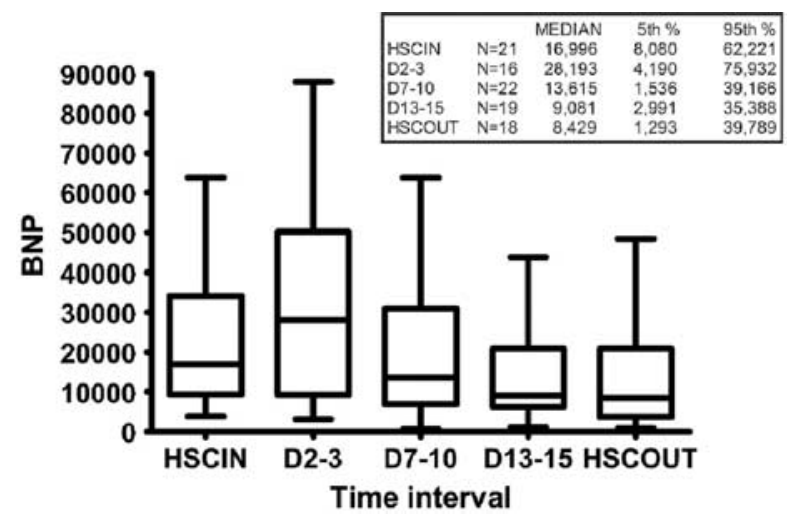

Conclusions: NT-proBNP levels are extremely elevated in acute pediatric CHF. NT-proBNP did not correlate with clinical measures 
of $\mathrm{CHF}$ and were not predictive of death or transplantation. Initial NT-proBNP did not predict the need for mechanical support. However, the rate of decline may be useful in determining which patients will eventually require mechanical support. Mechanical support results in a more rapid decrease in NT-proBNP, likely reflecting the volume unloading of the ventricle.

\author{
3048 \\ Effect of selective alpha 1 receptor agonist in the \\ treatment of postural orthostatic tachycardia syndrome in \\ children \\ Junbao Du, Department of Pediatrics, Peking University First Hospital, \\ Beijing 100034, China, China \\ Li Chen, Department of Pediatrics, Peking University First Hospital, \\ Beijing 100034, China, China \\ Li Wang, Health Science Center, Peking University, Beijing 100083, \\ China, China
}

Objective: The study was designed to examine the effect of selective alpha 1 receptor agonist midodrine hydrochloride in the treatment of children with postural orthostatic tachycardia syndrome. Methods Fifty nine children (23 male, 36 female, age $7-18$ yrs, mean age $12.3 \pm 2.4$ yrs) who came from Peking University First Hospital were included in the study and clinical investigations. They were randomally divided into group I (with midodrine hydrochloride and conventional therapy), group II (with metoprolol and conventional therapy) and group III (with conventional therapy only). Head-up tilt test under quiet circumstance was conducted. At the end of follow-up, the cure rate, improvement rate and effective rate of symptoms were compared among the three groups. Results The cure rate at the end of follow-up in group I was significantly higher than that of group II and group III $(60.87 \%$ vs $30.00 \%$ and $25.00 \%$, $\mathrm{P}<0.05)$. The effective rate at the end of follow-up in group I was also significantly higher than that of group II and group III (82.61\% vs $50.00 \%$ and $43.75 \%, \mathrm{P}<0.05)$. During follow-up, we discovered that the symptom recurrent rate in group I was significantly lower than group II and group III, respectively $(\mathrm{P}<0.05)$, but there was no significant difference between group II and group III. And the time of symptom improvement in group I was shorter than group II and III. Conclusion Selective alpha 1 receptor agonist-midodrine hydrochloride was effective in the treatment of children with postural orthostatic tachycardia syndrome.

\section{9}

Ethnic Group Differences in the Perinatal Antecedents of Early Childhood Obesity: A Population-Based Analysis Sarah E Messiah, University of Miami Miller School of Medicine, United States

Kristopher L Arheart, United States

Steven E Lipshultz, University of Miami Miller School of Medicine, United States

Tracie L Miller, University of Miami Miller School of Medicine, United States

Background: Approximately $26 \%$ of 2 - to 5 -year olds in the United States are overweight (body mass index [BMI] $>85$ th $\%$ ile for age and sex). Overweight preschool-aged children are more than four times as likely to become obese adults compared to their normal weight counterparts, placing them at high risk for cardiovascular disease (CVD) onset. Little is known about the perinatal etiology of early childhood obesity by ethnicity. We explored these relationships among US 3- to 6-year olds. Methods. Representative samples (1999-2004) of the National Health and Nutrition Examination Survey (NHANES) were used to examine the association between overweight, mother's smoking status during pregnancy, birthweight, and infant feeding method. $(\mathrm{N}=1,481)$. Results. Mexican American children were significantly more likely than their counterparts to be obese $(\mathrm{P}<0.001)$. Chi square analysis showed that breastfeeding had a modest protective effect against BMI $>95$ th\%ile for Hispanics $(\mathrm{p}<0.05)$. Mexican American and non-Hispanic black mothers who smoked during pregnancy were significantly more likely to have an obese preschool child than their non-smoking counterparts $(\mathrm{P}<0.01, \mathrm{P}<0.05$, respectively). Logistic regression analysis showed a protective effect for both mother quitting smoking during pregnancy (OR $0.74,95 \% \mathrm{CI}, 0.58,0.95)$ and normal birthweight (OR 0.53, 95\% CI, 0.30, 0.93) for obesity. Conclusions. Obesity in children as young as preschool age is associated with perinatal factors. Minority women should not smoke and should be encouraged to breastfeed as their children are disproportionately affected by the obesity epidemic, which in turn is a risk factor for later life onset of CVD.

\section{0 \\ Parent Well-Being Following Their Infant's Cardiac Surgery \\ Candice Franich-Ray, Murdoch Childrens Research Institute, Australia Brigid Jordan, 1. Royal Children's Hospital 2. Murdoch Childrens Research Institute 3. The University of Melbourne, Australia Vicki Anderson, 1. Murdoch Childrens Research Institute 2. The University of Melbourne 3. Royal Children's Hospital, Australia Elisabeth Northam, 1. Murdoch Childrens Research Institute 2. The University of Melbourne, 3. Royal Children's Hospital, Australia Andrew Cochrane, Monash Medical Centre, Australia \\ Samuel Menahem, 1. Monash Medical Centre 2. The University of Melbourne, Australia}

Objective: To investigate the emotional impact on parents of their infant's cardiac surgery. Surgery may result in parents being separated from their baby after birth. They experience uncertainty and anxiety about their baby's condition and prognosis. Many may witness their baby undergoing painful procedures.

Method: Mothers and fathers were recruited if their baby had cardiac surgery under three months of age. One month after their baby was discharged both parents were interviewed and asked to complete a questionnaire protocol that included the Edinburgh Postnatal Depression Scale, State-Trait Anxiety Inventory and Acute Stress Disorder Scale.

Results: Ninety nine families participated in the study. Seventy eight mothers and 57 fathers completed the questionnaires. Seventeen mothers (21\%) had possible depression and $11(14 \%)$ had probable depression. Nine mothers (12\%) and 4 fathers (7\%) were still experiencing clinically significant anxiety. Fifty-five mothers (72\%) and 32 fathers (58\%) experienced significant dissociation symptoms and 27 mothers (35\%) and 10 fathers $(18 \%)$ satisfied criteria for acute stress disorder.

Conclusion: The study found significant emotional trauma in parents in the context of their infant's cardiac surgery. It suggests that a trauma framework should be considered when assessing the impact on parents. Trauma reactions may impair parents' ability to process medical information, make informed decisions or develop a secure attachment relationship with their baby. The ongoing trauma reactions after hospital discharge suggests that parents may need to be assessed and offered treatment for this during their baby's admission with subsequent review after the baby is discharged. 


\section{1}

Heterotaxy Congenital Heart Disease Patients with Ciliary Dysfunction: Possible Role in Morbidity?

Nader J Nakhleh, Childrens National Medical Center and National Institutes of Health, United States

Linda Leatherbury, Childrens National Medical Center and National Institutes of Health, United States

Richard A Jonas, Childrens National Medical Center, United States

Rachel A Giese, National Institutes of Health, United States

Magaret W Leigh, University of North Carolina, Chapel Hill, United States

Michael R Knowles, University of North Carolina, Chapel Hill,

United States

Richard JB Francis, National Institutes of Health, United States

Cecilia $W$ Lo, National Institutes of Health, United States

Patients with congenital heart disease (CHD) associated with heterotaxy require high-risk surgical repairs. We recently showed such patients have increased post-surgical morbidity and respiratory complications. Heterotaxy is characterized by discordant abdominal/thoracic organ situs due to aberrant left-right patterning, a developmental process dependent on motile cilia in the embryonic node. As mucociliary clearance requires airway cilia motion, and we previously found a $40 \%$ incidence of CHD and heterotaxy in a mouse model of primary ciliary dyskinesia (PCD), we hypothesized PCD may have increased prevalence in heterotaxy patients. To test this hypothesis, we recruited 26 patients with CHD and heterotaxy, and evaluated for PCD with nasal nitric oxide (nNO) measurements and nasal biopsies for ciliary motion and electron microscopy (EM). Most CHD consisted of 9 poly/asplenia and in addition 7 non-HLHS single ventricles. Panel review of nNO, ciliary motion and EM data led to a consensus diagnosis of probable or possible ciliary dysfunction in $50 \%$ of patients. 6 with low nNO and ciliary dysmotility were designated probable PCD and 7 with borderline low $\mathrm{nNO}$ and/or aberrant ciliary motion distinct from classic PCD were designated possible PCD. Given heterotaxy and PCD are rare diseases each with incidences of $1 / 15,000$, these findings indicate some heterotaxy may be a ciliopathy. These findings suggest heterotaxy patients undergoing cardiac surgery should have a PCD work-up. Those with confirmed ciliary dysfunction may be offered pulmonary therapies to improve mucociliary clearance. Such change in the standard of care may improve outcomes for heterotaxy patients undergoing high-risk cardiac surgery.

\section{2}

Heterotaxy Patients with Congenital Heart Disease and Increased Postoperative Respiratory Complications

Linda Leatherbury, Childrens National Medical Center and National Institutes of Health, United States

Matthew W Swisher, National Institutes of Health, United States Richard A Jonas, Childrens National Medical Center, United States Xin Tian, National Institutes of Health, United States Gerard R Martin, Childrens National Medical Center, United States Cecilia W Lo, National Institutes of Health, United States

Patients with heterotaxy and complex congenital heart disease (CHD) undergo cardiac surgery with high mortality and morbidity. Recent studies have revealed an association between heterotaxy, CHD, and primary ciliary dyskinesia (PCD).

We undertook a retrospective review of patients undergoing cardiac surgery at Childrens National Medical Center between
2004 to 2008 to explore the hypothesis that postoperative complications associated with heterotaxy might be due to PCD. Reviewed were post-surgical outcomes of 87 patients with CHD and heterotaxy defined as any disruption in normal left-right asymmetry. As controls, 634 cardiac surgical patients with CHD, but without laterality defects, were selected. Surgical risks and complexities were similar between the two groups with both having median RACHS-1 scores of 3.0.

Our results showed the mean length of post-operative hospital stay (17 vs. 11 days) and mechanical ventilation (11 vs. 4 days) were significantly increased in heterotaxy compared to control patients. Also elevated were rates of tracheostomies ( $7 \%$ vs $2 \%$ : Odds Ratio 4.6), ECMO support (13\% vs 5\%: Odds Ratio 2.8), prolonged ventilatory course (23\% vs $12 \%$ : Odds Ratio 2.1$)$ and post-surgical deaths (16\% vs 5\%: Odds Ratio 3.9). All with $\mathrm{p}<0.01$.

In conclusion, our findings show heterotaxy patients had more complicated post-surgical courses with increased mortality despite being matched with controls for RACHS-1 surgical complexity score. This was associated with an increased risk for respiratory complications, consistent with the possible association of ciliary dysfunction or PCD with heterotaxy. Further studies are needed to explore the underlying causes for the increased surgical risks and respiratory complications for heterotaxy patients undergoing cardiac surgery.

\section{3}

Pulmonary venous anatomy and incidence of obstruction in total anomalous pulmonary venous connection (TAPVC) associated with heterotaxia syndrome

Makoto Ando, Sakakibara Heart Institute, Japan

Yukihiro Takahashi, Sakakibara Heart Institute, Japan

Naoki Wada, Sakakibara Heart Institute, Japan

Background: Repair of TAPVC associated with heterotaxia syndrome still carries a high mortality. Multiple factors are potentially involved, and acquired pulmonary venous obstruction (PVO) after a TAPVC repair may be an important one.

Methods: Retrospective review of patients undergoing repair of TAPVC associated with heterotaxia (H-TAPVC, $n=30)$ and with no other major cardiac or non-cardiac anomalies (Isolated TAPVC, $\mathrm{n}=63$ ) from 1997.7 to 2007.11.

Results: Normal PV anatomy (having non-obstructive PVs, wellformed confluence, and single vertical vein) was seen in 52/63 $(82.4 \%)$ of isolated TAPVC, and in 17/30 (56.7\%) of H-TAPVC (OR 3.7, $\mathrm{p}=0.006$ ). In 8 of the H-TAPVCs, PVs were converged to a small confluence, which necessitated an incision extending into the vertical vein, and mixed TAPVCs were seen in 6. Presence of PV anomaly was associated with a higher incidence of PVO (OR 7.0, $\mathrm{p}=0.03$ ). Reoperation for PVO was invariably seen in the acute postoperative period in isolated TAPVCs $(n=10)$, whereas it occurred constantly up to 3 years in H-TAPVC $(\mathrm{n}=8)$, with the interval to reoperation being $0.3 \pm 0.3$ and $1.2 \pm 1.0$ years $(p=0.012)$. Three mid-term mortality cases (occurred $>2$ years after repair) had acquired PVO.

Conclusions: PV anomaly was more frequently seen in H-TAPVC, and was associated with higher incidence of acquired PVO. The interval to onset of PVO is longer with H-TAPVC. A different mechanism of PVO may be involved in H-TAPVC, such vertical vein constriction, intrinsic obstruction, and orifice constriction caused by atrial enlargement/wall thickening. Modifications such as sutureless pericardial technique may be justified at initial repair. 
3055

Excellent Surgical Results of Extra-Cardiac Conduit Total Cavopulmonary Connection: Analysis of Consecutive 364 Cases

Toshihide Nakano, Fukuoka Children's Hospital, Japan

Hideaki Kado, fukuoka children's hospital, Japan

Kazuhiro Hinokiyama, Fukuoka Children's Hospital, Japan

Shin-ichiro Oda, Fukuoka Children's Hospital, Japan

Takahisa Sakurai, Fukuoka Children's Hospital, Japan

Kunihiko Joo, Fukuoka Children's Hospital, Japan

Objectives: Extra-cardiac conduit total cavopulmonary connection (EC-TCPC) has theoretical advantages over the other types of Fontan modification. In this study the clinical results of this procedure are assessed. Methods: Since 1994, 364 patients underwent EC-TCPC in Fukuoka Children's Hospital. Median age and body weight at operation were 3.5 years and $12.6 \mathrm{~kg}$. Most of the patients are receiving coumadin and aspirin postoperatively. Surgical results and hemodynamic variables were reviewed. Results: Follow-up time was $6.0 \pm 3.9$ years. There were no operative death and 13 late deaths (3.6\%). Actuarial survival rate was $95.5 \%$ at 10 years and $92.2 \%$ at 14 years. Eight patients had reoperation including fenestration closure in 1 , aortic valve replacement in 1 , ligation of major collateral vessel in 2 and pacemaker implantation in 4. Six patients had catheter intervention for branch pulmonary stenosis. Nineteen patients developed late complication including protein loosing enteropathy in 4, thromboembolism in 3, bleeding complication in 3, and new onset supraventricular arrhythmia in 9. Freedom from Fontan-related events was $85.6 \%$ at 10 years and $83.2 \%$ at 14 years. The incidence of late complication was not related to the size of the conduit. Late cardiac catheterization for 325 patients showed central venous pressure of $9.5 \pm 2.6 \mathrm{mmHg}$, cardiac index of $3.5 \pm 0.8 \mathrm{~L} / \mathrm{kg} / \mathrm{min}$ and arterial oxygen saturation of $94.1 \pm 2.3 \%$. No patient showed conduit stenosis. Plasma concentrations of atrial and brain natriuretic polypeptide $(\mathrm{pg} / \mathrm{ml})$ were $34.3 \pm 32.6$ and $26.0 \pm 48.3$. Conclusion: Early to midterm outcome of EC-TCPC was satisfactory with low incidence of late mortality and morbidity, and excellent hemodynamic state.

\section{6}

Biointegration and growth of porcine valved pulmonary conduits. Experimental model in a sheep

Glaucio Furlanetto, Instituto Furlanetto, Brazil

Carlos $H$ Passerino, Centro de Pesquisas Labcor Laboratórios, Brazil

José M Costa, Centro de Pesquisas Labcor Laboratórios, Brazil

Patricia M Dodd, Centro de Pesquisas Labcor Laboratórios, Brazil

Rolland Siegel, United States

David T Chueng, United States

Sidney Levitsky, Division of Cardiothoracic Surgery, Beth Israel

Deaconess Medical Center and, United States

Ivan S J Casagrande, Centro de Pesquisas Labcor Laboratórios, Brazil

Objective: To date, there is no suitable valved conduit for small children that demonstrates growth. Pulmonary conduits treated by the L-Hydro ${ }^{\mathbb{R}}$ process were implanted in newborn lambs to demonstrate growth potential.

Methods: The L-Hydro ${ }^{\circledR}$ process was used to treat porcine valved pulmonary conduits that were orthotopically implanted in 9 newborn lambs using extracorporeal circulation. Of the 8 survivors 7 were kept alive for 12 months after implantation. Diameter of the conduits and gradient across the valves at surgery, 3 and 9 months post-op were evaluated using bi-directional echocardiographic and angiographic methods before sacrifice. After necropsy, histological and radiological analysis were performed.
Results: The average weight of the animals was $4.2 \mathrm{~kg}$ at implantation, and $43.1 \mathrm{~kg}$ at sacrifice $(\mathrm{p}<0.05)$. There was significant increase in the average valve area, $139.9 \mathrm{~mm}^{2}$ at implantation and $443.4 \mathrm{~mm}^{2}$ at sacrifice $(\mathrm{p}<0.05)$. Pre-sacrifice angiographic examination showed absence of transvalvular gradient. The radiographic analysis did not reveal significant conduit wall or leaflet calcification in any of the animals. Histological analysis of the grafts demonstrated total integration with native-like intact valve leaflets.

Conclusion: Functional evaluation, echocardiographic analysis and histology demonstrated evidence of growth of the grafts without calcification and completely endothelialized and normal nativelike pulmonary valve leaflets.

\section{7}

An integrated model for interpreting missense variants/ mutations in the Long QT syndrome genes in families at risk of sudden cardiac death

Paul A James, Genetic Health Services Victoria, Australia

Ivan Macciocca, Genetic Health Services Victoria, Australia

Lavinia Gordon, Bioinformatics Department, Murdoch Childrens

Research Institute, Australia

Andrew Davis, Cardiology Department, Royal Childrens Hospital, Australia

Dean Phelan, Molecular Genetics Laboratory, Victorian Clinical

Genetics Service, Australia

Desiree du Sart, Molecular Genetics Laboratory, Victorian Clinical

Genetics Service, Australia

Background: Finding a mutation in an ion channel gene in Long QT syndrome allows the accurate identification of other family members at risk of sudden death. Interpretation of genetic investigations is complicated due to the majority of mutations in Long QT syndrome being missense mutations, frequently novel, and difficult to distinguish from rare polymorphisms.

Method: We present a new model that combines an analysis of 5 independent areas: the proband's clinical phenotype, familial segregation, evolutionary conservation, amino acid properties and data from control samples to generate an overall likelihood ratio that an observed variation is a pathogenic mutation. The ability of the model to improve the interpretation of DNA sequencing results was examined in 50 families with a clinical diagnosis of Long QT syndrome. Complete sequencing of six LQT genes (LQT1,2,3,5,6 and 7) was performed and the integrated model used to analyse any missense variants.

Results: Likelihood ratios strongly favouring pathogenicity were demonstrated for previously described mutations as well as novel mutations clinically suspected to be pathogenic. For recurrent mutations (e.g. KCNQ1 G314S) the LR exceeded 1000:1 in favour of causality while known polymorphisms analysed by the model showed LRs strongly against causation.

Conclusion: The described model provides an objective tool to analyse missense variants in long QT syndrome, allowing accurate interpretation of novel variants in a clinical time-frame and facilitating further of predictive testing in at risk families.

\section{8}

Asymptomatic ventricular preexcitation mimicking dilated cardiomyopathy: the location of the pathway is predictive of this association

Floris Udink ten Cate, Heart Center, University Hospital of Cologne, Germany 
Markus Kruessell, Germany

Mathias Emmel, Pediatric Cardiology, University of Cologne, Germany Narayanswami Sreeram, Heart Center, University Hospital of Cologne, Germany

Background: Ventricular preexcitation is common. Dilated cardiomyopathy is usually the result of longstanding tachyarrhythmia. We demonstrated a causal relationship between ventricular preexcitation and dilated cardiomyopathy in the absence of recorded tachyarrhythmias. This relationship appears to be accessory pathway (AP) location-specific.

Patients and Methods: 10 consecutive children, (age range 0.6 to 17 years) were studied. All had ventricular preexcitation on the ECG, with the vector of the AP suggesting a right sided paraseptal location. None had had a documented tachyarrhythmia; however all had evidence for dilated cardiomyopathy (DCM), with LVEDD $>97$ th centile for weight, and LV FS $<25 \%$ on M-mode echocardiography. Diagnostic cardiac catheterization was performed in the first 4 patients, in whom the LVEDP was normal. Myocardial biopsy findings did not suggest myocarditis. Metabolic screening, family history and viral screening were negative.

Procedures and results: Eight of the 10 patients underwent EP study, despite the absence of tachyarrhythmia (or symptoms suggestive thereof). The AP had a right sided septal location in 7 , and was a fasciculo-ventricular pathway in one (hitherto considered to be a benign finding). All APs were successfully ablated; 2 patients had spontaneous loss of preexcitation during follow-up. Loss of preexcitation (following ablation or spontaneously) was associated with complete recovery of LV function in $9 / 10$ patients, with the most recent patient showing improved function.

Conclusions: Right sided septal APs with overt preexcitation may result in marked ventricular dsysynchrony, which mimics DCM. A causal relationship between such APs and DCM is confirmed by normalisation of ventricular function following loss of preexcitation.

\section{9}

\section{Radiofrequency ablation procedures in children under} 2 years of age

Michal J Kantoch, University of Alberta, Canada

Shubhayan Sanatani, Division of Cardiology, Department of Pediatrics, British Columbia Children's Hospital and The University of British Columbia, Canada

Background: Radiofrequency ablation (RFA) has been shown to be effective in young children although significant complications were reported.

Methods: Thirty six procedures were done in 29 patients (16 female), age 11 days to 23 months (median 4 months), who were brought to the EP lab with the intention to perform RFA for tachycardia which was life-threatening or refractory to medical therapy. Diagnoses were atrio-ventricular bypass tracts (12), PJRT (5), ectopic atrial tachycardia (EAT) (7), atrial flutter (2), ventricular tachycardia (VT) (1), and congenital JET (2). There were 6 children with congenital heart disease and one with myocarditis. Fifteen presented with severe congestive heart failure ( 3 on ECMO support). In 3 patients RFA was not done because of complications (1) or technical difficulties.

Results: The overall success rate was 73\% (19/26) patients. Six patients underwent 2 procedures and 1 patient required 3 procedures. RFA was successful in $88 \%(14 / 16)$ children with AV bypass tracts, (failed in 1 with Ebstein anomaly and in 1 with bilateral epicardial tracts), in 2/4 patients with EAT, in 1 with LV $\mathrm{VT}$, and in 1 of 2 patients with congenital JET (1 cured,
1 improved). One infant with multifocal atrial tachycardia underwent $\mathrm{AV}$ node ablation and pacemaker implantation. The complication rate was $11 \%$ (4/36 procedures): complete heart block, hemopericardium requiring pericardiocentesis and right femoral artery occlusion in 2 patients.

Conclusion: RFA is effective in infants with tachyarrhythmia refractory to medical therapy. The procedure should be reserved for selected patients because of an increased risk of complications.

\author{
3060 \\ Homemonitoring in young patients with a congenital \\ heart disease and the need for pacemakers or \\ defibrillators \\ Peter A Zartner, German Paediatric Heart Center, Sankt Augustin, \\ Germany \\ Nicole Toussaint-Goetz, German Paediatric Heart Center, Sankt \\ Augustin, Germany \\ Martin BE Schneider, German Paediatric Heart Center, Sankt \\ Augustin, Germany
}

Subject: Children and young adults with a congenital heart disease (CHD) show a wide spectrum of cardiac arrhythmias. With the indication for pacemaker or defibrillator (ICD) implantation, decision has to be made whether an online monitoring system will reliably improve further therapy.

Method: 8 patients (age 4.1 to 37.6, mean 15.5 years) with a congenital heart disease received an implantable device with an integrated homemonitoring facility (Biotronik, Berlin). Followup time ranges between 168 and 896 days (mean 457 days, 10 patient years).

Results: In 7 of 8 patients specific event reports were received independently from follow up visits (figure) or perception of any clinically felt symptoms, which led to modification of antiarrhythmic medication, exercise tolerance, electrophysiological catheter ablation or system revision. Data tracking covered $94 \%$ of days during follow up. More than 1000 episodes were reported and evaluated. In three patients the reported events were of critical nature (ventricular fibrillation with shock not realised by the patient, lead failure, ventricular tachycardia in a Fontan patient). Conclusion: Patients with a CHD and progressive arrhythmia and patients with an ICD constitute a high risk group and should be followed as closely as possible. The integrated monitoring system reported decisive data on a daily basis, which led to early modifications of the actual therapy. Additionally it serves to control proper system function. For the patient's protection the safety and efficacy of the implanted systems are thus controllably improved. The online information system extends medical care especially in our young and active patient population.

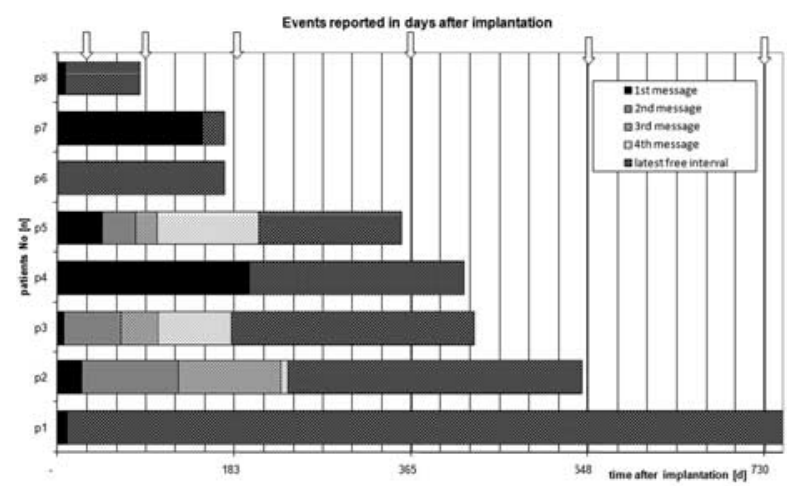


3061

Chronic dual chamber pacing improves relative aortic dilation in complete congenital heart block

Georgia Sarquella-BRugada, Hospital Sainte-Justine, Canada

Sylvia Abadir, Hospital Sainte-Justine, Canada

Nagib Dahdah, Hospital Sainte-Justine, Canada

Paul Khairy, Montreal Heart Institute, Canada

Anne Fournier, Hospital Sainte-Justine, Canada

Congenital complete heart block (CCHB) has been associated to the dilation of the ascending aorta (AsAo). Several mechanisms, such as increased stroke volume, have been proposed. We hypothesized that pacemaker (PM) implantation, known to reduce stroke volume in complete heart block, would help the regression of AsAo dilation in $\mathrm{CCHB}$.

We aim to describe our experience on the effects of chronic PM on aortic dilation in $\mathrm{CCHB}$ with structural normal hearts.

Methods: Retrospective review of echocardiograms was performed for patients with $\mathrm{CCHB}$ and structural normal heart in whom PM was implanted in the last 24 years at our institution. Z-scores of aortic segments were analyzed. Relative AsAo dilation was defined as AsAo/aortic annulus ratio $>2 \mathrm{SD}$ based on normal values from 250 controls.

Results: Twenty-five patients met inclusion criteria. Average age at PM was $4,0 \pm 3,8$ years with mean follow-up of 7,8 $\pm 5,6$ years. Z-score of the aortic valve annulus was similar before and after PM $(0,32 \pm 0,76$ vs $0,49 \pm 1,23, p=0,21)$. Increased AsAo/aortc valve ratio was observed in $29,4 \%$ of the patients without significant dilation of aortic root ratio. Relative AsAo Zscore regressed from $1,82 \pm 2,89$ to $1,65 \pm 1,64$ ( $p=0,001)$. While within normal limits, the Z-score of ortic root ratio regressed from $0,12 \pm 0,89$ to $0,005 \pm 0,51(p=0,001)$.

Conclusion: Ascending aorta dilation is present in a large proportion of patients with $\mathrm{CCHB}$. Our observations of relative AsAo regression following chronic dual chamber pacing support our hypothesis that large stroke volume in non-paced CCHB is the primum-movens of AsAo dilation.

\section{1}

Long-term effect of exercise-induced hypertension in patients with successfully repaired coarctation of the aorta Asle Hirth, Haukeland University Hospital, Department of Paediatrics, Bergen, Norway

Morten L Bøe, Haukeland University Hospital, Norway

Jagjit Bhamra, Haukeland University Hospital, Norway

Gottfried Greve, University of Bergen, Institute of Clinical Medicine,

Norway

Objective: Exercise-induced hypertension in patients with successfully repaired coarctation of the aorta is a well-known phenomenon. Its clinical importance is an ongoing debate, and the long-term clinical course has not been studied previously The aim of this study was to look at long-term outcome in patients successfully operated for coarctation of the aorta, with and without exercise-induced hypertension.

Methods: 44 patients were divided into two groups; one with normal blood pressure response to exercise $(\mathrm{G} 1, \mathrm{~N}=19)$ and one with abnormal response $(G 2, N=25)$. All underwent standard echocardiography and maximal cardiopulmonary retesting after a mean follow-up of 8.8 years. 30 healthy blood spenders served as controls. Results: There were no cardiovascular deaths during follow-up. The two groups remained as defined groups throughout the study period. The number of patients who had started antihypertensive treatment during follow-up was slightly higher in G1 than in G2. At follow-up the left ventricular mass divided by Body Surface Area was $99 \mathrm{gram} / \mathrm{m} 2$ in G1 and $114 \mathrm{gram} / \mathrm{m} 2$ in $\mathrm{G} 2$, both with a significant increase compared to the initial data ( $\mathrm{p} 0.001$ and 0.005 respectively) and significantly higher than in controls. Demographic data, resting systolic blood pressure and exercise performance were similar for both groups, both at initial work-up and at follow-up.

Conclusion: Regarding the risk of developing resting hypertension and/or left ventricular hypertrophy, we found no significant differences between patients with and without exercise induced hypertension, after successfully corrected coarctation of the aorta.

\begin{tabular}{lll}
\hline Variable & $\begin{array}{l}\text { Group I (Initial/ } \\
\text { Follow-up) }\end{array}$ & $\begin{array}{l}\text { Group II (Initial/ } \\
\text { Follow-up) }\end{array}$ \\
\hline Age at repair (months) & 107 & 117 \\
$\begin{array}{l}\text { Age at Follow-up (Yrs) } \\
\text { Systolic Blood Pressure, rest (mmHg) }\end{array}$ & 31.3 & 35.4 \\
$\begin{array}{l}\text { Systolic Blood Pressure, max exercise } \\
\text { (mmHg) }\end{array}$ & $183 / 135$ & $149 / 142$ \\
Arm-leg Blood Pressure gradient post & $6 / 0$ & $258 / 239$ \\
$\quad \begin{array}{l}\text { exercise (mmHg) 6/0 } \\
\text { Left Ventricular Mass/Body Surface Area } \\
\quad \text { (gram/m2) }\end{array}$ & $73 / 99$ & $18 /-1$ \\
$\begin{array}{l}\text { Maximal Oxygen Uptake (ml/kg/min) } \\
\text { aximal }\end{array}$ & $38 / 33$ & $88 / 114$ \\
\hline
\end{tabular}

3072

Quality of outcome for patients with double inlet left ventricle and similar morphologies - impact of coexisting aortic arch obstruction

Michael Seitz, The University of Sydney, Australia

Benedict Raj, The Children's Hospital at Westmead, Sydney., Australia

Andrew D Cole, The Children's Hospital at Westmead,

Sydney., Australia

Stephen G Cooper, The Children's Hospital at Westmead, Sydney., Australia

Gary F Sholler, The Children's Hospital at Westmead, Sydney., Australia

Ian A Nicholson, The Children's Hospital at Westmead, Sydney., Australia

Richard B Chard, The Children's Hospital at Westmead,

Sydney., Australia

David S Winlaw, The Children's Hospital at Westmead, Sydney., Australia

Double-inlet-left-ventricle (DILV) and similar pathologies are characterised by potential for systemic outflow tract obstruction at the bulboventricular foramen. This is best managed pre-emptively by performing a Damus-Kaye-Stansel (DKS) as part of a Norwood or when performing the superior cavopulmonary connection. We have shown that the latter strategy does not produce inferior mid term results. We evaluated late outcomes using this approach with a focus on the impact of aortic arch obstruction (AAO).

Methods: 60 consecutive patients with DILV, 1-TGA and similar were identified, 1990-2007. Quality indicators noted included number of procedures, adverse events, time to Fontan completion and late ventricular function.

Results: 19 had AAO. 12(63\%) achieved Fontan, 1 is waiting and $2(11 \%)$ are not suitable for completion, $4(21 \%)$ died. 1 patient required a Norwood and has achieved completion.

41 had no AAO. 30(73\%) achieved Fontan, 8(20\%) are waiting and 1 is not suitable, $1(2 \%)$ is lost, $1 \operatorname{died}(2 \%)$.

$70 \%$ achieved Fontan within 5 years in both groups. The AAO group required significantly more operations, were more likely to be on long-term diuretics and only $50 \%$ were in NYHA class I vs $77 \%$ in the remainder. 
Conclusions: Overall mortality for the group was acceptable, 5/ $60(8 \%)$, however the patients with AAO have a more protracted course with worse long-term outcome. This subset may be better served by a Norwood procedure to minimise ventricular dysfunction resulting from 'minor' residual arch gradients and PA banding whilst those without AAO can be well managed by a DKS as an infant rather than neonate.

\section{5}

Cardiac Surgery for children from developing countries: program evaluation and outcomes

Michael Weidenbach, Heart Center Leipzig, Germany

Rodney Franklin, Royal Brompton \& Harefield NHS Trust,

United Kingdom

Etsegenet Gedlu, Addis Ababa University, Ethiopia

Cornelia Woerner, Heart Center Leipzig, Germany

Jan Janousek, Heart Center Leipzig, Germany

Martin Kostelka, Heart Center Leipzig, Germany

Martin Kostelka, Heart Center Leipzig, Germany

Objectives: To evaluate the short term results of a single center program (Leipzig, Germany) for treating children since 2001 with cardiac diseases from developing countries. Treatment was offered without cost, was mediated through UK based charity Chain of Hope as part of a multifaceted approach to developing self sustaining local practice and included pediatric cardiology training (EG).

Methods: Retrospective chart review. Follow-up data gathered from referring institutions by email.

Results: 53 children referred for surgery (mean age 7.8 years). Predominant diagnoses were Tetralogy of Fallot (28\%), VSD/ AVSD (26\%), ASD (13\%) and complex lesions (9\%), 17\% had diagnostic modifications. 2 children, with complex lesions incorrectly diagnosed, were inoperable (fixed pulmonary hypertension, complex pulmonary atresia). 47 children underwent surgery, 4 transcatheter interventions and no early deaths. Followup data was available for 32 patients (62\%; mean follow-up 2.2 years): functional status NYHA I in 29, II in 2 and 1 late sudden death 5 years after surgery (subaortic stenosis with LV dysfunction). The program did not adversely effect running the 'routine' pediatric cardiac program, financially or logistically.

Conclusions: Cardiac surgery can be offered to children from developing countries with excellent short term results. Patient selection is of utmost importance: lesions should be 'curable' without the foreseeable need for further interventions, but severe enough to have a major impact on morbidity and mortality if untreated. Close co-operation between local referring centers and international organizations is vital to ensure correct prereferral diagnosis, safe transportation and long term outcomes of these often severely disabled children.

\section{6}

Difficulties and possible solutions in establishing pediatric cardiac care in a developing country

Masood Sadiq, The Children's Hospital \& Institute of Child Health, Lahore, Pakistan, Pakistan

Asim Khan, The Children's Hospital \& Institute of Child Health, Lahore, Pakistan, Pakistan

Background: Establishment of pediatric cardiac services in developing countries is a major challenge. Once established, it is even bigger a challenge to cater for the needs of very large populations. In Pakistan with population of 16 millions, about 4000 babies require urgent open heart surgery each year.

Objectives: To analyze difficulties and propose solutions, in establishing a pediatric cardiac program.

Methods: The Children's Hospital Lahore is the only public sector children's hospital in the country where we established a congenital cardiac program and neonatal interventions and open-heart surgeries are being performed. It is a multidisciplinary hospital, with all the support facilities. In a developing country, there are numerous challenges like paucity of centers and personnel, limited resources, overcrowding, illiteracy, and infection control.

Results: Pediatric cardiac program at $\mathrm{CH}$ started in 1999. The problems faced include recruitment of trained staff and equipment and lack of resources for ongoing financial needs. So far we have performed surgery on $>1500$ children, with an overall mortality of $7 \%$ in the year 2008 . The cost for an open heart surgery is about US $\$ 1200$ and for a closed heart surgery is US $\$ 250$. This is still too much for a poor patient. The current waiting list for routine open heart surgery is over 3 years.

Conclusions: Despite improved infrastructure and skills, the logistics, affordability, staffing, late presentation, nutritional status, infection, and unfavorable economics continue to negatively influence the overall results. With continued determination and philanthropic support, establishment of pediatric cardiac program is still possible.

\section{0}

Outcomes for the Fetus with Hypoplastic Left Heart Syndrome (HLHS): A Stratification Scheme for Prenatal Counseling

Jack Rychik, Fetal Heart Program, The Children's Hospital of Philadelphia, United States

Zhiyun Tian, Fetal Heart Program, The Children's Hospital of

Philadelphia, United States

Anita Szwast, Fetal Heart Program, The Children's Hospital of

Philadelphia, United States

Peter Gruber, Fetal Heart Program, The Children's Hospital of

Philadelphia, United States

J William Gaynor, Fetal Heart Program, The Children's Hospital of

Philadelphia, United States

Thomas Spray, Fetal Heart Program, The Children's Hospital of Philadelphia, United States

Objective: HLHS remains one of the most challenging forms of congenital heart disease to treat. Advances in fetal imaging have increased the frequency of prenatal identification of HLHS. Accurate prenatal counseling depends upon knowledge of the most current outcome statistics. We report on early outcomes for the fetus with HLHS, based on a large, single-institutional experience.

Methods: Fetuses with HLHS presenting between 7/04-7/08 were reviewed. Survival is defined as alive at discharge following Norwood operation. Risk factors analyzed were: presence of extracardiac or genetic/chromosomal anomaly (type I), cardiac factors such as intact atrial septum, severe valve regurgitation or ventricular dysfunction (type II) or prematurity $<34$ wks (type III). Subjects are stratified based on "high-risk" = presence of any risk factors, or "standard-risk" = absence of risk factors. Results: 210 fetuses were evaluated; 142 (68\%) standard-risk and 68 (32\%) high-risk. Risk factors were distributed as follows: 39 (19\%) type 1, 28 (13\%) type II, 7 (3\%) type III; 6 fetuses had $>1$ risk factor. Outcome from point of fetal diagnosis is shown in table. For all fetuses treated, survival was $84 \%$; standard-risk had significantly better survival outcome than high-risk fetuses $(93 \%$ vs $57 \%$, $\mathrm{p}<0.0001)$. Anatomical subtype of HLHS did not relate to survival. 
Conclusions: Early survival for the fetus with HLHS, with no additional anomalies and born at full term, is excellent in the current era. Our study provides for a means of stratifying outcome for the fetus with HLHS and has important implications for prenatal counseling.

\begin{tabular}{|c|c|c|c|c|c|c|c|}
\hline & Total & $\begin{array}{l}\text { Term- } \\
\text { inated }\end{array}$ & $\begin{array}{l}\text { No } \\
\text { Interven- } \\
\text { tion }\end{array}$ & $\begin{array}{l}\text { Fetal } \\
\text { Demise }\end{array}$ & $\begin{array}{l}\text { Trans- } \\
\text { plant }\end{array}$ & $\begin{array}{l}\text { Norwood } \\
\text { Candidate }\end{array}$ & Survivors \\
\hline High-Risk & 68 & 9 & 11 & 1 & 3 & 44 & $25(57 \%)$ \\
\hline $\begin{array}{l}\text { Standard- } \\
\text { Risk }\end{array}$ & 142 & 15 & 4 & 0 & 0 & 123 & $\begin{array}{l}115 \\
(93 \%)\end{array}$ \\
\hline
\end{tabular}

\section{1}

Fetal aortic valvuloplasty may reverse hydrops and enable postnatal biventricular circulation in selected fetuses with critical aortic stenosis

Gerald Tulzer, Children's Heart Centre Linz, Austria

Wolfgang Arzt, Maternity and Children's Hospital Linz, Institute of

Prenatal Medicine, Austria

Second trimester fetuses with critical aortic stenosis (AS), dilated left ventricles (LV) with endocardial fibroelastosis and reverse aortic arch flow will have a postnatal univentricular circulation. Since December 2001 we have attempted 15 fetal aortic valvuloplasties in 14 fetuses $(23+4$ to $32+5$ weeks) with these criteria. In 3 fetuses impaired right ventricular filling had resulted in advanced end-stage heart failure with hydrops. Percutaneous ultrasound guided valvuloplasty was technical successful in 9/15 procedures $(60 \%)$ or $9 / 14$ patients $(64 \%)$. Overall mortality was $3 / 15(20 \%)$, in the last 13 procedures $7,7 \%$. Of the 5 patients with no technical success 2 died in-utero and 3 ended up as hypoplastic left heart syndrome. Of the 9 fetuses with technical success $7(78 \%)$ patients now have a biventricular circulation after birth, 1 had a sudden IUD, 1 received a hybrid stage I. All of the 7 biventricular patients had to undergo another aortic valve dilation in the first week of life. In 4 of them this was the only procedure so far with a follow-up of 1,2, 24 and 29 months respectively. One child needed a coarctation repair, 3 underwent a Ross-Konno operation between 1 and 3 months of age, 1 of them had the mitral valve replaced at the age of 6 months. All 3 fetuses with hydrops had a successful procedure and hydrops resolved within 3-5 weeks.

In selected fetuses with critical AS in-utero valvuloplasty has the potential to reverse end-stage heart failure and to save the LV for a postnatal biventricular circulation.

\section{2}

Mid-term Result of Surgical Repair in Patients with Double Outlet Right Ventricle

Eun Young Choi, Sejong General Hospital, Korea, Democratic People's Rep Hong Gook Lim, Sejong General Hospital, Korea, Democratic People's Rep So Ick Jang, Sejong General Hospital, Korea, Democratic People's Rep Soo Jin Kim, Sejong general Hospital, Korea

Jin Young Song, Sejong General Hospital, Korea, Democratic People's Rep Woo Sub Shim, Sejong General Hospital, Korea, Democratic People's Rep Chang Ha Lee, Sejong General Hospital, Korea, Democratic People's Rep Cheul Lee, Sejong General Hospital, Korea, Democratic People's Rep

Objective: The aim of this study was to review characteristics and surgical result of double outlet right ventricle (DORV) and to assess risk factors for reoperation.
Methods: Between January 1995 and August 2008, 262 patients underwent repair of DORV. We reviewed their medical record retrospectively.

Results: 145 patients (55.3\%) were male and mean follow up period was 78.1 months (1 day 248.6 months). Patients were divided into four groups by their hemodynamic characteristics. Tetralogy of Fallot group was 96, ventricular septal defect (VSD) group was 49, transposion of great arteries group was 19 and complex group included 98 patients. Biventricular repair performed in 148 patients. Five-year survival rate was $95.6 \%$. There were 7 early and 3 late mortalities. Repaired mean age was 4.6 years in single ventricular repair group (SV group) and 3.4 years in biventricular repair group (BV group). 51 reoperations were done in 36 patients. In BV group, main causes of reoperation were conduit failure, severe pulmonary regurgitation, subaortic stenosis and residual VSD leakage. In SV group, they were pulmonary arteriovenous fistula, conduit failure and complete atrioventricular block. Freedom from reoperation at 5 and 10 years were $95.6 \%, 84.3 \%$ in SV group and $82.5 \%, 70.6 \%$ in $\mathrm{BV}$ group.

Conclusions: In spite of various features of DORV, their survival rate is very good but frequent reoperation is needed due to deformed ventricular out tracts. We conclude that single ventricular repair is the next best thing and more study about long term complications of single ventricular repair is needed.

\section{3}

Ross-Konno Procedure in Children - Mid-term Results

Viktor Hraska, German Pediatric Heart Centre, Asklepios Clinic Sankt Augustin, Germany, Germany

Christian Lillie, Eppendorf University Hamburg, Germany, Germany Andrea Kantorova, Pediatric Cardiac Center, Bratislava, Slovakia, Slovak Republic

Joachim Photiadis, German Pediatric Heart Centre, Asklepios Clinic

Sankt Augustin, Germany, Germany

Christo Fink, German Pediatric Heart Centre, Asklepios Clinic Sankt Augustin, Germany, Germany

Boulos Asfour, German Pediatric Heart Centre, Asklepios Clinic Sankt Augustin, Germany, Germany

Francois Lacour-Gayet, Denver Children's Hospital, USA,

United States

Christo Haun, German Pediatric Heart Centre, Asklepios Clinic Sankt Augustin, Germany, Germany

Objective: The aim of this study was to analyze mid-term results of Ross-Konno procedure in children.

Methods: Between 1997 and 2008, 27 patients (pts) with complex left ventricular outflow tract (LVOT) obstruction underwent Ross-Konno procedure. There were 11 (41\%) infants (group A) and 16 older pts (Group B). Median age at operation was 3.3 years (from 6 days to 16 years).

Results: At seven years of follow up survival was $96 \%$ (one late death) with no differences between groups A \& B. Freedom from AR $>$ mild was $80 \%$ with no differences between groups A \& B. No gradient on LVOT was noticed. Freedom from MR $>$ mild was $100 \%$ for group $\mathrm{B}$ and $67 \%$ for group A (P=0.007). MR was associated with development of endocardial fibroelastosis (EFE) after failed intervention during newborn period. Freedom from redo was $80 \%$ for group $\mathrm{B}$ and $25 \%$ for group $\mathrm{A}(\mathrm{P}=0.009)$. There has been a significant $(\mathrm{P}=0.07)$ reduction and normalization of $\mathrm{LV}$ mass index within the first year of follow up. $46 \%$ of pts are without medication. All pts are in sinus rhythm and one pt is on anticoagulation therapy because of mitral valve replacement.

Conclusions: With the technical aspects of this procedure well accomplished, mortality is low and functional outcome is 
encouraging. High incidence of $\mathrm{MR}$ in infants associated with development of EFE is worrisome. Earlier indication of RossKonno operation in infants might prevent development of EFE, thus preserving mitral valve function.

\section{5}

Transposition of the Great Arteries with Ventricular Septal Defect and Pulmonary Stenosis: Is the Rastelli Operation Still a Good Option?

John W Brown, Indiana University School of Medicine, Indianapolis, Indiana, United States

Mark Ruzmetov, Indiana University School of Medicine, Indianapolis, Indiana, United States

Daniel Huynh, St.Louis University School of Medicine, St.Louis, MO, United States

Mark D Rodefeld, Indiana University School of Medicine, Indianapolis, Indiana, United States

Mark W Turrentine, Indiana University School of Medicine,

Indianapolis, Indiana, United States

Andrew C Fiore, St.Louis University School of Medicine, St.Louis, MO, United States

Objectives: The optimal surgical treatment of patients with transposition of the great arteries (TGA), ventricular septal defect (VSD) and pulmonary stenosis is controversial. Although the Rastelli operation (RO) has been standard surgical management of this lesion, aortic root translocation with right ventricular outflow tract (RVOT) reconstruction is a recent surgical alternative. This report reviews our 20-years experience with the RO.

Methods: Between 1988 and 2008, 40 patients (median age, 4years) underwent $\mathrm{RO}$ at our institutions. Follow-up was available for all patients (mean follow-up, $8.6+5.6$ years). The RVOT was reconstructed with homograft $(n=25)$, bovine jugular vein $(n=8)$, nonvalved Gore-Tex tube $(\mathrm{n}=5)$, or a porcine valved conduit $(n=2)$. Three patients required a pacemaker.

Results: There was one early and two late deaths and one heart transplantation 12 years postoperative RO. Kaplan-Meier survival was $93 \%$ at 5,10, and 20 years. Univariate risk factors for death or transplantation included surgery before $1998(\mathrm{P}=0.03)$ and concomitant non-cardiac anomalies $(\mathrm{P}=0.001)$. Sixteen patients (40\%) had reoperation for RVOT conduit stenosis (mean; $7.8+3.8$ years) without mortality. Freedom from conduit replacement was $86 \%, 74 \%, 63 \%$, and $59 \%$ at $5,10,15$, and 20 years,respectively. Multivariate analysis revealed that the risks factors of conduit replacement were younger age at operation $(\mathrm{p}=0.001)$ and surgery before $1998(\mathrm{P}<0.001)$. One patient $(2.5 \%)$ required reoperation for left ventricular outflow tract obstruction (LVOTO).

Conclusion: The RO is a low-risk operation with regard to early and late mortality and reoperation for LVOTO. Conduit change operations will be required in all patients, but currently can be performed with low morbidity and mortality. These mid-term outcomes after the RO should serve as a basis for comparison with surgical alternatives more recently introduced for TGA, VSD with RVOTO.

\section{6}

Surgical treatment of the transposition of the great arteries with pulmonary stenosis: Mid-term results Katrin Weyand, Germany

Christoph Haun, German Pediatric Heart Centre, Sankt Augustin, Germany
Hedwig-Christine Blaschczok, German Pediatric Heart Centre, Sankt Augustin, Germany

Martin Schneider, German Pediatric Heart Centre, Sankt Augustin, Germany

Nicole Toussaint-Goetz, German Pediatric Heart Centre,

Sankt Augustin, Germany

Boulos Asfour, German Pediatric Heart Centre, Sankt Augustin, Germany

Nicodeme Sinzobahamvya, German Pediatric Heart Centre,

Sankt Augustin, Germany

Viktor Hraska, German Pediatric Heart Centre, Sankt Augustin, Germany

Objective: The purpose of this study was to evaluate midterm outcome of different surgical approaches for the treatment of the transposition of the great arteries with pulmonary stenosis (TGA + PS).

Methods: Between 1991 and 2008, 41 patients (pts) with well developed ventricles and TGA + PS were operated on. 23 pts had Rastelli operation, 8 pts underwent ASO with LVOTO surgery, 4 pts had REV procedure and Nikaidoh procedure was performed in 2 pts. In 4 pts a total cavopulmonary anastomosis (TCPC) was performed because of unfavorable intracardiac anatomy. The median age at final operation was 1.7 years (0.03-19.3).

Results: Overall survival rate was $97 \%$ (one early death after Rastelli op.) with the median length of FU 8.2 years. There were no differences in survival among the surgical groups. Event free interval was $100 \%, 83 \%$ and $41 \%$ at 1,5 and 10 years of FU respectively, being significantly worse for Rastelli group $(\mathrm{P}<0.047)$. On the last ECHO, all pts but one showed a good function of systemic ventricle, LVOTO $>30 \mathrm{~mm} \mathrm{Hg}$ was noticed in 2 pts $(5 \%)$ and RVOTO $>30 \mathrm{~mm} \mathrm{Hg}$ was detected in 11 pts $(27 \%)$. All pts are in sinus rhythm and $80 \%$ of them are without medication.

Conclusions: All surgical approaches are safe with excellent midterm functional outcome. The Rastelli operation suffers by a high reintervention rate; therefore alternative surgical methods are preferable. Nikaidoh operation has a potential to minimize utilization of TCPC used in the past for unfavorable intracardiac anatomy.

3087

Does the Mitral Annulus Grow in Children Following Early Mechanical Valve Replacement?

Lucian A Durham, The Mayo Clinic, Rochester, Minnesota, USA, United States

Harold M Burkhart, The Mayo Clinic, Rochester, Minnesota, USA, United States

Joseph A Dearani, The Mayo Clinic, United States

Francisco J Puga, The Mayo Clinic, Rochester, Minnesota, USA, United States

Patrick W O'Leary, The Mayo Clinic, Rochester, Minnesota, USA, United States

Hartzell V Schaff, The Mayo Clinic, Rochester, Minnesota, USA, United States

Introduction: Irreparable mitral pathology may lead to early mitral valve replacement (MVR) in children. Often, a small mechanical prosthesis $(<23 \mathrm{~mm})$ is required raising concerns for annular growth in patients who may require mitral valve re-replacement (MVRR). The aim of this study was to evaluate interval annular growth in this cohort.

Methods: Between 1972 and 2006, 164 children underwent MVR; 102 of these children (median age: 4 yrs., range: 
7 days -11 yrs.) required a small mechanical prosthesis $(<23 \mathrm{~mm})$. The most common diagnoses were congenital mitral stenosis/ regurgitation (48\%), AVSD (46\%), and other (6\%). This cohort was analyzed for age, body surface area (BSA), prosthesis size, annular size and Z-score at time of MVR and MVRR.

Results: At the time of MVR, 78 patients had a BSA of $0.77+0.06 \mathrm{~m} 2$, annular size $24+0.62 \mathrm{~mm}(Z$-score $2.91+0.23)$ and ultimately did not require MVRR. The cohort requiring MVRR $(\mathrm{n}=24)$ had BSA $0.62+0.05 \mathrm{~m} 2(\mathrm{p}=\mathrm{NS}$ vs. MVR only), annular size $20+0.49 \mathrm{~mm}$ (Z-score $1.85+0.22$ ) ( $p=0.008$ vs. MVR only) at time of initial implant. In the interval between MVR and MVRR $(7.8+1.1 \mathrm{yrs})$, BSA increased to $1.12+0.07 \mathrm{~m} 2$ and annulus size increased to $24+0.47 \mathrm{~mm}$ (Z-score $1.80+0.28)$.

Conclusions: The data support somatic growth of the mitral annulus following MVR with small mechanical prosthesis as evidenced by normal interval increase in BSA with unchanged Z-score. Additionally, there was a statistically significant difference in Z-scores between the cohorts requiring MVRR and those not needing re-replacement suggesting that placement of a slightly larger prosthesis may decrease the need for MVRR.

\section{8 \\ Relationship between Administration of Intravenous Immune-Globulin and Cardiac/Coronary Artery Leision in patients with Kawasaki Disease \\ Guo-Ying Huang, Children's Hospital of Fudan University, Shanghai, China \\ Li-Jian Xie, Children's Hospital of Jiaotong University, Shanghai, China Chen-Yan Yu, Children's Hospital of Fudan University, Shanghai, China Min Huang, Children's Hospital of Jiaotong University, Shanghai, China Shu-bao Chen, Shanghai Children's Medical Center, Shanghai, China Mei-rong Huang, Shanghai Children's Medical Center, Shanghai, China Rong-fa Wang, Xinhua Hospital of Jiaotong University, Shanghai, China Xiao-Jing Ma, Children's Hospital of Fudan University, Shanghai, China}

Objective: To evaluate the effectiveness of intravenous immunoglobulin (IVIG) in treating and preventing cardiac consequences (coronary artery lesion, CAL) of patients with Kawasaki disease (KD). Method A questionnaire form and guideline for KD were sent to 50 hospitals in Shanghai, which provided with pediatric medical care. 1682 KD patients were recoeded from January 1998 through December 2007, which included 1064 males and 618 females. The median age of KD patients was $2.57 \pm 2.33$ years old $(0.1 \sim 18.8$ years $)$. Six therapy schemes of IVIG were administered including $1 \mathrm{~g} / \mathrm{kg} \times$ once, $2 \mathrm{~g} / \mathrm{kg} \times$ once, $0.4 \sim 0.5 \mathrm{~g} / \mathrm{kg} \times$ five times, $1 \mathrm{~g} / \mathrm{kg} \times$ twice, $2 \mathrm{~g} / \mathrm{kg} \times$ twice and other. Results Among all, the lower incidence of CAL complications $(16.8 \%)$ was shown in patients with IVIG administration during $5 \sim 10$ day of illness. Lower incidence of CAL complications $(16.9 \%)$ was shown in patients with IVIG administration of $1 \mathrm{~g} / \mathrm{kg} \times$ twice. Conclusion The best therapy of IVIG in KD patients is $1 \mathrm{~g} / \mathrm{kg} \times$ twice and it should be used in $5 \sim 10$ day of KD illness.

\section{9}

Endothelial progenitor cells and arterial function in the late convalescence period of Kawasaki disease

Xiao-qin Liu, Pediatric Heart Center, Children's Hospital of Fudan University, China

Aim: To investigate endothelial progenitor cells level and ultrasound measurements of arterial function: flow-mediated dilation and carotid artery stiffness index in the convalescence of Kawasaki disease.
Methods: 63 children were studied, divided into coronary artery lesion group (Group1, $\mathrm{n}=21$ ), non-coronary artery lesion group (Group2, n = 20), and control group (Group3, n =22). Endothelial progenitor cells count and arterial function were measured by flow cytometry analysis and by ultrasound respectively.

Results: Flow-mediated dilation in three groups were $4.5 \% \pm 1.5 \%, 9.5 \% \pm 2.8 \%$ and $12.1 \% \pm 2.3 \%$ (Group 1 vs Group 2, Group 1 vs Group 3, Group 2 vs Group 3, all $\mathrm{p}<0.01$ ), Carotid artery stiffness index were $4.10 \pm 0.44$, $3.81 \pm 0.50$ and $3.59 \pm 0.46$ (Group 1 vs Group 2, p $<0.05$; Group 1 vs Group 3, p $<0.01$; Group 2 vs Group 3, p = 0.142) and the number of endothelial progenitor cells were $2.0 \pm 0.6 / \mathrm{ul}$, $4.2 \pm 0.8 / \mathrm{ul}, \quad 4.5 \pm 0.7 / \mathrm{ul}$ (Group1 vs Group2, $\mathrm{p}<0.01$; Group1 vs Group3, $p<0.01$; Group2 vs Group3, $p=0.292)$. Multiple linear regressions identified flow-mediated dilation and carotid artery stiffness index as significant determinants of endothelial progenitor cells level. Endothelial progenitor cells level had significant correlations with flow-mediated dilation and carotid artery stiffness index by Pearson correlation analysis.

Conclusion: Our findings suggest that systemic arterial dysfunction persist in the convalescence of Kawasaki disease. The level of endothelial progenitor cells could serve as a surrogate biologic measurement of vascular function.
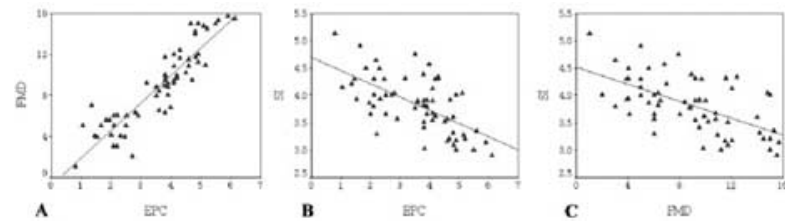

3090

In Vivo Plaque Composition and Morphology in Coronary Artery Lesions in The Adolescents and Young Adults Long After Kawasaki Disease: A Virtual Histology-Intravascular Ultrasound Study

Yoshihide Mitani, Mie University Graduate School of Medicine, Japan Hiroyuki Ohashi, Mie University Graduate School of Medicine, Japan Hirofumi Sawada, Mie University Graduate School of Medicine, Japan Yukiko Ikeyama, Mie University Graduate School of Medicine, Japan Hidetoshi Hayakawa, Mie University Graduate School of Medicine, Japan Yoshihiro Komada, Mie University Graduate School of Medicine, Japan

Background: Coronary artery lesions (CALs) late after Kawasaki disease (KD) were characterized by endothelial dysfunction and low-grade inflammation, surrogate markers for atherosclerosis. We tested the hypothesis that CALs in patients long after KD are accompanied by atheroma-like features, as assessed by virtual histology-intravascular ultrasound (VH-IVUS), a new method to assess coronary plaque composition and morphology in vivo. Methods and Results: VH-IVUS was performed in 13 Japanese KD patients (age: $19 \mathrm{y} 3 \mathrm{~m}$ (mean) $\pm 4 \mathrm{y} 6 \mathrm{~m}$ (SD); an interval after KD: $17 \mathrm{y} 4 \mathrm{~m} \pm 5 \mathrm{y} 0 \mathrm{~m})$. We investigated 6 sites with localized stenosis, 15 sites with an aneurysm, 29 sites with a regressed aneurysm, and 50 sites with a normal coronary segment. Plaque components were categorized into four parts: fibrous, fibrofatty, necrotic core (NC), and dense calcium (DC) areas. Qualitatively, the normal segment had no or trivial IVUS-visible plaque area, while the CAL exhibited a heterogenous plaque area with 4 components in different amounts and proportions. Quantitatively, a combined group of CALs had a higher absolute value of fibrous, DC, and NC areas than the normal segment $(\mathrm{p}<.05$, respectively). In further analyzing 3 subtypes of CALs, localized stenosis, an advanced lesion, exhibited higher absolute and relative values of DC and NC areas, 
and a lower relative value of the fibrous area than regressed and persistent aneurysms ( $p<.05$, respectively).

Conclusions: The present limited but initial VH-IVUS findings give a new insight into the potential role of atherogenesis in the evolution of CALs in the adolescents and young adults long after $\mathrm{KD}$, and therefore warrant further investigation.

\section{1}

Objective Measurement of Mitral Valve Thickness in Children with and without Rheumatic Heart Disease Rachel H Webb, Starship Children's Hospital, New Zealand Fiona Lean, Starship Children's Hospital, New Zealand Rachel Gatland, Starship Children's Hospital, New Zealand Sandy Long, Starship Children's Hospital, New Zealand Irene Zeng, Green Lane Cardiac Services, New Zealand Nigel J Wilson, Starship Children's Hospital, New Zealand

Objective: Mitral valve (MV) leaflet thickening is a well-recognised feature of rheumatic heart disease (RHD). Few publications describe methods for measuring MV thickness except in established mitral stenosis. We aimed to establish methodology for, and define ranges of, MV thickness in children with and without RHD. This has implications for RHD screening.

Methods: Prospective screening study conducted in a high risk population for RHD. Standard 2-dimensional and colourDoppler echocardiography on students aged 10-13 years using portable Vivid E machines (GE Healthcare) was performed. Anterior mitral valve leaflet (AMVL) thickness was measured in mid-diastole at the tip and the mid-point (parasternal) and the mid-point in systole (apical). Posterior mitral valve leaflet (PMVL) mid-point thickness was measured in mid-diastole (parasternal). Measurements from 280 students with normal screening echocardiograms were compared to 55 students with RHD according to modified WHO criteria. 28 with RHD had definite morphological changes of the mitral valve and 26 had mitral regurgitation without definite morphological changes.

\begin{tabular}{llllll}
\hline $\begin{array}{l}\text { Variable } \\
(\mathrm{mm})\end{array}$ & \multicolumn{2}{l}{ Normal $(\mathrm{N}=280)$} & $\mathrm{RHD}(\mathrm{N}=54)$ & P Value \\
\cline { 2 - 5 } & Median & $\begin{array}{l}\text { Inter-quartile } \\
\text { range }\end{array}$ & Median & $\begin{array}{l}\text { Inter-quartile } \\
\text { range }\end{array}$ & \\
\hline AMVL mid & 2.02 & $1.74-2.35$ & 2.88 & $2.62-3.20$ & $<0.0001$ \\
AMVL tip & 2.02 & $1.70-2.40$ & 2.79 & $2.44-3.29$ & $<0.0001$ \\
PMVL mid & 2.20 & $1.89-2.56$ & 2.72 & $2.42-3.00$ & $<0.0001$ \\
\hline
\end{tabular}

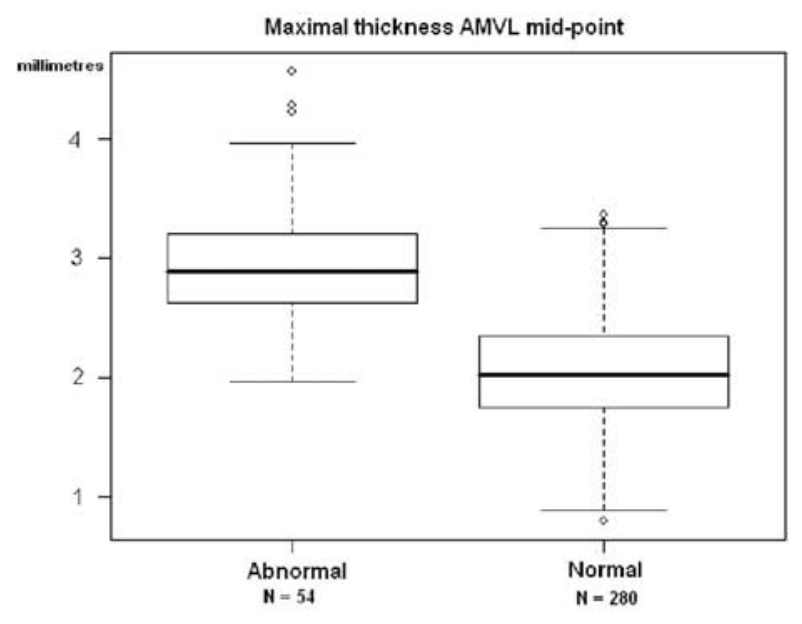

Results: There were statistically significant differences between the RHD group and normal children for AMVL and PMVL thickness (see graph). Measurements were reproducible with intra-class correlation co-efficients ranging between $0.76-0.90$. Conclusions:

1. We have developed an objective standardised method for measuring MV leaflet thickness.

2. Children with RHD had significantly thicker mitral valve leaflets than normal students.

3. Inter-observer comparison of the Vivid E measurements with other echocardiographic technology is needed.

3092

CRP-286 Gene Polymorphisms and Susceptibility to coronary arterial sequelae in Kawasaki Disease

Xiao-qin Liu, Center of cardiovascular disease, Children's Hospital of Fudan University, China

Objective: To investigate the correlation among single nucleotide polymorphisms (SNPs) of CRP gene -286 and susceptibility of $\mathrm{KD}$ in the late convalescence period of KD. Method: Genotyping were performed for the detection of SNP at positions -286 in $101 \mathrm{KD}$ patients and 173 healthy children. The serum hs-CRP levels were measured by enzyme-linked immunosorbent assay (ELISA). The correlations of SNPs of CRP gene and susceptibility of KD, serum hs-CRP levels in the late convalescence period of KD were analyzed. Results: (1) frequencies of genotypes of $\mathrm{A}$ and/or $\mathrm{T}$ at position -286 of CRP gene were $45.1 \%$ in CAL group and $28.9 \%$ in control group $(\chi 2=5.491$, $\mathrm{P}=0.019, \quad \mathrm{OR}=2.003,95 \% \mathrm{CI}, 1.116 \sim 3.594)$. The allele frequencies of the less common $\mathrm{A}+\mathrm{T}$ variant were $26.5 \%$ in $\mathrm{CAL}$ group and $14.7 \%$ in control group $(\chi 2=4.185, P=0.041$, $\mathrm{OR}=2.068,95 \% \mathrm{CI}, 1.023 \sim 4.180)$. (2) The serum of hs-CRP levels in CAL group, non-CAL group and control group were $3.42 \pm 1.66 \mathrm{mg} / 1,2.63 \pm 1.27 \mathrm{mg} / 1$ and $2.61 \pm 1.27 \mathrm{mg} / \mathrm{l}$, respectively $(\mathrm{P}<0.05, \mathrm{P}<0.01)$. (3) The serum of hs-CRP levels with CC, CA, CT, AT/AA/TT genotype at position -286 of $\mathrm{CRP}$ gene were $2.31 \pm 1.03 \mathrm{mg} / 1, \quad 3.55 \pm 1.59 \mathrm{mg} / \mathrm{l}$, $3.28 \pm 1.56 \mathrm{mg} / \mathrm{l}$ and $3.85 \pm 1.69 \mathrm{mg} / \mathrm{l}$, respectively $(\mathrm{P}<0.01)$. Conclusions: SNP at position -286 of CRP gene is associated with the susceptibility of KD. A and/or T alleles at position -286 of CRP gene might be regarded as genetic markers of risk factors for vascular lesions in late convalescence period of $\mathrm{KD}$.

\section{3}

Endothelial microparticles for assessment of vascular injury in Kawasaki disease

Suthep Wanitkun, Department of Pediatrics, Ramathibodi Hospital, Bangkok, Thailand, Thailand

Nongnuch Sirachainan, Department of Pediatrics, Ramathibodi Hospital, Bangkok, Thailand, Thailand

Kawasaki disease $(K D)$ is an arteritis syndrome affecting young children. Numerous reports demonstrated pathological and immunological mechanisms of vascular injury in KD. Endothelial microparticles (EMPs) are intact vesicles resulting from exocytic budding of activated or apoptotic endothelial cells in response to vascular injury. EMPs may play a role in diagnosis or monitoring vasculitis in KD.

Objective: We attempted to characterize the level of EMPs in acute $\mathrm{KD}$ patients with different clinical severity.

Methods: Blood samples from patients with Kawasaki disease were collected in acute phase just before the initiation of intravenous immunoglobulin (IVIG) therapy. Fresh heparinized venous blood 
were collected at room temperature and processed within 6 hours. The samples were incubated and immunolabelled with annexin V, CD105, and CD62E before flow cytometric immunophenotyping. Demographic data and other standard laboratory studies were also collected.

Results: There were 12 cases of acute KD with age ranging from 3-72 months. Comparing to age-match healthy infant, the EMPs level was high in KD patients in the range of $18-390 / \mathrm{mcL}$, especially higher in 3 IVIG-resistant cases. One patient with EMPs 99/mcL developed coronary artery ectasia. EMPs correlated well with clinical status and Kobayashi clinical severity scoring. However, ESR and CRP correlated poorly with EMPs.

Conclusion: Analysis of EMPs is a promising tool for assessing vascular injury in KD. The changes in the level of EMPs are more promptly reflecting the clinical status as compared to the conventional inflammatory markers.

\section{4}

Morphology of ventricular scoop in atrioventricular septal defect: relevance to "simplified" single-patch method Iki Adachi, Cardiac Morphology Unit, National Heart \& Lung Institute, Imperial College London, United Kingdom

Siew Yen Ho, Cardiac Morphology Unit, National Heart \& Lung Institute, Imperial College London, United Kingdom

Karen P McCarthy, Cardiac Morphology Unit, National Heart \& Lung Institute, Imperial College London, United Kingdom Hideki Uemura, Department of Cardio-Thoracic Surgery, Royal Brompton Hospital, United Kingdom

Background: The "simplified" single-patch repair for atrioventricular septal defect seems an attractive alternative to conventional methods despite controversies on its suitability in hearts with a large ventricular scoop. Since previous anatomical studies were conducted before the advent of this technique, we revisit this malformation with the aim to identify morphological markers that may aid patient selection.

Methods: We examined 43 heart specimens: 31 with complete form and 12 with partial form of the malformation.

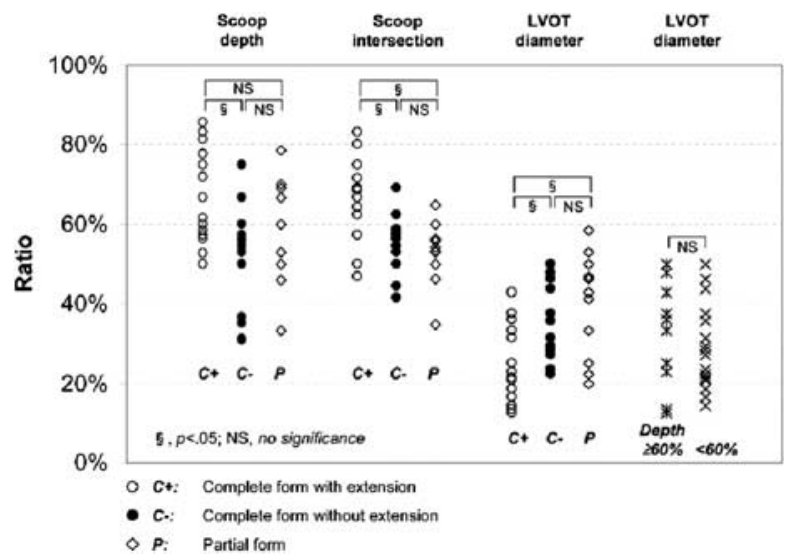

Results: In 16 hearts with complete form, the scoop extended antero-superiorly, beyond the atrioventricular junction, resulting in a skewed shape of the scoop. By contrast, none of the other hearts had such extension and the scoop was nearly symmetrical. Hearts with the extension had significantly narrower diameters of the left ventricular outflow tract [median (inter-quartile range); $22 \%(17 \%$ to $33 \%)]$ than complete form without the extension [38\% (29\% to $50 \%), \mathrm{p}=.01]$ and partial form [43\% (25\% to $50 \%)$, $\mathrm{p}=.01]$. However, when the diameters were stratified with scoop depth, no obvious difference was found between complete form with a deep scoop (defined as the depth of $\geqslant 60 \%$ ) and those with a shallower scoop [ $36 \%$ ( $24 \%$ to $49 \%$ ) vs $28 \%$ (21\% to $36 \%$ ), respectively, $\mathrm{p}=.146]$, indicating that antero-superior extension had more impact on the tract size than scoop depth.

Conclusions: The antero-superiorly extended and skewed scoop could lead to asymmetrical configuration of the valvar leaflets and outflow tract obstruction if the "simplified" technique is applied. Therefore, the antero-superior extension should require recognition when determining its suitability.

\section{5}

A study of Aortic Size in Cases with Bicuspid Aortic Valves Compared to Unicuspid or Tricuspid Ones Louise Calder, Starship Children's Hospital, Pediatric and Congenital Cardiology Department, New Zealand

Objective: Dilatation of the ascending aorta in adults with bicuspid aortic valves (BAV) is well recognized. Possible dilatation of the aorta in hearts with congenital heart defects and BAV compared to those with unicuspid (UAV) or tricuspid (TAV) ones was investigated. Methods: The aortas were measured at 6 sites in 89 cases with BAV, 35 with UAV and 66 with TAV. The TAV cases were selected to match the BAV ones with similar ages and diagnoses.

Results: The ages with BAV ranged from stillborn to 69 years, median 39 days, with TAV ranged from stillborn to 36 years, median 19 days (NS), and with UAV ranged from stillborn to 6 years, median 8 days. The deficient commissure in BAV cases was intercoronary in $62 \%$. The only commissure present with UAV was non coronary-left coronary in 75\%. Apart from smaller aortic valve size in UAV cases, all other measurements did not vary significantly between the 3 groups. The average ascending aortic diameters were 11.2 (7.5) in BAV compared to 9.3(6.4) in TAV ( $\mathrm{p}=0.05)$. The ascending aorta $\mathrm{Z}$ scores were -0.09 (0.61) in BAV cases, 0.13 $(0.58)$ in UAV and $-0.15(0.77)$ in TAV $(p=0.68$ and 0.16 comparing BAV or UAV with TAV respectively).

Conclusion: The aortas were not dilated in these cases with BAV. Further study is required to assess the histology of the aortas in different age groups.

\section{6 \\ Phenotypic features, cardiovascular manifestations and natural history in a unique cohort of infants with Arterial Tortuosity Syndrome \\ Mahesh Kappanayil, Amrita Institute of Medical Sciences and Research Center, Kochi, India, India \\ Gayathri Satish, Amrita Institute of Medical Sciences and Research Center, Kochi, India, India \\ Sheela Nampoothiri, Amrita Institute of Medical Sciences and Research Center, Kochi, India, India \\ Krishna Kumar, Amrita Institute of Medical Sciences and Research Center, Kochi, India, India}

Background: 'Arterial Tortuousity Syndrome (ATS)' is a rare autosomal recessive connective tissue disorder characterized by cutis laxa, elongation and tortuosity of large and medium arteries and typical phenotypic features. Many of the patients reported are older children and adults. Mutation of SCL2A10 gene (chromosome 20q13) is a proposed etiology.

Objectives: To describe phenotypic features, arterial manifestations and natural history of 5 infants diagnosed with cutaneous laxity and arterial tortuosity. 
Methods: Prospective, hospital-based, observational study conducted at a tertiary care hospital in India. Five consecutive patients with features of the syndrome were serially followed-up.

Results: All 5 patients presented in infancy and belonged to same geographical and ethno-religious background. Consanguinity was present in 2 families. Phenotypic features are depicted in Table, and figures 1,2 . All presented with respiratory symptoms, between 1-9 (average 3.5) months of age. Echocardiography and multislice CT revealed marked dilatation and tortuosity of ascending, descending and abdominal aorta, pulmonary arteries, and neck vessels, with narrowing at aortic isthmus, and biventricular hypertrophy in all the patients. Branch pulmonary artery stenosis was present in two. $3 / 5$ patients died in infancy (between 3.5-11 months) - terminal event being respiratory failure, which was accompanied by hemoptysis in one patient. Two surviving patients (10 and 14 months old) show significant progression of vascular dilatation with symptoms of resultant airway compression. SCL2A10 mutation has been ruledout in 3 patients.

\section{Common phenotypic features in patient cohort}

1. Cutis laxa (in all 5 patients)

2. Micrognathia (in 4 of 5 patients)

3. Hypertelorism (in 3 of 5 patients)

4. Downslanting palpebral fissures (in all 5 patients)

5. Generalized hypotonia (in all 5 patients)

6. Umbilical/inguinal herna (in 4 of 5 patients)

7. Gross-motor delay (in all 5 patients)

8. Prominent, visible arterial pulsations in the neck and axillary areas (in 3 of 5 patients)
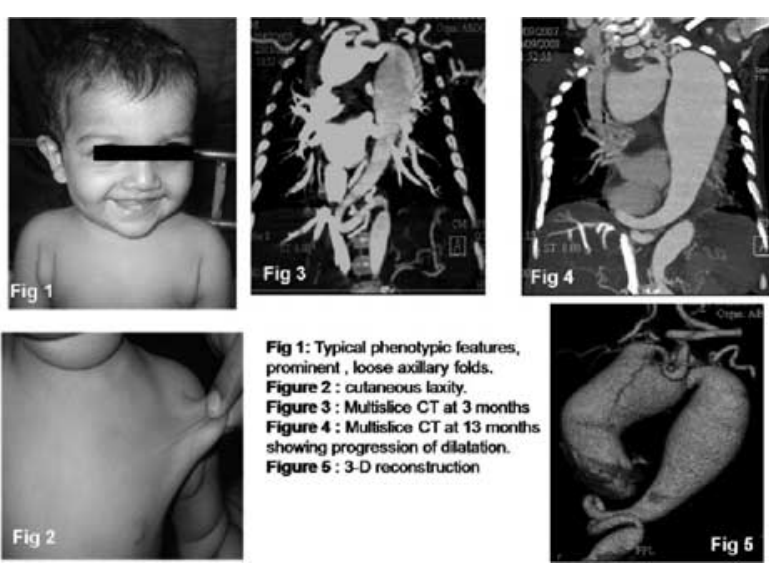

Conclusions: This cohort appears to be a unique, more lethal variant of ATS. Genetic studies are underway to identify the underlying mutation.

\section{7}

Clinical Screening for Congenital Heart Disease at Birth: A Prospective Study in a Community Hospital in

\section{Southern India}

Balu Vaidyanathan, Amrita Institute of Medical Sciences, Elamakkara P.O, Kochi, Kerala, India, India

$S$ Gayathri, Amrita Institute of Medical Sciences, Elamakkara P.O,

Kochi, Kerala, India, India

TM Sinimol, Amrita Institute of Medical Sciences, Elamakkara P.O, Kochi, Kerala, India, India

KR Sundaram, Amrita Institute of Medical Sciences, Elamakkara P.O,

Kochi, Kerala, India, India
KRR Warrier, Lakshmi Hospital, Diwan's road, Kochi, Kerala, India, India

Kumar Krishna R, Amrita Institute of Medical Sciences, Elamakkara P.O, Kochi, Kerala, India, India

Background: A large proportion of neonates born with congenital heart disease (CHD) are missed at birth in Indian hospitals. Objective: To examine the feasibility of development of a clinical strategy for detection of congenital heart disease (CHD) in the newborn through a combination of clinical signs and pulse oxymetry that best predicts the presence of CHD.

Methods: All consecutive newborns born in a secondary level hospital between June 2006 and June 2008 were prospectively screened for CHD 48 hours after birth. The on-site pediatrician did the clinical screening. A study nurse recorded pulse oxymetry. Echocardiography was performed on site in all newborns. A 6-week clinical follow-up evaluation was done for all.

Results and analysis: Of 4190 babies screened, 325 had CHD (prevalence: $7.7 \%)$. Nine $(0.21 \%$ ) had major CHD, two of whom (one ALCAPA and one large VSD) were missed during the initial evaluation; four of these had a normal clinical evaluation and only one had abnormal oxymetry. Univariate predictors of CHD included murmur, central cyanosis, precordial pulsation, and respiratory rate $>60 /$ minute. On multivariate analysis, murmur $(\mathrm{OR}=5.8 ; 95 \%$ C.I $3.44-9.76)$ and respiratory rate $>60 / \mathrm{min}(\mathrm{OR}=2.54 ; 95 \%$ C.I $1.0-6.47)$ were associated with presence of CHD. Pulse oxymetry did not appear to contribute to the detection of CHD. Conclusions: Clinical evaluation immediately after birth has a very low sensitivity for CHD detection in the newborns. Presence of murmur in a newborn warrants referral for echocardiography. A 6-week clinical evaluation is recommended to ensure that major CHD does not go undetected.

\section{8}

Mean platelet volume is a predictor of 22q11.2 deletion in congenital heart disease

Nitha Naqvi, Royal Brompton and Harefield Hospital NHS Trust, United Kingdom

Simon Davidson, Royal Brompton and Harefield Hospital NHS Trust, United Kingdom

Davina Wong, Royal Brompton and Harefield Hospital NHS Trust, United Kingdom

Paul Cullinan, National Heart and Lung Institute, Imperial College, United Kingdom

Rodney Franklin, Royal Brompton and Harefield Hospital NHS Trust, United Kingdom

Piers EF Daubeny, Royal Brompton and Harefield Hospital NHS Trust, United Kingdom

Objective: To investigate whether mean platelet volume may be a useful rapid indicator of the presence of $22 \mathrm{q} 11.2$ deletion.

Method: Design and setting: Retrospective analysis of mean platelet volume and 22q11.2 deletion status in a paediatric population undergoing cardiac surgery at the Royal Brompton Hospital, between August 1999 and June 2005. Genetic testing was performed in 166 children ( $<16$ years).

Main outcome measures: 22q11.2 deletion status and mean platelet volume. A mean platelet volume of $>10 \mathrm{fL}$ was considered abnormal.

Results: Twenty one children were $22 \mathrm{q} 11$ positive. The mean platelet volume was significantly larger $10.9 \mathrm{fL}$ for the $22 \mathrm{q} 11.2$ positive patient group compared to $8.6 \mathrm{fL}$ in the non-22q11.2 patients $(\mathrm{p}<0.001)$. Platelet counts were significantly lower in the 22q11.2 patient group (median $155 \times 109 / \mathrm{L}$ v $225 \times 109 / \mathrm{L}, \mathrm{p}=0.008)$. The area under 
the curve of the receiver operating characteristics curve of mean platelet volume was large enough (0.85) to enable the accurate prediction of 22q11.2 deletion using mean platelet volume.

Conclusion: Mean platelet volume $>10 \mathrm{fL}$ is a positive predictor of the presence of 22q11.2 deletion in children with congenital heart disease. This finding should aid rapid decision-making for ordering irradiated blood products to prevent potentially fatal transfusion-associated graft versus host disease and to alert clinicians to monitor serum calcium levels closely to prevent hypocalcaemic seizures.

\section{2}

The proposed founder mutation Y111C-KCNQ1- a substantial cause of LQT1 in Sweden

Annika Winbo, Dept of Clinical Sciences, Umeå University Hospital, Sweden

Ulla-Britt Diamant, Dept of Public Health and Clinical Medicine, Umeå University Hospital, Sweden

Steen M Jensen, Dept of Public Health and Clinical Medicine, Umeå University Hospital, Sweden

Annika Rydberg, Dept of Clinical Sciences, Umeå University Hospital, Sweden

Eva-Lena Stattin, Dept of Medical Biosciences, Umeå University

Hospital, Sweden

Objective: The long QT syndrome (LQTS) is a known cause of sudden death in young individuals. In Scandinavia, LQT founder mutations explain $73 \%$ of LQTS in Finland, while in Norway the mutational spectrum is diverse without founder mutations. This study investigates the occurrence of the Y111C-KCNQ1 mutation in the Swedish population, where the LQTS mutational spectrum is previously unknown.

Methods and Results: In Sweden we have, so far, identified 122 carriers of a LQT1 mutation (Y111C-KCNQ1) in 27 index families. Presently, Y111C constitutes almost a third of all identified LQTS mutations in probands analyzed at the Department of Clinical Genetics, Umeå University Hospital, Sweden.

The founder mutation concept is based on genealogical, geographical and genetic evidence. A common founder for 9 of the families, a woman who married twice, born in 1694 in northern Sweden has been identified. Interconnections between several other index families are being established. Ancestors of 19 probands originate from the same geographic region.

In March 2009, investigations to determine the age of the mutation is planned, and analysis of microsatellite markers will provide formal evidence as to whether the Y111C mutation is the first Swedish LQT founder mutation.

Conclusions: The Y111C mutation, the first Swedish LQT founder mutation by genealogical and geographical evidence, is a substantial cause of LQTS in Sweden. Genetic analysis will conclusively reveal whether the occurrence of the Y111C mutation in the Swedish population is "hot spot" or a founder effect.

\section{3}

Stress Echocardiography Using Semi-Supine Cycle Ergometry in Patients with Pediatric Heart Disease. Nearly 700 Tests Later!

George GS Sandor, Division of Cardiology, Department of Pediatrics, British Columbia Children's Hospital and The University of British Columbia, Canada

Astrid M De Souza, Division of Cardiology, Department of Pediatrics, British Columbia Children's Hospital and The University of British Columbia, Canada
Eustace S De Souza, Division of Cardiology, Department of Pediatrics, British Columbia Children's Hospital and The University of British Columbia

Mary T Potts, Division of Cardiology, Department of Pediatrics, British Columbia Children's Hospital and The University of British Columbia, Canada

Brian G Sinclair, Division of Cardiology, Department of Pediatrics, British Columbia Children's Hospital and The University of British Columbia, Canada

James E Potts, Division of Cardiology, Department of Pediatrics, British Columbia Children's Hospital and The University of British Columbia, Canada

Background: Stress testing poses problems in clinical practice in pediatrics: treadmill testing does not permit measuring most physiological parameters during staged-exercise; and pharmacologic testing is non-physiological. Performing echocardiography and Doppler with metabolic measurements during exercise overcomes these limitations.

Methods: Subjects exercised on a semi-recumbent cycle ergometer using a 3-minute step protocol of 20-40 watts at 60-70 rpm until volitional fatigue. Measurements were made at rest, during each stage of exercise, and post-exercise. The protocol was adapted to assess function in patients with special forms of congenital heart disease such as those with univentricular hearts or systemic right ventricles. Testing was not done routinely in children less than 8 years of age. RESULTS: Since 1997 we have performed 660 stress tests on 427 pediatric patients and 28 healthy controls. We have defined a normal response to exercise as an increase in heart rate $>70 \mathrm{bpm}$, systolic blood pressure $>50 \mathrm{mmHg}$, a sustained stroke volume index $>20 \%$, a cardiac index double the resting value, an increase in shortening fraction $>10 \%, \mathrm{MVCFC}>0.2 \mathrm{circ} / \mathrm{s}$, a fall in wall stress, an upward and leftward shift in MVCFc/stress, an increase in contractility of all myocardial segments, and a workload greater than $900 \mathrm{~J} / \mathrm{kg}$.

Conclusions: Our stress echocardiography protocol allows us to assess exercise capacity and oxygen consumption, ventricular function, myocardial reserve, segmental wall motion, valvar obstruction, ventricular synchrony, and unmasks systemic and pulmonary venous obstruction.

\section{4}

A case control study of neurodevelopmental outcome in infants undergoing cardiac vs non cardiac surgery Karen Walker, The Children's Hospital at Westmead, Sydney., Australia

David S Winlaw, The Children's Hospital at Westmead, Sydney., Australia

Andrew D Cole, The Children's Hospital at Westmead, Sydney., Australia

Rober Halliday, The Children's Hospital at Westmead, Sydney., Australia

Andrew Holland, The Children's Hospital at Westmead, Sydney., Australia

Gary Sholler, The Children's Hospital at Westmead, Sydney., Australia Megan Sherwood, The Children's Hospital at Westmead, Australia Nadia Badawi, The Children's Hospital at Westmead, Sydney., Australia

Introduction: Improvements in neonatal and infant surgery increasingly relate to quality of outcome rather than mortality. There is a well founded concern that a significant number of neonates and infants with congenital heart disease (CHD) display neurodevelopmental impairment (NDI). 
Causes and promoters of NDI are present at every stage from gestation to peri-procedural care and operation. Many factors and the over all burden of critical illness similarly affect children undergoing major non cardiac surgery such as repair of diaphragmatic hernia.

Method: This prospective population based case control study commenced enrolment in 2006. We recruited infants who had undergone cardiac or major non-cardiac surgery in the first 90 days and compared both with normal infants.

Circulatory arrest was not employed in the cardiac surgery group. Developmental outcomes were measured using the Bayley Scales of Infant and Toddler Development Version III. Clinical data, including brain imaging, were also documented.

Results: 329 case and control infants were assessed at one year of age. See table below.

Conclusion: Early results confirm a significant level of NDI in those undergoing cardiac and non cardiac surgery with a higher percentage of the cardiac surgery group being affected. The study highlights the need for i) evaluation of how medical and surgical approaches can be tailored to minimise the impact of both disease and treatment in those with CHD and ii) routine surveillance of these groups to provide early intervention where required.

\begin{tabular}{llll}
\hline & $\begin{array}{l}\text { Gross Motor } \\
\text { Subtest } \\
(\% \text { below } \\
\text { average })\end{array}$ & $\begin{array}{l}\text { Expressive } \\
\text { Language subtest }\end{array}$ & $\begin{array}{l}\text { Receptive Language } \\
\text { subtest } \\
(\% \text { below average })\end{array}$ \\
\hline $\begin{array}{l}\text { Cardiac surgery group } \\
\text { Non cardiac surgery }\end{array}$ & 49 & 41 & 25 \\
group & 34 & 22 & 25 \\
Control infants & 15 & 10 & 14 \\
\hline
\end{tabular}

\section{5}

Resting MRI does not predict ventricular dysfunction on exercise in children with repaired tetralogy of Fallot Lucy Roche, The Hospital for Sick Children Toronto, Canada Lars Grosse-Wortmann, The Hospital for Sick Children Toronto, Canada

Mark K Friedberg, The Hospital for Sick Children Toronto, Canada Derek Stephens, The Hospital for Sick Children Toronto, Canada Andrew N Redington, The Hospital for Sick Children Toronto, Canada Paul F Kantor, The Hospital for Sick Children Toronto, Canada

Objective: To investigate the potential for exercise to reveal occult left and right ventricular dysfunction in children with tetralogy of Fallot (TOF).

Methods: We conducted a prospective study of controls $(\mathrm{n}=25)$ and children with repaired TOF $(n=29)$. All children underwent echocardiography at rest and during bicycle ergometry, performed at increasing workloads until exhaustion. Those with TOF were also studied by MRI and cardiopulmonary stress testing.

Functional M-mode and tissue Doppler data was obtained for each ventricle and for the right ventricular outflow tract (RVOT) with images recorded at rest and peak exercise. Myocardial acceleration during isovolumic contraction (IVA) was measured at each $10 \mathrm{bpm}$ heart rate increase during exercise and forcefrequency curves were constructed. In those with TOF, ventricular volumes and flow data were calculated by offline analysis of the MRI.

Data were compared between groups using standard statistical techniques and software.

Results: By MRI, the children with TOF had RV dilatation (mean $\mathrm{RVEDVi}=153 \mathrm{mls} / \mathrm{m} 2 \mathrm{SD}=37.3$ ) with normal ventri- cular function $(\mathrm{LVEF}$ mean $=59.3 \% \mathrm{SD}=6.2, \mathrm{RVEF}$ mean $=$ $50.2 \% \mathrm{SD}=8.5$ ).

Resting echocardiography detected abnormalities of right but not left ventricular function (table). Exercise revealed abnormalities of LV contractility (table) and a highly abnormal forcefrequency relationship (figure) $\mathrm{p}<0.0001$.

There was a weak relationship between peak exercise LV IVA and VO2 at cardiopulmonary stress testing $\mathrm{R}=0.51 \mathrm{p}<0.02$.

Conclusions: The RV of patients with TOF has abnormal contractile properties at rest. Exercise reveals evidence of LV dysfunction and disruption of LV force-frequency relations, which may be related to impaired exercise performance. This occult dysfunction is not demonstrated by MRI.

\begin{tabular}{lccc}
\hline & $\begin{array}{l}\text { Controls (mean } \\
\text { \& SD) }\end{array}$ & $\begin{array}{l}\text { TOF (mean } \\
\text { \& SD) }\end{array}$ & p value \\
\hline Age at study (years) & $12.3(3.2)$ & $12.2(3.1)$ & $\mathrm{NS}$ \\
RV long axis FS at rest (\%) & $31.3(6.5)$ & $16.8(5.4)$ & $<0.0001$ \\
RVOT FS at rest $(\%)$ & $33.2(5.1)$ & $18.9(8.1)$ & $<0.0001$ \\
RV free wall peak S at rest $(\mathrm{cm} / \mathrm{sec})$ & $9.61(1.3)$ & $6.04(1.2)$ & $<0.0001$ \\
LV long axis FS at rest $(\%)$ & $20.44(4.5)$ & $18.74(5.3)$ & $\mathrm{NS}$ \\
LV free wall peak S at rest $(\mathrm{cm} / \mathrm{sec})$ & $6.85(1.3)$ & $5.62(1.3)$ & $\mathrm{NS}$ \\
LV free wall peak S exercise $(\mathrm{cm} / \mathrm{sec})$ & $9.90(1.8)$ & $8.06(2.4)$ & 0.002 \\
LV peak IVA $(\mathrm{cm} / \mathrm{sec} 2)$ & $9.52(2.9)$ & $3.72(1.7)$ & $<0.0001$ \\
\hline
\end{tabular}

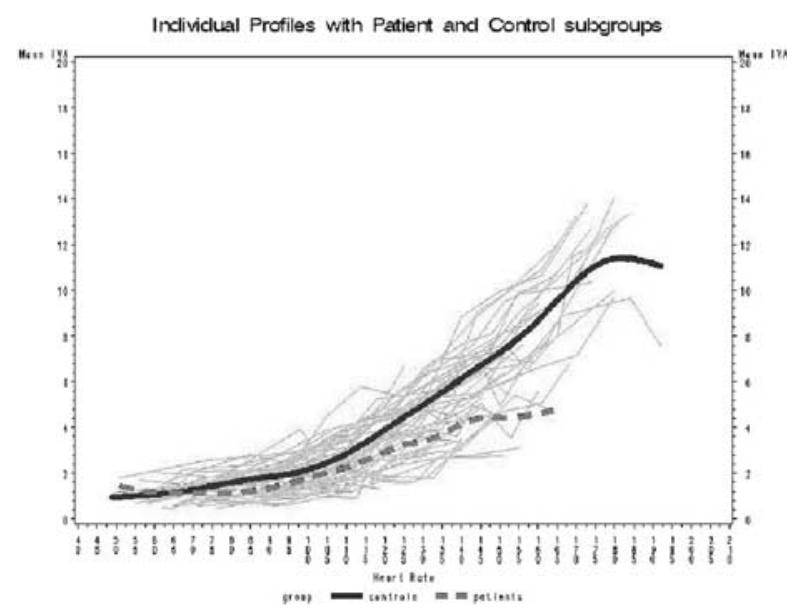

3116

Establishing a congenital cardiac surgery program in a developing country. The impact of International, Regional and National collaboration

Muneer M Amanullah, The Aga Khan University Hospital - Karachi, Pakistan

Asif Hasan, Freeman Hospital - Newcastle upon Tyne,

United Kingdom

Rajesh Sharma, Escorts Heart institute and Research Centre - Delhi, India

Asim M Khan, The Children's Hospital - Lahore, Pakistan

Mehnaz A Atiq, The Aga Khan University Hospital - Karachi,

Pakistan

Kelly Dilworth, Freeman Hospital - Newcastle upon Tyne, Australia Mansoor A Khan, The Aga Khan University Hospital - Karachi, Pakistan

Anwar U Haq, The Aga Khan University Hospital - Karachi, Pakistan

Incidence of CHD is $8 / 1000$ live births. Population of Pakistan 160 million, birth rate 3\%; therefore more than 50,000 children born each year with CHD. Lack of awareness, infra-structure, and experienced health care providers leads to delayed presentation 
and inadequate management of CHD. Multi-regional collaboration provides opportunities to enhance local expertise with satisfactory results.

Objective: To emphasize the importance of collaboration and share the progress of our program.

Material $\mathcal{E}$ Methods: Collaboration established in phases with several cardiac surgery centers to minimize the learning curve. To evaluate impact of the new program, data on selected variables were prospectively collected for consecutive cardiac surgeries from June 2006-December 2008.

Results: Phase One was training of personal, procurement of equipment and logistics. Phase Two saw collaboration with Freeman and Birmingham Children's Hospitals - UK, Escort Hospital - India, and Lahore Children's Hospital - Pakistan . Distribution of open heart surgeries (248): ToF 30\%, VSD 25\%, ASD $15 \%$, Single Ventricle palliation 5\%, TGA 3\%, TAPVD 5\% others $17 \%$. Distribution of closed heart surgeries (82): BTS $45 \%$, PDA $25 \%$, CoA $10 \%$, others $20 \%$. Median age at surgery, 4.6 (1 day to 67) years; weight $14(0.8-80) \mathrm{kg}$; CICU stay was 1.5 days. Mortality for open heart surgery was $22 / 248-8.8 \%$, and for closed heart surgery $7 / 82-8.5 \%$.

Conclusion: Our mortality is comparable to reports from established congenital cardiac surgery centers of the region, confirming that multi-regional collaboration helped achieve favorable results in the early phase of the program.

\section{7}

\section{Economic Evaluation of Palivizumab in Children with} Congenital Heart Disease: A Canadian Perspective

Derek G Human, Division of Cardiology, Department of Pediatrics, British Columbia Children's Hospital and The University of British Columbia, Canada

Kevin C Harris, Division of Cardiology, Department of Pediatrics, British Columbia Children's Hospital and The University of British Columbia, Canada

Aslam H Anis, Department of Health Care and Epidemiology,

The University of British Columbia, Canada

Marsha C Crosby, Division of Cardiology, Department of Pediatrics, British Columbia Children's Hospital and The University of British Columbia, Canada

Laurie M Cender, Division of Cardiology, Department of Pediatrics, British Columbia Children's Hospital and The University of British Columbia, Canada

James E Potts, Division of Cardiology, Department of Pediatrics, British Columbia Children's Hospital and The University of British Columbia, Canada

Background: Respiratory syncitial virus (RSV) is a common cause of bronchiolitis in infants. In children with congenital heart disease (CHD) it is associated with significant morbidity and mortality.

Objective: To assess cost-savings, cost-effectiveness and cost-utility of palivizumab in children $<2$ years with hemodynamically-significant CHD in a provincially-administered RSV prophylaxis program.

Methods: Children who received palivizumab from 2003-2007 were compared to a historical cohort of children from 1998-2003 who met the eligibility criteria for palivizumab before it was available. Direct costs were obtained from hospital administrative data. Indirect costs were estimated from government sources. We assessed the incremental cost of the program, the cost to prevent one day of hospitalization, the life-years gained and cost per quality-adjusted life-year. Discounting was applied to all future costs and benefits.
Results: The cost of palivizumab prophylaxis/patient/RSV season was $\$ 8,521$. Costs in the historical cohort were $\$ 838 /$ patient compared to $\$ 9,130 /$ patient in the palivizumab cohort. Hospital admissions were reduced by $58 \%$, and days in hospital were reduced by $88 \%$ in the palivizumab cohort. After accounting for the costs of hospitalization, the cost of the RSV prophylaxis program was $\$ 8,292 /$ patient and the cost to prevent one day of hospitalization was $\$ 15,737$. The cost per life-year gained was $\$ 111,118$ and the cost per quality-adjusted life-year was $\$ 126,621$.

Conclusion: Palivizumab is clinically-effective in terms of reducing RSV-related hospitalization. However, the cost of palivizumab is high. Given the financial constraints in public health, strict criteria for patient selection and reduced drug costs will improve the cost-effectiveness of RSV prophylaxis.

\section{8}

Neoaortic valve function 10-18 years after arterial switch operation (ASO) for transposition of the great arteries (TGA). One centre experience

Krzysztof W Michalak, Department of Cardiology, Polish Mother's Memorial Hospital, Research Institute, Lódz, Poland

Jadwiga A Moll, Department of Cardiology, Polish Mother's Memorial Hospital, Research Institute, Lódz, Poland

Katarzyna Mludzik, Department of Cardiology, Polish Mother's

Memorial Hospital, Research Institute, Lódz, Poland

Marek Kopala, Department of Cardiosurgery, Polish Mother's Memorial Hospital, Research Institute, Lódz, Poland

Andrzej Sysa, Department of Cardiology, Polish Mother's Memorial Hospital, Research Institute, Lódz, Poland

Jacek J Moll, Department of Cardiosurgery, Polish Mother's Memorial Hospital, Research Institute, Lódz, Poland

Arterial switch operation (ASO) is currently a procedure of choice for TGA treatment. During the procedure, the valves are not switched: the pulmonary valve remains within the systemic circulation, and the aortic valve within the pulmonary circulation. The aim of this paper is to evaluate neoaortic valve function, the degree and development of its regurgitation (NeoAR) in the long-term follow-up period (10-18 years).

From 519 children treated with ASO for TGA between 1990 and 2008 with overall mortality 7,1\%, 161 patients were classified for the study (average age at operation 9 days, $\mathrm{SD}=8$ days). Inclusion criteria: 10-year follow-up period, with at least two echocardiographic evaluations conducted at our institute. The subjects were divided into group 1 with TGA + IVS (109 pts) and group 2 with TGA + VSD (52 pts). Statistical analysis was performed using Statistica 8.0.

\begin{tabular}{llllll}
\hline NeoAR & $\begin{array}{l}\text { 0-3 years } \\
\text { after op. }\end{array}$ & $\begin{array}{l}4-7 \text { years } \\
\text { after op. }\end{array}$ & $\begin{array}{l}8-11 \text { years } \\
\text { after op. }\end{array}$ & $\begin{array}{l}12-14 \text { years } \\
\text { after op. }\end{array}$ & $\begin{array}{l}15-18 \text { years } \\
\text { after op. }\end{array}$ \\
\hline $\begin{array}{l}\text { None - 0 } \\
\text { Trivial and } \\
\text { Mild - I }\end{array}$ & $15 \%$ & $70 \%$ & $60 \%$ & $50 \%$ & $43 \%$ \\
\begin{tabular}{l} 
Moderate - II $0 \%$ \\
\hline
\end{tabular} & $26 \%$ & $31 \%$ & $37 \%$ & $45 \%$ \\
\hline
\end{tabular}

Within the analyzed group, the frequency of regurgitations increased from 15\% 0-3 years after the operation to $57 \%$ after $15-18$ years $(\mathrm{p}<0.01)$. None $(0) /$ trivial and mild $(\mathrm{I}) /$ moderate regurgitation(II): $85 \% / 15 \% / 0 \%$ [0-3 years after the operation]; $70 \% / 26 \% / 4 \%$ [ $4-7$ years]; $60 \% / 31 \% / 9 \%$ [8-11 years]; 50\%/ $37 \% / 11 \%$ [12-14 years]; 43\%/45\%/12\% [15-18 years] respectively) (Tabl). No severe regurgitations requiring a reoperation were observed. In $27 \%$ of cases (44 pts) NeoAR increased 
moderate (from 0 to I or I to II) and in $4 \%$ (6 pts) considerably (from 0 to II).

Conclusions:

1. Neoaortic valve regurgitation arises and develops gradually with time after ASO, with no significant hemodynamic consequences.

2. VSD associated with TGA didn't impact on NeoAR in the long-term follow-up period.

3119

Long-term Results of Modified Fontan Operation

for Single Ventricular Patients Associated with Atrioventricular Valve Regurgitation

Yuji Naito, Department of Cardiovascular Surgery, Heart Institute

of Japan, Tokyo Women's Medical University, Japan

Yasuharu Imai, Department of Cardiovascular Surgery, Heart Institute

of Japan, Tokyo Women's Medical University, Japan

Kazuaki Ishihara, Department of Cardiovascular Surgery, Heart

Institute of Japan, Tokyo Women's Medical University, Japan

Takeshi Hiramatsu, Department of Cardiovascular Surgery, Heart

Institute of Japan, Tokyo Women's Medical University, Japan

Kouta Agematsu, Department of Cardiovascular Surgery, Heart

Institute of Japan, Tokyo Women's Medical University, Japan

Masaki Sasoh, Department of Cardiovascular Surgery, Heart Institute

of Japan, Tokyo Women's Medical University, Japan

Toshio Nakanishi, Department of Pediatric Cardiology, Heart Institute of Japan, Tokyo Women's Medical University, Japan

Hiromi Kurosawa, Department of Cardiovascular Surgery, Heart

Institute of Japan, Tokyo Women's Medical University, Japan

Objective: To examine the long-term outcome of modified Fontan operation (mFO) concomitant with valve surgery against atrioventricular valve (AVV) regurgitation.

Patients and Methods: We retrospectively reviewed 555 children (median 6.2 years) who underwent $\mathrm{mFO}$ between 1974 and 2003, and they were subdivided into group P (With AVV plasty, $\mathrm{n}=210$ ) and group $\mathrm{N}$ (Without AVV plasty, $\mathrm{n}=345$ ).
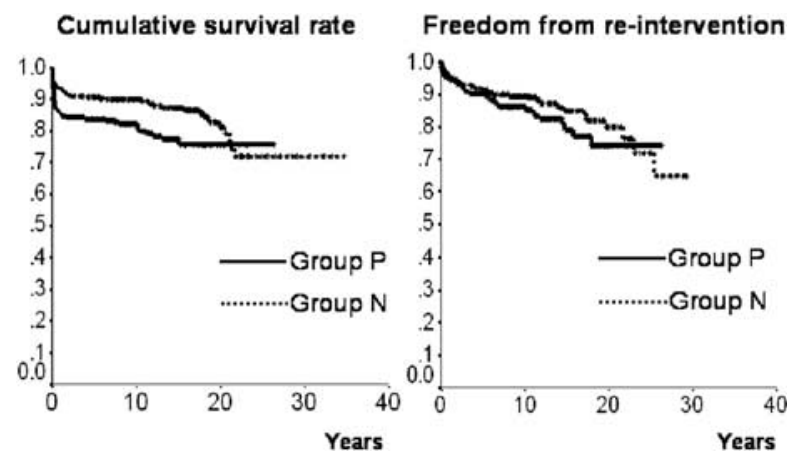

Results: Significantly higher proportion of patients have heterotaxic heart in group P (Group P vs N: 48\% vs 15\%, p <0.01), and the common AVV were significantly prevalent in group $\mathrm{P}$ $(49 \%$ vs $14 \%, p<0.01)$. Preoperative grading of AVV regurgitation was significantly higher in group $\mathrm{P}(1.7$ vs $0.1, \mathrm{p}<0.01)$. Surgical techniques to correct valve incompetence included circular annuloplasty, unilateral valve closure, annuloplasty with Kaye-Reed methods, and edge-to-edge repair methods, which were combined according to the etiology of lesion. There were 24 and 13 hospital deaths, plus 20 and 35 late deaths in group P and $\mathrm{N}$, respectively. Actuarial survival were $82.1 \%$ and $75.8 \%$ in group $\mathrm{P}$, and $90.0 \%$ and $81.2 \%$ in group $\mathrm{N}(\mathrm{p}<0.05)$ at 10 and
20 years, respectively. Freedom from re-intervention including all reoperation and balloon angioplasty for pulmonary stenosis were $86.1 \%$ and $74.1 \%$ in group $\mathrm{P}$, and $89.5 \%$ and $79.8 \%$ in group $\mathrm{N}$ (N.S.) at 10 and 20 years, respectively. Long-term survivor showed acceptable valve competence, and long-term BNP concentrations were comparable in both groups (mean 84 vs $95 \mathrm{pg} / \mathrm{ml}$ ).

Conclusion: Single ventricular patients with AVV regurgitation showed acceptable long-term result with no progression of lesion However, further improvement of valve surgery is required.

3120

Surgical repair for congenital mitral valve dysplasia: a single center 36 years experience

Giovanni Stellin, Pediatric and Congenital Cardiac Surgery Unit, University of Padua, Italy

Massimo A Padalino, Pediatric and Congenital Cardiac Surgery Unit, University of Padua, Italy

Giovanna Boccuzzo, Department of Statistics, University of Padua, Italy

Vadimiro L Vida, Pediatric and Congenital Cardiac Surgery Unit, University of Padua, Italy

Roberta Biffanti, Italy

Ornella Milanesi, Department of Paediatrics-University of Padua, Italy

Objective: to evaluate results of surgery of congenital mitral valve (MV) dysplasia and to determine predictors for late postoperative outcome.

Methods: preoperative, operative and postoperative data were obtained from institutional database; follow up data came from regular clinical evaluation at our institution or elsewhere. Patients were divided in simple and complex, according to the complexity of associated lesions.

Results: between 1972 and 2008, 93 patients (M/F=43/50) underwent MV repair ( median 4.5 years, 0.16-19.8 years,). Predominant MV regurgitation was present in 52\%. Associated cardiac anomalies were present in $72 \%$. Sixtyone patients were in the complex group. All patients underwent successful MV repair. Surgical repair was tailored to patient's valve anatomy. Early death was $7.5 \%$. Postoperative course was uneventful in $86 \%$. At a mean follow up of 10.3 years (median 8.4 years, completeness 94\%), late mortality is $8 \%$ (7 patients). Twelve patients underwent MV reintervention (11 replacements and 1 repair). Among the 80 survivors, $82,5 \%$ were in NYHA class I-II, $61,2 \%$ had some degree of persistent mitral regurgitation or stenosis, despite stable hemodynamics. Stenosis is a statistically significant risk factor for operation under 1 year of age and is related to higher overall mortality and incidence of late cardiac failure and mitral dysfunction; parachute MV is related to higher mortality and morbidity.

Conclusions: Conservative mitral surgery approach shows acceptable early mortality and reoperation rates. Complex malformations are statistically related to a significant higher risk of reoperation on $\mathrm{MV}$; parachute mitral valve is associated with a higher rate of early mortality.

\section{1}

Long-term survival and freedom from reintervention in patients with congenital aortic stenosis after balloon valvuloplasty vs operative commissurotomy Boris A Nasseri, Deutsche Herzzentrum Berlin, Germany

Peter Ewert, Deutsche Herzzentrum Berlin, Germany Hashim Abdul-Khaliq, Deutsche Herzzentrum Berlin, Germany Christof Stamm, Deutsche Herzzentrum Berlin, Germany Vladimir Alexi-Meskishvili, Deutsche Herzzentrum Berlin, Germany Yu-Guo Weng, Deutsche Herzzentrum Berlin, Germany 
Felix Berger, Deutsche Herzzentrum Berlin, Germany

Roland Hetzer, Deutsche Herzzentrum Berlin, Germany

Background: Congenital aortic stenosis (AS) in children is initially treated with operative commissurotomy (OC) or balloon valvuloplasty (BV). We compared freedom from re-intervention and long-term survival after these treatments.

Methods: We reviewed 168 patients with congenital AS who received OC or BV in 1984-2006. Number of reinterventions, time to reintervention, and survival were compared (Kaplan-Maier). Results: Mean age was 4.67 (range 0-20.51) years at first intervention. First intervention was OC in 47 patients and BV in 121 patients at mean age of 6.80 (range $0-19.29$ ) and 3.74 (range 0-20.51) years, respectively.

In the OC group, 2 received a second OC, 9 received $\mathrm{BV}$ and 26 aortic valve replacement (AVR) as a second intervention.

In the $\mathrm{BV}$ group, 22 received a second $\mathrm{BV}, 6$ received $\mathrm{OC}$ and 21 $\mathrm{AVR}$ as a second intervention.

There were 16 deaths, 5 in OC and 11 in BV group during follow-up. After OC 1, 5 and 10-year freedom from second intervention was $92.8 \pm 3.5 \%, 54.5 \pm 7.0 \%$ and $42.1 \pm 7.0 \%$. After BV 1,5 and 10 -year freedom from second intervention was $79.2 \pm 3.7 \%$, $56.2 \pm 5.0 \%$ and $50.5 \pm 5.3 \%$.

One, 5 and 10-year survival after OC was $89.7 \pm 4.0 \%$, $86.9 \pm 4.7 \%$ and $84.2 \pm 4.9 \%$. One, 5 and 10 -year survival after BV was $88.7 \pm 2.8 \%, 88.7 \pm 2.8 \%, 84.6 \pm 3.5 \%$.

Conclusions: In the first year after intervention there is a higher rate of reinterventions in the $\mathrm{BV}$ group. However, there were no significant differences between the OC and BV groups in survival time (log rank 0.63 ) or freedom from re-intervention (log rank 0.64), despite older age in the OC group.

\section{2}

Congenital Left-to-right Shunts with Severe Pulmonary Artery Hypertension: Comparison of Outcomes of Surgical or Non-surgical Treatment Adjusted by Propensity Score

Huili Gan, Cardiac Surgery Department, Beijing Anzhen Hospital, Capital Medical University (BAZH - CMU) 100029 Beijing China, China

Jian-qun Zhang, Cardiac Surgery Department, Beijing Anzhen Hospital, Capital Medical University (BAZH - CMU) 100029 Beijing China, China

Qi-wen Zhou, Cardiac Surgery Department, Beijing Anzhen Hospital, Capital Medical University (BAZH - CMU) 100029 Beijing China, China

Ping Bo, Cardiac Surgery Department, Beijing Anzhen Hospital, Capital Medical University (BAZH - CMU) 100029 Beijing China, China

Objective: Our aim was to investigate the relationship between the long-term survival of surgical treatment and preoperative pulmonary vascular resistance (PVR) and pulmonary to systemic flow ratio $(\mathrm{Qp} / \mathrm{Qs})$ in congenital left to right shunts with severe pulmonary hypertension $(\mathrm{PH})$.

Methods: 1212 cases of congenital left to right shunts with severe PH were treated non-surgically $(n=297)$ or surgically $(n=915)$. Propensity scores for inclusion into surgical group were calculated for each patient and were used to match 245 pairs between the two groups. Kaplan-Meier survival curves were constructed with PVR stratum at the level of 15 WU or with $\mathrm{Qp} / \mathrm{Qs}$ stratum at the level of 1.25.

Results: In the 245 propensity score matched pairs, the actuarial survival of the surgical group was significantly higher than that of the non-surgical group when PVR was less than $15 \mathrm{WU}$ or Qp/ Qs was larger than $1.25(\mathrm{P}=0.000$ and 0.001$)$, but the actuarial survival between the two groups had no difference when PVR was larger than $15 \mathrm{WU}$ or Qp/Qs was less than $1.25(\mathrm{P}=0.596$ and 0.424). Cox proportional hazard analysis revealed that hemoglobin higher than $160 \mathrm{~g} / \mathrm{L}$, PVR larger than $15 \mathrm{WU}$, and $\mathrm{Qp} / \mathrm{Qs}$ less than 1.25 were the independent risk predictors for the late death.

Conclusion: Surgical criteria for congenital systemic-to-pulmonary shunts with severe $\mathrm{PH}$ should be revised as preoperative PVR less than $15 \mathrm{WU}$ and Qp/Qs larger than 1.25. For patients with PVR greater than $15 \mathrm{WU}$ and/ or its Qp/Qs less than 1.25, surgical closure therapy provides no benefits.

\section{3}

Tricuspid Valve Replacement in Ebstein Anomaly: Improved Outcomes with Bioprostheses

Morgan L Brown, Mayo Clinic, United States

Joseph A Dearani, Mayo Clinic, United States

Gordon K Danielson, Mayo Clinic, United States

Frank Cetta, Mayo Clinic, United States

Heidi M Connolly, Mayo Clinic, United States

Carole A Warnes, Mayo Clinic, United States

David J Driscoll, Mayo Clinic, United States

Objectives: Our objective was to assess the outcomes in patients with Ebstein anomaly who required a tricuspid valve (TV) replacement with a porcine tissue bioprosthesis (BPV) or a mechanical prosthesis (MechV).

Methods: Between 1972 to 2006, 333 patients received a BPV and 45 received a MechV. Patient records were reviewed and patients were contacted by questionnaire.

Results: Early mortality was statistically similar in patients who received a MechV (11\%) or a BPV (5\%) inserted in the TV position $(\mathrm{P}=0.173)$. Greater than moderate left ventricular dysfunction (OR 3.1, P = 0.03) was independently associated with operative mortality. Twenty-year survival was better in patients who had a BPV (75\%) than for those who had a MechV $(43 \%, \mathrm{P}=0.003)$. After adjusting for ablation of accessory pathways, sinus rhythm at dismissal, and concomitant repair of pulmonary valve stenosis, a BPV remained a predictor of late survival (HR 0.42, P = 0.004). Survival free of reoperation on the TV at 20 years postoperatively was similar for patients who had a MechV (49\%) compared to those who had a BPV (42\%) inserted $(\mathrm{P}=0.941)$. A greater percentage of patients who had a MechV reported endocarditis ( $12 \%$ vs. $2 \%)$, bleeding requiring hospitalization ( $6 \%$ vs. $3 \%$ ), and thrombosis (12\% vs. $6 \%)$; however, none were statistically significant. All MechV patients and $17 \%$ of BPV patients were on warfarin at last follow-up. Conclusions: A BPV in the tricuspid position was an independent predictor of improved survival. For most patients with Ebstein anomaly, a porcine BPV is recommended when TV replacement is required.

\section{4}

Improved outcome of fetuses with congenital heart disease. Multicentre study

Vlasta M Fesslova, Centre of Fetal Cardiology, Policlinico San Donato, IRCSS, Milano, Italy

Annaclaudia Masini, Centre of Fetal Cardiology, Policlinico San Donato, IRCSS, Milano, Italy

Jelena Brankovic, Centre of Fetal Cardiology, Policlinico San Donato, IRCSS, Milano, Italy 
Daniela Prandstraller, Dpt. Ped. Cardiology, Policlinico S. Orsola, Bologna, Italy

Flavia Ventriglia, Dpt. Ped. Cardiology, Università alla Sapienza, Roma, Italy

Roberto Crepaz, Dpt. Cardiology, Azienda Ospedaliera Bolzano, Italy Francesco De Luca, Dpt. Ped. Cardiology, Università di Catania, Italy Gabriella Errico, Dpt. Ped. Cardiology, Ospedale Borgonuovo, Verona, Italy

Objectives: to analyse the outcome of fetuses with congenital heart disease (CHD) in the period 2000-2005 in comparison to the data of a multicentre study regarding the previous era.

Methods: Data of 7 centres operating in prenatal echocardiographic diagnosis were prospectively collected and analyzed; the inclusion criteria being a confirmed fetal diagnosis after birth or at autopsy and a known follow-up, for at least 6 months after birth.

Results: Data of 661 fetuses satisfied the inclusion criteria. Age at diagnosis varied between 15 and 37 weeks' gestation, median 24 . Chromosomal and extracardiac anomalies were associated in 67 and 60 cases respectively (10.1\% and 9.1\%).

Outcome: Hundred forty cases opted for the termination of pregnancy $(21.2 \%)$ versus the previous figure of $28.9 \%$. Five hundred twenty one fetuses continued pregnancy, 23 of them died in utero $(4.4 \%)$.

A total of $109 / 521(21 \%)$ died postnatally, postoperative death occurred in 58/285 (20.4.\%) versus previous total deaths in $43 \%$ and postoperative ones in 38\%. Surgical mortality was highest in cases with hypoplastic left heart and Ebstein's anomaly. The mortality rate was higher in presence of associated chromosomal or extracardiac anomalies (40\% and 31\% respectively versus $15.6 \%$ isolated CHD). A total of 389/521 (74.7\%) infants survived versus previous $45 \%$. Recurrence of $\mathrm{CHD}$ was $6 \%$.

Conclusions: Our data show an improved outcome of cases with prenatal diagnosis of CHD both as for the total and surgical mortality. These data reflect an improved perinatal management and improved results of cardiosurgery in the treatment of neonates with congenital heart lesions.

3125

Fetal Pulmonary Vasoreactivity in Response to Maternal Hyperoxygenation: A Useful Tool in the Prenatal Evaluation of Hypoplastic Left Heart Syndrome (HLHS)

Anita Swast, Fetal Heart Program, The Children's Hospital of Philadelphia, United States

Zhiyun Tian, Fetal Heart Program, The Children's Hospital of

Philadelphia, United States

Jack Rychik, Fetal Heart Program, The Children's Hospital of

Philadelphia, United States

Background: Limited left atrial egress due to a restrictive/intact atrial septum (RIAS) can lead to abnormal pulmonary vascular development, severe cyanosis at birth, and is a risk factor for survival in HLHS. Maternal hyperoxygenation $(\mathrm{MH})$ can lead to observable fetal pulmonary vasodilation in the third trimester of pregnancy and may offer a means for testing the fetal pulmonary vasculature.

Objective: To determine the utility of $\mathrm{MH}$ in assessing pulmonary vasoreactivity in the fetus with HLHS.

Methods: Doppler derived pulsatility index (PI), a measure of vascular resistance, was evaluated in the distal branch pulmonary artery (PA) of fetuses with HLHS before, during, and after $\mathrm{MH}$ with 20 minutes of $60 \%$ oxygen. 68 studies were performed; 56 had open atrial septum and 12 had RIAS, of which 7 required urgent intervention at birth due to severe cyanosis.
Results: $\mathrm{MH}$ resulted in a significant decrease in PA-PI in comparison to room air (RA) in open septum, but not in RIAS fetuses (see table). Absence of pulmonary vasoreactivity at $>30$ wks gestation defined as PA-PI change of $<10 \%$ with $\mathrm{MH}$ was highly predictive of severe cyanosis, discriminating within the RIAS group for those who required urgent intervention at birth. There was no significant change in umbilical or middle cerebral artery PI with $\mathrm{MH}$ and no ductal constriction.

Conclusions: $\mathrm{MH}$ is safe and useful in evaluating fetal HLHS and should be incorporated into routine prenatal assessment of this anomaly.

\begin{tabular}{lcc}
\hline & Open Septum: PA-PI & RIAS: PA-PI \\
\hline RA (21\%) & $4.06(0.66)$ & $4.01(0.74)$ \\
MH (60\%) & $3.37(0.57)$ & $3.8(0.67)$ \\
P Value & $<0.001$ & NS \\
\hline
\end{tabular}

3126

Effect of a regional training programme in fetal echocardiography on antenatal detection of major congenital heart disease

Angela McBrien, United Kingdom

Andrew Sands, United Kingdom

Brian Craig, The Royal Belfast Hospital for Sick Children,

United Kingdom

Frank Casey, United Kingdom

Objectives: Antenatal detection of major congenital heart disease (CHD) in Northern Ireland from 2003-2006 was 27\%. In 2007 we delivered a regional one-year fetal echocardiography training course (with particular emphasis on outflow tract views). We aimed to assess the effect of training on antenatal detection of major CHD. Methods: Training was offered to all obstetric radiographers performing routine anomaly scanning. The programme involved attendance at: a one-day fetal echocardiography foundation course, 2 regional fetal cardiology clinics, 2 supervised anomaly scanning sessions in each local hospital and 1 refresher afternoon. Antenatal detection rates were monitored during the training period. Data were obtained from: the regional fetal and paediatric cardiology database and post-mortem records. Defects were classified into 2 groups: those detectible on the 4 chamber view and those requiring outflow tract views for detection.

Results: Antenatal detection of major CHD improved from 27\% $(72 / 262)$ to $43 \%(36 / 84)$ during the year of training $(p=0.008)$. There was significant improvement in the detection of outflow tract view defects $(8 \%, 7 / 92$ vs. $21 \%, 6 / 28, \mathrm{p}=0.04)$ and also 4 chamber view defects $(38 \%, 65 / 170$ to $54 \%, 30 / 56, p=0.04)$. Conclusions: Training to include more detailed fetal echocardiography as part of routine anomaly scanning has significantly improved regional detection of major CHD. The majority of major defects visible on the four chamber view are now diagnosed antenatally. Training has also resulted in significantly greater detection of outflow tract view defects. There is room for further improvement and ongoing training has been implemented.

\section{7}

Evaluation of fetal cardiac function in gestational diabetes mellitus pregnancies

Chen Chu, Cardiovascular Center, Children's Hospital of Fudan University, Shanghai, China

Yonghao Gui, Cardiovascular Center, Children's Hospital of Fudan

University, Shanghai, China 
Yunyun Ren, Ultrasound Department, Gynaecology and Obstetrics Hospital of Fudan University, Shanghai, China

Liye Shi, Ultrasound Department, International Peace Maternity \& Child Health Hospital of China Welfare Institute, Shanghai, China

Objective: To evaluate the alterations of fetal cardiac sizes and function in gestational diabetes mellitus (GDM) pregnancies under different maternal glucose control.

Methods: In two tertiary obstetrics hospitals of Shanghai, 44 GDM mothers received 78 fetal echocardiography examinations in the study period and were divided into good (DM1) and poor (DM2) glucose control groups due to their glucose levels at examination. 70 uncomplicated mothers were as control. Parameters of cardiac sizes and function were measured and analyzed by gestational ages subgroups $(<28,28-34$ and $\geqslant 34$ weeks).

Results: In GDM fetuses the ventricular walls, especial interventricular septum were thicker than control, DM1 group more significantly than DM2 group. Peak flow velocities of aorta and pulmonary artery in GDM fetuses increased since $<28$ and 28-34weeks subgroups respectively, and right/left cardiac outputs ratio decreased slightly during gestation unlike its increase in control group. Left atrial shortening fraction and tricuspid E/A ratio showed smaller in GDM fetuses than control since $\geqslant 34$ and 28-34 weeks subgroups respectively with no significant differences between DM1 and DM2 group. Left and right ventricular Tei index were higher than control since $28-34$ and $<28$ weeks subgroups respectively in DM1 fetuses, but significantly increased till $\geqslant 34$ weeks subgroup in DM2 fetuses.

Conclusion: Fetal cardiac sizes and function changed in GDM pregnancy, mainly the thickening interventricular septum, increasing blood flow and left cardiac outputs, decreased diastolic and global ventricular function. Tei index changed earlier and right ventricle was influenced earlier than left ventricle. More earlier and significant changes were observed in fetuses of poor maternal glucose control.

\section{8}

Quantifying the Spectrum of Cardiovascular Findings in the Twin-Twin Transfusion Syndrome: The CHOP TTTS Cardiovascular Score

Zhiyun Tian, Fetal Heart Program, The Children's Hospital of Philadelphia, United States

Michael Bebbington, Center for Fetal Diagnosis and Treatment, The Children's Hospital of Philadelphia, United States

Jack Rychik, Fetal Heart Program, The Children's Hospital of Philadelphia, United States

Jack Rychik, Fetal Heart Program, The Children's Hospital of Philadelphia, United States

Background: Abnormalities of the cardiovascular (CV) system play an important role in the pathophysiology of twin-twin transfusion syndrome (TTTS). Currently there is no means of quantifying overall degree of CV derangement in TTTS.

Objective: To develop a tool that describes the magnitude of $\mathrm{CV}$ abnormality in TTTS.

Methods/Results: Fetal echocardiograms of 100 twin-pairs referred for TTTS were reviewed. We applied a novel 20 point Score to each set. The Score is designed to characterize the unique cardiovascular findings and is a composite of variables that include: (1) recipient assessment of heart size, ventricular hypertrophy, systolic function, valve regurgitation, pulmonary arterial size relative to aorta (as a measure of relative pulmonary stenosis) and diastolic filling properties, as well as (2) donor assessment of diastolic umbilical arterial flow as a measure of placental vascular resistance. Variables are recorded as normal $=0$, mildly abnormal $=1$, or severely abnormal $=2$. The Score correlates well with myocardial performance (Tei) index in the recipient $\mathrm{RV}$ and correlates with other measures of disease severity (Quintero) but is additive in informing upon the $\mathrm{CV}$ state. Thirty-two patients underwent placental laser photocoagulation treatment and exhibited a significant decrease in Score $(9+5$ versus $4+3, p<0.001)$ within 2 weeks after intervention. Conclusions: The CHOP Score characterizes the magnitude of CV abnormality in TTTS, is disease specific, and can be applied to (1) grade severity at initial presentation and serial follow up, (2) assess efficacy of treatment strategies and (3) prognosticate for development of residual long-term derangements that may present after birth, into adulthood.

\section{3}

IMPACT Registry: Improving Pediatric and Adult Congenital Treatment

Kathy Jenkins, Children's Hospital of Boston, United States

Jonathan Rome, Children's Hospital of Philadelphia, United States

Cardiac catheterization for congenital heart disease (CHD) has transitioned from a predominantly diagnostic procedure to therapy that may be performed by itself or in combination with surgery. Guidelines have been published providing recommendations for the appropriate use of diagnostic catheterization, interventional catheterization, and surgical treatments. However, because studies performed in CHD are generally small in number and because prior registries of CHD have been limited in scope, there is a paucity of evidence-based data relating to diagnostic catheterization and catheter-based interventions, and to the morbidity and mortality associated with these procedures. The IMPACT registry will be a multi-center registry collecting data on all diagnostic and interventional cardiac catheterizations in pediatric laboratories as well as in adult laboratories treating congenital heart disease. Data variables will be chosen to allow for measurement of variability in procedures. Variability will be linked to outcome measures, allowing a more meaningful measurement of performance and benchmarking. Performance improvement tools will be implemented and better outcomes will be pursued. The Registry will initially be based upon discharge data but will transition to a longitudinal database. Data variables will be collected to allow comparison with the STS surgical database. A working group has been assembled to outline the necessary demographics, nomenclature, procedure and complication lists and outcome measurements for diagnostic catheterizations, ASD closure, PDA closure, pulmonary valve stenosis, aortic valve stenosis, coarctation of the aorta, and pulmonary artery stenting. The registry will undergo pilot testing in 2009 and then be released to CHD centers in 2010 .

\section{7}

Quantification of Aortopulmonary Collateral Flow In Single Ventricle Patients With Cavopulmonary Connections Using Magnetic Resonance Imaging Velocity Mapping

Kevin K Whitehead, Children's Hospital of Philadelphia, United States Matthew J Gillespie, Children's Hospital of Philadelphia, United States Matthew A Harris, Children's Hospital of Philadelphia, United States Mark A Fogel, Children's Hospital of Philadelphia, United States Jonathan J Rome, Children's Hospital of Philadelphia, United States

Background: Systemic to pulmonary collateral flow (SPCF) is common in single ventricle patients. Because no validated method to quantify SPCF exists, neither its hemodynamic 
burden nor clinical impact can be systematically evaluated. We hypothesize that (1) the difference in total ascending aortic (Ao) and caval flow (SVC + IVC) and (2) the difference between pulmonary vein and artery flow (PV - PA) provide two independent estimators of SPCF. Methods and Results: We measured Ao, SVC, IVC, right (RPA) and left (LPA) PA, left (LPV), and right (RPV) PV flows in 23 single ventricle patients, 18 superior cavopulmonary (SCPC) and 5 Fontan, during routine cardiac MRI studies using through-plane phase contrast velocity mapping. Two independent measures of SPCF were obtained: (1) Ao - (SVC + IVC). (2) (LPV-LPA) + (RPV-RPA). Values were normalized to BSA, Ao, and PV and comparisons made using regression and Bland-Altman analysis. SPCF ranged from $0.2-2.1 \mathrm{~L} / \mathrm{min}$ for (1) and $0.2-2.2 \mathrm{~L} / \mathrm{min}$ for (2) (average indexed SPCF of $\left.0.4-5.8 \mathrm{~L} / \mathrm{min} / \mathrm{m}^{2}\right)$. SPCF in SCPC patients averaged $38 \%$ of Ao and $55 \%$ of PV, compared to $24 \%$ ( $p=0.04)$ and $28 \%(p=0.003)$ respectively in Fontans, both statistically significant differences. There was strong linear correlation between the two methods of estimating SPCF $(y=0.78 x+0.24$, $\mathrm{r}=0.79, \mathrm{p}<0.001)$.

Conclusions: We present a novel noninvasive method for the SPCF quantification that should prove an important clinical tool. We show that SPCF is often a significant hemodynamic burden, especially in SCPC physiology. Future investigations will allow objective study of the impact of collateral flow on outcome.

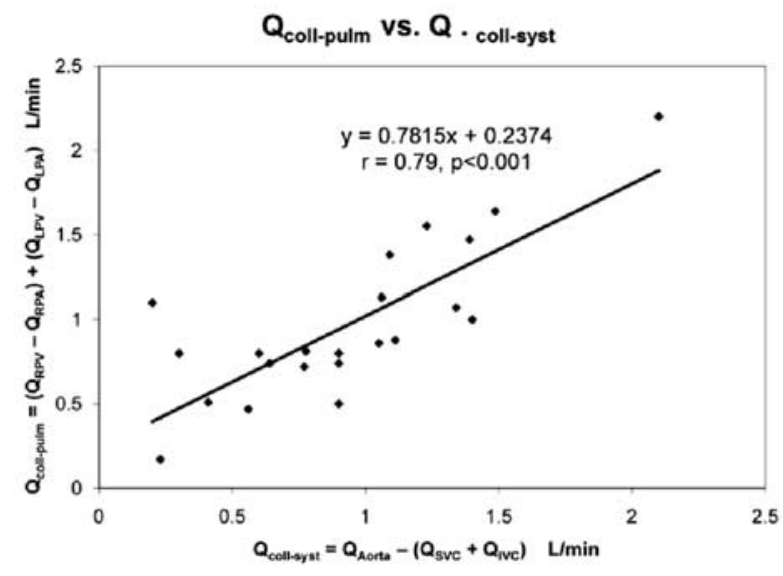

4012

Posthumous diagnosis of long QT syndrome from the neonatal screening card

Jonathan R Skinner, Starship Childrens Hospital, Auckland, New Zealand

Jackie Crawford, Starship Childrens Hospital, Auckland, New Zealand Anne Vaughan, Lap Plus, Auckland., New Zealand Carey-Anne Eddy, University of Auckland, New Zealand

Mark Rees, University of Wales, United Kingdom

Donald Love, Lap Plus, Auckland., New Zealand

Andrew Shelling, University of Auckland, New Zealand

Background: Neonatal screening (Guthrie) cards provide a potential source of DNA, but their use in diagnosing inherited cardiac conditions following sudden death has not been assessed. Aims: We report a consecutive series of posthumous genetic tests for long QT syndrome using the Guthrie card.

Methods: No post-mortem tissue suitable for DNA extraction was available from 23 families who approached our cardiac genetic clinic seeking a cause of death of their relative 2-14 years after the death. Deaths occurred at $<1$ year in $3,1-18$ yrs in 15 , and 19-35 yrs in 4. Informed consent from the next of kin was obtained. DNA was extracted and amplicons corresponding to the coding regions of the long QT (LQT) genes 1, 2, 3, 5, and 6 were subjected to DHPLC screening and/or direct sequencing. Results: Adequate DNA was extracted in every case. A full diagnostic screen was performed in 19 cases. Of these, variants were detected in four (21\%) cases: one mutation was detected in the KCNQ1 gene in a $12 \mathrm{yr}$ old boy; two mutations were found in the $\mathrm{KCNH} 2$ gene in 20 month and 27 year old females respectively; and a novel variant was found in the SCN5A gene in a three day old infant. Point mutation tests for familial mutations were performed on four cards with a positive result in all four. Conclusions: Guthrie cards, up to 23 years old, provide a valuable and reliable source of DNA for the posthumous diagnosis of long QT syndrome.

\section{6}

Fetal pulmonary wave generation augments mid-systolic forward flow across the ductus arteriosus

Joseph J Smolich, Murdoch Children's Research Institute, Australia Jonathan P Mynard, Murdoch Children's Research Institute, Australia Daniel J Penny, The Royal Children's Hopsital, Melbourne, Australia

Objective: Recent studies in lambs using wave intensity (WI) analysis indicate that a characteristic mid-systolic fall in fetal pulmonary artery (PA) blood flow is related to a large pressure-increasing, flowdecreasing backward-running compression wave (BCWms), which originates in the pulmonary microvasculature via cyclical vasoconstriction and reflection of the early-systolic forward-running compression wave (FCW) associated with ventricular ejection. This $\mathrm{BCWms}$ is partially transmitted into the pulmonary trunk $(\mathrm{PT})$ as a smaller BCWms. This study sought to determine if the PA BCWms was also transmitted into the ductus arteriosus (DA), and the hemodynamic effects of any transmission.

Methods: Simultaneous PT, left PA and DA WI analysis was performed in 8 anaesthetized late-gestation fetal sheep instrumented with PT, left PA and DA micromanometer catheters to measure pressure $(\mathrm{P})$ and transit-time flow probes to obtain blood velocity (U). The main PA was briefly occluded in a subgroup $(\mathrm{n}=5)$ to abolish wave transmission from the lungs. WI was calculated as the product of $\mathrm{P}$ and $\mathrm{U}$ rates of change.

Results: The left PA WI profile displayed a striking BCWms 4-fold larger than the PT BCWms $(\mathrm{P}<0.001)$, with both waves increasing $\mathrm{P}$ but decreasing U. By contrast, the DA WI profile demonstrated a prominent mid-systolic FCW, which increased both DA P and U. Main PA occlusion abolished not only the PT BCWms, but also the DA mid-systolic FCW.

Conclusion: These results suggest that transmission of the fetal PA $\mathrm{BCW}$ s into the DA as a FCW augments forward flow across this shunt, and thus descending aortic flow.

\section{7}

Novel strategies to improve myocardial performance and diminish capillary leak after cardiac surgery and cardiopulmonary bypass in children utilising $\mathbf{P} 188$

Jonathan R Egan, Kids Heart Research, The Children's Hospital at Westmead, Sydney, Australia, Australia

Tanya L Butler, Kids Heart Research, The Children's Hospital at Westmead, Sydney, Australia, Australia David S Winlaw, Kids Heart Research, The Children's Hospital at Westmead, Sydney, Australia, Australia

Background: Poloxomar 188(P188) is a non-ionic surfactant associated with improved blood viscosity and plasma membrane protection in human and experimental contexts respectively. We 
recently reported use of $\mathrm{P} 188$ in a lamb CPB model and demonstrated improved haemodynamics, reduced haemodilution and reduced membrane injury. We sought to elucidate its mechanisms of action utilising a Langendorff model.

Methods: Rat hearts were perfused using a crystalloid perfusate \pm P188. Flow was maintained for $120 \mathrm{~min}(\mathrm{n}=12)$ or temporarily suspended for $30 \mathrm{~min}(\mathrm{n}=12)$ to model ischaemia. A low dose $(0.42 \mathrm{mg} / \mathrm{ml})$ was followed by a clinically relevant high dose $(150 \mathrm{mg} / \mathrm{ml})$. Myocardial function was assessed by LV developed pressure. Myocardial water content (MWC) was measured.

Results: In low dose and non-ischaemic hearts, P188 was associated with a reduction in MWC, 0.78 vs. $0.82, p=0.03$. There was no effect on MWC in the ischaemic hearts and no difference in myocardial function between groups. At high dose in ischaemia, P188 was associated with a trend towards improved diastolic function, $\mathrm{p}=0.06$, but no difference in MWC.

Conclusion: Neither the diminished myocardial water accumulation or trend toward better diastolic function can be well explained by previously described rheological effects of P188 in this asanguinous model. Reduced MWC could be explained by diminished endothelial leak in the face of endothelial activation. Improved myocardial performance may relate to previously proposed mechanisms including membrane sealing to minimise intracellular calcium overload. The effects of P188 on the microcirculation and myocyte membrane as well as dose finding experiments are required with a view to using P188 in the context of cardiac surgery.

\section{0}

Use of Forensic Interview Techniques To Identify Important Handover Information In Post-Operative Paediatric Cardiac Surgery Patients

Leonie Crone, Erasmus MC, Rotterdam, The Netherlands Judith de Pont, Erasmus MC, Rotterdam, The Netherlands David Schell, Children's Hospital at Westmead, NSW, Australia Marino Festa, Children's Hospital at Westmead, NSW, Australia Mark Wiggins, MacQuarie University, NSW, Australia

Objective: To use forensic interview techniques to compare information used by expert clinicians at handover of critically ill children following cardiopulmonary bypass and cardiac surgery. Analysis of features from interviews is used to construct a handover checklist of important information for use at handover by non-expert staff. Methods: Approval was obtained from the Local Research and Ethics Committee for this study. Four groups (anaesthetists, surgeons, intensivists and nurses) expert in the care of postoperative cardiac cases at our institution were identified. Two researchers $(\mathrm{LC}, \mathrm{JdP})$ interviewed three experts from each group using a semi-structured cognitive interview protocol used previously in forensic investigations.

All interviews were recorded and later transcribed for analysis by two independent raters. Features relating to shared information during patient handover from theatre to intensive care were extracted. These were subsequently compiled into a checklist following similar lay-out guidelines used in other high-risk industries, eg aviation.

Results: Overall reliability of initial feature extraction between raters was satisfactory $(69.1 \%$ agreement). Raters achieved subsequent consensus on all remaining features. Anaesthetists generated the greatest number of features. Fourteen features were common to all expert groups. All identified features were incorporated into a chronologically-ordered checklist for use at handover by non-expert staff.

Conclusions: The cognitive interview technique demonstrates similarities and differences between expert groups caring for postoperative cardiac babies and children, and allows mapping of both common and distinctive features. Identified features are useful in developing a handover tool to assist transfer of necessary information. This may lead to improved patient safety.

\section{1}

Can Indigenous cardiac health "Close the Gap" through a state-wide capcity building approach? Rohan Corpus \& Toby Laakso

Rohan J Corpus, The Prince Charles Hospital, Australia

Toby Laakso, The Prince Charles Hospital, Australia

Background: Indigenous cardiac outreach program was established early 2007 answering the concerns of ever increasing incidences of cardiovascular disease within rural Queensland's Indigenous population.

Aim: Develop a culturally appropriate, accessible speciality service to enhance Indigenous cardiac health. Provide access; encourage community participation, ownership \& management, education \& diagnostic services to marginalised \& disadvantaged communities.

Method: Dialogue \& adoption of Indigenous community values defined community expectations in the program. In collaboration with community a holistic framework was established, incorporating tertiary, secondary and primary health interventions, cardiac specific information \& the development of assessment tools. Indigenous Health Care Professionals engaged community through a "body, mind \& spirit" approach. The specialist cardiac team includes cardiologist, sonographer with portable echo machine, \& Indigenous Program Coordinator;

- Introduction of specialist cardiac outreach services \& education to Community Health Centres.

- Prior to the clinics, local Indigenous Health Care Workers

- engage community through the completion of a pre-screen

- cardiac risk assessment tool.

- Indigenous Health Care Professionals in collaboration with

- the Cardiac Outreach Team coordinate the clinics.

- Lobbying Government \& private enterprise to fund a community

- managed cardiac prevention/rehabilitation program.

- Outcomes:

- Clinic attendance rate, approx $>90 \%$.

- Expansion of clinics from 11 to 17.

- Raised community awareness of individual modifiable risk

- factors including lifestyle behaviours \& dietary intake.

- Community satisfaction \& improved confidence in Queensland

- Health Services.

- New diagnoses ie, rheumatic, ischaemic heart disease \& heart

- failure.

- Interventions included referrals for balloon mitral

- valvotomy cardiac stress testing \& coronary angiograph.

4022

Does a new ultrasound flow modality improve visualisation of neonatal pulmonary veins?

Siri Ann Nyrnes, Norway

Lasse Lovstakken, Norwegian University of Science and Technology, Norway

Eirik Skogvoll, Norwegian University of Science and Technology/

St. Olavs Hospital, Norway

Hans Torp, Norwegian University of Science and Technology, Norway Bjorn Olav Haugen, Norwegian University of Science and Technology $/$ St. Olavs Hospital, Norway 
Objective: Echocardiography with conventional colour Doppler has technical limitations, which may impede flow visualisation in the pulmonary veins.

Blood Flow Imaging is a new ultrasound modality which supplements colour Doppler with angle-independent information of flow direction that is not influenced by velocity aliasing. This is done by visualising an additional blood speckle movement superimposed on the colour Doppler images. The aim of this study was to investigate if this method improves the visualisation of the pulmonary veins.

Methods: Twenty six neonates with suspected congenital heart disease were included prospectively and examined with echocardiography following parental consent. Imaging of the pulmonary veins with Blood Flow Imaging was added to the standard examination.

For each patient, Blood Flow Imaging and colour Doppler imaging cine loops were presented to four echo cardiographers (paediatric cardiologist/cardiologists) in a random fashion. Information regarding the venous connections, flow direction, and the overall quality of the image was evaluated on a visual analogue scale from 0 (worst) to 100 (best). The ratings were compared within each observers using Wilcoxon's exact signed-rank test.

Results: Blood Flow Imaging was consistently ranked as better than colour Doppler imaging. With respect to visualisation of the pulmonary venous connections, blood flow direction and the overall quality, the median difference in favour of blood flow imaging was 6,12 , and 22 on the visual analogue scale, respectively (all p-values $<0.01$ ).

Conclusion: Blood Flow Imaging may improve the visualisation of the pulmonary veins in neonates.

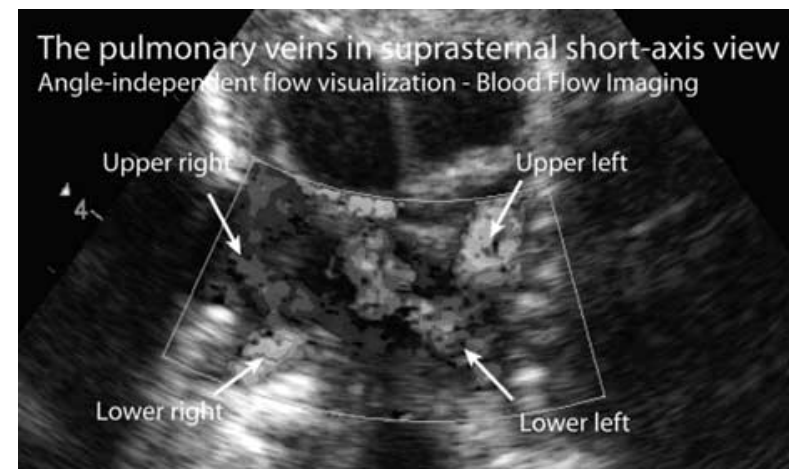

4023

Early experience with the Aquilion One 320 slice computerised tomography scanner in paediatric and congenital heart disease

Sarah A Hope, Monash Cardiovascular Research Centre, Australia Marcus Crossett, Australia

Arthur Nasis, Australia

Sujith Seneviratne, Australia

The Aquilion One 320 slice computerised tomography (CT) scanner provides $16 \mathrm{~cm}$ of coverage in a single rotation. Consequently cardiac imaging can be acquired in one rotation $(<350 \mathrm{~ms})$ and functional data over one heart beat. Additionally, unlike previous generations of CT scanners, functional data may be acquired over consecutive heart beats, more comparable with conventional angiography. The first Aquilion One CT scanner in the southern hemisphere was commissioned by MonashHeart in September 2008. MonashHeart manages both children and adults with congenital and acquired heart disease.
In the first 3 months 22 patients with congenital heart disease have been scanned, 5 paediatric and 17 adult, and 2 children with acquired heart disease (Kawasaki disease, now aged 7 and 10 years). Indications were delineation of anatomy prior to interventional or surgical procedures, post-operative assessment of anatomy and ventricular function and evaluation of coronary artery disease. Patients were aged between 3 months and 78 years. All studies have been diagnostic. Congenital diagnoses have included unoperated ASD, PDA and aortic valve or root abnormalities, post systemic to pulmonary artery shunt for tetralogy of Fallot, pulmonary atresia with VSD or intact ventricular septum, pre or post pulmonary homograft replacement, and post Fontan surgery or revision of Fontan. No patient has been anaesthetised or sedated for scanning. Radiation exposure has been low (as low as $0.2 \mathrm{mSv}$ in babies).

This technology eliminates the need for conventional diagnostic catheterisation in many patients, providing excellent anatomical and functional data, with low radiation exposure and without anaesthesia or sedation.

\section{4}

Restrictive right ventricular physiology after tetralogy of Fallot repair is associated with fibrosis of the right ventricular outflow tract visualized on MRI

Peter Munkhammar, Dep of Pediatric Cardiology, Lund University Hospital, Sweden

Marcus Carlsson, Dep of Clinical Physiology, Lund University

Hospital, Sweden

Hakan Arheden, Dep of Clinical Physiology, Lund University Hospital, Sweden

Erkki Pesonen, Dep of Pediatric Cardiology, Lund University Hospital, Finland

Objectives: The explanation for restrictive right ventricular (RV) physiology after tetralogy of Fallot (ToF) repair is not known. We hypothesized that restrictive RV physiology is associated with fibrosis of the right ventricular outflow tract (RVOT) as detected on cardiac MRI.

Methods: 29 patients $(10.5 \pm 2.8$ years, 14 females $)$ with ToF repair were included $9.6 \pm 2.9$ years after total correction. The subjects were examined with delayed contrast enhanced (DE) MRI to detect fibrosis of the RV and echo/Doppler-cardiography to detect and measure end-diastolic antegrade flow in the pulmonary artery. One patient was excluded due to suboptimal DE-MRI. Fisher's exact test was used to determine if the asscociation between restrictivity and fibrosis was significant and the odds ratio (OR) was calculated.

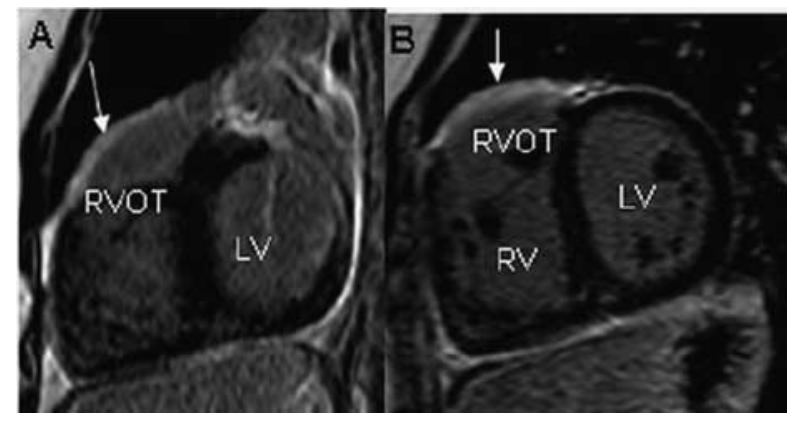

Results: Fourteen patients showed fibrosis in the RVOT on DEMRI and echo/Doppler showed restrictive physiology in 11 of these 14 patients. Of the remaining 14 patients without fibrosis in 
the RVOT on DE-MRI, two showed restrictive physiology on echo/Doppler. The OR of having RVOT fibrosis was 22 (CI $3.0-157.4, p=0.0018)$ in patients with restrictive RV physiology. In addition, fibrosis was seen at the place of the ventricular septum defect patch and in one patient there was a minor area of fibrosis separate from the RVOT.

Conclusion: Restrictive physiology of the RV is associated with fibrosis of the RVOT. Therefore, fibrosis of the RVOT may constitute the substrate that partly explains restrictive physiology in ToF patients after repair.

Figure: Example of DE-MRI in one patient showing fibrosis of the RVOT (white arrows).

\section{5}

Computer-assisted noninvasive estimation of pulmonary vascular resistance using color M-mode echocardiography Yu-qi Zhang, Department of Pediatric Cardiology, Shanghai Children's Medical Center, Shanghai Jiaotong University, Shanghai, China

Kun Sun, Department of Pediatric Cardiology, Shanghai Children's Medical Center, China

Shu-wen Zhong, Department of Pediatric Cardiology, Shanghai Children's Medical Center, Shanghai Jiaotong University, Shanghai, China Lan-Ping Wu, Department of Pediatric Cardiology, Shanghai Children's Medical Center, Shanghai Jiaotong University, Shanghai, China Guo-Zhen Chen, Department of Pediatric Cardiology, Shanghai Children's Medical Center, Shanghai Jiaotong University, Shanghai, China

Objective: The goal of this study was to seek a new method for noninvasive estimation of PVR by velocity propagation (VP) within the main pulmonary artery from color $\mathrm{M}$-mode Doppler imaging using custom software on a personal computer.

Methods: Color M-mode imaging of the pulmonary flow was obtained and transferred onto a computer. The flow wave front of the first aliasing velocity was identified with the custom software and regressed to a straight line. The slope representing $\mathrm{VP}$ of the pulmonary flow was automatically obtained. Comparative studies between this color M-mode Doppler echocardiography and cardiac catheterization for predicting PVR were performed in 20 children with congenital heart disease and 20 normal children.

Results: VP of children with congenital heart disease were significant lower than those of normal children obtained by color M-mode Doppler echocardiography $\quad(38.38 \pm 18.89$ vs $80.34 \pm 15.65 ; \mathrm{P}<0.01)$ and correlated well with invasive PVR measurements $(\mathrm{r}=-0.69 ; \mathrm{P}<0.01)$. The correlation and repeatability of the VP obtained by the custom software were better than the VP obtained by Doppler echocardiography $(\mathrm{r}=-0.78 ; \mathrm{P}<0.01)$. A VP cutoff value obtained by the custom software of 35.910 had a sensitivity of $92.9 \%$ and a specificity of $100 \%$ to determine PVR $>16 \mathrm{kPa} \cdot \mathrm{s} / \mathrm{L}$.

Conclusions: PVR could be accurately estimated by VP within the pulmonary artery obtained by color M-mode echocardiography using custom software on a personal computer.

\section{6}

Coronary flow dynamics in children after repair of Tetrology of Fallot by Transthoracic Doppler echocardiography

Elhadi H Aburawi, Lund University Hospital, Department of Peadiatrics, Section of Paediatric Cradiology, SE-221 85 Lund, Sweden, Sweden

Marcus Carlsson, Lund University Hospital, Department of Clinical Physiology, SE-221 85 Lund, Sweden, Sweden
Erkki Pesonen, Lund University Hospital, Department of Peadiatrics, Section of Paediatric Cradiology, SE-221 85 Lund, Sweden, Sweden

Objectives: Open-heart surgery has been reported to increase coronary flow (CF). Myocardial fibrosis and restrictive right ventricular physiology (RRVP) might also affect the magnitude of CF in postoperative patients with Fallot's tetrology (TOF).

Methods: Echocardiography was used in 32 patients anatomically corrected for TOF to measure the flow in the pulmonary artery, posterior descending coronary artery (PDCA) and left anterior descending artery (LAD). Magnetic resonance imaging (MRI) was used to detect fibrosis in the right ventricular outflow tract. Twenty age-matched healthy children served as controls.

Results: The median age at surgery was 7.5 months (1-24 months). RRVP was found in 16 of 32 patients. Coronary flow could be registered successfully from PDCA and LAD during the same study in $24 / 32$. In those, right ventricular fibrosis was registered in 10 and 11 had no fibrosis. Coronary flow parameters were increased in both coronary arteries. In PDCA peak flow velocity in diastole, velocity time integral and coronary flow were increased more in those with RRVP as compared with those without $(39 \pm 7$ versus $32 \pm 5, \mathrm{p}<0.02 ; 17 \pm 4$ versus $15 \pm 3$, $\mathrm{p}<0.0001 ; 48 \pm 5$ versus $32 \pm 5, \mathrm{p}<0.0007$, respectively). The flow parameters increased and distributed almost equally when the patients were grouped on the basis of fibrosis instead of RRVP. Conclusion: RRVP is associated with increased coronary flow.Increased systolic workload against stiff fibrotic myocardium and earlier surgery with cardiopulmonary by-pass increase the basal coronary flow. The reported decrease of coronary flow reserve after surgery might be due to increased basal flow.

\section{7}

The Value of Cardiovascular Imaging Methods in the Diagnosis of Complex Congenital Heart Disease Evaluated by ROC Analysis

Guo-Zhen Chen, Department of Pediatric Cardiology, Shanghai Children's Medical Center, Medical College of Shanghai Jiaotong University, China

Kun Sun, Department of Pediatric Cardiology, Shanghai Children's Medical Center, China

Yu-Min Zhong, Department of Pediatric Cardiology, Shanghai Children's Medical Center, Medical College of Shanghai Jiaotong University, China

Lan-Ping Wu, Department of Pediatric Cardiology, Shanghai Children's Medical Center, Medical College of Shanghai Jiaotong University, China

Ai-Min Sun, Department of Pediatric Cardiology, Shanghai Children's Medical Center, Medical College of Shanghai Jiaotong University, China Yu-Qi Zhang, Department of Pediatric Cardiology, Shanghai Children's Medical Center, Medical College of Shanghai Jiaotong University, China

Qian Wang, Department of Pediatric Cardiology, Shanghai Children's Medical Center, Medical College of Shanghai Jiaotong University, China

Min Zhu, Department of Pediatric Cardiology, Shanghai Children's Medical Center, Medical College of Shanghai Jiaotong University, China

Objective: To evaluate the diagnostic value of imaging methods in pathological morphology of complex congenital heart disease (CHD) using receiver operating characteristic (ROC) analysis. Methods: Twenty-eight children (aged $33.7 \pm 48.1$ months) with CHD were diagnosed by three clinical-data-blinded cardiologists with two-dimensional echocardiography (2DE), real-time threedimensional echocardiography (RT-3DE), magnetic resonance 
imaging (MRI) and digital subtraction angiography (DSA), respectively. With surgical findings as "Golden Standard", the results of cardiovascular malformations were compared and grouped by a five-point categorical scale ranged from definitely abnormal to normal and further analyzed by ROC curve.

Results: To diagnose the intracardiac malformations in atria, ventricles, great arteries, atrio-ventricular and ventricular-arterial continuity, ROC curve for RT-3DE was located left-superior to that for 2DE, MRI and DSA. The area under ROC curve for RT-3DE (0.96) was higher than that for 2DE (0.92), MRI (0.91) and DSA (0.89). There was significant difference by the method of $\mathrm{Z}$ test between RT-3DE and 2DE $(\mathrm{Z}=2.30, \mathrm{P}=0.0215)$, MRI $(Z=2.80, P=0.0050)$, DSA $(Z=3.18, P=0.0015)$. While for the diagnosis of extracardiac malformations, ROC curve for DSA was located left-superior to that for MRI and 2DE. The area under ROC curve for DSA (0.99) was higher than that for MRI (0.98) and 2DE (0.92). There was significant difference between DSA and 2DE $(Z=2.67, P=0.0075)$, but no significant difference between DSA and MRI $(Z=1.52$, $\mathrm{P}=0.1292$ ).

Conclusions: ROC analysis showed that RT-3DE had advantages in displaying spatial intracardiac structures, while DSA and MRI had advantages in displaying extracardiac structures, MRI could replace angiography to some extent.

\section{8 \\ Patient specific finite element analysis: a new device for percutaneous pulmonary valve implantation \\ Claudio Capelli, UCL Institute of Child Health \& Great Ormond Street Hospital for Children, United Kingdom \\ Andrew M Taylor, UCL Institute of Child Health \& Great Ormond Street Hospital for Children, United Kingdom \\ Philipp Bonhoeffer, UCL Institute of Child Health \& Great Ormond Street Hospital for Children, United Kingdom \\ Silvia Schievano, UCL Institute of Child Health \& Great Ormond \\ Street Hospital for Children, United Kingdom}

Objective: Since 2000, percutaneous pulmonary valve implantation (PPVI) has become a reality, with over 500 patients treated worldwide. However, due to morphological limitations, the current device is only suitable for about $15 \%$ of patients requiring treatment. Recently, a new PPVI device has been developed. This preliminary study aims to mimic implantation of this new device into a selected patient's right ventricular outflow tract (RVOT) using finite element (FE) modelling, to study its positioning and anchoring in situ.

Methods: The study involved 3 phases: 1) Computed tomography (CT) scan - 3D images of the patient's RVOT anatomy were acquired throughout 10 phases of the cardiac cycle; 2) Imaging postprocessing - CT data were elaborated to reconstruct 3D volumes of the patient's RVOT over time; 3) FE analysis - each reconstructed RVOT volume was discretized and exported into the FE software Abaqus. A model of the device was designed. Expansion of the stent was simulated inside each RVOT volume (all 10 phases) to evaluate areas of contact with the RVOT wall, the stent deployed diameters, and stress and strain distribution during the cardiac cycle.

Results: At the end of the expansion the stent was safely anchored in the patient's RVOT, in both the proximal and distal parts, throughout the cardiac cycle.

Conclusion: Patient specific 3D morphological reconstructions from CT images combined with FE analyses may help clinicians in the decision process for PPVI of a new device, thus accelerating the introduction of new devices into clinical practice and increasing patient safety.
4030

Pulmonary Artery Growth After Palliation Of Congenital Heart Disease With Duct-Dependent Pulmonary Flow: Arterial Duct Stenting Vs Surgical Shunt

Giuseppe Santoro, Cardiology, Monaldi Hospital, Naples, Italy

Gianpiero Gaio, Italy

Chiara Marrone, Italy

Maria Teresa Palladino, Italy

Marianna Carrozza, Italy

Carmela Morelli, Italy

Maria Giovanna Russo, Italy

Raffaele Calabrò, Cardiology, II Unversity of Naples, Naples, ITALY, Italy

Background: Arterial duct (AD) stenting is nowadays considered a reliable alternative to surgical shunt for palliation of congenital heart disease (CHD) with duct-dependent pulmonary circulation. We hypothesized that the stented duct might better adapt to the pulmonary artery (PA) anatomy than a surgical conduit, thereby promoting a more balanced PA growth. Thus, this study compared the PA growth after AD stenting vs surgical shunt.

Methods: In 34 patients submitted to AD stenting $(\mathrm{n}=18$, Group I) or surgical shunt $(n=16$, Group II), the PA growth was assessed as PA z-scores and the Nakata Index by pulmonary angiography before palliation and at surgical repair. The right-to-left PA diameter ratio was considered as index of growth balance.

Results: The $\mathrm{AD}$ stent diameter was $3.3+0.3 \mathrm{~mm}$ and the shunt size was $4.4+0.3 \mathrm{~mm}(\mathrm{p}<0.001)$. At control angiography, the Nakata Index had significantly grown in both groups (from $145+80$ to $293+92 \mathrm{~mm} / \mathrm{m} 2, \mathrm{p}<0.0001$, Group I; from $160+104$ to $320+232 \mathrm{~mm} / \mathrm{m} 2, \mathrm{p}<0.05$, Group II) without any difference between the groups $(146+120 \%$ vs $110+111 \%, p=N S)$. However, the surgical shunt caused a preferential growth of the contra-lateral PA compared to the $\mathrm{AD}$ stenting, thereby resulting in a significant difference of the final left-to-right PA diameter ratio between the groups $(0.9+0.1$ Group I vs $1.6+0.6$ Group II, $\mathrm{p}=0.0001)$.

Conclusions: Percutaneous AD stenting is as effective as surgical shunt in promoting the pulmonary artery growth in CHD with duct-dependent pulmonary blood flow. In addition, the pulmonary flow resulting from $\mathrm{AD}$ stenting promotes a more balanced growth of PAs.

\section{1}

Initial results and medium term follow up of PAIVS patients with moderate right ventricular hypoplasia following radiofrequency valvotomy and PDA stenting Kok Kuan Choo, National Heart Institute, Malaysia Mazeni Alwi, National Heart Institute, Malaysia Hasri Samion, National Heart Institute, Malaysia Haifa Abdul Latiff, National Heart Institute, Malaysia Geetha Kandavello, National Heart Institute, Malaysia

Objectives: To assess the initial results and medium-term outcome of pulmonary atresia-intact ventricular septum (PAIVS) patients with moderate right ventricular (RV) hypoplasia after radiofrequency valvotomy (RFV) with concomitant PDA stenting. Methods: Between January 2001 and June 2008, 33 PAIVS patients with moderate RV hypoplasia underwent RFV and concomitant PDA stenting. RV size was assessed by twodimensional echocardiography and RV angiography. The finding of 'moderate RV hypoplasia' and decision for concomitant PDA stenting was determined by 2 independent observers.

Results: RFV and concomitant PDA stenting were performed successfully in 33 patients. At time of procedure, the median age 
was 10 days, median weight $3.1 \mathrm{~kg}$, mean $\mathrm{z}$-score of tricuspid annulus $-3.9+/-2.5$, mean tricuspid/mitral ratio $0.6+/$ -0.18 . There was no procedure-related mortality. There were 2 early deaths from low cardiac output syndrome and 1 late death. Mean duration of follow up was 4.3 years. Of the 30 survivors, $9(30 \%)$ were on follow up less than one year. $9(30 \%)$ had $)(\$ 1 \backslash$ tfrac $\{1\}\{2\} \$$ ventricular circulation and received bidirectional cavopulmonary shunt.12 (40\%) had complete biventricular circulation, with 6 requiring no further reintervention whereas 2 required ASD closure, 2 RV outflow tract reconstruction, one PDA restenting and one balloon dilatation of RVOT. The freedom from reintervention was 70\%, 50\% and $40 \%$ at 1 year, 2 years and 3 years respectively.

Conclusions: Primary treatment with RFV and concomitant PDA stenting for PAIVS with moderate RV hypoplasia provides good initial palliation that obviates the need for early surgical intervention. However, a significant proportion eventually required $1 \frac{1}{2}$ ventricular repair.

\section{2}

Long Term Single Centre experience with the Amplatzer Perimembranous Ventricular Septal Occluder

Lucas J Eastangh, The Royal Children's Hospital, Melbourne, Australia

Kate Briggs, The Royal Children's Hospital, Melbourne, Australia Andrew M Davis, The Royal Children's Hospital, Melbourne/Murdoch Children's Research Institute, Australia

Samuel Menahem, The Royal Children's Hospital, Melbourne, Australia

TH Goh, The Royal Children's Hospital, Melbourne, Australia James L Wilkinson, The Royal Children's Hospital, Melbourne, Australia

Geoffrey K Lane, The Royal Children's Hospital, Melbourne, Australia

Introduction: The Amplatzer perimembranous ventricular septal occluder was developed as an alternative to open heart surgery. Appropriate device positioning and sizing are important factors for a successful outcome yet clinically significant atrio-ventricular block is known to occur.

Methods: We reviewed our institution's experience of the patients who underwent percutaneous transcatheter closure of PmVSDs. Procedures were performed using general anaesthesia, with transoesophageal echocardiography guidance. Procedural success was defined as accurate placement of a device. Pre and postprocedure echocardiograms and electrocardiograms were reviewed. Additional data collected included 24 hour Holter monitors, exercise tests, and pacemaker interrogations.

Results: 33 patients underwent device closure between Dec 2002 and May 2006, with excellent haemodynamic outcomes and appropriate device positioning. Age range at implantation was $1.95-15.9$ yrs, weight range between $10.2-87.3 \mathrm{~kg}$ and median follow up time of 2.5 years. 13 patients had pre-procedure incomplete right bundle branch block (IRBBB), and one had a low atrial rhythm. Post-procedure, one patient developed firstdegree AV block, three demonstrated new IRBBB and three have complete right bundle branch block. Three patients developed complete AV block (CHB), 6 days, 657 days (1.8 yrs) and 1191 days (3.3 yrs) respectively, post-procedure. One patient with $\mathrm{CHB}$ has returned to sinus rhythm.

Conclusions: Our experience has demonstrated excellent haemodynamic results but with a high incidence of post-procedure conduction abnormalities. For this reason we no longer advocate the use of these devices for closure of PmVSDs. The appearance of $\mathrm{CHB}$ greater than three years after implantation necessitates ongoing vigilance and regular clinical follow-up.

\section{3}

Balloon Expandable Stent Implantation for Native and Recurrent Coarctation of the Aorta - Prospective Assessment of Stent Integrity, Aneurysm Formation and Stenosis Relief

Gareth J Morgan, Bristol Congenital Heart Centre, United Kingdom Damien Kenny, Bristol Congenital Heart Centre, United Kingdom Shanta Chakrabarti, Bristol Congenital Heart Centre, United Kingdom Stephanie Curtis, Bristol Congenital Heart Centre, United Kingdom Mark Hamilton, Bristol Congenital Heart Centre, United Kingdom Andrew Tometzki, Bristol Congenital Heart Centre, United Kingdom Mark Turner, Bristol Congenital Heart Centre, United Kingdom Robin Martin, Bristol Congenital Heart Centre, United Kingdom

Introduction: We report comprehensive clinical, echocardiographic and prospective computerised tomography (CT) follow-up data following aortic stent procedures from a single centre.

Stenting for aortic coarctation is known to be effective in the medium term. Aneurysm formation following stent implantation is a known complication. However, data regarding aortic wall injury and stent integrity following stent placement are sparse. Methods: Full data analysis of all patients undergoing balloon expandable stent implantation and follow up procedures in a single tertiary congenital cardiac unit.

Results: From October 2002 till April 2008, we performed 102 coarctation stent procedures on 88 patients. Median age was 20.6 years (range 8.5-65) and median weight $65 \mathrm{Kgs}$ (range 34-101). Ninety-four stents $(26$ covered $)$ were implanted. Twelve procedures were re-dilatations. Stenting resulted in a reduction of the gradient across the site of coarctation, from a median of $20 \mathrm{~mm} \mathrm{Hg}$ to $4 \mathrm{~mm} \mathrm{Hg}$. There were no procedure related deaths. Four patients had immediate complications $(1$ requiring emergency surgery). During median follow-up of 34.5 months (range 4.2-72.8), 2 patients had late complications requiring additional procedures. Follow-up CT or MRI data are available in 85 patients $(96.5 \%)$. Only 1 patient developed a procedure related aortic aneurysm. Stent fractures were detected in 7 patients $(7.9 \%)$.

Conclusions: Stenting for aortic coarctation and recoarctation is effective with low immediate complication rates. Computerized tomography is useful in the longer term assessment of stent integrity and post-procedural aneurysm formation. Overall incidence of post-procedural aneurysm is rare and stent fractures were not seen with common with newer generation stents.

\author{
4034 \\ Protecting the lung and the heart in experimental acute \\ lung injury: Low tidal voulme ventilation in combination \\ with intravenous ET-1 receptor blockade by tezosentan \\ Ralf Geiger, Pediatric Cardiology and Pulmonology, Medical University \\ Innsbruck, Austria, Austria \\ Iris Pircher, Anesthesiology and Critical Care Medicine, Medical \\ University Innsbruck, Austria, Austria \\ Martin Schwienbacher, Pediatric Cardiology and Pulmonology, Medical \\ University Innsbruck, Austria, Austria \\ Joerg I Stein, Pediatric Cardiology and Pulmonology, Medical University \\ Innsbruck, Austria, Austria \\ Alex Loeckinger, Anesthesiology and Critical Care Medicine, Medical \\ University Innsbruck, Austria, Austria
}


Background: Acute respiratory distress syndrom/acute lung injury (ARDS/ALI) can lead to excessive pulmonary hypertension $(\mathrm{PH})$ which imposes strain on the right ventricle (RV). Low tidal volume ventilation (LTVV) might be detrimental to RV-function for its potential hypercapnia.

Objective: In a pig model of acute PH secondary to ALI we evaluated the cardiopulmonary effects of LTVV with and without pulmonary vasodilation by tezosentan (TEZO), a dual ET-1 receptor blocker. We hypothesized that ET-1 blockade would attenuate $\mathrm{PH}$ in this setting.

Method: Eighteen 4-week-old white farm piglets were randomly assigned to low tidal volume ventilation (LTVV) $(6 \mathrm{ml} / \mathrm{kg})$ $(\mathrm{n}=6)$, LTVV plus continous iv TEZO $(5 \mathrm{mg} / \mathrm{kg} / \mathrm{h})(\mathrm{n}=6)$, or to normal ventilation (controls, $n=6$ ). ALI was induced by bronchoalveolar lavage with normal saline and $2 \mathrm{ml} / \mathrm{kg} 0.1$ molar hydrochloric acid.

Results: After 4 hours of ALI mean pulmonary artery pressure (mPAP) and pulmonary vascular resistance index (PVRi) as well as ratios of pulmonary to systemic artery pressure (PAP/SAP), and resistance (PVR/SVR) were lower in the LTVV + TEZO group than in controls $(\mathrm{p}<0.0001$ for all parameters), not different from baseline values (before induction of ALI). Central venous saturation was higher in the treatment groups $(71 \% \pm 6$ vs. $55 \% \pm 13, \mathrm{p}<0.002)$, while partial arterial pressure of oxygen $(\mathrm{PaO} 2)$ did not differ.

Conclusion: In experimental ALI low tidal volume ventilation in combination with dual ET-1 receptor blockade by tezosentan completely blocks pulmonary vasoconstriction, thereby deminishing right ventricular strain. Combining lung and heart protective strategies without afflicting pulmonary gas exchange might be beneficial in the treatment of ALI/ARDS.

\section{5}

Right ventricular pressure during exercise in adolescents with atrial or ventricular septal defect

Thomas Möller, Pediatric Department, Vestfold Hospital Trust, Norway Henrik Brun, Norway

Per Morten Fredriksen, Division of Rehabilitation, Rikshospitalet University Hospital, Oslo, Norway

Henrik Holmstrom, Pediatric Cardiology, Pulmology and Allergy,

Rikshospitalet University Hospital, Oslo, Norway

Kari Peersen, Department of Cardiology, Vestfold Hospital Trust, Tonsberg, Norway

Eirik Pettersen, Department of Cardiology, Rikshospitalet University

Hospital, Oslo, Norway

Ekkehard Grünig, Thoraxklinik University Hospital, Heidelberg,

Germany

Erik Thaulow, Norway

Background: Prevalence of pulmonary arterial hypertension (PAH) in adults with either closed atrial septal defect (ASD) or closed ventricular septal defect (VSD) has been reported to be $18 \%$ and $26 \%$ respectively. Abnormal right ventricular systolic pressure (RVSP) response during exercise has been identified as a precursor of PAH. Our study investigated abnormal RVSP response in young patients with isolated ASD or VSD.

Methods and results: The study comprised a population-based selection of 45 patients (age 13-25 yrs) with either isolated VSD $(n=27)$ or ASD $(n=19)$ and with no right ventricular outflow tract obstruction. 30 defects had been closed (all ASD, 11 VSD). Cardiovascular exercise testing and echocardiography at rest and during exercise were performed in all patients and in 90 healthy controls. NT-pro-BNP and inflammation markers were analysed in the patient group. Aerobic capacity was significantly reduced in all patient groups. Parameters of right ventricular performance were significantly lower in closed ASD and closed VSD as compared to controls and to patients with open VSD. Four of 87 controls (5\%), one patient with ASD (6\%) and 9 patients with VSD (33\%), regardless defect closure, showed abnormal RVSP response above $50 \mathrm{mmHg}$ during exercise.
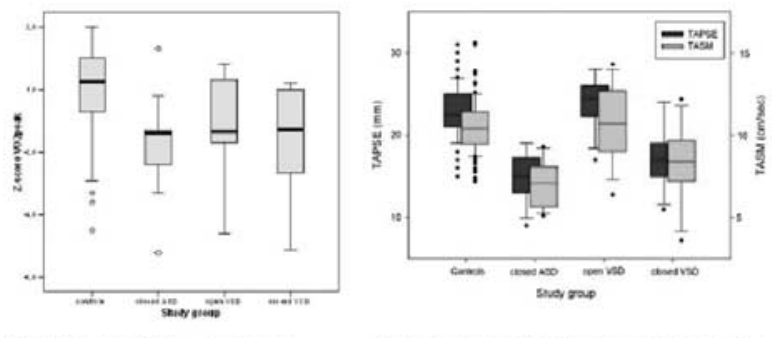

Figare 1: Z-score of $\mathrm{VO}_{2 \text { ene }}$ by diagnosis
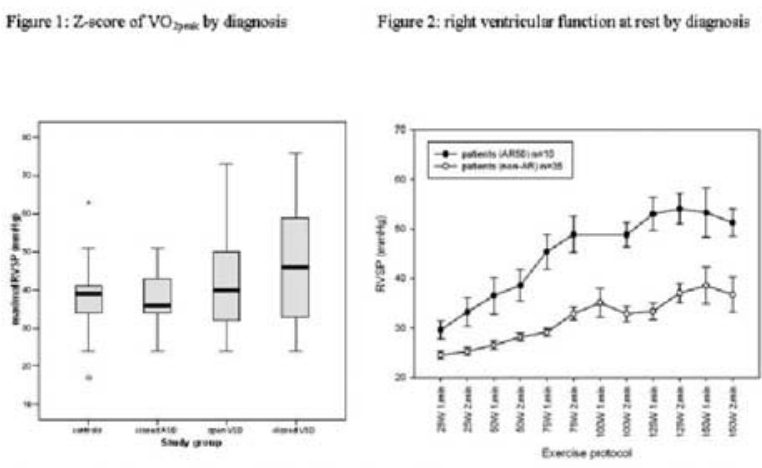

Figure 3: maximal RVSP during exercise by diagnosi

Figure 4: RVSP curves during exerise in patients
with maximal RVSP $>50$ mmHHg (ARS0) with maximal RVSP > $50 \mathrm{mmH} / \mathrm{g}$ (ARSO)
and maximal RVSP $\leq 50 \mathrm{mmH} / \mathrm{g}$ (non-

Conclusion: One third of young patients with isolated VSD have abnormal right ventricular systolic pressure response during exercise without signs of right ventricular outflow tract obstruction. Patients with isolated ASD or VSD have reduced aerobic exercise capacity and patients with closed defect also show reduced right ventricular performance at rest.

\section{6}

Role of caveolin-1 in pulmonary hypertension

Rajamma Mathew, New York Medical College, United States

Jing Huang, New York Meical College, United States

Narendra Dereddy, New York Medical College, United States

Umadevi S Katta, United States

John H Wolk, New York Medical College, United States

Michael H Gewitz, New York Medical College, United States

Objective: Our objective was to elucidate the role of caveolin-1 in human pulmonary hypertension $(\mathrm{PH})$. We have previously shown that monocrotaline (MCT)-induced $\mathrm{PH}$ in rats is preceded by a progressive reduction in endothelial caevolin-1 expression and rescue of caveolin-1 attenuates $\mathrm{PH}$. Caveolin-1, a major scaffolding protein of caveolae found on most cells including endothelial and smooth muscle cells (SMCs), interacts with several signaling molecules, and it inhibits cell proliferation. Methods: Histology, pulmonary arterial medial thickness; and endothelial cell membrane integrity and caveolin-1 expression using immunofluorescence technique were examined in lung biopsy specimens from children with $\mathrm{PH}(\mathrm{n}=6)$. In addition, rats were given $60 \mathrm{mg} / \mathrm{kg}$ MCT and examined 2 and 4 wks postMCT to evaluate $\mathrm{PH}$ and the expression of proteins of interest. Results: Clinical PH revealed not only loss of endothelial caveolin-1 but also caveolin-1 expression in SMCs. Neointima 
formation was seen in vessels with robust expression of caveolin1 in SMCs. MCT-treated rats showed progressive $\mathrm{PH}$ at 2 and 4 wks post-MCT (max.systolic pressure, $40 \pm 4 \mathrm{mmHg}$ ), loss of endothelial caveolin-1. However, neither caveolin-1 expression in SMCs nor neointima was observed.

Conclusions: Initial injury (mechanical or inflammatory) results in endothelial damage leading to cell proliferation and medial wall thickening. Robust expression of caveolin-1 in SMCs appears to be a prerequisite for neointima formation. It is likely that in $\mathrm{PH}$, caveolin-1 in SMC switches from antiproliferative to a proproliferative molecule, therefore, the use of statins to disrupt cell membrane and inhibit proproliferative activity of caveolin-1 may be beneficial in $\mathrm{PH}$.

\section{7}

\section{Does Anticoagulation in Eisenmenger Syndrome Alter} Long-Term Survival?

Julio Sandoval, Instituto Nacional de Cardiología Ignacio Chávez, Mexico

Luis Santos, Instituto Nacional de Cardiología Ignacio Chávez, Mexico Craig S Broberg, Oregon Health and Science University, United States Julio Cordova, Instituto Nacional de Cardiología Ignacio Chávez, Mexico

Tomas Pulido, Instituto Nacional de Cardiología Ignacio Chávez, Mexico Gerardo Gutierrez, Instituto Nacional de Cardiología Ignacio Chávez, Mexico

Edgar Bautista, Instituto Nacional de Cardiología Ignacio Chávez, Mexico Eulo Lupi-Herrera, Instituto Nacional de Cardiología Ignacio Chávez

Background: Strong arguments have been made both for and against anticoagulation for primary prevention in Eisenmenger syndrome, based on the known risk of hemoptysis and pulmonary artery thrombosis, though the issue has never been clinically studied.

Methods: After initial evaluation with laboratory, echocardiographic and hemodynamic data, anticoagulation in Eisenmenger patients was based on physician preference alone. We retrospectively identified patients who were started on anticoagulants (AC group) within 12 months of initial presentation and followed for at least 2 years, and compared them to non-anticoagulated patients (Non-AC).

Results: Out of 144 patients screened, We identified 48 anticoagulated and 44 non-anticoagulated patients with Eisenmenger physiology (mean $\mathrm{PAP}=73 \pm 19 \mathrm{mmHg}$, oxygen saturation $82 \pm 9 \%, \mathrm{PaO} 248 \pm 8 \mathrm{mmHg}$, hemoglobin $18.6 \pm 4 \mathrm{~g} / \mathrm{dL}$ ). There were more ASD patients in the AC group, but otherwise no baseline differences in clinical, functional, or hemodynamic data. After mean follow-up of $7 \pm 5.4$ years (range 1-31), 11 patients died in the $\mathrm{AC}$ and 10 in the Non-AC group. There was no survival difference $(\log$ rank test $=1.78 ; p=N S)$. For the entire cohort, mortality was significantly associated with NYHA class 3-4 $(\mathrm{HR}=4.2)$, evidence of right heart failure $(\mathrm{HR}=13.6)$, and a mean corpuscular volume $<80 \mu 3 \quad(\mathrm{HR}=3.8)$. Use of anticoagulation did not impact survival. Bleeding complications occurred in $7(16 \%)$ of AC patients, including two fatalities. Conclusion: Anticoagulation had no impact on long-term survival and was not benign. The data do not support the routine use of warfarin in these patients.

\section{8}

Vasoreactivity test in pulmonary arterial hypertension due to congenital heart disease (Eisenmenger syndrome): significance and impact on long term prognosis
Michele D'Alto, Chair of Cardiology, Second University of Naples, Monaldi Hospital, Naples, Italy, Italy

Paola Argiento, Chair of Cardiology, Second University of Naples, Monaldi Hospital, Naples, Italy, Italy

Emanuele Romeo, Chair of Cardiology, Second University of Naples, Monaldi Hospital, Naples, Italy, Italy

Giuliano D'Alterio, Chair of Cardiology, Second University of Naples, Monaldi Hospital, Naples, Italy, Italy

Berardo Sarubbi, Chair of Cardiology, Second University of Naples, Monaldi Hospital, Naples, Italy, Italy

Giuseppe Santoro, Chair of Cardiology, Second University of Naples, Monaldi Hospital, Naples, Italy, Italy

Maria Giovanna Russo, Italy

Raffaele Calabro', Chair of Cardiology, Second University of Naples, Monaldi Hospital, Naples, Italy

Objective: To evaluate the impact on the outcome of some invasive and non-invasive parameters in patients with unoperated Eisenmenger syndrome (ES) before starting specific treatment for $\mathrm{PAH}$. Methods. Patients, before starting specific therapy, underwent basal. At follow-up, we considered clinical worsening. Results: 29 consecutive patients (12 M, 17F, age $36.8 \pm 12.5)$ were followed-up for $33.1 \pm 11$ months. All were treated with oral bosentan. 10 patients (group A) had clinical worsening and 19 (group B) did not. At basal clinical evaluation there was no significant difference between group A and B (WHO class $3.4 \pm 0.5$ vs $3.1 \pm 0.4, p=n s)$. At $6 \mathrm{MWD}$ test group $\mathrm{A}$ and $\mathrm{B}$ were not significantly different in walking distance (310 \pm 128 vs $293 \pm 106 \mathrm{~m}, \mathrm{p}=\mathrm{ns})$ and $\mathrm{O} 2$ saturation at the end of the test $(75 \pm 6$ vs $73 \pm 8 \%, p=n s ; O 2$ saturation reduction $-11 \pm 2$ vs $-14 \pm 4 \% ; p=n s)$. At right heart catheterization there was no significant difference in $\mathrm{QP} / \mathrm{QS}(0.7 \pm 0.2$ vs $0.9 \pm 0.5)$, indexed cardiac output ( $2.9 \pm 1.1$ vs $3.2 \pm 1.21 / \mathrm{min} / \mathrm{m} 2, \mathrm{p}=\mathrm{ns})$, indexed pulmonary vascular resistances (iPVR: $19 \pm 7$ vs $23 \pm 15 \mathrm{WU} / \mathrm{m} 2$; $\mathrm{p}=\mathrm{ns})$, mean pulmonary arterial pressure $(72 \pm 18$ vs $79 \pm 14 \mathrm{mmHg} ; \mathrm{p}=\mathrm{ns}$ ). At vasoreactivity test, Group A (poorer prognosis) showed a significantly lower reduction of iPVR $(-41 \pm 13 \%$ vs $-62 \pm 18 \%, p<0.01)$. A reduction $<50 \%$ of iPVR had a sensitivity of $80 \%$ and a specificity of $79 \%$ for poorer prognosis at a long term follow-up. Only $1 / 29$ showed a positive response to the vasoreactivity test according to guidelines criteria.

Conclusions: A reduction of $\mathrm{PPVR}<50 \%$ at vasoreactivity test is associated with a poorer prognosis in Eisenmenger syndrome at a long term follow-up.

\section{9}

Bosentan Promotes Incorporation of Bone MarrowDerived Endothelial Progenitor Cells but Inhibits Infiltration of Macrophages into Pulmonary Vascular Lesions in Mice: A New Mechanism of Bosentan in Inhibiting Pulmonary Hypertension

Taichi Kato, Mie University Graduate School of Medicine, Japan Yoshihide Mitani, Mie University Graduate School of Medicine, Japan Junko Maruyama, Mie University Graduate School of Medicine, Japan Hiroyuki Ohashi, Mie University Graduate School of Medicine, Japan Masahiro Masuya, Mie University Graduate School of Medicine, Japan Hideto Shimpo, Mie University Graduate School of Medicine, Japan Kazuo Maruyama, Mie University Graduate School of Medicine, Japan Yoshihiro Komada, Mie University Graduate School of Medicine, Japan

Background: Recent studies demonstrated that administration of endothelial progenitor cells (EPCs) or suppression of several inflammatory pathways inhibited the development of pulmonary hypertension $(\mathrm{PH})$ in animals. We tested the hypothesis that 
bosentan modulates the incorporation of bone marrow (BM)derived EPCs and macrophages into pulmonary vascular lesions. Methods and Results: Lethally irradiated C57BL6 mice, transplanted with BM cells from littermates expressing eGFP, were kept in hypobaric hypoxia $(380 \mathrm{mmHg})$ or in the ambient air, and were injected with saline or bosentan sodium salt $(30 \mathrm{mg} / \mathrm{kg} /$ $\mathrm{d}$, ip), for 21 days. Compared with controls $(17.6 \pm 0.9 \mathrm{mmHg}$ ), right ventricular systolic pressure was increased in hypoxic mice $(26.6 \pm 1.0, \quad \mathrm{p}<.05)$, which was inhibited by bosentan $(22.9 \pm 1.1, \mathrm{p}<.05)$. Furthermore, compared with controls, percentages of CD31 + eGFP + BM-derived endothelial cells/ whole CD31+ cells $(4.3 \pm 0.3$ vs $1.4 \pm 0.1)$ and of MOMA2 + eGFP + BM-derived macrophages/ whole MOMA2+ cells $(38.4 \pm 5.5$ vs $17.4 \pm 2.6)$ were higher in hypoxic mice $(p<.05)$, either of which were promoted $(5.5 \pm 0.3)$ or inhibited $(18.6 \pm 2.4)$ by bosentan $(\mathrm{p}<.05)$, respectively. Consistently, compared with controls, percentages of EPCs/ whole mononuclear cells in the peripheral blood were increased in hypoxic mice, which were further increased by bosentan.

Conclusions: Bosentan promoted BM-derived EPC incorporation but inhibited macrophage infiltration into pulmonary vascular lesions. Modulation of BM-derived cells could be a new mechanism of bosentan in inhibiting $\mathrm{PH}$.

4051

Role of heme oxygenase- 1 and endothelial progenitor cells in the beneficial effects of erythropoietin on flow-associated pulmonary arterial hypertension

Rosa Laura E van Loon, Department of Pediatric Cardiology,

University Medical Center Groningen, Groningen,

The Netherlands

Beatrijs Bartelds, Department of Pediatric Cardiology, University

Medical Center Groningen, University of Groningen, Groningen,

The Netherlands

Frank ADTG Wagener, Department of Pharmacology and Toxicology,

Nijmegen Center for Molecular Life Sciences, University Nijmegen

Medical Center, The Netherlands

Mirjam E van Albada, Department of Pediatric Cardiology, University

Medical Center Groningen, University of Groningen, Groningen,

The Netherlands

Nada Affara, Department of Pediatric Cardiology, University Medical

Center Groningen, Groningen, The Netherlands

Hans Wijnberg, Department of Pediatric Cardiology, University Medical Center Groningen, Groningen, The Netherlands

Saffloer Mohaupt, Department of Pediatric Cardiology, University

Medical Center Groningen, Groningen, The Netherlands

Rolf M F Berger, Department of Pediatric Cardiology, University

Medical Center Groningen, Groningen, The Netherlands

Objectives: We previously reported that erythropoietin (EPO) improves pulmonary vascular remodeling in rats with flowassociated pulmonary arterial hypertension $(\mathrm{PAH})$. To test the hypothesis that EPO improves this remodeling through activation of heme oxygenase-1 (HO-1) and mobilization of endothelial progenitor cells (EPCs), we treated these rats with EPO with and without a HO-activity blocker (SnMP).

Methods: Flow-associated PAH was created in rats by injection of monocrotaline followed by an abdominal aorto-caval shunt. Rats were randomized to EPO $(\mathrm{PAH}+\mathrm{EPO}, \mathrm{n}=14), \mathrm{EPO}+\mathrm{SnMP}$ $(\mathrm{PAH}+\mathrm{EPO}+\mathrm{SnMP}, \mathrm{n}=13), \mathrm{SnMP}(\mathrm{PAH}+\mathrm{SnMP}, \mathrm{n}=11)$ or no treatment $(\mathrm{PAH}, \mathrm{n}=14)$. Three weeks later, hemodynamics, pulmonary vascular remodeling, number of EPCs in peripheral blood and pulmonary $\mathrm{HO}$-activity were evaluated.
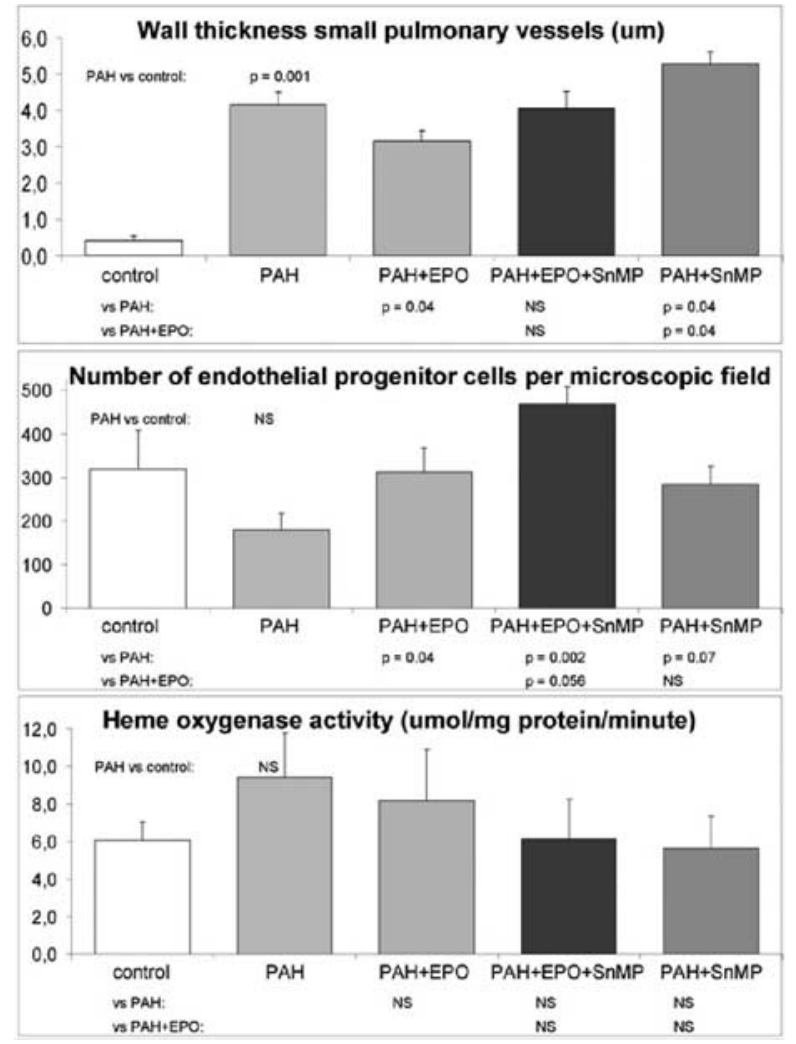

Results: Compared to PAH, wall thickness of small pulmonary vessels decreased after EPO, increased after EPO+SnMP and increased even more after SnMP treatment (Figure).

Number of EPCs increased after EPO and SnMP and increased even further after EPO+SnMP treatment. Pulmonary HO-activity remained stable after EPO and decreased after SnMP and EPO + SnMP. Pulmonary arterial pressure and right ventricular contractility remained unchanged.

Conclusion: In this rat model of flow-associated PAH, EPO improved pulmonary vascular remodeling, increased the number of EPCs in peripheral blood, but did not increase pulmonary $\mathrm{HO}$-activity. HO-activity blockade alone worsened pulmonary vascular remodeling, while increasing the number of EPCs. This indicates that both EPO and HO have beneficial effects on PAH, however, mediated via different mechanisms. The beneficial effects of EPO do not seem mediated by increased $\mathrm{HO}$-activity. Our data suggest, however, that HO may facilitate the homing of circulating EPCs to the diseased pulmonary vascular bed.

\section{2}

$\mathrm{X}$-chromosome inactivation patterns in left ventricular outflow tract obstruction: epigenetic factors of disease expression

Georgia Sarquella-Brugada, Hospital Sainte Justine, Canada

Oscar Campuzano, Hospital Sainte Justine, Canada

Roxanne Gendron, Hospital Sainte Justine, Canada

Jean Luc Bigras, Hospital Sainte Justine, Canada

Phillipe Chetaille, CHUL, Canada

Andrea Richter, Hospital Sainte Justine, Canada

Kathleen Riopel, Hospital Sainte Justine, Canada

Gregor Andelfinger, Hospital Sainte Justine, Canada

Left ventricular outflow tract obstructions (LVOTO) constitute a subgroup of congenital heart defects genetically determined. 
Significant male-gender bias is observed, pointing to involvement of X-chromosome. Skewed X-chromosome inactivation (XCI) is observed in heterozygous females carrying gene mutations involved in X-linked syndromes. We hypothesized that this nonrandom XCI may contribute to LVOTO in women.

Methods: We accessed provincial patient and DNA database to who echocardiography was performed. To assess XCI in females, we determined methylation status at human androgen receptor locus on $\mathrm{X}$ chromosome. Briefly, we assayed methylation of the different alleles by digestion of the DNA with a methylation sensitive enzyme (HpaII), followed by PCR amplification with fluoro-primers. Skewing was calculated as ratio of amplification of methylated versus unmethylated alleles. Individual data was analyzed in pedigree context. Affected status was defined as aortic stenosis, bicuspid aorta, coarctation or aortic root filation. Skewing was analyzed using three cutoffs: 70:30, 75:25 and 80:20.

Results: We identified 307 individuals distributed in 43 families; among them, 156 were females (48 affected). We found skewing in excess of 80:20 is significantly more prevalent in affected than unaffected females $(p=0.004$, odds-ratio $3.3[1.4-7.4])$. In 19 females $(11.3 \%)$, skewing seems to be protective based on possible segregation of an X-linked disease allele. When analyzing allele size, tendency is seen towards a protective or deleterious effect of certain allele-sizes action (allele-sizes 247bp and 236bp, $\mathrm{n}=18$ and $\mathrm{n}=10$, respectively; X2 $247=4.3, \mathrm{X} 2236=3.6 ; \mathrm{p}=0.08$ ).

Conclusions: Skewed $\mathrm{X}$ inactivation significantly contributes to LVOTO in affected females. In families compatible with X-linked inheritance, inactivation of affected allele can be protective against disease in women. These findings should facilitate search for LVOTO loci on X-chromosome and prospective use of XCI as clinical marker for disease progression.

\section{7}

Catheter closure of perforated sinus of valsalva

Ramesh Arora, Metro Heart Institute, India

Puroshattam Lal, Metro Heart Institute, India

Objectives: Perforated sinus of valsalva (PSOV), is usually congenital but the successful attempt to close the acquired PSOV following AVR in a high risk surgical candidate opened the possibility for transcatheter closure of PSOV.

Material \& Methods: The procedure was attempted in 14 patients, who were either not willing or were high risk candidates for surgical correction. The age range was 12 to 48 years, and all were in NYHA class III or IV. Echocardiography revealed rupture from right coronary sinus into right ventricle (RV) in 9 and noncoronary sinus into right atrium (RA) in 5 . The estimated size of the defect was $6-12 \mathrm{~mm}$. The defect was crossed retrogradely from the aortic side and an arteiovenous guidewire circuit was established. After balloon sizing, devices were successfully deployed by antegrade venous approach (Rashkind umbrella device in 2 and Amplatzer septal or ductus occluders in 12).

Results: There was complete abolition of shunt in 12, while 2 had residual shunt. One patient with residual shunt developed haemolysis, and was taken up for re-intervention but continued to have haemolysis, while the second was readmitted with IE. Both these patients were referred for surgical correction, which was done successfully. On follow-up of 4 to 146 months, there was no device embolisation and aortic regurgitation. One patient died of progressive congestive heart failure.

Conclusions: Transcatheter closure in a selective group of patients is safe and effective. Definitely, it has the advantage of obviating open heart surgery, but complete occlusion is mandatory to prevent complications.

\section{8}

Comparison of transcatheter closure of VSD between AmplatzerTM perimembranous VSD and Nit Occlud ${ }^{\circledR}$ Lê VSD Coil

Kritvikrom Durongpisitkul, Faculty of medicine Siriraj Hospital, Bangkok, Thailand, Thailand

Chodchanok Vijarnsorn, Faculty of medicine Siriraj Hospital, Bangkok, Thailand, Thailand

Jarupim Soongswang, Faculty of medicine Siriraj Hospital, Bangkok, Thailand, Thailand

Trong Phi Lê, University of Hamburg, Hamburg, Germany, Germany

Background: Ventricular septal defect (VSD) is the most common congenital heart disease. Perimembranous type (PmVSD) and doubly committed subarterial VSD (DCSA VSD) were by far the majority isolated defect in Thai children. Transcatheter device closure technique for both types of VSD has emerged as an alternative treatment.

Objective: We retrospectively reviewed our patients who underwent transcatheter closure of VSD using Amplatzer ${ }^{\mathrm{TM}}$ Perimembranous VSD device from AGA Medical Corp, USA (begin in 2003) in comparison with Nit Occlud ${ }^{\mathbb{R}}$ Le VSD Coil, Pfm AG, Cologne, Germany (begin in 2006 with Lê TP). We compared the demographic data and results of treatments including residual shunts and complication such as third degree AV block (3AVB) or pacemaker placement for both techniques in our VSD patients from 2003 to 2008. The diagnosis of PmVSD or DCSA VSD was confirmed by transesophageal echocardiography or angiographic method.

Results: There are 85 patients received closure during the study period. Device or coil was successfully implanted in 80 patients (94.1\%). The results of closure were shown in the table. Conclusions: It appeared that transcatheter closure of VSD in both Pm VSD and DCSA can be achieved by using either device. The Amplatzer ${ }^{\text {TM }}$ Perimembranous VSD had the advantage of closure a larger defect with some patients with moderate residual shunt but appeared to have a small number of $3 \mathrm{AVB}$ and required Pacemaker. The Nit Occlud ${ }^{\circledR}$ Le VSD Coil had the advantage of closure both type of defects with no 3AVB.

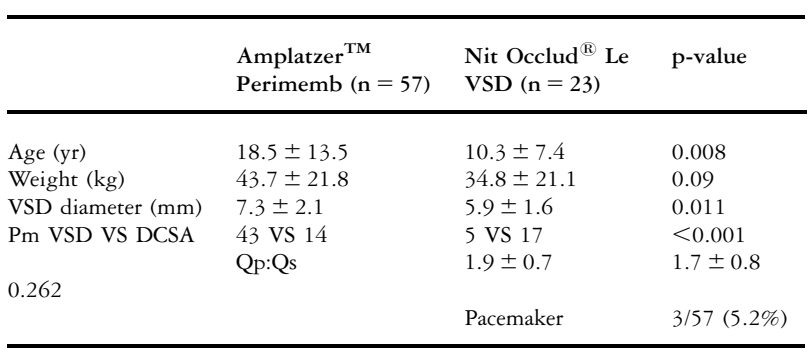

\section{9}

Percutaneous Pulmonary Valve Implantation in the Young: Medium Term Follow-up

Marko Vezmar, Labatt Family Heart Centre, SickKids ${ }^{\circledR}$, University of Toronto, Canada

Kyong-Jin Lee, Labatt Family Heart Centre, SickKids ${ }^{\circledR}$, University of Toronto, Canada

Rajiv Chaturvedi, Labatt Family Heart Centre, SickKids ${ }^{\circledR}$, University of Toronto, Canada

Claudia Almeida, McMaster University, Canada

Brian McCrindle, Labatt Family Heart Centre, SickKids ${ }^{\circledR}$, University of Toronto, Canada

Cedric Manlhiot, Labatt Family Heart Centre, SickKids ${ }^{\circledR}$, University

of Toronto, Canada 
Erick M Horlick, Peter Munk Cardiac Centre, University Health Network, Toronto General Hospital, Canada

Lee N Benson, Labatt Family Heart Centre, SickKids ${ }^{\mathbb{R}}$, University of Toronto, Canada

To investigate physiological and clinical consequence of percutaneous pulmonary valve implantation (PPVI) in patients with chronic right ventricular (RV) outflow tract obstruction and volume overload.

Between October 2005 and December 2008, 28 children underwent PPVI due to the RV outflow tract (RVOT) stenosis and/or regurgitation. The median age was 14.9 years (range 10.9-19 years) and weight $57.7 \mathrm{~kg}$ (range $40-90.6 \mathrm{~kg}$ ). The RVOT gradient was reduced $(36 \pm 15$ vs. $12 \pm 7 \mathrm{~mm} \mathrm{Hg}$, $\mathrm{p}<0.001), \mathrm{RV}$ systolic pressure fell $(61 \pm 16$ vs. $41 \pm 11 \mathrm{~mm}$ $\mathrm{Hg}, \mathrm{p}<0.001$ ), and pulmonary regurgitation (PR) improved from grade 2 or greater to none greater than grade 1 after PPVI $(\mathrm{p}<0.001)$. MRI studies demonstrated a reduction in RV enddiastolic volume $\left(148 \pm 48\right.$ vs. $\left.114 \pm 35 \mathrm{ml} / \mathrm{m}^{2}, \mathrm{p}<0.005\right)$, and increase in left ventricular stroke volume $(52 \pm 12$ vs. $\left.55 \pm 14 \mathrm{ml} / \mathrm{m}^{2}, \mathrm{p}<0.008\right)$. Aerobic exercise performance improved ( $24 \pm 5$ vs. $28 \pm 4 \mathrm{ml} / \mathrm{kg} / \mathrm{min}, \mathrm{p}<0.0001)$. RV function was preserved and PR was absent in majority of children 24 months after the implant by echocardiographic study: with RV to aortic pressure ratio $(0.75 \pm 0.16$ before PPVI, vs. after $0.54 \pm 0.13, p<0.001)$ and $\mathrm{RV}$ to PA peak pressure gradient (67 \pm 23 before, vs. after $32 \pm 11 \mathrm{~mm} \mathrm{Hg}, \mathrm{p}<0.001)$. Freedom from reoperation was $91 \%, 83 \%, 83 \%$, and from transcatheter reintervention $91 \%, 80 \%, 80 \%$, at 12,24 and 36 months respectively.

PPVI is feasible and safe in young patients with dilated RVs. There is an immediate improvement in hemodynamics and very low rate of surgical re-intervention. Long term follow-up demonstrates marked improvement in ventricular parameters, PR and objective improvement in exercise capacity.

\section{0}

Transcatheter fenestration creation and stenting of the Fontan circuit with "diabolo" stents

Vinay K Bhole, Birmingham Children's Hospital, United Kingdom Zdenka Reinhardt, Birmingham Children's Hospital, United Kingdom Joseph V DeGiovanni, Birmingham Children's Hospital, United Kingdom

John GC Wright, Birmingham Children's Hospital, United Kingdom Paul A Miller, Birmingham Children's Hospital, United Kingdom Rami Dhillon, Birmingham Children's Hospital, United Kingdom Arivalagan Kannivelu, Birmingham Children's Hospital, United Kingdom

Oliver Stumper, Birmingham Children's Hospital, United Kingdom

Introduction: The role of fenestration in the Fontan circuit in the early and late postoperative period remains controversial. We present our experience of fenestration creation or optimization using transcatheter techniques.

Materials and methods: Retrospective review of records of all the patients who underwent transcatheter fenestration of the Fontan circulation between $1 / 1998$ to $12 / 2008$.

Results: Nineteen patients underwent interventional creation/ augmentation of Fontan fenestrations (median interval - 29 days (2 days -5.5 years) postoperatively). Indication for fenestration was prolonged chylothorax in $9(47 \%)$, Low cardiac output state (LCOS) in 2 (11\%), Protein Losing Enteropathy (PLE) in 4(21\%) and plastic bronchitis in $4(21 \%)$ patients. All patients, except 3 had extracardiac Fontan. Transcatheter fenestration was created in 6 patients. We used peripheral Jostents with "diabolo configuration" (waist $4-5 \mathrm{~mm}$ ) for fenestration stenting in all. There was a significant drop in saturations $(p<0.001)$ of $9 \%$ (95\% CI: 6-12) and mean Fontan pressure of $3 \mathrm{~mm} \mathrm{Hg}$ (95\% CI: $1-4)$. There was no catheter related mortality. There was one episode of transient neurologic event and one pericardial effusion requiring drainage.

There was resolution of chylothorax, PLE and bronchial casts in all and cardiac output improved in both patients with LCOS. Stent waist size was reduced in 2 but none have occluded. Conclusions: Transcatheter creation and stenting of Fontan fenestration is a safe and effective procedure for a failing Fontan circulation. Stents with a diabolo configuration provide excellent palliation and long-term patency.

\section{1}

Aortic valvuloplasty in children: impact of rapid ventricular pacing

Georgia Sarquella-BRugada, Hospital Sainte-Justine, Canada

Sylvia Abadir, Hospital Sainte-Justine, Canada

Josep Rodés-Cabau, CHUL, Québec, Canada

Christine Houde, CHUL, Québec, Canada

Adrian Dancea, Montreal Children's Hospital, Canada

Nagib Dahdah, Hospital Sainte-Justine, Canada

Joaquim Miró, Hospital Sainte-Justine, Canada

Introduction: Ventricular pacing for balloon stabilization in aortic valvuloplasty is increasingly used. We sought to evaluate its safety and benefits in a children population.

Methods: Retrospective review of ten year experience in three pediatric centers. Control group was obtained based on age of patients and catheterizer in a match proportion of 2 non-paced to 1 paced procedure.

Results: Overall, 140 procedures were performed in 130 patients with mean follow-up time of 32,07 $\pm 34,98$ months . There was male predominance $(68,3 \%)$ with mean age of $5,6 \pm 6,97$ years, and mean weight of $24,24 \pm 26,68 \mathrm{~kg}$ at procedure. Ventricular pacing has been used in 23 aortic valvuloplasties $(16,4 \%)$ since 2003.

Paced patients and control group were similar for procedure duration, radiation time and final gradient relief measured by echocardiography and catheterization. Non-paced procedures were much more associated with multiple $(>3)$ balloon inflations $(41,3 \%$ vs. $4,34 \%, \mathrm{X} 210,174 \mathrm{p}=0,001)$, significant increase of aortic regurgitation at 1 year $(28,2 \%$ vs. $4,34 \%, \quad \mathrm{X} 2$ $5,42 \mathrm{p}=0,017)$ and last follow-up $(30,4 \%$ vs. $4,34 \%, \mathrm{X} 2$ $6,133 \mathrm{p}=0,011)$. Non-paced procedures were associated with a higher risk of reintervention $(23,9 \%$ vs. $4,34 \%, \mathrm{X} 24,086$ $\mathrm{p}=0,039)$.

There was no difference in arrhythmia inducibility due to ventricular pacing $(13,4 \%$ vs. $8,69 \%$, X2 $0,87 \mathrm{p}=0,7)$.

Conclusion: Rapid ventricular pacing for aortic valvuloplasty does not increase the risk of arrhythmias, simplifies the procedure and is associated with lower rates of reintervention possibly by diminishing the number of balloon inflations needed.

\section{2}

Long term results of pulmonary arteries rehabilitation in patients with pulmonary atresia, ventricular septal defect, pulmonary arteries hypoplasia and major aortopulmonary collaterals

Andreea Dragulescu, Timone Children's Hospital, Marseille, France Virginie Fouilloux, Timone Children's Hospital, Marseille, France 
Bernard Kreitmann, Timone Children's Hospital, Marseille, France Pascal Amedro, Arnaud de Villeneuve Hospital, Montpellier, France Dominique Metras, Timone Children's Hospital, Marseille

Alain Fraisse, Timone Children's Hospital, Marseille

Objectives: To report long-term results of pulmonary artery rehabilitation in pulmonary atresia, ventricular septal defect, hypoplastic pulmonary arteries and major aortopulmonary collaterals.

Methods: Since 1993, 20 patients with profound pulmonary arteries hypoplasia (mean Nakata index $26 \pm 14 \mathrm{~mm}^{2} / \mathrm{m}^{2}$ ) benefited from a medico-surgical strategy of native pulmonary arteries rehabilitation with an ultimate goal to achieve anatomical repair with satisfactory hemodynamics (right ventricle to aortic pressure ratio $<0.8$ ).

Results: The first step, right ventricle to pulmonary artery connection, was performed at a median age of 4.1(0.1-18.7) months with one operative death. After a median duration of 4.3(1.1-26) months, followed the 2nd step of interventional catheterizations (median: 2(1-7)/patient) consisting of 36 pulmonary angioplasties, 11 stent implantation and 20 collaterals occlusions. Significant pulmonary arteries growth was obtained in all cases with a Nakata index of $208 \pm 85 \mathrm{~mm}^{2} / \mathrm{m}^{2}$ before surgical correction $(\mathrm{p}<0.001)$. The 3 rd step of surgical repair was performed at a median age of 1.9(0.6-10.7) years, with right ventricular outflow reconstruction and ventricular septal defect patch closure, fenestrated in 3 cases. During a mean follow-up of $8.2 \pm 4.5$ years, pulmonary arteries rehabilitation was pursued in most patients, with 47 pulmonary angioplasties, 15 stent implantation and 11 collaterals occlusion. Three patients with poor hemodynamic result died. At last visit, the 16 survivors are in NYHA class I $(n=12)$ or II $(n=4)$ with satisfactory hemodynamics in 13 cases.

Conclusion: Medico-surgical pulmonary arteries rehabilitation is successful in the majority of cases allowing complete repair with an early and aggressive approach. However, long term management often requires pursuit of the rehabilitation process.

\section{3 \\ Is it Possible to Estimate the globe contractile function of right ventricular Using Ultrasound Strain Rate Imaging? Kun Sun, Department of Pediatric Cardiology, Shanghai Children's Medical Center, China \\ XiangChun Meng, Department of Pediatric Cardiology, Shanghai \\ Children's Medical Center, Shanghai Jiaotong University, China \\ YuQi Zhang, Department of Pediatric Cardiology, Shanghai Children's Medical Center, Shanghai Jiaotong University, China \\ LiPing Yao, Department of Pediatric Cardiology, Shanghai Children's Medical Center, Shanghai Jiaotong University, China \\ GuoZhen Chen, Department of Pediatric Cardiology, Shanghai \\ Children's Medical Center, Shanghai Jiaotong University, China}

Objective: To evaluate the validation of ultrasound-based strain rate imaging (SRI) in the quantitative assessment of the right ventricular (RV) globe contractile function. Methods Tissue Doppler images of whole longitudinal RV free wall were recorded from the apical 4-chamber view in 7 closed-chest anesthetized mongrel dogs during 5 different inotropic states. $\mathrm{dP} / \mathrm{dTmax}$ of $\mathrm{RV}$ in the isovolumic contraction period was obtained simultaneously in each inotropic state during the right cardiac catheterization, and served as the gold standard of RV contractility. Peak systolic velocities (V), peak systolic strain rates (SR), peak systolic strains (S) at the basal and middle segment of $\mathrm{RV}$ free wall from the longitudinal axis were quantitatively measured using commercial QLAB software system respectively.
The above peak systolic indices were compared against $\mathrm{dP} /$ dTmax by linear correlation. Results The peak systolic indices at the basal and middle segments of longitudinal RV free wall increased with the infusion of dobutamine and decreased with the infusion of esmolol. A significant correlation was found between RV dP/dTmax and peak systolic indices of RV free wall. But the most significant correlation was between the peak systolic SRs and $\mathrm{dP} / \mathrm{dTm} \max$ (with the peak systolic SR at the basal segment $r=0.9197, p<0.01$; with the corresponding one at the middle segment $\mathrm{r}=0.9033, \mathrm{p}<0.01)$. Conclusion Ultrasound-based SRI could quantitatively estimate RV globe contractile function. Peak systolic SRs determined at the longitudinal RV free wall might be a good noninvasive indices of RV globe contractility.

\author{
4064 \\ Assesment of Ventricular Function and Synchrony by \\ Tissue Dopler Echocardiography in Children with \\ Congenital Complete Heart Block and Ventricular Pacing \\ Patricia Alvarez, CLINICA ALEMANA - HOSPITAL \\ ROBERTO DEL RIO, Chile \\ Valeria Acevedo, CLINICA ALEMANA - HOSPITAL \\ ROBERTO DEL RIO, Chile \\ Rodrigo Neghme, CONGENITAL HEART INSTITUTE, \\ ORLANDO - FL., United States \\ Gilberto Palominos, HOSPITAL ROBERTO DEL RIO, Chile \\ Katty Quezada, HOSPITAL ROBERTO DEL RIO, Chile \\ Marcela Alburquenque, HOSPITAL ROBERTO DEL RIO, Chile \\ Daneil Aguirre, CLINICA ALEMANA - HOSPITAL \\ ROBERTO DEL RIO, Chile
}

Manuel Pinto, CLINICA ALEMANA, Chile

Ventricular pacing is an accepted therapy for congenital complete atrioventricular block (CCAVB). Electromechanical dyssynchrony may play a role in the development of cardiomyopathy. Objetive: To determine the effect of ventricular pacing in cardiac function and synchrony in paced patients with CCAVB.

Methods: We compared 25 patients with CCAVB with 30 healthy children with conventional echocardiography, TDI, TSI, strain, strain rate, maximal difference in $12 \mathrm{LV}$ segments for Ts and Ts-SD. Results: The median age at pacemaker implatation was 30 months with a median length of follow-up of 63 months. Our report compares findings between the paced and control groups. LV, RV and LA sizes were similar. A difference was not found in systolic and diastolic LV function. A significant difference was observed in tricuspid tissue Doppler: e' $0.13 \mathrm{v} / \mathrm{s} 0.15 \mathrm{~m} / \mathrm{s}(\mathrm{p}=0.021)$, E/e' $5.8 \mathrm{v} / \mathrm{s} 4.3(\mathrm{p}<0.001)$ and s' $0.08 \mathrm{v} / \mathrm{s} 0.12 \mathrm{~m} / \mathrm{s} \quad(\mathrm{p}<0.001)$. Maximal difference in $12 \mathrm{LV}$ segments was $109 \mathrm{v} / \mathrm{s} 89 \mathrm{~m} / \mathrm{s}$ $(p=0.004)$ for Ts and $36 \mathrm{v} / \mathrm{s} 30 \mathrm{~m} / \mathrm{s}$ Ts-SD $(p>0.05)$. Strain was similar in both groups. A significant difference $(\mathrm{p}<0.001)$ was found between septum and LV, septum and RV, and RV and LV when evaluating synchrony with TDI y TSI.

Conclusion: Conventional echocardiography did not find differences between groups. However, tissue Doppler demonstrated abnormalities in RV function and LV synchrony. These findings could play a role in the development of late cardiomyopathy. Doppler and strain rate imaging may become useful in the long term follow-up of CCAVB patients.

\section{5}

Abnormal Left Ventricular Torsion and Untwist Rate in Patients with Myocarditis

Nee Scze Khoo, Stollery Children's Hospital, Canada 
Andrew Mackie, Stollery Children's Hospital, Canada Jeffrey Smallhorn, Stollery Children's Hospital, Canada Ian Paterson, University of Alberta Hospital, Canada

Background: Conventional echocardiography has low sensitivity in detecting patients with myocarditis and normal left ventricular ejection fraction (LVEF). Speckle tracking imaging allows measurement of LV torsion and untwist, an alternative measure of LV function. Abnormalities of these parameters may identify patients with myocardial inflammation.

Method: We consecutively enrolled patients (10-45 yrs) with chest pain and elevated troponin when ischemia has been excluded on magnetic resonance imaging (MRI) or angiography. All patients had ECG, echocardiography and MRI. Angiography was performed if an ischemic cause was suspected. LV torsion parameters were compared with age-matched controls.

Results: We recruited 10 patients with 1 excluded for poor image quality. Of the 9 patients analyzed, 7 have features of myocarditis on MRI. Patient 1 to 3 had reduced torsion and untwist rate. Patient 1 and 2 on follow up showed recovery of torsion and untwist rate. Patient 4 to 8 had increased basal rotation rate (rotR) and Patient 8 had increased torsion. Details are summarized in a table. Tissue Doppler imaging, LVEF and other measured LV torsion parameters were non-differentiating. Conclusion: Abnormality in LV torsion, untwist rate or basal rotR identified all patients with myocarditis. Reduction in torsion and untwist rate normalized with patient recovery. Enhanced LV basal rotR and torsion may reflect early compensatory mechanism to maintain LV function in myocarditis with normal LVEF.

\author{
4066 \\ Rapid Three Dimensional Echocardiography Assessment \\ of Right Ventricular Volume and Function in Congenital \\ Heart Disease: Comparison with Cardiac Magnetic \\ Resonance Imaging \\ Nee Scze Khoo, Stollery Children's Hospital, Canada \\ Alistair Young, Department of Anatomy with Radiology, University of \\ Auckland, New Zealand \\ Christopher Occleshaw, Department of Radiology, Auckland Hospital, \\ New Zealand \\ Brett Cowan, Centre for Advanced MRI, University of Auckland, \\ New Zealand \\ Irene Zeng, Department of Cardiology, Auckland Hospital, New Zealand \\ Thomas L Gentles, Green Lane Paediatric \& Congenital Cardiac \\ Services, Starship Children's Hospital, New Zealand
}

Background: Quantitative assessment of right ventricular (RV) volumes with 2-dimensional echocardiography is unreliable. Three-dimensional echocardiography (3DE) is promising but limited by time consuming analysis. Utility of 3DE for RV volume estimation is uncertain in congenital heart disease where RV dilatation and dysfunction is common. This study compares 3DE measurements of RV volumes and ejection fraction (EF) obtained by manual tracing methods; disc summation (DS) and 3DE apical rotation (AR), with automated border detection (ABD) algorithm and with manual adjustment (MABD) in patients with congenital heart disease. 3DE measurements were compared with cardiac magnetic resonance imaging (MR).

Method and Results: Twenty-eight (52\%) of 54 consecutively recruited patients (median age, 17 years) with congenital heart disease had adequate 3DE imaging. All MRI images were adequate. Analysis were performed by separate observers for the different modalities. There was a wide range of RV size (MR EDVi $143 \pm 43 \mathrm{ml} / \mathrm{m}^{2}$ ) and function (MR EF $48 \pm 10 \%$ ). RT3DE systematically underestimated EDV $(\mathrm{p}<0.001)$ with greater underestimation in dilated RVs $(r=0.60, p<0.001)$. There was no significant difference for EF measurement in all methods except ABD $(-2.6 \% \pm 6, p=0.03)$. No significant difference in inter and intra-observer reproducibility for EF between $3 \mathrm{DE}$ and MR. Mean analysis time for ABD was 5 compared with 13-19 minutes for MABD, DS and AR $(p<0.0001)$

Conclusion: Approximately half of patients with congenital heart disease had adequate 3DE images. 3DE accurately estimates EF but underestimates volumes, particularly when the RV is dilated. ABD minimally underestimates EF but offers significant analysis time reduction.

\begin{tabular}{llll}
\hline Variables & $\begin{array}{l}\text { EDVi [\% difference], EF } \\
\text { [absolute difference] } \\
\text { mean, SD }\end{array}$ & $\begin{array}{l}\text { Difference } \\
\text { (3DE-MR) vs. MR, } \\
\text { p value }\end{array}$ & $\begin{array}{l}\text { 3DE vs. MR, } \\
\text { r value }\end{array}$ \\
\hline DS EDVi & $-19.3,14$ & $<0.001$ & 0.91 \\
AR EDVi & $-19.1,13$ & $<0.001$ & 0.92 \\
ABD EDVi & $-21.6,13$ & $<0.001$ & 0.89 \\
MABD EDVi & $-22.1,12$ & $<0.001$ & 0.91 \\
DS EF & $-0.5 \%, 6.9$ & 0.72 & 0.78 \\
AR EF & $-0.7 \%, 6.4$ & 0.52 & 0.79 \\
ABD EF & $-2.6 \%, 6.0$ & 0.03 & 0.83 \\
MABD EF & $+1.4 \%, 6.9$ & 0.31 & 0.78 \\
\hline
\end{tabular}

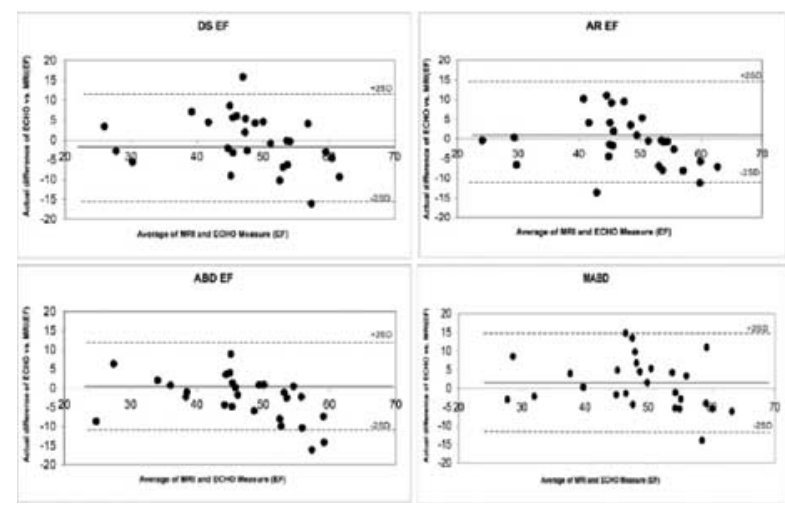

4067

Right Ventricular Ejection Fraction, Measured During Routine Inter-stage Cardiac Magnetic Resonance Imaging, Predicts Outcome for Patients with Hypoplastic Left Heart Syndrome

Marina L Hughes, Great Ormond Street Hospital for Children, London, UK, United Kingdom

Vivek Muthurangu, Great Ormond Street Hospital for Children, London, UK, United Kingdom

Andrew M Taylor, Great Ormond Street Hospital for Children, London, United Kingdom

Victor Tsang, Great Ormond Street Hospital for Children, London, UK, United Kingdom

Katherine Brown, Great Ormond Street Hospital for Children, London, UK, United Kingdom

Background: A protocol involving cardiac MRI for inter-stage assessment of all infants with hypoplastic left heart syndrome (HLHS) has been adopted by our unit since 2003 .

Methods: All locally followed survivors of the Norwood I procedure were included, following imaging under GA on a $1.5 \mathrm{~T}$ scanner. Coarctation (CoA) size was indexed against diaphragmatic descending aorta. The primary outcome measure was survival to analysis date (October 08). Secondary, functional outcome measures were RV ejection fraction (RVEF), and cardiac output. 
Results: The cohort comprised 30 patients; of which 15 were Sano-type, with median age and weight at scan 91 (33-291) days, and $5(3.2-11) \mathrm{kg}$.

The median CoA area index for all patients was $0.52(0.22-1.0)$. Twenty-one $(70 \%)$ had a CoA area index $<0.7$. There was significant correlation between CoA index and cardiac output $(\mathrm{p}=0.04)$, but CoA indices were not correlated with RVEF.

The median RVEF for this cohort was 53\% (30-81\%). There were 7 deaths, during total follow-up time 67 person-years.

The RVEF was strongly predictive of death, with hazard ratio $0.92(\mathrm{p}=0.02)$. Other factors, such as the CoA and PA indices, anatomical type, age at MRI and cardiac output, did not predict death.

Conclusions: Cardiac MRI provides comprehensive 3D imaging and gives functional indices that predict outcome. Death is more likely in HLHS patients with lower RV ejection fraction at interstage MRI. Measures to preserve RV systolic function are paramount in the complex management of these patients.

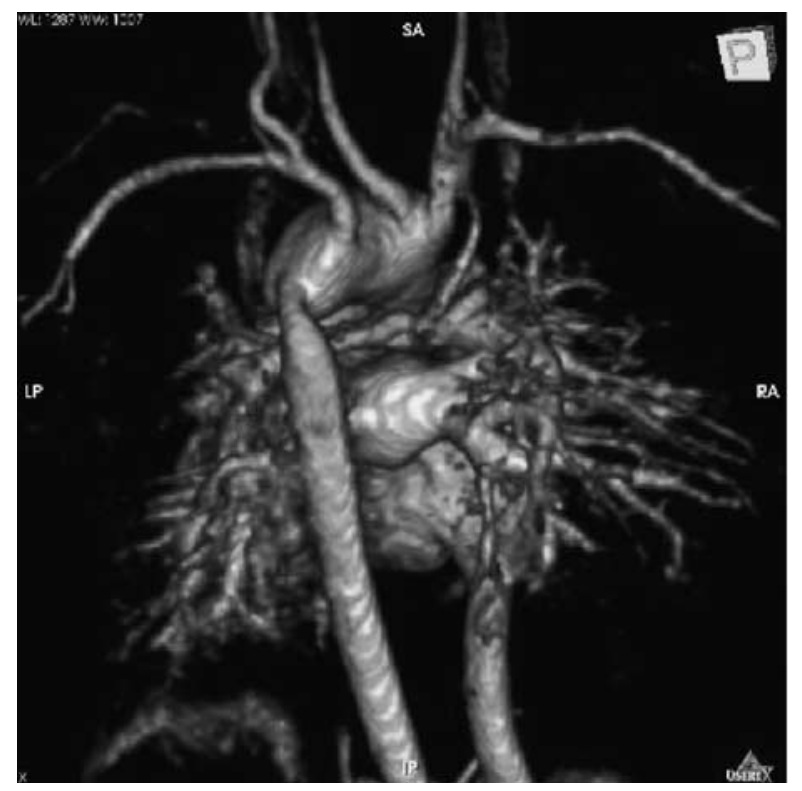

\section{8}

Cardiac Effects of Antiretroviral Therapy in HIV-Negative Infants Born to HIV-Positive Mothers: The Prospective NHLBI CHAART-1 Study

Steven E Lipshultz, University of Miami, United States

William T Shearer, Baylor College of Medicine, United States Bruce Thompson, Clinical Trials and Survey Corporation, United States Kenneth C Rich, University of Illinois- CHicago, United States Irene Cheng, Clinical Trials and Survey Corporation, United States James D Wilkinson, University of Miami, United States Steven D Colan, Harvard University, United States

Background: Antiretroviral therapy (ART) has reduced vertical transmission of HIV. However, long-term cardiotoxicity to children after in utero exposure to ART is unknown but has occurred in animals.

Methods: We compared echocardiograms taken between birth and 24 months in two groups of HIV-negative infants of HIVpositive mothers: one group of 136 infants who had been exposed to ART (ART + ) and the other of 216 infants who had not (ART-).

Results: Mean left ventricular (LV) mass z-scores ranged from -0.09 to -1.42 below normal in both groups and were consistently lower in ART + girls than in ART - girls: the differences in mean $\mathrm{Z}$-scores were -0.40 at birth $(\mathrm{P}=0.01)$, -0.99 at 6 months $(\mathrm{P}<0.001),-0.73$ at 12 months $(\mathrm{P}<0.001)$, and -0.86 at 24 months $(\mathrm{P}<0.001)$. Corresponding differences in $\mathrm{Z}$-scores for boys were smaller: 0.13 at 1 month $(\mathrm{P}=0.41)$, $-0.45(\mathrm{P}=0.01)$ at 6 months, $-0.19(\mathrm{P}=0.24)$ at 12 months, and -0.32 at 24 months $(P=0.05)$. Septal wall thickness and LV dimension were smaller than expected in ART + infants, but LV contractility was consistently about $1 \mathrm{SD}$ higher at all ages $(\mathrm{P}<0.001)$. In ART + infants, LV fractional shortening was higher than in ART - infants: girls showed a greater difference. Conclusion: Fetal exposure to ART is associated with reduced LV mass, LV dimension, and septal wall thickness Z-scores and increased LV fractional shortening and contractility during the first 2 years of life. These effects are more pronounced in girls than in boys. Fetal exposure to ART may impair myocardial growth while improving LV function.

\author{
4069 \\ Gender differences in long-term dexrazoxane \\ cardioprotection in doxorubicin-treated children with \\ acute lymphoblastic leukemia \\ Steven E Lipshultz, University of Miami Miller School of Medicine, \\ United States \\ Rebecca E Scully, University of Miami Miller School of Medicine, \\ United States \\ Stuart R Lipsitz, Brigham and Women's Hospital, United States \\ Steven E Sallan, Dana-Farber Cancer Institute, United States \\ Lewis B Silverman, Dana-Farber Cancer Institute, United States \\ Tracie L Miller, University of Miami Miller School of Medicine, \\ United States \\ E John Orav, Brigham and Women's Hospital, United States \\ Steven D Colan, Children's Hospital Boston, United States
}

Objective: To evaluate the long-term effect of the cardioprotectant dexrazoxane (DZR) in children undergoing doxorubicin (DOX) chemotherapy for leukemia.

Methods: We centrally remeasured echocardiograms from childhood leukemia survivors who were randomly assigned to treatment with DOX only $\left(\mathrm{n}=66 ; 30 \mathrm{mg} / \mathrm{m}^{2} /\right.$ dose for 10 doses) or DOX plus DZR ( $\mathrm{n}=68 ; 300 \mathrm{mg} / \mathrm{m}^{2} /$ dose).

Results: Five years post-treatment, mean LV end systolic dimension (ESD) z-score was significantly larger than predicted for body-surface area for DOX (mean $=0.46$, P-value deviation of $_{\text {. }}$ mean from normal $=0.01)$ but not for DZR/DOX $(0.06, \mathrm{P}=0.74)$; DOX LV fractional shortening (FS; $-0.78, \mathrm{P}=.001$; DZR/ $\mathrm{DOX}=-0.38, \quad \mathrm{P}=0.11$ ) and thickness to dimension ratio $(-0.96, \mathrm{P}<0.001 ; \mathrm{DZR} / \mathrm{DOX}=-0.32, \mathrm{P}=0.08)$ were also abnormal. LV end diastolic posterior wall thickness (EDPWT) was reduced in both groups $(1.19, \mathrm{P}<0.001)$ than DZR/DOX $(-0.74, \mathrm{P}<0.001)$.

By gender, LVESD z-score was significantly larger than normal for DOX males (mean $=0.48, \mathrm{P}=0.04$ ) but not DZR/DOX males $(0.19, \mathrm{P}=0.41)$ or females (DOX female $=0.38, \mathrm{P}=0.22$; DZR/ DOX female $=-0.17, \mathrm{P}=0.56)$. LVFS $\mathrm{z}$-score was significantly different from normal in DOX females (mean $=-1.29, \mathrm{P}<0.001$ ), but not DZR/DOX females $(-0.22, \quad \mathrm{P}=0.54)$ or males $(\mathrm{DOX}=-0.45, \mathrm{P}=0.15 ; \mathrm{DZR} / \mathrm{DOX}=-0.52, \mathrm{P}=0.09)$, as was LV thickness to dimension ratio (DOX female $=-1.03$, $\mathrm{P}<0.001 ;$ DZR/DOX female $=0.02, \mathrm{P}=0.93) . \mathrm{DZR} / \mathrm{DOX}$ females were the only group with normal LVEDPWT $z$-score (mean $=-0.43, \quad \mathrm{P}=0.07 ; \quad \mathrm{DOX} /$ female $=-1.43, \quad \mathrm{P}<0.001$; DOX male $=-1.05, \quad \mathrm{P}<0.001 ; \quad$ DZR/DOX male $=-0.94$, $\mathrm{P}<0.001)$. 
Conclusions: Though seen in both genders, primarily females drive the long-term DZR cardioprotective effect. DZR/DOX females exhibit more normal LV dimensions and more appropriate wall thickness for LV dimension, consistent with less LV remodeling.

\author{
4070 \\ Genetic Analysis in Patients with Left Ventricular \\ Noncompaction and Evidence for Genetic Heterogeneity \\ Fukiko Ichida, University of Toyama, Japan \\ Yanlin Xing, University of Toyama, Japan \\ Lishen Shan, University of Toyama, Japan \\ Rui Chen, University of Toyama, Japan \\ Sayaka Watanabe, University of Toyama, Japan \\ Shinichi Tsubata, University of Toyama, Japan \\ Neil E Bowles, University of Utah School of Medicine, United States \\ Jeffrey A Towbin, Bayler College of Medicine, United States
}

Left ventricular noncompaction (LVNC) is a cardiomyopathy characterized by numerous excessively trabeculations and deep intertrabecular recesses. This study was performed to investigate Japanese LVNC patients for disease-causing mutations in a series of selected candidate genes. DNA was isolated from the peripheral 103 patients including 44 cases from 20 families and 59 sporadic cases. DNA samples were screened for mutations in the genes encoding G4.5 (TAZ), $\alpha$-dystrobrevin (DTNA), $\alpha 1-$ syntrophin (STNA1), FK506 Binding Protein 1A (FKBP1A or FKPB12: FKBP1A), and LIM Domain Binding protein 3 (Cypher/ZASP: LDB3), using single-strand conformational polymorphism analysis (SSCP) and DNA sequencing. DNA variants were identified in 17 of the 103 patients, including 15 cases from 4 families and 2 sporadic cases. A splice acceptor mutation of intron 8 in TAZ (IVS8-1G $>$ C) was identified in one family with isolated LVNC, resulting in deletion of exon 9 from mRNA. In a sporadic case of isolated LVNC and Barth syndrome (BTHS), a 158insC in exon 2 of TAZ resulting in a frame-shift mutation was identified. A $1876 \mathrm{G}>\mathrm{A}$ substitution changing an aspartic acid to asparagine (D626N) was identified in LDB3 in four members of two families with LVNC. A $163 \mathrm{G}>\mathrm{A}$ polymorphism was identified in LDB3, which changed a valine to isoleucine (V55I) in one patient with isolated LVNC. In addition, in a family with nonisolated LVNC, a 362C $>\mathrm{T}$ mutation was identified in DTNA. LVNC, like other forms of inherited cardiomyopathy, is a genetically heterogeneous disease, associated with variable clinical symptoms and can be inherited as an autosomal or X-linked recessive disorder.

\section{1}

Endocardial Radiofrequency Septal Reduction-The 'other' treatment option for Hypertrophic Obstructive Cardiomyopathy (HOCM) in Children

Sujeev K Mathur, Birmingham Children's Hospital, United Kingdom Vinay K Bhole, Birmingham Children's Hospital, United Kingdom Arivalagan Kannivelu, Birmingham Children's Hospital, United Kingdom Rosemary A Edgar, Birmingham Children's Hospital, United Kingdom Jo-Anne M Timmins, Birmingham Children's Hospital, United Kingdom

Joseph V DeGiovanni, Birmingham Children's Hospital, United Kingdom

Objectives: To evaluate the efficacy and safety of endocardial radiofrequency septal reduction in the management of HOCM in children.

Method: Retrospective analysis of patient records over a ten year period (1998-2008).
Results: Twelve patients with median age of 10.2 years (range 4-17.2) and median weight of $31.3 \mathrm{~kg}$ (range 16-68.7) underwent 14 procedures. The mean (SD) peak systolic echo gradient across LVOT was 87.6( \pm 24.1$) \mathrm{mm} \mathrm{Hg}$. A mean (SD) of 20( \pm 9.7$)$ lesions were applied under fluoroscopic and trans-oesophageal echo guidance using a cooled tip ablation catheter whilst monitoring the cardiac electrogram for the presence of a His bundle potential. Mean (SD) fluoroscopy time was $25.8( \pm 6.4)$ minutes. Mean (SD) post-procedure Troponin-T level was $3.6( \pm 2.8) \mathrm{mcg} / 1$.

The mean $(95 \% \mathrm{CI})$ decrease in the peak systolic gradient in the LVOT was $22.7(9.8-35.5) \mathrm{mm} \mathrm{Hg}$ in peri- procedure transcatheter measurement $(\mathrm{p}=0.002)$, and $51.1(36-66.2) \mathrm{mm} \mathrm{Hg}$ on the trans- thoracic echo performed in the follow-up out-patient visit $(p<0.001)$. Two patients had transient heart block during the procedure and one, a groin haematoma. All but one patient reported symptomatic improvement post procedure.

Conclusion: Endocardial radiofrequency septal reduction is an effective alternative to surgical myectomy and alcohol septal ablation in children with HOCM. It is repeatable and minimally invasive. It can result in a significant reduction in gradient but needs to be performed systematically under imaging guidance and careful monitoring with intracardiac electrograms.

\section{2}

Prenatal echocardiographic diagnosis and follow-up of acid maltase deficiency with cardiomyopathy Anna Lamberti, R.DEBRE HOSPITAL PARIS, France Maurice Guirgis, R.DEBRE HOSPITAL PARIS, France Franck Iserin, R.DEBRE HOSPITAL PARIS, France Jean Marc Lupoglazoff, R.DEBRE HOSPITAL PARIS, France Hélène Ogier de Baulny, R.DEBRE HOSPITAL PARIS, France Suzel Magnier, R.DEBRE HOSPITAL PARIS, France André Denjean, R.DEBRE HOSPITAL PARIS, France Annabelle Azancot, R.DEBRE HOSPITAL PARIS, France

Enzyme replacement therapy (ERT) is a recent tool in treatment for cardiomyopathies (CMP) in Pompe's disease (PD). ERT by MYOZYME(alfa-alglucosidase)intravenously can reduce hypertrophy and improve cardiac function. We describe prenatal echocardiographic (E) diagnosis and follow-up of a case of familial PD.

In this 6 th pregnancy diagnosis was confirmed on 17 th week (W) by fetal blood analysis .Parents refused pregnancy interruption; ultrasound examination at third trimester evidenced prefrontal oedema and enlarged heart. Fetal E on 31st W established CMP with right and left ventricular (LV) enlargement, wall thickening, tricuspid regurgitation, moderate LV dysfunction (shortening fraction SF:25\%);one month later LV function was deteriorating and induced delivery planned. At birth the baby $4.2 \mathrm{kgs}$ b.w.at 37 th $\mathrm{W})$ required respiratory assistance, vasodilators and diuretics; he was breathing spontaneously and breast feeding in 2nd W. After pretreatment investigations and MRI he had first ERT injection in day 18th and was discharged two days later. He is now on MYOZYME $20 \mathrm{mg} / \mathrm{kg}$ twice monthly, doing well, active and beginning to stand up. Neonatal E confirmed CMP and follow-up showed regression of hypertrophy and cardiac function recovery: SF $13 \%$ at birth improved to $32 \%$ and interventricular septum thicker than 2SD at birth, normalized.

Conclusion: in our case of Pompe's CMP LV function dramatically worsened during last month of pregnancy but quickly recovered with ERT. Best results are obtained with ERT early in life therefore pregnancies at risk for PD should have fetal E monitoring. If cardiac dysfunction is assessed early delivery should be scheduled for optimal management and ERT. 
4073

Cardiac response to Enzyme replacement therapy (ERT) in infantile Pompe disease

Arul Narayanan, Royal Manchester Children's Hospital, United Kingdom

Alex Broomfield, Royal Manchester Children's Hospital,

United Kingdom

Chetan Mehta, Royal Manchester Children's Hospital, United Kingdom

Objectives: Infantile Pompe disease is a rapidly progressive lysosomal storage disease with fatality before 18 months. A deficiency of lysosomal alpha-glucosidase results in glycogen storage within many cells, including cardiac myocytes. The resultant cardiomyopathy presents in early infancy. Most patients die of cardiac or respiratory failure in the first year of life. The effects of ERT on cardiac remodelling have thus far suggested preservation of cardiac function during remodelling. We report our experience of the cardiac response of eight patients to ERT (myoenzyme).

Methods: Retrospective review of the echocardiograms of eight children with Pompe looking at the Left Ventricular mass, LVPWd and FS over time after initiation of ERT.

Results: Eight patients with median age of 43 months (IQR:13-63). All children except one had regression of ventricular hypertrophy in response to ERT, with near normalisation of posterior wall thickness, ventricular mass and ventricular size. Systolic function was preserved and their FS improved with ERT despite rapid changes in ventricular mass and size. The oldest patient in our series is eight years old, has no Left Ventricular Hypertrophy and has normal ECG. One child (No. 6 in the chart) who died while on Enzyme replacement had enzyme specific antibody response, which may have decreased the efficacy of ERT. He also differed as initially he had dilated Cardiomyopathy. This patient in fact had increase in LV mass during ERT.

Conclusions: During ERT therapy, there appears to be preservation and indeed improvement in left ventricular function while cardiac remodelling occurs.

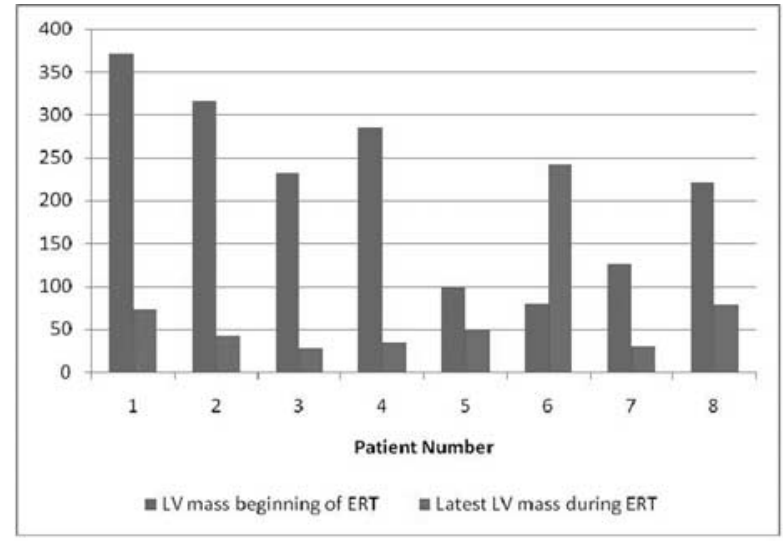

4080

Screening for Chromosomal Deletions and Duplications in Congenital Heart Disease using Multiplex Ligation-dependent Probe Amplification

Milad El-Segaier, Department of Paediatric Cardiology, Lund University Hospital, Lund, Sweden, Sweden

Karina Meden Sorensen, Statens Serum Institut, Copenhagen, Denmark, Denmark
Patrice Bouvagnet, Department of Pediatric Cardiology, Groupe

Hospitalier Est, Lyon, France, France

Jesper Stensbjerg, Department of Paediatric Cardiology, Copenhagen

University Hospital, Copenhagen, Denmark, Denmark

Ab Errami, MRC-Holland, Amsterdam, The Netherlands,

The Netherlands

Jan Schouten, MRC-Holland, Amsterdam, The Netherlands,

The Netherlands

Vibeke Hjortdal, Department of Cardiothoracic and Vascular Surgery, Skejby, Aarhus, Denmark, Denmark

Lars Allan Larsen, Wilhelm Johannsen Centre for Functional Genome Research, University of Copenhagen, Copenhagen, Denmark, Denmark

Objective: Recent studies have suggested that chromosomal deletions and duplications may be found in a proportion of patients with congenital heart disease (CHD).

Methods: We have designed an assay for simultaneous analysis of deletions and duplications in 26 genomic regions which have previously been associated with CHD. The assay is based on the Multiplex Ligation-dependent Probe Amplification (MLPA) technique. The assay is performed in a single reaction and targets the following genomic regions: $1 \mathrm{p} 36,1 \mathrm{q} 21,2 \mathrm{q} 37,2 \mathrm{p} 22$, 3q22, 4q32, 4p16, 5q35.2, 5q35.3, 6p25.3, 7q22, 7q11.23, $8 \mathrm{q} 12.2,8 \mathrm{p} 23.2,8 \mathrm{p} 23.1,8 \mathrm{q} 34.13,8 \mathrm{q} 34.11,10 \mathrm{q} 25.2,10 \mathrm{q} 25.3$, 11q25, 13q14, 15q26, 17p13, 17p11, 20p12, 22q11.

Results and conclusions: At the time of abstract submission we have screened 173 patients with CHD using the CHD-MLPA kit. The screening has revealed previously undetected chromosome abnormalities in eight patients; 22q11 duplication (4 patients), $22 \mathrm{q} 11$ deletion (one patient), 5q35 triplication (one patient), $2 \mathrm{p} 22$ duplication (one patient) and 2q37 deletion (one patient). Conclusion: Our data suggest that approximately $5 \%$ of patients with CHD are carriers of rare chromosomal abnormalities which can be easily detected using the current designed kit; CHD-MLPA.

\section{1}

Increased myocardial Met-Enkephalin is associated with reduced arterial oxygenation in Tetralogy of Fallot

Olivier $W$ van den Brink, Alfred Hospital, Australia

Andrew D Cochrane, The Royal Children's Hopsital, Australia

Franklin L Rosenfeldt, Alfred Hospital, Australia

Daniel J Penny, The Royal Children's Hopsital Melbourne E Murdoch Children's Research Institute, Australia

Salvatore Pepe, The Royal Children's Hopsital \& Murdoch Children's Research Institute, Australia

Enkephalins are co-released with catecholamines from sympathetic terminals in the heart and have recently been identified to play an important adaptive metabolic signalling role in cardioprotection against ischemia or hypoxia-related injury. The study aims were: a) to determine the myocardial levels of methionine-enkephalin in tetralogy of Fallot; and b) to correlate myocardial content of methionine-enkephalin with the extent of arterial oxygen desaturation. Tissue samples obtained during surgical correction of tetralogy of Fallot were assayed via radioimmunochemistry for their methionine-enkephalin content. Arterial oxygen saturation was measured on admission for surgery. The main finding was evidence of greater methionineenkephalin content in right ventricles of 21 patients suffering from cyanotic spells (cyanotic spells: $2418 \pm 844 \mathrm{pg} / \mathrm{g}$ wet weight tissue, $\mathrm{n}=6$; no spells: $1175 \pm 189 \mathrm{pg} / \mathrm{g}$ wet weight tissue, $\mathrm{n}=15, \mathrm{p}=0.045)$. A negative correlation was evident between the arterial oxygen saturation and myocardial methionineenkephalin content. In conclusion, myocardial methionine-enkephalin 
levels increase with the severity of cyanosis in congenital cardiac disease. Opioids, particularly methionine-enkephalin, the key bioactive end product of proenkephalin, may play an important adaptive role in hypoxia-related adrenergic over-activity.

\section{2}

Histone deacetylase inhibitor Trichostatin A promotes cardiomyocyte differentiation of mesenchymal stem cells Jie Tian, Department of Cardiology, Children's Hospital of Chongqing Medical University, China

Ge Yang, Department of Cell and Molecular Biology, Pediatric Institute of Chongqing Medical University, China

Jing Zhu, Department of Cell and Molecular Biology, Pediatric Institute of Chongqing Medical University, China

Yuan Chen, Department of Cardiology, Children's Hospital of

Chongqing Medical University, China

Chuan Feng, Department of Cardiology, Children's Hospital of

Chongqing Medical University, China

Lili Zhao, Department of Cardiology, Children's Hospital of Chongqing Medical University, China

Tewei Lv, Department of Cardiology, Children's Hospital of Chongqing Medical University, China

Background: This study was designed to investigate the effect of histone deacetylase (HDAC) inhibitor Trichostatin A (TSA) on the differentiation potential of mesenchymal stem cells (MSCs) into cardiomyocytes.

Methods: Adult rat bone marrow MSCs were co-cultured with rat cardiomyocytes in the presence of 5-azacytidine (5-aza), with or without the treatment of different concentrations of TSA $(100 \mathrm{nmol} /$ $\mathrm{L}, 300 \mathrm{nmol} / \mathrm{L}$ and $500 \mathrm{nmol} / \mathrm{L}$ ) for $72 \mathrm{hrs}$. mRNA expression of early transcription factors related to cardiac muscle cells - GATA-4, NKx2.5, MEF2c was evaluated using real time RT-PCR and the protein expression of cardiac troponin $\mathrm{T}$ ( $\mathrm{cTnT}$ ) was determined using immunofluorescence staining and Western blotting.

Results: One week after the co-culture of MSCs with pulsating cardiac muscle cells, the cardiomyocyte differentiation began to occur in MSCs. A significant amount of $\mathrm{cTnT}$ protein were detected in two kinds of induction including the co-culture with rat cardiomyocytes and 5-aza, cTnT protein levels were significantly increased after TSA intervention of $72 \mathrm{hrs}$ as the TSA concentration levels vary, the expression of cTnT was upregulated in a trend of concentration gradient, and associated with constitutive activation of early transcription factors of cardiac muscle cells - GATA-4, NKx2.5, MEF2c in MSCs. However, GATA-4, NKx2.5, MEF2c mRNA levels were significantly up-regulated after intervened $72 \mathrm{hrs}$ as the concentration levels of TSA vary in two kinds of induction.

Conclusion: Inhibition of HDAC facilities the ability of MSC differentiation into cardiomyocyte, which manifests an important role of acetylation in the regulation of cell differentiation.

\section{3}

Platelet GP IIb/IIIa receptor blockade versus selective inhibition of platelet signal transduction: Which approach is better to protect platelets during extracorporeal circulation?

Andreas Straub, Dept. of Thoracic, Cardiac and Vascular Surgery, University Hospital of Tübingen, Germany

Hans-Peter Wendel, Dept. of Thoracic, Cardiac and Vascular Surgery, University Hospital of Tübingen, Germany

Ruben Azevedo, Dept. of Thoracic, Cardiac and Vascular Surgery, University Hospital of Tübingen, Germany
Daniela Schiebold, Dept. of Thoracic, Cardiac and Vascular Surgery, University Hospital of Tübingen, Germany

Karlheinz Peter, Baker IDI Heart and Diabetes Institute,

Melbourne, Australia

Simone Schoenwaelder, Australian Centre for Blood Diseases,

Melbourne, Australia

Gerhard Ziemer, Germany

Objective: Blood contact with extracorporeal circulation (ECC) and hypothermia as used in cardiac surgery cause platelet dysfunction possibly followed by severe bleeding as well as thrombotic complications. Platelet glycoprotein (GP) IIb/IIIa blockers protect platelets during ECC but may cause prolonged platelet inhibition. An alternative may be selective inhibition of platelet signal transduction using the new phosphoinositide-3kinase-p110 $\beta$ inhibitor TGX-221 that inhibits shear-induced platelet activation without affecting the bleeding time.

Methods: Heparinized blood of healthy volunteers was treated invitro with GP IIb/IIIa blockers (tirofiban [half-life:1.5-2 hours], eptifibatide [half-life:1.5 hours], FK633 [half-life:0.52 hours]), TGX-221 or as control and circulated in an ECC model at normothermia $\left(37^{\circ} \mathrm{C}\right)$ and hypothermia $\left(18^{\circ} \mathrm{C}\right)$. Before and after circulation platelet aggregation and activation (P-selectin expression and $\beta$-thromboglobulin release) and platelet-ECC adsorption were investigated in flow cytometry and by ELISA.

Results: All investigated GP IIb/IIIa blockers and TGX-221 inhibited platelet activation and aggregation at normothermia. At hypothermia, however, platelet P-selectin expression was decreased by tirofiban to $19.5 \%$ of control values but augmented by FK633 and eptifibatide to $269 \%$ and $163 \%$ of control values respectively $(\mathrm{p}<0.0001)$. Compared to control values tirofiban and TGX-221 decreased platelet-ECC adsorption to $11.6 \%$ and $29.5 \%$ respectively $(\mathrm{p}<0.05)$.

Conclusions: FK633 is the most suitable GP IIb/IIIa blocker to protect platelets during normothermic ECC especially regarding its short half-life that may facilitate therapy control. However, at hypothermia prothrombotic side effects of GP IIb/IIIa blockers should be considered. TGX-221 is a promising alternative and may be safer for clinical use because of its selective mode of action.

\section{4}

Mechanistic insights into hypothermia-induced platelet dysfunction: P2Y12 receptor blockade protects platelets at hypothermia as employed in cardiac surgery

Andreas Straub, Dept. of Thoracic, Cardiac and Vascular Surgery, University Hospital of Tübingen, Germany

Erik Westein, Australian Centre for Blood Diseases, Melbourne, Australia Nicole Bassler, Baker IDI Heart and Diabetes Institute, Melbourne, Australia Shala Dezfouli, St. Vincent's Hospital, Department of Haematology, Melbourne, Australia

Harshal Nandurkar, St. Vincent's Hospital, Department of Haematology, Melbourne, Australia

Shaun Jackson, Australian Centre for Blood Diseases, Melbourne, Australia Michael J Hickey, Centre for Inflammatory Diseases, Monash Medical Centre, Melbourne, Australia

Karlheinz Peter, Baker IDI Heart and Diabetes Institute, Melbourne, Australia

Objective: Hypothermia is employed in cardiac surgery to inhibit ischemia-related organ damage. However, hypothermia causes platelet activation and dysfunction, which can result in bleeding as well as thrombotic complications.

Methods: Platelet adhesion and spreading on different physiologically relevant surfaces was investigated at normothermia 
$\left(37^{\circ} \mathrm{C}\right)$, hypothermia $\left(18-28^{\circ} \mathrm{C}\right)$ and after rewarming under ex vivo flow and static conditions. In mice with body temperatures of $37^{\circ} \mathrm{C}$ and $28^{\circ} \mathrm{C}$ tail bleeding times were assessed and platelet aggregation in mesenteric arteries examined using intravital microscopy. Platelet agonist ADP levels and ADPase CD39 activity were determined using bioluminometry and thin layer chromatography.

Results: Bleeding times as well as platelet-collagen and plateletvWF adhesion at physiological flow are reversibly prolonged by hypothermia. Furthermore, hypothermia causes a partially reversible platelet spreading defect.

In contrast, platelet thrombi formed in vivo at hypothermia are 2.2fold larger $(\mathrm{p}<0.001)$ and dissagregate 5-fold less $(\mathrm{p}<0.001)$ compared to normothermia. This is paralleled by a 2.2 -fold increase of platelet-fibrinogen adhesion $(\mathrm{p}<0.001)$.

Hypothermia reduces CD39-activity, subsequently impairing ADP degradation and increasing ADP-mediated platelet activation. Administration of platelet ADP receptor P2Y12 antagonists reverses hypothermia-induced platelet thrombus formation in vivo.

Conclusions: Most hypothermia induced platelet function-inhibiting effects are reversible on rewarming. Hypothermia-induced platelet activation can be explained by the observed reduction of CD39 ADP-metabolising activity. We present a novel concept of platelet protection against activation during hypothermia, which is based on P2Y12 inhibition. Because short-acting intravenously applicable P2Y12-blockers may soon be clinically available further clinical studies demonstrating the transferability of our concept into clinical practice are warranted.

\section{5}

Incidence of Significant Tricuspid Regurgitation in Patients with Right Ventricular Outflow Tract Dysfunction and Impact of Percutaneous Pulmonary Valve Implantation on Tricuspid Valve Function Philipp Lurz, UCL Institute of Child Health and Great Ormond Street Hospital for Sick Children, United Kingdom

Johannes Nordmeyer, UCL Institute of Child Health and Great

Ormond Street Hospital for Sick Children, Germany

Sachin Khambadkone, UCL Institute of Child Health and Great

Ormond Street Hospital for Sick Children, India

Vivek Muthurangu, UCL Institute of Child Health and Great Ormond

Street Hospital for Sick Children, United Kingdom

Silvia Schievano, UCL Institute of Child Health and Great Ormond Street Hospital for Sick Children, Italy

Philipp Bonhoeffer, UCL Institute of Child Health \& Great Ormond Street Hospital for Children, United Kingdom

Andrew M Taylor, UCL Institute of Child Health and Great Ormond Street Hospital for Sick Children, Germany

Background: Percutaneous pulmonary valve implantation (PPVI) is a less invasive strategy to treat right ventricle (RV) to pulmonary artery (PA) conduit dysfunction. However, in contrast to surgery, concomitant tricuspid valve lesions cannot be treated with this technique. Therefore, we analysed the incidence of significant tricuspid regurgitation in patients with $\mathrm{RV}$ to $\mathrm{PA}$ conduit dysfunction and the impact of PPVI on tricuspid valve function.

Methods: Magnetic resonance (MR) imaging including biventricular volumes and great vessel blood flow assessment was performed prior to and immediately after PPVI in 146 patients. Retrospectively, out of this patient population, we selected patients who had a tricuspid regurgitation fraction (TRF) $>10 \%$ on $\mathrm{MR}$ prior to valve implantation. TRF was calculated from right ventricular stroke volume and pulmonary artery forward flow.
Results: 16 patients had a tricuspid regurgitation fraction $>10 \%$ (incidence 10.9\%). After PPVI, the gradient across the conduit fell from $40.8 \pm 12.4 \mathrm{mmHg}$ to $15.9 \pm 5.3 \mathrm{mmHg}(\mathrm{p}<0.001)$. In addition, there was a decrease in RV end-diastolic volumes $(113.3 \pm 20.8 \mathrm{ml} / \mathrm{m} 2$ to $89.3 \pm 19.6 \mathrm{ml} / \mathrm{m} 2, \quad \mathrm{p}<0.001)$ and pulmonary regurgitation fraction $(16.6 \pm 14.6 \%$ to $1.9 \pm 2.7 \%$, $\mathrm{p}<0.024)$ on MR. Reduction in RV pressure and/or volume overload was associated with a significant reduction in TRF $(25.4 \pm 6.4 \%$ to $8.2 \pm 3.7 \%, \mathrm{p}<0.001)$.

Conclusion: Significant tricuspid regurgitation in the context of RV to PA conduit dysfunction is not common. Further, relief in RV volume and pressure overload by PPVI improves tricuspid valve function acutely. This is important, when patients are selected for either surgical or percutaneous treatment of RV to PA conduit dysfunction.

\section{6}

Are some ASD patients predisposed to developing heart block?

Dhaval R Patel, Hope children's Hospital, Advocate Christ Medical Center, United States

David A Roberson, Hope children's Hospital, Advocate Christ Medical Center, United States

Vivian Cui, Hope children's Hospital, Advocate Christ Medical Center, United States

Tarek Husayni, Hope children's Hospital, Advocate Christ Medical Center, United States

Alexander J Javois, Hope children's Hospital, Advocate Christ Medical Center, United States

Introduction: Complications of device closure of ASD are well known and device position has been implicated in the incidence of heart block. We suggest preclosure ASD electrophysiology may also predispose patients to permanent forms of heart block. Method: A retrospective analysis was performed on patients who underwent catheterization for closure of ASD or PFO between March'02 and October'08. Pre and post procedure electrocardiograms were reviewed.

Results: $16.5 \%(41 / 248)$ had preexisting prolonged PR interval (first degree block). 8 of these 41 (19.5\%) still had first degree block at 12 months. Of the 207 with normal PR pre closure, 5 had first degree block at 12 months (2.4\%).

ANOVA with post hoc Tamhane' T2 was performed to test the change in PR, HR over time. No change in HR was seen $(\mathrm{P}=0.083)$. Compared to baseline, $\mathrm{PR}$ significantly shortened by 6 months post procedure $(\mathrm{P}<0.01)$ and more notably by 12 months $(\mathrm{P}<0.001)$.

$2 \%(5 / 248)$ had atrial arrhythmia (not heart block) that completely resolved (2/248 required oral antiarrythmic therapy).

Conclusion: A high incidence of prolonged PR interval was observed prior to ASD closure (16.5\%); this has not been previously reported. After device closure, PR interval remained abnormal in a $19.5 \%$ of these patients at one year post implant. Denovo first degree block was seen in only $2.4 \%$ at one year. Preoperative right atrial enlargement with tricuspid annular enlargement may predispose patients to pericatheterization or post device heart block; heart block may not be entirely related to the device.

\section{7}

Pulmonary artery stents in small children $(<$ or $=12 \mathrm{~kg})$ : short and mid-term results

Jeffrey R Frazer, Rady Children's Hospital of San Diego and the

University of California San Diego, United States

Frank F Ing, Baylor College of Medicine, United States 
Objectives: Stent placement in branch pulmonary arteries(BPA) of small children, has been limited by the need for large sheaths and hemodynamic instability. Advances in delivery techniques and stent design have allowed the delivery of large stents through small sheaths. We report our short and mid-term results of BPA stents in patients $</=12 \mathrm{~kg}$.

Methods: Initial and follow-up cath data of all pts $</=12 \mathrm{~kg}$ who received stents in BPA from 1998-2006 was reviewed.

Results: 70 patients received 98 stents in 94 vessels. Median age was 1.2 yrs (0.1-3.2) and weight $8.6 \mathrm{~kg}$ (3.3-12.0). A subgroup of 33 patients (41 vessels) were $<8 \mathrm{~kg}$ at initial stent implant with median age 0.6 yrs (0.1-1.2) and median weight $5.9 \mathrm{~kg}(3.3-7.9)$. (Table I) Follow-up data was available for 57 pts (77vessels) and 125 caths, median 2 additional caths/patient (1-9 caths), over a median followup of 3.6 yrs (0.2-10.0). Further balloon/stent dilation was performed in $83 \%(64 / 77)$ of vessels and $17 \%$ of vessels $(13 / 77)$ required no further interventions to date. At the last cath, the vessels were dilated to $9.2 \pm 2.4 \mathrm{~mm}$ and the peak gradient decreased to $7.9 \mathrm{mmHg}$ $(p<0.001)$. Three major complications occurred-rupture of the LPA during intraoperative implantation, air embolus and stent entrapment of a dilator tip. There were no procedural deaths.

Conclusion: BPA can be stented safely and effectively in small patients. Redilation or further dilation to accommodate somatic growth with good hemodynamic results has been achieved at medium-term follow-up. Long term follow up is necessary to determine if these stents can be dilated to adult size.

\begin{tabular}{llll}
\hline Acute results & $\begin{array}{l}\text { Minimum } \\
\text { diameter }(\mathrm{mm})\end{array}$ & $\begin{array}{l}\text { Peak gradient } \\
(\mathrm{mmHg})\end{array}$ & $\begin{array}{l}\text { Right ventricle/ } \\
\text { Femoral artery } \\
\text { ratio (\%) }\end{array}$ \\
\hline$<=12 \mathrm{~kg}$ (pre) & 3.1 & 34 & 72 \\
$<=12 \mathrm{~kg}$ (post) & 6.9 & 11 & 52 \\
$<=8 \mathrm{~kg}$ (pre) & 2.6 & 37 & 71 \\
$<=8 \mathrm{~kg}$ (post) & 6.2 & 10 & 54 \\
All p values $<=0.01$ & & & \\
\hline
\end{tabular}

\section{8}

Severe Pulmonary Valve Stenosis With Right Ventricular Failure And Cyanosis : Results Of Valvoplasty

Najma Patel, National Institute of Cardiovascular Diseases, Pakistan

In Pakistan a large proportion of children with pulmonary valve stenosis (PS) present with cyanosis and severe right ventricular failure (RVF). Our objective is to present immediate and long term results of pulmonary valvoplasty (PVD) in these severely sick children.

Methods: 150 children presented with PS from 2000 to 2007, in paediatric cardiology unit of National Institute of Cardiovascular Diseases. out of these 79 children, mean age 6.4 years, had RVF often with ascitis (10) and peripheral oedema (34) and cyanosis (55). JVP raised in 48, hepatomegaly (38).

PVD was performed with sedation in most and general anesthesia in babies and unstable children.

Balloon dilatation wad performed using balloons 1.2-1.5 times the PV annulus diameter, in very sick children predilatation done with smaller balloons.

Results: Mean LV saturation predilatation was $70 \%$ increased to 90\% immediately after dilatation, 60 patients had Tricuspid Regurgitation (TR) predilatation and 45 pts had TR post dilatation. Mean peak pressure gradient (PPG) predilatation was $140 \mathrm{mmHg}$ decreased to $60 \mathrm{mmHg}$ postdilatation. 2 patients died one with pulmonary hemorrhage, one with bradycardia unresponsive to pacing and medication.
After 3 months of follow up mean PPG decreased to $30 \mathrm{mmHg}$ $(10-50 \mathrm{mmHg})$, mean $\mathrm{O} 2$ sat increased to $96 \%$

On follow up all are doing well except those with hypoplastic RV. One developed restenosis 5years after the successful dilation at 6 month of age with severe RV dysfunction and TR.

Conclusions:We conclude that safe and effective PVD can be achieved even in severely symptomatic children with PVS and RVF. One need to evaluate the right and left ventricular sizes in these children prior to dilatation, so that required degree of dilatation can be determined.

\section{9 \\ Closure of atrial septal defects in children with a biodrgradeble implant device \\ Gareth J Morgan, Hospital for Sick Children, Toronto, Canada Rajiv Chaturvedi, Hospital for Sick Children, Toronto, Canada Kyong-Jin Lee, Hospital for Sick Children, Toronto, Canada Lee Benson, Hospital for Sick Children, Toronto, Canada}

Device closure of atrial septal defects first took place in the 1970s and since then, has become an attractive alternative to surgery in the majority of secundum defects and patent foramen ovales. The design of various devices available has changed considerably, but in the majority of devices the presence of a significant amount of foreign material is still a concern, for many reasons.

We describe the first series of 10 paediatric atrial septal defect occlusions with the BioSTAR biodegradable implant device. All implants were carried out by one operator. The only inclusion criteria was a stretched diameter of $16 \mathrm{~mm}$ or less. We retrospectively matched a cohort of patients having defect closure using the Amplatzer Septal Occluder in the same institution and era and compared procedural data and acute and mid term closure rates and complications.

The Biostar acute and mid term closure rates were both 100\% compared to $90 \%$ acute and $100 \%$ midterm closure in the amplatzer patients. There were no significant complications in either group. Although the procedure and fluorocscopy times tended to be longer in the Biostar group, neither of these were statistically significant.

Although the BioSTAR device is only suitable for smaller defects and requires an $11 \mathrm{~F}$ sheath, it can achieve at least equivalent closure rates to the Amplatzer Septal Occluder with only a minimal skeleton of foreign material remaining in the septum after 6 months. Longer fluoroscopy and procedure times may be a drawback, however this may improve with familiarity with the device and deployment system.

\section{0 \\ Stent implantantation in aortic coarctation. Covered or bare? \\ Gianfranco Butera, Policlinico San Donato IRCCS, Italy \\ Luciane Piazza, Policlinico San Donato IRCCS, Italy \\ Massimo Chessa, Policlinico San Donato IRCCS, Italy \\ Raul Abella, Policlinico San Donato IRCCS, Italy \\ Angelo Micheletti, Policlinico San Donato IRCCS, Italy \\ Diana Negura, Policlinico San Donato IRCCS, Italy \\ Carmelo Arcidiacono, Policlinico San Donato IRCCS, Italy \\ Mario Carminati, Policlinico San Donato IRCCS, Italy}

Aim: To compare results and complications between bare stents (BS) and covered stents (CS) for the treatment of aortic coarctation. Methods and patients: Between January 2000 and October 2008, 144 consecutive patients (median age 15 years, range 6-66 years, 
64 males) underwent treatment for native (92 pts) or recurrent (52 pts) aortic coarctation. Bare stents were used in 78 patients, while covered stents were used in 66 subjects.

Results: There were no differences for age, gender, native coarctation/recoarctation rate, mean drop of peak systolic gradient, increase of diameter of coarcted segment, mean fluoroscopy and procedure times. Stents were placed in the correct position in all subjects in both groups. Long-sheath used for stent implantation was larger in CS compared to BS (median 12 vs 10 French; $p=0.01$ ). Total complication rate was higher in BS $(12 \%$ vs $0 \% ; p=0.03)$. The following complications occurred in BS group: early post-procedural death due to acute aortic dissection in 1 patient, stent embolization in 3 subjects, femoral artery pseudo-aneueysm needing vascular surgery in 3 pts, early periaortic hematoma in $1 \mathrm{pt}$, aneurysm formation during followup in 2 subjects. Follow-up was longer for BS (median 38 vs 13 months; $p=0.04$ ). A total of 6 subjects (4 in the BS group and 2 subjects in the CS group) needed re-dilation during follow-up. Finally, 10 subjects in BS (14\%) and 9 in CS (27\%) needed anti-hypertensive drugs during follow-up $(p=0.14)$.

Conclusions: Covered Cheatham-Platinum stents appears to be safer that bare metal stents. Long-term follow-up data are needed.

\section{1 \\ Using practice experience to develop nursing education programmes \\ Joy E Lyon, School of Health Sciences, United Kingdom}

Advanced practice nurses in the UK will be required to demonstrate Masters level competencies by 2010. This is challenging for specialist nurses caring for adults with congenital heart disease (ACHD), due in part, to the relative recent appearance in significant numbers of this patient group.

In the past nursing skills were learnt through experience gained through caring for children requiring surgery for congenital heart disease, and adults requiring surgery for acquired heart disease. Understanding of physiological principles contributed to appreciating the impact of pathological changes. While fulfilling a joint education/clinical role I gained experience of ACHD in practice and increased requests for teaching programmes related to ACHD.

Currently in an education role enables taking a strategic perspective to staff training related to minority patient groups such as ACHD. The evolution of government policies related to people with long term conditions has provided opportunities to explore common themes. Due to relatively small numbers of registered nurses involved in care for ACHD, taught courses are impractical. This difficulty has been overcome through offering work based learning programmes for health and social professionals completing undergraduate and post-graduate awards.

Masters and $\mathrm{PhD}$ level programmes are being devised to enable advanced practice nurses to explore patient care aspects related to their practice in relation to ACHD. Common areas may be identified with other patients with chronic childhood conditions who are now frequently surviving to adulthood.

\section{2}

Best Outcomes Nursing Children with Excellence

Sandra L Staveski, United States

Andreas Tsakistos, United States

Sandra Roodt, South Africa

The current nursing shortage in developed countries has escalated international nurse recruitment efforts and caused nurse migration away from developing countries. Dependence on foreign-trained nurses in developed countries has significant implications on global healthcare delivery. The Best Outcomes Nursing Children with Excellence (BOuNCE) project is aimed at developing a reproducible nursing retention and development model to help keep nurses in their homeland in order to meet the health care needs of local pediatric patients.

Across the world, successful health care depends upon on all nations having the ability to supply adequate nursing care to their sick. The goal of the BOuNCE project is to test the premise that by fostering nursing professional development at a specific institution, recruitment and retention of nurses will be enhanced. Our project's interventions focus on the following: 1) implementing practice-based nurse training, 2) exploring clinical leadership roles, and 3) team building. Central to the structure of this program is a "twinning" relationship between Red Cross Children's Hospital in Cape Town and Lucile Packard Children's Hospital at Stanford. We plan to explore our interventions, the outcomes, and the implications of the project for global health care. The project highlights the potential to improve a global problem when institutions in different nations come together to enhance health care delivery for pediatric patients.

\section{3}

Variation in Nursing Intensity and its Impact on Mortality for Congenital Heart Surgery Using Pediatric Health Information System (PHIS) Administrative Data

Patricia A Hickey, Children's Hospital Boston, United States Kimberlee Gauvreau, Children's Hospital Boston, United States

Jean A Connor, United States

Eileen M Sporing, United States

Kathy J Jenkins, Children's Hospital Boston, United States

Objective: To examine nursing's impact on risk adjusted mortality for pediatric cardiac surgical patients across 38 free standing children's hospitals in the USA.

Methods: A patient level analysis was conducted using secondary data from the PHIS and NACHRI datasets for the two year period of 2005-2006 and responses from Chief Nursing Officers to examine nursing indicators. Cases $<18$ years of age undergoing surgical repair of a congenital heart defect were identified using ICD-9-CM codes. Risk adjustment was performed using the RACHS-1 method. Analyses were performed using generalized estimating equations models to account for the intrainstitutional correlation among cases from the same hospital.

Results: 21,885 cases of congenital heart surgery were identified. There is variation in ICU nursing hours per patient day (15-32). Variation exists in ICU nursing skill mix (80-100\%)and 20 hospitals had $100 \%$ registered nurse staffing in ICUs. The association between hours of nursing care and mortality (odds ratio $=0.88, \mathrm{p}=0.23$ ) and between Magnet status and mortality (odds ratio $=0.90, p=0.42$ ) were not significant. ICU nursing worked hours tend to increase as hospital volume of congenital heart surgery increases. (Spearman rank correlation coefficient $=0.39 \mathrm{p}=0.027)$. Hospital volume was significantly associated with risk-adjusted mortality. There was significant variation in mortality across hospitals (median unadjusted mortality rate $3.9 \%$, range 1.6 to $15.8 \%$ ). An odds ratio of $0.93(p<0.001)$ was associated with each increase in volume of 100 cases.

Conclusion: Significant variation exists in nursing indicators and mortality across Children's Hospitals. Higher surgical volume centers have a reduced likelihood of death controlling for demographic and clinical variables. 


\section{4}

It's not just about the heart: focusing on the transition journey \& Life after paediatric care

Bronwyn Norman, The Royal Children's Hopsital, Australia

Felicity M Sloman, Private Consultant, Australia

Despite a clear recognition in the literature of the need for early preparation for transition including a well-coordinated transfer to adult health care, young people with congenital heart disease continue to receive ad hoc preparation for their transition to adult health care worldwide. The literature has long indicated that this issue is widely recognized as an essential component of the overall health care plan of any patient with a lifelong condition, yet many health services continue to be challenged to find an effective solution. A strategy has been developed and implemented in the Cardiology Department, Royal Children's Hospital (RCH) Melbourne to inform patients and their families about transition. Working closely with the $\mathrm{RCH}$ Transition coordinator for the hospital, international transition resources have been adapted for this group of young people. In addition, support and discussion opportunities are offered well in advance of the move to adult services. An experienced cardiac nurse was appointed specifically to facilitate transition processes by assisting staff to integrate transition into their current practice. This presentation will outline the issues, the new clinics, the challenges and the future plans. Following the implementation of this strategy at the $\mathrm{RCH}$ in Melbourne the hospital-wide Transition coordinator was given the opportunity to work within an innovative model of care newly developed in Toronto, Canada. This model, known as the LIFEspan model will be briefly outlined and its application to the transition of young people with congenital heart disease will be explored.

4101

Tbx1 Knock-down leads to reduced pharyngeal neural crest cells and cardiac performance in zebrafish

Lifeng Zhang, Cardiovascular center, Children's Hospital of Fudan University, China

Yuexiang Wang, Shanghai Medical School \& Key Laboratory of Molecular Medicine, Ministry of Education, Fudan University, China Qiu Jiang, Shanghai Medical School \& Key Laboratory of Molecular Medicine, Ministry of Education, Fudan University, China

Houyan Song, Shanghai Medical School \& Key Laboratory of Molecular Medicine, Ministry of Education, Fudan University, China Yonghao Gui, Cardiovascular center, Children's Hospital of Fudan University, China

Objective: Tbx1 is the most promising candidate gene for DiGeorge syndrome. The phenotypes of Tbx1 knock-down in some animal models have been well described morphologically; however, the cardiac performance analysis of Tbx1 knock-down is limited. We aim to explore the roles of Tbx1 in cardiac neural crest and myocardial development in zebrafish.

Methods: To elucidate these issues, Tbx1 specific morpholino was used to reduce the amount of Tbx1 in zebrafish. We studied cardiac differentiation in Tbx1 morphant embryos using whole mount in situ hybridization. Heart rates were counted in Tbx1 morphant embryos. The cardiac performance was analyzed by measuring VSF, ASF in Tbx1 morphant embryos.

Results: Tbx1 morphant embryos are characterized by defects in the pharyngeal arches, otic vesicle, aortic arches and thymus. The expression of crestin, hand 2 and $\mathrm{dl} \times 2 \alpha$ in neural crest cells are extremely reduced, especially in the posterior arches. Tbx1 knock down may reduce the amount of pharyngeal neural crest cells in zebrafish. At the meantime, heart rate was decreased in Tbx1 morphant embryo when compared to that in control embryo. Decreased cardiac performance was observed in Tbx1 morphant embryos, VSF and ASF are both repressed due to Tbx1 knock-down when compared to that in control embryos. Conclusions: Our results suggest that Tbx1 might play an essential role in the development of pharyngeal neural crest cells in zebrafish. Tbx1 Knock-down causes impaired cardiac performance in Tbx1 morphant embryos. These results will shed new insight into the roles of Tbx1 in the etiology of DGS.

\section{2}

Right ventricular response to abnormal loading conditions in mice

Beatrijs Bartelds, Dept of Pediatric Cardiology, CVC, GUIDE, University Medical Center Groningen, University of Groningen, The Netherlands

Annemiek Smit-van Oosten, University Medical Center Groningen, University of Groningen, The Netherlands

Janny Takens, Dept of Pediatric Cardiology, CVC, GUIDE, University Medical Center Groningen, University of Groningen, The Netherlands

Marcel Nederhoff, Department of Medical Physiology, UMCU, Utrecht, The Netherlands

Leon J de Windt, Department of Medical Physiology, UMCU, Utrecht, The Netherlands

Wiek H van Gilst, Dept of Cardiology, CVC, GUIDE, University Medical Center Groningen, University of Groningen, The Netherlands Rolf MF Berger, Dept of Pediatric Cardiology, CVC, GUIDE, University Medical Center Groningen, University of Groningen, The Netherlands

Background: Right ventricular (RV) dysfunction is an important determinant of long-term outcome of patients with congenital heart diseases, associated with abnormal ventricular loading conditions (pressure and/or volume load). The pathophysiological mechanisms are yet poorly understood. We aimed to characterize two mice models inducing different types of RV overload, using functional and pathophysiological outcome parameters.

Methods and Results: We developed a mouse model of RV pressure load by banding of the pulmonary artery (Banding) and of volume load by an aorto-caval shunt (Shunt). Exercise capacity, measured by voluntary cage-wheel exercise before and 4 wks after surgery, was reduced in Banding-mice, whereas it was unchanged in Shunt-mice (Control vs. Banding vs. Shunt: $-100 \pm 64$ vs. $-374 \pm 255^{\star}$ vs. $-150 \pm 174$ min., $\star=p<0.05$ vs. Control).

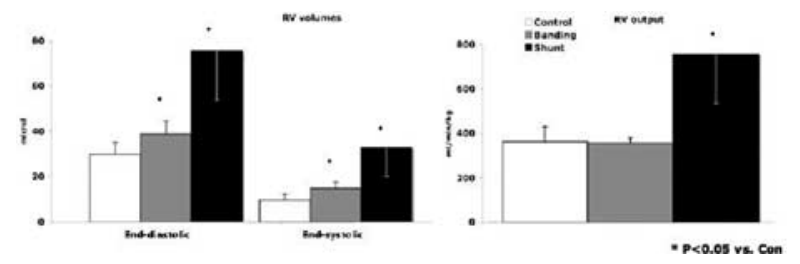

Cardiac MRI (9.4T-scanner) 4 wks after surgery revealed increased enddiastolic and -systolic RV volumes with preserved RV-stroke volume, -output and ejection fraction in Bandingmice (Figure). Shunt-mice also showed increased RV-volumes, but in contrast to Banding-mice, increased RV-stroke volume and -output, whereas RV ejection fraction was decreased (Control vs. Banding vs. Shunt: $67.9 \pm 4.3$ vs. $61.9 \pm 5.8$ vs. 
$\left.58.0 \pm 8.6^{\star}\right)$. At autopsy, banding- and shunt-mice showed similar degrees of severe RV-hypertrophy, expressed as RV/BW (Control vs. Banding vs. Shunt: $0.88 \pm 0.17$ vs. $1.61 \pm 0.25^{\star}$ vs. $1.49 \pm 0.27 \star)$.

Conclusions: Pressure vs. volume overload of the RV in these mice models induced similar degrees of RV-hypertrophy, but marked differences in functional characteristics. These mice models, with rodent-MRI and exercise-testing, allow for further unraveling the pathophysiological and molecular adaptation of the RV to different types of abnormal loading.

\author{
4103 \\ Cellular and humoral immune response to open heart \\ surgery in children is modulated by methylprednisolone \\ Jozsef Bocsi, Pediatric Cardiology, Cardiac Centre Leipzig, \\ University Leipzig,, Germany \\ Marie -Christin Haenzka, Pediatric Cardiology, Cardiac Centre \\ Leipzig, University Leipzig, Germany \\ Pavel Osmancik, Cardiocenter, Charles University and University Hospital \\ Kralovske Vinohrady, Prague, Czech Republic, Czech Republic \\ Joerg Hambsch, Pediatric Cardiology, Cardiac Centre Leipzig, \\ University Leipzig,, Germany \\ Ulrich Sack, Institute for Clinical Immunology, IKIT, University \\ Leipzig, Germany \\ Winfried Bellinghausen, Anaesthesiology, Cardiac Centre Leipzig, \\ University Leipzig,, Germany \\ Jan Janousek, Heart Center Leipzig, Germany \\ Attila Tarnok, Pediatric Cardiology, Cardiac Centre Leipzig, University \\ Leipzig,, Germany
}

Postoperative effusions, edema and capillary leak syndrome in children after cardiac surgery with cardiopulmonary bypass (CPB) constitute considerable clinical problems. In order to reduce overshooting immune response glucocorticoids are administered pre- and perioperatively. In a retrospective study we investigated the modulation of humoral and cellular immune response by methylprednisolone (MP).

Children undergoing cardiac surgery (mostly septum defect corrections) without (MP-, $\mathrm{n}=10)$ and with $\mathrm{MP}$ administration before $\mathrm{CPB}(\mathrm{MP}+, \mathrm{n}=23$, dose: $5-20 \mathrm{mg} / \mathrm{kg}$ body-weight $)$ were enrolled. Blood was obtained $24 \mathrm{~h}$ preoperatively, after anesthesia, CPB begin, CPB end, $4 \mathrm{~h}, 24 \mathrm{~h}, 48 \mathrm{~h}$ after surgery, at discharge and at out-patient follow-up $(9.1+7.0$ month after surgery). Serum analysis, clinical chemistry and flow cytometry were performed to determine the degree of activation of various leukocyte subpopulations.

The patients follow-up was uneventful. Effusion volume, intensive care unit and hospital days were similar with and without MP. Release of proinflammatory cytokines (IL-6, IL8) was reduced and of anti-inflammatory cytokines (IL-10) upregulated by MP. Significant peri- und postoperative increase of circulating neutrophils and monocytes was detected in both groups. However, infiltration of monocytes was delayed with MP. LFA-1 and Mac-1 expression were similar for MP+ and MP-. T-lymphocyte count decreased and the CD4+/CD8+ ratio was lower with MP+. Blymphocytes count increased significantly in the MP+ but remained constant in the MP- group.

MP treatment decreased the proinflammatory effect of CPBsurgery and induced antiinflammatory effect on the cellular and humoral level, but without obvious clinical advantages during surgery and at outcome.

Support: German Heart Foundation;

MSM-0021620817-Charles University Prague, Czech Republic(P.O.)

\section{4}

Connexin40 is expressed in the right ventricles of patients with congenital heart malformations

Giselle V Rowlinson, Royal Brompton Hospital, London/Imperial

College London, United Kingdom

Piers EF Daubeney, Royal Brompton Hospital, London,

United Kingdom

Emmanuel Dupont, Imperial College London, United Kingdom

Nicholas J Severs, Imperial College London, United Kingdom

Objective: Gap junctions allow direct cell-cell communication and electrical coupling between individual myocytes. The component proteins of these junctions are the connexins $(\mathrm{Cx})$. Adult working ventricular myocytes express $\mathrm{Cx} 43$ but normally lack detectable $\mathrm{Cx} 40$. Early in gestation, mice and humans express $\mathrm{Cx} 40$ in the developing trabeculated ventricle; this $\mathrm{Cx} 40$ disappears by birth. As studies on transgenic mice have demonstrated roles for connexins in cardiac morphogenesis, we investigated connexin expression in human congenital heart malformations.

Methods and Results: Connexin expression was studied in the right ventricles (RVs) of pre- and post-operative patients with tetralogy of Fallot ( $n=20$; age 3 months to 27 years), double chambered right ventricle ( $n=5$; age 10 months to 14 years) and controls without RV pathology ( $\mathrm{n}=5$; age 2 months to 11 years). Immunoconfocal microscopy demonstrated localisation of gap junctions to the intercalated disk by age 2 years. In contrast to control hearts, $\mathrm{Cx} 40$ was prominently and heterogeneously expressed in the working right ventricular myocytes of all the diseased hearts. Electron microscopy demonstrated co-localisation of $\mathrm{Cx} 40$ and $\mathrm{Cx} 43$ within the same gap junction. Quantitative Western blot analysis showed that Cx40 expression represented up to $10 \%$ of total connexin in the RV.

Conclusion: There was significant expression of $\mathrm{Cx} 40$ in patients with RV outflow malformations; none in those without RV pathology. These findings were independent of whether the patients were pre- or post-operative, thus with markedly different underlying haemodynamics. Therefore our results suggest a lack of $\mathrm{Cx} 40$ repression during development which may be pivotal in the morphogenesis of these cardiac malformations.

\section{6}

Percutaneous versus surgical ASD closure: meta-analysis of currently available clinical evidence

Gianfranco Butera, Policlinico San Donato IRCCS, Italy

Giuseppe Biondi-Zoccai, Università di Torino, Italy

Mario Carminati, Policlinico San Donato IRCCS, Italy

Raul Abella, Policlinico San Donato IRCCS, Italy

Zakhia Saliba, Univesrity of Beirut, Lebanon

Massimo Chessa, Policlinico San Donato IRCCS, Italy

Alessandro Giamberti, Policlinico San Donato IRCCS, Italy

Alessandro Frigiola, Policlinico San Donato IRCCS, Italy

Aim: To provide an evidence-based evaluation of all available studies comparing surgery and the transcatheter approach in ASD closure.

Methods: Electronic databases were systematically searched for pertinent clinical studies comparing the two methods of closure (percutaneous and surgical) published up to October 2008 and reporting on $>20$ patients. The primary endpoints were the occurrence of death, of total and major early complications. Pooled estimates for odds ratios (OR) were computed (random-effect method), with statistical inconsistency appraised with I2. 
Results: After excluding 4297 non-pertinent citations, we finally included 13 original studies (3082 patients). All studies were nonrandomized comparisons between percutaneous and surgical closure. One death was encountered in the surgical group $(0.08 \%$; $95 \%$ C.I. $0-0.23 \%$ ).

Quantitative synthesis of total complications after procedure showed a $31 \%(95 \%$ CI $21-41 \%)$ rate in patients treated surgically and a $6.6 \%$ (95\% CI 3.9-9.2\%) rate in subjects treated percutaneously.

Comparison of percutaneous closure versus surgery showed adjusted odds ratios for total complications of 5.4 (95\% CI 2.96-9.84; $\mathrm{p}<0.0001)$, significantly favouring percutaneous closure.

Quantitative synthesis for major complications after procedure showed a $6.8 \%$ (95\% CI 4-9.5\%) rate in patients treated surgically and a $1.9 \%(95 \%$ CI $0.9-2.9 \%)$ rate after percutaneous closure. Comparison of percutaneous closure versus surgery showed adjusted odds ratios for total complications of 3.81 (95\% CI 2.7-5.36; $\mathrm{p}=0.006)$, thus again favouring the percutaneous approach.

Conclusions: The largest cohort to date of patients with secundum ASD shows that treatment by a percutaneous approach has a significantly lower rate of either total or major early postprocedural complications.

\section{7}

Thrombosis Following Closure of Large Distal Type Coronary Artery Fistulae : To Close or not to Close? Srinath T Gowda, The Children's Hospital of Michigan, United States Daniel Turner, The Children's Hospital of Michigan, United States

Thomas Forbes, The Children's Hospital of Michigan, United States Lourdes Prieto, Cleveland Clinic Foundation, United States

Larry Latson, Cleveland Clinic Foundation, United States

Introduction: Coronary artery fistulae (CAF) are rare congenital coronary anomalies. We sought to describe the risk of coronary thrombosis based on the type and size of CAF.

Method: Patients medical records were retrospectively reviewed from two tertiary medical centers. To predict risk factors for thrombosis; CAF based on origin was classified into proximal and distal type and the size as small, medium and large.

Results: Of 24 patients with CAF; 16 had large, 7 medium and 1 small. Median age were 11.5 (0.3 to 56 yrs). Seventeen patients underwent transcatheter, 4 surgical closures and 3 medical observation. Follow-up was 3 years (0.1 to 41.6). Eleven patients with proximal type of CAF had successful closure, no residual leak and remained asymptomatic. However, none had follow-up angiograms. Of Thirteen patients with distal type CAF; 7 had follow-up angiograms, 4 had significant adverse events with evidence of coronary thrombosis. Of these, 3 pts had myocardial infarction $<24$ hrs and 1 had thrombosis 40 yrs post closure. All 4 had large size CAF. Three patients without intervention (medical) had distal type CAF. They were small, medium and large size each and were $0.5,10$ and 56 years of age, respectively. They have remained asymptomatic clinically and none have had coronary angiograms on follow-up. Conclusion: Patients with distal type and large size CAF may be at higher risk for coronary thrombosis post closure. Therefore, definitive treatment for distal CAF with either intervention vs. medical observation is unclear. A multi-center longitudinal study in CAF patients is warranted.

\section{8}

\section{Potential coronary complications after arterial switch operation}

Milad El-Segaier, Department of Paediatric Cardiology, Lund

University Hospital, Lund, Sweden
Erkki Pesonen, Department of Paediatric Cardiology, Lund University Hospital, Lund, Sweden

Anders Lundin, Department of Radiology, Lund University Hospital, Lund, Sweden

Peter Hochbergs, Department of Radiology, Lund University Hospital, Lund, Sweden

Peeter Jögi, Department of Paediatric Cardiac Surgery, Lund University Hospital, Lund, Sweden

Objectives: Even asymptomatic patients may need coronary angioplasty and stenting after arterial switch operation (ASO).

Methods: Since 1980, 282 patients were operated with ASO. The operative technique has not been modified since 1996. Selective coronary angiograms were done on 81 patients at a mean age of 8.6 years.

Results: Before 1996, 88 patients were operated. Five patients had myocardial infarction early postoperatively. The follow up coronary angiogram revealed five coronary complications. Two of them had a single coronary artery and one had circumflex coronary artery (CX) from right coronary artery (RCA). One patient had ostial stenosis and one had conus branch occlusion with good collaterals. One patient had left coronary artery (LCA) ostial obstruction. He was operated with coronary bypass graft at age of 16 years. Stenting of LCA ostium was done in two asymptomatic patients: one with Cx from RCA with drugeluting stent at 10 years of age and one with normal anatomy with Driver stent at 18 years of age.

In patients operated after 1996 there were no early postoperative complications. One of 36 investigated asymptomatic patients showed severe LCA ostial obstruction. He was treated with balloon dilation at 5 years of age which led to dissection. Later at age of 9 years he was treated with a drug-eluting stent.

Conclusions: Asymptomatic patients with an uneventful postoperative course after ASO may have coronary obstruction which indicates the necessity of coronary angiography in all patients. The examination should probably be performed before marked increase in physical.

\section{9}

Clinical Evaluation of the new Amplatzer Duct Occluder II (ADOII)

Vinay K Bhole, Birmingham Children's Hospital, United Kingdom Patrick M Noonan, Birmingham Children's Hospital, United Kingdom Paul A Miller, Birmingham Children's Hospital, United Kingdom Chetan C Mehta, Alder Hey Children's Hospital, Liverpool, United Kingdom

Oliver Stumper, Birmingham Children's Hospital, United Kingdom John GC Wright, Birmingham Children's Hospital, United Kingdom Zdenka Reinhardt, Birmingham Children's Hospital, United Kingdom Joseph V DeGiovanni, Birmingham Children's Hospital, United Kingdom

Background: Several devices including coils and Amplatzer Duct Occluder (ADO) are used for catheter closure of patent arterial ducts (PDA). These devices generally carry a high success rate but residual shunts, sub-optimal device orientation and technical problems can be overcome by better designed devices. The new ADOII is designed with these in mind.

Objectives: The aim of this study was to evaluate the safety and efficacy of the ADO II for closure of PDA.

Methods: This was a prospective multicentre study from February 2008 to January 2009. Twenty-seven patients (17 females) received the ADO II, The median age was 22 months (Range: 7 months - 68 years) and the median weight was 11.7 $(6.9-110) \mathrm{Kg}$. The mean PDA diameter was $3(1-4.4) \mathrm{mm}$. The 
approach was arterial in 13(48\%) and venous in 14(52\%) patients. Follow-up included echocardiography at 1 day and 1 month post-implantation

Results: All implantations were technically successful. Angiography soon after device release showed complete closure in $20(77 \%)$ and trivial contrast flow in 6 patients with complete occlusion on echocardiography by the next day. The median procedure time was $41(15-82)$ minutes and the fluoroscopy time 6(2.2-26.5) minutes. There were no complications.

Conclusion: The ADO II is a versatile and very effective device for catheter closure of PDA up to diameters of $5.5 \mathrm{~mm}$. The articulations, high early closure rate, arterial/venous approach options and small diameter delivery catheter $(4-5 \mathrm{~F})$ are all beneficial features of this new device.

\section{0}

Bailout stent for critical coarctation in premature/ critical/complex/syndromic neonates

Matthias Gorenflo, Dept. Paed. Cardiol., UZ - KU Leuven; B-3000 Leuven, Belgium, Belgium

Derize Boshoff, Dept. Paed. Cardiol., UZ - KU Leuven; B-3000

Leuven, Belgium, Belgium

Benedicte Eyskens, Dept. Paed. Cardiol., UZ-KU Leuven; B-3000

Leuven, Belgium, Belgium

Ruth Heying, Dept. Paed. Cardiol., UZ - KU Leuven; B-3000

Leuven, Belgium, Belgium

Felip Rega, Dept. Cong. Heart Surg., UZ - KU Leuven; B-3000

Leuven, Belgium, Belgium

Bart Meyns, Dept. Cong. Heart Surg., UZ - KU Leuven; B-3000

Leuven, Belgium, Belgium

Marc Gewillig, Dept. Paed. Cardiol., UZ - KU Leuven; B-3000

Leuven, Belgium, Belgium

Introduction: Surgical repair of critical coarctation can be problematic in premature-critical-early postoperative-syndromatic neonates. Primary stent implantation allows to buy time and postpone surgery.

Patients: 18 neonates with severe coarctation: 5 prematuredysmature (1400-2000 g), 5 syndromatic-complex malformation, 4 early $(<24 \mathrm{~h})$ and 4 late $(4-8$ weeks $)$ after surgical coarctectomy.

Methods: A 4F (in 2 pts 5F) short introducer sheath in femoral artery; heparin $100 \mathrm{U} / \mathrm{kg}$; angiogram; a 0.014 " wire in the arch (in 1 patient coarctation was crossed anterograde). Bare coronary stents (diameter $4.0 \quad[3.5-6.0] \mathrm{mm}$; length $12 \quad[8-16] \mathrm{mm}$ ) advanced through $4 \mathrm{~F}$ sheath; position controlled before deployment by retrograde sequential contrast-saline injection through sheath. In 2 patients an additional stent was put in the cross. Stents were removed surgically depending on clinical needs.

Results: Adequate aortic flow was obtained in 17 patients; additional $8 \mathrm{~mm}$ balloon dilation in 1 patient after 6 days; femoral artery preserved in $16 / 18$ patients; 2 deaths non-procedure related (1 severe syndromic, 1 endocarditis post sepsis). In patients with simple stented coarctation the stent was removed after 2.2 (0.2-4.0) months. In complex cardiac malformation, additional delay obtained by stent dilation with $7-8 \mathrm{~mm}$ balloon after 20(5-38) months; the stent finally removed 20(5-38) months after implantation. Surgical technique: end-to-end in 7, extensive arch patch reconstruction in 7; 2 pts are awaiting stent removal. Final gradient across arch $0-20 \mathrm{mmHg}$ Doppler in all patients.

Conclusions: In premature/critical/complex syndromic neonates with severe coarctation, bailout stenting followed by early or late surgical coarctectomy appears a promising concept.
4111

Patient specific study of stent fracture after percutaneous pulmonary valve implantation

Silvia Schievano, UCL Institute of Child Health \& Great Ormond Street Hospital for Children, United Kingdom

Andrew M Taylor, UCL Institute of Child Health \& Great Ormond Street Hospital for Children, United Kingdom

Claudio Capelli, UCL Institute of Child Health \& Great Ormond

Street Hospital for Children, United Kingdom

Johannes Nordmeyer, UCL Institute of Child Health \& Great Ormond Street Hospital for Children, United Kingdom

Philipp Lurz, UCL Institute of Child Health \& Great Ormond Street Hospital for Children, United Kingdom

Philipp Bonhoeffer, UCL Institute of Child Health \& Great Ormond Street Hospital for Children, United Kingdom

Background: Stent fracture is a recognized complication following percutaneous pulmonary valve implantation (PPVI).

Methods: Biplane fluoroscopy images from a PPVI patient who experienced stent fracture were used to reconstruct the 3D shape and deformation of the stent in situ. Asymmetries were measured in all 3 orthogonal directions, in early systole and diastole. Magnetic resonance data from the same patient was used to create a finite element (FE) model of the patient's implantation site. The expansion of the PPVI stent into this patient's right ventricular outflow tract (RVOT) geometry was compared with the free expansion of the PPVI stent up to a uniformly deployed configuration, using FE analyses.

Results: The 3D reconstructions from fluoroscopy images showed asymmetries in all directions and the most expanded cells of the stent corresponded to the fracture locations. Although a simplified FE modelling of stent/implantation site interaction was adopted, this analysis gave useful information about the influence of the RVOT on the final geometry and mechanical performance of the stent. When deployed into the RVOT, the FE stent showed an asymmetrical shape, similar to the geometry seen in the "real" reconstructed fluoroscopy images. This geometry, when compared to the free-expanded stent, resulted in higher stresses in the portion of the stent where fractures occurred. Furthermore, fatigue fracture which was not predicted in the free-deployed stent, developed in the asymmetrically expanded device.

Conclusion: The interaction between the PPVI device and the patient's RVOT is likely to be the crucial factor involved with this undesired event.

\section{2}

Comparison of transcranial contrast Doppler and transesophageal contrast echocardiography in the detection of patent foramen ovale in migrainer Guang-yi Wang, Department of cardiology, Chinese PLA general hospital, China

Zhi-feng Wang, China

Zhi-bin Zhou, China

Objective: Transesophageal echocardiography (TEE) and transcranial Doppler (TCD) examination with contrast injection can both be used to search for a patent foramen ovale (PFO). We compared the accuracy of these techniques for identifying a $\mathrm{PFO}$ in patients with migraine. Methods: PFO was assessed by performing TEE and TCD with IV injection of agitated saline in 41 subjects with migraine with aura $(\mathrm{n}=26)$ and without aura $(\mathrm{n}=15)$ in OPD. Results: We found 22 PFOs with both TCD and TEE. Both contrast tests were negative in 16 patients. 
For 1 patient, the contrast test was positive only with TEE. And 2 were positive only in TCD. In comparison with TEE, adopted as the "gold standard", TCD had a sensitivity of $94 \%$ (16 of 17), specificity of $92 \%$ (17 of 18). Conclusions: cTCD is a examination with high sensitivity and high specificity in detecting a PFO.

Key words: transcranial Doppler; transesophageal echocardiography; patent foramen ovale; migraine without aura; migraine with aura.

\section{3}

Subnormal Effective Forward Cardiac Index in Congenital Heart Disease with Right Ventricular Loading: Assessment by Magnetic Resonance Imaging

Boris S Lowe, McGill University Adult Unit for Congenital Heart

Disease Excellence (MAUDE Unit), Montreal, Canada

Ariane J Marelli, McGill University Adult Unit for Congenital

Heart Disease Excellence (MAUDE Unit), Montreal, Canada

Luc Jutras, McGill University Adult Unit for Congenital Heart Disease Excellence (MAUDE Unit), Montreal, Canada

Natalie Bottega, McGill University Adult Unit for Congenital Heart Disease Excellence (MAUDE Unit), Montreal, Canada

Giuseppe Martucci, McGill University Adult Unit for Congenital Heart Disease Excellence (MAUDE Unit), Montreal, Canada

Judith Therrien, McGill University Adult Unit for Congenital Heart Disease Excellence (MAUDE Unit), Montreal, Canada

Objective: We sought to assess the determinant variables of a low cardiac index among ambulatory patients with congenital heart disease (CHD) and haemodynamic loading of the right ventricle $(\mathrm{RV})$.

Methods: One hundred consecutive patients (mean age $32 \pm 14$ years, $48 \%$ female) aged $>18$ years with CHD referred for magnetic resonance imaging to assess the $\mathrm{RV}$ were included. Effective forward cardiac index (CIeff, subnormal defined as $<2.61 / \mathrm{min} / \mathrm{m}^{2}$ ) was derived from ventricular volumetric and great arterial phaseencoded velocity mapping, calculated by [end-diastolic volume (EDV) - end-systolic volume (ESV)) - valvular regurgitant volume] $\mathrm{x}$ heart rate (HR)/body surface area.

\begin{tabular}{|c|c|c|c|}
\hline & $\begin{array}{l}\text { LVCleff } \\
<2.61 / \mathrm{min} / \mathrm{m}^{2} \\
\mathrm{n}=28\end{array}$ & $\begin{array}{l}\text { LVCleff } \\
<=2.61 / \mathrm{min} / \mathrm{m}^{2} \\
\mathrm{n}=72\end{array}$ & $\mathrm{p}$ value \\
\hline Age & $30.5 \pm 15.8$ & $25.5 \pm 12.3$ & 0.03 \\
\hline Female gender & $18(64 \%)$ & $30(42 \%)$ & NS \\
\hline Reparative cardiac surgery & $17(46 \%)$ & $46(64 \%)$ & NS \\
\hline Beta-blocker medication & $4(14 \%)$ & $8(11 \%)$ & NS \\
\hline NYHA > 1 & $16(57 \%)$ & $18(25 \%)$ & $<0.01$ \\
\hline \multicolumn{4}{|l|}{ Loading condition } \\
\hline volume & $17(61 \%)$ & $45(63 \%)$ & NS \\
\hline pressure & $6(21 \%)$ & $15(21 \%)$ & NS \\
\hline mixed & $5(18 \%)$ & $11(15 \%)$ & NS \\
\hline $\operatorname{HR}(/ \min )$ & $63 \pm 13$ & $69 \pm 11$ & 0.02 \\
\hline LVCleff $\left(1 / \mathrm{min} / \mathrm{m}^{2}\right)$ & $2.3 \pm 0.2$ & $3.2 \pm 0.7$ & $<0.01$ \\
\hline $\operatorname{LVEDVI}\left(\mathrm{ml} / \mathrm{m}^{2}\right)$ & $75.8 \pm 13.7$ & $90.4 \pm 23.4$ & $<0.01$ \\
\hline LVEF (\%) & $53.5 \pm 9.4$ & $57.5 \pm 8.4$ & NS \\
\hline RVCleff $\left(1 / \mathrm{min} / \mathrm{m}^{2}\right)$ & $2.3 \pm 0.6$ & $3.4 \pm 1.1$ & $<0.01$ \\
\hline Rveff Stroke Volume index $\left(\mathrm{ml} / \mathrm{m}^{2}\right)$ & 37.8 & 52.4 & $<0.01$ \\
\hline $\operatorname{RVEDVI}\left(\mathrm{ml} / \mathrm{m}^{2}\right)$ & $134 \pm 45$ & $135.1 \pm 50.7$ & NS \\
\hline RVEF (\%) & $45.8 \pm 11.8$ & $51 \pm 9.8$ & NS \\
\hline Total RV regurgitant volume $(\mathrm{ml})$ & $39.5 \pm 46$ & $12 \pm 36.9$ & 0.03 \\
\hline
\end{tabular}

median \pm standard deviation

Results: More than one quarter of the study population had a low LVCIeff. Prior reparative surgery, beta-adrenergic receptor antagonist medication, RV pressure and volume loading conditions were similar between patients with low and normal CIeff. Almost half of the patients with subnormal CIeff were asymptomatic. Patients with subnormal LVCIeff were characterised by smaller indexed LVEDV, smaller RV forward stroke volume and larger total (tricuspid and pulmonic) RV regurgitant volume (table). Valvular regurgitation even of modest severity appears to be an integral contributor to low forward stroke volumes ejected from a RV already haemodynamically stressed by CHD. Smaller indexed LV cavity volumes may reflect consequential LV preload deprivation.

Conclusions: Low cardiac forward flow is not uncommon among ambulatory patients with CHD and is not identified by symptomatic status, RV cavity volumes and ejection fraction. Assessment for valvular regurgitation of any severity is requisite when evaluating effective cardiac function.

\section{4}

B-type Natriuretic Peptide in Pregnant Women with Cardiac Disease

David Tanous, Australia

Samuel C Siu, University Health Network and Mount Sinai Hospital, Toronto, Canada

Jennifer Mason, University Health Network and Mount Sinai Hospital, Toronto, Canada

Matthias Greutmann, University Health Network and Mount Sinai

Hospital, Toronto, Canada

Rachel M Wald, University Health Network and Mount Sinai

Hospital, Toronto, Canada

Mathew Sermer, University Health Network and Mount Sinai

Hospital, Toronto, Canada

Jack M Colman, University Health Network and Mount Sinai

Hospital, Toronto, Canada

Candice K Silversides, University of Toronto Pregnancy and Heart

Disease Research Program, University Health Network and Mount

Sinai Hospital, Toronto, Canada

Background: B-type natriuretic peptide (BNP) release occurs with hemodynamic stress. Although pregnancy can impose stress on the heart in women with heart disease, BNP levels during pregnancy in this group of women has not been studied. The objective of this study was to examine the neurohormonal response to pregnancy in women with heart disease.

Methods: We prospectively enrolled 66 women with structural heart disease (age $31 \pm 5$ years) and 12 healthy pregnant controls. Clinical data, echocardiographic data and plasma BNP levels during the 1st trimester, 3rd trimester and post-partum $(>6$ weeks) were obtained.

Results: The most common cardiac diagnoses were congenital cardiac lesions (74\%). Plasma BNP concentrations were significantly higher in women with cardiac disease (1st trimester $98 \pm 203$ vs. $28 \pm 14, \quad 3$ rd trimester $82 \pm 82$ vs. $24 \pm 13$, postpartum $102 \pm 144 \mathrm{pg} / \mathrm{mL}$ vs. $24 \pm 8 \mathrm{pg} / \mathrm{mL}, \mathrm{p}<0.05$ all time-points) compared to controls. Adverse cardiac events occurred in 8 patients (death $\mathrm{n}=1$, need for cardiac surgery $\mathrm{n}=1$, sustained arrhythmia $\mathrm{n}=3$; heart failure $\mathrm{n}=3$ ). Women with events had higher BNP levels $(518 \pm 442$ vs. $89 \pm 58 \mathrm{pg} / \mathrm{mL}, \mathrm{p}<0.001)$, lower $\mathrm{LVEF}$ $(54 \pm 4$ vs.62 $\pm 7, \mathrm{p}=0.006)$ and more commonly had a history of cardiac events before pregnancy $(63 \%$ vs.11\%, p = 0.003). A maximum BNP $>100 \mathrm{pg} / \mathrm{mL}$ was present in $100 \%$ (8/8) of patients with adverse events. Thirty-three percent $(8 / 24)$ of patients with a $\mathrm{BNP}>100 \mathrm{pg} / \mathrm{mL}$ had an adverse event.

Conclusions: Pregnant women with heart disease have increased BNP levels during pregnancy compared to pregnant women without heart disease. Elevated BNP levels during pregnancy and early postpartum are associated with increased risk of adverse cardiac events. 


\section{5}

Endothelin receptor antagonism is an effective and safe long term treatment modality in pulmonary arterial hypertension associated with complex congenital heart disease

Fiona D Kermeen, The Prince Charles Hospital, Australia

Cherie Franks, The Prince Charles Hospital, Australia

Kathy O'Brien, The Prince Charles Hospital, Australia

Helen Seale, The Prince Charles Hospital, Australia

Kathleen Hall, The Prince Charles Hospital, Australia

Keith McNeil, The Prince Charles Hospital, Australia

Dorothy J Radford, The Prince Charles Hospital, Australia

Introduction: Treatment options for patients with pulmonary arterial hypertension (PAH) associated with congenital heart disease (CHD) are limited. Bosentan has been shown to improve pulmonary haemodynamics and exercise tolerance short term in these patients but long term clinical studies are lacking.

Aim: To report long term efficacy and safety data with endothelin receptor antagonists (ERA) in patients with $\mathrm{PAH}$ associated CHD.

Methods: Prospective, single centre study of 44 patients (28 females, 15 Trisomy 21, mean age $32 \pm 11$ years) prescribed ERA (39 bosentan, 5 sitaxentan) from 2003 to 2008 . Outcome measurements of oxygen saturation $(\mathrm{SaO} 2)$, WHO class, 6MWD, echocardiographic parameters, BNP and adverse events were analysed.

Results: Mean duration of therapy was $16 \pm 13$ months in 27 simple CHD and 17 complex CHD (single ventricle physiology). Four patients failed ERA, three died ( 2 progressive RHF. No abnormal liver transaminases occured on bosentan, one case on sitaxentan). After 3 and 6 months of treatment significant improvement seen in WHO class (mean 3.16 vs 2.7 vs 2.47, $\mathrm{p}<0.01, \mathrm{p}<0.01)$ and 6MWD $(339 \pm 21$ vs $401 \pm 19$ vs $423 \pm 20, \mathrm{p}<0.01, \mathrm{p}<0.01)$ compared with baseline. Trisomy 21 and complex CHD subgroup significantly improved 6MWD at 6 months $(257 \pm 30$ vs $345 \pm 27, p=0.02 ; 307 \pm 26$ vs $356 \pm 34, \mathrm{p}=0.02)$ respectively. No changes in $\mathrm{SaO} 2, \mathrm{RV}$ or $\mathrm{LV}$ function (echocardiographic) were demonstrated.

Conclusion: This large single centre study shows ERA is an effective and safe treatment in PAH associated CHD with/ without Trisomy 21. The improvements in exercise tolerance are similar to reported benefits in other forms of $\mathrm{PAH}$.

\section{6}

Echographic Parameters Associated with Atrial Fibrillation in Adults Congenital Heart Disease Judith Bouchardy, McGill Adult Unit for Congenital Heart Disease Excellence (MAUDE Unit), Canada

Ariane J Marelli, McGill Adult Unit for Congenital Heart Disease

Excellence (MAUDE Unit), Canada

Natalie A Bottega, McGill Adult Unit for Congenital Heart Disease

Excellence (MAUDE Unit), Canada

Giuseppe Martucci, McGill Adult Unit for Congenital Heart Disease Excellence (MAUDE Unit), Canada

Judith Therrien, McGill Adult Unit for Congenital Heart Disease Excellence (MAUDE Unit), Canada

Background: In the general population, atrial fibrillation (AF) is thought to originate from the left atrium (LA). Left-sided echocardiographic parameters associated with AF have been described, including LA and right atrium (RA) enlargement, with a RA/LA ratio $<1$. Right-sided pathologies are frequent in congenital heart disease (CHD) and AF is a well known complication. Our objectives were to compare prevalence and echographic parameters associated with AF in CHD patients with right-sided pathologies versus left-sided pathologies.

Methods: All consecutive adult CHD patients with right-sided and left-sided pathologies were identified over a 12 months period. Diagnosis of AF was made on ECG or Holter recording by chart review. LA and RA length and area were obtained from the apical views, and volumes were calculated using $8 / 3 \pi \mathrm{A} 2 / \mathrm{L}$ where $\mathrm{A}=$ area and $\mathrm{L}=$ length.

\begin{tabular}{lllll}
\hline & \multicolumn{2}{l}{ Right-sided pathologies } & \multicolumn{2}{l}{ Left-sided pathologies } \\
\cline { 2 - 5 } & $\begin{array}{l}\text { No Atrial } \\
\text { Fibrillation }\end{array}$ & $\begin{array}{l}\text { Atrial } \\
\text { Fibrillation }\end{array}$ & $\begin{array}{l}\text { No Atrial } \\
\text { Fibrillation }\end{array}$ & $\begin{array}{l}\text { Atrial } \\
\text { Fibrillation }\end{array}$ \\
\hline $\begin{array}{l}\text { RA volume }(\mathrm{ml})(\text { Normal } \\
\text { values }=18-58)\end{array}$ & 50 & 84 & 39 & 53 \\
$\begin{array}{l}\text { LA volume (ml) (Normal } \\
\text { values }=18-58)\end{array}$ & 36 & 71 & 44 & 67 \\
RA/LA ratio & 1.38 & 1.20 & 0.88 & 0.80 \\
\hline
\end{tabular}

Results: Three hundred and ten patients were identified, 198 with right-sided lesions, 112 with left-sided lesions. Prevalence of atrial fibrillation was $16 \%$ in right-sided pathologies versus $7 \%$ in left-sided pathologies. In patients with $\mathrm{AF}$ and right-sided pathologies, the RA volume was enlarged and greater than the LA volume. In patients with AF and left- sided pathologies, the LA volume was increased and greater than RA volume.

Conclusion: Patterns of atrial dilatation differ according to sidedness of underlying cardiac pathologies. Different initiating mechanisms of AF in patients with right-sided heart lesion should be considered.

\section{2}

Anaesthetic management of a child with cyanotic congenital heart disease and functionally single ventricle for non-cardiac surgery

Aarti Agarwal, Sahara Hospital, Lucknow, India, India

Puneet Goyal, Sanjay Gandhi Post Graduate Institute of Medical

Sciences, Lucknow, India, India

Objective: Successful anaesthetic management of child with tricuspid atresia and functionally single ventricle, undergoing corrective surgery for ano rectal malformation.

Methods and results: 14 month old child, presented with ano-rectal malformation and cyanosis since birth. Echocardiography showed tricuspid atresia, large osteum secondum ASD with right to left shunt, dilated and hypertrophied LV with normal contractility, rudimentary RV, Pulmonary stenosis, and small inlet VSD. Child had undergone Modified BT shunt at the age of 4 months, which was patent and functioning well. SPO2 was $80-82 \%$ at rest and $65-70 \%$ during crying on room air. Haematocrit was $52 \%$ and other lab investigations were with in normal limits. Child was on oral ecosprin which was stopped 5 days preoperatively and heparin infusion was started and was stopped 6 hrs prior to surgery. The child was posted for definitive surgery of anorectal malformation, Anaesthetic technique included fentanyl, pancuronium and sevoflurane in air oxygen mixture. ECG, SPO2, Arterial blood pressure and central venous pressure were monitored. Arterial BP was maintained in the range of 80-85/40-45 mm Hg, Phenylephrine was kept ready to treat any episode of hypotension aggressively but was not required. CVP was maintained approx. $12 \mathrm{mmHg}$. Surgery lasted for $3 \mathrm{hrs}$ and child was extubated at the end of surgery. Hypoxemia, hypercarbia, acidosis and hypothermia were avoided.

Conclusion: Most important goal was to maintain shunt patency and flow as this was the only source of pulmonary blood flow and 
oxygenation. It was also important to maintain the delicate balance between PVR and SVR.

\section{3}

Brain Immaturity on MRI is Associated with Pre- and Postoperative Brain Injury in Neonates Undergoing Cardiac Surgery

Dean B Andropoulos, Texas Children's Hospital/Baylor College of Medicine, United States

Jill V Hunter, Texas Children's Hospital/Baylor College of Medicine, United States

David P Nelson, Texas Children's Hospital/Baylor College of Medicine, United States

Stephen A Stayer, Texas Children's Hospital/Baylor College of Medicine, United States

Ann R Stark, Texas Children's Hospital/Baylor College of Medicine, United States

Emmitt D McKenzie, Texas Children's Hospital/Baylor College of Medicine, United States

Jefrey S Heinle, Texas Children's Hospital/Baylor College of Medicine, United States

Introduction: Full-term neonates with CHD have brain immaturity on MRI scans.(1,2) This may explain the vulnerability of these neonates to injury. We hypothesized that neonates with brain immaturity are more likely to have both pre- and postoperative brain injury.

Methods: 68 neonates with CHD, 35+ weeks gestational age, had pre-, and 7-day postoperative MRI. Brain abnormality score was calculated, including white matter injury (WMI), infarction, and hemorrhage. Brain maturity was assessed from T1 and T2weighted MRI using the Total Maturity Score of Childs(3) scoring 4 features: myelination, cortical infolding, germinal matrix, and bands of migrating glial cells.

Results: Birthweight was lower in single ventricle patients, but there was no difference in gestational age, head circumference, or TMS for the entire group (Table).

\begin{tabular}{lll}
\hline Parameter & $\begin{array}{l}\text { Single Ventricle, } \\
\mathbf{n}=36\end{array}$ & $\begin{array}{l}\text { Two Ventricle, } \\
\mathbf{n}=32\end{array}$ \\
\hline Birthweight (kg) & $3.01 \pm 0.33$ & $3.27 \pm 0.56^{*}$ \\
Gestational age (weeks) & $38.3 \pm 1.3$ & $38.8 \pm 1.4$ \\
Head circumference (cm.) & $33.2 \pm 1.7$ & $33.9 \pm 1.3$ \\
MRI Total Maturity Score (TMS) & $11.8 \pm 1.5$ & $11.8 \pm 1.0$ \\
Patients with TMS $\leqslant$ 10, no., (\%) & $8(22)$ & $6(19)$ \\
Preoperative total MRI abnormality score & $1.8 \pm 2.6$ & $2.3 \pm 2.8$ \\
7-day postoperative total MRI abnormality & $3.8 \pm 4.2$ & $3.2 \pm 3.7$ \\
$\quad$ score & & \\
\hline
\end{tabular}

Seven of 13 patients with TMS $\leqslant 10$ (equal to brain maturity of 35 weeks gestational age or less) had postoperative brain injury score $\geqslant 6$ (moderate injury), vs. only 10 of 54 with TMS $>10$. $(\mathrm{p}=0.01)$. Preoperative WMI was more likely with TMS $<10$ (5/13 vs. 6/55, $\mathrm{p}=0.03)$, as was postoperative WMI $(6 / 13$ vs. $9 / 54, p=0.05)$. Discussion: Neonates with CHD who have a structurally immature brain are more likely to have both pre- and postoperative WMI, and moderate or severe postoperative brain injury score. This could explain the high rate of WMI in these patients, as the brain in CHD shares features with the premature neonates, who also has a high rate of WMI.

References:

1. Licht DJ. JTCVS 2008; in press.

2. NEJM 2007;357:1928

3. AJNR 2001;22:1577

\section{4}

EEG Seizures After Neonatal Cardiac Surgery with HighFlow Bypass and Maximized Oxygen Delivery Strategy Dean B Andropoulos, Texas Children's Hospital/Baylor College of Medicine, United States

David P Nelson, Texas Children's Hospital/Baylor College of Medicine, United States

Ann R Stark, Texas Children's Hospital/Baylor College of Medicine, United States

Emmitt D McKenzie, Texas Children's Hospital/Baylor College of Medicine, United States

Jeffrey S Heinle, Texas Children's Hospital/Baylor College of Medicine, United States

Charles D Fraser, Texas Children's Hospital/Baylor College of

Medicine, United States

Richard A Hrachovy, Texas Children's Hospital/Baylor College of

Medicine, United States

Eli M Mizrahi, Texas Children's Hospital/Baylor College of Medicine, United States

Introduction: EEG seizures are seen in 11-20\% of neonates undergoing cardiac surgery with $\mathrm{CPB}$ with low flow or circulatory arrest. $(1,2)$ The objective of this study was to assess neonates for EEG seizures after surgery with high flow $\mathrm{CPB}$ and maximized cerebral oxygenation. The hypothesis was that EEG seizures would be minimized.

Methods: CPB protocol was $150-\mathrm{ml} / \mathrm{kg} / \mathrm{min}$ flows, $\mathrm{pH}$ stat, hematocrit 30-35\%, NIRS monitoring. Antegrade cerebral perfusion was used, DHCA was minimized. 10 lead neonatal EEG was used for 6 hour baseline EEG preoperatively, and 72 hours of continuous postoperative EEG.

Results: 68 patients were studied: 36 single ventricle, and 32 two ventricle. In 4968 hours of postoperative EEG, no $2 \mathrm{~V}$ patients had seizures, and $2 \mathrm{SV}$ patients had brief seizures, for an incidence of $2.9 \%$. $(p=0.49)$ A 30 -second seizure occurred in a Norwood Stage I patient at hour 72 , and another SV patient had a 30 second seizure at hour 7, and 95 second seizure at hour 10 (See Table). Both had prolonged $\mathrm{rSO} 2<45 \%$ (total of 2105 and 668 minutes) although low $\mathrm{rSO} 2$ was not associated with EEG seizures $(\mathrm{p}=0.1)$.

\begin{tabular}{|c|c|c|}
\hline Parameter & $\mathrm{SV}(\mathrm{n}=36)$ & $2 \mathrm{~V}(\mathrm{n}=32)$ \\
\hline Postop EEG seizures (no. of patients, \%) & $2(6)$ & 0 \\
\hline Seizure burden (seizures $\mathrm{x}$ seconds) & $4.3 \pm 21.1$ & 0 \\
\hline $\begin{array}{l}\text { Midazolam }(\mathrm{mg} / \mathrm{kg}) \text {, intra- and } 72 \mathrm{~h} \\
\text { postop (\#pts) }\end{array}$ & $4.6 \pm 4.6(36 \mathrm{pts})$ & $2.3 \pm 2.4 *(32 \mathrm{pts})$ \\
\hline $\begin{array}{l}\text { Lorazepam (mg/kg), intra- and } 72 \mathrm{~h} \\
\text { postop (\#pts) }\end{array}$ & $1.0 \pm 1.4(15 \mathrm{pts})$ & $0.7 \pm 0.6(21 \mathrm{pts})$ \\
\hline
\end{tabular}

Discussion: Previous reports note duration of ictal activity of 139 minutes in one study, and mean 36 seizures in another. Our cohort exhibits dramatically reduced EEG seizure burden, likely due to improved perioperative oxygen delivery to the brain. Our patients did receive a significant dose of benzodiazepines; both patients who seized were SV patients receiving large doses of intra- and postoperative benzodiazepines.

References:

1. Circulation 1998; $97: 773$

2. JTCVS 2005;130:1278

6005

Anesthesiologist Administered Propofol Sedation for Children Undergoing Echocardiography

Hamish M Munro, Congenital Heart Institute, Arnold Palmer Hospital for Children, United States 
Donald E Felix, Congenital Heart Institute, Arnold Palmer Hospital for Children, United States

Kimberly A Hogan, Congenital Heart Institute, Arnold Palmer Hospital for Children, United States

Craig E Fleishman, Congenital Heart Institute, Arnold Palmer Hospital for Children, United States

Objectives: Given the variability and unpredictability of oral sedation, we aimed to determine the safety, efficacy and efficiency of intravenous propofol sedation in children with congenital heart disease undergoing transthoracic echocardiography.

Methods: Data from consecutive patients scheduled for echocardiograms from January 2005 to December 2008 were recorded prospectively. Sedation was initiated with sevoflurane, IV access secured and propofol infused at $100-200 \mathrm{mcg} / \mathrm{kg} / \mathrm{min}$. Patients breathed spontaneously with nasal cannula and end-tidal CO2 sampling. Vital signs were recorded at 5 minute intervals. Adverse events were documented, as were sedation time to echocardiogram, sedation duration and time to discharge.

Results: One hundred and ninety one records were analyzed. Median age was 24 months (3 months to 13 years) and mean weight $12.6 \mathrm{~kg}$. Fifty one patients $(27 \%)$ had a known genetic anomaly or developmental delay. Five had previously failed chloral hydrate sedation. Forty seven patients $(25 \%)$ had single ventricle physiology. In two patients IV access proved impossible and sedation was continued with sevoflurane. Echocardiograms were completed in all patients. Mean time from sedation to echo, sedation duration, recovery room time and total hospital stay was 7, 61, 49 and 115 minutes respectively. Adverse events included hypotension (2) and bradycardia (1). In two patients a laryngeal mask was inserted electively (one with sleep apnea, one with known difficult airway), no patients required intubation.

Conclusions: Propofol sedation is a safe effective alternative to oral sedation in children with heart disease. With a low incidence of adverse events, it provides ideal conditions for transthoracic echocardiography.

6006

Rare case of pulmonary AV fistula communicating with left atrium and presenting as cyanotic heart disease: Role of Pre-incision echocardiography in diagnosis and management

Puneet Goyal, Sanjay Gandhi Post Graduate Institute of Medical Sciences, Lucknow, India, India

Prabhat Tewari, Sanjay Gandhi Post Graduate Institute of Medical

Sciences, Lucknow, India, India

Surendra Kumar Agarwal, Sanjay Gandhi Post Graduate Institute of Medical Sciences, Lucknow, India, India

Objective: This report describes the diagnosis and management of a rare case of pulmonary AV fistula communicating with left atrium and presenting as cyanotic heart disease in a child.

Methods and results: 6 year old boy presented with recurrent respiratory tract infection since 6 months of age, dyspnoea on exertion and cyanosis since last 2 years, without any cyanotic spells. Echocardiography revealed $6 \mathrm{~mm}$ osteum secondum ASD with left to right shunt, large AV fistula with flow in Right Pulmonary Artery, contrast echo revealed early appearance of contrast in LA. Angiography showed giant pulmonary AV fistula arising from RPA and appearance of contrast in enlarged LA but the exact site of communication between the two could not be demonstrated. Cardiac catheterization data is shown in table 1.
Trans-thoracic echo done in Operation room after induction of anaesthesia showed a communication between right pulmonary artery and LA, which was entering from posterior wall of LA, also the giant aneurismal sac was lifting the LA from behind producing a camel hump (Fig 1. parasternal long axis view). Under standard cardiopulmonary bypass \& cardioplegia, aneurismal sac was seen arising from RPA and entering in the posterior wall of LA. LA was opened and the fistula repaired, ASD closed. Patient was weaned off from CPB. SPO2 returned to $100 \%$ after surgery. Patient was extubated 6 hrs after the surgery. Conclusion: Echocardiography in operation room was helpful in localizing the site of fistula so that surgeons could locate it quickly without wasting precious time on $\mathrm{CPB}$.

Table 1. Pressure \& Oxymetric data on cardiac catheterisation study

\begin{tabular}{lll}
\hline Site & Oxygen saturation & Systolic/diastolic pressure $(\mathrm{mmHg})$ \\
\hline SVC & $71.4 \%$ & $5 / 3$ \\
IVC & $65.9 \%$ & $6 / 4$ \\
PA Main & $71.2 \%$ & $12 / 10$ \\
LV & $79.5 \%$ & $125 / 0$ \\
Ascending Aorta & $84 \%$ & $110 / 70$ \\
\hline
\end{tabular}

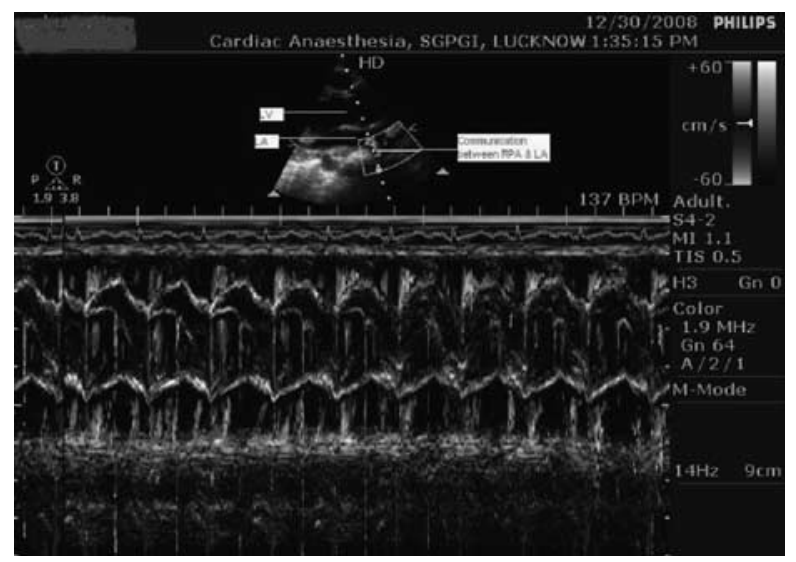

6007

Transesophageal Echocardiography Probe May Impede Cerebral Blood Flow

Samantha J Hill, Montreal Children's Hopsital, McGill University, Canada

Karen Brown, Montreal Children's Hopsital, McGill University, Canada Luc Jutras, Montreal Children's Hopsital, McGill University, Canada Christo I Tchervenkov, Montreal Children's Hopsital, McGill

University, Canada

Introduction: Transesophageal Echocardiography (TEE) is frequently used in pediatric cardiac surgery. Although widely accepted to be a safe procedure, compression of vascular structures by the TEE probe may occur. We present a previously undescribed phenomenon wherein insertion of a TEE probe significantly altered cerebral blood flow measured via transcranial doppler over the middle cerebral artery.

Methods: This was a case-control study of nine patients, using each patient's contralateral middle cerebral artery as the control. Cases were identified intra-operatively, on the basis of a $10 \%$ or larger decrease in cerebral blood flow velocity during insertion of the TEE. The contralateral side was employed as a control. Results were analysed using both parametric and non-parametric testing methods. 
Results: All events involved the left-sided blood flow; two were associated with significant regional cerebral desaturation. When compared to the right side, the decrease in cerebral blood flow seen with probe insertion was statistically significant $(\mathrm{p}<0.05)$. All events resolved with removal of the TEE probe.

Conclusion: This series indicates a need for due diligence. TEE may have deleterious effects on cerebral blood supply, and factors clearly identifying those at risk have yet to be identified. In the event of a significant change seen in cerebral blood flow with TEE insertion, which cannot be resolved by repositioning the head or probe, removal of the probe with subsequent postoperative re-insertion may be indicated. This affects a minority of patients, and seems appropriate to avoid potential hours of compromised cerebral perfusion.

\section{8}

A Prospective Audit of Paediatric Cardiac MRI under General Anaesthesia; Practise and Problems

Marina Hughes, Great Ormond Street Hospital, London, United Kingdom

Emma f Stockton, mater childrens hospital, brisbane, Australia Andrew Taylor, Great Ormond Street Hospital, London, United Kingdom Angus Mcewan, great ormond street hospital, London, United Kingdom

Background: Cardiac MRI (CMRI) is increasingly used to augment surgical planning and follow up of children with congenital heart disease. General anaesthesia (GA) is always required for imaging small children. We describe our experience of GA for paediatric CMRI, using data collected prospectively over 3 years.

Methods and Results: 120 patients presented for CMRI under GA from November 2005 to May 2008. Patients were aged 0-288 months (median 28 months), and weighed $2.8-64 \mathrm{~kg}$ (median $11.7 \mathrm{~kg})$.

Fifty-seven children $(47.5 \%)$ had a functionally single ventricle. Fifty patients $(42 \%)$ had normal oxygen saturations. Of the cyanosed patients, pulmonary blood flow was supplied through an aorto-pulmonary shunt in $33 \%$ and $13 \%$ had a cavopulmonary shunt. Sixteen patients (13\%) had a mixed circulation.

Six patients $(5 \%)$ had severely impaired systemic ventricular function. 14 patients $(11.6 \%)$ had at least moderate systemic outflow tract obstruction.

The majority of patients (90\%) were admitted as a day-case for CMRI. A senior, cardiac anaesthetist was continuously present during every case. Most patients (100/120, 83\%) received inhalational inductions with sevoflurane. The majority of children $(85 / 120,71 \%)$ had an uneventful CMRI under GA. Thirtythree (27.5\%) patients suffered minor adverse events. One major adverse event occurred; a patient with hypoplastic left heart syndrome (HLHS) became hypotensive and underwent successful resuscitation in the MRI scanner.

Conclusions: Despite the precarious physiology of children with congenital heart disease, GA for CMRI can be administered safely, with a senior multi disciplinary team. Inter-stage HLHS patients are at particularly risk of serious morbidity.

6009

Blood Conservation Techniques for cardiac surgery in a child of Jehovah's Witness: A case report

Jagdish M Shahani, KK Hospital for Women and Children, Singapore

The goal of safe, transfusion free pump case for a 4 years old child of Jehovah's Witness $(14 \mathrm{~kg})$ was acheived by employing various techniques by the surgical team. The techniques included preopeartive buildup of hematocrit with r-erythropoetin and iron supplements. The anesthetic techniques included hypotensive anaesthesia in the prebypass period, use of tranxeminic acid, intraopeartive collection and transfusion of autologous blood, appropraite reversal of heparin and minimizing blood sampling. The techniques employed by the perfusionist included reduction of priming volume by shortening the bypass circuit,maintaining lower perfusion pressures and continous ultrafiltration.

The lowest hematocrit during surgey was $25 \%$ and the surgery and recovery was uneventful.

Key Words: Jehovah's Witness, Cardiopulmonary bypass, blood transfusion

\section{0}

Non-invasive Cardiac Output Measurement Using Physioflow ${ }^{\mathrm{B}}$ in Anaesthetised Paediatric Patients- a comparison with mass spectrometry

Katherine Taylor, Department of Anesthesia,Hospital for Sick Children, Toronto, Canada

Helen Holtby, Hospital for Sick Children, Toronto, Canada

Gustavo La Rotta, Department of Cardiology, Labatt Family Heart

Centre, Hospital for Sick Children, Toronto, Canada

Cedric Manlhiot, Cardiovascular Clinical Research Unit, Labatt Family

Heart Centre,Hospital for Sick Children, Toronto, Canada

Andrew Redington, Labatt Family Heart Centre,Hospital for Sick

Children, Toronto, Canada

Objectives: The study objective is to evaluate the accuracy and reliability of cardiac output (CO) measurements using electrical bioimpedance cardiography, (Physioflow ${ }^{\circledR}$ ) (CO) compared to mass spectrometry $(\mathrm{CO})$, in children undergoing cardiac catheterisation.

Methods: After Institutional Ethics Committee approval and informed consent, eligible patients undergoing cardiac catheterization including oxygen consumption measurement by mass spectrometry were recruited for additional simultaneous monitoring using Physioflow ${ }^{\circledR}(\mathrm{PF})$. After anaesthesia induction, 6 Physioflow ${ }^{\circledR}$ ECG electrodes were applied. An Amis 2000 quadrupole mass spectrometer (Innovision A/S. Odense, Denmark) was adapted for patients ventilated with the Drager Primus ventilator (Drager, Germany). Measurements of CO were made after $\mathrm{VO}$ and heart rate stability and before angiography. The Physioflow ${ }^{\circledR}$ measured continuous real time measurements. CO measurements were averaged, indexed and compared with the indexed CO measurements using Bland-Altman analysis.

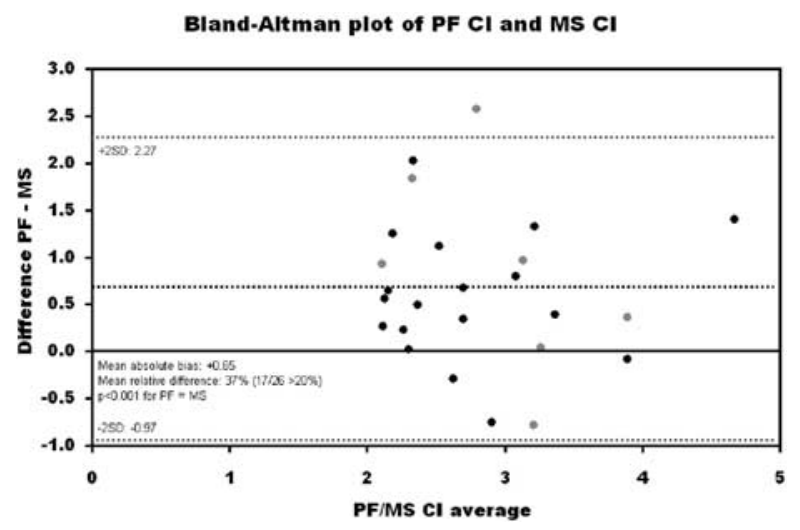

Results: Preliminary results of 26 patients (10 males/16 females) are presented. Median (range) age was 5.5 years (1.4-16.3 years) 
and median (range) BSA was $0.74 \mathrm{~m}$ (0.41-1.71 m). 5958/8435 (71\%) measurements were valid. Median measurements per patient $=316(40-616)$. Physioflow omitted 11\% CI measurements and identified $27 \%$ as artifact. A further $3 \%$ were identified by investigators as biologically implausible, Physioflow missed $30 \%$ of these. Mean bias $(\mathrm{PF}-\mathrm{MS})=+0.65 \mathrm{p}<0.001$, limits of agreement $(95 \%)=-0.97-2.27$. In $35 \%$ of patients $\mathrm{CO}$ was within $20 \%$ of CO and $69 \%$ of measurements were within $40 \%$. Impact: This represents the first study using Physioflow ${ }^{\mathbb{R}}$ to assess $\mathrm{CO}$ in anaesthetised children, and is an attempt to formally assess the utility of new equipment in the paediatric population. FICKPFFICKFICK2PFFICKPFFICK

\section{1}

Combined central venous catheter versus single-lumen catheter for monitoring left atrial pressure following pediatric cardiac surgery

Fuxia Yan, Cardiovascular Institute and Fu Wai Hospital, Chinese Academy of Medical Sciences, China

Rong Wang, Cardiovascular institute and $\mathrm{Fu}$ Wai hospital, China Lin Lin, Cardiovascular institute and Fu Wai hospital, China

Objective: To compare a new combined central venous catheter for monitoring left atrial pressure with a single-lumen catheter in pediatric cardiac patients. The combined catheter is made of a 5Fr triple-lumen catheter and a longer microtube inserted within. Method: 40 cases were observed following complex congenital heart defect repair. After anesthetic induction the combined catheter and single-lumen catheter were placed into right atrium in the same patient through jugular vein. The distal ends of the microtube and of the single-lumen catheter were repositioned transseptally and advanced into the left atrium after the repair of intracardiac defect. The ease of catheter placement and pressure measured by both were compared.

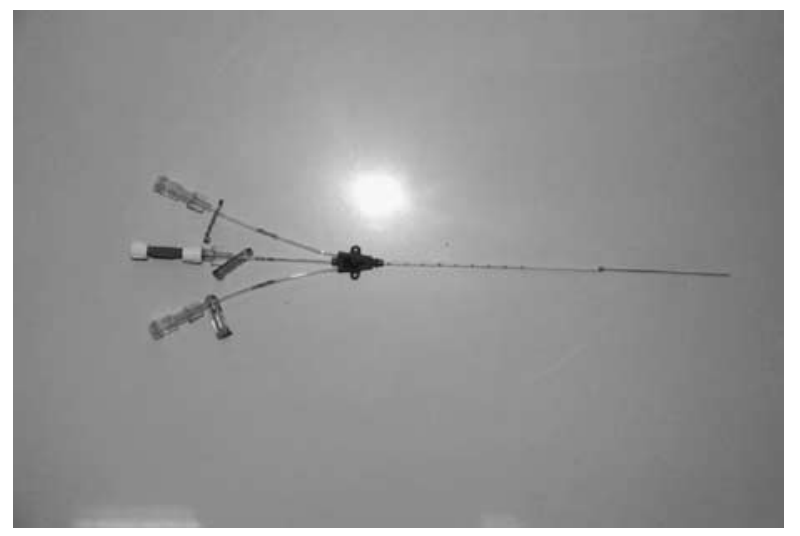

Results: Left atrial pressure measured by microtube and by singlelumen catheter were closly related. Both microtube insertion and blood sampling were quite easy. No mortality or catheter-related complication were observed.

Conclusion: Microtube inserted within the combined central venous catheter might be an alternative to single-lumen catheter for monitoring left atrial pressure and drug administration to left atrium. Catheterization time and costs might also be reduced.

\section{2}

The Development of a CDROM: An Aide to Fetal Cardiac Counseling

Kalhara Caldera, Faculty of Medicine, Dentistry and Health Sciences, University of Melbourne, Melbourne, Australia, Australia
Samuel Menahem, Fetal Cardiac Unit, Monash Medical Centre, Melbourne, Australia, Australia

Objective: Advances in ultrasound technology in prenatal scanning have led to increased detection of congenital heart defects. In a setting of great emotional distress affected parents need understand the abnormality so as to make an informed decision concerning pregnancy continuation. A computer learning aide may allow such parents to review the information provided at a time of their choosing.

Methods: A CDROM was developed to include information on normal heart structure and function, and ultrasound technology. Common abnormalities and their management options were developed, further enhanced by colour animations modified from diagrams on the Royal Children's Hospital, Melbourne, website. The diagrams showed direction of blood flow with varying colouration showing the degree of oxygenation. Five abnormalities were covered: ventricular septal defect, transposition of the great arteries, Fallot's tetralogy, hypoplastic left heart syndrome and tricuspid atresia. Once the abnormality was diagnosed, parents were offered the CDROM, and then asked to complete a questionnaire. They were only provided the code to review the specific diagnosis in their fetus. Results: To date, a total of 11 patients have been recruited. The responses highlighted strengths and weaknesses of the information provided but uniformly suggested that the CDROM was helpful, especially the diagrams provided, though there was a varying response as to the amount of medical detail thought appropriate.

Conclusions: The results are indeed promising and suggest such an aide can be developed further to include other congenital heart abnormalities for use in clinical practice.

\section{3}

\section{What do we know about isolated VSD in fetuses?}

Anna Kaplanska, Perinatology and Perinatal Cardiology Unit, 2nd Department of Obstetrics and Gynaecology, Medical University of Warsaw, Poland

Pawel Wlasienko, Perinatology and Perinatal Cardiology Unit, 2nd Department of Obstetrics and Gynaecology, Medical University of Warsaw, Poland

Joanna Kuran, Perinatology and Perinatal Cardiology Unit, 2nd Department of Obstetrics and Gynaecology, Medical University of Warsaw, Poland

Joanna Dangel, Perinatology and Perinatal Cardiology Unit, 2nd Department of Obstetrics and Gynaecology, Medical University of Warsaw, Poland

Objective: To evaluate prevalence of types of isolated VSDs in fetuses in relation to chromosomal aberrations and extracardiac malformations and assess perinatal follow-up.

Methods: Single-centre retrospective analysis of computer database of reference perinatal cardiology unit between 1994-2008. Out of 883 fetuses with congenital heart defects, 169(19\%) with isolated VSD were found. We divided pts into diagnosed before and after 2002 due to different organization of perinatal centre. Results: VSD was diagnosed in $29+/-6$ weeks of gestation before 2002, and in $26+/-6$ after 2002. Types of VSD: perimembranous-58(34\%), muscular-53(31\%), inlet-47(28\%), multiVSD-5(3\%), malalignment-3(2\%), unknown-3(2\%). Karyotype was performed in 87 pts; in 34 was normal, trisomy 18(T18)-27 fetuses, trisomy 21(T21)-15, trisomy13(T13)-8, other aberrations-4. 22(81\%) fetuses with T18 had inlet VSD, $11(73 \%)$ fetuses with T21-perimembranous VSD. Among muscular VSDs there were no chromosomal abnormalities. Most 
common extracardiac malformations were: CNS(19), facial(12), GI(11). Extracardiac malformations were mostly in T18, T13 and also with normal karyotype. Follow-up: table. Neonatal deaths were caused by lethal conditions, not VSD as such. In those cases perinatal palliative care was provided.

Conclusions:

1 Prognosis of fetal isolated VSD is different to postnatal due to chromosomal aberrations and extracardiac malformations.

2 Fetal large inlet and perimembranous VSD are strong markers of chromosomal abnormalities. Such diagnosis is an indication for karyotyping.

3 Fetal muscular isolated VSD with otherwise normal US is a benign lesion. Other investigations are unnecessary.

\begin{tabular}{cccccc}
\hline VSD type & TOP & $\begin{array}{c}\mathrm{I} / \mathrm{U} \\
\text { death }\end{array}$ & $\begin{array}{c}\text { Born } \\
\text { alive }\end{array}$ & $\begin{array}{c}\text { Neoatal death and } \\
\text { perinatal palliative } \\
\text { care }\end{array}$ & $\begin{array}{c}\text { Un- } \\
\text { known }\end{array}$ \\
\hline
\end{tabular}

\begin{tabular}{lccccc}
\hline Perimembranous $(\mathrm{n}=58)$ & 5 & 3 & 35 & 9 & 15 \\
Muscular $(\mathrm{n}=53)$ & 1 & 0 & 42 & 2 & 10 \\
Inlet $(\mathrm{n}=47)$ & 6 & 11 & 27 & 19 & 3 \\
Multi $(\mathrm{n}=5)$ & 0 & 0 & 5 & 3 & 0 \\
Malalignment $(\mathrm{n}=3)$ & 0 & 0 & 3 & 0 & 0 \\
Unknown $(\mathrm{n}=3)$ & 2 & 1 & 0 & 0 & 0 \\
Total $(\mathrm{n}=169)$ & 14 & 15 & 112 & 33 & 28 \\
\hline
\end{tabular}

6014

Spectrum of Primary Cardiac Tumors Diagnosed by Fetal Echocardiography

Nawal A AlAbdulkarim, Prince Sultan Cardiac Center, Saudi Arabia

Introduction: True Primary Cardiac Tumors (PCT) are rare, however they are associated with serious complications. They can be diagnosed and followed by fetal echocardiography (FE) to plan proper management.

Objective: To determine accuracy of diagnosis and outcome of PCT diagnosed by FE.

Methods: We retrospectively evaluated 1460 consecutive FE performed during March 1990-December 2008 followed by chart review of mothers and newborns with PCT at a tertiary care facility. Results: PCT were diagnosed in 14 Patients [0.001\%] referred by obstetrics because of cardiac mass in 12 and 2 with large isolated pericardial effusion (PE). The gestational age at referral was 25-34 weeks. 12 patients had rhabdomyoma (RM), 9 had Tuberous Sclerosis (TS) including all patients with multiple masses. 1 patient with single RM underwent palliation with Blalock-Taussig shunt for right ventricular inflow obstruction with subsequent regression. 1 patient with multiple RM died 12 days postnatally due to ventricular arrhythmias in addition to right ventricular outflow obstruction. The 2 Patients with PE had single right atrial mass; one fetus died in utero due to congenital anomalies and the other underwent successful resection postnatally preceeded by antenatal and postnatal pericardiocentesis.

Conclusions: 1. Fetal echocardiography is accurate in assessment of PCT 2. PCT should be suspected in fetuses with large isolated PE. 3. The most common fetal PCT is multiple RM which is associated with TS. 4. PCT can lead to fatal arrhythmias and critical cardiac obstruction.

\section{5}

Fetal Echocardiography and Arteriovenous

Malformartions (AVM)

Nawal A AlAbdulkarim, Prince Sultan Cardiac Center, Saudi Arabia
Introduction: (AVM) are important noncardiac cause of heart failure in neonates and young infants, therefore antenatal detection and monitoring are essential for proper management.

Objective: Assess fetal cardiac findings in patients diagnosed antenatally with (AVM).

Method: Retrospective Review of consecutive Fetal Echocardiograms then Patients diagnosed antenatally with (AVM)/ Aneurysm of vein of Galen (AVG) at a tertiary care cardiac center.

Results: Total number of Fetal echocardiograms were 1460, 4 patients had (AVG) two underwent Successful occlusion by catheter intervention and two died \#1 and \#4, patient \#5 had Large Pulmonary fistula which was occluded successfully by catheter intervention, as illustrated in Table 1, all patients had progressive course with increased combined cardiac output,

Conclusions: 1. Fetal Echocardiography is useful in diagnosis and prediction of management and outcome of patients with (AVM). 2. Presence of severe multiple congenital anomalies/hydrops/ pseudocoarctation/ marked increase in combined cardiac output or (MR with AVG) could be predictors for poor prognosis of patients with $(\mathrm{AVM}) /(\mathrm{AVG})$.

\begin{tabular}{|c|c|c|c|c|c|c|c|c|}
\hline Patient \# & Sex & $\begin{array}{c}\text { Type of } \\
\text { AVM }\end{array}$ & $\begin{array}{l}\text { Cardio- } \\
\text { megaly }\end{array}$ & $\begin{array}{c}\text { Pericardial } \\
\text { Effusion/ } \\
\text { Hydrops/ } \\
\text { Pseudo- } \\
\text { coarctation }\end{array}$ & TR & MR & $\begin{array}{l}\text { Chamber } \\
\text { dilatation }\end{array}$ & $\begin{array}{c}\text { Foramen } \\
\text { Flow }\end{array}$ \\
\hline$\# 1$ & $\mathrm{~F}$ & $\begin{array}{l}\text { Aneurysm } \\
\text { of vein of } \\
\text { Galen } \\
\text { (AVG) }\end{array}$ & $\begin{array}{r}+>24 \\
\text { weeks }\end{array}$ & - & + & - & SVC/RA/RV & $\mathrm{R} \gg>\mathrm{L}$ \\
\hline$\# 2$ & M & (AVG) & $\begin{array}{r}+>24 \\
\text { weeks }\end{array}$ & - & + & - & SVC/RA/RV & $\mathrm{R}>>\mathrm{L}$ \\
\hline$\# 3$ & $\mathrm{~F}$ & (AVG) & $\begin{array}{r}+>24 \\
\text { weeks }\end{array}$ & - & + & - & SVC/RA/RV & $\mathrm{R} \gg>\mathrm{L}$ \\
\hline$\# 4$ & $\mathrm{~F}$ & $(\mathrm{AVG})$ & $\begin{array}{r}+>24 \\
\text { weeks }\end{array}$ & + & + & + & $\mathrm{SVC} / \mathrm{RA} / \mathrm{RV}$ & $\mathrm{R}>>\mathrm{L}$ \\
\hline$\# 5$ & M & $\begin{array}{l}\text { Pulmonary } \\
\text { (AVM) }\end{array}$ & $\begin{array}{r}+>24 \\
\text { weeks }\end{array}$ & - & + & + & $\begin{array}{l}\text { Pulmonary } \\
\text { veins/LA/LV }\end{array}$ & $\mathrm{L}>>\mathrm{R}$ \\
\hline
\end{tabular}

\section{6}

Prenatal diagnosis of anomalous systemic venous return: a case series

Catherine Barrea, University Clinic Saint Luc, Belgium

Stéphane Moniotte, University Clinic Saint Luc, Belgium

Corinne Hubinont, University Clinic Saint Luc, Belgium

Pierre Bernard, University Clinic Saint Luc, Belgium

Objective: Increasing experience in fetal ultrasound leads to more frequent diagnoses of isolated anomalous systemic venous return (ASVR). Our objectives were to describe the spectrum and the echocardiographic features of ASVR not associated with other structural heart defect, and to evaluate associated extracardiac and chromosomal anomalies.

Methods: From 2003 to 2009, 340 consecutive fetuses were diagnosed with a cardiac malformation, including 25 cases of isolated ASVR. The following variables were collected: gestational age (GA) et diagnosis, type of ASVR, associated extracardiac anomalies, karyotype and outcome.

Results: The diagnosis was obtained at a mean GA of $27+3$ weeks. The most common forms of ASVR were: 1) persistence of a left superior vena cava (SVC) draining into the coronary sinus with the presence of a normal right SVC $(\mathrm{N}=15)$ and 2) presence of an interrupted inferior vena cava (IVC) with azygos continuation $(\mathrm{N}=5)$. The other fetuses had a persistent left SVC without a right $\mathrm{SVC}(\mathrm{N}=3)$, bilateral agenesis of the SVC with 
cephalic venous drainage towards the IVC through the azygos $(\mathrm{N}=1)$ or total ASVR to the coronary sinus $(\mathrm{N}=1)$. Genetic disorders were confirmed in 4 fetuses, all with a persistent left SVC, with associated extracardiac anomalies in 3 of them. Seven other fetuses had associated extracardiac anomalies. When available, cardiac diagnosis was confirmed postnatally in all but one. Echographic findings are summarized in the Table. Conclusions: Detailed diagnosis of isolated ASVR can be accurately obtained prenatally. The majority of fetuses with a genetic anomaly presented also with extracardiac malformation.

\begin{tabular}{|c|c|c|c|c|}
\hline & $\begin{array}{c}\text { Abnormal } \\
\text { '3-vessel view' }\end{array}$ & $\begin{array}{l}\text { Dilated } \\
\text { coronary } \\
\text { sinus }\end{array}$ & $\begin{array}{l}\text { Retro-aortic } \\
\text { vessel }\end{array}$ & $\begin{array}{c}\text { Abnormal } \\
\text { RSVC -IVC } \\
\text { view }\end{array}$ \\
\hline $\begin{array}{l}\text { Persistent LSVC } \\
\text { to coronary sinus }\end{array}$ & $\begin{array}{c}+(4 \text { vessels, } \\
\text { LSVC on the } \\
\text { left })\end{array}$ & + & - & - \\
\hline $\begin{array}{l}\text { Interrupted IVC } \\
\text { with azygos } \\
\text { continuation }\end{array}$ & - & - & + (feet-to-head) & $\begin{array}{l}+(\text { no IVC } \\
\text { continuation) }\end{array}$ \\
\hline $\begin{array}{l}\text { Persistent LSVC } \\
\text { to coronary sinus } \\
\text { without RSVC }\end{array}$ & $\begin{array}{l}+(3 \text { vessels, } \\
\text { large LSVC on } \\
\text { the left })\end{array}$ & ++ & - & - \\
\hline $\begin{array}{l}\text { Bilateral absent } \\
\text { SVC }\end{array}$ & $\begin{array}{c}+(2 \text { vessels } \\
\text { anterior } \\
\text { without } S V C, \\
\text { (hemi)-azygos } \\
\text { posterior) }\end{array}$ & - & + (head-to-feet) & + (dilated IVC) \\
\hline $\begin{array}{l}\text { Total ASVR to } \\
\text { coronary sinus }\end{array}$ & $\begin{array}{l}+(3 \text { vessels, } \\
\text { large LSVC on } \\
\text { the left })\end{array}$ & +++ & - & - \\
\hline
\end{tabular}

6017

Evaluation of fetal echocardiography and postnatal follow up in $\mathbf{1 1 0}$ fetuses with hypoplastic left heart syndrome single reference fetal cardiology center experience

Joanna H Dangel, Perinatal Cardiology Department, 2nd Department of Obstetrics and Gynecology, Medical University of Warsaw, Poland Zbigniew Kordon, Pediatric Cardiology Department, Collegium Medicum, Cracow, Poland

Anna Kaplanska, Perinatal Cardiology Department, 2nd Department of Obstetrics and Gynecology, Medical University of Warsaw, Poland Pawel Wlasienko, Perinatal Cardiology Department, 2nd Department of Obstetrics and Gynecology, Medical University of Warsaw, Poland Maria M Dangel, Student's Scientific Club of Perinatal Cardiology, Medical University of Warsaw, Poland

Joanna Kuran, Perinatal Cardiology Department, 2nd Department of Obstetrics and Gynecology, Medical University of Warsaw, Poland

Out of 883 fetuses with CHD, fetal echocardiographic results in $110(12 \%)$ with HLHS were correlated with postnatal outcome. Fetal echocardiography was performed in one fetal cardiology reference center. Neonates were operated on in two different pediatric cardiac surgery centers. There were just 8 fetuses who had severe AS leading to HLHS at the end of pregnancy. 21 fetuses had severe Fo restriction(no survivors) and 8 moderate restriction(survived). Since 2002 more precise prenatal diagnosis was made, with special interest in Fo communication. In 3 progression of Fo restriction was observed. The study was divided into 2periods: (1) 1994-2001(22fetuses) and (2) 2002-2008(88fetuses). Follow up in the (1) period: TOP-2(9\%), IUD-1, live born-19. Neonatal death -10 : ECM-3, compassionate care due to parents' request - 7. 1 newborn died after unsuccesfull aortic valvuloplasty, 7 after classic Norwood operation, it was 1 survivor after modified Norwood. Follow up in the (2)period: TOP-13(15\%); IUD-3; neonatal death-15: prematurity-2, severe restriction Fo-11, 2 after unsucesfull BAS, 3-compas- sionate care due to severe RV dysfunction, 3-ECM. 2 babies died after oesophageal atresia operation, 9 after Norwood procedure, in whom 4 had restrictive Fo. There were 29 survivors: $33 \%$ of all, but $40 \%$ of live-borns, all after modified Norwood procedure.

Conclusions: Results of HLHS treatment improved significantly, but still prenatal diagnosis is the risk factor. Fetal echocardiography must be repeated monthly due to precise counseling and organizing optimal perinatal care. As a restrictive Fo is a real risk factor for adverse outcome, prenatal or very quick postnatal intervention must be taken into consideration.

\begin{tabular}{|c|c|c|c|c|c|c|c|}
\hline & $\begin{array}{c}\mathrm{GA} \\
\text { mean }\end{array}$ & $\begin{array}{c}\text { Isolated } \\
\text { HLHS }\end{array}$ & $\begin{array}{l}\text { Fo } \\
\text { restr }\end{array}$ & $\begin{array}{c}\text { Extracardiac } \\
\text { malformations } \\
\text { ECM }\end{array}$ & $\begin{array}{l}\text { Abnormal } \\
\text { karyotype }\end{array}$ & $\begin{array}{l}\text { Heart } \\
\text { Failure }\end{array}$ & \\
\hline $\begin{array}{r}1994- \\
2001\end{array}$ & 29 & 11 & 5 & 3 & 2 (T13, other) & 1 & 22 \\
\hline $\begin{array}{r}2002- \\
2008\end{array}$ & 25,7 & 52 & 16 & 7 & $\begin{array}{c}8 \text { (T18-2; } 45 \times 0- \\
5 ; \text { other } 1)\end{array}$ & 5 & 88 \\
\hline Total & & 63 & 21 & 10 & 10 & 6 & 110 \\
\hline
\end{tabular}

6018

The prevalence of dextrocardia and dextroposition of the heart in fetuses

Maria M Dangel, Student's Scietific Club Perinatal Cardiology, Medical University of Warsaw, Poland

Justyna Papierz, Student's Scietific Club Perinatal Cardiology, Medical University of Warsaw, Poland

Pawel Wasienko, Perinatal Cardiology Clinic, Medical University of Warsaw, Poland

Objective: How to distinguish right from the left side of the fetus and what is the difference between dextrocardia and dextroposition of the heart? The aim of the study, prepared by the Student's Scientific Club, was to evaluate the prevalence of the abnormal position and/or the axis of the fetal heart.

Material and methods: Retrospective evaluation of 42 fetuses between 2004-2008 whose heart was shifted to the right. The reason for referral was: abnormal heart view, abnormal US or different fetal malformations. The mean age of fetal echo was 24 (dextrocardia) and 30.5 (dextroposition) weeks.

Results: Dextroposition (shifting of the heart to the right, apex to the left) was found in 35(83\%) fetuses. 30(85\%) fetuses had left-diaphragmatic hernia (DH), 3(9\%)-congenital cystic adenomatoid malformation (CCAM), 1(3\%) - pleural effusion, 1(3\%) left pulmonary artery agenesis. Secondary hypoplasia of the left heart due to DH was seen in 4 cases. In 9 fetuses extracardiac malformations existed: hypotrophy 4 , cleft-lip 1 , shortening of the long bones 1 , myelocystocele 1 .

Dextrocardia (apex to the right) was found in $7(17 \%)$ fetuses, all with CHD: $1(14 \%)$ situs inversus (SV, TGA); 6 (86\%) situs solitus: right isomerism - 1 (AVSD, AP), left isomerism - 1 (TGA, VSD, PS), SV - 1, HLHS - 1, other - 2 .

Conclusion: Dextroposition is more common than dextrocardia, due to extracardiac malformations. Dextrocardia was much less frequent and was associated with multiple and complex congenital cardiac anomalies. It was difficult to distinguish between dextrocardia and dextroposition during a screening US exam.

\section{9}

Secondary left heart hypoplasia in fetuses with diaphragmatic hernia - is that a risk factor?

Maria M Dangel, Student's Scientific Club of Perinatal Cardiology, Medical University of Warsaw, Poland 
Pawel Wasienko, Perinatal Cardiology Department, Medical University of Warsaw, Poland

Joanna H Dangel, Perinatal Cardiology Department, Medical University of Warsaw, Poland

Objective: Congenital diaphragmatic hernia $(\mathrm{CDH})$ is diagnosed by obstetricians who often described HLHS in such cases. The aim of this study was to evaluate heart abnormalities coexisting with $\mathrm{CDH}$.

Material and methods: This study was prepared by the Student's Scientific Club of Perinatal Cardiology. We evaluated echocardiograms of 35 fetuses examined between 2002-2008 with left sided $\mathrm{CDH}$. Lung development, heart size, disproportion between LV, RV, pulmonary artery and aorta, co-existing heart defects were checked. The mean age of fetal echo was 29 weeks. Karyotype was done in 29 fetuses: 25 had normal and 4 abnormal.

Results: 26 fetuses had isolated CDH and 8(23\%) with associated heart defects. In all heart to chest area ratio was smaller than 0.25 . Secondary left ventricular hypoplasia (LV less that $50 \%$ of RV), described by obstetricians as HLHS, was in 4 cases, none of them survived. Additional heart defects were: VSD 6, TOF - 1, critical pulmonary valve stenosis -1 , they didn't survive either.

Conclusion: Heart defects complicated $\mathrm{CDH}$ is serious risk factor for survival, even if lungs were not severely hypoplastic. There were not fetuses with real HLHS, but secondary left ventricular hypoplasia caused low cardiac output what complicated respiratory failure in neonates with pulmonary hypoplasia due to $\mathrm{CDH}$. There is still unknown reason for which just some fetuses with $\mathrm{CDH}$ developed secondary left ventricular hypoplasia so further studies will include the role of growth factors (TGF- $\beta$ ), transcription factors (Fog2, COUP-TFII, Gata4), genes (STRA6).

6021

Outcome in utero and at long-term postnatal follow-up of cases with tricuspid valve anomaly presenting in fetal age Vlasta ME, Fesslova, Centre of Fetal Cardiology, Policlinico San Donato IRCCS, Milan, Italy

Jelena Brankovic, Centre of Fetal Cardiology, Policlinico San Donato IRCCS, Milan, Italy

Angelo Micheletti, Dpt. Pediatric Cardiosurgery, Policlinico San Donato IRCCS, Milan, Italy

Massimo Chessa, Dpt. Pediatric Cardiosurgery, Policlinico San Donato IRCCS, Milan, Italy

Gianfranco Butera, Dpt. Pediatric Cardiosurgery, Policlinico San

Donato IRCCS, Milan, Italy

Carmelo Arcidiacono, Dpt. Pediatric Cardiosurgery, Policlinico San Donato IRCCS, Milan, Italy

Mario Carminati, Dpt. Pediatric Cardiosurgery, Policlinico San Donato IRCCS, Milan, Italy

Alessandro Frigiola, Dpt. Pediatric Cardiosurgery, Policlinico San

Donato IRCCS, Milan, Italy

Objectives of the study: to analyse retrospectively the characteristics and outcome of cases with tricuspid valve anomaly diagnosed at fetal echocardiography.

Material: Sixteen fetuses with Ebstein (Ebst) and 16 with non Ebstein anomaly (NEbst) of the tricuspid valve (TV), one with associated mitral dysplasia) diagnosed at 21-36 (median 30) week's gestation (wg), between 1986-2008, were followed up in utero and postnatally for 6 months- 21 years, median 5 years.

Results: Echocardiographic features: Moderate-severe tricuspid regurgitation (TR) was present in 11/16 Ebst fetuses (with severe displacement of TV) and in 10/16 NEbst; 4 cases had associated pulmonary stenosis and 4 functional-organic pulmonary atresia. Six fetuses (4 Ebst, 2 Nebst) had fetal hydrops (FH). Outcome: Two cases opted for termination of pregnancy, 3 Ebst died in utero at 31 wg, ( 2 with FH, 1 in supraventricular tachycardia). Five cases died spontanouesly at 1-7 days. Three infants Ebst and 3 NEbst were operated, 2 surviving; the case with associated mitral dyplasia improved the TR but required a mitral valve plasty and is alive at 8 yrs. One Ebst died at 3 years due to arrhythmia and severe worsening of TR. Total mortality was $13 / 29$ (44.8\%) cases that continued pregnancy, 4/6 with FH,. 9/16 Ebst (56\%), 4 /16 NEbst $25 \%$. Sixteen cases are alive at $6 \mathrm{~m}-21$ years, stable or improved. Conclusions: Our data confirm a variable spectrum of tricuspid valve anomalies presenting in utero. Negative prognostic factors for outcome were heart failure and arrhythmias, milder forms of both variants stabilized after birth.

\section{2}

Premature fetal closure of the arterial duct : clinical presentations and outcome

Marc Gewillig, UZ Lewven, Belgium

Stephen Brown, Bloemfontein, South Africa

Luc de Catte, UZ Leuven, Belgium

Benedicte Eyskens, UZ Leuven, Belgium

Derize Boshoff, UZ Leuven, Belgium

Introduction: Intra-uterine dysfunction of the duct occurs; incidence and clinical consequences are poorly understood.

Patients and methods: Retrospective analysis of fetal $(\mathrm{n}=602)$ and neonatal echocardiographic databases $(n=1477)$ between 1998 and 2008. Clinical and imaging studies were reviewed for pathology due to or associated with premature closure of the duct.

Results: Twelve cases were identified. Eight (1.3\%) were diagnosed prenatally at 29.0 weeks (range: $20.0-37.5 \mathrm{w}$ ). Four neonates $(0.3 \%)$ : all presented with significant cyanosis and absence of duct. Echo: RV hypertrophy $(\mathrm{n}=12)$, more than usual tricuspid regurgitation $(\mathrm{n}=12)$ and pulmonary regurgitation $(\mathrm{n}=11)$, and right atrial dilation $(\mathrm{n}=8)$; pulmonary artery dilation in $42 \%$, dysplastic pulmonary valve in $25 \%$, hydrops $8 \%$. Premature delivery was advised for 5 patients. Neonatal therapy: observation and oxygen 7 , ventilation with pulmonary vasodilators 5, ECMO in 1. Respiratory failure with severe pulmonary hypertension was the cause of death in 3. During follow-up two children required additional right heart procedures, 1 developed non-compaction cardiomyopathy at 4 years.

Conclusion: The incidence of ductal dysfunction may be grossly underestimated. Fetal premature closure of the arterial duct causes stress at different fetal ages and many different levels of the right heart and pulmonary circulation, resulting in a wide range of secondary pathology. Clinical outcomes range from antenatal hydrops and right heart damage to lethal respiratory insufficiency. Premature delivery may be indicated in selected patients.

6023

Assessment of fetal atrioventricular time interval by Doppler tissue imaging (DTI) in the normal fetal heart Anita Hamela-Olkowska, Perinatal Cardiology Department, Medical University of Warsaw, Poland

Joanna H Szymkiewicz - Dangel, Perinatal Cardiology Department, Medical University of Warsaw, Poland

Introduction: Accurate assessment of atrioventricular conduction time (AV) is crucial for early detection of envolving fetal heart block. DTI-derived AV intervals correlate better with PR intervals in ECG than do other fetal echocardiographic approaches. 
Objective: To establish gestational age-specific reference values of normal fetal AV intervals by spectral DTI in healthy singleton fetuses.

Methods: AV intervals were measured from atria contraction (Am) to isovolumic contraction (IV) at the right ventricular free wall in 140 subjects with normal cardiac and extracardiac anatomy between 18 and 40 week's gestation. All fetuses had sinus rhythm. Examinations were performed using Sequoia 512, with cardiological probe of $2.5-5 \mathrm{MHz}$ and pediatric cardiology software. The sample volumes were placed in the basal part of RV in the 4-chamber view.

Results: There was a negative correlation between AV intervals and FHR $(\mathrm{p}<0,001, \mathrm{r}=-0,318)$ and positive correlation with gestational age $(p=0,049 ; r=0,167)$. The table shows reference values for $\mathrm{AV}$ intervals by gestational age group.

Conclusions: The study establishes gestational age-specific reference data for DTI-derived AV intervals, which can be used for detection of subtle AV conduction anomalies in fetuses with maternal anti-Ro and anti-La autoantibodies.

\begin{tabular}{lllll}
\hline AV (ms) & \multicolumn{4}{l}{ Gestational age (weeks) } \\
\cline { 2 - 5 } percentile & $\mathbf{1 8 - 2 2}$ & $\mathbf{2 3 - 2 8}$ & $\mathbf{2 9 - 3 4}$ & $\mathbf{3 5 - 4 0}$ \\
\hline 3 & 75 & 69 & $\mathbf{7 3}$ & $\mathbf{7 3}$ \\
10 & 78 & 78 & 80 & 84 \\
50 & 92 & 91 & 93 & 95 \\
90 & 108 & 106 & 103 & 113 \\
97 & 123 & 116 & 113 & 120 \\
No of fetuses & 36 & 35 & 35 & 34 \\
\hline
\end{tabular}

\section{4}

Screening of prolonged AV interval in the fetus by Doppler echocardiography

Akiko Hirose, Department of Pediatrics, Kurume University school of medicine, Japan

Yasuki Maeno, Department of Pediatrics, Kurume University school of medicine, Japan

Hiroshi Nishino, Department of Pediatrics, Kurume University school of medicine, Japan

Shintarou Kishimoto, Department of Pediatrics, Kurume University school of medicine, Japan

Motofumi Iemura, Department of Pediatrics, Kurume University school of medicine, Japan

Kenji Suda, Department of Pediatrics, Kurume University school of medicine, Japan

Toyojirou Matsuishi, Department of Pediatrics, Kurume University school of medicine, Japan

Background: Early detection of atrio-ventricular (AV) block caused by maternal anti-SSA antibody is important to start early treatment to the affected fetuses. To examine suitability to screen prolonged mechanical AV interval by fetal echocardiography, 3 different Doppler measurement method, 1) simultaneous recording of the superior vena cava and the ascending aorta (SVC-aAo), 2) simultaneous recording of mitral inflow and aortic outflow (mitral A-wave/aortic outflow), and 3) simple mitral inflow (mitral Awave/mitral valve closure) are compeared.

Methods: All 34 fetuses with normal cardiac structure with normal rhythm and without maternal anti-SSA antibody who underwent fetal echocardiography in our institution from March to November 2008 were included.

Results: The mean value and the standard deviation of the measurement value in all cases: SVC-aAo : $111.5 \pm 13.96 \mathrm{msec}$, mitral A-wave/aortic outflow : $123 \pm 8.66 \mathrm{msec}$, mitral A-wave/ mitral valve closure : $88 \pm 5.92 \mathrm{msec}$. There was no correlation to the gestational age. Measurement using mitral A-wave/mitral valve closure is the most easily obtained measurement and has smallest standard deviation.

Conclusion: Although all three measurements of mechanical AV interval can be reliably obtained by fetal Doppler echocardiography, mitral A-wave/mitral valve closure seems to be the most suitable one to screen first degree AVB.

\section{5}

Does antenatal diagnosis affect clinical course in neonates with simple transposition of the great arteries?

Caroline B Jones, Alder Hey Children's Hospital, Liverpool. UK, United Kingdom

Helen C Michael, Alder Hey Children's Hospital, Liverpool. UK, United Kingdom

Nelson Alphonso, Alder Hey Children's Hospital, Liverpool. UK, United Kingdom

Gordon Gladman, Alder Hey Children's Hospital, Liverpool. UK, United Kingdom

Joyce SL Lim, Alder Hey Children's Hospital, Liverpool. UK, United Kingdom

Objective: To determine whether neonates with an antenatal (AN) diagnosis of simple transposition of the great arteries (TGA) have a different clinical course to those diagnosed in the postnatal (PN) period.

Methods: Retrospective analysis of notes of all neonates with simple TGA (with or without ventricular septal defect) seen in our institution between January 2001 and June 2008. Those whose anatomy was not suitable for a primary arterial switch operation (ASO) were excluded from the study.

\begin{tabular}{llll}
\hline & AN group (14) & PN group (81) & P value \\
\hline Birth weight $(\mathrm{kg})$ & $3.6 \pm 0.7$ & $3.4 \pm 0.6$ & $\mathrm{NS}$ \\
Age at presentation (hours) & $6.1 \pm 2.3$ & $167.5 \pm 278.5$ & $<0.0001$ \\
Prostin dose on arrival (ng/kg/min) & $13.2 \pm 29$ & $30.9 \pm 37$ & 0.0038 \\
Oxygen saturation on arrival (\%) & $82 \pm 9$ & $67 \pm 15$ & 0.0013 \\
Total time spent on ICU (days) & $6.3 \pm 3.5$ & $9.1 \pm 5.5$ & 0.028 \\
Length of stay post ASO (days) & $12.1 \pm 4.3$ & $14.1 \pm 5.6$ & 0.17 \\
Duration follow-up (months) & $12.3 \pm 18.1$ & $37.4 \pm 26.4$ & 0.0013 \\
\hline
\end{tabular}

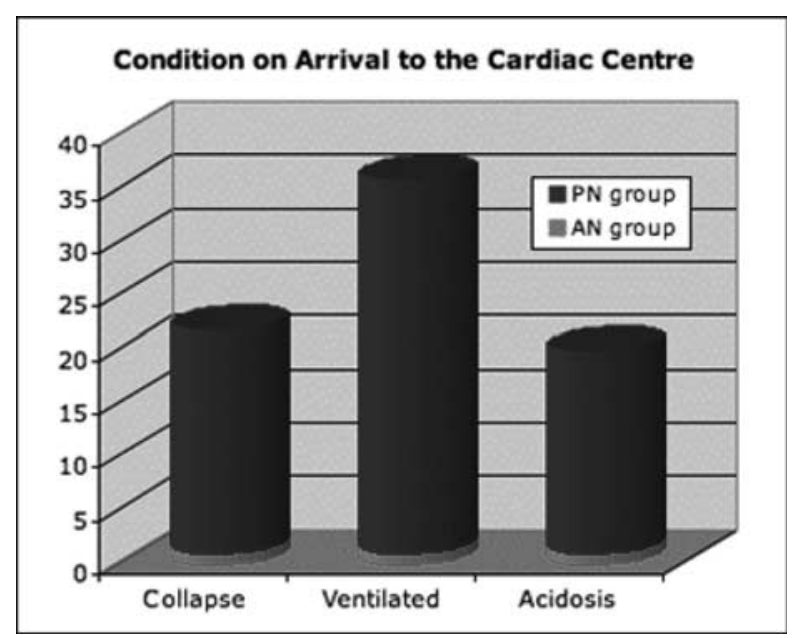

Results: We identified 95 neonates with simple TGA within the given time period. Mean gestational age was $39.7 \pm 1.1$ weeks. Fourteen of the 95 neonates $(14.7 \%$ ) had an antenatal diagnosis. Three neonates in 
the PN group died, two prior to the ASO and one shortly following the operation. All the neonates in the AN group survived.

Conclusions: All neonates diagnosed with TGA antenatally were transferred promptly the cardiac centre within the first 12 hours of a life facilitating early expert management and septostomy where appropriate. All but one in this group were in good condition at presentation, not requiring ventilation. It is likely that this improved pre-operative state lead to a smoother post-operative course and slightly shorter length of stay $(p=0.17)$. None of the patients in the antenatal group died as compared to 3 in the postnatal group. None of the patients have died in long-term follow-up (32.6 \pm 26.4 months).

6026

Antenatal cardiac screening practice within Northwest of England and North Wales - detection rates for simple Transposition of Great Arteries (TGA)

Helen C Michael, Alder Hey Children's Hospital, United Kingdom Caroline B Jones, Alder Hey Children's Hospital, United Kingdom Denise Kitchiner, Alder Hey Children's Hospital, United Kingdom Gordon Gladman, Alder Hey Children's Hospital, United Kingdom Joyce SL Lim, Alder Hey Children's Hospital, United Kingdom

Objectives: To survey antenatal cardiac screening practice within the Northwest of England and North Wales, and to ascertain antenatal detection rate for simple transposition of great arteries (TGA).

Methods: A questionnaire was sent to all obstetric units within the region, which refer to the foetal cardiac clinics at Manchester and Liverpool. All infants with simple TGA (with or without VSD) presenting to Alder Hey Children's Hospital between January 2001 and June 2008 were identified; the referring obstetric unit was ascertained where possible and the group subdivided according to antenatal (AN) or postnatal (PN) diagnosis.

Results: Twenty-seven obstetric units responded to the questionnaire survey. Responses indicated that 12 units (44\%) assessed only the standard four chamber view and 15 units (56\%) included outflow views. 113 patients (14 AN, 99 PN) with simple TGA were treated at our institution during this period.

Conclusion: $56 \%$ of obstetric units in our region satisfy the United Kingdom national guidelines to include outflow tract views in the antenatal cardiac screening protocol. Isolated cardiac outflow tract anomalies are not well detected antenatally with only $12.3 \%$ infants with TGA diagnosed in-utero. It is anticipated that with increasing adherence to the antenatal screening national guidelines, antenatal diagnosis rates for outflow tract anomalies will improve.

\begin{tabular}{lll}
\hline & $\begin{array}{l}\text { Antenatal } \\
\text { diagnosis (14 } \\
\text { patients; 12.3\%) }\end{array}$ & $\begin{array}{l}\text { Postnatal } \\
\text { diagnosis (99 } \\
\text { patients; 87.7\%) }\end{array}$ \\
\hline $\begin{array}{c}\text { Four chamber view with } \\
\text { outflow tract views }\end{array}$ & 12 & 44 \\
$\begin{array}{c}\text { Standard four chamber } \\
\text { view only }\end{array}$ & 2 & 47 \\
Variable practice & 0 & 8 \\
\hline
\end{tabular}

6027

Pprenatal echocardiographic findings of MAPCAs(Major Aortopulmonary Collaterals) : Two Case Reports

Da-Hye Ju, Department of Obstetrics and Gynecology, University of Ulsan College of Medicine, Asan Medical Center, Seoul, Korea, Korea Hye-Sung Won, Department of Obstetrics and Gynecology,University of Ulsan College of Medicine, Asan Medical Center, Korea
In-Sook Park, Department of Pediatric Cardiology, Asan Medical Center, University of Ulsan College of Medicine, Korea

Pil Ryang Lee, Department of Obstetrics and Gynecology, University of Ulsan College of Medicine, Asan Medical Center, Seoul, Korea, Korea Ahm Kim, Department of Obstetrics and Gynecology, University of Ulsan College of Medicine, Asan Medical Center, Korea

Pulmonary atresia (PA) and ventricular septal defect (VSD) associated major aortopulmonary collateral arteries (MAPCAs) is uncommon complex congenital heart anomaly as a severe form of tetralogy of Fallot. This anomaly occurs in $1.5 \%$ of patients with congenital heart disease, and $15 \sim 20 \%$ of tetralogy of Fallot. MAPCAs are wide connection arteries originated from the descending aorta, the arcus aorta or the subclavian artery. The majority of untreated patients present with severe congestive heart failure and respiratory distress. Prenatal diagnosis of PA and VSD associated with MAPCAs is important to counsel parents to outcome. We present two cases of PA and VSD associated with MAPCAs examined prenatally by fetal echocardiography.

\section{8}

Prenatal echocardiography of Total Anomalous Pulmonary Venous Connection (TAPVC)

Hye-Sung Won, Department of Obstetrics and Gynecology, University of Ulsan College of Medicine, Asan Medical Center, Korea

Da-Hye Ju, Department of Obstetrics and Gynecology, University of

Ulsan College of Medicine, Asan Medical Center, Seoul, Korea, Korea Dong-Man Seo, Asan medical center, Korea

In-Sook Park, Department of Pediatric Cardiology, Asan Medical

Center, University of Ulsan College of Medicine, Korea

Objective: TAPVC has deteriorate neonatal condition. It is usually accompanied by other cardiac anomalies. It is important to detect TAPVC prenatally and the associated pulmonary venous stenosis (PS) as well.

Methods: We reviewed fetal echocardiography from July 1996 to december 2008 at our center. We had 2895 congenital heart anomaly and suspected TAPVC in 73. We suspected TAPVC if pulmonary vein to left atrium was not definite or extrachamber was shown in 2D and color Doppler ultrasound.

Results: Among 73 fetuses, we could identify TAPVC in 50, 28 through postnatal echocardiography and 22 in autopsy record, 23 have not shown up again. Out of 28 deliveres, 13 were confirmed as TAPVC, 2 PAPVC, and 1 PS. Through autopsy, 9 out of 22 were confirmed as TAPVC and 1 PAPVC. The type of TAPVC showed 7 supracardiac, 3 cardiac, 2 infracardiac, and 1 mixed connection. Four deaths occurred due to postoperative acidosis, septic shock and sudden collapse of pulmonary vein. We suspected isolated TAPVC in 5 prenatally, which were proven as 4 TAPVC and 1 PS. The isolated TAPVCs are good state after surgery, but the fetus with PS has expired one day after birth. Conclusions: In echocardiography prenatally, absence of typical pulmonary drain to left atrium or presence of extrachamber could provide simple clue for anomalous pulmonary venous connection. Indefinite extrachamber could not rule out anomalous pulmonary venous connection. On the contrary, in that case, pulmonary venous stenosis should be suspected, which seemed most important in prognosis.

\section{9}

Maternal Smoking and Congenital Heart Disease in Fetus Yukiko Kawazu, Department of Pediatric Cardiology, Osaka Medical Center for Maternal and Child Health, Osaka, Japan 
Noboru Inamura, Department of Pediatric Cardiology,

Osaka Medical Center for Maternal and Child Health, Osaka, Japan, Japan

Akane Kadota, Department of Pediatric Cardiology,

Osaka Medical Center for Maternal and Child Health,

Osaka, Japan, Japan

Shu Maekawa, Department of Pediatric Cardiology,

Osaka Medical Center for Maternal and Child Health,

Osaka, Japan, Japan

Yuji Hamamichi, Department of Pediatric Cardiology,

Osaka Medical Center for Maternal and Child Health,

Osaka, Japan, Japan

Futoshi Kayatani, Department of Pediatric Cardiology,

Osaka Medical Center for Maternal and Child Health,

Osaka, Japan, Japan

Background: Maternal smoking is thought to be one of risk factors of problems in fetus. Most congenital heart diseases (CHD) are of unknown etiology. This study examined the association between maternal smoking during the periconceptional period and the risk of CHD in fetus.

Methods: We performed the fetal echocardiogram for 254 times, 172 maternal cases between January and December 2007. The all cases are divided into three groups. A: 40 cases of CHD fetus (without chromosomal anomaly), B: 117 cases with no CHD fetus (without chromosomal anomaly), C: 15 cases with chromosomal anomaly fetus. Diagnoses of all cases were confirmed postnatally. Information on maternal smoking was obtained via medical records of the mothers.

Results: GroupA had 14 maternal smoking cases (35\%). GroupB had 21 maternal smoking cases (17.9\%). GroupC had 1 maternal smoking case $(0.07 \%)$. Maternal smoking during the periconceptional period was associated with CHD (without chromosomal anomaly) of their fetuses $(p=0.027)$. On the other hand, groupA, B and C had 22(55\%), 59(50.4\%), and 5(33.3\%) paternal smoking cases, respectively. There was no increased risk of CHD fetus with paternal smoking.

Conclusion: This study observed that maternal smoking during the periconceptional period increased the risk for CHD in the fetus. Further study are needed to elucidate this association.

\section{0 \\ Clinical and imaging characteristics of primary fetal cardiac tumors \\ Maria G Kiaffas, Onassis Cardiac Surgery Center, Greece \\ Cleo Laskari, Onassis Cardiac Surgery Center, Greece \\ Spyridon Rammos, Onassis Cardiac Surgery Center, Greece}

Objective: Presentation of our experience with the clinical and imaging characteristics of fetal cardiac tumors

Methods: Seven cases of fetal cardiac tumors, at the gestational age (GA) of 22-32 weeks (wks), were diagnosed echocardiographically, between September of 2003 and December of 2008.

Results: Two of the fetuses had multiple tumors in both the right and left ventricles (RV \& LV). Five had solitary tumors; in two the mass was located at the left ventricular outflow tract (LVOT) causing obstruction, in two it was located in the ventricular septum (VS) and in one in the left atrium (LA). Termination was elected in four pregnancies; the remaining three were carried to full-term. One of the tumors (located in the VS) was histologically proven to be a fibroma; the remaining are considered to be rhabdomyomas due to histology (two), association with tuberous sclerosis (one) multiple location (one) and regression of size (one). Association with tuberous sclerosis was identified in three of the cases. Follow-up ranges from 8 months to 3.5 years (Table).

\begin{tabular}{|c|c|c|c|c|}
\hline Diagnosis & Location & Tumor type & $\begin{array}{l}\text { Associated } \\
\text { problems }\end{array}$ & Outcome \\
\hline 22 wks & Single - VS & Rhabdomyoma & - & Termination \\
\hline 23 wks & Single - VS & Fibroma & $\begin{array}{l}\text { Myocardial } \\
\text { dysfunction/ } \\
\text { Pericardial effusion }\end{array}$ & Termination \\
\hline 23 wks & Single - LA & Rhabdomyoma & Tuberous sclerosis & Termination \\
\hline 29 wks & $\begin{array}{l}\text { Multiple - } \\
\text { RV \& LV }\end{array}$ & Rhabdomyoma & Tuberous sclerosis? & $\begin{array}{l}\text { Term delivery } / 8 \\
\text { mos TS? }\end{array}$ \\
\hline $31 \mathrm{wks}$ & $\begin{array}{c}\text { Multiple - } \\
\text { RV \& LV }\end{array}$ & Rhabdomyoma & Tuberous scerosis & Termination \\
\hline $32 \mathrm{wks}$ & $\begin{array}{l}\text { Single - } \\
\text { LVOT }\end{array}$ & Rhabdomyoma & LVOT obstruction & $\begin{array}{l}\text { Term delivery/ } 3.5 \\
\text { yrs healthy }\end{array}$ \\
\hline $32 \mathrm{wks}$ & $\begin{array}{l}\text { Single - } \\
\text { LVOT }\end{array}$ & Rhabdomyoma & LVOT obstruction & $\begin{array}{l}\text { Term delivery/ } 2 \text { yrs } \\
\text { Renal lesions - } \\
\text { Arrhythmias }\end{array}$ \\
\hline
\end{tabular}

Conclusions: Echocardiography is a vital diagnostic tool for fetal cardiac tumors identifying their number, location, size and echogenic characteristics usually indicative of possible histology. Rhabdomyomas are the most common tumors; they can be solitary or multiple and have a high association with TS. Fibromas, the second commonest, are usually massive with preference to the VS and often associated with myocardial dysfunction. Termination of pregnancy usually follows prenatal diagnosis of myocardial tumors, especially in an early GA and when TS is suspected.

\section{1}

Usefullness of Spatio-Temporal Image Correlation (STIC) echocardiography in assessment of the fetal atrioventricular septum

Adam Kolesnik, Department of Anatomy, Center of Biostructure Research, Warsaw, Poland, Poland

Joanna Szymkiewicz-Dangel, Perinatology and Perinatal Cardiology Unit, 2nd Department of Obstetrics and Gynecology, Medical University of Warsaw, Poland, Poland

Background: Three-dimensional fetal echocardiography is now a routine method of examination. Spatio-Temporal Image Correlation (STIC) modality allows to obtain four-dimensional reconstruction of the single fetal cardiac evolution basing on series of heart rate-gated images. The atrioventricular septum (AVS) is affected in numerous congenital cardiac malformations. Aim: Our study aimed to check whether STIC volume files can be used for morphometric analysis of the atrioventricular septum.

Material and methods: Material for the study consisted of 50 STIC volume files acquired during routine echocardiographic examinations of fetuses aged 18-34 weeks of gestation using GE Voluson 730 Expert equipment. Files were processed offline using 4D-View software. Section Planes and Tomographic Ultrasound Imaging options were used for visualization and measurements. Hight, lenght and thickness of the AVS were measured basing on 5 parallel TUI images distributed equally throughout the lenght of the AVS.

Results: TUI allowed for obtaining 5 parallel slices of AVS in three phases of the cardiac cycle - isovolumetric diastole, isovolumetric systole and when atrioventricular valves were completely open (Fig. 1). Quality of reconstructed images was satisfactory in all cases. Measurements were the easiest to perform in the middle of the AVS and in isovolumetric diastole. Measured values were repeatable in different section planes.

Conclusions: We conclude that STIC four-dimenional echocardiography is a valuable method of assessment of visualization and morphometric analysis of normal fetal atrioventricular septum 
in vivo. It can be helpful to standardize methods of echocardiographic measurements.

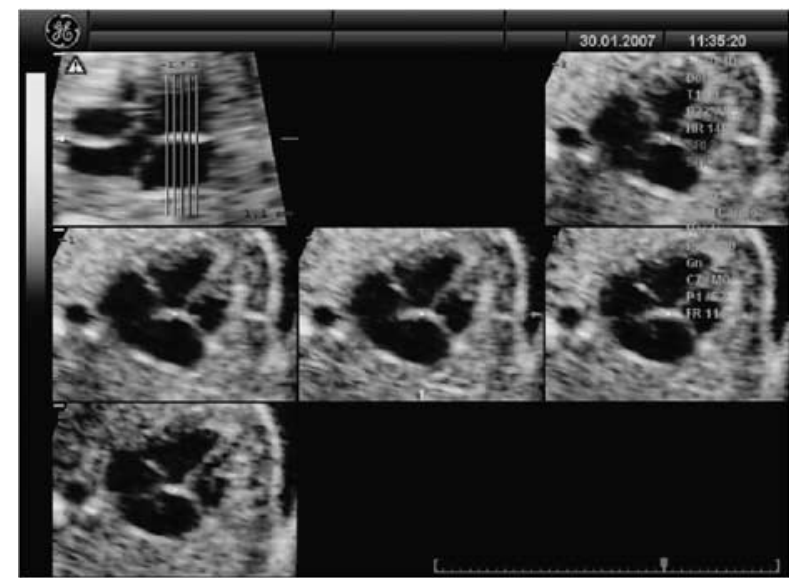

6032

Fetal inappropriate sinus tachycardia

Nathalie Jeanne Magioli Bravo-valenzuela, University of Taubaté (UNITAU), Brazil

Lilian Lilian Maria Lopes, São Paulo University Medical School (USP), Brazil

Introduction: Inappropriate sinus tachycardia (IST) is rare in the general population and its etiology is unknown. First described by Bauernfeind et al, it is characterized by a persistently high heart rate (HR) and an exaggerated response to physical activities. It has non-defined mechanisms. The IST is suggested to be a primary abnormality of the sinus node. Tachyarrhythmias in fetal life may cause heart failure and intrauterine death.

Objectives: Report an IST case in a seven-week gestation fetus emphasizing the importance of fetus echocardiography in early phase of gestation.

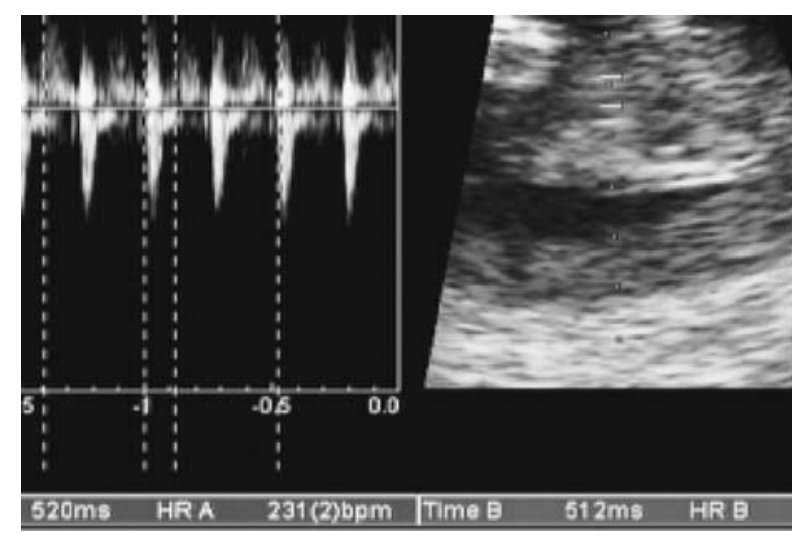

Methods: Pregnant patient referred due to persistent fetal tachycardia following obstetric ultrasonography. The fetus presented an initial frequency of around 240 beats/min (bpm) with 1:1 atrioventricular conduction with no hydropsy in the transvaginal echocardiogram. It was about an IST, being the differentiated supraventricular tachycardia diagnostic initially difficult. A digital therapy with the mother was started with no success. Flecainide was used next, again to no avail. Solatol oral use was then chosen, there initially being a reduction of fetal myocardium HR to tolerable levels $(180 \mathrm{bpm})$. The number of heartbeats normalized at thirty six weeks gestation $(140 \mathrm{bpm})$. It showed a persistent high basal HR in a reassessment after the neonatal period $(180 \mathrm{bpm})$, increasing excessively at minimum efforts and $\mathrm{P}$ wave in sinus rhythm morphology on ECG. Possible sinus tachycardia causes were dismissed, thus confirming the above diagnostic.

Conclusion: The authors report the importance of fetal echocardiography for the diagnosis and early therapy of fetal arrhythmias to avoid complications.

\section{3}

Comparison of antenatally versus postnatally diagnosed major congenital heart disease

Angela McBrien, United Kingdom

Andrew Sands, United Kingdom

Brian Craig, The Royal Belfast Hospital for Sick Children, United Kingdom

Frank Casey, United Kingdom

Objective: To compare outcomes and characteristics of patients with antenatally diagnosed major congenital heart disease (CHD) with postnatally diagnosed cases. Northern Ireland has 22000 live births per year. There is a low termination rate; providing the opportunity to study the natural history of fetal CHD.

Methods: Patients born between $1 / 1 / 3$ and $31 / 12 / 8$ were included. Cases were identified using the regional fetal and paediatric cardiology database and post-mortem records. Data recorded includes - outcome (termination of pregnancy, intrauterine death, neonatal death, neonatal survival), karyotype and physiology (uni- or bi-ventricular).

Results: 31\% (108/346) of major CHD was diagnosed antenatally. Of the antenatal diagnoses, $80 \%$ (86) were live born, $12 \%$ (13) died in-utero and $8 \%$ (9) pregnancies were terminated. $22 \%$ (19) of antenatally diagnosed live births died during the neonatal period. $62 \%(67 / 108)$ reached 28 days, compared with $93 \%$ of the postnatal group $(221 / 238, p<0.0001)$. There was no significant difference in the proportion of karyotype defects in the antenatal group $(25 \%, 27 / 108)$ versus the postnatal group $(23 \%, 54 / 238, p=0.64)$. Excluding lethal karyotypes (trisomy 13 and trisomy 18), mortality for livebirths was not significantly different $(12 \%, 9 / 75$ vs $7 \%, 15 / 229, p=0.13) .46 \%(50 / 108)$ of the antenatal group had functionally univentricular physiology, compared with $8 \%(18 / 238)$ of the postnatal group $(\mathrm{p}<0.001)$. Conclusions: Antenatally diagnosed cases have significantly higher early mortality than those with a postnatal diagnosis. However, the mortality difference is negligible for liveborn infants without lethal trisomies. This is despite the significantly higher proportion of these cases having functionally uni-ventricular physiology.

\section{4}

Fetal Diagnosis of Congenital Heart Disease by Telemedicine

Brian A McCrossan, Royal Belfast Hospital for Sick Children, United Kingdom

Andrew J Sands, Royal Belfast Hospital for Sick Children, United Kingdom

Frank A Casey, Royal Belfast Hospital for Sick Children, United Kingdom

Introduction: There remains a disparity between, and within, regions in access to fetal cardiology and detection rates of congenital heart disease (CHD). We aimed to evaluate the feasibility, accuracy and patient acceptability of remote fetal echocardiograms (FE). 
Methods: Prospective study over 20 month period. An initial FE was performed by a radiographer in the district general hospital (DGH) (D1) followed by a second FE transmitted to the regional centre, in real time, via a telemedicine link (384Kbps) with live guidance by a fetal cardiologist (D2). A FE was performed later at the regional centre (D3, gold standard). Identical questionnaires were completed at DGH and regional centre relating to satisfaction with the consultation and preferred method of FE (5-point Likert scale).

Results: 69 remote FE have been performed: 58 normal hearts and 11 with CHD (3 duct dependent) confirmed by D3. D2 was accurate in $97 \%$ cases compared with D3 (kappa score $=0.90$ ) indicating excellent agreement. Respondents preferred to have the FE performed by tele-link at the DGH: Mean difference $=1.6(p<0.01)$. The mean satisfaction scores for telemedicine and "hands-on" consultations were high, 46.6/50 and 46.5/50 respectively.

Discussion: To date this is the largest study of its kind. CHD can be confidently diagnosed and excluded by remote FE. Pregnant women prefer to have the FE performed at their local hospital via telemedicine. This application of telemedicine could improve access to fetal cardiology, support radiographers screening for CHD and reduce the burden on the limited capacity of tertiary centres.

\section{5}

Prenatally Diagnosed Hypoplastic Left Heart Syndrome With Intact Atrial Septum and Ventriculocoronary Arterial Fistula

Masayuki Nakada, Shizuoka Children's Hospita, Japan

Masaki Nii, Shizuoka Children's Hospita, Japan

Masaki Osaki, Shizuoka Children's Hospita, Japan

Noriko Kitamura, Shizuoka Children's Hospita, Japan

Kisaburou Sakamoto, Shizuoka Children's Hospita, Japan

Yasuo Ono, Shizuoka Children's Hospita, Japan

Background: Intact-atrial-septum (IAS) and left-ventriculocoronary-arterial-fistula (LVCAF) are the significant risk factors in hypoplastic-left-heart-syndrome (HLHS) and the prognosis of their association is considered grim. Although there has been no survival report of patient with this association, prenatal diagnosis and postnatal aggressive approach may have potential to save patient.

Method and Results: We report a case of prenatally diagnosed HLHS with IAS and LVCAF who were treated aggressively with surgical atrial septostomy and bilateral pulmonary-arterialbanding immediately after birth followed by planned extracorporeal-membrane-oxygenation support. Echocardiogram clearly showed the change of flow direction of left coronary artery before and after atrial septostomy (from retrograde to antegrade). Although the patient died on 5th postoperative day due to sepsis, hemodynamic markers were stable until the deterioration of sepsis. Postmortem histology revealed no myocardial ischemia nor significant change of pulmonary arteriolar lumen, suggesting prenatal planning and aggressive approach may lead to a survival of this ultimate association.

Conclusion: Prenatal planning and postnatal aggressive approach may lead to the survival of patient with this association in future.

\section{6}

Cardiovascular profile score in fetal right heart defects

Ana L Neves, Hospital S. João, Portugal

Leigh Mathias, University of South Florida, United States

Jenny Leshko, All Children's Hospital, United States

Kersti Linask, University of South Florida, United States
José C Areias, Hospital S. João, Portugal

James C Huhta, Congenital Heart Institute of Florida, Pediatric

Cardiology Associates, United States

Introduction: Congestive heart failure (CHF) in fetuses with congenital heart diseases is associated with high perinatal mortality. CHF may be assessed by the cardiovascular profile (CVP) score that comprises hydrops, cardiothoracic ratio, cardiac function, arterial and venous Doppler. Fetal right heart defects (critical pulmonary stenosis-7, pulmonary atresia with intact ventricular septum-18, and Ebstein's anomaly-3) were assessed. Our hypothesis was that outcome of these may be related to prenatal condition and LV function.

Objective: Our aim was to assess the value of CVP-score in evaluating risk of death in right heart defects diagnosed prenatally. Methods: In a retrospective study, reviewed medical records of 28 fetuses, evaluated in our perinatal care center, between 2004-2008, with right heart defects. Correlation between death and the CVPscore, presence of RV-coronary fistula, Doppler and tissue Doppler parameters of LV performance was performed using chi-square tests for homogeneity and measures of association.

Results: There were 13 patients who died prior to 30-postnatal days-of-life. Statistical analyses of the data supports the hypothesis that the CVP-score is related to risk of death $(p=0.04)$. The chi-square test also indicated a marginal statistical association between the presence of a fistula in a fetal echo and risk of death $(p=0.08)$. LV parameters didn't correlate with outcome.

Conclusions: Fetal echocardiography is useful in predicting outcome in right heart defects. There is an association between the CVP-scores assessed prenatally and the risk of fetal or neonatal death in this group. Further analyses will be performed to determine how the CVP-score could be weighted to more accurately predict the outcome.

\section{7}

Autopsy routine for the fetal heart in the 1st trimester of pregnancy

Cezary S Niszczota, Department of Anatomy, Center of Biostructure Research, Medical University of Warsaw, Poland, Poland

Adam Kolesnik, Department of Anatomy, Center of Biostructure

Research, Medical University of Warsaw, Poland, Poland

The examination of fetal heart in the 1st trimester of pregnancy is difficult for echocardiographers as well as for pathologists. Pathologists use different approaches to visualize cardiac anatomy. Some of them prefer histological methods whereas others try to follow routine dissection method. We aimed to develop our own autopsy routine for small fetal hearts. Twenty normal and malformed fetal hearts aged from 8 until 12 weeks (heart length from $4.2 \mathrm{~mm}$ to $8.00 \mathrm{~mm}$ ), fixed and unfixed, were submerged in water and dissected using microsurgical instruments. SMZ 1500 microscope, Nikon DS-Fi1 camera and NIS-Elements 3.00 software were used for observation and documentation. The chest was opened by medial sternotomy and the heart was dissected in situ and sequential segmental analysis was applied. External morphology of atrial appendages, great arteries were determined and heart measurements were done. Right atrium was opened by horizontal cut from base of appendage to orifice of inferior vena cava. Left atrium was opened between right and left pulmonary veins. Detailed anatomy of atriums, interatrial septum, pulmonary and systemic veins drainage was examined. Ventricles were opened along interventricular grooves (figure). Posterior right ventricle section was extended through the annulus of tricuspid valve to the right atrium for better 
visualization of its structures, e.g. right sinus venosus valve. In all cases morphology of chambers, atrioventricular and ventriculoarterial connections were possible to determine. We concluded that microautopsy of fetal heart is possible using classical pathological methods. Detailed structures are best visible when specimen is unfixed and submerged in water.

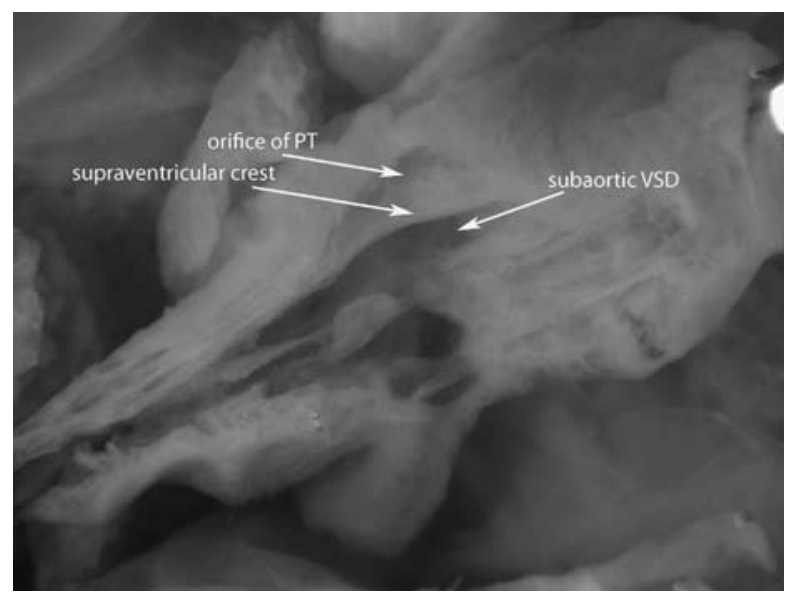

6038

Comprasion of Prevalance and Distribution of Structural Heart Defects in High and Low Risk Pregnancies Nazan Ozbarlas, Department of Pediatric Cardiology, Faculty of Medicine, Cukurova University, Adana Turkey, Turkey Sevcan Erdem, Department of Pediatric Cardiology, Faculty of Medicine, Cukurova University, Adana Turkey, Turkey Osman Kucukosmanoglu, Department of Pediatric Cardiology, Faculty of Medicine, Cukurova University, Adana Turkey, Turkey Cansun Demir, Department of Gynecology and Obstetrics, Faculty of Medicine, Cukurova University, Adana Turkey, Turkey Cuneyt Evruke, Department of Gynecology and Obstetrics, Faculty of Medicine, Cukurova University, Adana Turkey, Turkey Fatma Tuncay Ozgunen, Department of Gynecology and Obstetrics, Faculty of Medicine, Cukurova University, Adana Turkey, Turkey

Objective: To evaluate the intrauterin prevelance and distribution of congenital heart defects (CHD) in high and low risk pregnancies. Methods: The records of 3782 patients who underwent fetal echocardiography (FE) at our pediatric cardiology unit between 1998 and 2008 years were reviewed for reason for referral and results of echocardiography. The patients were grouped by reason for referral into high and low risk groups.

Results: Number of patients in high and low risk group was 2172 and 1610 , respectively. Structural heart defects were found in 210 fetuses in both groups with the $5.6 \%$ prevelance. Most common defects were VSD (37.1\%), AVSD (14.3\%), HLHS (9.5\%), abnormality of tricuspid valve (7.1\%) and single ventricle $(5.2 \%)$. Sensitivity of FE was $96 \%$ and specifity was $99 \%$ in our center. Prevelance of CHD was 7.6\% (166/2172) in high risk group (90 complex, 50 significant, 26 minor).

Prevelance of CHD was $2.7 \%(44 / 1610)$ in low risk group. (9 complex, 13 significant, 22 minor). In this group CHD prevelance was high in particular conditions; such as intrauterin death in previous pregnancy $(6.3 \%)$, abnormal first or second trimester screening tests (4.3\%), multiple gestations (3.4\%).

Conclusion: We found the prevelance of CHD is higher in fetuses than birth prevelance in both groups. CHD rate is increased in high risk group as expected. However, the CHD prevelance found to be also increased in following groups: intrauterine deaths in previous pregnancies, abnormal screening tests and multiple gestation. We think there may be some additions to the well known high risk group.

\section{1}

Right ventricular non compaction diagnosed by fetal echocardiography

Funda Oztunc, Istanbul University Cerrahpasa Medical Faculty, Department of Pediatrics, Division of Pediatric Cardiology, Turkey Ayse Guler Eroglu, Istanbul University Cerrahpasa Medical Faculty, Department of Pediatrics, Division of Pediatric Cardiology, Turkey Selman Gokalp, Turkey

Bulent Koca, Turkey

Selman Gokalp, Turkey

Safa Baris

Ayse Yurt

Isolated non-compaction of the ventricular myocardium is a rare cardiomyopathy characterized by numerous prominent trabeculations and deep intertrabecular recesses. This rare disease is due to an arrest of myocardial morphogenesis. Most cases, when seen in children, are associated with obstructive malformations. We noticed that the diagnosis of this disease is very rare in fetal life. In this report we present a case of right ventricular non compaction diagnosed by fetal echocardiography at 33 weeks of gestation Diagnosis of ventricular noncompaction was made according to the characteristic appearance of a two-layered structure of the myocardial wall consisting of a thin compacted epicardial layer and a thick noncompacted endocardial layer (ratio of noncompacted to compacted layers $>2$ ) with numerous, excessively prominent trabeculations and deep intertrabecular recesses that filled with blood from the ventricular cavity visualized on color Doppler imaging on echocardiography.

In contrast with postnatal experience, we found isolated noncompaction in the right ventricle. We discuss prenatal diagnosis and natural history of isolated non-compaction of right ventricle.

\section{2}

Does the prenatal diagnosis of severe heart disease facilitate the developing mother-infant relationship following surgery for congenital heart disease?: Two months assessment Jennifer M Re, School of Psychology, Psychiatry and Psychological Medicine, Monash University, Melbourne; Murdoch Children's Research Institute, Australia

Amichay Rotstein, Department Paediatric Cardiology and MonashHeart, Royal Children's Hospital \& Monash Medical Centre (MMC)., Australia Samuel Menahem, Foetal Cardiac Unit and MonashHeart, Monash Medical Centre, Melbourne., Australia

Suzanne Dean, School of Psychology, Psychiatry and Psychological Medicine, Monash University, Melbourne., Australia John Taffe, School of Psychology, Psychiatry and Psychological Medicine, Monash Medical Centre, Monash University, Melbourne., Australia Andrew Cochrane, Department of Cardiac Surgery, Monash Medical Centre, Melbourne., Australia

Congenital heart disease (CHD) carries a significant morbidity and mortality, bringing severe distress to parents. Prenatal diagnosis allows many critical issues to be identified prior to birth. Objective: The study aimed to explore whether the timing of the diagnosis of a major CHD (prenatal or postnatal), affects the early mother-infant relationship and outcomes. 
Methods: As part of a three-stage longitudinal study, assessment being at 2, 9 and 18-months of age, infants with CHD requiring early surgery and diagnosed prenatally or postnatally were recruited following delivery. This paper reports preliminary data for stage one. Medical and surgical outcomes were reviewed. Mothers were asked to complete a questionnaire and were interviewed. Observations were made of infant development, mother-infant and researcher-infant interaction. The questionnaire included standardised measures of maternal distress, (depression, stress and anxiety), parent-child attachment and adult attachment. Infant measures included the Bayley-111 Screening Test and Infant Social Withdrawal Scale.

Results: There were 14 mother-infant dyads, where the diagnosis was made prenatally and 11 postnatally. The cardiac diagnoses varied from an isolated VSD to HLHS. No significant differences were found between the groups on measures of maternal distress, adult attachment or infant social withdrawal. Across both groups, mothers manifested marked distress and infants were likely to be more socially withdrawn.

Conclusion: While no differences between the groups emerged, both mothers and infants in both groups were found to experience considerable difficulties that may have implications for the developing relationship. These findings have implications for planned interventions for the mother and infant.

6043

The convexity of the great vessel arising from the anterior ventricle- an aide in the fetal diagnosis of transposition of the great arteries (TGA)

Amichay Rotstein, Department of cardiology and cardiac surgery,

The Royal children's hospital Melbourne, Australia

Samuel Menahem, Fetal diagnostic unit, Monahs Medical center,

Melbourne Australia, Australia

Simon Meagher, Department of Obstetrics and Gynaecology, Monash

University, Clayton, Victoria, Australia, Australia

Objective: The fetal diagnosis of conotruncal abnormalities including TGA remains problematic. The diagnosis of TGA is commonly based on demonstrating the bifurcation of the great vessel arising from the posterior left ventricle and by the parallel course of the great arteries.

Methods: We retrospectively reviewed fetal studies where a postnatal diagnosis of TGA was confirmed, specifically images of the great vessel arising from the anterior chamber as it courses cranially to the three vessel tracheal view (3VTV). We compared these images to those in the fetus with a structurally normal heart (SNH).
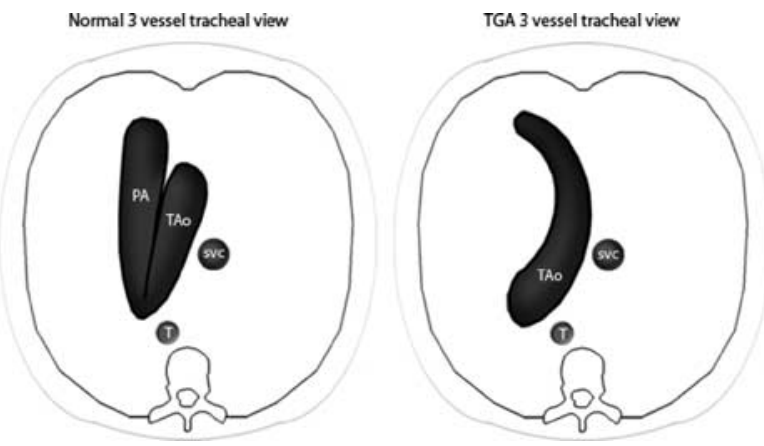

Results: In all 9 recent TGA cases, scanning the great vessel (aorta) cranially following it from the anterior ventricle to the 3VTV demonstrated a convexity to the right, as opposed to a convexity to the left of the great vessel (pulmonary artery), in all the cases with
SNH. We note that only 2 vessels were demonstrated in the 3VTV in patients with TGA, (SVC and transverse aorta), as opposed to 3 vessels, in the fetus with a SNH (SVC, transverse aorta and pulmonary artery). Figures 1 illustrates these findings.

Discussion: The 3VTV as a routine part of fetal cardiac scanning has been well described. Determining the direction of the convexity of the great vessel arising from the anterior right ventricle may aid in the fetal diagnosis of TGA. The relative ease, in which this view may be obtained, may prove to be the view of choice in the fetal diagnosis and screening of TGA.

\section{4}

Relationship between left and right ventricular performance during fetal life assessed by tissue Doppler imaging

Masamichi Tamura, Akita University School of Medicine, Japan Manatomo Toyono, Akita University School of Medicine, Japan Mieko Okazaki, Akita University School of Medicine, Japan Shunsuke Shimada, Akita University School of Medicine, Japan Jun Oyamada, Akita University School of Medicine, Japan

Objectives: The aim was to compare the indices between right (RV) and left (LV) ventricle obtained by tissue Doppler imaging (TDI) and evaluate the relationship between them in fetuses with structurally normal heart.

Methods: This was a cross-sectional study on 20 normal fetuses between 18 and 38 weeks of gestation. Two-dimensional echocardiography was used to examine fetal anatomy. TDI was used to assess diastolic velocities (peak velocity of early (E') and late (A') diastolic filling) and peak systolic velocity (S') at the lateral margins of both mitral (MVa) and tricuspid (TVa) annulus. The RV and LV Tei indices were calculated.

Results: A total of 30 echocardiographic examinations were performed. The mean gestational age was $32.1 \pm 4.3$ weeks. Among the peak velocity indices obtained by TDI, MVa-S' $(r=0.62)$, TVa-S' $(r=0.56)$, MVa-E' $(r=0.68)$, and TVa-E' $(r=0.57)$ showed positive correlation with gestational age, while neither MVa-A' nor TVa-A' correlate with the age. Negative correlation was demonstrated only between gestational age and LV-Tei index $(\mathrm{r}=0.36)$. Comparing the indices obtained from $\mathrm{LV}$ to that from RV, a significant positive correlation was present only between MVa-S' and TVa-S' $(r=0.53)$.

Conclusions: In the normal fetus, longitudinal myocardial performance in systole appears to be developed in proportion to the gestational age in contrast to that in diastole. Additionally, systolic performance in LV being correlated to that in RV may suggest that proportional development in the both ventricle is the must in normal fetus.

\section{5}

Prenatal Diagnosis of Congenital Absence of Aortic or Pulmonic Valve Leaflets

Zhiyun Tian, Fetal Heart Program, The Children's Hospital of Philadelphia, United States

Jack Rychik, Fetal Heart Program, The Children's Hospital of Philadelphia, United States

Background: Absence of semilunar valves is a rare congenital anomaly. Absent pulmonary valve leaflets (APVL) is associated with tetralogy of Fallot (ToF), pulmonary insufficiency, pulmonary artery dilation and respiratory failure. APVL can occur with other malformations, and little is known about absent aortic valve leaflets (AAVL). 
Objective: Review our experience with prenatal diagnosis and outcome of absent semilunar valves.

Methods/Results: Eighteen cases were identified between 2001-8; two AAVL, 16 APVL. Initial evaluation occurred at $26 \pm 6$ wks. Of the AAVL, 1 had cystic hygroma with Turner's syndrome and 1 had VSD with interrupted aortic arch - both died in-utero at 15 and 37 wks, respectively. Of APVL, 10 had ToF and 6 had non-ToF anatomy including 5 with unique right-sided disease: tricuspid/RV hypoplasia, intact ventricular septum and subaortic stenosis due to bulging septum (3); tricuspid atresia, VSD and complete heart block (1); tricuspid atresia, intact ventricular septum (1). One fetus with APVL had two good size ventricles, aorto-pulmonary window and intact ventricular septum. Of the 10 ToF group, 3 terminated pregnancy, and 5 are alive after surgery, 1 with tracheostomy. Of the 6 non-ToF group, 1 died in-utero, 2 died after birth, 2 survived surgical palliation - both with tracheostomy - and 1 received a heart transplant. Of 12 liveborn APVL, only 4 are repaired and breathing spontaneously. Conclusions: Congenital absence of semilunar valves can be diagnosed prenatally. AAVL is rare and lethal. APVL with nonToF anatomy is commonly associated with right heart hypoplasia and has high morbidity and mortality.

\section{6}

Nation-wide Prenatal Ultrasound Screening of Congenital Heart Defects in an Unselected Population: 22-year experience

Viktor Tomek, Kardiocentrum and Cardiovascular research center, University Hospital Motol, Prague, Czech Republic

Jan Škovránek, Kardiocentrum and Cardiovascular research center, University Hospital Motol, Prague, Czech Republic

Jiri' Gili'k, Kardiocentrum and Cardiovascular research center, University Hospital Motol, Prague, Czech Republic

Jan Marek, Kardiocentrum and Cardiovascular research center, University Hospital Motol, Prague, Czech Republic

The aim of prospective study was to analyze prevalence, spectrum and effectiveness of antenatal cardiac nation-wide screening program of congenital heart disease (CHD) in a region with unselected population between 1986 and 2007. Prenatal echocardiographic examinations were performed in 10027 fetuses between 13 to 41 weeks of gestation (median 23 weeks). CHD was found in 1828 fetuses (545 had additional extra cardiac anomalies). Early termination was performed in $1040(56.9 \%)$ cases, 60 (7.6\%) died in utero, and 728 (92.4\%) were born alive. Detection rate increased from $0.6 \%$ for all CHD and $2.3 \%$ for critical lesions in 1986, to $30.7 \%$ for all and $83 \%$ for critical CHD in 2007. The highest detection was in double outlet right ventricle (74\%), hypoplastic left heart (51.2\%), Ebstein anomaly (51.0\%) and atrioventricular septal defect $(43.2 \%)$. The lowest detection rate was in ventricular septal defects (2.6\%) and pulmonary stenosis (3.2\%). Antenatal detection rate increased significantly comparing two periods, 1987-1999 and 2000-2007. In the second decade of screening program, hypoplastic left heart was diagnosed prenatally in $95.2 \%$, transposition of great arteries in $26.2 \%$, and coarctation of aorta in $20.3 \%$. In double outlet right ventricle, prenatal detection rate exceeded estimated postnatal prevalence.

Conclusion: The nation-wide prenatal ultrasound program has enabled detection of cardiac abnormalities in 30\% of patients born with CHD and more than $80 \%$ of those with critical forms. Prenatal ultrasound spectrum of CHD differed from that seen postnatally with high association of non-cardiac lesions.

Supported by grants - NR9451-3/2007 and MZO 00064203

\section{7}

Treatment of Fetal Intrapericardial Teratoma using pericardio-amniotic shunting

Viktor Tomek, Kardiocentrum and Cardiovascular research centre, University Hospital Motol, Prague,, Czech Republic

Radek Vlk, Department of Obstetrics and Gynecology, University Hospital Motol, Prague, Czech Republic

Tomás Tláskal, Kardiocentrum and Cardiovascular research centre, University Hospital Motol, Prague, Czech Republic

Jan Skovránek, Kardiocentrum and Cardiovascular research centre, University Hospital Motol, Prague,, Czech Republic

Ivana Spálová, Department of Obstetrics and Gynecology, University Hospital Motol, Prague, Czech Republic

Intrapericardial teratoma is rare prenatal condition. A diagnosis is based on the observation of intrapericardial heterogenous mass accompanied by pericardial effusion. It is the second most frequent primitive cardiac tumor in neonate and is usually benign. It may lead prenatally to rapid hydrops progression with cardiac tamponade and lung compression. We present a prenatal intervention using the first successful pericardio-amniotic drainage.

Case report: Teratoma $35 \times 25 \mathrm{~mm}$ accompanied by a large pericardial effusion was diagnosed at 28 weeks of gestation. Our strategy was to avoid cardiac tamponade and possible pulmonary hypoplasia using pericardio-amniotic shunting. The procedure was performed together with amniodrainage contributing to a delay in delivery. That procedure led to significant decrease of pericardial effusion and expansion of lungs. Cardiothoracic index dropped from 0,67 to 0,47 . After 10 days, however, the shunt was released from pericardial sac to pleura. Pericardial effusion gradually increased, size of teratoma progressively extended to $50 \times 45 \mathrm{~mm}$ and compressed right atrium and superior vena cava. Neonate was delivered in 33rd week of gestation by Caesarean section. The surgery was performed within 6 hours in a baby without haemodynamic compromise. The tumor arising from the ascending aorta was carefully dissected and histology confirmed diagnosis of intrapericardial immature teratoma. The recovery was uneventful and at 6 month the baby is healthy. Conclusion: Pericardio-amniotic shunt seems to be a promising treatment option in fetuses with large pericardial effusion to prevent endouterine death and/or severe respiratory distress in neonates. Supported by grants - NR9451-3/ 2007 and MZO-00064203.

\section{8}

Fetal heart anomalies detected during the first trimester screening examination - what we cannot assess? Pawel Wlasienko, Perinatology and Perinatal Cardiology Unit, 2nd Department of Obstetrics and Gynaecology, Medical University of Warsaw, Poland

Anita Hamela-Olkowska, Perinatology and Perinatal Cardiology Unit, 2nd Department of Obstetrics and Gynaecology, Medical University of Warsaw, Poland

Joanna Dangel, Perinatology and Perinatal Cardiology Unit, 2nd Department of Obstetrics and Gynaecology, Medical University of Warsaw, Poland

Objective: To assess possibility of fetal heart evaluation between 11,0-13,6 weeks.

Methods: Prospective echocardiography in 1170 fetuses between 2004-2008, CRL45-84 mm, heart anatomy was verified in the second trimester.

Results: Mean maternal age was 30 years. 237(20\%) mothers were $>35$. In $87(7 \%)$ pts NT was $>95$ centile. All 17(1,5\%)fetuses with abnormal karyotype, except 1 with trisomy 18, had increased 
NT. 4-chamber view was visualized in $93 \%$ and outflow tract in $80 \%$ fetuses.

In 22 pts CHD was detected in first trimester: Cantrell pentalogy, HLHS, left isomerism with CHB, PS/VSD with trisomy 18, AVSD with trisomy 21 . In 2 the picture was unclear and in repeated scan in 16/18 weeks TOF was diagnosed. In 13 pts heart anomaly was not detected in the first trimester scan-they had small VSDs. NT $>95$ centile was in 17(44\%) fetuses with CHD. 19 fetuses with CHD were karyotyped and in $8(20 \%)$ it was abnormal: 3-trisomy 21, 4-monosomy $\mathrm{X}$ and 1-trisomy 18. There were early rhytm disturbances in 2 cases: $(\mathrm{CHB} /$ normal karyotype/left isomerism and tachycardia(FHR-187)/monosomy $\mathrm{X} /$ normal heart anatomy) - both fetuses died in utero. Conclusions: Fetal heart can be assessed in a reference perinatal cardiology center since 11 th week. In fetuses with CHD there is a high risk of abnormal karyotype. HLHS can be detected since the first trimester screening examination. Conotruncal anomalies may present dubious scan in first trimester, leading to early rescan in early second trimester. Due to difficulties in visualization of VS a control echocardiographic examination must be performed in each case in the second trimester.

\section{9}

Why TGA is so difficult in prenatal screening?

Pawel Wasienko, Perinatology and Perinatal Cardiology Unit, 2nd Department of Obstetrics and Gynaecology, Medical University of Warsaw, Poland

Joanna Dangel, Perinatology and Perinatal Cardiology Unit, 2nd Department of Obstetrics and Gynaecology, Medical University of Warsaw, Poland

Objective: To evaluate the importance of fetal heart axis assessment and the efficacy of prenatal US screening for conotruncal anomalies.

Methods: Evaluation of fetal heart axis in pts diagnosed in the reference perinatal cardiology center between 2005-2008. Out of 531 fetuses with CHD data of $83(15,6 \%)$ with conotruncal anomalies (TOF, DORV, CAT, TGA) was reviewed.

Results: Most pts were referred due to suspicion of CHD by obstetricians who were trained in screening program. Mean maternal age was 29 and mean gestational age at the time of diagnosis (GA) was $27.58(70 \%)$ patients were in a low risk group. There were TOF-42, DORV-24, TGA-13 and CAT-4. Karyotyping was performed in 53(64\%) fetuses, abnormal-15(18\%). The fetal heart axis was assessed in $80(96 \%)$ cases. Among TOF fetuses mean GA was 25 , the mean heart axis was $63^{\circ} .17(40 \%)$ were from high risk group. Among DORV fetuses mean GA was 30 , the mean heart axis was $60^{\circ}$. Only $5(21 \%)$ pts were from high risk group. All CAT pts were from the low risk group. The mean GA was 29 and the mean fetal heart axis was $88^{\circ}$. In TGA pts mean GA was 27 , the mean heart axis was $46^{\circ} .3(23 \%)$ pts were from the high risk group.

Conclusions: Fetal heart axis is helpful in diagnosis of CAT, TOF and DORV, but in fetuses with TGA it was close to normal. Detection of TGA in screening US exam is still difficult due to normal fetal heart axis and 4-ch view, so assessment of outflow tracts should be recommended.

\section{0}

Does the left ventricle of a fetus twist?

Satoshi Yasukochi, Nagano Children's Hospital, Japan

Gengi Satomi, Nagano Children's Hospital, Japan

Kiyohiro Takigiku, Nagano Children's Hospital, Japan
Ichige Kajimura, Nagano Children's Hospital, Japan

Kouta Takei, Nagano Children's Hospital, Japan

Seiichi Tazawa, Nagano Children's Hospital, Japan

Nao Inoue, Nagano Children's Hospital, Japan

Yuusuke Nakano, Nagano Children's Hospital, Japan

Background: Little was known about the regional wall motion of the left ventricle (LV) in fetus, especially whether a fetal myocardium twist.

Aim: The purpose of this study is to determine the feasibility of measurement of the twist of LV in fetus, by using velocityvector-imaging (VVI), as a new imaging modality of twodimensional tissue tracking.

Subjects: 9 normal fetuses with normal ejection fraction from 29 to 38 weeks of gestation.

Methods: Diagnostic apparatus was Sequoia 512 (Siemens Medical Systems) with $8-3 \mathrm{MHz}$ probe. The short axial B-mode images of LV each at cardiac base and at apex (frame rate $>80 \mathrm{fps}$ ) were obtained with maternal ECG-triggering then digitally stored. Data were analyzed by off-line Syngo program (Siemnes MS). Endodiastolic frame of fetal LV was determined as the frame at the longest internal diameter by M-mode. The border of endocardium (End) and epicardium (Epi) was manually traced then automatically tracked by Syngo sytem to calculate the rotational angles. LV twist was calculated by subtracting the averaged rotational angle of cardiac apex from that of cardiac base along a cardiac cycle.

Results: In fetuses, the peak-twist was 7.16 \pm 3.1 degree in End and $5.6 \pm 2.7$ degree in Epi, respectively. The peak twist was not changed along gestational age.

Conclusion: The LV of fetus does twist in same fashion as that of an adult but the peak-twist of the LV in fetus is about a half of that in adult $(13.3+/-0.3$ degree $)$ and is not changed along the gestational age.

\section{1}

Familial recurrence of congenital heart defect detected by fetal echocardiography: result from one institution in Korea Sin-Weon Yun, Department of Pediatrics Chung-Ang University, Korea Kyung-Sook Choi, Department of Nursing, Chung-Ang University, Korea Jung-Yun Choi, Department of Pediatrics Seoul national University, Korea

Background: Congenital heart disease (CHD) is generally assumed to have a multifactorial etiology. The purpose of this study was to investigate pattern and recurrence of familial aggregation in CHD. Methods: From Jan 1988 to Dec 2003, 822 fetal echocardiograms whose familial history of CHDs in Seoul National University Hospital was evaluate and enrolled. We excluded syndromic disease, chromosomal abnormalities, associated multiple anomalies.

Results: Among 822 exams, 772 had family with CHD in first degree relatives. We enrolled 735 fetal exams with at least 1 siblings had CHD. If the first baby had CHD, recurrent incidence in consecutive pregnancy was $2.73 \%(18 / 659)$. When two siblings have CHD, the risk for a subsequent pregnancy reachs $10.5 \%(2 / 19)$. This recurrence rate was highly dependent on the type of lesion in the 1 st baby. Truncal abnormalities were 25\%(3/12). AVSD were 11\%(2/ 18 ), right sides lesions (tricuspid atresia, pulmonary atresia, pulmonary stenosis etc) were $6.67 \%(3 / 45)$, situs and positional abnormalities were $4.34 \%(2 / 46)$, outflow lesions (DORV, complete TGA, corrected TGA) were $2.27 \%(2 / 88)$, TOF were $1.72 \%(2 /$ 116), VSD were $2.66 \%(4 / 150)$. Most recurrences were concordant with index case. In families with two more recurrences, the exact concordance rate was very high. Exact concordant rate was particularly higher in isolated AVSD and truncus arteriosus. 
Conclusions: Our findings suggest the recurrence and concordance rate is high in some family and some type of CHD. We could select families with a "tendency' to develop CHD, and analyze the genetic pattern to establish abnormalities and the bases of CHD.

\section{3}

Double Inferior Vena Cava; A Case Report

Vehbi Dogan, Sami Ulus Pediatric Research and Traning Hospital Departman of Pediatric Cardiology, Turkey

Utku Arman Orun, Sami Ulus Pediatric Research and Traning

Hospital Departman of Pediatric Cardiology, Turkey

Filiz Senocak, Sami Ulus Pediatric Research and Traning Hospital Departman of Pediatric Cardiology, Turkey

Cigdem Unver, Sami Ulus Pediatric Research and Traning Hospital Departman of Radiology, Turkey

Selmin Karademir, Sami Ulus Pediatric Research and Traning Hospital Departman of Pediatric Cardiology, Turkey

Burhan Ocal, Dr. Sami Ulus Pediatric Research and Traning Hospital Departman of Pediatric Cardiology, Turkey

Murat Koc, Dr. Sami Ulus Pediatric Research and Traning Hospital Departman of Cardiovascular Surgery, Turkey

Ali Kutsal, Sami Ulus Pediatric Research and Traning Hospital, Turkey

A 12 year old male admitted to our hospital with a complaint of poor weight gain. Abdominal ultrasonography suggested double inferior vena cava that the left one draining into right inferior vena cava (IVC) immediately after giving origin to right renal vein. Echocardiography revealed a drop-out appearance on interatrial septum and a second venous structure located at the left side of the abdominal aorta. Diagnosis of patent foramen ovale and double IVC was further confirmed by conventional angiography. Although rarely suspected, recent studies have revealed that IVC anomalies are not rare if anticipated and evaluated. The prevalence of double IVC is reported between $0.2 \%$ and $3 \%$ in general poulation. The left IVC typically ends at the renal vein which crosses anterior to the aorta in the normal fashion to join to the right IVC. In routine conditions, conventional angiography is not indicated in case of isolated double IVC. For our patient, we planned device closure of PFO because of reportedly increased risk of trombosis and embolism due to this rare venous anomaly.

\section{4}

Assessment of Ventricular Function with Conventional Echocardiography and Tissue Doppler Imaging (TDI) in Obese Children

Patricia Alvarez, CLINICA ALEMANA/ HOSPITAL

ROBERTO DEL RIO, Chile

Marcela Alburquenque, HOSPITAL ROBERTO DEL RIO, Chile Valeria Acevedo, CLINICA ALEMANA/ HOSPITAL ROBER-

TO DEL RIO, Chile

Katty Quezada, HOSPITAL ROBERTO DEL RIO, Chile

Daneil Aguirre, CLINICA ALEMANA - HOSPITAL

ROBERTO DEL RIO, Chile

Rodgrigo Nehgme, CONGENITAL HEART INSTITUTE,

ORLANDO-FL., United States

Maria Herrera, HOSPITAL ROBERTO DEL RIO, Chile

Ignacio Hernandez, HOSPITAL ROBERTO DEL RIO, Chile

Obesity is a well established risk factor for cardiomyopathy and congestive heart failure. Our objective was to investigate structural and functional LV abnormalities by echocardiography in obese children.
Forty-six obese and 46 normal children were included in the study. All patients were asymptomatic. Evaluation included EKG, conventional echocardiography and TDI, Strain and strain rate (SR). Demographic data for the obese vs. control groups revealed a median age of 11.5 vs. 12 years, body mass index (BMI) 27 vs. 18 , male gender 22 vs. 23. Metabolic syndrome and elevated blood pressure was seen in 30\% of obese patients. EKG was normal in both groups. Echocardiography data in obese vs. control groups revealed increased LV mass $(p=0.003)$ and LA dimension $(p=0.012)$. TDI data showed elevated $a^{\prime}(p=0.012)$, elevated $\mathrm{E} / \mathrm{e}^{\prime}(\mathrm{p}=0.001)$ and decreased $\mathrm{s}^{\prime}(\mathrm{p}=0.02)$ in the septal mitral ring when comparing obese vs. normal children. The TEI index of the LV was abnormal as well $(\mathrm{p}=0.021)$. There were no differences in Strain and SR between groups.

Conclusion: Obese children have structural and functional heart abnormalities detected by echocardiography despite the absence of symptoms. Tissue Doppler was useful in detecting early functional involvement. These finding could represent an early stage of the cardiomyopathy reported in obese patients in long term follow-up. Tissue Doppler should be part of the routine evaluation of these patients.

\section{5}

Exercise Testing and Echocardiographic Evaluation Following Complete Repair of Tetralogy of Fallot Berna Saylan Cevik, Dr. Behçet Uz Childrens' Hospital Pediatric Cardiology Department IZMIR, Turkey

Intracardiac repair of tetralogy of fallot (TOF) can be performed with an acceptably low morbidity and mortality. However, the procedure may adversely affect myocardial function and compromise long-term results. Impairment of right ventricular function has been reported in patients with TOF after repair. Pulsed doppler tissue imaging (DTI) is an ultrasound tool that provides quantitative assessment of myocardial velocities by its high spatial and temporal resolution. It has been reported to be a valuable tool for the prediction of myocardial functional response to physical exercise. The aim of this study was to evaluate changes in RV function in response to exercise using DTI and conventional Doppler echocardiography.

Method: We examined 20 patients(mean age $8.05 \pm 3.00$ years), with a mean follow-up of $4.5 \pm 2.7$ years after surgery. Two patients were excluded, one because of incooperation, the other because of viral illness. another one had to finish the test at the first minute because of viral illness.

Results: The test was noted as submaximal in 12 patients (\% 66.6). The functional parameters of the test and RV echocardiographic pulse doppler and DTI parameters were not statistically significant. The total correction age was negatively significant with exercise time and METS index $(p=0.015, p=0.010)$ Conclusion: The exercise test should be performed in this group of patients to determine the maximal heart rate and maximal blood pressure.

\section{7}

Assessment of Pulmonary Artery Morphology: Conventional versus Computed Tomogram Angiography

Haifa Abdul Latiff, Institut Jantung Negara, Kuala Lumpur, Malaysia Amin Itam, Institut Jantung Negara, Kuala Lumpur, Malaysia Yang Rafidah Abdul Aziz, University Malaya Medical Center, Malaysia

Pui Ding Chan, University Malaya Medical Center, Malaysia Mazeni Alwi, Institut Jantung Negara, Kuala Lumpur, Malaysia 
Hasri Samion, Institut Jantung Negara, Kuala Lumpur, Malaysia Geetha Kandavello, Institut Jantung Negara, Kuala Lumpur, Malaysia

Objective: To determine the reliability of Computed Tomogram angiography (CTA) in evaluating pulmonary artery (PA) morphology compared to conventional angiogram.

Methods: This prospective observational study involved 36 patients with cyanotic heart disease from July 2006 to June 2008. The CTA and conventional angiogram were performed in patients in whom the PA was not well visualized on conventional angiogram or; as part of routine pulmonary artery assessment post PDA stenting. The size of right (RPA) and left (LPA) pulmonary artery diameters were measured on both modalities. Branch pulmonary arteries were classified as either confluent, stenosed (defined as $\geqslant 50 \%$ narrowing) or absent. The investigators reporting the CTA are blinded of the angiogram findings. The findings on both modalities were compared. The sensivity and specificity of CTA were mesured based on the the ability to demonstrate the significant stenosis or absent pulmonary artery. Results: Branch PA was not visualized (absent) in 12 patients on conventional angiogram, but all were visualized on CTA. Branch PA stenosis was reported in 3 patients on CTA, 3 patients of conventional angiogram and 4 patients on both modalities. The mean RPA diameter measured by angiogram and CTA were $10.1 \pm 4.04 \mathrm{~mm}$ and $9.53 \pm 4.67 \mathrm{~mm}$ respectively $(\mathrm{p}=0.22)$ The mean LPA size measured by angiogram and CTA were $7.51 \pm 4.16 \mathrm{~mm}$ and $7.95 \pm 4.74 \mathrm{~mm}$ respectively $(\mathrm{p}=0.61)$. The sensitivity and specificity of CTA were $70 \%$ and $94 \%$ respectively.

Conclusions: CTA is superior to conventional angiogram in evaluating pulmonary artery morphology.
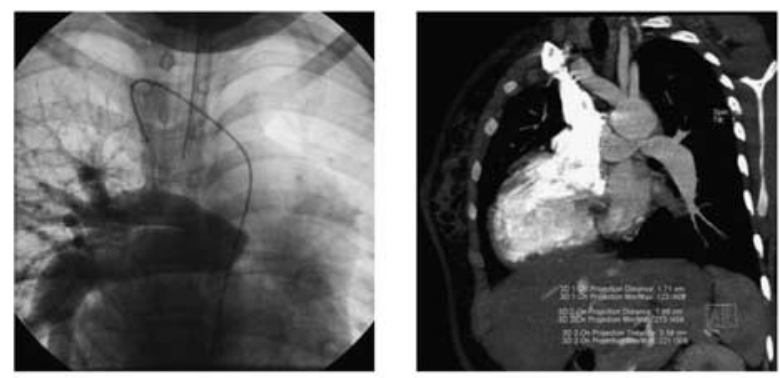

Conventional angiogram (left) failed to delineate the left pulmonary artery (LPA) but CT angiogram in the same patient (right) demonstrates the LPA clearly

\section{8}

Pathophysiology of Coronary Blood Flow in Congenital Heart Disease

Elhadi H Aburawi, Department of Paediatrics, Section of Paediatric Cardiology, Lund University Hospital, Sweden

Petru Liuba, Department of Paediatrics, Section of Paediatric

Cardiology, Lund University Hospital, Sweden

Erkki Pesonen, Department of Paediatrics, Section of Paediatric

Cardiology, Lund University Hospital, Sweden

Objective: Pressure and volume overload of cardiac chambers increase coronary flow but the magnitude of their effect is not known.

Methods: Sixty-four patients with acyanotic congenital heart diseases (CHD) were included in the study: 12 neonates with coarctation of aorta (CoA), 7 neonates with severe pulmonary valve stenosis (PS), 18 infants with ventricular septal defect (VSD), and 27 children with atrial septal defect (ASD). Coronary flow (CF) parameters were measured in the left anterior descending artery in CA and VSD and in the posterior descending artery in PS and ASD patients with transthoracic Doppler echocardiography. Age-matched healthy controls $(\mathrm{n}=49)$ were included. Patients were studied 1-2 days before the treatment with either surgery or interventional cardiac catheterization. Coronary flow data in each patient group are expressed as percent change from the controls.

Results: Cardiac output and fractional shortening were decreased in patients as compared with controls $(p<0.05$ in all patient groups). The median of peak flow velocity was $600 \%$ in patients with CoA and $225 \%, 225 \%$ and $118 \%$ in patients with PS, VSD and ASD, respectively. The increase in CF was relatively larger than peak flow velocity (773\% in CoA, 233\% in PS, 172 in VSD, and 185 in ASD).

Conclusions: Coronary flow and peak flow velocity appear to be markedly increased in patients with CHD. Increased preductal blood pressure, myocardial strain and ventricular hypertrophy due to CoA seem to have maximal effect on coronary flow, but volume and pressure overload of the ventricles have also significant increasing effects.

\section{9}

Atrial septal angulation as predictor for poor outcome of atrioventricular septal defect

Zaheer Ahmad, Congenital Cardiac Centre, Southampton University Hospital NHS Trust, United Kingdom

Zek Lim, Congenital Cardiac Centre, Southampton University Hospital NHS Trust, United Kingdom

Hunaid Vohra, Congenital Cardiac Centre, Southampton University Hospital NHS Trust, United Kingdom

Kevin Roman, Congenital Cardiac Centre, Southampton University Hospital NHS Trust, United Kingdom

Marcus Haw, Congenital Cardiac Centre, Southampton University Hospital NHS Trust, United Kingdom

Robert H Anderson, Institue of Child Health, London, United Kingdom Joseph Vettukattil, Congenital Cardiac Centre, Southampton University Hospital NHS Trust, United Kingdom

Objective: Three-dimensional echocardiography offers new insights into the morphology of atrioventricular septal defects (AVSD). In this setting, the alignment of the atrial and ventricular septums is disrupted leading to variation in the venous return in to corresponding ventricles. We attempted to establish the orientation of the atrial and ventricular septums relative to each other and the atrioventricular junction to study its impact on septatability.

Methods: We evaluated 31 patients using 3-dimensional echocardiography. Off-line analysis using Tometec was used for multiplanar review. In 4 chamber view crux of the heart was identified to define the relation of the muscular ventricular septum to the crux. We drew a line along long axis of the ventricular septum to join the leading edge of the atrial septum thus defining the plane of AVSD. A second line was constructed along long axis of the atrial septum. The angle formed between these two lines was measured.

Results: Biventricular repair proved possible in 21 patients. The atrial septum was parallel to ventricular septum in 14 while 7 had angulation (mean angle 11.8). Patients with unbalanced ventricles had significant angulation ( $\mathrm{n}=10$ mean $29 \mathrm{p}=<0.0002)$ One patient had 1-1/2 ventricular repair while another had biventricular repair incorporating a prosthetic left atrioventricular valve. Three died and five had successful single ventricle palliation. Atrial septal angles more than 26 predicted adverse outcome.

Conclusions: Multiplanar review of three-dimensional data sets is valuable for assessing the septatability of AVSD. Malalignment of 
septal structures may be a substrate for underdevelopment of the ventricular volume.

\author{
6060 \\ Echocardiographic study of superior inferior ventricles \\ and criss-cross hearts \\ Dursun Alehan, Hacettepe University Faculty of Medicine Division of \\ Pediatric Cardiology, Ankara, Turkey \\ Suheyla Ozkutlu, Hacettepe University Faculty of Medicine Division of \\ Pediatric Cardiology, Ankara, Turkey
}

Objective: Superior-inferior ventricles are characterized by abnormal relationship of the ventricles, in which the ventricles lye one above the other instead of side-by-side. Consequently the interventricular septum is more or less horizontal. The anomaly, that is almost always associated with complex cardiac abnormalities, is very rarely seen and little information exists about it. We aimed to investigate the feasibility of echocardiohraphy in the diagnosis of this complex malformation.

Methods: Patients with superior-inferior ventricles were assessed by segmental and sequential echocardiography study.

Results: 12 patients aged 4 months to 12 years were studied. Two of the patients had in utero diagnosis. Eight patients had usual atrial arrangement (2 of them have isolated dextrocardia), 3 had mirror-imaged arrangement and 1 had left atrial isomerism. Two of the patients had isolated levocardia. Seven patients had discordant atrioventricular connections, and 5 had discordant ventriculoarterial connections. Criss-cross hearts were present in 5 patients. Frequently associated anomalies included ventricular septal defect (10 patients), pulmonary outflow tract stenosis or atresia (7 patients), and double outlet right ventricle (5 patients). One patient had severe left ventricular outflow tract obstruction. Conclusion: Superior-inferior ventricles are rare anomalies associated with complex cardiac malformations. Correct diagnosis is feasible with echocardiographic study.

\section{1}

Should Adult CMRI Measures of Ventricular Volumes be used for Children?

Luis A Altamirano, The Prince Charles Hospital, Canada

Wendy Strugnell, The Prince Charles Hospital, Australia

Harry Bartlett, Queensland university of technology, Australia

Richard Slaughter, Australia

Tameeka Parry, The Prince Charles Hospital, Australia

Robyn Riley, The Prince Charles Hospital, Australia

Andrew Trotter, The Prince Charles Hospital, Australia

Robert Justo, Mater Children's Hospital, Australia

Objectives: To compare the cardiac magnetic resonance imaging (CMRI) Body surface area (BSA)-indexed values of left and right ventricular volumes obtained in adults with those of children aged $8-12$ yrs.

Background: CMRI is increasingly recognised as the reference standard for the non-invasive assessment of ventricular size and function. While normal values have been established in an adult population, there is a paucity of published values in children. To our knowledge, no study has been undertaken to determine if values obtained in an adult population can be reliably extrapolated to children.

Methods: Normal values and ranges of left and right ventricular function were established in adults (50 males (age range 19-60; mean 38) and 50 females (age range 18-59; mean 40)) and children (44 boys (age range 8.0-12.1; mean 9.9) and 45 girls (age range 8.2-12.0; mean 9.6)). CMRI imaging was performed on a $1.5 \mathrm{~T}$ GE Twinspeed without sedation using ECG-gating and suspended respiration. Steady state free precession cine MR images were acquired and analysis was performed off-line using Mass Analysis software. Left and right ventricular end-diastolic volumes (EDV), end-systolic volumes (ESV) were obtained. Results were indexed to (BSA).

Results: RVEDVi women $113 \mathrm{ml} / \mathrm{m} 2$ girls $101 \mathrm{mls} / \mathrm{m} 2$ difference of $12 \mathrm{mls}(10.6 \%)$. Men $131 \mathrm{ml} / \mathrm{m} 2$ boys $124 \mathrm{ml} / \mathrm{m} 2$ difference of $7 \mathrm{mls}$ (5.3\%). RVESVi women $54 \mathrm{ml} / \mathrm{m} 2$ girls $46 \mathrm{ml} / \mathrm{m} 2$ difference $8 \mathrm{ml}(14 \%)$, Males $72 \mathrm{ml} / \mathrm{m} 2$ and boys $61 \mathrm{ml} / \mathrm{m} 2$ difference of $11 \mathrm{ml}(15 \%)$.

Conclusion: There is significant difference in RVEDVi between women and girls and RVEDVi and RVESVi between men and boys.

\section{2}

Relation of Mean Right Atrial Pressure to Doppler Parameters of Right Atrial and Hepatic Venous Flow in Pediatric Patients with Congenital Heart Disease

Hamid Amoozgar, Shiraz University of Medical Sciences, Iran khobiar Zare, Shiraz University of Medical Sciences, Iran Mohammad Borzoee, Shiraz University of Medical Sciences, Iran Gholamhossein Ajami, Shiraz University of Medical Sciences, Iran Saiid Abtahi, Shiraz University of Medical Sciences, Iran

Background: A paucity of data exists regarding the relation of mean right atrial pressure (RAP) to Doppler parameters of right atrial and ventricular filling in pediatric patients with congenital heart disease.

Methods: fifty patients ( 30 male and 20 female) with mean age of $4.96+-4.05$ who were admitted in the pediatric cardiology ward of Shiraz medical university affiliated hospital, Nemazee Hospital, Shiraz, Iran were included in this study. Data gathered from hepatic venous flow, tricuspid diastolic flow and Pulse tissue Doppler of lateral tricuspid annulus of each patient were then compared with right atrial pressure obtained by cardiac catheterization.

Results: If change of peak $\mathrm{S}$ wave velocity of hepatic vein in respiration was more than $63.1 \%$, sensitivity and specificity of a RA pressure more than $8 \mathrm{mmHg}$ were $90 \%$ and $51.3 \%$ respectively with likelihood ratio (LR) equal to 1.85; A peak S wave velocity of less than $70 \mathrm{~mm} / \mathrm{Sec}$ also showed a RA pressure more than $8 \mathrm{mmHg}$ with sensitivity and specificity of 70 and 82.1 respectively $(\mathrm{LR}=3.9)$. A peak $\mathrm{D}$ wave velocity of hepatic vein more than $63 \mathrm{~mm} / \mathrm{sec}$ was indicator of RA pressure more than $8 \mathrm{mmHg}$ with sensitivity and specificity of $60 \%$ and $92.3 \%$ respectively $(\mathrm{LR}=7.8)$.

Conclusion: This study showed that hepatic venous flow can be valuable for estimation of mean RA pressure in pediatric patients with congenital heart disease.

\section{3}

Correlation between Echocardiography and cardiac angio TAC to diagnose unusual Supracardiac total anomalous pulmonary venous drainage

Lucrecia Avila Rosales, Hospital para el Niño IMIEM, Mexico JM Yañez Sanchez, Hospital Para el Niño IMIEM, Mexico Humberto Rodríguez Saldaña, Hospital Para el Niño IMIEM, Mexico G Melendez Ramirez, Hospital Para el Niño IMIEM, Mexico Manuel Ochoa, Hospital Para el Niño IMIEM, Mexico Felipe Arizmendi Tapia, Hospital Para el Niño IMIEM, Mexico Lidia Rodriguez Hernandez, Hospital Para el Niño IMIEM, Mexico

Correlation between Echocardiography and cardiac angio TAC to diagnose unusual Supracardiac total anomalous pulmonary venous drainage. 
Objective: Utility of Echocardiography and AngioTAC, to obtain a better view of anatomic structures.

18 years old patient with heart failure grade II of the New York Heart Association.

Chest Radiography: Cardiomegaly grade III, due to enlarge right cavities, collector of the right pulmonary veins "Scimitar sign" to the right of the right atrium.

\begin{tabular}{lll}
\hline & ECHO & ANGIOTAC \\
\hline left & small & Similar \\
atrium & $28 \mathrm{~mm}$ & \\
DDVR & $41 \mathrm{~mm}$ & $53 \mathrm{~mm}$ \\
DDVL & $24 \mathrm{~mm}$ & $27 \mathrm{~mm}$ \\
TAP & $32 \mathrm{~mm}$ & $32 \mathrm{~mm}$ \\
VCS & $72 \mathrm{~mm}$ & $72 \mathrm{~mm}$ \\
Artery cocronary origin & Normal & Normal \\
PSAP & $77 \mathrm{mmHg}$ & - \\
RT & $62 \mathrm{mmHg}$ & - \\
RDAP & $18 \mathrm{~mm}$ & $18 \mathrm{~mm}$ \\
RIAP & $18 \mathrm{~mm}$ & $18 \mathrm{~mm}$ \\
\hline
\end{tabular}

Echocardiography: Situs solitus, concordance atrioventricular and ventriculoarterial, supracardiac anomalous venous drainage, atrial septal defect, dilated superior vena cava, and right atrium and ventricle. It could be seen with the angiotac that a large collector was bifurcated only behind the lower and middle right atrium it was again only one collector in the upper part of the right atrium and drained into the superior vena cava in its union with the right atrium.

Conclusion: Neither the echocardiogram nor cardiac cath didn't showed this detail and was reported as "drainage to the acigos", the studies were complementary and should be done in cases that have some doubt, in spite this arrangement of the collector don't change the surgery, this presentation hasn't been reported before.

6064

Impact of vascular rings on tracheal geometry: multidetector computed tomography study

Oleksandr Kondrachuk, Ukrainian Children's Cardiac Center, Ukraine Tetyana Yalynska, Ukrainian Children's Cardiac Center, Ukraine Oleksandr Bablyak, Ukrainian Children's Cardiac Center, Ukraine Illya Yemets, Ukrainian Children's Cardiac Center, Ukraine

Objective: Double aortic arch (DAA), right aortic arch with aberrant left subclavian artery (RAA/ALSA) and left aortic arch with aberrant right subclavian artery (LAA/ARSA) are the most common vascular rings. DAA and RAA/ALSA are usually symptomatic and form a complete vascular ring. LAA/ARSA typically is asymptomatic and does not cause a complete vascular ring. The aim of this study was to define the difference in tracheal geometry between patients with complete vascular rings, subjects with incomplete vascular rings and individuals with normal left aortic arch.

Methods: In total, 57 patients underwent multidetector computed tomography (MDCT). Patients were divided in three groups. Group A patients $(\mathrm{n}=19)$ had complete vascular rings; group B patients $(\mathrm{n}=19)$ had incomplete vascular rings; group $\mathrm{C}$ patients $(\mathrm{n}=19)$ had normal left aortic arch. Percentage of maximum area (PMA) was defined as the smallest tracheal cross-sectional luminal area divided by the largest area $\times 100 \%$.

Results: In group A, significant tracheal narrowing (PMA $<50 \%$ ) was observed in 8 (42.1\%) of 19 patients. There were no cases of significant tracheal narrowing in groups B and C. Percentages of maximum area were $53.0 \pm 23.8 \%, 78.5 \pm 11.1 \%, 82.2 \pm 7.2 \%$ for groups $\mathrm{A}, \mathrm{B}$ and $\mathrm{C}$, respectively. There were significant differences between groups A and B $(p<0.01)$ and between groups $A$ and $C(p<0.01)$. There was no significant difference between group $\mathrm{B}$ and group $\mathrm{C}$.

Conclusions: Patients with complete vascular rings have significantly altered tracheal geometry compared to subjects with LAA/ ARSA. Tracheal geometry in patients with incomplete vascular rings is similar to normal.

\section{6}

Assessment of left and right ventricular function with Speckle Tracking Echocardiography in patients late after repair of tetralogy of Fallot: reproducibility and comparaison to Magnetic Resonance Imaging

Laurent Bonnemains, Centre Chirurgical Marie Lannelongue, France

Bertrand Stos, Centre Chirurgical Marie Lannelongue, France

Julie Blanc, Hopital Necker Enfants Malades, France

Andre Capderou, Centre Chirurgical Marie Lannelongue, France

Fabien Labombarda, Hopital Necker Enfants Malades, France

Jean Francois Paul, Centre Chirurgical Marie Lannelongue, France

Damien Bonnet, Hopital Necker Enfants Malades, France

Younes Boudjemline, Hopital Necker Enfants Malades, France

Background: Two-dimensional (2D) speckle tracking echocardiography (STE) is anew ultrasound technique allowing the assessment of global and segmental myocardial strain and strain rate. We assessed the feasibility and reproducibility of this technique within a paediatric population. We quantified the correlation between global strains and MRI computed ejection fraction.

Methods and Results: 40 post-operative tetralogy of Fallot adolescents wereprospectively included. All patients underwent 2D STE and a cardiac MRI. Globalcircumferential (GCS) and longitudinal (GLS) strains of both ventricles weremeasured using STE modality. We established a new Functional three-dimensional Index (FI) combining GCS and GLS. Ejection fractions were measured by MRI andcompared to FI. Feasibility of STE and MRI analysis in our population were similar(range $=83-88 \%)$. GLS and GLS' rates for the left ventricle were respectively $18.5 \%+/-5.4$ and $1.02 \mathrm{~s}-$ $1+/-0.4$ as compared with $20 \%+/-5$ and $0.97 \mathrm{~s}-1+/-0.32$ for theright ventricle. Strain intra and interobserver variations were low (range $=1.4-2.7 \%$ ). Strain rates had a low reproducibility (range $=0.22-0.25 \mathrm{~s}-1)$. FI and ejection fractionwere well correlated for the left $(\mathrm{r}=0.61, \mathrm{p}=0.001$, see $=4.0, \mathrm{n}=30)$ and right $(\mathrm{r}=0.51, \mathrm{p}=0.03$, see $=4.8, \mathrm{n}=19)$ ventricles.

Conclusions: STE is a new echocardiographic technique allowing a reproduciblemyocardial function assessment. A simple combination of strains obtained in several dimensions allows a correct left and right ventricular function assessment well correlated to MRI ejection fraction in the specific clinical setting of our study.

\section{7}

Loeys-Dietz syndrome: Comprehensive Assessment by Whole Body MRI

Timothy J Bradley, The Hospital for Sick Children, Canada

Brian Grant, The Hospital for Sick Children, Canada

Michael T Seed, The Hospital for Sick Children, Canada

Susan Blaser, The Hospital for Sick Children, Canada

Lars Grosse-Wortman, Canada

Shi-Joon Yoo, The Hospital for Sick Children, Canada

Objectives: Loeys-Dietz syndrome is a recently described autosomal dominant connective tissue disorder caused by heterozygous mutations in the genes encoding for transforming growth factor $\beta$ receptors 1 and 2. It is characterized by aggressive vascular pathology with aortic and arterial tortuosity, aneurysm formation and a 
propensity for early dissection. We describe a single study, whole body MR approach for paediatric assessment of the known associated cardiovascular, skeletal, brain and spine abnormalities.

Methods: Eight children (age range 0.5-14 years) with known Loeys-Dietz syndrome had MRI studies performed on a $1.5 \mathrm{~T}$ scanner. General anaesthesia was utilized less than 6 years of age with initial X-rays to exclude cervical spine instability. The imaging sequences included: cardiac cine anatomical and functional imaging, phase-contrast imaging of the aortic valve and major vessels, whole body MR angiography (using a single injection of $0.4 \mathrm{cc} / \mathrm{kg}$ intravenous gadolinium), brain and spinal anatomical imaging and 3D time-of-flight imaging of the circle of Willis.

Results: Vascular abnormalities demonstrated included: 5 moderate to severe aortic root dilatation; 5 tortuous aortic arch, descending thoracic or abdominal aorta; 7 tortuous innominate, carotid, vertebral or intracranial arteries; and 2 with abdominal aortic branch involvement. Associated skeletal, brain and spine abnormalities included: 1 craniosynostosis; 2 hypertelorism; 1 pectus excavatum; 1 anterior corpus callosal hypoplasia; 1 arachnoid cysts; 3 cervical spine anomalies; 2 scoliosis; and 3 lumbosacral dural ectasia. Conclusion: Our whole body MR approach allows for comprehensive assessment of the complex range of cardiovascular, skeletal, brain and spine abnormalities associated with LoeysDietz syndrome.

\section{8 \\ Device closure of atrial septal defects with Amplatzer septal occluder devices in patients where interatrial septum is not in its usual plane \\ Ramyashri Chandrasekaran, MIOT Hospital, Chennai, India, India Kothandam Sivakumar, MIOT Hospital, Chennai, India, India Shrinivas V Chakravarthi, MIOT Hospital, Chennai, India, India Anpon Bhagyavathy, MIOT Hospital, Chennai, India, India Radhakrishnan Satish, MIOT Hospital, Chennai, India, India Arunkumar Govindarajan, MIOT Hospital, Chennai, India, India}

Objectives: Catheter closure of secundum defects using Amplatzer septal occluders utilises fluoroscopic and echocardiographic guidance. We report our experience in patients with altered anatomy of interatrial septal plane (IASP) who pose challenges. Methods: Patients who underwent device closure of secundum defects, with altered IASP were analyzed. Interatrial septal plane was altered in severe kyphoscoliosis, atrial septal malalignment with multiple atrial septal defects, juxtaposed atrial appendages with altered IASP, anatomically corrected malposition with coronal IASP, interupted inferior venacava with azygos continuation (Figure). Multiple pulmonary venous angiogram were done in these patients to orient the IASP on fluoroscopy; different transesophageal planes were recorded to get the best visualisation of IASP.

Results: 8 patients with secundum atrial septal defects (age range 2-30 years) had a different IASP, four were patients with malalignment between the primum and secundum septum with multiple defects, one with severe thoracic kyphoscoliosis, one with juxtaposed atrial appendage, one with coronal IASP associated with anatomically corrected malposition of great arteries, one with interupted inferior venacava with azygos continuation. Flouroscopic and transesophageal view were initially selected to clearly delineate IASP and these unusual angles guided device deployment. Post deployment, levophase of pulmonary arteriogram was routinely observed to visualize IASP and alignment of the device to the septum. Device closure was successful in all patients with no complications. On a follow up ranging 3 months to 5 years, there were no residual flows.
Conclusions: Safe device closure of atrial septal defects in altered IASP proves the versatility of the nitinol septal occluders.
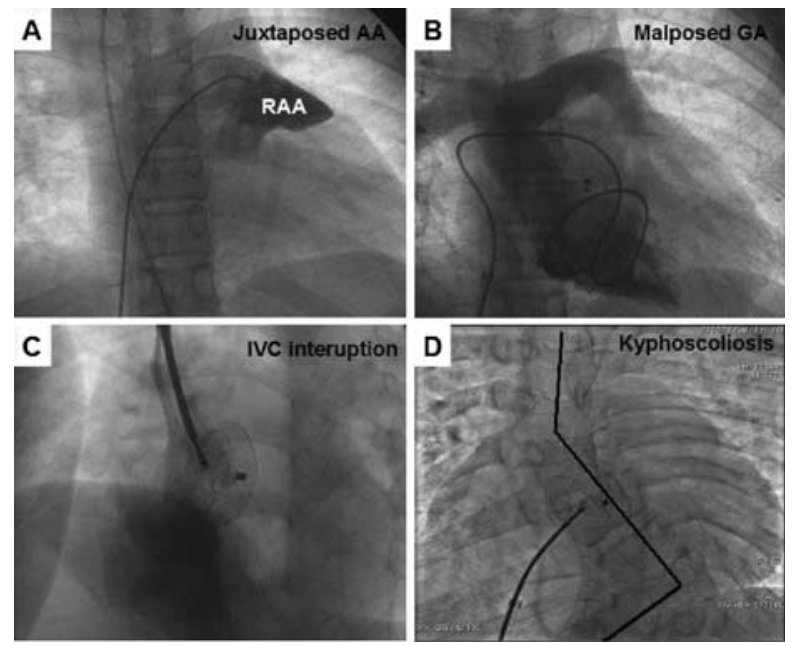

6069

Quantification of Right Ventricular Function using Tricuspid and Mitral valve annular descent by Tissue Deformation Imaging in patients with Tetralogy of Fallot James Charlton, University of Southampton, United Kingdom Zaheer Ahmad, Congenital Cardiac Centre, Southampton University Hospital NHS Trust, United Kingdom

Kevin Roman, Congenital Cardiac Centre, Southampton University Hospital NHS Trust, United Kingdom

Sheena Toyot, University of Southampton, United Kingdom

Zek Lim, Congenital Cardiac Centre, Southampton University Hospital NHS Trust, United Kingdom

Joseph Vettukattil, Congenital Cardiac Centre, Southampton University Hospital NHS Trust, United Kingdom

Objectives: There are no reproducible and clinically applicable echocardiographic parameters to assess right ventricular (RV) function. We used Tissue Motion Annular Displacement (TMAD) with Speckle Tracking Echocardiography (STE) to quantify RV function.

Methods: Fourteen Tetralogy of Fallot (TOF) patients and 9 controls were enrolled. Images were acquired using Philips iE33 and S5 probe. Apical 4 chamber (AP4) views and mid-level short-axis views were obtained for both patients and controls. Offline analysis was done with QLab 6.0. TMAD was used as a surrogate marker for assessing the longitudinal fibre shortening. Tissue traction points were assigned to the hinge points of the corresponding atrioventricular valve leaflets and ventricular apices. In short-axis view with superimposed colour kinesis, circumferential contraction was assessed from end-diastole to end-systole. Multiple measurements were taken and analysed with SPSS16.

Results: Longitudinal shortening of the RV was significantly reduced in TOF patients $(17.9+/-2.8 \mathrm{vs} 24.0+/-3.3 \mathrm{p}=$ 0.0001). Impairment of the tricuspid valve descent was more pronounced in the RV free wall $(15.4+/-3.4 \mathrm{vs} 20.6+/-2.4$ $\mathrm{p}=0.0007)$ than the interventricular septum $(11.9+/$ $-3.1 \mathrm{vs} 12.8+/-2.3 \mathrm{p}=0.5)$. There was a significant difference in longitudinal shortening between the right and left ventricles in controls $(24.0+/-3.3$ vs $17.9+/-4.0 \mathrm{p}=0.003)$ and patients with TOF. However, this difference was less pronounced in patients with TOF $(17.8+/-2.8$ vs $15.0+/-4.1 \mathrm{p}=0.05)$. Longitudinal shortening of the left ventricle was not significantly 
reduced in TOF patients $(15.1+/-4.1$ vs $17.9+/-4.0 \mathrm{p}=0.1)$ when compared with the control group.

Conclusion: RV function can be reliably quantified using STE. Impaired longitudinal shortening seen in TOF predominantly affects the RV free wall while the inter-ventricular septum is spared.

\section{0}

Implications of Restrictive Right Ventricular Physiology in Right Ventricular Adaptation to Chronic Pulmonary Regurgitation in Patients with Repaired Tetralogy of Fallot: Assessment with Cardiovascular Magnetic Resonance and Tissue Doppler Imaging

Chun-An Chen, Department of Pediatrics, National Taiwan University Hospital, Taipei, Taiwan, Taiwan

Wen-Yih Tseng, Department of Medical Imaging, National Taiwan University Hospital, Taipei, Taiwan, Taiwan

Jou-Kou Wang, National Taiwan University Hospital, Taiwan

Chung-I Chang, Department of Surgery, National Taiwan University Hospital, Taipei, Taiwan, Taiwan

Ing-Sh Chiu, Department of Surgery, National Taiwan University Hospital, Taipei, Taiwan, Taiwan

Yih-Sharng Chen, Department of Surgery, National Taiwan University Hospital, Taipei, Taiwan, Taiwan

Hsi-Yu Yu, Department of Surgery, National Taiwan University Hospital, Taipei, Taiwan, Taiwan

Mei-Hwan Wu, Department of Pediatrics, National Taiwan University Hospital, Taipei, Taiwan, Taiwan

Objectives: To explore the role of restrictive right ventricular (RV) physiology (antegrade pulmonary arterial flow in late diastole) in $\mathrm{RV}$ adaptation to pulmonary regurgitation (PR) in repaired tetralogy of Fallot.

Methods: We studied 80 patients (aged $22.7 \pm 7.8$ years) without significant residual pulmonary stenosis using cardiovascular magnetic resonance and tissue Doppler imaging. Restrictive RV physiology was assessed by Doppler echocardiography.

\begin{tabular}{lcccr}
\hline Variables & All $(\mathbf{n}=\mathbf{8 0})$ & $\begin{array}{c}\text { With restrictive } \\
\text { RV physiology } \\
(\mathbf{n}=32)\end{array}$ & $\begin{array}{c}\text { Without restrictive P value } \\
\text { physiology } \\
(\mathbf{n}=48)\end{array}$ & \\
& & & & \\
& & & & \\
& & & & \\
TAP repair & $38 / 73(52 \%)$ & $9 / 30(30 \%)$ & $(67 \%)$ & 0.001 \\
QRS duration (ms) & $147.8 \pm 20.3$ & $139.2 \pm 18.3$ & $153.5 \pm 19.8$ & 0.002 \\
RV MPI & $0.49 \pm 0.11$ & $0.46 \pm 0.10$ & $0.51 \pm 0.11$ & 0.030 \\
Aa $(\mathrm{cm} / \mathrm{s})$ & $7.9 \pm 2.8$ & $8.8 \pm 3.0$ & $7.4 \pm 2.6$ & 0.029 \\
RVEDVi $(\mathrm{ml} / \mathrm{m} 2)$ & $119.5 \pm 35.2$ & $96.9 \pm 20.9$ & $134.5 \pm 34.9$ & $<0.001$ \\
RVESVi $(\mathrm{ml} / \mathrm{m} 2)$ & $63.1 \pm 26.6$ & $48.6 \pm 14.1$ & $72.8 \pm 28.6$ & $<0.001$ \\
PRF $(\%)$ & $28.8 \pm 17.3$ & $18.3 \pm 15.5$ & $35.8 \pm 14.7$ & $<0.001$ \\
RV outflow tract & $17(21 \%)$ & $1(3 \%)$ & $16(33 \%)$ & 0.002 \\
aneurysm & & & & \\
\hline
\end{tabular}
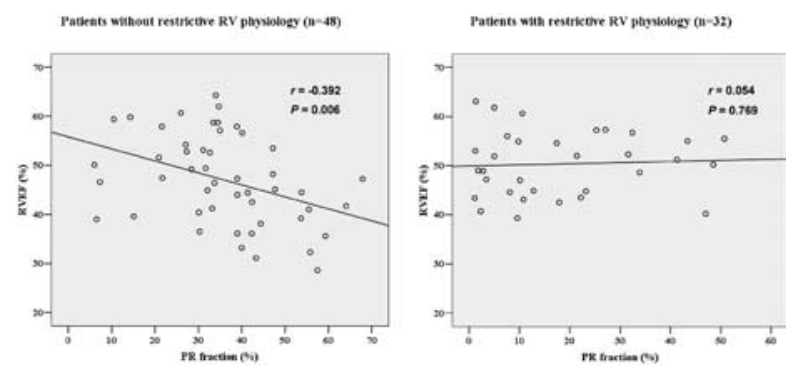

Results: Patients with restrictive RV physiology (32 patients, 40\%) had lower PR fraction (PRF), RV end-diastolic volume index
(RVEDVi), myocardial performance index (MPI), higher late diastolic annular velocity, less RV outflow tract aneurysm and transannular patch (TAP) than those without (Table). Age at repair (OR: $0.61, P=0.042$ ), TAP (OR: $0.20, P=0.031$ ), RVEDVi (OR: 0.93, $P=0.001$ ), and MPI (OR: 0.001, $P=0.027)$ were independently associated with restrictive RV physiology on multivariate analysis. Furthermore, PRF correlated negatively with RV ejection fraction (RVEF) in patients without restrictive RV physiology, whereas such relationship was absent in those with restrictive RV physiology, either in overall patients (Figure) or in those with PRF $>20 \%$ (55 patients). Subgroup analysis in patients with PRF $>20 \%$ revealed smaller RVEDVi $\left(111.7 \pm 14.8\right.$ versus $\left.139.2 \pm 34.2 \mathrm{ml} / \mathrm{m}^{2}, \mathrm{p}<0.001\right)$ and better RVEF $(51.2 \pm 5.5$ versus $46.8 \pm 9.2 \%, P=0.042)$ in patients with restrictive RV physiology (13 patients) than those without despite similar PRF.

Conclusions: Patients with restrictive RV physiology had better $\mathrm{RV}$ performance, probably through mitigating the detrimental effect of PR on RV remodeling.

\section{1}

The value of real-time three-dimensional echocardiography in the assessment of pre- and post-operative left ventricular function in children with conotruncal defects Guo-Zhen Chen, Department of Pediatric Cardiology, Shanghai Children Medical Center, Medical College of Shanghai Jiaotong University, China

Kun Sun, Department of Pediatric Cardiology, Shanghai Children's Medical Center, China

Mei-Rong Huang, Department of Pediatric Cardiology, Shanghai Children Medical Center, Medical College of Shanghai Jiaotong University, China

Yu-Qi Zhang, Department of Pediatric Cardiology, Shanghai Children Medical Center, Medical College of Shanghai Jiaotong University, China

Objective: This study is aimed to evaluate the value of real-time three-dimensional echocardiography (RT-3DE) in the assessment of pre- and post-operative left ventricular function in children with conotruncal defects (CTD).

Methods: Forty-six consecutive children (mean aged 2.0 \pm 2.7 years) with CTD were examined by Phillips RT-3DE System. Their pre- and post-operative left ventriclular function parameters (one month after surgical operation) such as volume, stroke volume and ejection fraction were estimated by Tomtec RT-3DE apical longitudinal 8-plane method. The results were compared and assessed. using paired t-test and Pearson's correlation coefficient analysis.

Results: Compared with pre-operative end-diastolic and endsystolic volume $(18.7 \pm 14.9 \mathrm{ml}$ and $9.0 \pm 7.3 \mathrm{ml})$ of left ventricle, their post-operative end-diastolic volume $(22.2 \pm 17.6 \mathrm{ml})$ was increased, while their post-operative end-systolic volume $(9.5 \pm 7.8 \mathrm{ml})$ was not obviously changed in one month after surgical operation. Furthermore, there were good correlations between pre- and postoperative end-systolic volume $(\mathrm{r}=0.97)$, and between the pre- and postoperative end-diastolic volume $(\mathrm{r}=0.95)$. Accordingly, compared with pre-operative stroke volume $(9.6 \pm 7.6 \mathrm{ml})$ and ejection fraction $(0.52 \pm 0.03)$, their post-operative stroke volume $(12.7 \pm 9.8 \mathrm{ml})$ and ejection fraction $(0.58 \pm 0.03)$ were markedly increased $(\mathrm{p}<0.05)$. Moreover, compared with pre-operative end-diastolic wall mass (19.4 \pm $11.4 \mathrm{~g})$, post-operative end-diastolic wall mass $(21.1 \pm 11.0 \mathrm{~g})$ was increased. Thus, after cardiovascular deformities of CTD were surgically corrected, cardiac function was early improved and gradually normalized completely. 
Conclusions: By making precise quantitative assessments without geometric assumptions, RT-3DE could be helpful in providing more information for preoperative diagnosis, post-operative follow-up and prognostic prediction in CTD.

\section{2}

Three dimensional echocardiography used for evaluation of the structure of tricuspid valve in hypoplastic right ventricle

Sun Chen, Shanghai Children's medical center,Medical school of Jiaotong university, China

Kun Sun, Department of Pediatric Cardiology, Shanghai Children's Medical Center, China

Shubao Chen, Shanghai Children's medical center,Medical school of

Jiaotong university

Objectives: To reveal the characteristics of tricuspid valve in hypoplastic right ventricular heart syndrome (HPRVHS) using 3-D echocardiography.

Methods: Twenty eight patients with hypoplastic right ventricle were included in the study. These patients were divided into two groups according to the degree of tricuspid regurgitation. Eighteen normal children were included as control. The Volume of right ventricle (Vrv), the area of tricuspid annulus (A TVA) and leaflets, the distance from tips of leaflets to the nearest papillary muscles were measured in three dimensional echocardiogram using Q-lab image processing software. These data were standardized by body surface area (BSA).

Results: The A-TV was significantly correlated with the volume of right ventricle $(r=0.90, p<0.0001)$. In the tricuspid regurgitation group, the ratio of the area of anterior leaflet (A an) to the area of tricuspid annulus (A TVA) was significantly higher than that in non-regurgitation group and normal group (ratio $=0.55 \pm 0.044, \mathrm{P}<0.0001)$. The ratio of the Area of the posterior leaflet (A po) to the area of tricuspid annulus (A TVA) in regurgitation group was significantly less than that in normal and non-regurgitation groups. The distance from tips of leaflets was significantly shorter in HPRVHS than in normal group.Distance from the tip of septal leaflet to the nearest papillary muscle is shorter in regurgitation group than in non-regurgitation group $(\mathrm{p}=0.0023)$.

Conclusions: 3-dimensional echocardiography could be applied for evaluating the pathologic changes of tricuspid valve. In HPRVHS, hypoplasty of posterior leaflet and shortness of the cords of septal leaflet could be the causes of tricuspid regurgitation.

\section{4}

Results of PDA occlusion with device in ISFAHAN,IRAN Ali Reza Ahmadi, Medical University of Isfahan, Israel

Objective: The aim of the study was to assess the results of PDA occlusion with device in children.

Background: Patent Ductus Arteriosus remain a major congenital heart disease in children. PDA occlusion with device is a dramatic advances in the management have been made. Data from one of the important center of heart center is presented.

Methods: A total of 25 patients (age range 1-16 yrs) that PDA occlusion were performed in one year period ( from may 2007-may 2008) were studied for age, sex, PA pressure, residual shunt and type and size of device.
Results: All of the above - mentioned patients under went occlusion of PDA after the evaluation of anatomical and hemodynamical status by echocardiography and catheterization and angiography. Forty percent of patients were under two years of age. Mean age of patient was 4 years .Girls were seventy percent of patients. PA pressure in sixty percent was near normal. In ninety one percent Nit occlude coil was used. PDA size in forty four percent was small and fifty six percent were medium size. In forty percent no residual shunt was remained immediately, and fifty eight percent has no residual shunt after 24 hours. Conclusion: PDA occlusion with device in children was performed successfully at our center.

\section{5}

Reliability of measuring the abdominal aortic diameter in neonates

Jean H Du Plessis, Dept of Cardiology, Princess Margaret Hospital for Children, Perth, Western Australia, Australia

Paul Norman, School of Surgery, University of Western Australia,

Perth, Australia, Australia

James $M$ Ramsay, Dept of Cardiology, Princess Margaret Hospital for Children, Perth, Western Australia, Australia

Andy Gill, Dept. of Neonatology, King Edward Memorial Hospital, Perth, Western Australia, Australia

Karen Simmer, University of Western Australia, Perth, Western Australia, Australia

Joan A Sharpe, Dept. of Cardiology, Princess Margaret Hospital for Children, Perth, Western Australia, Australia

Elaine Pascoe, Clinical Research and Education, Princess Margaret

Hospital for Children, Perth Western Australia, Australia

Background: Intra-uterine growth restriction influences aortic wall thickness and may predict cardiovascular disease. The possibility that adult aneurysmal arterial disease has fetal origins has attracted little attention. We explored the feasibility and repeatability of measuring infrarenal aortic diameter in neonates.

Aim: 1) Determine reliability and repeatability of infrarenal aortic diameter measurements in neonates. 2) Determine whether Two-dimensional or M-mode measurements are superior.

Method: Twenty-six neonates ranging from 25 to 41 (median $=38.3$ ) weeks had their infrarenal aorta scanned in coronal views obtained from either the left or right flanks. Twodimensional and $\mathrm{M}$-mode images were recorded and measured in both systole and diastole. Scans were re-measured at a later stage by a sonographer blinded to previous measurements. Inter-rater agreement was measured using an intra-class correlation coefficient (ICC) and intra-rater reliability with Pearson's correlation coefficient $(\mathrm{r})$.

Results: Inter-rater agreements were high for M-mode images in both systole $(\mathrm{ICC}=0.78,95 \%$ CI $0.58-0.90)$ and diastole $(\mathrm{ICC}=0.75$, 95\% CI $0.52-0.88)$ and very high for twodimensional images in both systole (ICC $=0.94,95 \%$ CI $0.87-0.97$ ) and diastole ( $\mathrm{ICC}=0.93,95 \%$ CI $0.85-0.97$ ). Intra-rater reliability was high for $\mathrm{M}$-mode images in systole $(\mathrm{r}=0.79,95 \%$ CI $0.49-0.92)$ and diastole $(\mathrm{r}=0.83,95 \% \mathrm{CI}$ $0.56-0.94)$ and very high for two-dimensional images in systole $(\mathrm{r}=0.92,95 \%$ CI $0.78-0.97)$ and diastole $(r=0.91,95 \% \mathrm{CI}$ $0.77-0.97)$.

Conclusion: This study describes a new technique to reliably and repeatibly measure the infrarenal aortic diameter in neonates. Two-dimensional measurements are superior to $\mathrm{M}$-mode measurements and future studies measuring infra-renal aortic diameter in neonates should use two-dimensional measurements. 
6077

Differential Branch Pulmonary Artery Regurgitant Fraction Identifies Patients with Unilateral Stenosis Associated With Relatively Elevated Pulmonary Vascular Resistance in the Contralateral Lung After Repair of Conotruncal Anomalies

Matthew A Harris, The Children's Hospital of Philadelphia, United States

Kevin K Whitehead, The Children's Hospital of Philadelphia, United States

Paul M Weinberg, The Children's Hospital of Philadelphia, United States

Matthew J Gillespie, The Children's Hospital of Philadelphia, United States

A Fogel, United States

Objective: To determine in postoperative conotruncal anomalies patients if: (1) residual branch pulmonary artery (BPA) stenosis or size discrepancy is associated with differential BPA regurgitation (2) differential BPA regurgitation correlates with differential pulmonary vascular resistance (PVR).

Methods: We retrospectively reviewed 71 consecutive cardiac magnetic resonance (CMR) studies for BPA size and PhaseContrast Magnetic Resonance (PCMR) data. We also reviewed 13 consecutive pts who underwent both CMR and catheterization.

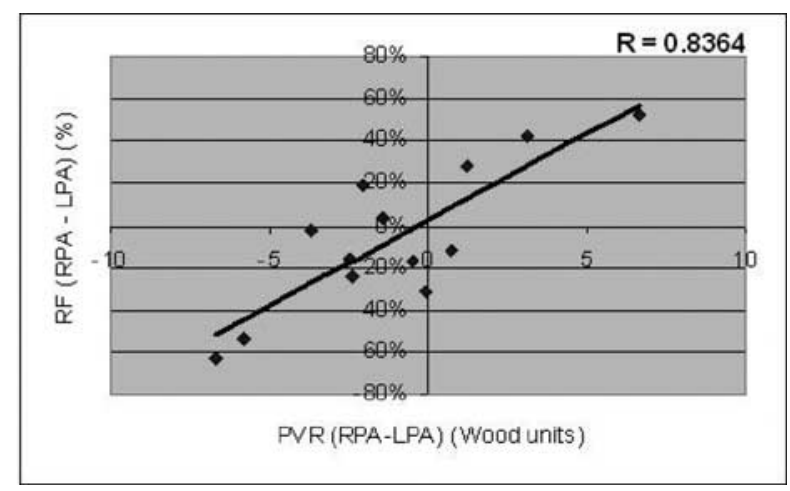

Results: 27 of the 71 pts had either BPA stenosis or one BPA cross-sectional area (CSA) comprise $<33 \%$ of the total BPA CSA. Among these $27 \mathrm{pts}$, there was no significant difference between RPA and LPA regurgitant fraction (RF) (28 vs 32\%, $p=0.49)$, however, there was a significantly increased RF of the larger vs smaller BPA (39 vs $21 \%, \mathrm{p}<0.001)$. In contrast, the 44 pts without BPA stenosis or size discrepancy showed a significant difference between RPA and LPA RF (31 vs 38\%, p <0.001), without a significant difference in the larger vs smaller BPA RF (36 vs $33 \%, p=0.14$ ). Retrospective review of pts who underwent both CMR and catheterization demonstrates that differential BPA RF strongly correlates with differential PVR $(\mathrm{R}=0.8364, \mathrm{p}<0.001)$ (See figure).

Conclusions: BPA stenosis or size discrepancy outweighs the increased LPA RF of pts without stenosis or size discrepancy. Since differential BPA RF correlates with differential PVR, PCMR can serve as an important screening tool for identifying pts with stenosis or size discrepancy who may have developed relatively increased PVR in the contralateral larger pulmonary artery.

6078

Three-Dimensional "Fly-Through" Post-Processing of Magnetic Resonance Images Identifies Coronary Ostial Stenosis in Patients With Anomalous Origin of a Coronary Artery
Matthew A Harris, Children's Hospital of Philadephia, United States Kevin K Whitehead, Children's Hospital of Philadephia, United States Matthew J Gillespie, Children's Hospital of Philadephia, United States Michael T Cosulich, Children's Hospital of Philadephia, United States Paul M Weinberg, Children's Hospital of Philadephia, United States Mark A Fogel, Children's Hospital of Philadephia, United States

Objective: Anomalous coronary artery orgin - both coronaries from the same sinus of valsalva - has been identified as a cause of sudden death. Coronary arteries arising from the contralateral sinus often have a tangential origin from the aorta with an elliptical ostium resulting in ostial stenosis. MRI identifies the sinus of origin of each coronary artery. Yet, distinguishing single versus separate ostia from the same sinus, and ostial stenosis is challenging with 2-dimensional imaging. This study evaluates a 3-dimensional imaging possibility.

Methods: We retrospectively reviewed 6 pediatric cardiac MRI $3-$ dimensional data sets of pts with anomalous left (ALCA) $(n=2)$ or anomalous right (ARCA) $(n=4)$ coronary arteries. The data were analyzed with "Fly-Through" software allowing one to view the dataset as if within the vessel.

Results: Of 4 pts with ALCA, 2 have a single right coronary artery without ostial stenosis. Of the remaining 2 pts, 1 had an elliptical ostial opening of the left coronary artery (Fig. A) suggesting stenosis, (compared to normal right ostium [Fig. B]) and the other had 2 adjacent ostia without evidence of ostial stenosis. Both pts with ARCA had 2 separate ostia: 1. was identified with ostial stenosis related to a tangential origin. Three of the 6 pts had their imaging findings confirmed at the time of surgery (Fig C), and the remainder did not undergo surgical intervention.

Conclusion: "Fly-Through" technique noninvasively identifies ostial stenosis associated with ALCA and ARCA. This data may become important in stratifying a pt's risk for sudden death.

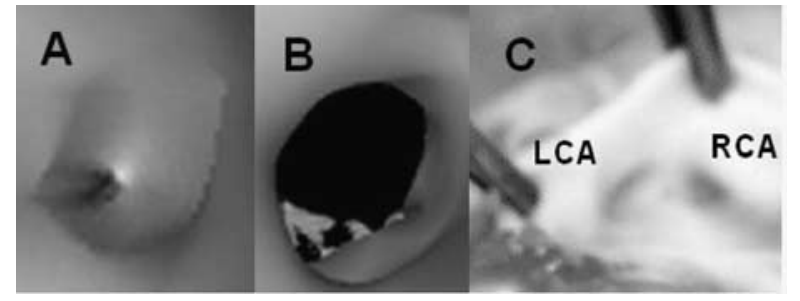

6080

Routine Performance of Intraoperative Transoesophageal Echocardiography in Children with Congenital Heart Disease: 1546 Cases in One Center

Guo-ying Huang, Pediatric Heart Center, Children's Hospital of Fudan University, Shanghai, China

Xiao-jing Ma, Pediatric Heart Center, Children's Hospital of Fudan University, Shanghai, China

Xue-cun Liang, Pediatric Heart Center, Children's Hospital of Fudan University, Shanghai, China

Xiao-qin Liu, Pediatric Heart Center, Children's Hospital of Fudan University, Shanghai, China

Bing Jia, Pediatric Heart Center, Children's Hospital of Fudan University, Shanghai, China

Zhang-gen Chen, Pediatric Heart Center, Children's Hospital of Fudan University, Shanghai, China

Objective: To evaluate the value of routine intraoperative transoesophageal echocardiography (TEE) in children with congenital heart disease (CHD). 
Methods: In recent 5 years, intraoperative TEE was performed in 1546 cases of CHD aged $(3.5 \pm 2.6)$ years old in our pediatric heart center. The cases with weight $<4 \mathrm{~kg}$ were excluded for the reason of safety. TEE was performed both before and after cardiopulmonary bypass in each case.

Results: (1) Preoperative TEE added additional findings or changed the diagnoses made by transthoracic echocardiography in 123 cases $(8.0 \%)$, among which the findings had therapeutic significance in 93 cases $(6.0 \%)$ that altered the planned surgical procedures. (2) Residual problems or sequels were detected by postoperative TEE in 270 cases (17.5\%), with 57 patients $(3.7 \%)$ requiring instant intervention or return to bypass for modifications of the surgical procedures. (3) We encountered no severe complications due to the performance of TEE. Mild complications occurred in only 3 patients $(0.2 \%)$.

Conclusions: TEE is a useful and safe imaging tool with which to determine the strategies for treatment in the perioperative period, and to improve the quality of surgical procedures in children with CHD.

\section{1}

How and where to measure? Comparing contemporary noninvasive assessment of pulmonary artery size in children with congenital heart disease

Marina L Hughes, Great Ormond St Hospital for Children NHS

Trust, United Kingdom

F Bailliard, University of North Carolina Children's Hospital, United States

Andrew M Taylor, Great Ormond St Hospital for Children NHS

Trust, United Kingdom

J Marek, Great Ormond St Hospital for Children NHS Trust, United Kingdom

Objectives: Knowledge of the size and nature of the proximal PAs is crucial for planning surgical management of infants with CHD. Because contemporary clinical data on their relative merits is scant, we compared PA measurements using gadoliniumenhanced 3D cardiac magnetic resonance angiography (CMRA) and 2Dechocardiography.

Methods: A prospective, observational study of 56 consecutive children undergoing CMR and 2Decho under GA, between 2006-2008.

Two independent observers measured optimised 2Decho images of PAs and 2 independent observers measured cross-sectional area, shortest and orthogonal PA diameters with Siemens 3D software from isotropic CMRA images.

Results: The full spectrum of pre-surgical CHD was represented. $29 / 56(52 \%)$ had functionally univentricular hearts. The median age was 1.1 years (range $0.2-8.5$ ) and median weight $9.4 \mathrm{~kg}$ (range 4.0-24.1).

CMRA assessed the 3D form of all vessels. 2Decho measurements were lacking from the distal LPA in 39 patients.

Inter-technique assessment showed significant differences between narrowest CMRA measurement and the 2Decho measurement of the proximal PAs, (mean difference $1.0 \mathrm{~mm}$, paired 2-tailed $\mathrm{P}<0.01$ ) but when shortest and orthogonal CMRA diameters were averaged, measurements compared well to single-plane 2Decho.

There was reasonable correlation of 2Decho (calculated) Nakata index and measured CMRA Nakata index $(\mathrm{r}=0.54, \mathrm{p}<0.01)$. Interobserver variability was significant (mean difference $>0.3 \mathrm{~mm}, \quad \mathrm{P}<0.01$ ) for 2Decho measurements of the proximal LPA and distal RPA, but was insignificant for all paired CMRA measurements.
Conclusions: 2Decho is limited and may fail to visualise the narrowest PA dimension, but when acoustic windows permit there is good correlation of 2Decho and CMRA measurements of PAs.

6082

The Role of Computed Tomogram (CT) angiography in the assessment of pulmonary venous anomaly Ijaz Hussain, Institut Jantung Negara, Kuala Lumpur, Malaysia Haifa Abdul Latiff, Institut Jantung Negara, Kuala Lumpur, Malaysia Abdul Samad Sakijan, Institut Jantung Negara, Kuala Lumpur, Malaysia Mazeni Alwi, Institut Jantung Negara, Kuala Lumpur, Malaysia Hasri Samion, Institut Jantung Negara, Kuala Lumpur, Malaysia Geetha Kandavello, Institut Jantung Negara, Kuala Lumpur, Malaysia

Objective: To examine the role and safety of CT angiography in the diagnosis of pulmonary vein anomalies and its impact on the management.

Methods: 41 patients who had CT angiography from July 2007 to October 2008 for the assessment of pulmonary veins were retrospectively reviewed. The indication for the CT angiogram, diagnosis, management and surgical findings were studied. The radiation dose was calculated.

Results: Median age is 6 months and median weight is $5 \mathrm{~kg}$. All but 5 required light sedation; only one patient required general anaesthesia. There was no procedure related complication. 11/41 were postoperative patients with persistent respiratory distress including 2 with post repair of pulmonary venous anomaly, and $30 / 41$ were preoperative patients diagnosed to have anomalous pulmonary venous drainage (APVD) on echocardiogram. Of 41 patients, 11 patients had total APVD (4 supracardiac, 3 intracardiac, 3 infracardiac and one mixed type), 8 had partial APVD, 10 had hypoplastic pulmonary veins, 2 had pulmonary veins stenosis and 10 were normal. Five TAPVD patients had right and one with partial APVD had left isomerism. 14/31 $(45.2 \%)$ patients with abnormal pulmonary veins underwent surgical repair and the findings were confirmed at surgery in all patients; $16 / 31(51.6 \%)$ patients were treated conservatively due to severe associated cardiac lesions in 5, hypoplastic pulmonary veins not amenable to surgery in 11 and in one parents refuse surgical treatment. Mean total radiation dose was 2.5 msev. Conclusion: CT angiography is safe and reliable in delinating pulmonary veins pathology which helps in the planning of patients' management.
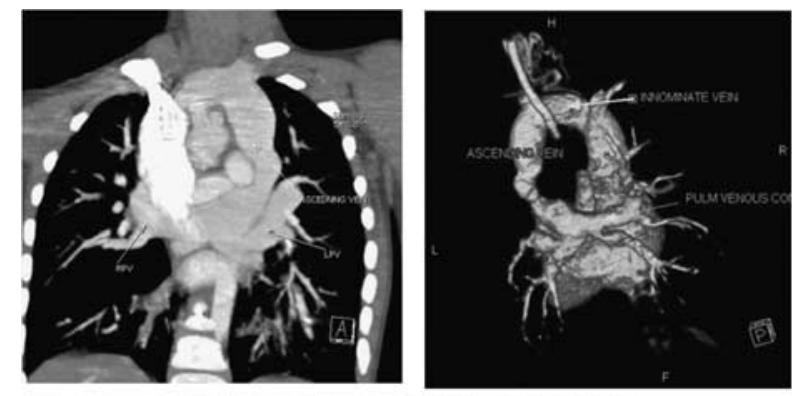

Suprac ardiac TAPVD on CT angiogram MIP (left) and VRT (right). All pulmonary veins drain into the innominate vein via an ascending vein

6084

Comparison of Multidetector-Row Computed

Tomographic Coronary Angiography and Invasive Coronary Angiography to Identify Coronary Abnormalities in Children and Adolescent Chien-Chang Juan, Department of Pediatrics, National Yang-Ming University Hospital, l-Lan, Taiwan. R.O.C, Taiwan 
Betau Hwang, Taiwan

Bing-Yao Wang, Department of Pediatrics, National Yang-Ming University Hospital, I-Lan, Taiwan. R.O.C, Taiwan

Pi-Chang Lee, Department of Pediatrics, Taipei Veterans General Hospital, Taipei, Taiwan, R.O.C, Taiwan

Objective: This study is comparing the accuracy of MDCT with invasive contrast coronary angiography (ICA) identifying coronary abnormalities in children and adolescents.

Methods: We reviewed all patients, from Jan. 2002 to Dec. 2008, with congenital or acquired coronary abnormalities underwent either ICA, MDCT or both studies for assessment of coronary anatomy. We analyzed the coronary abnormalities and discriminations between ICA and MDCT.

Results: Thirty-three patients (20 males, 13 females) (mean age: 10.3 years) have coronary abnormalities including coronary artery aneurysm in Kawasaki disease $(\mathrm{KD})(\mathrm{n}=15)$, coronary artery fistula $(n=12)$, myocardial bridge $(n=2)$, anomalous left coronary artery from pulmonary artery (ALCAPA, $n=4$ ). Seventeen patients referred for ICA detected 5 coronary aneurysms (4 LCA, 1 RCA), 11 coronary artery fistulas and 2 ALCAPAs. Sixteen patients received MDCT study and 11 coronary artery aneurysms (7 LCA, 4 RCA), 2 myocardial bridges, 2 coronary artery fistulas and 2 ALCAPAs were assessed. Due to complex coronary anatomy, 5 patients with 3 large coronary aneurysms, 1 single LCA accompanied with coronary fistula, and 1 ALCAPA underwent both MDCT and ICA. Finally, 11 patients with indication of ICA spared invasive cardiac catheterization under accurate results of MDCT. Only one patient with small RCA aneurysm identified by ICA had different results of normal coronary anatomy examined by MDCT.

Conclusion: We conclude that MDCT is a good and accurate modality for assessment of congenital or acquired coronary abnormalities in children and adolescents. However, MDCT can't replace invasive cardiac catheterization and ICA due to lack of therapeutic role.

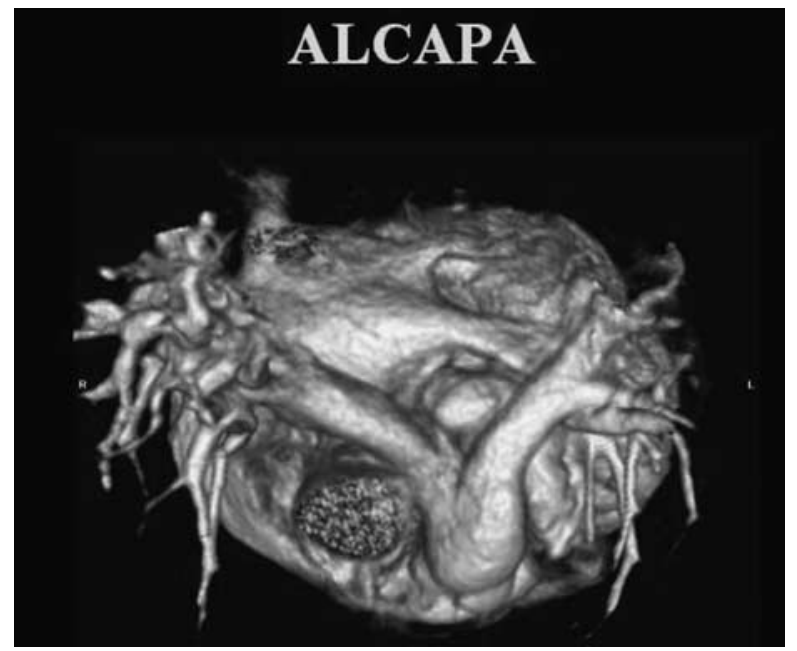

6085

The Relationship Between Cardiac Events and Diastolic Function in Adolescents With Severe Coronary Involvements After Kawasaki Disease

Hiroshi Kanamaru, Department of Pediatrics and Child Health, Nihon University School of Medicine, Japan

Kensuke Karasawa, Department of Pediatrics and Child Health, Nihon University School of Medicine, Japan

Takahiro Nakamura, Department of Pediatrics and Child Health, Nihon University School of Medicine, Japan
Junji Fukuhara, Department of Pediatrics and Child Health, Nihon University School of Medicine, Japan

Mamoru Ayusawa, Department of Pediatrics and Child Health, Nihon University School of Medicine, Japan

Naokata Sumitomo, Department of Pediatrics and Child Health, Nihon University School of Medicine, Japan

Tomoo Okada, Department of Pediatrics and Child Health,

Nihon University School of Medicine, Japan

Hideo Mugishima, Department of Pediatrics and Child Health,

Nihon University School of Medicine, Japan

Background: The purpose of this study was to clarify relations between cardiac events and diastolic function evaluated by quantitative gated SPECT (QGS) in adolescents with severe coronary involvements after Kawasaki disease (KD).

Methods: Sixteen patients with mean age 20.6 year-old who had severe coronary involvements after KD were performed QGS using Thallium-201 at rest and Tecnetium-99m tetrofosmin at erogometer stress. Ejection fraction (EF), peak ejection rate (PER), peak filling rate (PFR) and time to peak filling (TTPF) was evaluated by QGS (Philips Auto QUANT Ver.6.0) at rest, at stress and as the ratio of stress/rest. Severity score (SS) and extent score (ES) were evaluated by the myocardial perfusion image. We compared parameters between patients with cardiac events defined as the status resulted in coronary obstruction based on the findings of coronary angiogram and without events.

Results: There was no significant difference in SS, ES, EF, PER. $\mathrm{PFR}$ at stress resulted $2.58 \pm 0.44 \mathrm{EDV} / \mathrm{s}$ and $3.19 \pm 0.36 \mathrm{EDV} / \mathrm{s}$ $(p<0.05)$, but no significant difference in PFR at rest, in TTPF at rest and stress. The ratio of stress/rest resulted $1.03 \pm 0.19$ and $1.28 \pm 0.29$ in PFR $(p<0.05), 1.19 \pm 0.24$ and $0.92 \pm 0.08$ in TTPF $(\mathrm{p}<0.01)$.

Conclusion: Cardiac events that relate to coronary obstruction may cause diastolic dysfunction rather than decreased perfusion in adolescents with severe coronary involvements after KD.

6086

3-D Digital Rotational Angiography As A Diagnostic Aid In Congenital Heart Disease

Carlos E B Kapins, Escola Paulista de Medicina - UNIFESP - SP, Brazil

Ralph B Coutinho, Escola Paulista de Medicina - UNIFESP - SP,

Brazil

Fabiane B Barbosa, Escola Paulista de Medicina - UNIFESP - SP, Brazil

Celia M C Silva, Escola Paulista de Medicina - UNIFESP - SP,

Brazil

Antonio C C Carvalho, Escola Paulista de Medicina - UNIFESP - SP, Brazil

Joao Saba Chaker, Brazil

Introduction: Treatment and diagnosis advances in congenital heart disease are dependent on the improvement of anatomic imaging. Images obtained with 3D computerized tomography, magnetic resonance and echocardiography have helped, but they cannot determine precise haemodynamic parameters. 3D-rotational angiography (3DRA) is a new 3D computerized imaging method used in the catheterization laboratories, and has proved to be an important tool in neurologic and urologic procedures.

Objectives: Assess of the use and benefits of 3D-RA in congenital heart disease.

Material and Methods: A total of 34 congenital heart disease patients with mean age of $8,96 \pm 9,11$ years, and mean weight of $24,94 \pm 14,44 \mathrm{~kg}$ underwent hemodynamics study and subsequent 
3D-RA examination using Philips Allure FD10 equipment and low osmolarity contrast injection.

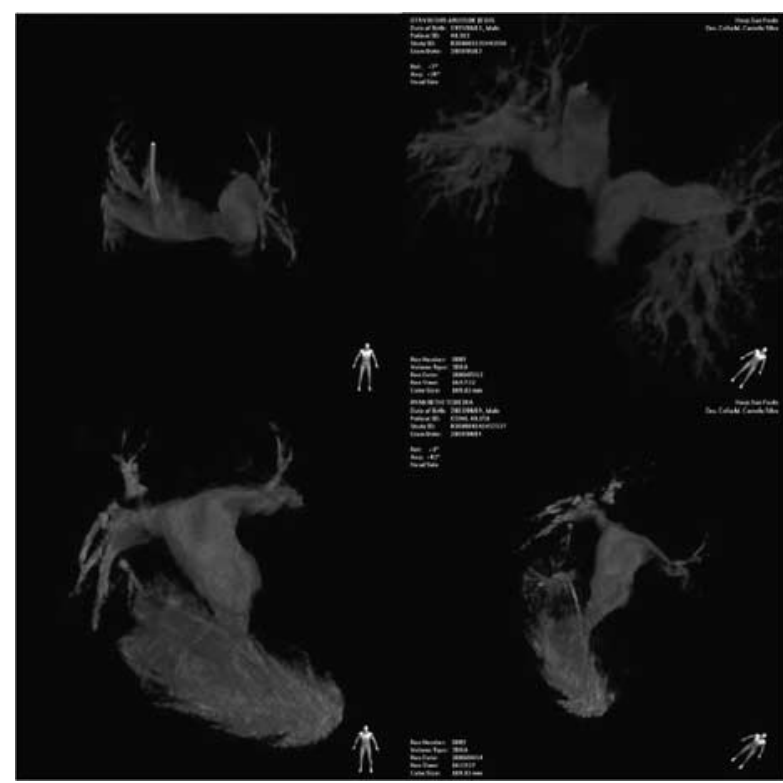

Results: A total of 43 3D-RA injections was performed with $80 \%$ of success in obtain a clear $3 \mathrm{D}$ image. Mean of contrast used in 3D-RA acquisition was $2,41 \pm 1,10 \mathrm{ml} / \mathrm{kg}$ and total exam contrast was $5,27 \pm 2,29 \mathrm{ml} / \mathrm{kg}$. Mean KV was $88,72 \pm 15,63$, $\mathrm{mAs}$ of $476 \pm 165$, and $\mathrm{ms}$ of 7,8 $\pm 1,4$. Air Kerna from 3D-RA injection was $40,48 \pm 22,36 \mathrm{mS}$ and Dose-Air Product was $501,47 \pm 1172,70 \mathrm{mS} . \mathrm{cm}^{2}$. There was no statistically significant difference between regular and 3D-RA injections.

Conclusions: Improvement is needed. Although larger contrast volume being used in 3D-RA than CT and regular haemodynamics study, less radiation was used compared with $\mathrm{CT}$. This new method could help in the treatment and diagnosis of congenital heart disease.

\section{7}

Remote analysis of 3 Dimensional Echocardiography Datasets against the 2D echocardiography for diagnosis of structural heart defects: A unique alternative

Varvara Karagkiozaki, Congenital cardiothoracic centre, Southampton University Hospital UK, United Kingdom

Saad Khoshhal, Paediatric Echocardiography Division, Prince Sultan Cardiac Center, Riyadh, Saudi Arabia, Saudi Arabia

Zek Lim, Congenital cardiothoracic centre, Southampton University Hospital UK, United Kingdom

Zaheer Ahmad, Congenital cardiothoracic centre, Southampton University Hospital UK, United Kingdom

Joseph Vettukattil, Congenital cardiothoracic centre, Southampton University Hospital UK, United Kingdom

Objective: The feasibility in diagnosing congenital heart diseases using 3 dimensional (3D) echocardiography datasets at a remote setting has yet not been investigated. We aimed to evaluate the accuracy and efficacy of 3D findings against the conventional twodimensional (2D) echocardiography and intra operative findings. Methods and Results: Thirty patients age ranged from 11 months to 18 years, underwent $2 \mathrm{D}$ and $3 \mathrm{D}$ echocardiography assessment at centre A (Kingdom of Saudi Arabia). 2D images were analysed while 3D full volume datasets were stored and exported for detailed analysis by independent experts in centre B (United
Kingdom). All data from baseline characteristics, diagnostic findings on 2D and 3D echocardiography, and intra operative findings were evaluated. The $3 \mathrm{D}$ echocardiographic findings were measured against the 2D findings to determine the feasibility and accuracy of using 3D datasets for remote analysis. Our results show that independant and remote analysis of 3D echocardiography provide similar diagnostic information to that of 2D. 3D echocardiography is especially superior in anatomic delineation of complex cardiac abnormalities, providing good spatial orientation and the dynamic morphology of valves. 3D echocardiography also supplements 2D in assessment of functional haemodynamics, through its ability to measure the effective orifice area, vena contracta and ventricular volumes. Conclusions: Remote analysis of congenital heart diseases by 3D echocardiography is highly feasible and accurate, complementing $2 \mathrm{D}$ echocardiography examinations in the diagnosis and management of complex cardiac defects. It aids in decision making and discussion between professionals at diffrent geographic locations in the managemet of complex congenital heart defects.

\section{8}

Segmental Contraction Pattern in infants with Hypoplastic Left Heart prior to Bidirectional Cavopulmonary Connection

Nee Scze Khoo, Stollery Children's Hospital, Canada

Edythe Tham, Canada

Akio Inage, Stollery Children's Hospital, Canada

Andrew Mackie, Stollery Children's Hospital, Canada

Jeffrey Smallhorn, Stollery Children's Hospital, Canada

Background: Mechanical dyssynchrony is described in hypoplastic left heart (HLH). However the "normal" contraction pattern at each stage of palliation is not well understood. This prospective study aims to describe the ventricular contraction pattern in $\mathrm{HLH}$ prior to bidirectional cavopulmonary connection (BCPC). Method: Fifteen infants (mean age $4.7 \pm 2.1 \mathrm{mths}$ ) prior to BCPC $($ HLHS $=11$, unbalanced AVSD $=4)$ with ECG, echocardiography and magnetic resonance imaging were analyzed. Longitudinal and circumferential global and segmental time to peak strain (tmS), time to peak strain rate (tmSR) and time to peak early systolic lengthening (tmEL) were measured from the 4 chamber and basal short axis view with speckle tracking imaging on commercially available software. All time variables were reported as a percentage of systole, and MRI volume and mass were indexed to body size. Standard deviation (SD) of tmS, tmSR and tmEL were calculated as a measure of synchrony.
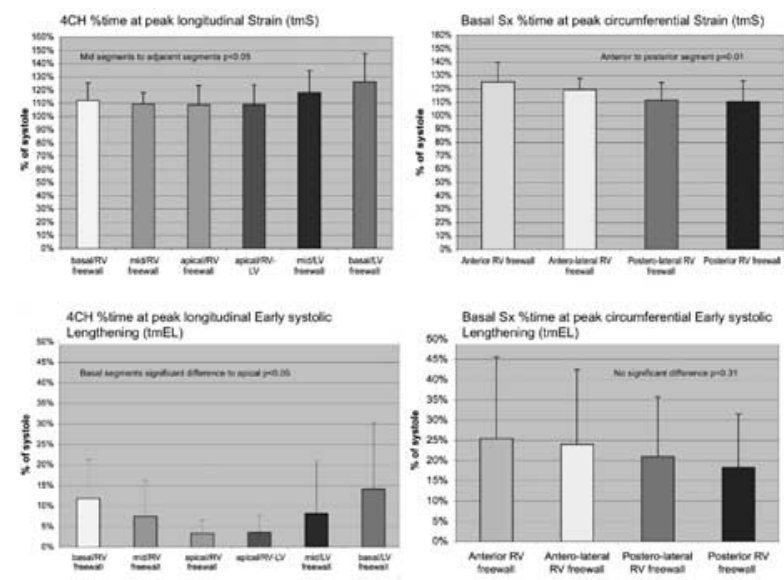
Results: Ventricular contraction pattern is displayed in Figure 1. Significant linear relationship exists between RV mass/volume ratio and SD of both longitudinal tmSR $(r=0.81, p<0.01)$ and tmS $(\mathrm{r}=0.52, \mathrm{p}<0.05)$. Mass/volume ratio correlated to mass $(\mathrm{r}=0.69, \mathrm{p}<0.01)$ but not to EDV. Systolic blood pressure was negatively related to stroke volume $(\mathrm{r}=-0.55, \mathrm{p}<0.05)$ but no other RV volumetric parameters. QRS duration (77 $\pm 13 \mathrm{~ms})$ had no significant correlations.

Conclusion: The sequence of systemic RV contraction is from apex to base, and at the basal level, from posterior to anterior. Longitudinal SD of tmSR and tmS may be a useful measure of dyssynchrony. Increased mass/volume ratio may reflect compensatory hypertrophy secondary to mechanical dyssynchrony in HLH.

\section{9}

The clinical utility of real-time three-dimensional echocardiography in patients with congenital heart disease: structural and volume study

Sumito Kimura, Kitasato univ. department of pediatrics, Japan Yayoi Nakahata, Kitasato univ. department of pediatrics, Japan Hisashi Andou, Kitasato univ. department of pediatrics, Japan Natsuko Oowada, Kitasato univ. department of pediatrics, Japan Masahiro Ishii, Kitasato univ. department of pediatrics, Japan

Aim: Evaluation of anatomical structure is important for management of the patients with congenital heart disease (CHD). It was usually required for an accurate measurement by 2 -dimentional echocardiography (2DE). Recently, transthoracic 3-dimentional echocardiography (3DE) is useful for understanding anatomical structure before surgical operation. We assessed anatomical structure in patients with congenital heart disease using real time 3DE. We provide the clinical utility of Left ventricular (LV) volume measurement using $3 \mathrm{D}$ data set.

Method: We performed transthoracic 3DE on 10 patients (2months to 5years old) with CHD include in complex CHD.

Transthoracic 3DE was performed using a Philips iE33 system with an X7-2 probe. The 3DE images were acquired full-volume $3 \mathrm{DE}$ data sets and reconstructed using an offline computer system (QLAB Ver.5.0, Philips Medical Systems Inc. Andover, MA, USA).

Result: There were 10 patients with CHD include ventricular septal defect(VSD), patent ductus arteriosus(PDA), Ebstein's anomaly, complete transposition of the great arteries (TGA), double outlet right ventricle (DORV), tetralogy of Fallot (TOF), coarctation of aorta complex, congenital mitral valve regurgitation, pulmonary atresia with VSD and supra mitral ring. 3DE described accurate images of various CHD. Valve disease and shunt were diagnosed by 3DE, which could not be diagnosed by 2DE. The LV volume was determined by 3DE tend to be correlation those measured by cine angiography $(r=0.88)$.

PDA, CoA and shunt were provided an accurate description as well as 3D computed tomography.

Conclusion: 3DE is useful of understanding the morphology and physiology of the CHD. 3DE is a valuable noninvasive method in clinical setting

\section{0}

Myocardial Deformation in Isolated Left Ventricular Noncompaction: a Speckle Tracking Echocardiographic Analysis

Carline Koh, Department of Paediatric and Adolescent Medicine, Princess Margaret Hospital, Hong Kong
WJ Hong, Shanghai Children's Medical Center, Shanghai JiaoTong University, China

SJ Wong, Department of Paediatric and Adolescent Medicine, Queen Mary Hospital, The University of Hong Kong, Hong Kong YF Cheung, Department of Paediatric and Adolescent Medicine, Queen Mary Hospital, The University of Hong Kong, Hong Kong

Objective: We sought to test the hypothesis that myocardial deformation is abnormal in children with isolated left ventricular (LV) noncompaction.

Methods: Longitudinal and circumferential myocardial deformation was determined using speckle tracking echocardiography in 9 patients aged $5.6 \pm 5.5$ years and compared with those of 9 agematched controls. Their LV ejection fraction and systolic dyssynchrony index (SDI) were assessed by 3-dimensional echocardiography. Relationships between myocardial deformation and LV dyssynchrony with LV ejection fraction were further determined in patients.

Results: Noncompaction involved both LV apex and mid lateral wall in 8 patients, and LV apex alone in 1. Compared with controls, patients had lower LV global longitudinal systolic strain $(p=0.007)$, systolic strain rate $(S R)(p=0.041)$, and early diastolic SR $(p<0.001)$, and global circumferential strain $(p=0.033)$, systolic SR $(p=0.17)$, and early diastolic SR $(\mathrm{p}=0.043)$, but similar RV longitudinal myocardial deformation parameters (all $\mathrm{p}>0.05$ ). Regionally, the longitudinal systolic strain of the basal $(\mathrm{p}=0.009)$, mid $(\mathrm{p}=0.005)$, and apical $(p=0.086)$ segments of LV lateral wall, and basal $(p=0.015)$, mid $(p=0.016)$ and apical $(p=0.13)$ septum was lower in patients than controls. Three (33\%) patients exhibited LV mechanical dyssynchrony (SDI $>4.9 \%$ ). In patients, LV ejection fraction correlated significantly with SDI $(r=-0.98, p<0.001)$, global LV longitudinal systolic strain $(r=0.70, p=0.035)$ and SR $(r=0.78, p=0.013)$, and global LV circumferential strain $(\mathrm{r}=0.92, \mathrm{p}<0.001)$ and SR $(\mathrm{r}=0.83, \mathrm{p}=0.006)$.

Conclusions: Abnormal myocardial deformation of the left ventricles extends beyond the noncompacted regions and contributes probably to global LV systolic dysfunction and mechanical dyssynchrony.

\section{1}

Relationship between central pulmonary arteries sizes and differential perfusion of the lungs in conotruncal heart defects: magnetic resonance imaging study

Oleksandr Kondrachuk, Ukrainian Children's Cardiac Center, Ukraine Tetyana Yalynska, Ukrainian Children's Cardiac Center, Ukraine Illya Yemets, Ukrainian Children's Cardiac Center, Ukraine Nadiya V Rokitska, Ukrainian Children's Cardiac Center, Ukraine

Objective: The purpose of this study was to determine the relationship between central (proximal branch) pulmonary arteries sizes and differential perfusion of the lungs in conotruncal heart defects using magnetic resonance imaging (MRI).

Methods: In total, 26 patients with conotruncal heart defects underwent cardiovascular MRI. The right/left lung perfusion ratio between 65:35 and 40:60 was considered as normal.

Results: The mean right/left lung perfusion ratio was 63:37, while the mean right/left pulmonary artery cross-sectional area ratio was 54:46 $(\mathrm{P}<0.05)$. Regurgitation fraction $(\mathrm{RF})$ was significantly greater in the left pulmonary artery (LPA) $(12.2 \pm 16.8 \%)$ than in the right pulmonary artery (RPA) $(3.2 \pm 5.5 \% ; \mathrm{P}=0.014)$. There was no significant correlation between right/left pulmonary artery cross-sectional area ratio and the right/left lung perfusion ratio $\left(\mathrm{R}^{2}=0.089, \mathrm{P}=0.139\right)$. 
The large difference $(>10 \%)$ between right/left pulmonary artery cross-sectional area ratio and the right/left lung perfusion ratio was found in $16(61.5 \%)$ cases. Abnormal right/left lung perfusion ratio was observed in $10(38.5 \%)$ of 26 patients. Central pulmonary arteries sizes predicted the asymmetry of lung perfusion only in 2 of 10 cases.

Conclusions: Morphological evaluation of the proximal branch pulmonary arteries cannot be used to predict differential perfusion of the lungs in patients with conotruncal heart defects. Asymmetric pulmonary perfusion is not related to the size of the proximal branch pulmonary arteries in most cases. The higher RF observed in the LPA compared with the RPA suggest that the pulmonary vascular resistance greatly dominates differential perfusion of the lungs over the anatomy of the central pulmonary arteries.

\section{2}

Our experience in evaluation extracardiac congenital vascular anomalies by computed tomography and magnetic-resonance angiography

Tetyana A Yalynska, Children's cardiac centre, Ukraine

Raad Tammo, Children's cardiac centre, Ukraine

Yevgeniya B Yershova, Children's cardiac centre, Ukraine

Nadiya V Rokitska, Ukrainian Children's Cardiac Center,

Ukraine

Olexandr S Kondrachuk, Children's cardiac centre, Ukraine

Objective: The objective of this study was to show the usefulness of CT and MRA for more accurate definition of various congenital thoracic vascular anomalies in paediatric patients.

Methods: 259 patients (from 1 day to 13 years) with suspected thoracic vascular anomalies underwent CT (203) and MRA (56). Results: CT and MRA accurately demonstrated total anomalous pulmonary venous connections in $24(9,3 \%)$ patients, partial anomalous pulmonary venous in $23(8,9 \%)$ patients, double aortic arch were in $13(5,5 \%)$ patients. Interrupted aortic arch type A was in 4 patients $(1,6 \%)$, type B in $1(0,4 \%)$ patient. 28 $(11,0 \%)$ patients had abnormalities in arch position: a right aortic arch with mirror image branching was present in 19 patients, a right aortic arch with an aberrant left subclavian artery in 9. A left aortic arch with aberrant right subclavian artery in $11(4,2 \%)$. Coarctation of aorta in $81(31,3 \%)$ patients. Truncus arteriosus was in $13(5,0 \%)$ patients, aortopulmonary window was in 4 $(1,5 \%)$ patients. $56(21,7 \%)$ patients had pulmonary atresia with major aortopulmonary collaterals arteries, pulmonary artery sling -1 patient $(0,4 \%)$. In $68 \%$ cases were combined congenital heart disease. Accurate diagnosis was obtained in 99,2\%.

Conclusion: CT and MRA provided an excellent evaluation of the vessel anatomy. We prefer to use MRA for the evaluation of aortic coarctation, TA, CT is suitable for the evaluation of anomalous pulmonary venous connections, pulmonary atresia with MAPCAs and other vascular anomalies. CT better demonstrated the spatial relationship of the vascular anomalies and nonvascular structures.

\section{3}

Peri operative transoesophageal echo guidance in the arterial switch-high impact and safe

Vikram Kudumula, Queen's Medical Centre, Nottingham, United Kingdom

Sujeev Mathur, Glenfield Hospital, Leicester, United Kingdom Justiaan Swanevelder, Glenfield Hospital, Leicester, United Kingdom Frances A Bu'Lock, Glenfield Hospital, Leicester, United Kingdom
Abdul K Duke, Glenfield Hospital, Leicester, United Kingdom Giles J Peek, Glenfield Hospital, Leicester, United Kingdom Richard K Firmin, Glenfield Hospital, Leicester, United Kingdom Ranjit Leanage, Glenfield Hospital, Leicester, United Kingdom

Aim: To assess the role of peri-operative transoesophageal echocardiography (TOE) during the arterial switch operation. Methods: Case note review of 22 babies undergoing arterial switch operation between April 2005 \& September 2008 (41 months). Correlation of pre and post-operative trans-thoracic echo (TTE) with TOE findings, impact of intra-operative TOE and complications with use of TOE were noted. Standard paediatric multi-plane TOE probes were used.

Results: 20/22 patients had a peri-operative TOE. Median weight was $3.69 \mathrm{Kg}$ (range $3.2-4.3 \mathrm{Kg}$ ). Mean age at surgery was 22 days. The preoperative TOE in two patients demonstrated a previously undetected origin of the circumflex from the right coronary artery. Cardiopulmonary bypass was reinstituted based on the initial post-operative study in 2 cases, where RV dyskinesia \& significant branch pulmonary stenosis were noted. One of these had a satisfactory RVOT reconstruction which was confirmed on TOE. The second patient continued to have elevated RV pressures after reconstruction of the RVOT although the postoperative TOE did not demonstrate an abnormality. Subsequent scans (TTE and TOE) demonstrated branch PA stenosis which was relieved by further surgery. One patient had mild branch pulmonary artery stenosis noted on TOE and confirmed on subsequent TTE's. The post-op TOE findings correlated well with subsequent TTE's in all cases. No problems were encountered in introducing the probe and there were no complications.

Conclusion: Peri-operative TOE can be a valuable and safe tool for the surgeon during the arterial switch. Branch PA stenosis can be detected but not excluded on TOE.

\section{6}

The Role of Transesophageal Echocardiography in Evaluating the Perioperative Tetralogy of Fallot in Pediatric Patients

Xiao-jing Ma, Pediatric Heart Center, Children's Hospital of Fudan University, Shanghai, China

Guo-ying Huang, Pediatric Heart Center, Children's Hospital of Fudan University, Shanghai, China

Bing Jia, Pediatric Heart Center, Children's Hospital of Fudan

University, Shanghai, China

Zhang-gen Chen, Pediatric Heart Center, Children's Hospital of Fudan University, Shanghai, China

Objective: To elucidate the role of transesophageal echocardiography (TEE) in evaluating tetralogy of Fallot (TOF) during perioperative period in pediatric patients.

Methods: One hundred and four consecutive cases of TOF aged $(2.7 \pm 3.4)$ years old were included. Intraoperative TEE and transthoracic echocardiography (TTE) before and after operation within 1 week were performed.

Results: (1) All cases were diagnosed as TOF by both TEE and TTE. According to surgical exploration, doubly-committed VSD was verified in 11 cases and subaortic VSD in 93 cases. The diagnostic accordance rate was $99.0 \%$ by TEE and $98.1 \%$ by TTE. Stenosis was mainly at infundibulum, pulmonary arteries and both sites in 40, 11 and 53 cases, respectively. There's no significant difference between TEE and TTE in defining the sites of stenosis (98.1\% vs. 96.2\%). (2) Residual shunt was found in 32 cases $(30.8 \%)$ by TEE. Instant intervention was performed in only 1 patient with shunt of $4 \mathrm{~mm}$.Residual obstruction was 
found in 18 cases $(17.3 \%)$ by TEE. Instant intervention was performed in 3 patients with pressure gradient over $36 \mathrm{mmHg}$. However, the obstruction of distant pulmonary arteries was not seen by TEE in 4 patients $(3.8 \%)$, but detected by TTE at followup. Pulmonary regurgitation was found in 70 cases $(67.3 \%)$ by TEE. Conclusions: TEE was reliable to diagnose TOF before operation and detect residual problems after surgical repair, except for the residual obstruction of distant pulmonary arteries.

\section{7}

Methodological Study on Volumetric Views of

Three-dimensional Echocardiography and Its

Applications in Diagnosing Tetralogy of Fallot

Xiao-jing Ma, Pediatric Heart Center, Children's Hospital of Fudan

University, Shanghai, China

Guo-ying Huang, Pediatric Heart Center, Children's Hospital of Fudan

University, Shanghai, China

Xiao-qin Liu, Pediatric Heart Center, Children's Hospital of Fudan

University, Shanghai, China

Xue-cun Liang, Pediatric Heart Center, Children's Hospital of Fudan

University, Shanghai, China

Bing Jia, Pediatric Heart Center, Children's Hospital of Fudan

University, Shanghai, China

Objective: To establish the methodology for volumetric views of three-dimensional echocardiography (3DE) and to test the feasibility of the examination protocol in tetralogy of Fallot (TOF), one of complex congenital heart disease.

Methods: The images were post-processed with online QLAB workstation after the 3DE datasets acquired. Cropping functional keys were used to display the volumetric views for the region of interest (ROI) from any angle. Based on Van Praagh sequential segmental approach, TOF was diagnosed in 36 cases aged $(2.6 \pm 3.9)$ years old. Two-dimensional echocardiography (2DE), $3 \mathrm{DE}$ and surgical repair were performed within one week.

Results: (1) The examination protocol for 3DE was established. The volumetric views of 3DE were displayed by consecutive orthogonal cropping, including coronal, saggital and horizontal directions. The views of ROI at non-orthogonal planes were also displayed by appropriate cropping. In addition, the typical volumetric views of cardiovascular structures were nominated. (2) TOF was diagnosed with volumetric views by consecutive orthogonal cropping and typical volumetric views of main malformations from 3DE datasets. Compared with surgical exploration, the accordance rate of $3 \mathrm{DE}$ in diagnosing TOF was $97.2 \%$. The rate was $97.2 \%$ and $94.4 \%$ in determining the positions of VSD and RVOTO, respectively, both were higher than 2DE. However, compared with 2DE, 3DE had limitations in detecting co-existed extracardiac anomalies and minute structures $(90.6 \%$ vs. $71.9 \%)$.

Conclusions: Volumetric views of $3 \mathrm{DE}$ is a promising imaging modelity in diagnosing TOF with normalized examination protocol, although improvements should be made to better display extracardiac anomalies and minute structures.

\author{
6098 \\ Assessment of Pulmonary Blood Supply in Children with \\ Tetralogy of Fallot by Atrial Volume Measured with \\ Three-dimensional Echocardiography \\ Xiao-jing Ma, Pediatric Heart Center, Children's Hospital of Fudan \\ University, Shanghai, China \\ Guo-ying Huang, Pediatric Heart Center, Children's Hospital of Fudan \\ University, Shanghai, China
}

Fang Liu, Pediatric Heart Center, Children's Hospital of Fudan University, Shanghai, China

Lin Wu, Pediatric Heart Center, Children's Hospital of Fudan University, Shanghai, China

Bing Jia, Pediatric Heart Center, Children's Hospital of Fudan University, Shanghai, China

Zhang-gen Chen, Pediatric Heart Center, Children's Hospital of Fudan University, Shanghai, China

Objective: To evaluate the feasibility of using atrial volume with three-dimensional echocardiography (3DE) in assessing pulmonary blood in children with tetralogy of Fallot (TOF).

Methods:Twenty cases of TOF aged $(3.2 \pm 4.7)$ years old were recruited, but plentiful shunts through atrial septum, severe valvular regurgitation and problems at atrial inlet or outlet were all excluded. Atrial volume was measured by $3 \mathrm{DE}$ and pulmonary blood was evaluated by angiography. Follow-up of atrial volume by 3DE was conducted within 1 week after surgical repair.

Results: (1) According to McGoon index derived from angiography, the cases were divided into 2 groups. Compared with the the group with McGoon index $>1.5(\mathrm{n}=11)$, the volume of left atrium $(\mathrm{LAV})$ was smaller in the group with McGoon index $<1.5(\mathrm{n}=9)$, $[(20.2 \pm 2.1) \mathrm{ml} / \mathrm{m} 2$ vs. $(16.7 \pm 1.2) \mathrm{ml} / \mathrm{m} 2, \mathrm{t}=4.414, \mathrm{p}=0.000]$ While, the volume of right atrium (RAV) was larger, [(23.5 \pm 2.2$)$ $\mathrm{ml} / \mathrm{m} 2$ vs. $(27.7 \pm 1.3) \mathrm{ml} / \mathrm{m} 2, \mathrm{t}=5.120, \mathrm{p}=0.000]$. (2) After surgical repair, LAV was increased from $(17.2 \pm 3.2) \mathrm{ml} / \mathrm{m} 2$ to $(22.4 \pm 6.2) \mathrm{ml} / \mathrm{m} 2,(\mathrm{t}=3.941, \mathrm{p}=0.001)$. However, no significant changes occurred with RAV, [(26.9 \pm 3.4$) \mathrm{ml} / \mathrm{m} 2$ vs. $(27.9 \pm 7.3) \mathrm{ml} / \mathrm{m} 2, \mathrm{t}=0.532, \mathrm{p}=0.602]$. The atrial volumetric ratio, defined as $\mathrm{LAV} / \mathrm{RAV}$, was increased from $(0.8 \pm 0.2)$ to $(1.0 \pm 0.3),(\mathrm{t}=2.935, \mathrm{p}=0.009)$

Conclusions: Atrial volume measured with 3DE is an index, which is able to reflect the pulmonary development in children with TOF. Smaller LAV and Larger RAV were observed in the group with less pulmonary blood supply. With the relief of obstruction after surgical repair, the increased LAV indicates the improved pulmonary blood supply.

\section{9}

Value of the magnetic resonance imaging for the early brain injury define in term neonates with transposition of great arteries on the preoperative study

Yevgeniya B Yershova, Children's cardiac centre, Ukraine

Tetyana A Yalynska, Children's cardiac centre, Ukraine

Andrey V Maksimenko, Children's cardiac centre, Ukraine

Illya N Yemets, Children's cardiac centre, Ukraine

Objective: Early detection of the brain injury in neonates with TGA in the pre-surgery is crucial for both anesthetic and surgical strategies. The objective was to define the ballon atrioseptostomy invasiveness degree, identify emerged lesions.

Methods: The total of 36 term newborns, mean age 6,4 days (range 1-17) with d-TGA underwent brain MRI before arterial switch. Group 1: 22 newborns had brain MRI after BAS within 4,5 days average, 6 of them underwent additional MRI on the planned surgery day. Group 2: 14 neonates had MRI before BAS and 8 of them underwent MRI also after BAS. None of the neonates had evident clinical neurological deficit after BAS.

Apparent diffusion coefficient(ADC) was calculated before BAS in 7 visually non-affected brain regions most sensitive to hypoxic-ischemic injury and additionally in ischemic damaged brain regions after BAS.

Results: In group 1-17 newborns $(77 \%, \mathrm{p}<0,001)$ and 3 newborns $(37,5 \%, p=0,062)$ in group 2 had brain injury after BAS. No brain 
injury in group 2 before BAS. White matter injury and focal strokes are the two types of lesions that often occur (53\% and 18\% accordingly, coexisting injury 29\%). ADC of the BAS caused ischemic stroke, regardless location, remained low within 0,4$0,5 \times 10-3 \mathrm{~mm} 2 / \mathrm{s}(\mathrm{p}<0,05)$ during the first $4-5$ days.

Conclusions: Acquire brain injury is caused by the BAS. The acute ischemia of the brain can be reversible so requires the surgery postponing and pharmacological neuroprotection. All newborns with TGA have to undergo brain MRI after BAS prior to arterial switch.

\section{0}

Peri-operative transoesophageal echo guidance in cardiac surgery on babies- safe and effective

Sujeev K Mathur, Glenfield Hospital, United Kingdom

Vikram Kudumula, Glenfield Hospital, United Kingdom

Frances A Bu'lock, Glenfield Hospital, United Kingdom

Magdi A Tofeig, Glenfield Hospital, United Kingdom

Justiaan Swanevelder, Glenfield Hospital, United Kingdom

Richard K Firmin, Glenfield Hospital, United Kingdom

Giles J Peek, Glenfield Hospital, United Kingdom

Ranit U Leanage, Glenfield Hospital, United Kingdom

Objective: To assess impact of peri- operative transoesophageal echocardiography (TOE) on surgery for congenital heart disease in patients weighing less than 10 kilograms.

Methods: Retrospective analysis of patient records over an 18 month period.

Results: 77 patients weighing less than $10 \mathrm{Kg}$, (Median 5.95, range $3.2-9.97 \mathrm{Kg}$ ) aged between $3 \& 826$ days (Mean age 251 days) had peri-operative TOE using standard multiplane paediatric TOE probes. The procedures included patch closure of VSD (20), repair of tetralogy of Fallot (TOF) (13), repair of complete atrio-ventricular septal defect (cAVSD) (10), arterial switch operation (ASO) (9) and Pulmonary Artery banding (4). Cardiopulmonary bypass was reinstituted in $5(6.4 \%)$ cases based on the initial postoperative TOE findings. These included 2 ASO's and one patient each with sub-aortic stenosis, repair of cAVSD with tetralogy of Fallot and repair of cAVSD. Other postoperative TOE findings included a small residual VSD in 2 cases, mild branch pulmonary artery stenosis in one patient after ASO and mild left AV valve regurgitation in one patient after cAVSD repair. All of these were corroborated on subsequent trans-thoracic echocardiography (TTE). 2 patients had mild residual lesions on TTE, not detected on the postoperative TOE. There were no complications resulting from peri-operative TOE during any of the surgical procedures.

Conclusion: Even in small infants, peri-operative TOE can safely be performed with modern equipment and is safe and effective in providing intra-operative surgical guidance.

\section{1}

Exercise echocardiography in adolescents with aortic coarctation

Thomas Möller, Vestfold Hospital Trust, Norway

Elisabeth Getz, Rikshospitalet University Hospital, Norway

Mona Knutsen, Rikshospitalet University Hospital, Norway

Erik Thaulow, Norway

Background: Detection of recoarctation is a main focus in followup programs for aortic coarctation and reinterventions are frequent. Both arm-leg-gradient in systolic blood pressure and exercise hypertension are known to be influenced by general changes in arterial reactivity and aortic stiffness and these are inappropriate tests to indicate recoarctation. Catheterization, MRI and CT-scan are technical demanding and expensive. We investigated if exercise echocardiography can give additional indications of recoarctation.

Methods: Thirty-two patients (male/female 19/13) age 15 to 17 years formerly treated for coarctation without clinical symptoms of recoarctation were examined by echocardiography at rest and during supine incremental cycling until exhaustion (WHOprotocol).

Results: Mean of systolic peak velocity in the descending aorta (VDAo) at rest was $2.2 \mathrm{~m} / \mathrm{s}$ (SD 0.52). Mean of maximal VDAo during exercise was $3.5 \mathrm{~m} / \mathrm{s}$ (SD 0.78). Mean of maximal systolic blood pressure during exercise was $211 \mathrm{mmHg}$ (SD 34.3). Maximal VDAo was positively correlated to maximal systolic blood pressure $(\mathrm{r}=0.533, \mathrm{p}=0.002)$. Nineteen individuals had a positive arm-leg-gradient $(>0 \mathrm{mmHg})$. All individuals with maximal VDAo $\geqslant 4.0 \mathrm{~m} / \mathrm{s} \quad(\mathrm{n}=8)$ had a positive arm-leggradient and six (75\%) had prominent diastolic flow during peak exercise. Of 24 individuals with maximal VDAo $<4.0 \mathrm{~m} / \mathrm{s} 11$ (46\%) had a positive arm-leg-gradient and six (25\%) had prominent diastolic flow.

Conclusion: Exercise echocardiography gives additional data in adolescent patients treated for aortic coarctation. Exercise echocardiography may facilitate discrimination between general vascular changes and recoarctation when a positive arm-leggradient of systolic blood pressure at rest is found.
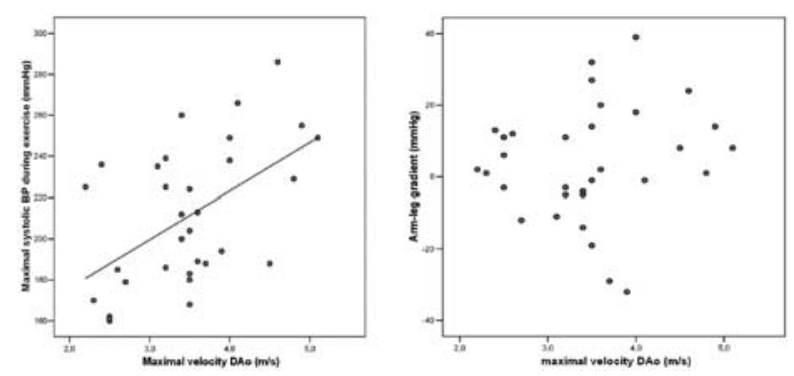

6102

ALCAPA single centre experience

Nitha Naqvi, Royal Brompton Hospital, United Kingdom

Objectives: Retrospective review of single centre experience of ALCAPA cases.

Method: Retrospective case note review and echocardiographic images review.

Results:

\section{3}

Improved synchronicity of left ventricular contraction after transcatheter ASD closure

Masaki Nii, Shizuoka Children's Hospital, Japan

Kennichi Kurosaki, National Cardiovascular Center, Japan, Japan

Masataka Kitano, National Cardiovascular Center, Japan, Japan

Satoshi Yazaki, National Cardiovascular Center, Japan, Japan

Shigeyuki Echigo, National Cardiovascular Center, Japan, Japan

Background: ASD associated with significant right ventricular (RV) volume overload causes left ventricular (LV) dysfunction, manifested by decreased peak LV systolic pressure, peak dp/dt, 
prolonged Tau and decreased filling rate. Marked LV deformity in ASD patients causes heterogeneous wall stress, which may lead to heterogenecity of wall strain and dyssynchronicity. Furthermore, heterogenecity of LV wall stress may impair LV torsion. Objectives of this study are: 1) to evaluate the wall strain of all LV-segments before and after trans-catheter ASD closure (ASDCL), 2) to evaluate synchronicity of LV wall, 3) to evaluate the change of LV torsion.

Methods: Twenty ASD patients (Age 17.2 years (6.9-57.2) (median (range)) were included. All patients had successful ASDCL. Echo data were obtained before, $48 \mathrm{Hr}$ after and 3 months after ASDCL. Radial and circumferential strains of 18 segments of LV were measured in short-axis at base, mid and apex. Peak strain and torsion were measured. Synchronicity was assessed as a standard deviation of time from QRS to peak strain of all segments.

Results: 1) Apical circumferential strains were significantly improved $(\mathrm{P}<0.05)$. However, ejection fraction did not change. 2) Synchronicity was significantly improved after ASDCL: pre: $51.4 \mathrm{~ms}(34.2-78.5) ; 48 \mathrm{Hr} 39.9 \mathrm{~ms}(30.9-60.3)(\mathrm{P}<0.05) ; 3 \mathrm{M}$ $40.4 \mathrm{~ms}(22.3-57.8)(\mathrm{P}<0.01)$. 3) Torsion did not change: pre 11.0 degree (2.3-15.6); 48 Hr 12.0 (5.3-16.9); 10.9 (2.2-17.6). Conclusion: Although the ejection fraction did not change, apical circumferential strains and synchronicity of LV were improved, suggesting improved efficiency of LV contraction by removal of RV volume overload.

6104

A novel three-dimensional endocardial surface image in congenital heart defects

Hisayuki Oda, Pediatric cardiology division, Fukuoka Children's

Hospital, Japan

Hiroya Ushinohama, Pediatric cardiology division, Fukuoka Children's

Hospital, Japan

Shirou Ishikawa, Pediatric cardiology division, Fukuoka Children's

Hospital, Japan

Yuichi Ishikawa, Pediatric cardiology division, Fukuoka Children's

Hospital, Japan

Makoto Nakamura, Pediatric cardiology division, Fukuoka Children's

Hospital, Japan

Konichi Sagawa, Pediatric cardiology division, Fukuoka Children's

Hospital, Japan

Toshihide Nakano, Cardiac surgery division, Fukuoka Children's

Hospital, Japan

Hideaki Kado, Cardiac surgery division, Fukuoka Children's Hospital

Background: Three-dimensional (3D) images with CT/MRI only show us the extra-cardiac structure. We addressed to slice novel 3D endocardial surface images in congenital heart defects and to compare them with surgical findings.

Methods and Results: First, patients with heart defects were imaged with a 16-slice multidetector CT scanner. Helical CT scanning was performed from the head to the diaphragm after intravenous injection of contrast media. Using the image data (DICOM 3) burned onto a CD directly at the scanner, postprocessing of the axial CT images was performed with an electro-anatomic mapping system (CartoTMXP, Biosense Webster Inc). Image segmentation, the extraction of the $3 \mathrm{D}$ anatomy of individual structures from the cardiac CT images, was performed with an image segmentation and integration module (CartoMergeTM, Biosense Webster Inc). The chambers and vessels of interest were identified with semi-automatic process. Cutting and punching tools were available for fine tuning. With the clipping plane function, we could slice images in any plane to visualize the endocardial surface. In 1 year-old girl with DORV after PA banding, the 3D endocardial surface images from the RV clearly showed a position and shape of non-committed VSD. And in 9 month-old boy with DORV and PS, the 3D endocardial surface images from the LV suggested small apical muscular VSD. The findings of two patients were entirely consistent with the surgical findings.

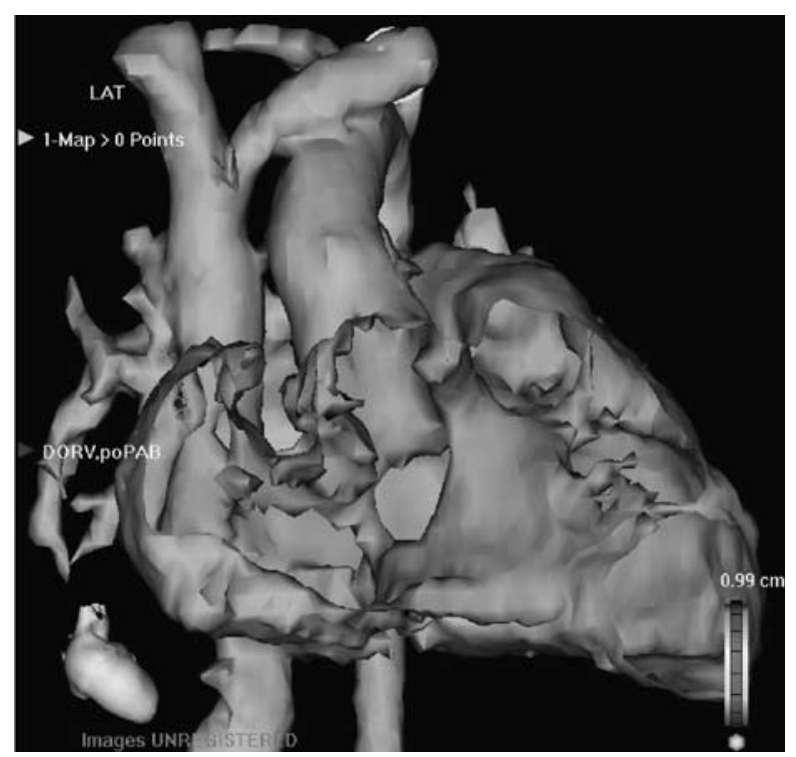

Conclusion: The three-dimensional endocardial surface images using the CartoTMXP system and CartoMergeTM Image Integration Module have a potential of diagnostic tool for congenital heart defects.

\section{5}

Assessment of left ventricular cardiac dysfunction by tissue Doppler imaging in the time-course of Kawasaki disease

Kenichi Okumura, department of pediatrics, Osaka Medical College, Takatsuki City, Osaka, Japan

Kanta Kishi, Japan

Yasuhiko Mori, Department of pediatrics, Osaka Medical College, Takatsuki City, Osaka, Japan

Naohisa Kawamura, Department of pediatrics, Osaka Rosai Hospital, Sakai City, Osaka, Japan

Hiroshi Tamai, Department of pediatrics, Osaka Medical College, Takatsuki City, Osaka, Japan

Background: In Kawasaki disease (KD), myocardial inflammation has been shown to cause aberrations in cardiac function. Several recent studies have discussed the usefulness of tissue Doppler imaging (TDI) to evaluate left ventricular function. In this study, we used TDI to evaluate disturbance of myocardial function in the time-course of KD.

Subjects: The KD patients group consisted of 59 children, aged 0.3-8.0 years. Age-matched control group consisted of 30 children.

Methods: Echocardiographic examination was performed in the acute phase (before treatment for KD), the subacute phase (after treatment for $\mathrm{KD}$ ), the convalescent phase and the late phase (3-14 months after KD onset). From the apical 4-chamber view, sample volume was placed at the lateral corner of the mitral annulus. The following measurements were obtained from the 
TDI recordings: peak systolic velocity (Sm), early (Em) and late diastolic velocities, isovolumic contraction time, ejection time, isovolumic relaxation time. Mitral inflow velocity (E)/Em and Tei index (TI) were also calculated.

Results: (1) E/Em in the acute phase was significantly more increased than those in control and the other phase. (2) Sm in the subacute and convalescent phase were significantly more decreased than those in control and the late phase. (3) TI in the acute, subacute and convalescent phase were significantly more increased than those in control and the late phase.

Conclusion: Latent abnormal tissue Doppler profiles, possibly reflecting systolic and diastolic dysfunction have been noted in KD patients. These findings suggest that TDI may be useful method for the detection of impaired myocardium in $\mathrm{KD}$ patients.

\section{6 \\ Assessment of right ventricular function with tissue Doppler imaging in children after tetralogy of Fallot total correction \\ Radoslaw Pietrzak, Department of Children's Cardiology and General Pediatrics in Medical University of Warsaw, Poland \\ Bozena Werner, Department of Children's Cardiology and General Pediatrics in Medical University of Warsaw, Poland \\ Piotr Perdeus, Department of Children's Cardiology and General \\ Pediatrics in Medical University of Warsaw, Poland}

The aim of the study was to asses right ventricular function by tissue Doppler imaging (TDI) in asymptomatic patients with repaired tetralogy of Fallot.

Methods: The study group consisted of 20 children 7-18 years after surgical treatment, in whom echocardiographic parameters and TDI values were analyzed. Patients were divided into two groups according to the level of pulmonary regurgitation (PR). The group 1. consisted of 9 children with mild/moderate PR, the group 2 consisted of 11 patients with severe PR.

Results: Patients in the group 1. had significantly higher systolic velocity, early diastolic velocity and late diastolic velocity comparing to the patients from the group 2. $(14,1+/-1,3$ vs $8,9+/-1,46 ; 13,8+/-0,9$ vs $8,7+/-1,1 ; 15,3+/-1,2$ vs $10,3+/-1,3 \mathrm{~cm} / \mathrm{s}$; respectively, $\mathrm{p}<0,05)$. Isovolumic relaxation time and isovolumic contraction time in the group 2 were significantly longer than those in children in the group 1 $(85,6+/-15$ vs $57,6+/-12 ; 110+/-34$ vs $78+/-19 \mathrm{~ms}$, respectively; $p<0,05)$. Duration of ejection time did not differ between both groups. Tei index was significantly greater in children of the group 2. than in patients of the group 1. $(0.73+$ / -0.09 vs $0.54+/-1.0$, respectively, $\mathrm{p}<0.05)$.

Conclusion: Tissue Doppler imaging can be useful diagnostic tool of right ventricular function in children after tetralogy of Fallot correction. Systolic velocity, early diastolic velocity, late diastolic velocity are significantly higher and Tei index is lower in children with mild/moderate pulmonary regurgitation than in patients with severe pulmonary regurgitation, which can indicate right ventricular function disturbances.

\section{7}

Echocardiography in Ligation of Coronary Fistulae in Pulmonary Atresia with Intact Ventricular Septum Lee A Pyles, University of Minnesota Department of Pediatrics, United States

Shanthi Sivanandam, University of Minnesota Department of Pediatrics, United States
Edward C Martin, CentraCare Health System, United States Julia Steinberger, University of Minnesota Department of Pediatrics, United States

John L Bass, University of Minnesota Department of Pediatrics, United States

Adriana L Gittenberger-De Groot, University of Leyden, The

Netherlands

John E Foker, University of Minnesota Department of Surgery,

United States

Objectives: Coronary artery to right ventricular (RV) fistulous connections are often thought to preclude decompression of the small hypertensive RV in pulmonary atresia with intact ventricular septum (PAIVS). With RV decompression, fistulae may cause myocardial steal, hypoxic myocardial perfusion, and wall motion abnormalities (WMA). We report the first series of echo assessment of coronary fistula ligation, a critical step to hypoplastic RV decompression to allow post-natal RV growth and 2 ventricle repair.

Methods: Coronary fistulae were identified with transthoracic echo plus angiocardiographic confirmation. Intraoperatively, fistulae were localized and ligation assessed with TE and high frequency epicardial surface echo. Follow-up TE and transthoracic echo assessed completeness of fistulae ligation and WMAs.

Results: Five consecutive patients with severe PAIVS and multiple fistulae were studied. 27 fistulous connections were identified pre-op (mean 5.4/patient). Significance was judged by 1) enlargement of the right or left coronary, 2) size of fistulae, 3) retrograde coronary flow. In 3 of 5 , right and left coronaries were enlarged. No interruptions were seen but stenoses were observed on angio. Coronary to aortic root connection was best seen with echo flow. Ligation of 25/27 fistulae produced no WMAs. All had RV decompression. Two small fistulae not ligated were well tolerated. All 5 patients were discharged.

Conclusions: Pre and intra operative echo provided essential surgical guidance and prompt evaluation of fistula ligation. Significance of the fistulae was determined by echo and angio agreement on coronary size, dilatation, retrograde/bidirectional flow. Coronary fistula analysis is important to RV rehabilitation in PAIVS treatment.

\section{8}

Ventricular Septal Defects in Children in Fiji - an Echocardiographic Study

Malcolm E Richardson, Women's and Children's Hospital, Adelaide, Australia

Kerrie A Richardson, Women's and Children's Hospital, Adelaide, Australia

Joseph Kado, Colonial War Memorial Hospital, Suva, Fiji

Objective: To describe the spectrum of ventricular septal defects seen in children in Fiji.

Methods: We reviewed the clinical records and echocardiograms of children attending a screening clinic that was part of an annual visiting cardiac surgical team.

Results: A total of 519 children were examined between 2002 and 2008. Ages ranged from 1 day to18 years. Of the 136 with ventricular septal defects, 108 had perimembranous defects including 4 with an additional muscular defect, 24 had doubly committed juxta-arterial defects including 2 with an additional muscular defect and 4 had muscular defects, including 1 with multiple defects. Outlet extension was seen in $41 \%$ of perimembranous defects. Aortic valve prolapse was seen in $32 \%$ of perimembranous defects and in $67 \%$ of juxta-arterial defects. 
Aortic regurgitation occurred in $24 \%$ of all ventricular septal defects; $22 \%$ of these were graded as moderate or severe. We frequently observed a distorted right coronary cusp bulging into and partially closing a large defect. In the most extreme cases, the aorta appeared to be displaced anteriorly through the defect. Conclusion: Doubly committed juxta-arterial and perimembranous ventricular septal defects with outlet extension are common in this group. Aortic valve prolapse and regurgitation frequently complicate these lesions. It is especially important to consider the aortic valve in surgical decision making in this population.

\section{9}

Tissue Doppler Imaging in Evaluating Transposition of Great Arteries (TGA)

S Ramakrishnan, All India Institute of Medical Sciences, New Delhi, India

Indra Kuladhipati, All India Institute of Medical Sciences, New Delhi, India

Sandeep Seth, All India Institute of Medical Sciences, New Delhi, India Rajnish Juneja, All India Institute of Medical Sciences, New Delhi, India

Shyam S Kothari, All India Institute of Medical Sciences, New Delhi, India

Anita Saxena, All India Institute of Medical Sciences, New Delhi, India Vinay K Bahl, India

Background: Tissue Doppler and Strain rate imaging are relatively load independent. We studied the usefulness of Tissue Doppler and Strain rate imaging in detecting temporal changes in regional and global function of left and right ventricle in transposition of great arteries (TGA).

Methods: Standard echocardiography including Doppler studies was performed with 5 or $10 \mathrm{MHz}$ probe (GE Vivid 7 dimension Machine). The images were analyzed offline by a customized software package (Echo Pac PC,GE Vivid Ultrasound). Intra and inter-observer variability was assessed to be within standard limits. The correlation with surgical outcome will be presented. Results: A total of 35 patients (aged 3-123 days) were included in this study. Twelve LV and 2 segments of RV in each patients were analyzed for peak systolic velocity, systolic strain rate and strain. There were no significant intergroup differences in of any of these parameters in patients with or without VSD, or in the groups with regressed or prepared LV. Paradoxically, peak systolic velocity and peak strain rates were statistically significantly higher in patients older than 28 days.

Conclusion: Conventionally classified as regressed LV do not show reduced tissue velocities and strain rates. Peak systolic velocity and peak strain are higher in patients more than 28 days. These findings suggest that possibly intrinsic contractility of the LV myocardium is preserved in a majority of patients with TGA.

\section{0}

Role of F-18 Fluorodeoxyglucose Positron Emission Tomography in Children with Active Rheumatic Carditis and Chronic Rheumatic Heart Disease

CM Nagesh, All India Institute of Medical Sciences, New Delhi, India, India

Anita Saxena, All India Institute of Medical Sciences, New Delhi, India, India

Chetan Patel, All India Institute of Medical Sciences, New Delhi, India, India

M Nadig, All India Institute of Medical Sciences, New Delhi, India, India
Arun Malhotra, All India Institute of Medical Sciences, New Delhi, India, India

Objective: We report use of F-18 Fluorodeoxyglucose Positron Emission Tomography (FDG-PET) scan in children with rheumatic heart disease (RHD), to see if it helps in diagnosis of carditis, since FDG, a glucose analogue is taken up by the inflammatory cells. Methods: 36 children (age 6-17 years, mean 10.2 years) with significant rheumatic mitral regurgitation (MR) underwent FDGPET scanning of the heart after fasting for 6 hours. They were divided into two groups based on Jones criteria - active carditis (AC) and non active. 12 age and sex matched children with normal hearts, undergoing FDG-PET scan for other reasons, served as controls. The primary end point was to compare the pattern of FDG-PET scanning in AC, chronic RHD and in controls.

Results: AC was present in 19/36 cases, 17 were not active. Diffuse FDG uptake was seen in 14/19 cases with AC, all 17 non active cases and 12/12 controls. 5 cases with AC did not show any uptake. Therefore, no uptake pattern on FDG PET scan had a sensitivity of $26 \%$ and positive predictive value of $100 \%$ for diagnosing AC. Conclusion: FDG PET scan in children shows diffuse myocardial uptake in cases with RHD, similar to that seen in controls. In 1/ 4th of AC cases, no myocardial uptake is seen. This finding may be related to the inability of myocytes to shift from fatty acids to glucose substrate for their metabolism. F -18 FDG PET may help to diagnose $\mathrm{AC}$ in equivocal situations, since its positive predictive value is high.

\section{1}

Novel applications of non-ECG-gated multi-slice CT angiography in the diagnosis of complicated congenital heart disease

Isao Shiraishi, National Cardiovascular Center (Osaka), Kyoto Prefectural University of Medicine, Japan

Objectives: To minimize radiation exposure during a multi-slice computed tomography (MSCT) examination in children with congenital heart disease (CHD), we assessed diagnostic value and the various applications of MSCT without electrocardiography (ECG)-gated image acquisition, including making plastic replicas by stereolithography.

Methods: Between 1997 and 2008, we performed 885 MSCT examinations in children with CHD. 3D images were reconstructed with differential color-coding of the arteries and veins. The vessel diameter was measured with $\mathrm{CT}$ attenuation profiles. Ventricular volumetry was initially validated by phantom experiment and followed by comparison with that of cineangiography. Simulation for surgical operation was performed to evaluate the possibility of intracardiac rerouting in patients with double outlet right ventricle (DORV). Stereolithographic biomodels of CHDs were manufactured with MSCT imaging. Results: Differential color-coding was able to provide accurate anatomical structure of CHD. The vessel diameter in MSCT well correlated with that obtained by angiography $(\mathrm{r} 2=0.86)$. Based on the phantom experiments, ventricular volume was studied in patients with heart rate $>120$ beat/min. The ventricular volume in MSCT well correlated with taht obtained by cine-angiography $(\mathrm{Y}=0.77 \mathrm{X}, \mathrm{r} 2=0.98$ for $\mathrm{RV} ; \mathrm{Y}=0.90 \mathrm{X}, \mathrm{r} 2=0.94$, for $\mathrm{LV})$. Simulative operation provided appropriate indication for biventricular repair in patients with DORV. Stereolithographic biomodels made of the both solid epoxy and flexible urethane were successfully manufactured by using multi-slice CT imaging, which reproduced fine anatomical structure and allowed simulation surgery. 
Conclusions: Non-ECG-gated MSCT angiography is applicable for precise anatomical diagnosis, ventricular volumetric study, and simulation surgery, and making stereolithography biomodels in children with CHD.

6112

Estimation of abnormalities of the left ventricle diastolic function in children with juvenile idiopathic arthritis Krzysztof K Truszkowski, Children's Cardiology Department, 2nd Chair of Pediatrics Medical University of Lodz, Poland

Katarzyna Niewiadomska-Jarosik, Children's Cardiology Department, 2nd Chair of Pediatrics Medical University of Lodz, Poland

Elzbieta Smolewska, Children's Cardiology Department, 2nd Chair of Pediatrics Medical University of Lodz, Poland

Malgorzata Biernacka-Zielinska, Children's Cardiology Department, 2nd Chair of Pediatrics Medical University of Lodz, Poland

Henryka Brozik, Children's Cardiology Department, 2nd Chair of Pediatrics Medical University of Lodz, Poland

Jerzy A Stanczyk, Children's Cardiology Department, 2nd Chair of Pediatrics Medical University of Lodz, Poland

One of the most prevalent systemic autoimmune connective tissue diseases in children is juvenile idiopathic arthritis (JIA). In children with JIA, the heart is involved in $30-80 \%$ of cases, with clinical manifestation in 3-9\%.

The aim of the study was to check if patients with JIA have the left ventricle (LV) diastolic dysfunction.

The study involved 50 children with JIA. The control group consisted of 20 healthy children. Each patient had echocardiography examination; the systolic and diastolic function of left ventricle were analyzed.

Statistically significant differences were noted between E/A ratio, $(1.456+/-0.353$, and $1.864+/-0.385$ in the studied and in the control group, respectively). MPI was approximately $0.476+/-0.13$ and $0.411+/-0.075$ in the groups. Mean AEF value in the groups was $12.09+/-6.04 \mathrm{kdyn}$ and $6.17+/$ $-1.9 \mathrm{kdyn}$. Mean VpMV was $0.7+/-0.14 \mathrm{~m} / \mathrm{s}$, and $0.81+/$ $-0.13 \mathrm{~m} / \mathrm{s}$ in the groups, respectively. All values also differed in a statistically significant way. In the studied group, there was no LV systolic function impairment observed.

Conclusions:

1. In evaluation of LV diastolic dysfunction in patients with JIA, use of several parameters is recommended. Total myocardial performance index (MPI), and velocity of early mitral inflow wave propagation (VpMV) seem to be the most valuable, due to their independence from other factors.

2. Atrial ejection force (AEF) is a useful parameter in evaluation of left ventricle diastolic function.

3. The presence of LV diastolic dysfunction did not correlate with disease duration.

\section{3}

Agreement of left ventricular mass in steady state free precession and delayed enhancement MR images: Implications for quantification of fibrosis in congenital and ischemic heart disease

Sigurdur S Stephensen, Department of Clinical Physiology and Department of Pediatric Cardiology, Lund University Hospital, Lund, Sweden., Sweden

Marcus Carlsson, Department of Clinical Physiology, Lund University Hospital, Lund, Sweden., Sweden

Peter Munkhammar, Department of Pediatric Cardiology, Lund

University Hospital, Lund, Sweden., Sweden
Håkan Arheden, Department of Clinical Physiology, Lund University Hospital, Lund, Sweden., Sweden

Objective: MRI is being established as the reference method for quantification of myocardial infarction using delayed contrast enhanced imaging (DE). Left ventricular mass (LVM) is used when expressing infarct as percentage of the left ventricle (LV). However, quantification of LVM is interchangeably carried out in steady state free precession cine (SSFP) and DE MRI. These techniques may yield different LVM. The aim of the present study was to compare LVM determined by SSFP and DE MRI. Methods: SSFP and DE images were acquired in 60 patients $(20$ with infarction, 20 without infarction and 20 children). Ex vivo MRI was used as a reference method for LVM in 19 pigs and compared to in vivo SSFP and DE.

Results: LVM in humans measured with SSFP $(126.2 \pm 52.1 \mathrm{~g})$ was significantly higher compared to DE $(120.5 \pm 50.6 \mathrm{~g})$ and bias was $5.0 \% \pm 6.7 \%$. LVM in pigs was significantly higher on SSFP $(100.9 \pm 15.9 \mathrm{~g})$ than on DE $(93.6 \pm 14.5 \mathrm{~g})$ and bias was $7.5 \% \pm 6.9 \%$. Bias for SSFP and DE images compared to ex vivo LVM was $0.2 \% \pm 9.0 \%$ and $-7.7 \% \pm 8.5 \%$ respectively.

Conclusions: LVM was higher when measured with SSFP compared to DE. Thus, the percentage infarction of the LV will differ whether SSFP or DE are used to determine LVM. Opposed to DE there was no significant difference between LVM on SSFP and ex vivo, suggesting that SSFP is more accurate for quantification of LVM.

6114

A New Scoring System for the Prediction of Spontaneous Closure of Perimembranous Ventricular Septal Defect in Children

Jing Sun, Department of Pediatric Cardiology, Shanghai Children's

Medical Center, China

Kun Sun, Department of Pediatric Cardiology, Shanghai Children's

Medical Center, China

Lanping Wu, Department of Pediatric Cardiology, Shanghai Children's Medical Center, China

Liping Yao, Department of Pediatric Cardiology, Shanghai Children's Medical Center, China

Xiao Tan, Department of Pediatric Cardiology, Shanghai Children's

Medical Center, China

Yuqi Zhang, Department of Pediatric Cardiology, Shanghai Children's Medical Center, China

Juan Qian, Department of Pediatric Cardiology, Shanghai Children's

Medical Center, China

Zhifang Zhang, Department of Pediatric Cardiology, Shanghai

Children's Medical Center, China

Objective: Perimembranous ventricular septal defect (PMVSD) has a relative high rate of spontaneous closure, but there are still a lot of patients with PMVSD could not get closed spontaneously. Our aim was to develop a new scoring system for the prediction of spontaneous closure of PMVSD.

Methods: Eleven variables of 1873 well followed patients diagnosed with PMVSD were analyzed with logistic regression for their effects on the rate of spontaneous closure. The variables confirmed to significantly contribute to spontaneous closure of PMVSD were selected and their coefficients were calculated to establish the scoring system for predicting spontaneous closure of PMVSD. The chances of spontaneous closure of 406 cases diagnosed with PMVSD were predicted prospectively using the scoring system to evaluate its feasibility.

Results: Seven variables (first contact age, defect size, extending orientation, shunt flow, aneurysm of the ventricular membranes 
septum, complications, left ventricular diastolic dimension) were found to have significant effects on spontaneous closure of PMVSD according to multivariate analysis and selected to establish a new scoring system based on their coefficients. Patients with higher scores seemed to have higher spontaneous closure rate in the new scoring system. For the 406 cases, the accuracy of the scoring system was $78.6 \%$.

Conclusions: The probability of spontaneous closure of PMVSD could be predicted by this scoring system. Patients with low probability could be informed earlier and scheduled for intervention appropriately. However, patients having high probability could be followed intensively waiting for spontaneous closure and the excessive operations could be avoided.

\section{5}

Real Time Three-dimensional Echocardiography Provides New Insight into the Mechanisms of Left Atrioventricular Valve Regurgitation in Patients with Atrioventricular Septal defect

Ken Takahashi, Dept of Pediatrics, University of Alberta and Stollery Children's Hospital, Edmonton, AB, Canada, Canada Ghassan Al-Naami, Dept of Pediatrics, University of Alberta and Stollery Children's Hospital, Edmonton, AB, Canada, Canada Akio Inage, University of Alberta and Stollery Children's Hospital, Canada

Andrew S Mackie, Dept of Pediatrics, University of Alberta and Stollery Children's Hospital, Edmonton, AB, Canada, Canada

Ivan M Rebeyka, University of Alberta and Stollery Children's Hospital, Canada

David Ross, Dept of Surgery, University of Alberta and Stollery Children's Hospital, Edmonton, AB, Canada

Richard Thompson, Dept of Biomedical Engineering, University of Alberta, Edmonton, AB, Canada

Jeffrey F Smallhorn, Dept of Pediatrics, University of Alberta and Stollery Children's Hospital, Edmonton, AB, Canada

Background: Although left atrioventricular valve (LAVV) regurgitation in patients with atrioventricular septal defect (AVSD) has an important negative impact on outcome, the mechanisms are still unclear.

Methods: Real time 3-dimensional (3D) echocardiography was performed in 59 patients with AVSD (aged 3.3-32.6 years) (28 partitioned and 31 common AVV orifice) and 40 normal controls (aged 3.4-31.5 years). 30 points around the LAVV annulus, the tip of the papillary muscles (PM) and the surfaces of the LAVV leaflets were converted into 3D (x,y,z) coordinates. Deformities of the LAVV were evaluated by $3 \mathrm{D}$ re-constructed images. Results: There were 28 patients with mild (Group 1) and 31 with moderate (Group 2) LAVV regurgitation. 15/31 (48.4\%) patients in Group 2 had a deformity of the inferior bridging leaflet (IBL) and a commissural abnormality between IBL and mural leaflet (ML) compared to $2 / 28(7.1 \%)$ and 5/28 (17.9\%) patients in Group $1(\mathrm{p}<0.001)$, respectively. Annular area and the prolapsed volume were greater in Group 2 than Group 1 and controls $(p<0.001)$. In group 2 , the anterolateral PM was more laterally displaced than in group 1 and controls $(\mathrm{p}<0.001$ and $<0.05$ respectively). Older age, larger annular area and lateral displacement of the anterior PM were significantly correlated with moderate LAVV regurgitation by multivariable analysis.

Conclusion: In AVSD, significant LAVV regurgitation is associated with aging, larger annular area, lateral papillary muscle displacement, prolapse and structural abnormalities of the IBL. These findings may impact on future surgical management.

\section{6}

Assesment of Left Ventricle Wall Motion Beforre and After Transcatheter Closure of Atrial Septal Defect Using 2-D Speckle-tracking Analysis

Kohta Takei, Nagano Children's Hospital, Japan

Satoshi Yasukochi, Nagano Children's Hospital, Japan

Kiyohiro Takigiku, Nagano Children's Hospital, Japan

Ichige Kajimura, Nagano Children's Hospital, Japan

Seiichi Tazawa, Nagano Children's Hospital, Japan

Nao Inoue, Nagano Children's Hospital, Japan

Yusuke Nakano, Nagano Children's Hospital, Japan

Objective: The impact of transcatheter closure of ASD by Amplatzer septal occluder (ASO) on LV wall motion remains uncertain. We investigate to evaluate the changes of LV wall motion before and after ASO.

Methods: 19 patients with ASD who underwent ASO (ASO-Gr, aged $13.2 \pm 6.9$ years) included in this study. $2 \mathrm{D}$ images of short axis and apical four-chamber view were obtained by Vivid 7 with S3 probe (GE) at before, 1day, 1, 3, 6 and 12 months after ASO, respectively. The acquired images were analyzed by EchoPAC (GE) to calculate radial (SR), circumferential (SC) and longitudinal (SL) strain, radial (DR) and longitudinal (DL) displacement and LV Torsion. LV was divided into 6 segments to evaluate the regional wall motion. These data was compared to those of 6 normal children ( $\mathrm{N}-\mathrm{Gr}$, aged $5.5 \pm 0.5$ years).

Results: (1) The preoperative DR of anterior-septal and septal segments of ASO-Gr was significantly lower than those of N-Gr: mid anterior-septal $(3.6 \pm 1.5$ vs. $4.7 \pm 0.8 \mathrm{~mm} / \mathrm{m})$, mid septal $(2.5 \pm 0.9$ vs. $4.0 \pm 0.8 \mathrm{~mm} / \mathrm{m})$. (2) There was no significant difference between ASO-Gr and N-Gr in SR, SC, SL, DL, LV Torsion. (3) There was no significant change in SR, SC, SL, LV Torsion before and after ASO until 12 months, however, DR of anterior-septal and septal segments were significantly increased (mid anterior-septal; $2.5 \pm 1.0 \rightarrow 3.7 \pm 0.7 \mathrm{~mm} / \mathrm{m}, \mathrm{p}<0.001)$.

Conclusions: Strain and torsion of LV in ASD patients were preserved before and after ASO. Displacement of LV septum was reduced and recovered by ASO as improving RV volume overload, which associates with the improvement of paradoxical septal wall motion.

\section{7}

Global apical strain and strain rate measured by speckle tracking imaging reflects ventricular volumes in hypoplastic left heart

Edythe B Tham, Stollery Children's Hospital, Canada

Nee Scze Khoo, Stollery Children's Hospital, Canada

Akio Inage, Stollery Children's Hospital, Canada

Andrew Mackie, Stollery Children's Hospital, Canada

Jeffrey Smallhorn, Stollery Children's Hospital, Canada

Objective: Long-term preservation of ventricular function is becoming increasingly important as the survival of patients with single ventricle physiology improves. Strain and strain rate (SR) imaging is a relatively load independent measure of ventricular function. In this prospective study, we measured strain and SR pattern in a homogeneous group of infants with hypoplastic left heart (HLH). Method: Fifteen infants $($ HLHS $=11$, unbalanced AVSD $=4)$ underwent a sedated echocardiogram and cardiac MRI prior to the bidirectional cavopulmonary anastomosis (BCPA) at a mean of $27 \pm 19$ days apart. Global longitudinal (apical 4 chamber) and circumferential (basal and apical short axis) strain and SR were measured with speckle tracking imaging using commercially available software. MRI ventricular volumes and mass were indexed to body surface area. 
Results: Mean age at echocardiography was $4.7 \pm 2.1$ months. One had severe and 3 had moderate tricuspid regurgitation. Mean strain and SR are summarized in Table 1. Apical circumferential strain and SR decreased with increasing ventricular volumes and showed significant linear correlations (Figure 1). Increased RV mass significantly correlated with decreased apical strain $(r=0.68, p<0.01)$ and SR $(r=0.7$, $\mathrm{p}<0.01)$. RV volumes and mass had no relationship to basal circumferential or longitudinal strain or SR.

Conclusion: Apical strain and SR were the best indicators of ventricular size in infants with $\mathrm{HLH}$ prior to BCPA, and may provide a serial marker for which to quantify ventricular function. Further longitudinal studies are required to determine the applicability of these results to all stages of surgical palliation.

\begin{tabular}{lll}
\hline & Strain $(\%)$ & Strain rate $(1 / \mathbf{s})$ \\
\hline Apical circumferential & $-12.3 \pm 2.7$ & $-0.9 \pm 0.23$ \\
Basal circumferential & $-18.7 \pm 6.4$ & $-1.2 \pm 0.45$ \\
Longitudinal & $-17.9 \pm 2.5$ & $-1.1 \pm 0.23$ \\
\hline
\end{tabular}
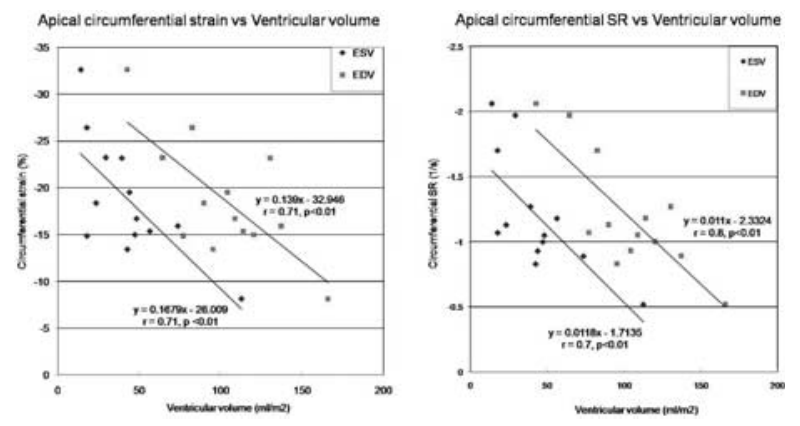

6119

3D Velocity-Encoded Magnetic Resonance Imaging: a new tool for the assessment of right ventricular diastolic function in children with pulmonary regurgitation after corrected Tetralogy of Fallot

Annelies E van der Hulst, Division of Pediatric Cardiology, department of Pediatrics, Leiden University Medical Center, The Netherlands Jos JM Westenberg, Division of image processing, department of Radiology, Leiden University Medical Center, The Netherlands Lucia JM Kroft, Department of Radiology, Leiden University Medical Center, The Netherlands

Jeroen J Bax, Department of Cardiology, Leiden University Medical Center, The Netherlands

Albert de Roos, Department of Radiology, Leiden University Medical Center, The Netherlands

Nico A Blom, Division of Pediatric Cardiology, department of Pediatrics, Leiden University Medical Center, The Netherlands

Arno AW Roest, Division of Pediatric Cardiology, department of

Pediatrics, Leiden University Medical Center, The Netherlands

Background: In patients with corrected Tetralogy of Fallot (cToF) assessment of diastolic function of the right ventricle (RV) is complicated by pulmonary regurgitation (PR). Two-dimensional one-directional velocity-encoded MRI (2D VE-MRI) can assess diastolic function by summation of flow through the tricuspid (TV) and pulmonary valve (PV) during diastole. However, 2D VE-MRI of the TV is hampered by heart motion. Furthermore, heartrate variability influences accurate summation of $2 \mathrm{D}$ MRI TV and PV flow. Three-dimensional (3D) three directional VE-MRI overcomes these problems with velocity-encoding in three orthogonal directions of all valves during one acquisition and retrospective valve tracking. This study evaluates 3D VE-MRI for assessing PV and TV flow in children with cToF.

Methods: 12 children (mean age $13.7 \pm 2.7$ year) with cToF and PR underwent MRI with 2D and 3D VE-MRI flow mapping of the PV and TV. Summation of the 3D VE-MRI curves yielded RV time-volume curves. RV dimensions were calculated from transversal cine planimetry images.

Results: A good correlation was found between planimetry stroke volume and 3D pulmonary forward flow (Pearson's correlation coefficient $0.84, \mathrm{p} 0.001)$, and between $2 \mathrm{D}$ and $3 \mathrm{D}$ pulmonary forward flow (0.86, p<0.0001) PR (0.95, p<0.0001). 3D VE-MRI peak early filling rate was $483 \pm 171 \mathrm{ml} / \mathrm{s}$, peak atrial filling rate $254 \pm 99 \mathrm{ml} / \mathrm{s}, \mathrm{E} / \mathrm{A} 2.3 \pm 1.5$, and deceleration time $271 \pm 88 \mathrm{~ms}$.

Conclusion: 3D VE-MRI is a reliable new tool for the assessment of PV flow and PR in patients with cToF. 3D VE-MRI could be a new tool for the assessment of diastolic function in $\mathrm{cToF}$ patients with PR.

\section{0}

The first experience with the high-resolution multi-slice CT-angiography in pediatric cardiology in Slovakia

Peter Olejnik, Department of pediatric cardiology, Children's University Hospital Bratislava and Children's Cardiac Center, Slovak Republic Peter Boruta, Slovak Republic

Anna Glezlova, Slovak Republic

Jozef Masura, Slovak Republic

Zuzana Venczelova, Slovak Republic

Objectives: The high-resolution multi-slice computed tomography angiography (HRMS-CTA) is a new imaging method characterized by a precise isotropic imaging of any cardiovascular system structure. The purpose of this study was to review the first experience with the high-resolution multi-slice HRMS-CTA in pediatric patients with congenital heart defects as well as with acquired cardiac diseases in the Slovak Republic.

Methods: We retrospectively reviewed benefits of HRMS-CTA and its influence on the subsequent choice of the most appropriate management in 20 patients with pulmonary atresia with ventricular septal defect and multiple aortopulmonary collaterals (PA, VSD, MAPCAs) and in 71 patients with different diagnoses with expected benefit from HRMS-CTA in terms of an exact delineation of extra-cardiac vascular structures.

Results: The delineation of the examined extra-cardiac structures was very detailed and clear in all 91 patients. All findings definitely contributed to the choice of the most appropriate management not only in PA, VSD, MAPCAs patients (20), but also in 71 patients with different diagnoses.

Conclusions: HRMS-CTA is a new complementary imaging method with the potential to replace invasive catheterization procedures in large number of patients who need extra-cardiac vascular structure imaging.

\section{1}

\section{Effectiveness of simulator based training in congenital} heart disease

Robert Wagner, Heart Center Leipzig, Department for Pediatric Cardiology, Leipzig, Germany, Germany

Florentine Wild, Heart Center Leipzig, Department for Pediatric Cardiology, Leipzig, Germany, Germany

Vit Razek, Heart Center Leipzig, Department for Pediatric Cardiology, Leipzig, Germany, Germany 
Jan Janousêk, Heart Center Leipzig, Germany

Michael Weidenbach, Heart Center Leipzig, Department for Pediatric Cardiology, Leipzig, Germany, Germany

Objectives: Although simulator based training is increasingly used in medical training, there is paucity of studies proving its effectiveness. We have studied the effectiveness of our previously described and validated echocardiography simulator EchoCom.

Methods: The simulator consists of a life-sized neonatal manikin, an electromagnetic tracking system and a computer application. The application is linked with a data base of 3D echocardiographic data sets of congenital heart lesions. For the present study we have chosen nine pairs of data sets with the following diagnoses: VSD, ASD, AVSD, TOF, TGA, ccTGA, TAC, HLHS and normal heart. The first set was presented to 10 beginners in echocardiography without clinical information. Participants were asked to scan the data and come up with a presumed diagnosis. All participants underwent a structured simulator based echocardiography training session by an expert afterwards. After training the study was repeated using different data sets of identical lesions. Pre- and post-training results were compared to evaluate the effectiveness of simulator based training. Results: Post-training results were significantly better than pre-training results. All participants improved the number of correct diagnoses. Conclusion: Simulator based training improves beginner's skills in echocardiography in congenital heart lesions. We have chosen data sets with identical lesions to have the same level of complexity. It is unclear if the result would be the same for previously unknown lesions. We have also not compared simulator based training with traditional learning like bed-side teaching or lectures. Bed-side teaching however is often impractical for hands-on training in critical lesions.

\section{2}

Diagnosis Accuracy of Ebstein's Anomaly by

Echocardiography

Lianyi Wang, First Hospital of Tsinghua University, China

Ping Lu, First Hospital of Tsinghua University, China

Guangyu Pan, First Hospital of Tsinghua University, China

Qingyu Wu, First Hospital of Tsinghua University, China

Object: To evaluate the diagnostic ability and accuracy of echocardiography in Ebstein's anomaly (EA).

Methods: 117 patients were diagnosed with EA from Mar. 2003 to Oct. 2008 in our hospital. Among them, 64 patients received cardiac surgery and all pathology was confirmed. We compare the main pathology finding intraoperatively with echocardiography reports.

Results: In 64 patients with surgery, the diagnosis of EA was confirmed in all. Concomitant defects reported by echo were ASD in 31 cases, PFO in 6, VSD in 3, LSVC in 1 and PS in 1 . There were tricuspid regurgitation in all patients, 57 were severe, 6 were moderate and 1 was mild. All findings were confirmed during surgery. There were 62 septal, 35 anterior and 62 posterior leaflets of tricuspid valve involved in regarding of downward displacement. Echocardiography was able to identify all septal and posterior leaflet displacements, yet identified only $40 \%$ of anterior leaflet displacements. During surgery, septal leaflet was found absent in 19 patients, reported by echo in only 12(63.1\%); posterior leaflet was absent in 18, reported by echo in 9 (50\%). There was a big variety of leaflet hypoplasty. Echcardiography was able to identify moderate to severe cases, yet under evaluated mild cases, especially if the leaflet was partially involved.

Conclusion: In EA, all 3 leaflets of tricuspid valve can be involved. Though echocardiography is a very useful diagnostic tool,there is some limitation in accurate diagnosis, especially for hypoplasty of the leaflets and partial anterior leaflet displacement. It is easy to under evaluate the pathology.
6123

Implementation of simulator based training for pediatric echocardiography

Michael Weidenbach, Heart Center Leipzig, Germany

Vit Razek, Heart Center Leipzig, Germany

Floretine Wild, Heart Center Leipzig, Germany

Robert Wagner, Heart Center Leipzig, Germany

Thomas Berlage, Fraunhofer Institute for Applied Information

Technology, Germany

Jan Janousek, Heart Center Leipzig, Germany

Objectives: Testing the feasibility of implementing simulator training into courses for pediatric echocardiography.

Methods: We used EchoCom, a simulator for echocardiography that we have developed at our institution, as an additional tool during a board certified echocardiography course at the Heart Center Leipzig. EchoCom consists of a manikin, a electromagnetic tracking system and a computer application (see figure). The application visualizes two-dimensional echocardiographic images derived from stored 3D data sets according to the tracker position and represents them sideby-side with a virtual 3D scene of the heart and ultrasound probe. It is linked with a data base of 3D data sets covering most congenital heart diseases. The course is divided into a theoretical and a practical part. Within the practical part children (with/without cardiac disease) volunteer as training models. Participants were divided into groups of 5 and had two training sessions with children and one simulator session. After completion they filled out a standardized questionnaire regarding the usefulness of the simulator.

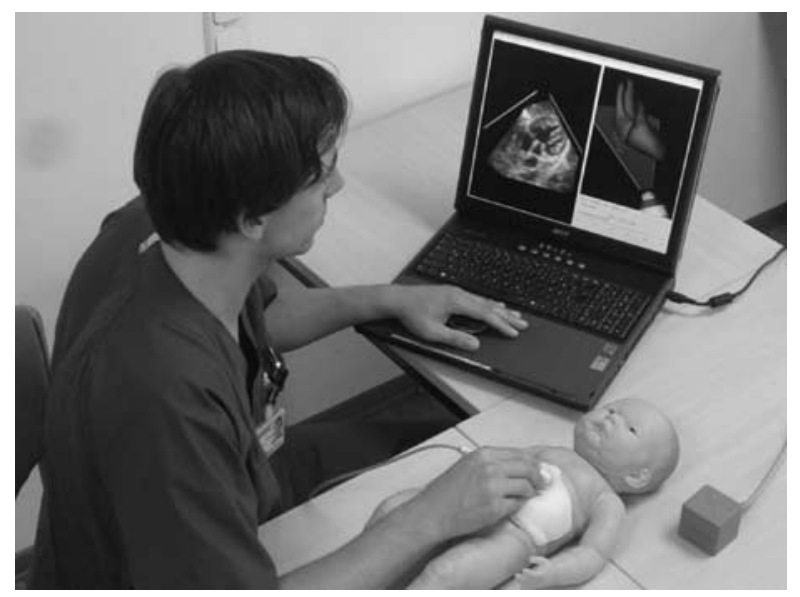

Results: A total of 30 physicians participated in the study. All regarded the implementation of the simulator as useful and said it has advantages compared to training on volunteers. $92 \%$ said the simulator could impart aspects they would have not acquired without the simulator. $53 \%$ voted to have more simulator training, while $47 \%$ said that the extent was adequate. No one wanted to have less simulator training.

Conclusion: Implementing simulator training in addition to traditional learning methods in echocardiography courses is useful and rated positively by course participants.

6124

Assessment of patent ductus arteriosus among adult patients with multi-slice computed tomography angiography before transcatheter closure Martin NL Wong, Sarawak General Hospital, Malaysia Boonhock Choo, Sarawak General Hospital, Malaysia Kuihian Sim, Sarawak General Hospital, Malaysia 
Objectives: Accurate assessment of size and morphology of patent ductus arteriosus (PDA) is crucial in selecting best device for transcatheter closure. This often can only be achieved by invasive angiography among adult patients because of poor transthoracic echocardiographic windows. We aimed to determine accuracy of multi-slice computed tomography (MSCT) angiography in assessing PDA among adults before transcatheter closure.

Methods: 24 patients (mean age $25.4 \pm 1.3$ years) with PDA underwent both MSCT and invasive angiography assessment before transcatheter PDA closure. Measurements and determination of morphological type were compared between the two methods.

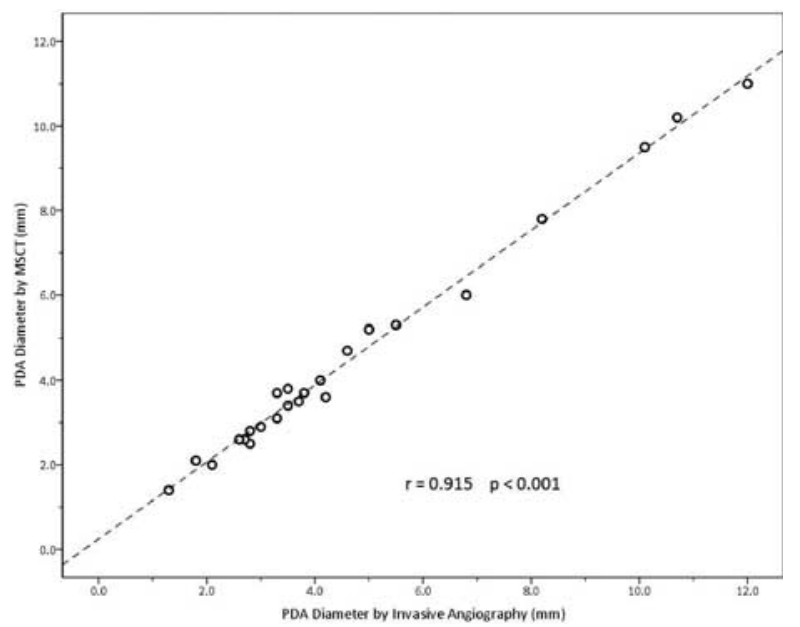

Results: The mean diameter of PDA was $4.6 \pm 2.9 \mathrm{~mm}$ (range 1.3 to $12.0 \mathrm{~mm}$ ) and the morphology types were: Type A (20 patients), Type B (1 patient), Type C (3 patients). Transcatheter PDA closure was successful in 22 patients. Types of devices used were: Amplatzer Duct Occluder 8/6 (9 patients), 10/8 (10 patients) and 16/14 (1 patient), Gianturco coil (1 patient), Amplatzer Muscular VSD Occluder $12 \mathrm{~mm}$ (1 patient). The remaining 2 patients had Eisenmenger syndrome. The PDA diameters measured on MSCT correlated highly with measurements by invasive angiography (Pearson correlation coefficient $=0.915, p<0.001$ ). Morphological classification were concordant in all cases. Type of closure devices used could be correctly predicted by MSCT before the procedure in all cases.

Conclusions: Assessment of PDA in adults by MSCT is accurate in guiding device selection for transcatheter closure.

6127

The Value of Three-dimensional Echocardiographic Virtual Endoscopy for the Diagnosis of Congenital Heart Disease in Children: A Novel Visualization Technique Using Virtual Reality

Hai-Hong Xue, Shanghai Children's Medical Center, Xinhua Hospital, School of Medicine, Shanghai Jiaotong University, China

Kun Sun, Shanghai Children's Medical Center, Xinhua Hospital, School of Medicine, Shanghai Jiaotong University, China

Jian-Guo Yu, Department of Electronic Engineering, Fudan University, Shanghai, China, China

Bin-Jin Chen, Department of Electronic Engineering, Fudan University, Shanghai, China, China

Guo-Zhen Chen, Department of Pediatric Cardiology, Shanghai

Children's Medical Center, School of Medicine, Shanghai Jiaotong University, China

Wen-Jing Hong, Department of Pediatric Cardiology, Shanghai Children's Medical Center, School of Medicine, Shanghai Jiaotong University, China Li-Ping Yao, Department of Pediatric Cardiology, Shanghai Children's Medical Center, School of Medicine, Shanghai Jiaotong University, China
Lan-Ping Wu, Department of Pediatric Cardiology, Shanghai Children's Medical Center, School of Medicine, Shanghai Jiaotong University, China

Objective: The purpose of this study was to evaluate the feasibility and value of our newly developed three-dimensional echocardiographic intracardiac endoscopic simulation system (3DE IESS) in the diagnosis of congenital heart disease in children.

Methods: Three experienced pediatric cardiologists blinded to the patients' diagnoses separately reviewed 40 two-dimensional echocardiographic (2DE) data-sets and 40 corresponding VE datasets, judging whether abnormal intracardiac anatomy was present on a 5-point scale ( 1 = definitely absent; $2=$ probably absent; $3=$ cannot determine; $4=$ probably present; and $5=$ definitely present).

Results: Three-dimensional echocardiographic intracardiac endoscopic imaging could be reconstructed successfully by $3 \mathrm{DE}$ IESS in all 40 patients. The three observers' independent examination of $2 \mathrm{D}$ and VE datasets provided 162 diagnostic evaluations of each method. The receiver operating characteristic (ROC) curve for VE was closer to the optimal performance point than that for 2DE. The area under the ROC curve was 0.96 for $\mathrm{VE}$ and 0.93 for 2DE, respectively. Kappa values (range, 0.73-0.79) for VE and 2DE indicated substantial agreement.

Conclusion: 3D echocardiographic VE can enhance our understanding of intracardiac structures and provide intuitional, reproducible and clinically useful visualizations. This may open a new door for the diagnosis of congenital heart diseases.

\section{9}

The Value of Three Dimensional Echocardiography in the Diagnosis and Surgical Design for Children with Atrioventricular Septal Defect

Kaihua Yang, Shanghai Children's Medical Center, Shanghai Jiaotong University, China

Kun Sun, Department of Pediatric Cardiology, Shanghai Children's Medical Center, China

Lanping Wu, Shanghai Children's Medical Center, Shanghai Jiaotong University, China

Lisheng Qiu, Shanghai Children's Medical Center, Shanghai Jiaotong University, China

Objective: To evaluate the value of three dimensional echocardiography (3DE) in the diagnosis for atrioventricular septal defect (AVSD), and explore new quantitative parameters for pre-surgical design.

Methods: 32 consecutive patients with AVSD were randomly enrolled, 23 of them had surgery after 3DE. QLAB and selfdesigned Cadio4DViewer software were used for analysis. The anatomical information, projective area of common atrioventricular valve annulus $(\mathrm{CVa})$, post-operative area of left atrioventricular valve (LAVV) annulas (MVa), area of bridge valves of LAVV $(\mathrm{A}+\mathrm{P})$ and corresponded total area of $\operatorname{LAVV}(\mathrm{T})$ were measured. The solid configuration of common valve annulus was demonstrated by Cadio4DViewer.

Results: Both $\mathrm{A}+\mathrm{P} / \mathrm{T}$ and $\mathrm{MVa} / \mathrm{CV}$ a had significant relationship with the change of degree of LAVV regurgitation $\left(\mathrm{MR}_{\mathrm{after}}{ }^{-}\right.$ $M R_{\text {pre }}$ ), the value of $\mathrm{R}$ was 0.90 and -0.73 . There was distinct separation of the value of $\mathrm{A}+\mathrm{P} / \mathrm{T}$ and $\mathrm{MVa} / \mathrm{CVa}$ between patients had or not have improvement of LAVV function postoperatively.

Conclusion: 3DE is useful in the pre-surgical design for AVSD. It is important to make sure the percentage of the area of bridge valves is greater than $50 \%$ of the total area of the LAVV when separating the bridge valve. Finally, the annulus of common valve is not planar, it's more solid and complex than ever thought before. 
6130

Tetralogy of Fallot: any correlation between echocardiographic measures, radionuclide angiography and (gold standard) MRI?

D Zentner, Royal Melbourne Hospital, Australia

A Ellims, Royal Melbourne Hospital, Australia

D Sivaratnam, Royal Melbourne Hospital, Australia

Michael Cheung, The Royal Children's Hopsital, Melbourne, Australia

L Grigg, Royal Melbourne Hospital, Australia

Objective: To determine whether correlations exist between echocardiography (TTE), radionuclide angiography (RNA) and magnetic resonance imaging (MRI) assessment of the right ventricle $(\mathrm{RV})$ in adults post TOF repair.

Methods: Prospective MRI assessment and data comparison with most recent TTE and RNA. Data is presented as median (interquartile range).

Results: MRI has been performed 28.8 (23.6-33.8) years post repair (y) in 33 patients, of whom all have had TTE (29.6 (25.2-35.0) y) and 26 have had RNA (26.4 (22.3-31.8) y). The cohort are 30.3 (27.0-39.0) years old; with a gender ratio18:15 (F:M) and MRI RVEDVI of $152 \mathrm{mLs} / \mathrm{m} 2$ (120-187). Data collection and analyses are ongoing, with interim analysis demonstrating moderate correlation between TTE RV size and MRI RV volume and pulmonary regurgitation (PR) (mLs) (Table 1).

Conclusion: TTE measures of RV size correlate with MRI RVEDI and PR volume. No correlation was found between pulse wave TDI and MRI RVEF, despite the premise that increasing RV size would be associated with decreasing function. Objective assessment of RV function remains a challenge.

\begin{tabular}{lll}
\hline Correlation variables & TTE RVOT size & TTE RV base size \\
\hline MRI RVEDVI & $\mathrm{r}=0.62 \mathrm{p}=0.002$ & $\mathrm{r}=0.42 \mathrm{p}=0.019$ \\
MRI PR & $\mathrm{r}=0.62 \mathrm{p}=0.01$ & $\mathrm{r}=0.62 \mathrm{p}=0.003$ \\
\hline
\end{tabular}

\section{1}

Echocardiographic assessment of children with

juxtaposition of the right atrial appendages : 33 cases from a tertiary children's medical center

Yu-qi Zhang, Department of Pediatric Cardiology, Shanghai Children's Medical Center, Shanghai Jiaotong University, China

Kun Sun, Department of Pediatric Cardiology, Shanghai Children's

Medical Center, China

Shu-wen Zhong, Department of Pediatric Cardiology, Shanghai Children's Medical Center, Shanghai Jiaotong University, China Guo-Zhen Chen, Department of Pediatric Cardiology, Shanghai Children's Medical Center, Shanghai Jiaotong University, China

Objective: The objective of this study was to analyze echocardiographic characteristics of juxtaposition of the right atrial appendages (JRAA) and to evaluate the diagnostic value of color Doppler echocardiography.

Methods: The echocardiographic characteristics of children with JRAA from 1998 to 2008 in our heart center were analyzed and compared with the findings of selective angiocardiography, magnetic resonance imaging (MRI) and surgical operation.

Results: JRAA was diagnosed in 33 of 10880 patients by angiocardiography.Juxtaposed atrial appendages and abnormal spatial orientation of atrial septum were visualized by color doppler echocardiography in all patients from the parasternal short-axis scan at the level of the great arteries, parasternal longaxis view, apical four chamber view, and subcostal four chamber view. 28 cases were diagnosed correctly by echocardiography, in the remaining 5 cases, 3 cases were suspected with JRAA, but it could not be determined by echocardiography; 2 cases were missed. The most common associated anomalies were conotruncal malformations (16 cases with double outlet of right ventricle, 9 cases with pulmonary atresia, 5 cases with transposition of the great arteries, 1 case with tetralogy of Fallot) and tricuspid malformations( 6 cases with tricuspid straddle, 3 cases with tricuspid atresia, 2 cases with tricuspid valve stenosis). Surgical operation was performed in 21 patients with 2 death.

Conclusions: Based on the characteristic alteration of the plane of the atrial septum and visualization of the malpositioned right atrial appendages, JRAA could be accurately diagnosed by color doppler echocardiography.

\section{2}

Diagnosis of Congenital Pulmonary Venous stenosis by Color Doppler Echocardiography

Zhi-fang Zhang, Department of Pediatric Cardiology, Shanghai Children's Medical Center, Shanghai Jiaotong University, China Yu-qi Zhang, Department of Pediatric Cardiology, Shanghai Children's Medical Center, Shanghai Jiaotong University, China

Kun Sun, Department of Pediatric Cardiology, Shanghai Children's Medical Center, China

Yi-qing Liu, Department of Pediatric Cardiology, Shanghai Children's Medical Center, Shanghai Jiaotong University, China

Rong Shen, Department of Pediatric Cardiology, Shanghai Children's Medical Center, Shanghai Jiaotong University, China

Objective: To evaluate the diagnostic value of color doppler echocardiography for the diagnosis of congenital pulmonary venous stenosis (PVS).

Methods: A retrospective echocardiography review of 15 patients with PVS confirmed by catheterization, Magnetic Resonance Imaging (MRI) or surgical operation.

Results: Among 15 PVS patients, six patients had right pulmonary veins stenosis, four patients $(66.7 \%)$ were diagnosed correctly by echocardiography, and 2 patients (33.3\%) were missed. Four patients had left pulmonary vein stenosis, all patients were diagnosed correctly. Five patients had both side pulmonary vein stenosis, four patients (80\%) were diagnosed correctly and one patient with left pulmonary vein stenosis was missed Four patients (26.7\%) were simplex PVS, eleven patients $(73.3 \%)$ were found to accompany with other cardiovascular anomalies. The most common associated anomalies were atrial septal defect, ventricular septal defect, patent ductus arteriosus, completely atrioventricular septal defect and double outlet of right ventricle.

Conclusion: By fully aware and understanding of this anomaly, PVS could be diagnosed accurately by color doppler echocardiography.

\section{3}

The relationship between plasma ionized concentration of calcium and magnesium with cardiac energetics and systemic oxygen transport in neonates after the Norwood procedure

Hargen Zhang, Heart Center, Hospital for Sick Children, Canada Helen Holtby, Heart Center, Hospital for Sick Children, Toronto, Canada Sally Cai, Data Center, Congenial Heart Surgeon's Society, Canada Jia Li, Department of Cardiology, Stollery Children's Hospital, Canada

Objective: We examined the relationship between $\mathrm{Ca} 2+$ and $\mathrm{Mg} 2+$ and systemic oxygen transport in neonates after the Norwood procedure. 
Methods: Oxygen consumption (VO2) was measured using respiratory mass spectrometry for $72 \mathrm{hrs}$ in 17 neonates. Arterial, superior vena cava and pulmonary venous gases and pressures with $\mathrm{Ca} 2+$ and lactate were measured $2-4$ hourly to calculate cardiac output $(\mathrm{CO})$, systemic vascular resistance (SVR), rate pressure product (RPP), cardiac power output (CPO), systemic oxygen delivery (DO2) and oxygen extraction ratio (ERO2). $\mathrm{Mg} 2+$ was measured 4-12 hourly.

Results: Ca2+ decreased in 8 hrs from $1.08 \pm 0.13$ to $0.98 \pm$ $0.08 \mathrm{mmol} / \mathrm{L}$, followed by an increase to $1.10 \pm 0.26 \mathrm{mmol} / \mathrm{L}$ at 72 hrs $(\mathrm{p}<0.0001) . \mathrm{Mg} 2+$ decreased in $24 \mathrm{hrs}$ from $1.57 \pm 0.29$ to $0.94 \pm 0.14 \mathrm{mmol} / \mathrm{L}$, followed by a slow decrease to $0.67 \pm$ $0.16 \mathrm{mmol} / \mathrm{L}(\mathrm{p}<0.0001) . \mathrm{Mg} 2+$ positively correlated with $\mathrm{CO}$ $(p=0.008)$ and CPO $(p=0.01)$, and negatively with heart rate $(p=0.05)$. It trended to positively correlate with DO2 $(p=0.08)$, and negatively with $\mathrm{VO} 2(\mathrm{p}=0.08), \mathrm{ERO} 2(\mathrm{p}=0.04)$ and lactate $(\mathrm{p}=0.05)$. Negative trends were found between $\mathrm{Mg} 2+$ and SAP $(p=0.16), \operatorname{RPP}(p=0.14)$ and SVR $(p=0.64)$. Ca2+ showed opposite trends without achieving significance in these variables except lactate $(\mathrm{p}=0.007)$. For a given $\mathrm{CPO}, \mathrm{Mg} 2+$ negatively correlated with RPP $(p=0.01)$, and Ca2+ showed a positive trend $(p=0.50)$. Conclusion: $\mathrm{Mg} 2+$ exerts favorable effects on myocardial energetics and systemic oxygen transport in neonates after the Norwood procedure, whereas $\mathrm{Ca} 2+$ might be harmful. Maintaining a relatively high $\mathrm{Mg} 2+$ and low $\mathrm{Ca} 2+$ may promote the efficiency of myocardial work and improve oxygen transport in neonates after $\mathrm{CPB}$.

\section{4}

Short Term Outcome in Patients with Hypoplastic Left Heart Syndrome and Ventriculo-Coronary Connections Shyam K Sathanandam, Hope Children Hospital, United States Nguyen Vu Nguyen, Hope Children Hospital, United States David A Roberson, Hope Children Hospital, United States Vivian Cui, Hope Children Hospital, United States Alisa Niksch, Hope Children Hospital, United States Andrew Van Bergen, Hope Children Hospital, United States Bettina F Cuneo, Hope Children Hospital, United States Michel N Ilbawi, Hope Children Hospital, United States

Objective: To report incidence and assess outcome of hypoplastic left heart syndrome (HLHS) patients with ventriculo-coronary connections (VCC) following stage1 palliation (S1P). Impaired coronary perfusion and mitral stenosis/aortic atresia (MS/AA) subtype have both been implicated to cause early post-operative and interstage mortality in HLHS. We seek to determine if presence of VCC affects outcome of patients with MS/AA.

Methods: We reviewed 86 patients with HLHS between September 2005 and December 2008. Besides VCC, age at $\mathrm{S} 1 \mathrm{P}$, birth weight $(\mathrm{BW})$, ascending aorta size (AAo), anatomic type, associated lesions, surgical technique, cardiopulmonarybypass time, cross-clamp time (XCT), circulatory arrest time and post-operative complications were reviewed.

Results: Overall survival of MS/AA group to stage 2 palliation (S2P) was $71 \%$; 12 of 24 patients with MS/AA had VCC (50\%). Eleven of twelve patients with VCC survived to S2P (92\%). Presence of VCC with MS/AA did not contribute to higher mortality $(p<0.05)$. Interstage mortality between S1P and S2P was not different among the 3 subtypes of HLHS $(p<0.05)$. Higher incidence of restrictive atrial communication, RV dysfunction, arrhythmia and ECMO in the mitral atresia/aortic atresia group was due to chance. Multivariate analysis demonstrated that low BW, longer XCT, AAo $<2 \mathrm{~mm}$ and arrhythmia contributed to higher mortality and need for ECMO.
Conclusion: VCC occurs exclusively with MS/AA subtype. Incidence of VCC was $50 \%$ of MS/AA. The presence of VCC was not associated with higher morbidity or mortality after S1P. We postulate this to be due to our institutional preference of performing RV-PA conduits for patients with aortic atresia during S1P.

\section{5}

Incidence, Risk Factors and Outcomes from Necrotizing Enterocolitis in Congenital Heart Disease

Prakul Chanthong, Thailand

Helena Frndova, Canada

Cedric Manlhiot, Cardiovascular Clinical Research Unit, Labatt Family

Heart Centre, Hospital for Sick Children, Toronto, Canada

Brian W McCrindle, Canada

Venkatesan B Sivarajan, The Hospital for Sick Children, Canada

Background: Infants with congenital heart disease(CHD) are at risk for necrotizing enterocolitis (NEC). We performed a case-control study of infants admitted to the cardiac critical care unit during a five year period in order to determine the incidence, risk factors and outcomes for NEC in patients with congenital heart disease.

Methods: NEC cases were identified by ICD-10 codes and matched with random CHD controls. Cases and controls were compared for NEC risk factors; factors associated with NEC were tested in a multivariate logistic regression model.

Results: Sixty-six NEC cases occurred in 1,185 consecutive admissions with an incidence of 5.7 [4.3-7.0]\%; 30 (45\%) developed NEC postoperatively. Lower gestational age at birth, $\mathrm{OR}=1.2[1.1-1.4], \mathrm{p}=0.02$; lower birth weight, $\mathrm{OR}=[1.1$ $1.05-1.2], \quad p=0.006 ;$ non-singleton pregnancy, $\quad O R=3.3$ $[1.1-10.8], \mathrm{p}=0.05$; acidosis, $\mathrm{OR}=3.1$ [1.4-6.7], $\mathrm{p}=0.004$ and hypotension, $\mathrm{OR}=\left[\begin{array}{ll}1.1 & 1.05-1.2\end{array}\right], \mathrm{p}=0.002$ were significantly associated with the subsequent development of NEC. Median intensive care unit length of stay was significantly higher in the NEC group versus controls (10 vs 5 days, $p=0.003$ ). Death occurred in 11 of $66(17 \%)$ patients with NEC compared with 6 of 66 controls (9.1\%), OR $=2.0$ [0.7-5.8], $\mathrm{p}=0.09$. Conclusions: NEC is a frequent complication of CHD with significant implications for morbidity and mortality. Prevention of acidosis and hypotension may modify these risks.

\section{7}

\section{Peripheral Venous Pressure as a Predictor of Central Venous Pressure in Continues Monitoring, in Children}

Hamid Amoozgar, Shiraz University of Medical Sciences, Iran Parisa Ebrahimi, Divisions of Pediatric Cardiology Department of Pediatrics, Shiraz University of Medical Sciences, Shiraz, Iran., Iran Mohammad Borzoee, Divisions of Pediatric Cardiology Department of Pediatrics, Shiraz University of Medical Sciences, Shiraz, Iran., Iran Gholamhossein Ajami, Divisions of Pediatric Cardiology Department of Pediatrics, Shiraz University of Medical Sciences, Shiraz, Iran., Iran

Background: Central venous pressure (CVP) measurement is a reliable method for evaluating intravascular volume status and cardiac function, however it is an invasive and expensive method that may result in some complications such as arterial puncture, pneumothorax, and development of infections.

Methods: The current study was performed to compare CVP measurements between central and peripheral catheters in infant and children with congenital heart disease during a period of time and to show if the change in CVP and peripheral venous 
pressure (PVP) is constant. The CVP and PVP in 30 patients were measured simultaneously within 10 consecutive hours. Results: The mean difference between CVP and PVP was $1.48 \pm 0.98 \mathrm{mmHg}$. The linear regression equation showed that $\operatorname{cvp}=0.374+0.774 \operatorname{pvp}(\mathrm{r}=0.725)$.

Conclusion: PVP measured from a peripheral intravenous catheter in infants and children with congenital heart disease is an accurate estimation of CVP and its changes has good concordance with CVP over a long time period.

\section{8}

Supraventricular tachycardia hard to management. A case report

Lucrecia Avila Rosales, Hospital para el Niño IMIEM, Mexico

Olga Montiel Galvan, Hospital para el Niño IMIEM, Mexico

Female, 10 years old. History: Two days with emesis, diarrhea, palpitations and thoracic pain.

Cardiovascular examination. $32 \mathrm{~kg}$ weight, heart rate: 250 beats/ min, respiratory frecuency 64 breaths $/ \mathrm{min}$, Temp: $36^{\circ} \mathrm{C}, \mathrm{BP}$ : 120/100 mmHg. Diagnosis: Supraventircular tachycardia. She received adenosine $0.1 \mathrm{mcg} / \mathrm{kg}$ for 3 doses without response, then it was administrated esmolol in bolus $100 \mathrm{mcg} / \mathrm{kg}$ following for another bolus with $500 \mathrm{mcg} / \mathrm{kg}$ until $15 \mathrm{mg}$ as a total dose and then $25 \mathrm{mcg} / \mathrm{kg} / \mathrm{min}$ as a infusion until $50 \mathrm{mcg} / \mathrm{kg} / \mathrm{min}$. Tachycardia reverts to sinus rhythm without cardiac failure and patient continues with amiodarone $150 \mathrm{mg}$ oral, daily.

Chest X-ray: minimus cardiomegaly.

Elecrocardiogram: Supraventricular tachycardia, QRS narrow, Heart rate 250 beats/min.

Sinus rhythm, Heart rate 80 beats $/ \mathrm{min}, \mathrm{AQRS}+45^{\circ}, \mathrm{rS}$ in V1-2, $\mathrm{R}$ in V4-6. Delta wave in DII and V3 with short PR, it sugests Accessory pathway.

Evolution: consciousness, without cyanosis or precordial deformity, protosystolic left paraesternal murmur I/VI with normal second sound and normal peripheric pulses.

Echocardiography: M-mode, 2D, Doppler echocardiography and color flow imaging: situs solitus, AV and VA concordance, mild degree tricuspid regurgitation, pulmonary annulus $14 \mathrm{~mm}$, bicuspid aortic valve, peak aortic valve velocity $1.47 \mathrm{~m} / \mathrm{s}$. Aortic root $19 \mathrm{~mm}$, LA $19 \mathrm{~mm}$, IVS $8 \mathrm{~mm}$, PW $7 \mathrm{~mm}$, LVDD $39 \mathrm{~mm}$, LVSD $28 \mathrm{~mm}$, EDLVV $56 \mathrm{ml}$, ESLVV $33 \mathrm{ml}$.EF 52\% SF 26\%. It was descarted mitral valve prolapse and Ebstein anomaly. Conclusion: Preexcitation syndrome tipe Wolf-Parkinson-White through Kent pathway. Bicuspid aortic valve. Mild tricuspid regurgitation.

\section{1}

Extracorporeal Membrane Oxygenation (ECMO) As Rescue Therapy for severe enterovirus 71 infection with cardiogenic shock

Jeng-Sheng J Chang, Dpt. of Ped., Div. of Ped. Cardiology, China Medical University Hospital, Taiwan

Yu-Chih Huang, Dpt. of Ped., Div. of Ped. Cardiology, China Medical University Hospital, Taiwan

Shiou-Jeng Lin, Dpt. of Ped., Div. of Infection, China Medical University Hospital, Taiwan

Bing-Tsun Lee, Dpt. of Cardiovascular Surgery, China Medical University Hospital, Taiwan

Background: Ever since the spring 1998, the severe enterovirus 71 (EV71) infection in Taiwan has resulted in fatality of 240 children, mostly under 3-years-old. Aside from hand-foot-mouth syndrome or herpangina, a full-blown severe EV71 infection tends to invade the brain stem and presents itself by 3 devastating stages as encephalomyelitis, hypercatecholamine and cardiogenic shock. Case fatality rates in those patients complicated with cardiogenic shock and/or pulmonary hemorrhage are as high as $80 \%$.

Design/Objective: Use ECMO to rescue patients of severe EV71 infection complicated with cardiogenic shock.

Methods: There were 5 cases of severe EV71 infection who received urgent ECMO therapy during Aug. 2004 through July 2008 when conventional inotropes and vasodilators were not effective. All ECMO were VA cannulated between the right atrium and the ascending aorta via sternotomy. An atrioseptectomy was also created for satisfactory left heart venting.

Results: The 5 patients were between 13 to 33 -months of age. All had positive virus cultures of EV71. Their brain stem injuries were proved by MRI studies. Their left ventricle ejection fractions were only $24.9 \pm 7.4 \%$ when manifesting cardiogenic shock. All ECMO were successfully weaned off in 48-94 hours. Their LVEF returned to $73.8 \pm 3.9 \%$ at discharge. One became vegetative state, two received tracheostomy and gastrostomy and two were normal. Histologically, the atrial septectomised tissues showed only apoptosis and minimal leukocytes infiltrates.

Conclusions: Cardiogenic shock in severe EV71 infection can be stabilized by ECMO therapy, though these patients are still high risk for neurological sequelae.

6142

Role of atrial septostomy as salvage treatment for severe low output syndrome secondary to restrictive right ventricular physiology following surgical repair of Tetralogy of Fallot

Boonhock Choo, Sarawak General Hospital, Malaysia Martin NL Wong, Sarawak General Hospital, Malaysia

Prashant Joshi, Sarawak General Hospital, Malaysia

Kuihian Sim, Sarawak General Hospital, Malaysia

Objectives: Restrictive right ventricular (RV) physiology is a major cause of low output syndrome following surgical repair of Tetralogy of Fallot (TOF). Treatment includes preload volume optimization, inotropes, inhaled nitric oxide and respiratory manipulation to enhance forward pulmonary flow. We investigated the role of atrial septostomy as salvage treatment in patients who failed these measures.

Methods: Retrospective review of all TOF operated between years 2005-2008 in our institution. Cases requiring atrial septostomy were identified and their postoperative notes and ICU charts were reviewed.

Results: 5 patients (mean age $3.2 \pm 0.6$ years) had severe low output syndrome and underwent atrial septostomy at median time 20 hours (ranged 17 to 50 hours) after surgery. All had restrictive RV physiology by echocardiography criteria and did not respond to medical treatment. 4 were performed transcatheterly and 1 surgically under cardiopulmonary bypass. Haemodynamic parameters improved following the procedure: mean systolic $\mathrm{BP}$ (from $57 \pm 3 \mathrm{mmHg}$ to $68 \pm 8 \mathrm{mmHg}$ ), urine output (from $0.8 \pm 0.3 \mathrm{ml} / \mathrm{kg} / \mathrm{h}$ to $2.4 \pm 2.1 \mathrm{~m} / \mathrm{kg} / \mathrm{h}$ ), base excess (from $-9.5 \pm 2.3 \mathrm{mmol} / \mathrm{L}$ to $-5.7 \pm 2.1 \mathrm{mmol} / \mathrm{L}$ ). It resulted in slight drop of oxygen saturation from $96 \pm 3 \%$ to $92 \pm 2 \%$. All patients survived without neurological sequelae although they required prolonged ventilation $(8.0 \pm 2.1$ days $)$ and ICU stay (11.4 \pm 1.1 days).

Conclusions: Atrial septostomy improves haemodynamics and is effective as salvage treatment for patients with severe low output syndrome and restrictive RV following TOF repair. 


\section{3}

Interrelationships between plasma $\mathrm{N}$-terminal pro-brain natriuretic peptide ( $\mathrm{Nt}$-proBNP), troponin I ( TnI), and high sensitivity C -Reactive Protein (hs- CRP) in children and adolescent submitted to surgical repair of atrial septal defect ( ASD)

Massimo Colaneri, Cardiochirurgia e Cardiologia Pediatrica e Congenita - Ospedali Riuniti - Ancona, Italy

Maria G Bettuzzi, Cardiochirurgia e Cardiologia Pediatrica e

Congenita - Ospedali Riuniti - Ancona, Italy

Alessandra Baldinelli, Cardiochirurgia e Cardiologia Pediatrica e

Congenita - Ospedali Riuniti - Ancona, Italy

Roberto Ricciotti, Cardiochirurgia e Cardiologia Pediatrica e Congenita Ospedali Riuniti - Ancona, Italy

Andrea Quarti, Cardiochirurgia e Cardiologia Pediatrica e Congenita Ospedali Riuniti - Ancona, Italy

Alessandro D' Alfonso, Cardiochirurgia e Cardiologia Pediatrica e

Congenita - Ospedali Riuniti - Ancona, Italy

Marco Pozzi, Cardiochirurgia e Cardiologia Pediatrica e Congenita -

Ospedali Riuniti - Ancona, Italy

Background: Nt-proBNP, TnI and hs-CRP are circulating markers of ventricular function, myocardial damage and inflammation.

Objective: To determine the interrelationships between preoperative and postoperative levels of these markers in pts undergone ASD surgical repair.

Methods: Markers blood levels were measured before surgery and after $1,24,48,72,96,120$ and 144 hours in 45 pts (median age 5.1 years, range $0.5-15.8$ years, 24 boys ) submitted to surgical repair of ostium secundum ASD.

Results: Preoperative and post-operative peak values of $\mathrm{Nt}-$ proBNP, TnI and hs-CRP are showed in table 1.

Preoperative Nt-proBNP was significantly correlated to postoperative Nt-proBNP $(\mathrm{r}=0.57, \mathrm{p}<0.001)$ and postoperative TnI $(r=0.30, p<0.05)$.

Postoperative Nt-proBNP was highly correlated to postoperative TnI $(\mathrm{r}=0.63, \mathrm{p}<0.001)$.

No significant correlations were observed between preoperative $\mathrm{Nt}$-proBNP and postoperative Nt-proBNP / postoperative TnI versus postoperative hs-CRP

Conclusions: Nt-proBNP is significantly elevated before surgery in pts with ASD.

Nt-proBNP, TnI and hs-CRP significantly increase after repair of ASD.

After surgery there is a strong interplay between neurohormonal activation and myocardial damage.

On the contrary inflammatory activation appears uncorrelated to neurohormonal activation and myocardial damage.

Table 1. Preoperative and postoperative peak values of NtproBNP, TnI and hs-CRP

\begin{tabular}{lccc}
\hline & $\begin{array}{l}\text { Nt-proBNP (pg/ml) } \\
\text { Median (range) }\end{array}$ & $\begin{array}{l}\text { TnI (ng/ml) } \\
\text { Median (range) }\end{array}$ & $\begin{array}{l}\text { hs-CRP (mg/dL) } \\
\text { Median (range) }\end{array}$ \\
\hline Time & $147^{\circ}(27-1096)$ & $0.01(0.01-0.04)$ & $0.3(0.3-0.5)$ \\
Preoperative & $2.71^{\star}(1.19-15.63)$ & $9.1^{\star \star}(2.9-18.3)$ \\
Postoperative peak & $3799 \star \star(546-21239)$ & & \\
\hline${ }^{\circ} \mathrm{p}<0.05$ versus normal values; ${ }^{\star} \mathrm{p}<0.05$ and $\left.{ }^{\star \star} \mathrm{p}<0.001\right)$ & versus & preoperative values \\
(Wilcoxon test) & & &
\end{tabular}

6144

Biphasic temporal pattern of plasmatic N-terminal pro-brain natriuretic peptide ( $\mathrm{Nt}$-proBNP) in children and adolescent undergoing surgical repair of ostium secundum atrial septal defect (ASD)
Massimo Colaneri, Cardiochirurgia e Cardiologia Pediatrica e Congenita - Ospedali Riuniti - Ancona, Italy

Maria G Bettuzzi, Cardiochirurgia e Cardiologia Pediatrica e Congenita - Ospedali Riuniti - Ancona, Italy

Alessandra Baldinelli, Cardiochirurgia e Cardiologia Pediatrica e Congenita - Ospedali Riuniti - Ancona, Italy

Roberto Ricciotti, Cardiochirurgia e Cardiologia Pediatrica e Congenita Ospedali Riuniti - Ancona, Italy

Andrea Quarti, Cardiochirurgia e Cardiologia Pediatrica e Congenita Ospedali Riuniti - Ancona, Italy

Alessandro D' Alfonso, Cardiochirurgia e Cardiologia Pediatrica e Congenita - Ospedali Riuniti - Ancona, Italy

Marco Pozzi, Cardiochirurgia e Cardiologia Pediatrica e Congenita -

Ospedali Riuniti - Ancona, Italy

Background: Nt-proBNP is a circulating marker of ventricular function produced within cardiomyocites in response to pressure and volume overload.

Methods: Plasmatic Nt-proBNP levels were measured before surgery and after $1,8,16,24,48,72,96,120,144,168$ hours in 45 pts ( median age $5.1(0.5-15.8)$ years ) submitted to ASD surgical repair.

Results: Pre and post-operative peak values of Nt-proBNP are shown in tab 1.

Nt-proBNP level reached the peak of 3799 (546-21239) pg/mL 24 hours after surgery.

The level decreased and then again increased forming the second smaller peak of 779 (535-2940) pg/mL on the fifth day.

Right/left ventricle end-diastolic dimension ratio measured by echocardiography on the second peak time was significantly lower than the first peak $(0.85+/-0.10$ vs $1.25+/-0.12$, $\mathrm{P}<0.05)$.

On multivariate regression analysis using age, postoperative peak Tn I, preoperative,, first peak, second peak Nt-proBNP as variables the only determinant of the postoperative length of stay (LOS) was the second peak value of $\mathrm{Nt}$-proBNP $(\mathrm{R} 2=0.46$, $\mathrm{p}<0.05$

Conclusion: A biphasic pattern of plasma Nt-proBNP is seen after surgical closure of ASD. The second peak values of Nt-proBNP may reflect the amount of the left ventricle overload after L-R shunt closure.

Secondary elevation of Nt-proBNP is a strong determinant of postoperative LOS.

Table 1. Pre and postoperative peak values of Nt-proBNP

Nt-proBNP $(\mathrm{pg} / \mathrm{mL})$

Time

Preoperative

Postoperative first peak ( $24 \mathrm{~h})$

Postoperative second peak (144h)

$* \mathrm{P}<0.05$ and $* * \mathrm{P}<0.001$ vs

preoperative

6146

Peri-transport Clinical Presentation and Management of Previously Undiagnosed Congenital Heart Disease in Neonates

Alejandro A Floh, Hospital for Sick Children, Canada

Johanne L'Herault, Hospital for Sick Children, Canada

Kyong-Jin Lee, Hospital for Sick Children, Canada

Kyong-Soon Lee, Hospital for Sick Children, Canada 
Objective: Analysis of the clinical presentation and management of $\mathrm{CHD}$ in neonates to guide improvements in transport stabilization. Methods: A retrospective review of neonates transported with a postadmission diagnosis of CHD during April 2005 to June 2006. Results: 62 neonates with CHD (7.5\% of all transports) presented to regional transport in our region. Mean BW was $3305+/$ $-703 \mathrm{~g}$, GA $38.7+/-1.8$ weeks, and median age of presentation was 0 (IQR 0,1) days. Acyanotic CHD accounted for 61\%, including TGA 19, PA/IVS/VSD 5, TOF 4, PS 4,TAPVD 3, Ebsteins 2, truncus 1; 39\% were acyanotic (coarctation 9, HLHS 7, interrupted aortic arch 3, AVSD 2, AS 1, cardiomyopathy 1). Most common presenting symptoms were cyanosis $76 \%$, murmur $53 \%$, respiratory distress $53 \%$ and shock $18 \%$. Fifty (81\%) had duct-dependent lesions and PGEs were started during stabilization in $90 \%$ of cyanotic and $88 \%$ of acyanotic lesions. Acyanotic lesions received more support compared with cyanotic lesions: $79 \%$ vs $47 \%$ ventilated $(p=0.04)$; inotropes $33 \%$ vs $5 \%$ $(\mathrm{p}=0.01)$. Acyanotic lesions presented with lower $\mathrm{pH}$ and this difference in $\mathrm{pH}$ increased at NICU admission. Mean $\mathrm{pH}$ on initial presentation was: acyanotic $7.30+/-0.14$ vs cyanotic $7.33+/-0.10 \quad(\mathrm{p}=0.14) ; \mathrm{pH}$ on NICU admission was: acyanotic $7.28+/-0.11$ vs cyanotic $7.35+/-0.12(\mathrm{p}=0.03)$. Mean lactate on NICU admission was: acyanotic $6.9+/-7.5$ vs cyanotic: $3.2+/-6.9(\mathrm{p}=0.02)$.

Conclusions: These data highlight the challenges transport teams face when managing undifferentiated infants with unexpected significant CHD. Acyanotic lesions were less stable on presentation, required more support during stabilization, and arrived in less stable condition.

\section{7}

\section{The risk factors of VAP following pediatric cardiac} operation

Li Juan Fu, Heart Center of Shanghai Children's Medical Center, China

Jun Li, Shanghai JiaoTong University, School of Nursing, China Zhou ming Xu, Thoracic \& Cardiovascular Surgery of Shanghai Children's Medical Center, China

Jun Bian, Thoracic \& Cardiovascular Surgery of Shanghai Children's Medical Center, China

Objective: Ventilator-associated pneumonia (VAP) is the most common and lethal form of hospital-acquired pneumonia. It is to determine the risk factors of VAP after the pediatric cardiac operation and provide the evidence how to prevent it.

Methods: Between January 2007 and December 2007, 283 patients with congenital heart disease underwent mechanical ventilation more than 48 hours after pediatric cardiac operation in a heart center. There were two groups. One with VAP, the other without VAP (Table 1). Gender, weight, prolonged cardiopulmonary bypass, delayed sternal closure, reintubation, the ways of the artificial, chest physical therapy, heads up, nutrition, ways of feeding, mouth care and surgery-related complications were analyzed between the two groups.

Results: The LOGISTIC regression showed that prolonged cardiopulmonary bypass, delayed sternal closure and reintubation were associated with increased incidence of VAP. Intra-enteral nutrition and heads up were associated decreased incidence of VAP (Table 2).

Conclusion: Based on this study, it showed that intra-enteral nutrition and heads up which we recommend following pediatric cardiac, especially in patients with prolonged cardiopulmonary bypass, delayed sternal closure or reintubation, VAP raises the cost of care and increases patient-care time, hospital length of stay, and patient morbidity, Progressive nursing care should be taken to prevent the VAP, therefore to decrease the in stay and the cost of therapy. The burden of parents then can be decreased and the quality of health care would be raised in the end.

\section{0}

The Efficacy of Sildenafil Therapy in Children with Pulmonary Hypertension Postoperatively

Feiqiong Huang, Singapore

Xu Wang, Pediatric Cardiac Center, Fuwai Cardiovascular Hospital, Chinese Academy of Medical Science, Beijing Union Medical University, China

Yanbo Zhang, Pediatric Cardiac Center, Fuwai Cardiovascular Hospital, Chinese Academy of Medical Science, Beijing Union Medical University, China

Yan He, Pediatric Cardiac Center, Fuwai Cardiovascular Hospital, Chinese Academy of Medical Science, Beijing Union Medical University, China

Yajuan Zhang, Pediatric Cardiac Center, Fuwai Cardiovascular Hospital, Chinese Academy of Medical Science, Beijing Union Medical University, China

Objective: Sildenafil is a Phosphodiesterase-5 inhibitor which is a potent pulmonary vasodilator. Some reports have been shown that it could decrease the pulmonary vascular resistance in adult patient with pulmonary hypertension. Few data in pediatrics postoperative pulmonary hypertension were established. In this study we try to evaluate the effect of oral Sildenafil in children with pulmonary hypertension postoperatively.

Methods: Forty patients with congenital heart diseases who underwent pulmonary hypertension postoperatively were included in this study. All patients were ventilated with sedation and muscle relaxed. The pulmonary artery systolic pressure (PASP) was 58.22 \pm $8.31 \mathrm{mmHg}, \quad \mathrm{Pp} / \mathrm{Ps}$ was $0.73 \pm 0.15, \quad \mathrm{SO} 2$ was $68.24 \pm 9.34$ $(\mathrm{FiO} 2>60 \%)$. After ventilation, using vasodilator, inhaling $\mathrm{NO}$ and $\mathrm{O} 2$, the decreased of pulmonary pressure was not satisfied, then Sildenafil was given from $0.3 \mathrm{mg} /$ dose, four times per day. If it didn't work, the dosage increased to $0.5-2.0 \mathrm{mg} / \mathrm{dose}$ (the largest dosage is $2.5 \mathrm{mg} / \mathrm{kg} /$ dose). If pulmonary pressure began to decrease, the dosage was maintained till patients were stable.

Result: The outcome of all the patients was good. The pulmonary pressure decreased $(\mathrm{p}<0.01), \mathrm{Pp} / \mathrm{Ps}$ was decreased $(\mathrm{p}<0.01)$, SO2 increased to $98 \%-100 \%$ (Fio $2<40 \%$ ), and all patients were extubated. Twelve patients had systemic hypotension, but made comeback soon without any intervention treatment.

Conclusion: When pediatric patients with congenital heart disease suffered pulmonary hypertension postoperatively, sildenafil could decrease the pulmonary resistant and make an effect on pulmonary hypertension.

\section{1}

Therapeutic mild hypothermia on pediatric patients with low cardiac output syndrome after open heart surgery

Tatsuo Iwasaki, Okayama University Graduate School of Medicine and Dentistry, Japan

Yuichiro Toda, Okayama University Graduate School of Medicine and Dentistry, Japan

Kazuyoshi Shimizu, Okayama University Graduate School of

Medicine and Dentistry, Japan

Tomohiko Suemori, Okayama University Graduate School of Medicine and Dentistry, Japan

Satoshi Suzuki, Okayama University Graduate School of Medicine and Dentistry, Japan 
Kiyoshi Morita, Okayama University Graduate School of Medicine and Dentistry, Japan

Low cardiac output syndrome (LOS) following open heart surgery is not rare in pediatric patients. Mild hypothermia might be one of the useful therapeutic options to control LOS because myocardial contractility depends on temperature. But only a few report are available and we conducted this retrospective chart review to Material and method: This was a retrospective study based on medical chart review of admissions to a university hospital. Twenty patients of 226 children who admitted to ICU after open heart surgery from January to August 2008 were enrolled to this study.

Results: All 20 patients were infants. Core temperature was controlled between 34 to 36 degrees C. Diagnoses included TAPVD(6), HLHS(5), IAA(2), CoA complex(2), others(5). The mean body weight of patients was 2.7(1.7-3.2)(median (range)) $\mathrm{kg}$, systolic blood pressure and base excess on admission to ICU were $55(35-110) \mathrm{mmHg},-1.1(-15.5-10.1) \mathrm{mmol} / \mathrm{L}$ respectively. Five patients required peritoneal dialysis, and 8 cases include 5 children of ECMO underwent delayed sternal closure. Two patients suffered from infectious complication. Two infants died from cardiac failure with infectious complication. Duration of mechanical ventilation was 182 (61-2575) hours. Conclusion: Therapeutic mild hypothermia might be useful to control LOS after open heart surgery, and required to pay attention to infectious comploication.

\section{4}

Transport of Sick neonates with Congenital Heart Defects Snehal Kulkarni, India

Pankaj Kasar, India

Swati Garekar, Wockhardt Pediatric and Congenital Heart Center, India

Zakiya Khan, India

Amit Mhatre, Wockhardt Pediatric and Congenital Heart Center,

Mumbai, India

Sachin Patil, Wockhardt Pediatric and Congenital Heart Center,

Mumbai, India

Prasanna Salvi, India

Suresh Joshi, India

We present our retrospectively collected data on transporting neonates with suspected or diagnosed CHD.

36 patients were transferred emergently from November 2006 to September 2008. 27 (75\%) were neonates. Our transport team comprised of a pediatric cardiac registrar and a trained nurse. The mean age of neonates was $7.85 \pm 7.1$ days; mean weight was $2.89 \pm 0.55 \mathrm{~kg}$. $17 / 27$ neonates $(63 \%)$ were on PGE1 infusion during transport. $12 / 27$ neonates were on Ionotropes. Prior to transport, our team attempted metabolic and hemodynamic stability. 23/27 newborns were on ventilator prior to transfer. During transfer, the intubated babies were placed on the neonatal resuscitator. The mean duration of transport from and to our PICU $2.5 \pm 2.3$ hrs (range 1.5-13).

There was no mortality during transport. There were no cases of emergency resuscitation in the ambulance. All neonates were found to be hemodynamically stable upon arrival to the PICU. 3/27 neonates were mildly hypothermic.

The major CHDs were TGA (6), large PDA (3), Duct dependant pulmonary circulation (8), coarctation (3).

In the same period 7 neonates came to our PICU without our transport team. PGE1 infusion was turned off inappropriately in 2 cases during transport. There were 2 tube dislodgments and 2 cases of metabolic acidosis. $5 / 7$ babies were hypothermic.
Despite resource constraints, effective pre transfer management, a trained transport team and use of neonatal resuscitator enabled us to transport neonates safely. Efforts must be made to have uniform transport guidelines across the country for sick neonates with CHD.

\section{5}

How much is the sufficient pressure for arterial-line flushing system?

Ming-Chih Lin, Taichung Veterans General Hospital, Taiwan Mi-Shu Huang, Taichung Veterans General Hospital, Taiwan Yun-Ching Fu, Taichung Veterans General Hospital, Taiwan Sheng-Ling Jan, Taichung Veterans General Hospital, Taiwan

Background: Arterial lines (A-line) insertion is a routinely performed procedure in intensive care units and operation rooms. The manufacturer's recommendation is to keep the external pressure at about $300 \mathrm{mmHg}$. However, to keep the pressure at such level can be hazardous to pediatric patients. The literature about the impact of keeping lower pressure is limited. Thus, we conduct a retrograde cohort study to explore this topic. Material and methods: We retrospectively reviewed patients who received radial arterial cannulation in a pediatric intensive unit from July 2007 to July 2008. During this period, nursing routine was changed by nursing administrations at February 2008. The pressurized bag level was reduced from $300 \mathrm{mmHg}$ to $200 \mathrm{mmHg}$. Kaplan-Meier survival analysis was applied for Aline clot and all causes A-line survival. Cox regression model was applied for searching potential confounding factors.

Results: During the study period, 123 A-lines with pressure $300 \mathrm{mmHg}$ and 98 A-lines with pressure $200 \mathrm{mmHg}$ were recorded. The mean survival time for A-line clot was 10.0 and 24.0 days for 300 and $200 \mathrm{mmHg}$ groups. The difference was statically significant. When analyzing all-causes A-line survival, the mean survival time is 6.7 days and 13.6 days for 300 and $200 \mathrm{mmHg}$ groups. It also reached statistically significant. Conclusion: Keeping lower pressure in the pressurized bag might be a safe and efficient alternative. It can reduce the potential central nervous system hazard and the cost of maintaining A-line system for pediatric patients. Further randomized trials might be needed to confirm it.

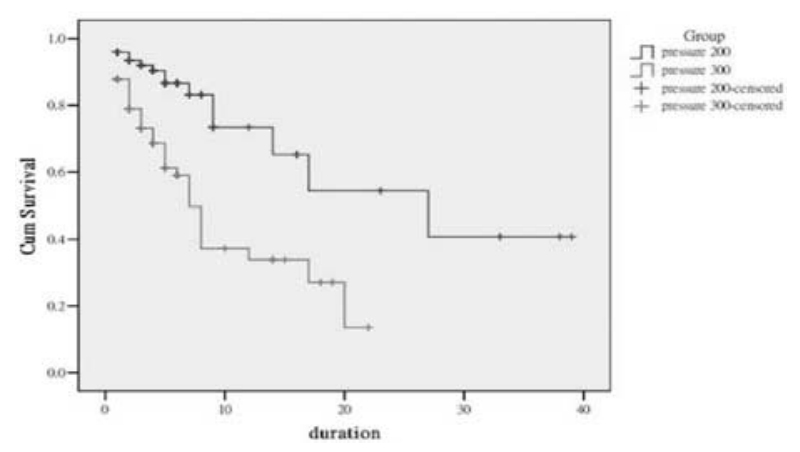

6156

The effect of duration of cardiopulmonary bypass on hemodynamics and sedative status after postoperative administration of dexmedetomidine in pediatric patients with congenital heart disease

Mitsugi Nagashima, Japan

Fumiaki Shikata, Department of Surgery, Stroke and Cardiovascular Center, Ehime University Hospital, Japan 
Masahiro Ryugo, Department of Surgery, Stroke \& Cardiovascular Center, Ehime University Hospital, Japan

Hiroshi Imagawa, Department of Surgery, Stroke \& Cardiovascular Center, Ehime University Hospital, Japan

Eiichi Yamamoto, Department of Pediatric Cardiology, Stroke E Cardiovascular Center, Ehime University Hospital, Japan Masaaki Ohta, Department of Pediatric Cardiology, Stroke \& Cardiovascular Center, Ehime University Hospital, Japan Takashi Higaki, Department of Pediatric Cardiology, Stroke E Cardiovascular Center, Ehime University Hospital, Japan Kanji Kawachi, Department of Surgery, Stroke \& Cardiovascular Center, Ehime University Hospital, Japan

Purpose: The aim of this study was to evaluate of the effect of the duration of cardiopulmonary bypass (CPB) on hemodynamics, respiratory condition and sedative state after continuous dexmedetomidine administration.

Method: Dexmedetomidine $(0.5 \mu \mathrm{g} / \mathrm{kg} / \mathrm{hr})$ was continuously administrated for postoperative sedation in 55 pediatric patients (range 1 months to 5 years) who underwent cardiac surgery. Patients were divided into two groups depending on the duration of $\mathrm{CPB}$. Twenty eight patients who had undergone $\mathrm{CPB}$ over 2 hours were eligible into long $\mathrm{CPB}$ duration group (Group L) and the remaining 27 patients were placed into short duration group (Group S). Arterial systolic and diastolic pressure, heart rate, respiratory rate and sedative score (Ramsay score) were measured pre, 1 and 3 hours after administration.

Results: Average duration of CPB were $180 \pm 41$ minutes in group L and $68 \pm 25$ minutes in group S. Average age was 1.4 years old in group L and 1.8 in group S. Percent change in systolic but not diastolic arterial pressure from pre-administration was significantly $(\mathrm{p}<0.05$, repeated measured ANOVA) decreased in group L compared with group S. Percent change in heart rate was significantly $(p<0.05)$ decreased in group $S$ compared with group L. Percent change of respiratory rate increased along time course in both groups. Sedative state did not change in both groups during administration

Conclusion: Postoperative continuous administration of dexmedetomidine could modify hemodynamics depending on the duration of $\mathrm{CPB}$ in pediatric patients and it did not suppress respiratory function and maintained good sedative status postoperatively.

\begin{tabular}{|c|c|c|c|c|c|c|}
\hline & & $\begin{array}{l}\% \text { in sys } \\
\text { AP }\end{array}$ & $\begin{array}{l}\% \text { in diast } \\
\mathrm{AP}\end{array}$ & $\%$ in $\mathrm{HR}$ & $\%$ in $\mathrm{RR}$ & $\begin{array}{l}\text { Ramsay } \\
\text { score }\end{array}$ \\
\hline \multirow[t]{3}{*}{ Group L } & pre & $100 \pm 0$ & $100 \pm 0$ & $100 \pm 0$ & $100 \pm 0$ & $4.4 \pm 1.1$ \\
\hline & $1 \mathrm{hr}$ & $95 \pm 11$ & $96 \pm 14$ & $94 \pm 6$ & $103 \pm 21$ & $4.9 \pm 0.8$ \\
\hline & $3 \mathrm{hr}$ & $91 \pm 13$ & $94 \pm 13$ & $93 \pm 7$ & $109 \pm 23$ & $406 \pm 0.9$ \\
\hline \multirow[t]{3}{*}{ Group S } & pre & $100 \pm 0$ & $100 \pm 0$ & $100 \pm 0$ & $100 \pm 0$ & $4.6 \pm 0.9$ \\
\hline & $1 \mathrm{hr}$ & $98 \pm 9$ & $99 \pm 11$ & $90 \pm 9$ & $104 \pm 28$ & $4.7 \pm 0.8$ \\
\hline & $3 \mathrm{hr}$ & $100 \pm 13$ & $102 \pm 16$ & $86 \pm 10$ & $113 \pm 40$ & $4.4 \pm 0.8$ \\
\hline \multicolumn{2}{|c|}{$\begin{array}{l}\text { Repeated measured } \\
\text { ANOVA }\end{array}$} & $p<.05$ & NS & $p<.05$ & NS & NS \\
\hline
\end{tabular}

\%: precent change, sys AP: systolic arterial pressure, diast AP: diastolic arterial pressure, HR: heart rate, RR: respiratory rate

\section{8}

Tetralogy of Fallot: Outcome of surgical repair in a resource-limited country

George Ofori-Amanfo, The Cardiac Center, Children's Hospital of Philadelphia, United States

Michael L O'Byrne, United States

Josiah AM Ruturi, Kenya
Vernon M Gracii, Kenya

Elizabeth Olaly-Nyakundi

Background: TOF accounts for $19 \%$ of unrepaired cyanotic CHD in children in developing countries. Limited access to surgery accounts, in part, for the increased prevalence of TOF. Such patients have variable life expectancy and poor quality of life. Due to resource limitation, diagnosis and surgery are delayed. Complications of prolonged cyanosis, malnutrition, endocarditis and stroke may increase risk of perioperative morbidity and mortality. We report early results and determinants of outcome in patients undergoing surgery for TOF in a developing country.

Methods and Results: Patients who underwent surgery for TOF at Mater Hospital, Kenya between March, 2005 and July 2007 were reviewed. Forty two patients were identified, 24 (57\%) males and $18(43 \%)$ females. Median age at surgery was 58 (9-204) months, $50 \%(21 / 42)$ were below 3rd percentile for weight and 59\% (19/ 32)had Oxygen saturation less than $80 \%$. Twenty five patients had hematocrit $>60 \%$ and 2 patients had Blalock-Taussig shunts. Mean CPB time was $66+/-28$ minutes, cross-clamp time was $102+/-37$ minutes. Median ICU length of stay was 3 (1-34) days, hospital LOS was 10 (1-168) days. Median intubation days was $1(0-15)$ days and duration of inotropic support was $1(0-12)$ days. Post-operative complications were pleural effusions, $24 \%$, surgical bleeding, 10\%, wound infection, 10\%. JET occurred in 1 patient. Survival to hospital discharge was $83 \%$ (35/42). Preoperative saturation $<80 \%$ was a risk factor for complications, $\mathrm{p}=0.036$. Malnutrition was not a risk factor for morbidity, $\mathrm{p}=0.75$ nor blood products requirement, $\mathrm{p}=0.5$. Shorter intubation was associated with shorter ICU stay, $\mathrm{p}>0.0001$ and hospital stay, $\mathrm{p}=0.0002$.

Conclusion: TOF repair can be performed in developing countries with good outcomes.

\section{9}

Neutrophil gelatinase-associated lipocalin (NGAL): Is it a good predictor of radiocontrast nephrotoxicity in children undergoing angiography?

Deniz A Oguz, Gazi University Medical Faculty Pediatric Cardiology Department, Turkey

Rana F Olgunturk, Gazi University Medical Faculty Pediatric Cardiology Department, Turkey

Sedef F Tunaoglu, Gazi University Medical Faculty Pediatric Cardiology Department, Turkey

Oguz Soylemezoglu, Gazi University Medical Faculty Pediatric

Nephrology Department, Turkey

Cihat Sanli, Gazi University Medical Faculty Pediatric Cardiology

Department, Turkey

Abdulkadir Eren, Gazi University Medical Faculty Pediatric Cardiology Department, Turkey

Objective: Radiocontrast nephrotoxicity ( $\mathrm{RCN})$ is a common and important cause of hospital-acquired renal insufficiency and a well-recognized complication of cardiac angiography. RCN is generally mild and reversible but can lead to prolonged hospitalization, increased health care costs, and substantial morbidity and mortality. Neutrophil gelatinase-associated lipocalin (NGAL) is an early predictive biomarker of renal failure unlike serum creatinine levels.

Method: We prospectively enrolled 46 children (age 0-16 years, median: 3 yrs) with congenital heart disease (9 cyanotic, 37 acyanotic) undergoing elective cardiac catheterization and angiography with non-ionic contrast (IOPROMID $769 \mathrm{mg} /$ 
ml) administration in our study. Serial urine and plasma samples (baseline, $4 \mathrm{hr}, 24 \mathrm{hr}$, and $48 \mathrm{hr}$ after the procedure) were analyzed for NGAL and creatinine.

Results: The volume of contrast medium injected was $4.20 \pm 2.18 \mathrm{cc} / \mathrm{kg}$. $(0,65-10,6 \mathrm{cc} / \mathrm{kg})$. RCN, defined as a $25 \%$ increase in serum creatinine from baseline, was found in 17 subjects (34\%). However, in 36 patients (72\%) serum NGAL levels were increased (in some up to 9 fold) after 4 hours of contrast administration. The volume of contrast medium injected between the patient group who had increased NGAL levels and who had not differed significantly $(4,77 \pm 2,04$ vs $3,00 \pm 2,01 \mathrm{cc} / \mathrm{kg}, \mathrm{p}<0,01)$.

Conclusion: The dependency of creatinine to some factors like age, gender, muscle mass, muscle metabolism, medications, hydration status, and delayed response to renal function loss which make take several days are important disadvantages. According to the results of our study plasma NGAL levels have emerged as sensitive, specific and highly predictive early biomarker of RCN in children.

\section{0 \\ Right atrial thrombus successfully treated with tissue plasminogen activator and heparin in two neonates \\ Ji-Ae Park, Department of Pediatrics, Pusan National University Children's Hospital, Korea \\ Hyoung-Doo Lee, Department of Pediatrics, Pusan National University Children's Hospital, Korea \\ Min Jung Cho, Department of Pediatrics, Pusan National University Children's Hospital, Korea}

Introduction: Intracardiac thrombosis is rare in neonates. Mostly thrombi occur in right heart and are associated with central venous catheter (CVC), congenital heart disease, sepsis and peripartum asphyxia. Anticoagulants, fibrinolytic drugs or surgical removal are used for the treatment of intracardiac thrombi in neonates, but are still controversial. We report that CVC-related right atrial (RA) thrombus were treated successfully with tissue plasminogen activator (t-PA) and heparin in two neonates with transposition of the great arteries (TGA).

Cases: The first neonate, aged 22 days, developed a RA thrombus $(2 \times 1.2 \mathrm{~cm})$ following arterial switch operation(ASO) with CVC insertion. The second, aged 32days, developed a RA thrombus $(1 \times 1.2 \mathrm{~cm})$ following ASO with CVC insertion. Both were treated by combination with t-PA and heparin. T-PA was given intravenously in 6 hour infusion with daily dosage being steadily increased $(0.10 \sim 0.25 \mathrm{mg} / \mathrm{kg} / \mathrm{h})$ and heparin was continuously infused at $50 \mathrm{U} / \mathrm{kg} / \mathrm{h}$. We treated for 14 days with daily echocardiography and echocardiography showed progressive thrombus reduction. During and after treatment, major bleeding was not.

Conclusion: T-PA and heparin was safe and effective in neonates with CVC related RA thrombus.

\section{2}

Renal insufficiency in young children is uncommon after cardiac surgery despite aggressive administration of nephrotoxic drugs

Christina M Phelps, The Children's Hospital of Denver, United States Jennifer C Eshelman, The Children's Hospital of Denver, United States Jill Ibrahim, The Children's Hospital of Denver, United States

Jon Kaufman, The Children's Hospital of Denver, United States

The development of renal insufficiency (RI) following cardiac surgery can lead to a prolonged stay in the intensive care unit and has been linked with increased mortality. This study was undertaken to evaluate the incidence of RI after cardiac surgery and the routine administration of nephrotoxic drugs. A retrospective review of the EMR of patients $<2$ years-old admitted to the CICU after undergoing cardiac surgery from 3/1/07 until 9/30/08 was conducted with IRB approval. Patients receiving intravenous furosemide ( $+/$-additional diuretics) and ACE inhibition in the form of captopril or enalapril/enalaprilat were included.

322 patients $<2$ were admitted to the CICU during the review period. Of those patients, $176(54 \%)$ received both furosemide and ACE inhibitors following surgery. RI (defined as a creatinine as $>0.8 \mathrm{mg} / \mathrm{dl}$ and a BUN of $>20 \mathrm{mg} / \mathrm{dl}$ ) occurred in less than $10 \%$ of this population (25 patients had 26 episodes). Peak creatinine occurred from 32 days prior to 8 days after the initiation of ACE inhibitors with 17/26 episodes occurring prior to initiation of ACE inhibition. Elevated creatinine correlated more closely with diuretic use (6 days prior to 12 days following peak furosemide administration) with all patients receiving loop diuretic therapy during the period of RI. Peak BUN also related more closely to diuretic use than to initiation or up-titration of ACE inhibition. RI occurred in $<10 \%$ of patients in our study and appears to be more closely correlated with aggressive diuretic use and the patient's peri-operative hemodynamics rather than renal toxicity from the use of ACE inhibitors.

\section{3}

Use of inhaled Nitric Oxide in the treatment of post operative pulmonary arterial hypertension

$R$ Suresh Kumar, ICVD, Madras Medical Mission, Chennai, India Roy Varghese, ICVD, Madras Medical Mission, Chennai, India G Selvakumar, ICVD, Madras Medical Mission, Chennai, India G Madhusudan, ICVD, Madras Medical Mission, Chennai, India G Ganeshkumar, ICVD, Madras Medical Mission, Chennai, India Atul Prabhu, ICVD, Madras Medical Mission, Chennai, India P Sreeja, ICVD, Madras Medical Mission, Chennai, India Udaycharan Murmu, ICVD, Madras Medical Mission, Chennai, India

Objective: Evaluation of the role of inhaled Nitric Oxide (iNO) in critical peri-operative pulmonary hypertension (PAH).

Methods: This was an observational study of 41 consecutive patients (age 15 days -28 years, median- 4 months) who required iNO for management of critical peri-operative $\mathrm{PAH}$ from January 2001 to August 2008.

Results: The diagnostic categories and outcome are shown in table 1. iNO (10 to $80 \mathrm{ppm}$, mean duration 79.6 hours) was added to a regimen of Adrenaline/Dopamine/Milrinone for systemic/suprasystemic pulmonary pressures causing inability to come off bypass (n33,80\%), inability to be weaned off ventilator $(\mathrm{n} 6,15 \%)$ or persistent hypoxemia (n2, 5\%). Outcome measures were successful management of the crisis, decrease in PA pressure and discharge from hospital. Mean PA pressure of the group decreased by $10.5 \mathrm{~mm}$ of $\mathrm{Hg}(\mathrm{p}<0.05)$, post iNO (Figure 1). All the patients who were started on iNO were weaned-off bypass successfully. Ten (24.4\%) patients (age 1 month - 28 years, median 4 months) showed no response to iNO and died in the ICU (TGA/VSD 4, TAPVC 4). 2 responders died later from septicemia. All others were extubated and discharged. The survivors had significantly lower mean PA pressures compared to those who died $(\mathrm{p}<0.05)$. Serum methemoglobin levels were $>1.5 \%$ in $11(27 \%)$ patients.

Conclusion: Addition of iNO to a conventional drug regimen is a useful strategy for salvaging critically ill patients with perioperative PAH especially when there is difficulty to come off bypass or ventilator. 


\begin{tabular}{lllll}
\hline Diagnosis & $\begin{array}{l}\text { Number } \\
(\mathbf{N}=41)\end{array}$ & $\begin{array}{l}\text { Mean PA pre } \\
\text { iNO (mm Hg) }\end{array}$ & $\begin{array}{l}\text { Mean PA post iNO } \\
(\mathrm{mm} \mathrm{Hg})\end{array}$ & $\begin{array}{l}\text { Mortality } \\
(\%)\end{array}$ \\
\hline AV canal defect & 6 & 51 & 35 & $1(16.6)$ \\
TGA/VSD & 5 & 54 & 36 & $4(80)$ \\
Truncus & 5 & 36 & 32 & NIL \\
VSD & 9 & 45 & 35 & $1(11)$ \\
AP Window & 1 & 32 & 36 & NIL \\
TAPVC & 14 & 47 & 34 & $5(35.7)$ \\
Miscellaneous & 1 & 52 & 72 & $1(100)$ \\
\hline
\end{tabular}

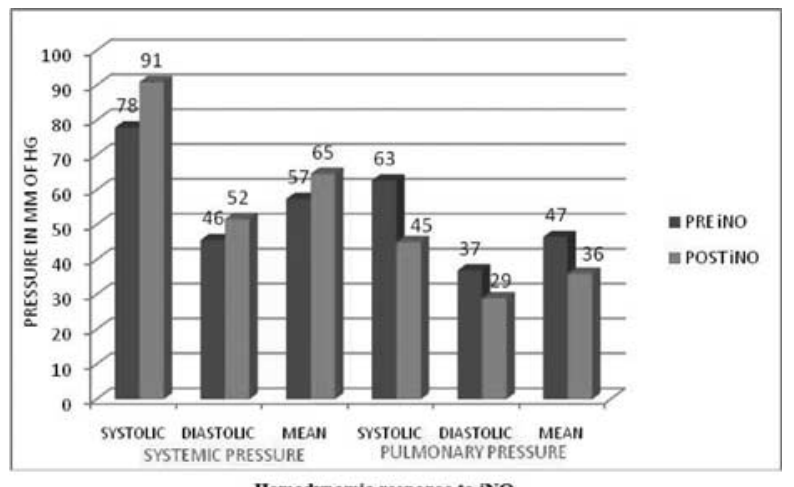

6164

A Pilot study to assess the safety and efficacy of stem cell therapy in paediatric population with cardiac problems refractory to conventional surgical or medical management

Premsekar Rajasekaran, Frontier Lifeline Hospital (Dr.K.M.Cherian

Heart Foundation), India

Anuradha Sridhar, India

Shanthi Chidambaram, India

Subramaniam Raghavan, India

Ravi Agarwal, Frontier Lifeline \& Dr. K M Cherian Heart

Foundation, Chennai, India

Soma Guhathakartha, India

Kotturathu M Cherian, India

Objectives: Stem cell therapy has been reported to improve cardiac function, reduce pulmonary pressure and improve exercise tolerance in adult patients. The aim of this study is to assess the safety and efficacy of stem cell therapy in patients $<18$ years of age with end stage cardiac failure and severe primary or secondary pulmonary hypertension.

Method: 8 patients underwent stem cell therapy. The age ranged from 0.4 to 11 years (Mean age 5.7). Transcoronary implantation of autologous peripheral blood endothelial cells was done in 3 patients with DCM. Transcatheter implantation of autologous bone marrow derived mononuclear cell suspension into RPA and LPA was undertaken in 2 patients with primary pulmonary hypertension and in 1 patient with pulmonary hypertension operated for VSD. Peroperative transmyocardial injection of stem cells was undertaken in 2 infants, the first receiving parent donated peripheral blood endothelial progenitor cells at the time of ALCAPA repair and the second, autologous bone marrow derived mononuclear cells during VSD closure for associated noncompaction of LV.

Results: (As in Table 1)

Conclusion: Stem cell therapy in the pediatric population is safe in the short term period. Intra coronary stem cell implantation for dilated cardiomyopathy results in transient improvement in symptoms and cardiac function. Intrapulmonary stem cell implantation appears to have contributed to significant reduction in pulmonary hypertension in one patient with primary pulmonary hypertension. Intramyocardial implantation of stem cells may have contributed to the improved postoperative cardiac function and recovery in an otherwise high risk substrate of noncompaction of myocardium.

\begin{tabular}{|c|c|c|c|}
\hline Diagnosis & $\begin{array}{l}\text { Number of } \\
\text { patients }\end{array}$ & Clinical outcome & $\begin{array}{l}\text { Follow up } \\
\text { details }\end{array}$ \\
\hline $\begin{array}{l}\text { Dilated } \\
\text { Cardiomyopathy }\end{array}$ & 3 & $\begin{array}{l}2 \text { (Transient improvement of } \\
\text { LVEF and reduction of serum } \\
\text { BNP at } 3 \text { month follow up } \\
\text { but failed to sustain the } \\
\text { improvement over a longer } \\
\text { period.) }\end{array}$ & $\begin{array}{l}\text { No mortality. } 1 \\
\text { patient lost } \\
\text { to follow-up. }\end{array}$ \\
\hline $\begin{array}{l}\text { Severe primary } \\
\text { pulmonary } \\
\text { hypertension }\end{array}$ & 2 & $\begin{array}{l}1 \text { (awaiting repeat cardiac } \\
\text { catheterization to assess } \\
\text { pulmonary pressure) }\end{array}$ & 1 mortality. \\
\hline $\begin{array}{l}\text { Persisting pulmonary } \\
\text { hypertension post } \\
\text { VSD surgical } \\
\text { closure }\end{array}$ & y & $\begin{array}{l}1 \text { (awaiting repeat cardiac } \\
\text { catheterization to assess } \\
\text { pulmonary pressure) }\end{array}$ & No mortality. \\
\hline \multirow[t]{2}{*}{$\begin{array}{l}\text { Anomalous origin of } \\
\text { left coronary artery } \\
\text { from pulmonary } \\
\text { artery }\end{array}$} & 1 & $\begin{array}{l}\text { Significant and rapid resolu- } \\
\text { tion of the LV dilatation and } \\
\text { dysfunction with transient } \\
\text { hypertrophic response of } \\
\text { the LV myocardium. }\end{array}$ & No mortality. \\
\hline & & $\begin{array}{l}\text { Noncompaction of the left } \\
\text { ventricle with associated VSD }\end{array}$ & 1 \\
\hline
\end{tabular}

\section{6}

Methylprednisolone in neonates following cardiac surgery $\sim$ Potent rapid vasopressor?

Kazuyoshi Shimizu, Dept. of Anesthesiology and Resuscitology, Okayama University Medical School, Japan

Hiroshi Morimatsu, Dept. of Anesthesiology and Resuscitology,

Okayama University Medical School, Japan

Yuichiro Toda, Dept. of Anesthesiology and Resuscitology, Okayama University Medical School, Japan

Satoshi Suzuki, Dept. of Anesthesiology and Resuscitology, Okayama University Medical School, Japan

Tomohiko Suemori, Dept. of Anesthesiology and Resuscitology,

Okayama University Medical School, Japan

Tatsuo Iwasaki, Dept. of Anesthesiology and Resuscitology, Okayama University Medical School, Japan

Kiyoshi Morita, Dept. of Anesthesiology and Resuscitology, Okayama University Medical School, Japan

Background: We have investigated retrospectively the global hemodynamic effects of methylprednisolone (MP) in 3 hours after administration in the neonates after cardiac surgery.

Methods: A retrospective chart review was conducted for 67 neonates with congenital heart disease, who underwent cardiac surgery from 2004 to 2005 at Okayama University Hospital. We collected their demographics, operative variables, ICU admission variables, and outcomes. They were divided into two groups, steroid (S) group and non-steroid (NS) group, whether received steroid treatment in the ICU period. Comparisons have been done between the groups using Student's t-test. Among S group, we also assessed 5 global hemodynamic changes including the dose of epinephrine, mean arterial pressure (MAP), base excess (BE), lactate concentration (Lac), and urine output (UO) between pre- 3 hours and post- 3 hours of steroid administration. Data were expressed as mean with $95 \%$ confidence interval, and a $\mathrm{p}$ value $<0.05$ was considered as statistically significant.

Results: Of 67 neonates, $40(57.1 \%)$ received MP with a mean dose of $10.0 \mathrm{mg} / \mathrm{kg}(8.3,11.7)$. The $\mathrm{S}$ group received more 
complex operation. On ICU admission, the $\mathrm{S}$ group was severer than the NS group, although their ICU survival did not differ. Three hours after the administration of MP, MAP was significantly increased, however, BE, Lac, and UO did not improve significantly (Table).

Conclusion: Steroid was administered in neonates who received more complex surgery, and who were hypotensive. The effect of methylprednisolone has already occurred within 3 hours after administration as "vasopressor" rather than "inotrope".

\begin{tabular}{lccc}
\hline & Pre steroid & Post steroid & P value \\
\hline Epinephrine dose $(\mathrm{mcg} / \mathrm{kg} / \mathrm{min})$ & $0.05(0.03,0.07)$ & $0.056(0.036,0.075)$ & 0.23 \\
MAP (mmHg) & $52.7(49.9,55.5)$ & $56.7(53.8,59.6)$ & 0.001 \\
Base excess $(\mathrm{mmol} / \mathrm{L})$ & $0.2(-1.3,1.6)$ & $-0.3(-1.6,1.1)$ & 0.24 \\
Lactate $(\mathrm{mmol} / \mathrm{L})$ & $3.8(2.7,5.0)$ & $4.4(2.6,6.2)$ & 0.35 \\
Urine output $(\mathrm{ml} / \mathrm{kg} / \mathrm{h})$ & $3.3(2.5,4.2)$ & $3.9(2.8,5.0)$ & 0.31 \\
\hline
\end{tabular}

6167

Clinical impact of vasopressin infusion in cardiac arrest and refractory hypotension in the pediatric cardiac intensive care unit

Vishal K Singh, ESCORT'S HEARTINSTITUTE AND

RESEARCH CENTRE, NEW DELHI, INDIA, India

Amit Agrawal, ESCORT'S HEART INSTITUTE AND

RESEARCH CENTRE, NEW DELHI INDIA, India

Amit Varma, ESCORT'S HEART INSTITUTE AND

RESEARCH CENTRE, NEW DELHI INDIA, India

Rajesh Sharma, Escorts Heart Institute and Research Centre,

New Delhi., India

Objective: To assess the effect of vasopressin(AVP) on hemodynamics when used as an infusion for refractory hypotension and post cardiac arrest.

Material And Methods: A prospective study conducted from February 08 to august 08 in a tertiary pediatric cardiac critical care unit.

Twenty patients (median age 4.5 months ) underwent surgery for complex congenital heart disease and required AVP infusion for more than 60 mins were included. AVP infusion was started following cardiac arrest in 9 patients \& in 11 cases was instituted for refractory hypotension. Vasopressin was administered as a bolus during cardiac arrest at 0.4 units $/ \mathrm{kg} /$ dose, and the infusion was administered within the dose range 0.0001 to 0.003 units $/ \mathrm{kg} / \mathrm{min}$. Results: The mean arterial blood pressure (ABP) improved from $42 \pm 15.3 \mathrm{~mm}$ hg to $58 \pm 16.8 \mathrm{~mm}$-hg after one hour and after 24 hours the mean $\mathrm{ABP}$ improved to $68 \pm 19.3 \mathrm{~mm}$ hg. No significant increase in the heart rate was observed . Inotrope score decreased from $34 \pm 13$ at baseline to $26 \pm 7$ after one hour and $22 \pm 8$ after 24 hours of AVP infusion There was no significant alteration observed in urine output, and serum sodium levels. The median duration of vasopressin infusion was $55.5 \mathrm{hrs}$ (range $12 \mathrm{hrs}-237 \mathrm{hrs}$ ).

Conclusion: Vasopressin infusion does have a role as a rescue therapy in cases with refractory hypotension and cardiac arrest. However further trials are required to validate efficacy, adverse effects and dosage and interaction with other inotropic agents in pediatric intensive care units.

\section{8}

Extracorporeal membrane oxygenation as a evolving treatment modality for low cardiac output state in developing countries

Vishal K Singh, ESCORT'S HEART INSTITUTE AND

RESEARCH CENTRE, NEW DELHI, INDIA, India
Priya Poonkodi, ESCORT'S HEART INSTITUTE AND

RESEARCH CENTRE, NEW DELHI, INDIA, India

Rajesh Sharma, Escorts Heart Institute and Research Centre,

New Delhi., India

Amit Varma, ESCORT'S HEART INSTITUTE AND

RESEARCH CENTRE, NEW DELHI, INDIA, India

Objective: To assess the efficacy of bridging with rescue venoarterial extracorporeal membrane oxygenation (ECMO) after repair of congenital cardiac defects

Methods: From September 07 to December 08, 16 cases underwent ECMO and all cases had intra thoracic cannulation. Ten cases underwent ECMO post arterial switch operation for D-Transposition of great arteries (D-TGA) with severe LV dysfunction. Four cases required ECMO following prolonged cardiac arrest after intracardiac repair for Tetralogy of Fallot (TOF) and Rastelli procedure for TOF and pulmonary atresia . Two cases required emergency ECMO following prolonged cardiac arrest after severe pulmonary artery hypertensive $(\mathrm{PAH})$ crisis with severe right ventricular (RV) dysfunction.

Results: The mean age and weight were $184.45 \pm 181.69$ days and $6.79 \pm 3.91 \mathrm{Kg}$ ECMO was successfully weaned off in $69.2 \%(11 / 16)$ and the mean duration of ECMO was 97 hours. One case required premature weaning off ECMO due to excessive bleeding. Eleven cases were successfully discharged and the mean intensive care unit (ICU) stay was 22 days after weaning off ECMO. Five patients expired. One patient had ventricular dysrhythmia on ECMO and three cases had choreoathetoid movements following ECMO weaning which responded very well to oral sodium valproate. The average cost of ECMO per day in our unit was approximately 300 US $\$$, besides standard ICU costs. Conclusion: With a rising trend of complex CHD's being operated in developing countries, ECMO as a rescue therapy holds promise, with a small increment in ICU costs.

\section{9}

Fate of ductal stent deployed in pulmonary circulation on a critically closing ductus arteriosus

Kothandam Sivakumar, MIOT Hospital, Chennai, India, India Hariprakash Suriyanarayanapillai, MIOT Hospital, Chennai, India, India

Radhakrishnan Satish, MIOT Hospital, Chennai, India, India Arunkumar Govindarajan, MIOT Hospital, Chennai, India, India Robert Coelho, MIOT Hospital, Chennai, India, India

Anpon Bhagyavathy, MIOT Hospital, Chennai, India, India

Objective: Ductal stent (DS) offers an alternative to Blalock Taussig shunt (BTS) in cyanotic diseases. We analyze procedural results and followup till the next palliation.

Methods: Cyanotic infants were offered DS instead of BTS. After aortogram under sedation with ketamine or general anesthesia, Prostaglandin E1 stopped, ducts were cannulated with cut pigtail catheter and coronary guidewires and entire duct stented with 3.5 or $4 \mathrm{~mm}$ (former in infants under $2.5 \mathrm{~kg}$ ) coronary stents. Associated procedures included pulmonary valve perforation and balloon dilatation. Subsequent care included 48 hours heparinization, aspirin and clopidogrel, monthly monitoring for reappearance of hypoxia. Results: 19 patients aged 3 days to 1year had DS, weight ranged 2.1-6 kg. 17 were neonates. Pulmonary valve perforation was done in 4 patients, others had DS alone. Duct anatomy was vertical in 8 patients, tortuous in 7, contralateral in 3 and bilateral in one. The mean oxygenation improved from $58 \%$ to $84 \%$, mean hospital stay was $3.1+/-0.8$ days. There was one procedural death due to acute stent thrombosis when guidewire 
was removed; two hospital deaths due to pulmonary overcirculation, hypotension and myocardial failure, one late sudden death at home. Next palliation in 15 patients included bidirectional glenn surgery in 5, conduit repair of Fallot's tetralogy in 6, BTS in one. The mean duration till next palliation was $4.6+/-3.1$ months ( 2 weeks to 11 months). Four patients who had balloon pulmonary dilatation had no further surgery. Conclusions: DS offers a less morbid alternative to BTS in duct dependent patients; however with considerable procedural and interstage mortality.

\section{0}

Hybrid neonatal stage I management of hypoplastic left heart syndrome in India, a developing economy

Hariprakash Suriyanarayanapillai, MIOT Hospital, Chennai, India, India

Kothandam Sivakumar, MIOT Hospital, Chennai, India, India

Robert Coelho, MIOT Hospital, Chennai, India, India

Arunkumar Govindarajan, MIOT Hospital, Chennai, India, India

Radhakrishnan Satish, MIOT Hospital, Chennai, India, India

Ramyashri Chandrasekaran, MIOT Hospital, Chennai, India, India

Objective: Stage I palliation of hypoplastic left heart syndrome (HLHS) is morbid in developing countries. A less invasive hybrid approach was adopted in a low birth weight baby.

Methods: HLHS was diagnosed in one of twin delivered with birth weight of $2.2 \mathrm{~kg}$. Echocardiogram showed aortic atresia, restrictive foramen ovale, adequate right ventricular and tricuspid valve function. After ProstaglandinE1 (PGE1) and dopamine infusions for 5 days, bilateral pulmonary artery banding to $3 \mathrm{~mm}$ was done through sternotomy while ventilating in $21 \%$ oxygen. After a pursestring supported insertion of a 6 French introducer sheath through the main pulmonary artery, angiogram was done in lateral view with cranial angulation. The entire duct length was stented with $8 \mathrm{~mm}$ balloon expandable stent. The retrograde aortic arch flows were uninterupted. After stent placement, the PGE1 infusion was stopped. Adequate Rashkind balloon atrial septostomy was done through a right femoral venous access.

Results: Systemic pressures stabilised after bilateral pulmonary artery banding and ductal stent. Baby was extubated in less than 24 hours, and given oral aspirin. On two month follow up, the baby weighed $4.1 \mathrm{~kg}$, there was a $15 \mathrm{mmHg}$ gradient across the distal end of ductal stent, which was dilated during a cardiac catheterization done at 75 days. The baby awaits second stage Norwood procedure. At the last follow up right ventricular and tricuspid valve function are good.

Conclusion: Stage I hybrid management of HLHS offers a less morbid and more predictable approach than conventional Norwood especially in the developing world.

6171

Relative plasma Brain Natriuretic Peptide (BNP) level may predict ability to wean from extracorporeal life support (ECLS)

Timothy $R$ Thiruchelvam, Intensive Care Unit, Royal Childrens

Hospital, Melbourne, Australia

Bryn Jones, Department of Cardiology, Royal Childrens Hospital, Melbourne, Australia

Michael Cheung, The Royal Children's Hopsital, Melbourne, Australia Yves D'Udekum, Department of Cardiac Surgery, Royal Childrens Hospital, Melbourne, Australia

Lara S Shekerdemian, Intensive Care Unit, Royal Childrens Hospital, Melbourne, Australia
W W Butt, Intensive Care Unit, Royal Childrens Hospital, Melbourne, Australia

Objective: To determine whether the relative change in plasma BNP during weaning from ECLS predicts successful separation from mechanical support in children.

Methods: A prospective cohort study, in children receiving ECLS for cardiac support in a tertiary paediatric intensive care unit between December 2007 and November 2008. Weaning duration was calculated $\left(t_{1}-t_{0}\right)$ where $t_{0}$ was the time at which ECLS was maximal, immediately prior to commencing weaning, and $t_{1}$ was the time of the final decision whether or not to decannulate. Plasma BNP samples were taken times $t_{0}$ and $t_{1}$ $\left(\mathrm{BNP}_{0}, \quad \mathrm{BNP}_{1}\right.$ respectively)and the plasma $\mathrm{BNP}$ ratio $\left(\mathrm{BNP}_{1}: \mathrm{BNP}_{0}\right)$ was calculated for each weaning attempt. The ECLS team were blind to plasma BNP levels.

Results: Nineteen weaning attempts were made in eighteen children, median age 101 days (range 1 day-15.7 years). Thirteen patients required ECLS after surgery for congenital heart disease, two had uncontrolled arrhythmia and three had refractory septic shock. The overall median duration of weaning $\left(\mathrm{t}_{1}-\mathrm{t}_{0}\right)$ was 18.5 hours (range 3-92.5 hours). Median $\mathrm{BNP}_{0}$ was $600 \mathrm{pg} / \mathrm{ml}$ (IQR 253-635 pg/ml) and $\mathrm{BNP}_{1}$ was $555 \mathrm{pg} / \mathrm{ml}$ (IQR $353-763 \mathrm{pg} / \mathrm{ml}$ ). Twelve weaning attempts were successful, and seven were not. Weaning time $t_{1}-t_{0}$ was 19.8 hours and 16.0 hours for successful and unsuccessful groups respectively $(\mathrm{p}=0.23) . \quad \mathrm{BNP}_{1}: \mathrm{BNP}_{0}$ was lower in patients that were successfully weaned (median 0.86 , range $0.1-2.0$ ), than for those who could not be weaned median $2.0(0.9-3.3)(\mathrm{p}=0.009)$. Conclusions: $\mathrm{BNP}_{1}: \mathrm{BNP}_{0}$ may be a useful adjunctive predictor of successful weaning from ECLS in children.

6172

Acute kidney injury following cardiac surgery in children -application of pRIFLE-

Yuichiro Toda, Department of Anesthesia, Okayama University Medical School, Japan

Tatsuo Iwasaki, Department of Anesthesia, Okayama University

Medical School, Japan

Kazuyoshi Shimizu, Department of Anesthesia, Okayama University Medical School, Japan

Satoshi Suzuki, Department of Anesthesia, Okayama University

Medical School, Japan

Tomohiko Suemori, Department of Anesthesia, Okayama University

Medical School, Japan

Kiyoshi Morita, Department of Anesthesia, Okayama University

Medical School, Japan

Shingo Kasahara, Department of Cardiac Surgery, Okayama University

Medical School, Japan

Shunji Sano, Department of Cardiac Surgery, Okayama University

Medical School, Japan

Objective: To identify incidence of Acute kidney injury(AKI) and outcome of this complication in children following cardiac surgery.

Methods: A retrospective cohort study using the prospectively collected electronic database. Children under 18 years old admitted to cardiac care unit (CCU) during 2007 were included. Only creatinin component of pRIFLE criteria(Akcan-Arikan, Kidney Int 71, 2007) was used to classify AKI in this study. Then patients were categorized into Normal (N), Risk (R), Injury (I), or Failure $(\mathrm{F})$ by change in Estimated creatinin clearance.

Results: Our data identified 322 children under 18 years of age. Their median age was 20.5 months, body weight was $8.6 \mathrm{~kg}$ and 
gender male was $183 / 322(56.8 \%)$. By the pRIFLE criteria, there were 100 children with AKI on admission to CCU. Forty-four children were classified as R, 26 were I, and 33 were F. Some of them developed to worse category judged by $\max$ serum creatinin during stay. These children with AKI had significantly higher mortality $(7.0 \%$ vs $0.45 \%, p=0.0014)$, longer duration of mechanical ventilation (75 vs 8 hrs, $\mathrm{p}<0.0001$ ), longer duration of CCU stay (7 vs 4 days, $\mathrm{p}<0.0001$ ), and higher incidence of peritoneal dialysis requirement $(16.0$ vs $2.3 \%)$ compared with children without AKI.

Conclusion: About one third of children under 18 years of age following cardiac surgery developed AKI within $24 \mathrm{hrs}$ after admission to intensive care unit when classified by the creatinin component of pRIFLE. These children with AKI required more ventilatory and/or renal supports.

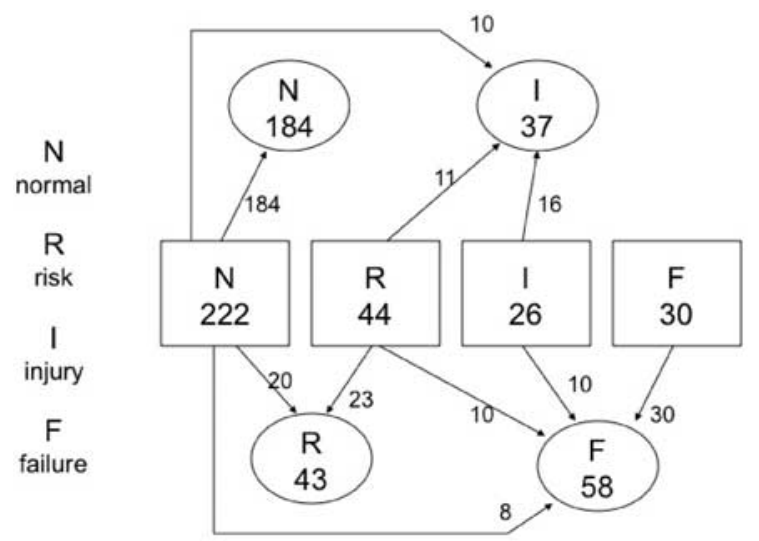

criteria durig first $24 \mathrm{hrs}$ after admission

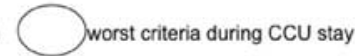

6174

Response to Bosentan in Children with Functional Single Ventricle

Zhuoming Xu, Department of cardiovascular surgery, Shanghai Children's Medical Center, Medical college of Shanghai Jiaotong University, China

Limin Zhu, Department of cardiovascular surgery, Shanghai Children's Medical Center, Medical college of Shanghai Jiaotong University, China

Xiaoman Cai, Department of cardiovascular surgery, Shanghai Children's Medical Center, Medical college of Shanghai Jiaotong University, China

Gang Ji, Department of cardiovascular surgery, Shanghai Children's Medical Center, Medical college of Shanghai Jiaotong University, China

Jinfen Liu, Department of cardiovascular surgery, Shanghai Children's Medical Center, Medical college of Shanghai Jiaotong University,

China

Zhaokang Su, Department of cardiovascular surgery, Shanghai Children's Medical Center, Medical college of Shanghai Jiaotong University, China

Objective: To describe an early experience of treating 14 children with the dual endothelin receptor antagonist bosentan, which is known to be safe and effective in adults with pulmonary hypertension.

Background: In cavopulmonary anastomoses (Glenn shunt and its variants, Fontan palliation and its variants), even slight increases in pulmonary vascular resistance can significantly lower the transpulmonary blood flow, therefore, leading to circulation failure. Similar unknowns exist regarding potential for benefit of administration of pulmonary vasoactive agents in functional single ventricle (FSV).

Design: In this prospective, observational study, 14 children with FSV of high-risk factors undergoing cavopulmonary anastamosis received bosentan therapy (Table). The median age was 11 months (range 5 months -15 years).

Interventions: Bosentan was given as $2-4 \mathrm{mg} / \mathrm{kg} /$ day, twice daily. At the cutoff date, children were treated for a mean of 3.4 months (range 2-5 months).

Main outcome measures: Response to treatment was judged by WHO functional class, six minute walk test, and oxygen saturation.

Results: Bosentan was well tolerated. WHO class, six minute walk test, and oxygen saturation improved significantly (Figure 1, 2). Conclusion: These data suggest that bosentan, an oral endothelin ETA/ETB receptor antagonist, is efficacious for the treatment of high-risk FSV children. Confirmation of these results in a randomized, controlled trial is essential.

\begin{tabular}{llllll}
\hline $\begin{array}{l}\text { Patient } \\
\text { No. }\end{array}$ & Age & Sex & Weight $(\mathrm{kg})$ & Diagnosis & Procedure \\
\hline 1 & & & & & \\
& $24 \mathrm{~m}$ & $\mathrm{~F}$ & 10 & $\begin{array}{l}\text { TA/TGA/VSD/ } \\
\text { ASD/PH S/P PAB }\end{array}$ & R-BDG \\
2 & $24 \mathrm{~m}$ & $\mathrm{~F}$ & 8.5 & SV/SA/PA & L-BDG \\
3 & $12 \mathrm{~m}$ & $\mathrm{M}$ & 6.5 & HLHS S/P Norwood? & Bi-BDG \\
4 & $5 \mathrm{~m}$ & $\mathrm{M}$ & 5 & L-TGA/VSD/SA/PS & Bi-BDG \\
5 & $7 \mathrm{~m}$ & $\mathrm{M}$ & 5.5 & HRHS & R-BDG \\
6 & $11 \mathrm{~m}$ & $\mathrm{M}$ & 8.5 & SV/SA/CAVSD/PS & R-BDG \\
7 & $3 \mathrm{y}$ & $\mathrm{F}$ & 12 & PA/VSD & Bi-BDG \\
8 & $8 \mathrm{~m}$ & $\mathrm{~F}$ & 7 & SV/SA/PS & R-BDG \\
9 & $7 \mathrm{y}$ & $\mathrm{F}$ & 12 & SV/PS & R-BDG \\
10 & $18 \mathrm{~m}$ & $\mathrm{M}$ & 10 & Ebstein's anomaly & 1 1/2 repair \\
11 & $3 \mathrm{y}$ & $\mathrm{M}$ & 13 & PA/IVS & 1 1/2 repair \\
12 & $3 \mathrm{y}$ & $\mathrm{M}$ & 13 & TA/PA/VSD & Staged Fen. Fontan \\
13 & $4 \mathrm{y}$ & $\mathrm{F}$ & 13 & SV/CAVSD/PS & Fen. Fontan \\
14 & $15 \mathrm{y}$ & $\mathrm{M}$ & 35 & SV/SA/PS & Fen. Fontan \\
\hline
\end{tabular}

\begin{tabular}{llllll}
\hline & \multirow{2}{*}{$\begin{array}{l}\text { Initials of } \\
\text { Bosentan }\end{array}$} & \multicolumn{2}{l}{ Conventional treatment } & $\begin{array}{c}\text { Duration of } \\
\text { Patient No. }\end{array}$ \\
\cline { 3 - 6 } & & diuretics & ACEI & Digoxine & \\
\hline & & & & & \\
1 & 3m post-op & Y & N & N & $3 \mathrm{~m}$ \\
2 & 1w post-op & Y & N & N & $5 \mathrm{~m}$ \\
3 & 1w post-op & Y & N & N & $2 \mathrm{~m}$ \\
4 & 1w post-op & Y & N & Y & $3 \mathrm{~m}$ \\
5 & 4d post-op & Y & N & N & $3 \mathrm{~m}$ \\
6 & 1w post-op & Y & Y & N & $3 \mathrm{~m}$ \\
7 & 1m post-op & Y & Y & N & $5 \mathrm{~m}$ \\
8 & 1w post-op & Y & N & N & $3 \mathrm{~m}$ \\
9 & 2w post-op & Y & N & N & $4 \mathrm{~m}$ \\
10 & 1w post-op & Y & Y & N & $3 \mathrm{~m}$ \\
11 & 8m post-op & Y & N & N & $3 \mathrm{~m}$ \\
12 & 1m post-op & Y & N & N & $3 \mathrm{~m}$ \\
13 & 2w post-op & Y & N & N & $4 \mathrm{~m}$ \\
14 & 8y post-op & Y & N & N & $3 \mathrm{~m}$ \\
\hline & & & & &
\end{tabular}

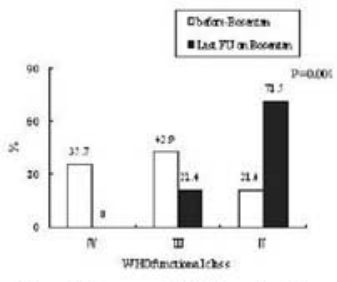

Figure 1 Changes of WHO functional class

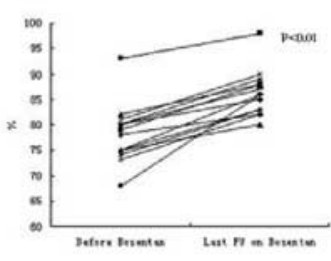

Figure 2 Changes of oxygen saturation 
6175

Effect of epinephrine injection via left atrium versus right atrium on resuscitation of cardiac arrest

Fuxia Yan, Cardiovascular Institute and Fu Wai Hospital, Chinese Academy of Medical Sciences, China

Rong Wang, Cardiovascular Institute and Fu Wai Hospital, Chinese Academy of Medical Sciences, China

Yue Tang, Cardiovascular Institute and Fu Wai Hospital, Chinese

Academy of Medical Sciences, China

Wenjing Xiao, Cardiovascular Institute and Fu Wai Hospital, Chinese Academy of Medical Sciences, China

Qian Lei, Cardiovascular Institute and Fu Wai Hospital, Chinese

Academy of Medical Sciences, China

Qinhua Xue, Cardiovascular Institute and Fu Wai Hospital, Chinese

Academy of Medical Sciences, China

Yuhong Wang, Cardiovascular Institute and Fu Wai Hospital, Chinese Academy of Medical Sciences, China

Lihuan Li, Cardiovascular Institute and Fu Wai Hospital, Chinese

Academy of Medical Sciences, China

Objective: The purpose of this study was to determine if delivery of epinephrine to heart via left atrial line is more efficient than right atrial epinephrine administration during cardiac resuscitation.

Methods: This randomized, blind, crossover controlled study, undertaken at an institute research laboratory, involved 6 mixedbreed dogs. Animals had two catheters placed into right atrium under anesthesia by ketamine, diazepam and relaxant with mechanical ventilation. Asphyxial cardiac arrest was induced by stopping ventilation, After 10 minutes of ventricular fibrillation, the dogs were randomized to two groups, each comprising 3 dogs. The dogs were given either left atrial or right atrial epinephrine $(0.05 \mathrm{mg} / \mathrm{kg})$, and followed by 3 minutes cardiopulmonary resuscitation. 2 direct current countershocks $(0.5 \mathrm{~J} / \mathrm{kg})$ were attempted over one minute and, if unsuccessful, repeating resuscitative efforts were given by the same way. After two times cardiac resuscitation, if still unsuccessful, crossing over the left atrial and right atrial administration of epinephrine between two groups, and repeating the same resuscitation for another two times.

Results: In the first left atrial epinephrine group, return of spontaneous circulation was faster, and peak aterial pressure and peak heart rate were higher after epinephrine administration, and no animal needed subsequent epinephrine administration via right atrium. In the first right atrial epinephrine group, all the animals needed subsequent epinephrine administration via left atrium. Two animals restored spontaneous circulation and one didn't response to epinephrine during four times resuscitations. Conclusion: Administration of epinephrine via left atrium is more efficient than via right atrium for resuscitation of cardiac arrest.

\section{6}

Post-cardiac Surgery Handover: A Simple Tool Improves Quality by Reducing Omissions in Information Exchanged

Samara Zavalkoff, Montreal Children's Hospital, Canada

Saleem Razack, Canada

Josee Lavoie, Canada

Arian Dancea, Canada

Objective: To improve quality of care of complex, post-cardiac surgery patients by reducing omissions in information exchanged during handover, without significantly prolonging handover duration.

Methods: An interdisciplinary group developed a fill-in-theblank, one-page tool to guide information exchange between the $\mathrm{OR}$ and PICU teams, during handover of post-cardiac surgery patients. The tool consists of four categories of information: preoperative (7 elements), medical intra-operative (14 elements), surgical intra-operative (11 elements) and current status (11 elements). We conducted a prospective, interventional study to establish the tool's impact on information exchanged overall(total score) and in the four categories (4 subscores). One investigator directly observed all handovers, scored them with a checklist (1 point per element if discussed) and timed their duration.

Results: The pre and post-intervention groups were similar at baseline. There was a significant improvement in the total handover score after the implementation of the handover tool $(28.2+/-4.6$ vs. $33.5+/-3.7$ points, $\mathrm{p}=0.002)$. In addition, there was a significant improvement in the medical $(8.3+/-2.6$ vs $10.3+/-2.1$ points, $\mathrm{p}=0.024)$ and surgical $(7.5+/-1.4$ vs $9.3+/-1.6$ points, $\mathrm{p}=0.002)$ intraoperative subscores. The preoperative and current status scores did not change. There was no considerable increase in handover duration when using this tool $(8.3+/-4.6$ vs $11.1+/-3.9$ minutes, $\mathrm{p}=0.1)$.

Conclusions: Use of a simple tool, during handover of post-cardiac surgery patients, resulted in a more complete exchange of critical information with no significant prolongation of the handover duration.

\section{7}

The Hybrid Procedure in the Management of Neonates with Large Left Ventricular Tumours: Intermediate Term Follow Up

Saadeh B Jureidini, Saint Louis University/Cardinal Glennon

Children's Medical Center, United States

Grace A Freire, Saint Louis University/Cardinal Glennon Children's

Medical Center, United States

Kenneth O Schowengerdt, Saint Louis University/Cardinal Glennon Children's Medical Center, United States

Kathy Tinker, Cardinal Glennon Children's Medical Center, United States

Andrew C Fiore, Saint Louis University/Cardinal Glennon Children's Medical Center, United States

Neonates with large, space-occupying left ventricular (LV) tumours present with ductal dependent systemic circulation. Their management with total or subtotal resection or Norwood palliation have been associated with disappointing and often fatal outcome. We present our short-term follow-up of two such neonates after the hybrid procedure.

The two patients were diagnosed prenatally with large tumours occupying the majority of the LV. Both patients had moderate to severe mitral regurgitation and heart failure scores which improved on Digoxin. At birth, ductal patency with prostaglandin was deemed necessary because the following findings were noted by echocardiography: retrograde aortic arch flow, bidirectional ductal flow, reduced antegrade aortic flow, poor LV function (in one patient) and significant mitral regurgitation.

After stabilization with intravenous inotropes and diuretics, each patient underwent the Hybrid approach at one week of life. Following sternotomy self expandable 8 and $9 \mathrm{~mm}$ stents were implanted into the ductus arteriosus following bilateral $3.5 \mathrm{~mm}$ branch pulmonary artery bands. One week later they underwent balloon atrial septostomy. At 5 months of age, both patients demonstrated almost total resolution of mitral regurgitation, improvement to normal systolic LV function and clinical resolution of heart failure symptoms. After repeat catheterization, they underwent surgical Norwood, removal of stent and Glenn shunt with satisfactory results. Both patients are thriving well at 15 and 16 months of age. 
We conclude that the hybrid approach followed by staged cavopulmonary avastomosis is feasible and an effective alternative in the management of neonates with large LV tumours

6178

The Advancement of Nursing Care for Children with HLHS in Western Canada

Lois Hawkins, Stollery Children's Hospital, Edmonton, Canada Sharon Chow, Western Canadian Children's Heart Network, Edmonton, Canada

Gwen Rempel, University of Alberta, Edmonton, Canada Lea Legge, Variety Children's Heart Centre, Winnipeg, Canada Marie Penner, Royal University Hospital, Saskatoon, Canada Patty Knox, Alberta Children's Hospital, Calgary, Canada Laurie Cender, BC Children's Hospital, Vancouver, Canada Mary Spencer, BC Children's Hospital, Vancouver, Canada

Purpose: The Western Canadian Children's Heart Network (WCCHN) serves the pediatric cardiac population of Canada's four western provinces and two northern territories.

The Network's goals: sharing clinical knowledge and expertise, improving care for children with heart disease, and providing support for these children and their families. Nursing's goal is evidence-based practice - one target group has been infants with hypoplastic left heart syndrome (HLHS) and their families.

Process: Monthly Allied TeleHealth session provide a forum for collaboration, discussion and implementation regarding WCCHN nursing practice. Expertise \& Leadership - With doctoral support from the Faculty of Nursing at the University of Alberta, advanced practice nurses at the Network's two surgical sites (Edmonton's Stollery Children's Hospital and Vancouver's BC Children's Hospital) have developed core patient education material, teaching strategies and nursing interventions that have been adopted by the Network. Education - HLHS families receive a detailed information package. Teaching includes scale and oximeter use, infant assessment, CPR, and decision-making when their infant is ill. Consultants - TeleHealth case conferences are utilized for complex discharges, particularly those from remote locations. Network nurses are key resources for primary caregivers and families once infants are home. Research - The Network conducts annual quality reviews and family satisfaction surveys. Neurodevelopmental outcomes continue to be tracked, and parenting research continues.

Conclusion: The Network nursing approach has proven successful. A conceptual model of parenting and nursing's role in preparing and caring for HLHS families is evolving. A focussed nursing database would further enhance Network nursing research and practice.

\section{9}

Valvulate stent implantation on pulmonary valve Manuela Asenjo, Hospital General Gregorio Marañon, Spain Raquel Martinez, Hospital General Gregorio Marañon, Spain Cristina Relaño, Hospital General Gregorio Marañon, Spain Susana Cobos, Hospital General Gregorio Marañon, Spain

Objetive: We present two patients, 11 and 14 years old, carriers of valve prosthesis lung failure and are candidates for surgery to change the prosthesis to be implanted stent valvulado through femoral catheterization.

Methods: For two patients, 11 and 14 years, patients with congenital heart disease and had been made through implant artery cow to replace the pulmonary valve. Valve implanted is MELODY (biological membrane and suture mounted on a stent) through femoral catheterization with a device that incorporates a $24 \mathrm{Fr}$ introducer of low profile for ease of introduction, transportation and deployment of the native valve, without losing its properties as valve Native long.

Results: The procedure was between 30 and 90 minutes. without complications. Patients were discharged within 24 hours of intervention.

Conclusion: The implant of the Melody valve is an alternative to surgery, avoiding the risks inherent in it. Another advantage is the reduction of hospitalization and reduction in the critical care units.

\section{0}

Angioplasty of lung branch with cutting-ballon in pediatric patients

Manuela Asenjo, Hospital General Gregorio Marañon, Spain Raquel Martinez, Hospital General Gregorio Marañon, Spain Susana Martinez, Hospital General Gregorio Marañon, Spain Susana Cobos, Hospital General Gregorio Marañon, Spain Cristina Relaño, Hospital General Gregorio Marañon, Spain

Introduction: Angioplasty branches of underlying lung or lobares is a technique in the laboratory of hemodynamics in children. The causes of these strictures can be congenital and surgical treatment but his ball conventional high pressure do not get satisfactory results because the elasticity of injuries thus began the use of cutting-Ballon (CB). These balls shrink avoid making small cuts longitudinal tissue inside the artery.

Methodology: Since 2006 we have performed 129 lung angioplasties and 19 of them since we started with CB use in 2005. The ages of patients ranged between 1 month and 6 years. The most common diseases that the patients presented were: Syndrome of left ventricular hypoplasiaPulmonary stenosis CIVTruncusTransposition of great arteries.

Results: In 80\% of the cases was an increase in the size needed in 6 of them stent implantation to maintain the desired diameter and 3 of them coated stent to cover dissection successfully angiographic later.

Conclusion: Angioplasty of the pulmonary branches with CB is a relatively simple technique, which compared with the use of conventional balls, improved performance, without increasing risks or complications.

\section{1}

\section{Puncture transhepátic in neonatal patient}

Manuela Asenjo, Hospital General Gregorio Marañon, Spain Raquel Martinez, Hospital General Gregorio Marañon, Spain Susana Martinez, Hospital General Gregorio Marañon, Spain Susana Cobos, Hospital General Gregorio Marañon, Spain Cristina Relaño, Hospital General Gregorio Marañon, Spain

Introduction: A case of neonatal patients with congenital heart disease, surgery, ventricular support and membrane oxygenator (ECMO) via femoral vein and right internal presented impossibility of channeling femoral veins.

Methodology: Patient 50 days and $3.2 \mathrm{~kg}$, diagnosed with pulmonary atresia and hypoplastic pulmonary extreme branches. Unífocalización of surgery and interposition of homoingerto. Hemodynamic study is done by branches of pulmonary stenosis and inability to puncture the femoral or jugular vein was decided channeling inferior vena cava puncture transhepática

Results: After a puncture failed the liver vein was canalized and progresses $5 \mathrm{Fr}$ introducer of Pediatric and inferior vena cava to the study without complications within it. 
For the withdrawal of the introducer is accurate placement plug Ampltzer $5 \mathrm{~mm}$ puncture closure of the channel.

Conclusions: The transhepática puncture is a useful and viable access in those patients who present impossibility of puncture and channeling of more frequent use due their pathology even in patients of less than six months of age.

6183

Growth and nutritional intervention in infants and young children with congenital heart disease: a snapshot of the Queensland Paediatric Cardiac Service population Claire Costello, QLD Paediatric Cardiac Service, Mater Children's Hospital, Brisbane, Australia, Australia

Marcelee Gellatly, QLD Paediatric Cardiac Service, Mater Children's Hospital, Brisbane, Australia, Australia

Jane Daniel, QLD Paediatric Cardiac Service, Mater Children's

Hospital, Brisbane, Australia, Australia

Robert Justo, QLD Paediatric Cardiac Service, Mater Children's

Hospital, Brisbane, Australia, Australia

Kelly Weir, Royal Children's Hospital, Brisbane, Australia, Australia

Infants and young children with congenital heart disease (CHD) commonly experience growth retardation, causes are multifactorial including decreased oral intake secondary to feeding difficulties.

The aim of this investigation was to assess the degree of growth failure in children attending the Queensland Paediatric Cardiac Service to inform further research and treatment within this service.

Growth parameters, feeding practices and previous nutritional intervention information was collected on 76 patients aged $0-3$ years with CHD on admission to hospital for cardiac surgery. Data were collected via parents/carers completion of written questionnaires, reported growth and feeding history on admission. Data were examined using descriptive statistics, CDC 2000 growth charts and EpiInfo software.

In this population $(n=77), 58 \%$ were male with a median age of 4 months (range 0-33 months). Median hospital length of stay was 10.5 days (range $4-50$ days). Poor growth was demonstrated in this group with a mean weight $\mathrm{z}$ score $-1.4(\mathrm{SD} \pm 1.2)$, mean height $\mathrm{z}$ score $-0.9(\mathrm{SD} \pm 1.1)$ and mean weight for height $\mathrm{z}$ score -0.9 (SD \pm 1.5$)$. Using WHO cut-off's for $\mathrm{z}$ scores $(<-2$ SD), $32 \%$ had wasting, $12 \%$ had stunting and $28 \%$ malnutrition. A significant proportion of patients were $\leqslant 3$ rd percentile for weight (48\%); height (25\%) and combined weight for height (35\%) with $39 \%$ crossing down weight percentiles pre-admission. $21 \%$ of patients had a naso-gastric tube for feeding and $28 \%$ were on fortified feeds on admission.

Growth failure is common in infants and children with CHD. Further research is underway linking cardiac diagnosis, oral feeding difficulties and growth failure. 23/1 DG.

\section{4}

Oral feeding difficulties and growth failure in infants and young children with congenital heart disease: what is the clinical evidence?

Marcelee Gellatly, Queensland Paediatric Cardiac Service, Mater

Children's Hospital, Australia

Claire Costello, Queensland Paediatric Cardiac Service, Mater

Children's Hospital, Australia

Jane Daniel, Queensland Paediatric Cardiac Service, Mater Children's Hospital, Australia

Robert Justo, Queensland Paediatric Cardiac Service, Mater Children's Hospital, Australia
Kelly Weir, Royal Children's Hospital, Brisbane, Australia

Oral feeding difficulties and growth failure are common in infants and young children with congenital heart disease (CHD). Current literature linking these two factors is limited.

The aim of this investigation was to identify the incidence of oral feeding difficulties and growth failure associated with specific cardiac diagnoses in children attending the Queensland Paediatric Cardiac Service.

Oral feeding skills, anthropometric data and cardiac diagnoses (cyanotic/acyanotic) were collected from 57 children with CHD on admission to hospital for cardiac surgery. Data collected on admission were growth parameters, clinical feeding assessments and parents'/carers' completion of written questionnaires. Data were examined using descriptive statistics and CDC 2000 growth charts.

In this population $(n=57), 58 \%$ were male with a median age of 4 months (range 0-33 months). Oral feeding difficulties were identified in $61 \%(\mathrm{n}=35)$ of patients, of which $19 \%$ were nil by mouth pre-surgery. A significant proportion of patients demonstrated growth failure ( $\leqslant=3$ rd percentile) for weight $(50 \%)$; height $(33 \%)$ and combined weight for height (33\%). Additionally, patients with oral feeding difficulties $(n=35)$ were more likely to have growth failure for weight (59\%); height (36\%) and weight for height (39\%). Growth failure was more evident in acyanotic (24\%) versus cyanotic (9\%) patients for combined weight for height. There was no relationship between diagnosis and presence of oral feeding difficulties.

A relationship exists between oral feeding difficulties and growth failure. Future prospective studies are required to identify effective intervention strategies to minimise the impact of oral feeding difficulties and growth failure.

\section{6 \\ Perioperative needs of parents with congenital heart disease \\ Yongmei Guan, Heart Center, Shanghai Children's Medical Center, Shanghai, China, China \\ Pingping He, Heart Center, Shanghai Children's Medical Center, Shanghai, China, China \\ Xiaorong Gu, Heart Center, Shanghai Children's Medical Center, Shanghai, China, China}

Objective: To describe the parents' perioperative needs of children with congenital heart disease, and to identify the factors influencing parents' needs.

Methods: Descriptive comparative design was used in this study. Purposive sampling was used to recruit 802 parents. Parents' needs of children with congenital heart disease in self-developed questionnaire included 34 items, 6 domains which are decrease anxiety, seek information, help children, accompany children, seek support, environment and equipment.

Results: The mean score of the parents' needs was 3.98, seek information, decrease anxiety and help children ranked the top three needs of the parents. The factors influencing the needs of the parents were gender, age, timing of children's diagnosis, parents' occupation and family income.

Conclusion: Nurses should apply their knowledge and skills to communicate with the parents to meet the information needs of the parents. Nurses should provide age-appropriate care to different kids, provide family centered nursing care and help the parents to participate in taking care of their child, and seek resource to provide financial support to the poor family. 
6187

The Expert Congenital Cardiac Nursing Model: The Advancement of Borderless Expertise

Jeanette M Guimond, Hospital For Sick Children, Canada

Collaboration between an interprofessional team from the Cardiac Critical Care Unit, at SickKids hospital in Toronto, Canada, and Children's HeartLink, a non-profit organization from the United States, has resulted in several medical exchanges to China. To date SickKids and Children's HeartLink have collaborated three international exchanges to Chengdu. The overall goal of each exchange was to work in partnership with the local hospital to improve its capacity to advance the care of pediatric cardiac patients.

Objective: To discuss how care can be advanced through professional exchanges. To meet the challenge to provide expert care in an environment where there are modest resources and sensitivity to culture diversity is expected.

Methods: This presentation will highlight the role of the interprofessional team within these medical exchanges. These exchanges provide an opportunity for teaching, coaching, and mentoring the local team both at the bedside and in a more formal classroom environment.

Results: The SickKids interprofessional team worked closely with their Chinese colleagues, as a unified team, to advance the care of pediatric cardiac patients.

Conclusion: Through an interprofessional, collaborative approach, expert models of care can be incorporated into an already existing practice, advancing overall patient care.

\section{8}

The challenge of respiratory therapy to delayed sternal closure after surgical repair of congenital heart defect

Pingping He, Department of cardiovascular surgery, Shanghai Children's Medical Center, Medical college of Shnaghai Jiaotong University, China

Liping Liu, Department of cardiovascular surgery, Shanghai Children's Medical Center, Medical college of Shnaghai Jiaotong University, China

Jun Bian, Department of cardiovascular surgery, Shanghai Children's Medical Center, Medical college of Shnaghai Jiaotong University, China Gang Ji, Department of cardiovascular surgery, Shanghai Children's Medical Center, Medical college of Shnaghai Jiaotong University, China Limin Zhu, Department of cardiovascular surgery, Shanghai Children's Medical Center, Medical college of Shanghai Jiaotong University, China

Objective: Delayed sternal closure (DSC) is one of useful technique to prevent cardiac tempnade resulted from myocardial edema during the early period after open heart surgery. The aim was to establish the respiratory effect of DSC, and to guide ventilation management after closure immediately.

Methods: Thirty-five infants with the median age 6 weeks (ranged from 2 days to 13 weeks) and median weight $4.0 \mathrm{~kg}$ (ranged from 1.7 to $9 \mathrm{~kg}$ ) were involved in his study. Respiratory function and hemodynamic was measured $30 \mathrm{~min}$ before and after sternal closure prospectively in paralyzed ventilated infants.

Results: After operation about $(2.9 \pm 0.9) \mathrm{d}$ were successful to close without mediastinal infection. The change of hemodynamic, air way mechanism and oxygenation $30 \mathrm{~min}$ before and after DSC were shown in table 1 , which showed a significant increase of peak airway pressure (PIP) $(\mathrm{P}<0.01,11.2 \%)$ and a significant decrease of dynamic compliance (Cdyn ) $(\mathrm{P}<0.01$, $32.2 \%) 30 \mathrm{~min}$ after DSC. At the mean while, there was a significant decrease of $\mathrm{PaO} 2 / \mathrm{FiO} 2$ ratio $(279.4 \pm 78.3$ versus $241.6 \pm 101.1, \mathrm{P}<0.05), \mathrm{DA}-\mathrm{aO} 2(166.7 \pm 70.3$ versus $203.8 \pm$ $82.3, \mathrm{P}<0.01)$ and respiratory index $(1.7 \pm 1.4$ versus $2.4 \pm 1.9$, $\mathrm{P}<0.01)$. Also, there was no significant change on hemodynamic. Fifty-four $\%$ of the patients developed pulmonary atelectasis or effusion. Three cases developed ventilation associated pneumonia account for the incidence of $12 \%$.

Conclusion: The respiratory function and oxygenation may be compromised after DSC and the mechanical ventilation should be very carefully. The best opportunity peri-DSC should improve the outcomes for the patients.

Table 1 The effect of hemodynamic, air way mechanism and oxygenation $30 \mathrm{~min}$ before and after DSC

\begin{tabular}{|c|c|c|c|c|}
\hline & & pre-DSC & post-DSC & $P$ value \\
\hline & HR (bpm) & $140.3 \pm 23.9$ & $143.4 \pm 19.8$ & $P>0.05$ \\
\hline \multirow[t]{3}{*}{ hemodynamic } & $\mathrm{BP}(\mathrm{M})(\mathrm{mmHg})$ & $69.0 \pm 11.7$ & $70.5 \pm 14.8$ & $P>0.05$ \\
\hline & $\mathrm{CVP}(\mathrm{mmHg})$ & $10.4 \pm 3.2$ & $11.3 \pm 3.1$ & $P>0.05$ \\
\hline & $\mathrm{PIP}\left(\mathrm{cmH}_{2} \mathrm{O}\right)$ & $18.7 \pm 3.6$ & $20.8 \pm 3.6$ & $P>0.01$ \\
\hline \multirow[t]{3}{*}{ airway mechanism } & $\mathrm{MAP}\left(\mathrm{cmH}_{2} \mathrm{O}\right)$ & $8.0 \pm 2.2$ & $8.7 \pm 2.2$ & $P>0.05$ \\
\hline & $\mathrm{Cdyn}\left(\mathrm{ml} / \mathrm{cmH}_{2} \mathrm{O}\right)$ & $3.7 \pm 1.7$ & $3.1 \pm 1.1$ & $P>0.01$ \\
\hline & $\mathrm{P} / \mathrm{F}$ & $279.4 \pm 78.3$ & $241.6 \pm 101.1$ & $P>0.05$ \\
\hline \multirow[t]{2}{*}{ oxygenation } & $\mathrm{DA}-\mathrm{a} \mathrm{O}_{2}(\mathrm{mmHg})$ & $166.7 \pm 70.3$ & $203.8 \pm 82.3$ & $P>0.01$ \\
\hline & RI & $1.7 \pm 1.4$ & $2.4 \pm 1.9$ & $P>0.01$ \\
\hline
\end{tabular}

\section{9}

Transition to Adult Care in the Pediatric McMaster Lipid Clinic

Liz Helden, McMaster Children's Hospital, Canada

Geraldine Cullen-Dean, Hospital for Sick Children, Canada

William Conner, McMaster Children's Hospital, Canada

Katherine Morrison, McMaster Children's Hospital, Canada

Andrew Don-Wauchope, Hamilton Health Sciences, Canada

The benefit of ongoing treatment of Familial Hypercholesterolemia is to prevent cardiovascular disease in mid-adulthood. As young adults with Familial Hypercholesterolemia move away from a Pediatric setting their level of support changes and these changes may disrupt lifestyle interventions and adherence to medications. There are a number of factors that make the transition easier for young adults. One of these factors can be addressed by a formal transition clinic.

The Pediatric Lipid clinic at McMaster Children's Hospital has recently introduced a transition Lipid Clinic for patients 17-20 years of age.

It is critical to start the transition process early in the clinic to encompass the different stages of development from child to adulthood. At the Pediatric Lipid Clinic self-management skills for a heart healthy lifestyle is introduced early in life to increase the likelihood of health behaviours and, for some, cholesterol lowering medication continuing into adolescence and young adulthood.

For many years, the clinic has already incorporated some of the concepts of the shared management model described by the Toronto's Hospital for Sick Children Good 2 Go Transition Program. Education and care has gradually shifted from combined clinic visits with parent and youth to visits only with the youth. In addition, the clinic utilizes tools such as clinical adolescent assessment forms, Readiness checklist, group education, Good 2 Go MyHealth Passport modified for dyslipidemia and graduation certificates. The clinic will be evaluated to determine if this approach improves the effectiveness of transition and improves medical follow-up in adulthood. 


\section{0}

Readiness to Change in Parents and Obese Youth Commencing a Pediatric Weight Management Program Liz Helden, Department of Pediatrics, McMaster University, Canada Susan M Docherty-Skippen, Department of Pediatrics-McMaster University, Canada

Katherine M Morrison, Department of Pediatrics-McMaster University, Canada

Readiness to change (RTC) is important in counseling families regarding behaviour modification, although assessment of RTC in families and youth with obesity remains under investigated. Objective: To assess RTC at commencement of a pediatric weight management program, in parent and youth, and their confidence in maintaining those changes over the next 6 months.

Method: Parents and youth (8-16 years) enrolled in a research study to assess the Determinants of Change in Childhood Obesity (DECCO) identified their RTC at baseline using a 10point visual analog scale. The study visit included assessment of demographics, family history, lifestyle, anthropometry measurements, and body composition by DXA.

Results: Of the 268 children (123 males) enrolled in the study, (BMI Z-score 2.26 \pm 0.39 ; body fat $39.9 \pm 5.08 \%$ in boys, $42.1 \pm 4.43 \%$ in girls), 244 completed the RTC questions. Parent scores for current RTC were higher than youth ((mean \pm SD); $8.3 \pm 1.51$ vs. $7.5 \pm 1.98, p<0.0001)$, although the range was broad for both $(3-10$ vs. $1-10)$. Confidence of maintaining change for 6 months was also higher in parents $(7.9 \pm 1.56$ vs. $7.5 \pm 2.23, \mathrm{p}<0.0001)$.

Summary: At the onset of presentation to a pediatric weight management program, RTC in parents and youth with obesity varies greatly. Parents' RTC and confidence that they can maintain these changes exceeds that of their children. Different strategies for weight management may need to be implemented for those at different RTC and confidence levels.

6191

The Healthy Hearts Study: Recruitment and Consenting Issues in a School-based Rheumatic Heart Disease Screening Study

Heather Kellas, KidzFirst Public Health Nursing, Counties Manukau District Health Board, New Zealand

Brenda Luey, KidzFirst Public Health Nursing, Counties Manukau

District Health Board, New Zealand

Rachel H Webb, Starship Children's Hospital and the University of

Auckland, New Zealand

Diana Lennon, School of Population Child and Youth Health, University of Auckland, New Zealand

Adrian A Trenholme, KidzFirst Children's Hospital, Counties

Manukau District Health Board, New Zealand

Nigel J Wilson, Department of Paediatric and Congenital Cardiac

Services, Starship Children's Hospital, New Zealand

Objectives and methods:A screening study using portable echocardiography was developed to determine the prevalence of Rheumatic Heart Disease (RHD) in children 10-13 years in Counties Manukau, South Auckland. The study population consisted of seven socio-economically deprived schools, where the majority of students are Pacific Islanders or Maori. KidzFirst Public Health Nurses (KFPHN) deliver nursing services to these schools and have experience recruiting participants into community research programmes (eg Meningococcal B Vaccine Trials).

The KFPHN team, cultural advisors and investigators developed materials including information pamphlets, and consent forms.
Pacific translations were produced. School principals were approached and educational talks to students delivered by nurses. Initially a two-stage process was used with families given an invitation flyer and responders then sent a consent form. After piloting this process in two schools a simplified single-stage process was adopted. Nurses worked in partnership with Community Workers making home visits, telephoning families and liaising with school staff.

Results: 1292 of the 1759 students returned consent forms: the overall response rate was $74 \% .1158$ consented: the overall consent rate was $66 \%$. Using the two-step process the consent rate was $53 \%$; consent increased to $79 \%$ using the one-step process. Consent rates at individual schools varied between $44 \%$ and $90 \%$. Community Workers made 2050 phone calls and 491 home visits.

Conclusions: Higher consent rates were achieved using the onestep process. Despite standardised methods variable consent rates were observed across the 7 study schools. Culturally-appropriate community workers appear to be essential for successful recruitment and community engagement.

6192

Using a 'sense of coherence' as a way to situate adults' understanding of their congenital heart disease Joy E Lyon, University of Southampton, United Kingdom

Aim: to explore how adults understand their congenital heart disease; this study explored adults' views of living well with CHD.

Research design: a convenience sample of twenty-eight people aged 20-52 years was recruited from an adult congenital cardiac outpatient clinic at a UK tertiary cardiac centre. Participants took part in qualitative, one-to-one, focused interviews in which they provided their personal story of living with their heart condition. Findings: the key themes emerging from the stories were health within illness and positive health. These themes are related to the sense of coherence outlined by Antonovsky in 1987, which is used to situate the adults' understanding of their heart condition within existing theories of health behaviour change. Events that are understandable, meaningful and manageable contributed to participants' perceptions of being well.

Conclusions: information about the heart condition was understandable when it was related to daily life; this was in contrast to medical, textbook explanations.

Engaging in meaningful activities contributed to participant's well being. This included making their own decisions regarding career options, relationships, sporting activities.

Events were considered to be manageable if support was available, when necessary, from family, partners, friends or health professionals. Participants appreciated opportunities to provide support for themselves and others, demonstrating a reciprocal partnership, rather than dependency.

Implications for practice: structuring clinic activities using principles of motivational interviewing may contribute towards preparing ACHD patients to engage actively in the clinic visit.

\section{3}

Development of specialist Adult Congenital Heart Unit at The Prince Charles Hospital- trialling a unique Queensland model of Transition care

Theresa Malpas, The Prince Charles Hospital Chermside Brisbane, Australia

Jillian Kaisar, The Prince Charles Hospital Chermside Brisbane, Australia 
Background: Since 1968 this hospital has successfully treated the paediatric and ever-increasing adult population with congenital heart conditions. In 2005 the Adult Congenital Heart Unit was established. In 2008 paediatric cardiology relocated elsewhere. A Transition policy, model of care and evaluation process has commenced. Current psychological research within the unit includes state-wide psychosocial profiling of the adult congenital heart population. Qualitative research explores the experiences of young Australian adults with congenital heart conditions and parental perspectives.

Aims: Our unit aims to provide specialist inpatient and outpatient services to adolescents and adults; seamless transition from paediatric to adult services; and multidisciplinary response to psycho-social problems. Research and psychological screening aims to inform Transition related interventions and increase awareness of psychosocial issues for Australians living with congenital heart conditions. Method: The Director, Staff Specialist, Fellow in medicine, Psychologist and Nurse Coordinator facilitate the Transition Clinic, training of medical and nursing staff, and management of adult congenital heart patients. Patients receive ongoing access to progressive surgery, the heart transplant unit, specialist cardiology interventions and diagnostic services located at the hospital.

Outcomes: Increased clinic attendance rates, treatment compliance and patient satisfaction.

Decreased inpatient occurrence and decreased length of hospital stays. Improved quality of life for patients. Better management of adults through continuous co-ordinated specialist care. Teaching, training and research within nursing, medical and allied health disciplines, maintaining best-practice standards.

\section{4}

Access to central vascular in pediatric patients

Raquel Martinez, Hospital General Gregorio Marañon, Spain Cristina Relaño, Hospital General Gregorio Marañon, Spain Manuela Asenjo, Hospital General Gregorio Marañon, Spain Susana Cobos, Hospital General Gregorio Marañon, Spain Laura Cervantes, Hospital General Gregorio Marañon, Spain Soledad Ferrer, Hospital General Gregorio Marañon, Spain Gloria Pinero, Hospital General Gregorio Marañon, Spain Teresa Martinez, Hospital General Gregorio Marañon, Spain

Objetive: The hemodynamic studies in pediatric patients may require alternative pathways to the femoral vascular access.

Methods: Quantify the number of vascular access by nurses in pediatric patients in the hemodynamic laboratory as alternative via the femoral. Results: Patients registered between 2007 and 2008 were 775. 106 is not used in the femoral access.

Jugular 81 humeral 14 Subclavia 2, hepatic 1, axillary artery 2, carotid artery 6 . The percentage of patients who benefited from not femoral vascular access was $13.6 \%$.

The alternative to the femoral vascular access is essential for the realization hemodynamics in pediatric patients. Nurses working in the laboratory of hemodynamics should know alternative ways to access the femoral.

No complications from the technique applied.

\section{5}

To Beat or not to Beat. The role of the Fetal Cardiac Nurse Specialist

J M McKirkle, Great Ormond Street Hospital, London, UK, United Kingdom

M Mc Laughlin, Royal Brompton, London, UK., United Kingdom

Service delivery within the NHS is changing with improved access to care, clinical outcomes and patient experience of care as primary objectives. Within secondary healthcare in particular, there has been movement towards sub-specialisation in nursing, backed up by evidence that access to specialist opinion and skills improves quality of clinical outcomes.

Advances in modern medicine and technology have led to an increase in the number of foetuses being diagnosed with anomalies in the ante-natal period, thus allowing for preparation and support of parents for delivery of a baby who may require immediate, long term medical or surgical intervention. In some case it could be argued that in-utero intervention has increased the chances of fetal survival and allows for a better long-term outcome in this client group.

To meet the potential changes in survival the current services provided need to be changed and updated. The outcomes of a UK based audit will be presented on how Fetal Cardiology is currently managed, with recommendations to develop this service further to ensure holistic care is provided for the family and the foetus. The role of the Fetal Cardiology Specialist Nurse will be further explored enlight of the outcomes of a parental audit, as we delve into the needs and perceptions of such a service in its infancy.

\section{6}

Predictors of oral feeding difficulty in cardiac surgical infants in Korea

Ju Ryoung Moon, Cardiac \& Vascular Center, Samsung Medical Center, Korea

Joo Min Hwang, Cardiac \& Vascular Center, Samsung Medical Center, Korea

Yu Kyung Lee, Department of Thoracic and Cardiovascular surgey, Samsung Medical Center, Korea

June Huh, Department of Pediatrics, Samsung Medical Center, Sungkyunkwan University School of Medicine, Korea

I-Seok Kang, Department of Pediatrics, Samsung Medical Center, Sungkyunkwan University School of Medicine, Korea

Ji-Hyuk Yang, Department of Thoracic and Cardiovascular surgey, Samsung Medical Center, Sungkyunkwan University, Korea Tae-Gook Jun, Department of Thoracic and Cardiovascular surgey, Samsung Medical Center, Sungkyunkwan University, Korea Heung Jae Lee, Department of Pediatrics, Samsung Medical Center, Sungkyunkwan University School of Medicine, Korea

Purpose: The purpose of this study was to identify factors contributing to postoperative feeding difficulties in infants following cardiac surgery.

Methods: A retrospective cohort study used chart audit of 555 consecutive infants who underwent cardiac surgery. Ten variables were analyzed as possible predictors of postoperative feeding difficulties including; diagnosis, demographics, details of surgery and postoperative course. Results; Mean patient age was 40.0days (range; $1.2 \sim 98.0$ days) and mean patient body weight was $3.9 \mathrm{~kg}$ (range; $1.2 \sim 7.7 \mathrm{~kg}$ ). At hospital discharge, 496 (89.3\%) infants were orally fed and $58(10.7 \%)$ were not. Overall mean hospital length of stay was $20.0( \pm 22.5)$ days. Mutilvariate logistic regression analysis revealed vocal cord palsy (odds ratio 9.50), length of postoperative intubation (odds ratio 5.73), neurologic complication after surgery (odds ratio 3.50 ) and weight at surgery (odds ratio 0.38 ) as independent predictors of failure to feed orally at discharge from hospital.

Conclusion: Risk factors for feeding difficulties in the postcardiac surgery infant are vocal cord injury, prolonged intubation, neurologic complication after surgery and low weight at surgery. Early identification of neonates at risk for feeding difficulties may lead to development of strategies to reduce morbidity, improve patient care. 
6197

Family Stress, Coping, and Outcomes Following the Diagnosis of Single Ventricle Congenital Heart Disease in a Newborn

Kathleen A Mussatto, Children's Hospital of Wisconsin, United States

Lisa Young-Borkowski, Medical College of Wisconsin, United States Jane Leske, University of Wisconsin - Milwaukee, United States Nancy Ghanayem, Medical College of Wisconsin, United States Kathleen Sawin, Children's Hospital of Wisconsin, United States Victoria Pemberton, National Institutes of Health, United States Judith Geva, Children's Hospital Boston, United States Cheryl Nowak, University of Michigan, United States

Objective: To describe stress, coping, and outcomes for parents of infants with single ventricle congenital heart disease (SVCHD). Survival for one of the most complex forms of congenital heart disease, SVCHD, has improved dramatically. Little is known about family stress, coping, and adaptation following the diagnosis of SVCHD.

Methods: A descriptive, repeated-measures design using survey techniques is utilized. Parents of affected infants complete study measures at three time points (A-4-6 weeks, B-4-6 months, C-13-15 months of infant age).

Results: 87 mothers and 43 fathers have participated; time $\mathrm{A}=90$, $\mathrm{B}=65, \mathrm{C}=35$. Parents were 19.7 to 52.9 years old. Nearly half were first-time parents. Mothers reported higher post-traumatic stress disorder symptoms, greater impact on the family, more frequent stressful experiences, greater use of coping skills, and lower individual well-being than fathers (t-test, $\mathrm{p}<0.05)$. Family function was in the clinical range for $13 \%$ of mothers and $10 \%$ of fathers. High risk of stress-related illness due to cumulative life changes was present for $24 \%$ of mothers and $30 \%$ of fathers. Perceptions of stress for both parents decreased from time A to time C, however family resources for management of stress also decreased (ANOVA with Tukey post-hoc comparisons, $\mathrm{p}<0.05$ ).

Conclusion: Parents demonstrate a complex response to the stress of SVCHD in an infant. Mothers and fathers exhibit different patterns of responses. Most families adapt well over time, however resources for managing the stress of chronic illness may decline. Plans for psychosocial support should be in place to help parents face this challenge.

\section{8}

Aortic valvuloplasty with cardiac stimulation in pediatric patients

Cristina Relaño, Hospital General Gregorio Marañon, Spain Manuela Asenjo, Hospital General Gregorio Marañon, Spain Raquel Martinez, Hospital General Gregorio Marañon, Spain Susana Cobos, Hospital General Gregorio Marañon, Spain

Objetive: We present 10 patients with valvular stenosis to aortic valvuloplasty is accelerated by stimulation with cardiac pacemaker in the right ventricle

Methods: Patients aged 1 month and 15 years. Is performed with aortic balloon valvuloplasty. By pacemaker catheter inserted into the right ventricle undergoes heart overstimulation at 200 beats per minute.

Results: Access via femoral. 7 cases without complications. 2 cases with mild insufficiency and 1 case with mild to moderate corrected within 24 hours

Conclusions: The acceleration by cardiac stimulation in the right ventricle during aortic valvuloplasty is a useful technique. avoiding high-risk situations for patients pediatrico.la training and coordination of all staff is important to ensure the success of the technique.

\section{9}

Angioplasty ballon-cutting of lung in pediatric patients Cristina Relaño, Hospital General Gregorio Marañon, Spain Manuela Asenjo, Hospital General Gregorio Marañon, Spain Susana Cobos, Hospital General Gregorio Marañon, Spain Raquel Martinez, Hospital General Gregorio Marañon, Spain Laura Cervantes, Hospital General Gregorio Marañon, Spain Ana Isabel Fernandez, Hospital General Gregorio Marañon, Spain

Paola Alcaide, Hospital General Gregorio Marañon, Spain

Objetives: Angioplasty branches of underlying lung or lobares is a technique in the laboratory of hemodynamics in children. The causes of these strictures can be congenital and surgical treatment but his ball conventional high pressure do not get satisfactory results because the elasticity of injuries thus began the use of cutting-Ballon (CB). These balls shrink avoid making small cuts longitudinal privacy glass.

Methods: Since 2006 we have performed 129 lung angioplasties and 19 of them since we started with CB use in 2005.

The ages of patients ranged between 1 month and 6 years.

The most common diseases that the patients presented were:

Syndrome of left ventricular hypoplasiaPulmonary stenosis CIVTruncusTransposition of great arteries.

Results: In $80 \%$ of the cases was an increase in the size needed in 6 of them stent implantation to maintain the desired diameter and 3 of them coated stent to cover dissection successfully angiographic later.

Conclusions: Angioplasty of the pulmonary branches with $\mathrm{CB}$ is a relatively simple technique, which compared with the use of conventional balls, improved performance, without increasing risks or complications

\section{0}

The Creation and Use of a Pediatric Cardiac Catheterization Database to Improve Efficiency of Patient Flow

Heather M Shockley RN, BSN, CCRN, The Children's Hospital

Aurora, Colorado, United States

Denise Kaufholz, The Children's Hospital Aurora, Colorado, United States

Objective: The scheduling of patients through a pediatric cardiac catheterization lab is subjective. The unpredictability of case length due to subjective scheduling can cause unnecessary delay or cancellation of cases, unwarranted overnight admissions and staff overtime. These issues directly impact patients, families, and staff satisfaction. A database was created to pursue the creation of an objective scheduling tool for the pediatric cardiac catheterization lab. Results from the database will be evaluated to implement changes in the scheduling process to ensure proper scheduling times which will ultimately improve patient, family and staff satisfaction.

\begin{tabular}{|c|c|c|c|}
\hline $\begin{array}{l}\text { Variables } \\
\text { procedure date and } \\
\text { room }\end{array}$ & diagnosis & $\begin{array}{l}\text { actual length of } \\
\text { time of case }\end{array}$ & $\begin{array}{l}\text { time procedure } \\
\text { began }\end{array}$ \\
\hline $\begin{array}{l}\text { ASA and Sedation } \\
\text { or anesthesia }\end{array}$ & $\begin{array}{l}\text { type of interven- } \\
\text { tion or diagnostic } \\
\text { procedure }\end{array}$ & $\begin{array}{l}\text { patient arrival time } \\
\text { to procedure lab }\end{array}$ & $\begin{array}{l}\text { time all access } \\
\text { achieved }\end{array}$ \\
\hline $\begin{array}{l}\text { height, weight, and } \\
\text { date of birth }\end{array}$ & $\begin{array}{l}\text { scheduled amount } \\
\text { of time for case }\end{array}$ & $\begin{array}{l}\text { time patient } \\
\text { preperation } \\
\text { complete }\end{array}$ & $\begin{array}{l}\text { time procedure } \\
\text { complete and } \\
\text { time patient } \\
\text { out of lab }\end{array}$ \\
\hline
\end{tabular}



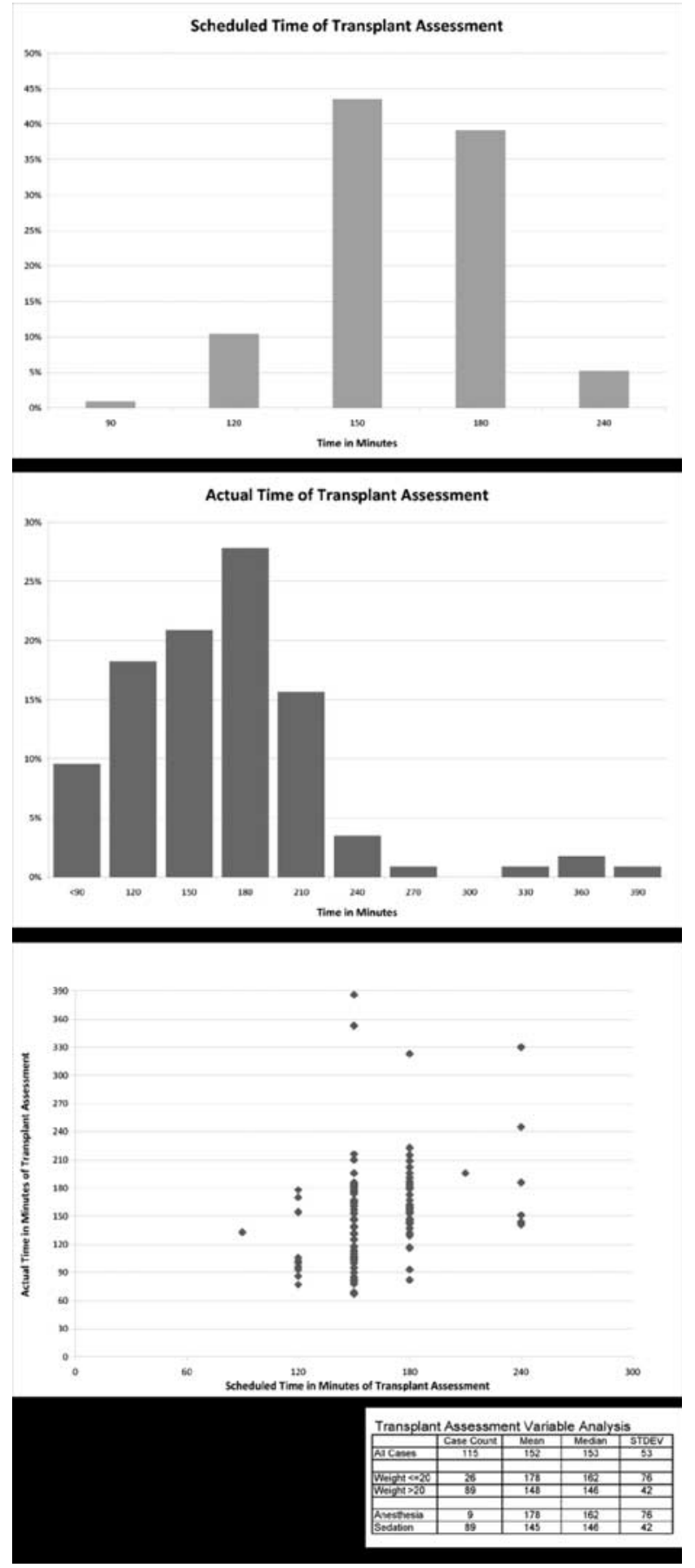

Methods: A database was created in Microsoft Access to retrospectively analyze data from all patients undergoing a cardiac catheterization procedure beginning January 1, 2008. (Please see table for variables.) Queries were run from the documented data to find correlations between the variables. Information was then transferred into Microsoft Excel to perform the statistical analysis and relevance.

Results: Currently there are 777 enrolled into the database. Queries and statistical analysis are in process for the diagnostic and interventional procedures performed. To date transplant assessments have been analyzed with a sample size of 115 patients. (Please see Transplant Assessment graghs.) Analysis of all procedures performed will be completed with the same method.
Conclusions: Analysis of results will lead to changes in the scheduling process utilizing evidence based practice. Increased objectivity will allow improved scheduling accuracy and efficiency and thereby patient, family and staff satisfaction. Development of an objective scheduling tool is currently in process with collaboration from the medical director of the catheterization $\mathrm{lab}$

\section{1}

Creation of a Marfan and ConnectiveTissue Disorders Clinic

Nancy R Slater, Hospital for Sick Children, Canada

Nicole Macbeth-Curtis, Canada

Timothy Bradley, Canada

Marfan syndrome is a disorder of connective tissue primarily involving the heart, eyes and skeleton. The incidence is approximately $2-3 / 10,000$, with no known gender or race predisposition. This may be considered relatively rare, but the major heart-related complication is progressive enlargement of the aorta, with the risk of rupture, dissection and sudden cardiac death. For children with Marfan syndrome, as many of the diagnostic criteria are adult based and sometimes without a definite family history, the evolving clinical diagnosis can be difficult, leaving families unsure and anxious about the diagnosis. Other connective tissue disorders also prone to progressive aortic root enlargement, like Loeys-Dietz and Ehlers-Danlos syndromes, are being increasingly described. The specialized needs of these families were recognized at the Hospital for Sick Children in Toronto, Canada and a Marfan and Connective Tissue Disorders Clinic was created. This clinic is now well established and continues to evolve as the patient population increases towards the development of a multidisciplinary clinic. The role of the Clinical Research Nurse Coordinator has been developed to assist in screening these families, to improve patient care and quality of life, to provide up-to-date information and education, to liaise with the relevant support organizations and to promote clinical research in the clinic. Areas for continued development for the clinic are providing more access to webbased information for these families and to their caregivers to help increase awareness of Marfan syndrome and related connective tissue disorders in the community.

6202

A New Nursing Challenge ( Non medical prescribing in an acute paediatric tertiary referral centre )

Elisabeth C Smith, Great Ormond Street Hospital for Children NHS Trust, London, UK, United Kingdom

Non-medical prescribing (NMP) for nurses in the United Kingdom (UK) has been in the making for 20 years. "Independent prescribing" is described by the Department of Health as a process in which the "nurse prescriber" takes responsibility for the clinical assessment, establishing a diagnosis, clinical management and prescribing where necessary, commenced in May 2006. In the UK there is also "supplementary prescribing (March 2003)" which is described as a voluntary partnership between the independent prescriber (doctor or dentist), the supplementary prescriber (registered nurse) and the patient.

However when these qualifications are achieved, what challenges does an experience nurse at advanced practitioner level, working in a large tertiary cardiac referral unit, face with implementation 
of these skills, in a large multiprofessional team setting such as paediatric intensive care.

Aims:

- Factors which challenge this new role within the intensive care environment

- What challenges \& benefits does the nurse practitioner face in this new role?

- Benefits and non benefits to children, families and the clinical team

Outcome:

As when learning all new skills the greater your skill base developed through clinical practice, based within your team, with a medical mentor, the greater the understanding and the ability to integrate into a team with a new a skill base to the advantage of children and families.

6203

Transition of children's care out of the intensive care area supported on a mechanical device

Elisabeth C Smith, Great Ormond Street Hospital, United Kingdom

As a tertiary heart transplant centre the use of mechanical devices to bridge children to heart transplantation is logical and necessary service. However the use of such a device has challenges for the team, the family and the child. With increased pressures for cost savings, service provision and quality of care, what environment should these children be cared and who should provide that care.

Aims:

- To meet cost pressures, service provision and quality of care

- To assess which environment is optimal for these children

- To decide who can provide their daily care

This presentation addresses the issues raised to effect this transition.

6204

Novalung: A Bridge to Transplantation for Paediatric End Stage Pulmonary Hypertension

Cecilia St George-Hyslop, Labatt Family Heart Centre, Hospital for

Sick Children, Toronto Canada, Canada

Ben Sivarajen, The Hospital for Sick Children, Canada

Colleen Gruenwald, The Hospital for Sick Children, Toronto Canada, Canada

Shaf Keshavjee, Toronto General Hospital, Toronto Canada, Canada

Tilman Humpl, The Hospital for Sick Children, Toronto Canada, Canada

Steve Jarvis, The Hospital for Sick Children, Toronto Canada, Canada Celeste Foreman, The Hospital for Sick Children, Toronto Canada, Canada

Anne-Marie Guerguerian, The Hospital for Sick Children, Toronto

Canada, Canada

At the Hospital for Sick Children in Toronto, Canada, the Novalung extracorporeal membrane ventilator, a pumpless device, was successfully used as a bridge to lung transplantation in a paediatric patient.

Objectives: We share our experience with the support of a $15 \mathrm{yr}$ old female, with life threatening pulmonary hypertension secondary to pulmonary veno-occlusive disease. Building on 20 years of experience with ECMO, our team was guided by the expertise of our Lung Transplant Program, pioneers in lung transplantation.

Methods: Case report. Patient and device management are explained for a month of Novalung support.

Results: Following a brief cardiac arrest and interval of ECMO, using a novel trans-thoracic cannulation arrangement, the Novalung was used as an oxygenating pulmonary artery to left atrial (PA-LA) shunt. Bypassing the lungs and offloading the heart, the Novalung provided $\mathrm{CO} 2$ removal and oxygenation. Post operative care and monitoring, and transition to lung transplantation are described. The clinical course of the patient including the anticipation of ventilator failure and its exchange in the operating room are reported. Characteristic safety considerations and their management, including actual and potential complications are illustrated.

Conclusion: The Novalung proved to be a successful device as a bridge to transplantation and may become a key component to paediatric pre-lung transplant care given the paucity of organs available. The interprofessional approach enabled this team to achieve a successful outcome and transition this patient from a certain death to bilateral lung transplantation while optimizing nutrition and physiotherapy.

6205

Comprehensive Interdisciplinary Care Coordination for

Berlin Heart VAD Patients

Sandra L Staveski, United States

Stephen J Roth, United States

David Rosenthal, Stanford University, United States

Gail E Wright, United States

Pediatric nurse practitioners (PNPs) play integral roles in the development and implementation of programs to optimize care coordination and delivery in complex pediatric patients. We describe the development of an interdisciplinary program for young children with heart failure requiring a Berlin Heart ventricular assist device (VAD) and the contributions of a PNP in the intensive care setting.

Young children supported on VADs often have prolonged hospitalizations while awaiting heart transplantation. Providing critical care specifically oriented toward long-term stays requires a significant culture shift in the ICU. A PNP-led interdisciplinary group evaluated our ICU practices and identified areas for improvement. The work group introduced specific interventions to optimize interdisciplinary care coordination and enhance practice standards. These included: 1) interdisciplinary care guidelines for chronic patients, 2) assigning a primary physician and PNP to each patient, 3) psychological interventions for families to reduce the impact of extended stays, 4) early jejunal feedings to optimize nutritional status, growth and healing, 5) a protocol for developmental and rehabilitative services, 6) a strategy to minimize blood draws and transfusions, 7) increased structure for interdisciplinary rounds to optimize team and family communication, 8) comprehensive nursing education for VAD patients that included wound care, anticoagulation, and blood drawing practices, and 9) a protocol for off-unit rehabilitative/ developmental interventions. Our team found that comprehensive, interdisciplinary care coordination and standardized practice was achieved. Overall care for our Berlin Heart patients was improved, and the ICU PNP was integral to the initiation and implementation of the interdisciplinary care process we developed. 


\section{6}

Pulmonary physiotherapy of infants with congenital heart diseases - a pilot study

Agnieszka Adameczek, Department of Pediatric Cardiology and

General Pediatrics, Medical University of Warsaw, Poland

Bozena Werner, Department of Children's Cardiology and General

Pediatrics in Medical University of Warsaw, Poland

Introduction: Infants with congenital heart diseases before cardiosurgery often demonstrate pathological respiratory symptoms. The aim of this study was to analyze the effectiveness of vibrations as a rehabilitation method.

Materials and Methods: The study population consisted of 22 infants. Congenital non cyanotic heart defects with increased pulmonary blood flow were diagnosed in 13 children (6 complete atrioventricular septal defect, 7 ventricular septal defect including 5 with coexisting atrial septal defect) in 9 cyanotic heart diseases ( 7 tetralogy of Fallot, 1 transposition of the great arteries, 1 total anomalous pulmonary venous drainage).

Vibrations in gravity-assisted positioning were used in every patient. Saturation, respiratory rate and heart rate were controlled before and after the procedure, as well as after a 15-minute-break. Results: In infants with non cyanotic lesions with increased pulmonary blood flow the average heart rate (HR) before the procedure was $154,3 / \mathrm{min}$, after the procedure $\mathrm{HR}$ was 154,2/ $\min$ and after the 15-minute-break was $140,3 / \mathrm{min}$. Average respiratory rate before the procedure was $41,98 / \mathrm{min}$, after procedure was $23,1 / \mathrm{min}$ and after the 15 -minute-break was $33,62 / \mathrm{min}$

In infants with cyanotic heart diseases the average saturation before the procedure was $91 \%$, after the procedure $93 \%$ and after the15-minute-break 94,1\%. Differences were not statistically significant $(p \geqslant 0,05)$. Research is still in progress.

Conclusions: 1. Pulmonary physiotherapy has a positive effect on heart rate and breathing in infants with congenital heart diseases with the left-to-right shunt. 2. Improved saturation after the physiotherapy in infants with cyanotic lesions is observed.

\section{7}

Employing rotation thromboelastometry to analyse coagulation disturbances in congenital heart surgery Andreas Straub, Dept. of Thoracic, Cardiac and Vascular Surgery, University Hospital of Tübingen, Germany

Daniela Schiebold, Dept. of Thoracic, Cardiac and Vascular Surgery, University Hospital of Tübingen, Germany

Hans Peter Wendel, Dept. of Thoracic, Cardiac and Vascular Surgery, University Hospital of Tübingen, Germany

Carole Hamilton, Dept. of Thoracic, Cardiac and Vascular Surgery, University Hospital of Tübingen, Germany

Thomas Wagner, Dept. of Anesthesiology and Intensive Care Medicine, University Hospital of Tübingen, Germany

Eckhard Schmid, Dept. of Anesthesiology and Intensive Care Medicine, University Hospital of Tübingen, Germany

Gerhard Ziemer, Germany

Objective: Cardiac surgery employing cardiopulmonary bypass (CPB) and deep hypothermic circulatory arrest (DHCA) can induce a coagulopathy, which may be especially severe in infants. Patient management may be improved by quickly finding causes for coagulation disturbances peri-operatively. This study aimed to monitor coagulation defects during congenital heart surgery using rotation thromboelastometry $\left(\right.$ ROTEM $\left.^{\circledR}\right)$.

Methods: Ten infants underwent surgery for congenital heart disease using $\mathrm{CPB}$ and DHCA. Blood was sampled at skin incision (baseline), during, and after CPB. In ROTEM ${ }^{\mathbb{R}}$ the clot amplitude at 10 minutes (A10) and clotting time (CT) were analysed with heparin-sensitive in-TEM ${ }^{\mathbb{R}}$, heparinase-containing hep-TEM ${ }^{\mathbb{R}}$, tissue factor-activated ex-TEM ${ }^{\mathbb{R}}$, and fibTEM $^{\mathbb{R}}$, which analyses the fibrin component of coagulation. Standard coagulation tests (including fibrinogen levels and platelet counts) were also performed.

Results: The following changes occurred after $\mathrm{CPB}$ compared to baseline:

In-TEM $^{\mathbb{R}}-\mathrm{A} 10$ and hep-TEM ${ }^{\mathbb{R}}-\mathrm{A} 10$ decreased by mean \pm SEM $38 \pm 5 \%(\mathrm{p}<0.0001)$ and $34 \pm 3 \%(\mathrm{p}<0.0001)$ respectively, ex-TEM ${ }^{\mathbb{R}}-\mathrm{a} 10$ by $27 \pm 4 \%(\mathrm{p}<0.0001)$, and fib-TEM ${ }^{\mathbb{R}}$ a10 by $22 \pm 11 \%(\mathrm{p}=0.034)$. In-TEM ${ }^{\mathbb{R}}$-CT and hep-TEM ${ }^{\mathbb{R}}$ CT were prolonged by $214 \pm 54 \mathrm{sec}(\mathrm{p}=0.002)$ and $206 \pm 77$ $\sec (p=0.013)$ respectively. These findings indicate clotting factor depletion, platelet dysfunction and exclude excessive heparin. After targeted blood product administration coagulation was satisfactory.

Significant correlations of in-TEM ${ }^{\circledR}-\mathrm{a} 10$ with platelet counts $(\mathrm{r}=0.63 / \mathrm{p}=0.049)$ and A10 changes with fibrinogen levels $(r \geqslant 0.69 / p \leqslant 0.026)$ were observed.

Conclusions: During congenital heart surgery ROTEM ${ }^{\mathbb{R}}$ detects causes of CPB- and DHCA-associated coagulopathy. ROTEM ${ }^{\circledR}$ results are quickly available and reflect changes in plasmatic and cellular components of haemostasis. ROTEM ${ }^{\mathbb{R}}$ can thereby contribute to specific blood product treatment and may decrease bleeding complications.

\section{8}

Challenges in International Heart Surgery: Massive Air Embolism of Unusual Origin and Requirement for Modification of Standard Algorithms and Procedural Checks

Peter Allen, London Health Sciences center, London, Ontario, Canada Qasim Simmons, St. Mary's Hospital, Passaic, New Jersey.,

United States

Carmen Giacomuzzi, Oregon Health \& Sciences University, Portland, Oregon, United States

Karl Welke, Oregon Health \& Sciences university, Portland, Oregon, United States

Victor Carcioppolo, Southwest General Health Center, Middleburg Heights, Ohio, United States

Scott Snider, Acron City Hospital, Acron, Ohio, United States

Dan Woodward, Oregon Health \& sciences University, Portland, Oregon, United States

Aubyn Marath, Oregon Health \& Sciences University, Portland, Oregon, United States

Objective: In conducting international cardiac surgery, an unanticipated intraoperative perfusion complication encouraged us to review procedure and equipment checks for perfusion management when standards of excellence are not available. We describe the index case, contrast it to a similar case in a mature program and detail our response: pediatric perfusion protocols, specific for developing programs.

Methods: Case 1 occurred in an advanced cardiac center: bloodair foam penetrated the oxygenator's air/oxygen inflow line. During hypothermic arrest, oxygenator substitution permitted continuation of the surgical procedure without adverse outcome. An oxygenator design moulding fault was confirmed.

Case 2, occurred during an international start up program, using donated equipment that included a single air circuit alarm. Suddenly, massive air entered the oxygenator. Despite retrograde cerebral perfusion and satisfactory heart procedure, brain damage 
and death occurred. The oxygenator was faultless; a malfunctioning oxygen tank regulator valve was confirmed.

Results: These experiences with divergent outcomes in contrasting settings motivated us to develop a modified algorithm for pediatric perfusion management which specifically addresses: numerical unit value differences, sterility, un-contaminated blood availability, room temperature and humidity, atmospheric pressure differences in various geographical locations, quality checks on donated equipment, safety protocols, documenting non-availability of equipment, and counter measures to prevent air/oxygen inflow disasters using modified circuitry to ensure controlled delivery when tank gas air/oxygen is used.

Conclusion: Protocols assist compliance with international standards in developing programs. They also reduce ethical concerns about operating or withholding surgery, team dynamics, unfamiliar settings, language barriers and when procedural missteps are possible.

\section{0}

The Cerebrovascular Endothelial Cell Injury After Cardiopulmonary Bypass In Rats

Gang Chen, Cardiovascular Center, Children's Hospital of Fudan University, China

Bing Jia, Cardiovascular Center, Children's Hospital of Fudan University, China

Ye Liu, Department of Pathology, Medical College of Fudan University, China

Xiangang Yan, Cardiovascular Center, Children's Hospital of Fudan University, China

Chunlan He, Cardiovascular Center, Children's Hospital of Fudan

University, China

Ming Ye, Cardiovascular Center, Children's Hospital of Fudan

University, China

Qilin Tao, Cardiovascular Center, Children's Hospital of Fudan

University, China

Objectives: To investigate the functions and microstructure changes of cerebrovascular endothelial cell (EC) after different CPB mode.

Methods: 24 S.D. rats $(469.0 \pm 45.2 \mathrm{~g})$ were equally divided into four groups, which were underwent normothermic CPB for $120 \mathrm{~min}$ (group A), DHLF for $60 \mathrm{~min}$ (B), DHCA for $60 \mathrm{~min}$ (C) and sham group (D) via right carotid and jugular cannulation. The internal jugular vein blood examples were taken before and after bypass to test plasma NO concentration. The middle cerebral artery (MCA) was harvested to evaluate the EC response induced by different concentration of acetylcholine. The eNOS protein expression was determined by Western-immunoblotting. The hippocampus was harvested to observe the ultramicrostructure of EC.

Results: All the rats were successfully established and weaned from bypass. CPB reduced the plasma NO concentration compared with sham group. Plasma NO levels were lower in group B \& C compared with A $(3.94 \pm 0.15 \mathrm{mg} / \mathrm{L} \& 2.93 \pm 0.33 \mathrm{mg} / \mathrm{L}$ vs $4.33 \pm 0.17 \mathrm{mg} / \mathrm{L}, \mathrm{P}=0.002)$. Acetylcholine induced a dosedependent MCA vasodilation in sham group $(24.26 \pm 1.90 \%$ increase in diameter) that was attenuated in all $\mathrm{CPB}$ groups $(9.60 \pm 1.09 \%, \quad 5.97 \pm 0.68 \% \& 5.72 \pm 0.67 \%, \quad \mathrm{P}<0.01)$. The hypothermic bypass correlated with significantly reduced eNOS expression compared with group A $(\mathrm{P}<0.01)$, whilst eNOS expression was even lower in group $\mathrm{C}$ compared with group $\mathrm{B}$ $(\mathrm{P}=0.002)$. The ultramicrostructure of $\mathrm{EC}$ and capillary observed by electron microscope found normal in group A \& $\mathrm{D}$, but the $\mathrm{V}-\mathrm{R}$ space widened in group $\mathrm{B}$ and structural injury in $\mathrm{EC}$ and cerebral edema in group C.
Conclusion: $\mathrm{CPB}$ can induce the cerebrovascular EC injury, particularly in DHCA/DHLF bypass mode. The severity of the EC dysfunction maybe associated with the degree of brain damage after CPB.

\section{1}

Outcome of cardiac Extra-Corporeal Membrane Oxygenation

Rebecca Hill, Alder Hey Foundation NHS Trust, Liverpool, United Kingdom

Nelson Alphonso, Alder Hey Foundation NHS Trust, Liverpool, United Kingdom

Edmund J Ladusans, Alder Hey Foundation NHS Trust, Liverpool, United Kingdom

Steven Kerr, Alder Hey Foundation NHS Trust, Liverpool,

United Kingdom

Philip D Arnold, Alder Hey Foundation NHS Trust, Liverpool,

United Kingdom

Robert A Johnson, Alder Hey Foundation NHS Trust, Liverpool, United Kingdom

Antonio F Corno, Alder Hey Foundation NHS Trust, Liverpool, United Kingdom

Antonio F Corno, Alder Hey Foundation NHS Trust, Liverpool, United Kingdom

Objectives: To benchmark mortality of cardiac Extra-Corporeal Membrane Oxygenation (ECMO) in our institution, against the Extracorporeal Life Support Organisation (ELSO) Registry's survival data.

Methods: Prospective audit data from all ECMO cases January 2004 to November 2008 were reviewed for age, weight, operation, indication for ECMO, and duration of mechanical support.

Primary outcome measure was survival to hospital discharge. This was compared to ECLS Registry survival statistics for neonatal cardiac ECLS (38\%) and paediatric cardiac ECLS survival (44\%) (ELSO Registry, 2006).

Results: 20 patients underwent ECMO, representing 1.6\% of all cardiopulmonary bypass cases undertaken during this period. 10 patients $(50 \%)$ were neonates (6\% of neonatal bypass cases). Median age was 36.5 days (range 4 days-10 years) with a neonatal mean body weight of $3.25 \mathrm{~kg}$ (range $2.4-4.1 \mathrm{~kg}$ ).

Median duration of support was 4 days (range 3-7). The main diagnostic groups requiring ECMO support were Transposition of Great Arteries, Aortic Stenosis, Hypoplastic Left Heart Syndrome and Tetralogy of Fallot.

ECMO was instituted in theatre in 15 cases, and on PICU in 5 cases ( 3 cardiac arrest, 2 persistent low cardiac output state).

Two patients were transferred on ECMO support for ongoing care in ECMO centres.

There were one PICU death, two hospital deaths, and 17 patients (85\%) surviving to hospital discharge (90\% neonatal and $80 \%$ pediatric survival).

Conclusion: Despite the infrequent use of cardiac ECMO in our institution, survival compares favourably with ELSO registry survival data. However, small patient numbers, patient selection and short ECLS runs may bias our results.

6212

Congenital Heart Surgery: Changes In Blood Pressure And Blood Viscosity After Modified Ultrafiltration Guillermo Lema, Pontificia Universidad Católica de Chile, Chile Waldo Merino, Pontificia universidad Católica de Chile, Chile Roberto Canessa, Pontificia Universidad Católica de Chile, Chile 
Becker Pedro, Pontificia Universidad Católica de Chile, Chile Carvajal Claudia, Pontificia Universidad Católica de Chile, Chile Aeschlimann Nicolas, Pontificia Universidad Católica de Chile, Chile Gonzalez Rodrigo, Pontificia Universidad Católica de Chile, Chile

Introduccion: Modified ultrafiltration (muf) in children undergoing cardiac surgery, improves cardiac function after cardiopulmonary bypass (cpb). Increase in cardiac output and blood pressure, has been described so far. The exact mechanism underlying these hemodynamic changes remains unclear. We hypothesize that changes in blood pressure after muf are mostly related to changes in viscosity, due to a rise in hematocrit. The aim of this study is to assess blood viscosity pre and post muf, and correlate those measurements with changes in both systolic and diastolic blood pressures simultaneously. Methods: After written consent and institutional approval, 23 children scheduled for cardiac surgery with $\mathrm{cpb}$ and muf were prospectively studied. Plasma and blood viscosity were measured with a digital viscometer brookfield ${ }^{\mathbb{R}}, 37^{\circ} \mathrm{C}(100$ and $50 \mathrm{rpm})$. Systolic and diastolic blood pressures were recorded. All measurements were performed, immediately after $\mathrm{cpb}$ before and after muf. Anesthesia and $\mathrm{cpb}$ technique were uniform for all patients.

Results: (Graphics)

Conclusion: Blood pressures, and plasma and blood viscosity increases significantly after muf. However there is no correlation between changes in blood pressure and changes in blood viscosity after muf. Hemodynamic improvement after muf are not primary related to changes in viscosity, but probably to other mechanisms, not well studied as yet.

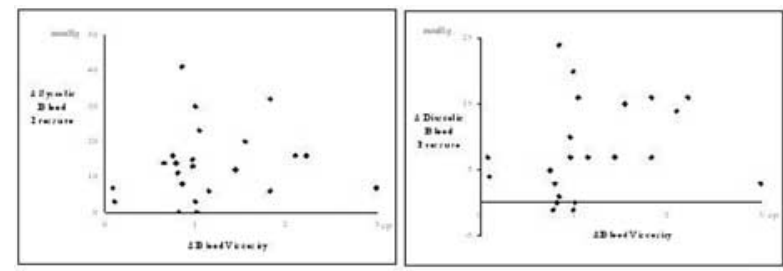

$\Delta$ Systolic Blood Pressure $=$ (Post Mut - Pre Mut) $\Delta$ Diastoic Biood Pressure $=($ Post Mut - Pre Muif) DBlood Viscosty $=$ (Post Mut - Pre Mut)

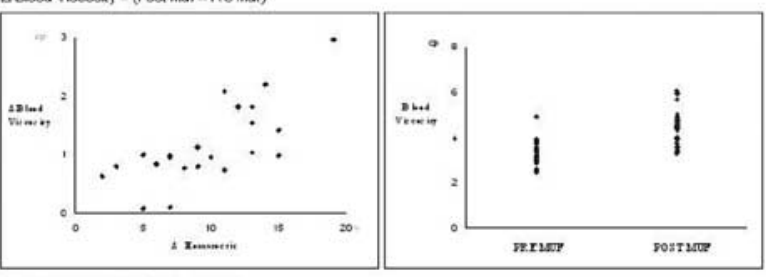

$\triangle$ Henatocit = (Post Mut - Pre Mut)

\section{3}

Hypothermic Circulatory Arrest increases Permeability of Blood Brain Barrier in Watershed Area

Toru Okamura, Cardiac Surgery, Children's National Heart Institute, Children's National Medical Center, United States

Nobuyuki Ishibashi, Cardiac Surgery, Children's National Heart

Institute, Children's National Medical Center, United States

TK Susheel, Cardiac Surgery, Children's National Heart Institute, Children's National Medical Center, United States

David Zurakowski, Cardiac Surgery, Children's National Heart

Institute, Children's National Medical Center, United States

Richard A Jonas, Cardiac Surgery, Children's National Heart Institute, Children's National Medical Center, United States

Objective: The integrity of blood brain barrier (BBB) after cardiopulmonary bypass (CPB) with hypothermic circulatory arrest (HCA) is controversial, especially in children. We tested the hypothesis that $\mathrm{BBB}$ is disrupted by HCA.
Methods: 25 piglets (mean weight $12 \mathrm{~kg}$ ) were randomly assigned to 5 groups (5 per group): anesthesia alone (control); $\mathrm{CPB}$ at $37^{\circ} \mathrm{C}$ with full-flow (FF); CPB at $25^{\circ} \mathrm{C}$ with very low flow (LF) and $\mathrm{HCA}$ at 15 or $25^{\circ} \mathrm{C}$. Evans blue dye was infused two hours after end of CPB. Brain was removed after one hour observation. Evans Blue (EB) extravasation in brain tissue was measured using the following two methods; 1) extraction method of $\mathrm{EB}$ with homogenate for cortex and cerebellum; ratio of EB of brain tissue/plasma was compared in all groups and 2) imaging methods; i) macroscopic method for whole brain and ii) microscopic method with immunohistochemistry for cortex, caudate nucleus, thalamus, hippocampus and cerebellum using measuring density of EB.

Results: 1) The quantity of $\mathrm{EB}$ in cortex and cerebellum was significantly higher in $25^{\circ} \mathrm{C}$ HCA than control. 2-i) BBB disruption was obvious macroscopically around watershed areas in $25^{\circ} \mathrm{C}$ HCA. 2-ii) Microscopic data showed that the leakage of $\mathrm{EB}$ in $25^{\circ} \mathrm{C}$ HCA was significantly more severe than control in the cortex, caudate nucleus, thalamus, hippocampus and cerebellum. $(\mathrm{p}<.05)$

Conclusion: $\mathrm{BBB}$ is impaired around watershed areas by $25^{\circ} \mathrm{C}$ $\mathrm{HCA}$ for one hour. $15^{\circ} \mathrm{C} \mathrm{HCA}$ and $25^{\circ} \mathrm{C} \mathrm{LF}$ were more effective in maintaining $\mathrm{BBB}$ integrity than $25^{\circ} \mathrm{C}$ HCA. Images data show clear evidence of $\mathrm{BBB}$ leakages in $25^{\circ} \mathrm{C}$ HCA.

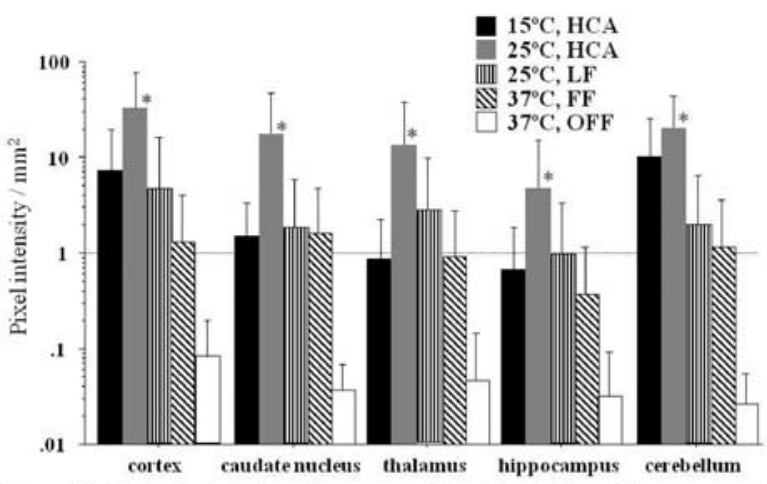

Figure: Blood brain barrier leakage for cortex, caudate nucleus thalamns, hippocampus and cerebellum using Evans blue dye at three hour later from end of cardiopulmonary bypass. Pixel intensity in $25^{\circ} \mathrm{C}$, HCA group was significantly higher than control at each brain area. $(p<0.05$; one way ANOVA)

\section{4}

Our experience with intraoperative cell salvage in children undergoing open heart surgery

Mila S Stajevic, Mother and Child Health Institute of Serbia, Yugoslavia

Lazo S Eremija, Mother and Child Health Institute of Serbia, Yugoslavia

Igor N Sehic, Mother and Child Health Institute of Serbia, Yugoslavia Vladimir B Milovanovic, Mother and Child Health Institute of Serbia, Yugoslavia

Objective: Intraoperative cell salvage for children undergoing open heart surgery has been enabled by the recent introduction of smaller reservoirs. We are showing the effectiveness of this method in paediatric open heart surgery.

Methods: Between November 2004 and December 2008, 28 patients underwent open heart surgery with the use of a intraoperative cell saver system. Patients weight ranged from 3.4 to $80 \mathrm{~kg}$ with mean $\mathrm{BW}$ of $33.8 \mathrm{~kg}$. 22 patients underwent different re-do operations, 3 patients were Jehowa's witnesses and 3 patients had preoperative haematological disorders. In all patients conventional or modified ultrafiltration was used. Residual volume of the circuit was salvaged. Laboratory tests 
(haematocrit, platelet count, fibrinogen, ACT, PT, APTT), postoperative bleeding and blood products administration were compared with a control group.

Results: The use of blood and blood products and postoperative bleeding was significantly decreased in the cell saver group. Haematocrit was the only parameter which showed significant difference in the compared laboratory results. Jehowa's witnesses received salvaged blood and their haematocrit was above 35 in all 3 cases.

Conclusion: The use of the cell saver system can be safely used in the paediatric open heart surgery. It can decrease the administration of allogenic transfusion and other blood products, increase intraoperative safety in redo operations and can minimize intraoperative risks in specific cases.

6215

Platelet reactivity in paediatric cardiac surgery: Should pharmacological platelet protection be performed during cardiopulmonary bypass and deep hypothermic circulatory arrest in young infants?

Andreas Straub, Dept. of Thoracic, Cardiac and Vascular Surgery, University Hospital of Tübingen, Germany

Joseph Smolich, Australia and New Zealand Children's Heart Research Group, Murdoch Children's Research Institute, Melbourne, Australia Hans Peter Wendel, Dept. of Thoracic, Cardiac and Vascular Surgery, University Hospital of Tübingen, Germany

Gerhard Ziemer, Germany

Yves d'Udekem, Cardiac Surgical Unit, Royal Children's Hospital, Melbourne, Australia

Christian Brizard, Cardiac Surgical Unit, Royal Children's Hospital, Melbourne, Australia

Karlheinz Peter, Baker IDI Heart and Diabetes Institute, Melbourne, Australia

Stephen B Horton, Cardiac Surgical Unit, Royal Children's Hospital, Melbourne, Australia

Objective: Cardiopulmonary bypass $(\mathrm{CPB})$ and hypothermia cause platelet activation and dysfunction, which can contribute to thrombotic events as well as bleeding during cardiac surgery. Short-acting platelet inhibitor administration can protect platelets against CPB- and hypothermia-induced damage. In young infants, however, platelets are immature and may therefore be hyporeactive during CPB. Hence, it is unclear whether pharmacological platelet protection may be beneficial in these patients. This study aimed to measure effects of CPB and hypothermia on platelets in young infants.

Methods: Congenital cardiac defects were repaired in young infants using hypothermic $\mathrm{CPB}$ in centre $1[\mathrm{n}=15$; median age: 9 (range: 1-622) days; lowest temperature: $26^{\circ} \mathrm{C}$ ] and using deep hypothermic circulatory arrest (DHCA) in centre $2[\mathrm{n}=5$, median age: 84 (range: 20-99) days; lowest temperature: $15^{\circ} \mathrm{C}$.

Using flow cytometry, the following platelet activation parameters were assessed before (baseline), during, and after CPB: percentages of platelet $\mathrm{P}$-selectin expression and aggregatebound platelets (all patients) and platelet GPIb $\alpha$ internalisation (centre 1).

Results: In centre 1 the following significant $(\mathrm{p}<0.01)$ median changes of platelet parameters were observed: Compared to baseline platelet P-selectin expression and aggregation were increased 6-fold and 2.3-fold respectively directly after CPB. Compared to baseline GPIb $\alpha$-expression was decreased 1.9-fold directly after CPB.

In centre $2 \mathrm{P}$-selectin expression was increased 4-fold directly after DHCA compared to pre-DHCA values $(p=0.031)$.
Conclusion: In young infants distinct platelet activation occurs in both CPB with hypothermia and DHCA. Both patient groups may therefore benefit from pharmacological platelet protection.

\section{6}

Modified ultrafiltration have a hemostatic effect in pediatric cardiac surgery

Kazuto Yokoyama, Mie University Graduate School of Medicine, Japan Shin Takabayashi, Mie University Graduate School of Medicine, Japan Noriko Yodoya, Mie University Graduate School of Medicine, Japan Hiroyuki Ohashi, Mie University Graduate School of Medicine, Japan Yoshihide Mitani, Mie University Graduate School of Medicine, Japan Hideto Shimpo, Mie University Graduate School of Medicine, Japan

Objective: Although it is suggested that modified ultrafiltration appears to have a hemostatic function, the effects of modified ultrafiltration on the coagulation and fibrinolysis system is unknown. We have investigated the effect of modified ultrafiltration on the coagulation and fibrinolysis system in pediatric cardiac surgery.

Methods: Ten consecutive patients $(23.7 \pm 23.6$ month old, $9.3 \pm$ $5.5 \mathrm{~kg}$ ) who underwent cardiac surgery with modified ultrafiltration were investigated. We measured hematocrit, platelet count, coagulation factors (fibrinogen, factor V, VII, X, and XIII), and fibrinolytic factors (alpha 2 plasmin inhibitor and plasmin-alpha 2 plasmin inhibitor complex) before and after modified ultrafiltration. Results: Hematocrit and platelet count were increased after modified ultrafiltration (hematocrit; $27.5 \pm 6.7 \rightarrow 40.8 \pm$ $6.1 \%$, platelet count; $\left.9.7 \pm 3.6 \rightarrow 14.2 \pm 3.6 \times 10^{4} / \mu 1, \mathrm{p}<0.05\right)$. Additionally, fibrinogen $(78.8 \pm 12.5 \rightarrow 95.3 \pm 14.8 \mathrm{mg} / \mathrm{dl})$, factor $\mathrm{V} \quad(25.1 \pm 7.0 \rightarrow 37.3 \pm 13.1 \%)$, VII $\quad(23.3 \pm 8.1 \rightarrow 44.3 \pm$ $19.9 \%)$, X $(24.2 \pm 6.5 \rightarrow 33.0 \pm 8.8 \%)$, and XIII $(30.9 \pm 9.9 \rightarrow$ $40.7 \pm 7.6 \%)$ levels were increased after modified ultrafiltration $(\mathrm{p}<0.05)$. Although plasmin-alpha2 plasmin inhibitor complex was same level during modified ultrafiltration $(24.6 \pm 47.5 \rightarrow$ $28.0 \pm 53.1 \mu \mathrm{g} / \mathrm{ml}, \quad \mathrm{p}=0.9)$, alpha2 plasmin inhibitor was increased after modified ultrafiltration $(42.8 \pm 8.7 \rightarrow 52.9 \pm$ $8.2 \%, \mathrm{p}<0.05)$, that means fibrinolysis system was not activated during modified ultrafiltration.

Conclusion: Coagulation factor levels were increased same as hemoconcentration, and fibrinolysis system was not activated during modified ultrafiltration. It suggests modified ultrafiltration have an intraoperative hemostatic effect in pediatric cardiac surgery.

\section{7}

The Effect of Tranexamic Acid on Hemostasis in Pediatric Cyanotic Cardiac Surgery

De Ming Zhu, Shanghai Children's Medical Center, Medical College, Shanghai Jiao tong University, China

Jia Shen, Shanghai Children's Medical Center, Department of Cardiothoracic Surgery, China

Objective: Tranexamic acid is an antifibrinolytic agent to reduce blood loss in cardiac surgery. We investigated its effect of different dose in pediatric cyanotic cardiac surgery.

Method: Using a prospective, randomized, double-blind study design, we examined 45 patients of cyanotic congenital heart disease of 6 to $22 \mathrm{Kg}$. In group $1(\mathrm{n}=15)$, patients acted as the control group and receive saline. In group $2(\mathrm{n}=15)$, the low dose group, patients received tranexamic acid $(10 \mathrm{mg} / \mathrm{Kg}$ after induction of anesthesia, $10 \mathrm{mg} / \mathrm{Kg}$ in the pump prime and $1 \mathrm{mg} / \mathrm{Kg}$ per 30 minutes during $\mathrm{CPB}$ period ). In group $3(\mathrm{n}=15)$, the high dose group, tranexamic acid was given at the same time point in a fivefold dose. Total blood lose and transfusion requirements during the period from protamine administration until 24 hours after admission 
to the ICU were recorded. In addition, platelet account and coagulation studies were recorded.

Results: The control group had the longest sterna closure time, maximum blood loss at 24 hours, but there is no difference between the two tranexamic acid groups. No significant statistical difference was found in requirements of blood and blood products. In the low dose group, the fibrin account and the platelet account were significantly higher during $\mathrm{CPB}$ period and at 4 hours after surgery. No patient in this study required dialysis for renal failure after CPB. Conclusion: Tranexamic acid can reduce perioperative blood loss in pediatric cyanotic heart disease. The low dose group $(10 \mathrm{mg} /$ $\mathrm{Kg}$ ) performed the best effect on the protection of platelet and coagulation parameter.

\section{8}

Assessment of the general knowledge of congenital cardiac defects in parents of first nations children in Saskatchewan

Edwin Bolster, University of Saskatchewan, Canada

Ashok Kakadekar, Canada

Parents require a basic level of understanding about congenital cardiac defects to care for children with cardiac disease. We assessed general knowledge of congenital cardiac defects in parents of First Nations children in Saskatchewan.

The study was conducted in the pediatric outpatient clinic and wards. A questionnaire was designed to assess parental knowledge of their child's heart condition as well as to identify any erroneous knowledge. At the end of the survey participants were asked how information could have been better communicated. A descriptive analysis of the results was performed.

The study was conducted from June 2004 to May 2007 and the parents of 44 patients were surveyed. The education level of those surveyed varied with $34 \%$ having completed primary school, 23\% had completed some high school, 23\% had completed high school, and $20 \%$ had completed some college. Only 57\% listed English as their first language. Only 27\% could correctly name the child's heart condition. Fifty-nine percent were able to explain in lay language the nature of the child's heart condition. When given a diagram of the heart, only $32 \%$ marked their defect correctly. Only 10 of 16 parents could describe the indication for the medication their child was prescribed.

First Nations parents of children with congenital heart defects in Saskatchewan have poor knowledge of their children's heart defects. Patient education needs to be emphasized as an important part of clinic visits and ward consultations in order for parents to fully participate in the health care of their children.

6219

Activity and Health Behaviour of Children after surgery for Congenital Heart Disease

Frank A Casey, Royal Belfast Hospital for Sick Children, United Kingdom

Chris G McCusker, United Kingdom

Bernadette Molloy, United Kingdom

Nicola Doherty, United Kingdom

Brian Craig, Royal Belfast Hospital for Sick Children, United Kingdom

Andrew Sands, United Kingdom

Nichola Rooney, United Kingdom

Moira Stewart, United Kingdom

Objectives: To examine the physical and psychosocial determinants of activity and health behaviour in children after surgery for congenital heart disease (CHD).
Methods: Health Behaviour outcomes were assessed in 91 children (mean age 4.6 years) with surgically treated CHD. Subjects were classified into 4 groups according to severity: The three main outcome measures were. 1. Presence of residual symptoms. 2. Frequency of visits to General Practitioner (G.P.) or Accident and Emergency (A\&E) Department. 3. Ability to participate in physical activity using a calculated "Activity Score". Predictor variables examined included socio-economic and maternal factors in addition to medical status.

Results: Children had very few residual symptoms post "corrective surgery". Those with complex CHD post "Fontan"repair had symptoms on average 18.2 days per month. Surprisingly the complex group had less days "sick" from non cardiac causes and made less visits to G.P. or A\&E departments. Regression analysis indicates that 3 variables had significant relevance to G.P./A\&E. visits: Complex CHD- less visits $(p=0.046)$, Townsend Score- more deprivation-more visits $(p=0.002)$ Maternal worry-higher maternal worry score-more visits $(p=0.015)$. Regression analysis indicates lower activity score is significantly related to complex heart disease $(p=0.034)$ and higher maternal worry score $(\mathrm{p}<0.001)$

Conclusions: The majority of this group of children reported few residual symptoms and had good exercise tolerance. Maternal worry is a significant factor in influencing both activity levels and frequency of unscheduled health service demands. Identification of "non cardiac" factors influencing health behaviour helps inform management plans aimed at care of the whole child and family.

\section{0}

The impact of cardiac surgery on infants' emotional development and the infant parent relationship

Brigid Jordan, 1. Royal Children's Hospital, Melbourne 2. Murdoch Childrens Research Institute 3. The University of Melbourne, Australia Candice Franich Ray, Murdoch Childrens Research Institute, Australia Vicki Anderson, 1. Murdoch Childrens Research Institute 2. The University of Melbourne 3. Royal Children's Hospital, Melbourne, Australia

Elisabeth Northam, 1. Murdoch Childrens Research Institute 2. The University of Melbourne 3. Royal Children's Hospital, Melbourne, Australia

Andrew Cochrane, Monash Medical Centre, Melbourne, Australia Samuel Menahem, 1. Monash Medical Centre, Melbourne 2. The University of Melbourne, Australia

Objective: To describe the emotional impact of cardiac surgery on infants. Optimal early emotional experiences and a secure mother-infant attachment relationship are vital for cognitive, social and emotional development. Emotional trauma can compromise brain development. Few studies have focused on the impact of cardiac surgery and hospitalization on emotional wellbeing and the infant-parent relationship during infancy.

Methods: Ninety nine infants subjected to cardiac surgery in the first 3 months of life were recruited. One month after discharge from hospital parents (92 mothers, 64 fathers) completed a study questionnaire which included the Post Natal Attachment Scale and 79 mothers and 57 fathers completed a semi structured interview about their perception of the impact of the surgery and hospitalization on their baby, and the infant-parent relationship. Results: Mothers reported that infants were clingy, hyper-vigilant, and alert to traumatic reminders. A quarter of the infants still had feeding problems, $20 \%$ were unsettled and 14\% had sleeping problems. Seventy three percent (67/92) of mothers and 40/ $64(62 \%)$ of fathers reported impacts on the infant parent relationship including delayed 'bonding', feeling estranged from the baby, fear 
and distress affecting interactions or heightened attachment feelings and behaviours. Although the distribution of scores on the Post Natal Attachment Questionnaire did not differ from community samples $(M=84.47$ SD:7.5), 9/65 (13\%) had scores indicating significant distress in the mother infant relationship.

Conclusion: The care of infants post cardiac surgery may benefit from an assessment and interventions to support the infant's emotional wellbeing and the infant parent relationship.

6221

Riding the rollercoaster: The experiences of parents caring for the child with hypoplastic left heart syndrome Marion E Hamer, Paediatric and Congenital Cardiac Service, Starship Children's Hospital, Auckland, New Zealand

Objective: This study explored the experiences of parents caring for the child with hypoplastic left heart syndrome (HLHS); from diagnosis to the child's second surgery occurring at approximately four months of age.

Methods: A qualitative study using a phenomenological approach in the form of semi-structured interviews of seven mothers and six fathers $(n=13)$. Interviews were analysed using a general inductive approach allowing the dominant and significant themes to emerge from the findings.

Results: Consistent themes described the unrelenting emotional anxiety experienced by parents caring for their child with HLHS. Significant impacts were felt throughout the entire family unit, with life revolving around ensuring the survival of the child. Parents faced significant challenges in navigating a complex and fragmented health care environment, with geographical remoteness from the tertiary cardiac centre placing some families at a disadvantage. Parents sought to have control over their lives through knowledge acquisition, developing and accessing supports, managing family and home environment, as well as, through developing successful relationships with health professionals.

Conclusion: The study identified the extraordinary achievements of parents as they faced the challenges of caring for their child with HLHS within the New Zealand setting. They needed support from knowledgeable and empathetic health professionals who were responsive not only to the needs of the child, but to the concerns of the parents themselves. Education of health professionals, resource development, care coordination and collaborative practice across the health care continuum are key areas for change to ameliorate the identified impacts and pressures for these families.

6222

Biopsychosocial Determinants of Quality of Life in Adolescents and Adults with Congenital Heart Disease Yu-Chuan Hua, Cardiac Children's Foundation, Taiwan, Taiwan Chun-An Chen, Department of Pediatrics, National Taiwan University Hospital, Taipei, Taiwan, Taiwan

Shih-Cheng Liao, Department of Psychiatry, National Taiwan University Hospital, Taipei, Taiwan, Taiwan

Jou-Kou Wang, National Taiwan University Hospital, Taiwan Chung-I Chang, Department of Surgery, National Taiwan University Hospital, Taipei, Taiwan, Taiwan Ing-Sh Chiu, Department of Surgery, National Taiwan University Hospital, Taipei, Taiwan, Taiwan

Yih-Sharng Chen, Department of Surgery, National Taiwan University Hospital, Taipei, Taiwan, Taiwan

Mei-Hwan Wu, Department of Pediatrics, National Taiwan University Hospital, Taipei, Taiwan, Taiwan
Objectives: To assess the quality of life (QoL) in an Asian cohort of adolescents and adults with congenital heart disease (CHD) and to identify its biopsychosocial determinants.

Methods: 278 patients ( $>12$ years) with CHD (Figure) were investigated at a mean age of 25.8 years. QoL was measured using the Quality of Life questionnaire of the World Health Organization (WHOQOL-BREF). Personality traits, psychological characters and family support were assessed by the Maudsley Personality Inventory (MPI), the Brief Symptom Rating Scale (BSRS) and the Family APGAR, respectively. CHD severity was evaluated according to initial diagnosis, illness course, and CHD functional index.

\begin{tabular}{|c|c|c|c|c|}
\hline Variables & QoL: physical & $\begin{array}{l}\text { QoL: } \\
\text { psycholog- } \\
\text { ical } \\
\text { P value }\end{array}$ & Coefficients & $\begin{array}{l}\text { QoL: } \\
\text { environmental } \\
\text { P value }\end{array}$ \\
\hline Depression & $-0.027 \pm 0.009$ & 0.002 & $-0.059 \pm 0.010$ & $<0.001$ \\
\hline Extroversion & $0.098 \pm 0.026$ & $<0.001$ & $0.123 \pm 0.026$ & $<0.001$ \\
\hline Family APGAR & $0.074 \pm 0.032$ & 0.022 & $0.151 \pm 0.031$ & $<0.001$ \\
\hline $\begin{array}{l}\text { CHD functional } \\
\text { index }\end{array}$ & $-0.399 \pm 0.103$ & $<0.001$ & - & NS \\
\hline Age & $-0.027 \pm 0.009$ & 0.005 & - & NS \\
\hline
\end{tabular}

\begin{tabular}{lllll}
\hline Variables & Coefficients & P value & Coefficients & P value \\
\hline Depression & $-0.035 \pm 0.009$ & $<0.001$ & $-0.029 \pm 0.010$ & 0.006 \\
Extroversion & $0.148 \pm 0.031$ & $<0.001$ & $0.096 \pm 0.031$ & 0.002 \\
Family APGAR & $0.115 \pm 0.038$ & 0.003 & $0.216 \pm 0.037$ & $<0.001$ \\
$\begin{array}{l}\text { CHD functional } \\
\quad-\end{array}$ & NS & - & NS \\
Andex & - & NS & $-0.037 \pm 0.010$ & $<0.001$ \\
\hline
\end{tabular}

Results: CHD patients had worse QoL compared to healthy population. On multivariate analysis (Table), depressive mood significantly predicted QoL in each domain (physical, psychological, social, and environmental), and $20 \%$ patients had significantly lower depression scores. Better family support and higher extroversion scores were most closely associated with better QoL. CHD functional index determined only the physical domain of $\mathrm{QoL}(\beta=-0.399, P<0.001)$, and the correlation was marginal $(r=-0.162, P=0.007)$. The initial diagnosis and illness course of CHD had no significant influence on QoL. Conclusions: In this Asian cohort, lower QoL was found in adolescents and adults with CHD and all the dimensions were predominantly determined by patient's depressive symptoms, level of extroversion, and family function. The fact that functional status of CHD patients affected only physical domain of QoL highlighted the limitation of traditional therapies targeting somatic functioning in improving overall QoL.

Multiple linear regression models of sub-dimensions of WHOQOL scale by stepwised forward method with clinical and psychosocial variables as regressors

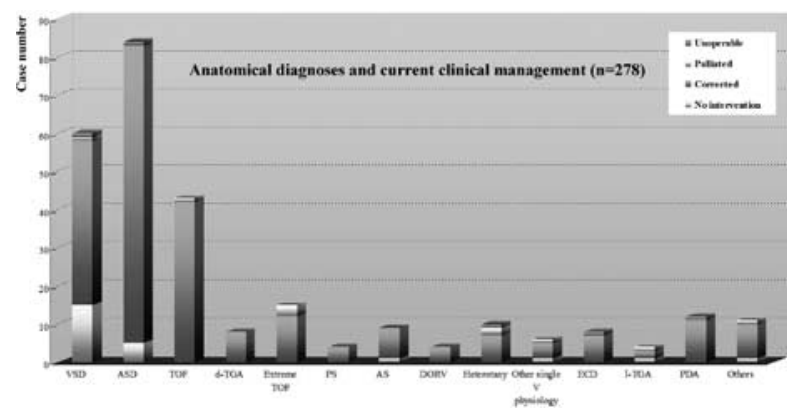


6223

Impaired neuroanatomic development in infants with congenital heart disease

Kazuhiro Watanabe, University of Toyama, Japan

Mie Matsui, University of Toyama, Japan

Naoki Yoshimura, University of Toyama, Japan

Keiich Hirono, University of Toyama, Japan

Fukiko Ichida, University of Toyama, Japan

Yoshihiro Oshima, Hyogo Children's Hospital, Japan

Tachiyo Matsuzaki, National Cardiovascular Center, Japan

Toshikatsu Yagihara, National Cardiovascular Center, Japan

Objectives: Although most infants with critical congenital heart disease (CHD), especially hypoplastic left heat syndrome (HLHS), have a reduced head circumference, there are no data regarding exact brain volume. We hypothesized that infants with critical CHD have impaired anatomic development of the brain, and this is associated with functional impairment.

Methods: We performed a regional volumetric study of the brain using

3-dimensional magnetic resonance imaging (3D-MRI) in 40 infants with heterogeneous CHD including 17 infants with single ventricle physiology (SV), 5 with transposition of great arteries (TGA), and 18 with ventricular septal defect (VSD) and performed neurodevelopmental assessment using the Bayley Scales of Infant Development II.

Results: The global volume of gray matter was significantly reduced in the patients with CHD compared with normal controls $(\mathrm{P}<0.001)$, whereas no significant difference in the volume of white matter was observed. Further, the decrease in gray matter volume was more apparent in the frontal lobe than in the temporal lobe, especially in infants with SV physiology or TGA. Multivariate analysis revealed that preoperative hypoxia is strongly associated with decreased frontal gray matter volume $(\mathrm{P}<0.01)$, as well as a diagnosis of HLHS $(\mathrm{P}<0.05)$. Of note, frontal gray matter volume, which includes the motor area, correlated weakly with psychomotor developmental index scores $(\mathrm{P}<0.01)$.

Conclusions: Brain developmental impairment occurs in many infants with CHD, especially in those who have preoperative hypoxia and critical CHD. This quantitative volumetric study encourages larger scale and longitudinal follow-up to elucidate the significance of impaired neuroanatomic development on functional outcome.

\section{4}

Establishment of a European parent- and patient-oriented information and communication platform on Congenital Heart Defects

Marte A Jystad, Norwegian association for children with congenital heart disease, Norway

Ulrike Bauer, Nationales Register Fuer Angeborene Hersfehler EV,

Germany

Thomas Pickardt, TUV NORD EnSys Hannover GmbHECoBiotechnologie, Germany

Peter Nordqvist, Hjertenbarnsforeningen, Sweden

Hermine Nock, Bundesverband Herzkranke Kinder, Germany

Maria Escudero, Menudos Corazones, Spain

Anne Keatley-Clarke, Chilrden Heart Federation, United Kingdom

Wiebke Lesch, Lesch Communication Consultancy, Germany

We are a consortium consisting of five European patient organisations (conjoint under the ECHDO umbrella), a communication/PR-consultancy, and the German National Registry for CHD. In 2007 we successfully applied for a project within the 7th EU Framework Programme (FP7) that establishes a European web-based information and communication platform that should help to build up a large and powerful European network on congenital heart defects. The project should horizontally and vertically crosslink patients, parents, physicians, scientists, medical organisations etc., i.e. all concerned stakeholders. We intend an increased spirit of co-operation as well as strengthening science and research in this area. Our project called Corience started in February 2008. The website www.corience.org was officially launched in September 2008.

Corience provides a comprehensible and target group optimised supply of information and initiates discussion and dialogue between all groups. Background information about new medical research is processed in close cooperation between scientists, physicians, editors, patients and parents. The CoHD platform also provides media-oriented elements such as news, interviews, fact sheets etc.

The website will be offered in at least four European languages. Information workshops for all concerned groups are arranged in order to foster dialogue and knowledge-transfer. We also organize press conferences in order to inform the public, politics, non-governmental organisations etc.

We will present our concept, strategy, stage of work, and our vision.

6225

Psychological responses following the diagnosis of congenital heart disease: A systematic review of the literature and recommendations for supportive care during the perinatal period

Nadine A Kasparian, School of Women's and Children's Health, Faculty of Medicine, University of New South Wales, Kensington, NSW, Australia, Australia

Bryanne E W Barnett, Karitane Early Parenting Services, Carramar, NSW, Australia, Australia

Gary F Sholler, Adolph Basser Cardiac Institute, The Children's Hospital at Westmead, Westmead, NSW, Australia, Australia David S Winlaw, Adolph Basser Cardiac Institute, The Children's Hospital at Westmead, Westmead, NSW, Australia, Australia Edwin P E Kirk, Department of Medical Genetics, Sydney Children's Hospital, Randwick, NSW, Australia, Australia

Introduction: Advances in paediatric cardiac surgery have resulted in increasing numbers of children surviving congenital heart disease (CHD), yet despite this medical success, CHD is widely recognised as a major source of stress and trauma within families. Objectives: To conduct a systematic review of the literature reporting on psychological responses, and/or parent-infant interaction, following CHD diagnosis. To then apply this information to the development of evidence-based recommendations for the supportive care of families affected by CHD.

Methods: Relevant studies were identified via the MEDLINE, PsycINFO, EMBASE and CINAHL databases (1988-2008), supplemented by citation lists in retrieved articles and expert consultation. A total of 277 articles were critically appraised to assess eligibility, with 41 studies meeting all inclusion criteria.

Results: Studies have documented a range of psychosocial challenges experienced by parents of infants with CHD, including: fears about medical prognosis, frequent hospitalisations and periods of family separation, financial and care-giving burdens, marital breakdown, and grief. A substantial proportion of parents report unmet support needs; however, there are no controlled trials of psychological or supportive care interventions in this setting. 
Conclusions: To address this deficiency, we have embarked upon a prospective study examining the predictors of resilience or vulnerability in parents of infants with a major heart abnormality, with particular focus on responses following prenatal versus postnatal diagnosis. The perinatal period is a critical time to identify, and offer appropriate intervention to, parents at risk of adverse psychological outcomes, thus supporting the development of a secure attachment pattern between infant and parent.

\section{6}

Quality of life in children with congenital heart disease after surgery

Signe H Larsen, Department of Cardiothoracic Surgery, Aarhus University Hospital -Skejby, Denmark

Elisabeth B Jacobsen, Department of Cardiothoracic Surgery, Aarhus University Hospital -Skejby, Denmark

Kristian Emmertsen, Department of Cardiology, Aarhus University

Hospital -Skejby, Denmark

Soren P Johnsen, Department of Clinical Epidemiology, Aarhus

University Hospital, Denmark

Vibeke E Hjortdal, Department of Cardiothoracic Surgery, Aarhus

University Hospital -Skejby, Denmark

Objective: Quality of life has become a part of outcome assessment in the health system. We examined quality of life in groups of children with congenital heart disease after surgery according to the Risk Adjusted Classification for Congenital Heart Surgery (RACHS-1).

Methods: We identified 878 children operated from 1996 to 2002 at Aarhus University Hospital, Skejby.

The Child Health Questionnaire (CHQ-87) was send to all patients aged 10 to 20 years $(\mathrm{N}=441)$. Low RACHS-1 categories (one and two) were compared with high categories (three and four) using the Wilcoxon-Mann-Whitney test. CHQ87 scores are presented as mean $\pm \mathrm{SD}$.

Results: Fifty-one percent answered the questionnaire $(\mathrm{N}=215)$. The distribution of RACHS- 1 categories was: One and two: $66 \%$, three and four: $31 \%$ (3\% could not be assigned to a category). Children in high RACHS-1 categories reported significant lower scores in several CHQ-87 subscales: General Health Perceptions $(68.2 \pm 21.0$ versus $79.4 \pm 16.4, \mathrm{p}<0.001)$, Physical Functioning $(91.5 \pm 10.8$ versus $96.1 \pm 8.8, \mathrm{p}<0.001)$, Self Esteem $(81.7 \pm 14.2$ versus $87.9 \pm 11.2, \mathrm{p}<0.05)$, Mental Health $(78.5 \pm 15.1$ versus $84.1 \pm 11.6, \mathrm{p}<0.05)$, Behavior $(78.1 \pm 15.1$ versus 83.6 $\pm 11.2, \mathrm{p}<0.05)$ Role/Social Limitations -Emotional $(87.2 \pm 23.1$ versus $96.3 \pm 11.1, \mathrm{p}<0.05)$ and Role/Social Limitations -Behavioral (87.7 \pm 23.1 versus $96.6 \pm 12.4, p<0.001)$. There were no differences in General Health, Bodily Pain, Global Behavior and Family Activities between groups.

Conclusion: Children in high RACHS-1 categories have lower scores in several components of health related quality of life using the CHQ-87, when compared with children in low RACHS-1 categories. Further studies are needed to explore this difference and compare these results with children from the general population.

\section{7}

Telemedicine home support program for infants with major congenital heart disease: a randomised control trial Brian A McCrossan, Royal Belfast Hospital for Sick Children, United Kingdom

Gareth J Morgan, Royal Belfast Hospital for Sick Children, United Kingdom
Brian Grant, Royal Belfast Hospital for Sick Children, United Kingdom Andrew J Sands, Royal Belfast Hospital for Sick Children, United Kingdom

Brian Craig, Royal Belfast Hospital for Sick Children, United Kingdom Frank A Casey, Royal Belfast Hospital for Sick Children, United Kingdom

Introduction: Bringing home a child with major congenital heart disease (CHD) is difficult and anxiety levels are often high. As a regional service, patients may be distant from specialist help if problems arise. This study aimed to assess the effect of home support by videoconferencing compared with telephone support on: parental anxiety, patient management, health service utilization and cost implications.

Methods: Prospective, randomized, control trial: video-conference support, telephone support and control group. Patients with major CHD were included if significant support was anticipated post-discharge. Home support provided for 10 weeks. Parental anxiety was assessed using standardised questionnaires.

Results: Over 4 year study period, 105 families included. Age range $=8-490$ days. Video-conference group- $\mathrm{n}=36$, telephone group $=32$, control group $=37$. Median reduction in anxiety score: video-conference group $=6$, telephone group $=3$ $(p<0.05)$. Clinicians felt able to adequately address families' concerns more often in video-conference group (91\% vs $64 \%$, $\mathrm{p}<0.05)$ and were more likely to advise seeking direct health service assessment in telephone group ( $4 \%$ vs $20 \%$, p < 0.05 ). Mean number of health service episodes per patient per week was significantly lower in video-conference group compared with both telephone and control groups ( 0.48 vs 0.81 vs $0.72, \mathrm{p}=0.02)$. The sum of providing home support and health service episodes was significantly lower in the videoconference group compared with both telephone and control groups $(p<0.01)$.

Discussion: Home support for children with major congenital heart disease via video-conferencing decreases parental anxiety compared with telephone support, affects patient management, decreases health service utilization and may be cost-saving.

6228

Attachment, Parenting Style, Coping and Self-Esteem: A comparison between mothers with and without congenital heart disease (CHD)

Smadar Shaleve, Australia

Margaret Hay, Monash University, Australia

Samuel Menahem, Fetal diagnostic unit, Monahs Medical center, Melbourne Australia, Australia

Objectives: This study assessed, through retrospective reporting, parent-child bonding of children with congenital heart disease (CHD) during their own childhood, and who have subsequently become mothers themselves. It also assessed their current psychosocial wellbeing.

Method: Mothers with CHD varying from post-operative transposition of the great arteries to non-operated aortic stenosis were compared to healthy mothers on retrospectively reported parentchild bonding and current self-esteem, adult attachment, coping with motherhood, and child-rearing practices. Sixteen mothers with $\mathrm{CHD}$ (mean age 31.4 years, $\mathrm{SD}=5.5$ ) were recruited through a tertiary centre. A comparison sample of 48 healthy mothers (mean age 37.5 years, $\mathrm{SD}=4.6$ ) were recruited from the community. All participants completed a set of questionnaires assessing their experiences in childhood, adulthood, and as current mothers. Results: A series of independent measures t-tests with Bonferroni adjustment for multiple comparisons found no significant 
differences between the two groups for retrospective child-parent bonding measures. There was a slight trend for mothers with CHD to score higher on both mother and father care in their retrospective reports of received parenting. Mothers in the CHD group also scored higher on current attachment anxiety, and these differences were significant at $\alpha=.05$, but became nonsignificant after Bonferonni adjustment. There was a tendency for mothers with CHD to score higher on current emotion-focused coping, and lower on problem-focused coping; however, these differences were not significant.

Conclusion: These trends indicate that further studies with a larger sample of mothers with CHD are required to accurately determine the health care needs of mothers with CHD.

\section{9}

Tell me your story: An extended interview with mothers of infants with serious heart disease

Jennifer M Re, School of Psychology, Psychiatry and Psychological

Medicine, Monash University, Calyton, Melbourne; Murdoch

Children's Research, Australia

Suzanne Dean, School of Psychology, Psychiatry and Psychological

Medicine, Monash University, Calyton, Melbourne., Australia

Samuel Menahem, Monash Cardiovascular Research Centre,

MonashHeart, Monash University Department of Medicine, Monash

Medical Centre (MMC), Feta, Australia

Serious Congenital heart disease (CHD) requiring early surgical intervention, leads to severe distress on the part of the affected families, poorly addressed by the limited mental health resources available.

Objective: To explore the mothers' experience of diagnosis and treatment of their infant with CHD by means of an in-depth interview and to investigate the consequences of this interview. Methods: As part of a longitudinal study, a carefully structured indepth interview was conducted with mothers of two-month old infants, asking them to reflect on their infant's experience and their own experience of the diagnosis and treatment. Mothers were asked for feedback following the interview.

Results: Of the twenty-eight mother-infant dyads recruited, one mother declined an interview following her infant's death. Themes emerging from feedback indicated that mothers found the interview helped them to put together what had happened to them and their infant.

Discussion: The researchers reflected on this unexpected outcome and suggest several reasons for this. It was the first time mothers told the story from the beginning; it provided an opportunity to review their experience with a 'third eye'; they had been asked to reflect on their own and their infant's internal world in a way that was novel to many mothers. There may also be an element of timeliness, as preliminary 9-month data suggest these issues may have closed off by then.

Conclusion: The extended interview may offer a valuable intervention for mothers and families, especially where resources are limited requiring efficient use of skilled professionals.

\section{0}

\section{Psychological problems of adults with congenital heart} disease

Takeaki Shirai, Department of Adult Congenital Heart

Disease and Pediatric Cardiology, Chiba Cardiovascular Center, Japan

Yoshiko Mizuno, Department of Adult Congenital Heart Disease and Pediatric Cardiology, Chiba Cardiovascular Center, Japan
Yumi Shina, Department of Adult Congenital Heart Disease and Pediatric Cardiology, Chiba Cardiovascular Center, Japan

Yuuko Wakisaka, Department of Adult Congenital Heart Disease and Pediatric Cardiology, Chiba Cardiovascular Center, Japan

Tomohiko Toyoda, Department of Adult Congenital Heart Disease and Pediatric Cardiology, Chiba Cardiovascular Center, Japan

Shigeru Tateno, Department of Adult Congenital Heart Disease and Pediatric Cardiology, Chiba Cardiovascular Center, Japan

Yasutaka Kawasoe, Department of Adult Congenital Heart Disease and Pediatric Cardiology, Chiba Cardiovascular Center, Japan

Koichiro Niwa, Department of Adult Congenital Heart Disease and

Pediatric Cardiology, Chiba Cardiovascular Center, Japan

Background: Recently, psychological distress is thought to be common in patients with cardiac disease. Management of psychological issues in adults with congenital heart disease (ACHD) has been emphasized in US and Euro. However, the priority of mental health care for these patients has not been well recognized in Japan.

Aim: The aim of this study is to investigate current psychological problems in ACHD.

Methods: Patients' history regarding psychological issues was investigated from clinical records in our ACHD clinic from January to March in 2008.

Results: Psychological problems were recognized in 14.1\% (33 patients ; 13males, 20females) of 234 total patients. The median age of them is 26 years old (male 24 , female 34.5 ). The problems consists of depression 12, mental retardation 11, hyperventilation 7 , counseling for anxiety 2 , schizophrenia 1 . There was a wide variety of underlying cardiac diseases. 16 were in NYHA class 1 , 11 in class 2, 6 in class 3. The proportion of NYHA class 2 and 3, residual cyanosis and un- or palliative operation, were significantly higher in patients with psychological problems than the other patients respectively $(\mathrm{p}<.01)$.

Conclusions: Psychological problems of ACHD were inclined to associate with the severity of cardiac functional status. The number of adults with CHD and psychological problems is also not small in Japan.

\section{1}

Anxiety and Depression in Adolescents with Heart Disease Qi Feng Wang, Monash University, Australia

Margaret Hay, Monash University, Australia

David M Clarke, Australia

Samuel Menahem, Fetal diagnostic unit, Monahs Medical center, Melbourne Australia, Australia

Objectives: To evaluate the prevalence and contributory factors of depression and anxiety in adolescents with heart disease (HD). Methods: A cohort of adolescents with HD recruited from an ambulatory setting at a tertiary centre and a private service completed questionnaires further supplemented by clinical information provided by the attending cardiologists. The questionnaires included the Hospital Anxiety and Depression Scale (HADS), Multidimensional Scale of Perceived Social Support (MSPSS), Life Orientation Test-Revised (LOT-R), Sense of Coherence-13 and a researcher-designed questionnaire to assess the adolescent's knowledge of their specific cardiac condition.

Results: 121 patients were recruited between May 2007 and August 2008. They were aged from 12 to 20 years. The majority (98\%) were in NYHA I, having minor abnormalities or residual lesions following surgery or catheter intervention. The abnormities included congenital HD (92\%), acquired HD and arrhythmias. A third $(35 \%, 42 / 121)$ of patients were above the cut-off score for 
probable depression or anxiety, another $21 \%(25 / 121)$ scored in the range for possible depression or anxiety. Scores on the HADS were significantly negatively associated with optimism (Pearson correlation, $\mathrm{r}=-0.496, \mathrm{p}<0.01)$, social support $(\mathrm{r}=-0.316, \mathrm{p}<0.01)$ and sense of coherence $(r=-0.274, p<0.01)$. No relationship was found between patient's scores on the HADS and their knowledge and understanding of their cardiac problem, the severity of their $\mathrm{HD}$, their age or gender.

Conclusions: Depression and anxiety may be common in adolescents with HD. Feelings of optimism, a strong sense of coherence and good social support are protective factors.

\section{2}

Adolescents' Knowledge and Understanding of Their Cardiac Abnormality and Their Influence on Their Quality of Life

Qi Feng Wang, Monash University, Australia

Margaret Hay, Monash University, Australia

David Clarke, Australia

Samuel Menahem, Fetal diagnostic unit, Monahs Medical center, Melbourne Australia, Australia

Objectives: To assess if the adolescents' knowledge and understanding of their congenital heart disease (CHD) influence their quality of life.

Methods: A cohort $(\mathrm{n}=108)$ of adolescents with CHD was recruited from an ambulatory setting at a tertiary centre and a private service. Patients completed self-report questionnaires, supplemented by clinical information provided by the attending cardiologists. Additional questionnaires included The Paediatric Quality of Life Inventory (HRQoL) 4.0-cardiac model, and a questionnaire assessing adolescents' knowledge of their cardiac condition.

Results: 108 patients aged from 12 to 20 years were recruited between May 2007 and August 2008. 98\% (106/108) of patients were in NYHA class I. Abnormities included minor CHD and generally non-haemodynamically significant residual lesions following surgery for conditions such as TGA, TOF and VSD etc. Two adolescents had had a Fontan procedure. Adolescents generally had a poor understanding of their cardiac conditions; $53 \%(57 / 108)$ had no anatomical knowledge of the structurual abnormalities. 32\% (34/108) provided an incorrect anatomical drawing. Only $10(9 \%)$ patients drew reasonably accurate anatomical diagrams of their abnormalities. Of these, 6 completed their questionnaires at home with possible assistance. A greater knowledge and understanding of the cardiac abnormality correlated with increasing age (Pearson correlation, $\mathrm{r}=.19, \mathrm{P}<0.05)$ and improved health related quality of life (HRQoL; $r=.23, \mathrm{P}<0.05$ ). Correlation of severity of CHD and HRQoL was non-significant.

Conclusions: Adolescents' cardiac knowledge and understanding of their cardiac abnormalities have a positive influence on HRQoL in adolescents with CHD. This influence is of greater significance than the severity of their abnormality.

\section{1}

Unique detection of SCN5A deletion by MLPA in teenager with Brugada Syndrome

Lucas J Eastaugh, The Royal Children's Hopsital, Australia Dean G Phelan, VCGS Pathology/Murdoch Children's Research Institute, Australia

Paul James, The Royal Children's Hopsital, Australia

Desiree DuSart, VCGS Pathology/Murdoch Children's Research

Institute, Australia

Andrew M Davis, The Royal Children's Hopsital, Australia
Mutations in SCN5A account for approximately 25\% of cases of Brugada Syndrome (BS). There are no previous reports of SCN5A deletions in BS detected only by multiplex ligationdependant probe amplification (MLPA).

Objective: Demonstration that classic sequencing can miss major deletions in BS and sodium channelopathies.

Methods: A 14 year old boy who presented with atrial flutter had a Brugada type 2 pattern after cardioversion to sinus rhythm. He also had significant sinus node dysfunction. At electrophysiological study a type I pattern appeared under general anaesthesia (Figure). The HV interval was prolonged. Polymorphic VT was induced with triple stimuli. Standard sequencing of SCN5A was performed with no abnormalities detected. Given classic clinical features and HV prolongation MPLA was performed.

Results: MLPA demonstrated a large deletion (c.999424_1338 + 81del0). It was predicted that this deletion would result in SCN5A haploinsufficiency.

Conclusions: In patients in whom there is a high index of suspicion for sodium channelopathy and who have normal sequencing for SCN5A, strong consideration should be given to performing MLPA. This method enables demonstration of large deletions that would have otherwise not have been appreciated and can oocur in childhood.

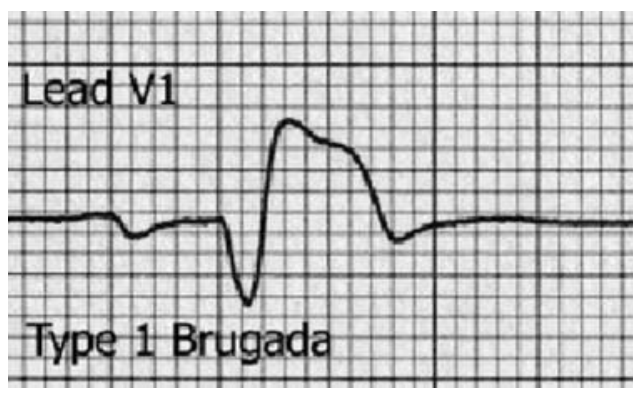

7002

Exercise Stress Testing in Patients With Normal Hearts and $\mathbf{T}$ Wave Inversion on Electrocardiogram

Kipp Ardoin, Ochsner for Children, United States

Sarah Helmig, Ochsner for Children, United States

Terry D King, Ochsner for Children, United States

Christopher S Snyder, Ochsner for Children, United States

Background: T wave inversion on an electrocardiogram (ECG) is suspicious of cardiac disease, yet normal pediatric patients with this abnormality have been identified. The purpose of this study is to evaluate the $\mathrm{T}$ wave response of patients with normal hearts and $\mathrm{T}$ wave inversion on baseline ECG during exercise stress testing (EST). Methods: IRB approved retrospective review of the EST database was performed. Inclusion criteria included a normal heart and age $>18$. Data included: baseline ECG, peak heart rates and METS achieved, and heart rates at which T waves "normalized" during EST. All were exercised using Modified Bruce Protocol with continuous monitoring of ECG's.

Results: Thirteen patients met inclusion criteria (8 males/5 females). Race included Caucasian (6), African American (6), and Hispanic (1). Median age was 15.5 years (range $8-18$ ). Eleven patients $(85 \%)$ experienced complete $\mathrm{T}$ wave reversion (normalization) and 2 (15\%) experienced partial (3/6 limb leads) reversion during EST. Mean peak heart rate was 192 beats per minute (bpm)(range 169-214) and mean maximum METS achieved was 13.2 (range 9-18.5). The median heart rate at which T wave normalization was $153 \mathrm{bpm}$ (range 113-187) and median METS was 4.5 (range 1-12.3). 
Conclusion: Exercise Stress Testing patients with normal hearts with $\mathrm{T}$ wave inversion on resting ECG may result in complete reversion or normalization of their $\mathrm{T}$ waves. This phenomenon occurs in all sexes and ethnicities encountered. A larger study is needed to improve the power and garner long-term follow up information.

7003

Fetal diastolic ventricular function after cardioversion of supraventricular tachycardia (SVT) and atrial flutter (AF) Anita Hamela-Olkowska, Perinatal Cardiology Department, 2nd Department of Obstetrics and Gynecology, Medical University of Warsaw, Poland

Joanna H Szymkiewicz-Dangel, Perinatal Cardiology Department, 2nd Department of Obstetrics and Gynecology, Medical University of Warsaw, Poland

Objective: To assess diastolic ventricular function by establishing E/A ratio (in pulsed Doppler-PD) and Em/Am ratio(in Doppler tissueDTI) in fetuses with SVT and AF after conversion to sinus rhythm. Methods: We analyzed 15 fetuses with SVT and 4 with AF with transplacental antiarrhythmic treatment between 22 and 38 week's gestation. All of them had conversion to sinus rhythm. E/A ratio was evaluated in 15 objects with SVT and 4 with AF and Em/Am ratio in 10 and 4 respectively. Examinations were performed using Sequoia 512, with transabdominal $(3,5-6 \mathrm{MHz})$ and cardiological probe $(2-5 \mathrm{MHz})$. Sample volumes were placed in the tricuspid valve (PD) and the basal part of RV (DTI) in the 4-chamber view. Results: During early phase after conversion to sinus rhythm the $\mathrm{E} / \mathrm{A}$ and $\mathrm{Em} / \mathrm{Am}$ ratio was normal $(<1)$ only in 2 fetuses with SVT. E/A ratio was abnormal $(>1)$ in 13 fetuses with SVT and 4 with AF. Em/Am ratio was abnormal $(>1)$ in 8 with SVT and 4 with AF. The normalization of E/A ratio took from 3 to 8 days, and $\mathrm{Em} / \mathrm{Am}$ ratio - from 3 to 22 days.

Conclusions: To the best of our knowledge our observation is the first report of abnormal fetal $\mathrm{E} / \mathrm{A}$ and $\mathrm{Em} / \mathrm{Am}$ ratio at early recovery after drug-induced cardioversion of SVT and AF. The observed phenomenon may be caused by the transient improvement of ventricular diastolic function and myocardial blood flow, which returns to typical fetal features with individual differences. Further studies to confirm our observations are warranted.

\section{4}

Heart rate variability in children with Fontan circulation lateral tunnel and extracardiac conduit

Jenny Alenius Dahlqvist, Dept Clinical Sciences, Umeå University Hospital, Sweden

Marcus Karlsson, Dept Radiation Sciences, Umea University, Sweden Urban Wiklund, Dept Radiation Sciences, Umeå University, Sweden Rolf Hörnsten, Dept Radiation Sciences, Umeå University, Sweden Eva Strömvall-Larsson, Dept Pediatric Cardiology, Sahlgrenska University Hospital, Gothenburg, Sweden

Katarina Hanséus, Dept Pediatric Cardiology, Lund University

Hospital, Sweden

Annika Rydberg, Dept Clinical Sciences, Umeå University Hospital, Sweden

Objective: The technique in Fontan surgery has developed from lateral tunnel towards extracardiac conduit in order to reduce long-term complications. Earlier studies have shown lower heart rate variability (HRV) in patients with Fontan circulation palliated with lateral tunnel (LT) as compared to healthy controls. This study also investigates HRV parameters in children with Fontan circulation palliated with extracardiac conduit (EC).
Methods: 102 patients (61 LT and $41 \mathrm{EC)}$ and 64 healthy controls underwent Holter-ECG. Analysis included RR-intervals and power spectrum analysis of HRV (Total power TP, Low frequency LF, high frequency HF).

Results: The mean age at Holter for the whole study group was 9.1 years, LT 9.9 years and EC 7.1 years. Children with Fontan circulation (both LT and EC) showed significantly longer RRinterval as compared to controls $(p=0,0015)$. Furthermore, the children with Fontan circulation showed significantly lower total power $(p=0,0076)(F i g), \operatorname{LF}(p=0,0013)$ and $\mathrm{HF}(p=0,00023)$ as compared to healthy controls, respectively. There was no significant difference in HRV between the children operated with LT and EC, although there was a tendency of lower HRV parameters for the patients with EC compared to patients with LT.

Conclusions: A new finding in this study was the significantly lower HRV parameters found in children with extra cardiac conduit as compared to healthy controls. Another interesting finding was the tendency to lower HRV among patients with extra cardiac conduit as compared to those with lateral tunnel - a finding that needs to be further investigated.

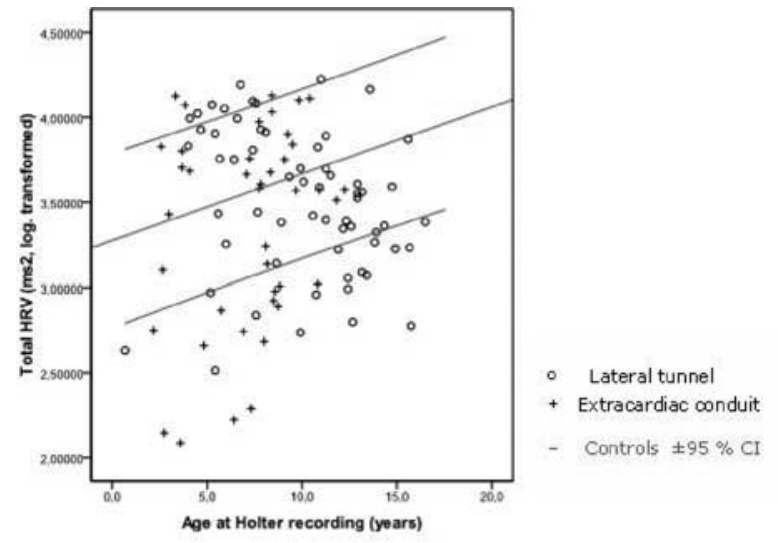

7005

Protein S deficiency associated with acute myocardial infarction (AMI) and cardiac sudden cardiac death (SCD) in adolescents: description of cases in a family

Rogerio B Andalaft, Instituto Dante Pazzanese de Cardiologia, Brazil Danielle D Lima, Instituto Dante Pazzanese de Cardiologia, Brazil Ricardo G Habib, Instituto Dante Pazzanese de Cardiologia, Brazil Cesar A Lima, Instituto Dante Pazzanese de Cardiologia, Brazil Claudia S Fragata, Instituto Dante Pazzanese de Cardiologia, Brazil Christian M Luize, Instituto Dante Pazzanese de Cardiologia, Brazil Georgina V Abreu, Instituto Dante Pazzanese de Cardiologia, Brazil Dalmo R Moreira, Instituto Dante Pazzanese de Cardiologia, Brazil

Introduction: Protein S deficiency is a disorder associated with increased risk of venous thrombosis,but arterial events are rare. Events in the coronary arteries are extremely rare and can cause AMI and SCD.

Objective: Describe a family (three brothers and father) carrying of deficiency of protein S associated with the familiar history of SCD. Case report: A) Patient of 14 years admitted in emergency room with ventricular fibrillation terminated by a 360 joule shock. The ECG (after shock) shows a ST - Elevation MI. The patient denied use of illegal drugs. The coronary angiography resulted normal. The magnetic resonance evidenced signals of necrosis in the apex of the left ventricle. The electrophysiologic study do not induced ventricular arrhythmias. The patient received ICD therapy, amiodarone and beta-blockade therapy and AAS. Two months later the patient had near syncope and 
sensation of shock. Evaluation of the ICD disclosed recurrent VF with 3 appropriate therapies; B) Patient, male (18 years) with syncope presented ECG, echocardiography, Holter and ergometric test without significant finds. The patient presented acute chest pain followed by pulmonary edema and death (autopsy); C) Patient, male (11years) followed by palpitations. ECG, echocardiography, ergometric test, Holter and signal average ECG results normal. The father and two patients had $\mathrm{C}$ present protein deficiency S (13\%, 29\% and 20\% respectively) and currently receive warfarin and AAS therapy.

Conclusion: 1) The thrombophilia research must be considered in young patients with AMI of undetermined origin; 2) The ICD therapy must be used for treatment of new arrhythmic events.

\section{6}

Transesophageal electrophysiologic study in infancy and adolescence: paper in the inquiry of tachycardia

Rogério B Andalaft, Instituto Dante Pazzanese de Cardiologia, Brazil Danielle D Lima, Instituto Dante Pazzanese de Cardiologia, Brazil Ricardo G Habib, Instituto Dante Pazzanese de Cardiologia, Brazil Claudia S Fragata, Instituto Dante Pazzanese de Cardiologia, Brazil Christian M Luize, Instituto Dante Pazzanese de Cardiologia, Brazil Cesar A Lima, Instituto Dante Pazzanese de Cardiologia, Brazil Fabiano M Pegas, Instituto Dante Pazzanese de Cardiologia Dalmo R Moreira, Instituto Dante Pazzanese de Cardiologia, Brazil

Introduction: The supraventricular tachycardia (SVT) are common in infancy and adolescence, however the not invasive diagnosis depends of the register of the tachycardia crisis. The transesophageal electrophysiologic study (TES) can help in the clarification of the palpitations in highly suggestive cases of STV (reentry mechanism)or in the cases where routine (ECG, Holter) failed in show the episodes.

Objective: Describe the behavior of 49 children and adolescents with palpitations submitted to TES where non invasive methods failed in register the crisis.

Results: 49 children and adolescents $(25$ girls, average of $14,36 \pm 3,7$ years -between 7 and 20 years) with palpitation without ECG crisis register had been evaluated. The protocol involved extra stimulus and fast atrial stimulation. Nobody had congenital heart disease with repercussion. Eleven patients can not stand the TES, or had presented difficulty technique in realization of the same. We observed presence of dual behavior of the junction in 7 cases, accessory pathways in 4 cases, and SVT for reentry in 14 cases ( 6 atrioventricular nodal reentrant tachycardia and 8 atrioventricular reentry tachycardia) totalizing $36.7 \%$ in TES. One case presented laboratorial atrial fibrillation. The TES follow-up provided reduce the use of amiodarone $(25 \%$ for $2 \%$ of the patients) and improved the use of the beta blockade treatment of symptoms.

Conclusions: 1) The TES is important method in the diagnosis of the SVT in infancy and adolescence being option to the invasive methods for diagnosis. 2) Children and adolescents have good response to the TES with low indices of complications.

\section{7}

Maternal complication after sotalol treatment for fetal supraventricular tachycardia: case report

Merna M Atiyah, Prince Sultan Cardiac Center, Saudi Arabia Saleh S Al Ghamdi, Prince Sultan Cardiac Center, Saudi Arabia

We report a case of previously healthy mother who developed postpartum tachyarrhythmia as complication after she received oral sotalol for prenatal diagnosis of fetal supraventricular tachycardia (SVT).

The fetal SVT detected at 21 weeks gestation complicated with congestive heart failure and ascites. There was no structural cardiac defect

Before the initiation of sotalol therapy, maternal electrolytes and ECGs were performed to exclude preexisting arrhythmias

The sotalol dose started with $80 \mathrm{mg}$ twice a day and increased to $160 \mathrm{mg}$ three times per day, the fetus successfully converted to sinus rhythm without relapse, and the cardiac function was restorted.The maternal QT interval prior and after increases dose were normal.

The sotalol dose was decreased to $160 \mathrm{mg}$ twice a day at 27 weeks gestation and further weaned gradually one week prior to delivery because of maternal fatigability and dizziness. She had elective cesarean section at 37 weeks gestation and delivered healthy baby girl in normal sinus rhythm, the mother post delivery she was well however six hr post delivery she developed SVT with HR $200 \mathrm{bpm}$ confirmed and converted to sinus rhythm after two doses of adenosine and maintained on verapamil.

Conclusion: This report suggests that sotalol is a potent antiarrhythmic agent in the treatment of fetal SVT because of the excellent placental transfer, especially in hydrops fetalis. However, of more concern are the possible pro-arrhythmic effect both in the mother and the fetus still outweighs it is benefits. The possibility of an adverse effect post withdrawal of these medications must be considered.

\section{8}

Normative data for $\mathrm{QT} / \mathrm{QTc}$ in the recovery phase after exercise in children

Wouter R Berger, Universitair Medisch Centrum Groningen/Royal Children's Hospital Melbourne, The Netherlands

Suleman Kamberi, The Royal Children's Hopsital, Australia

Vanessa Connell, The Royal Children's Hopsital, Australia

Ivan Macciocca, The Royal Children's Hopsital, Australia

Michael MH Cheung, The Royal Children's Hopsital Melbourne/

Murdoch Children's Research Institute, Australia

Andrew M Davis, The Royal Children's Hopsital, Australia

Congenital long QT syndrome (LQTS) is an enigmatic disease that can be difficult to diagnose. Examination of repolarisation in the recovery phase of exercise enhances diagnostic accuracy. There is however no normative data available for children.

Objective: To establish normative data for QT/QTc in children in the recovery phase of exercise.

Methods: Bicycle exercise testing was performed by 88 volunteers, aged 8-17. 12 lead monitoring was continued for 10 minutes into recovery. Four consecutive QT and preceding RR intervals were measured in lead II by a standardized manual method under magnification and averaged for each minute of recovery.QTc was calculated using Bazett's formula. In addition four consecutive QT intervals in V5 were measured and averaged at recovery heart rates of $100 / 110 / 120$ and 130 .

Results: QTcII for the first 10 minutes of recovery and QTV5 at HR 100-130 are shown in table. Maximum QTcII was at 4-7 minutes. During the first 2 minutes of recovery females had a longer QTc: 0.43 vs 0.41 at 1 minute $(p<0.0005)$ and 0.44 vs 0.43 at 2 minutes $(p<0.05)$, thereafter there was no significant difference. Younger volunteers $(8-12)$ had a significantly shorter QTc at 1 minute: 0.41 vs 0.43 and therafter there was no significant difference. 
Conclusions: Normative data for QT/QTc will enhance diagnostic accuracy for LQTS in childhood.

\begin{tabular}{llll}
\hline $\begin{array}{l}\text { Minute/s of } \\
\text { recovery }\end{array}$ & $\begin{array}{l}\text { QTc II (seconds) mean } \\
\pm \text { SD }\end{array}$ & $\begin{array}{l}\text { Heart } \\
\text { rate }\end{array}$ & $\begin{array}{l}\text { QTV5 (seconds) mean } \\
\pm S D\end{array}$ \\
\hline 1 & $0.42 \pm 0.02$ & 100 & $0.33 \pm 0.02$ \\
2 & $0.43 \pm 0.02$ & 110 & $0.32 \pm 0.01$ \\
3 & $0.44 \pm 0.02$ & 120 & $0.30 \pm 0.02$ \\
$4-7$ & $0.45 \pm 0.02$ & 130 & $0.28 \pm 0.01$ \\
$8-10$ & $0.44 \pm 0.02$ & & \\
\hline
\end{tabular}

7009

Extraction of nonfunctional endocardial leads in children Katarzyna Bieganowska, Department of Cardiology The Children's Memorial Health Institute, Warsaw, Poland

Andrzej Kutarski, Department of Cardiology, Medical University, Lublin, Poland

Maria Miszczak-Knecht, Department of Cardiology The Children's

Memorial Health Institute, Warsaw, Poland

Monika Brzezinska-Paszke, Department of Cardiology The Children's Memorial Health Institute, Warsaw, Poland

Agnieszka Kaszuba, Department of Cardiology The Children's

Memorial Health Institute, Warsaw, Poland

Joanna Rekawek, Department of Cardiology The Children's Memorial Health Institute, Warsaw, Poland

Mariusz Birbach, Department of Cardiac Surgery, The Children's

Memorial Health Institute, Warsaw, Poland

Wanda Kawalec, Department of Cardiology The Children's Memorial

Health Institute, Warsaw, Poland

Introduction: Extraction of pacemaker and implantable cardioverter-defibrillator (ICD) leads in children is difficult clinical problem. Abandoned nonfunctional endocardial lead is an risk of venous obstruction or infection and makes new leads implantation more difficult. The purpose of this study was to sum up our experience in endocardial lead removing.

Material and methods: From January 2007 do December 2008 we extracted 11 leads (9 pacemaker leads: 7 ventricular, 2 atrial and 2 ICD leads)from 11 children age from 5 to 17,2 (mean 13,1) years. The implant duration ranged from 0,9 to 11,4 (mean 4,9) years. The main reason for extraction was lead failure (7 leads), in free children with long permanent pacing pacemaker leads were dangerous tight because of the patients' growth. In one case the ICD lead was removed because of sepsis. In all children the lead extraction procedure was performed under general anaesthesia by the same experienced team, the extracorporeal oxygenation and circulation system was available. In all children the Cook Medical system was used during the procedure.

Results: Ten leads $(90,9 \%)$ were completely removed. In one case a short fragment of pacing atrial lead strongly connected with ventricular lead was failure to extract. In this patient massive subclavian venous bleeding occurred as a main our complication. None required cardiac operation.

Conclusion: Extraction of en endocardial lead in children is possible even after long pacing duration, but experienced team and proper facilities are necessary. In children the main indication to remove is en endocardial lead failure.

7010

Markers of myocardial damage after percutaneous radiofrequency catheter ablation in children with tachyarrhythmias
Katarzyna Bieganowska, Department of Cardiology, The Children's Memorial Health Institute, Warsaw, Poland

Maria Miszczak-Knecht, Department of Cardiology, The Children's Memorial Health Institute, Warsaw, Poland

Lukasz Szumowski, Cardiac Arrhythmias Department, Institute of Cardiology, Warsaw, Poland

Monika Brzezinska-Paszke, Department of Cardiology, The Children's Memorial Health Institute, Warsaw, Poland

Joanna Rekawek, Department of Cardiology, The Children's Memorial Health Institute, Warsaw, Poland

Roman Kepski, Cardiac Arrhythmias Department, Institute of Cardiology, Warsaw, Poland

Agnieszka Kaszuba, Department of Cardiology, The Children's

Memorial Health Institute, Warsaw, Poland

Franciszek Walczak, Cardiac Arrhythmias Department, Institute of

Cardiology, Warsaw, Poland

Introduction: Radiofrequency (RF) catheter ablation produces endomyocardial necrosis by way of resistive heating of the tissue in contact with the catheter tip electrode. It may result in release biochemical markers of myocardial cell damage. The aim of the study was to check the levels of markers of myocardial tissue damage in children undergoing ablation RF.

Methods and Results: In 168 children with tachyarrhythmias (mean age 14,9 years) blood samples were taken before ablation (baseline), and next 6-8 hours and 3 days after the procedure. The lactate dehydrogenase (LDH), creatine kinase - MB (CK$\mathrm{MB})$, creatine kinase (CPK) and troponin levels were measured. Results: Mean baseline enzyme levels were: $\mathrm{LDH}-170,1 \mathrm{U} / 1$, CPK-95,1U/1, CK-MB-4,12 U/1, troponin - $0 \mathrm{ng} / \mathrm{ml}$, early hours post ablation: LDH - $165 \mathrm{U} / 1$, CPK-112,6 U/1, CKMB-5,66 U/1, troponin - $1,244 \mathrm{ng} / \mathrm{ml}, 3$ days after the procedure: $\mathrm{LDH}-167,7 \mathrm{U} / 1, \mathrm{CPK}-79,5 \mathrm{U} / 1$, CKMB-4,28 U/1, troponin $-0,168 \mathrm{ng} / \mathrm{ml}$. In children CPK, CK$\mathrm{MB}$ and troponin levels significantly increased early after catheter ablation, then after 3 days CK-MB normalised, troponin level decreased slowly, CPK level was lower compare to baseline level, LDH level was stabile after the procedure.

Conclusion: In children undergoing catheter ablation level of $\mathrm{CPK}, \mathrm{CK}-\mathrm{MB}$ and troponine significantly increased after the procedure as a marker of myocardial damage.

\section{1}

RF ablation of incessant atrioventricular tachycardia after Carpentier operation with Kalangos ring due to Ebstein anomaly

Artur Baszko, Pediatric Electrophysiology Laboratory and

Department of Cardiology, University of Medical Sciences,

Poznan, Poland

Waldemar Bobkowski, Department of Pediatric Cardiology, University of Medical Sciences, Poznan, Poland

Malgorzata Pawelec-Wojtalik, Department of Pediatric Cardiology,

University of Medical Sciences, Poznan, Poland

Krzysztof Czyz, Department of Pediatric Cardiology, University of

Medical Sciences, Poznan, Poland

Mariusz Wojtalik, Department of Pediatric Cardiac Surgery, University of Medical Sciences, Poznan, Poland

Aldona Siwinska, Department of Pediatric Cardiology, University of

Medical Sciences, Poznan, Poland

Stefan Grajek, Poland

We present a 15-year old girl with Ebstein anomaly, significant tricuspid valve (TV) regurgitation, ASD II and initially asymptomatic WPW syndrome. Biodegradable Kalangos Ring 
was used for TV plasty with very good hemodynamic effect. Several weeks after the operation she presented persistent atrioventricular tachycardia (AVRT) with RBBB morphology (130-160/min). Tachycardia did not respond to antyarrhythmic therapy.

Before ablation echocardiography showing good function of the TV with thick folding of the myocardium over the ring at the posterolateral wall. EPS confirmed the presence of atrioventricular pathway (AP) with slow bi-directional conduction. Single stimulus induced AVRT with long RP' interval. The mapping of the posterior-to-lateral wall of the TV showed the broad zone of low amplitude potentials. The $4 \mathrm{~mm}$ tip RF ablation electrode was used and after 2 applications the tachycardia was terminated, but antegrade conduction over the AP was preserved. Several additional applications were only transiently successful thus the decision was made to use irrigated catheter. Linear application was performed with repeated cessation of conduction over the pathway, however the slow tachycardia was inducible. After several minutes the pathway become silent. During 6 months follow-up she did not experience tachycardia despite no antyarrhythmic therapy.

This case reveals that implantation of biodegradable Kalangos Ring may change the electrical properties of the preexisting AP leading to incessant AVRT. In this situation the ablation of the pathway can be difficult due to a myocardial folding covering the pathway. Integrating echocardiography, angiography with irrigated electrode can be successful in abolition of the pathway.
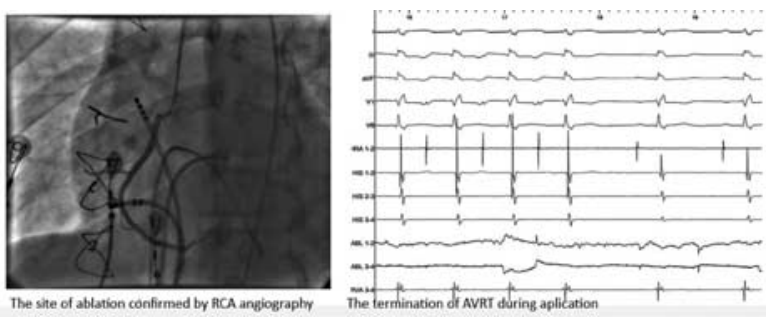

7012

Incessant atrioventricular tachycardia in an infant with postero-septal accessory pathway and coronary sinus diverticulum

Artur Baszko, Pediatric Electrophysiology Laboratory and Department of Cardiology, University of Medical Sciences, Poland

Waldemar Bobkowski, Department of Pediatric Cardiology, University of Medical Sciences, Poland

Malgorzata Pawelec-Wojtalik, Department of Pediatric Cardiology, University of Medical Sciences, Poland

Krzysztof Czyz, Department of Pediatric Cardiology, University of

Medical Sciences, Portugal

Alicja Bartkowska-Sniatkowska, Department of Pediatric Anesthesiology and Intensive Theraphy, University of Medical Sciences, Poland Aldona Siwinska, Department of Pediatric Cardiology, University of Medical Sciences, Poland

Stefan Grajek, Poland

RF ablation of WPW syndrome is not recommended treatment for infants and small children, but cases of incessant tachycardia. The procedure can be further complicated by the presence of anatomical abnormalities, like diverticulum of the coronary sinus.

We present an infant 1 month old with WPW syndrome and incessant orthodromic tachycardia who was unsuccessfully treated with amiodarone, flecainide, propranalol and digoxine. After intensive treatment including deep sedation, intubation and ventilation the tachycardia slowed down from 330 to $260 / \mathrm{min}$., however the signs of heart failure were still present.

The ablation was performed with $5 \mathrm{~F}$ diagnostic and ablation (Marinr, 5F) electrodes. The ECG suggested left posteroseptal pathway (AP). This region was mapped through PFO, but local potentials were later in comparison to right sided location. We performed 2 applications in RPS region resulting in interruption of AP conduction and noninducibility of AVRT. However, the tachycardia recurred after the child returned to the ward, but without signs of preexcitation. The next day the procedure was repeated, starting with CS angiography which showed diverticulum. Retrograde AP conduction was abolished by compression with catheter. 90 minutes later, the retrograde AP conduction recurred and AVRT was induced. The CS and diverticulum were explored and two RF applications resulted in cessation of AP conduction. The follow-up of the child was uneventful, despite frequent atrial premature beats.

The AP in posterosptal region can be associated with large diverticulum which may impede ECG interpretation. The save and successful ablation can be performed after angiographic visualization of the diverticulum.
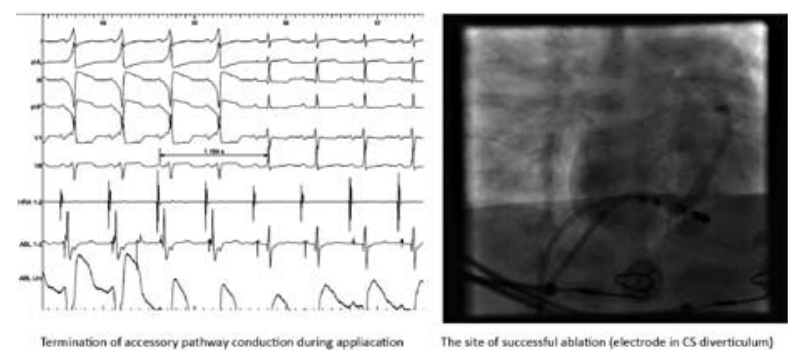

7013

The Evaluation of Ventricular Hypertrophy in Right Ventricular Pressure Overload Rat Model by Electrocardiogram

Young Kuk Cho, Korea

Hwa Jin Cho, Department of Pediatrics, Chonnam National University Medical School, Chonnam National University Hospital, Gwangju, Korea Woo-Yeon Choi, Department of Pediatrics, Chonnam National University Medical School, Chonnam National University Hospital, Gwangju, Korea Gwang-Hyun Eum, Department of Pharmacology, Chonnam National University Medical School, Gwangju, Korea

Hyun Kook, Department of Pharmacology, Chonnam National

University Medical School, Gwangju, Korea

Jae-Sook Ma, Korea

Objective: As cardiac surgery advances, congenital heart patients might have long-term complications such as right ventricular (RV) failure. RV hypertrophy in animal models are evaluated by measuring the heart and by Western or PCR. The authors used electrocardiogram (ECG) to evaluate RV hypertrophy in rat model. Method: 3-week-old rats $(\mathrm{n}=52)$ were divided into control $(\mathrm{n}=24)$ and RV pressure overload group $(\mathrm{n}=28)$. Of latter group, 14 were injected monocrotaline $(30 \mathrm{mg} / \mathrm{kg})$ to obtain pulmonary hypertension (MCT group), and the remaining 14 had pulmonary artery banding (PAB group). ECG was performed 3 times over 1 week.

Results: The ratio of RV free wall and the body weight of MCT group $(1.56 \pm 0.32 \mathrm{mg} / \mathrm{g}) \quad(\mathrm{p}<0.01)$ and $\mathrm{PAB}$ group $(1.97 \pm 0.41 \mathrm{mg} / \mathrm{g})(\mathrm{p}<0.01)$ were increased compared to control group $(0.87 \pm 0.09 \mathrm{mg} / \mathrm{g})$. The 7 th day ECG showed reversed $\mathrm{T}$ wave in lead V1 and the 14th day ECG showed high amplitude of $\mathrm{P}$ 
wave in lead II and reversed T wave in lead V1 in the PAB group compare to control. The 21th day ECG revealed high amplitude of T wave in lead I, of $\mathrm{P}$ wave in lead II, of $\mathrm{R}$ wave in lead aVL, of $\mathrm{S}$ wave in lead aVF, of P and S wave in lead V1, of Twave in lead V6 and it also revealed reversed $\mathrm{T}$ wave in lead $\mathrm{V} 1$ in the $\mathrm{PAB}$ group. Conclusion: ECG may determine the RV hypertrophy in RV pressure overload rat model.

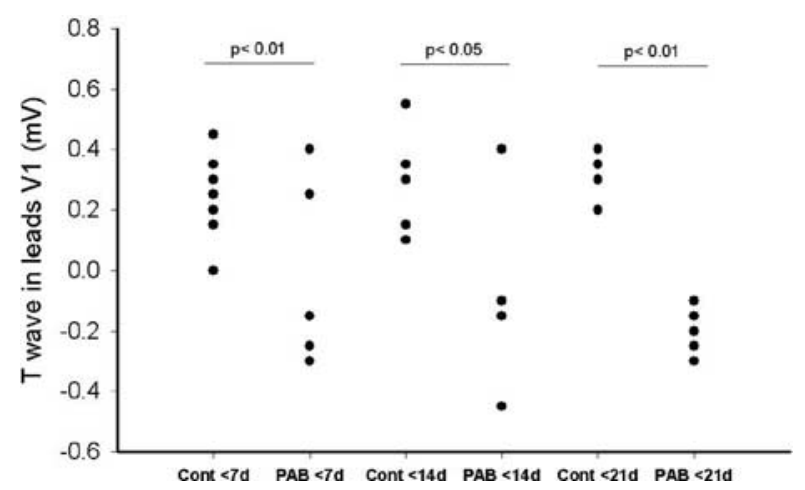

\section{4}

Vectorcardiography may predict long QT syndrome in children

Ulla-Britt Diamant, Dept of Public Health and Clinical Medicine, Umeå University Hospital, Sweden

Annika Winbo, Dept of Clinical Sciences, Umeå University Hospital, Sweden

Annika Rydberg, Dept of Clinical Sciences, Umeå University Hospital, Sweden

Eva-Lena Stattin, Dept of Medical Biosciences, Umeå University

Hospital, Sweden

Steen M Jensen, Dept of Public Health and Clinical Medicine, Umeå University Hospital, Sweden

Objectives: Electrocardiography (ECG) with manual measurements of QT intervals for diagnosing long QT syndrome (LQTS), is burdened with inter-and intra-individual variation. Measurement of QT interval in a pediatric population is even more difficult. The aim of this study was to investigate if vectorcardiographic (VCG) recordings could determine the QT interval with high precision and predict LQTS in a pediatric population.

Methods: ECG and VCG were performed in a pediatric population consisting of 24 children with genetically characterized LQTS and 24 children matched for age and gender in pairs. Mean age was $8 \pm 4$ years (1-15 y). The 12-lead ECG automatic measurement and interpreting of the QT interval corrected for heart rate $(\mathrm{QTc})$ where performed with a $\mathrm{Mac}^{\mathbb{R}} 5000$ (GE Medical system), the VCG automatic measurement were assessed with Mida ${ }^{\mathrm{B}} 1000$ and CoroNet (Ortivus AB). One experienced observer with small intra-observer variability $(1.3 \%)$ performed the manual measurements of QTc in lead II, V5 and V6. Bazett's formula was used for rate correction. QTc were categorized as normal $<440 \mathrm{~ms}$, borderline $440-460 \mathrm{~ms}$, prolonged $>460 \mathrm{~ms}$, QTc values categorized as borderline and prolonged QTc were counted as having LQTS.

Result: The number of diagnosed mutation-carriers and healthy controls with different electrographic methods are shown in the table below.

Conclusion: The VCG measurement of QTc seems to be a better predictor of LQTS in children than manual or automatic measurement and interpreting in scalar ECG. This will be further studied in larger series.

\begin{tabular}{|c|c|c|}
\hline & $\begin{array}{l}\text { Children } \\
\text { Mutation- } \\
\text { carriers }<15 y \\
n=24\end{array}$ & $\begin{array}{l}\text { Children } \\
\text { Non- } \\
\text { carriers }<15 \\
n=24\end{array}$ \\
\hline \multicolumn{3}{|l|}{ Diagnosed mutation-carrier for LQTS by } \\
\hline $\begin{array}{l}\mathrm{QT}_{\mathrm{VCG}}=\text { automatic algorithm computes QT } \\
\text { interval in VCG }\end{array}$ & 22 & 5 \\
\hline $\begin{array}{l}\mathrm{QT}_{\mathrm{ECG}}=\text { automatic algorithm computes } \mathrm{QT} \\
\text { interval in ECG }\end{array}$ & 17 & 6 \\
\hline $\begin{array}{l}\mathrm{QT}_{\text {Interpret }}=\text { automatic algorithm interpreting } \\
\text { QT interval in ECG }\end{array}$ & 13 & 6 \\
\hline $\begin{array}{l}\mathrm{QT}_{\text {manual }}=\text { automatic measurement of QT } \\
\text { interval in ECG }\end{array}$ & 18 & 4 \\
\hline \multicolumn{3}{|l|}{ Diagnosed non-carrier for LQTS by } \\
\hline $\mathrm{QT}_{\mathrm{VCG}}$ & 2 & 19 \\
\hline $\mathrm{QT}_{\mathrm{ECG}}$ & 7 & 18 \\
\hline $\mathrm{QT}_{\text {Interpret }}$ & 11 & 18 \\
\hline $\mathrm{QT}_{\text {manual }}$ & 6 & 20 \\
\hline
\end{tabular}

7015

The diagnostic protocol in children and adolescents with syncope: a multi-centre prospective study

Junbao Du, Department of Pediatrics, Peking University First Hospital, Beijing, China, China

Qingyou Zhang, Department of Pediatrics, Peking University First

Hospital, Beijing, China, China

Cheng Wang, Department of Pediatrics, Xiangya Second Hospital of

Zhongnan University, Changsha, China, China

Zhongdong Du, Department of Cardiology, Beijing Children's

Hospital, Capital Medical University, Beijing, China, China

Li Wang, Health Science Center, Peking University, Beijing, China, China Chaoshu Tang, Key Laboratory of Molecular Cardiology, Ministry of Education, Beijing, China, China

Objective: The appropriate diagnostic protocol for children with syncope has not been well established. A diagnostic protocol was developed and prospectively implemented to improve the diagnostic performance of paediatricians.

Methods: The study population included 474 consecutive patients (range 6-17 years) presenting with a syncopal spell in one of the five participating hospitals of China. In step 1, all patients underwent initial evaluation for history, physical examination, standing test and standard electrocardiography (ECG). In step 2, priority was given to cardiographic tests for possibly cardiogenic syncope, or electroencephalographic examination and brain imaging for suspected neurological syncope, or psychiatric tests for suspected psychiatric syncope. Patients with unexplained syncope underwent head-up tilt testing (HUT).

Results: The initial evaluation gave a definite diagnosis in 59 $(12.4 \%)$ and possible diagnosis in 54 of the 474 patients. Further testing gave a definite diagnosis for 326 patients (69.7\%). After the entire diagnostic protocol, definite diagnosis was established in 385 patients (81.1\%). Autonomic-mediated reflex syncope (AMS) accounted for $73.0 \%$ of cases. The average cost of diagnostic results per patient was RMB 1030.24 \pm 150.09 $(\$ 118.42 \pm 17.25)$.

Conclusion: The use of a simplified diagnostic protocol for children and adolescents with syncope improves diagnostic yield.

\section{6}

\section{RF Ablation of Mahaim Pathways in children and} adolescents

Roman Gebauer, Department of Pediatric Cardiology, University of Leipzig, Heart Center, Leipzig, Germany, Germany 
Peter Kubus, Kardiocentrum and Cardiovascular Research Center, University Hospital Motol, Prague, Czech Republic, Czech Republic Jan Janousek, Heart Center Leipzig, Germany

Objective: Atriofascicular (Mahaim) pathways (MP) are a rare reason for supraventricular tachycardia in childhood. We analyzed results of RF ablation and further clinical course of patients with AV reentrant tachycardia due to MP.

Methods: Data from 18 consecutive pts. from 2 centers aged 9.1-19.6 (median 14.9) years, who underwent RF ablation of a MP between 1998-2008 (follow-up: median 14.1, range $0.2-50.6$ months) were analyzed.

Results: MP was located at right lateral (9 pts.) or posterolateral $(9$ pts.) tricuspid annulus. Mapping was based on the finding of a specific Mahaim potential in 14/18 pts. and/or on intermittent catheter-induced mechanical conduction block in the MP in 9/18 pts. Acute ablation success rate was $100 \%$. Of the 18 patients with initially successful ablation, 4 pts. had arrhythmia recurrence and 3 of them were successfully re-ablated carrying a total long-term ablation success rate of $94 \%(17 / 18$ pts.). The remaining pat. is on antiarrhythmic medication. In $5 / 18$ pts. additional arrhythmogenic substrates were found: WPW syndrome in 2 pts., concealed atrioventricular pathway in 1 pat. and AVNRT in 2 pts. All of these substrates were successfully ablated. Mean fluoroscopy time was 22.4 (range 5.9-64.3) minutes. There was one minor procedure-related complication, no major complications were observed.

Conclusions: Mapping of MP may be hampered by frequent mechanical conduction block. Ablation therapy is effective and incidence of MP recovery approaches figures reported for conventional right free wall pathways. (Supported by Research Project No 00064203 of University Hospital Motol, Prague, Czech Republic).

\section{7}

PJRT - a rare but potentially life threatening arrhythmia Anita Hiippala, Pediatric Cardiology, Helsinki University Hospital for Children and Adolescents, Finland

Olli Pitkänen, Pediatric Cardiology, Helsinki University Hospital for Children and Adolescents, Finland

J-M Happonen, Pediatric Cardiology, Helsinki University Hospital for Children and Adolescents, Finland

Objective: Permanent junctional re-entrant tachycardia (PJRT) is a rare arrhythmia due to a concealed accessory pathway with decremental retrograde conduction properties. The arrhythmia, often incessant, may cause dilated cardiomyopathy, especially in infants. The objective was to analyze PJRT incidence, response to medication and catheter ablation results.

Methods: Thirteen PJRT patients under 15 years of age were identified for a retrospective study.

Results: The incidence of PJRT in Finland is approximately 1/ 100000 children/year. PJRT was diagnosed prenatally or during neonatal period in 7 patients $(54 \%)$. The tachycardia was incessant in three infants causing cardiomyopathy, which resolved in two after conversion to sinus rhythm. One infant died of neurological complications after treatment with an LV assisting device one week after presenting with arrhythmia and severe heart failure. The remaining 6 patients were diagnosed at median 12 years (range 2-14) and had normal LV function.

In five infants, 3-8 medications or combinations were needed before achieving arrhythmia control. At least 2 antiarrhythmic agents were needed in 9/13 patients. Flecainide, propafenone and sotalol were the most effective drugs.

Nine patients (median age 12.7 years, range 5.4-15.6) underwent catheter ablation. Accessory pathway location was right posteroseptal in 6 , left lateral in 2 , and left posterolateral in 1 . The primary procedure was successful in $7 / 9$ patients. A redo ablation was needed in one case after arrhythmia recurrence.

Conclusions: PJRT often manifests in infancy with incessant arrhythmia and tachycardia induced cardiomyopathy. The response to antiarrhythmic medication can be unsatisfactory. Older children benefit of catheter ablation.

\section{8}

Permanent pacing in childrer-long term results Anna I Kaneva-Nencheva, National Heart Hospital, Bulgaria Margarita R Tzonzarova, National Heart Hospital, Bulgaria Georgi v Christov, National Heart Hospital, Bulgaria Ivailo R Kojucharov, National Heart Hospital, Bulgaria Stojan D Lazarov, National Heart Hospital, Bulgaria

Between 1975-2007, 101 patients, aged $8 \pm 5,6$ years, 92 AV block and 9 sick sinus syndrome, underwent pacemaker (PM) implantation with 58 endocardial and 43 epicardial leads mode of pacing VVI-77, VVRR-5, VAT-2, VDD-13, DDD-4. The dysrithmia was postoperative in 36 pts, congenital in 58 and acquired in 7. Patients were followed up $8.9 \pm 7.6(0,1-27 q 4)$ years. Dilated cardiomyopathy (CMP) developed after PM implantation in 3 cases and caused death in all of them. During the follow-up 137 reimplantations were performed with 132 generators and 55 leads were replaccad. After implantation 19 patients died (mortality 18,8\%). No relationship was found between mortality and tye type of dysrhythmia, mode of pacing or lead. Fourty seven complications needed reimplantations were observed in 38 pts - exit block (EB)-22; lead fracture (FL)-9, lead dislodgement-3; pocket infection -8 , decubitus-6, generator migration-2. The 1-, 5- and 10-year freedom from generator reipmlantation is $84 \%, 50 \%, 8 \%$. Multivariate risk factor for generator reimplamtation (Cox regression analysis) is the age below 7 years (RR-3,13). The 1-, 5-, 10- and 12 year freedom from lead reimplantation is $88 \%, 72 \%, 46 \%, 32 \%$. Multivariate risk factors for lead replacement are age below 7 years, epicardial lead and complications (RR-6,7).

Conclusion: the main factor for the choice of the lead and type of stimulation is the patient's age. The age below 7 years, epicardial lead and complications are risk factors for reimlantation.

\section{9}

Prevention of thrombotic complications in children undergoing radiofrequency procedures in the left heart Michal J Kantoch, University of Alberta, Canada

Reeni Soni, Canada

Frank Dicke, Canada

Michael Giuffre, Canada

Ashok Kakadekar, Canada

Shubhayan Sanatani, Division of Cardiology, Department of Pediatrics, British Columbia Children's Hospital and The University of British

Columbia, Canada

Background: Radiofrequency ablation (RFA) in the left heart is associated with thromboembolic complications. Thrombus formation on the transseptal sheaths and at the ablation sites has been reported.

Methods: All prospectively evaluated patients were prescribed ASA $81 \mathrm{mg}$ for 3 days before RFA and for the following 6-8 weeks. A 100 units $/ \mathrm{kg}$ heparin bolus was given as the first flush through the transseptal sheath followed by continuous heparin infusion 20 units $/ \mathrm{kg}$ (maximum 1000 units $/ \mathrm{kg}$ ). All patients were 
scheduled for transthoracic echocardiograms on day 1 and 2-3 weeks later.

Results: Between March 2006 and December 2008, 67 patients (28 female), age $0.8-17$ years (mean 12.2 years) underwent RFA. All patients had accessory pathways except for 3 patients with ectopic atrial tachycardia. A single patient had mild Ebstein's anomaly of the tricuspid valve. Transseptal puncture was done in 49 patients (43 with TEE guidance). Eighteen patients had a PFO. The LA catheter time was $10-120$ min (mean $38 \mathrm{~min}$ ). The RF application time was $30-794 \mathrm{sec}$ (mean $210 \mathrm{sec}$ ). The ACT was $165-377 \mathrm{sec}$ (mean $254 \mathrm{sec}$ ). The permanent RFA success rate was $88 \%$. No patient developed thromboembolic complications. No patient had a recognizable thrombus by echo on day 1 (4 patients had small pericardial fluid) and none of 56 patients on follow up, mean 17 days post RFA. One patient had new mild/ moderate mitral regurgitation.

Conclusion: Thorough anticoagulation with heparin combined with administration of low dose ASA may prevent thrombotic complications.

\section{0}

The incidence and risk factors for supraventricular tachyarrhythmia induced by cardiac catheterization in Thai children and adult with congenital heart disease Apichai Khongphatthanayothin, Division of Cardiology, Department of Pediatrics, Faculty of Medicine, Chulalongkorn University, Thailand Pornthep Lertsapcharoen, Division of Cardiology, Department of Pediatrics, Faculty of Medicine, Chulalongkorn University, Thailand

Objective: To find the incidence and risk factors for supraventricular tachyarrhythmia (SVT) occurring during cardiac catheterization at our institution.

Methods: Occurrence of SVT during diagnostic and interventional cardiac catheterization for children and adult with congenital heart disease (age $3.7 \pm 4.7$ years, $54 \%$ male) between the year 2000-2004 was prospectively recorded. SVT was defined as tachyarrhythmia of non-sinus origin with narrow or similar QRS complex as during the sinus rhythm. The incidence and type of tachyarrhythmia was determined for each diagnostic category. Chart reviews were done in 2008 for arrhythmic events after the catheterization procedure.

Results: SVT occurred in 41 patients out of 835 catheterization procedures (incidence $=4.9 \%$ ). The mechanisms were thought to be atrial flutter or atrial tachycardia (AT/AF) in 16, junctional tachycardia in 1 and reentry via the AV node (AVRT/AVNRT) in 24 patients. Age and sex were not associated with SVT occurrence. Patients with functional single ventricle were more likely to have SVT (mostly AVRT/AVNRT, incidence 13.4\%, relative risk 3.5, $\mathrm{p}<0.001$ ) and infants with PS were more likely to develop AT/AF during balloon valvuloplasty procedure (incidence $14 \%$, relative risk 3.2, $\mathrm{p}<0.01$ ). During follow-up, clinical tachyarrhythmia were rare, although the actual recurrence rate cannot be determined because many patients were taking medications with antiarrhythmic property (such as digoxin).

Conclusion: The diagnosis of single ventricle was a risk factor for SVT during diagnostic cardiac catheterization in children. Balloon pulmonary valvuloplasty in infant was a risk factor for AT/AF during the procedure. In our clinical setting, recurrence of SVT was rare.

7021

Long QT interval - Implantable cardioverter defibrillator in infant - case report
Ivan Malcic, University Hospital Center Zagreb, Croatia, Croatia Bruno Buljevic, University Hospital Center Zagreb, Croatia, Croatia Hrvoje Kniewald, University Hospital Center Zagreb, Croatia, Croatia Dalibor Saric, University Hospital Center Zagreb, Croatia, Croatia Ranko Ugljen, University Hospital Center Zagreb, Croatia Bozidar Ferek-Petric, Medtronic, Croatia

Milivoj Novak, University Hospital Center Zagreb, Croatia, Croatia

Introduction: Long QT interval syndrome (LQTS) is caused by an abnormality of the hearts' electrical system although the mechanical function of the heart is basically normal. Electric problems occur because of the pathological changes on the level of cellular ionic canals.

Case study: S.L. was admitted in the age of 5 months after cardiac arrest and successful resuscitation. She was resuscitated again in our institution. Ventricular tachycardia patterns which concur with TdP was registered. She had previously been treated with beta-blocators for 2 months. Rhythm normalized after an intravenous application of magnesium sulphates. We started with mexiletine and $\mathrm{MgSO} 4$ for 3 months but still had several of ventricular tachycardia crises of the TdP type with loss of conscience. ECG revealed LQTS as definite diagnosis (QTc was $0.54 \mathrm{sec}$ ). She had continously been monitored before the implantable cardioverter defibrillator was implanted (ICD) En TrustTM D 154 VCR Eurin Eucomed with epicardial electrodes transvenous SCV 6397 - 58 with a «dual chamber pacing». ICD was implanted subcutaneously in the left abdomen area and is well tolerated. This is the first infant with an ICD implanted in this way in infant age. Genetic analysis of the 10 known genes which cause LQTS interval was negative.

Conclusion: Case study presents an infant with a Long QT interval syndrome and threatening TdP incidents with lethal outcome, who had an ICD with epicardial electrodes subcutaneouslly implanted. This seems to be the youngest child with an ICD implanted in this way.

\section{2}

Congestive heart failure or left ventricular dysfunction is frequent manifestation in infants with chaotic atrial tachycardia

JaeKon Ko, Division of Pediatric Cardiology, Asan Medical Center, University of Ulsan College of Medicine, Korea

HyungSoon Choi, Division of Pediatric Cardiology, Asan Medical

Center, University of Ulsan College of Medicine, Korea

YongBum Kim, Division of Pediatric Cardiology, Asan Medical Center, University of Ulsan College of Medicine, Korea

YoungHwue Kim, Division of Pediatric Cardiology, Asan Medical Center, University of Ulsan College of Medicine, Korea

InSook Park, Division of Pediatric Cardiology, Asan Medical Center, University of Ulsan College of Medicine, Korea

Objectives: Chaotic atrial tachycardia (CAT) is a rare tachyarrhythmia in infants and it may resolve spontaneously in months without evidence of hemodynamic compromise, however, some infants exhibit signs of heart failure or cardiomyopathy. The purpose of this study was to review the clinical characteristics and late outcome in children with CAT.

Methods: During last 17 years, 36 patients were diagnosed as CAT by the electrocardiographic criteria of CAT. Clinical presentations, echocardiograms and long-term courses of these patients were reviewed. Particular attention was paid to evidence of cardiac dysfunction and follow-up cardiac status.

Results: 23 boys and 13 girls presented with CAT. The age at presentation were all below $1 \mathrm{yr}$ (median age 1 month) and 
$13.6(36 \%)$ patients presented as fetal arrhythmia. 11 (30\%) had structural heart diseases. 14 infants (39\%) showed signs of congestive heart failure or left ventricular dysfunction at echocardiogram. There was no difference in atrial rates or ventricular rates between patients with and without ventricular dysfunction. Structural heart disease did not have influence on the exhibition of clinical heart failure or left ventricular dysfunction. Ventricular function was recovered in all with dysfunction after resolution of CAT. Sinus rhythm was restored in 4.1 months on the average (1-9 months). During follow-up, there were 2 deaths, not related with tachyarrhythmia.

Conclusions: CAT in children usually occurs in the first months of life and preterm. Significant number of patients exhibited overt congestive heart failure or left ventricular dysfunction. However, long-term outcome was excellent, irrespective of the presence of ventricular function or structural heart disease.

7023

Clinical Characteristics and Outcomes of Catecholaminergic Polymorphic Ventricular Tachycardia in Children Bo Sang Kwon, Korea

Gi Beom Kim, Department of Pediatrics, College of Medicine, Seoul National University, Seoul, Korea

Eun Jung Bae, Department of Pediatrics, College of Medicine, Seoul National University, Seoul, Korea

Chung Il Noh, Department of Pediatrics, College of Medicine, Seoul National University, Seoul, Korea

Jung Yun Choi, Department of Pediatrics, College of Medicine, Seoul National University, Seoul, Korea

Yong Soo Yun, Department of Pediatrics, College of Medicine, Seoul National University, Seoul, Korea

Introduction: Catecholaminergic polymorphic ventricular tachycardia (CPVT) is a highly malignant and unclear arrhythmia. The objectives were to characterize the clinical and electrocardiograhpic features of CPVT.

Methods: We studied retrospective analysis of 11 patients (M:F = 9:2) diagnosed as CPVT from 1984 to 2008 in a tertiary center.

Results: The ages at the first event and at diagnosis were $6.8 \pm 2.8$ (1.3-10.0) years and $9.5 \pm 2.2(6.8-12.0)$ years. The interval of diagnostic delay was $2.0 \pm 1.6(0-5)$ years. The most common misleading diagnosis was epilepsy. Initial presentation of all was syncope related to exercise or emotional stress. All 5 early onset patients (less than 7 years of age) showed accompanying learning disability and developmental delay. Resting ECG showed sinus bradycardia, $55 \pm 7(48-76) \mathrm{bpm}$, and QTc was $0.42 \pm 0.03$ $(0.40-0.49) \mathrm{sec}$. There were $U$ wave in 9 patients, $\mathrm{J}$ wave in 5 , sinus pause in 2, and wandering pacemaker in 2. On ECG during CPVT, mean heart rate, QRS duration, coupling interval, QT and QTc immediately before CPVT, and VT index were $184 \pm 21(169-240) \mathrm{bpm}, \quad 0.12 \pm 0.03(0.08-0.18) \mathrm{sec}, \quad 0.33 \pm 0.04$ $(0.26-0.40) \mathrm{sec}, \quad 0.31 \pm 0.06(0.22-0.44) \mathrm{sec}, \quad 0.45 \pm 0.07(0.37-$ $0.59) \mathrm{sec}$, and $1.11 \pm 0.17(0.78-1.29)$, respectively. Sinus rate before the onset of CPVT was $131 \pm 24(95-160) \mathrm{bpm}$. Polymorphic and bidirectional VT was documented in all patients. Five patients also had atrial tachycardia. All patients took beta-blocker. The Six out of 11 patients died suddenly (4/6) or had rescued cardiac arrest (2/6) during $7.1 \pm 4.2(0.6-14.3)$ years of follow-up. Aborted cardiac arrest was a risk factor of sudden death. Although 1 patient underwent ICD implantation, he suffered from several episodes of electrical storm. RyR2 mutation was documented in $1 / 5$ patients.

Conclusion: CPVT patients have still had unsatisfactory and limited prognoses despite medical therapy.
7024

Conductions abnormalities (AV block) and cardiac arrhythmias in children with laminopthies

Maria V Lafuente, Hospital de Pediatría Juan P Garrahan, Argentina Soledad Monges, Hospital de Pediatría Juan P Garrahan, Argentina Alberto M Sciegata, Hospital de Pediatría Juan P Garrahan, Argentina Horacio A Capelli, Hospital de Pediatría Juan P Garrahan, Argentina

Objetives: To describe conduction abnormalities and cardiac arrhythmias in four patients (pts) with neuromuscular disorders and confirmed mutations in LMNA/C gene.

Material and Methods: EKG and Holter alterations were evaluated in 4 pts.

The cardiac events appeared at age between 9 and 13 years.

Results: Patients were referred to the cardiologist with sustained atrial tachycardia (AT) with cardiac collapse in two case and non sustained AT with palpitations in one pts.

The EKG findings in 3 pts were: very low-amplitude $\mathrm{P}$ wave, progressive first degree AV block and left anterior hemiblock. Two of the latter evolved to complete LBBB. Only one patient still persist with a normal EKG.

In Holter monitoring all pts showed premature atrial beats and episodes of atrial tachycardia. Frequent single premature ventricular contractions (PVC) were detected in 3 pts, multiform PVC in 2, couplets in 2 and non sustained ventricular tachycardia in 1 case.

Two patients with progressive AV block and associated LBBB received permanent pacemakers.

At present all pts have normal ventricular diameters and function (ejection fraction more than 66\%).

Conclusions: The first clinical manifestation of cardiac involvement in patients with diagnosis of EDMD caused by a mutation in the LMNA gene could be the presence of palpitations due to symptomatic atrial tachycardia.

In Holter recording early detection of premature atrial/ ventricular contractions usually with repetitive forms is the rule. Disorders of atrioventricular and intraventricular conduction were rapidly progressive.

In the initial course of the disease systolic ventricular function was not affected.

\section{6}

Implantation of Transvenous Leads After Transvenous Closure of Intracardiac Septal Defects

Christopher S Snyder, Ochsner for Children, United States

Bryan Cannon, Texas Children's Hospital, United States

Maria Dortan, Ochsner for Children, United States

Victor Lucas, Ochsner for Children, United States

Ian Law, University of Iowa, United States

Robert Hamilton, Hospital for Sick Children, Canada

Randall Bryant, University of Florida, United States

Background: Transvenous lead (TVL) implantation in patients with a septal defect can result in complications. With the advent of the transvenous septal occlusions(TVSO) risks can be minimized. The purpose of this study is to evaluate the outcomes of transvenous lead implants after device closure of a septal defect.

Methods: IRB approved multi-center review. Data collected included:cardiac diagnosis, indication and complications of septal closure,indication, initial and long-term follow up of leads.

Results: We identified 11 patients who underwent TVSO followed by implantation of TVL(s). Eleven TVSOs ( 9 atrial, 1 ventricular and 1 both) were placed. Median age was 13 years (range 4-21). No complications resulted [median 60 months (range 2-153).] Leads were implanted: same stay (6), $<3$ months 
later (2) or $>3$ months (3). Indication for device: [pacer (7)/ICD (4)] was complete atrio-ventricular block (5), sinus node dysfunction (2) and aborted sudden death (4). No complications occurred with leads [average 44 months (range 5-84).] No significant difference were noted in thresholds (average: Atrium; implant $=0.5 \mathrm{~V}$ at $0.4 \mathrm{~ms}$, follow-up $=0.6 \mathrm{~V}$ at $0.4 \mathrm{~ms}$, Ventricle; implant $=0.6 \mathrm{~V}$ at $0.5 \mathrm{~ms}$, follow-up $0.9 \mathrm{~V}$ at $0.5 \mathrm{~ms}$.)

Conclusion: Implantation of TVL can be successfully performed after TVSO. TVL can be implanted during the same hospital stay as TVSO is performed. Acute and chronic thresholds are not affected by septal occlusion device placement in short-term follow-up. A large study with long term follow-up is warranted to evaluate lead and device longevity.

\section{7}

Postnatal course and long-term prognosis of fetal tachycardia

Yasuki Maeno, Department of Pediatrics, Kurume University School of Medicine, Japan

Akiko Hirose, Department of Pediatrics, Kurume University School of Medicine, Japan

Hiroshi Nishino, Department of Pediatrics, Kurume University School of Medicine, Japan

Shintaro Kishimoto, Department of Pediatrics, Kurume University

School of Medicine, Japan

Motofumi Iemura, Department of Pediatrics, Kurume University School of Medicine, Japan

Kenji Suda, Department of Pediatrics, Kurume University School of

Medicine, Japan

Toyojiro Matsuishi, Department of Pediatrics, Kurume University

School of Medicine, Japan

Effective pre-natal treatment was reported in the fetal tachycardia. However, postnatal course and long-term prognosis is not well understood. Some of the infants with atrioventricuar reentrant tachycardia are known to have recurrence after about several years.

Objective: We examined the postnatal course of tachycardia started at prenatal period.

Subjective: From 22 cases with fetal tachycardia during 25 years from 1983 to 2007, 21 cases (supra ventricular tachycardia (SVT), 7 cases; atrial flutter (AFL), 8 cases, others 6 cases) were included. Postnatal course were collected from the hospital chart or phone call.

Results: Follow-up period is ranging from 1 month to 23 years (median 7.0 years). Of the 8 cases with fetal AFL, no case had AFL during postnatal period or follow-up period. Of the 7 cases with fetal PSVT, 5 cases had PSVT during neonatal period and 4 of them were refractory cases requiring multiple medication. Two cases had recurrent PSVT after 10 and 13 years, respectively. Of the other 6 cases, one died at neonatal period, and another still require medication at the last follow-up in 18 years old. Conclusions: Fetal PSVT requires special attention at postnatal period and during follow-up period.

\section{8}

The electrophysiological change of fast pathway property after slow pathway ablation in children with atrio-ventricular nodal reentrant tachycardia

Masaharu Matsumura, Japan

Naokata Sumitomo, Department of Pediatrics and Child health, Nihon University School of Medicine, Tokyo, Japan

Hiroya Ushinohama, Department of Cardiovascular system, Fukuoka children's Hospital \& Medical Center for Infectious Disease,

Fukuoka, Japan
Kazuo Taniguchi, Department of Pediatrics and Child health, Nihon University School of Medicine, Tokyo, Japan

Junji Fukuhara, Department of Pediatrics and Child health, Nihon University School of Medicine, Tokyo, Japan

Hiroshi Kanamaru, Department of Pediatrics and Child health, Nihon University School of Medicine, Tokyo, Japan

Mamoru Ayusawa, Department of Pediatrics and Child health, Nihon University School of Medicine, Tokyo, Japan

Hideo Mugishima, Department of Pediatrics and Child health, Nihon University School of Medicine, Tokyo, Japan

Background: Electrophysiological property of the fast pathway (FP) may change after slow pathway (SP) ablation in patients with atrio-ventricular nodal reentrant tachycardia (AVNRT).

Methods: A consecutive 42patients (mean \pm SD age $=13.2 \pm 5.7$ years; $M / F=23 / 19$ ) with AVNRT, who performed successful SP ablation were included in this study from August 1998 to December 2008. The AH interval, HV interval, the effective refractory period (ERP), functional refractory period (FRP), and Wenckebach rate of FP were compared before and after ablation of SP.

Results: HV interval was not changed before $(50 \pm 10 \mathrm{~ms})$ and after $(52 \pm 10 \mathrm{~ms})$ ablation. However, mean ERP of the FP was decreased from $346.8 \pm 86.5 \mathrm{~ms}$ to $253.5 \pm 68.9 \mathrm{~ms} \quad(\mathrm{p}<0.001)$; mean FRP of the FP was decreased from $422.2 \pm 87.1 \mathrm{~ms}$ to $371.4 \pm 72.0 \mathrm{~ms}(\mathrm{p}<0.001)$; mean Wenckebach rate was increased from $167.7 \pm 35.2 / \mathrm{min}$ to $188.2 \pm 29.3 / \mathrm{min}(\mathrm{p}=0.005)$; and $\mathrm{AH}$ interval was decreased from $98 \pm 24 \mathrm{~ms}$ to $91 \pm 28 \mathrm{~ms}(\mathrm{p}=0.026)$. Conclusions: The values of ERP, FRP of FP and AH interval were decreased, and Wenckebach rate of atrio-ventricular node was increased in patients of AVNRT after slow pathway ablation. These finding may indicate not only functional interaction of FP and SP but autonomic nerves interaction is existed in patients with AVNRT.

\section{9}

Problems with ablation treatment for fascicular tachycardia in young patients

Maria Miszczak-Knecht, The Children's Memorial Health Institute, Poland

Katarzyna Bieganowska, The Children's Memorial Health Institute, Poland

Lukasz Szumowski, Cardiology Institute, Poland

Franciszek Walczak, Cardiology Institute, Poland

Joanna Rekawek, The Children's Memorial Health Institute, Poland Monika Brzezinska-Paszke, The Children's Memorial Health Institute, Poland

Wanda Kawalec, The Children's Memorial Health Institute, Poland

Fascicular tachycardia (FT) is uncommon form of left ventricular tachycardia in young patients. This tachycardia is usually initiated by programmed ventricular or atrial stimulation. The electrophysiology study and RF ablation were preformed in 5 boys with FT, mean age 14 yr 6 months (ranged 11 yr 3 month-18 yr 6 month). All patients had normal heart morphology. The clinical arrhythmia was triggered by exercise in all patients. The EPS and RF ablation were preformed under general anesthesia in 2 patients and in local in three. In all but one tachycardia was difficult to induced, so Isoproterenol was used. Tachycardia started with right atrium rapid pacing $(360 \mathrm{~ms})$ in one, coronary sinus pacing $8 \times 460+450 \mathrm{~ms}$ in one, coronary sinus pacing $8 \times 460+360 \mathrm{~ms}$ in 1 patient. In 2 boys coronary sinus pacing $8 \times 460 \mathrm{~ms}+300+300 \mathrm{~ms}$ (1 pts), and $8 \times 460 \mathrm{~ms}+240+$ $210 \mathrm{~ms}$ (1pts) initiated atrio-ventricular nodal tachycardia which switch to fascicular tachycardia. In 2 boys also RV 
pacing $460 \mathrm{~ms}+300 \mathrm{~ms}(1 \mathrm{pts})$ and $8 \times 460 \mathrm{~ms}+340 \mathrm{~ms}(1 \mathrm{pts})$ initiated FT.

RF energy was delivered on posterior fascicle area during FT with the local Purkinji potential preceding QRS $18-28 \mathrm{~ms}$ in 3 patients. In one boy the RF ablation wasn't done because of short runs of tachycardia. In 1 pts RF ablation wasn't done due to slow pathway ablation complication.

In patients with AVNRT and FT ablation of slow pathway was also performed.

Conclusions: fascicular tachycardia is difficult to induce. It is easier to initiat it by atrial pacing. It may be provoked by atrioventricular nodal tachycardia.

\section{0}

Incidence of arrhytmogenic events after extra-cardiac conduit type Fontan

Aya Miyazaki, Department of Pediatric Cardiology, National Cardiovascular Center, Japan

Hideo Ohuchi, Department of Pediatric Cardiology, National Cardiovascular Center, Japan

Heima Sakaguchi, Department of Pediatric Cardiology, National Cardiovascular Center

Masaki Yamamoto, Department of Pediatric Cardiology, National Cardiovascular Center

Ken-ichi Kurosaki, Department of Pediatric Cardiology, National Cardiovascular Center

Toshikatsu Yagihara, National Cardiovascular Center, Japan

Osamu Yamada, Department of Pediatric Cardiology, National Cardiovascular Center, Japan

Background: The extra-cardiac conduit type Fontan procedure (ECC) has a lower incidence of arrhythmogenic events compared with lateral tunnel and atriopulmonary connection. However some intrinsic arrhythmogenic patients, such as isomerism heart have shown a high incidence of arrhythmias.

Methods: Two hundred twelve patients (age at ECC: $4.2 \pm 5.7 \mathrm{yrs}$ ) underwent ECC since 1995. We retrospectively reviewed the incidence and the clinical course of arrhythmias after ECC (followup period after ECC: $5.6 \pm 3.1 \mathrm{yrs}$ ).

Results: Atrial situs was solitus (SS) in 144 patients, inversus (SI) in 8, right isomerism (RI) in 38 and left isomerism (LI) in 15. The Supraventricular tachycardias (SVT) occurred in 26 patients (12\%) after perioperative period, and 18 patients (8\%) was required antiarrhythmic medication during follow-up. Two patients (RI: 1, SI: 1) died related with SVT in early postoperative period, and 2 (RI: 2) died later. The freedom from SVT after perioperative period of ECG in SS/SI, RI, LI was $93 \%, 70 \%, 70 \%$ at 5 years, and $93 \%, 65 \%$, $70 \%$ at 10 years $(\mathrm{p}<0.0001)$. Pacemaker implantation $(\mathrm{PMI})$ was performed after ECC in 17 patients $(8 \%)$, caused by sinus node dysfunction in 12, atrioventricular block in 4 and atrioventricular block creation as the treatment of SVT in 1. The freedom from PMI in SS/SI, RI, LI was $95 \%, 90 \%, 57 \%$ at 5 years $(\mathrm{p}=0.0003)$.

Conclusion: Because postoperative arrhythmogenic events are not uncommon in ECC patients, preoperative comprehensive therapeutic strategy, including EPS, should be considered in some ECC patients, especially in isomerism heart.

\section{1}

HAV pattern in Pediatric Patients with Typical AV Node Reentry

Jose M Moltedo, IntraMed, Argentina

Christopher S Snyder, Ochsner Children's Hospital, United States

Alejandro Ithuralde, FLENI, Argentina
Mariano Ithuralde, FLENI, Argentina

Mauricio S Abello, FLENI, Argentina

Objectives: The purpose of this study was to assess the prevalence of an HAV pattern (i.e. the atrial electrogram following the His bundle -HB- electrogram and preceding the ventricular one) in the $\mathrm{HB}$ channel in pediatric patients with atrioventricular node reentry (AVNRT).

Matherial And Methods: The pediatric eplectrophysiology databases of two separate institutions were analysed, and 33 pediatric patients with a diagnosis of AVNRTwere identified. Demographic and clinical data were assessed, as well as the electrophysiology study (EPS) information, including induction manouvers, tachycardia cycle length and the presence of an HAV pattern in the HB channel.

Results: Of the 33 patients, 11 were male. Thirty of them were caucasian and 4 were hyspanic The average age at the time of the EPS was $12,11 \pm 3,77$ years. Induction was achieved with atrial pacing in 16 patients, with one atrial extrastimulus in 8 and with 2 atrial extrastimuli in 5. Isoproterenol was necessary to induce tachycardia in 17 patients. During the EPS tachycardia cycle length $328 \pm 50 \mathrm{~ms}$. An HAV pattern was present in 35 (80\%) of the patients. All the pts. under 10 years of age had an HAV pattern.

Conclusion: An HAV pattern in the His bundle channel is a common finding in pediatric patients with AVNRT. It's presence in patients under 10 years of age is pathognomonic of this tachyarrhythmia.

\section{2}

Radiofrequency Catheter Ablation (RFCA) of Tachycardia In Children Under 5 Years of Age Yoshihide Nakamura, Department of Pediatric Cardiology, Japanese Red Cross Society, Wakayama Medical Center, Japan

Keiko Toyohara, Department of Pediatric Cardiology, Japanese Red Cross Society, Wakayama Medical Center, Japan

Jun Yoshimoto, Department of Pediatric Cardiology, Japanese Red Cross Society, Wakayama Medical Center, Japan

Noriyasu Ozaki, Department of Pediatric Cardiology, Japanese Red Cross Society, Wakayama Medical Center, Japan

Hitoo Fukuhara, Department of Pediatric Cardiology, Japanese Red Cross Society, Wakayama Medical Center, Japan

Backgrounds: Successful and safe ablation has been made possible by various three-dimensional mapping systems. However retrograde approach and use of three-dimensional mapping catheter was limited in infant and young children.

Purpose: The purpose of this study is to show the results of radiofrequency ablation for pediatric patients under 5 years of age. We performed RFCAs in 104 young children (14:0 y, 29:1y, 22:2y, 19:3y, 20:4y). from Oct. 2001 to Dec.2008.

The arrhythmias included atrioventricular pathway (AP: $n=53$ ), atrioventricular nodal reentrant tachycardia (AVNRT: $\mathrm{n}=14$ ), atrial tachycardia (AT: $n=16$ ), ventricular tachycardia (VT: $\mathrm{n}=9$ ), twin atrioventricular nodes(TAVN: $\mathrm{n}=4$ ) and combined mechanisms $(\mathrm{n}=4)$.

51 patients (49\%) have congenital heart disease ( $n=40: 7$ Ebstein's anomaly, 6 right isomeric syndrome, etc) or cardiac myopathy $(\mathrm{n}=11)$.

We used routinely three-dimensional mapping except for very low weight $(<7 \mathrm{~kg})$ infant. Results

Complete success was achieved in 52(/55) AP and 19(/20) AVNRT, 12(/16) AT, 6 VT(2/4LVVT, 4/4 RVVT, 0/1 polymorphic VT) and 4(/4) twin atrioventricular nodes. Unsuccessful cases were 3 AP (1: cardiac tumor, 1: complete 
atrioventricular septal defect, 1: permanent junctional reentrant tachycardia), 1 AVNRT with complete atrioventricular septal defect), 4 AT and 3 VT (2 left ventricular cases and 1 polymorphic VT). Procedure-related complications were infrequent (1 complete AVB, 1 incomplete AVM and 1 reversible brachial plexus injury).

Conclusion: RFCA was shown to be effective and safe method in even children under 5 years of age.

\section{3}

Cryo ablation of congenital Junctional Ectopic

Tachycardia with medium term follow-up

Mohammed T Numan, University of Texas, United States

Elsayed Mahmud, Hamad Hospital, Qatar

Congenital Junctional Ectopic tachycardia (JET) is a rare SVT. We are reporting our experience in cryo ablation of JET with follow up to three years.

We performed cryo ablation for four children. Their median age $5.5+0.7$ years and median weight $19.4+1.1 \mathrm{Kg}$. Two patients had dilated LV. The automaticity nature of the arrhythmia was documented and reentrant circuits were excluded.

We performed cryo-mapping $\left(-30^{\circ} \mathrm{C}\right)$ for 30 seconds in each location prior to full cryo lesion $\left(-60\right.$ to $\left.-75^{\circ} \mathrm{C}\right)$ for 3-4 minutes if it shows potential success. CS pacing was performed during lesions to document intact AV conduction. Cessation or slowing by more than $20 \%$ of the JET was used as a marker for potential success.

The total procedural and fluoro time were $152+41$ minutes and $18.8+15.3$ minutes. All patients had acute procedural success and ended with sinus rhythm. One patient had slow Junctional rate $85 /$ min with Isuprel sinus rhythm took over. The total cryo lesions were $6+3$. All the lesions were around the area of compact AV node. Occasionally transient AV block was observed during the lesion that resolved few minutes after immediate cessation of cryo energy.

Echo at 6 months follow up showed return LV to normal function in the four. Three patients' Holter after 3 years of ablation showing no recurrence of JET.

Cryo ablation can safely terminate and treat drug resistant congenital JET with good long term follow up and outcome.

\section{4}

Exercise treadmill test in children with ventricular arrhythmias and no structural heart disease

Beata J Pietrucha, Children Cardiology Department, University Children Hospital Jagiellonian University, Cracow, Poland

Agata Sulik, Children Cardiology Department, University Children

Hospital Jagiellonian University, Cracow, Poland

Jolanta Oko-Lagan, Children Cardiology Department, University

Children Hospital Jagiellonian University, Cracow, Poland

Krystyna Gut, Children Cardiology Department, University Children

Hospital Jagiellonian University, Cracow, Poland

Andrzej Rudzinski, Children Cardiology Department, University

Children Hospital Jagiellonian University, Cracow, Poland

The aim of our study was assessment of changes in ECG trace during treadmill exercise test in patients with ventricular arrhythmias.

Study group consists of 30 pts aged 7,1 to 17,8 years (mean 14,2 years): 19 boys and 11 girls with ventricular ectopics (diagnosed in Children Cardiology Department) and normal heart anatomy - Group I and 27 patient without heart disease age and sex matched to the study group - Group II.
Treadmill exercise test with Bruce protocol was performed in all patients with assessment of maximal heart rate $-\% \mathrm{HR}$; achieved metabolic equivalent of exercise - MET; presence of ventricular ectopics, ventricle tachycardia during test.

Results: There was no difference between maximal heart rate in both groups (\%HR - 103,8 vs 104,5) and achieved metabolic equivalent of exercise - MET (respectively 13,7 vs 13,28 $\pm 1,76$ ). Positive test result was noticed in 2 pts $(13,3 \%)$ from Group I ( in 1 pts right bundle branch block and non-sustained ventricle tachycardia were present during recovery time and in $1 \mathrm{pt}$ with diabetes also non-sustained ventricle tachycardia was present).

Conclusions: In majority of children with ventricle contractions diminishing of ectopics was observed during exercise treadmill testing.

Children with ventricular arrhythmia have comparable exercise capacity with healthy subjects.

\section{5}

Implantable Cardioverter Defibrillator Therapy for Life-Threatening Arrhythmias in children: experiencie in a single center

Katty Quezada, Hospital Roberto Del Rio, Chile Valeria Acevedo, Hospital Roberto Del Rio, Chile Patricia Alvarez, Hospital Roberto Del Rio, Chile Gilberto Palominos, Hospital Roberto Del Rio, Chile Rodrigo Neghme, Congenital Heart Institute, Orlando-Fl, Chile Rodrigo Gonzalez, Hospital Roberto Del Rio, Chile

The implantable cardioverter- defibrillator (ICD) is the primary therapeutic option for survivors of sudden cardiac death (SCD) in children.

The objective of this study was to evaluate the impact of ICDs on the pediatric population in a single pediatric cardiac center. We studied the clinical characteristics and outcomes of 50 children who underwent ICD implantation.

The patients age were 4 months to 16 years (mean 9.5 years) and weighted $6-67 \mathrm{~kg}, 22$ females. The indications were long QT syndrome 27 (54\%), 10 hypertrophic (20\%) and 3 dilated cardiomyopathies (6\%), congenital heart defects $4(8 \%)$, ventricular tachycardia $3(6 \%)$ and idiopathic ventricular fibrillation in $3(6 \%)$. Sixteen patients were survivors of SCD. Three were epicardiac.

The follow-up was 2 to 60 months, at least one ICD discharge occurred in 15 of the $50(30 \%)$ patients. Twelve received one appropriate ICD discharge, and 3 patients $(6 \%)$ had one or more inappropriate discharges. The causes of inappropriate discharges were lead failure in $2(4 \%)$ and sinus tachycardia in $1(2 \%)$.

There were 2 deaths during the study period: one secondary to ventricular storm, and one pulmonary infection. None died suddenly.

Conclusions: ICDs provide an important therapeutic option to prevent sudden arrhythmic death in high-risk patients. These data demonstrate that ICDs provide safe and effective therapy in young patients. The complication rate is low and there are a high incidence of appropriate ICD therapy for malignant ventricular arrhythmias in this highly selected group of patients in this single center study.

\section{6}

Current challenges in implantable cardioverterdefibrillator (ICD) device implantation in children Tonglin Su, The Children's Hospital at Westmead, Sydney., Australia David S Winlaw, The Children's Hospital at Westmead, Sydney., Australia 
Richard B Chard, The Children's Hospital at Westmead, Sydney. Australia

Ian A Nicholson, The Children's Hospital at Westmead, Sydney., Australia

Gary F Sholler, The Children's Hospital at Westmead, Sydney., Australia

K C Lau, The Children's Hospital at Westmead, Sydney., Australia

Introduction: As awareness of sudden death syndromes grows, increasing numbers of children are being referred for ICD which can be challenging in infants and small children. We review our experience with focus on those $<5$ year old.

Methods and Results: Retrospective chart review. Since 1996, 24 children received an ICD. Indications included: 12/24(50\%) long QT syndrome, 4/24(16\%) hypertrophic cardiomyopathy, 4/ $24(16 \%)$ VT/VF (1 idiopathic, 3 related to structural heart disease); 3/24(12\%) catacholaminergic polymorphic VT, 1/24 Brugada syndrome.

The majority were for primary prevention. None have required shocks since insertion. 8/24(33\%) patients were for secondary prevention, many have required device discharge.

36 procedures were performed on 24 patients. Breakdown of lead integrity accounted for the majority of lead revisions/ replacement. Abrasion and damage from external forces were the major causes.

Age at first ICD ranged between 6d and 15y. 5/24(21\%) were<5y. The youngest child had an ICD-implanted was for LQTS diagnosed on the day of birth after ventricular fibrillation arrest.

In the patients under five years of age, $4 / 5$ patients had the shocking coil tunnelled subcuaneously from the device heading laterally, then superiorly toward the left axilla. The defibrillation threshold in these patients was between $3 \mathrm{~J}$ and 35J. A pericardialdefibrillator-patch was used in one patient.

Conclusion: ICD-implantation has been safe but there is a significant rate of late lead revision. Subcutaneous placement of shocking coils in the very young is an effective temporising measure. Better definition of the genetic and molecular basis underlying many conditions allows better discrimination of who will benefit.

\section{7}

Treatment of junctional ectopic tachycardia (JET) before and during TCPC procedure in Asplenic heart

Shigeru Tateno, Department of Adult Congenital Heart Diease and Pediatrics Cardiology, Chiba Cardiovascular Center, Japan Yumi Shiina, Department of Adult Congenital Heart Diease and Pediatrics Cardiology, Chiba Cardiovascular Center, Japan Yuko Wakisaka, Department of Adult Congenital Heart Diease and Pediatrics Cardiology, Chiba Cardiovascular Center, Japan Takeaki Shirai, Department of Adult Congenital Heart Diease and Pediatrics Cardiology, Chiba Cardiovascular Center, Japan Tomohiko Toyoda, Department of Adult Congenital Heart Diease and Pediatrics Cardiology, Chiba Cardiovascular Center, Japan Yasutaka Kawasoe, Department of Adult Congenital Heart Diease and Pediatrics Cardiology, Chiba Cardiovascular Center, Japan Kozo Matsuo, Department of Adult Congenital Heart Diease and Pediatrics Cardiology, Chiba Cardiovascular Center, Japan Koichiiro Niwa, Department of Adult Congenital Heart Diease and Pediatrics Cardiology, Chiba Cardiovascular Center, Japan

Objective: Non-surgically related JET in heterotaxy syndrome has been rare, however if it occurs, such tachycardia make serious influence for morbidity and mortality in patients after Fontan type operation. Our aim of this study was to evaluate efficacy and risk of DDD pacemaker implantation and AVN modification during TCPC procedure.

Methods: A retrospective study was performed on the medical records and electro-physiologic studies.

Results: A total of 3 patients with asplenic heart were diagnosed as paradoxical JET prior to TCPC (Total Cavo-Pulmonary Connection) procedure. First onset of JET was from 9 day to 7 years of age. JT was terminated by combination of breath holding method, intravenous administration of adenosine triphosphate and verapamil in all 3 patients. On electro-physiologic studies, narrow QRS tachycardia with irregular RR interval was induced and terminated by ventricular pacing, and no retrograde conduction via atrio-ventricular node (AVN) and no twin atrioventricular node were observed in any patient. DDD pacemaker implantation and Cryo-ablation for AVN were performed during TCPC procedure in all 3 patients. Accelerated junctional rhythm occurred immediately after the operation and terminated by overdrive pacing in one patient. Transient complete atrioventricular block occurred in 2 patients. JT have never recurred after the operation in all patients with mean follow up of 27 months. Conclusion: Electro physiologic study and their drug efficacy suggested that triggered activity was the mechanism of JET in patients with asplenic heart. DDD pacemaker implantation and AVN modification during TCPC procedure could be possible method for elimination of the JET.

\section{8}

Electrophysiological studies and radiofrequency catheter ablation in patients with asplenia syndrome

Keiko Toyohara, Department of Pediatric Cardiology, Japanese Red

Cross Society, Wakayama Medical Center, Japan

Yoko Yoshida, Department of Pediatric Cardiology, Japanese Red Cross Society, Wakayama Medical Center, Japan

Noriyasu Ozaki, Department of Pediatric Cardiology, Japanese Red Cross Society, Wakayama Medical Center, Japan

Hitoo Fukuhara, Department of Pediatric Cardiology, Japanese Red Cross Society, Wakayama Medical Center, Japan

Jun Yoshimoto, Department of Pediatric Cardiology, Japanese Red Cross Society, Wakayama Medical Center, Japan

Yoshihide Nakamura, Department of Pediatric Cardiology, Japanese

Red Cross Society, Wakayama Medical Center, Japan

Objectives: We characterized the mechanism of supraventricular tachycardia (SVT) in patients with asplenia syndrome.

Patients and Methods: We performed electrophysiological studies (EPS) in 17 patients (pre total cavo-pulmonary connection $(T C P C)=11$, post $T C P C=6)$ who had a history of SVT.

Results: The type of SVT was atrioventricular nodal reentrant tachycardia (AVNRT): 5, atrioventricular reciprocating tachycardia (AVRT) involving twin atrioventricular nodes (twin AVNs): 4, atrial tachycardia (AT): 4, AVRT with accessory pathway (WPW): 1, atrial flutter (AFL): 1, and junctional tachycardia (JT): 1. 8/17 had twin atrioventricular nodes (twin AVNs). Five patients had AVRT involving twin AVNs. We could ablate the anterior AVN via the atrium in four cases (fenestrated TCPC 2, pre TCPC 2), but were unable to ablate the posterior AV node via the ventricle in one case (post TCPC). In five cases with AVNRT, we could ablate the posterior AVN in two cases with twin AVNs, ablate slow pathway of the anterior AVN in two cases with one AVN, and could not ablate the posterior AV node in one case (post TCPC). In six pre TCPC cases (AT:4, WPW:1, AFL:1) ablation was successful. JT was documented in one case with twin AVNs after TCPC. We were unable to 
eliminate the JT. We could ablate the substrates in all pre TCPC cases $(11 / 11)$, but only $3 / 6$ with post TCPC.

Conclusion: EPS are essential for complete arrhythmic evaluation before TCPC. After TCPC, it is difficult to manipulate catheters, but it is still possible to ablate the substrates in some patients.

\section{9}

Radiofrequency catheter ablation for the treatment of idiopathic ventricular arrhythmia in children: single center experience

Yoko Yoshida, Japan

Hitoshi Nisida, Department of pediatrics, Kitano hospital, Osaka, Japan

Noriyasu Ozaki, Department of pediatric cardiology, Japanese Red Cross Society Wakayama medical center, Wakayama, Japan

Jun Yoshimoto, Department of pediatric cardiology, Japanese Red Cross Society Wakayama medical center, Wakayama, Japan

Hitoo Fukuhara, Department of pediatric cardiology, Japanese Red Cross Society Wakayama medical center, Wakayama, Japan

Keiko Toyohara, Department of pediatric cardiology, Japanese Red Cross Society Wakayama medical center, Wakayama, Japan

Ken Watanabe, Department of pediatrics, Kitano hospital,

Osaka, Japan

Yoshihide Nakamura, Department of pediatric cardiology, Japanese

Red Cross Society Wakayama medical center, Wakayama, Japan

Introduction: Idiopathic ventricular arrhythmia is rare in children. Treatment by radiofrequency catheter ablation (CA) is being applied; however, limited data exist regarding the outcome in such patients.

Purpose: To review the clinical picture and evaluate the efficacy and safety, we report a single center's experience with CA for the treatment of idiopathic ventricular ablation in children.

Methods And Results: The subjects were 37 pediatric patients with idiopathic ventricular arrhythmia. The median age at CA was 12.4 years (range 2.2-16.6). Sustained ventricular tachycardia was observed in $9(24 \%)$, non-sustained ventricular tachycardia in $22(59 \%)$, and premature ventricular contractions in $6(16 \%)$. Initially 22 received antiarrhythmic drugs and in 31 exercise was restricted. Nine had symptoms, syncope or heart failure was in 4. Serum brain natriuretic peptide was elevated $(>20 \mathrm{pg} / \mathrm{ml})$ in 15 . Thirty six patients underwent CA and 41 sites of origin were detected, specifically, in the right outflow 22(54\%), right inflow 4(9.8\%), right epicardium 1(2.4\%), Tricuspid annulus 1(2.4\%), pulmonary artery $1(2.4 \%)$, left ventricular septum $5(12 \%)$, left epicardium 4(9.8\%), and the left outflow 3(7.1\%). The acute success rate was 28/36(78\%). CRBBB occurred in 2 patients. After 3 months 29 patients were evaluated by Holtor ECG. Complete success was noted in 19, partial effect in 3 , recurrence in 4 , and no change in 3 . Of the 35 patients followed up more than 3months, 32 were free of drugs and 30 were free of exercise restriction. Conclusions: CA is a safe and effective therapy for pediatric idiopathic ventricular arrhythmia and enables patients to live an active social and physical life.

\section{0}

Long term follow-up of transvenous leads in young infants solving complications with epicardial systems

Peter A Zartner, German Paediatric Heart Center, Sankt Augustin, Germany

Joachim Will, Humboldt University, Berlin, Germany

Martin BE Schneider, German Paediatric Heart Center, Sankt

Augustin, Germany
Aims: In the first instance epicardial leads and an abdominal pacemaker are implanted in newborns and small infants. In case of recurrent complications, early conversion to transvenous leads might improve long-term outcome and reduce frequent revisions.

Methods: We report on three children with post surgical atrioventricular block and an epicardial pacemaker system. All three infants underwent several revisions within 3 years because of lead complications, local infection and early battery depletion due to a high threshold. To avoid further complications and recurrent epicardial surgery, all three systems were successfully replaced (bodyweight 6.1, 6.8, 9.8 kg) using a 4.5 French bipolar transvenous lead and a small pacemaker.

Results: During the mean follow-up time of 9.1 years no further complications occurred. After a mean implantation period of 6.6 years all pacemakers were replaced with continuing use of the prior implanted ventricular leads. Increase in weight and growth were adequate to the clinical conditions in all three patients.

Conclusion: Transvenous lead implantation showed fewer complications than epicardial leads did and a reduction in reoperations. Also for patients with a weight between 6 and $10 \mathrm{~kg}$ the long term results of the transvenous approach with a thin lead is of benefit.

\section{1}

The treatment and follow-up studies of arrhythmia after transcatheter closure of membranous ventricular septal defects

Lifeng Zhang, Cardiovascular center, Children's Hospital of Fudan University, China

Lin Wu, Cardiovascular center, Children's Hospital of Fudan University, China

Fang Liu, Cardiovascular center, Children's Hospital of Fudan University, China

Ying Lu, Cardiovascular center, Children's Hospital of Fudan University, China

Feng Sheng, Cardiovascular center, Children's Hospital of Fudan University, China

Objective: To summarize the revalence, causes, prevention and treatment of arrhythmia after transcatheter closure of ventricular septal defects, and assess the clinical results.

Methods: Fifty two cases accepting successful transcatheter closure were analyzed. 12 leads ECG and 24-hour record of Holter were taken before and 1-4 days after transcatheter closure. The results were automatically analyzed by computer and then reviewed by professional electrocardiogram technician.

Results: 17.5 percent of patients had different kinds of arrhythmia before transcatheter closure. Except bundle branch block other kinds of arrhythmia were resolved in all the patients prior to discharge. The arrhythmia was significantly increased post closure. The arrhythmias occurring in the patients who underwent transcatheter closure of VSD included complete right bundle branch block (CRBBB, 3.5\%), incomplete right bundle branch block (IRBBB, 12\%), left anterior fascicular block (LAFB, 3.8\%). 2 patient developed transient complete atrioventricular block after closure, and returned to normal sinus rhythm in two days.

Conclusion: The transcatheter closure of VSD is a safe and feasible method in pediatric patients. Conduction blockades are the common complications after transcatheter closure of VSD, incomplete right bundle branch block (IRBBB) caused by interventional therapy is benign, the majority of which may resolve itself 6-12 months after the procedure. 
7042

Comparison of Bruce treadmill test and Dobutamnine test in follow-up examination of children after surgical repair of the aorta coarctation in children

Pawel Banaszak, Poland

Malgorzata Szkutnik, Poland

Jacek Kusa, Poland

Jacek Bialkowski, Silesian Center for Heart Diseases, Poland

Background: Exercise induced hypertension is a well known problem after surgical repair of coarctation of the aorta $(\mathrm{CoA})$. The cause of this complication is still uncleare. Comparison of two methods: Bruce treadmill stress test (TT) and dobutamine echo stress test (DT) was performed to assess the results of surgical repair of CoA. Methods: Twenty nine patients (pts) ): 20 male and 9 female aged from 4,5 to 18 (mean 12) years were included in the study. All of them had both TT and DT performed with assessment of such parameters as systolic blood pressure (SBP), residual gradient (RG) through the isthmus and the heart rate (HR) at the peak of exercise/dobutamine test.

Results: Positive correlation between both tests was documented in case of SBP and RG (see table I - Legend: Mean value and range (in brackets) are presented, $\mathrm{r}-$ coefficients of Pearson correlation, NS - difference not significant statistically.)

Conclusion: Dobutamine echo stress test is useful for evaluation of the results of surgical repair of CoA in children.

\begin{tabular}{llll}
\hline & SBP $(\mathrm{mmHg})$ & $\mathrm{RG}(\mathrm{mmHg})$ & HR (min) \\
\hline TT & $163,7(120-230)$ & $59,8(29-109)$ & $169(140-188)$ \\
DT & $164,7(123-215)$ & $55,4(29-113)$ & $111(76-155)$ \\
r & $\mathrm{r}=0,68$ & $\mathrm{r}=0,82$ & $\mathrm{r}=0,421$ \\
$\mathrm{p}$ & $<0,001$ & $<0,001$ & $\mathrm{NS}$ \\
\hline
\end{tabular}

\section{3}

Anomalous Left Coronary Artery from the Pulmonary Artery: Mid and long-term results after surgery Solana Villafañe Molina, Argentina

Maria L Pilan, Hospital de Pediatria Prof. Dr. J. P. Garrahan, Argentina

Gladys Salgado, Hospital de Pediatria Prof. Dr. J. P. Garrahan, Argentina

Horacio Capelli, Hospital de Pediatria Prof. Dr. J. P. Garrahan, Argentina

Between 1988 and 2008, 35 consecutive patients with anomalous left coronary artery from the pulmonary artery (ALCAPA) underwent surgery. The anomalous left coronary artery (LCA) was reimplanted in 29 and it was ligated in 6 . Patients were evaluated during a median follow-up of 82 months. There was one early death in the reimplanted group. (3,4\%) All survivors showed significant improvement of LV function. Shortening fraction increased from $\mathrm{X}=21 \%(10-44 \%)$ to $\mathrm{X}=39 \%$ (29-49\%). MR was identified in $80 \%$ of patients preoperatively and it improved in $53 \%$ of them after the LCA reimplantation. Fifteen patients were cathetherized. Five showed obstruction of the LCA. Three of these a had normal exercise test and only two showed abnormal ECG changes. Four of the five patients with occluded LCA had abnormal scintigraphy. Ninety one percent are asymptomatic. Only two patients with the LCA occluded had chest pain.

Two of the 6 patients with a ligated LCA died. One had severe LV disfunction and died shortly after surgery and the other died suddenly at the age of 15 . All 4 survivors have normal shortening fraction.
Conclusion: Reestablishment of a dual coronary artery system showed less early and late mortality than ligation. LV systolic function improved in all survivors despite some had occlusion of the LCA. Echocardiography and exercise test were good tools to assess the potential myocardial insult of survivors but did not predict the two coronary artery patency. Thus, coronary angiography is mandatory during follow-up.

\section{4}

Cardiac diastolic dysfunction in cardiovascular asymptomatic HIV-infected children

M L Silva, Joana de Gusmao Children's Hospital, Florianopolis, Brazil M M Pires, Joana de Gusmao Children's Hospital, Florianopolis, Brazil $S$ Nassar, Joana de Gusmao Children's Hospital, Florianopolis, Brazil M E Silva, Joana de Gusmao Children's Hospital, Florianopolis, Brazil M Zadinello, Joana de Gusmao Children's Hospital, Florianopolis, Brazil

Objectives: To verify the frequency of cardiac diastolic dysfunction in vertically HIV-infected symptomatic and clinically stable children, but asymptomatic from the cardiovascular point of view. To explore the association of the diastolic dysfunction with the immunological status and to evaluate malnutrition and anaemia as associated causes.

Methodology: This observational, cross-sectional and exploratory study was carried out at a regional outpatient centre for Paediatric AIDS care. Ninety four (94) children, from 20.3 to 170.6 months of age, consisting of a convenient and nonprobabilistic sample, had their weight, blood hemoglobin level, CD4+, T-lymphocytes percentage and diastolic biventricular function (by Doppler echocardiography) evaluated. The study was approved by the institution ethics committee, and written informed consent was obtained from the children's parents or relatives.

Results: Fifty (50) children (54.3\%, 95\% CI, $44.1 \%$ to $64.5 \%)$ showed diastolic dysfunction. Left ventricular dysfunction occurred in $38.4 \%$ of the studied group (95\% CI, $28.8 \%$ to $48.6 \%$ ), with a prevalence of decreased myocardial compliance. Right ventricular dysfunction occurred in $29.4 \%$ of the children (95\% CI, $20.1 \%$ to $38.7 \%$ ), with an incidence of abnormal relaxation. Simultaneous biventricular dysfunction occurred in $14.1 \%$ of the group ( $95 \%$ CI, $7.0 \%$ to $21.2 \%$ ). No association with the immunological status was observed.

Conclusions: Cardiac diastolic dysfunction occurs in HIV-infected children, asymptomatic from the cardiovascular point of view, with no association with the immunological status. Myocardial compliance impairment and abnormal relaxation are the most common dysfunctions associated with the left and right ventricles, respectively. No relationship was found between diastolic dysfunction and malnutrition and anaemia.

\section{5}

Aortic Coarctation and Arch Hypoplasia Secondary To Twin-Twin Transfusion Syndrome

Jutta van den Boom, Auckland City Hospital, New Zealand Malcolm Battin, Auckland City Hospital, New Zealand

Tim S Hornung, Starship Hospital, Auckland, New Zealand

Objective: The twin-twin transfusion syndrome (TTTS) complicates $10-30 \%$ of monochorionic pregnancies. The incidence of pulmonary stenosis and endocardial fibroelastosis is especially high in the recipient twin. We report a novel finding of four cases of coarctation of the aorta and hypoplastic aortic arch in the donor twin. 
Methods: Retrospective review of tertiary referral neonatal database and mortality data from 2002 to 2007 with crossvalidation from the local tertiary paediatric cardiology unit data (1998-2006) to identify children presenting with coarctation who were also twins.

Results: We identified four monochorionic twin pairs affected by the TTTS, delivered between 25 weeks to 36 weeks gestation, where the donor twin was found to have coarctation of the aorta or aortic arch hypoplasia. In addition, two of the four recipients also had cardiac abnormalities. There was a high mortality rate of $30 \%$ for both twins, and a high morbidity rate, especially for neurological sequelae.

Conclusion: We believe that the increased incidence of aortic arch abnormalities in the donor twins may be explained by the altered fetal blood flow and haemodynamics in TTTS with a relative decrease in left ventricular output due to decreased placental return. Given the increased prevalence of congenital heart disease in TTTS, with an increased risk for coarctation in the donor twin and pulmonary stenosis in the recipient, intrauterine surveillance and a postnatal comprehensive cardiac assessment for both twins is warranted.

\begin{tabular}{|c|c|c|c|c|}
\hline & $\begin{array}{l}\text { Gestation } \\
\text { (weeks) }\end{array}$ & $\begin{array}{l}\text { Birthweight } \\
\text { (g) }\end{array}$ & Diagnosis & Oucome \\
\hline Twin 1 & 27 & 1200 & PPHN & Death \\
\hline Twin 2 & 27 & 725 & PDA, coarctation & $\begin{array}{l}\text { Persistent } \\
\text { neurological } \\
\text { problems }\end{array}$ \\
\hline Twin 1 & 28 & 1180 & PDA & Discharged \\
\hline Twin 2 & 28 & 850 & PDA, coarctation & Discharged \\
\hline Twin 1 & 25 & 713 & Coarctation & Discharged \\
\hline Twin 2 & 25 & 937 & $\begin{array}{l}\text { Dysplastic Ao valve, } \\
\text { LVH, severe MR }\end{array}$ & Death \\
\hline Twin 1 & 33 & 1520 & Coarctation, PDA & $\begin{array}{l}\text { Transferred to } \\
\text { referring hospital }\end{array}$ \\
\hline Twin 2 & 33 & 2490 & $\begin{array}{l}\text { PPHN, Ebstein's } \\
\text { anomaly }\end{array}$ & $\begin{array}{l}\text { Transferred to } \\
\text { referring hospital }\end{array}$ \\
\hline
\end{tabular}

\section{7}

Haemodynamic and Right Ventricular Mechanical Response to Exercise in Atrial Repaired Transposition of the Great Arteries

Meera Ramani, Division of Cardiology, Department of Pediatrics, British Columbia Children's Hospital and The University of British Columbia, Canada

George GS Sandor, Division of Cardiology, Department of Pediatrics, British Columbia Children's Hospital and The University of British Columbia, Canada

Astrid M De Souza, Division of Cardiology, Department of Pediatrics, British Columbia Children's Hospital and The University of British Columbia, Canada

Mary T Potts, Division of Cardiology, Department of Pediatrics, British Columbia Children's Hospital and The University of British Columbia, Canada

Eustace S De Souza, Division of Cardiology, Department of Pediatrics, British Columbia Children's Hospital and The University of British Columbia

Michael WH Patterson, Division of Cardiology, Department of Pediatrics, British Columbia Children's Hospital and The University of British Columbia, Canada

Marla Kiess, Division of Cardiology, Department of Medicine, St. Paul's Hospital and The University of British Columbia, Canada James E Potts, Division of Cardiology, Department of Pediatrics, British Columbia Children's Hospital and The University of British Columbia, Canada
Introduction: Patients who have had atrial repair of Transposition of the Great Arteries (TGA) have decreased exercise tolerance. The mechanisms and factors for exercise intolerance are not clear. This study was performed to determine the different factors that may be involved in the impaired exercise response of TGA patients.

Methods: Patients exercised in 3 minute stages of 20-40 watts at 60-70 rpm on a semi-recumbent cycle ergometer until volitional fatigue. Echo-Doppler measurements were taken prior to, during, and after exercise. Segmental wall motion was assessed. Doppler velocities were measured. Right Ventricular (RV) dimensions and area change, time to peak contraction, and synchrony were expressed as ratios. RV strain was assessed using speckle tracking. There were 49 patients (female $=17$ ); mean age $=24.2$ years; 15 were post-intervention for SVC syndrome and 10 had pacemakers.

Results: Heart rate increased $(70-160 \mathrm{bpm})$ while Stroke Volume Index (SVI) tended to fall $\left(44-41 \mathrm{~mL} / \mathrm{m}^{2}\right)$ with incremental exercise. The increase in Cardiac Index $\left(3.0-6.3 \mathrm{~L} / \mathrm{min} / \mathrm{m}^{2}\right)$ was chronotropically-driven. Mean work was $870 \mathrm{~J} / \mathrm{kg}$, slightly lower than normal. Pacemakers had a negative effect on these variables, but previous SVC syndrome did not. Longitudinal and circumferential strain fell at peak exercise. Septal motion was biphasic and RV synchrony improved with exercise.

Conclusion: Patients with atrial repair of TGA have decreased exercise capacity due to a number of factors including an inability to increase or sustain SVI, chronotropic dependence, decreased myocardial contractility, and ventricular dyssynchrony. Previous SVC obstruction did not influence exercise capacity.

\section{8 \\ Body image perception, dietary habits and physical activity of middle school children Vincent PR Aluquin, Penn State Childrens Hospital, United States Bobby Kumar, Penn State Childrens Hospital, United States}

Background: Obesity is a growing epidemic in the country. It is linked to a greater risk of developing coronary artery disease, hypertension, hyperlipidemia, and diabetes among others. It is important to initiate preventive strategies at an early age since majority of overweight school-age children remain obese as adults. Our objective was to determine the body perception, diet, and level of physical activity of middle school children in central Pennsylvania and compare responses of overweight and normal weight children.

Methods: A 22-item survey questionnaire was provided to 126 children aged 11-14 years whose body mass indices were computed. As part of the survey, we validated the modified Baecke questionnaire which assesses each child's participation in sports and leisure time physical activities.

Results: $39.7 \%(50 / 126)$ of the middle school children were overweight (BMI $>85$ th percentile). Compared to normal weight children, overweight children felt less happy with their bodies and had less energy the past four weeks $(\mathrm{p}=<.001$ and $0.03)$. They also had a tendency to miss breakfast $(p=0.07)$. The frequency of eating out and television viewing was not statistically different between the two groups. Although both groups had similar leisure time physical activities, the normal weight children were found to have a higher sports participation index $(\mathrm{p}=0.049)$.

Conclusion: Overweight middle school children had a more negative attitude towards body image and participated less in sports compared to normal weight children. Efforts should be focused on increasing vigorous physical activities in children especially in the school setting. 


\section{9}

Postoperation Electrocardiographic Changes in Children with Fallot's Tetralogy (FT)

Vivien YJ Lim, National University of Singapore, Singapore

William CL Yip, Gleneagles Medical Centre, Singapore

Wenxia Wu, Gleneagles Medical Centre, Singapore

Ting Fei Ho, National University of Singapore, Singapore

Objective of study: Patients with FT are known to have increased risk for dysrhythmias and sudden death during long term followup after surgical repair of the defect. The aim of this study is to assess the postoperative changes in electrocardiographic parameters in children after repair of FT.

Method: Twenty one children (mean age: 3.7 years; 10 males, 11 females) with complete electrocardiographic recordings $\geqslant 3$ years post-operation were evaluated in comparison with healthy controls. Resting 12-lead ECG, 24-hour Holter, signal averaged ECG (SAECG) of these patients were assessed. From SAECG we determined the durations of total filtered QRS (TQRS), high frequency, low amplitude signals in terminal portion of QRS (HFLA) and root-mean-square voltage in last $40 \mathrm{msec}$ of QRS (RMS). From Holter we assessed the occurrence of ventricular and atrial dysrhythmias.

Results: During the $\geqslant 3$ years post-operation period, 20 and 12 of the FT children had significantly prolonged mean TQRS (174.9 vs 103.0 msec, $\mathrm{p}<0.01)$ \& HFLA (70.4 vs $23.0 \mathrm{msec}, \mathrm{p}<0.01$ ), respectively, as compared to controls. $38 \%$ had $1,14 \%$ had 2 and $43 \%$ had 3 abnormal SAECG parameters. Of all patients $(n=20)$ who had abnormal SAECG parameters, $65 \%$ and $90 \%$ had ventricular and atrial ectopics, respectively. $65 \%$ had both ventricular and atrial ectopics and $85 \%$ had heart block detected on Holter.

Conclusion: Children after corrective surgery for FT had abnormal SAECG indicating abnormal cardiac conduction patterns. In addition they had increased incidence of ventricular or atrial dysrhythmias and heart block in the postoperative period.

\section{0}

The value of serum NT-proBNP levels in the differential diagnosis and follow-up of congestive heart failure and respiratory distress due to pulmonary etiologies in infants and children

Melike Evim Sezgin, Eskisehir Osmangazi University Faculty of Medicine, Department of Pediatrics, Eskisehir, Turkey, Turkey

Birsen Ucar, Eskisehir Osmangazi University Faculty of Medicine, Department of Pediatric Cardiology, Eskisehir, Turkey, Turkey Zubeyir Kilic, Eskisehir Osmangazi University Faculty of Medicine, Department of Pediatric Cardiology, Eskisehir, Turkey, Turkey Omer Colak, Eskisehir Osmangazi University Faculty of Medicine, Department of Biochemistry, Eskisehir, Turkey, Turkey

Mehmet Arif Aksit, Eskisehir Osmangazi University Faculty of Medicine, Department of Neonatology, Eskisehir, Turkey, Turkey

Objective: We aimed to determine whether NT-proBNP can differentiate cardiac or pulmonary etiologies of dyspnea, if NTproBNP can be used for evaluating the effect of treatment in heart failure and for predicting severe pulmonary diseases which are complicated with heart failure.

Methods: Seventy-six children with dyspnea between 1 month17.5 years old were enrolled. Forty-one of them had heart failure ( 25 caused by heart disease, 16 caused by pulmonary disease) and 35 had dyspnea due to pulmonary disease. Control group consisted of 32 healthy children. Ross scoring was made, serum NT-proBNP levels were analyzed and left ventricular systolic functions were evaluated by echocardiography.
Results: Serum NT-proBNP levels were significantly higher in children with heart failure than those with pulmonary disease and controls (median 7,321 pg/ml, $241 \mathrm{pg} / \mathrm{ml}, 87.71 \mathrm{pg} / \mathrm{ml}$, respectively), were higher in children with heart failure due to pulmonary diseases than those with pulmonary diseases $(2,728 \mathrm{pg} / \mathrm{ml}, 241 \mathrm{pg} /$ $\mathrm{ml}$, respectively), and were higher in children who died from heart failure than the survivors $(\mathrm{p}<0.05)$. After treatment of heart failure, serum NT-proBNP levels significantly decreased $(p<0.001)$. The cutoff level of NT-proBNP for differentiating heart failure from pulmonary disease was $726.8 \mathrm{pg} / \mathrm{ml}$ (sensitivity $100 \%$, specificity 94.3\%). NT-proBNP levels didn't correlate with left ventricular systolic functions or Ross scores.

Conclusions: Serum NT-proBNP levels can differentiate dyspnea due to heart failure from pulmonary diseases. It can also be used to monitor the effects of treatment of heart failure and to estimate the prognosis, and for predicting pulmonary diseases which are complicated with heart failure.

\section{1}

Pulmonary Thromboembolism in Children with Nephrotic Syndrome

Deepti Suri, Post Graduate Institute of Medical Education \& Research - chandigarh India, India

Surjeet Singh, Post Graduate Institute of Medical Education \& Research - chandigarh India, India

B R Mittal, Post Graduate Institute of Medical Education E Researchchandigarh India, India

Jasmina Ahluwalia, Post Graduate Institute of Medical Education E Research - chandigarh India, India

Manojkumar Rohit, Post Graduate Institute of Medical Education E Research - chandigarh India, India

Nephrotic syndrome (NS) is an acquired hypercoagulable state in which thromboembolic phenomena and pulmonary thromboembolism [PTE] are well recognized complications that can contribute to mortality if not managed well in time.

Material \& Methods: We retrospectively analyzed the clinical course and outcome of children with NS who were admitted in the, PGIMER with a diagnosis of PTE from January 2005 to April 2008. The children were clinically suspected to have PTE if they developed sudden onset respiratory distress, cough, wheeze or hypoxemia. The diagnosis of PTE was confirmed by ventilation perfusion scan showing moderate to high probability \& CT scan confirmed the diagnosis.

Results: A total of 9 children developed PTE; seven were diagnosed ante-mortem while two at necropsy. PTE occurred mostly as an inpatient complication between day 1 to day 9 of hospitalization. All children had presented in relapse with edema, heavy proteinuria and hypoalbuminemia. Ventilation perfusion scans revealed high probability of PTE in 5 and intermediate probability in 2 patients. Co-existing infections were identified in four patients (spontaneous bacterial peritonitis 3, meningitis 1). Doppler scan did not reveal co-existent deep venous thrombosis in any child. Surrogate marker of thrombosis (D-dimer test) was found to be positive in four patients. All the seven patients were anticoagulated using conventional heparin and later overlapped with warfarin as a single daily dose titrating with the internationalized normalized ratio of $2-4$ with good outcome. Conclusion: High index of clinical suspicion for diagnosing PTE in children with NS is necessary for a favorable outcome.

\section{2}

The Hybrid Procedure for the Borderline Left Heart Christopher Davis, Rady Children's Hospital San Diego, United States 
Peter Pastuszko, Rady Children's Hospital San Diego, United States John Lamberti, Rady Children's Hospital San Diego, United States John Moore, Rady Children's Hospital San Diego, United States Howaida El Said, Rady Children's Hospital San Diego, United States

Objectives: In patients with varying degrees of left heart hypoplasia, it is often difficult to determine if the left heart structures are adequate in size to support a biventricular circulation. Historically, the decision to pursue a univentricular (UV) or biventricular (BV) repair needed to be made early and was often irreversible. We sought to determine if the Hybrid Procedure is a better initial approach in patients with left ventricles that are borderline in size.

Methods: We describe a series of four patients with various congenital malformations, all of whom had borderline left ventricles. Based on preoperative echocardiograms, several published scoring systems were used to predict whether UV or BV repair would be optimal.

Results: Calculations with three scoring systems were performed and left ventricular volume was calculated for each patient. $20 \mathrm{ml} / \mathrm{m} 2$ was used as the minimum cutoff value for adequacy of BV repair. The LV volumes for the patients were 17.1, 23.7, 25.4, and $25.8 \mathrm{ml} /$ $\mathrm{m} 2$. In none of the four patients were the calculations unanimous in the recommendation to pursue either a UV or BV repair. All four patients underwent the Hybrid Procedure and then eventual UV (2 patients) or BV (2 patients) repair. All four survived.

Conclusions: The Hybrid Procedure may be the best option in patients born with a borderline left ventricle. It can serve as a bridge to more definitive repair when patients are older, larger, and for whom the decision between UV and BV repair can be more easily made.

\section{3}

Parental awareness of endocarditis prophylaxis in children with congenital heart disease in Korea

Mi Kyung Park, Department of Pediatrics, Presbyterian Medical Center, Joenju, Korea, Korea

Kang Wook Lee, Department of Pediatrics, Presbyterian Medical Center, Joenju, Korea, Korea

Sin Ae Park, Department of Pediatrics, Presbyterian Medical Center,

Joenju, Korea, Korea

Soo Jin Kim, Department of Pediatrics, Sejong Genaral Hospital, Bucheon, Korea, Korea

Infectife endocarditis remains an important cause of morbidity and mortality in children with structural heart disease. The aim of this study is to determine the parental knowledge of bacterial endocarditis prophylaxis (BEP).

Parents of 141 healthy children and 135 congenital heart disease patients attending the Pediatric Clinics at Presbyterian Medical Center, Jeonju, Korea and Sejong General Hospital, Bucheon, Korea were asked to complete a questionaire pertaining to their konwledge of their child's cardiac disease, medication, BEP, dental attitudes and dental health practicies. Each patients in the study group was matched by age, gender and social class. The patients' need for BEP was determined according to the American Heart Association recommendations.

All patients were asked to complete a questionaire. The mean age of all children was 3 years and 11 months. 19(14.1\%) parents correctly defined endocarditis. $72(53.3 \%)$ parents knew the correct name of their child's cardiac condition, and $12(50 \%)$ of those on medication knew the names of their current medications. Only 29(21.5\%) parents whose children were at risk were aware of measures to prevent endocarditis. The patient group had significantly poorer dental health practices than the healthy group. $77.8 \%$ of the patient group had never visited the dentist compared with only $47.5 \%$ for the healthy group.

While most patents know the name of their child's heart disease and current medication, their general knowledge of endocarditis and $\mathrm{BEP}$ was limited. Intensified education and awareness programs are needed in order to prevent potential major morbidity and mortality in pediatric patients with congenital heart disease.

\section{4}

The incidence of persistent left superior vena cava in children with other congenital heart defects

Serdar Kula, Gazi University, Faculty of Medicine, Department of Pediatric Cardiology, Ankara, Turkey

Rana Olguntürk, Gazi University, Faculty of Medicine, Department of Pediatric Cardiology, Ankara, Turkey

Sedef Fatma Tunaoglu, Gazi University, Faculty of Medicine,

Department of Pediatric Cardiology, Ankara, Turkey

Deniz Ayse Oguz, Gazi University, Faculty of Medicine, Department of Pediatric Cardiology, Ankara, Turkey

Cihat Sanli, Gazi University, Faculty of Medicine, Department of Pediatric Cardiology, Ankara, Turkey

Ayhan Cevik, Gazi University, Faculty of Medicine, Department of

Pediatric Cardiology, Ankara, Turkey

Gökçe Zeynep Gayretli, Gazi University, Faculty of Medicine,

Department of Pediatrics, Ankara, Turkey

Objective: This study was planned to investigate the incidence of persistent left superior vena cava of all congenital heart defects and common congenital heart anomalies coexisting with persistent left superior vena cava.

Methods: We reviewed retrospectively the medical records of 1205 patients. Cardiac catheterization and angiography were performed from the left femoral vein percutaneously. In order to determine persistent left superior vena cava existance all the subjects underwent routine superior vena cava injection during angiography.

Results: $55 \%$ of patients (664) were male and 45\% (541) were female. Mean age of the patients was $52.69 \pm 50.67$ months. Persistent left superior vena cava was determined in $6 \%(74)$ of patients. Ventricular septal defect $(n=470,39 \%)$ and atrial septal defect $(n=312,26 \%)$ were the most frequently encountered cardiac anomalies during angiography. Connection between two superior vena cava was detected in $1.7 \%(n=20)$. In our study, the type and frequency of encountered congenital heart defects coexisting with persistent left superior vena cava were VSD 57\% $(n=42)$, ASD $42 \%(n=31)$, pulmonary stenosis $26 \%(n=19)$, atrioventricular septal defect 14\% $(n=10)$, PDA $8 \%(n=6)$, cortriatriatum dextrum $4 \%(n=3)$ respectively. These associations were found statistically significant $(\mathrm{p}<0,05)$.

Conclusion: The prevalance of persistent left superior vena cava is between $0.5-2 \%$ in general population. Although transthoracic ecocardiography diagnoses persistent left superior vena cava with a high accuracy, in order to prevent incidental diagnosis of persistent left superior vena cava and mortality during cardiovascular surgery, patients should undergo routine superior vena cava injection during angiography.

\section{5}

Register of CHD, population and hospital - Prospective Croatian study 2002-2007

Ivan Malcic, University Hospital Center Zagreb, Croatia, Croatia Danijel Dilber, University Hospital Center Zagreb, Croatia, Croatia 
Hrvoje Kniewald, University Hospital Center Zagreb, Croatia, Croatia Dalibor Saric, University Hospital Center Zagreb, Croatia, Croatia Sanja Dorner, University Hospital Osijek, Croatia, Croatia

We present results of national multicentric prospective epidemiologic study of the register of congenital heart defects as a population and hospital register. The data have been collected between 2002-2007. During that 5 year period 205917 children have been born and 1965 congenital heart defects have been found $(9.7$ incidence) with similar prevalence among male and female gender. Distribution of congenital heart diseases are same as referred in literature. 94\% diagnosis of CHD in Croatia are made during first year of life.

There are 14 medical centers practicing pediatric cardiology in Croatia that are involved in data collecting. Most of the patients (64\%) are examined in Referral Center for Pediatric Cardiology, University Medical Center Zagreb. The ratio of surgically treated to total number of patients is $430 / 1956$ that is $22 \%$. 283 of all surgically treated patients $(66 \%)$ have been treated abroad, and there have been performed 341 surgical procedures.

During that 5 year period of time, $57(2.9 \%)$ children of all the children born with congenital heart defect have died and 31 $(1.5 \%)$ of them were previously surgically treated.

This study reveals teamwork insufficiencies at cardiac surgery level, most of the children are still being treated abroad and that is the fact that has to be improved.

\section{6}

Diastolic function and valvar involvement in Mucopolysaccharidosis and Mucolipidosis June Huh, Samsung Medical Center, Department of Pediatrics, Sungkyunkwan University School of Medicine, Korea Soo In Jeong, Samsung Medical Center, Department of Pediatrics, Sungkyunkwan University School of Medicine, Korea I-Seok Kang, Samsung Medical Center, Department of Pediatrics, Sungkyunkwan University School of Medicine, Korea Heung Jae Lee, Samsung Medical Center, Department of Pediatrics, Sungkyunkwan University School of Medicine, Korea Kyung Hoon Paik, Samsung Medical Center, Department of Pediatrics, Sungkyunkwan University School of Medicine, Korea

Dong Kyu Jin, Samsung Medical Center, Department of Pediatrics, Sungkyunkwan University School of Medicine, Korea

Hye Sun Hyun, Department of Pediatrics, Samsung Medical Center, SungKyunKwan University School of Medicine, Seoul, Korea

Objective: This study was aimed to evaluate ventricular diastolic function as well as valvar involvement in Mucopolysaccharidosis (MPS) and Mucolipidosis.

Methods: Forty six patients were enrolled (10 patients with MPS I, 27 with MPS II, 2 with MPS III, and 2 with MPS IV, 5 with Mucolipidosis). The mean age was 11.8 years with a range of 2.5-32 years. Echocardiographic measurements were preformed. Results: Mitral regurgitation was common (mild degree 27, moderate 7, severe 1) and mitral stenosis in 7 patients. Aortic valve involvement were noted in 19 with aortic regurgitation (minimal 11, mild 7, moderate 1), and 7 with aortic stenosis. Mitral valve prolapse was detected in 23 with variable insufficiency. Thickening of mitral valves was noted in 44 and thickening of aortic valves in 40 . There was no significant difference of valvar involvement according to subtypes. All showed hypertrophy of both ventricles with normal ventricle dimensions. Deceleration time of left ventricle $>150 \mathrm{msec}$ was recorded in 28 patients. E/A ratio $>1.5$ was recorded in 8 patients. E/E' ratio was high in 31 patients (11 patients with $8<\mathrm{E} / \mathrm{E}^{\prime}<15,20$ patients with $\mathrm{E} / \mathrm{E}^{\prime}>=15$ ).

Conclusions: Diastolic dysfunction of left ventricle as well as valvar involvement in MPS and Mucolipidosis is common. Regular echocardiography is necessary to assess valvar and ventricular function and subsequently to provide early appropriate treatment.

\section{7}

Diagnosis and management of symptomatic congenital ductus arteriosus aneurysm in neonates

Nageswara Rao Koneti, CARE Hospital, The Institute of Medical

Sciences, India

Vasudevan Kanchi, CARE Hospital, The Institute of Medical

Sciences, India

Pramod Reddy, Fernandez Hospital, Hyderabad, India

Objective \& Method: We report four neonates presented with stridor and diagnosed as Ductus Arteriosus Aneurysm (DAA) and managed successfully. All four neonates presented within 24 hours with stridor, respiratory distress and weak cry. Cardiovascular system examination revealed no abnormality. There was prominent ductus shadow in the chest skiagram in two neonates. The echocardiogram revealed a large DAA with tortuous patent ductus arteriosus. The suprasternal and high parasternal views showed triple star sign and rabbit ear appearance. All babies were closely observed and assessed by echocardiogram. Two babies underwent CT scan in view of significant obstructive symptoms to decide further management. Results: One neonate underwent surgical intervention as DAA was compressing the left bronchus. There was significant thrombus formation in the aneurysm observed in the remaining three neonates who were followed till the disappearance of DAA. All babies are doing well during the follow-up.

Conclusion: Ductus arteriosus aneurysm is an important cause for the stridor in neonates. Majority of the babies improve without any cardiac intervention however surgery may be needed if obstructive symptoms persist.

\begin{tabular}{|c|c|c|c|c|c|c|c|}
\hline $\begin{array}{l}\text { Serial } \\
\text { no. }\end{array}$ & Sex & $\begin{array}{l}\text { Gesta- } \\
\text { tional } \\
\text { age }\end{array}$ & $\begin{array}{l}\text { Risk } \\
\text { factors }\end{array}$ & $\begin{array}{l}\text { Birth } \\
\text { weight } \\
\text { (kgs) }\end{array}$ & $\begin{array}{l}\text { Saturation } \\
(\%)\end{array}$ & $\begin{array}{l}\text { Ventila } \\
\text { tion }\end{array}$ & $\begin{array}{l}\text { Manage- } \\
\text { ment }\end{array}$ \\
\hline Case1 & $\mathrm{M}$ & Term & Nil & 2.8 & 78 & Yes & Operated \\
\hline Case2 & $\mathrm{F}$ & $\begin{array}{l}36 \\
\quad \text { weeks }\end{array}$ & Nil & 2.1 & 92 & No & Conservative \\
\hline Case3 & $\mathrm{F}$ & Term & $\begin{array}{c}\text { Gestational } \\
\text { Diabetes }\end{array}$ & 3.5 & 60 & Yes & Conservative \\
\hline Case 4 & M & Term & Nil & 2.7 & 95 & No & Conservative \\
\hline
\end{tabular}

\section{8}

Autonomic control of the cardiovascular system is changed in children with mitral valve prolapse Krzysztof Czyz, Department of Pediatric Cardiology, Poznan University of Medical Sciences, Poland, Poland

Waldemar Bobkowski, Department of Pediatric Cardiology, Poznan University of Medical Sciences, Poland, Poland Przemyslaw Guzik, Department of Cardiology and Intensive Therapy, Poznan University of Medical Sciences, Poland, Poland Tomasz Krauze, Department of Cardiology and Intensive Therapy, Poznan University of Medical Sciences, Poland, Poland Jaroslaw Piskorski, Institute of Physics, University of Zielona Gora, Poland, Poland

Aldona Siwinska, Department of Pediatric Cardiology, Poznan University of Medical Sciences, Poland, Poland 
Introduction: Mitral valve prolapse (MVP) is one of the most common form of cardiac disease in children and may be accompanied by some autonomic abnormalities. Analysis of heart rate variability $(\mathrm{HRV})$, resting heart rate $(\mathrm{HR})$ or baroreflex sensitivity (BRS) allow studying autonomic modulation of cardiovascular system.

Aim: The aim of this study was to investigate frequency domain HRV, BRS and resting HR to assess the changes in sympathovagal balance in children with MVP.

Methods: 43 children with MVP (29 girls; 5-18 years) and 52 healthy children ( 28 girls; $7-18$ years) were included. 30 -minute resting ECG (Porti 5, TMSI, Netherlands) and blood pressure (Portapres 2, FMS, Netherlands) signals were recorded noninvasively in supine. The HRV was evaluated by spectral analysis: total power (TP), power of very low (VLF), low (LF) and high frequency (HF) and LF/HF ratio. The BRS was measured using a cross-correlation method.

Results: Patients with MVP had significantly reduced TP $\left(5207 \pm 704\right.$ vs $\left.9083 \pm 1036 \mathrm{~ms}^{2} ; \mathrm{p}=0.003\right), \operatorname{VLF}(1841 \pm 141$ vs $\left.3776 \pm 582 \mathrm{~ms}^{2} ; \mathrm{p}=0.009\right)$, LF $\left(1420 \pm 221\right.$ vs $2126 \pm 261 \mathrm{~ms}^{2}$; $\mathrm{p}=0.022)$, HF $\left(1772 \pm 376\right.$ vs $\left.2876 \pm 435 \mathrm{~ms}^{2} ; \mathrm{p}=0.035\right)$ and reduced mean duration of cardiac cycles $(764 \pm 20$ vs $832 \pm 20 \mathrm{~ms}$; $p=0.028)$ as compared with healthy children. There was no significant reduction in prolongation of delay of baroreflex $(2.01 \pm 0.06$ vs $1.87 \pm 0.06 \mathrm{~s} ; \mathrm{p}=0.069)$ and no significant differences in BRS and LF/HF ratio between both groups.

Conclusions: Children with MVP have a changed autonomic control of the cardiovascular system. Our study demonstrates that children with MVP characterized by sympathetic hyperactivity and/or reduced vagal tone.

\section{9 \\ Deceleration and acceleration capacity in children with mitral valve prolapse \\ Krzysztof Czyz, Department of Pediatric Cardiology, Poznan University of Medical Sciences, Poland, Poland \\ Waldemar Bobkowski, Department of Pediatric Cardiology, Poznan University of Medical Sciences, Poland, Poland \\ Przemyslaw Guzik, Department of Cardiology and Intensive Therapy, \\ Poznan University of Medical Sciences, Poland, Poland \\ Tomasz Krauze, Department of Cardiology and Intensive Therapy, \\ Poznan University of Medical Sciences, Poland, Poland \\ Jaroslaw Piskorski, Institute of Physics, University of Zielona Gora, Poland, Poland \\ Rafal Surmacz, Department of Pediatric Cardiology, Poznan University of Medical Sciences, Poland, Poland \\ Aldona Siwinska, Department of Pediatric Cardiology, Poznan \\ University of Medical Sciences, Poland, Poland}

Introduction: Mitral valve prolapse (MVP) is a common cardiovascular abnormality in children. It is suggested that MVP can be accompanied by some autonomic abnormalities. Deceleration capacity and acceleration capacity are two, newly recognized ECG-derived descriptors of autonomic modulation of cardiovascular system. Both deceleration and acceleration capacity have been shown to predict mortality in survivors after myocardial infarction. Deceleration and acceleration capacity has not been evaluated in children with MVP so far.

Aim: Comparison of deceleration and acceleration capacity in children with MVP and in healthy children.

Methods: 43 children with MVP (29 girls; age \pm SD: $13.3 \pm 3.5$ years) and 52 healthy children (28 girls; age \pm SD: $13.8 \pm 2.7$ years) were examined. The chest ECG (Porti 5, TMSI, The Netherlands) was recorded at rest in a recumbent position for
30 minutes in children breathing spontaneously. Deceleration capacity and acceleration capacity were calculated by means of phase rectified signal averaging. Comparisons were made using the Mann-Whitney U test. A probability value of $<0.05$ was considered statistically significant.

Results: There was not significant reduction in deceleration capacity $(19.8 \pm 1.8$ vs $25.1 \pm 2.0 \mathrm{~ms} ; \mathrm{p}=0.090)$ and acceleration capacity $(-18.3 \pm 1.5$ vs $-22.7 \pm 1.6 \mathrm{~ms} ; \mathrm{p}=0.092)$ in children with MVP as compared with the control group.

Conclusion: Both deceleration capacity and acceleration capacity are similar in children with MVP and in healthy children.

\section{0}

Transcatheter balloon dilation versus surgical commisurotomy in neonates with critical aortic stenosis Laureen Cohen, Timone Children's Hospital, Marseille, France Andreea Dragulescu, Timone Children's Hospital, Marseille, France Virginie Fouilloux, Timone Children's Hospital, Marseille, France Beatrice Bonello, Timone Children's Hospital, Marseille, France Bernard Kreitmann, Timone Children's Hospital, Marseille, France Dominique Metras, Timone Children's Hospital, Marseille Alain Fraisse, Timone Children's Hospital, Marseille

Objective: To compare neonates with cardiac failure treated either by surgical commisurotomy or transcatheter balloon dilation for critical aortic stenosis.

Methods: Between 1998 and 2008, 16 neonates with aortic valve stenosis underwent surgical commisurotomy $(\mathrm{n}=7)$ or transcatheter balloon dilation $(n=9)$. Two of them who underwent balloon dilation were excluded because they had severe aortic valve stenosis without cardiac failure. All the 14 remaining patients had signs of heart failure with either severe left ventricular dysfunction and/or need for prostaglandin E1 infusion.

Results: Median age and weight at intervention were 8 (1-30) days and 3.1(2.5-4.5) kg, respectively. Median aortic valve Zscore was $-1.1(-4$ to +0.9$)$. Both surgery and balloon valvuloplasty were successful in reducing transvalvular mean Doppler gradient from $56.3 \pm 25.2$ to $21.3 \pm 12.3 \mathrm{mmHg}$ with no significant difference between the two methods $(p=0.4)$. Two patients died, one after balloon dilation from respiratory failure due to tracheal stenosis and one after surgery with cardiac failure and persistent left ventricular dysfunction. Postprocedural aortic regurgitation was absent in 7 patients, minimal in 4 and moderate in 3 , with no difference between transcatheter and surgical therapy $(p=0.7)$. Mean intensive care stay was significantly shorter for patients with balloon dilatation $(2.2 \pm 1.6$ versus $13.8 \pm 12$ days, $\mathrm{p}=0.05)$. There were two late reinterventions on the aortic valve (Ross procedure) after surgical commisurotomy.

Conclusion: Transcatheter balloon dilation and surgical commisurotomy have similar short term results, with earlier recovery and shorter intensive care stay with balloon dilation. Longer follow-up is needed to compare long-term results for the two procedures.

\section{1}

Non-Invasive Assessment of the Biophysical Properties of the Aorta and Ventricular-Vascular Coupling in the Fontan Circulation

Kim A Myers, Division of Cardiology, Department of Pediatrics and The University of British Columbia, Canada

James E Potts, Division of Cardiology, Department of Pediatrics and The University of British Columbia, Canada 
Mande T Leung, Division of Cardiology, Department of Pediatrics and The University of British Columbia, Canada

Mary T Potts, Division of Cardiology, Department of Pediatrics and The University of British Columbia, Canada

George GS Sandor, Division of Cardiology, Department of Pediatrics and The University of British Columbia, Canada

Introduction: The Fontan circulation is prone to deterioration in functional status over time. Invasive studies have shown that Fontan patients require maximum ventricular power to maintain cardiac output at the expense of lower ventricular efficiency.

Objectives: To non-invasively assess the biophysical properties of the aorta and determine ventricular-vascular interaction and efficiency in the Fontan circuit.

Methods: Twenty-five patients with a Fontan circulation and 17 Controls were assessed prospectively using echocardiography/ Doppler methods. The carotid pressure waveform was recorded using applanation tonometry.

Results: Fontan patients were older $(15.0$ vs 10.4 yrs; $\mathrm{p}<0.002)$. Their aortic valve cross-sectional area $\left(3.8\right.$ vs $2.0 \mathrm{~cm}^{2}$, $\mathrm{p}<0.0001)$ was larger and peak left ventricular outflow tract flow (356 vs $244 \mathrm{~cm}^{3} / \mathrm{s} ; \mathrm{p}<0.003$ ) higher, and their cardiac index $\left(3.64\right.$ vs $\left.4.68 \mathrm{~L} / \mathrm{min} / \mathrm{m}^{2}, \mathrm{p}<0.05\right)$ lower than Controls. Pulse wave velocity $(455$ vs $337 \mathrm{~cm} / \mathrm{s})$, elastic pressurestrain modulus (300 vs $173 \mathrm{mmHg}, \mathrm{p}<0.0006)$ and arterial stiffness index (3.36 vs 2.10, p $<0.0004)$ were higher in Fontan patients. Total $(1019$ vs $743 \mathrm{~mW}, \mathrm{p}<0.02)$ and mean $(878$ vs $657 \mathrm{~mW}, \mathrm{p}<0.04)$ ventricular hydraulic power were higher and efficiency lower $(84$ vs $89 \%, p<0.0003)$ in Fontan patients. The Tei-Index $(0.49$ vs $0.29, \mathrm{p}<0.001)$ was also higher in Fontan patients.

Conclusions: The biophysical properties of the aorta and ventricular-vascular coupling in patients with a Fontan circulation are abnormal. These contribute to increased power expenditure, reduced ventricular efficiency and global function in these patients.

\section{2}

Treatment of full-term infants with patent ductus arteriosus based on preterm infants

Kang-wook Lee, Presbyterian Medical Center, Korea

Sin-ae Park, Presbyterian Medicatl Center, Korea

Mi-kyung Kim, Presbyterian Medical Center, Korea

O-kyung Lee, Korea

Purpose: Indomethacin treatment is successful in about $90 \%$ of patent ductus arteriosus (PDA) in preterm infants. But, treatment of PDA in full-term infants is not established yet. The object of this study is to compare full-term infants to preterm infants with PDA and to determine the treatment of full-term infants with PDA.

Methods: The 174 neonates, admitted to neonatal intensive care unit of Presbyterian medical center from January 2003 to December 2007 were diagnosed of PDA. The comparison fullterm to preterm infants with PDA were studied retrospectively. Results: The treatment group was 36 patients $(42.9 \%)$ in preterm and 24 patients $(26.7 \%)$ in full-term. In preterm, closure rate of PDA was significantly higher $(88.9 \%$ vs $62.5 \%, \mathrm{p}<0.05)$ than full-term. But in full-term, closure rate was high. In nontreatment group, the age of PDA closure was significantly shorter in full-term $(4.2 \pm 1.3$ days vs $4.9 \pm 2.0$ days $\mathrm{p}<0.05)$. In fullterm infants with 2nd cycle-administered(indomethacin) or surgery, the diameter of PDA was significantly larger than 1st cycle-administered $(4.1 \pm 0.9 \mathrm{~mm}$ vs $2.7 \pm 0.5 \mathrm{~mm} \mathrm{P}<0.05)$.
Conclusion: Although closure rate of PDA in preterm infants is more higher than full-term, indomethacin treatment is effective for PDA in full-term infants prior to considering surgical treatment.

\section{4}

Pulmonary artery diverticulum:? An angiographic marker of Williams Syndrome

Zaheer Ahmad, Congenital Cardiac Centre, Southampton University Hospital NHS Trust, United Kingdom

Joseph Vettukattil, Congenital Cardiac Centre, Southampton University Hospital NHS Trust, United Kingdom

Objectives: We evaluated the presence of a pulmonary artery diverticulum in patients with Williams syndrome in comparison with other conditons causing peripheral pulmonary artery stenosis (PPS).

Methods: Angiographic characteristics of patients with a definitive diagnosis of Williams Syndrome, by fluorescence in situ hybridization, between 1990 and 2008 were reviewed. This data was compared with those diagnosed with PPS without Williams syndrome. Differentiating morphological features on angiography were compared between the groups along with demographic and echocardiographic data.

Results: Twelve patients with chromosomal diagnosis of Williams syndrome who underwent cardiac catheterization were identified. Seven were male. Eleven patients (91\%) had supravalvar aortic stenosis and eight (66\%) had PPS. Pulmonary valve stenosis was seen in two patients. Eight patients who were negative for Williams syndrome and had PPS were identified during the same period. Two had Alagille syndrome and one had Noonan syndrome. Mean age at catheterization was five years in Williams group versus eight years in non Williams group. Pulmonary artery diverticulum involving the main pulmonary artery was documented in all the patients with Williams syndrome while none of the patients in other group had it. It originated at bifurcation of the pulmonary artery in all.

Conclusion: Angiographic appearance of a diverticulum as an extension of the main pulmonary artery is a consistent finding in patients with Williams Syndrome. In addition to the classically described findings of supravalvar aortic stenosis and PPS, it can be considered as pathognomonic feature of Williams syndrome.

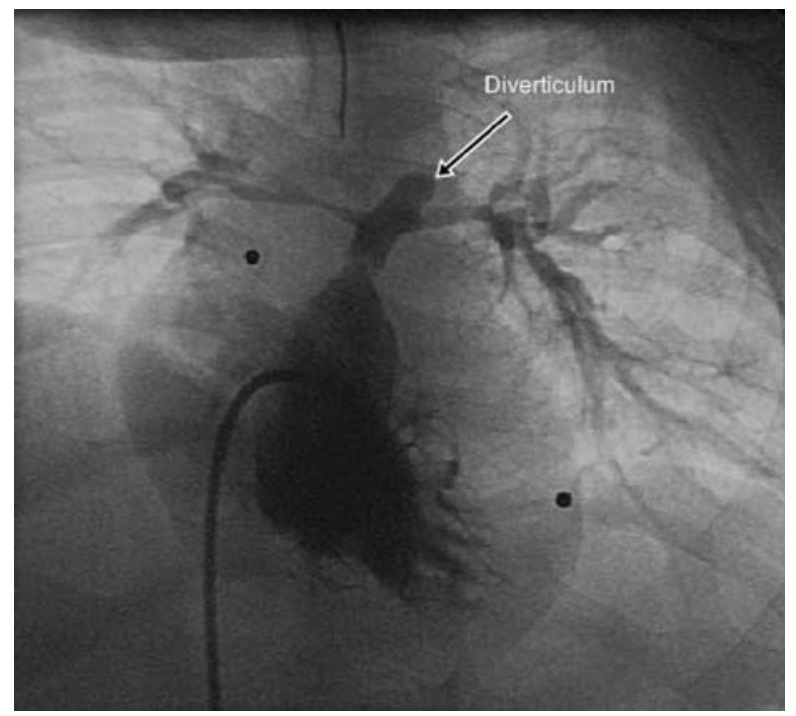




\section{6}

Coarctation of the aorta - still a missed diagnosis

Silvia Alvares, Centro Hospitalar do Porto, EPE, Unidade Maria Pia, Portugal

Marisa Carvalho, Centro Hospitalar do Porto, EPE, Unidade Maria Pia, Portugal

Marilia Loureiro, Centro Hospitalar do Porto, EPE, Unidade Maria Pia, Portugal

Carmen Carmo, Centro Hospitalar do Porto, EPE, Unidade Maria

Pia, Portugal

Celia Madalena, Centro Hospitalar do Porto, EPE, Unidade Maria

Pia, Portugal

Ricardo Machado, Centro Hospitalar do Porto, EPE, HGSA, Portugal Conceição Mota, Centro Hospitalar do Porto, EPE, Unidade Maria Pia, Portugal

Aortic coarctation is the most common correctable cardiovascular cause of secondary hypertension. We present two clinical cases of unusual presentation. Case 1: A 12 years old girl was referred for cardiac evaluation in the setting of hypertension difficult to control. Diagnosis of hypertension was established at the age of ten. Anterior investigation was inconclusive. The father had been operated for aortic coarctation. She presented a precordial systolic ejection murmur with irradiation to the back, no abdominal murmur and a differential arm-leg blood pressure of $50 \mathrm{mmHg}$. 2D/Doppler Echocardiogram showed concentric LV hypertrophy, Doppler investigation of the aorta from the suprasternal view was normal, but the flow in the abdominal aorta was typical of coarctation. MR angiography confirmed the diagnosis and surgical correction was performed. Case 2: A 14 years old girl with hypertension was referred for cardiac investigation. The abdominal auscultation revealed a systolic peri-umbilical murmur and the differential arm-leg blood pressure was $40 \mathrm{mmHg}$. Echo/Doppler was consistent with abdominal coarctation. MR angiography revealed a stenotic area in the abdominal portion of the aorta. She underwent surgery. Comments: Aortic coarctation is still a missed and late diagnosis. The first case is unusual as only the flow in the abdominal aorta was consistent with aortic coarctation. Abdominal coarctation is a rare disease. These cases are examples of the importance of a complete physical examination in the presence of arterial hypertension in children, as well as a thorough echocardiographic evaluation, so that a treatable cause of hypertension can be detected.

\section{7}

Does the size of pulmonary artery (PA) still affect the Fontan circulation after completion of the procedure? Tatsuya Ando, The Jikei University School of Medicine, Japan Ryota Saito, The Jikei University School of Medicine, Japan Takashi Urashima, The Jikei University School of Medicine, Japan Masako Fujiwara, The Jikei University School of Medicine, Japan Makoto Nakazawa, The Jikei University School of Medicine, Japan Hiroyuki Ida, The Jikei University School of Medicine, Japan Kiyozo Morita, The Jikei University School of Medicine, Japan

Objective: The aim of this study is to evaluate cardiac function in Fontan circulation, and investigate the implications of postoperative PA size in Fontan circulation.

Methods: The subjects were 17 consecutive patients with Fontan circulation. Cardiac output (CO) was measured by the pulse contour cardiac output monitoring system. Dobutamine was infused to analyze cardiac functional reserve.

Results: The mean age of the subjects was 8 years (range 2-34). The mean age when the patients underwent the Fontan procedure was 3.0 years (1-19). The mean value of Nakata index (PAI) and lower lobe index (LLI) was 262 (121-403) and 193 (75-283), respectively. Baseline value of pulmonary arterial pressure was $10.9 \mathrm{mmHg}(8-16)$, and cardiac index(CI) was $3.2 \mathrm{l} /$ $\mathrm{min} / \mathrm{m}^{2}(2.2-4.7)$. Heart rate and $\mathrm{CO}$ were increased by dobutamine infusion. We divided the patients into two groups based on baseline $\mathrm{CI}\left(3.01 / \mathrm{min} / \mathrm{m}^{2}\right)$ and maximal $\mathrm{CI}(4.0)$. PAI was significantly higher in groups with the higher baseline $\mathrm{CI}$ and maximal CI $(p=0.003$ and $p=0.03)$. LLI was not a significant factor regarding CI in this study.

Conclusion: Nakata index is speculated to correlate with the total amount of pulmonary blood flow. Thus it may represent cardiac output in Fontan circulation. Recently, some authors suggested small preoperative PA size did not matter in the completion of Fontan procedure. However, if PA size limits cardiac output in Fontan circulation, the PA size is still remaining important. The options such as additional PA flow, or PA enlargement before Fontan procedure might be helpful to the patients with small PA, regarding $\mathrm{CO}$.

\section{8}

Right ventricular dysfunction and B-type natriuretic peptide in asymptomatic patients after repair of tetralogy of Fallot

Christian Apitz, Pediatric Cardiology, University Children's Hospital, Tuebingen, Germany, Germany

Ludger Sieverding, Pediatric Cardiology, University Children's Hospital, Tuebingen, Germany, Germany

Heiner Latus, Pediatric Cardiology, University Children's Hospital, Tuebingen, Germany, Germany

Anselm Uebing, Pediatric Cardiology, University Children's Hospital, Kiel, Germany, Germany

Stephan Schoof, Pediatric Cardiology, University Children's Hospital, Hanover, Germany, Germany

Michael Hofbeck, Germany

Background: Early detection of right ventricular (RV) dysfunction is essential in the assessment of patients with repaired tetralogy of Fallot (TOF). Purpose of this study was to assess latent RV dysfunction in asymptomatic patients with TOF, and to determine the predictive value of B-type natriuretic peptide (BNP).

Methods: Pressure-volume loops were recorded in 16 young patients (NYHA class I) (mean age 15.9 years) by conductance catheter technique. All patients had moderate to severe pulmonary regurgitation (PR) after surgical repair of TOF (mean PR 36.2\%) and an increased enddiastolic RV volume of $129.7 \mathrm{ml} / \mathrm{m} 2$ on magnetic resonance imaging. Indexes of RV function were derived at baseline level and during dobutamine infusion. Contractility was calculated by the slope of the endsystolic pressure-volume relation (ESPVR). An increase in ESPVR during dobutamine infusion was considered to indicate contractile reserve as a marker for latent RV dysfunction.

Results: Mean ESPVR was significantly increased from $0.34 \mathrm{mmHg} / \mathrm{ml}$ at baseline to $0.60 \mathrm{mmHg} / \mathrm{ml}$ during dobutamine $(\mathrm{p}=0.005)$. However, in 5 patients no relevant increase in contractility was found indicating impaired RV contractile reserve. There was only a weak, and not significant inverse correlation between contractile reserve and BNP $(r=-0.28)$. Conclusions: Even asymptomatic patients with an only mildly enlarged right ventricle can have impaired RV function. BNP is not able to predict early RV dysfunction accurately. 


\section{0}

Effectiveness of a home self-tilt training (TT) program for the prevention of recurrent neurally mediated syncope ( NMS ) in children and adolescents

Massimo Colaneri, Cardiochirurgia e Cardiologia Pediatrica e Congenita, Ospedali Riuniti, Ancona, Italy

Maria G Bettuzzi, Cardiochirurgia e Cardiologia Pediatrica e Congenita, Ospedali Riuniti, Ancona, Italy

Alessandra Baldinelli, Cardiochirurgia e Cardiologia Pediatrica e

Congenita, Ospedali Riuniti, Ancona, Italy

Roberto Ricciotti, Cardiochirurgia e Cardiologia Pediatrica e Congenita, Ospedali Riuniti, Ancona, Italy

Marco Pozzi, Cardiochirurgia e Cardiologia Pediatrica e Congenita, Ospedali Riuniti, Ancona, Italy

Background: Recurrent NMS can be a disabling disorder poorly responding to medical therapy. Orthostatic training has been reported to be effective in reducing recurrence of NMS. We evaluated the effectiveness of a home orthostatic self-training program to prevent NMS in a pediatric population.

Methods: We studied 20 pts (12 females, mean age 10, 6-16 years) with recurrent $(3.5+/-2.5$ mean number of syncopes in the last year) NMS and a positive head-up tilt test.

The patients performed the first 3 sessions in hospital by standing against a wall with their feet $20 \mathrm{~cm}$ away from the wall ( TT ) for 10 minutes. Then TT was performed at home twice a day progressively increasing its duration up to 30 minutes. Home training lasted 30 days and was repeated twice at interval of 1 month. Head-up tilt test was reevaluated at 6 months and the clinical effect was noted over a mean follow-up period of $11+/-5.4$ months.

Results: Compliance to TT was $90 \%(18 / 20$ pts ). Positivity to 6 month head-up tilt test was $6.7 \%(1 / 15)$ with a $93.3 \%$ reduction $(\mathrm{p}<0.01$ before $\mathrm{v}$ after TT). Spontaneous syncope recurrence was $5 \%(1 / 20 \mathrm{pt})$ with a $95 \%$ reduction $(\mathrm{p}<0.01$ before $\mathrm{v}$ after TT) No adverse events were observed during the home training. Conclusions: Home self-TT is feasible, safe and effective in preventing recurrence of NMS in children and adolescents.

7072

Pulse oximetry as a screening tool for detecting Congenital Heart Defects in the West Midlands, United Kingdom - An Interim Analysis

Abhay Bhoyar, University of Birmingham, Edgbaston, B15 2TT, Birmingham, UK Cardiology Department, Birmingham Children's NHS Foundation Trust,, United Kingdom

Alexandra Furmston, University of Birmingham, Edgbaston, B15

2TT, Birmingham, UK, United Kingdom

Jane Daniels, University of Birmingham, Edgbaston, B15 2TT,

Birmingham, UK, United Kingdom

Lee Middleton, University of Birmingham, Edgbaston, B15 2TT,

Birmingham, UK, United Kingdom

John G C Wright, Cardiology Department, Birmingham Children's

NHS Foundation Trust, Steelhouse Lane, B4 6NH, Birmingham,UK, United Kingdom

Khalid Khan, University of Birmingham, Edgbaston, B15 2TT,

Birmingham, UK and Birmingham Women's NHS Foundation Trust, Metchley Park Road, E, United Kingdom

Andrew Ewer, University of Birmingham, Edgbaston, B15 2TT,

Birmingham, UK and Birmingham Women's NHS Foundation Trust, Metchley Park Road, E

Objective: To determine the accuracy, psychosocial effects and cost effectiveness of Pulse oximetry (PO) for detecting critical and clinically significant congenital heart disease (CHD) in newborns.
Design: Population based prospective multicentre delayed cross sectional study in 6 hospitals in the West Midlands. Asymptomatic newborns $\geqslant 35$ weeks of gestation had PO performed within 24 hours of birth. Low saturations $(<95 \%$ in either limb or a difference of $\geqslant 3 \%$ between the limbs) on two separate occasions lead to referral. Echocardiography was performed in all the referrals. Up to one year after completion of recruitment, systematic interrogation of clinical databases will identify all CHD cases in the study population.

Results: Of the 24657 deliveries from February 2008 to December 2008, 18000 (73\%) babies have been screened. Parental consent was lower amongst the Asian and the Black populations, $80 \%$ and $75 \%$ respectively Vs White population of $>90 \%(\mathrm{p}<0.0001)$. Of the infants screened, $185(1.02 \%)$ were referred. Thirty four (18.4\%) had CHD, 22 (11.9\%) were critical CHD. Pulmonary or other disorders were present in 65 (35.1\%). Of the 34 CHD, 11 were antenatally diagnosed. The prevalence of critical and clinically significant CHD in our population was 1.9 per 1000. Final accuracy data will be published on completion of study.

Conclusion: Pulse oximetry appears to promote early detection of CHD. Our initial experience is encouraging and may support introducing this as a screening tool in the newborn nursery in order to improve the outcome of babies with critical CHD.

\section{3}

\section{Hepatopulmonary syndrome and intrapulmonary} vasodilatation in children with chronic liver diseases Mohammad Borzouee, shiraz university of medical sciences, Iran Gholamhosein Ajami, Iran

Heart function, pulmonary artery pressure (PAP) and also pulmonary vascular bed status should be assessed before liver transplantation. The hepato-pulmonary syndrome (HPS) is defined as the triad of liver disease, arterial desaturation, and intrapulmonary vascular dilatation (IPVD). The reported prevalence of HPS in adult cirrhotic patients varies between $4 \%$ and $19 \%$, and various threshold values defining arterial desaturation have been used and recommended previously. We studied 114 pediatrics patients with chronic liver diseases $(\mathcal{E} 18 \mathrm{y})$ in waiting list for liver transplantation. Conventional and contrast enhanced echocardiography was performed for detection of pulmonary vasodilatation and blood gas analysis for hypoxia. Cardiac function and PAP estimation were normal in all. Sixty patients $(52 \%)$ had positive contrast enhanced echocardiography which means $52 \%$ of patients had IPVD. Twenty five of 71 patients which had arterial blood gas analysis revealed positive contrast enhanced echocardiography with $\mathrm{Pao} 2<70 \mathrm{mmhg}$ which means $35.5 \%$ of our patients fulfilling the criteria of HPS.

Conclusion: We concluded which the heart function and PAP were in acceptable range. There was no indirect evidence(s) of pulmonary arterial hypertension. Pulmonary venous dilatation and HPS are common findings in children with chronic liver disease independent of causes. The prevalence seems to be higher among children than those reported in adulthood which may hypothesize more vascular responsiveness or angiogenesis in children.

\section{4}

The effects of congenital heart disease in children with Down syndrome

Nathalie J M Bravo-valenzuela, Faculdade de Ciencias Medicas da Santa Cas de Sao Paulo, Brazil

Maria L Passarelli, Faculdade de Ciencias Medicas da Santa Casa de Sao Paulo, Brazil 
Objectives: To identify malnutrition in children with Down Syndrome (DS) and congenital heart disease (CHD). To examine the long-term effects of correction intervention.

Methods: Data $(\mathrm{n}=181)$ were obtained from the health records (from 1984 to 2007) of all children (0-172 months) with DS followed at the Santa Casa de São Paulo Hospital, Brazil. Cases with mosaicism and/or coexistent major pathology were excluded. Detailed anthropometry was performed and z-scores calculated. Malnutrition was defined as weight height $\mathrm{z}$-score $<2$, 0. Patients with CHD admitted for corrective intervention were evaluated for nutritional status before and after surgery (6 months; 1,2 , and 5 years).

Results: 165 children with DS were analysed, the others were excluded. $67,8 \%(\mathrm{n}=112)$ was a sample of children with CHD and $32,1 \%(\mathrm{n}=53)$ without heart disease.58 children were admitted for corrective intervention. Mean age of correction was 25,4 months $(+/-35,495)$. Before the surgery the mean weight $\mathrm{z}$-score was $-2,6448(+/-1,68)$ and mean height $\mathrm{z}$-score was $-3,037(+/-1,862)$. The time elapsed to weight height was on average one to two years after surgery (Table I/ group I = age of correction until 84,05 months and Group II after this age). The patients with greater growth disturbance present better recovery on postoperative growth (Tables I and II/ Groups I, II and IIII = age of correction until 56,03, between 56,04 and 112,07 months after this age).

Conclusions: Malnutrition is common in children with CHD and SD. Corrective intervention results in significant improvement nutritional status.

\section{5}

Evaluation of a Humanitarian Cardiovascular Screening Program - Use of Clinical Parameters and NonCcardiologist in the Screening Process

David M Bush, San Antonio Military Medical Center, United States Ariuntsatsral Erdenebileg, Mongolia

Anneke C Bush, San Antonio Military Medical Center, United States Kirk A Milhoan, San Antonio Military Medical Center, United States

Pediatric humanitarian cardiovascular screening often poses unique challenges in both time and patient numbers. The most effective process remains unclear.

As part of ongoing CHD screening in Mongolia, a team (PS) of PNPs, internists, pediatricians and medical students evaluated available children in the Hovskul region over five days. Demographic and clinical information was collected. A pediatric cardiologist (PC) evaluated a 5\% random sample of those screened. Children with pathologic murmurs (PM) were referred for ECHO.

Of 1171 children evaluated, $48 \%$ were male, aged $9.2 \pm 4.4$ years. A murmur was detected in $14.9 \%$, whereas a PM was found in $4.4 \%$. Pulse oximetry did not significantly discriminate among those with $\mathrm{CHD} \quad(\mathrm{SaO} 2=94.2 \pm 6.6 \quad \mathrm{CHD}+$, $96.8 \pm 1.8 \%$ CHD-), though it did identify those with cyanotic forms $(\mathrm{SaO} 2=86.5 \pm 7.7 \%, \mathrm{p}<0.01)$. Likewise, family history of CHD and prior notation of a murmur (innocent or pathologic), did not aid in CHD identification. PC review of 60 children screened by PS showed $80 \%$ agreement. For the 12 children in whom disagreement occurred, 10 were felt to have a PM by PS, but an innocent murmur by PC. For two patients with an innocent murmur by PS, but PM by PC, one had bicuspid aortic valve and one had a moderate-sized ASD.

PS may be used effectively to screen large cohorts of children for CHD. The added utility of pulse oximetry or history, in this screening setting, appears minimal. Most concerning among missed diagnoses is ASD; additional testing (such as ECG) may be warranted.

\section{6}

Use of Enteral Budesonide for the Treatment of Protein-Losing Enteropathy following Fontan Pallation A Case Report

David M Bush, San Antonio Military Medical Center, United States Mary E Porisch, San Antonio Military Medical Center, United States Michael Serwacki, San Antonio Military Medical Center, United States Kirk A Milhoan, San Antonio Military Medical Center, United States

Protein-losing enteropathy (PLE) remains a feared complication of Fontan palliation (FP), with few effective treatment options. While systemic corticosteroids aide some patients, side-effects may be prohibitive. A patient with FP and severe PLE refractory to standard therapies is presented. Use of oral budesonide (OB, Entocort ${ }^{\mathbb{R}}$ EC) resulted in significant improvement.

E.F. is a 9 y.o. black female, born with a hypoplastic left heart syndrome variant. She underwent Stage I palliation in infancy, Glenn anastamosis at age 6 months and extracardiac, unfenestrated FP at age $2 \frac{1}{2}$ years. Post-operative pleural effusions resolved after three weeks. Fifteen months after FP, E.F. presented with abdominal and facial swelling. Serum albumin levels (SAL) were $<2 \mathrm{mg} / \mathrm{dL}$; stool alpha-1-antitrypsin levels were increased. Cardiac catheterization revealed central venous pressures of $13 \mathrm{mmHg}$ and good ventricular function. Narrowing of the LPA was successfully stented. Symptoms nonetheless persisted; the patient was subsequently lost to follow-up. She represented at 7 years of age with severe edema, refractory to heparin, diuretics, systemic steroids and diet. Albumin was required every 48 hours. A RAST panel revealed allergies to wheat, egg and dairy products. These were eliminated without improvement. OB was added and SAL increased $>2.5 \mathrm{mg} / \mathrm{dL}$ for three weeks. OB was discontinued three months later, with return to her pretreatment state. It was restarted after 9 months, with prompt improvement.

We hypothesize that food allergies may play a role in refractory PLE patients. Use of $\mathrm{OB}$, with limited systemic absorption, may offer a therapeutic option for selected patients.

\section{7}

Effect of digoxin in Neonates with Ventricular Septal Defect and Congestive Heart Failure using Tissue Velocity Imaging

Jia-Kan Chang, Department of Pediatrics, Cheng-Hsin General Hospital, Taipei, Taiwan

Kai-Sheng Hsieh, Department of Pediatrics, Veterans General Hospital, Kaohsiung, Taiwan

Po-Yen Liu, Department of Pediatrics, Veterans General Hospital, Kaohsiung, Taiwan

Chu-Chuan Lin, Department of Pediatrics, Veterans General Hospital, Kaohsiung, Taiwan

Da-Cheng Hwang, Department of Pediatrics, Veterans General

Hospital, Kaohsiung, Taiwan

Ken-Pen Wong, Department of Pediatrics, Veterans General Hospital, Kaohsiung, Taiwan

Background: Ventricular septal defect VSD is a congenital heart disease often cause congestive heart failure (CHF) in neonates or early infancy necessitating digoxin therapy. Therefore, we use new Doppler myocardial imaging to evaluate the effects of digoxin. 
Methods: The Doppler myocardial images were obtained from 8 infants with ventricular septal defect. Their ages were from 2 to 28 days mean 13.8 days. Doppler myocardial images were recorded before digoxin administration and after digitalization. All images were obtained with standard parasternal and apical views. From the extracted strain rate curves, peak values for systole, early diastole, and late diastole were calculated.

Results: We summarize the effect of digoxin on neonates with VSD and CHF in the following table. Abbreviations: $\mathrm{BS}=$ basal septal, MS = mid septal, AS = apical septal, AL = apical lateral, $\mathrm{ML}=$ mid lateral, $\mathrm{BL}=$ basal lateral segments at apical 4 chamber view.

Conclusion: Based on the objective, quantitative Doppler myocardial images, we found that digoxin did not show benefit for neonates with VSD and CHF.

\begin{tabular}{|c|c|c|c|c|c|c|c|}
\hline & BS & MS & AS & $A L$ & ML & $\mathrm{BL}$ & $\begin{array}{c}P- \\
\text { value }\end{array}$ \\
\hline \multicolumn{8}{|l|}{ velocity } \\
\hline Before-mean & $\begin{array}{r}4.32 \pm \\
1.06\end{array}$ & $\begin{array}{r}3.42 \pm \\
1.35\end{array}$ & $\begin{array}{r}1.88 \pm \\
1.32\end{array}$ & $\begin{array}{r}2.11 \pm \\
1.22\end{array}$ & $\begin{array}{r}3.81 \pm \\
1.30\end{array}$ & $\begin{array}{r}5.48 \pm \\
2.05\end{array}$ & \\
\hline After-mean & $\begin{array}{r}4.21 \pm \\
1.17\end{array}$ & $\begin{array}{r}2.69 \pm \\
1.88\end{array}$ & $\begin{array}{r}1.71 \pm \\
2.67\end{array}$ & $\begin{array}{r}2.04 \pm \\
1.26\end{array}$ & $\begin{array}{r}3.63 \pm \\
0.99\end{array}$ & $\begin{array}{r}5.20 \pm \\
0.74\end{array}$ & 0.71 \\
\hline \multicolumn{8}{|l|}{ strain } \\
\hline Before-mean & $\begin{array}{c}-14.17 \pm \\
12.76\end{array}$ & $\begin{array}{c}-19.02 \pm \\
6.08\end{array}$ & $\begin{array}{c}-24.22 \pm \\
8.57\end{array}$ & $\begin{array}{c}-22.17 \pm \\
12.04\end{array}$ & $\begin{array}{c}-17.10 \pm \\
10.85\end{array}$ & $\begin{array}{c}-10.59 \pm \\
11.43\end{array}$ & \\
\hline After-mean & $\begin{array}{c}-25.61 \pm \\
36.84\end{array}$ & $\begin{array}{c}-16.29 \pm \\
10.95\end{array}$ & $\begin{array}{c}-24.83 \pm \\
9.80\end{array}$ & $\begin{array}{c}-21.86 \pm \\
13.49\end{array}$ & $\begin{array}{c}-18.76 \pm \\
12.33\end{array}$ & $\begin{array}{c}-18.67 \pm \\
13.37\end{array}$ & 0.51 \\
\hline strain rate & & & & & & & \\
\hline Before-mean & $\begin{array}{c}-2.12 \pm \\
0.85\end{array}$ & $\begin{array}{c}-2.36 \pm \\
1.05\end{array}$ & $\begin{array}{c}-3.15 \pm \\
1.27\end{array}$ & $\begin{array}{c}-2.71 \pm \\
1.39\end{array}$ & $\begin{array}{r}-2.31 \pm \\
0.84\end{array}$ & $\begin{array}{c}-3.01 \pm \\
1.07\end{array}$ & \\
\hline After-mean & $\begin{array}{c}-1.97 \pm \\
1.35\end{array}$ & $\begin{array}{c}-2.10 \pm \\
0.90\end{array}$ & $\begin{array}{c}-3.25 \pm \\
1.06\end{array}$ & $\begin{array}{c}-3.21 \pm \\
0.92\end{array}$ & $\begin{array}{c}-2.37 \pm \\
0.80\end{array}$ & $\begin{array}{c}-2.36 \pm \\
0.63\end{array}$ & 0.58 \\
\hline
\end{tabular}

\section{8}

The Natural History of Ventricular Septal Defects: Followed from Less Than Six Months of Age

Jia-Kan Chang, Department of Pedaitrics, Cheng-Hsin General

Hospital, Taiwan

Wen-Yen Jen, Department of Pedaitrics, Cheng-Hsin General Hospital, Taiwan

Kai-Sheng Hsieh, Department of Pedaitrics, Veterans General HospitalKaohsiung, Taiwan

Aim: To determine the natural history and timing of spontaneous closure of ventricular septal defect (VSD) in patients followed from less than six months of age in our cardiac clinic.

Methods: Using color Doppler flow mapping, a total of 84 infants were diagnosed of isolated VSD: 41 (48.8\%) muscular type, 33 (39.3\%) perimembranous, 7 (8.3\%) subpulmonary, and $3(3.6 \%)$ mal-aligned. The patients were divided into two groups. Sixtyfour patients were diagnosed at the age of less than or equal to one month (group I) and 20 were diagnosed at the age of more then one month (group II). Spontaneous closure of VSD was confirmed by color Doppler flow mapping.

Results: Surgical closure was performed in four patients $(4.8 \%)$. Spontaneous closure occurred in 52 patients (61.9\%) of the whole group, $73.4 \%$ of group I and $25 \%$ of group II, respectively. Spontaneous closure occurred only in patients with perimembranous type of VSD $(48.5 \% ; 16 / 33)$ and in patients with muscular type $(87.8 \% ; 36 / 41)$. None of the subpulmonary or mal-aligned types of VSD closed spontaneously.

Conclusions: Muscular type VSD was the most common type of VSD in group of very young infants. Spontaneous closure of VSD is age-dependent, occurring mainly in muscular and perimembranous types of VSD.
7079

Aberrant Right Subclavian Artery Causes Esophageal Compression in Children As Severe As Complete Vascular Rings and Can be Restored by Aortopexy : An Esogaphageal Manometry Study

Fong-Lin Chen, Chung Shan Medical Unversity Hospital, Taiwan Shu-Min Kuo, Chung Shan Medical Unversity Hospital, Taiwan Yi-Ching Li, Chung Shan Medical Unversity, Taiwan

Shiang-Suo Huang, Chung Shan Medical Unversity, Taiwan

Shuping Ge, Texas Heart Institute/St. Luke's Episcopal Hospital, United States

Aberrant right subclavian artery(ARSCA)is believed to have a benign natural history with no significant compression of the trachea and esophagus, in contrast to complete vascular ring(CVR). However, our clinical observation is that ARSCA can cause significant swallowing difficulties. In this study, we prospectively performed esophageal manometry to evaluate the severity of esophageal function in subjects with ARSCA and CVR and efficacy of surgical intervention.

From January, 2002 to December, 2007, 46 children of $\operatorname{ARSCA}(8.5+/-2.9$ years $)$ and 40 with $\operatorname{CRV}(6.9+/-4.1$ years, $\mathrm{p}>0.05)$ were enrolled after confirmation of diagnosis by a history of dysphagia, barium swallow, echocardiography, and computed tomography. Patients with developmental delay or mental retardation were excluded. Esophageal manometry was performed preoperatively and postoperatively to measure the severity of esophageal compression.

Esophageal manometric measurement was successfully obtained for all subjects. All had symptomatic relief of dysphagia. All subjects had a high-pressure zone at the site of vascular compression. The maximal and mean preoperative esophageal pressures were not statistically different between ARSCA $(77.4+/-36.9 \mathrm{mmHg}$ and $45.8+/-25.8 \mathrm{mmHg})$ and CVR $(98.6+/-37.1 \mathrm{mmHg}, \mathrm{p}>0.05$ and $53.1+/-28.9 \mathrm{mmHg}, \mathrm{p}>0.05)$, respectively. Postoperatively, both maximal and mean pressures were significantly reduced for ARSCA $(41.8+/-25.8 \mathrm{mmHg}$ and $23.8+/-15.3 \mathrm{mmHg})$ and for CVR $(53.1+/-37.1 \mathrm{mmHg}$ and $28.9+/-15.1 \mathrm{mmHg}$ ), respectively.

This is the first study to demonstrate that ARSCA has a significant impact on esophageal function as CVR has. Esophageal manometry revealed a typical high pressure zone that is useful to quantitatively evaluate the severity of obstruction and the efficacy of surgical intervention. Left thoractomy with aortopexy may provide an effective surgical approach for ARSCA with restoration of esophageal function.

\section{0}

The Prevalence of Cardiovascular Disease in School-aged Children: A Large-scale Prospective Screening Study in 276,332 Subjects

Fong-Lin Chen, Chung Shan Medical University Hospital, Taiwan Shu-Min Kuo, Chung Shan Medical University Hospital, Taiwan

Yi-Ching Li, Chung Shan Medical University, Taiwan

Shiang-Suo Huang, Chung Shan Medical University, Taiwan

Chung-Pin Liao, Chung Shan Medical University Hospital, Taiwan Chih-Yun Wen, Chung Shan Medical University Hospitalr, Taiwan

Background: Unlike the newborn and adult population, there is little large-scale screening data and statistics in cardiovascular disease (CVD) in school-aged children, which may accurately define the impact of CVD on growth, development, school performance, sport participation, risks for sudden cardiac death (SCD), and to develop effective screening and preventive strategies to improve the cardiovascular health for this population. 
Methods: From 2001 to 2006, we screened 276,332 school-aged children in primary and middle schools in central Taiwan by $2 \mathrm{D}$ echocardiography.

Results: The prevalence of CVD in this population is 17.82/ 1000. A total of $69.9 \%$ of CVD in this population are new diagnosis. Among these new cases, severe congenital CVD are rare and simple congenital CVD are more common. A total of $43.0 \%$ of these cases warranted surgical or transcatheter intervention. CVD that may lead to significantly high risk for sport participation and possible SCD, including hypertrophic cardiomyopathy, coronary anomalies, Marfan syndrome, etc. The largest under-diagnosed CVD that may cause morbidity is congenital vascular anomalies. In this group, only $48.0 \%$ of the patient has clinical signs and symptoms.

Conclusions: This study suggests that CVD in the school-aged children are more common than in the literature. Although severe congenital CVD and those with a high risk for SCD are rare, a significant number of children will need intervention for CVD. Congenital vascular anomalies are significantly underdiagnosed by clinical evaluation. Echocardiography is a useful tool for CVD screening for this population but the costeffectiveness needs to be further evaluated.

\section{1}

Early Surgical Management of Anatomically Complete Vascular Rings: Screening for Anatomically Complete Vascular Rings

Fong-Lin Chen, Chung Shan Medical University Hospital, Taiwan Jung-Min Yu, Chung Shan Medical University hospital, Taiwan Tsung-Po Tsai, Chung Shan Medical University hospital, Taiwan Shu-Min Kuo, Chung Shan Medical University Hospital, Taiwan Zen-Chung Weng, Taipei Veterans General Hospital, Taiwan

Background: Adults with potentially life-threatening congenital vascular anomalies due to tracheoesophageal compression and aneurismal rupture are often misdiagnosed as having asthma or recurrent bronchopulmonary infection. However, the early recognition and treatment of these anomalies can help to achieve a low mortality rate.

Methods: Two-dimensional echocardiography (2DE) was used to diagnose anatomically complete vascular rings (including double aortic arch and right aortic arch with left ligamentum arteriosum) in 96 patients. They underwent a barium esophagogram, spiral $\mathrm{CT}$ and/or MRI to confirm the existence of the vascular rings. Demographics, historical objectives and the results of physical examinations were all taken into account when performing the clinical diagnosis, as opposed to the conventional methods that are used when diagnosing ACVR. Those patients with overt clinical symptoms of tracheoesophageal compression were then earmarked for surgical treatment.

Results: Among the patients diagnosed with ACVR, 64 received surgical treatment after ACVR was confirmed. Right aortic $\operatorname{arch}(\mathrm{RAA})$ with Kommerell's divertiuculum (KD) with left ligamentum arteriosum was found to be the most common type of $\operatorname{ACVR}(80.3 \%$ of cases). $2 \mathrm{DE}$ was found to have a $100 \%$ overall sensitivity and a $100 \%$ specificity with regard to its ability to diagnose ACVR. Recurrent bronchopulmonary infections and asthmatic cough were the dominant symptoms of tracheal compression and all of these patients who underwent surgical treatment were well without morbidity and mortality.

Conclusion: 2DE is an effective primary diagnostic method with a high sensitivity and specificity. Early surgical management of ACVR is safe and easily achieved.

\section{2}

Current status in the management of pediatric infective endocarditis: a national survey

Shubao Chen, Shanghai Children's Medical Center, China

Yume Xie, Gaungdong Cradiovascular Institute, China

Jinghui Sun, The First Hospital of Jilin Universit, China

Po Zhang, Shen Yaung general Hospital of PLA, China

Yue Yuan, Beijing Children's Hospital, China

Shanliang Zhu, Nanjing Children's Hospital, China

Xiufen Hu, Tongii Hospital of Huazhong University of Science technology, China

Guoying Huang, Children's Hospital of Fudan University, Cocos

(Keeling) Islands

Objective: To understand current practice pattern in the management of pediatric IE in China.

Methods: This retrospective, multicenter study was conducted in 13 hospitals. Clinical data of 268 patients diagnosed as IE according to IE criteria between 2000 and 2006 were analysed. The mean age of patients was 8.94 years (18d-18y).

Results:56 antibiotics were used to treat children with IE in our group. The most commonly used antibiotics in patients with streptococci detected were penicillin G, cephalosporin, vancomycin, penicillin \& beta-lactamase inhibitor and aminoglycoside, in patients with staphylococci detected were cephalosporin, oxicillin, vancomycin, aminoglycoside, and quinolones. Only one antibiotic agent was used in 33(12.3\%), two in 83(31.1\%), 3 and more in 151(56.6\%). Duration of antibiotic treatment was from 1 day to 98 days, $<2$ weeks in $19(7 \%)$, 2weeks to $<4$ weeks in $74(27.7 \%), 4-6$ weeks in $122(45.7 \%),>6$ weeks in 52 (19.4\%). 123 patients simultaneously underwent surgical management. In antibiotics and surgical treatment group, 111(90.2\%) were cured, 4 referred to surgery, $5(4 \%)$ died, 3 refused medical advice, in antibiotics alone treatment group, 75(51.7\%) were cured, $13(8.96 \%)$ died, 57 refused medical advice. There was a significantly difference in outcomes between two groups $(p=0.000)$. The results of multivariable logistic regression analysis showed that staphylococci aureus as a pathogen, children with repaired congenital heart disease, and complicated with heart failure were risk factors related to death.

Conclusion: There were a wide range antibiotics and regimen used to manage children with IE in China.

\section{3}

Differential Impact of Right Ventricular Pacing on Three-Dimensional Global Left Ventricular Dyssynchrony in Children and Young Adults with Congenital and Acquired Heart Block

Wen-Jing Hong, Department of Paediatrics and Adolescent Medicine, Queen Mary Hospital, The University of Hong Kong., Hong Kong Tak-Cheung Yung, Department of Paediatrics and Adolescent Medicine, Queen Mary Hospital, The University of Hong Kong., Hong Kong Kin-Shing Lun, Department of Paediatrics and Adolescent Medicine, Queen Mary Hospital, The University of Hong Kong., Hong Kong Yiu-Fai Cheung, Department of Paediatrics and Adolescent Medicine, Queen Mary Hospital, The University of Hong Kong., Hong Kong

Objective: We sought to determine the effect of long-term right ventricular (RV) pacing on left ventricular (LV) mechanical dyssynchrony in children and young adults with congenital and acquired heart block.

Methods: Eighteen patients aged $19.5 \pm 7.0$ years with congenital heart block (group I), 9 aged $20.7 \pm 11.1$ years with acquired heart block post congenital heart surgery (group II), and 15 healthy 
control subjects (group III) were studied. The LV volumes, ejection fraction, and systolic dyssynchrony index (SDI), as determined by three-dimensional echocardiography, were compared among groups.

Results: Groups I $(6.68 \pm 2.44 \%)$ and II $(9.43 \pm 4.44 \%)$ had significantly greater SDI compared with group III $(3.88 \pm 0.63 \%$, $\mathrm{p}=0.011$ and $<0.001$, respectively). The prevalence of $\mathrm{LV}$ mechanical dyssynchrony (SDI $>5.14 \%$, mean +2 SD of controls) in group I and II was $72.2 \%$ and $66.7 \%$, respectively. In 27 patients with RV pacing, the LV SDI correlated negatively with LV ejection fraction $(r=-0.744, p<0.001)$. The times-to-minimum regional volume were significantly longer in lateral, posterior, and inferior segments in group I and septal and inferior segments in group II compared with those in group III $(p<0.05)$. Compared to patients without LV dyssynchrony, patients with LV dyssynchrony had lower LV ejection fraction $(\mathrm{p}<0.001)$, shorter $\mathrm{R}-\mathrm{R}$ interval $(p<0.001)$, and tended to have dual chamber pacing $(p=0.088)$, but had similar duration of pacing, QRS duration, and position of pacing wire (epicardial vs endocardial).

Conclusions: Permanent RV pacing in childhood has a negative effect on LV systolic dysfunction through induction of mechanical dyssynchrony, the magnitude and pattern of which differ between congenital and acquired heart block.

\begin{tabular}{lllll}
\hline & $\begin{array}{l}\text { Group I } \\
(\mathbf{n}=18)\end{array}$ & $\begin{array}{l}\text { Group II } \\
(\mathbf{n}=9)\end{array}$ & $\begin{array}{l}\text { Group III } \\
(\mathbf{n}=15)\end{array}$ & p value \\
\hline $\begin{array}{c}\text { LVEDVi } \\
(\mathrm{ml} / \mathrm{m} 2)\end{array}$ & $49.9 \pm 9.2$ & $46.0 \pm 8.2$ & $42.2 \pm 7.1$ & 0.04 \\
$\begin{array}{c}\text { LVESVi } \\
(\mathrm{ml} / \mathrm{m} 2)\end{array}$ & $25.0 \pm 6.0$ & $24.6 \pm 6.8$ & $17.7 \pm 3.5$ & 0.001 \\
$\begin{array}{l}\text { LV EF (\%) } \\
\text { SDI (\%) }\end{array}$ & $49.9 \pm 10.0$ & $45.8 \pm 11.1$ & $58.0 \pm 4.5$ & 0.004 \\
\hline
\end{tabular}

7084

Impact of Temporary Interruption of Long-Term Right Ventricular Pacing on Left Ventricular Function and Dyssynchrony in Children and Young Adults with Congenital and Acquired Heart Block

Wen-Jing Hong, Department of Paediatrics and Adolescent Medicine, Queen Mary Hospital, The University of Hong Kong., Hong Kong Tak-Cheung Yung, Department of Paediatrics and Adolescent Medicine, Queen Mary Hospital, The University of Hong Kong., Hong Kong Kin-Shing Lun, Department of Paediatrics and Adolescent Medicine, Queen Mary Hospital, The University of Hong Kong., Hong Kong Yiu-Fai Cheung, Department of Paediatrics and Adolescent Medicine, Queen Mary Hospital, The University of Hong Kong., Hong Kong

Objective: We sought to determine the effect of temporary interruption of long-term right ventricular (RV) pacing on left ventricular (LV) function and mechanical dyssynchrony in children and young adults with complete heart block.

Methods: Twelve patients aged $20.0 \pm 7.4$ years with congenital heart block (group I) and six aged $22.7 \pm 11.0$ years with surgically acquired heart block (group II) with RV pacing were studied. The pacing rate was reduced to less than patient's intrinsic heart rate and maintained for 5 minutes. The LV ejection fraction (EF) and systolic dyssynchrony index (SDI) as determined by three-dimensional echocardiography, two-dimensional global LV systolic strain and strain rate (SR), and Dopplerderived isovolumic acceleration (IVA) before and after interruption of RV pacing were compared.
Results: The LV EF, strain, SR, and IVA increased and QRS duration decreased significantly after pacing interruption in both groups (all $\mathrm{p}<0.05$ ). While SDI decreased significantly in both groups I $(6.78 \pm 2.30$ to $3.79 \pm 0.83, \mathrm{p}<0.001)$ and II $(9.17 \pm 4.08$ to $5.03 \pm 1.64, \mathrm{p}=0.001)$, it remained higher in group II than in group I $(\mathrm{p}=0.046)$ after pacing interruption. The prevalence of LV dyssynchrony (SDI $>4.7 \%$ ) decreased significantly in group I $(83.3 \%$ to $25.0 \%, \mathrm{p}=0.006)$ but not group II $(66.7 \%$ to $50.0 \%, p=0.50)$. The percent increase in LV ejection fraction correlated positively with percent reduction in LV SDI $(\mathrm{r}=0.80, \mathrm{p}<0.001)$

Conclusions: Achievement of LV synchronization after interruption of RV pacing occurs more commonly in patients with congenital than those with surgically acquired heart block, which has implications on the benefit of cardiac resynchronization therapy.

\section{5}

Total Anomalous Pulmonary Venous Connection in a

\section{Sibling}

Woo-Yeon Choi, Department of pediatrics, Chonnam National University Medical School, Chonnam National University Hospital, Gwang-Ju, Korea, Korea

Young-Jin Lee, Department of pediatrics, Chonnam National University Medical School, Chonnam National University Hospital, Gwang-Ju,

Korea, Korea

Sun-Ju Park, Department of pediatrics, Chonnam National University Medical School, Chonnam National University Hospital, Gwang-Ju,

Korea, Korea

Young-Kuk Cho, Korea

Jae-Sook Ma, Korea

Objective: Total anomalous pulmonary venous connection (TAPVC) is a rare anomaly, and the risk of recurrence in siblings has been considered to be negligible. A few reports of familial recurrence, however, have been published. We report TAPVC to superior vena cava (SVC) and coronary sinus in a sibling.

Results:

case1. : The first baby of non-consanguineous parents was admitted to hospital because of tachypnea at birth date. Echocardiogram revealed TAPVC to SVC (Fig. 1). An operation was performed the next day. The vertical vein was ligated and an anastomosis was made between the common pulmonary venous chamber and the left atrium. At follow-up at 5 years of age, he was healthy.

Figure 1

Echocardiogram reveals TAPVC to SVC

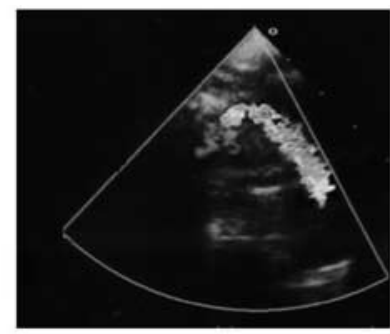

Figure 2.

Echocardiogram reveals TAPVC to coronary sinus

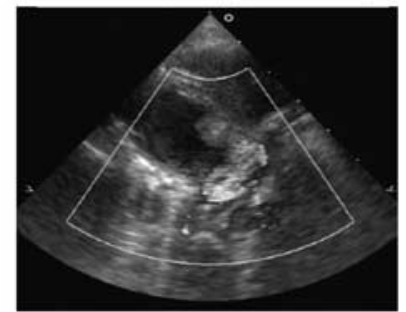


case 2: The second baby in the family also admitted to hospital because of irritability at 1 month of age. Echocardiogram revealed TAPVC to coronary sinus (Fig. 2). Unroofing the coronary sinus and patch closure of the atrial septum were performed the next day. At follow-up at 2 months of age, he was healthy.

Conclusion: Autosomal dominant inheritance with incomplete penetrance or rather an autosomal dominant gene mutation parents have been suggested as a possible pattern of inheritance. The connection between pulmonary venous return and some portions of the primitive foregut venous plexus are accidental. That is shown by the fact that even in siblings different types of venous return are seen. Even slight abnormalities on chest X-ray should alert the pediatrician to the possibility of TAPVC, especially in relatives of known cases.

\section{6}

\section{Imipramine Induced Toxicity with Brugada Electrocardiographic Pattern in a Toddler: a Case Report}

Woo-Yeon Choi, Department of pediatrics, Chonnam National University Medical School, Chonnam National University Hospital, Gwang-Ju, Korea, Korea

Ui-Jeong Han, Department of pediatrics, Chonnam National University Medical School, Chonnam National University Hospital, Gwang-Ju, Korea, Korea

Young-Nam Kim, Department of pediatrics, Chonnam National University Medical School, Chonnam National University Hospital, Gwang-Ju, Korea, Korea

Young-Kuk Cho, Korea

Jae-Sook Ma, Korea

Object: Clinical toxicity with the treatment of tricylclic antidepressants (TCAs), including imipramine, is anticholinergic effects and central nervous system toxicity. Of these, the most life-threatening toxicity remains the development of cardiac dysrhythmias. Conduction delays such as QRS and corrected QT prolongation, wide QRS complex tachycardia, and the Brugada electrocardiographic pattern (BEP) have been reported. Sodium bicarbonate decreases QRS widening and suppresses dysrhythmias by providing excess sodium to reverse the TCAinduced sodium-channel blockade and possibly by binding directly to the myocardium.

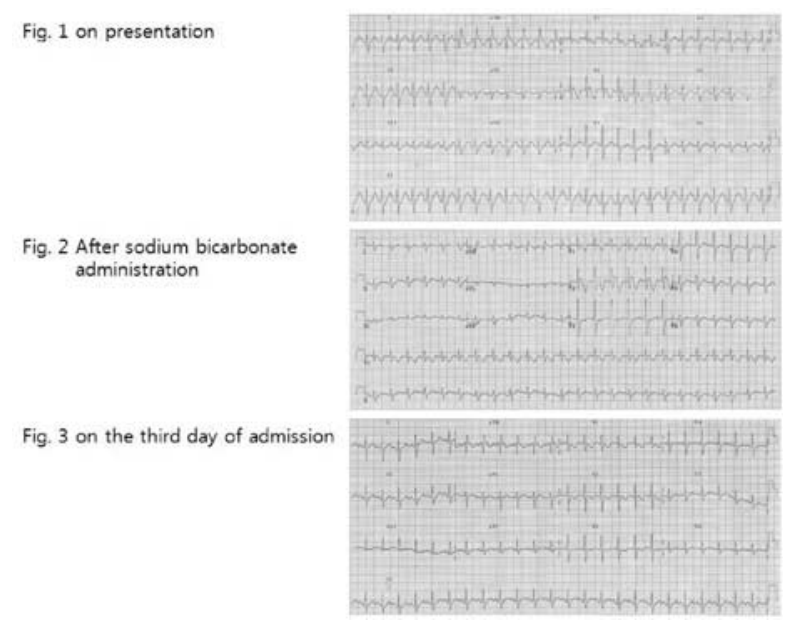

Result: A 3-year-old boy presented to the emergency department with generalized tonic-clonic movements, fever and vomiting. The history was significant for the ingestion of four imipramine tablets (one tablet contains $25 \mathrm{mg}, 7.69 \mathrm{mg} / \mathrm{kg}$ ) obtained from a kindergarten friend, 15 hours previously. The patient was successfully treated with intravenous diazepam, one dose, for the generalized seizures. An electrocardiogram displayed a wide QRS complex tachycardia, with a down slopping ST segment elevation and a prominent $\mathrm{J}$ wave in lead V2, characteristic of the BEP found in the Brugada syndrome (Fig. 1). The patient was treated with a sodium bicarbonate infusion. However, the BEP persisted (Fig. 2). Three days after admission the BEP resolved (Fig. 3). The patient had a complete clinical recovery, and was discharged to home on the seventh hospital day.

Conclusion: We describe a patient that presented with convulsions, tachycardia with a wide QRS complex, a BEP, and anuresis associated with an accidental over dose ingestion of imipramine, and the outcome of treatment with sodium bicarbonate.

\section{7}

Excercise Induced Chest Pain In Pediatric Patients

Devyani Chowdhury, Penn State Hershey Children's Hospital, United States

Bobby Kumar, Penn State Hershey Children's Hospital, United States Marie Shaner, Penn State Hershey Children's Hospital, United States Mathew Dean, Penn State Hershey Children's Hospital, United States Steven Cyran, Penn State Hershey Children's Hospital, United States

Objective: Exercise-induced chest pain in pediatric patients with structural heart disease can be secondary to life-threatening conditions like ischemia and arrhythmias. The aim of this study was to evaluate exercise induced chest pain in pediatric patients. Methods: A retrospective chart review study was conducted. 350 children with chest pain presented to pediatric cardiology outpatient clinic at Penn State Hershey Children's hospital (7/04-1/08). All patients had resting cardiac echocardiogram and exercise stress test (EST). Deep sternal palpation was performed on 313 patients, pre- and post-stress test to identify costochondritis. (4 patients on Digoxin were excluded)

Results: Of these 313 patients (mean age 14 years), 175 (56\%) experienced skeletal chest pain pre- and/or post-EST. 114 (65\%) patients complained of palpable chest pain pre- and post-EST and 6 $(5 \%)$ had complaints of chest pain pre but not post-EST. 30\% (55) of patients denied reproducible chest pain pre-stress test but complained of chest pain with palpation post-test. Of these 55 subjects with posttest chest pain, $69 \%$ had normal cardiac anatomy, 16\% had significant structural heart disease and 15\% had minor structural anomalies. Significant ST segment changes were identified in 2 of the 55 patients, both had structurally normal hearts with no regional wall motion abnormalities on stress echocardiogram.

Conclusion: These data indicate that the most common etiology of exercise-induced chest pain in patients even with complex cardiac structure is non-cardiac. Exercise-induced costochondritis should be considered in the differential diagnosis regardless of cardiac anatomy and may easily be identified in the exercise laboratory

\section{8}

Evaluation of perinatal cardiac function in diabetic pregnant fetuses by brain natriuretic peptide (BNP) in cord blood

Chen Chu, Cardiovascular Center, Children's Hospital of Fudan University, Shanghai, China

Yonghao Gui, Cardiovascular Center, Children's Hospital of Fudan University, Shanghai, China

Yunyun Ren, Ultrasound Department, Gynaecology and Obstetrics Hospital of Fudan University, Shanghai, China 
Objective: To approach the possibility of evaluating perinatal cardiac function in fetuses of diabetic mothers by measuring brain natriuretic peptide (BNP) in umbilical cord plasma.

Methods: 24 gestational diabetic mothers were divided into good and poor control groups due to their blood glucose before delivery. Fetal cardiac sizes and function were measured in their late pregnant periods by fetal echocardiography. 25 uncomplicated mothers were as control. Cord bloods were sampled for detecting the BNP concentrations.

Results: Among the groups the fetuses' gestational weeks and weights at birth had no significant differences, and the BNP concentrations had no correlations with them. The BNP concentrations in diabetic group were significantly higher than control $(114.0 \pm 39.0$ vs. $80.6 \pm 13.7 \mathrm{pg} / \mathrm{ml}, \mathrm{p}<0.001)$, among which the poor control group higher than the good control group $(142.1 \pm 44.1$ vs. $102.4 \pm 31.2 \mathrm{pg} / \mathrm{ml}, \mathrm{p}<0.01, \mathrm{n}=7$ and 17 respectively). No significant difference was found between the gestational diabetes mellitus and impaired glucose tolerance mothers, and no correlation of the BNP concentrations to these mothers' blood glucose at diagnosis. The BNP concentrations positively correlated to the fetal left ventricular walls thicknesses and the peak velocities of mitral A wave, and negatively correlated to the mitral E/A ratios $(\mathrm{r}=0.715,0.491$ and -0.507 respectively, $\mathrm{p}<0.05)$.

Conclusions: BNP in cord blood is possible to evaluate perinatal cardiac function. diabetic pregnant fetuses have an increased BNP level in cord blood, which accords with maternal glucose control and changes in fetal diastolic function. Good glucose control may decrease the impact of abnormal maternal blood glucose on fetal hearts.

\section{9}

Estimation of left ventricular filling pressure

Milan M Djukic, University Childrens Hospital Belgrade, Yugoslavia Estimation of left ventricular filling pressure in children on hemodyalisis M. Djukic, I. Jovanovic, Lj. Sulovic, I. Stefanovic, M. Dabetic,

T. Ilisic. University Children's Hospital, Belgrade, Serbia

Introduction: The ratio of the early transmitral inflow velocity and early diastolic mitral annular velocity $(\mathrm{E} / \mathrm{Em})$ can be used to estimate LV filling pressure (LVFP).

Left ventricular filling pressure is increased in children undergoing hemodialysis, due to left ventricular pressure and volume overload.

Objective: To compare LVFP using E/Em ratio in healthy children and children undergoing HD (before and after procedure) and to estimate influence of volume and pressure changes after HD on LVFP.

Method: Forty children were studied (20 undergoing HD and 20 healthy), of similar age and body composition.

Doppler echocardiography was used to measure E velocity. Em was measured using pulsed tissue Doppler echocardiography. $\mathrm{E} / \mathrm{Em}$ was then calculated.

\begin{tabular}{llll}
\hline & $\mathbf{t}$ & $\mathrm{df}$ & $\mathrm{p}$ \\
\hline Before HD and health & 3.56 & 38 & $0.001^{*}$ \\
Before and after HD & 1.66 & 19 & 0.114 \\
After HD and health & 1.05 & 38 & 0.299 \\
\hline
\end{tabular}

Results: E/Em ratio before haemodyalisis was $8.58+/-1.47$, $8.01+/-2.02$ after hemodyalisis, and $7.11+/-0.97$ in healthy children. Differences in E/Em values among three groups were shown in table.
Conclusion: LVFP was significantly higher in children before HD comparing to healthy children. There was no significant diffrence in LVFP between children after HD and healthy children. Diastolic function of LV was significantly improved after HD due to volume and systolic unload of LV.

\section{0}

Monozygotic twins discordant for congenital heart defects Sanja Dorner, Department od Pediatrics, University Hospital Osijek, Croatia

Dalibor Saric, Department of Pediatrics, University Hospital Centre Zagreb, Croatia

Jasenka Wagner, Medical School Osijek, University J.J. Strossmayer

Osijek, Croatia

Vesna Milas, Department od Pediatrics, University Hospital Osijek, Croatia

Hrvoje Kniewald, Department of Pediatrics, University Hospital Centre Zagreb, Croatia

Ivan Malcic, Department of Pediatrics, University Hospital Centre

Zagreb, Croatia

Cardiac malformations are the commonest anomalies with astonishing similarity in reported birth rates in different centres. Searching for causative factors is still ongoing process. Results of Baltimore Washington Infant Study and New England Study and presents that cardiac defects are remarkably constant in time and place, which tends to argue against hidden teratogens as a major cause of malformations. Despite this, many authors have favoured a major environmental contribution on the basis of twin studies. There is well-known that cardiac defects are common in twin pregnancies, occurring more commonly in monozygotic twins and usually affecting one twin in more than $90 \%$ of cases. The mechanism for the increase malformation remains unknown, although several explanations have been proposed. One possibility is that monozygotic twinning process itself increases the incidence of congenital heart disease, with postzygotic unequal division of the inner cell mass being responsible for discordant cardiovascular anatomy. Another contributor might be phenotypic variability of the same genome or possibly even genetic inter-twin differences caused by de novo mutations.

Here we report case of monozygotic male twins discordant for cardiac malformations presenting with pulmonary atresia and intact ventricular septum (PS/IVS) in one of them. Another twin presents with subaortic ventricular septal defect (VSD) and many small muscular ventricular defects. According to the developmental-mechanistic approach this cardiac defects belong in different groups. Twin pairs with congenital heart defects generally have concordant defects by mechanistic group. To our knowledge the occurrence of PS/IVS and VSD in monozygotic twins has never been reported.

\section{1}

Spontaneous closure of fistula between right coronary artery and right ventricle in infant

Ivan Malcic, University Hospital Center Zagreb, Croatia, Croatia Drazen Belina, University Hospital Center Zagreb, Croatia, Croatia Roland Gitter, AKH Linz, Austria, Austria

Hrvoje Kniewald, University Hospital Center Zagreb, Croatia, Croatia Mladen Viljevac, General Hospital Virovitica, Croatia, Croatia Dalibor Saric, University Hospital Center Zagreb, Croatia, Croatia

We present a rare case of spontaneous closure of fistula between right coronary artery and right ventricle in female infant. At the age of 7 days continuous murmur intensity $3 / 6$ was registered in 
3rd intercostal space along right sterna border. Echocardiography indicated, with high probability, a coronary artery fistula. Definitive diagnose was made at the age of 1 month with aortography. Radioscintiography and oxymetric analysis revealed $47 \%$ of left-to-right shunting at level of fistula. Because of the size of the shunting a surgical correction had been planned, but in mean-time the murmur had gone. At the age od 6 months control aortography showed spontaneous closure of fistula, but with significant morphological changes in the right coronary artery (stenosis and aneurisms). Exact mechanism of spontaneous closure is unknown, nor is the timing of therapeutic intervention. Control coronarography after 1 year showed regression of pathological changes in right coronary artery. Based on the literature and own experiences we consider invasive therapy indicated in children with significant clinical symptoms. In cases were clinical symptoms are absent expectative state should be taken, because there is chance of spontaneous closure of fistulas, even larger ones, as it is in our case.

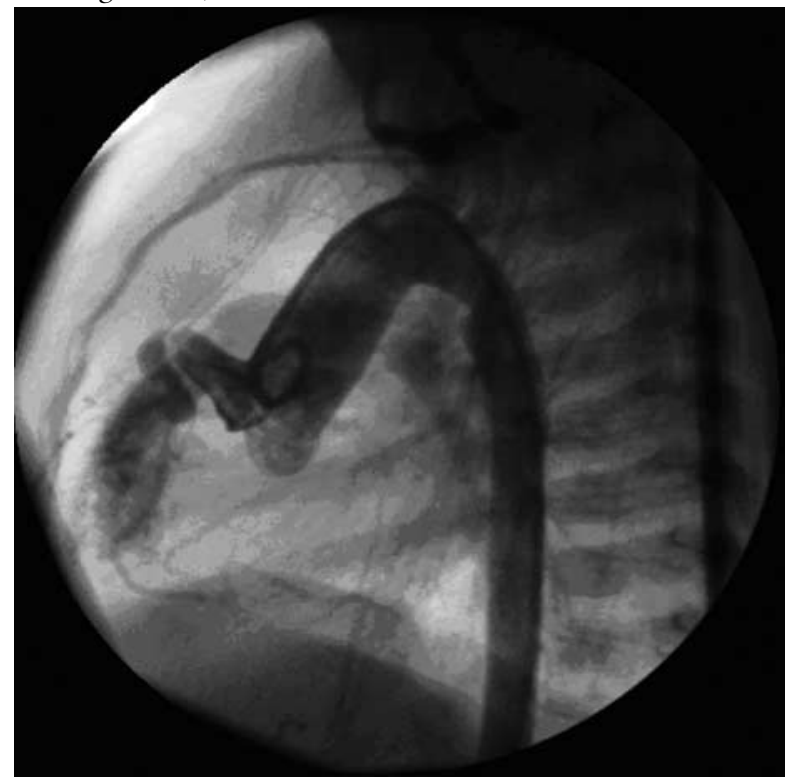

7092

Williams' syndrome: an unusual cause of neonatal coarctation

Andreea Dragulescu, Timone Children's Hospital, Marseille, France Issam Kammache, Timone Children's Hospital, Marseille, France Phillipe Acar, Children's Hospital, Toulouse, France

Pascal Amedro, Arnaud de Villeneuve Hospital, Montpellier, France Virginie Fouilloux, Timone Children's Hospital, Marseille, France Bernard Kreitmann, Timone Children's Hospital, Marseille, France Dominique Metras, Timone Children's Hospital, Marseille

Alain Fraisse, Timone Children's Hospital, Marseille

Objective: To determine the frequency, clinical profile and outcome of patients with Williams syndrome and neonatal coarctation.

Patients and Results: Among 135 reviewed patients less than 3 months of age who underwent aortic coarctation repair in our institution between January 1996 and June 2007, Williams syndrome was found in 3 cases $(2,2 \%)$. They presented with congestive heart failure and absent femoral pulses, the diagnostic being confirmed by echocardiography. One case had an associated small ventricular septal defect. The 3 patients were operated at a mean age and weight of $51.6 \pm 32.7$ days and $3.5 \pm 0.6 \mathrm{~kg}$, respectively. All had resection of the coarctation segment with end-to-end anastomosis, through left thoracotomy in 2 cases, and sternotomy under cardiopulmonary bypass with large patch enlargement of the aortic arch and ventricular septal defect closure in one. The diagnosis of Williams syndrome was confirmed postoperatively by FISH analysis. All patients remained hypertensive and presented with recoarctation $2,2.3$, and 14.3 months after initial repair. Transcatheter balloon dilation was attempted with failure to proceed in one and partial success in 2 cases. All had redo surgery at $0.6,1.2$ and 10.9 years after initial repair with patch enlargement of the aorta in two cases and an extra-anatomic bypass in the third, with good results and discontinuation of antihypertensive treatment.

Conclusion: Williams syndrome presenting as neonatal coarctation is unusual, being characterized by more complex repair and recurrent aortic obstruction. Balloon dilation seems disappointing to treat successfully these recoarctations. Reoperations are often necessary with patch enlargement and/or exclusion of the stenotic aortic segment.

\section{3}

Serum levels of natriuretic peptides decrease during treatment protocol for univentricular heart (UVH)

Anneli Eerola, Department of Pediatrics, University Hospital of Tampere, Finland

Eero Jokinen, Hospital for Children and Adolescents, University of Helsinki, Finland

Jaana I Pihkala, Hospital for Children and Adolescents, University of Helsinki, Finland

Purpose: Prospective evaluation of serum levels of N-terminal proatriopeptide (ANPN) and N-terminal pro-brain natriuretic peptide (NT-proBNP) in children treated for UVH.

Methods: Samples were analyzed in 20 children before first operation, before bidirectional Glenn shunt, at the age of one year, and before total cavopulmonary connection (TCPC). Additionally, we measured peptide levels in 21 children before and 11 after TCPC.

Results: Between first and second operation, peptide levels decreased (Table). By TCPC, further reduction had taken place. Throughout follow-up, levels were higher than in controls. There was no difference between patients with right and left ventricular morphology. In cross-sectional study, before TCPC, median ANPN concentration measured 0.37 (range 0.18-1.00) $\mathrm{nmol} / \mathrm{l}(\mathrm{p}=0.001$, compared with controls) and NT-proBNP 156 (range 13-955) ng/l ( $<<0.001)$. After TCPC, median ANPN concentration measured 0.39 (range 0.09-0.98) nmol/1 $(\mathrm{p}=0.037)$ and NT-proBNP 184 (range 76-1406) ng/l $(\mathrm{p}<0.001)$.

\begin{tabular}{|c|c|c|c|c|c|c|}
\hline & Baseline & preGlenn & 1 year & preTCPC & $\begin{array}{c}\text { controls } \\
<6 \mathrm{mos}\end{array}$ & $\begin{array}{l}\text { controls } \\
>6 \mathrm{mos}\end{array}$ \\
\hline $\mathrm{n}$ & 20 & 19 & 12 & 11 & 12 & 64 \\
\hline age (years) & $\begin{array}{c}0.0(0.0- \\
0.1)\end{array}$ & $\begin{array}{c}0.3(0.1- \\
0.4)\end{array}$ & $\begin{array}{c}1.0(0.7- \\
1.1)\end{array}$ & $\begin{array}{c}2.1 \\
(1.7-3.5)\end{array}$ & $\begin{array}{c}0.1(0.0- \\
0.4)\end{array}$ & $\begin{array}{c}6.8(0.6- \\
15.6)\end{array}$ \\
\hline $\begin{array}{l}\text { ANPN } \\
\quad(\mathrm{nmol} / \mathrm{l})\end{array}$ & $\begin{array}{l}16.80 \\
(0.73- \\
40.20)^{*}\end{array}$ & $\begin{array}{c}1.80(0.69- \\
6.10) \dagger \S\end{array}$ & $\begin{array}{r}0.76(0.34- \\
10.10)^{*} \|\end{array}$ & $\begin{array}{c}0.31 \\
(0.26- \\
0.48) \ddagger \| \#\end{array}$ & $\begin{array}{c}0.88(0.51- \\
3.70)\end{array}$ & $\begin{array}{l}0.28 \\
(0.10- \\
0.61)\end{array}$ \\
\hline $\begin{array}{l}\text { NT- } \\
\text { proBNP } \\
(\mathrm{ng} / \mathrm{l})\end{array}$ & $\begin{array}{c}24503 \\
(924- \\
35001)^{*}\end{array}$ & $\begin{array}{c}3020(119- \\
1751) * \|\end{array}$ & $\begin{array}{l}311(225- \\
26393)^{*} \infty\end{array}$ & $\begin{array}{c}196 \\
(79-718) \\
* \| \#\end{array}$ & $\begin{array}{c}281(122- \\
3224)\end{array}$ & $\begin{array}{c}57 \\
(14-157)\end{array}$ \\
\hline
\end{tabular}

Conclusion: Natriuretic peptide levels decrease but remain higher than in controls throughout treatment protocol for UVH. They reflect reduction of volume overload of the single ventricle and can be used for hemodynamic monitoring. 
Table Levels of natriuretic peptides in children during treatment for univentricular heart.

- ${ }^{\star} \mathrm{p}<0.001 ; \uparrow \mathrm{p}<0.01 ; \ddagger \mathrm{p}<0.05$ compared with controls

- $\S \mathrm{p}<0.001 ;|| \mathrm{p}<0.01 ; \infty \mathrm{p}<0.05$ compared with baseline

- \# $\mathrm{p}<0.01$ compared with preGlenn

7094

Paternal Fibrillin 1 and TGFBR2 Mutation Transmitting to an Affected Son with Neonatal Marfan Syndrome: The Importance of Early Recognition

Huda Elshershari, University of Illinois Medical Center at Chicago,

Division of Pediatric Cardiology, United States

Catharine Harris, University of Illinois Medical Center at Chicago,

Division of Genetics, United States

Introduction: Marfan syndrome is an autosomal dominant connective tissue disorder in which abnormalities occur in the cardiovascular, skeletal, ocular and pulmonary systems. Neonatal Marfan syndrome (nMFS) is the rare and severe form caused by mutations in the fibrillin 1 gene (FBN1) located on chromosome $15 q 21.1$. The relative infrequency of this syndrome hampers the early diagnosis. We describe a case of nMFS diagnosed because of family history, dysmorphic features and cardiac abnormality.

Case Description: This infant was the third child of nonconsanguineous parents. Father had Marfan syndrome for many years. He has had aortic valve replacement and multiple eye surgeries. The mother and their other 2 children are healthy. The infant was born at term by spontaneous vaginal delivery with a birth weight of 3200 gram (25th percentile) and a length of $51 \mathrm{~cm}$ ( $>50$ th percentile). Physical examination revealed long and protruding ears, micrognathia, high palate, arachnodactyly and undescended testicles. No heart murmur. Echocardiogram showed aortic root dilatation measured $1.6 \mathrm{~cm}(\mathrm{Z}=2.94)$. Molecular genetic studies showed a mutation in exon 31 of the FBN1 gene in the infant and Father. The infant is treated with losartan, an angiotensin II type 1 receptor blocker that has potential role in treatment of Marfan syndrome.

Conclusion: Neonatal Marfan syndrome is associated with poor outcome, $50 \%$ of affected infants dying during the first year of life due to cardiac complications. Therefore, it is important to recognize nMFS in utero and shortly after birth to initiate the appropriate investigation and management.

\section{7}

Use of Ambulatory Blood Pressure Monitoring in Children and Adolescents with Vasovagal Syncope Nai Chung Fong, Department of Paediatrics, Princess Margaret Hospital, Hong Kong

Wai Yau Mak, Department of Paediatrics, Princess Margaret Hospital, Hong Kong

Chi Keung Li, Department of Paediatrics, Princess Margaret Hospital, Hong Kong

Objective: To study the ambulatory blood pressure profile of paediatric pateints with vasovagal syncope and assess its role in the diagnosis of vasovagal syncope.

Methods: This study was done in a tertiary Paediatric referral centre in Hong Kong. Patients with recurrent syncope confirmed to be vasovagal in origin by tilt table test were recruited. Ambulatory blood pressure monitoring was done after tilt test.
Results: 40 patients were recruited between 2005 to 2007. Male to Female ratio was 10:30. The mean age was 15.7 year (range 9-22.5). Blood pressure result was interpreted with reference to data by Soergel etal. Several interesting findings were noted. $68 \%$ $(12-100 \%)$ and $39 \%(37-100 \%)$ of daytime systolic blood pressure readings were less than the 50 and 10 centile age reference. $76 \%(37-100 \%)$ and $42 \%(5-86 \%)$ of daytime diastolic pressure readings were less than the 50 and 10 centile age reference. The mean systolic blood pressure load was $2.5 \%$ (range 0-24\% with 27 patients having 0\%) and the mean diastolic pressure load was $3.6 \%$ (range $0-21 \%$ with 21 patients having $0 \%$ ). The daytime systolic and diastolic blood pressure range were $34.7 \mathrm{mmHg}$ (range $17-61 \mathrm{mmHg}$ ) and $30.5 \mathrm{mmHg}$ (range $12-64 \mathrm{mmHg}$ ) respectively. With the use of event recording and patient-triggered instantaneous blood pressure measurement during dizziness and presyncope, vasovagal syncope could be diagnosed in a more physiological way.

Conclusion: Ambulatory blood pressure monitoring is a useful tool in diagnosing vasovagal syncope.

\section{8}

Hypoplastic Left Heart Complex: A Play on Z-Scores Matthias Freund, Pediatric Cardiology, University Medical Centre Utrecht, The Netherlands

Casper Bollen, Pediatric Hospital, University Medical Centre Utrecht, The Netherlands

Jan Erik Freund, Pediatric Hospital, University Medical Centre

Utrecht, The Netherlands

Felix Haas, Pediatric Cardiovascular Surgery, University Medical Centre Utrecht, The Netherlands

Jan Strengers, Pediatric Cardiology, University Medical Centre Utrecht, The Netherlands

Introduction: Hypoplastic Left Heart Complex (HLHC) is characterized by a mitral (MV) or aortic (AoV) valve z-score $<-2$, antegrade flow in the ascending aorta, ductal dependency, possible coarctation or aortic arch hypoplasia, and no significant valvar or subvalvular stenosis (Tchervenkov 2000). The z-scores of the valves are the leading features; therefore the algorithm of the z-score-calculation has a fundamental impact. However, in all HLHC-publications different equations were used. The aim of the study was to evaluate and apply existing $\mathrm{z}$-score-calculations in HLHC.

Method: The literature dealing with $\mathrm{z}$-score-calculations and HLHC were compared. An own cohort of 10 newborns diagnosed with a HLHC was evaluated.

Results: Five different methods are published for z-scorecalculation of the MV and AoV. The correlation between the different regression equations is vague. In our cohort, the maximal z-values of the MV and AoV were $-3,7 /-4,4$ using the equations published by Zilberman 2005 and Petterson 2008 but $-8,5 /-7,5$ while using the formula published by Dauberney 1999, indicating a high standard error. The z-score-formulas published by Kirklin 1985 and Serraf 1999 used either not echo-derived or m-mode-derived normal values which results in an overestimation of the $z$-value.

Conclusion: The MV and AoV z-scores calculated with different equations vary enormously. Comparing patients with HLHC is therefore not possible when using different methods. The published z-score-calculation-algorithms from Zilberman and Petterson seem to be the most appropriate to use in HLHC. The database project hypoplastic left heart syndrome (Tchervenkov) should implement a uniform use of z-score-calculation in HLHC. 
7099

Evaluation of congenital cardiac care in disadvantaged healthcare systems: A database for developing nations Cecily Froemke, Portland State University, Portland, Oregon, United States

Janine Henson, Fairview Hospital, University of Minnesota, Minneapolis, Minnesota, United States

Kelly Hattabaugh, Axis Clinical Software Inc., Portland, Oregon, United States

Veronica Swanson, Oregon Health \& Sciences University, Portland, Oregon, United States

Mary Minette, Oegon Health \& Sciences University, Portland, Oregon, United States

Michael Silverbach, Oregon Health \& Sciences University, Portland, Oregon, United States

Karl Welke, Oregon Health \& Sciences university, Portland, Oregon, United States

Aubyn Marath, Oregon Health \& Sciences University, Portland, Oregon, United States

Objectives: Although many patients with congenital and adult heart diseases are tracked in Europe and North America, most live elsewhere. We examined unique risk factors and comorbidities of populations in nations with disadvantaged healthcare systems to track related diseases, functional status, quality of life, morbidity, short and long-term mortality, outcome and uniformity of surgical performance.

Methods: We constructed a database with elements and their definitions leveraged from available databases for cardiology, surgery, anesthesia and perfusion to ensure international comparisons and benchmarking. Additional elements capture the socioeconomic conditions of healthcare in the developing world and their impact on congenital cardiac care; 325 questions, adjudicated by expert consensus, focus on culture-specific demographics, socioeconomic status, quality of life and nutritional status.

Results: Now available in English \& Spanish, (www.cardiostart. org) this comprehensive database examines most aspects of perioperative care and long term follow up. It is compatible with the European Association of Cardiothoracic Surgery and Society of Thoracic Surgeons' Congenital Heart Databases, and provides a quality improvement and educational tool for evolving cardiac surgery programs in developing nations.

Conclusion: Although mature databases exist for the collection of congenital cardiac surgical data in the developed world, these databases do not capture many of the unique qualities of disadvantaged health care systems. With this new database, medical standards which drive decision making, trends in poor community disease processes, the influence of family and cultural settings, cost-benefit of equipment and personnel, and the outcomes of local and visiting surgical teams can now be more fully examined.

\section{0}

\section{Systemic Venous Collateral Development after the Fontan} Procedures

Takanari Fujii, Department of Pediatric Cardiology, Tokyo Women's Medical University, Japan

Yoshiki Mori, Department of Pediatric Cardiology, Tokyo Women's Medical University, Japan

Kannta Kishi, Department of Pediatric Cardiology, Tokyo Women's

Medical University, Japan

Hiromi Kurosawa, Department of Cardiovascular Surgery, Tokyo

Women's Medical University, Japan
Toshio Nakanishi, Department of Pediatric Cardiology, Tokyo Women's Medical University, Japan

Background: It is known that systemic venous collaterals develop and that they can lead an unanticipated cyanosis after the Fontan procedures. However, there have been a few reports focusing on this problem.

Objective: To determine the incidence, clinical course and factors associated with venous collaterals after the Fontan procedures.

Methods: We reviewed angiograms and clinical data in 171 Fontan patients who underwent cardiac catheterization. Venous collaterals with $<95 \%$ systemic desaturation were defined as significant. Anatomic and hemodynamic variables as the predictive factors were compared between the groups with or without decompressing venous collaterals.

Results: Fifty-four venous collaterals were identified in 36 patients $(21 \%)$. In 19 patients (53\%), the collaterals were detected within 1 year after the Fontan procedures. The follow-up catheterizations $(n=25)$ showed that the arterial oxygen saturation at rest $(90 \pm 4 \%)$ did not change during follow-up periods ( $5.3 \pm 3.3$ years), although the size of vessels increased in some cases. The morphology of ventricle, hemodynamic variables including pulmonary artery pressure, resistance, Nakata index before the Fontan procedures and transpulmonary pressure after the procedures were similar between the groups. The only anatomic factor of heterotaxia syndrome was a predictor for venous collaterals $(53 \%$ in heterotaxia vs. $22 \%$ in non-heterotaxia, $\mathrm{p}<0.001)$.

Conclusions: Systemic venous collaterals are common in the Fontan patients. They increase in size but do not hemodynamically deteriorate with time. These collaterals are more likely to occur in the heterotaxia syndrome.

\section{1}

Congenital Heart Block and Dilated Ascending Aorta

Swati Garekar, Wockhardt Pediatric and Congenital Heart Center, India

Snehal Kulkarni, India

Kaushik Bhojani, India

Pankaj Kasar, India

Zakiya Khan, India

Suresh Joshi, India

Shreepal Jain, India

Congenital Complete heart block has a well known association with maternal systemic lupus erythematosus. We present a case series of 5 consecutive patients of CCHB from Nov 2006 to August 2008. Three out of the five cases had severe dilatation of the ascending aorta.

See table.

There is a strong association between $\mathrm{CCHB}$ and maternal lupus. Congenital AV block occurs in association with maternal antibodies in 1/15000-20000 live births. Specific antibodies directed against ribonuclear proteins are transmitted across the placenta, often in absence of maternal symptoms. However little is known about $\mathrm{CCHB}$ in association with connective tissue disorders in the child. The presence of significantly dilated ascending aorta in 3 out of 5 patients possibly indicates a connective tissue disorder in the children. To the best of our knowledge, ours is only the second report of occurrence of dilated ascending aorta in children with CCHB. A detailed echocardiogram is warranted in every case of CCHB. A dilated ascending aorta may prove to be a marker of morbidity and mortality in such children and may necessitate further work up. 


\begin{tabular}{|c|c|c|c|c|}
\hline Age & $\begin{array}{l}\text { Ventricular rate } \\
\text { (per min) }\end{array}$ & $\begin{array}{c}\text { Mother's Antibody } \\
\text { Status }\end{array}$ & $\begin{array}{l}\text { Initial LVIDd } \\
\quad(\mathrm{z} \text { score })\end{array}$ & FS $\%$ \\
\hline 1 day & 50 & $\begin{array}{l}\text { Anti Ro La negative, } \\
\text { ANA positive, 1:320; } \\
\text { speckled }\end{array}$ & $2.1 \mathrm{~cm}(0.67)$ & 34 \\
\hline 3 day & 50 & Anti Ro/LA positive & $2 \mathrm{~cm}(0.5)$ & 45 \\
\hline $3.5 \mathrm{mo}$ & 80 & $\begin{array}{c}\text { Anti Ro/La postive, ANA } \\
\text { positive }(1: 10000) \\
\text { speckled nucleolar }\end{array}$ & $2.2 \mathrm{~cm}(0.8)$ & 36 \\
\hline $4.5 y r s$ & 50 & ANA postive & $3.5 \mathrm{~cm}(1.23)$ & 23 \\
\hline $13 \mathrm{mo}$ & 45 & ANA positive & $3.3 \mathrm{~cm}(+3.9)$ & 36 \\
\hline
\end{tabular}

\begin{tabular}{|c|c|c|c|c|}
\hline Age & CHD & $\begin{array}{l}\text { Initial Ascending } \\
\text { Aorta ( } \mathrm{z} \text { score) }\end{array}$ & Pacemaker & $\begin{array}{l}\text { Mosst recent } \\
\text { Follow Up }\end{array}$ \\
\hline 1 day & PDA & $1.3 \mathrm{~cm}(+4)$ & $\begin{array}{l}\text { epicardial } \\
\text { VVIR }\end{array}$ & $\begin{array}{c}\text { Dilated Cardiomyopathy, } \\
\text { asc aorta } 1.5 \mathrm{~cm}\end{array}$ \\
\hline 3 day & $\mathrm{PDA} / \mathrm{ASD}$ & normal & Epicardial VVIR & lost to follow up \\
\hline $3.5 \mathrm{mo}$ & PDA & normal & - & 2nd degree heart block \\
\hline $4.5 \mathrm{yrs}$ & ASD & $2.1 \mathrm{~cm}(+4.3)$ & - & posted for pacemaker \\
\hline $13 \mathrm{mo}$ & ASD & $2.6 \mathrm{~cm}(+12.8)$ & - & posted for pacemaker \\
\hline
\end{tabular}

\section{2}

Outcome of the LV in Children and Young Adults with Severe Combined Aortic and Mitral Valve Regurgitation Compared to Isolated Lesions

Thomas L Gentles, Starship Children's Hospital, New Zealand Kirsten A Finucane, Starship Children's Hospital, New Zealand Jonathan Mervis, Starship Children's Hospital, New Zealand

Nigel J Wilson, Starship Children's Hospital, New Zealand

Aortic and mitral regurgitation (AR and MR) expose the left ventricle (LV) to abnormal load, and carry a risk of progressive contractile dysfunction. In childhood, LV mechanics and predictors of postoperative LV dysfunction are poorly defined. Methods: Retrospective identification of children and young adults (age $<23$ years) who underwent surgery for severe combined AR\&MR (Group I, $\mathrm{n}=13$ ), isolated severe AR (Group II, $n=14$ ) or MR (Group III $n=21$ ), and had suitable echocardiograms for analysis before and 4-18 months after surgery. Cardiac size and function were expressed as z-scores normalised to body surface area and age. The stress velocity index (SVI) was expressed as a $\mathrm{z}$-score normalising shortening to afterload.

\begin{tabular}{lccc}
\hline $\begin{array}{l}\text { Preoperative LV } \\
\text { Mechanics }\end{array}$ & $\begin{array}{c}\text { Group I } \\
\text { (AR\&MR) }\end{array}$ & $\begin{array}{c}\text { Group II } \\
\text { (AR) }\end{array}$ & $\begin{array}{c}\text { Group III } \\
\text { (MR) }\end{array}$ \\
\hline EDV z-score & $6.3 \pm 2.1 *$ & $5.8 \pm 1.4^{*}$ & $4.5 \pm 1.8^{*}$ \\
EF z-score & $-1.2 \pm 1.1^{*}$ & $-1.1 \pm 1.2^{*}$ & $-0.1 \pm 1.9$ \\
Fibre stress z-score & $1.3 \pm 1.7^{*}$ & $1.5 \pm 1.6^{*}$ & $0.1 \pm 1.5$ \\
SVI & $-1.7 \pm 1.3^{*}$ & $-2.0 \pm 1.0^{*}$ & $0.0 \pm 1.4$ \\
\hline
\end{tabular}

Results: LV dilatation in Group I was similar to Group II and greater than Group III $(\mathrm{p}<0.05)$. Likewise afterload (fibre stress) was elevated and function depressed to a degree similar to Group II. These parameters were normal in Group III (Table; $\star=\mathrm{p}<0.05$ vs. normal). After operation LV dysfunction (EF zscore $<-2$ ) was more prevalent in Group I than in Groups II and III $(85 \%$ vs. $36 \%$ vs. $57 \%, p=0.04)$. The preoperative endsystolic volume $\mathrm{z}$-score independently predicted postoperative LV dysfunction in Group II and III patients ( $p=0.03$, z-score cut-off 4.8 and 2.5 respectively), but not in Group I where moderate LV dysfunction (EF z-score $<-3$ ) was related to the preoperative SVI $(p=0.04)$.

Conclusions: LV mechanics in severe combined AR\&MR are similar to those of severe AR but the outcome of the LV is worse. Surgery should be considered at the earliest opportunity.

\section{3}

A new category of VSD - Silent VSD !

Tiow $H$ Goh, MONASH MEDICAL CENTRE, MELBOURNE, Australia

Objective: As is commonly understood, the clinical course of small peri-membranous VSD is an initial loud pan systolic murmur that progressively softens and eventually disappears as the VSD closes. These signs are confirmed echocardiographically. However the condition where the murmur becomes silent clinically yet a VSD persists echocardiographically has not been described before.

Methods \& Results: I reviewed more than 120 of my perimembranous VSD pts.on serial follow up and describe 8pts (aged8mths-10yrs) that have had spontaneous clinical closure of small peri-membranous VSD with the progressive disappearance of the pan systolic murmur, (independently observed) but have persistent echocardiographic features of VSD (based on 2D,colour flow and continuous wave Doppler with complete or incomplete envelope). These pts. have now been followed up for 9mths-6yrs and remain well.

Conclusion: Like silent PDA, silent VSD cannot be heard clinically but exist on echocardiography. Because anatomically a VSD remains, it's clinical significance needs to be further established.

\section{4}

Corrected QT Interval in Children with Brain Death

Anita M Grady, University of California, San Francisco, United States Jennifer M Plymale, University of California, Davis, United States Jeanny Park, University of California, Davis, United States

Background: Prolongation of the QT interval occurs in adults with severe brain injury. However whether there is significant QT prolongation in children with brain injury is unknown. The objective of this study was to test the hypothesis that the QT interval corrected for heart rate (QTc) is longer in this population than expected for a normal population. Mechanism of brain injury, electrolytes, cardiac function, age, and gender were also evaluated.

Methods: All previously healthy children $(<18$ years) cared for in our hospital from 1995-2007 with brain death who had at least one 12-lead ECG and normal anatomy by echo were included. Admission details, past medical/family history, demographics, medications and laboratory data were collected. The QTc was measured by two independent observers blinded to clinical history. QTc was calculated using the Bazett method.

Results: 37 patients met inclusion criteria. Five patients had event histories concerning for possible Long QT Syndrome; data analysis was performed with and without these patients. The QTc data were normally distributed. The mean (SD) QTc for the entire cohort was $452(61) \mathrm{ms}$; excluding the five patients, 449(62)ms. On multivariate analysis, gender (QTc female $<$ male), hypokalemia, and injury type (QTc traumatic $<$ ischemic) were associated with worsening QTc prolongation. Glucose, magnesium, calcium, age, systolic function, $\mathrm{pH}$ and temperature demonstrated no significant effect.

Conclusions: QTc in children with brain death is normally distributed but higher than expected for normal children. This 
information is important for clinicians attempting to determine the cause of death and/or if the patient is being considered for organ donation.

\section{5}

B-type Natriuretic Peptide in Japanese Obese Children Hisanori Haniu, Division of Pediatrics, Niigata Graduate School of Medical and Dental Sciences., Japan

Hiroshi Suzuki, Division of Pediatrics, Niigata Graduate School of Medical and Dental Sciences., Japan

Satohshi Hasegawa, Division of Pediatrics, Niigata Graduate School of

Medical and Dental Sciences., Japan

Fujito Numano, Division of Pediatrics, Niigata Graduate School of Medical and Dental Sciences., Japan

Toru Kikuchi, Division of Pediatrics, Niigata Graduate School of Medical and Dental Sciences., Japan

Makoto Uchiyama, Division of Pediatrics, Niigata Graduate School of Medical and Dental Sciences., Japan

Background: B-type Natriuretic Peptide (BNP) has emerged as a hormone with many favorable physiological properties. Several researchers have demonstrated that BNP is lower level in obese adult than in non-obese ones, suggesting that Lower BNP may be involved in developing cardiovascular disease in obese adult. However, there have been few reports to exam relations between BNP and obesity in children. This study aimed to assess BNP level, and the relationship among BNP and obesity-related factors in obese children.

Methods and Results: The subjects were 131 obese and 20 non-obese boys aged 6 to 15 years. Obese boys were defined as the over middle-class obesity (obesity rate $>30 \%$, obesity rate was calculated: body weight-standard weight/standard weight $\star 100$ ). Body height, weight, blood pressure and pulse rate were measured. Serum BNP, blood sugar, insulin, HbA1c, triglyceride, leptin and adiponectine were examined as obesity-related factors. BNP levels $(11.38+/$ $-11.42 \mathrm{pg} / \mathrm{ml}$ ) were significantly lower in obese boys than in nonobese boys $(14.76+/-9.98 \mathrm{pg} / \mathrm{ml}) \quad(\mathrm{p}<0.05)$. And the mean blood pressure were higher in obese boys than in non-obese ones $(p<0.001)$. BNP was inversely associated with obesity rate, systolic blood pressure and pulse rate. There were positive association between BNP and insulin, leptin.

Conclusions: BNP decreased in obese children similarly to adults. Moreover, BNP level was related to insulin and leptin. Lower level of BNP persisted since children may be one of the factors of the metabolic syndrome and cardiovascular disease in adult.

\section{6}

Physiologic Response to Feeding in Infants with Transposition

Tondi M Harrison, University of Minnesota, United States

Objective: The purpose of this study was to compare physiologic regulation before, during, and after feeding between 15 infants with transposition of the great arteries (TGA) and 16 healthy infants matched for age, gender, and feeding type.

Methods: Data were collected post-surgically at 2 weeks and 2 months of age. High frequency heart rate variability (HF-HRV) was used as an index of physiologic regulation and examined across three phases of feeding: 30 minutes pre-feeding, duringfeeding, and 60 minutes post-feeding. Data were analyzed using fixed occasions, multiple condition, linear and logistic regression analyses and event history analysis.

Results: At 2 weeks, infants with TGA demonstrated lower HFHRV pre- and post-feeding when compared with healthy infants.
Infants with TGA were less likely to demonstrate adaptive reductions in HF-HRV during-feeding and adaptive increases in HF-HRV post-feeding. Post-feeding recovery to pre-feeding HFHRV levels was similar between groups. At 2 months, no group differences were found in HF-HRV with feeding phases. However, twice as many TGA infants as healthy infants failed to recover to pre-feeding HF-HRV levels within the post-feeding observation. Conclusions: These findings suggest that when infants with TGA are surgically corrected and have had time to recover from surgery, physiologic regulation may be comparable to that of healthy infants. However, the prolonged recovery time observed in infants with TGA at 2 months raises questions about the equivalence of these groups. Further research in this area is needed to describe mechanisms involved in the physiologic response to feeding and its development over time.

\section{7}

First Database of Arterial Function (Aix, PWVao, SBPao) from healthy children and adolescents

Erzsébet Hidvégi, Dr. Jakab Ltd., Outpatient Dept. of Paediatric

Cardiology, Szolnok, Hungary

János Huberth, 5th GP District, Tapolca, Hungary

Györgyi Jákli, 3rd GP District, Dabas, Hungary

Hajnalka Klemencz, 4th GP District, Dabas, Hungary

Miklós Illyés, Heart Institute, Faculty of Medicine, Unversity of Pécs, Hungary

Introduction: The clinical value of non-invasive measurement of augmentation index (Aix), aortic pulse wave velocity (PWVao) and central systolic blood pressure (SBPao) to assess arterial function have been proven in several pathological conditions. However we missed database of these parameters from healthy chidren and adolescents.

Aim: To assess the physiological changes of Aix, PWVao and SBPao in healthy subjects aged 3-18 years.

Methods: We used a new oscillometric method (Arteriograph, TensioMed Ltd., Hungary), which is simple (upper arm cuff), fast (2-3 minutes) and user independent. Data were collected from healthy subjects with normal peripherial BP and BMI ( 889 boys, 1042 girls).

Results: Aix decreased with growing-up age from $-20.7 \%$ to $-65.5 \%$ in boys $(\mathrm{R}=0.57)$, and from $-17.7 \%$ to $-57.7 \%$ in girls $(\mathrm{R}=0.43)$. PWVao increased from $5.6 \mathrm{~m} / \mathrm{s}$ to $6.8 \mathrm{~m} / \mathrm{s}$ in boys $(\mathrm{R}=0.45)$, and from $5.9 \mathrm{~m} / \mathrm{s}$ to $6.7 \mathrm{~m} / \mathrm{s}$ in girls $(\mathrm{R}=0.37)$. Peripherial SBP increased yearly in both gender, from 101.7 to $128.1 \mathrm{mmHg}$ (boys) and from 103.8 to $119.3 \mathrm{mmHg}$ (girls). However central SBPao only slightly increased in boys (from 104.0 to $110.3 \mathrm{mmHg}$ ), and even decreased in girls (from 108.7 to $105,2 \mathrm{mmHg}$ ).

Conclusions: We set-up the first database of arterial stiffness parameters in healthy population aged 3-18 years using a new oscillometric method (Arteriograph). We described first the relationship between central and peripherial SPB in this population (increasing peripherial SBP and relatively constant central SPBao during the adolescent age). Our fidings may play important role to define the border between normal and abnormal arterial function parameters.

\section{8}

NT-pro-BNP is sensitive to latent chronic heart failure in the patients with single ventricle physiology and Tetralogy of Fallot

Keiichi Hirono, Department of Pediatrics, Faculty of Medicine, University of Toyama, Japan 
Keijirou Ibuki, Department of Pediatrics, Faculty of Medicine, University of Toyama, Japan

Kazuyoshi Saito, Department of Pediatrics, Faculty of Medicine, University of Toyama, Japan

Kazuhiro Watanabe, Department of Pediatrics, Faculty of Medicine, University of Toyama, Japan

Sayaka Watanabe, Department of Pediatrics, Faculty of Medicine, University of Toyama, Japan

Naoki Yoshimura, 1st department of surgery, Faculty of Medicine, University of Toyama, Japan

Fukiko Ichida, Department of Pediatrics, Faculty of Medicine, University of Toyama, Japan

Toshio Miyawaki, Department of Pediatrics, Faculty of Medicine, University of Toyama, Japan

Background: Plasma levels of brain natriuretic peptide (BNP) and $\mathrm{N}$-terminal pro-BNP (NT-pro-BNP) are useful as diagnostic objective markers of chronic heart failure $(\mathrm{CHF})$ due to systolic and diastolic dysfunction in adults.

Objective: The aim of our study was to examine whether plasma levels of NT-pro-BNP correlate with clinical severity of chronic heart failure (CHF) in children with single ventricle (SV) physiology and Tetralogy of Fallot (TOF).

Method: Eighty-four patients, 50 males and 34 females, aged 1 to 26 years (median 5 year) were enrolled. Patients were divided two groups; 46 patients with SV physiology and 38 patients with TOF. We examined NT-pro-BNP, BNP, ejection fraction and severity of heart failure in the two groups, comparing with healthy controls.

Results: Plasma levels of NT-pro-BNP were $874 \pm 1306 \mathrm{pg} / \mathrm{m}$ in SV group and $581 \pm 721 \mathrm{pg} / \mathrm{ml}$ in TOF group, and which in both groups were significantly higher than healthy controls $(61.9 \pm 32.1 \mathrm{pg} / \mathrm{ml})$. The plasma levels of BNP were significantly higher in groups of SV group $(105.8 \pm 157.0 \mathrm{pg} / \mathrm{ml})$ and TOF group $(73.5 \pm 59.5 \mathrm{pg} / \mathrm{ml})$ than healthy controls $(12.8 \pm 4.2 \mathrm{pg} /$ $\mathrm{ml})$, not strong correlation than NT-pro-BNP. NT-pro-BNP had better correlations with ejection fraction and the severity of heart failure in each group.

Conclusions: Our study showed that the serum levels of NT-proBNP were more sensitive to detect heart failure than the plasma levels of BNP in children, and NT-pro-BNP is a good biomarker to monitor the $\mathrm{CHF}$ in the young patients with SV physiology and TOF.

\section{0}

Management of transposition of the great arteries in southern France: impact of antenatally diagnosis Nicolas Hugues, Children's hospital La Timone, Marseille, France Pascal Amedro, Arnaud de Villeneuve Hospital, Montpellier, France Jean-René Lusson, Gabriel Montpied hospital, Clermont-Ferrand, France

Philippe Acar, Children's hospital, Toulouse, France

François Bourlon, Cardio-Thoracic center, Monaco, Monaco

Francis Rouault, St-Joseph hospital, Marseille, France

François Heitz, Clinique Pasteur, Toulouse, France

Alain Fraisse, Children's hospital La Timone, Marseille, France

Introduction: Few multicentric data are available regarding the management of transpositions of the great arteries (TGA). We report a multi-centric experience in southern France.

Materials and Methods: From June 2002 to June 2007, 154 neonates presented with TGA to 8 centres.Among them, 66(42,8\%) were diagnosed prenatally (Group A) and 57,2\% post-natally (Group B).Group B was only studied for mortality statistics.
Results: An amniocentesis was performed in 37(56\%) cases with one premature delivery. Standard karyotypic analysis was normal in all the cases. 53 patients (80\%) were delivered in a level III maternity and the rest in a level II. An on-site surgeon was available in $25(37,8 \%)$ cases. A balloon atrial septostomy (BAS) was performed within 24 hours postnatally in 59(94\%) patients. There were $2(3 \%)$ deaths prior to surgery. The surgical repair through the arterial switch operation was performed at a median age of $11(5-40)$ days. There were 6 postoperative deaths $(9.4 \%)$ due to myocardial ischemia in 4 patients ( 2 with intramural coronary arteries). Factors influencing post-operative survival were: The degree of technical difficulty in re-implanting the coronary artery $(\mathrm{p}=0.006)$, Post-oprative ECG anomalies $(\mathrm{p}<0.0001)$, Time on extra-corporeal circulation ( $\mathrm{p}-0.0004)$, Aortal clamping time $(\mathrm{p}=0.02)$. Factors influencing reanimation time included:Prematurity $(\mathrm{p}=0.007)$, Extra-cardiac anomalies $(p=0.036)$, Place of delivery $(p=0.023)$ and Post-operative ECG anomalies $(p=0.005)$. The incidence of TGA did not appear to be affected by seasonal variations. When comparing group A to group B, there was no statistically significant difference in pre- or global operative mortality.

Conclusion: TGA remains undiagnosed prenatally in the majority of the cases. Intramural coronary anatomy seems to remain the main risk factor for surgical death.

\section{1}

Cardiovascular malformations in Down Syndrome: spectrum, management and survival over 22 years

Claire A Irving, Freeman Hospital, Newcastle upon Tyne, United Kingdom

Valerie Sutherland, Freeman Hospital, Newcastle upon Tyne, United Kingdom

Milind P Chaudhari, Freeman Hospital, Newcastle upon Tyne, United Kingdom

Objectives: The incidence of cardiovascular malformations in Down syndrome (DS) is well described however there remains little data on spectrum, management and outcome. We aimed to describe this in a defined population over 22 years.

Methods: Data were obtained on all live-births and live-births with DS from 1985-2006 in the Northern region of the UK (population 3 million) with all congenital heart disease referred to a single centre.

Results: In 1985-2006 there were 754,486 live-births. 821 were live-born with DS (1.08 per 1000 with no change in live birth prevalence over time). 343(42\%) had a cardiovascular malformation (Figure 1). One patient had pulmonary atresia/intact ventricular septum and one had an unbalanced complete atrioventricular septal defect requiring completion of Fontan circulation. $21 \%$ had two or more abnormalities, the commonest additional being a persistent ductus arteriosus.

In 1985-1995, 101/163(62\%) had surgery of which 30 died (30\% operated on in this time), 14 within 30 days post-operatively. $17(5 \%)$ patients in the total group developed pulmonary hypertension. In 1996-2006, 129/180(72\%) had surgery of which 7 died ( $5 \%$ operated on during this time), 2 within 30 days. There was 1 Fontan completion and 2 cardiac transplants. 1 year survival in DS with cardiac diagnosis improved from $78 \%$ in 1985-1995 to 91\% in 1996-2006 (Figure 2).

Conclusions: Although the spectrum of cardiovascular malformations in DS has remained unchanged there has been a reduction in post-operative mortality. Other treatment modalities such as single ventricle palliation and transplantation are offered. Long term survival has improved over time. 


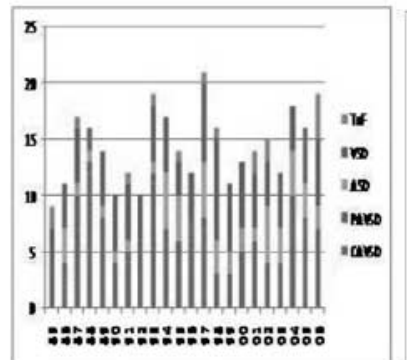

Figure 1: Total numbers of DS patients with cardiac diagnoses by year of birth

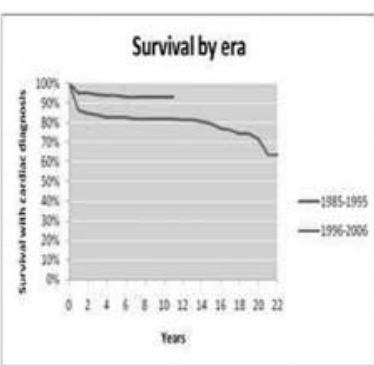

Figure 2: Long term survival

\section{2}

Mortality in infants with cardiovascular malformations Claire A Irving, Freeman Hospital, Newcastle upon Tyne, United Kingdom Christopher Wren, Freeman Hospital, Newcastle upon Tyne, United Kingdom

A Hasan, Freeman Hospital, Newcastle upon Tyne, United Kingdom JRL Hamilton, Freeman Hospital, Newcastle upon Tyne, United Kingdom J H Smith, Freeman Hospital, Newcastle upon Tyne, United Kingdom $S$ R Haynes, Freeman Hospital, Newcastle upon Tyne, United Kingdom M P Chaudhari, Freeman Hospital, Newcastle upon Tyne, United Kingdom JJ O'Sullivan, Freeman Hospital, Newcastle upon Tyne, United Kingdom

Objectives: Mortality in infants with cardiovascular malformations (CVMs) may be due to cardiac defects, their treatment or noncardiac causes. We investigated causes of death in infants with CVMs, and contribution of "surgical" mortality to total infant mortality over 20 years.

Methods: We obtained details of all deaths of infants with CVMs in the Northern Region of England. Two independent panels reviewed anonymised data and categorised the cause of death as non-cardiac, non-surgical or surgical.

Results: In 1987-2006 there were 676,927 live-births in the population. Total infant mortality fell from 8.1 to 4.9 per 1000 . CVMs were found in 4652 infants (6.9 per 1000) and 464(10\%) died before age 12 months. Of these 103(22\%) had a chromosomal abnormality and $78(17 \%)$ had a major non-cardiac malformation. Infant mortality with a CVM decreased from $23 \%$ in 1987 to $5 \%$ in 2006.

Of 464 deaths, 152(32\%) were non-cardiac, 148(32\%) nonsurgical, 37(8\%) intra-operative, 106(23\%) post-operative, and $21(5 \%)$ late and sudden. Median age at death was 27 days (0-390). Death was non-cardiac in $60(58 \%)$ infants with a chromosomal abnormality, 42(54\%) with a non-cardiac malformation and $52(18 \%)$ with isolated CVM.

$225 / 464(49 \%)$ who died underwent intervention. "Surgical" mortality was 10/75(13\%) operations in 1989 compared with 3/ $172(2 \%)$ in 2006 . The contribution of "surgical" mortality to total mortality fell from $10 / 32(31 \%)$ to $3 / 14(21 \%)$ in that time. Conclusions: Mortality in live born infants with CVMs has declined over 20 years. The majority of deaths in infants with a chromosomal or major non-cardiac malformation are noncardiac in origin. Surgical mortality declined markedly.

\section{3}

Effects of Carvedilol for Heart Failure in Patients with Functionally Univentricular Heart

Naoko Ishibashi, Department of Pediatrics and Cardiovascular Surgery Sakakibara Heart Institute, Japan

In-Sam Park, Department of Pediatrics and Cardiovascular Surgery Sakakibara Heart Institute, Japan
Tadashi Waragai, Department of Pediatrics and Cardiovascular Surgery Sakakibara Heart Institute, Japan

Tadahiro Yoshikawa, Department of Pediatrics and Cardiovascular Surgery Sakakibara Heart Institute, Japan

Mitsunori Nishiyama, Department of Pediatrics and Cardiovascular Surgery Sakakibara Heart Institute, Japan

Yasuo Murakami, Department of Pediatrics and Cardiovascular Surgery Sakakibara Heart Institute, Japan

Makoto Ando, Department of Pediatrics and Cardiovascular Surgery Sakakibara Heart Institute, Japan

Yukihiro Takahashi, Department of Pediatrics and Cardiovascular Surgery Sakakibara Heart Institute, Japan

Objective: Our purpose was to evaluate the clinical effects of carvedilol for heart failure in patients with functionally univentricular heart.

Methods: Between 2002 and 2008, we used carvedilol in treating patients with functionally univentricular heart exhibiting heart failure $(n=51)$. We classified them into three groups according to the status of each patient including status post Fontan operation(F), status post bidirectional Glenn $(\mathrm{G})$, and those undergoing neither Glenn nor Fontan (NF). We compared clinical parameters before and after implementing carvedilol therapy. The parameters included cardiothoratic ratio (CTR), ventricular end-diastolic pressure (EDP), central venous pressure (CVP), ventricular ejection fractio$\mathrm{n}(\mathrm{EF})$,serum brain natriuretic peptide level (BNP), and dosages of diuretics. We also investigated the New York Heart Association (NYHA) classification, degree of artrioventricular valvular regurgitation (AVVR), and presence of arrhythmia.

Results: Carvedilol therapy was started at the mean age of $10.1 \mathrm{y}$ (range, $1 \mathrm{~m}$ to $34.8 \mathrm{y}$ ). The initial dose was 0.04 (range, 0.01 to $0.18) \mathrm{mg} / \mathrm{kg} /$ day and the maximum dose was 0.42 (range, 0.01 to $0.92) \mathrm{mg} / \mathrm{kg} /$ day. The main reason for starting carvedilol was heart failure associated with ventricular contractile dysfunction $(\mathrm{n}=35)$, pleural effusion $(n=11)$, ascites $(n=1)$, arrhythmia $(n=10)$, AVVR ( $n=11)$, protein-losing enteropathy $(n=1)$ and frequent hospitalization $(\mathrm{n}=3)$. The patients were on diuretics (furosemide, hydrochlorothiazide or spironolactone; $n=43)$, enalapril $(n=34)$, pimopendan $(n=9)$, digoxin $(n=19)$ and losartan potassium $(n=1)$. The CTR improved significantly from 59 to $46 \%$ $(p<0.01)$, and the dosage of diuretic reduced significantly $(\mathrm{p}<0.01)$. The EF also significant improved in all patients (from 35 to $40 \% ; p<0.05$ ). This improvement was especially prominent in the Fontan group (from 35 to $45 \%$; $<<0.05$ ). Clinical signs,symptoms and NYHA classes were also improved.

Conclusions: We think that carvedilol might play an important role in treating heart failure associated with functionally univentricular heart.

\section{4}

Angiotensin II blockade diminished plasma BNP levels in surgically corrected tetralogy of Fallot patients with moderate-to-severe pulmonary regurgitation

Yuichi Ishikawa, Pediatric cardiology division, Fukuoka Children's Hospital, Japan

Shirou Ishikawa, Pediatric cardiology division, Fukuoka Children's Hospital, Japan

Kouichi Sagawa, Pediatric cardiology division, Fukuoka Children's Hospital, Japan

Hiroya Ushinohama, Pediatric cardiology division, Fukuoka Children's Hospital, Japan

Makoto Nakamura, Pediatric cardiology division, Fukuoka Children's Hospital, Japan

Naoki Fusazaki, Neonatal cardiology division, Fukuoka Children's Hospital, Japan 
Objective: In patients (pts) with tetralogy of Fallot (TOF) after surgical correction, pulmonary regurgitation (PR) is one of major risk factors of the poor prognosis. Up to now, there is no effective treatment for the $\mathrm{PR}$ expect pulmonary valve replacement (PVR). The aim of the study is to evaluate the effects of an angiotensin II receptor blocker (ARB) olmesartan on the postoperative PR in pts with TOF.

Methods: Forty-three pts of TOF and others after surgery with moderate-to-severe PR (12.2 \pm 6.8 years; $9.5 \pm 5.7$ years after surgery; New York Heart Association functional class I) were enrolled. Forty-one pts were orally given olmesartan $(0.2 \mathrm{mg} / \mathrm{kg}$, orally, once daily) and 38 had tolerated for 6 months and more. We examined alterations on the systolic and diastolic blood pressures (SBP and DBP, respectively), the cardio thoracic ratio (CTR) of a chest X-ray film, and plasma natriuretic peptide type $\mathrm{A}$ and type B levels (ANP and BNP, respectively) before and after the treatment of olmesartan.

Results: Olmesartan decreased the SBP significantly $(97 \pm 16$ to $87 \pm 17 \mathrm{mmHg})$, and also showed a tendency of the decrease in DBP $(61 \pm 8$ to $56 \pm 12 \mathrm{mmHg}, \mathrm{p}=0.11)$. Furthermore, olmesartan decreased the CTR $(57 \pm 4$ to $56 \pm 4 \%$ ) and the plasma BNP levels $(59 \pm 52$ to $41 \pm 23 \mathrm{pg} / \mathrm{mL}$ ) significantly. It also showed a tendency of the decrease in plasma ANP levels $(57 \pm 42$ to $48 \pm 27 \mathrm{pg} / \mathrm{mL}, \mathrm{p}=0.17$ ).

Conclusions: The present small cohort study suggested that olmesartan might attenuate the development of cardiac remodeling by PR to postpone the PVR in pts with TOF after surgery.

\section{5}

Intermediate-term follow-up after end-to-end operation for coarctationof the aorta - one centre experience Dorota Jagiellowicz, Poland

Grazyna Brzezinska-Rajszys, Heart Catheterization Laboratory, The Children's Memorial Health Institute, Warsaw, Poland, Poland Wanda Kawalec, Cardiology Clinic, The Children's Memorial Health Institute, Warsaw, Poland, Poland

Mieczyslaw Litwin, Nephrology Clinic, The Children's Memorial Health Institute, Warsaw, Poland, Poland

Anna Niemirska, Nephrology Clinic, The Children's Memorial Health Institute, Warsaw, Poland, Poland

Introduction: The aim of the study was to evaluate the 6-12 yrs follow-up after end-to-end anastomosis in children with coarctation of the aorta.

\begin{tabular}{|c|c|c|c|c|}
\hline & & Group I & Group II & p-value \\
\hline \multirow{2}{*}{$\begin{array}{l}\text { Average systolic } \\
\text { pressure }(\mathrm{mmHg})\end{array}$} & day-time & $118 \pm 9$ & $120 \pm 10$ & 0.610 \\
\hline & night-time & $102 \pm 9$ & $105 \pm 11$ & 0.415 \\
\hline \multicolumn{2}{|l|}{ 24-hours pressure laod } & $30.80 \pm 26.94$ & $27.40 \pm 21.56$ & 0.882 \\
\hline \multicolumn{2}{|l|}{ Arterial hypertension (pts) } & $5(33 \%)$ & $9(26 \%)$ & \\
\hline \multicolumn{2}{|l|}{ LV mass (gram) } & $62.42 \pm 12.35$ & $111.21 \pm 58.24$ & 0.003 \\
\hline \multicolumn{2}{|l|}{ LVMI } & $70.25 \pm 15.10$ & $83.59 \pm 27.81$ & 0.036 \\
\hline \multirow[t]{3}{*}{ IVS } & $S^{\prime}$ & $7.63 \pm 1.03$ & $7.85 \pm 1.64$ & 0.871 \\
\hline & $\mathrm{E}^{\prime}$ & $-13.11 \pm 1.86$ & $-12.74 \pm 2.77$ & 0.307 \\
\hline & $A^{\prime}$ & $-4.87 \pm 3.69$ & $-6.03 \pm 1.64$ & 0.451 \\
\hline \multirow[t]{3}{*}{ LWLV } & $S^{\prime}$ & $8.29 \pm 2.33$ & $8.76 \pm 2.36$ & 0.529 \\
\hline & $\mathrm{E}^{\prime}$ & $-16.27 \pm 3.04$ & $-16.58 \pm 4.31$ & 0.775 \\
\hline & $A^{\prime}$ & $-5.41 \pm 1.48$ & $-5.99 \pm 1.86$ & 0.253 \\
\hline \multirow[t]{3}{*}{ PWLV long axis } & $\mathrm{S}^{\prime}$ & $7.42 \pm 7.84$ & $6.74 \pm 1.55$ & 0.454 \\
\hline & $\mathrm{E}^{\prime}$ & $-11.33 \pm 2.80$ & $-11.68 \pm 3.03$ & 0.707 \\
\hline & $A^{\prime}$ & $-4.05 \pm 1.49$ & $-4.23 \pm 1.49$ & 0.698 \\
\hline \multirow[t]{4}{*}{ IMT } & RCA & $0.449 \pm 0.04$ & $0.421 \pm 0.053$ & 0.075 \\
\hline & LCA & $0.407 \pm 0.066$ & $0.436 \pm 0.051$ & 0.476 \\
\hline & RFA & $0.273 \pm 0.039$ & $0.286 \pm 0.052$ & 0.397 \\
\hline & LFA & $0.295 \pm 0.026$ & $0.293 \pm 0.036$ & 0.866 \\
\hline
\end{tabular}

Material/methods: In 50pts operated between 1995-2000 ABPM, IMT measurements and echocardiography (TDI) were performed. Patients were divided into two groups. Group I-15pts operated below $3 \mathrm{mths}$ of age $(1.2 \pm 0.766)$, the current age $6.4-11.2 \mathrm{yrs}$ (mean 7.9yrs), the follow-up $7.8 \pm 1.28 \mathrm{yrs}$. Group II-35pts operated after $3 \mathrm{mth}$ of age $(30.1 \pm 29.6)$, the current age 6.65-18yrs (mean 11.9yrs), the follow-up $9.3 \pm 2$ yrs.

Results: No significant differences were found with respect to parameters in ABPM, left ventricle function and IMT between two groups. Age at surgery influence on index mass.

Conclusion: There is a substantial incidence of arterial hypertension in mid-term follow-up in patients after end-to-end operation.

Age of children operated for coarctation of the aorta by end-toend anastomosis has no significant influence on the IMT and left ventricle function.

\section{6}

Prediction of intravenous immunoglobulin unresponsiveness in patients with Kawasaki disease by a new vasculitis biomarker, pentraxin 3

Yasuhiro Katsube, Department of Pediatrics, Nippon Medical School, Japan

Masanori Abe, Department of Pediatrics, Nippon Medical School, Japan Mitsuhiro Kamisago, Department of Pediatrics, Nippon Medical School, Japan

Ryuji Fukazawa, Department of Pediatrics, Nippon Medical School, Japan

Scunichi Ogawa, Department of Pediatrics, Nippon Medical School, Japan

Background: It is important to predict the degree of vasculitis in order to prevent coronary artery lesions (CAL) in Kawasaki disease. Several reports have attempted to predict the occurrence of coronary artery lesions based on biochemical data. At present, however, there are no clear criteria. Pentraxin 3 (PTX3) is the prototypic member of the long pentraxin family synthesized at inflammatory sites such as small vessel vasculitis. Purpose: 1) To investigate levels and dynamics of PTX3 in patients with Kawasaki disease. 2) To attempt to predict the intravenous immunoglobulin unresponsiveness and the occurrence of CAL by PTX3.

Methods: Thirteen patients with Kawasaki disease were enrolled. As a treatment for Kawasaki disease, high dose $\boldsymbol{\gamma}$-globulin $(2 \mathrm{~g} / \mathrm{kg})$ was administered. Blood samples were collected before and at 2 days after $\gamma$-globulin therapy and at one month after onset.

Results: The values (all cases) for PTX3 were 29.0 \pm 6.4, 13.8 \pm 2.2 , and $4.9 \pm 0.6 \mathrm{ng} / \mathrm{ml}$, respectively at the above-mentioned time points. The mean values of responder $(\mathrm{n}=11)$ and non-responder $(\mathrm{n}=2)$ group to $\gamma$-globulin were 20.5 and $63.0 \mathrm{ng} / \mathrm{ml}$. The mean value in age-matched controls was $3.2 \mathrm{ng} / \mathrm{ml}$.

Conclusions: PTX3 levels are very high, particularly in nonresponder group in the acute phase of Kawasaki disease, and they decline rapidly with $\gamma$-globulin therapy. Even in the recovery phase of Kawasaki disease (one month after onset), PTX3 levels remain high when compared with controls, thus suggesting that silent inflammation in vessels continues.

\section{9}

\section{QT Dispersion in Childhood Obstructive Sleep Apnea Syndrome}

Anant Khositseth, Faculty of Medicine Ramathibodi Hospial, Mahidol University, Thailand

Palinee Nantarakchaikul, Faculty of Medicine Ramathibodi Hospial, Mahidol University, Thailand 
Teeradej Kuptanon, Faculty of Medicine Ramathibodi Hospial, Mahidol University, Thailand

Aroonwan Preutthipan, Faculty of Medicine Ramathibodi Hospial, Mahidol University, Thailand

The difference between maximal and minimal QT interval and corrected QT (QTc) defined as QT dispersion (QTd) and QTc dispersion (QTcd) may reflect inhomogeneity of repolarization and myocardial electrical instability. Many studies in demonstrated that QTd was significantly higher in obstructive sleep apnea syndrome (OSAS). However, there was no study in childhood OSAS.

Objective: This study was to evaluate QTd and QTcd in childhood OSAS.

Patients \& Methods: Forty-four children with OSAS (34 males), mean $+/-$ SD of $6.18+/-3.49$ yrs along with 38 age-, sexmatched healthy children (25 males), $6.62+/-2.11$ yrs underwent 12-lead ECG. The QT and RR intervals were blinded measured by two independent observers.

Results: Means QTd and QTcd were significantly higher in OSAS than controls, $51.59+/-27.18$ vs $39.73+/-14.42 \mathrm{~ms}$, $(\mathrm{p}=0.014)$, and $71.16+/-28.70$ vs $57.23+/-18.86 \mathrm{~ms}$, $(\mathrm{p}=0.010)$, respectively. Interestingly, QTd and QTcd in OSAS with obesity $(57.04+29.97$ and $73.18+/-30.78 \mathrm{~ms})$ were significantly higher than in control $(39.74+/-14.42 \mathrm{~ms}$ and $57.23+/-18.86 \mathrm{~ms}), \mathrm{p}=0.009$ and 0.043 , respectively. However, QTd and QTcd in OSAS without obesity $(42.94+$ / -19.92 and $67.94+/-52.62 \mathrm{~ms}$ ) were not significantly different. Moreover, there was a correlation between QTd and body mass index (BMI) $(r=0.437, \mathrm{p}<0.001)$.

Conclusions: QTd and QTcd were significantly increased in childhood OSAS. Obesity may be the factor affected the increased QTd and QTcd in childhood OSAS.

\section{0}

Influence of the Fontan modification on systemic venous and pulmonary arterial flow pattern according to the respiratory cycle - assessment by intravascular Doppler echocardiography

Yuria Kim, National Health Insurance Corporation Ilsan Hospital, Korea

Jae Young Choi, Severance Cardiovascular Hospital, Yonsei University Health System, Korea

Nam Kyun Kim, Severance Cardiovascular Hospital, Yonsei University Health System, Korea

Byung Won Yoo, Severance Cardiovascular Hospital, Yonsei University Health System, Korea

Jun Hee Sul, Severance Cardiovascular Hospital, Yonsei University Health System, Korea

Objective: To assess the effect of Fontan modification on systemic venous and pulmonary artery flow and on respiratory dependance of flow.

Method: We performed intravascular Doppler echocardiography in the systemic veins and pulmonary artery in 9 patients with atriopulmonary connection (APC group), 12 patients with total cavopulmonary connection (TCPC) by lateral tunnel (LT group), and 13 patients with TCPC by extracardiac conduit (ECC group). We obtained intravascular Doppler spectrals under simultaneous respirometer recording and compared flow characteristics in each group, according to the cardiac and respiratory cycles.

Result: In APC group, velocity-time integrals (VTI) at systole (VTIS) of caval and hepatic veins were greater than VTI at diastole (VTID) in contrast to LT and ECC groups. VTI of retrograde flow (VTIR) of caval and hepatic veins were significantly greater in APC group than the other two groups. Net antegrade flow integrals (NAFIs) of hepatic vein and pulmonary artery were significantly greater in LT and ECC groups. The respiratory variation index (RVI) of NAFI at superior vena cava and RVI of VTID at pulmonary artery were significantly greater in ECC group than the other two groups. Conclusion: In LT and ECC groups, the retrograde flows in systemic veins are decreased and the antegrade flows in hepatic vein and pulmonary artery are increased. In ECC group, the flows of superior vena cava and pulmonary artery are more dependent on the respiration than the other two groups. These physiologic differences should be taken into account in respiratory care for patients after Fontan-type repair.

\section{1}

Native Infective Endocarditis in 111 Children: Predictors of morbidity and mortality

Maria V Lafuente, Hospital de Pediatría J P garrahan, Argentina

Claudia N Villalba, Argentina

María G Rossi, Hospital de Pediatría J P garrahan, Argentina

Horacio A Capelli, Hospital de Pediatría J P garrahan, Argentina

Objectives: To determinate predictors of morbidity and mortality in children with native infective endocarditis (EI).

Methods: 111 patients (pts) with definitive IE according to the Duke criteria were evaluated.

Two groups of pts were identified according to age: younger than $(<) 1$ year (21pts) and older than $(>) 1$ year (90pts).

Results: The most common microorganisms in both groups were: staphylococcus aureus. In pts $<1$ year: candida albicans ( $p$ 0.0000003), enterococcus ( $p 0.003$ ) meanwhile in pts $>1$ year the second most frequent microorganism was streptococcus viridans ( $p$ 0.006).

A congenital heart defect was found in 64 pts and no heart lesion was documented in 47.

The localization of the vegetations were predominantly in the right heart in pts $<1$ year and in the left heart in the older group (p 0.0000001)

Adverse events were diagnosed in $70 \%$. In pts $<1$ year: uncontrolled infection ( $\mathrm{p}$ 0.001) related to candida albicans (p 0.001). In the older group: progressive heart failure ( $p$ 0.003) and systemic embolizations ( $\mathrm{p}$ 0.008).

Overall mortality was $9 \%$. It was greater in the younger group $19 \%$ than in the older $6 \%$ ( $\mathrm{p} 0.07$ ). Eight pts had no previous heart lesion ( $p$ 0.001), and the microorganism found was a staphylococcus aureus (p 0.02)

Conclusions: Predictors of mortality were:

- Abscence of previous cardiac lesion

- Presence of a staphylococcus aureus.

In pts $<1$ year morbidity was related to uncontrolled infection and to the presence of candida albicans. In the older group morbidity was related to progressive heart failure, systemic embolizations and the presence of vegetations in the left heart.

\section{2}

The Incidence of the 22q.11.2 Deletion Syndrome in children in Cape Town, South Africa

John Lawrenson, Western Cape Paediatric Cardiac Services, South Africa

Rik De Decker, Western Cape Paediatric Cardiac Services, South Africa Liesl Zuhlke, Western Cape Paediatric Cardiac Services, South Africa 
Zandre De Decker, Division of Human Genetics, Faculty of Health Sciences, University of Cape Town, South Africa Mardelle Schoeman, 2Division of Human Genetics, Faculty of Health Sciences, University of Cape Town, South Africa

Glynis Schutte, 3National Health Laboratory Service, Groote Schuur Hospital, Cape Town, South Africa

Previous estimates of the prevalence of the 22q11.2 deletion syndrome in a paediatric cardiac clinic population were based on a retrospective review at our institution. This prevalence had increased in 2006 to a peak of $1.8 \%$ of the new patients seen at this centre. However, in the face of marked phenotypic variability, concern existed that the diagnosis may be missed in many patients. Aims:

1. To determine the incidence of the 22q11.2 deletion syndrome in a cohort of patients with congenital heart disease presenting to a tertiary cardiology referral centre.

2. To assess an international scoring system for clinical recognition of the 22q11.2 deletion syndrome in cardiac patients.

Methods: All new patients with a significant congenital cardiac lesion were assessed for recruitment to the study. Once consent was obtained, all children were tested for the 22q11.2 deletion by the standard TUPLE1 fluorescent-in-situ-hybridisation (FISH) probe. All children were assigned a clinical "O score" at presentation (Oskarsdöttir 2005)

Results: To date 88 patients have been recruited and FISH tested; 69 (78.4\%) FISH tests have been reported, and $4(5.8 \%)$ are deleted. The mean $\mathrm{O}$ score of these 4 positive patients on presentation was 2.5.A cohort of seven patients was identified with clear clinical features of 22q deletion syndrome but FISH-negative.

Conclusions: Preliminary results of this study suggest that the $22 \mathrm{q} 11.2$ deletion is almost three times more than anticipated. The international clinical scoring system used to assess the need for FISH testing was not to apply to our patient population.

7123

Correlation between Six- Minute Walk Test and Exercise Stress Test in Healthy Children

Alisa Limsuwan, Division of Pediatric Cardiology, Ramathibodi

Hospital, Mahidol University, Thailand

Ratthapon Wongwandee, ivision of Pediatric Cardiology, Ramathibodi Hospital, Mahidol University, Thailand

Pongsak Khowsathit, ivision of Pediatric Cardiology, Ramathibodi

Hospital, Mahidol University, Thailand

Background: Cardiovascular fitness is nowadays considered one of the most important health markers. Despite a well established maximum incremental cardiopulmonary exercise test, six minute walk test (SMWT) has been proposed as an indicator of functional capacity. We investigate the correlate between SMWT and incremental treadmill exercise stress test. We also provide a contemporary set of results of the SMWT in our cohort of healthy children in comparison with the standard reference previously published for children.

Methods: Healthy children aged 9 to 12 years were included in this study. The anthropometric data, SMWT distance and exercise stress test were prospectively measured using the standard protocols. Various parameters were analyzed to define the correlation between SMWT and stress exercise test.

Results: A total of 100 subjects (53 boys) were recruited and the SMWT distance was $586.1 \pm 44.0 \mathrm{~m}$. The maximal oxygen consumption obtained during the exercise stress test tends to be greater in boys than girls $(47.5 \pm 10.2 \mathrm{ml} / \mathrm{kg} / \mathrm{min}$ VS $45.8 \pm 9.7 \mathrm{ml} / \mathrm{kg} / \mathrm{min}$ ) nevertheless there was no statistically significant. Among parameters obtained during exercise stress test, maximal heart rate and the maximal oxygen consumption were found to have significant correlation with SMWT distance. Conclusions: SMWT distance is significantly correlated with the oxygen consumption obtained during the treadmill exercise stress test. This indicates that SMWT is also one of the predictive markers for exercise stress test performance.

\section{4}

\section{Long-term Survival in Children born 1973 - 1997 with} Complex AVSD in Sweden

Bo Lundell, Pediatric Cardiology, Dept. of Woman and Child Health, Karolinska University Hospital, Stockholm, Sweden

Christina Frid, Pediatrics, Dept. of Woman and Child Health, Karolinska University Hospital, Stockholm, Sweden

Anders Jonzon, Pediatric Cardiology, Dept. of Women's and Children's Health, Uppsala University Hospital, Uppsala, Sweden

Gudrun Bjorkhem, Pediatric Cardiology, Dept. of Pediatrics,

Lund University Hospital, Lund, Sweden

Jan Sunnegardh, Pediatric Cardiology, Sahlgrenska University Hospital, Gothenburg, Sweden

Goran Annerén, Dept. of Genetics and Pathology, Uppsala University Hospital, Uppsala, Sweden

Complex atrioventricular septal defect (complexAVSD) with outflow obstructions, heterotaxia, unbalanced ventricular volumes and systemic and pulmonary venous abnormalities remains a challenge with complex staged surgical procedures.

Objectives: To study long-term survival in complexAVSD, in a national Swedish cohort.

Methods: National birth-, malformation- and death registers were searched for the AVSD diagnosis. A retrospective study of hospital charts and databases for the AVSD diagnosis was made at all centers for pediatric cardiology and heart surgery in Sweden. ComplexAVSD was defined as any combination of AVSD (with a common atrioventricular valve) with outflow obstructions to the pulmonary or systemic circulations or unbalanced ventricular volumes. Age at death, age at surgery, type of palliative or corrective procedure and other cardiac and extracardiac malformations were also recorded. All infants born alive with complexAVSD from 1973 to 1997 (25 years) were included and recorded as dead or alive on May 28, 2008.

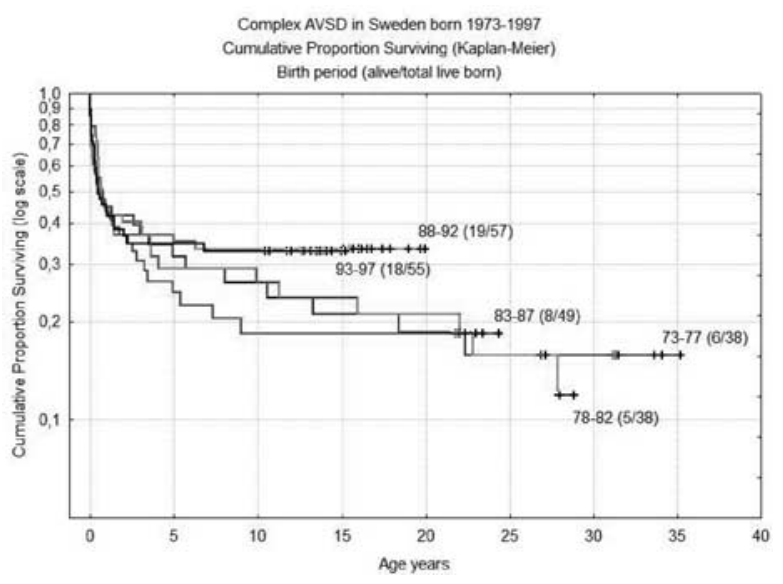

Results: 237 infants with complexAVSD were born alive 1973-1997 in Sweden. Down syndrome was diagnosed in $84(35 \%)$ and heterotaxia in $71(30 \%)$. Surgery was performed in 
$139 / 237(59 \%)$ and age at first operation decreased significantly from $4,9 \pm 4,0$ to $0,5 \pm 0,8$ years during the study period $(\mathrm{p}<0,01) .17$ were palliated with TCPC. Corrective surgery was completed in 69 of 139 operated. Only 56/237 subjects were alive at end of study with a median follow-up time of $16,6(10,4-35,2)$ years. Total mortality decreased from $85 \%$ to $67 \%(p<0.01)$ Conclusion: Mortality has been significantly reduced in complexAVSD but long-term survival is still poor at only $33 \%$.

7127

Correction of imbalanced pulmonary blood flow is effective in plastic bronchitis associated with Fontan operation: A case report

Yoichi Mino, Division of Pediatrics and Perinatology, Tottori University, Japan

Yasuhiro Tsuji, Division of Pediatrics and Perinatology, Tottori

University, Japan

Hiroki Kuranobu, Division of Pediatrics and Perinatology, Tottori

University, Japan

Yuichiro Hashida, Division of Pediatrics and Perinatology, Tottori

University, Japan

Hiroaki Funata, Division of Pediatrics and Perinatology, Tottori

University, Japan

Susumu Kanzaki, Division of Pediatrics and Perinatology, Tottori

University, Japan

Yoshio Aragaki, Department of Pediatrics, Kurashiki Central Hospital, Japan

Syunji Sano, Department of Cardiovascular Surgery, Okayama

University, Japan

Plastic bronchitis is a rare fatal disease characterized by the airway obstruction due to bronchial casts. Though causes of plastic bronchitis remain unclear, some cases of plastic bronchitis have occurred after Fontan operation. We present a patient with plastic bronchitis associated with Fontan operation in whom correction of imbalanced pulmonary blood flow (PBF) is effective.

This patient was a 6-year-old boy with hypoplastic left heart syndrome. He had been treated a modified Norwood operation with RV-PA shunt at 7 days of age; a bidirectional Glenn operation at 6 months; a fenestrated, lateral tunnel Fontan operation at 2 years. At the age of 6 years he was admitted to our hospital because of acute episode of dyspnea. During admission he coughed up a dendritic mucoid bronchial cast. This cast consisted of fibrin and mucin, and contained eosinophils as well as inflammatory cells. He was diagnosed as plastic bronchitis, and was treated with both inhaled and oral corticosteroid (CS). Though CS therapy was effective, plastic bronchitis often recurred shortly whenever CS therapy was reduced. Bronchoscopic examination during dyspnea episode revealed bronchial cast located in right bronchus. By cardiac catheter examination left pulmonary artery stenosis was confirmed, and lung perfusion scintigram showed relative high right PBF. We implanted a stent percutaneously for the left pulmonary artery stenosis, and then high right PBF decreased. After stenting, plastic bronchitis had no recurrence only use of a low dose CS therapy.

We suggest correction of imbalanced PBF may benefit patients in whom other therapies have failed.

7128

Nationwide Prospective Study of a Contemporary Cohort of Patients with Tetralogy of Fallot - Analysis of 407 patients correlating imaging to exercise tolerance and quality of life

Samir Sarikouch, Hannover Medical School, Germany
Karl O Dubowy, Department for Congenital Heart Disease, Heart and Diabetes Centre Bad Oeynhausen, Germany

Petra Boettler, Department for Congenital Heart Disease, University of Freiburg, Germany

Thomas S Mir, Pediatric Cardiology, University Heart Center

Hamburg, Germany

Alfred Hager, Department for Pediatric Cardiology and Congenital Heart Disease, Deutsches Herzzentrum München, Technische Universität München, Germany

Brigitte Peters, Institute for Biometry and Medical Informatics,

University of Magdeburg, Germany

Titus Kuehne, Unit of Cardiovascular Imaging - Congenital Heart

Diseases, German Heart Centre Berlin, Germany

Philipp Beerbaum, Division of Imaging Sciences, King's College, Guy's

\& St Thomas' Hospital, London, United Kingdom

Background: The "Competence Network for Congenital Heart Defects" in Germany initiated a nationwide study in Tetralogy of Fallot (TOF) with emphasis on noninvasive imaging, exercise tolerance and quality of life.

Methods: Prospective, longitudinal study in repaired TOF, age $>8$ yrs including physical examination, ECG, Holter, echocardiography (ECHO), tissue-doppler-imaging (TDI), magnetic resonance imaging (MRI), body plethysmography, cardiopulmonary exercise test and age adjusted quality of life questionnaire. Standardisation for all diagnostic modalities, central core laboratory evaluation of ECHO, TDI, MRI and exercise tests.

Results: 407 patients were included in 14 sites, mean age $17.87 \pm 8.31$ yrs, weight at corrective surgery $11.55 \pm 6.57 \mathrm{~kg}$. Transvalvular patch in 50.9\%. 67.2\% were in NYHA class I, $30.8 \%$ NYHA II and 2\% in NYHA III. $90.9 \%$ were in sinus rhythm, heart rate $73.3 \pm 14.5 \mathrm{bpm}$, max. QRS $145.6 \pm$ $23.6 \mathrm{msec}$. Right ventricular enddiastolic volume $120 \pm 33 \mathrm{ml} /$ $\mathrm{m}^{2}$, right ventricular ejection fraction $50 \pm 9 \%$, mean pulmonary regurgitation $26 \pm 18 \%$. Correlations between MRI and exercise tolerance revealed highly significant correlations, e.g. left ventricular ejection fraction and peak oxygen uptake. Results were related to age matched reference populations (Figure 1peak oxygen uptake for female study members). The quality of life of paediatric patients did not differ in any dimension from the quality of life of a healthy comparison group

Conclusions: A large data base for a contemporary cohort after correction of Tetralogy of Fallot was established for robust statistical analysis and further longitudinal studies.

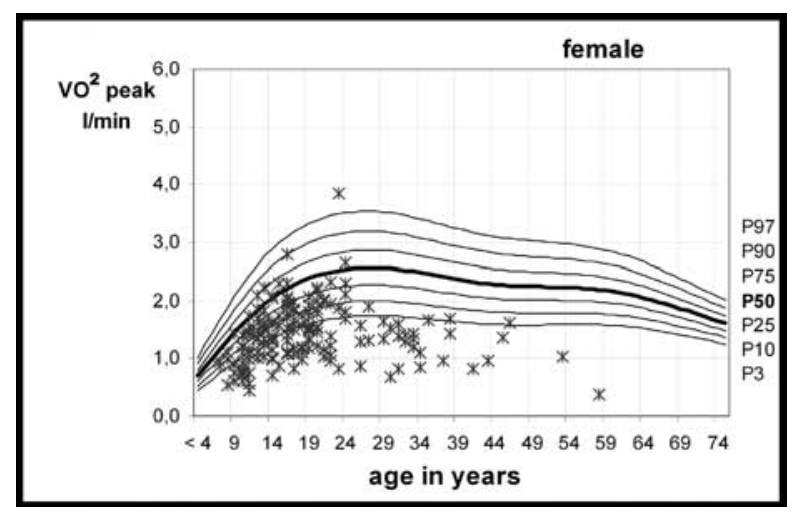

7130

Minimally invasive surgery for congenital heart disease: a gender differentiated approach

Vladimiro L Vida, Pediatric and Congenital Cardiac Surgery Unit, Italy 
Massimo A Padalino, Pediatric and Congenital Cardiac Surgery Unit, University of Padua, Italy

Altin Veshti, Pediatric and Congenital Cardiac Surgery Unit, University of Padua, Italy

Simone Speggiorin, Pediatric and Congenital Cardiac Surgery Unit, University of Padua, Italy

Gianclaudio Falasco, Department of Anesthesia, University of Padua, Italy

Giovanna Boccuzzo, Department of Statistics, University of

Padua, Italy

Giovanni Stellin, Pediatric and Congenital Cardiac Surgery Unit, University of Padua, Italy

Objectives: Since 1996 we have routinely used minimally invasive gender differentiated surgical approaches (GDSA) for surgical repair of various simple congenital heart diseases, mostly including a right anterior mini-thoracotomy in females and a mid-line mini-sternotomy in males.

Methods: Between January 1996 and December 2004, all patients who underwent a GDSA were included. Hospital results were compared to a full sternotomy group (controls). Patient's clinical condition and satisfaction at follow-up were evaluated.

Results: Three-hundred-eight patients had GDSA: 1) minithoracotomy in 147 (47.7\%), 2) mini-sternotomy in 161 (52.3\%). Thirty patients had a "full sternotomy" for atrial septal defect closure. The most common diagnosis was an atrial septal defect (231 patients, $75 \%$ ). None of the patients required an extension of the surgical access. There were neither major complications nor hospital deaths. All patients were discharged home without residual defects. Median follow-up time was 71.5 months (48.2-85.7 months). There were no late deaths. No scoliosis, asymmetric breast development or lactation problems were reported in the mini-thoracotomy group. Twenty-five of 147 patients $(17 \%)$ with mini-thoracotomy complained a trivial persistent $(<6$ months) sensitive skin deficit in the mammary area, most often localized at the infero-medial quadrant. The vast majority of patients $(295 / 308,96 \%)$ were in NYHA class I and $282 / 308$ patients $(91.5 \%)$ were satisfied with the cosmetic result of surgery.

Conclusions: GDSA for simple congenital heart disease is a safe procedure, providing both excellent functional and cosmetic results. Antero-lateral mini-thoracotomy is a valid and highly appreciated procedure in female patients.

7131

Is there an optimal timing for surgical ligation of patent ductus arteriosus in preterm infants?

Vladimiro L Vida, Department of Pediatric and Congenital Cardiac

Surgery, University of Padua, Italy

Paola Lago, Department of Pediatrics, University of Padua, Italy

Sabrina Salvadori, Department of Pediatrics, University of

Padua, Italy

Giovanna Boccuzzo, Department of Statistics, University of

Padua, Italy

Massimo A Padalino, Department of Pediatric and Congenital Cardiac Surgery, University of Padua, Italy

Ornella Milanesi, Department of Pediatrics, University of Padua, Italy Simone Speggiorin, Department of Pediatric and Congenital Cardiac

Surgery, University of Padua, Italy

Giovanni Stellin, Department of Pediatric and Congenital Cardiac

Surgery, University of Padua, Italy

Objective: We sought to define the variables associated with hospital outcome in preterm infants with patent ductus arteriosus
(PDA), and also to identify the optimal timing for PDA closure, in order to improve hospital outcome.

Patients and Methods: From January 2001 to June 2007, all premature babies with PDA who were $\leqslant 32$ weeks of gestational age who received primary medical treatment with ibuprofen, were included. Number of Ibuprofen's cycles, gestational age, body weight and presence of symptomatic hypotension requiring vasoactive/inotropic drugs were related to hospital outcome, including hospital mortality, presence of necrotizing enterocolitis, acute renal failure, intra-ventricular haemorrhage, retinopathy and bronco-pulmonary dysplasia at 36th week. A logistic regression model was used to analyze the data.

Results: There were 201 consecutive patients. Medical treatment was effective in 149 patients (75\%). Fifty-two patients (25\%) required surgical ligation after failed medical treatment; they had lower gestational age $(25 \pm 2.1$ vs $27 \pm 2.3$ weeks, $\mathrm{p}<0.0001)$, lower body weight $(745 \pm 186$ vs $892 \pm 257$ grams, $p=0.0002)$ and higher incidence of symptomatic hypotension $(38 / 52,73 \%$ vs $56 / 149,38 \%, \mathrm{p}<0.0001)$ than patients who respond to ibuprofen. The prolonged medical treatment $(>2$ cycles of Ibuprofen) was significantly associated with an increased risk for bronco-pulmonary dysplasia $(\mathrm{OR}=2.81, \mathrm{p}=0.03)$ and acute renal failure $(\mathrm{OR}=3.81, \mathrm{p}=0.09)$.

Conclusions: The prolonged patency of the ductus arteriosus in preterm infants is related to an increased morbidity. Surgical ligation of PDA is a safe and effective treatment and should be done soon after 2 complete cycles of ibuprofen, especially in selected patients, to improve clinical outcome.

\section{2}

The Scimitar Syndrome: An Italian Multicentric Study Vladimiro L Vida, Department of Pediatric and Congenital Cardiac Surgery, University of Padua, Italy

Simone Speggiorin, Department of Pediatric and Congenital Cardiac Surgery, University of Padua, Italy

Massimo A Padalino, Department of Pediatric and Congenital Cardiac Surgery, University of Padua, Italy

Giovanni Stellin, Department of Pediatric and Congenital Cardiac Surgery, University of Padua, Italy

Background: Scimitar syndrome is a rare congenital heart disease. To evaluate the surgical results of this malformation we have embarked on a multi-centric Italian study involving 7 different Centres.

Methods: From June 1982 to December 2007, 28 patients who had scimitar syndrome and underwent surgery were included. Primary outcome was the efficacy of repair at follow-up.

Results: Median age at surgery was 7 years (IQR 47 days-64 years). Twenty-one patients (75\%) presented with symptoms including upper respiratory tract infections $(n=14)$, recurrent pneumonia $(n=10)$, cardiac failure $(n=6)$, and cyanosis $(n=2)$. Associated cardiac anomalies included: atrial septal defect (ASD) in 18 patients (64\%), dextrocardia in 9 (32\%), right lung hypoplasia in $9(32 \%)$ and aorto-pulmonary collaterals in 5 (18\%). Surgical repair included: intra-atrial baffle repair in 18 patients $(69 \%)$ (group 1) and re-implantation of the "scimitar vein" into left atrium in 8 patients (31\%)(group 2). Two patients (6.4\%) had a right pneumectomy and ASD closure (group 3). One patient died in hospital (3.5\%). Median follow-up time was 4 years (IQR 1.8-9.7 years). There were 2 late deaths $(7.4 \%)$. Four patients $(16 \%)$ presented an occlusion of the scimitar drainage ( 2 in group 1, 12\% and 2 in group 2, 25\%) and three patients $(12 \%)$ required balloon dilation/stenting for stenosis of the scimitar vein (1 in group 1, 6\% and 2 in group 2, 25\%). 
Conclusions: The intra-atrial baffle repair is the most used surgical technique in our population and seems to offer better results in the mid-term.

\section{3}

Rastelli repair: impact of a remote ventricular septal defect on outcome

Claudia N Villalba, Argentina

Marcela I Woloszyn, Hospital de pediatría J.P. Garrahan, Argentina Mariela Mouratian, Hospital de pediatría J.P. Garrahan, Argentina

Jorge Barreta, Hospital de pediatría J.P. Garrahan, Argentina

Horacio Faella, Hospital de pediatría J.P. Garrahan, Argentina

Juan P Laura, Hospital de pediatría J.P. Garrahan, Argentina

Horacio A Capelli, Hospital de pediatría J.P. Garrahan, Argentina

Objective: To evaluate the impact of the anatomic localization of ventricular septal defect (VSD) on the Rastelli procedure.

Methods: 47 patients(pts) after a Rastelli procedure were studied during a follow-up period X:6 years(ys).

The VSD was related to the great arteries in 29, Group I, and it was a remote defect in 18 , Group II.

Results: 22 pts required 27 new surgical procedures after a followup period of $\mathrm{X}: 3$ ys. The right ventricle to pulmonary artery conduit was replaced in 12 pts at X:5 ys.

A VSD had to be closed in 13 pts at X: 1 month. It was a remote septal defect in 11 pts $\mathrm{p} 0.001$.

A subaortic stenosis was relieved in 6 pts at X: 5 years. Four were in group II p 0.31

Overall mortality was $17.2 \%$ (8pts). Seven of them had a remote VSD p 0.003 . Five pts died at X: $5 \mathrm{~m}$ after the inicial operation During the same period 5 pts were referred for a Nikaido procedure. Four had a remote VSD. All survived and none required a redo after 3 years of follow-up.

Conclusions: In the Rastelli procedure, the presence of a remote VSD predisposed to:

- Higher rate of early reoperation p 0.05

- Higher mortality rate p 0.003

- In this complex subset of pts, the Nikaido operation or a univentricular approach may be a better surgical option.

\section{4}

Cardiac surgery for congenital heart disease in premature infants: a good midterm follow up

Audrey Rolland, Centre Chirurgical Marie Lannelongue, France

Bertrand Stos, Centre Chirurgical Marie Lannelongue, France

Virginie Lambert, Centre Chirurgical Marie Lannelongue, France

Andre Capderou, Centre Chirurgical Marie Lannelongue, France

Emre Belli, Centre Chirurgical Marie Lannelongue, France

Oswin Grollmuss, Centre Chirurgical Marie Lannelongue, France

Jean Losay, Centre Chirurgical Marie Lannelongue, France

Alain Serraf, Centre Chirurgical Marie Lannelongue, France

We reviewed a large population of premature infants operated in the preterm period with a special attention to the midterm follow-up.

Methods: Between 2000 and 2007, 67 infants born before 38 weeks of gestation (WG) were operated for a congenital heart disease. Patients with isolated patent ductus arteriosus were excluded.

Results: mean gestational age at surgery 36.6 WG (range 34 to $37.8 \mathrm{WG}$ ), age was 14 days (range 1 to 66 days), and weight $2270 \mathrm{~g}$ (range 1075 to $4020 \mathrm{~g}$ ). Complete repair was achieved in one stage in $78.4 \%$ of the 51 patients with repairable cardiac anomaly; palliative surgery during the neonatal period was performed in $27 / 67$ patients $(40.3 \%)$. Four patients among them had a repair after a median delay of 6.8 months. Hospital death was $15 \%$, related to the duration of preoperative assisted ventilation. Mean duration of follow up was 26 months (range 1 day to 7 years). Actuarial survival at 1 year and 3 years was respectively 72 and $70 \%$. A reoperation was required in 28 pts. Preoperative lung function is the main factor for survival. Immediate and midterm mortality were neither related to age, nor term and weight at operation, type of surgery, complexity of the disease and syndrome. At the end of FU, clinical status was good for the most of patients. Two had a long term tracheotomy.

Conclusion: Cardiac surgery performed in premature infants is feasible with an acceptable survival and good midterm clinical status. Complete repair should be performed as the first surgery.

\section{6}

Proyecto "De Todo Corazón": A Pediatric Heart

Program evolves changing paradigms

José R Iribarren, Fundación de Todo Corazón Richard Gibson, Venezuela

Iván A Machado, Fundación de Todo Corazón Richard Gibson, Venezuela

Luis Ravelo, Fundación de Todo Corazón Richard Gibson, Venezuela José A Figueira, Fundación de Todo Corazón Richard Gibson, Venezuela

José M Castejón, Fundación de Todo Corazón Richard Gibson, Venezuela Brigida Aguerrevere, Fundación de Todo Corazón Richard Gibson, Venezuela

Claudia von Scanzoni, Fundación de Todo Corazón Richard

Gibson, Venezuela

Richard Gibson, Fundación de Todo Corazón Richard Gibson, Venezuela

Congenital heart defects (CHD) are considered one of the leading death causes in childhood and continue to be a major public health problem worldwide. In our country, the complexity of CHDs and a cost-constrained healthcare environment makes their care challenging.

In 1996 "Proyecto de Todo Corazón" started as a pediatric cardiac program. Its mission: provide the best quality of medical and nursing care at the lowest possible cost. Clinical, economic, and human resource factors based on family-centered care principles were considered to ensure quality outcomes. Clinical pathways were applied to analyze procedures and protocols. Funding could be obtained from external private sources.

From April 1999 until December 2005, 3445 children were evaluated, 662 underwent surgery.

On February 2006, the new goal was to transfer our experience to another Pediatric facility. A satellite program was started in Maracaibo. A team of fully trained cardiac physicians, nurses and perfussionists travelled monthly from Caracas. Nurses adopt different roles (cross-training between operating room, Anesthesia and Pediatric ICU). Special care guaranteed early extubation, short-term Intensive Care and hospital stay.

From February 2006 to December 2008, 204 surgeries were performed in 80 surgical days (mean 2,5 patients/day). Mortality 7/204(3,4\%): on-pump: 140 (69\%) peripherals: 64(31\%).

Ongoing review of clinical pathways, evidence-based modifications of clinical paradigms and good teamwork leads to cost reduction, new solutions and improvement of a pediatric cardiac program in a cost-constrained environment. It allows to create satellite programs to perform heart surgery for a broader population in the same country. 
7137

Transpulmonary Direct Surgical Closure of Restrictive Subarterial Ventricular Septal Defects

Kelly K Nagasawa, University of Hawaii School of Medicine, Honolulu, HI, United States

Carlos E Moreno-Cabral, University of Hawaii School of Medicine, Honolulu, HI, United States

John J Lamberti, Rady Children's Hospital of San Diego, San Diego, $C A$, United States

Venu D Reddy, Kapiolani Medical Center for Women and Children, Honolulu, HI, United States

Lance K Shirai, Kaiser Permanente Medical Center, Honolulu, HI, United States

James Y Sim, Kapiolani Medical Center for Women and Children, Honolulu, HI, United States

Objective: Subarterial VSD are uncommon defects. Our preferred technique is direct closure through the pulmonary artery. We are reporting our updated surgical experience and its effect on aortic regurgitation (AR).

Materials and Methods: From March 1993 through March 2008, 197 children underwent operations for isolated VSD. Thirtyeight (19\%) had subarterial defects. Mean age was 6.2 years, $63 \%$ were male and 94\% were Asian or Pacific Islanders. Preoperative echocardiograms showed mild AR in 24 patients (63\%), trivial in $3(8 \%)$, and none in $11(29 \%)$. The operation is done through a longitudinal arteriotomy, pledgeted mattress sutures are placed at the crest of the VSD and exit at the annular insertion of the pulmonary valve. This results in direct approximation of the lower rim of the VSD to the intervalvar fibrous tissue.

Results: There were no deaths, valve complications or reoperations. To assess the impact of the operation on the 24 patients with mild AR, post-discharge echocardiograms were evaluated. These were available in 22 patients. Of these, 13 patients (59\%) showed improvement and 9 patients (41\%) showed no change. We found a positive correlation between age and the presence of AR. Patients with AR were older (mean age 8.2 years; 24 patients) than the patients without AR (mean age 2.6 years; 11 patients) $\mathrm{p}<0.005$.

Conclusion: Transpulmonary closure of subarterial VSD is a safe and effective method that results in the improvement or elimination of AR in the majority of patients, effectively reversing the trend of progression of the aortic disease.

\section{8}

Ventricular Septation for double inlet ventricle patients

Qingyu Wu, First Hospital of Tsinghua University, China

Hongyin Li, First Hospital of Tsinghua University, China

Xingpeng Chen, First Hospital of Tsinghua University, China

Lianyi Wang, First Hospital of Tsinghua University, China

Hui Xue, First Hospital of Tsinghua University, China

Ping Lu, First Hospital of Tsinghua University, China

Objective: To report the experience of septation operation in double inlet ventricle patients.

Method: Four patients aged from 10 to 19 years old underwent ventricular septation procedure for double inlet ventricle from Jul. 2005 to Oct. 2008. There were two male patients with double inlet ventricle; one of them complicated pulmonary artery stenosis but both patients with normal relationship of the great arteries and two female with corrected transposition of great arteries and pulmonary stenosis. There were not any muscular components of ventricular septum in all patients. Their mean pulmonary artery pressure was over $20 \mathrm{mmHg}$, not suitable for Fontan and Glenn procedure. Isolated septation procedure was performed in one patient. Septation procedure, pulmonary valvulotomy and reconstruction of RVOT were in one patient. Sepation and Restalli procedures were in the other two patients. Result: Three patients survived and are doing well. One patient died 17days after the operation because of the bleeding of gastrointestinal tract and renal failure.

Conclusion: Septation procedure can get good result for some patients with double inlet ventricle and PA pressure not suitable for Fontan or Glenn procedures.

\section{0}

Performance of Synergraft Decellularized Pulmonary Heart Valve in Patients Undergoing a Ross Procedure: A Single Institution Experience

John W Brown, Indiana University School of Medicine, Indianapolis, Indiana, United States

Mark Ruzmetov, Indiana University School of Medicine, Indianapolis, Indiana, United States

Mark D Rodefeld, Indiana University School of Medicine, Indianapolis, Indiana, United States

Mark $W$ Turrentine, Indiana University School of Medicine, Indianapolis, Indiana, United States

Background: In the Ross procedure a valved conduit is used in place of autotransplanted pulmonary valve. Despite Ross procedure' advantages, there is a certain risk of structural valve deterioration, especially of the cryopreserved pulmonary allograft as a result of shrinkage and subsequent stenosis predominantly. This study analyzed our results of the CryoValve SynerGraft decellularized pulmonary heart valve (SGDPV) implanted in patients undergoing a Ross procedure.

Methods: Between 2000 and 2008, 27 consecutive patients received a SGPV during the Ross procedure. Patient age ranged from 4 mo to 58 years, mean age at implant was 28.2 years. Retrospective data included reported adverse events and recent hemodynamic results.

Results: There was no operative mortality and late mortality or morbidity during the mean follow-up of $4.1+2.1$ years (range 3 mo to 8 years). No patients required reoperation. The median gradient at discharge was $12 \mathrm{~mm} \mathrm{Hg}$, which remained uncharged at last follow-up. No deterioration in conduit or conduit valve function was noted.

Conclusions: The new SGDPV conduit can be a viable alternative to a cryopreserved homograft in the Ross procedure. The early clinical and hemodynamic results are encouraging. SG technology may provide a more durable, low risk option for those patients needing heart valve replacements.

\section{1}

Modifications in surgical technique and protocol improve Norwood outcomes in a low volume institution

Janeth C Ejike, Loma Linda Univeristy Children's Hospital, United States

Jennifier B Newcombe, Loma Linda Univeristy Children's Hospital, United States

Sarah Heinonen, Loma Linda Univeristy Children's Hospital, United States

Corey Fuller, School of Medicine, Loma Linda University, United States Neda F Mulla, Loma Linda Univeristy Children's Hospital, United States

James Fitts, United States

Leonard L Bailey, Loma Linda Univeristy Children's Hospital, United States 
Anees J Razzouk, Loma Linda Univeristy Children's Hospital, United States

Background: Low volume institutions ( $<16$ cases per year) may produce less favorable outcomes of the Staged Norwood procedure using Blalock Taussig shunt (BT) or right ventricle to pulmonary conduit (Sano).

Objectives: To compare Norwood BT/Sano outcomes after implementing a protocol in a single institution.

Methods: A retrospective review of all patients having staged Norwood BT/Sano reconstruction for $\star \star$ HLHS or its variants from 2002-2008. Modifications in the protocol included surgical techniques, post-operative care and follow-up surveillance.

Results: From 2002-2004, 48.3\% had the Norwood/Sano procedure compared to $54.5 \%$ in $2005-2008$ ( $\mathrm{p}=0.600)$ Modified interventions resulted in improvements in 48-hr and 30-day mortality, and survival to Stage II Glenn procedure. Age at death between groups did not reach statistical significance $(\mathrm{p}=0.385)$.

Conclusions: Implementation of a multidisciplinary protocol improved survival to Stage II Glenn in patients undergoing the Norwood BT/Sano operation in a low volume institution.

\begin{tabular}{|c|c|c|c|c|}
\hline & & $\begin{array}{c}2002-2004 \\
\text { Median (range) } \\
\mathrm{n}=29\end{array}$ & $\begin{array}{c}2005-2008 \\
\text { Median (range) } \\
n=44\end{array}$ & P value \\
\hline Demographics & Age (days)/weight $(\mathrm{Kg})$ & $\begin{array}{l}8(1-64) / \\
3.3(2-4.4)\end{array}$ & $\begin{array}{l}9(3-55) / \\
3.1(2-45)\end{array}$ & $\begin{array}{r}0.138 / \\
0.217\end{array}$ \\
\hline \multirow[t]{5}{*}{ Intervention } & $\begin{array}{c}\# \text { of follow-up phone } \\
\text { calls }\end{array}$ & $0.0(0-9)$ & $6.0(0-23)$ & $*<0.001$ \\
\hline & $\begin{array}{l}\text { \# of outpatient } \\
\text { appointments }\end{array}$ & $f 4.69(6.11)$ & f9.05 (5.62) & $* 0.003$ \\
\hline & \# of readmissions & $1.0(0-10)$ & $2.0(0-6)$ & $* 0.010$ \\
\hline & $\begin{array}{l}\text { Pre-stage II Glenn } \\
\text { catheterization age } \\
\text { (months) }\end{array}$ & $5.05(2.2-10.8)$ & $5.3(1.5-7.4)$ & 0.706 \\
\hline & $\begin{array}{l}\text { Age at stage II Glenn } \\
\text { (months) }\end{array}$ & $6.5(4.4-11.7)$ & $6.25(4.2-8.7)$ & 0.886 \\
\hline \multirow[t]{2}{*}{$\begin{array}{l}\text { Norwood BT/ } \\
\text { Sano outcomes }\end{array}$} & $\begin{array}{l}\text { 48-hr/30-day Mortality } \\
(\%)\end{array}$ & $27.6 / 31.0$ & $4.5 / 4.5$ & $\begin{array}{r}* 0.005 / \\
* 0.002\end{array}$ \\
\hline & $\begin{array}{c}\text { Survival to Stage II } \\
\text { Glenn }(\%)\end{array}$ & 39.0 & 83.0 & $*<0.001$ \\
\hline
\end{tabular}

7144

Isolated Congenital Mitral Valve Regurgitation Presenting in the First Year of Life

Anusha Ganeshalingham, Starship Hospital, Auckland, New Zealand Kirsten Finucane, Starship Hospital, Auckland, New Zealand Tim S Hornung, Starship Hospital, Auckland, New Zealand, New Zealand

Objectives: Isolated congenital mitral regurgitation is rare and when presenting in infancy, often represents a severe malformation. The natural history may be fatal and early management remains a therapeutic challenge. These infants are poorly understood largely due to limited experience in each institution and a lack of reporting in the medical literature.

Methods: We performed a review of children presenting with isolated congenital mitral regurgitation at our centre over a 10 year period. Patients were identified from our echocardiography database.

Results: Seven patients were identified with isolated congenital mitral regurgitation: an associated patent ductus arteriosus was present in 2. Median age at referral was 12 weeks, (1 day to 43 weeks). Median weight was $6.9 \mathrm{~kg}$, (3.7 to $10.4 \mathrm{~kg})$. Two infants were managed conservatively and 1 underwent surgical ligation of a patent ductus arteriosus. Following ductal closure, no further intervention was required in two infants. The remaining four infants underwent 3 valve repairs and 3 valve replacements. The in-hospital mortality was $29 \%$ (2 patients), in both cases occurring following emergency mitral surgery under one year of age. Two re-operations occurred following mitral repair and no significant complications or late deaths occurred.

Conclusions: Our experience suggests closure of a patent ductus arteriosus should be undertaken prior to mitral valve surgery. There may be a poorer prognosis in those under one year of age requiring emergency mitral valve surgery. Those who can be managed conservatively or undergo mitral valve surgery as an elective procedure tend to have a better outcome.

\section{5}

The Relation of Nt-proBNP Values with Mortality and Morbidity in Congenital Heart Surgery

Murat Koc, Dr.Sami Ulus Pediatric Research and Training

Hospital,Departments of Cardiovascular Surgery, Turkey

Olcay Murat Disli, Dr.Sami Ulus Pediatric Research and Training

Hospital,Departments of Cardiovascular Surgery, Turkey

Rusen Melih Nebigil, Dr.Sami Ulus Pediatric Research and Training Hospital,Departments of Cardiovascular Surgery, Turkey

Utku Arman Orun, Dr.Sami Ulus Pediatric Research and Training Hospital,Departments of Pediatric Cardiology, Turkey

Selmin Karademir, Dr.Sami Ulus Pediatric Research and Training

Hospital,Departments of Pediatric Cardiology, Turkey

Ali Kutsal, Dr.Sami Ulus Pediatric Research and Training Hospital, Departments of Cardiovascular Surgery, Turkey

Type-B natriuretic peptides are synthesized by the cardiac miyosits as a response to increased wall stress,and after being excreted as prohormone, they are separated as an active hormone (BNP) and Nt-proBNP which includes the inactive N-terminal part biologically.

In this study, our aim is to see the Nt-proBNP values of the patients who had congenital cardiac surgery and also if there is any relationship between their levels and the postoperative progress, mortality and morbidity.

For this purpose, the patients who had left-right shunt lesion and ventricular outflow tract obstruction were seperated in two groups as the ones who were in need of high dose $(>7,5 \mathrm{mcg} /$ $\mathrm{kg} / \mathrm{min}$ ) inotropic treatment and as the ones who were not in need or low dose inotropic treatment during the postoperative intensive care followup. The Nt-proBNP values of the patients were measured before the surgery and after the second (T1) and the twenty-fourth (T2) hours of the surgery. Additionally, postoperative inotropic treatment needs, and duration ; cardiopulmonary by- pass (CPBS), cross-clamp (KK), and mechanical ventilation (MVZ) time of the patients were recorded.There was statistically high meaningfull among the inotropic treatment period, mechanical ventilation period, and staying in intensive care period.

In our study, it was observed that there was a correlation between the inotropic treatment dose and duration time and the Nt-proBNP level; the average level of serum Nt-proBNP level was found as $1009,10 \pm 1345,43 \mathrm{pg}$ / in patients who were in need of high dose inotropic treatment.

\section{6}

Long-term outcome of surgery for supravalvar aortic stenosis

Arjuna Somanathan, University of New South Wales, Australia 
Andrew D Cochrane, Monash Medical Centre, Australia Christian P Brizard, The Royal Children's Hopsital, Australia Yves d'Udekem, The Royal Children's Hopsital, Australia

Objective: Supravalvar aortic stenosis is an uncommon form of left ventricular outflow tract obstruction, with a typical localized form of the disease, and a less frequent diffuse form involving the ascending aorta and beyond.

Methods: A retrospective review of 41 patients undergoing surgery between 1980 and 2007 at the Royal Children's Hospital, Melbourne.

Results: There were 41 patients (27 males, 14 females) with a median age at operation of 50 months. William's syndrome was diagnosed in $63 \%$ of patients. There were 33 (80\%) with localised stenosis. Surgery provided effective relief, reducing the gradient from $84 \mathrm{~mm} \mathrm{Hg}$ to $15 \mathrm{~mm} \mathrm{Hg}$ post-operation $(p<0.001)$. The long term peak gradient remained low, and at 5,10 , and 20 years, the mean peak gradient was $20 \mathrm{~mm} \mathrm{Hg}$, $28 \mathrm{~mm} \mathrm{Hg}$ and $23 \mathrm{~mm} \mathrm{Hg}$ respectively. Three different types of repair were used. There were 6 deaths (2 early and 4 late), 4 reoperations, and the survival at 5, 10 and 20 years was $86 \%$ (95\% CI: 70-94\%), 86\% (95\% CI: 70-94\%), and 79\% (95\% CI: 55-91\%). Diffuse stenosis, residual post-operative gradient and younger age at presentation were univariate risk factors for mortality. The residual gradient was a risk factor for re-operation. There was no difference between the surgical techniques for mortality, reoperation or gradient.

Conclusion: Surgical management results in immediate and effective relief of the stenosis and provides a stable long term result. However, the diffuse type of stenosis is associated with a poorer outcome. Residual gradient confers a significant risk of death and reoperation.

\section{7}

End-to-End Anastomosis versus Subclavian Flap Repair for Aortic Coarctation in Infants $<6$ Months: Single Centre Experience

Damien P Kenny, Bristol Congenital Heart Centre, University Hospitals Bristol, UK., United Kingdom Giri Dhandayuthadani, Bristol Congenital Heart Centre, University Hospitals Bristol, UK., United Kingdom Christopher Gillett, Bristol Congenital Heart Centre, University Hospitals Bristol, UK, United Kingdom

Robin P Martin, Bristol Congenital Heart Centre, University Hospitals Bristol, UK., United Kingdom

Andrew J Parry, Bristol Congenital Heart Centre, University Hospitals Bristol, UK., United Kingdom

Claire Skerritt, Bristol Congenital Heart Centre, University Hospitals Bristol, UK., United Kingdom

Massimo Caputo, Bristol Congenital Heart Centre, University

Hospitals Bristol, UK., United Kingdom

Objectives: We compared outcomes in infants undergoing end-toend anastomosis (EEA) or subclavian flap repair (SFR) for aortic coarctation in a single centre.

Methods: This was a retrospective study of 177 consecutive infants undergoing aortic coarctation repair between December 1995 and January 2008. Infants with associated complex cardiac defects or undergoing concomitant cardiopulmonary bypass were excluded.

Results: Ninety-seven infants underwent EEA and 80 infants SFR with non-significant differences in median ages and weights. Median hospital stay was 8 (4-56) and 10 (4-188) days in the EEA and SFR groups respectively $(p=0.37)$. One hospital death occurred in each group (1.1\%) and was due to non-cardiac pathology. Mean follow-up for all patients was $6.3 \pm 3.3$ years. Post-operative morbidity occurred in $14.3 \%$ of the EEA and $7.5 \%$ of the SFR group $(p=0.17)$.

Five-year survival was $97 \%$ (CI $86.7 \%-99.2 \%$ ) in those undergoing EEA and $98.3 \%$ (CI 88.7\%-99.7\%) in patients with SFR $(p=0.5)$. Cardiac event-free survival (catheter intervention for restenosis) including death at 5 years was $89.9 \%$ (CI 80\%-94.9\%) and $92.2 \%$ (CI $83.4 \%-96.4 \%$ ) in the EEA and SFR group respectively $(\mathrm{p}=0.4)$.

At latest follow-up, mean echocardiographic descending aortic Doppler velocities were $1.9 \pm 0.5 \mathrm{~m} / \mathrm{s}$ and $2.0 \pm 0.6 \mathrm{~m} / \mathrm{s}$ in the EEA and SFR group respectively $(p=0.7)$. Medically treated systemic hypertension was present in $12.3 \%$ of EEA and $15 \%$ of SFR patients $(\mathrm{p}=0.4)$.

Conclusions: Both techniques achieve excellent comparable early and longer term outcomes in infants undergoing aortic coarctation repair. With negligible mortality, continued evaluation of surgical technique on relevant major pathology (reintervention and hypertension) in later life is warranted.

\section{8}

Long-term outcomes of total surgical correction for complete atrioventricular septal defect in infants: Alive but well?

Yan Li, National Heart Center and Fuwai Hospital, China

Shoujun Li, National Heart Center and Fuwai Hospital, China

Xiangdong Shen, National Heart Center and Fuwai Hospital, China

Jun Yan, National Heart Center and Fuwai Hospital, China Yinglong Liu, National Heart Center and Fuwai Hospital, China Shengshou Hu, National Heart Center and Fuwai Hospital, China Zhe Zheng, National Heart Center and Fuwai Hospital, China

Objective: Correction of complete atrioventricular septal defect (AVSD) in infants achieves satisfactory early results. This study outlines the long-term outcomes of all infants at our institution who underwent correction for complete AVSD.

Methods: A total of 104 consecutive infants underwent surgical repairs for complete AVSD between 1991 and 2006. 44 infants were associated with Down syndrome. Health and neurodevelopment (Bayley Scales of Infant Development II) were assessed at 18 to 24 months of age. Multivariate analyses were determined to identify risk factors associated with longterm outcomes.

Results: There were 5 peri-operative deaths. At the average 8year follow-up, 4 late deaths occurred and reoperation was required in 9 patients for left atrioventricular valve (AVV) regurgitation. At last follow-up, no or only mild left AVV and right AVV regurgitation was present in $79.8 \%$ and $92.9 \%$ of patients, respectively, and $69.7 \%$ of patients were medicationfree. Risk factors for reoperation were younger age ( $<6$ months), lower weight $(<6 \mathrm{~kg})$, and in non-Down children. 57 nonDown children were assessed for neurodevelopment. The mean scores were $86(45-110)$ for mental skills and 90 (47-121) for motor skills. Low gestational age, length of postoperative ventilation, and high postoperative lactate was associated with mentally and/or motor-delayed non-Down children.

Conclusion: Complete AVSD can be corrected with good longterm survival; postoperative moderate or more AVV regurgitation could be anticipated in patients $<6$ months and weighing $<6 \mathrm{~kg}$. Neurodevelopmental outcome is a concern, and there may be potentially modifiable postoperative factors that can be optimized to improve developmental outcomes. 


\section{9}

Congenital Heart Surgery in Hawaii, Outcome Analysis Using the Risk Adjustment Method (RACHS-1)

Jennifer Sasaki, United States

James Y Sim, Kapiolani Medical Center for Women and Children, United States

Carlos E Moreno-Cabral, Kapiolani Medical Center for Women and Children, United States

D Venu Reddy, Kapiolani Medical Center for Women and Children, United States

Lance K Shirai, Kaiser Permanente Clinic - Honolulu, United States Christopher B Mahnke, Tripler Army Medical Center, United States John W Moore, Rady's Children's Hospital of San Diego, United States John J Lamberti, Rady's Children's Hospital of San Diego, United States

Objective: Pediatric heart surgery in Hawaii faces many challenges due to its geographic isolation and small surgical volume. Since 1995, a surgical program was adopted at Kapiolani Medical Center (KMC) in Honolulu. Elective cases of intermediate complexity were performed assisted by an experienced visiting pediatric heart surgeon on regular interval. We review the surgical outcome using Risk Adjustment in Congenital Heart Surgery (RACHS-1) model.

Methods: Patient under18 year's old undergoing heart surgery at KMC was reviewed from January 1st, 2001 to June 22nd, 2007. Each case was classified according to RACHS-1. Additional risk factors: age, prematurity, combination procedures and major structural anomalies, were included in the final multivariable analysis. Outcome was compared with two multi-institutional data sets: the Pediatric Cardiac Care Consortium (PCCC) and Hospital Discharge (HD).

Results: 494 surgical cases were reviewed. 205 cases were excluded (age over 18, prematurity, missing data, unlisted risk category). 289 cases were assigned to RACHS-1 risk categories, (77 level I, 127 level II, 84 level III, and 1 level IV). There were three (1\%) in hospital mortality (95\% CI, 0.23-7.16). The RACHS-1 multivariable model predicted mortality of 6.72 and 5.69 using PCCC and HD data respectively.

Conclusion: The mortality of pediatric heart surgery in Hawaii is lower than the predicted number based on RACHS-1 model, but did not reach statistical significance due to the small sample size. However, the data demonstrate satisfactory clinical outcome can be achieved in a small, self contained pediatric heart surgery program such as in Hawaii.

7150

Epicardial Echocardiography: A Safe and Accurate Alternative

Claudio Ramaciotti, UT SouthWestern Medical Center Dallas, Texas, USA, United States

Timothy H Thomas, UT SouthWestern Medical Center Dallas, Texas, USA, United States

David Troendle, UT SouthWestern Medical Center Dallas, Texas, USA, United States

Joann Dudley, Children's Medical Center of Dallas, Texas, USA, United States

Joseph M Forbess, UT SouthWestern Medical Center Dallas, Texas, USA, United States

Objective: Transesophageal echocardiography (TEE) in the immediate postoperative period is standard of care for most congenital heart operations. However, patient size, esophageal anatomy, or surgical field interference may prevent its use. We hypothesize that epicardial echocardiography (EPI) is a safe and accurate alternative.
Methods: 98 consecutive neonates underwent EPI(group OR EPI). Discrepancies between these EPIs and the first complete post-operative transthoracic echocardiogram (TTE)were compared. A second group of 22 consecutive and contemporaneous neonates (group OR TEE) underwent intraoperative TEE, not EPI. Discrepancies between those TEEs and the first complete post-operative TTE were also compared.

Results: EPI views were obtained in all patients without hemodynamic compromise. Group OR EPI had lower weight (median 2.9, range $1-4.2 \mathrm{~kg}$ ) vs. OR TEE patients (3.6, $3.2-4.5 \mathrm{~kg}),(\mathrm{p}<0.0001)$, but similar age at surgery (OR EPI median 5, range $1-29$ days, OR TEE $5,1-28$ days, $p=0.5)$. There was no difference between the frequency of minor discrepancies with TTE in group OR EPI (24\%) vs. group OR TEE $(32 \%)$ $(p=0.36)$, as shown in the Table. One patient required TEE after EPI to diagnose tricuspid stenosis related to a ventricular septal hematoma. No minor discrepancies required further intervention. EPI was diagnostic in 2 cases of total anomalous pulmonary venous return that required additional surgery.

Conclusions: Intraoperative epicardial echocardiography is a safe and accurate alternative to transesophageal echocardiography in neonates.

Minor discrepancies: OR EPI /OR TEE

vs. first post-op TTE

OR EPI OR TEE

$(\mathrm{n}=98) \quad(\mathrm{n}=22)$

Change in valvar regurgitation (1 degree)

Residual Patent Foramen Ovale

Residual VSD

Mild branch pulmonary artery stenosis

$\begin{array}{cc}17 & 4 \\ 2 & 2 \\ 3 & 2 \\ 1 & 0\end{array}$

\section{1}

A case of truncus arteriosus succesfully operated at the age of 12 years old

Ayse Guler Eroglu, Istanbul University Cerrahpasa Medical Faculty,

Department of Pediatrics, Division of Pediatric Cardiology, Turkey

Bulent Koca, Turkey

Selman Gokalp, Turkey

Levent Saltik, Turkey

Funda Oztunc, Istanbul University Cerrahpasa Medical Faculty,

Department of Pediatrics, Division of Pediatric Cardiology, Turkey

We report a 12 years old male patient who has truncus arteriosus, large ventricular septal defect, pulmonary hypertension and successfully underwent corrective surgery.

This patient was admitted to another Pediatric Cardiology Center, diagnosed as truncus arteriosus and pulmonary stenosis $(60 \mathrm{mmHg}$ systolic gradient between the truncal artery and the pulmonary artery) when he was three months old and lost the following. The patient was evaluated with echocardiography and cardiac catheterization at 12 years old in our center and diagnosed as truncus arteriosus, large ventricular septal defect and pulmonary hypertension with echocardiography. Pulmonary stenosis was not detected and pulmonary vascular resistance showed significant reversibility with oxygen administration at cardiac catheterization. The patient successfully underwent surgical correction. Postoperative cardiac catheterization showed normal pulmonary arterial pressure. The patient is in good condition after one-year follow-up.

The reversibility of pulmonary vascular resistance and successful surgical correction may be due to the pulmonary stenosis during infancy. 
7152

Repair Of Anomalous Left Coronary Artery from the Pulmonary Artery on a beating heart

Sriram Shankar, K K Women and Children's Hospital, Singapore

Anna Mani, K K Women and Children's Hospital, Singapore

Teng Hong Tan, K K Women and Children's Hospital, Singapore

Keng Yean Wong, K K Women and Children's Hospital, Singapore

Introduction: Patients with anomalous origin of the left coronary artery from pulmonary artery (ALCAPA) have myocardial ischaemia resulting in left ventricular dysfunction. Both direct re-implantation and the tunnel procedure that are conventionally done require a period of global myocardial ischaemia.

Objective: To evaluate the outcome of patients with ALCAPA who underwent aortopulmonary tunnel (Takeuchi) repair on a beating heart in K K Women and Children's Hospital.

Methods: The hospital records of patients who underwent aortopulmonary tunnel (Takeuchi) repair on a beating heart for ALCAPA were retrospectively reviewed.

Results: From October 2001 to March 2007 eight patients underwent Takeuchi repair on beating heart. The age ranges from 2 months to 24 months (median 3months), weight ranges from $3.9 \mathrm{~kg}$ to $10.6 \mathrm{Kg}$ (median $5.49 \mathrm{Kg}$ ). Two patients were on ventilatory support prior to surgery. The cardiopulmonary bypass time ranges from 102 minutes to 228 minutes (median 149.5 minutes). None of the patients were on assist device support post-operatively. There were no operative mortalities. Post-operative hospital stay ranges from 11 days to 38 days (mean 23.5 days, median 22 days). One patient had baffle leak and underwent correction 18 days after the first operation. On follow-up 2 patients had moderate pulmonary stenosis and underwent balloon dilatation 11 months and 24 months after the initial surgery respectively.

Conclusion: In critically ill patients surgical correction on a beating heart will reduce peri-operative myocardial injury, possibly reduce the need for temporary assist and improve outcomes.

\section{3}

Results and indications of coronary artery bypass graft operation for coronary artery lesion after Kawasaki disease Masanori Abe, Nippon Medical School, Japan

Ryuji Fukazawa, Nippon Medical School, Japan

Ei Ikegami, Nippon Medical School, Japan

Mitsuhiro Kamisago, Nippon Medical School, Japan

Yasuhiro Katsube, Nippon Medical School, Japan

Shunichi Ogawa, Nippon Medical School, Japan

Masami Ochi, Nippon Medical School, Japan

Background: Coronary artery lesion (CAL) in children caused by Kawasaki disease (KD) can lead to serious complications including myocardial ischemia and infarction. We tested whether coronary artery Bypass Graft operation (CABG) could be effective for KD patients with CAL.

Methods: Thirty cases with 50 branches had undergone CABG. The age at CABGs was $12.1 \pm 8.1 \mathrm{y}$. For $47 \mathrm{LCA}$ branches and 3 RCA branches, CABGs were performed. The details are next; Y type anastomosis using RITA for 1 case and 2 branches, sequential anastomosis between LITA to LAD and D1 for six cases and 12 branches, anastomosis of GEA graft for 2 cases, anastomosis of GEA free graft for 1 case. CALs are in the followings:1) 18 cases had Giant Aneurysms with stenosis or occlusion, 2) four had uncontrollable thrombus in Giant aneurysms, 3) four had Giant Aneurysms without stenosis, 4)
2 had with stenosis without PCI indication. All cases showed myocardial ischemia.

Results: All 30 cases are survived without exercise limitation and are classified under NYHA1. Coronary angiography showed that 46 of 50 branches were patent without anastomosis stenosis. But, 3 branches in Y type graft and 1 branch anastomosised by GEA were occluded. According to growth, diameter and length in bypass graft were grown.

Conclusions: We recommend the following methods of CABG in KD patients, especially infant and young child. 1. On-pump, 2. End to side anastomosis of one graft using LITA or RITA to one branch, 3. Sequential anastomosis could be allowed in more than young-adult.

\section{4}

Early results of indigenous Decellularised Porcine

Xenograft for RVOT reconstruction

Ravi Agarwal, Frontier Lifeline \& Dr. K M Cherian Heart

Foundation, Chennai, India

Karthik Vaidyanathan, Frontier Lifeline \& Dr. K M Cherian Heart

Foundation, Chennai, India

Soma Guhathakurta, Frontier Lifeline \& Dr. K M Cherian Heart

Foundation, Chennai, India

Kotturathu M Cherian, India

Objective: To evaluate the early results of indigenously developed decellularised porcine xenograft conduit for RVOT reconstruction in patients with congenital heart disease.

Methods and Results: Prior to clinical use the xenograft was subjected to extensive tests to ensure preservation of collagen structure and tissue strength. Clinical use was initiated in February 2006. Till date this xenograft has been implanted in 46 patients (aged 5months to 29 years, mean- 8.9 years) with congenital heart disease. The clinical conditions encountered were TOF with pulmonary atresia $(n=23)$ or pulmonary stenosis $(n=6)$ or absent pulmonary valve $(n=4)$; truncus arteriosus $(\mathrm{n}=2)$; DORV with VSD and pulmonary stenosis or atresia $(n=7)$; TGA with left ventricular outflow tract obstruction $(\mathrm{n}=2)$; homograft replacement $(\mathrm{n}=1)$ and right ventricular outflow tract reconstruction following tetralogy repair $(n=1)$. The median xenograft size used was $16 \mathrm{~mm}$ (range 13-23 mm). Follow up was complete for all domestic patients $(\mathrm{n}=20)$ and ranged from 3-18 months. There was no incidence of conduit thrombosis, one patient developed infective endocarditis after one month of surgery and was managed conservatively. One patient required conduit replacement five months after truncus repair. Among the remaining survivors xenograft function was excellent in the majority of patients. Mild to moderate obstruction was noted at the distal end of the conduit in two patients and mild to moderate regurgitation was seen in four patients. There seemed to be no progress of pulmonary regurgitation on serial follow up.

Conclusions: Indigenously developed decellularised xenograft offers excellent hemodynamics with a low re-operation rate in the short term follow up.

\section{5}

Minimally invasive Trans-ventricular pulmonary valve replacement: report of $\mathbf{1 3}$ consecutive cases

Howaida M O Al Qethamy, Head of Cardiac surgery Prince Sultan Cardiac Centre, Saudi Arabia

Mohamed E A Ibrahim, Research \& Delopment, Prince Sultan Cardiac Centre, Sudan 
Yahya M Al Faraidi, Senior Consultat Ped. Cardiac Surgery, Prince Sultan Cardiac Centre, Saudi Arabia

Hatim Mohamed, Prince Sultan Cardiac Centre, Sudan

Patients and methods: Between August 2007 to February 2008 for the first time, we performed minimally invasive trans-ventricular pulmonary valve replacement operations in 13 patients in series. Patient's ages ranged from 3 to 26 years all of whom complained of pulmonary valve complications. All the cases had a previous operation for the Tetralogy of Fallot repair except one. Median sternotomy with limited exposure of the right ventricle is performed, then the Contegra 200 pulmonary valve (Medtronic, Inc. USA) is transfixed inside a CP stent (NuMed, Inc. USA) using multiple $5 / 0$ prolene sutures. A Cristal balloon catheter (Balt, France) crimps the valve/stent composite. A small pursestring then introduces the valve/stent and the balloon via a placed at the RVOT. The stent/valve composite is then expanded in the pulmonary valve position.

Results: Hospital stay ranged from 4 to 9 days and no mortalities are reported so far. The mean follow up was 6 months $(2-12$ months range). 53\% had no PV insufficiency postoperatively, $31 \%$ had mild or trivial insufficiency while $15 \%$ had moderate regurgitation. $70 \%$ of the cases had mild to moderately depressed RV function with good LV function except for one case that had mildly depressed LV function as well. In the post-operatively follow up period all the cases showed good RV and LV functions except one that his RV remained dilated after 4 months. One case had a tilted valve that was corrected through percutaneous balloon.

Discussion: Trans-ventricular PVR can be performed with high survival and minimal complications.

\section{6}

Outcome of surgical treatment Neonatal Aortic Arch

Obstruction

Abdulraoof AH Al Saeedi, Saudi Arabia

Amina Hassan, Saudi Arabia

Jassim Abdul Hamed, Saudi Arabia

Background: This report deal with our surgical experience at PSCC in neonatal aortic arch obstruction (369) cases.

Methods: 729 Cases underwent Coarctation or Interrupted Arch repair at PSCC, in the Period between Oct.1982 to Dec 2006. only cases $<3$ months of age were reviewed. 369 were neonate and small infants $<3$ months, median age was 16 days.

Male to Female ratio for COA 1:1.5. Male to Female ratio for IAA 0.9:1

Weight is 1.5 to $4.7 \mathrm{~kg}$, median weight is $2.6 \mathrm{~kg}$. Associated cardiac problems were detected in 232cases(62.8\%).VSD's 133(36\%), Single Ventricle were 17 cases(4.6\%). Complex lesions were 58 cases (15.7\%), One case of complete congenital heart block.

Result: Extended arch repair were done in 140 pts (38\%). 111 pts $(30 \%)$ had resection and end to end at anastomosis. 118 pts(32\%) had Subclavian flap. Hospital Mortality were 17 cases (4.6\%), 11 COA (3.5\%), 6 IAA. (11\%). Operative complications: Immediate post operative 24-36 hrs, hypertension were noted in $117(32 \%)$, NEC 41 cases, 2 case had paraplegia, Hemorrhage need reexploration in 4 cases, Chylothorax 21 cases, Diaphragmatic palsy 7 cases, Hoarseness of voice was noted in 3 cases, Periapism in one case. Re-coarctation that needed intervention occurs in 23 cases (6.2\%). Recoarctation is related to $\mathrm{BW}<3 \mathrm{Kg}$ ( $\mathrm{P}$ value 0.04 ), and not to Type of Surgery $(\mathrm{p}$ value $=0.30$ ).

Conclusion: Surgery For Aortic Arch Obst. Is safe reproducible with low mortality and recoarctation rate. Recoarctation is related to BW., and Surgical experience and not to the Type of Surgery.
7157

Intra-to-extracardiac Fontan

Abdul Raoof AH Alsaeedi, Prince Sultan Cardiac Centre, Saudi

Arabia

Abdulrahman E Redhyan, Saudi Arabia

Reida M El Oakley, Saudi Arabia

Background: Since its introduction in 1970s, Fontan operation underwent several modifications, including the most commonly used extracardiac technique. Extracardiac extracardiac Fontan may not be suited for some cases such as

1. Abnormal pulmonary venous drainage to lower SVC

2. Hepatic veins draining to a left sided IVC, controlateral to the side of previous Kawashiama shunt

3. Abnormal separate drainage of hepatic veins.

We present an early experience of novel Fontan modification in seven such cases. Video recording of the surgical technique will also be presented.

Methods and Results: Between January 2000 and August 2007, 181 Cases underwent Fontan operation at the PSCC, Riyadh. Seven of them underwent fenestrated Intra-to-extracardiac Fontan.Three patients had an abnormal pulmonary venous drainage to lower SVC. Two pateints previously underwent Kawashima shunt for interrupted IVC with azygous continuation, they were found to have hepatic veins draining to a left sided IVC, controlateral to the side of Kawashiama shunt. Two patients with abnormal separate drainage of hepatic veins. Short term clinical outcome of these cases was compared to other cases who underwent the usual extracardiac Fontan.

No deaths in the intra to extra cardiac group. No significant difference was found between Intra-to-extracardiac and extracardiac groups in terms of post operative saturation (85-92\%), duration of chest drainage (10.14 vs 8.6 days), and hospital stay (15.4 vs 13.3 days).

Conclusions: Intra-to-extracardiac Fontan is a useful technique in cases with difficult anatomy. Due to the small number of patients in the Intra-to-extracardiac Fontan, comparison with other Fontan modification may be difficult.

\section{9}

Sutureless pericardial repair of total anomalous pulmonary venous connection in patients with asplenia syndrome

Masaya Aoki, First Department of Surgery, University of Toyama, Graduate School of Medicine, Japan

Naoki Yoshimura, First Department of Surgery, University of Toyama, Graduate School of Medicine, Japan

Hironori Matsuhisa, First Department of Surgery, University of

Toyama, Graduate School of Medicine, Japan

Junichiro Kitahara, First Department of Surgery, University of Toyama,

Graduate School of Medicine, Japan

Keiichi Hirono, Department of Pediatrics, University of Toyama, Graduate School of Medicine, Japan

Kazuhiro Watanabe, Department of Pediatrics, University of Toyama, Graduate School of Medicine, Japan

Fukiko Ichida, Department of Pediatrics, University of Toyama,

Graduate School of Medicine, Japan

Takuro Misaki, First Department of Surgery, University of Toyama, Graduate School of Medicine, Japan

Objective: Asplenia syndrome associated with total anomalous pulmonary venous connection (TAPVC) has remained a clinical challenge. Surgical relief of pulmonary venous obstruction (PVO) is a difficult clinical problem associated with significant risk of recurrent PVO, which makes difficult to achieve right heart bypass operation. Prevention of this problem remains elusive. 
Methods: Between November 2007 and July 2008, 5 patients, including 3 neonates, with asplenia syndrome underwent sutureless pericardial repair of TAPVC. Age at operation ranged from 7 days to 2 years and body weight ranged from 1.9 to $9.5 \mathrm{~kg}$. The sutureless repair was used in 3 naonates as an initial procedure and in 2 infants as a second procedure. Under deep hypothermic circulatory arrest or low flow cardiopulmonary bypass, pulmonary vein was incised as long as possible. Atrial wall was partially resected and anastomosed to the pericardial wall around the incised pulmonary vein.

Results: There were no early deaths. No patients showed recurrence of PVO. There was 1 late death. This patient died from congestive heart failure due to the progression of the common atrioventricular valve insufficiency. One patient underwent bilateral bidirectional Glenn and common atrioventricular valvuloplasty 12 months after the primary sutureless repair. Pulmonary venous confluence was confirmed to be left open at the time of Glenn surgery.

Conclusions: The sutureless technique may be useful not only for postrepair PVO but also for non-operated TAPVC in neonates with asplenia syndrome.

\begin{tabular}{|c|c|c|c|}
\hline & Case 1 & Case 2 & Case 3 \\
\hline Age & 12 days & 19days & 2 years \\
\hline Body weight & $1.9 \mathrm{~kg}$ & $2.5 \mathrm{~kg}$ & $9.5 \mathrm{~kg}$ \\
\hline Type of TAPVC & supracardiac & infracardiac & supracardiac \\
\hline Indications & primary & primary & $\begin{array}{l}\text { branch PVO } \\
\text { after Fontan }\end{array}$ \\
\hline Follow up & 13 months & 13 months & 10 months \\
\hline Echocardiography & PVO(-) & PVO(-) & $\mathrm{PVO}(-)$ \\
\hline Results & alive & alive & alive \\
\hline
\end{tabular}

\begin{tabular}{|c|c|c|}
\hline & Case 4 & Case 5 \\
\hline Age & 7 months & 7 days \\
\hline Body weight & $6.4 \mathrm{~kg}$ & $2.7 \mathrm{~kg}$ \\
\hline Type of TAPVC & supracardiac & infracardiac \\
\hline Indications & $\begin{array}{l}\text { branch PVO after TAPVC } \\
\text { repair }\end{array}$ & primary \\
\hline Follow up & 10 months & 3 months \\
\hline Echocardiography & PVO(-) & PVO(-) \\
\hline Results & alive & late death \\
\hline
\end{tabular}

\section{0}

\section{The Faith of Small Contegra ${ }^{\circledR}$-Grafts in RVOT}

Boulos Asfour, Congenital Cardiac Center, Dpt. of Cardiac Surgery, 53757 Sankt Augustin, Germany, Germany

Nicodeme Sinzobahamvya, Congenital Cardiac Center, Dpt. of Cardiac Surgery, 53757 Sankt Augustin, Germany, Germany

Christoph Haun, Congenital Cardiac Center, Dpt. of Intensive Care Medicine, 53757 Sankt Augustin, Germany, Germany

Martin Schneider, Congenital Cardiac Center, Dpt. of Cardiology, 53757 Sankt Augustin, Germany, Germany

Ehrenfried Schindler, Congenital Cardiac Center, Dpt. of Anesthesiology, 53757 Sankt Augustin, Germany, Germany

Victor Hraska, Congenital Cardiac Center, Dpt. of Cardiac Surgery, 53757 Sankt Augustin, Germany, Germany

Background: Early conduit failure is typically seen in small sized grafts used for reconstruction of RVOT. We analyzed the freedom from explantation and catheter-reintervention of small Contegra ${ }^{{ }^{\mathbb{B}}}$-grafts (CG) in relation to the underlying malformation.

Methods: Between 10/2002 and 12/2007 37 patients (pts.) underwent reconstruction of RVOT with either $12 \mathrm{~mm}$ CG (29 pts.) or $14 \mathrm{~mm}$ CG (8 pts.). Pts were divided in three groups: group I: PA/VSD/MAPCA - suboptimal pulmonary artery architecture $(\mathrm{n}=13)$; group II: truncus arteriosus (TAC) $(\mathrm{n}=11)$; groupIII others $(\mathrm{n}=13)$. Mean age at operation was 136 days $(5-780)$.

Results: At the mean FU of 26 months, overall survival rate was $86 \%$, and $81 \%$ at one and two years of FU respectively. Deaths were not conduit related. Six grafts were explanted due to failure. Freedom from CG redo was $87 \%$ and $82 \%$ at 12 and 24 months of FU with no differences among groups. No catheter interventios were needed for CG but pulmonary artery stenosis. At 24 months of FU, freedom from catheter-intervention was $22 \%, 39 \%, 80 \%$ in I ( $p=0.01)$, II and III group respectively. Conclusion: Longevity of small Contegra ${ }^{\mathbb{R}}$-grafts is not effected either by the underlying diseases or small pulmonary arteries (group I). Therefore the use of small CG seems to be justified even for pts with suboptimal pulmonary artery architecture.

\section{1}

Median sternotomy - the preferred surgical approach to achieve complete unifocalization in pulmonary atresia with major aortopulmonary collateral arteries Oleksandr Bablyak, Ukrainian Children's Cardiac Center, Ukraine Roman Sekelyk, Ukrainian Children's Cardiac Center, Ukraine Illya Yemets, Ukrainian Children's Cardiac Center, Ukraine

Objective: To analyze the results of one-stage unifocalization (UF) performed through the median sternotomy in the surgical treatment of pulmonary atresia with major aortopulmonary collateral arteries (MAPCAs).

Methods: Since 2007 we operated on 16 consecutive patients with pulmonary atresia, ventricular septal defect, hypoplastic central pulmonary arteries and MAPCAs. Median age was 8 months. Median weight was $7.1 \mathrm{~kg}$. Median MAPCAs quantity was 4 . All patients received one stage UF through the median sternotomy. Only native tissues were used. Total repair preferentially was done at the same operation either central shunt was performed.

Results: There were no early or late deaths. Total repair was performed at the time of UF in 13 of 16 patients, in 1 more patient - 6 months later. Two more patients with UF and central shunt are awaiting total repair. Early postrepair peak systolic right to left ventricular pressure ratio ranged from 0.45 to 0.8 (median -0.5 ). All patients were followed postoperatively with routine CT angiography and echocardiography. Median follow-up period was 9 months (range - 2-18 months). Two patients required balloon dilation of the pulmonary arteries at 5 and 13 months after surgery and 1 patient required conduit replacement at 9 months.

Conclusions: Complete UF was achieved in all patients through the median sternotomy and was followed by successful total repair in $88 \%$ of them. Presently, one-stage UF through the median sternotomy is our preferred strategy in pulmonary atresia with ventricular septal defect, hypoplastic central pulmonary arteries and MAPCAs.

\section{2}

A twenty year experience of surgery for truncus arteriosus - improved outcomes are not related to younger age at operation

Levi Bassin, The Children's Hospital at Westmead, Sydney, Australia Andrew D Cole, The Children's Hospital at Westmead, Sydney, Australia Gary F Sholler, The Children's Hospital at Westmead, Sydney, Australia 
Ian A Nicholson, The Children's Hospital at Westmead, Sydney, Australia Richard B Chard, The Children's Hospital at Westmead, Sydney, Australia David S Winlaw, The Children's Hospital at Westmead, Sydney, Australia

Introduction: The approach to repair of truncus arteriosus (TA) has changed over the last 20 years, with most units now preferring to operate in the neonatal period. There is a perception that the older patients did better after surgery although delaying operation risks consequences of heart failure. Refinement of perioperative practice and a tendency to now homografts rather than Hancock and non valved conduits also complicate analysis of outcomes.

Methods: We assessed the impact of these factors in our singleinstitution historical cohort. 73 consecutive patients underwent repair of TA $(n=66)$ or hemitruncus $(n=7)$ between 1986 and 2005. Interrupted aortic arch was present in 6 . Era $1(\mathrm{n}=37)$ was 1985 - 1993, era $2(n=36)$ was 1993-2005.

Results: Follow up was complete in 63. There were 21 perioperative deaths ( 30 day), $28 \%$, in the entire cohort with 3 late deaths. Early mortality was 16/37(43\%) in era 1 vs $5 / 36$ $(14 \%)$ in era $2(p<0.05)$. The was no difference in age at operation between eras ( 34 and $28 \mathrm{~d}$, range $0-2.6 \mathrm{y}$ ) although the median age of those that died was $15 \mathrm{~d}$ vs survivors at $34 \mathrm{~d}$ $(p=n s)$. Time to first conduit replacement was 3.4 years for the entire group and was not influenced by conduit choice.

Discussion: Survival in neonates undergoing repair of TA improved significantly between eras. Numerous factors may explain this improvement but age at operation and choice of RVPA conduit do not explain this difference. Neonatal repair with a homograft RV-PA connection remains our current approach.

\section{4}

Results of Modified Norwood's Operation for Hypoplastic Left Heart Syndrome, Chulalongkorn Experience Vichai Benjacholamas, Cardiothoracic Unit, Department of Surgery, King Chulalongkorn Memorial Hospital., Thailand Apichai Khongphatthanayothin, Cardiac Unit, Department of Pediatrics, King Chulalongkorn Memorial Hospital., Thailand Pornthep Lertsapcharoen, Cardiac Unit, Department of Pediatrics, King Chulalongkorn Memorial Hospital., Thailand

Objective: The first-stage palliation for hypoplastic left heart syndrome was analyzed.

Methods: Between August 1996 and November 2008, Modified Norwood's operation with right modified Blalock-Taussig shunt was performed in 26 patients with hypoplastic left heart syndrome. All of them were operated on by modified Norwood's procedure using homograft for arch reconstruction (group 2) except the first four patients that using only autologous great vessel tissue (group 1). Results: Overall hospital mortality was $23.07 \%$. Hospital mortality in group 1 and group 2 were $50 \%$ and $18.18 \%$, respectively. There were four of twenty survivors who reached Fontan type operation (20\%).

Conclusions: Only a limited number of pediatric cardiac centers in our region could offer surgical treatment of hypoplastic left heart syndrome. The survival rate in our study was in ecceptable range despite limited resource.

7165

Complete atrioventricular canal repair less than 1 year with mono- patch techniques in (Australian Technique) chamran open heart center in Isfahan Hamid Bigdelian, CHAMRAN OPEN HEART CENTER ISFAHAN UNIVERSITY, Iran
Abolghasem Mirdehghan, CHAMRAN OPEN HEART CENTER ISFAHAN UNIVERSITY, Iran

Background: The improved repair techniques that have resulted from the increased understanding of atrioventricular Valve anatomy and physiology, In 1999 Nicholson from Australia, described new technique for rapier at complete Av canal, we choice Australian technique in our study.

Methods: Between May 2008 and September 2008, 9 patients with complete atriventricular canal underwent monopatch procedure. All patients were operated on same technique, under hypothermic cardio pulmonary bypass and arrested with blood cardioplegia 4/1 and all patients have same ICU managements.

Results: mean age at surgery was $6 / 81 / 2$ months, mean weight was $.5 .41 \mathrm{~kg}$, trisory 21 was present in 7 patients Early survival was $86 \%$, no patients had residual ventricular septel detect and 3 patients had mild mitral valve regurgitation and no patients had LV outflow obstruction and all patients had increased weight after two months. Conclusions: mono-patch repair in complete atrioventricular canal is a safe and fast technique. Among survival in short time was good a but we need to evaluation in future.

\section{6}

Coronary artery translocation with total autogeneous composit tunnel technique

Mehmet S Bilal, Medicana Hospitals, Istanbul, Turkey

Ece Salihoglu, Medicana Hospitals, Istanbul, Turkey

Numan A Aydemir, Siyami Ersek Hospital, Istanbul, Turkey

Yahya Yildiz, Medicana Hospitals, Istanbul, Turkey

Ali R Karaci, Siyami Ersek Hospital, Istanbul, Turkey

Yalim Yalcin, Medicana Hospitals, Istanbul, Turkey

Ahmet Çelebi, Siyami Ersek Hospital, Istanbul, Turkey

Objective: Coronary artery translocation is a critical stage of arterial switch procedure and surgery of ALCAPA. In some coronary abnormalities direct translocation can be impossible and different surgical techniques has been described to solve this problem. We used total autogenous composit tunnel to faciliate coronary transfer in 7 patients.

Material: This technique consist of elongation of coronary button by creating a tube using part of the wall of pulmonary artery and aorta. Three of the patients have ALCAPA with left coronary artery arising remotely from aorta, other 4 patients were TGA with unusual coronary patterns. Age of the patients ranged between 5 days to 12 years.

Results: All patients had uneventful postoperative course. All patients were followed with echocardiography and ECG. Mean follow up time was $2.6 \pm 1.5$ years. All patients are alive and had NYHA class1 functional status. Nonewly deleopped ischemic finding were presented after surgery until present. Three patient had control angiograhy which demonstrated widely open tunnel and patent coronary circlation.

Conclsion: Total autogenous composit tunnel can be performed succesfully to faciliate coroanary tranfer in some cases. Althought we had limited experience we believe that this technique is a good surgical alternative. We suggest that in situ utilization of autogeneous tissues had advantages of preventing trombosis and restenosis and had growth potentiel.

\section{7}

Double switch operation for congenitally corrected transposition of great arteries: 13 year experience in 11 patients Mehmet S Bilal, Medicana Hospitals, Istanbul, Turkey 
Ece Salihoglu, Medicana Hospitals, Istanbul, Turkey

Yahya Yildiz, Medicana Hospitals, Istanbul, Turkey

Numan A Aydemir, Siyami Ersek Hospital, Istanbul, Turkey

Ali Riza, Siyami Ersek Hospital, Istanbul, Turkey

Yalim Yalçin, Medicana Hospitals, Istanbul, Turkey

Ahmet Çelebi, Siyami Ersek Hospital, Turkey

Objective: Double switch operation (DSO) to restore an anatomic correction seems to be more appealing for long-term prognosis We evaluated our results to determine the advantages and potential future complications of this operation.

Material: Between1995 and 200814 patients were accepted as candidates for DSO. In 4 patients with systemic atrioventricular valve dysfunction pulmonary artery banding was performed first. Of these one had completion of DSO 18 months after the banding procedure. Total of 11 patients had anatomical correction which 8 of them had arterial switch combined with Senning procedure and 3 patients with pulmonary stenosis underwent atrial switch plus a Rastelli type procedure. The median age was 59.8 months, (6 months -8 years).

Results: Median follow up was 57 months, (6 months-13 years). There was no in hospital or late mortality. One patient required early pacemaker implantation after the surgery for complete heart block There was one early (3rd postoperative day) and one late (8 th year) postoperative reintervention for pulmonary venous baffle obstruction. In the latter patient simultaneous surgical radiofrequency ablation for WPW was also performed. Two patients who underwent Rastelli type procedure needed reoperation for conduit obstruction (early thrombectomy for Contegra thrombosis in one and late conduit replacement in the other at postoperative 6th year).

Conclusion: Anatomic correction in C-TGA can be performed with acceptable mortality and morbidity. We believe this procedure could have better prognosis compared to conventional repair. Close follow-up is needed for occurrence of supraventricular arrythmias, atrial baffle and conduit obstruction.

\section{8}

Arterial switch operation - recent experience of 188 cases from a single institution

Lyubomyr Y Bohuta, Ukrainian Children's Cardiac Center, Ukraine

Oleksandr M Romaniuk, Ukrainian Children's Cardiac Center,

Ukraine

Iryna O Aksjonova, Ukrainian Children's Cardiac Center, Ukraine Oleksandr D Bablyak, Ukrainian Children's Cardiac Center, Ukraine Igor G Lebid', Ukrainian Children's Cardiac Center, Ukraine Illya M Yemets, Ukrainian Children's Cardiac Center, Ukraine

Objective: To evaluate our recent experience with arterial switch operation (ASO) for transposition of the great arteries (TGA). Methods: All patients undergoing an ASO at our institution from January 2006, to November 2008, were reviewed. During that period we performed 188 ASO's. There were 119 patients with TGA-IVS (63.2\%), 58 with TGA-VSD (30.9\%) and 11 with TGA-CoA (5.9\%). Taussig-Bing anomaly $(n=9,4.8 \%)$ was referred to the TGA-VSD group. Aortic arch anomalies $(n=11)$ were managed during ASO in all cases. Eight patients underwent ASO after prior PA banding and systemic-to-pulmonary shunt for LV retraining. Coronary pattern other than usual was seen in $63(33.5 \%)$ cases - circumflex from right in $36(19.1 \%)$; single in $8(4.3 \%)$; inverted coronaries in $11(5.9 \%)$; intramural in 2 (1.1\%), inverted circumflex and right in $6(3.1 \%)$.

Results: There were 8 hospital deaths (4.3\%) with only 3 of them among last 120 cases. Coronary and aortic arch anatomy could not be shown to affect survival. Postoperative period was uncomplicated in $163(86.7 \%)$ cases. Median ICU stay was 6.8 (range 4-38) days. There were 2 late deaths due to non-cardiac causes.

Conclusions: In the current era ASO can be safely performed for both TGA-IVS and TGA-VSD. Complex anatomical patterns including unusual coronaries and aortic arch anomalies were not found to affect early survival in our study as well.

\section{9}

Is vertical vein patency beneficial after repair of total anomalous pulmonary venous connection?

Lyubomyr Y Bohuta, Ukrainian Children's Cardiac Center, Ukraine Evgen V Segal, Ukrainian Children's Cardiac Center, Ukraine Illya M Yemets, Ukrainian Children's Cardiac Center, Ukraine

Objective: Surgical approach to vertical vein (VV) during repair of total anomalous pulmonary venous connection (TAPVC) remains controversial. We aimed to evaluate the effect of different approaches on immediate surgical outcomes.

Methods: Between January 2000 and September 2008, 93 patients underwent isolated TAPVC repair at our institution. Fifty-seven patients who had ascending or descending VV were included in this study. Twenty five patients $(43.9 \%)$ were younger than 1 month, and 27 (47.4\%) patients were between 1 and 12 months. There were 41 patients $(71.9 \%)$ with supracardiac type TAPVC, $12(21.1 \%)$ - with infracardiac type and $4(7 \%)$ - with mixed type. VV was ligated in 45 patients (group I) and left patent in 12 (group II). There was no significant difference between patient groups in terms of age, weight, anatomical pattern and preoperative condition. In all the cases decision to ligate VV was left to discretion of the operating surgeon.

Results: Postoperative period was uncomplicated in 34 (75.6\%) patients in group I and only in $2(16.7 \%)$ patients in group II. Twelve patients $(21.1 \%)$ died in the postoperative period with only 2 deaths among last 30 cases $(6.7 \%)(p=0.007)$. Patent VV was shown to negatively affect survival and degree of heart failure during both univariate and multivariate analyses.

Conclusions: Results of surgery for TAPVC have significantly improved recently. In our study patent VV in the postoperative period was found to be an independent risk factor for early death.

\section{0}

The outcome of pulmonary artery banding as used in a staged approach to defer primary definitive biventricular repair in perceived high-risk cases

Andre Brooks, Christiaan Barnard Department of Cardiothoracic Surgery, University of Cape Town, Red Cross Children's Hospital, South Africa

Agnetha Geldenhuys, Christiaan Barnard Department of Cardiothoracic Surgery, University of Cape Town, Red Cross Children's Hospital, South Africa

Paul Human, Christiaan Barnard Department of Cardiothoracic

Surgery, University of Cape Town, South Africa

Liesl Zuhlke, Department of Paediatric Cardiology, University of Cape Town, Red Cross Children's Hospital, South Africa

Objective: Intra-cardiac defects causing large left-right shunts may lead to severely ill patients with potential high risk for cardiopulmonary bypass and definitive repair. We test the hypothesis that surgical risk may be lowered by a staged approach when a pulmonary artery band $(\mathrm{PAB})$ is first applied.

Method: A retrospective review of all $\mathrm{PAB}$ operations done at our institution between January 2002 and December 2007 was done. 
Data was analyzed further if the aim was to later achieve a biventricular repair.

Results: Of the 187 files that were reviewed 144 had a PAB with a view to later achieve a bi-ventricular repair(30 excluded due to uni-ventricular pathway, 13 files lost). Of these $(\mathrm{N}=144)$ the total mortality, defined as all cause mortality until the time of definitive repair, was $24 \%(\mathrm{~N}=35)$. The in-hospital mortality was $8 \%(\mathrm{~N}=12)$ and the inter-stage mortality was an additional $16 \%$ $(\mathrm{N}=23)$. In the majority positive growth was not documented following PAB application. The mean weight at PA banding was $5 \pm 3 \mathrm{~kg}$ (range, 1.8 to $25 \mathrm{~kg}$ ). The mean time interval between PA banding and definitive repair was $22 \pm 13$ months. The mean ICU stay at PAB was $7 \pm 15$ days with a mean hospital stay of $21 \pm 34$ days.

Conclusion: Despite an acceptable in-hospital mortality, the practice of deferring bi-ventricular repair by the application of a pulmonary artery band carries such a high total mortality that consideration should be given to early definitive repair even in perceived high risk cases.

7171

Modification of the Ross Aortic Valve Replacement to Prevent Late Autograft Dilatation

John W Brown, Indiana University School of Medicine, Indianapolis, Indiana, United States

Mark Ruzmetov, Indiana University School of Medicine, Indianapolis, Indiana, United States

Ali P Shahriari, Indiana University School of Medicine, Indianapolis, Indiana, United States

Mark D Rodefeld, Indiana University School of Medicine, Indianapolis, Indiana, United States

Mark $W$ Turrentine, Indiana University School of Medicine, Indianapolis, Indiana, United States

Background: Aortic root dilatation with and without aortic regurgitation is seen in up to $20 \%$ of patients undergoing the Ross aortic root replacement at late follow-up. We present our early experience with reduction annuloplasty in combination prosthetic Dacron graft replacement of the aorta above the autograft to prevent late dilation after the Ross aortic root replacement.

Methods: Since 2001, 27 of 81 adult and pediatric patients (mean age $28.8+14.8$ years; range $8-53$ years) with bicuspid aortic valve and dilatation of the ascending aorta underwent a modified Ross procedure with reduction annuloplasty in combination prosthetic Dacron graft replacement of the ascending aorta. The diameter of the ascending aorta was measured before and early after surgery and then between 3 months and 7 years (mean, $2.1+2.0$ years) .

Results: There were no early or late deaths. Reduction annuloplasty combined with ascending aortic graft replacement decreased the ascending aortic diameters from $41.9+7.4 \mathrm{~mm}$ preoperatively to $25.1+2.8 \mathrm{~mm}$ early after surgery $(\mathrm{P}<0.001)$. During follow-up, there was no significant increase of the aortic diameter compared with the postoperative period $(26.9+4.3 \mathrm{~mm} ; \mathrm{P}=0.08)$. The root diameter increased in only $3(11 \%)$ of 27 patients. No patient underwent reoperation. At last follow-up, mild $(\mathrm{n}=14)$ or trivial $(\mathrm{n}=13)$ aortic regurgitation was observed.

Conclusions: Ross aortic root replacement combined with reduction annuloplasty and Dacron graft replacement of the ascending aorta demonstrated excellent early- to mid-term results in patients with bicuspid aortic valve and dilatation of the ascending aorta. Continued use and long-term follow-up imaging is necessary to further demonstrate the value of this technique.
7172

Mitral Valve Replacement with Ross II Technique: Intermediate-Term Outcome and Autograft Function

John W Brown, Indiana University School of Medicine, Indianapolis, Indiana, United States

Mark Ruzmetov, Indiana University School of Medicine, Indianapolis, Indiana, United States

Mark $W$ Turrentine, Indiana University School of Medicine, Indianapolis, Indiana, United States

Background: Pulmonary autograft mitral valve replacement (PAMVR) was introduced clinically by Ross in 1967 and recently modified by Kabbani, but has been rarely utilized in North America and Europe. The aim of this study is to review our continued experience with PA-MVR.

Methods: Since June 2002, 9patients between 12 to 46 years of age with outgrown mechanical valves $(n=3)$, failed $M V$ repair for rheumatic or congenital MV disease $(n=5)$ and irreparable bileaflet myxoid prolapse $(n=1)$ underwent PA-MVR. The pulmonary autograft was harvested and replaced using a cryopreserved pulmonary homograft.

Results: There were no early or late deaths. Intraoperative echocardiography confirmed a trivial $(n=8)$ or mild $(n=1)$ regurgitation. Follow-up (range 2months to 6years) echocardiography in 5patients showed no increase in MV gradient or regurgitation. Four patients have required reoperation:due to progression of trivial-to-moderate regurgitation due to stretching of a single autograft leaflet producing prolapse $(n=3)$, and a moderate gradient due to retention of excessive native mitral leaflet and subannular chordal tissue $(n=1)$. All 4 patients had an uneventful PA-MVR using a mechanical prosthesis. Freedom from reoperation is $56 \%$ at 6 years.

Conclusions: PA-MVR offers selected patients a potentially lifelong autologous valve without the need for long-term anticoagulation. The PA-MVR technique deserves careful consideration in younger patients in sinus rhythm. Postoperative systemic hypertension should be treated aggressively to prevent excessive stress on the PA particularly in the early postoperative months. The Dacron external support should not be oversized. Long term follow-up of more patients is required to determine the long-term utility of this very attractive alternative to prosthetic MVR in young patients.

\section{3}

Transapical Myectomy Provides Effective Relief of Midventricular Obstruction in Hypertrophic Cardiomyopathy Morgan L Brown, Mayo Clinic, United States Joseph A Dearani, Mayo Clinic, United States Steve R Ommen, Mayo Clinic, United States Rick A Nishimura, Mayo Clinic, United States Patrick W O'Leary, Mayo Clinic, United States Hartzell V Schaff, Mayo Clinic, United States

Objective: Hypertrophic cardiomyopathy (HCM) is a congenital condition with a broad spectrum of phenotypic variability. A small group of patients may develop symptomatic midventricular obstruction which can be difficult to eliminate through a transaortic approach alone. Our objective was to evaluate the early outcomes of a transapical approach for relief of midventricular obstructive HCM.

Methods: Between 1985 and 2007, 13 symptomatic patients with obstructive midventricular HCM underwent transapical midventricular myectomy. Information from a prospective database was reviewed. 
Results: The median age was 40 years (range 11-65); 58\% were female. Twelve patients (92\%) had fatigue and shortness of breath, $9(69 \%)$ had presyncope or syncope, $7(54 \%)$ had palpitations, and $5(46 \%)$ had angina. The mean preoperative EF was $74 \pm 7 \%$. All patients were on medical therapy: calcium channel blockers $(\mathrm{n}=9)$, beta-blockers $(\mathrm{n}=8)$, and disopyramide $(\mathrm{n}=2)$. Atrial arrhythmias were present in 2 patients, ventricular arrhythmias in 3 , and 5 patients had an AICD. The median preoperative resting midcavitary gradient was $36 \mathrm{mmHg}$; provoked gradient was $52 \mathrm{mmHg}$. All patients had a transapical midventricular myectomy and 8 patients had concomitant transaortic septal myectomy; $9.9 \pm 3.7 \mathrm{~g}$ of muscle was removed. There were no iatrogenic injuries to the aortic or mitral valve and there were no early ventricular arrhythmias. All patients had a successful reduction in midcavitary gradients (postoperative $7 \pm 11 \mathrm{mmHg}$ ) with a mean EF of $67 \pm 11 \%$.

Conclusions: Midventricular myectomy through an apical incision effectively eliminates midventricular obstruction in symptomatic patients without significant change in left ventricular function. Further follow up is needed to determine if this approach impacts late left ventricular function or arrhythmogenic potential.

7174

Early experience in surgical treatment of complete atrioventricular septal defect with patient's own tissue Piotr Burczynski, Cardiothoracic Department, Childrens' Health Memoral Hospital Warsaw, Poland

Krzysztof Mozol, Cardiothoracic Department, Childrens' Health Memoral Hospital Warsaw, Poland

Michal Kozlowski, Cardiothoracic Department, Childrens' Health Memoral Hospital Warsaw, Poland

Mariusz Birbach, Cardiothoracic Department, Childrens' Health

Memoral Hospital Warsaw, Poland

Andrzej Kansy, Cardiothoracic Department, Childrens' Health

Memoral Hospital Warsaw, Poland

Malgorzata Malek, Cardiothoracic Department, Childrens' Health

Memoral Hospital Warsaw, Poland

Wanda Kawalec, Cardiology Department, Childrens' Health Memoral Hospital Warsaw

Bohdan Maruszewski, Cardiothoracic Department, Childrens' Health Memoral Hospital Warsaw

Background: In current practice, correction of CAVSD can be performed using one of the three techniques: single patch, two patch or modified single patch. We summarize our experience with two patch technique using glutarladehyde-treated autologous pericardial patch for closure of CAVSD.

Materials and Methods: 30 children (11 males, 19 females) underwent CAVSD repair using glutaraldehyde-treated autologous pericardium between January 2006 and October 2008. The age and weight at the time of surgery were: 63-362 days (median - 179 days), and $3.3-8.0 \mathrm{~kg}$ (median $5.0 \mathrm{~kg}$ ). After establishing cardiopulmonary bypass, a strip of pericardium was harvested from the patient, bathed in $0,6 \%$ glutharaldehyde solution for between 10 and 12 minutes, and subsequently washed out with $0,9 \%$ saline solution. Follow-up time varied from 20 to 773 days (median - 369 days).

Results: There was a 90\% 1-year survival, with no 30-day mortality found. Of the two children who died of non-cardiac reasons, one was in critical condition in pre-operative period. Postoperative 2D echocardiograms have shown moderate left $\mathrm{AV}$ valve incompetence in $7(24.1 \%)$, and severe in $4(13.8 \%)$. 3 patients required reoperation due to residual shunt (2), and/or severe left AV valve incompetence. One patient required pacemaker.
Conclusions: Glutharaldehyde-treated autologous pericardium is an excellent material for surgical repair of CAVSD. Due to the flexibility and compliance of the pericardium we believe it prevents LVOT obstruction and diminishes AV incompetence. Although our results are promising, further follow-up is required to assess long-term efficacy of this method.

\section{7}

Surgical correction of an anomalous origin of the left pulmonary artery branch from the ascending aorta in an infant with sub aortic obstruction

Jorge L Cervantes, "Ignacio Chávez” National Cardiology Institute of Mexico, Mexico

Pedro J Curei-Curi, "Ignacio Chávez" National Cardiology Institute of

Mexico, Mexico

Samuel E Ramirez-Marroquin, "Ignacio Chávez" National

Cardiology Institute of Mexico, Mexico

Antonio Razo, "Ignacio Chávez" National Cardiology Institute of Mexico, Mexico

Juan Calderon-Colmenero, "Ignacio Chávez” National Cardiology Institute of Mexico, Mexico

Objective: The aim of this study is to describe a clinical case of anomalous origin of the left pulmonary artery branch from the ascending aorta, and to present a current literature review of this rare disease.

Methods: A 2 year-old infant was referred to our institution for surgical correction of sub aortic obstruction. The non invasive investigation disclosed a right aortic arch anatomy with left ventricle outflow tract obstruction due to sub aortic membrane associated with an anomalous origin of the left pulmonary artery branch from the ascending aorta. Immediately after diagnosis, the patient underwent a total surgical correction of the defects, including simultaneous anastomosis of the left pulmonary artery to the pulmonary trunk.

Results: Results of the surgical repair of this case were successful. We also present a literature review of the incidence, pathophysiological, clinical, diagnostic, and surgical characteristics of this rare disease.

Conclusions: Although anomalous origin of the left pulmonary artery branch from the ascending aorta is a rare disease, successful results can be obtained when early diagnosis and opportune total surgical corrections are performed.

\section{8}

Biventricle Repair of Double Outlet Right Ventricle with Non-Committed Ventricular Septal Defect

Chung-I Chang, National Taiwan University Hospital, Taiwan Ing-Sh Chiu, National Taiwan University Hospital, Taiwan Yih-Sharang Chen, National Taiwan University Hospital, Taiwan Jou-Kou Wang, National Taiwan University Hospital, Taiwan Mei-Hwan Wu, National Taiwan University Hospital, Taiwan

Materials and Methods: From Oct 1996 to Jane 2008, twenty patients of DORV with non-committed VSD underwent biventricular repair. The age distribution is from 13 days to 23 years old (Mean 2.8 years old). The sex ratio of male to female is equal. The associate anomalies included pulmonary stenosis and atresia 6 ,coarctation of aorta 4 ,straddling of atrioventricular valve 3, ECD 2, juxtaposed atrial appendage 2, LV diverticulum 1, anatomic corrected malposition (ACM) 2, TAPVR 1. Eleven patients had palliative procedure (systemic-pulmonary shunt 4 , PA banding 7, CoA repair 4). The definite surgical procedures 
were performed in 20 patients. The definite surgical procedures divided into two group. Group I, Five patents underwent arterial switch opeation. Three of them had VSD enlargement. Group 2, Fifteen patients underwent intraventricular rerouting procedure thirteen of them had VSD enlargement. Only one had extracardiac conduit.

Results: There are three early mortality, 2 in group 1 and 1 in group 2, and no late mortality. All of them are neonates and small infant ( $\leq 3$ months). The follow-up period was 3 months to 10 years. The postoperative complications are AV block 1, residual LVOTO 3 (pressure gradient $<20 \mathrm{mmHg}$ ), residual PS 3 (pressure gradient $\leq 45 \mathrm{mmHg}$ ) and mitral regurgitation 1 .

Conclusion: Biventricular repair in feasible in DORV with noncommitted VSD and certain anatomical features. Neonatal definite repair will be high risk in our series. Intra-ventricular LVOT rerouting procedure is recommended. Precise preoperative evaluations will determined the type of the definite repair.

\section{9}

Double outlet right ventricle (DORV) with subpulmonary ventricular septal defect (the Taussig-Bing heart): analysis of surgical outcome and predictors of prognosis Chung-I Chang, National Taiwan University Hospital, Taiwan Ing-Sh Chiu, National Taiwan University Hospital, Taiwan Yih-Sharang Chen, National Taiwan University Hospital, Taiwan Jou-Kou Wang, National Taiwan University Hospital, Taiwan Mei-Hwan Wu, National Taiwan University Hospital, Taiwan

Materials and Methods: From August 1995 to August 2008. There were 34 Taussig-Bing complex in NTUH, 10 female and 24 male. The location of VSDs were subpulmonary, diameter ranging from $3 \mathrm{~mm}$ to $20 \mathrm{~mm}$. Fourteen cases were associated with coarctation of aorta or hypoplastic aortic arch (41.1\%). Twenty-nine cases received definite surgery $(85.3 \%)$, either arterial switch operation (ASO) with VSD repair $(26,89.9 \%)$, or Kawashima procedure with Rastelli conduit (3, 10.1\%). The rest 5 partiers had complex intracardiace anomalies(1 with severe LVOTO and 3 hypoplastic LV) and Eisemenger reaction. They all were excluded. Patients with definite repairs were separated into 2 groups: before 1999 (Group I) and after 1999 (group II). The operation results and long-term follow-up were compared among group I and II. Factors such as VSD size, associated anomaly, age at operation, presence of subaortic or subpulmonary stenosis were analyzed.

Results: Before 1999, there were 14 cases in Group I receiving definite surgery, including ASO(12) or Kawashima procedure with Rastelli conduit (2), and there were 7 early mortality within 30 days post-op (50\%). After 1999, there were 15 Taussig-Bing cases receiving definite surgery: ASO in 14 cases and Kawashima procedure with Rastelli conduit in 1 case. There was 2 early mortality case (13.3\%). The long-term survival was steady in OPD follow-up, except for one sudden death case in groupI 4 years after operation. Hypoplastic LV and restrictive VSD are the risk factors. Conclusion: Arterial switch operation will be the surgical treatment of choice with satisfactory result for the Taussig-Bing anomaly.

\section{0}

Transposition of the Great Arteries with Posterior Aorta Chung-I Chang, National Taiwan University Hospital, Taiwan Ing-Sh Chiu, National Taiwan University Hospital, Taiwan Jou-kon Wang, National Taiwan University Hospital, Taiwan Mei-Hwan Wu, National Taiwan University Hospital, Taiwan
Transposition of the great arteries (TGA) with posterior aorta was firstly reported in 1971 and the first successful arterial switch operation was not accomplished until 1990. We reported 6 infants of TGA with posterior aorta during 1993 and 2005 of which underwent arterial switch operation. The other died before operation. The age at operation ranged 8 days to 7 months old. All of them received cardiac catheterization and balloon arterial septostomy before operation. Typical picture of ventricular arterial discordance with aorta located posterior to the main pulmonary artery was delineated. We performed arterial switch operation without Lecompte maneuver in all the five patients. The ventricular septal defects (VSD) were closed through the native aortic root after aortic transaction. All but one of the five patients were doing well during follow-up except mild to moderate pulmonary stenosis. We concluded TGA with posterior aorta could be successfully corrected by arterial switch operation during early infancy. VSD closure through native aortic root and no Lecompte maneuver were recommended. There is no late mortality.

\section{1}

Truncus Arteriosus with intact ventricular septum (Van Praagh Type B1) - delayed presentation and innovative septation without conduit

Maitri Chaudhuri, WOCKHARDT HOSPITALS,

BANGALORE, INDIA, India

N S Devananda, WOCKHARDT HOSPITALS,

BANGALORE, INDIA, India

$M$ Jarranganth, WOCKHARDT HOSPITALS, BANGALORE, INDIA, India

Subash V Chandra, WOCKHARDT HOSPITALS, BANGALORE, INDIA, India

Type B1 Truncus Arteriosus (Van Praagh) was reported before as autopsies or fetuses with hypoplastic ventricle. We report a unique patient who presented late, had two adequate ventricles and successfully underwent conduitless repair into separate ventricular outflows.

Case Report: A 4 years old girl presented with growth retardation and frequent pneumonias. She was acyanotic with hyperdynamic precordium, single S2, prominent early diastolic murmur. ECG, ChestX-ray showed moderate cardiomegaly (LV contour), plethoric lungs. Echocardiography revealed intact cardiac septae, normal systemic venous return, dilated left heart, adequate right heart, common outlet (type I truncus arteriosus), redundant truncal valve, moderate regurgitation and severe $\mathrm{PAH}$. Our dilemmas were absence of intracardiac shunt and RV connection to truncus. Cardiac catheterization revealed operable hemodynamics but failed to distinguish from proximal aortopulmonary window.

Peroperatively, a single semilunar valve seen with annulus directly attached to crest of IVS without VSD. It had three leaflets: anterior, rightlateral and posterior (latter had pseudocommissure). Rightlateral leaflet was directly committed to Aorta and other leaflets were doubly committed to both great vessels. Utilising the pseudocommissure, the common valve was septated by Goretex patch into separate pulmonary and aortic outflows. PApressure normalized immediately.Postoperative echo showed laminar RV and LVOTs, mild AR, moderate PR, normal ventricular function.

Conclusion: Type B1 Truncus with this particular morphology allowed survival without VSD and septation. Echocardiography correctly identified the lesion,angiography was inconclusive. This unique conduitless strategy hopefully means decreased reoperations. Selective incomplete development of distal conal cushion 
with normal development of proximal and middle cushions is a possible embryological explanation.

\section{2}

Which is the best approach to repair tetralogy of Fallot ten years' experience in Shanghai Children Medical Cente Huiwen Chen, Department of Pediatric Cardiothoracic Surgery, Shanghai Children's Medical Center, Shanghai Jiaotong University, Shanghai, China

Zhiwei Xu, Department of Pediatric Cardiothoracic Surgery, Shanghai Children's Medical Center, Shanghai Jiaotong University,

Shanghai, China

Objective: Transatrial repair of tetralogy of Fallot (TOF) has become a standard procedure at any age.Elective repair is generally recommended between 3 and 6 months of life. Delayed presentation in China further compound disease severity. Secondary hypertrophy in the right ventricule and small pulmonary valve ring make difficulty by transatrial approach. Transatrial with minimal right ventriculotomy approach (RA-RV) was performed since 2000. To compare the perioperative outcomes with tranvebtricular and transatrial-transventricular approach to TOF.

Methods: Fifteen hundreds and fifteen consecutive patients who underwent complete repair of tetralogy of Fallot between January 1998 and December 2007 were reviewed.

Results: Patients by transventricular approach decreased from $88 \%$ in 1998 to $57 \%$ in 2002. Transatrial-transventricular approach increased from $38 \%$ in 2002 to $61 \%$ in $2003,77 \%$ in 2007. Patients by transatrial-transventricular approach had less aortic clamp time and $\mathrm{CPB}$ time than transventricular approach. No difference in transannular patch ratio. Patients by transatrialtransventricular approach were better in the median ICU length of stay and mechanical ventilation time 3 ICU, residual VSD, RVMPA pressure gradient, TR, PI and JET than transventricular approach. No difference in MOF. Patients by transatrialtransventricular approach had lower morbidity in one to two organ systems dysfunction than transventricular approach. No difference in 3 or more organ systems dysfunction between them. Reoperative and mortality in patients by transatrial-transventricular approach less than transventricular approach.

Conclusions: Transatrial-transventricular approach to repair TOF fits to actual conditions in China.

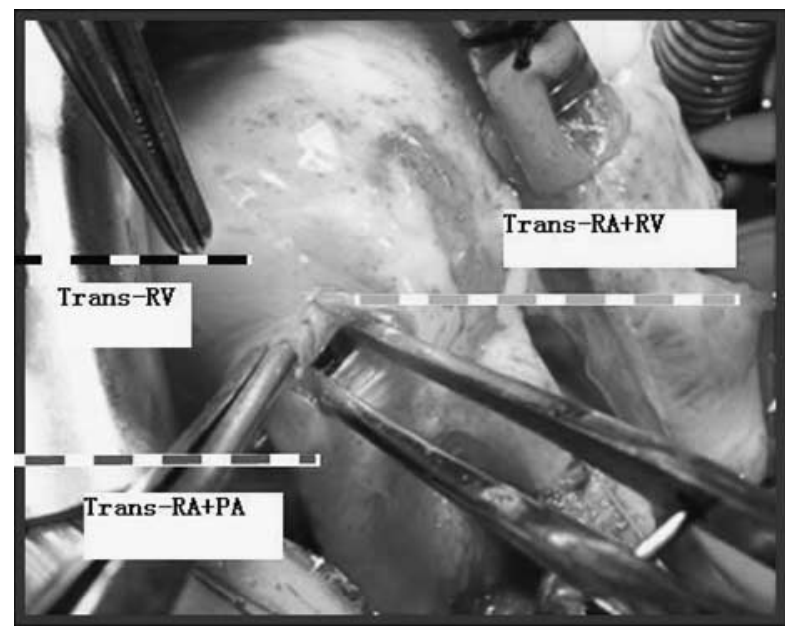

7183

Individual or institutional learning curves : the arterial switch operation in a mid-size center model
Caroline Chenu, Timone University Hospital, Marseille, France, France Virginie Fouilloux, Timone University Hospital, Marseille, France, France Olivier Ghez, Timone University Hospital, Marseille, France, France Dominique Metras, Timone University Hospital, Marseille, France, France Bernard Kreitmann, Timone University Hospital, Marseille, France, France

Objectives: Concept of "learning curve" is well known. But currently, suboptimal results due to learning curve are less tolerated by professional and public, especially in pediatric cardiac surgery. Furthermore, relationship between patient volume and results are matter of concern.

The aim of this study is the assessment of outcomes after arterial switch operation (ASO) in a mid-size center over time, then outcomes for each surgeon, to analyze relationship between institutional and individual surgical learning curves, and its consequences on patient safety.

Methods: We took ASO for transposition of great arteries (TGA) as a model of complex procedure requiring experience. We analyzed and compared mortality and morbidity rates at the institutional level and for each surgeon over time.

Results: From January 1985 to December 2008, 319 ASO were performed in our institution. Institutional mortality rate decreased from $27,7 \%$ to $3 \%(\mathrm{p}<0,05)$ over these 23 years, with a rapid decrease in the 5 first years, time of "learning curve" of surgery and post-operative care. The same evolution was found for the first surgeon, whom performed ASO since 1985. On the other hand, a second surgeon, with the mentorship of the first, and subsequently a third surgeon, experienced a low rate of mortality since their beginnings, in 1990 and 2003 respectively, and institutional mortality rate continued to decrease.

Conclusions: Mentorship, even in a mid-size institution, can offset the lack of experience of young surgeons, allowing them to perform their learning curve without adverse effects on patient safety.

\section{5}

Is low weight in congenital cardiac surgery still a risk factor - a retrospective single institution early mortality analysis

Mi-Young Cho, Department of Cardiac Surgery, Heart Center, University of Leipzig, Struempellstr. 39, 04289 Leipzig,

Germany, Germany

Thomas Walther, Department of Cardiac Surgery, Heart Center, University of Leipzig, Struempellstr. 39, 04289 Leipzig,

Germany, Germany

Ardawan Rastan, Department of Cardiac Surgery, Heart Center, University of Leipzig, Struempellstr. 39, 04289 Leipzig,

Germany, Germany

Jörg Kempfert, Department of Cardiac Surgery, Heart Center,

University of Leipzig, Struempellstr. 39, 04289 Leipzig,

Germany, Germany

Friedrich Wilhelm FW Mohr, Department of Cardiac Surgery,

Heart Center, University of Leipzig, Struempellstr. 39, 04289 Leipzig,

Germany, Germany

Martin Kostelka, Herzzentrum Leipzig, Germany

Objective: Due to tremendous improvements congenital cardiac surgery in neonates can be performed with reasonable results. But it is reported that low weight remains a risk factor for cardiac surgery.

Methods: We analyzed early mortality of patients weighing $4 \mathrm{~kg}$ and less who underwent cardiac surgery at our centre between September 1998 and February 2008. Of this total of $n=564$ consecutive patients we formed two groups according to their weight. Group1 included patients weighing less or equal $2.5 \mathrm{~kg}$ 
$(\mathrm{n}=87)$ and was compared with Group2 with weight at operation 2.6 to $4 \mathrm{~kg}(\mathrm{n}=477)$. Furthermore we analyzed the predictive value of the Aristotle Basic Complexity scores.

Results: Overall $\mathrm{n}=23$ (4\%) died early, whereas the early mortality in this challenging Group1 was $10.3 \% \quad(14.8 \%$ on-pump, 3\% off-pump)compared to Group2 with 2.9\% (3.4\% on-pump, $1 \%$ off-pump). Median age were nearly the same with $11 \mathrm{~d}$ rather $12 \mathrm{~d}$. Higher mortality was associated with extended bypass time. Early mortality was high after palliation in Group1 $\mathrm{n}=7$ (22.6\% of all palliations), compared to Group $2 \mathrm{n}=6$ $(5.6 \%)$. The highest mortality was recognizeable in Group 1 with functional single ventricle after on-pump palliation (40\%). $22 \%$ of infants in Group1 with with the highest Aristotle Complexity Score 4 died early in comparison just 3\% in Group 2 with the same scoring.

Conclusion: Congenital cardiac surgery in low weight infants can be performed with reasonable results but with elevated mortality compared to normal weight patients. Especially on-pump palliation in single ventricle morphology is associated with higher mortality in low weight neonates.

\section{6}

Outcome of single stage total correction of coarctation of aorta with ventricular septal defect

SeongHo Cho, Department of Thoracic and Cardiovascular Surgery

Samsung Medical Center, Sungkyunkwan University School of

Medicine, Korea

Tae-Gook Jun, Department of Thoracic and Cardiovascular Surgery

Samsung Medical Center, Sungkyunkwan University School of

Medicine, Korea

Ji-Hyuk Yang, Department of Thoracic and Cardiovascular Surgery

Samsung Medical Center, Sungkyunkwan University School of Medicine, Korea

Pyo Won Park, Department of Thoracic and Cardiovascular Surgery Samsung Medical Center, Sungkyunkwan University School of Medicine, Korea

Kiick Sung, Department of Thoracic and Cardiovascular Surgery Samsung Medical Center, Sungkyunkwan University School of Medicine, Korea

Wook Sung Kim, Department of Thoracic and Cardiovascular Surgery Samsung Medical Center, Sungkyunkwan University School of Medicine, Korea

Young Tak Lee, Department of Thoracic and Cardiovascular Surgery Samsung Medical Center, Sungkyunkwan University School of Medicine, Korea

Objective: The optimal surgical strategy for the repair of coarctation of aorta with ventricular septal defect (VSD) is controversial. This study was undertaken to determine outcome of single stage total correction of coarctation of aorta with VSD in the single institution. Method: Retrospective review of patients undergoing single stage total correction between January 1, 1995 and December 31, 2007.

Results: Single stage total correction was performed in 72 patients (43 males) at a median age of 28 days (range: $3 \sim 188$ days) and median weight of $3.7 \mathrm{~kg}$ (range: $2.16 \sim 5.6 \mathrm{~kg}$ ). The VSD was perimembranous in 42 , subarterial in 26 and muscular in 4 patients. Arch hypoplasia was present in 63 patients. Repairs were performed via a median sternotomy. Total circulatory arrest was performed in 22 patients and selective antegrade cerebral perfusion was performed in 49 patients. There was no operative death and one late death at a median follow-up of 38.2 months (range; $0 \sim 158.1$ months). Left main bronchus compression which needed aortopexy occurred in 1 patient. Postoperative subaortic stenosis occurred in 4 patients and surgical repair were performed in 2 patients. Recoarctation of aorta occurred in 2 patients and intervention with balloon dilatation was performed in 1 patient.

Conclusion: The advantages of single stage total correction over 2 stage repair of coarctation of the aorta with VSD include an earlier age at completion of repair and it can be performed with low mortality and morbidity as in this study.

\section{7}

Total Anomalous Pulmonary Venous Connection: 12 Years Experience in a Medical Center

Tzu-Yao Chuang, Pediatric Cardiology, China Medical University Hospital, Taichung, Taiwan, Taiwan

Ping-Chun Li, Cardiovascular Surgery, China Medical University Hospital, Taichung, Taiwan, Taiwan

Jeng-Sheng Chang, Pediatric Cardiology, China Medical University Hospital, Taichung, Taiwan, Taiwan

Background: Among all correctable congenital heart disease, total anomalous pulmonary venous connection (TAPVC) is known for its high rates of mortality and morbidity.

Methods: During the period of 1996 through 2008, twenty-four patients of TAPVC without heterotaxy syndrome received total correction surgery in our hospital. Their charts were reviewed. Results: Twelve were male and 12 were female. Supracardiac, cardiac and infracardiac types of TAPVR appeared in 6, 12 and 6 patients. Their age of diagnosis ranged from one-day-old to 20year-old (median: 7-days-old). Fourteen patients (58\%) received urgent operation soon after birth, most were infracardiac or supracardiac type. Pulmonary hypertension occurred in 13 patients after operation and were managed with inhale NO. Early death ( $<30$ days) occurred in 2 patients $(8 \%)$. Six patients $(25 \%)$ developed postoperative progressive pulmonary vein stenosis (PPVS), including 3 infracardiac, 1 supracardiac, 2 cardiac type (directly to RA). Five of them were found postoperatively less than 3 months. Two of the 6 patients had anastomic stenosis, 1 ostial stenosis, and the other 3 had both anastomic and ostial stenosis. Reoperation trying to relieve PPVS by pericardial patch augmentation were performed on 2 patients and 1 died. Overall, 2 patients (33\%) expired lately due to PPVS. One patient who had unilateral PPVS survived.

Conclusion: Urgent operation to rescue neonates with obstructed TAPVC is associated with high rates of mortality and complications, especially PPVS.

\section{8}

Right Ventricular Outflow Tract Obstruction after the Arterial Switch Operation - can we prevent it? Julie Cleuziou, Department of Cardiovascular Surgery, German Heart Centre Munich, Germany

Christian Schreiber, Department of Cardiovascular Surgery, German

Heart Centre Munich, Germany

Jürgen Hörer, Department of Cardiovascular Surgery, German Heart Centre Munich, Germany

Zsolt Prodan, Department of Cardiovascular Surgery, German Heart Centre Munich, Germany

Manfred Vogt, Department of Paediatric Cardiology and Congenital

Heart Disease, German Heart Centre Munich, Germany

Rüdiger Lange, Department of Cardiovascular Surgery, German Heart Centre Munich, Germany

Objectives: The arterial switch operation (ASO) shows excellent early and long-term results. The aim of the present study was to 
evaluate the incidence of right ventricular outflow tract (RVOT) obstructions after the ASO and determine its risk factors.

Methods: A total of 513 arterial switch operations were performed since 1983. Simple transposition of the great arteries (TGA) was present in 319 patients, 152 patients had an additional VSD and 42 patients had a Taussig-Bing (TB) anomaly.

Results: Out of 480 early survivors, 44 (9\%) required an intervention for RVOT obstruction (surgical intervention, $\mathrm{n}=31$ ) at a mean time of 3 years after the ASO. Most obstructions were beyond the pulmonary bifurcation $(n=16,36 \%)$, whereas 10 patients $(22 \%)$ had a predominantly supravalvar obstruction, 6 patients $(14 \%)$ had a subvalvar obstruction, 1 patient $(2 \%)$ had a valvar obstruction and 11 patients (25\%) had obstructions at different levels of the RVOT. Freedom from reintervention was better for simple TGA, compared to patients with TGA+VSD $(p=0.02)$, and for patients with TB $(p<0.001)$. The multivariate analysis identified only the repair of an additional aortic obstruction $(\mathrm{p}<0.001)$ as an independent risk factors.

Conclusion: A concern about the right ventricular outflow tract after the ASO is justified. Its development can affect different levels of the RVOT and might be avoided. Patients with an aortic obstruction have a higher risk of developing a RVOT obstruction and might benefit from a subaortic resection at time of ASO. Peripheral stenosis can be prevented by thorough dissection of the pulmonary arteries.

\section{9}

\section{Assisted Fontan procedure}

Antonio F Corno, Alder Hey Children Foundation NHS Trust, Liverpool, United Kingdom

Chellappan Subramanian, Alder Hey Children Foundation NHS

Trust, Liverpool, United Kingdom

Nelson Alphonso, Alder Hey Children Foundation NHS Trust, Liverpool, United Kingdom

Gordon Gladman, Alder Hey Children Foundation NHS Trust, Liverpool, United Kingdom

Jonathan C Jarvis, Human Anatomy \& Cell Biology, University of

Liverpool, Liverpool, United Kingdom

Objectives: The main limitation for indication to a Fontan procedure is the presence of elevated Pulmonary Vascular Resistance (PVR).

A skeletal muscle (Latissimus Dorsi = LD) intermittently stimulated with a pulse-generator has been proven to become a powerful fatigue-resistant muscle.

We tested the hypothesis that an extra-cardiac Fontan connection including a uni-directional valve with the LD wrapped around, intermittently compressing the conduit, would: a) increase the baseline flow; b) overcome increased P.V.R.

Methods: The LD was prepared in 4 pigs by three weeks of activation via the thoraco-dorsal nerve $(210 \mu$ s pulses at $30 \mathrm{~Hz}$ frequency, amplitude $1.5-2.5 \mathrm{~V}$, on 0.19 seconds and off 6 seconds, 24 hrs/day).

The pigs (mean body weight $63 \mathrm{~kg}$, range $57-68 \mathrm{~kg}$ ) then underwent connection of the inferior vena cava to the pulmonary artery (PA) by implantation of combined valved conduit (Contegra and Dacron prosthesis).

After weaning from cardiopulmonary bypass (mean duration $=136$ ', range 132-143') the prepared distal LD was wrapped around the extracardiac cavo-pulmonary valved conduit.

Results: The extrinsic conduit compression by intermittent stimulation of the LD provided a mean 7\% (range 3.7-10.0\%) decrease of the baseline PA pressure, with simultaneous increase of the flow through the conduit of $2 \%$ (range -6 to $+12.5 \%$ ).
Conclusions: The preliminary results confirmed the feasibility of the technique. More studies are required to further test our hypothesis and to overcome technical problems related to the poor perfusion of the mobilized LD, the timing of LD transfer, and the low "pre-load" within the wrapped conduit.

\section{0}

Surgical Repair of Congenital Mitral Valve Malformations Pedro J Curi-Curi, "Ignacio Chávez" National Cardiology Institute of Mexico, Mexico

Samuel E Ramirez-Marroquin, "Ignacio Chávez" National

Cardiology Institute of Mexico, Mexico

Jorge L Cervantes, "Ignacio Chávez" National Cardiology Institute of Mexico, Mexico

Mauricio Soule, "Ignacio Chávez" National Cardiology Institute of

Mexico, Mexico

Julio Erdmenger, "Ignacio Chávez" National Cardiology Institute of

Mexico, Mexico

Juan Calderon-Colmenero, "Ignacio Chávez" National Cardiology

Institute of Mexico, Mexico

Objective: Surgical developement of mitral valve repair techniques in pediatric patients has been slow because of the great variety in the presentation of congenital mitral valve malformations and the still unknown growing effect over the complex mitral valve apparatus. The aim of this study is to review our early an mid term institutional outcomes in surgical repair of congenital mitral valve malformations.

Methods: We studied retrospectively 14 patients with surgical repair of congenital mitral valve malformations in a 5 year period. Clinical and echocardiographic follow-up at a mean of 25 months was performed in all cases.

Results: Operative morbility was $77 \%$ and operative mortality $7 \%$. There were no late deaths. Clinical functional class stratification in the mid term improved in $73 \%$ of the survivors and persisted in the restant $27 \%$. Freedom from reoperation for mitral valve prosthetic replacement survival rate because of mitral valve repair failure was $84 \%$ at 30 days and $77 \%$ at 3.5 years.

Conclusions: Surgical reapair is the best technique option in the treatment of congenital malformations of the mitral valve, and transesophageal intraoperatory echocardiography is highly recommended for evaluation of the results.

\section{1}

Early and mid term results of an alternative procedure to homografts in primary repair of Truncus Arteriosus Communis

Pedro J Curi-Curi, "Ignacio Chávez" National Cardiology Institute of Mexico, Mexico

Samuel E Ramirez-Marroquin, "Ignacio Chávez” National Cardiology Institute of Mexico, Mexico

Jorge L Cervantes, "Ignacio Chávez” National Cardiology Institute of Mexico, Mexico

Mauricio Soule, "Ignacio Chávez" National Cardiology Institute of Mexico, Mexico

Julio Erdmenger, "Ignacio Chávez" National Cardiology Institute of Mexico, Mexico

Carlos Zabal, "Ignacio Chávez" National Cardiology Institute of Mexico, Mexico

Juan Calderon-Colmenero, "Ignacio Chávez” National Cardiology Institute of Mexico

Objective: Early repair of truncus arteriosus communis (TAC) has become a standard practice in many centers. We report our 
experience on primary repair of TAC predominantly with the use of a pericardial valved Woven Dacron conduit as an alternative procedure to homografts, with a focus on early and midterm results. Methods: We studied 18 patients in a 6-year period, with a median age of 2.5 years that underwent primary repair of TAC. Cases with cardiogenic shock, complex associated cardiac lesions or adverse anatomy of the truncal valve were excluded. Collett and Edwards classification was type I, 12 (66\%); type II, 5 (28\%) and type III, 1 (6\%). Right ventricular outflow tract was reconstructed in 15 cases $(83.3 \%)$ with a pericardial valved Woven Dacron conduit.

Results: The overall hospital mortality was $6 \%$. At a mean followup of 28 months there were no deaths (5-year actuarial survival $94 \%)$. Of the 17 midterm survivors, 3 developed stenosis of the pericardial valved Woven Dacron conduit, but only one underwent interventional procedure. For the group of patients that received a pericardial valved Woven Dacron conduit, the freedom from reintervention in the mid term (5 years) was $76 \%$. Conclusions: Truncus arteriosus communis repair with a pericardial valved Woven Dacron conduit can be performed with a very low perioperative mortality and satisfactory midterm morbidity, favorably compared with that reported for the use of homografts. Interventional cardiac catheterization may delay the time of reoperation for inevitable conduit replacement.

\section{2}

\section{FloWatch ${ }^{\circledR}$ : Anatomical limitations}

Alessandro D'Alfonso, Pediatric Cardiac Surgery Unit, Ancona, Italy, Italy

Marco Pozzi, Pediatric Cardiac Surgery Unit, Ancona, Italy, Italy Andrea Quarti, Pediatric Cardiac Surgery Unit, Ancona, Italy, Italy Maria Grazia Bettuzzi, Pediatric Cardiac Surgery Unit, Ancona, Italy, Italy

Massimo Colaneri, Pediatric Cardiac Surgery Unit, Ancona, Italy, Italy Alessandra Baldinelli, Pediatric Cardiac Surgery Unit, Ancona, Italy, Italy

Pier Luigi Colonna, Pediatric Cardiac Surgery Unit, Ancona, Italy, Italy

Roberto Ricciotti, Pediatric Cardiac Surgery Unit, Ancona, Italy, Italy

Objectives: We report our experience with telemetrically adjustable PAB FloWatch ${ }^{\circledR}$ and assess its limitations.

Methods: Eleven infants, mean age 2.4 months (10 days to 10 months), mean weight $3.8 \mathrm{~kg}(2.3$ to $7.0 \mathrm{~kg})$ underwent implantation of FloWatch ${ }^{\circledR}$ through median sternotomy from May 2006 to June 2008. Diagnosis was complex/multiple ventricular septal defects (VSDs) (8), atrio-ventricular septal defect (2), congenitally Corrected TGA (1).

Results: Neither death nor device-related complication occurred during a mean follow-up of 15.4 months (1 day to 30 months). A mean of 3.1 adjustments/patient were required, in $85 \%$ of cases $(29 / 34)$ to narrow the pulmonary artery and in $15 \%(5 / 34)$ to release it. Four infants had removal of device (one at repair for atrio-ventricular septal defect, two for VSD closure, eight, six and eight months later respectively, and one for chest encumbrance the same day of implantation in a patient weighing $2.8 \mathrm{Kg})$.

Conclusion: In this initial series we experienced a single haemodynamic compromise due to the bulk of the device in the small chest of a patient. In another series, one of the authors (MP) met two other cases requiring immediate removal of FloWatch ${ }^{\circledR}$ : one for coronary and one for left superior vena cava compression. Despite that, this technology may obviate the need for reoperations and shorten the stay in ICU, and appears a suitable surgical option for left ventricular re-training. However it is not suitable for all anatomic conditions and, given the cost of the device, it could be advisable use a test device prior to implantation.

\section{3}

End to Side repair for Neonatal Aortic Coarctation: Long and Mid Term Result

Dan Spiegelstein, Department of Congenital Heart Surgery, Edmond and Lily Safra Children's Hospital, Chaim Sheba Medical Center, Israel Joseph Danieli, Department of Congenital Heart Surgery, Edmond and Lily Safra Children's Hospital, Chaim Sheba Medical Center, Israel Uriel Katz, Department of Congenital Heart Surgery, Edmond and Lily Safra Children's Hospital, Chaim Sheba Medical Center, Israel Sergey Preisman, Department of Congenital Heart Surgery, Edmond and Lily Safra Children's Hospital, Chaim Sheba Medical Center, Israel Dan Loberman, Department of Congenital Heart Surgery, Edmond and Lily Safra Children's Hospital, Chaim Sheba Medical Center, Israel David Mishali, Department of Congenital Heart Surgery, Edmond and Lily Safra Children's Hospital, Chaim Sheba Medical Center, Israel

Background: Many surgical techniques to fix a coarctation of the aorta incorporate abnormal tissue in the final repair with a significant incidence of recurrent obstruction requiring intervention. By connecting the descending aorta to the proximal aortic arch (end-to-side aortic anastomosis) we eliminate the isthmus and hypoplastic distal arch tissue from the anastomotic site. We retrospectively analyzed our experience in repair of aortic coarctation by using end to side aortic anastomosis technique in respect to the re-coarctation complication.

Methods: From January 1999 to August 2008, 117 patients underwent end to side anastomosis repair of aortic coarctation, via left thoractomy approach. Mean age was $28 \pm 34$ days (1-217 days), with 86 patients $(74 \%)$ in the neonate period ( $<30$ days). Mean weight was $3.3 \pm 0.9 \mathrm{~kg}(2-7 \mathrm{~kg})$, with $86 \%$ less then $4.0 \mathrm{~kg} .68$ patients had other cardiac anomalies, and 20 patients underwent concomitant cardiac procedure at the time of aortic coarctation repair. Late follow up was completed in 112 patients (96\%).

Results: There was no operative mortality, nor immediate complications related to the repair technique (Major bleeding, Re-open, vocal cord paralysis, distal organ ischemia etc.). Mean follow up was $18 \pm 23$ month (3-110 month). None of the patients needed re-intervention for recurrent aortic coarctation, none of them had a peak gradient over $20 \mathrm{mmHg}$ by EchoDoppler. At follow up mean systolic pressure gradient across the repair site was $4.7 \pm 10.1 \mathrm{mmHg}$.

Conclusions: End to side anastomosis for aortic coarctation, provide excellent short and mid term results, for neonatal coarctation in respect to coarctation recurrence.

\section{5}

Pulmonary Artery Banding in Atrio-Ventricular Septal Defects

Ramana RV Dhannapuneni, Alder Hey Foundation NHS Trust, Liverpool, United Kingdom

Natasha Prior, Alder Hey Foundation NHS Trust, Liverpool, United Kingdom

Andreas Hoschtitzky, Alder Hey Foundation NHS Trust, Liverpool, United Kingdom

Nelson Alphonso, Alder Hey Foundation NHS Trust, Liverpool, United Kingdom

Diana Mathioudakis, Alder Hey Foundation NHS Trust, Liverpool, United Kingdom 
Edmund J Ladusans, Alder Hey Foundation NHS Trust, Liverpool, United Kingdom

Antonio F Corno, Alder Hey Foundation NHS Trust, Liverpool, United Kingdom

Objectives: To review the results of Pulmonary Artery Banding (PAB) in Complete Atrio-Ventricular Septal Defects (cAVSD). Methods: From 2000 to 200819 patients with cAVSD underwent $\mathrm{PAB}$ because of unsuitable intra-cardiac anatomy (unbalanced ventricles and/or associated lesions) or clinical condition (late presentation with pulmonary hypertension, infection, chronic lung disease, non-cardiac malformation).

Since 2005 FloWatch PAB was introduced in our institute. Patients were retrospectively divided in Group A (conventional $\mathrm{PAB}): \mathrm{n}=14(74 \%)$, median age 67 days (range 6-635 days), median weight $3.4 \mathrm{Kg}$ (range $2.1-8.6 \mathrm{Kg}$ ) and Group B (FloWatch PAB): $n=5(26 \%)$, median age 120 days (range 81-181 days, N.S.), median weight $4.5 \mathrm{Kg}$ (range $3.2-6.1 \mathrm{Kg}$, N.S.).

Results: $10 / 14$ patients in Group A died $(=71.4 \%)$ versus $0 / 5$ in Group B $(=0 \%, \mathrm{P}<0.005)$.

Median ICU stay was 10 days (range 1-61 days) in Group A and 4.5 days (range 2-9 days) in Group B $(\mathrm{P}<0.05)$, while median Mechanical Ventilation duration was 9 days (range 0-61 days) in Group A and 3 days (range 1-8 days in Group B, P $<0.05$ ).

6/8 survivors (1 from Group A and 5 from group B) underwent repair after a median interval of 125 days (range 34-871 days), $2 / 8$ are waiting for repair.

Conclusions: In patients with cAVSD complicated by intra-cardiac anatomy unsuitable for repair and/or high-risk clinical condition, $\mathrm{PAB}$ followed by late repair is a viable alternative surgical option. In this group of patients the introduction of adjustable FloWatch $\mathrm{PAB}$ not only improved survival but made later repair feasible in better clinical conditions.

\section{6 \\ New hybrid strategy for hypoplastic left heart syndrome with intact or highly restrictive atrial septum \\ Hironori Ebishima, Department of Cardiovascular surgery, Okayama University, Japan \\ Shingo Kasahara, Okayama University Hospital, Japan \\ Yasuhiro Kotani, Dept. of Cardiovascular surgery. Okayama University Graduate School of Medicine and Dentistry, Pharmaceutical Science., Japan Yasuhiro Fujii, Department of Cardiovascular Surgery, Okayama University Hospital, Japan \\ Masami Takagaki, Department of Cardiovascular Surgery, Okayama University Graduate School of Medicine, Dentistry, and Pharmaceutical Sciences, Japan \\ Shunji Sano, Okayama University Hospital, Japan}

Objective: The presence of intact or highly restrictive atrial septum (HLHS/IAS or HLHS/HRAS) has been recognized as one of the risk factor for the treatment of hypoplastic left heart syndrome (HLHS). We have introduced a new hybrid strategy to the patients with HLHS associated with IAS/HRAS. This new technique included bilateral pulmonary artery banding (PAB) and balloon atrial septostomy (BAS) without using cardiopulmonary bypass (CPB).

Patients and Methods: Bilateral PAB was performed as a first palliation to 9 high risk neonates with HLHS from October 2005 to October 2008. Four patients out of 9 had a IAS/HRAS and bilateral PAB and BAS was applied under echo-guidance without CPB. There were 3 IAS and 1 HRAS. All 4 patients underwent re-BAS with 1 failure and 1 patient required stent to restenosed atrial septum. Two of them had a prenatal diagnosis and underwent this new technique within 1 and 2 hours after birth. Both of them survived and underwent rapid stage Norwood with RV-PA and BDG successfully. Other 2 patients had no pre-natal diagnosis and emergency surgery was applied at day1 and day3 with a same technique. Both of them died after rapid stage Norwood with RV-PA due to pulmonary hypertension.

Conclusion: Mortality to neonates with HLHS/IAS or HLHS/ HRAS remains high, however new hybrid strategy including bilateral $\mathrm{PAB}$ and $\mathrm{BAS}$ without $\mathrm{CPB}$ may improve long term survival if it is done before irreversible pulmonary vascular damage.

\section{7}

\section{Outcome of Mechanical Heart Valve Replacement in Children}

Frank Edwin, National Cardiothoracic Centre, Korle Bu Teaching Hospital, Accra, Ghana

Lawrence Sereboe, National Cardiothoracic Centre, Korle Bu Teaching Hospital, Accra, Ghana

Mark Tettey, National Cardiothoracic Centre, Korle Bu Teaching

Hospital, Accra, Ghana

Ernest Aniteye, National Cardiothoracic Centre, Korle Bu Teaching

Hospital, Accra, Ghana

David Kotei, National Cardiothoracic Centre, Korle Bu Teaching

Hospital, Accra, Ghana

Martin Tamatey, National Cardiothoracic Centre, Korle Bu Teaching

Hospital, Accra, Ghana

Kow Entsua-Mensah, National Cardiothoracic Centre, Korle Bu

Teaching Hospital, Accra, Ghana

Kwabena Frimpong-Boateng, National Cardiothoracic Centre, Korle Bu Teaching Hospital, Accra, Ghana

Objective: Cost constraints and the need to avoid reoperation make valve replacement an attractive option for a durable valve substitute in developing nations. This study was carried out to evaluate the outcome of mechanical valve replacement of the left heart in children ( $\leqslant 18$ years) at Ghana's National Cardiothoracic Centre.

Method: A retrospective analysis of all consecutive left heart valve replacements performed from January 1993-November 2008 was carried out. The study end-points included mortality, valverelated morbidity, and re-operation.

Results: In the 16 year study period, 114 patients underwent mitral valve replacement (MVR), aortic valve replacement (AVR) or mitral and aortic valve replacements (MAVR). There were 48 males and 66 females (ratio of 1:1.4). Their ages ranged from 6-18 years (median 14 years). Follow up was $94.7 \%$ complete. Median follow up was 9.1 years. MVR was performed in 91 (79.8\%) patients, AVR in $13(11.4 \%)$ and MAVR in $10(8.8 \%)$ patients. Tricuspid valve repair was performed concomitantly in $45(39.5 \%)$ patients.

There were 6 early deaths (5.3\%) and 6 late deaths (5.3\%). Actuarial survival at 1 and 15 years were $98.1 \%$ and $94.0 \%$ respectively. Prosthetic valve thrombosis (PVT) occurred in 4 patients $(0.45 \%$ per patient-year). Two reoperations were performed $(0.22 \%$ per patientyear). There was $1(0.9 \%)$ each of major bleeding event, nonstructural dysfunction and prosthetic valve endocarditis.

Conclusion: Mechanical valve replacement in this age group has acceptable early and late mortality. PVT is the main cause of morbidity and so measures to prevent PVT must be intensified.

\section{8}

Complete Heart Block Following COngenital Heart Repairs - A 16 Year Review

Frank Edwin, National Cardiothoracic Centre, Korle Bu Teaching Hospital, Accra, Ghana 
Ernest Aniteye, National Cardiothoracic Centre, Korle Bu Teaching Hospital, Accra, Ghana

Lawrence Sereboe, National Cardiothoracic Centre, Korle Bu Teaching Hospital, Accra, Ghana

Mark Tettey, National Cardiothoracic Centre, Korle Bu Teaching

Hospital, Accra, Ghana

David Kotei, National Cardiothoracic Centre, Korle Bu Teaching

Hospital, Accra, Ghana

Martin Tamatey, National Cardiothoracic Centre, Korle Bu Teaching

Hospital, Accra, Ghana

Kow Entsua-Mensah, National Cardiothoracic Centre, Korle Bu

Teaching Hospital, Accra, Ghana

Kwabena Frimpong-Boateng, National Cardiothoracic Centre, Korle Bu

Teaching Hospital, Accra, Ghana

Background: The incidence of post-operative complete heart block $(\mathrm{CHB})$ in patients undergoing intra-cardiac repair for congenital heart disease in Ghana has not been reported. This information is important in pre-operative counseling of patients and parents and facilitates the process of obtaining informed consent for patients undergoing such repairs.

Objectives: This study was undertaken to determine: 1) the incidence of permanent post-operative $\mathrm{CHB}$ in the setting of congenital heart disease, 2) the intra-cardiac anatomic risk factors in such patients and 3) the significance of the pacemaker in terms of morbidity after intra-cardiac repair.

Design: This is a retrospective descriptive study.

Setting: The National Cardiothoracic Centre (NCTC), Korle Bu Teaching Hospital, Accra, Ghana.

Method: This was a review of patients who had intra-cardiac repair of congenital heart disease known to predispose to postoperative complete heart block from January 1992 to November 2008. Analysis of results was performed using the SPSS program. Results: Six patients out of $216(2.8 \%)$ developed permanent post-operative CHB. All underwent closure of a large perimembranous ventricular septal defect (VSD) either as an isolated defect or in the setting of tetralogy of Fallot. The dominant morbidity relating to the implanted device was parental concern regarding the financial implications of future multiple surgeries to replace a depleted pulse generator.

Conclusion: Permanent post-operative CHB occurred in $2.8 \%$ of patients undergoing intracardiac repair for congenital heart disease on the ventricular septum. The dominant anatomic risk factor was a large perimembranous VSD. Pacemaker-associated morbidity is acceptable in this setting.

\section{9}

Outcome of Double Valve Replacement in Children in Ahmed Gasim Cardiac Center

Hani Eltayeb Elamin, Ahmed Gasim Cardiac Center, Khartoum

North, Sudan, PO Box:992, Sudan

Objectives: To study the outcome of aortic and mitral valves replacement with mechanical valves in children.

Methodology: Retrospective descriptive hospital based study. The data was collected manually by the authors and analyzed by a computer programe(SPSS) in the period between Jan 2003-Dec 2008, Ahmed Gasim Cardiac Center, Sudan.

Results: Nine patients underwent mitral and aortic valve replacement,six of them were males. Seven of patients were in the age group $12-15$ years. NYHA II was 33\%, NYHA III $44 \%$ and NYHA IV constituted 22\%. The mean left atrial dimension was $5.3 \mathrm{~cm}$. Mitral regurgitation with Aortic regurgitation constituted $55.6 \%$ of cases, combined mitral disease with aortic regugitation and combined aortic disease with mitral regurgitation had equal percentages of $22.2 \%$. Eight of the cases had LVEF of $>40 \%$. Pulmonary artery pressure of $>50 \mathrm{mmHg}$ was detected in three and less than $50 \mathrm{mmHg}$ in six cases. The mean intubation time was 17.3 hours and the mean intensive care stay was 37 hours. It was difficult to control the INR in one patient. There was one late mortality caused by CCF.

Conclusion: Early outcome of double valve replacement is excellent, however,anticoagulation remains an important issue in developing countries.

\section{2}

Anomalous Aortic Origin of a Coronary artery: Extended Experience With Surgical Treatment

Eldad Erez, Cookchildrens Medical Center, United States

Vincent KH Tam, Cookchildrens Medical Center, United States

Nancy A Doublin, Cookchildrens Medical Center, United States

Lisa Roten, Cookchildrens Medical Center, United States

Deborah Schutte, Cookchildrens Medical Center, United States

Objective: Anomalous aortic origin of a coronary artery with subsequent coursing between the great vessels is a rare congenital heart defect with increased risk for sudden death. We present our experience with the diagnosis and surgical treatment of this coronary anomaly.

Methods: Since June 2003, 27 patients were diagnosed with anomalous aortic origin of a coronary artery coursing between the great vessels. Age ranged from 4 months to 23 years. The right coronary artery was involved in 20 patients. Six patients had a slit opening and 8 patients had an intramural proximal segment. 15 patients presented with exertional chest pain, 8 experienced syncope and one patient had an acute myocardial infarction.

Diagnosis was made using echocardiography. 18 patients had coronary artery re-implantation in the correct aortic sinus, 5 patients had unroofing of the intramural coronary segment, 3 patients had pericardial patch enlargement of the coronary artery.

Results: There were no postoperative deaths. One patient underwent heart transplantation. 8 patients had transient ST segment changes postoperatively. Hospital stay ranged from 4 to 16 days.

Follow-up ranged from 6 months to 5.5 years. All patients underwent exercise myocardial perfusion scan 3 months postoperatively without evidence of myocardial ischemia and all patients remain clinically well.

Conclusion: Echocardiography imaging of young patients with exertional chest pain or syncope is reliable for the diagnosis of this coronary anomaly. The surgical approach may vary based on individual coronary anatomy. Early and mid- term results are favorable; however, long term coronary anatomy changes should be monitored.

7203

A Comparison of Cerebral Protection using Alpha-Stat and $\mathbf{P h}-$ Stat Strategies in an Animal Model of Pediatric Cardiopulmonary Bypass

Jacob Ostrowsky, Department of Pediatric Cardiothoracic Surgery, Rainbow Babies and Children's Hospital, Case Western Reserve University School, United States

Mark Henderson, Department of Pediatric Cardiothoracic Surgery, Rainbow Babies and Children's Hospital, Case Western Reserve University School, United States

Ahmed Farouk, Department of Pediatric Cardiothoracic Surgery, Rainbow Babies and Children's Hospital, Case Western Reserve University School, United States 
Shivaprakash Seshadri, Department of Pediatric Cardiothoracic Surgery, Rainbow Babies and Children's Hospital, Case Western Reserve University School, United States

Steve Schomisch, Department of Pediatric Cardiothoracic Surgery, Rainbow Babies and Children's Hospital, Case Western Reserve University School, United States

Hani A Hennein, Department of Pediatric Cardiothoracic Surgery, Rainbow Babies and Children's Hospital, Case Western Reserve University School, United States

Objective: To compare the effects of blood-gas management using either alpha-stat (temperature-uncorrected blood-gas management) or $\mathrm{pH}$-stat (temperature-corrected blood-gas management) strategies on cerebral oxygenation during cardiopulmonary bypass.

Methods: Six piglets, ranging from $8-12 \mathrm{~kg}$, were placed on cardiopulmonary bypass, and cooled to $12 \mathrm{o} \mathrm{C}$ utilizing alpha-stat $(\mathrm{N}=3)$ or $\mathrm{pH}$-stat blood gas management $(\mathrm{N}=3)$, then warmed to $40^{\circ} \mathrm{C}$. At every 2 degrees Celsius, parameters were maintained for 10 minutes, and both arterial and venous blood gas data, pressure data, heart rate and cerebral oximetry data were recorded. Regional cerebral oximetry was monitored using near infrared reflective spectroscopy (NIRS). Continuous in-line arterial blood gases were monitored and confirmed every 10 minutes using a blood gas analyzer.

Results: Alpha-stat animals had temperature-uncorrected arterial blood gases of; $\mathrm{pH} 7.45 \pm 0.08, \mathrm{PaCO} 240 \pm 3 \mathrm{mmHg}, \mathrm{PaO} 2$ $226 \pm 63 \mathrm{mmHg}$. PH-stat animals had temperature-corrected arterial blood gases of; $\mathrm{pH} 7.42 \pm 0.06, \mathrm{PaCO} 240 \pm 4 \mathrm{mmHg}$, $\mathrm{PaO} 2214 \pm 54 \mathrm{mmHg}$. Both alpha-stat and $\mathrm{pH}$-stat temperature management techniques demonstrated an inverse correlation to cerebral oximetry $(\mathrm{R} 2=0.64, \mathrm{r}=-0.71, \mathrm{P}<0.0001)$. However, separating the two groups, the $\mathrm{pH}$-stat population showed a more significant increase in cerebral oximetry, compared to Alpha-stat, across the entire hypothermic temperature range $(\mathrm{R} 2=0.89, \mathrm{r}=-0.92, \mathrm{P}<0.0001$, Fig).

Conclusion: PH-stat blood gas management, as compared to alpha-stat, is associated with higher cerebral oxygenation during cardiopulmonary bypass. This may provide better cerebral protection, which is the goal of successful pediatric cardiac surgery in the current era.

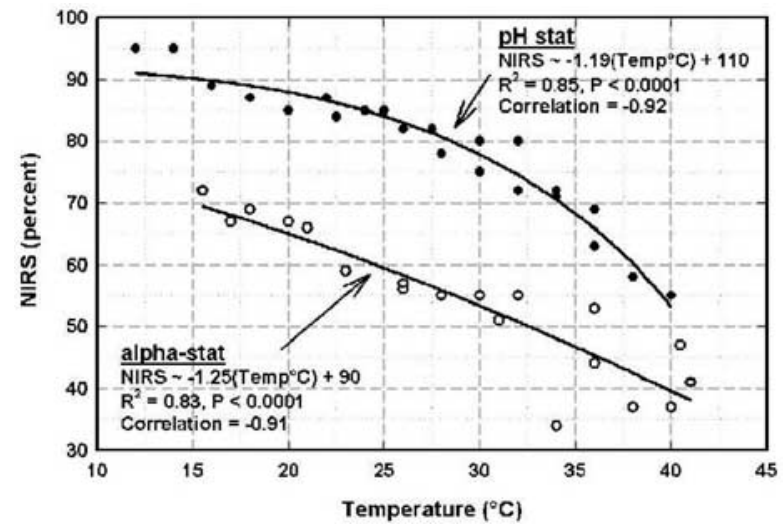

7204

Aortic Root Dilatation after repair of Tetralogy of Fallot: Hemodynamics and Demographics are not the Answer Ahmed Farouk, Pediatric Cardiothoracic Surgery, Rainbow Babies and Children's Hospital, Case Western Reserve University, Cleveland, $\mathrm{OH}$, United States

Kenneth Zahka, Pediatric Cardiology, Rainbow Babies and Children's Hospital, Case Western Reserve University, Cleveland, OH, United States
Francine Erenberg, Pediatric Cardiology, Rainbow Babies and Children's Hospital, Case Western Reserve University, Cleveland, $\mathrm{OH}$, United States

Yasser Al-Khatib, Pediatric Cardiology, Rainbow Babies and Children's Hospital, Case Western Reserve University, Cleveland, OH, United States Alex Golden, Pediatric Cardiology, Rainbow Babies and Children's Hospital, Case Western Reserve University, Cleveland, $\mathrm{OH}$, United States

Ernest Siwik, Pediatric Cardiology, Rainbow Babies and Children's Hospital, Case Western Reserve University, Cleveland, $\mathrm{OH}$, United States

Hani A Hennein, Pediatric Cardiothoracic Surgery, Rainbow Babies and Children's Hospital, Case Western Reserve University, Cleveland, $\mathrm{OH}$, United States

Objectives: To determine the prevalence of aortic root dilation and aortic regurgitation (AR) after tetralogy of Fallot (TOF) repair, as well as associated risk factors.

Methods: Aortic dimensions, adjusted for body surface area and expressed as Z-scores, and AR were assessed by echocardiography in 24 patients at $7.4 \pm 7.5$ years after repair of TOF.

Results: The Z-scores of the aortic dimensions at the levels of the annulus, sinus of Valsalva, sinotubular junction, and ascending aorta were $3.9 \pm 2.5, \quad 4.1 \pm 1.5, \quad 3.9 \pm 1.7, \quad 4.1 \pm 2.1$ respectively, all of which were significantly greater than the reference mean of $Z=0 \pm 2(p<0.001)$. The annulus, sinus of Valsalva, sinotubular junction, and ascending aorta were dilated in $91.6 \%, 91.6 \%, 91.6 \%$, and $87.5 \%$ of patients respectively. However, there was no significant association between the enlarged aortic roots in the TOF patients to the age at surgical repair, age at follow-up, duration of follow-up after repair, body surface area, gender, or previous palliation. Five of the 24 patients (20\%) had AR, which was trivial or mild in four, and moderate-to-severe in one. There were no statistically significant differences in the aortic Z-scores of patients with or without AR.

Conclusion: Although there is a significant prevalence of aortic root dilation following repair of TOF, the presence and progression of aortic root dilation was not associated with any of the hemodynamic or demographic variables measured here. Aortic root dilatation appearing after TOF repair may be solely due to individual histopathological changes and does not appear to be hemodynamically or surgically significant at $7.4 \pm 7.5$ years follow-up.

\section{5}

\section{Mentoring Pediatric Cardiac Surgery in Developing} Countries

Kathleen N Fenton, International Children's Heart Foundation, United States

Sergio Hernandez, Nicaragua

William Novick, United States

Aiding the formation of cardiac surgical centers in developing countries includes remote training of personnel, medical team visits, and donation of equipment and supplies. When a program fails to progress, the donating organization often decides that its efforts could be more useful elsewhere. We have instead placed a US-trained pediatric heart surgeon in such a situation in order to be a catalyst and a mentor for the growth of the program.

Methods: Following a "scout" trip with a small medical team, one surgeon relocated with the intention of starting an open heart surgery program in a country that had failed to develop one despite ten years of team visits. Results of the first 
year of this experience (Group IIa) are compared with a concurrent series of patients operated by the pre-existing local team (Group I), and with the second year's experience (Group 1Ib).

Results: Fifty-six patients were operated in Group IIa with 6 deaths $(11 \%)$, five in infants undergoing palliative operations. There was one complication: a pleural effusion requiring thoracentesis. In Group I, 40 patients were operated with a mortality of $15 \%(p<0.02)$. Complications included four $(10 \%)$ nontherapeutic operations (two died), one paraplegia after coarctation repair, and one reexploration for hemorrhage. Mortality in Group IIb was further decreased at $1.7 \%$ $(p<0.02)$. Conclusions: Placement of a "mentoring" surgeon can effectively promote the safe growth of pediatric cardiac surgery in a developing country, and rapid improvement in results can be obtained. Infants undergoing palliative procedures remain high-risk surgical candidates.

\begin{tabular}{|c|c|c|c|c|c|}
\hline \multirow{2}{*}{$\begin{array}{l}\text { Complexity } \\
\text { (RACHS) }\end{array}$} & \multirow{2}{*}{$\begin{array}{l}\text { Group I } \\
\text { N Deaths (\%) }\end{array}$} & \multicolumn{2}{|c|}{ Group IIa } & \multicolumn{2}{|c|}{ Group IIb } \\
\hline & & $\mathbf{N}$ & Deaths $(\%)$ & $\mathbf{N}$ & Deaths $(\%)$ \\
\hline 1 & $302(6.7 \%)$ & 34 & $1(2.9 \%)$ & 45 & $1(2.2 \%)$ \\
\hline 2 & $11(100 \%)$ & 2 & $0(0 \%)$ & 8 & $0(0 \%)$ \\
\hline 3 & $83(37.5 \%)$ & 8 & $5(62.5 \%)$ & 6 & $0(0 \%)$ \\
\hline Unclassified & $10(0 \%)$ & 4 & $1(25 \%)$ & 1 & $0(0 \%)$ \\
\hline
\end{tabular}

7206

Medium-Term Outcome for Interrupted Aortic Arch: Results of a Selective Single Stage Strategy

Kirsten A Finucane, Starship Children's Hospital, New Zealand

Julia Flint, Starship Children's Hospital, New Zealand

Judith D MacCormick, Starship Children's Hospital,

New Zealand

Thomas L Gentles, Starship Children's Hospital, New Zealand

Objectives: Biventricular repair of the interrupted aortic arch (IAA) in neonates involves an early choice between a single or two-stage strategy, with the risk factors for each not yet fully investigated. We assessed outcome of a selective single stage strategy at our institution.

Methods: A retrospective review to assess the outcome of neonates undergoing repair of IAA repair and associated congenital heart defects between 2000 and 2006. Pre-operative assessment, operative management and outcome were examined.

Results: Eighteen consecutive patients were identified. Associated congenital cardiac defects included VSD (17), truncus arteriosus (3), AP window (2), aortic valve atresia (2) and TGA (1). Seventeen underwent a single stage procedure including 2 Norwood-Rastelli and one arterial switch and IAA repair. The other patient, born at 32 weeks gestation weighing $970 \mathrm{~g}$ underwent a two stage procedure with aortic arch repair and pulmonary artery banding at 3 weeks of age. There were no early deaths. One patient required a pacemaker for complete heart block. There are no late deaths and there was normal functional status with mean follow-up 4.5 years. The premature infant having a staged repair required a redo arch repair at the time of VSD closure. Three others required late reoperation, one Ross-Konno at 6 months and two pulmonary conduit changes both at 35 months.

Conclusions: Good functional status and low re-operation rates can be achieved with a single stage repair however an individualised approach is necessary for neonates with risk factors including low birth weight and prematurity.
7207

Cerebral Near Infrared Spectroscopy during Cardiopulmonary Bypass Accurately Predicts Superior Vena Cava Oxygen Saturation

Joseph M Forbess, University of Texas Southwestern and Childrens Medical Center Dallas, United States

Richard M Ginther, niversity of Texas Southwestern and Childrens Medical Center Dallas, United States

Rong Huang, Childrens Medical Center Dallas, United States

Steven $R$ Leonard, niversity of Texas Southwestern and Childrens Medical Center Dallas, United States

Ronald M Gorney, niversity of Texas Southwestern and Childrens Medical Center Dallas, United States

Kristine J Guleserian, niversity of Texas Southwestern and Childrens Medical Center Dallas, United States

Background: Cerebral (CNIRS) and Flank (FNIRS) nearinfrared spectroscopy are employed to monitor tissue oxygenation during cardiopulmonary bypass(CPB) in pediatric patients. We sought to validate these non-invasive measurements as predictors of oxygen saturation in the superior and inferior vena cava during $\mathrm{CPB}$.

Methods: 8 patients underwent elective repair of congenital heart defects with bicaval cannulation. Ultrasonic flow probes and oximetric catheters were placed in the superior and inferior vena caval limbs of the perfusion circuit. CNIRS, FNIRS, and 13 additional variables were recorded each minute on CPB. Relationships between these variables and superior vena cava oxygen saturation (SVCsat) and inferior vena cava saturation(IVCsat) were analyzed by linear mixed modeling. To assess NIRS as a trend indicator, the difference between consecutive vena cava oxygen saturations was regressed with the difference between consecutive NIRS oxygen saturations.

Results: The mean number of observation time points per patient was 86 (median 72, range 34 to 194) for 690 total observations. CNIRS was the strongest single predictor of SVCsat $(\mathrm{p}<0.0001)$. CNIRS predicted SVCsat value within $\frac{1}{2}$ standard deviation (SD) at the rate of $47.3 \%$. CNIRS was also a powerful indicator of changes in SVCsat $(p<0.0001)$. FNIRS $(p<0.0001)$ was the strongest single predictor of IVCsat, though it predicted IVCsat value within $\frac{1}{2} \mathrm{SD}$ at a rate of $26.4 \%$, much lower than the CNIRS/SVCsat relationship. FNIRS also predicted IVCsat changes $(\mathrm{p}=0.02)$

Conclusion: CNIRS accurately predicts SVCsat and changes in SVCsat on CPB. The relationship between FNIRS and IVCsat is not as strong. Further investigation is warranted to determine the value of FNIRS measured during CPB.

7208

A Novel Strategy for Flow Control Right Ventricle to Pulmonary Artery Shunt in Infants with Hypoplastic Left Heart Syndrome

Yasuhiro Kotani, Okayama University Hospital, Japan

Shingo Kasahara, Okayama University Hospital, Japan

Sadahiko Arai, Okayama University Hospital, Japan

Yasuhiro Fujii, Okayama University Hospital, Japan

Masami Takagaki, Okayama University Hospital, Japan

Shin-ichi Otsuki, Okayama University Hospital, Japan

Teiji Akagi, Okayama University Hospital, Japan

Shunji Sano, Okayama University Hospital, Japan

Background: Appropriate pulmonary blood flow is important for addressing systemic circulatory instability in infants with hypoplastic left heart syndrome (HLHS). To address such 
problems, we have developed a novel strategy to control shunt flow, which is partial clipping on the PTFE graft.

Methods: Since 2005, we conducted this strategy in infants with HLHS who had right ventricle to pulmonary (RV-PA) shunt at the time of Norwood operation for HLHS if the patients fulfilled at least one of the following conditions; 1) high pulmonary blood flow (SpO2 $>85 \%$ in $0.2-0.4$ of $\mathrm{FiO} 2), 2$ ) low cardiac output, 3) more than moderate tricuspid valve regurgitation, 4) low body weight. A 5 or $6 \mathrm{~mm}$ PTFE tube were used for the establishing RV-PA shunt, and partially clipped (approximately $1 / 4$ to $1 / 3$ of diameter) using hemoclip under the careful monitoring of systemic oxygen saturation.

Results: In 71 patients with HLHS who underwent Norwood operation, 7 infants (age, 3 days to 3 months) received this technique. Four of 7 infants developed cyanosis and required of balloon dilatation of RV-PA shunt and removal of clip at $5.5 \pm 0.4$ (range, $4-6$ ) months after the initial surgery. Systemic oxygen saturation increased from $78 \pm 1$ to $86 \pm 3 \%$ after the intervention. Remaining 3 infants have reached to the bidirectional Glenn procedure without requiring any additional interventions. Conclusions: This novel strategy contributes to appropriate RV-PA shunt flow in infants with Norwood operation of HLHS. Development of cyanosis related with patient's somatic growth can be avoided by subsequent balloon dilatation of the clipping site.

7209

Early outcome of the arterial switch (Jatene) operation in 173 consecutive patients. A single centre experience in a development country

Beatriz H S Furlanetto, Instituto Furlanetto, Brazil

Sandra S Henriques, Instituto Furlanetto, Brazil

Patricia M V P Medeiros, Instituto Furlanetto, Brazil

Marcus V L Medeiros, Instituto Furlanetto, Brazil

Lilian M Lopes, Instituto Furlanetto, Brazil

Salvador A B Cristovão, Instituto Furlanetto, Brazil

Grace C V L Bichara, Instituto Furlanetto, Brazil

Gláucio Furlanetto, Instituto Furlanetto, Brazil

Objective: The accomplishment of Jatene's operation needs a tertiary hospital with specialized team in pediatric cardiology to obtain a good result. We examined the immediate results of 176 consecutive arterial switch during last 15 years.

Methods: Between December 1993 and December 2008, 173 children with transposition of great arteries (TGA) were submitted to Jatene operation; $114(65 \%)$ were simple TGA and $59(34,1 \%)$ were TGA with ventricular septal defect; 5 $(2,8 \%)$ had association with coarctation of aorta. The current arterial switch is made with maneuver of LeCompte and initial anastomoses of the neoaorta and posterior implants of the coronary arteries. The restoration of the neopulmonary is accomplished with fresh autograft pericardium. Bypass is accomplished with hypothermia to $25^{\circ} \mathrm{C}$ and normoflow and myocardium protection with blood cardioplegic solution and modified ultrafiltration. We divided the pacients following a chronological order: Group A (57 children), B (58 children) and C (58 children) and we evaluated the surgical mortality.

Results: The surgical mortality was: Group A mortality $=36,8 \%$; Group B mortality $=34,4 \%$; Group C mortality $=8,6 \%$. There was statistical difference among the groups $\mathrm{C}$ and $\mathrm{B}$ and also $\mathrm{C}$ and $\mathrm{A}$. In Group $\mathrm{C}$ the mortality with standard risk profile to coronary pattern was 3,7\% and with high risk was $75 \%$ (right coronary with looping and intramural).
Conclusion: After 173 consecutives arterial switch was possible the improvement in the surgical result. The translocation of right coronary with looping still presents high risk.

\section{0}

Which is the ideal age to take place the correction of tetralogy of Fallot?

Beatriz H S Furlanetto, Instituto Furlanetto, Brazil

Sandra S Henriques, Instituto Furlanetto, Brazil

Patricia MV P Medeiros, Instituto Furlanetto, Brazil

Grace C V L Bichara, Instituto Furlanetto., Brazil

Lilian M Lopes, Instituto Furlanetto, Brazil

Salvador A B Cristovão, Instituto Furlanetto, Brazil

Carlos E B Kapins, Instituto Furlanetto, Brazil

Gláucio Furlanetto, Instituto Furlanetto, Brazil

Objetive: Compare the immediate results of children who underwent correction of TF in different age groups.

Methods: Between January 1995 and September 2008, 332 children with tetralogy of Fallot were submitted to surgical correction. They were contained according to the age in 3 groups: Group $\mathrm{A}=$ age $>$ 1 year; Group B $=4$ months $<$ age $<1$ year and Group $C$ age $<4$ months. The hospital mortality was analyzed in each group and also according to the phase. The incidence of atriopulmonary correction (APC) was also analyzed.

Results: Group A: $\mathrm{N}=197$, mortality $\mathrm{N}=7$ (3,5\%), phase1 (5\%), phase2 (2\%), APC N=61 (30,9\%). Group B: $N=88$, mortality $\mathrm{N}=3(3,4 \%)$, phase1 (6,8\%), phase2 (0\%), APC $\mathrm{N}=19(21,5 \%)$; Group C: $\mathrm{N}=47$ (neonates $\mathrm{N}=23(48,9 \%)$, mortality $\mathrm{N}=4$ $(8,5 \%)$, phase1 (6,8\%), phase2 (0\%), APC $\mathrm{N}=5(10,6 \%)$. The general hospital mortality was $4,2 \%$, phase $1=7,1 \%$ ( $\mathrm{N}$ inicial $=166)$ and phase $2=1,2 \%(\mathrm{~N}$ final $=166)$. There was not statistical difference in the hospital mortality among the groups $\mathrm{B}$ and $C(p=0,312)$. There was not difference in the accomplishment of APC among the groups A and B $(p=0,150)$, but there was difference between groups $\mathrm{A}$ and $\mathrm{B}$ with $\mathrm{C}(\mathrm{p}=0,011)$.

Conclusion: The correction of tetralogy of Fallot can be accomplished in precocious age, besides in the neonatal period, without difference in the mortality, with larger atriopulmonary correction in age superior to 4 months.

\section{1}

Porcine valved pulmonary conduit with potential for biointegration. A new graft option in the reconstruction of the right ventricle outflow. Early results

Glaucio Furlanetto, Instituto Furlanetto, Brazil

Sandra S Henriques, Instituto Furlanetto, Brazil

Patricia MV P Medeiros, Instituto Furlanetto, Brazil

Grace C V L Bichara, Instituto Furlanetto, Brazil

Lilian M Lopes, Instituto Furlanetto, Brazil

Salvador A B Cristovão, Instituto Furlanetto, Brazil

Mario C C Olmos, Instituto Furlanetto, Brazil

Beatriz H S Furlanetto, Instituto Furlanetto, Brazil

Objective: To date, there is no suitable valved conduit for small children with anomaly of the right ventricle outflow. Experimental study accomplished in sheep with the L-Hydro porcine valved pulmonary conduit (PVPC) revealed long term great results with biointegration and growth besides the valvular leaflets. The L-Hydro is an alternative method of non-aldehyde tissue preservation and have been attempted to encourage implants to re-cellularize with host endothelial cells which allows potential regeneration and growth of the valvular tissue, transforming it in a living structure. The purpose of this 
investigation is to analyze the immediate results of PVPC in children with right ventricle outflow anomalies (RVA).

Methods: Between October 2006 and December 2008, 25 children with RVA undergoing PVPC implants (15 Tetralogy of Fallot (TF); 3 Pulmonary Atresia with Ventricular Septal Defect; 2 Double Outlet Right Ventricle; 1 reoperation of TF; 2 Truncus Arteriosus (TAC); 1 reoperação TAC); 14 was male and 16 had age below 1 year. The implants were done with bypass circulation at $27^{\circ} \mathrm{C}$, normoflow and use of cardioplegic solution with blood. The size of the grafts varied from $11 \mathrm{~mm}$ to $19 \mathrm{~mm}$. Results: There was 1 surgical death (4\%). A color ecodopplercardiografic control done at immediate surgical period showed moderate valvar insuficience in 2 children and important in 1 child.

Conclusion: The use of porcine valved pulmonary conduit L-Hydro showed a good immediate result and a late follow-up is necessary to evaluate the advantages and disadvantages on the other substitutes available for the pulmonary valve.

7212

Unidirectional valve patch provides no survival benefits to ventricular septal defect with severe pulmonary hypertension

Huili Gan, Cardiac Surgery Department, Beijing Anzhen Hospital, Capital Medical University (BAZH - CMU) 100029 Beijing China, China

Objective: To test the hypothesis that unidirectional valve patch (UVP) would provide benefit to early and actuarial survival for the ventricular septal defect (VSD) complicated with sever pulmonary artery hypertension (PAH).

Methods: 876 cases of VSD with severe PAH were closed with or without UVP and were retrospectively classified as UVP group $(\mathrm{n}=195)$ and NVP group $(\mathrm{n}=681)$. Propensity scores of inclusion into UVP group were estimated and 138 pairs were get with the propensity score matching between the two groups. Results: For the 138 propensity matched pairs, there were 7 and 9 early deaths for the UVP and NVP group respectively, of which, 14 were due to $\mathrm{PAH}$ crisis and 2 were due to the reperfusion injury. The difference of the early mortality between UVP and NVP did not reached statistically differences, $\chi^{2}=0.265$, $\mathrm{P}=0.6064$. With a mean of $9.2 \pm 4.92$ years and a total of 2511 patient-years follow-up, there were 6 late deaths in UVP group and 7 late deaths in NVP group. The actuarial survival at $5,10,15$ and 18 yrs of the two groups reached no significantly difference (Log rank test, $\chi^{2}=0.565, \mathrm{P}=0.331$ ). The 6MWD assessed at the last follow-up of the two group were 525.9 \pm 88.0 meters for the UVP group and $536.5 \pm 95.8$ meters, according to the t-test, $\mathrm{F}=1.550, \mathrm{P}=0.214$, the difference between the two groups did not reach a statistically significance.

Conclusion: UVP provides no benefits to early and actuarial survival when it is used to deal with VSD complicated with severe PAH.

\section{3}

Current surgical management of ascending aortic aneurysm in children and young adults

Heidi Goerler, Hannover Medical School, Germany

Masamichi Ono, Hannover Medical School, Germany

Dietmar Boethig, Hannover Medical School, Germany

Mechthild Westhoff-Bleck, Hannover Medical School, Germany

Thomas Breymann, Hannover Medical School, Germany

Objective: The aim of this study was evaluation of different surgical strategies of ascending aortic aneurysm repair in children and young adults.
Methods: From August 2003 to August 2008, 35 patients aged 7 to 35 (mean 21) years presented with ascending aortic aneurysm. In 18 patients, the aneurysm was a primary lesion (Group A), whereas in 17 patients, aortic aneurysm developed after surgical and/or catheter interventions for congenital heart disease (Group B).

Results: Surgical procedures included aortic valve sparing operation (6 patients in Group A vs.2 patients in Group B), conduit replacement (10 vs.11), and ascending aortic replacement (2 vs.4). Mean cardiopulmonary bypass and aortic cross-clamp time were $137 \pm 24$ vs. $241 \pm 116 \mathrm{~min} \quad(\mathrm{p}=0.02)$ and $86 \pm 20$ vs. $98 \pm 32 \mathrm{~min}(\mathrm{p}=0.19)$ in Group A and Group B, respectively. One patient in Group B died on the 16th postoperative day. The other 34 patients survived the operation and are in New York Heart Association functional class II or less at maximum of 5 years follow-up. Actuarial survival was $97.3 \%$ at 5 years, and all patients were free from reoperation. There was only one thromboembolic complication. Aortic valve function was good in all 8 patients after valve sparing operation.

Conclusions: Ascending aortic aneurysm in children and young adults was surgically treated with excellent mid-term outcome. Conduit implantation is the gold standard in children and young adult and showed good results. Aortic valve sparing operation showed encouraging results with acceptable valve function for selected patients.

7215

Aortic Arch Reconstruction Using Cardiopulmonary Bypass and Moderate Hypothermia Without Circulatory Arrest

Kristine J Guleserian, Children's Medical Center/UT Southwestern

Medical Center Dallas, United States

Richard M Ginther, Children's Medical Center/UT Southwestern

Medical Center Dallas, United States

Ronald M Gorney, Children's Medical Center/UT Southwestern Medical Center Dallas, United States

Steven R Leonard, Children's Medical Center/UT Southwestern

Medical Center Dallas, United States

Joseph M Forbess, Children's Medical Center/UT Southwestern

Medical Center Dallas, United States

Background: Aortic arch reconstruction requiring cardiopulmonary bypass $(\mathrm{CPB})$ is usually performed with circulatory arrest (CA) or selective cerebral perfusion at profound hypothermia (H-SCP). We describe outcomes of patients who underwent aortic arch reconstruction using only moderate hypothermia with selective cerebral perfusion (M-SCP).

Results: There were 54 patients (19 two-ventricle, 35 singleventricle) identified. Single-ventricle (1V) diagnoses included: hypoplastic left heart syndrome (HLHS) or variant HLHS $(\mathrm{N}=24)$, unbalanced AV canal $(\mathrm{N}=5)$, DILV with arch hypoplasia $(\mathrm{N}=3)$, and DORV with mitral atresia and arch hypoplasia $(\mathrm{N}=3)$. Two-ventricle $(2 \mathrm{~V})$ diagnoses included: coarctation of the aorta (CoA) with VSD $(\mathrm{N}=11)$, CoA with arch hypoplasia $(\mathrm{N}=4)$, interrupted aortic arch (IAA, $\mathrm{N}=3$ ) and recurrent CoA late $\mathrm{s} / \mathrm{p}$ IAA repair $(\mathrm{N}=1)$. Median age at surgery was 5 days (range, 1 day-10yrs) and median weight $3.2 \mathrm{~kg}$ $(2-32 \mathrm{~kg})$. There were 4 operative deaths in the $1 \mathrm{~V}$ group $(11.4 \%)$ and no deaths in the $2 \mathrm{~V}$ group. There were no instances of multiorgan failure referable to renal, hepatic, gastrointestinal, or spinal cord injury in either group. Delayed sternal closure was performed in all of the $1 \mathrm{~V}$ group and 2/19 (10.5\%) of the 2V group. Median intubation time was 4 days (range, $0.5-95 \mathrm{~d}$ ), median ICU stay was 8 days (range, 1-110d) and median hospital stay was 20 days (range, 3-177d) for the entire cohort. Median follow-up was 22.5 months 
(range, 9 months -3.7 yrs) with recurrent arch obstruction occurring in 2 patients (4\%), both of whom were in the $1 \mathrm{~V}$ group. Conclusions: Aortic arch reconstruction with M-SCP is feasible and safe for $1 \mathrm{~V}$ and $2 \mathrm{~V}$ patients, with a low incidence of recurrent arch obstruction. Avoidance of prolonged cooling and rewarming using this technique may also reduce overall cardiopulmonary bypass support time.

\section{6}

Case Report : Anomalous Right Coronary Artery from Pulmonary Artery with Aorto-Pulmonary Window and Ventricular Septal Defect

Hariadi Hadibrata, Resident of Indonesian Cardiothoracic and Vascular Surgery Program, Indonesia

Budi Rahmat, Staf of Pediatric \& Congenital Heart Surgery, National Cardiovascular Center Harapan Kita, Jakarta,, Indonesia Pribadi $W$ Busro, Staf of Pediatric \& Congenital Heart Surgery, National Cardiovascular Center Harapan Kita, Jakarta, Indonesia Dicky Fakri, Staf of Pediatric \& Congenital Heart Surgery, National Cardiovascular Center Harapan Kita, Jakarta, Indonesia

Poppy S Roebiono, Pediatric Cardiologist, National Cardiovascular Center Harapan Kita, Jakarta, Indonesia

Novik Budhiwardana, Pediatric Intensive Care Unit, National Cardiovascular Center Harapan Kita, Jakarta, Indonesia

Eva M Harahap, Pediatric Intensive Care Unit, National Cardiovascular Center Harapan Kita, Jakarta, Indonesia

Introduction: Anomalous Right Coronary Artery from Pulmonary Artery (ARCAPA) is a very rare cardiac anomaly, where the right coronary artery is origin from pulmonary artery. It's incidence has been estimated as $0,002 \%$ of the population. Anatomically there are 4 variation of the origin coronary artery from pulmonary artery : Left coronary artery, (most frequently), Right coronary artery, both coronary artery from pulmonary artery (most lethally) and accessory coronary artery. Frequently, ARCAPA is found occasionally at coronary examination or surgery. We report a child with ARCAPA, AortoPulmonary Window (APW) and ventricular septal defect,(VSD) undergoing surgical repair.

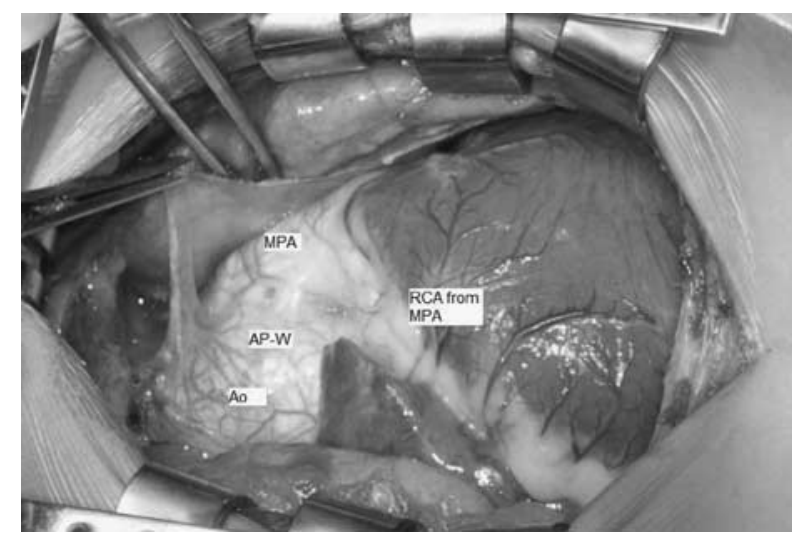

Case: A 3-year old girl weighing $8500 \mathrm{gr}$, was admitted to the hospital with chief complaint cough, and fever and recurrent dyspnoe. Echocardiography and catheterization demonstrated an ARCAPA with APW and VSD as well as Pulmonary Hypertension. A surgical repair of the AortoPulmonary septal defect and reimplantation of right coronary artery from pulmonary artery to aorta as well as closure of VSD was conducted. Postoperative Ekokardiografi revealed no residual of APW or VSD. Follow-up at4 0 days, there was no complaint, better activity and increase of body weight
Conclusion: ARCAPA is a rare congental cardiac diseases. Simptom and severity of the disesase depends on variation of origin of the coronary artery. Patient can grow and develop well after the adequate surgical treatment.

\section{7}

Ventricular septal defect repairs in infants with left ventricular noncompaction and severe pulmonary hypertension

Masanori Hara, Department of Cardiovascular Surgery, Toho University Omori Medical Center, Japan

Tsukasa Ozawa, Department of Cardiovascular Surgery, Toho University Omori Medical Center, Japan

Satoshi Hamada, Department of Cardiovascular Surgery, Toho

University Omori Medical Center, Japan

Hiroshi Masuhara, Department of Cardiovascular Surgery, Toho

University Omori Medical Center, Japan

Chikao Teramoto, Department of Cardiovascular Surgery, Toho

University Omori Medical Center, Japan

Tsutomu Saji, Department of Pediatrics, Toho University Omori

Medical Center, Japan

Yoshinori Watanabe, Department of Cardiovascular Surgery, Toho

University Omori Medical Center, Japan

Nobuya Koyama, Department of Cardiovascular Surgery, Toho

University Omori Medical Center, Japan

Background: Left ventricular noncompaction (LVNC) is an unclassified cardiomyopathy. Intracardiac repair (ICR) for congenital heart disease complicated by LVNC has been only rarely described.

Case 1: A 2-month-old boy was referred for treatment. An echocardiogram revealed a large perimembranous ventricular septal defect (VSD) with LVNC and pulmonary hypertension $(\mathrm{PH})$. Cardiac catheterization indicated that PA pressure and aortic pressure were almost equal. To relieve cardiac volume overload and progressive PH, ICR was performed. However, low cardiac output syndrome developed. Decreased urine output required peritoneal dialysis (PD), and a $\mathrm{PH}$ crisis-like event necessitated nitric oxide (NO) inhalation. Intensive treatment improved hemodynamic and respiratory parameters and he was weaned from PD. Nitric oxide inhalation therapy was replaced by sildenafil administration. Three years after the repair, he is doing well without sildenafil therapy.

Case 2: A 5-month-old girl was admitted for a perimembranous VSD with LVNC and $\mathrm{PH}$ that had been revealed on echocardiography. Cardiac catheterization showed that PA pressure was $90 \%$ of aortic pressure. The malaligned VSD was closed with a patch. She required PD and NO inhalation after repair; however, both were successfully discontinued within 1 week. One month after surgery, she is receiving sildenafil and doing well.

Summary: Reports of VSD repair in patients with severe $\mathrm{PH}$ and LVNC are very rare. We described 2 such cases that were similar. Our findings indicate that the severity of PH might be increased by LVNC. Because our patients remain at risk for heart failure, arrhythmia, and thromboembolism, careful monitoring will continue.

\section{8}

Surgical experience with Ebstein anomaly and variants in neonates

Yorikazu Harada, Department of Cardiovascular Surgery, Nagano

Children's Hospital, Japan 
Takahiko Sakamoto, Department of Cardiovascular Surgery, Nagano Children's Hospital, Japan

Kentaro Umezu, Department of Cardiovascular Surgery, Nagano

Children's Hospital, Japan

Satoshi Yasukochi, Department of Pediatric Cardiology, Nagano

Children's Hospital, Japan

Kiyohiro Takigiku, Department of Pediatric Cardiology, Nagano Children's Hospital, Japan

Ichige Kajimura, Department of Pediatric Cardiology, Nagano

Children's Hospital, Japan

Gengi Satomi, Satomi Clinic, Japan

Objective: Neonates with critical heart failure soon after birth resulting from Ebstein anomaly and variants were very difficult to manage. We report our surgical experience with these malformations in severely symptomatic neonates.

Methods: A retrospective study of all patients $(\mathrm{n}=8)$ who underwent neonatal surgical intervention for Ebstein anomaly and varients between 1995 and 2008 was carried out. In view of preoperative assessment including chest X-ray and echocardiography, we adopted univentricular strategy in all patients. RV exclusion $(n=6)$ as well as reduction RA plasty were performed. Tricuspid valve was closed with a perforated patch. Pulmonary blood flow was secured by a modified Blalock Taussig shunt using 3.0 or $3.5 \mathrm{~mm}$ EPTFE graft. Delayed sternal closure was done in all patients.

Results: Median age and weight at operation were 6 days and 2800 g. Cardio thoracic ratio was nealy $100 \%$ in all patiets. Hospital survival in this cohort was $75 \%(6 / 8)$ and 1 patient died suddenly at home after discharge. Prenatal diagnosis was established among the survivor $(6 / 8)$, whereas no prenatal diagnosis was available in the hospital death patients $(2 / 8)$. Three patients are waiting for bidirectional Glenn shunt, and 2 patients completed Fontan procedure.

Conclusion: Modified Blalock Taussig shunt and RV exclusion with RA plasty combined with tricuspid valve closure by a fenestrated patch seemed to be a choice of procedure in the surgical treatment of severely symptomatic neonates with this malformations. Prenatal diagnosis was important in order to achieve good clinical results.

\section{9}

\section{Surgical treatment of ectopia cordis}

István Hartyánszky, Gottsegen György National Institute of Cardiology, Budapest, Hungary, Hungary

Tibor Verebély, Semmelweis University, I. Paediatric Department, Budapest, Hungary, Hungary

Krisztina Kádár, Gottsegen György National Institute of Cardiology, Budapest, Hungary, Hungary

Valéria Oprea, Gottsegen György National Institute of Cardiology, Budapest, Hungary, Hungary

Gábor Bodor, Gottsegen György National Institute of Cardiology,

Budapest, Hungary, Hungary

Krisztián Fischer, Gottsegen György National Institute of Cardiology,

Budapest, Hungary, Hungary

András Szatmári, Gottsegen György National Institute of Cardiology, Budapest, Hungary, Hungary

Ectopia cordis is classified into five types: cervical, cervicothoracic, thoracic, abdominal and thoracoabdominal. Prognosis (especially complete forms) is very poor.

Methods: We wish to present three cases: 1 . Closure of the chest wall defect, repair of omphalocele and resection of right ventricular diverticulum were performed, followed by correction of tetralogy of Fallot 22 months later. 2. After intrauterine diagnosis, repair of sternal defect and omphalocele, left modified Blalock-Taussig shunt (tetralogy of Fallot) was performed, followed by bidirectional Glenn shunt and pulmonary arterioplasty, later fenestrated TCPC, and closure of fenestration. 3. At a newborn (2700gr) with complete thoracic ectopia cordis replaceing the heart into the thoracic cavity was possible. Goretex patch was first placed to close the sternum defect, later the anterior chest wall was reconstructed successfully with bone cement.

Results: First patient died after reconstruction of tetralogy of Fallot. Second patient (now 17 years old) is doing well. To the authors's knowledge, he has the longest follow-up period after the reconstruction of ectopia cordis and complex heart defects. Third patient died 6 months after the surgical repair due to an acute pulmonary infection. This is the first reported case of Kryptonite bone cement used for sternal reconstruction.

Conclusions: Successful surgical management of this rare anomaly is possible. In all our cases replacement of the heart in the thoracic cavity was successful. The Kryptonite bone cement is a valid option for reconstruction of the thoracic cavity with ectopia cordis, and other types on anterior chest wall defects.

\section{0}

An attempt for prediction of postoperative drainage volume in pediatric cardiac surgery using parameters of preoperative global coagulation test

Tamaki Hayashi, Department of Pediatrics, Nara Medical University, Japan

Yoshihiko Sakurai, Department of Pediatrics, Nara Medical University, Japan

Kazuyoshi Fukuda, Department of Pediatrics, Nara Medical University, Japan

Midori Shima, Department of Pediatrics, Nara Medical University, Japan

Yoshiro Yoshikawa, Department of Thoracic and Cardiovascular Surgery, Nara Medical University, Japan

Coagulopathies after cardiac surgery are complex and its influences to the clinical condition remain unclear. The aim of this study was to determine if there is a direct relationship between coagulation parameters and postoperative chest tube drainage (CTD).

Methods: The coagulation factors were analyzed before and 24 hours after cardiac surgery in 20 patients with congenital cardiac disease. The parameters included platelet count, hematocrit, prothrombin time, activated partial thrombin time, thrombinantithrombin complex (TAT), Rotation thromboelastometry (ROTEM) parameters (clotting time (CT), clot formation time (CFT), maximum clot firmness (MCF), max velocity (maxVel), time to max velocity (t-maxVel), area under curve (AUC)), thrombin generation test (TGT) parameters (peak height (Peak),time to peak (ttPeak), endogenous thrombin potential (ETP), and operation time.

Results: Platelet count decreased and TAT increased after operation significantly. MCF and AUC altered significantly but there were no obvious change in TGT. Results of classical coagulation tests had no correlation with CTD. However, preoperative ETP and CFT, postoperative TAT, Peak, ETP, CFT, $\alpha$, maxVel, MCF, and AUC, and operation time correlate with CTD. Furthermore, there was a correlation between operation time and ROTEM parameters.

Conclusion: Our findings suggested that these parameters would be useful in predicting the postoperative bleeding risk. 


\section{1}

Right Ventricular Morphology is a Risk Factor for Patients undergoing Extracardiac Fontan Procedure Hani A Hennein, Pediatric Cardiothoracic Surgery, Rainbow Babies and Children's Hospital, Case Western Reserve University, Cleveland, OH, USA, United States

Ahmed Farouk, Pediatric Cardiothoracic Surgery, Rainbow Babies and Children's Hospital, Case Western Reserve University, Cleveland, $\mathrm{OH}$, USA, United States

Francine Erenberg, Pediatric Cardiology, Rainbow Babies and Children's Hospital, Case Western Reserve, United States

Yasser Al-Khatib, Pediatric Cardiology, Rainbow Babies and Children's Hospital, Case Western Reserve, United States

Alex Golden, Pediatric Cardiology, Rainbow Babies and Children's

Hospital, Case Western Reserve, United States

Ernest Siwik, Pediatric Cardiology, Rainbow Babies and Children's

Hospital, Case Western Reserve, United States

Kenneth Zahka, Pediatric Cardiology, Rainbow Babies and Children's Hospital, Case Western Reserve, United States

Objective: To evaluate the effect of univentricular morphology, as well as other perioperative risk factors, on mid-term outcomes following extracardiac Fontan (ECF) procedure.

Methods: Over a ten-year period ending in 2007, 128 consecutive patients (mean age of $4.4 \pm 3$.2years) underwent an ECF using an aortic homograft for the inferior cava-to-pulmonary artery conduit. The single functional ventricle was of left ventricular morphology in $70 / 128$ patients (55\%), and right in 58/128(45\%). Right univentricular morphology was associated with low age at operation (age $<2 \mathrm{yrs}, \mathrm{p}=0.0004)$.

Results: $124 / 128$ patients (97\%) survived to hospital discharge. $117 / 128$ patients $(91 \%)$ were discharged on aspirin, and $4 / 128$ $(5 \%)$ on warfarin. $38 / 128$ patients $(29 \%)$ had prolonged pleural effusions $(>10$ days) with risk factors being lack of a patent fenestration $(30 / 51 \mathrm{vs} .8 / 77, \mathrm{p}<0.0001)$, and a low pulmonary artery index ( PAI, $187 \pm 25 \mathrm{vs} .218 \pm 24 \mathrm{~mm} 2 / \mathrm{m} 2, \mathrm{p}=0.003$ ). $20 / 128$ patients $(15 \%)$ had postoperative arrhythmias with right (vs. left) univentricular morphology being the most important risk factor $(18 / 58 \mathrm{vs} .2 / 70, \mathrm{p}<0.0001)$, in addition to a low PAI (176 $\pm 16 \mathrm{vs} .213 \pm 26 \mathrm{~mm} 2 / \mathrm{m} 2, \mathrm{p}=0.0162)$, and increased atrioventricular valvular regurgitation (AVR, $1.50 \pm 0.75$ vs. $0.29 \pm 0.53, p=0.0217)$. Risk factors for death included increased AVR (2.50 $\pm 0.58 \mathrm{vs} .0 .55 \pm 0.36, \mathrm{p}<0.0001)$ and increased pulmonary vascular resistance $(3.17 \pm 0.25 \mathrm{vs} .2 .43 \pm 0.54$ Wood units, $\mathrm{p}=0.0397)$. At $4.9 \pm 2.1$ years follow-up, there have been no documented thromboembolic events, circuit revisions, conduit replacements, or late deaths.

Conclusion: The ECF is a safe and effective operation. Using a homograft eliminates the need for long-term anticoagulation with warfarin in most patients. Right univentricular morphology was associated with a greater risk for developing postoperative arrhythmias, which may have an affect on long term survival.

\section{2}

\section{The Status of Pediatric Cardiac Medicine in Africa}

John P Hewitson, Chris Barnard Division Of Cardiothoracic Surgery, University Of Cape Town, South Africa

Peter Zilla, Chris Barnard Division Of Cardiothoracic Surgery, University Of Cape Town, South Africa

John Lawrenson, Western Cape Paediatric Cardiac Service, Red Cross Children's Hospital, South Africa

In Africa, thousands of children with untreated but correctable cardiac defects die each year because they live in poor countries where more is spent on servicing debt than on health and education. Despite outside aid there has been little progress in improving infrastructure and training in cardiac services until recently. Some services struggled for years (e.g. Nigeria: 122 cardiac operations in 34 years); others are slowly growing (e.g. Kenya: 2230 operations in 35 years). But unless supplied free by visiting teams, most facilities must exclude the indigent by charging for surgery.

The creditable focus on primary healthcare in Africa has often been at the expense of tertiary service development. Teams from developed nations have little impact unless local expertise is built. There needs to be better collaboration between groups to develop local centres. To this end, we have embarked on an audit of what is currently being done in Africa, to facilitate cooperation. Preliminary results have been collated, showing very little pediatric cardiac work being done.

Recent intergovernmental agreements within Africa are seeing plans laid to begin new cardiac units. The first such unit was recently inaugurated in Namibia.

Whilst equipment donations and team visits have value, the greatest need is always trained local personnel, who tend to be constantly lost to developed countries; methods must be sought to address this.

In Cape Town we aim to build a training base, and have already trained 5 specialist surgeons and cardiologists for other African countries.

\section{3}

Neonatal repair of Type C Interrupted Aortic Arch, Aberrant Right Subclavian Artery, Aortic Atresia and Ventricular Septal Defect

Samantha J Hill, Montreal Children's Hospital, McGill University, Canada

Gordan Samoukovic, Montreal Children's Hospital, McGill University, Canada

Ting Zhang, Montreal Children's Hospital, McGill University, Canada Noritaka Ota, Montreal Children's Hospital, McGill University, Japan Christo I Tchervenkov, Montreal Children's Hospital, McGill University, Canada

Introduction: Interrupted aortic arch (IAA) represents $1 \%$ of all congenital cardiac abnormalities. Type $\mathrm{C}$, wherein the interruption occurs between the left common carotid and the innominate artery, is the rarest variant, accounting for $1-5 \%$ of all IAA. Aberrant subclavian arteries are present in only $25 \%$ of IAA. When present, the combination appears to be associated with left ventricular outflow tract obstruction.

Case presentation: We report a rare case of neonatal biventricular repair of an interrupted aortic arch (type C) with an aberrant right subclavian artery and aortic atresia. Concomitant with these pathologies, a ventricular septal defect, an atrial septal defect, and a patent ductus arteriosus were identified. Both coronary arteries and the left carotid artery were perfused via a fistula linking the main pulmonary artery to the right coronary artery. Repair consisted of baffling the ventricular septal defect to include both semilunar roots, anastamosing the main pulmonary artery to the distal aortic arch, and placing a Contegra conduit to establish continuity between the right ventricle and the left and right pulmonary arteries. The patient did well post-operatively and was transferred to the ward within 2 weeks. We discuss the anatomy of this rare congenital anomaly, its surgical implications and issues surrounding the adequacy of the left ventricular outflow tract.

Conclusion: Type $\mathrm{C}$ interrupted aortic arch with an aberrant right subclavian artery and aortic atresia is a rare phenomenon which 
poses a challenge to the neonatal cardiac surgeon. It is however feasible to offer a single-stage biventricular neonatal repair.

\section{4}

Interventions for aortic arch recoarctation after Norwood palliation

Keiichi Hirose, Department of cardiovascular surgery, Shizuoka prefectual children's Hospital, Japan

Yasufumi Fujimoto, Department of cardiovascular surgery, Shizuoka prefectual children's Hospital, Japan

Tomohiro Nakata, Department of cardiovascular surgery, Shizuoka prefectual children's Hospital, Japan

Yuko Tosaka, Department of cardiovascular surgery, Shizuoka prefectual children's Hospital, Japan

Yujiro Ide, Department of cardiovascular surgery, Shizuoka prefectual children's Hospital, Japan

Maiko Tachi, Department of cardiovascular surgery, Shizuoka prefectual children's Hospital, Japan

Masaki Osaki, Department of cardiac intensive care unit, Shizuoka prefectual children's Hospital, Japan

Kisaburo Sakamoto, Department of cardiovascular surgery, Shizuoka prefectual children's Hospital, Japan

Background: Aortic arch recoarctation (reCoA) is one of the complications after Norwood palliation and may lead to severe cardiac failure. Especially in univentricular repair cases such as HLHS, aggressive interventions should be needed because pressure load for single ventricle from reCoA may influence late quality after Fontan operation. We investigated interventions for reCoA after Norwood palliation and evaluated midterm cardiac function.

Methods: From 1998, interventions for reCoA were needed in 13 cases (21.3\%) among 61 Norwood palliations. For this study, we evaluated cardiac function in 19 cases which passed more than one year after Fontan operation.

Results: Mean follow-up period is 64.6 months (max 110 months) after Norwood palliation. All 14 interventions were needed in 7 reCoA cases (36.8\%). 7 balloon angioplasty (BAP) was performed in 5 cases and 3 operations with 4 BAP were performed in 2 cases. Mean pressure gradient before interventions were $16.5 \pm 13.9 \mathrm{mmHg}$ for 11 BAP and $34.7 \pm 16.7 \mathrm{mmHg}$ for 3 operations. Patch plasty were performed in 3 operations. Timing for interventions were 9 cases before bidirectional Glenn, 1 case before Fontan and 4 cases after Fontan. There was no significantly differences in cardiac function between with $(\mathrm{n}=7)$ or without $(\mathrm{n}=12)$ intervention (reference Figure, grading for TR was that none was 0 , trivial was 1 , mild was 2 , moderate was 3 , and severe was 4 ).

Conclusions: Interventions for reCoA were needed in 13 cases (21.3\%) after Norwood operations. Aggressive BAP and adequate surgical interventions for reCoA cases may maintain good cardiac function after Fontan operation.

\begin{tabular}{lccc}
\hline & Intervention (-) & Intervention $(+)$ & $\mathrm{p}$ \\
\hline $\mathrm{EDV}(\%$ of Normal) & $168.0 \pm 53.7$ & $158.3 \pm 42.4$ & 0.69 \\
$\mathrm{EF}(\%)$ & $54.9 \pm 10.5$ & $54.2 \pm 5.8$ & 0.88 \\
$\mathrm{EDP}(\mathrm{mmHg})$ & $5.2 \pm 3.1$ & $5.6 \pm 1.6$ & 0.75 \\
$\mathrm{CVP}(\mathrm{mmHg})$ & $11.8 \pm 2.5$ & $12.3 \pm 1.7$ & 0.62 \\
$\mathrm{CI}$ & $3.25 \pm 0.6$ & $3.31 \pm 0.61$ & 0.83 \\
$\mathrm{SaO} 2(\%)$ & $95.3 \pm 2.2$ & $93.7 \pm 2.1$ & 0.16 \\
$\mathrm{Tei}$ index & $0.39 \pm 0.11$ & $0.44 \pm 0.13$ & 0.44 \\
$\mathrm{TR}$ & $0.58 \pm 0.60$ & $0.86 \pm 0.63$ & 0.36 \\
\hline
\end{tabular}

7225

Surgical Repair of Coarctation of the Aorta and Ventricular Septal Defect in Infancy

Betau Hwang, Taiwan

Pi-Chang Lee, Taipei Veterans General Hospital, Taiwan

Pei-Chen Hsu, Taiwan

Zeng-Chung Weng, Taiwan

Chung-Chang L Meng, Taiwan

Background: Although one-stage complete correction had been reported to be feasible in infants with coarctation of aorta (CoA) with ventricular septal defect (VSD), the two-stage correction is still effectively to diminish mortality and morbidity. The purpose of this study is to compare the surgical outcome of infants with CoA and VSD undergoing two-stage correction between with and without pulmonary artery banding (PAB) in the first stage operation.

Methods: A retrospective review of the patients with CoA and VSD undergoing two-stage correction was made. We divided these patients into 2 groups: Group I included the patients undergoing initial $\mathrm{COA}$ repair with $\mathrm{PAB}$, and then VSD repair; Group II included the patients undergoing initial CoA repair without $\mathrm{PAB}$, and then VSD repair. The clinical and surgical characteristics were evaluated and compared.

Results: Sixteen patients (male: 6, female: 10) with a mean age of $3.8 \pm 5.1$ months and a mean body weight of $3.9 \pm 1.0 \mathrm{~kg}$ were enrolled. The Group I patients had a longer aortic clamping time $(118 \pm 26$ vs. $42 \pm 12$ minutes, $\mathrm{p}<0.001)$ and a longer bypass time $(162 \pm 44$ vs. $85 \pm 25$ minutes, $\mathrm{p}<0.001)$ in the second stage operation.

Conclusion: The two-stage correction is a feasible and safe method to manage newborns and infants with CoA and VSD. The initial $\mathrm{CoA}$ repair without $\mathrm{PAB}$ may decrease the aortic clamping time and the bypass time of the second stage operation.

\section{6}

Staged right ventricular outflow tract reconstruction and pulmonary valve replacement for patient with congenital heart disease

Yujiro Ide, Shizuoka Children's Hospital, Japan

Yoshifumi Fujimoto, Shizuoka Children's Hospital, Japan

Keiichi Hirose, Shizuoka Children's Hospital, Japan

Masaki Osaki, Shizuoka Children's Hospital, Japan

Yuko Tosaka, Shizuoka Children's Hospital, Japan

Tomohiro Nakata, Shizuoka Children's Hospital, Japan

Maiko Tachi, Shizuoka Children's Hospital, Japan

Kisaburo Sakamoto, Shizuoka Children's Hospital, Japan

Background: Pulmonary valve function is a major concern after right ventricular outflow tract reconstructions (RVOTR). As homografts and xenogragfts are hardly available in Japan, we used handmade -monocusp or -valved conduit for RVOTR and planned pulmonary valve replacement (PVR) after their growth. We sought to clarify midterm outcome of this strategy.

Method: Retrospective chart review of patients who had PVR from Jan/1999 to Dec/2008 after definitive repair.

Results: Forty-one had PVR $5.8 \pm 4.0$ years after the last RVOTR. (bioprosthesis 40, mechanical valve 1 ) with mean age $10.2 \pm 5.7$ years, and mean body weight $29.2 \pm 16.6 \mathrm{~kg}$, respectively. Primary diagnosis was pulmonary atresia with/ without MAPCAs in 6/14, tetralogy of Fallot in 10, transposition of great arteries in 6, and others in 5. In 36, handmademonocusp or -valved conduit were used in the last RVOTR. In others, their own valves were preserved. One hospital death was observed during follow-up of $3.8 \pm 1.9$ years. 
Cardiac catheterization $1.5 \pm 0.76$ years after PVR showed no change of RVEF $(49.37 \pm 10.55$ vs $49.26 \pm 10.76)$, but significant improvement of RVEDVI (286.43 \pm 144.35 vs $177.48 \pm 57.74, \quad \mathrm{P}<0.05)$ and $\mathrm{CVP} \quad(8.38 \pm 3.36 \quad$ vs $6.82 \pm 2.88, \mathrm{P}<0.05)$. Neither LVEDVI $(201.15 \pm 71.08$ vs $183.53 \pm 55.98)$ nor LVEF $(60.93 \pm 9.01$ vs $57.36 \pm 9.68)$ showed change. Freedom from reoperation after 5 -years and 8 years were $100 \%$ and $41.7 \%$, respectively. Three required reoperation $7.4 \pm 1.8$ years after PVR due to pulmonary stenosis. Conclusions: PVR contributes to the reduction of right ventricular volume and the decrease of CVP. Long-term durability of bioprosthesis valve needs to be determined.

7227

Factors That May Improve the Long-Term Outcome of the Ross Prosedure in Children

Chawki F elZein, Heart Institute for Children, United States

Teressa Taylor, Heart Insitute fir Children, United States

MaryJane Barth, Heart Insitute for Children, United States

Anastasios Polimenakos, Heart Institute for Children, United States

Michel Ilbawi, Heart Institute for Children, United States

Introduction: Ross procedure(RP) is a good alternative for the management of aortic valve disease in children. Its main potential drawback, however, is progressive neoaortic root dilatation and neoaortic valve regurgitation necessitating reoperation.

Objective: To evaluate long-term results of selective approach,and technical modifications of RP in a cohort of pediatric patients.

Material and methods: From 11/1994 till 6/2006,37pts ( $\leqslant 18$ yrs) underwent modified RP (MRP). Primary pathology was aortic stenosis (AS) in 6pts (16\%), combined AS and aortic insufficiency (AI) in 31 pts $(84 \%)$.

Patient Selection: All pts with an aortic annulus $\mathrm{Z}$ value (AVZ) $<-2$ and pts with failed valvuloplasty were offered modified RP (MRP). Pts with AVZ $\geqslant-2$ had initial valvuloplasty.

Techinical Modifications: The pulmonary autograft was harvested with a generous margin distal to the sinotubular bar and with intact adventitial lining, was secured to the LV-aortic junction with uniplanar interrupted mattress sutures placed through the aortic wall, providing external support. The mobilized coronary arteries were reimplanted. The distal autograft anastomosis was re-inforced with multiple pericardial strips.

Results: Mean age was $10.67 \pm 5.35 \mathrm{yrs}(0.35-17)$, and weight $37.94 \pm 27.15 \mathrm{Kg}$ (6-95). Preoperative AVZ was $0.79 \pm 3.28$, and sino-tubular $Z$ value (STZ) was $0.61 \pm 2.40$. Eleven pts $(26 \%)$ needed concomitant Konno procedure. There was no morbidity or mortality. One pt $(2.7 \%)$ had aortic autograft disruption secondary to SBE 3 months postoperatively. Mean follow-up was $5.9 \pm 3.3$ years (2.3-11.7). On last follow-up echo, 6pts (16\%) had mild AI, $1 \mathrm{pt}(2.7 \%)$ had moderate AI, and $2 \mathrm{pts}(5.4 \%)$ had mild AS. Three pts $(8.1 \%)$ needed neopulmonary valve replacement and one pt $(2.7 \%)$ had repair of supravalvar aortic stenosis. On last follow-up echo, AVZ was $1.03 \pm 2.41$, STZ was $2.77 \pm 3.02$, and sinus of valsalva $Z$ value was $2.77 \pm 2.95$. Overall actuarial freedom from reoperation was $87 \%$, and freedom from autograft operation was $97 \%$ at 10 years. None of the autografts needed to be replaced. Conclusions: The described technical modifications may improve the long-term outcome of RP in appropriately selected pediatric patients.

\section{8}

Provision of congenital heart surgery ans catheter interventions in Moscow

Vladimir N Ilyin, Filatov Children's Hospital, Moscow, Russia
Marat V Tarayan, Filatov Children's Hospital, Moscow, Russia

Objective: The efficiency of the system of medical and surgical care in children and adults with congenital heart disease (CHD) residents of Moscow has not been analysed until now.

Methods: Moscow is a large megapolis with more than 11 million inhabitants. In 2007 there were approximately 100000 live births, and 1192 new cases of CHD were diagnosed in children. There are 2 centres of pediatric cardiac care, employing 15 surgeons and 6 interventional cardiologists. Adult CHD patients are looked after in 6 clinics; however only 1 clinic perform adult congenital heart surgery over 20 procedures a year. All these clinics perform surgery on patients coming from other regions of Russia as well.

Results: Last year 305 primary procedures and 15 re-operations were done in children with CHD. Of these, 233 were "open heart" and 87 "closed heart" operations. 108 interventional catheter procedures were also done. 31 patients were neonates. 220 children diagnosed with CHD are currently on the waiting list, of them 122 are awaiting definitive surgery following earlier palliations. At the same time there were 37 surgeries and 23 catheter interventions performed for GUCH-patients.

Conclusions: It appears that significant increase in levels of provision of congenital cardiac surgical services for residents of Moscow is needed, in order to reduce the waiting list for children, and increase volume of interventions for adults with CHD.

7229

Mid-term results of arterial switch procedure in cases with complex coronary anatomy

Vladimir N Ilyin, Filatov Children's Hospital, Russia

Oleg J Kornouhov, Filatov Children's Hospital, Russia

Vladislav A Krjukov, Filatov Children's Hospital, Russia

Asija A Valitova, Filatov Children's Hospital, Russia

Background: Intermediate results of the ASO in patients with complex coronary anatomy represent a great interest.

Methods: We investigated 11 patients who underwent ASO at a median age of 73 (6-273) days. Mean follow up duration was 28 $(6-84)$ months. Anatomic diagnoses were: simple TGA $(n=6)$, TGA/VSD $(n=4)$ and Taussig-Bing anomaly $(n=1)$. Coronary anatomy variants were: single CA $(\mathrm{n}=5)$, CA with intramural course $(n=5)$, mixed $(n=1)$. During follow up, all patients had ECG, Doppler-Echocardiography and Holter monitoring; one patient underwent coronary angiography.

Results: No patients died or required re-intervention during the foloow-up period. All patients were in NYHA Class 1. No ECG abnormalities or significant arrhythmias were registered. In 2 cases there was moderate systolic pressure gradient between RV and PA; 2 patients has trivial aortic regurgitation and 1 had mild mitral regurgitation. Size and systolic function of the ventricles were within normal limits in all patients exept one who had LV end-diastolic volume $z$-score $+3,5$ and $\mathrm{EF}=0,42$. This patient underwent coronary angiography, which showed no CA stenosis. In two patients with single and intramural CA anatomy types, one coronary ostium was reported as narrowed, but no ventricular dysfunction was found. Conclusion: In the group of patients with complex coronary anatomy good intermediate results of ASO were achieved. However, CA stenosis in these patients dictates the need for careful further observation.

\section{0}

Predictors of Redo Mitral Valve Replacement due to Outgrowth of the Valve in Children

Takeshi Inoue, Department of Cardiovascular Surgery, Kobe Children's Hospital, Japan 
Yoshihiro Oshima, Department of Cardiovascular Surgery, Kobe Children's Hospital, Japan

Ayako Maruo, Department of Cardiovascular Surgery, Kobe Children's Hospital, Japan

Chikashi Shimazu, Department of Cardiovascular Surgery, Kobe Children's Hospital, Japan

Tomonori Higuma, Department of Cardiovascular Surgery, Kobe Children's Hospital, Japan

Masahiro Yamaguchi, Department of Cardiovascular Surgery, Akashi Medical Center, Japan

Aim: We investigated the appropriate timing of redo mitral valve replacement (re-MVR) in children who underwent MVR with mechanical prosthesis.

Methods: Of 47 MVRs in 36 children at our institution since 1979, 11 were re-MVRs in 10 children aged 4.6 17.6 years (mean 11.6 years) due to outgrowth of the valve or pannus formation. The average age and body weight at initial MVR was 2.0 years and $8.5 \mathrm{~kg}$. Interval from previous MVR was $1.8 \sim 15.5$ years (mean 7.9 years). We analyzed the following data obtained from 36 children with bileaflet valves ; maximum transprosthesis flow velocity (Vmax) or transprosthesis mean pressure gradient (mPG) estimated by Doppler echocardiography, pulmonary artery wedge pressure (PAWP) assessed by cardiac catheterization, and GOAI (geometric valve orifice area indexed for body surface area (BSA)). In redo patients, the mean GOAI at previous MVR was $5.0 \mathrm{~cm}^{2} / \mathrm{m}^{2}$.

Results: All valves at re-MVR were larger in size than the previous MVR (mean valve size, $20.8 \mathrm{~mm}$ to $25.7 \mathrm{~mm}$ ). There were no hospital or late death on mean follow-up of 7.3 years after re-MVR. PAWP had a significant correlation with Vmax, $\mathrm{mPG}$ and GOAI $(\mathrm{p}<0.05)$. At the obtained linear regression (Figure), when PAWP was $18 \mathrm{mmHg}, \mathrm{Vmax}, \mathrm{mPG}$ and GOAI were predicted as $2.5 \mathrm{~m} / \mathrm{s}, \quad 11.4 \mathrm{mmHg}$ and $2.1 \mathrm{~cm}^{2} / \mathrm{m}^{2}$, respectively.

Conclusion: GOAI, Vmax and $\mathrm{mPG}$ are useful predictors of PAWP. Redo MVR should be considered, when BSA achieves twice that at initial MVR, Vmax exceeds $2.5 \mathrm{~m} / \mathrm{s}$ or $\mathrm{mPG}$ exceeds $10 \mathrm{mmHg}$.

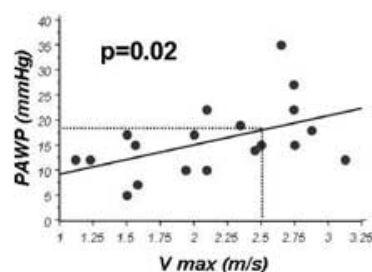

$V \max (\mathrm{m} / \mathrm{s})$
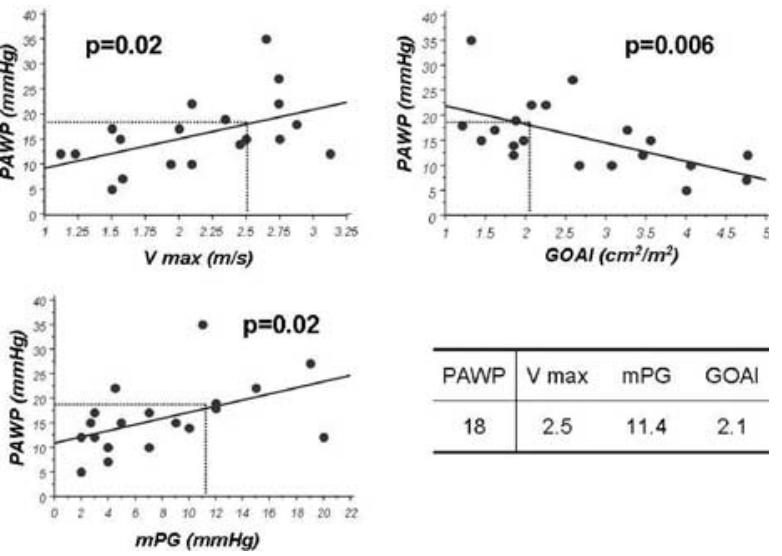

7231

Single Coronary Artery with Right Ventricular Fistula: Case Report and Literature Review

Yoichiro Ishii, Department of Cardiology, Gunma Children's Medical Center, Gunma, Japan

Takahiro Suzuki, Department of Pediatrics, Gunma University

Graduate School of Medicine, Gunma, Japan
Tohru Kobayashi, Department of Pediatrics, Gunma University Graduate School of Medicine, Gunma, Japan

Tomio Kobayashi, Department of Cardiology, Gunma Children's Medical Center, Gunma, Japan

Hirokazu Arakawa, Department of Pediatrics, Gunma University Graduate School of Medicine, Gunma, Japan

Introduction: Coronary artery fistula (CAF) and single coronary artery (SCA) are rare congenital heart diseases. Here, we report a rare case of CAF associated with a left SCA and review the literature regarding CAF associated with SCA.

Case: A 6-year-old boy was referred to us when a grade $3 / 6$ continuous murmur in the fourth intercostals space at the right sternal border was detected at a routine medical examination. On cardiac catheterization, the intracardiac and intravascular pressures were normal and the pulmonary/systemic flow ratio was 1.1:1. The ejection fraction of the left ventricle was $65.0 \%$, but slight apical hypokinesia was present. Selective coronary angiography revealed a left SCA arising from the left coronary sinus. An anomalous vessel arose from the left anterior descending artery and crossed the anterior surface of the right ventricular outflow tract. This vessel was dilated and very tortuous, and flowing into the right ventricle after communicated with the distal right coronary artery. At operation, the two CAFs were ligated after test clamping without any electrocardiographic changes. On follow-up catheterization, no residual fistulous communications were detected and the apical hypokinesia improved.

Discussion: We reviewed 19 cases in the literatures to assess the clinical characteristics, the types of SCA and CAF, and the treatment provided. Adult cases often have symptoms and suffer from complications. Therefore, we believe elective closure of the fistula during childhood in patients who have SCA combined with CAF.

\section{2}

The long term results of Senning operation

Shizuko Iwasa, Tokyo Women's Medical University, Japan Takashi Miura, Nagasaki University, Japan

Toru Okamura, Tokyo Women's Medical University, Japan Yuki Ichihara, Tokyo Women's Medical University, Japan Kazuaki Ishihara, Tokyo Women's Medical University, Japan Toshio Nakanishi, Tokyo Women's Medical University, Japan Yasuharu Imai, Tokyo Women's Medical University, Japan Hiromi Kurosawa, Tokyo Women's Medical University, Japan

Objective: Senning operation has been performed for the patient with transposition of the great arteries (TGA) before the arterial switch operation widespread in 1980s, and still now some cases need this operation for TGA with abnormal coronary artery and decreased left ventricular pressure and complex congenital heart disease. We examined the long term outcomes and associated risk factors after Senning operation.

Methods: 78 patients (male: 53, female: 25) underwent Senning operation from 1979 to 2008 in our hospital were reviewed. 44 patients were followed up in hospital and 9 patients were by phone interview.

Results: Early mortality was 5.13\% (4/78) and late mortality was $15.4 \%(12 / 78)$. The main causes of death were congestive heart failure, sudden death and pulmonary venous obstruction. Reoperations were performed in 11 patients (12.8\%) including tricuspid valve plasty or replacement, arterial switch conversion and pulmonary venous occlusion. The survival rate after Senning operation at 20 years was $65.4 \%(51 / 78)$. Senning operation 
using autologous pericardial flap were performed in 17 patients and their mortality was $58.8 \%(10 / 17)$. Reoperation was mandatory due to pulmonary venous obstruction in 4 patients and their mortality was $75 \%(3 / 4) .75 \%$ patients show sinus rhythm and 5 patients have had supraventricular arrhythmia. All survived patients are good condition in NYHA class 1 or 2 . Conclusions: Senning operation using autologous pericardial flap frequently causes pulmonary venous obstruction. Otherwise long-term outcome of this procedure is acceptable.

7233

Primary arterial switch beyond 3 weeks of age : What is feasible without ECLS?

Krishna S Iyer, Escorts Heart Institute \& Research Centre, New Delhi, India

Girish Kumar, Escorts Heart Institute \& Research Centre, New Delhi, India

Reetesh Gupta, Escorts Heart Institute \& Research Centre, New Delhi, India

Sunil K Kaushal, Escorts Heart Institute \& Research Centre,

New Delhi, India

Sameer Girotra, Escorts Heart Institute \& Research Centre, New Delhi, India

Sitaraman Radhakrishnan, Escorts Heart Institute \& Research Centre, New Delhi, India

Parvathi U Iyer, Escorts Heart Institute \& Research Centre, New Delhi, India

Savitri Shrivastava, Escorts Heart Institute \& Research Centre,

New Delhi, India

Objective: To evaluate the outcome of primary arterial switch in infants with dTGA.IVS beyond 3 weeks of age without mechanical support for rescue.

Design: A prospective pilot observational study.

Patients and Methods: 47 infants underwent primary arterial switch for dTGA.IVS during this period. 22 were $>3$ weeks of age (late Group) - median age 55 days, 22-149 days. Preop ECHO in these 22 infants showed varying LV mass $(30-43 \mathrm{gm} / \mathrm{m} 2)$ and LV morphology ranging from favorable to biventricular dysfunction. Post repair ECHO revealed varying degrees of LV dysfunction in 20/22. Perioperative low cardiac output was managed premptively using multiple, simple, inexpensive conventional strategies. Differences in primary (mortality) and secondary (measures of morbidity) outcome between the 2 groups were analyzed using Fisher's exact test, chi square, Mann-Whitney U and student $\mathrm{t}$ test as appropriate.

Results: In-hospital mortality was similar in both groups (late: 1/22 and early : $2 / 25, p=0.6$ ). Median ventilatory requirement (late 72.5 hours versus early - 95 hours), length of stay (late - 11.5 days versus early - 11.5 days) and inotrope score were similar in both groups respectively. There were no differences in the incidence of renal failure, lung injury and sepsis in the two groups. Conclusion: Preliminary observations suggest that primary arterial switch in infants $>3$ weeks of age using simple, inexpensive ICU strategies is feasible. These observations need to be validated in larger numbers of older infants to decide "how old is safe?".

\section{4}

Initial clinical experience with double shunt technique for hybrid palliation of hypoplastic left heart syndrome Marcelo B Jatene, Heart Hospital - ASS - São Paulo, Brazil Patricia M Oliveira, Heart Hospital - ASS - São Paulo, Brazil Fabiana P Succi, Heart Hospital - ASS - São Paulo, Brazil
Ieda B Jatene, Heart Hospital - ASS - São Paulo, Brazil Carlos A Pedra, Heart Hospital - ASS - São Paulo, Brazil

Simone Fontes-Pedra, Heart Hospital - ASS - São Paulo, Brazil

Elaine Cavalcanti, Heart Hospital - ASS - São Paulo, Brazil

Carlos R Ferreiro, Heart Hospital - ASS - São Paulo, Brazil

Objective: Hybrid approach with right and left PA banding and PDA stenting for surgical treatment of HLHS is being applied for many centers with improving results. Our objective is to present initial clinical experience with a new technique (double shunt), in hybrid treatment of HLHS.

Methods: 4 neonates ( 3 to 30 days), with HLHS were submitted to midline sternotomy, right and left PA banding, followed by a $4 \mathrm{~mm}$ reverse BT shunt from pulmonary artery to innominate artery; a second $7 \mathrm{~mm}$ BT shunt was done from PA to descending aorta. Distal edge of the 2nd shunt is closed and inserted in left pleural space; chest is closed and by left lateral 4th space thoracotomy the shunt is anastomosed to descending aorta. No PDA stenting was inserted.

Results: Initial success was obtained in 3 children, with one intraoperative death, during left thoracotomy. After initial favourable evolution, 2 children died (12th and 22nd PO), after infeccious complications. One child was operated on in the 5th month, with PA bandings removal and Norwood - Glenn operation. The initial evolution was good, being extubated in 2nd PO day, but with infection and sepsis detected in 10th PO day, with death in 15 th PO day.

Conclusion: Double shunt technique showed low surgical complexity, with minimal heart manipulation, good hemodynamic and ventilatory recovery. The 2 thoracotomies presented a disadvantage, but PTFE grafts facilitated CPB cannulation. Infection and sepsis were the major problems. More cases and experience should help to define real benefits of the technique.

\section{5}

Aorto pulmonary window: clinical management and surgical repair

Marcelo B Jatene, Heart Institute - University of São Paulo - São Paulo - SP, Brazil

Anderson Dietrich, Heart Institute - University of São Paulo - São

Paulo - SP, Brazil

Carla Tanamati, Heart Institute - University of São Paulo - São Paulo - SP, Brazil

Arlindo A Riso, Heart Institute - University of São Paulo - São Paulo - SP, Brazil

Juliano Penha, Heart Institute - University of São Paulo - São Paulo SP, Brazil

Marcos G Tiveron, Heart Institute - University of São Paulo - São Paulo - SP, Brazil

Antonio Augusto Lopes, Heart Institute - University of São Paulo São Paulo - SP, Brazil

Miguel Barbero Marcial, Heart Institute - University of São Paulo São Paulo - SP, Brazil

Objective: Aortopulmonary window (APW) is an uncommum congenital heart disease, with communication between the pulmonary artery (PA) and the ascending aorta in the presence of two separate semilunar valves. The objective of this sutdy is to show our institution experience, considering clinical and surgical aspects. Methods: Retrospective study, with patients diagnosed between 1986 and 2008; 33 patients with mean age of 19,6 months (13 days to 31 years) were admitted and diagnosed with APW, being $19(57,6 \%)$ females. Isolated APW was present in $17(51,5 \%)$ and with associated defcts in $16(48,5 \%)$ patients. Diagnosis was confirmed by echocardiogram in $21(63,6 \%)$, by angiographic 
study in $6(18,2 \%)$, by surgery in $5(15,2 \%)$ and in necropsy in $1(3 \%)$ patient. Moderate to severe mitral regurgitation was present in $9(27,3 \%)$ patients and $412 \%$ ) had cyanosis. Type I APW was detected in $24(72,7 \%)$ and type II in $9(27,3 \%)$.

Results: Twenty four $(85,7 \%)$ patients were submitted to surgical treatment with $2(6 \%)$ deaths. All other patients had a favourable outcome, with good long term follow-up. All patients, but one, were operated on with $\mathrm{CPB}$ support, closing the APW with heterologous patch and correcting associated defects; most commom associated lesions were VSD in 6 , ASD in 4, PDA and subaortic stenosis in 3 .

Conclusion: The surgical results are satisfactory, with low morbidity; recomendation after initial diagnosis is to operate soon to avoid pulmonary irreversible sequelae. The presence of associated congenital heart lesions could facilitate the diagnosis.

\section{6}

Neo-aortic valve function after arterial switch operation for transposition of great arteries

Won-Kyoung Jhang, University of Ulsan, Asan medical center, Korea Jeong-Jun Park, University of Ulsan, Asan medical center, Korea Tae-Jin Yun, University of Ulsan, Asan medical center, Korea Young-Hwue Kim, University of Ulsan, Asan medical center, Korea Jae-Kon Ko, University of Ulsan, Asan medical center, Korea In-Sook Park, University of Ulsan, Asan medical center, Korea Dong-Man Seo, University of Ulsan, Asan medical center, Korea

Objectives: Aortic insufficiency (AI) are major concern after the arterial switch operation (ASO) but there are still many controversies about incidence and risk factors for these. The aim of this study was to evaluate the fate of neo aorta and the neo-aortic valve function.

Methods: Between April 1997 and July 2008, 239 patients had an ASO for TGA using trap door technique in our institution. A retrospective review of 216 hospital survivors was performed.

Results: Upon the time of discharge, grade I AI was present in 19 $(19 / 216,8.7 \%)$ patients and grade II AI in $3(3 / 216,1.3 \%)$ patients. At a mean follow-up time of $70.5 \pm 53.5$ months, there was grade I AI in $31(31 / 216,14.3 \%)$ patients and grade II in $6(6 / 216,2.7 \%)$ patients. The $\mathrm{Z}$-value of neo-aortic valve, mid-sinus, and sinotubular junction at the lastest follow-up echocardiography were significantly larger in patients with grade II AI than others $(p=0.001)$. Freedom from at least grade II AI was $95.0 \%$ at 10 years. Reoperation for $\mathrm{AR}$ was done in only 1 patient with AI grade II because of LV dysfunction. None of perioperative and operative variables was significantly associated with the development of severe AI.

Conclusions: The prevalence of AI had an increasing tendency over time but significant $\mathrm{AI}$ and the need for reoperation are rare. No definite risk factors for AI was revealed from our data. However, more long term follow - up and meta analysis is needed to compair with other surgical technique and discover risk factors.

\section{8}

Surgical repair of aortic coarctation: a single institutional experience of 383 patients

Sune B Johansson, Department of Pediatric Cardiac Surgery,

Lund University Hospital, Sweden

Pia S Sjöberg, Department of Pediatric Cardiac Surgery,

Lund University Hospital, Sweden

Torsten Malm, Department of Pediatric Cardiac Surgery,

Lund University Hospital, Sweden

Jens Johansson, Department of Pediatric Cardiac Surgery,

Lund University Hospital, Sweden
Peeter Jogi, Department of Pediatric Cardiac Surgery,

Lund University Hospital, Sweden

Milad El-Segaier, Department of Pediatric Cardiology, Lund University Hospital, Sweden

Objective: To study outcome of surgical experience in infants and children with coarctation of the aorta. A special focus is given to the rate of re-coarctation, the impact of younger age at operation and mortality.

Methods and patients: Retrospective study of all 383 patients who have been operated for coarctation of the aorta at our institution between 1991 and 2005. Median follow-up time was 7.5 years (range 3.5 months - 15.8 years). Median age and weight at operation was 44 days (range $2-15.9$ years) and $4.0 \mathrm{~kg}$ (range $1.1-82.4 \mathrm{~kg}$ ), respectively. 182 patients were younger than one month and 18 weighed less than $2.5 \mathrm{~kg}$.

Results: There was no early mortality. Late mortality was $9 / 383$ $(2.3 \%)$, none associated with the coarctation. 31 patients needed re-intervention due to recoarctation. The recoarctation rate for children younger than one month has decreased from $17.1 \%$ during the first 5 years of the study period to $7.8 \%$ (7/89) during the last 5 . Although $71 \%$ of the patients with recoarctation were younger than 2 weeks at primary surgery, weight less than $2.5 \mathrm{~kg}$ was not a risk factor for recoarctation. After 5 months of age at operation, the risk for recoarctation is minimal.

Conclusions: Primary surgical repair of coarctation in newborns and infants is still a reasonable approach with low mortality and recoarctation risk. During the study period, technical improvements in both surgery and perioperative care have had major impact on the results.

\section{9}

Minimally Invasive Surgery through right anterior small thoracotomy (RAST) for Congenital Heart Defects Suresh Joshi, India

Niranjan Kumar, Wockhardt Pediatric and Congenital Heart Center, Mumbai, India

Swati Garekar, Wockhardt Pediatric and Congenital Heart Center, India

Snehal Kulkarni, India

Zakiya Khan, India

Pankaj Kasar, India

Sachin Patil, Wockhardt Pediatric and Congenital Heart Center,

Mumbai, India

Prasanna Salvi, India

Minimally Invasive open heart surgery (MIS) is a well accepted technique with various possible approaches. We describe our method and our results.

A retrospective analysis of patients who underwent MIS of congenital heart defects at our center was done.

From November 2006 to August 2008, 26 patients were identified. $15 / 26$ were male. The mean age was $9.09 \pm 6.18$ years. The mean weight was $25.65 \pm 13.7 \mathrm{~kg}$. The defects included secundum ASD (10), sinus venosus ASD (6) and perimembranous VSD (10). A short incision was made in the fifth intercostal space. Femoro -Bicaval cannulation was done. The mean cardiopulmonary bypass time was $62.87 \pm 15.73$ minutes (Range $34-106)$. The mean cross clamp time was $40.52 \pm 15.4$ minutes (Range 17-81). The intercostal drain was brought out through the same incision site used for bicaval cannulation. 16 patients (64\%) were extubated in operating room while the rest were extubated within 6 hours post surgery. Mean duration of IV analgesic use was $22 \pm 8.1$ hours. Drains were removed at a mean of $28 \mathrm{hrs}$. The average intensive care 
unit stay was $38.43 \pm 14.09$ hours. The mean in-hospital stay was $5 \pm 0.8$ days. In all cases post-operative echocardiogram suggested a complete closure of defects. There were no reexplorations. There was no early or late mortality.

The RAST technique involves less thoracic tissue handling, minimizes trauma to structures; is less painful and more cosmetic. The ICU and ward stay is reduced. The RAST is a safe and effective alternative to a posterior/ lateral thoracotomy for correction of congenital heart defects.

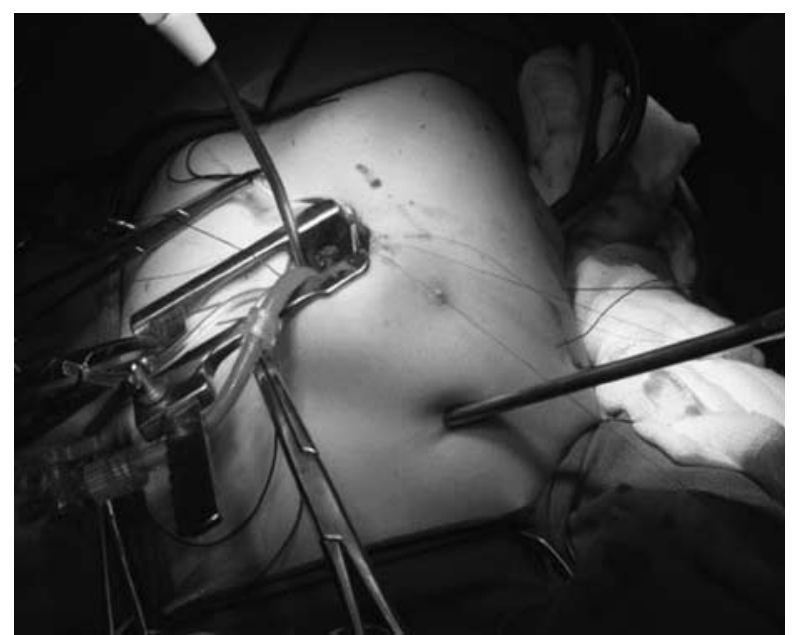

7240

Previous bidirectional cavopulmonary shunt may reduce postoperative morbidity after fenestrated extracardiac Fontan procedure

Tae-Gook Jun, Samsung Medical Center, Sungkyunkwan University School of Medicine, Korea

Ji-Hyuk Yang, Samsung Medical Center, Sungkyunkwan University School of Medicine

Pyo Won Park, Samsung Medical Center, Sungkyunkwan University School of Medicine

I-Seok Kang, Samsung Medical Center, Sungkyunkwan University School of Medicine

June Hurh, Samsung Medical Center, Sungkyunkwan University School of Medicine

Heung Jae Lee, Samsung Medical Center, Sungkyunkwan University School of Medicine

Objective: Although mortality and morbidity after the Fontan operation is low in the current era. factors related to prolonged postoperative morbidity are not clearly defined. The aim of this study is to evaluate and analyze the perioperative variables related postoperative morbidity, and the fate of fenestration during follow up period.

Methods: We performed a retrospective review of 52 patients underwent fenestrated extracardiac Fontan procedure from January 1996 to June 2008. The median age at surgery was 34.8 (range: $18.5 \sim 156$ ) months and the median body weight 13.2 (range: $9.5 \sim 33$ ) kg. A multivariable logistic regression model was used to compare demographic, anatomic, and physiological variables for the postoperative morbidity.

Results: Early mortality was 1.9\% (1/52). Median duration of respiratory support was 13 hours (range: 4-328 hours). Median duration of chest tube drainage was 6 days (range: 2-45 days), and median duration of hospital stay was 16 days(range: 7-444 days). In a multivariable model operation without previous bidirectional cavopulmonary shunt (OR 30, 95\% CI 3.1-289) was the only independent risk factor for prolonged pleural effusion. Aorta cross clamp time was identified as a risk factor of prolonged respiratory support. During follow-up ranged from 6 to 51 months (median; 45 months), there was one late death (1.9\%). Twenty two patients (43\%) underwent intervention for the fenestration closure.

Conclusions: The fenestrated extracardiac Fontan procedure, performed as a staged procedure, can be performed with low mortality and morbidity. Previous bidirectional cavopulmonary shunt may reduce postoperative morbidity after fenestrated extracardiac Fontan procedure.

\section{1}

Repair of Anomalous Pulmonary Venous Return to the Right Atrium

Mari Kakita, Osaka Medical College Hospital, Takatsuki, Japan, Japan

Shintaro Nemoto, Osaka Medical College Hospital, Takatsuki, Japan, Japan

Tomoyasu Sasaki, Osaka Medical College Hospital, Takatsuki, Japan, Japan

Hideki Ozawa, Osaka Medical College Hospital, Takatsuki, Japan, Japan

Takahiro Katsumata, Osaka Medical College Hospital, Takatsuki, Japan, Japan

Background: Diversion of the pulmonary venous (PV) blood into the left atrium (LA) through an atrial septal defect (ASD) is a mainstay of surgical repair for anomalous PV return to the right atrium (RA). We have used a pedicle RA wall flap to construct a wide diversion chamber. Early and midterm results of our surgical modification were assessed retrospectively.

Methods: Diagnosis; partial anomalous pulmonary venous return: 3 , total anomalous pulmonary venous return (TAPVR): 7 (Darling's classification; Ib: 1, IIa: 4, IIb: 2). Age distribution; neonate: 2, 1-3 months: 5, 4-12 months: 1 , and over 1 year: 2 . Operation: A wide RA incision was made vertically $1 \mathrm{~cm}$ posterior and parallel to the right atrioventricular groove. Two transverse parallel incisions a few millimeters down to the terminal crest were made in the middle portion of the posterior RA wall to create a pedicle RA wall flap. The tissue between the PV orifice or recess and the ASD was cut-back (5) or resected (2) in TAPVR. The rectangular flap was applied as a bulging roof to close the interatrial communication, covering PV and ASD, using absorbable polydioxanone suture. The remaining RA incision was closed directly.

Results: There was no death or atrial arrhythmia occurred in all cases during follow-up (up to 45 months) with a wide pathway from the PV to the LA by echocardiography.

Conclusion: Since the pedicle RA wall flap exhibits somatic growth, this method may be an alternative to achieve adequate long-term diversion for anomalous PV return to the RA.

7242

Indication of DKS procedure for Fontan candidate- Simplified anatomical discussion

Shingo Kasahara, Dept. of Cardiovascular surgery. Okayama University Graduate School of Medicine and Dentistry, Pharmaceutical Science., Japan

Yasuhiro Fujii, Dept. of Cardiovascular surgery. Okayama University Graduate School of Medicine and Dentistry, Pharmaceutical Science., Japan Yasuhiro Kotani, Dept. of Cardiovascular surgery. Okayama University Graduate School of Medicine and Dentistry, Pharmaceutical Science., Japan 
Yoshinori Miyahara, Dept. of Cardiovascular surgery. Okayama University Graduate School of Medicine and Dentistry, Pharmaceutical Science, Japan

Sadahiko Arai, Dept. of Cardiovascular surgery. Okayama University Graduate School of Medicine and Dentistry, Pharmaceutical Science., Japan

Teiji Akagi, Okayama University Hospital, Japan

Shunji Sano, Okayama University Hospital, Japan

Objective: Fontan circulation is the long-term goal in palliation for those with a functionally single ventricle. Systemic ventricular outflow obstruction (SVOO) has been widely recognized as an important risk factor for poor outcome in Fontan candidate. For this reason we have attempted following strategies: 1. Damus-Kaye-Stansel (DKS) procedure is a first choice for relieving obstructions. 2. DKS should be done not only for the patients having SVOO but also for the patients having bulvoventricular foramen (BVF) as outflow tract without SVOO. 3. DKS should be done at same time of BDG. This report summarizes the midterm results of our experience with the DKS for Fontan candidates in children, and examine about the indications.

Patients and Method: Forty-six consecutive patients who underwent DKS procedure between June 1993 and December 2008 were retrospectively reviewed. Thirty-nine patients had the systemic ventricular outflow tract (SVOT) depending on BVF. Result: Early mortality rate was $4.3 \%$ (2cases), although those were not related DKS procedure. The timing of DKS was at the time of Glenn operation in 36, Fontan operation in 5, Others in 5. The reason of requiring DKS were the SVOT depending on BVF with SVOO in 27, without SVOO in 12 and no depending on BVF in17. The mean preoperative maximum SVOT blood flow was $1.86 \mathrm{~m} / \mathrm{s}$, and it was improved in the all of the cases to $1.08 \mathrm{~m} / \mathrm{s}$. Conclusion: DKS should be done not only for the patients having SVOO but also for the patients having BVF dependent circulation without SVOO.

\section{3}

Stent graft insertion using novel technique for recurrent pulmonary venous obstruction

Shingo Kasahara, Department of Cardiovascular Surgery, Okayama University Graduate School of Medicine, Dentistry and Pharmaceutical Sciences, Japan

Yoshinori Miyahara, Department of Cardiovascular Surgery, Okayama University Graduate School of Medicine, Dentistry and Pharmaceutical Sciences, Japan

Takuya Kawabata, Department of Cardiovascular Surgery, Okayama University Graduate School of Medicine, Dentistry and Pharmaceutical Sciences, Japan

Teiji Akagi, Department of Cardiovascular Surgery, Okayama University Graduate School of Medicine, Dentistry and Pharmaceutical Sciences, Japan

Shunji Sano, Department of Cardiovascular Surgery, Okayama University Graduate School of Medicine, Dentistry and Pharmaceutical Sciences, Japan

Pulmonary venous obstruction (PVO) occurs rarely in infants undergoing correction of total anomalous pulmonary venous connection (TAPVC). And the surgical outcome of recurrent $\mathrm{PVO}$ is poor, especially for the patients required multiple reinterventions. Recently limited published data suggest that the stent insertion may improve that outcome in such the situation. We report the successful case of surgical stent graft insertion using stent in stent technique against multiple surgical repairs. A 5- year-old girl underwent surgery for supra-cardiac TAPVC. Despite three times surgical intervention, serious PVO was developed for her with obstructed total left PVs and right lower PV but right upper PV. Neo-intimal proliferation was caused obstruction of right upper PV, despite she had then a bare stent insertion. Through a median sternotomy, cardiopulmonary bypass was established with aorto-bicaval cannulaton. Under cardioplegic circulatory arrest, previous bare stent was totally removed through inter-atrial septum. At first bare stent was inserted and expanded, then PTFE tube graft was inserted at the inside of that. After that another bare stent was inserted at the inside of PTFE tube graft and expanded, as like stent-in-stent technique. Through these procedures, sterilized flexible fiber was very useful and helpful for stent positioning. She had a very smooth postoperative course with weaning from ventilator on post operative day 1. Echocardiography showed unobstructed pulmonary venous pathway with mild pulmonary hypertension. On follow-up 18 months, her CT confirmed unobstructed pulmonary venous flow.

\section{4}

Total Right Ventricular Exclusion Procedure for Neonatal Ebstein anomaly

Takuya Kawabata, Department of Cardiovascular Surgery, Okayama University Graduate School of Medicine and Dentistry, Japan

Shingo Kasahara, Department of Cardiovascular Surgery, Okayama

University Graduate School of Medicine and Dentistry, Japan Sadahiko Arai, Department of Cardiovascular Surgery, Okayama University Graduate School of Medicine and Dentistry, Japan Hideya Mitsui, Department of Cardiovascular Surgery, Okayama University Graduate School of Medicine and Dentistry, Japan Masami Takagaki, Department of Cardiovascular Surgery, Okayama University Graduate School of Medicine and Dentistry, Japan Sano Shunji, Department of Cardiovascular Surgery, Okayama University Graduate School of Medicine and Dentistry, Japan

We have created the total right ventricular exclusion procedure for the patients with Ebstein anomaly and arrhythmogenic right ventricular dysplasia since 1996 . We present a successful surgical case of a neonatal Ebstein anomaly that underwent this procedure.

The baby was born at 38week-gestation by planed normal vaginal delivery with his birth weight of $2.55 \mathrm{~kg}$. His fatal echocardiogram revealed the massive tricuspid regurgitation with plastering of the septal tricuspid leaflet, embranous pulmonary atresia and decompression of the left ventricle by the enlarged right ventricle, which were confirmed by UCG after birth. He was intubated and lipo-PGE1 was administered immediately after birth. His chest roentrogram showed 0.94 of the cardiothoracic ratio.

On the seventh day, a semi-urgent surgery was performed; resection of the most part of the right ventricular free wall and right atrium, closure of tricuspid valve orifice with fenestrated polytetrafluoroethylene patch and modified Blalock-Taussig shunt. The coronary sinus was included in the atrium and membranous septum was left above the patch. On the third postoperative day, for further reduction of the cardiac size, additional plications of the right atrium and right ventricle were performed during the delayed sternal closure. The postoperative $\mathrm{X}$-ray showed the decrease in cardiothoracic ratio from 0.69 to 0.59 . The postoperative UCG showed the good left ventricular function without septal asnynergy. Now he is doing well without respiratory nor inotropic support. We believe that early reduction of right ventricle volume overload will prevent possible deleterious effects on cardiorespiratory function. 


\section{6}

Intravenous Omega-3 pre-operatively protects against cardiopulmonary dysfunction following paediatric cardiac surgery

Niamh M Keenan, Department of Surgical Research, Royal College of Surgeons in Ireland, Dublin, Ireland., Ireland

Jonathan McGuinness, Our Lady's Children's Hospital, Crumlin, Dublin 12, Ireland., Ireland

John S Byrne, Department of Surgical Research, Royal College of

Surgeons in Ireland, Dublin, Ireland., Ireland

Darren McLoughlin, Department of Surgical Research, Royal College of Surgeons in Ireland, Dublin, Ireland., Ireland

Colin Canavan, Department of Surgical Research, Royal College of Surgeons in Ireland, Dublin, Ireland., Ireland

David Bouchier-Hayes, Department of Surgical Research, Royal College of Surgeons in Ireland, Dublin, Ireland., Ireland

J Mark Redmond, Our Lady's Children's Hospital, Crumlin, Dublin 12, Ireland., Ireland

Introduction: Improvements in paediatric cardiac surgical techniques have resulted in increasingly successful complex procedures. The systemic inflammatory response(SIR) to cardiac surgery is common and can result in multiple organ dysfunction (MOD) post-operatively.

Aim: We aimed to determine if a pre-operative infusion of omega-3 fatty acids could attenuate SIR induced early organ dysfunction.

Method: We used a juvenile piglet model of cardiopulmonary bypass and deep hypothermic circulatory arrest followed by an $8 \mathrm{hr}$ recovery period. Five animals received a $4 \mathrm{hr}$ infusion of omega-3 pre-operatively, five received a normal saline infusion serving as controls. Results are reported as mean $+/-$ standard error of mean, $\mathrm{p}<0.05$ on ANOVA.

Results: Significant early organ dysfunction was demonstrated in control animals. This manifested as reduced dynamic lung compliance (baseline: $12.7+/-1.2 \mathrm{ml} / \mathrm{cmH} 20 ; \quad 8 \mathrm{hrs}: 8.4+/-$ $1.1 \mathrm{ml} / \mathrm{cmH} 20, \quad \mathrm{p}=0.028$ ); troponin rise (baseline:0.1 $+/-$ $0.03 \mathrm{ng} / \mathrm{mL} ; \quad 3 \mathrm{hrs}: 12.1+/-1.8 \mathrm{ng} / \mathrm{mL}, \quad \mathrm{p}<0.0001)$; and left ventricular diastolic dysfunction $(\mathrm{dp} / \mathrm{Dtmin}$ baseline: $-2143+/$ -303 ; 4 hrs: $-4197+/-856, \quad \mathrm{p}=0.05)$. Omega-3 protected significantly against this injury, with improved pulmonary compliance at $8 \mathrm{hrs}$ (controls: $8.38+/-1.06 \mathrm{ml} / \mathrm{cmH} 2 \mathrm{O}$; omega$3: 10.22+/-1.04 \mathrm{ml} / \mathrm{cmH} 2 \mathrm{O}, \mathrm{p}=0.045)$ and reduced left ventricular diastolic dysfunction at $4 \mathrm{hrs}(\mathrm{dp} / \mathrm{Dtmin}$ : controls: $-4197+/$ -856 ; omega-3: $-2148+/-561, \mathrm{p}=0.047)$. With regard to the mechanism of protection, we demonstrated a reduced white cell count at $6 \mathrm{hrs}$ (controls:26.93 $+/-3 \times 10^{9} / \mathrm{L}$; omega$\left.3: 14.99+/-2.55 \times 10^{9} / \mathrm{L}, \mathrm{p}=0.02\right)$, and a significant increase in thrombomodulin in our omega- 3 group at $8 \mathrm{hrs}$ (controls: $0.31+/$ -0.05 ; omega-3:0.57 $+/-0.3, \mathrm{p}=0.04)$, indicative of a reduction in SIR.

Conclusion: We demonstrated a significant protective effect of omega-3 on cardiopulmonary function post cardiac surgery in a juvenile piglet model through a reduction in SIR. We conclude that this therapy could provide protection against the early MOD seen in paediatric cardiac surgical patients.

7247

Total-Anomalous-Pulmonary-Venous-Connection: Outcome in a developing unit

Asim Khan, The Children's Hospital \& Institute of Child Health, Lahore, Pakistan, Pakistan

Masood Sadiq, The Children's Hospital \& Institute of Child Health, Lahore, Pakistan, Pakistan
Objective: We report a retrospective analysis of the demographic, morphological and clinical profiles of patients along with results of operative repair for total anomalous pulmonary venous connection from an evolving paediatric cardiac unit.

Methods and Results: From January 2005 to December 2008, 38 patients (24 males \& 14 females) underwent repair for total anomalous pulmonary venous connection. Ages ranged from 2 days to 9 years (median $6 \&$ mean 17 months) and 26 of them were less than 1 year. Weight ranged from 2.4 to $18 \mathrm{~kg}$ (median $5 \mathrm{~kg}$ ). The anomalous connection was supracardiac in $18(47 \%)$, cardiac in 8 $(21 \%)$, infracardiac in $5(13 \%)$ and mixed in $7(18 \%)$ patients. Thirteen (34\%) patients had obstructed drainage and 21 (55\%) had moderate or severe pulmonary hypertension. Eleven patients (29\%) were operated on emergency basis. In hospital mortality was $21 \%$, more in obstructed group (46\%) than non-obstructed group (8\%), $\mathrm{p}=0.006$. The factors associated with early mortality were weight below 10th centile (OR 1.1; 95\% CI: 0.1-6.5, p-0.009), obstruction (OR 9.8; 95\% CI: 1.6-60, p-0.006) and sepsis (OR 23.3; 95\% CI: 3-177, p-0.002). Follow-up ranged from 1 to 45 months (median 24 months). There was one late death due to pulmonary vein stenosis that was re-operated upon.

Conclusions: In an evolving unit in a developing country, mortality continues to be high in patients with total anomalous pulmonary venous connection. Weight below 10th centile, presence of obstruction and sepsis appears to be the most important predictor of early operative mortality.

\section{8}

Surgical Management of Infracardiac Total Anomalous Pulmonary Venous Return: A Primary Sutureless Strategy Siho Kim, Dong-A University Medical Center, Korea

Glen S Van Arsdell, The Hospital for Sick Children's, Canada

John G Cols, Canada

Christopher A Caldarone, Canada

Objective: Obstructed total anomalous pulmonary venous return with infracardiac connection is frequently associated with a small pulmonary venous confluence and individual pulmonary veins. Surgically creating a traditional confluence or pulmonary vein to left atrial anastomosis with no flow disturbance can be challenging. We initiated a primary sutureless repair for all infracardiac TAPVD. As such there is no suturline on the confluence of pulmonary veins allowing maximal flow performance for an individuals intrinsic pulmonary venous drainage size. We sought to define the mid term result of a priamry sutureless repair technique for infracardiac type total anomalous pulmonary venous connection.

Methods: Seven patients with the primary obstructive infracardiac type total anomalous pulmonary venous connection were operated between Oct 2003 and Mar 2006. They comprised 2 male and 5 female patients (mean age 7.1 \pm 9.9 days). Associate anomalies were turncus arteriosus, atrial septal defects and right coronary anomaly. All patients underwent repair of the anomalous pulmonary venous connection by a sutureless technique with in situ pericardium. Four of seven patients (57.1\%) underwent concomitant procedures, including right ventricle to pulmonary artery conduit, arteriosus, atrial septal defect repairs and relieving right coronary artery stenosis. Results: No patient developed subsequent pulmonary vein stenosis. There was no operative or late death.

At the time of follow-up (mean $46.1 \pm 16.5$ months), echocardiography demonstrated a patent anastomosis in all patients without any evidence of stenosis.

Conclusion: The present data shows that a sutureless technique for obstructive infracardiac type total anomalous pulmonary venous connection was feasible and an effective surgical method. 
7249

Successful repair in multiple muscular VSDs with a sandwich patch technique

Siho Kim, Dong-A University Medical Center, Korea

Young seok Lee, Korea

Objectives: It is demandable to deal with muscular VSDs especially when those develop in the trabecular portion. Finding the exact locations of the defects and securely applying the patch on the multiple holes are challengeable.

Methods and results: The patient was 6-year old girl with a large perimembranous type VSD in conjunction with multiple muscular VSDs presenting with mild dyspnea caused by increased the right-to-left shunt.

We addressed the defects using two sandwich-like patches leaving one in the LV side of the interventricular septum without the continuous or interrupted suture techniques.

There was no operation related complication. The Follow-up echocardiogram shows none specific finding until now.

Conclusion: We believe the sandwich technique is a simple and secure method on for repairing a multiple VSD.

\section{0}

A simple patch technique of VSD with the progression

of aortic cusp prolapse or less than mild aortic regurgitation

Takashi Kitaichi, Department of Cardiovascular Surgery,

The University of Tokushima Graduate School, Japan

Homare Yoshida, Department of Cardiovascular Surgery,

The University of Tokushima Graduate School, Japan

Masahisa Urata, Department of Cardiovascular Surgery, The University

of Tokushima Graduate School, Japan

Tatsuo Motoki, Department of Cardiovascular Surgery, The University

of Tokushima Graduate School, Japan

Hirotsugu Kurobe, Department of Cardiovascular Surgery,

The University of Tokushima Graduate School, Japan

Tamotsu Kanbara, Department of Cardiovascular Surgery,

The University of Tokushima Graduate School, Japan

Yasunobu Hayabuchi, Department of Pediatrics, The University of

Tokushima Graduate School, Japan

Tetsuya Kitagawa, Department of Cardiovascular Surgery,

The University of Tokushima Graduate School, Japan

Objective: Optimal surgical technique and the timing of ventricular septal defect (VSD) with aortic cusp prolapse (ACP) remains to be determined. We have used a simple patch technique at the progression of ACP or the initial appearance of the aortic regurgitation (AR). We reviewed the patients with VSD and ACP to evaluate our tactics, retrospectively.

Patients: 24 patients with VSD and ACP, who underwent surgical repair during 1986 and 2008, enrolled in this study. The age at surgery was $6.0 \pm 4.5(0.3-17)$ years old. Among all patients, the location of VSD was perimembranous (2), midconus (5), and subpulmonary (16). Preoperative AR was observed at the mild grade (13), at the moderate grade (2), and at the zero grade (9). We performed simple patch technique for 22 patients with VSD, ACP and less than mild AR by the right atrial or pulmonary arterial approach. Two patients with VSD, ACP and moderate AR underwent both the above-mentioned VSD closure and the transaortic aortic valvuloplasty.

Results: There were no early and late deaths. All patients have been free from reoperation (re-aortic valve repair, aortic valve replacement). ACP improved in 17 patients, and unchanged in 7 cases one month after repair. However, among two of the latter,
ACP disappeared spontaneously during long-term follow-up. Consequently, mild AR before operation improved in 12 patients (92\%) after operation.

Conclusion: A simple patch technique of VSD with the progression of ACP or less than mild AR is a reasonable tactics for the patients with VSD and ACP.

7251

Double Valve (aortic and mitral) replacement in Children: Early Outcome

Bhagawan Koirala, Shahid Gangalal National Heart Centre,

Kathmandu, Nepal

Anil Acharya, Shahid Gangalal National Heart Centre, Kathmandu, Nepal

Samjhana Shakya, Shahid Gangalal National Heart Centre,

Kathmandu, Nepal

Ramesh Raj Koirala, Shahid Gangalal National Heart Centre,

Kathmandu, Nepal

Siddhartha Pradhan, Shahid Gangalal National Heart Centre,

Kathmandu, Nepal

Bijoy Gopal Rajbanshi, Shahid Gangalal National Heart Centre,

Kathmandu, Nepal

Rabindra Bhakta Timala, Shahid Gangalal National Heart Centre,

Kathmandu, Nepal

Jyotindra Sharma, Shahid Gangalal National Heart Centre, Kathmandu, Nepal

Objective: This study was carried out to evaluate the early surgical outcome of double valve (aortic and mitral) replacement (DVR) in children with rheumatic valve disease in Nepal.

Methods: Between 2002 and 2008 over 2000 valve replacements have been done in our institution. Of these 58 children under the age of 15 had undergone double valve replacement. Standard cardiopulmonary bypass techniques with mild hypothermia for perfusion and cold blood cardioplegia was used for myocardial protection. Bileaflet mechanical valves (St. Jude Medical ${ }^{\circledR}$, St Paul, MN, USA) were used to replace the valves.

Results: Average age of the children was 13.4 years. Indication for operation was heart failure despite medical therapy. Majority (87\%) of the children were in New York Heart Association (NYHA) functional class 3 or 4 . Mean preoperative diastolic left ventricular internal dimension (LVIDd) in was $6.2 \mathrm{~cm}$. Average $\mathrm{x}$-clamp time was 92 minutes and CPB time was 127 minutes. Operative mortality was $2 \%$. Most frequently implanted sizes were $19 \mathrm{hp}$ for aortic and $27 \mathrm{~mm}$ for mitral position. Seventeen percent of the children underwent tricuspid valve repair also. At 3 months all surviving patients who completed follow up studies $(\mathrm{n}=30)$ had significantly lower average postoperative LVIDd at three months than at the time of surgery $(4.5 \mathrm{~cm}$ vs $6.2 \mathrm{~cm})$. All of them were at functional class 2 or less. Conclusions: Double valve replacement in children with rheumatic disease has excellent early surgical outcome and may offer longer term palliation than repair.

7252

Influence of single ventricle morphology on the hemodynamics and exercise capacity in patients after modified Fontan procedure

Jacek Kolcz, Department of Pediatric Cardiac Surgery, Polish American Children's Hospital, Jagiellonian University Krakow, Pol, Poland

Lidia Tomkiewicz-Pajak, Jahn Paul II Hospital Krakow, Poland, Poland

Piotr Podolec, Jahn Paul II Hospital Krakow, Poland, Poland 
Janusz Skalski, Department of Pediatric Cardiac Surgery, PolishAmerican Children's Hospital, Jagiellonian University Krakow, Pol, Poland

The purpose of this study was to investigate the influence of ventricular morphology on efficiency of circulatory system and exercise capacity in patients after Fontan operation (FO).

Between 1991 and 2007, 270 patients with SV underwent staged FO. There were 134 (49.6\%) children with left ventricle (group I) and 136 (50.4\%) with right ventricle (group II). Retrospective analysis was performed (clinical status, echocardiography, biochemical markers, hemodynamics, cardiopulmonary exercise test).

The mortality after FO was $6,2 \%$. There was no difference in mortality rate between groups. Fifteen patients (5.9\%) were in NYHA class III, the others in NYHA class II or I. No difference in NYHA classification was noted between groups. There was no difference in oxygen uptake at the anaerobic threshold or peak exercise among groups. There were significant differences in hemodynamic response to exercise in group $\mathrm{I}$ : increase in heart rate $(\mathrm{p}=0.002)$, blood pressure $(\mathrm{p}=0.001)$ and tidal volume (0.001) during the test. The ventilatory equivalent for carbon dioxide (VE/VCO2) at anaerobic threshold was higher in group II $(p=0.049)$. In RV patients there was significant correlation of proANP with $\mathrm{VE} / \mathrm{VCO} 2(\mathrm{r}=0.88, \mathrm{p}=0.04)$ at an anaerobic threshold.

The survival rate and normal activity of patients after FO is satisfactory regardless of ventricular morphology. The hemodynamic response to exercise is better preserved in patients with systemic left ventricle. Higher VE/VCO2 in RV patients may be related to perfusion/ventilation mismatch. ProANP level may be predictor of unfavourable Fontan physiology.

\begin{tabular}{lccc}
$\begin{array}{l}\text { Differences in } \\
\text { hemodynamic data }\end{array}$ & Group I & Group II & p \\
\hline VEDP $(\mathrm{mmHg})$ & $6.97 \pm 1.8$ & $8.7 \pm 2.3$ & 0.002 \\
PA P $(\mathrm{mmHg})$ & $10.75 \pm 1.9$ & $12.07 \pm 2.3$ & 0.029 \\
CVP $(\mathrm{mmHg})$ & $11.82 \pm 1.8$ & $13.23 \pm 2.2$ & 0.01 \\
CI $(1 / \mathrm{min} / \mathrm{m} 2)$ & $3.93 \pm 0.89$ & $4.79 \pm 1.7$ & 0.005 \\
\hline
\end{tabular}

7253

Establishing a Rheumatic Heart Disease Biorepository for Multidisciplinary Research

Mark E Engel, University of Cape Town, South Africa

Liesl J Zühlke, Red Cross Children's Hospital, South Africa

Gasna Shaboodien, University of Cape Town, South Africa

Bongani M Mayosi, University of Cape Town, South Africa

Rheumatic heart disease (RHD) results from repeated attacks of acute rheumatic fever (ARF). Together, ARF and RHD are the leading cause of acquired heart disease in children in the world, affecting 15.6 million people worldwide. ARF is closely related to a preceding streptococcal pharyngitis which, if left untreated, carries up to a $3 \%$ risk of causing ARF. The pathogenetic mechanisms are largely unknown; studies suggest that host factors may play a role in individual susceptibility to ARF.

Advancements in molecular biology have necessitated the need for establishing biorepositories. Storage of biological samples with linkage to accurate clinical and epidemiological information will enable the determination of the role of genetic and nongenetic factors' interaction in determining ARF/RHD, the knowledge of which could aid in patient management.
In the report, we present our approach to developing a biorepository and data management procedures (including transfer agreements concerning confidentiality, use, and security of specimens and associated data; authorship and dissemination of research results, and ownership of future intellectual property) with discussion of the challenges needed to be considered in establishing such a bank. We also discuss the development of guidelines for specimen collection, processing, and storage in the context of participant confidentiality.

To our knowledge, no biorepository currently exists for RHD, and thus our intention was to create a resource to serve as a longterm national and international resource for the study of RHD. We anticipate that the biorepository will facilitate collaborative cross-disciplinary research as, and when, newer technologies become available and affordable.

\section{5}

Carotid artery intima-media thickness (CIMT) as a vascular subclinical marker of cardiovascular risk Vesna Herceg-Cavrak, Children's Hospital Zagreb, Department of Pediatrics, Cardiology Unit, Klaiceva 16, Zagreb, Croatia Maja Batinica, Children's Hospital Zagreb, Department of Pediatrics, Cardiology Unit, Klaiceva 16, Zagreb, Croatia Igor Boric, Children's Hospital Zagreb, Department of Pediatrics, Cardiology Unit, Klaiceva 16, Zagreb, Croatia

Jadranka Bozikov, Andrija Stampar School of Public Health, Croatia

Background: Child with coronary artery disease evolve as a new specialty. Assessment of traditional cardiovascular risk factors is mainstay of evaluation. Subclinical markers, cardiac and especially vascular, are helpful because they are sensitive to early and less atherosclerotic load. CIMT is an independent predictor of future cardiovascular events.

Objective: In children with esential hypertension determine CIMT and investigate whether there exists an association between CIMT and left ventricle mass index (LVMI).

Methods: A cross-sectional study of 32 subjects (mean age $15.3 \mathrm{yr}$, SD $1.7 \mathrm{yr}$ ) was conducted. The inclusion criteria were: (1) children ages 10-18 years with hypertension, defined as average systolic and/ or diastolic blood pressure that is $\geqslant 95$ th percentile for gender, age, and height on 3 or more separate occasions; (2) secondary hypertension exclusion; (3) no drugs with effect on blood pressure. CIMT measurements made of far wall from proximal and distal common carotid on both the right and left sides (mean of 4 measuraments). The correlation among CIMT and LVMI was determined using the Pearson correlation coefficient.

Results: The prevalence of left ventricle hypertrophy (LVMI $>38.6 \mathrm{~g} / \mathrm{m} 2.7$ ) was $31.3 \%$. CIMT was positively correlated with LVMI $(\mathrm{r}=0.30 ; \mathrm{p}=0.09)$.

Conclusions: Positive correlation between CIMT and LVMI is probably due to the same adaptational mechanisms and, as assessment of CIMT in adults is widely accepted as a valid and reliable measure of atherosclerotic burden, based on this and many other studies in children, these markers represent the end result of risk exposures in children as well.

\section{8}

Streptococcus beta hemolyticus endocarditis during hospital stay in a girl with acute rheumatic fever Najib Advani, Dept of Pediatrics, University of Indonesia, Jakarta, Indonesia

Endocarditis due to Streptococcus beta haemolyticus occurring during hospitalization in patients with acute rheumatic fever is not common and never reported in our institution. 
A-ten-year-old-girl was admitted with palpitation and a previous history of swollen left knee. On admission she was alert, no fever, with a grade $3 / 6$ pansystolic murmur at the apex spreading to the left axilla. No abnormalities seen on the left knee.Initial echocardiogram revealed moderate to severe mitral insufficiency with mildly dilated LA and LV. ECG showed a sinus rhythm with prolonged PR interval. Chest X-ray did not show cardiomegaly. Blood test showed ESR $71 \mathrm{~mm}$, CRP 96, ASTO $400 \mathrm{U}$.

She was given Benzathin Penicillin 1.2 million units for eradication/prohylaxis and asetosal. No IV line was inserted nor any intervention done. On day ten of hospitalization, fever developed, increasing gradually to $39 \mathrm{C}$. No significant changes of the pansystolic murmur was noted. On day 14 fever still persisted and a Janeway lesion seen on the sole. Another echocardiogram was carried out, which revealed two vegetations on the anterior and posterior leaflet of the mitral valve. Blood cultures from three different sites were taken over 24 hours, which later showed Streptoccus beta haemolyticus in every sample. In accordance with sensitivity test, Ceftriaxone was administered for 6 weeks. She was discharged in good condition.

We can conclude that in acute rheumatic fever, even after administration of benzathin penicillin in a hospitalized patient without any intervention, Streptococcus beta haemolyticus endocarditis may still be a threat.

\section{9}

When are the ECG changes significant in Kawasaki Disease?

Hak Lee Ang, Hospital Kuala Lumpur, Malaysia

Liang Choo Hung, Hospital Kuala Lumpur, Malaysia

Background: Kawasaki Disease (KD) is a common cause of acquired heart disease in children and may present with life threatening cardiovascular complications. Electrocardiogram (ECG) changes in KD are usually non-specific. Coronary artery (CA) abnormalities may lead to myocardial infarction and manifest as ST segment elevation, T wave inversion or abnormal Q waves.

Methods: We describe 4 children with significant ECG changes during the acute phase of illness but different severity of CA aneurysms. Q wave is considered significant if $>5 \mathrm{~mm}$ in depth and $>0.04$ second, ST segment elevation $>1 \mathrm{~mm}$ is significant. Results: We had 2 infants with KD and bilateral multiple large CA aneurysms (risk level IV) with thrombus in their right CA aneurysms. Both had deep Q wave of $7 \mathrm{~mm}$ and one also had ST segment elevation in leads II, III, and aVF. Both had raised Troponin $\mathrm{T}$ levels and normal creatinine kinase. They received antithrombotic agents in addition to antiplatelet treatment. Thrombi resolved after treatment. Two other patients had bilateral moderate multiple CA aneurysms (risk level III)without thrombosis. Their cardiac enzymes were normal. However, they had significantly deep Q wave or ST segment elevation in leads II, III, and aVF. All 4 patients had normal ventricular function throughout the course of their illness. One patient had myocardial perfusion scan and was normal. Their ECGs normalized on follow-up.

Conclusion: Deep Q waves and mild ST segment elevation in children with KD and moderate to large CA aneurysms are nonspecific and may not indicate myocardial infarction.

\section{0}

Characteristics of Fatal Atypical Kawasaki Disease - Less Contribution of Coronary Artery Aneurysm ?

Mamoru Ayusawa, Department of Pediatrics and Child Health, Nihon University School of Medicine, Japan
Takahiro Nakamura, Department of Pediatrics and Child Health, Nihon University School of Medicine, Japan

Junji Fukuhara, Department of Pediatrics and Child Health, Nihon University School of Medicine, Japan

Masaharu Matsumura, Japan

Osamu Abe, Department of Pediatrics and Child Health, Nihon University School of Medicine, Japan

Hiroshi Kanamaru, Department of Pediatrics and Child Health, Nihon

University School of Medicine, Japan

Naokata Sumitomo, Department of Pediatrics and Child Health, Nihon University School of Medicine, Japan

Yoshikazu Nakamura, Department of Public Health, Jichi Medical College, Japan

Objectives: Atypical Kawasaki disease (AKD) occasionally present serious course and fatal outcome despite of 4 or less principle signs. To clarify characteristics of fatal cases with AKD, demographic data of them reported to Japan Nationwide Surveillance for Kawasaki Disease (JNSKD) were summarized.

Methods: Ten fatal cases were reported as AKD (fAKD) including 3 females among 69,221 registrations in JNSKD during 1993-2002, while 34 fatal cases including 6 females were reported as 'typical' (fTKD). Age, duration until death, intravenous immunoglobulin (IVIG), coronary artery aneurysm (CAA) and reported clinical cause of death were compared between fAKD and fTKD.

Results: Average age and duration until death in fAKD and fTKD were 13.4 vs. 16.3 months (NS) and 74.5 vs. 151 days $(p<0.05)$, respectively. Initial IVIG day from onset and rate of IVIG treatment in fAKD and fTKD were 4.0 vs. 4.9 days (NS), and $4(40 \%)$ vs. $2(5.9 \%)$ cases $(\mathrm{p}<0.05)$, respectively. CAA was reported 3 cases $(30 \%)$ in fAKD and 16 cases $(47.1 \%)$ including 13 cases of giant CAA. Reported causes of fAKD were 2 encephalopathy's, 2 myocarditis', 1 aortic insufficiency, 1 heart failure, 1 multi-organ failure, 1 SIDS with moderate coronary aneurysm, and 1 drowning and 1 sudden death, without CAA. In contrast, 9 cases $(26.4 \%)$ in fTKD were reported as death of acute myocardial infarction.

Conclusion: Duration until death from onset of fAKD was shorter than that of fTKD. Indication of IVIG was insufficient. Although mild or moderate CAA complicate with AKD, its fatal contribution is considered to be negative.

\section{1}

Acute Viral Myocarditis: Role of Immuno Suppression Kalimuddin Aziz, National Institute of Cardiovascular Diseases Najma Patel, National Institute of Cardiovascular Diseases, Pakistan Tareen Saadullah, National Institute of Cardiovascular Diseases, Pakistan Habiba Tasneem, National Institute of Cardiovascular Diseases, Pakistan

Hasina Thawerani, National Institute of Cardiovascular Diseases, Pakistan

Saeed Talpur, National Institute of Cardiovascular Diseases, Pakistan

Our objective was to assess role of Prednisolone in Acute Myocarditis. We hypothesized that Predniosolone, administered at the 3 month of the onset of AM, will cause greater improvement and cure in comparison to the controls .

Methods: Inclusion criteria were duration of AM of 3 months. 173 children, age 3,7 \pm 2.9 years were registered, during July 2001 to Feb 2007. Randomization done at the completion of 3 months of disease. Patients excluded were those not showing at 3 months of disease or who had ejection fraction (EF) of left ventricle $>50 \%$ at randomization. 68 children were randomized, 
44 in Prednisolone treated group (Group A) and 24 in control group (Group B). Follow up $(\mathrm{f} / \mathrm{u})$ period for 38 children of group A was $14.6 \pm 9.1$ months and for 19 control group children was $13.3 \pm 8.6$ months. Prednisolone was given in $2 \mathrm{mg} / \mathrm{kg} / \mathrm{day}$ for 1 month with anti failure therapy (AFT) in Group A and only AFT in Group B.

Results: Comparisons were made at conclusion of 1 month after randomization. In group A, 23 children had EF greater than $40 \%$ and 21 less than $40 \%$, while in group B 18 had $\mathrm{EF}<40 \%$ and 6 had $>40 \%$, so group A showed greater improvement in $\mathrm{EF}(\mathrm{P}=0.029)$. Discrete analysis of change in EF of $<10 \%$ and $>10 \%$ or no change between groups showed significantly greater improvement in group A ( $P=0.019)$, after 1 month of randomization. At conclusion of $f /$ $\mathrm{u}$, more children in group A had EF of $>60 \%$ compared to group B $(\mathrm{P}=0.049)$. Mean $\mathrm{EF}$ on $\mathrm{f} / \mathrm{u}$ between the groups showed higher mean $\mathrm{EF}$ in group $\mathrm{B}(\mathrm{P}=0.028)$.

Conclusion: Immune suppression with Prednisolone administered at 3 month of onset of AM is effective in improving EF and cure of persistent left ventricular failure in comparison to children treated with AFT alone.

\section{4}

Severe Cardiac Infections in Infancy - a Reason to Suspect Immunodeficiency?

Jonathan TL Choo, KK Women's and Children's Hospital, Singapore Teng Hong Tan, KK Women's and Children's Hospital, Singapore Sriram Shankar, KK Women's and Children's Hospital, Singapore Keng Yean Wong, KK Women's and Children's Hospital, Singapore

Severe cardiac infections are rare. We describe 2 cases of fulminant cardiac infections, presenting in term infants with features associated with primary immunodeficiency.

The first patient presented at age 15 days with poor feeding. Auscultation revealed soft heart sounds and 2D-echocardiogram showed a large pericardial effusion. The intracardiac structures were normal. The infant underwent open pericardiotomy. Cultures from the pericardial fluid were positive for Staphylococcus Aureus. The infant also had an ulcer over the left hip which was positive for Mycobacterium Bovis. He was strongly suspected to have Severe Combined Immunodeficiency. He died at 5 months of age with parainfluenzae pneumonitis.

The second patient presented at age 2 months with fever and an irritable left hip. Left hip ultrasound scan revealed an effusion and open drainage yielded pus, positive for Staphylococcus Aureus. 2D-echocardiography revealed right ventricular outflow tract vegetations. She underwent debridement of the vegetations. Tissue cultures were also positive for Mycobacterium Bovis. She was diagnosed with Severe Combined Immunodeficiency and underwent bone marrow transplantation at 6 months of age.

Risk factors for severe cardiac infections remain the presence of underlying congenital heart disease. The first patient had pericarditis in the presence of a structurally normal heart. As the second patient had presented acutely with endocarditis, one could not determine whether the valves had been structurally normal before the onset of endocarditis. These 2 infants, did not have any septal defects or patent arterial ducts. We propose that in infants with fulminant cardiac infections, one should look for associated immunodefiency.

\section{7}

The Evolution of an Exercise Prescription Clinic for the Treatment of Pediatric Obesity

Astrid M De Souza, Division of Cardiology, Department of Pediatrics, British Columbia Children's Hospital and The University of British Columbia, Canada
Kristin M Houghton, Division of Rheumatology, Department of Pediatrics, British Columbia Children's Hospital and The University of British Columbia, Canada

Mary Hinchliffe, Shapedown Program, Centre for Healthy Weights, British Columbia Children's Hospital, Canada

Shubhayan Sanatani, Division of Cardiology, Department of Pediatrics, British Columbia Children's Hospital and The University of British Columbia, Canada

Walter J Duncan, Division of Cardiology, Department of Pediatrics, British Columbia Children's Hospital and The University of British Columbia, Canada

Derek G Human, Division of Cardiology, Department of Pediatrics, British Columbia Children's Hospital and The University of British Columbia, Canada

George GS Sandor, Division of Cardiology, Department of Pediatrics, British Columbia Children's Hospital and The University of British Columbia, Canada

James E Potts, Division of Cardiology, Department of Pediatrics, British Columbia Children's Hospital and The University of British Columbia, Canada

Background: Obese children are less active than their non-obese peers. The establishment of an exercise prescription clinic may provide families with the support they need to change their child's exercise habits. PURPOSE: To describe the evolution of and results for our exercise prescription clinic.

Methods: An initial cardiopulmonary exercise test (CPX) was performed to determine absolute and relative $\mathrm{VO}_{2}$ peak. A clinical exercise physiologist performed a baseline assessment of the child's current exercise regimen, habitual activity and screen time, and constructed a daily activity timeline. Perceived barriers to exercise and goals were also reviewed. Exercise prescription was child-specific and incorporated a periodized model. Children were asked to record their daily physical activity for 1 month to allow for modifications to the initial exercise prescription. Families were to return to the clinic on a monthly basis for continuous monitoring of the child's activity levels. Program length was a minimum of 6 months, with a repeat CPX performed afterwards.

Results: Fifty-three children were seen between January 2007 and February 2008. Twenty-eight patients (53\%) were seen at 6 month follow-up, while twenty-two patients (42\%) discontinued contact. Three patients did not need to be followed further. At follow-up, there was a significant increase in absolute and relative $\mathrm{VO}_{2}$ peak $(\mathrm{p}<0.001$ and $\mathrm{p}<0.002$, respectively), despite no change in weight $(p=0.086)$. Peak HR and treadmill time increased significantly from baseline $(\mathrm{p}<0.006$ and $\mathrm{p}<0.001$, respectively) suggesting an increased ability to tolerate exercise. Conclusion: Individualized exercise prescription is an important component of family-based behavioral programs directed at reducing pediatric obesity.

\section{8}

Fit or Unfit: Are overweight and obese children and adolescents really unfit or are we using the wrong assessment tools?

Astrid M De Souza, Division of Cardiology, Department of Pediatrics, British Columbia Children's Hospital and The University of British

Columbia, Canada

Kristin M Houghton, Division of Rheumatology, Department of Pediatrics, British Columbia Children's Hospital and The University of British Columbia, Canada

Mary Hinchliffe, Shapedown Program at the Centre for Healthy

Weights, British Columbia Children's Hospital, Canada 
Shubhayan Sanatani, Division of Cardiology, Department of Pediatrics, British Columbia Children's Hospital and The University of British Columbia, Canada

Walter J Duncan, Division of Cardiology, Department of Pediatrics, British Columbia Children's Hospital and The University of British Columbia, Canada

Derek G Human, Division of Cardiology, Department of Pediatrics, British Columbia Children's Hospital and The University of British Columbia, Canada

George GS Sandor, Division of Cardiology, Department of Pediatrics, British Columbia Children's Hospital and The University of British Columbia, Canada

James E Potts, Division of Cardiology, Department of Pediatrics, British Columbia Children's Hospital and The University of British Columbia, Canada

Background: Assessment of physical fitness is important in the management of overweight and obese children to modify long-term cardiovascular risk. Fitness has been shown to be lower in these children; however, the degree of impairment is unclear. $\mathrm{VO}_{2}$ peak is obtained from a maximal cardiopulmonary exercise test (CPX), but this method is expensive and requires technical expertise. The six minute walk test (6MWT), a submaximal test, can be used to predict $\mathrm{VO}_{2}$ peak, is easy to conduct, and requires minimal equipment. There are no data for the $6 \mathrm{MWT}$ in this population.

Objective: To determine the fitness of a cohort of overweight and obese children using a CPX and a 6MWT.

Methods: We studied 81 patients (46 male) between the ages of 9-17 years. All patients completed a treadmill CPX test. The $6 \mathrm{MWT}$ was completed by 49 patients. Results were compared to published normals.

Results: All males and 97\% of females had a median z-score within $( \pm 2 \mathrm{SD})$ or above the normal range for absolute $\mathrm{VO}_{2}$ peak (males $=1.0$; females $=1.4$ ). Median relative $\mathrm{VO}_{2}$ peak z-score was -1.8 for both males and females, with $63 \%$ and $60 \%$ within the normal range. Using the $6 \mathrm{MWT}, 65 \%$ of males and $86 \%$ of females had a walking distance below the normal range.

Conclusion: The CPX test suggests that a large proportion of overweight and obese children have normal fitness levels, while the submaximal 6MWT suggests that a large percentage are unfit. Depending on how fitness is assessed, these children may appear above, within, or below the normal range.

\section{0}

Hidden Systemic Diseases With Pericardial Effusion

Rasmia H Feituri, Libya

Naema I Ghobhaa, Libya

Ibrahim A Mekraz, Libya

Musa A Ghathi, Libya

Salma $M$ Ezwae, Libya

Salem M Erhuma, Libya

Objective: to prove that pericardial effusion could be a sign of serious diseases.

Design: 5 years prospective study.

Setting: Medical and cardiac departments of EL Fateh children hospital in Benghazi/Libya. Method All cases referred to cardiac clinic in this hospital with cardiomegally in the chest $\mathrm{x}$-ray, and whom Echocardiogram showed pericardial effusion in the period between 1st January 2003 to 1st December 2007 were included in the study. Results 87 patients had pericardial effusion, 56 $(64.36 \%)$ were males, and $31(35.64 \%)$ were females, so male: female ratio is $1.8: 1$.causes: $33(37.93 \%)$ was due to viral infection, $11(12.65 \%)$ with congenital heart diseases, 8 (9.2\%) rheumatoid arthritis, 7 (8.05\%) malignancy, 7 (8.05\%) postpericardiotomy, $6(6.89 \%)$ renal diseases, 3 (3.44\%) Bacterial infection, 3 (3.44\%) Rheumatic fever, 2 (2.3\%) skin lesions, 2 (2.3\%) Tuberculosis, 2 (2.3\%) infective endocarditis, 1 (1.15\%) Systemic lupus erythromatosis, 1 (1.15\%) Kawasaki disease, 1 $(1.15 \%)$ diagnosed intrauterine.

Treatment: Surgical drainage was performed on 3 (3.45\%), 5 (5.75\%) pericardio_ centesis, $21(24.14 \%)$ treated with small dose of diuretics. $19(21.85 \%)$ treated by steroid \pm aspirin, $5(5.75 \%)$ by intravenous antibiotics, 7 (8.05\%) treatment of malignancy, $6(6.89 \%)$ treatment of renal disease, the rest $21(24.12 \%)$ were small amount and resolve spontaneous without treatment.

Conclusions: Echocardiography is a good method for diagnosis and follow up of pericardial effusion and cardiac tamponade. the pericardial effusion could be a sign of serious systemic diseases.

7271

Natriuretic Peptide as an Adjunctive Diagnostic Test in the Acute Phase of Kawasaki Disease

Anne Fournier, Ste-Justine Hospital, Canada

Nagib Dahdah, Ste-Justine Hospital, Canada

Ana Siles, Ste-Justine Hospital, Canada

Jocelyne Cousineau, Ste-Justine Hospital, Canada

Edgar Delvin, Ste-Justine Hospital, Canada

Linda Spigelblat, Maisonneuve-Rosemont Hospital, Canada

Martine Montigny, Cité de la Santé Hospital, Canada

Background: There is a great emphasis on coronary artery involvement in Kawasaki disease (KD) but much less emphasis on the myocardial inflammation during the acute phase. Moreover, the clinical criteria and the biological markers utilized during the febrile days of the disease oversee the importance of a cardiac-specific marker. Some researchers have suggested that the B-type natriuretic peptide (BNP) increases upon $\mathrm{KD}$ onset, reflecting a subclinical myocardial dysfunction.

Objectives: To evaluate blood concentrations of BNP and NTproBNP during the acute and subacute phases of KD.

Methods: We conducted a prospective study comparing newly diagnosed KD patients to non-KD febrile controls. Blood specimen were collected at presentation, 6-12 hours after IVIG therapy, 1-2 weeks and 2-3 months later, and only upon reenrollment for controls.

Results: Forty-there KD and 19 control patients were consecutively enrolled. The mean age was $47.1 \pm 34.3$ and $62.2 \pm 44.9$ months respectively $(p=N S)$. Pre-IVIG NT-proBNP was significantly higher in KD than in controls $(923.6 \pm 1361.7$ v.s. $186.2 \pm 198.0 \mathrm{ng} / \mathrm{l} ; \mathrm{p}<0.001)$, with no statistical difference for BNP $(141.9 \pm 227.5$ v.s. $59.9 \pm 72.4 \mathrm{ng} / \mathrm{l} ; \mathrm{p}=0.112)$. Samples obtained 1-2 weeks and 1-3 months following the acute febrile status, returned to low values, comparable to controls.

Conclusion: This preliminary study suggests that NT-proBNP represents a hopeful adjunctive diagnostic method to support myocardial involvement in KD. As coronary artery dilatation may not be detected in all KD patients upon early presentation, this biochemical marker may prove valuable to support the diagnosis. Furthermore, NT-proBNP appears to have a significant advantage over BNP.

\section{3}

Long-term follow up the clinical outcome of Kawasaki disease patients with Giant Coronary Aneurysms Takashi Higaki, Department of Pediatric Cardiology, Stroke \& Cardiovascular Center Ehime University Hospital, Japan 
Eiichi Yamamoto, Department of Pediatric Cardiology, Stroke \& Cardiovascular Center Ehime University Hospital, Japan Yoshitaka Murakami, Department of Pediatrics, Ehime University Graduate School of Medicine, Japan

Osamu Matsuda, Department of Pediatrics, Ehime University Graduate School of Medicine, Japan

Kyoko Konishi, Department of Pediatrics, Ehime University Graduate School of Medicine, Japan

Koji Nagatani, Department of Pediatrics, Ehime University Graduate School of Medicine, Japan

Mitsugi Nagashima, Department of Cardiovascular Surgery, Stroke E Cardiovascular Center Ehime University Hospital, Japan

Eiichi Ishii, Department of Pediatrics, Ehime University Graduate School of Medicine, Japan

Background: The incidence of coronary artery involvement has markedly fallen following early gamma-globulin administration in Kawasaki disease (KD). Nevertheless coronary involvement may still occur and giant coronary aneurysms (GA) over $8 \mathrm{~mm}$ in diameter are more likely to lead to myocardial ischemia. However, long term cardiovascular complications and therapeutic strategy of KD with GA does not still remain well-known. Therefore, we retrospectively investigated the clinical outcome of KD patients with GA.

Patients and Methods: Between 1977 and 2008, we studied 27 patients with 53 branches. Patients' ages ranged from 4 to 31 years old and ages at onset ranged from 0.2 to 8.7 years old. All of the patients with GA were received a combination of low-dose aspirin and warfarin.

Results: 10 patients had no stenotic lesion and 10 patients were complicated with stenosis or spontaneous recanalization. In 5 patients, aneurysm-size decreased gradually without stenosis, and In 2 patients, coronary aneurysms completely regressed which confirmed by coronary angiography.

Four patients complicated acute thrombotic occlusion of coronary aneurysms. One had sudden death, one had acute myocardial infarction, one had angina pectoris and the other was silent. The age of three in these 4 patients were over 20 years old, and two of them had no stenosis under strict control of warfarin. Conclusion: To prevent coronary thrombosis, additional treatment may be needed in adult period even though having no stenosis. All patients who develop GA require diligent long-term review and re-consideration of therapeutic strategy.

\section{4}

Epidemiologic Research of Kawasaki Disease in Shanghai From 2003 Through 2007

Guo-ying Huang, Pediatric Heart Center, Children's Hospital of Fudan University, Shanghai, China

Chen-yan Yu, Pediatric Heart Center, Children's Hospital of Fudan

University, Shanghai, China

Min Huang, Children's Hospital of Jiaotong University, Shanghai,

China

Shu-bao Chen, Shanghai Children's Medical Center, Shanghai, China

Mei-rong Huang, Shanghai Children's Medical Center, Shanghai,

China

Rong-fa Wang, Xinhua Hospital of Jiaotong University, Shanghai, China

Xiao-jing Ma, Pediatric Heart Center, Children's Hospital of Fudan University, Shanghai, China

Fang Liu, Pediatric Heart Center, Children's Hospital of Fudan

University, Shanghai, China

Objective: To investigate the epidemiologic feature of Kawasaki disease (KD) in Shanghai from 2003 through 2007. Methods All patients in this survey were identified by the discharge diagnosis code in medical records with $\mathrm{KD}$ in 50 hospitals providing pediatric care in Shanghai during January 2003 through December 2007. Results A total of 1187 qualified patients with $\mathrm{KD}$ were reported. The incidence rate for each year: 43.06 (2003), 53.28 (2004), 50.35 (2005), 48.12 (2006), and 36.78 (2007) per 100,000 children under 5 years of age. The age of onset ranged from 12 days to 13.6 years (median 1.75 years), and 30\% of patients are under 1 year of age. The male/female ratio was 1.70:1. Fever (98.5\%) was the most common clinical symptom, followed by oral changes $(86.4 \%)$, extremities desquamate $(82.7 \%)$, conjunctive congestion (81.5\%), rash (75.8\%), lymphadenopathy (63.4\%), and crissum desquamate $(42.5 \%)$. Cardiac abnormalities were found in $24.2 \%$ of patients by echocardiography. The most common cardiac abnormality was coronary artery lesions including ectasia (64.1\%) and aneurysm (17.6\%). The fatality rate at acute stage of the disease was $0 \%$ in this study. A second onset of the disease occurred in $1.2 \%$ of patients. Conclusions Although the incidence of Kawasaki disease in Shanhai tends to increase, it is still lower than that reported in Japan. Sex and age distributions, clinical symtoms and rates of recurrence and cardiac lesion are similar to those in previous reports. Keywords: Kawasaki disease; Shanghai; Epidemicology; Cardiac sequelae

\section{5}

Peripheral Vascular Dysfunction in Patients with Coronary Artery Aneurysms Caused by Kawasaki Disease Using Laser Doppler Monitoring

Motofumi Iemura, Cardiovascular Research Institute, Kurume University, Japan

Tetsu Sugimura, Sugimura Child Clinic, Japan

Shintaro Kishimoto, Department of Pediatrics, Kurume University,

Japan

Hiroshi Nishino, Department of Pediatrics, Kurume University, Japan yusuke Koteda, Department of Pediatrics, Kurume University, Japan yoshiyuki Kudo, Department of Pediatrics, Kurume University, Japan Kenji Suda, Department of Pediatrics, Kurume University, Japan Toyojiro Matsuisi, Department of Pediatrics, Kurume University, Japan

Background: Endothelial dysfunction of coronary aneurysms $(\mathrm{CAL})$ and regressed coronary arteries after Kawasaki disease $(\mathrm{KD})$ is widely recognized. However, peripheral vascular function, especially small and micro sized vessels, is still uncertain. PURPOSE: To clarify the peripheral vascular function by using laser Doppler monitoring system (PeriFlux system 5000).

Methods: Subjects were 23 patients (CA) with KD (18.7 \pm 6.4 years old) who were still existed CAL and 17 healthy controls $(\mathrm{CONT})(20.4 \pm 6.2$ years old). All the measurements were performed in the supine position resting on a bed in a temperature-controlled room. After attachment of a sphygmomanometric cuff above the forearm, the laser Doppler probe was fixed to the skin of the thenar. The resting perfusion unit (rest PU) was monitored from 3 minutes and then forearm cuff was inflated up to $200 \mathrm{mmHg}$. After 3 minutes occlusion was suddenly released, the maximum perfusion unit (max PU) and time to max PU were recorded till hyperemia subsided.

Results: There were no significant differences in rest $\mathrm{PU}$ (mean $\pm \mathrm{SD}=188.5 \pm 108.9$ in $\mathrm{CA}$ vs. $179.5 \pm 163.4$ in CONT) and max PU (315.1 \pm 83.6 in CA vs. $329.9 \pm 128.7$ in CONT). However, Time to max PU in CA was significantly longer than Time to max PU in CONT $(37.4 \pm 30.5$ seconds in CA vs. $17.0 \pm 10.8$ seconds in CONT, $\mathrm{p}<0.05)$. 
Conclusion: These data suggested that peripheral vascular function, including endothelium, was impaired in patients with CAL. We considered that this laser Doppler monitoring system was non-invasive, practical, and useful system for measurement of vascular function.

7276

Long-term Follow-up Results of Percutaneous Catheter Intervention and Coronary Artery Bypass Graft Surgery for Kawasaki Disease in Japan

Masahiro Ishii, Dept. of Pediatrics Kitasato Universirt, Japan

Hiromi Muta, Japan

The aim of this study is to clarify the long-term results of percutaneous coronary intervention $(\mathrm{PCI})$ and coronary artery bypass graft (CABG) surgery for Kawasaki disease (KD) patients. The subject of this survey is KD patients were treated by PCI or CABG surgery in 1,637 hospitals in Japan. For the survey, a questionnaire was sent to pediatric departments in these hospitals, the type of procedure, age at the time of PCI or CABG surgery, gender, acute result, follow-up periods, follow-up results, complication of PCI and CABG surgery. A total of 76 PCI of 67 stenotic lesions ( 32 in RCA and 35 in LCA) in 62 patients ( 49 males and 13 females) were reported. Median age at PCI was 15.9 years (range: 2.2 to 35$)$ and median follow-up period was 5.2 years (0.2 to 19.0). The types of PCI included percutaneous transluminal coronary angioplasty (PTCA) $(n=15)$, stent implantation $(n=11)$, percutaneous transluminal coronary rotational ablation (PTCRA) $(\mathrm{n}=49)$, and direct coronary atherectomy $(\mathrm{n}=1)$. Of 76 PCIs, $68(90 \%)$ were successfully dilated. A total 156 grafts including 12 venous grafts used in CABG surgery ( 30 RCA and 66 LCA) for 84 $\mathrm{KD}$ patients (62 male and 13 female). Median age at CABG surgery was 12.8 years (range: 2.5 to 40 ) and median follow-up period was 11.5 years (0.1 to 21.2.0). Long term results of $\mathrm{PCI}$ and the results of CABG surgery were not differ and both methods are favorable. Conclusions long-term result of PCI and CABG surgery for KD patients is satisfactory.

7277

Asymmetric Dimethylarginine and the Effect of Folate Substitution in Children with Familial Hypercholesterolemia and Diabetes Mellitus Type1

Petr Jehlicka, Charles University Faculty Hospital, FN Plzen, Czech Republic

Frantisek Stozicky, Charles University Faculty Hospital, FN Plzen, Czech Republic

Otto Mayer, Charles University Faculty Hospital, FN Plzen, Czech Republic

Jaroslav Racek, Charles University Faculty Hospital, FN Plzen, Czech Republic

Jana Varvarovska, Charles University Faculty Hospital, FN Plzen, Czech Republic

Konrad Siala, Charles University Faculty Hospital, FN Plzen, Czech Republic

Objective: a recently discussed cardiovascular risk factor is asymmetric dimethylarginine (ADMA), which is known to act as an endogenous inhibitor of endothelial nitric oxide synthase. The aim of the study: to establish the relationship between ADMA and ultrasonographically or biochemically determined endothelial dysfunction in children, and furthermore to ascertain the effect of folate supplementation on this parameter. The study cohort included 32 children with familial hypercholesterolemia (FH), 30 with diabetes mellitus type 1 (DM1) and 30 age matched healthy children as a control group. Furthermore, twenty eight randomly selected FH and DM1 children were reexamined after 3-months supplementation with folic acid.

Results: baseline levels of ADMA (0.94 $\pm 0.18,0.84 \pm 0.18$, $0.77 \pm 0.14$, in $\mu \mathrm{mol} / \mathrm{L}$, respectively) and oxidized low density lipoproteins oxLDL $(73.69 \pm 24.9,51.71 \pm 23,55.7 \pm 30$, in $\mathrm{mU} / \mathrm{L}$, respectively) were significantly higher in $\mathrm{FH}$ group than in DM1 and healthy children. Children in the DM1 group had significantly lower homocysteine concentrations, nevertheless ADMA levels were normal. Folic acid supplementation significantly lowered homocysteine and hsCRP levels in both FH and DM1 groups; however, ADMA and oxLDL concentrations remained unchanged.

Conclusions: ADMA and oxLDL appear to be associated with ED in children with $\mathrm{FH}$ and thus may support early diagnosis of preclinical atherosclerosis in these children. Administration of folic acid does not appear to influence these markers in both $\mathrm{FH}$ and DM1 children.

\section{8}

External validation of a risk score to predict intravenous immunoglobulin resistance in patients with Kawasaki disease

Tohru Kobayashi, Gunma University Graduate School of Medicine, Japan Tomio Kobayashi, Gunma Children's Medical Center, Japan Akihiro Morikawa, Gunma University Graduate School of Medicine, Japan Tstsuya Otani, National Research Institute for Child Health and Development, Japan

Mitsuru Seki, Gunma University Graduate School of Medicine, Japan Keiji Tsuchiya, Japan Red Cross Medical Center, Japan Tomohiro Takeda, Japan Red Cross Medical Center, Japan Hirokazu Arakawa, Gunma University Graduate School of Medicine, Japan

Background: We previously reported a simple risk score to predict intravenous immunoglobulin (IVIG) resistance in patients with Kawasaki disease (KD) before initiation of treatment. However, the IVIG dosage used in this study $(1 \mathrm{~g} / \mathrm{kg} /$ day for each of 2 days) differs from the single infusion of $2 \mathrm{~g} / \mathrm{kg}$. Our aim was to assess the validity and applicability of our risk score in KD patients treated with a single infusion of $2 \mathrm{~g} / \mathrm{kg}$.

Methods: We utilized a database of $1448 \mathrm{KD}$ patients given IVIG ( $1 \mathrm{~g} / \mathrm{kg} /$ day IVIG for 2 consecutive days, $962 \mathrm{KD}$ patients; $2 \mathrm{~g} / \mathrm{kg} /$ day within a day, $486 \mathrm{KD}$ patients) at 15 hospitals in Gunma, Saitama, and Nagano prefectures, and metropolitan Tokyo from August 2000 to August 2008. We compared the accuracy of prediction by receiver operation characteristic (ROC) analysis. Result: Risk score points and clinical outcomes were similar in both groups. The area under the ROC curve to predict IVIG resistance were 0.82 (95\% confidence interval (CI), $0.78-0.85)$ in $1 \mathrm{~g} / \mathrm{kg} /$ day IVIG for 2 consecutive days, and 0.80 (95\% CI, 0.75-0.85) in $2 \mathrm{~g} / \mathrm{kg} /$ day IVIG within a day. Similarly, The area under the ROC curve to predict coronary artery lesions until a month were 0.78 (95\% CI, $0.71-0.84)$ in $1 \mathrm{~g} / \mathrm{kg} /$ day IVIG for 2 consecutive days and 0.78 (95\% CI, 0.72-0.85) in $2 \mathrm{~g} / \mathrm{kg} /$ day IVIG within a day. Conclusions: These findings indicated that we were able to apply our risk score to a regime of a single IVIG infusion of $2 \mathrm{~g} / \mathrm{kg}$.

\section{9}

Incidence of Rheumatic Fever and Prevalence of Rheumatic Heart Disease in a Underserved Population in South India Raman K Kumar, Amrita Institute of Medical Sciences and Research Center, India 
S Shylaja, Amritakripa Charitable Hospital, India

Ladish Krishnan, Amrita Institute of Medical Sciences and Research Center, India

Sanjeev Vasudevan, Amrita Institute of Medical Sciences and Research Center, India

K Ajithakumari, India

Background: Hospital records and selected population based surveys suggest that parts of the developing world may have experienced a substantial decline in the prevalence of rheumatic fever (RF) and rheumatic heart disease (RHD) and this is generally attributed to improving health care and human development.

Objective: To study the prevalence of RHD and incidence of RF in the population of Wayanad District in Kerala, South India, that has the highest tribal population and least sophisticated health infrastructure and lowest human developmentindex among all districts in the state (one doctor/3148 people).

Methods: The entire district (population: 0.79 million) was selected. Information on RF/RHD patients was gathered over 28 months (2006-2008) through passive reporting using the health care infrastructure of the district after thorough orientation of all health care professionals through structured workshops, teaching sessions, distribution of educational material. Additionally active surveillance was performed in a population proportionate sample of 11172 school children between 5-15 years. Echocardiography was performed for diagnostic confirmation all suspected cases.

Results: Of 953 cases of suspected RF/RHD who were reported, 373 had RHD (overall prevalence of $0.47 / 1000 ; 36 \pm 18$ years; 267 females) and 41 had previous history of RF and 27 instances of acute RF were recorded (incidence of 1.14-1.27/100,000/ year). Of the 11172 schoolchildren surveyed, only one child had RHD (prevalence of $0.09 / 1000$ ), and one child was on penicillin prophylaxis for a history of RF.

Conclusions: The incidence of RF and prevalence of RHD appear relatively low in this population in spite of limitations in health infrastructure.

\section{0}

\section{Coronary Arteriovenous Fistula and Kawasaki Disease}

Bo Sang Kwon, Korea

Gi Beom Kim, Department of Pediatrics, College of Medicine, Seoul National University, Seoul, Korea, Korea

Eun Jung Bae, Department of Pediatrics, College of Medicine, Seoul National University, Seoul, Korea, Korea

Chung Il Noh, Department of Pediatrics, College of Medicine, Seoul National University, Seoul, Korea, Korea

Yong Soo Yun, Department of Pediatrics, College of Medicine, Seoul National University, Seoul, Korea, Korea

Jung Yun Choi, Department of Pediatrics, College of Medicine, Seoul National University, Seoul, Korea, Korea

Introduction: Coronary arteriovenous fistula (CAVF) from the coronary artery to the pulmonary artery is a rare anomaly; however, its natural history and clinical course, especially related to Kawasaki disease (KD), have been still unclear. The purpose of this study was to identify the natural history and the relationship to Kawasaki disease.

Methods: We studied retrospective analysis of the patients diagnosed as CAVF from 1996 to 2007 in a tertiary center, excluding the iatrogenic cases.

Results: The mean age of 36 patients (M:F $=20: 16)$ was $5.4 \pm 5.4$ (range:0.1-22.4) years. The mean age at diagnosis and duration of follow-up was $3.9 \pm 4.8(0.1-18.4)$ years and $1.8 \pm 1.8$
(0.1-5.3) years, respectively. The fistulous opening sites were the main pulmonary artery in 35 and the left pulmonary artery in 1. Initial presentations were cardiac murmur in 7 , incidental detection in 24 including $6 \mathrm{KD}$ patients $(17 \%)$, chest pain in 2, and congestive heart failure in 1 . Fistulous opening in 8 patients spontaneously disappeared after $2.4 \pm 2.2(0.67-5)$ years but that in 28 has persisted for $1.6 \pm 1.8(0.05-4)$ years. In $\mathrm{KD}$, the duration of febrile illness was $6.3 \pm 2.4(5-10)$ days and coronary artery size was nearly $\operatorname{normal}(\mathrm{n}=3)$ and $3 \sim 3.5 \mathrm{~mm}$-sized dilatation $(\mathrm{n}=3)$. All KD patients were typical Kawasaki diseases. Although only 1 patient had abnormal myocardial SPECT findings, decreased anterior wall perfusion defect, he had no ischemic symptoms and normal ECG.

Conclusion: CAVF was frequently observed in $\mathrm{KD}$ patients. Although KD might be one of causes in CAVF, further studies are needed in order to search the etiology or causal relationship with KD.

\section{1}

\section{Echocardiographic study of pediatric patients with} Mucopolysaccharidosis

Gabriela N Leal, Instituto da Criança do Hospital das Clínicas da Faculdade de Medicina da Universidade de São Paulo, Brazil

Alessandro C Lianza, Instituto da Criança do Hospital das Clínicas da Faculdade de Medicina da Universidade de São Paulo, Brazil Ana Carolina de Paula, Instituto da Criança do Hospital das Clínicas da Faculdade de Medicina da Universidade de São Paulo, Brazil

Cláudio Leone, Instituto da Criança do Hospital das Clínicas da Faculdade de Medicina da Universidade de São Paulo, Brazil Chong A Kim, Instituto da Criança do Hospital das Clínicas da Faculdade de Medicina da Universidade de São Paulo, Brazil

Objective: To characterize the echocardiographic abnormalities in children and adolescents with Mucopolysaccharidosis, a glycosaminoglycan storage disease.

Method: Echocardiograms and medical records of 28 patients aged 2 to 14 years, seen from 2003 to 2005, were revised: 6 with MPS type I, 2 with type II, 6 with type III, 7 with type IV, 5 with type VI and 2 with type VII. At that time, the enzymatic replacement therapy was still not available at our institution.

Results: Echocardiographic alterations were detected in 26 patients (93\%), whereas only $16(57 \%)$ had abnormal auscultation, and $6(21 \%)$ cardiovascular complaint. Mitral valve thickening with dysfunction (regurgitation, stenosis or double lesion) was diagnosed in $60.8 \%$, left ventricular hypertrophy in $43 \%$ and aortic valve thickening with regurgitation in $35.8 \%$. There was no systolic dysfunction and mild left diastolic dysfunction was demonstrated in $21.5 \%$. Pulmonary hypertension was present in $32 \%$, causing the only two deaths recorded. A strong association was observed between accumulation of dermatan sulfate and presence of mitral valve dysfunction $(\mathrm{p}=0.0003)$, aortic valve dysfunction $(\mathrm{p}=0.006)$ and pulmonary hypertension $(\mathrm{p}=0.0032)$.

Among individuals with two or more exams, $82 \%$ had a worsening evolution.

Conclusions: Echocardiographic alterations in pediatric patients with Mucopolysaccharidosis are frequent and have a progressive character. Left valve lesions, ventricular hypertrophy and pulmonary hypertension were the most common findings and there was association between accumulation of dermatan sulfate and cardiovascular involvement. Unlike in adults, pulmonary hypertension was the main cause of death, not left ventricle systolic dysfunction. 
7282

Echocardiographic findings after enzyme replacement therapy for Mucopolysaccharidosis type I: initial results Gabriela N Leal, Instituto da Criança do Hospital das Clínicas da Faculdade de Medicina da Universidade de São Paulo, Brazil Alessandro C Lianza, Instituto da Criança do Hospital das Clínicas da Faculdade de Medicina da Universidade de São Paulo, Brazil Ana Carolina de Paula, Instituto da Criança do Hospital das Clínicas da Faculdade de Medicina da Universidade de São Paulo, Brazil Cláudio Leone, Instituto da Criança do Hospital das Clínicas da Faculdade de Medicina da Universidade de São Paulo, Brazil Chong Ae Kim, Instituto da Criança do Hospital das Clínicas da Faculdade de Medicina da Universidade de São Paulo, Brazil

Objective: To report the echocardiographic findings in children with Mucopolysaccharidosis type I, who received recombinant human alfa-L-iduronidase.

Method: Echocardiographic data from 3 male patients aged 2.1, 2.7 and 10.1 years at the initiation of therapy were revised. They received iaronidase $0.58 \mathrm{mg} / \mathrm{kg}$ weekly and a control examination was obtained after $2.3 \pm 0.5$ years.

Comparison were made using Student's t test, with $p \leqslant 0.05$ considered significant.

Results: Before treatment, all patients had mitral valve thickening and regurgitation was noted in two. Aortic thickening was present in two, accompanied by regurgitation in only one. Pulmonary hypertension was diagnosed in two. There was no left ventricle systolic dysfunction but mild diastolic dysfunction was detected in two. The $\mathrm{Z}$-score of the left ventricle diastolic diameter was $-0.06 \pm 0.49$, of the interventricular septum $+3.44 \pm 0.32$ and of the posterior wall $+3.63 \pm 1.25$.

After treatment, there was mitral thickening and regurgitation in all three. Aortic valvar thickening was universal, with regurgitation in two of them. Nevertheless, there was no patient with pulmonary hypertension and mild diastolic dysfunction was present in only one. Significant reduction in septal $(+1.70 \pm 0.46)$ and posterior wall $(+1.00 \pm 0.72)$ Z-score was detected $(p=0.05$ and $p=0.02$, respectively). The average left ventricle diastolic diameter was unchanged $(Z$-score $=+0.23 \pm$ $0.25 ; \mathrm{p}=0.3)$.

Conclusion: Enzyme replacement can reduce ventricular hypertrophy and improve diastolic feeling pattern, besides lowering pulmonary pressure. Unfortunately, the valve pathology doesn't seem to be altered in a short period of observation.

\section{4}

The tendency toward atopic diseases among children with past history of Kawasaki disease

Ming-Chih Lin, Taichung Veterans General Hospital, Taiwan

Yun-Ching Fu, Taichung Veterans General Hospital, Taiwan

Sheng-Ling Jan, Taichung Veterans General Hospital, Taiwan

Lin-Shien Fu, Taichung Veterans General Hospital, Taiwan

Introduction: Kawasaki disease is the leading cause of acquired heart disease in children. There are some evidences to support that Kawasaki disease comes from immune system dysfunction. However, there are very limited reports exploring the association between them. Thus, we conducted a cross-sectional study to investigate the correlation between Kawasaki disease and allergic diseases in Taiwan children.

Materials and Methods: Patients with past history of documented Kawasaki disease will be enrolled in the study. The standard questionnaire designed by International Study of Asthma and Allergic Childhood (ISSAC) were adopted in this study. Body weight, height, sex, family history of allergic diseases, social economical status, and residential areas will were collected along with the questionnaires.

Results: A total of 157 questionnaires were obtained. As high as $79.2 \%$ of these children reported that they had suffered from nasal problems in the past 12 months (relative risk 2.94). Furthermore, nasal problem "ever", associated itching eyes, and diagnosed rhinitis were all greater than $50 \%$. Wheeze "ever" (RR 1.86), wheezing in last 12 months (RR 2.50), and noctural cough (RR 1.77) reached statistical significance. Patients of Kawasaki disease also showed higher prevalence of atopic dermatitis than general population.

Conclusion: Children with past history of Kawasaki disease had higher risk for developing atopic diseases than general population. Hypersensitivity response might play a role in the pathogenesis of Kawasaki diseases. Further larger scale epidemiological survey would be needed to prove the hypothesis.

\begin{tabular}{lccccc}
\hline & $\begin{array}{c}\text { KD patients } \\
(\%)\end{array}$ & $\begin{array}{c}\text { Reference } \\
\text { group (\%) }\end{array}$ & $\begin{array}{c}\text { Relative } \\
\text { Risk }\end{array}$ & 95\% C.I. \\
\hline Nasal problem "ever" & 68.9 & 31.7 & 2.17 & 1.94 & 2.43 \\
Nasal Problem last 12 & 79.2 & 26.9 & 2.94 & 2.66 & 3.25 \\
$\quad$ months & 54.0 & 24.6 & 2.20 & 1.88 & 2.56 \\
Diagnosed rhinitis & 19.4 & 10.4 & 1.86 & 1.34 & 2.59 \\
$\quad$ Wheeze "ever" & 12.5 & 5.0 & 2.50 & 1.39 & 4.50 \\
$\quad$ Wheeze in last 12 months & 23.3 & 13.2 & 1.77 & 1.31 & 2.38 \\
Noctural cough & 24.3 & 5.8 & 4.20 & 3.13 & 5.65 \\
recurrent rash "ever" & 39.1 & 7.0 & 5.59 & 4.27 & 7.31 \\
recurrent rash last 12 & & & & & \\
$\quad$ months & & & & & \\
\hline
\end{tabular}

\section{5}

Severe Coronary Artery lesions in Children with Kawasaki Disease

Fang Liu, Cardiac Center, Children's Hospital of Fudan University, China

Lin Wu, Cardiac Center, Children's Hospital of Fudan University, China

Guo ying Huang, Cardiac Center, Children's Hospital of Fudan University, China

Ming Ye, Cardiac Center, Children's Hospital of Fudan University, China

Bing Jia, Cardiac Center, Children's Hospital of Fudan University, China

Ying Lu, Cardiac Center, Children's Hospital of Fudan University, China

Shou Bao Ning, Cardiac Center, Children's Hospital of Fudan

University, China

Severe coronary artery lesions (CALs) in affected children with Kawasaki disease (KD) usually need further assessment and coronary artery bypass grafting. Six cases were recruited in this report, including 4 boys and 2 girls, aged $12 \mathrm{~m}, 3 y 6 \mathrm{~m}, 4 y 6 \mathrm{~m}$, $7 \mathrm{y} 4 \mathrm{~m}, 8 \mathrm{y}, 10 \mathrm{y} 4 \mathrm{~m}$ respectively. CALs were found by echocardiography (ECHO) during the 15th to 21th day of the course. All patients were followed up with ECHO and ECG regularly, among whom 2 had angina and 3 had heart failure. Five patients had ST-T changes in ECG. In addition to multiple coronary aneurysms, ECHO also showed enlargement of left atrium and left ventricle with low ejection fraction and shortening fraction in 3 patients, and ventricular wall movement disconcordance in 2 patients. The remaining one had multiple ischemic focus in $99 \mathrm{mTc}-\mathrm{MIBI}$. Coronary artery angiography was performed in all patients from 6 months to 65 months after diagnosis of KD, and showed more than one coronary aneurysm formation and at least 
one aneurysm was giant in all patients, with thrombogenesis and distal coronary artery blocked in 5 patients. All patients underwent coronary artery bypass grafting and giant coronary aneurysm size-reducing repair. Five patients had satisfactory immediate results. However, the 22-month-old girl died in the operation. During follow-up, LVEF gradually improved, but not to normal. In conclusion, CALs should be followed up in patients with KD regularly. ECG, ECHO and 99mTc-MIBI and even MSCT if available should be combined for examination in those severe cases. CABG should be considered when myocardial ischemia was detected.

\section{6}

Morganella morganii pericarditis in a child with X-linked agammaglobulinemia

Jae-Sook Ma, Korea

Woo-Yeon Choi, Department of pediatrics, Chonnam National University Medical School, Chonnam National University Hospital, Gwang-Ju, Korea

Young Kuk Cho, Department of pediatrics, Chonnam National University Medical School, Chonnam National University Hospital, Gwang-Ju, Korea

Objective: X-linked agammaglobulinemia (XLA) is a relatively rare form of an immunodeficiency disease that is characterized by a profound deficiency of B lymphocytes. Morganella morganii, tribe Proteeae, is a Gram-negative bacillus that is ubiquitous in the environment and may cause opportunistic infections. Two cases of $\mathrm{M}$. morganii pericarditis after splenectomy following allogeneic bone marrow transplantation for acute lymphoblastic leukemia and mantle cell lymphoma have been described.

Results: A 5-year, 3-month-old boy was evaluated for fever, tachypnea, and chest retraction of 1 day duration and transferred to our hospital because of cardiomegaly on chest radiography. $\mathrm{He}$ had been hospitalized frequently for pneumonia since 1 year of age and had recurrent acute otitis media since 2 years of age. On physical examination, a cardiac rub was heard. An echocardiography showed a large circumferential pericardial effusion. he underwent emergency transthoracic pericardiocentesis. The results of the pericardial fluid studies were as follows: white blood cell count, 2,800 cells $/ \mathrm{mm} 3$ with neutrophilia of $81.3 \%$, and Gram-negative bacilli present. M. morganii was isolated from the pericardial and blood cultures. Flow cytometry of a blood sample revealed that CD19, a marker of mature B lymphocytes, was decreased to $0.02 \%$. Using direct sequencing of genomic DNA from peripheral blood leukocytes, point mutations were shown to affect splice sites of the BTK gene $(c .894+1 \mathrm{G}>\mathrm{C})$. $\mathrm{He}$ was therefore diagnosed with XLA.

Conclusion: When a child has pericarditis caused by uncommon bacteria, such as M. morganii, the physician should suspect and evaluate if the child is immunocompromised.

\section{7}

Cardiovascular Risk Factors Associated with Atherosclerosis in Children who are HIV-seropositive Tracie L Miller, University of Miami Miller School of Medicine, United States

John Orav, Harvard School of Public Health, United States Gabriel Somarriba, University of Miami Miller School of Medicine, United States

Daniella Neri, University of Miami Miller School of Medicine Natasha Schaefer, University of Miami Miller School of Medicine, United States
Armando Mendez, University of Miami Miller School of Medicine, United States

Lawrence B Friedman, University of Miami Miller School of Medicine, United States

Gwendolyn B Scott, University of Miami Miller School of Medicine, United States

Objective: HIV + children have cardiovascular risk factors associated with atherosclerosis. We compared biomarkers of vascular dysfunction among HIV + children to a similar group of HIV- children and determined factors associated with these biomarkers.

Methods: Biomarkers of vascular dysfunction (inflammation; hsCPR, IL-6. MCP1: coagulant dysfunction; fibrinogen, P-selectin: endothelial dysfunction; sICAM, vICAM, E-selectin: and metabolic dysfunction; leptin) were measured in $\mathrm{HIV}+$ and HIV - children. We simultaneously measured height, weight, waist and hip circumference, body fat, CD $4 \%$, HIV viral load, and antiretroviral therapy.

Results: $105 \mathrm{HIV}+$ (age 14.8y; SD4.3) and $55 \mathrm{HIV}$ - (age 12.3y; SD3.8) children were evaluated. Sex and BMI z-scores (.51 both groups) were similar. sICAM (240 vs 185); vICAM (1141 vs 685); MCP1 (154 vs 106); IL-6 (1.26 vs 0.88); and fibrinogen (376 vs 324 ) were all significantly elevated in the HIV + group. E-selectin $(p=0.07)$, and CRP $(p=0.08)$ trended to be greater yet leptin, and P-selectin were similar. The HIV + children were on HAART for a mean of $6 y \quad(\mathrm{SD}=3.1)$. In multivariable analyses, a 1 SD increase in waist/hip ratio was significantly related to a $17 \%$ increase in sICAM ( $p=0.01)$, a $19 \%$ increase in MCP1 ( $p<0.001)$, an 18\% increase in IL6 $(p=0.04)$ and a $59 \%$ increase in CRP $(p=0.01)$. Low CD $4 \%$ was independently associated with higher levels of biomarkers (vICAM 12\%; $\mathrm{p}=0.03$ : MCP1 18\%; $\mathrm{p}<0.001$ : IL6 22\%; $\mathrm{p}=0.004$ : fibrinogen $5 \% ; p=0.03$ : CRP 34\%; $\mathrm{p}=0.02)$.

Conclusion: HIV + children have higher levels of atherosclerotic biomarkers compared to sociodemographically-similar HIVchildren. Measures associated with biomarkers include waist/ hip ratios and HIV disease severity.

\section{8}

The spectrum of presentation and long term evaluation of valvar lesions in 1066 acute episodes of rheumatic fever in children and adolescents

Cleonice CC Mota, Division of Paediatric and Foetal Cardiology/ Department of Paediatrics / Hospital das Clinicas - Federal University of Minas Ge, Brazil

Zilda MA Meira, Division of Paediatric and Foetal Cardiology/ Department of Paediatrics / Hospital das Clínicas - Federal University of Minas Ge, Brazil

Fátima DM Rocha, Division of Paediatric and Foetal Cardiology/ Department of Paediatrics/ Hospital das Clinicas - Federal University of Minas Ge, Brazil

Objectives: To analyse the pattern of clinical presentation and evolution of valvar lesions.

Methods: This study investigated 1066 acute episodes of rheumatic fever and 585 episodes of carditis were seen in 489 patients, followed-up from 1.3 to 16.9 years (mean $=6.8 \pm 2.6$ years), resulting in 3325 patients-year (1977-2000). The following variables were analysed: frequency, type, association, degree of involution of valvar lesions, recurrences, and response to anti-inflammatory medication. To compare recurrences and progress of valvar disease the Fisher and the Qui-Square Tests were used, $\alpha<0,05$.

Results: The mean age at the first episode was $9.1 \pm 4.1$ years. Regurgitation valvar lesions were observed in $72.9 \%$ and mixed 
lesions in $\quad 27.1 \% \quad(\mathrm{MR}=44.2 \%, \quad \mathrm{MR}+\mathrm{AR}=65.0 \%$; $\mathrm{MR}+\mathrm{MS}=8.8 \% ; \mathrm{MR}+\mathrm{MS}+\mathrm{AR}=6.1 \%)$. Carditis was classified as severe in $39.8 \%$, moderate in $28.6 \%$, and mild in $31.6 \%$ Severe carditis was more prevalent in children with two or more recurrences, and valvar sequels more significant in those with severe carditis $(p<0,0001)$. The period of improvement using prednisone ranged from 2.0 to 42.0 days $($ mean $=14.0$; mode $=14.2$. Total regression in valvar lesions was seen in $34.4 \%$ of the patients, mostly mild ones and without recurrences. The pattern/frequency/association of the valvar lesions remained unchanged after the control of recurrences following the implementation of the Prophylactic Programme; however, significant reductions were observed in the occurrence of severe carditis, surgery and death $(\mathrm{p}<0.001)$.

Conclusions: Cardiac involvement was frequent and the sequels influenced by the severity of the carditis and by the number recurrences, reinforcing the need for effective strategies of secondary prophylaxis to reduce morbimortality.

\section{1}

Combinatorial Use of High Mobility Group Box 1 Values and Gunma Scores Improve The Accuracy of Predicting Non-Responders to Intravenous Immunoglobulin Treatment in Patients with Kawasaki Disease

Yuichi Nomura, Division of Pediatrics, Kagoshima University Graduate School of Medical and Dental Sciences, Japan

Kentaro Ueno, Division of Pediatrics, Kagoshima University Graduate School of Medical and Dental Sciences, Japan

Kiminori Masuda, Department of Pediatrics, Kagoshima Medical Association Hospital, Japan

Yasuko Morita, Department of Pediatrics, Kagoshima Medical

Association Hospital, Japan

Daisuke Hazeki, Division of Pediatrics, Kagoshima University

Graduate School of Medical and Dental Sciences, Japan

Taisuke Eguchi, Division of Pediatrics, Kagoshima University Graduate School of Medical and Dental Sciences, Japan

Yoshifumi Kawano, Division of Pediatrics, Kagoshima University

Graduate School of Medical and Dental Sciences, Japan

Objective: Patients with Kawasaki disease (KD) who do not respond to intravenous immunoglobulin (IVIG) treatment show a high incidence of coronary artery abnormalities (CAA). How to predict non-responders and then provide a specific treatment to prevent CAA is a current topic of discussion. Kobayashi et al. have reported the usefulness of a predictive score for nonresponders (Gunma score; sensitivity $86 \%$, specificity $68 \%$ ). In respect to non-responders, we have reported that an elevated value of high mobility group box 1 (HMGB1) is a potential marker. Combinational use of HMGB1 values with Gunma scores may improve the accuracy of predicting non-responders. Thus, we examined this possibility.

Methods: Serum HMGB1 values were measured in KD patients admitted to the Kagoshima City Medical Association Hospital. Clinical data and Gunma scores were obtained from these patients.

Results: Compared with responders $(\mathrm{n}=85)$, non-responders $(n=16)$ showed significantly elevated values of WBC and CRP, along with decreased values of albumin and sodium. Gunma scores were significantly higher in non-responders than responders $(5.1 \pm 2.9,2.7 \pm 2.1, p=0.0002)$. Sensitivity (score $>3$ ) was $63 \%$ and specificity $68 \%$. HMGB1 values before IVIG were also significantly higher in non-responders (14.8 \pm 11.3 , $9.7 \pm 5.5, p=0.0064)$. Sensitivity with high HMGB1 $(>15.2$ : mean + SD of responders) was $50 \%$ and specificity $71 \%$. When a predicted non-responder is defined as a patient with a Gunma score of 5 or over or a high HMGB1 value, sensitivity was $81 \%$ and specificity $71 \%$.

Conclusion: Combinatorial use of HMGB1 values and Gunma scores improve the accuracy of predicting non-responders.

7292

Rheumatic Fever and Rheumatic Heart Disease: A Geographic area of origin, referral rate, disease severity and outcome in patients presenting at $\mathrm{CH}$ Baragwanath Hospital in South Africa

Hopewell Ntsinjana, CH Baragwanath Hospital and University of the Witwatersrand, South Africa

Antionette Cilliers, CH Baragwanath Hospital and UniVersity of the Witwatersrand, South Africa

Lungile Pepeta, CH Baragwanath Hospital and University of the Witwatersrand, South Africa

Paul Adams, CH Baragwanath Hospital and University of the Witwatersrand, South Africa

Firoza Motara, Johannesburg Hospital and the University of the WitwatersrandDr, South Africa

Background: Acute Rheumatic fever (ARF) and its common complication Rheumatic heart disease (RHD) remains a major cause of mortality and morbidity amongst children and young adults in the developing countries. Poor socioeconomic status is of the predisposing factors to the development of ARF. New emphasis on primary health care might have played a role in the decrease in cases presenting at our unit.

Objective: To assess the demographics and referral rate of the patients seen at the above institution since 1993 and to evaluate whether the change in health policy in South Africa has had an impact on the picture of affected patients at presentation with regard to acute versus chronic disease including disease severity and outcome.

Method and results: We conducted a retrospective record review of all patients seen at our institution with rheumatic fever and rheumatic heart disease for the period of January 1993 to December 2005. The total number of patients reviewed was 391 with $46 \%$ males and $54 \%$ Females. The mean age at presentation was 9.8 years. There were $64 \%$ of patients originating from outside the Gauteng province and within Gauteng 70\% of the patients were from the informal settlement and townships. Conclusion: Although there is a drop in the number of RF and RHD patients presenting to our hospital there still remains a burden to health resources due to poor socioeconomic status of certain parts of our country.

7293

Doppler tissue imaging and myocardial performance index of right ventricle in children with type I Diabetes mellitus Deniz A Oguz, Gazi University Medical Faculty Pediatric Cardiology Department, Turkey

Sedef F Tunaoglu, Gazi University Medical Faculty Pediatric Cardiology Department, Turkey

Rana F Olgunturk, Gazi University Medical Faculty Pediatric Cardiology Department, Turkey

Serdar Kula, Gazi University Medical Faculty Pediatric Cardiology Department, Turkey

Oya Balci, Gazi University Medical Faculty Pediatric Cardiology

Department, Turkey

Introduction: Diabetes Mellitus (DM) Type I is a chronic autoimmune disorder. We aimed to determine the annular 
Doppler tissue imaging (DTI) and myocardial performance index (MPI) of children with DM.

Methods: 18 patients and 16 healthy children as control group were enrolled in the study. All children had chest X-ray and ECG. DTI was measured at the mitral and tricuspid annulus and systolic wave (S), early diastolic wave (E') and late diastolic wave (A') were determined. MPI was calculated by DTI.

Results: The mean age was 14,78 $\pm 3,73$ and 9,01 $\pm 3,38$ years in the patient and control group respectively. All children were asymptomatic with normal physical examination, chest-ray and ECG findings. Duration of the disease was 1,5-15 years (median: 4 years) and $\mathrm{HbA} 1 \mathrm{C}$ levels were 7,56 $\pm 0,85$. Right ventricle end diastolic diameter and vena cava inferior measurements were significantly higher in the DM group. Systolic functions of left ventricle (LV) showed no difference in both groups (EF:66,28+7,35 vs $66,50 \pm 6,57 \%$ and FS:36,28 $\pm 6,30$ vs $38,50+6,71)$.

MPI measurements for LV were similar in both groups; $0,41 \pm 0,08$ vs $0,43+0,09$ in the diabetic and control group respectively.

However MPI values for right ventricle were significantly higher in the patient group. $(0,35+0,14$ vs $0,26 \pm 0,09$, p:0,034) Conclusion: Though LV functions in DM had been thoroughly studied, right ventricle had been neglected in the past. Abnormalities in microvascular circulation in the pulmonary capillary bed can explain our DTI and MPI results which shows that right heart should also be evaluated in DM patients.

\section{4}

Cardiovascular profile in childhood obesity

Nikhil S Patankar, B.J.Wadia Hospital For Children, Mumbai, India, India

Shakuntala S Prabhu, B.J.Wadia Hospital For Children, Mumbai, India, India

Sumitra Venkatesh, India

Aims: To study cardiovascular profile in childhood obesity. Methods: 30 children with obesity were selected based on inclusion criteria. Dietary details, activity and sedentary hours, family history, general and cardiovascular examination were recorded. Body Mass Index Percentile (as per BMI \% tile NCHS/CDC charts) was assessed. An Electrocardiogram (ECG), six minute walk test for cardiac endurance and Echocardiographic evaluation was done in all.

Results: $66.7 \%$ were males and $33.3 \%$ were females. $23.3 \%$ were overweight and $76.7 \%$ obese. Exercise intolerance in $56.7 \%$, lethargy in $33.3 \%$ and snoring in $16.7 \%$ were associated symptoms. Decreased physical activity and sedentary hours positively correlated with BMI (P value $<0.01)$. Cardiac endurance testing revealed a statistical correlation between increments in mean heart rate per minute in the overweight group vs. the obese group ( $\mathrm{P}$ value $<0.043)$. 46.7\% had blood pressure between 90th -95 th percentile (for age, sex and height matched norms) and $26.7 \%$ had $>95$ th percentile. Pulse oximetry was normal in all. $26.7 \%$ had ECG abnormalities of which $23.3 \%$ had left ventricular hypertrophy for age. Left ventricular mass index by echocardiography showed a positive correlation with both, systolic and diastolic blood pressure and BMI (LVMI $>38.6 \mathrm{gm} / \mathrm{ht} 2.7)$. Left ventricular functions including myocardial performance index (normal $0.33 \pm 0.05$ ) were within normal limits, indicating preserved left ventricular functions.

Conclusions: Exercise intolerance, hypertension, ECG abnormalities, Left ventricular hypertrophy with high left ventricular mass index are seen in childhood obesity.
7295

Takayasu Arteritis in Children and Young Adults

Manojkumar Rohit, Post Graduate Institute of Medical Education

E Research - chandigarh India, India

Pooja Aggrawal, Post Graduate Institute of Medical Education

E Research - chandigarh India, India

Nidhi Narula, Post Graduate Institute of Medical Education

$\&$ Research - chandigarh India, India

Rajiv Mahajan, Post Graduate Institute of Medical Education

$\mathcal{E}$ Research - chandigarh India, India

Surjit Singh, Post Graduate Institute of Medical Education

$\mathcal{E}$ Research - chandigarh India, India

Objectives: Takayasu's arteritis (TA) is a rare chronic inflammatory disease of the aorta and its major branches. We aimed to describe clinical features and outcome of TA in children and young adults. Material and Method: All patients of TA admitted in the Department of Pediatrics, PGIMER, and Chandigarh were retrospectively studied.

Results: The mean age of presentation was 14 years (range 5-20 years) with 8 males and 12 females. Hypertension was the commonest mode of presentation seen in 65.5\% (13/20) of patients followed by systemic symptoms. Eight patients presented with congestive heart failure with severe Left ventricular systolic dysfunction were present in $50 \%$ patients. 2 children died during hospital admission. The cause of mortality was heart failure in both the patients. Inflammation markers were abnormal in $65 \%$ cases. CT angiography and conventional angiography showed that abdominal aorta was involved in 30\% cases, renal artery was involved in $45 \%$ cases, subclavian artery in $25 \%$, Superior mesenteric artery in 20\%, carotid and celiac artery in 15\% cases. Treatment modalities included antihypertensive drugs $(n=15)$, anti-tubercular therapy $(n=9)$, prednisone $(n=15)$ and methotrexate $(n=7)$. Thoraco abdominal angioplasty was performed in 4 patients, renal angioplasty in 2 patients, carotid angioplasty in 2 patients, Superior mesenteric artery and subclavian artery angioplasty in 1 patient. All had excellent results except one who had persisting severe LV systolic dysfunction.

Conclusion: Hypertension \& heart failure are the commonest presenting features of Takayasu arteritis in children.

\section{6}

S100 proteins could not be suppressed by infliximab treatment in refractory Kawasaki disease

Kazuyoshi Saito, Department of Pediatrics, Faculty of Medicine, University of Toyama, Japan

Keiichi Hirono, Department of Pediatrics, Faculty of Medicine, University of Toyama, Japan

Yasushi Kemmotsu, The First Department of Pediatrics, Toho

University School of Medicine, Japan

Keijirou Ibuki, Department of Pediatrics, Faculty of Medicine,

University of Toyama, Japan

Hirokazu Kanegane, Department of Pediatrics, Faculty of Medicine, University of Toyama, Japan

Fukiko Ichida, Department of Pediatrics, Faculty of Medicine,

University of Toyama, Japan

Tsutomu Saji, The First Department of Pediatrics, Toho University

School of Medicine, Japan

Toshio Miyawaki, Department of Pediatrics, Faculty of Medicine,

University of Toyama, Japan

Background: Kawasaki disease (KD) is the most common systemic vasculitis syndrome primarily affecting the coronary artery. Infliximab is a monoclonal antibody that binds specifically to 
human TNF- $\alpha-1$, and is effective in disorders in which inflammation is mediated by TNF- $\alpha$. S100 proteins, such as myeloid-related protein (MRP) 8/ MRP14 and S100A12, levels closely correlate with disease activity in acute $\mathrm{KD}$, and potential biomarker to predict coronary artery sequelae in the acute stage of $\mathrm{KD}$.

Objective: Our aim of study was to treat patients with refractory $\mathrm{KD}$ and examine the dynamic changes of $\mathrm{S} 100$ proteins during infliximab treatment.

Methods: We treated $11 \mathrm{KD}$ patients who did not respond to IVIG and/or IVMP with infliximab $(5 \mathrm{mg} / \mathrm{kg})$ after median 10 illness days.

We examined in sequential changes of serum levels of MRP 8/ MRP14 and S100A12 before and after infliximab treatment using by ELISA and compared these data with IVIG responders. Results: In 8 of 11 patients fever was subsided immediately responded to infliximab treatment. Four patients, who started infliximab after 12 days of illness, developed coronary artery lesions. Each of the cytokines was elevated before infliximab treatment in all patients. Although serum levels of proinflammatory cytokines such as IL-6 decreased dramatically after infliximab treatment, MRP8/MRP14 and S100A12 were not suppressed. In contrast, in IVIG responders all cytokines decreased markedly after IVIG treatment.

Conclusions: Different behaviors of pro-inflammatory cytokines and MRP8/MRP14 and S100A12 after infliximab treatment suggest that infliximab is effective for suppression of cytokinemediated inflammation, but could not completely block local vasculitis.

7297

Does Echocardiography Aid in the Diagnosis of Rheumatic Fever with Carditis in Children? Experience from a Country with High Prevalence of Rheumatic Heart Disease

Anita Saxena, All India Institute of Medical Sciences, New Delhi, India, India

Khyai Pandya, All India Institute of Medical Sciences, New Delhi, India, India

Shyam S Kothari, All India Institute of Medical Sciences, New Delhi, India, India

Rajnish Juneja, All India Institute of Medical Sciences, New Delhi, India, India

Neeraj Agarwal, All India Institute of Medical Sciences, New Delhi, India, India

Objective: The incremental value of echocardiography with Doppler (echo) in diagnosing acute rheumatic carditis (ARC) has been highlighted in several reports, but it is still not included in Jones criteria. We studied the role of echo in 175 children $<12$ years of age with ARC.

Methods: Hospital records of consecutive children diagnosed as rheumatic heart disease (RHD) over the last 5 years at this government funded, tertiary care centre were reviewed for clinical, laboratory and echo data.

Results: A total of 500 children had RHD. 197 had ARC $(39.4 \%), 175$ of these had echo and form the basis of this report. Their age ranged from 2 to 12 years (mean $9.7 \pm 2$ years), 112 were males. 71 were in NYHA class III or IV and 14 were in heart failure. Mitral regurgitation (MR) was present in 161, significant in 87 . Based on the modified Jones criteria, a diagnosis of ARC could be made in 152/175 cases (87\%). In addition to $\mathrm{MR} / \mathrm{AR}$, echo revealed multiple small nodules on mitral valve leaflets in 43 (24.5\%), including 6/23 suspected ARC cases. Mild pericardial effusion was seen in $13(7 \%)$, including $4 / 23$ suspected ARC cases. These two findings were not observed in the inactive group of 249 children who underwent echo.

Conclusion: Our data shows that echo is required in a minority of patients to diagnose carditis in communities with high prevalence of RHD. In addition to MR/AR, small nodules on the mitral valve and pericardial effusion are helpful pointers to carditis.

\section{8}

Delayed Diagnosis of Kawasaki Disease

Rekwan Sittiwangkul, Department of Pediatrics, Chiang Mai University, Thailand, 50200, Thailand

Yupada Pongprot, Department of Pediatrics, Chiang Mai

University, Thailand, 50200, Thailand

Suchaya Silviliarat, Department of Pediatrics, Chiang Mai

University, Thailand, 50200, Thailand

Charlie Phornphutkul, Department of Pediatrics, Chiang Mai

University, Thailand, 50200, Thailand

Background: Most common complications of Kawasaki disease (KD) are coronary artery abnormalities. The incidence of coronary artery aneurysms for the treatment beyond day 10 of illness are three times greater than the treatment within 10 days. The efficacy of treating patients with KD by using IVIG after 10 days of illness is unknown.

Objectives: We analyzed delayed-diagnosis KD patients' clinical manifestations and outcomes after treatment with IVIG.

Methods: A retrospective review was made of the medical records of KD patients at King Chulalongkorn hospital between 1996 and 2007. Delayed diagnosis of KD was defined as the diagnosis that was made 10 days after the onset of the fever.

Results: Twenty-seven (20 males and 7 females) of a total of $170 \mathrm{KD}$ children were delayed diagnosed. Of which 11 cases were incomplete $\mathrm{KD}$, eight cases were complete, and eight cases were atypical KD. Twenty cases (74.1\%) had the age of over one year. There were 16 cases (59.3\%) with coronary artery abnormalities at the time of diagnosis. Mean durations of fever were $14.07+3$ days. Seventeen patients were treated with IVIG. There was no statistical difference between IVIG group and non-IVIG group in terms of coronary arteries regression. However, the patients receiving IVIG treatment had significantly relieved the clinical symptoms.

Conclusions: Patients with delayed diagnosis of $\mathrm{KD}$ had high incidence of coronary abnormalities. There was no statistical difference in terms of coronary arteries regression between IVIG group and non-IVIG group.

\section{9}

Cyclosporin A treatment for refractory Kawasaki disease Masaru Terai, Tokyo Women's Medical University, Yachiyo Medicail Center, Japan

Hiromichi Hamada, Tokyo Women's Medical University, Yachiyo Medicail Center, Japan

Takafumi Honda, Tokyo Women's Medical University, Yachiyo

Medicail Center, Japan

Ayako Muto, Tokyo Women's Medical University, Yachiyo Medicail Center, Japan

Kaori Sasaki, Tokyo Women's Medical University, Yachiyo Medicail Center, Japan

Kitami Hayashi, Tokyo Women's Medical University, Yachiyo Medicail Center, Japan

Yoshihiro Onouchi, SNP Research Center, RIKEN, Japan

Akira Hata, Chiba University, Graduate School of Medicine, Japan

Kawasaki disease (KD) is a leading cause of acquired heart disease in children of developed countries. Intravenous immune globulin 
(IVIG) at $2 \mathrm{~g} / \mathrm{kg}$ is effective in the rapid resolution of $\mathrm{KD}$ inflammation. However, approximately 15 to $20 \%$ of patients had persistent or recurrent fevers after IVIG completion and are considered to have a higher risk of developing coronary aneurysms. The management of these IVIG-resistant patients is unestablished. Recently, Onouchi Y et al. identified a significant functional SNP in the inositol 1,4,5-triphosphate 3-kinase C (ITPKC) gene which was associated with KD susceptibility. ITPKC acts as a negative regulator of T-cell activation through the Ca2+/NFAT signaling pathway. Importantly, Cyclosporin A (CsA) suppresses T-cell activation by blocking downstream molecule of $\mathrm{Ca} 2+/ \mathrm{NFAT}$ pathway. Then, we sought to determine effects of CsA on clinical outcome in IVIG-resistant patients. Sixty-two patients admitted to our hospital from January 2008 through December 2008 were initially treated with IVIG plus aspirin. Of these patients, 12 patients $(19.3 \%)$ were resistant to initial treatment. Then, all 12 patients received re-IVIG at $2 \mathrm{~g} / \mathrm{kg}$. Consequently, 6 patients $(9.6 \%)$ resistant to re-IVIG were additionally treated with CsA at $4 \mathrm{mg} / \mathrm{kg}$ orally for 3 weeks. Five of the 6 patients responded to CsA and had normal coronaries, but the remaining one had persistent fever despite of increased dose of CsA up to $8 \mathrm{mg} / \mathrm{kg}$ and developed moderate size $(4-5 \mathrm{~mm})$ of aneurysms in both coronary arteries. Significant adverse effects of CsA have not been documented. CsA may be one of effective treatment for refractory KD.

\section{0}

Delayed Diagnosis of Kawasaki Disease and Outcomes after Treatment with IVIGMemorial hospital

Kanyalak Vithessonthi, King Chulalongkorn memorial Hospital, Thailand

Pornthep Lertsapchareon, King Chulalongkorn memorial Hospital, Thailand

Apichai Khongphattanatothin, King Chulalongkorn memorial Hospital, Thailand

Vidhavas La-orkhun, King Chulalongkorn memorial Hospital, Thailand

Background: Most common complications of Kawasaki disease (KD) are coronary artery abnormalities. The incidence of coronary artery aneurysms for the treatment beyond day 10 of illness are three times greater than the treatment within 10 days. The efficacy of treating patients with KD by using IVIG after 10 days of illness is unknown.

Objectives: We analyzed delayed-diagnosis KD patients' clinical manifestations and outcomes after treatment with IVIG.

Methods: A retrospective review was made of the medical records of KD patients at King Chulalongkorn hospital between 1996 and 2007. Delayed diagnosis of KD was defined as the diagnosis that was made 10 days after the onset of the fever.

Results: Twenty-four (20 males and 7 females) of a total of $170 \mathrm{KD}$ children were delayed diagnosed. Of which 11 cases were incomplete $\mathrm{KD}$, eight cases were complete, and eight cases were atypical KD. Twenty cases (74\%) had the age of over one year. There were 18 cases $(66.7 \%)$ with coronary artery abnormalities at the time of diagnosis. Mean durations of fever were $14.07+3$ days. Seventeen patients were treated with IVIG. There was no statistical difference between IVIG group and non-IVIG group in terms of coronary arteries regression. However, the patients receiving IVIG treatment had significantly relieved the clinical symptoms.

Conclusions: Patients with delayed diagnosis of $\mathrm{KD}$ had high incidence of coronary abnormalities. There was no statistical difference in terms of coronary arteries regression between IVIG group and non-IVIG group.

\section{1}

Is BCG Red in Kawasaki Disease with Coronary Artery Abnormality?

Chin Fu Wang, Department of Pediatrics, E-Da Hospital/I-Shou University, Taiwan

Kai Sheng Hsieh, Department of Pediatrics, Kaohsiung Veterans Genreal Hospital, Taiwan

It is common to find the red BCG (rBCG) in Kawasaki disease $(\mathrm{KD})$ in asian children. The exact mechanism of pathogenesis of $\mathrm{rBCG}$ is not clear at present. However, we find that $\mathrm{KD}$ with coronary artery abnormality (KD-CAA) is some related to $\mathrm{rBCG}$. From March, 2004 to December, 2008, we erolled children with $\mathrm{KD}$ in E-Da hospital in south Taiwan. The relationship of KD$\mathrm{CAA}$ and $\mathrm{rBCG}$ was discussed and literatures were reviewed.

7302

Analysis of the echocardiographic features in patients with ventricular septal rupture complicating acute myocardial infarction

Guang-yi Wang, Department of cardiology, Chinese PLA general

hospital, China

Hai-jun Hou, China

Zhi-feng Wang, China

Objective: To provide the evidence for treatment and evalution of prognosis of the ventricular septal rupture after acute myocardial infarction by analyzing clinical and echocardiographical characteristics. Methods: Clinical datas of seventeen patients with ventricular septal rupture complicating acute myocardial infarction in our hospital between 1994 and 2007 were analyzed retrospectively. Result: Among 2027 patients with acute myocardial infarction, 17 patients were proved to have ventricular septal rupture. The incidence of ventricular septal rupture after AMI was $0.83 \%$. the average age of 17 patients was $64.9 \pm 9.5$ years. 9 patients $(53 \%)$ had hypertension. 12 patients were diagnosised as acute anterior wall infarction. Coronary arteriography and ventriculography were done in 11 patients. Two coronary artery at least were found stenosis. Total occlusion of infarct related arteries were found in 10 case out of 11 patients (90.9\%). The average size of left ventricular was $54.1 \pm 6.2 \mathrm{~mm}$. Average ejection fraction was $40.4 \pm 10.8 \%$. The sites of septum rupture mostly existed in septum adjacent to cardiac apex. The average size of rupture was $9.5 \pm 3.9 \mathrm{~mm}$. In addition, the mortality was very high in patients receiving medical treatment (82\%).

Conclusion: The septum rupture after acute myocardial infarction mostly happened in aged patients with anterior wall infarction. Mortality was high in patients who received conservative medical treatment. Echocardiography was a sensitive and simple method for diagnosis.

\section{3}

Sivelestat sodium treatment for intravenous immunoglobulin-resistant Kawasaki disease

Kumi Yasukawa, Department of Pediatrics, Graduate School of Medicine, Chiba University, Japan

Ryota Ebata, Department of Pediatrics, Graduate School of Medicine, Chiba University, Japan

Kouji Higashi, Department of Pediatrics, Graduate School of Medicine, Chiba University, Japan

Background: Treatment of acute Kawasaki disease (KD) with intravenous immunoglobulin (IVIG) reduces the risk of coronary artery abnormalities. However, patients who are resistant to IVIG are at risk for developing coronary artery aneurysms. It was reported that the neutrophils were identified in the coronary 
arterial lesions of the patients who died 10 days after the onset of $\mathrm{KD}$, suggesting that neutrophil elastase might play an important role in the early stage of KD. Sivelestat sodium (Sivelestat-Na) is a small molecular weight neutrophil elastase inhibitor. The purpose of this study is to investigate the safety and efficacy of Sivelestat sodium for IVIG-resistant KD.

Methods and Results: Seventy patients of KD were initially treated with IVIG at $2 \mathrm{~g} / \mathrm{kg}$ and aspirin $(30 \mathrm{mg} / \mathrm{kg} /$ day) from January 2006 through December 2008. Of 70 patients, 18 patients who had persistent or recurrent fever after initial treatment were treated with re-IVIG at $2 \mathrm{~g} / \mathrm{kg}$ in a single dose plus Sivelestat-Na. Sivelestat-Na treatment at $0.2 \mathrm{mg} / \mathrm{kg} / \mathrm{hr}$ for 5 consecutive days. Six of the 18 patients had persistent fever, so then they were treated with Prednisolone at $2 \mathrm{mg} / \mathrm{kg} /$ day. Four of the 18 patients had coronary aneurysms, but 3 of 4 patients already had coronary abnormalities before Sivelestat-Na treatment. Ultimately, only one patient with Sivelestat-Na treatment started before 10 days of illness had moderate size of coronary aneurysm. Significant side effects of Sivelestat-Na have not been documented.

Conclusions: Sivelestat-Na treatment may be one of effective treatment for IVIG-resistant patients.

\section{5}

Rheumatic Fever/Rheumatic Heart Disease In South Africa: The Global Registry Initiative

Liesl J Zühlke, Red Cross Childrens' Hospitals, South Africa

Mark E Engel, University of Cape Town, South Africa

Bongani M Mayosi, University of Cape Town, South Africa

Introduction: Rheumatic fever (RF) and rheumatic heart disease (RHD) together affect about 15.6 million people worldwide, 2.4 million of whom are children in developing countries. There is a paucity of information on the prevalence of RHD in Africa. In South Africa, anecdotal information suggests that RHD is the leading cause of acquired heart disease in children and young adults.

The absence of contemporary, systematically collected data is a gap needed to be bridged in order to effectively treat patients with RHD in developing countries, where the rate of RF/RHD has not mirrored the decline in developed countries. Experience elsewhere has provided evidence of registers realising notable successes in reducing RF recurrence. We report the development and proposed mechanism for a RHD Registry.

Methods: We discuss steps involved in developing the global registry: meetings with various stakeholders enabled the honing of objectives, a universally-acceptable case record form (CRF) was developed and, an operations committee was established to manage the process. Institutions will be invited to participate in piloting the CRF to enable evaluation thereof.

Results \& Conclusion: It is envisaged that the Registry will be successfully established, and can be rolled out at country level in order to prevent RF among children and thus avoid RHD and cardiac surgery. As an initiative of the A.S.A.P Programme, together with the National Department of Health guidelines for the prevention and treatment of RF/RHD, it is certainly feasible that both RF and RHD could be eradicated from SA within one or two generations.

\section{6}

An Iceberg in Africa: Rheumatic Heart Disease requiring Surgery in Children

Liesl J Zuhlke, Western Cape Pediatric Cardiac Services, Red Cross and Tygerberg Hospitals, South Africa
Mark Engel, University of Cape Town, Cape Town, South Africa John Lawrenson, Western Cape Pediatric Cardiac Services, Red Cross and Tygerberg Hospitals, South Africa

Rik De Decker, Western Cape Pediatric Cardiac Services, Red Cross and Tygerberg Hospitals, South Africa

Kathie Walker, Western Cape Pediatric Cardiac Services, Red Cross and Tygerberg Hospitals, South Africa

Roeland Kraan, Medical Student, Rijks Universiteit Groeningen, The Netherlands

wendy Matthiassen, Western Cape Pediatric Cardiac Services, Red Cross and Tygerberg Hospitals, South Africa

Bongani Mayosi, Department of Medicine, University of Cape Town., South Africa

Introduction: RF/RHD affects about 15.6 million people worldwide, 2.4 million of whom are children between five and fourteen years old living in developing countries. RF/RHD leads to an estimated 350,000 deaths annually, and survivors are left disabled without access to the expensive medical and surgical care that the disease requires. Africa, which contains $10 \%$ of the world's population, has a disproportionately high share of people living with RF/RHD. Of the 2.4 million children with RF/RHD living in developing countries, nearly half ( $>1$ million) live in sub-Saharan Africa, making the continent the major hotspot of RF/RHD. However, there is a paucity of information on the prevalence, effect and outcomes of RHD in South Africa.

Aim: To review patients within a tertiary paediatric cardiac unit that have undergone either surgery for valve repair or replacement or catheter intervention for valve disease. Parameters that were reviewed included age, age at diagnosis and at surgery, incidence of significant complications and mortality.

Results: Within the past 15 years, more than 35 patients under the age of 18 have undergone valve repair/replacements or catheter valvuloplasty. The youngest patient at the time of intervention was 60 months old. There were 8 mitral valve and 5 double valve replacements, 3 catheter mitral valvotomies and 21 mitral valve repairs.

Conclusions: We report on a cohort of Rheumatic Heart Disease patients in Cape Town, South Africa. These patients represent the most severely affected and we reflect on the admission criteria, intervention as well as short-and medium term outcomes for these patients.

\section{7}

\section{Submitral left ventrcular aneurysms in a paediatric} population

Firoza Motara, University of the Witwatersrand and Johannesburg Academic Hospital, South Africa

Antoinette M Cilliers, University of the Witwatersrand and $\mathrm{CH}$

Baragwanath Hospital, South Africa

Deliwe P Ngwezi, University of the Witwatersand and Johannesburg Academic Hospitall, South Africa

Introduction: Submitral left ventricular aneurysms are widely recognized but mainly described in Sub Saharan Africa. These aneurysms occur in relation to the annulus of the mitral valve around the posteromedial commissural line. Patients may be asymptomatic or present with symptoms due to valvar regurgitation or from compression of cardiac structures. The aetiology is usually congenital although co existent pathology such as rheumatic carditis or tuberculosis has been described.

Methods: This retrospective review describes the clinical, anatomical and histological features of submitral aneurysms seen in the paediatric population at a South African academic hospital complex between 2001 and 2007. 
Results: There were six patients, aged 13 months to 15 years. Five of the patients presented with mitral regurgitation and congestive cardiac failure. The diagnosis was made in all instances on transthoracic echocardiography and supplemented with transoesophageal echocardiography or angiograms selectively. Five of the six patients underwent surgery and in 4 of these patients the aneurysm was related to the posterior mitral leaflet and in one patient the aneurysm was related to the anterior mitral leaflet. Histological diagnosis revealed features of rheumatic carditis in 2 patients, features of tuberculosis in one patient and nonspecific features in 2 patients. The outcome in all patients who underwent surgery was favourable. The sixth patient died soon after the initial diagnosis.

Conclusion: Submitral aneurysms are not uncommon. Surgery is the treatment of choice and ususlly has a good outcome.

\section{9}

Three cases of multiple coronary arterial fistulae

Hiromichi Nakajima, Chiba children's Hospital, Japan

Hiroyuki Aotsuka, Chiba children's Hospital, Japan

Shunsuke Tatebe, Chiba children's Hospital, Japan

Ryouta Ebata, Chiba children's Hospital, Japan

Mitsuru Aoki, Chiba children's Hospital, Japan

Tadashi Fujiwara, Chiba children's Hospital, Japan

Multiple coronary arterial fistulae (CAF) is a very rare malformation. We present three cases of this anomaly.

The first patient was a 7-year-old girl with tricuspid atresia type I-A and persistent left superior vena cava (PLSVC). After Modified Blalock-Taussig shunt in neonatal period, total cavopulmonary connection (TCPC) was done at 1.5 years of age. The coronary sinus (CS) was confirmed and the PLSVC was ligated. Catheter examination after TCPC showed multiple diffuse CAF from both coronary arteries to the left ventricle. The coronary diameter was enlarged up to $5 \mathrm{~mm}$. LVEDV was $192 \%$ of normal. She was stable using ACE inhibitor.

The second patient was a 4-year-old girl with large muscular VSD. PAB was performed at the age of 8 months. Catheter examination showed diffuse CAF from both coronary to the right ventricle. She was died soon after VSD patch closure because of left ventricular dysfunction. An autopsy revealed septal infarction and stenotic orifice of CS.

The third patient was 2-year-old male with double outlet right ventricle and PDA. PAB and PDA ligation was performed at neonatal period. He also had minor chromosomal anomaly (46XY (add(9) (q34,3)). Angiography showed diffuse CAF mainly to the right ventricle. Concerning about possible ischemia by right ventricular decompression, we avoided biventricular repair and performed bidirectional Glenn procedure successfully.

Conclusion: The etiology of diffuse type CAF is still unknown. Stenosis of CS might be a cause of this disease. A risk of ischemia after the pressure reduction of CAF should be considered in planning intra-cardiac surgery.

\section{0}

B-type natriuretic peptide as a marker of latent left ventricular impairment in pediatric patients with bronchial asthma

Tsuneyuki Nakamura, Department of Pediatrics, Kanazawa Medical University, Japan

Chika Kitaoka, Japan

Chisato Kitaoka, Japan

Hitoshi Sato, Japan

Toshimi Nakamura, Japan

Yutaka Saikawa, Japan
We investigated the changes of plasma B-type natriuretic peptide (BNP) levels in the pediatric asthma as a representative model of the pediatric obstructive lung disease. In 50 patients with bronchial asthma, the plasma BNP levels were determined at their attacks and convalescent phase. Cardiac function of the patients was simultaneously evaluated by echocardiography. The levels of plasma BNP in the acute phase were significantly elevated compared to those in the convalescent phase (Figure, $30.6 \pm 31.2$ vs $7.7 \pm 6.9 \mathrm{pg} / \mathrm{ml}, \mathrm{p}<0.0001$ ). The left ventricle Tei-indexes in the acute phase presented significantly higher values than in the convalescence phase $(0.44 \pm 0.26$ vs $0.35 \pm 0.13, p<0.05)$. Furthermore, the higher levels of plasma BNP were positively correlated with the asthmatic severity-duration scores $(\mathrm{p}<0.0001, \mathrm{r}=0.53)$ and inversely correlated with peripheral oxygen saturation levels $(p<0.001$, $r=-0.38)$ in the acute phase. Hypoxia-induced myocardial oxygen demand under asthma attack may contribute to BNP secretion. The plasma BNP levels could be a useful marker of latent cardiac impairment in pediatric patients with asthma attack.

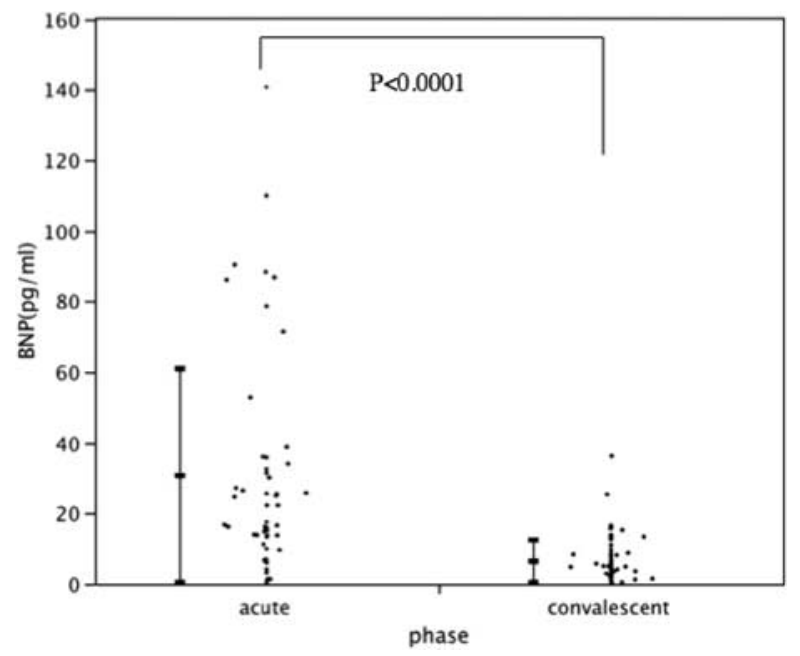

7311

Benign heart tumors present with a large spectrum of clinical features

Susanne Navarini-Meury, Department of Pediatric Cardiology, University Hospital Berne, Switzerland

Mladen Pavlovic, Department of Pediatric Cardiology, University

Hospital Berne, Switzerland

Alexander Kadner, Department of Heart Surgery, University Hospital Berne, Switzerland

Thierry Carrel, Department of Heart Surgery, University Hospital

Berne, Switzerland

Jean-Pierre Pfammatter, Department of Pediatric Cardiology, University Hospital Berne, Switzerland

Objective: Primary cardiac tumors are rare in the pediatric population and symptoms vary widely. We report on 5 children with a benign heart tumor presenting with different clinical symptoms.

Methods: We analysed retrospectively all patients presenting with a benign heart tumor at our pediatric cardiology department in the past 10 years.

Results: 5 patients were seen in the past 10 years for benign heart tumors. 2 patients were diagnosed with rhabdomyoma, 2 with fibroma and 1 patient with myxoma. Patients age varied from newborns up to a 14-year-old boy. Two patients presented with 
arrhythmia, one with supraventricular and one with ventricular tachycardia. Both were found to have rhabdomyoma and diagnosis of tuberous sclerosis. One patient presented with impaired sportive performance, dyspnea, coughing and syncope due to tricuspidal stenosis caused by a myxoma. Another patient was seen due to unclear dysmorphic features. Echocardiography revealed pericardial fibroma and a large ventricular septal defect, with a genetic analysis resulting in Gorlin syndrome. The second patient with fibroma presented with failure to thrive as the only symptom.

Conclusions: Pediatric primary cardiac tumors are extremely rare, and most of them are histologically benign. Depending on size and location of the tumor, patients present with a great variety of clinical symptoms. Besides hemodynamic obstruction, arrhythmia is an important sign and the first episode can be a life-threatening arrhythmia or sudden death. This case series underlines the diversity of clinical features in children with benign cardiac tumors.

\section{2}

\section{Left Ventricular diastolic function indices in healthy} children

Nobutoshi Nawa, Department of Pediatrics, Osaka Kosei-Nenkin

Hospital, Japan

Tetsuya Sano, Department of Pediatrics, Osaka Kosei-Nenkin Hospital, Japan

Yoshinobu Takada, Department of Pediatrics, Osaka Kosei-Nenkin

Hospital, Japan

Akira Taniguchi, Department of Pediatrics, Osaka Kosei-Nenkin

Hospital, Japan

Keiko Wada, Department of Pediatrics, Osaka Kosei-Nenkin Hospital, Japan

Hiroko Kashiwagi, Department of Pediatrics, Osaka Kosei-Nenkin

Hospital, Japan

Tetsuzo Tagawa, Department of Pediatrics, Osaka Kosei-Nenkin

Hospital, Japan

Yoshiki Seino, Department of Pediatrics, Osaka Kosei-Nenkin

Hospital, Japan

Objectives: Echocardiographic assessment of the left ventricular (LV) diastolic function in the pediatric populations has not been established. The aim of this study was to propose the reference values for the echocardiographic indices of the LV diastolic function in children.

Methods: We evaluated 79 children (2 months to 19 years of age, Median 5 years), who were confirmed no significant cardiac defects by echocardiographic examinations. In addition to routine cardiac assessments, LV diastolic function was evaluated by measuring parameters including the transmitral early (E) and late (A) inflow velocities, E wave deceleration time (DcT), and early (E') and late (A') diastolic tissue Doppler velocities of the septal mitral annulus. Results: E was $89+/-15 \mathrm{~cm} / \mathrm{s}$, A $53+/-14 \mathrm{~cm} / \mathrm{s}$, E/A ratio $1.8+/-0.63$, E' $10+/-1.8 \mathrm{~cm} / \mathrm{s}$, A' $5.9+/-1.7 \mathrm{~cm} / \mathrm{s}$, DcT $121+/-28.1 \mathrm{msec}$ and $\mathrm{E} / \mathrm{E}^{\prime}$ ratio $8.7+/-1.9$. Most of all diastolic parameters except E/E' ratio closely correlated with age, body surface area (BSA) and heart rate. E/E' ratio, however, was not influenced by these factors. None of diastolic parameters correlated with LV end-diastolic dimension, left atrium to aorta ratio or ejection fraction.

Conclusions: E/E' ratio may be a useful index to assess the LV diastolic function in children without influences of age, BSA, or heart rate. On the other hand, other diastolic parameters should be applied in consideration of influences of these factors. These results provide the reference values to evaluate $\mathrm{LV}$ diastolic function parameters in pediatric populations.
7313

Homozygous Familial Hypercholesterolemia Diagnosed in Childhood in Hungary

Agnes Nemeth, Semmelweis University Faculty of Medicine 2nd

Department of Pediatrics, Budapest, Hungary, Hungary

Lorand Kerkovits, Szent Imre Teaching Hospital, Budapest,

Hungary, Hungary

Judit Szabolcs, Semmelweis University Faculty of Medicine 2nd

Department of Pediatrics, Budapest, Hungary, Hungary

Elizabeth Horvath, Semmelweis University Faculty of Medicine 1st

Department of Pediatrics, Budapest, Hungary, Hungary

Tamas Szamosi, emmelweis University Faculty of Medicine 2nd

Department of Pediatrics, Budapest, Hungary, Hungary

Type of II/A familial hyperlipoproteinaemias are inherited autosomal disorders with incomplete penetrance. The manifestation of the homozygous form is 1:1,000,000. Two cases of homozygous form of familial hypercholesterolemia were confirmed in childhood in Hungary. Authors present a currently 28 years old young man whose valvular aortic stenosis discovered at age of two. In 1989 patient was examined due to the xanthomatous skin alterations; a familial hyercholesterolemia was diagnosed. The extended examination of lipid metabolism revealed normal level of receptors; however its function were decreased. His aortic stenosis was operated at Munich (Germany) in 1994, followed by regular Heparin induced Extracorporeal LDL-cholesterol aPheresis (H.E.L.P.) and oral statin treatment. $\mathrm{He}$ has been on this combined treatment from 9 years of age. Patient has no sign of any cardiovascular disease.

A currently 15 years old girl was examined first at 7 years of age due to the granulomatous and unusual xanthomatous tendon lesions. The extended examination of lipid metabolism confirmed the homozygous form of familial hypercholesterolemia. Her treatment was started with cholesterol plasmapheresis using H.E.L.P. system combined with ezetimibe and rosuvastatin therapies. She is on this combined treatment without any sign of cardiovascular disease.

Familial hypercholesterolemias represent an important group of diseases responsible for significant cardiovascular morbidity and mortality. Sustained elevation of serum cholesterol levels lead to early onset of acquired atherosclerotic heart disease. Authors consider worthwhile to present these cases due to the young age of the patients, the unusual presentations of the disease and the long term successful therapy by H.E.L.P.

\section{4}

Efficacy of cardiac resynchronization therapy in Fontan candidates with interventricular dyssynchrony

Kosuke Nishiguchi, Sakakibara Heart Institute, Japan

In-Sam Park, Sakakibara Heart Institute, Japan

Tadahiro Yoshikawa, Sakakibara Heart Institute, Japan

Junichiro Sato, Sakakibara Heart Institute, Japan

Yasuo Murakami, Sakakibara Heart Institute, Japan

Katsuhiko Mori, Sakakibara Heart Institute, Japan

Makoto Ando, Sakakibara Heart Institute, Japan

Yukihiro Takahashi, Sakakibara Heart Institute, Japan

Objective: We sought to evaluate the hemodynamic benefit and efficacy of cardiac resynchronization therapy (CRT) in Fontan candidates with interventricular dyssynchrony.

Methods: CRT was applied in three patients aged 5 months to 32 years with single ventricle physiology. Diagnoses included double outlet right ventricle in two patients and single right ventricle in one. Two patients had completed a total cavopulmonary connection, whereas the remaining one had a bidirectional 
Glenn shunt. In all patients, echocardiographic analysis demonstrated the existence of interventricular dyssynchrony and the flow regurgitation from right ventricle to (rudimentary) left ventricle through the ventricular septal defect.

Results: The mean QRS duration decreased from $175 \pm 41$ to $135 \pm 41 \mathrm{msec}$. Echocardiograpy showed improvement of interventricular dyssynchrony, which accompanied decreased flow regurgitation through the ventricular septal defect. The mean cardiothoracic ratio decreased from $72 \pm 5$ to $61 \pm 6 \%$. Clinical improvement was seen in all patients, with decrease of diuretics dosage.

Conclusions: CRT resulted in improved interventricular dyssynchrony and clinical status in patients with functional single ventricles. CRT may be a useful adjunct in the treatment of cardiac dysfunction in Fontan candidates, who's one ventricle has become a damping chamber.

\section{5}

\section{Effects of chronic Fluorosis on cardiovascular system in} children

Seref Olgar, Osmangazi Univercity Faculty of Medicine, Pediatric Cardiology Depatment, Eskisehir, Turkey, Turkey

Ayca Esra Kuybulu, Suleyman Demirel Univercity, Faculty of

Medicine, Department of Pediatrics, Isparta, Turkey, Turkey

Selmin Karademir, Dr. Sami Ulus Children's Hospital, Pediatric

Cardiology Department, Ankara, Turkey, Turkey

Tansu Sipahi, Ufuk UnivercityFaculty of Medicine, Pediatric

hematology Department, Ankara, Turkey, Turkey

Deniz A Oguz, Gazi University Medical Faculty Pediatric Cardiology

Department, Turkey

Ahmet Rifat Örmeci, Turkey

Fluoride is an essential trace element. Fluoride is taken mainly by drinking water beside various nutrition and beverages (e.g., tea, toothpaste, mouth rinse, drugs, fluoride containing salt, dust, etc.). Excessive intake of fluoride for a prolonged period can induce chronic fluorosis. A few studies was conducted to investigate effects of fluoride on cardiovascular system in human beings and animals.

In this study, we investigated the effect of sodium fluoride on cardiovascular system in children. Thirty-five children with dental fluorosis were included in this study. A pedodontist performed the oral examinations and study group was divided into two groups as mild-moderate (group I) and severe (group II). Twenty-six healthy children were included as control group. Written consents were taken. The medical histories were noted and physical examinations were performed. Blood samples were analyzed for renal functions, electrolytes, liver functions, lipid profile, and whole blood count. The fluoride levels in urine were measured by using the ion electrode method. Electrocardiography, echocardiography and 24-hour ambulatory holter monitorization were applied to all of the children.

We found statistically significant low T4, calcium and sodium levels, increased QT and QTc intervals in children severely affected with dental fluorosis $(p<0.05)$. These results were same with the literature. No statistically significant difference was noted concerning electrocardiography, echocardiography and heart rate variability. We concluded that endemic fluorosis is a risk factor for hypocalcaemia, hypernatremia, low T4 levels and long QT. Patients with fluorosis must be controlled intermittently for these side effects of fluorosis.

\section{6}

\section{Cases of Coronary Artery Fistula detected with Heart murmurs}

Masao Ohtaka, Juntendo University School of Medicine, Department of Pediatrics, Japan
Masahiko Kishiro, Juntendo University School of Medicine, Department of Pediatrics, Japan

Ken Takahashi, Juntendo University School of Medicine, Department of Pediatrics, Japan

Katsumi Akimoto, Juntendo University School of Medicine, Department of Pediatrics, Japan

Masahiro OHtsuki, Juntendo University School of Medicine, Department of Pediatrics, Japan

Keiko Sato, Juntendo University School of Medicine, Department of Pediatrics, Japan

Yoshie Negishi, Juntendo University School of Medicine, Department of Pediatrics, Japan

Toshiaki Shimizu, Juntendo University School of Medicine, Department of Pediatrics, Japan

Background: Coronary artery fistula (CAF) is a rare lesion that was in $0.2-0.4 \%$ of congenital heart disease. In childhood, most of patients have no symptoms. However, CAF may cause serious complications such as congestive heart failure, myocardial infarction and infectious endocarditis. We experienced 3 interesting cases with congenital CAF and discussed the management and treatment. CASE 1: A 4years-old boy with no symptom was referred to our hospital for evaluation of a heart murmur found at 3 years of age. The diagnosis by echocardiography and angiography was the right CAF drained to the right ventricle. There were no coronary artery lesions. CASE 2: A 1-years-old, asymptomatic, boy who had diagnosed right CAF (RCA-RV) at the age of 4 days was referred to our hospital. The giant aneurysm was noticed by echo. CASE 3: A girl was found to have a heart murmur on routine examination at the age of 6 months and diagnosed the right CAF that was drained to the right atrium. There were no signs of cardiac failure, but when aged 17 months, echocardiography and angiography revealed that there was the giant aneurysm. Discussion: In present cases, we experienced that the aneurysm could enlarge progressively in short term in 2 patients. These lesions may raise sudden death in their later life, and should be cured early.

Conclusion: Surgical correction has very low mortality and is curative, so early surgical closure was recommended. Case $2 \& 3$ were immediately intended to schedule of surgical closure.

\section{7}

Congenital cyanotic cardiac diseases and $\mathrm{R} 78 \mathrm{~W}$ polymorphism frequency of CFC1 gene in patients with situs anomaly

Rana Olguntürk, Gazi University, Faculty of Medicine, Department of Pediatric Cardiology, Ankara, Turkey

Ilknur Aktas, Gazi University, Faculty of Medicine, Department of Pediatrics, Ankara, Turkey

Mehmet Ali Ergün, Gazi University, Faculty of Medicine, Department of Genetic, Ankara, Turkey

Ferda Perçin, Gazi University, Faculty of Medicine, Department of

Genetic, Ankara, Turkey

Sedef Fatma Tunaoglu, Gazi University, Faculty of Medicine,

Department of Pediatric Cardiology, Ankara, Turkey

Deniz Ayse Oguz, Gazi University, Faculty of Medicine, Department

of Pediatric Cardiology, Ankara, Turkey

Serdar Kula, Gazi University, Faculty of Medicine, Department of

Pediatric Cardiology, Ankara, Turkey

Cihat Sanli, Gazi University, Faculty of Medicine, Department of

Pediatric Cardiology, Ankara, Turkey

Objective: There is evidence that most congenital cardiac anomalies are associated with failures during heart development or process of determination of left-right axis. CFC1 gene, which 
belongs to EGF-CFC gene family, is a gene responsible for leftright axis. Also, mutation of CFC1 gene has been detected in individuals with complex cardiac disease without heterotaxia. The purpose of this study is to determine the $\mathrm{R} 78 \mathrm{~W}$ polymorphism frequency of CFC1 gene in congenital cyanotic cardiac diseases and patients with dextrocardia and situs anomaly. Methods: Hundred and seven patients with cyanotic congenital heart disease at the age range of 0-16 years were included in the study. The diagnoses were confirmed by clinical and laboratory (ECG, chest X-ray, echocardiography, cardiac catheterisation) examinations. Age matched 100 normal children were included as controls. R78W polymorphism of CFC1 gene was evaluated using PCR with restriction analysis technique in both groups.

Results: R78W polymorphism frequency of CFC1 gene could not be shown in patient and control groups.

Conclusion: In this study, R78W polymorphism of CFC1 gene was not detected in 207 events which include patient and control groups. This result is parallel to the findings of the literature which indicates that this polymorphism is rare in caucasians and usually detected in African-Americans and Asians. As this mutation is rarely detected in congenital cardiac defects, it may not be directly related to the etiology of this disease group. Following investigations with larger groups and/or meta-analysis of the previous studies will bring solutions to this issue.

\section{8}

Regional Epidemiology of Congenital Heart Diseases According to Geographical Districts in Turkey Rana Olguntürk, Gazi University, Medical Faculty, Department of Cardiology, Ankara, Turkey

Serdar Kula, Gazi University, Medical Faculty, Pediatric Cardiology Department, Turkey

Ayhan Cevik, Gazi University, Faculty of Medicine, Department of Pediatric Cardiology, Ankara, Turkey

Fatma Sedef Tunaoglu, Turkey

Deniz Oguz, Gazi University, Medical Faculty, Department of

Pediatric Cardiology, Ankara, Turkey

Cihat Sanli, Gazi University, Faculty of Medicine, Department of

Pediatric Cardiology, Ankara, Turkey

Mustafa Ilhan, Turkey

Aim: To predict distrubition of congenital heart disease in Turkey and comparison of the geograhical regions.

Material Method: The medical records of 1300 patients with congenital heart disease admitted to Gazi University Department of Pediatric Cardiology during 1997-2007 were reviewed. The patients were divided into 7 groups according to the their origins and each group were divided into two groups as simple (Group 1) and complex (Group 2) congenital heart disease.

Results: Among the 1300 cases, there was no difference between the regions regarding mean age and male/female ratio. In three region had high incidence of complex cardiac malformations then others $(\mathrm{p}<0.05)$

Conclusion: This is the first epidemiological study about distrubition of congenital heart disease in our country. The incidence of Group 2 patients was high in three regions which had smilar socioeconomically and geographic specialities. In fact, these regions are affected more sunlight through the summer session then others. Additionally, the frequency of the intermarriage (29.1\%) and child count per family is high (5-15) in these regions. This is the limitation of our study because of these parameters were not available in our records. Nevertheless, this study may play an important role for further prospective studies in this subject.
7319

Retroapective Evaluation of the Patients with Pericarditis and Pericardial Effusion

Berna Saylan Cevik, Dr. Behçet Uz Childrens' Hospital Pediatric Cardiology Department IZMIR, Turkey

A total of 22 (13 girls and 9 boys) patients,mean age was 5,9 years was followed with the diagnosis of pericarditis-pericardial effusion were retrospectively evaluated from January 2003-2007 at Behçet Uz Childrens' Hospital. \%10 of the patients have fever and respiratory distress, 6 of them additionally have productive coughing. Chest pain was the main complaint. 3 patients had malaise and 1 patient had dysphagia. Patients who were 3-24 months had feeding difficulty. Only one patient was attended with generalized edema. All patients went under hematological, biochemistry and microbiological evaluation all the telecardiograms shows the typical "water-bottle". Electrocardiography and follow up were made with Spodic criteria, all of them have low voltage. Classification with echocardiography of pericardial effusion made with Horowitz criteria. Two of the four patients, microbiologically proved tuberculosis had constructive pericarditis and had pericardiectomy on follow up. Half of the 14 patients that underwent pericardiocentesis, have fibrinous pericarditis and three of them healed with antibiotics. Three patients with staphylolococcus aureus medical treatment was unsuccesful. Five patients have transuda. Serological Coxsackievirus was verified in one child. After surgical closure of an ASD, patient attended with respiratory distress had a very good result with pericardiocentesis and NSAID. A secondary pericardial effusion due to autoimmune hepatitis treated with pericardiocentesis. Two of the trisomy 21 patients with hypothyroidism have an excellent prognosis with medical treatment,only one of them with immunodeficiency managed with antibiotics is. Another patient with pulmonary hydatit cyst treated with mebendazole. A pericardial effusion in a SLE patient responds to steroids.

7320

Aggressive medical and surgical management improves outcome of children with right atrial isomerism - A 30-year experience in a single institution

Masaki Osaki, Shizuoka Children's Hospital, Japan

Yasuhiko Tanaka, Shizuoka Children's Hospital, Japan

Yasuo Ono, Shizuoka Children's Hospital, Japan

Kisaburou Sakamoto, Shizuoka Children's Hospital, Japan

Objective: To describe clinical outcomes of right atrial isomerism (RAI) and to clarify the impact of our aggressive strategy.

Methods: Retrospective chart review of RAI from 1978 to 2008. Patients older than 6-months or patients who had surgery before referral were excluded.

Results: There are 114 children enrolled (68 male). Median age at initial presentation was 9 days. Major cardiac abnormalities included atrioventricular septal defect in 109 (96\%), doubleoutlet right ventricle in 101 (89\%), pulmonary atresia in 57 $(50 \%)$, and extracardiac total anomalous of pulmonary venous connection (TAPVC) in $52(46 \%)$. None was suitable for biventricle repair. Among 62 RAI in the early era (1978 to 1995), no surgical intervention was planned in 12,10 of whom died. Fourteen (23\%) completed Fontan, 11 of whom alive (79\%). Survival was $59 \%$ at 1 year and $31 \%$ at 5 years. Multivariate analysis revealed TAPVC repair at initial surgery and atrioventricular valve regurgitation (AVVR) as significant risk factors for mortality $(p<0.05)$. Since mid-90s, we have changed our 
strategy more aggressively, which included earlier referral to surgery, repair of AVVR at initial palliation, and modification of TAPVC repair. Among 52 RAI in the modern era (1996 to present), all but one were referred to surgery. Twenty-three (43\%) completed Fontan, 21 of whom alive (91\%). Survival was $70 \%$ at 1 year and $54 \%$ at 5 years, which were improved significantly from the early era $(\mathrm{p}<0.05)$. AVVR remains as a significant risk factor for mortality.

Conclusion: Aggressive medical and surgical management has improved survival of RAI.

\section{1}

Double orifice tricuspid valve: rare or frequently missed? Ramesh C Parmar, The Children's Hospital at Westmead, Australia David S Winlaw, The Children's Hospital at Westmead, Australia Stephen G Cooper, The Children's Hospital at Westmead, Australia

Double-orifice-tricuspid-valve (DOTV) is rare with only 22 cases described. Only one incidence of preoperative diagnosis has been reported whereas double orifice mitral valve is routinely identified preoperatively. Embryological origins of the lesion are not well characterised. We detail our institutional experience with this defect.

Methods: A chart review was conducted with focus on echocardiographic assessment, coexisting lesions, surgical intervention and outcomes for cases diagnosed preoperatively in 2007-2008.

Results: Six cases were identified. Associated pathologies included inlet ventricular septal defect (VSD) (2), atrioventricular septal defect (1), d TGA/VSD (1), pulmonary atresia with VSD with major aortopulmonary collaterals (1) and univentricular connection with ccTGA, mitral atresia, hypoplastic left ventricle, VSD with infundibular and valvar pulmonic stenosis (1).

Modification of the subcostal-long axis view, apical-fourchamber and parasternal views were helpful. In cases with VSD, malalignement of interventricular and interatrial septae was a feature with the accessory orifice covering the defect created by the offset.

All but one underwent surgical correction involving the closure of the minor orifice and patch closure of the VSD, often requiring temporary detachment of tricuspid supports for both the orifices. Postoperative complete heart block (CHB) was observed two with one needing permanent pacemaker. One had residual trivial TI.

Conclusions: In complex pathology the diagnosis of DOTV may be overlooked if unaware of its possibility. CHB may occur given the position of the bundle on the left side of the septum and need to attach VSD patches close to this area. Repair may be complex but the outcomes are good.

7322

Infections and endothelial cell apoptosis

Erkki Pesonen, Lund University Hospital, Sweden

Malene Muusfeldt Birck, Faculty of Life Sciences, University of

Copenhagen, Denmark

Petru Liuba, Lund University Hospital, Sweden

Michal Odermarsky, Sweden

Kenneth Persson, Malmö University Hospital, Sweden

Axel Kornerup Hansen, Faculty of Life Sciences, University of

Copenhagen, Denmark

Antti Saraste, Turku University Hospital, Finland

Objectives: Endothelial cells of coronary arteries undergo apoptosis in patients with coronary artery disease. Carotid artery ultrasound studies show that after acute infections intima media thickness is increased. The possible effects of infections on endothelial cell apoptosis and the presence of apoptosis in early life remain unknown.

Methods: Chlamydia pneumonia was inoculated three times at three weeks intervals to half of 32 piglets starting at the age of 8 weeks. Half of the piglets received cholesterol feeding. Morphological studies were done at the age of 19 weeks. Right coronary arteries were carefully excised and trimmed for excess tissue. The vessels were cut perpendicular to the long axis and sectioned at $5-\mu \mathrm{m}$ thickness. TUNEL-assay was based on nick end labelling and staining of internucleosomal DNA fragments that are the biochemical hallmark of apoptosis. The percentage of apoptotic endothelial cells from intact endothelial cells was calculated using light microscopy. Results. Endothelial apoptosis appeared in $0.13 \%$ (SD 0.30\%) of endothelial cells of non-infected piglets but in $0.75 \%$ (SD $0.62)$ of infected piglets $(p<0.005$, t-test). In piglets fed with normal diet apoptosis appeared in $1.1 \%$ but in those on cholesterol rich diet the prevalence of apoptosis was lower $0.43 \%$ (SD 0.42).

Conclusions: Infections amplify endothelial cell apoptosis. The finding supports the notion that infection has a pro-atherogenic effect particularly in early life. Hypercholesterolemic diet did not further stimulate apoptosis.

7323

Usefulness of N-terminal pro-B-type natriuretic peptide as a marker of right ventricular function in children after tetralogy of Fallot total repair

Radoslaw Pietrzak, Department of Children's Cardiology and General Pediatrics, Medical University of Warsaw, Poland

Bozena Werner, Department of Children's Cardiology and General

Pediatrics, Medical University of Warsaw, Poland

The aim of the study was to determine $\mathrm{N}$-terminal proB-type natriuretic peptide (NT-pro-BNP) value in assessment of right ventricular function in children after Tetralogy of Fallot (TOF) correction. Methods: In 20 patients after TOF correction aged from 10 to 17 years NT-pro-BNP level and echocardiography were performed. In the control group NT-pro-BNP level was assessed.

Results: The mean values of NT-pro-BNP level in the study and control group were respectively $11,0+/-12,0 \mathrm{fmol} / 1$ and $5,4+/-7,5 \mathrm{fmol} / 1 \quad(\mathrm{p}<0,05)$. Right ventricular diastolic diameter ranged from $96,7 \%$ to $200 \%$ (mean $142,2 \%$ ) of limit and correlate positively with follow-up time $(p=0,004$, $r=+0,353)$. Severe pulmonary regurgitation $(P R)$ was visualized in 9 and mild/moderate PR in 11 patients. Statistically significant differences in NT-pro-BNP levels between children with severe $\mathrm{PR}$ and mild/moderate PR were detected (mean values 18,6 +/ $-15,0 \mathrm{fmol} / 1$ vs $4,2+/-3,9 \mathrm{fmol} / 1, \quad \mathrm{p}<0,05)$. In patients repaired with transannular patch the mean value of NT-proBNP level was higher than in children operated on without transannular patch $(18,3+/-16,5 \mathrm{fmol} / 1$ vs $6,8+/-7,9 \mathrm{fmol} /$ 1), $(\mathrm{p}<0,05)$. Right ventricular diameter correlated positively with NT-pro-BNP values $(\mathrm{p}<0,05, \mathrm{r}=+0,50)$.

Conclusions: The NT-pro-BNP level in patients after TOF correction is higher than in healthy children. NT-pro-BNP level is higher in children with right ventricle function disturbances expressed by the level of pulmonary regurgitation and value of right ventricle diameter. The NT-pro-BNP level is higher in children repaired with transannular patch than in children operated without transannular patch. 
7324

Effect of planned teaching of parents in care of children with Congenital Heart Disease

Shakuntala S Prabhu, B.J. Wadia Hospital For Children, India

Panchali Datta, India

Sumitra Venkatesh, India

Aim: Effect of planned teaching in the knowledge of care of children with Congenital Heart Diseases (CHD).

Methods: Either parent of 50 children diagnosed with CHD following-up at the cardiac clinic and informed about their child's condition were included. A structured questionnaire (in 2 vernacular languages) was used for the interview of the parents. All underwent a pretest followed by planned teaching (using audiovisual aids) which was concluded by a post test 15 days later. Knowledge of the parents about pathophysiology, signs and symptoms of the cardiac condition, infective endocarditis (IE) prophylaxis, dental hygiene and special care during complications were assessed.

Results: $36 \%$ of parents had high school education and $28 \%$ belonged to the middle socioeconomic strata. Planned teaching improved the understanding of the parents regarding the CHD in their child from $70 \%$ to $82 \%$. Knowledge regarding appropriate feeding increased from $58 \%$ to $92 \%$ and care of children during complications of the disease from $52 \%$ to $74 \%$ in the post test evaluation; all of which were statistically significant. Also $72 \%$ were aware of the need for IE prophylaxis during dental manipulation, though $74 \%$ of them were unaware of signs and symptoms of the same. The post test awareness of proper dental hygiene increased to $88 \%$ from $60 \%$.

Conclusion: Planned teaching is an effective and significant tool to improve the knowledge of parents of children with CHD and such education should be a part of all pediatric cardiac clinics.

\section{5}

Motivating Forces for Parenting Infants with a Complex Congenital Heart Defect and Feeding Interaction

Karen A Pridham, University of Wisconsin-Madison, School of Nursing, United States

Tondi M Harrison, University of Minnesota, School of Nursing, United States

Mary Krolikowski, Herma Heart Center, Children's Hospital of Wisconsin, United States

Roger Brown, University of Wisconsin-Madison, School of Nursing, United States

Raymond Fedderly, Herma Heart Center, Children's Hospital of

Wisconsin, Medical College of Wisconsin, United States

Objective: Examine parents' motivations for parenting a 1-monthold infant with a complex congenital heart defect and the difference made by motivation classes for parent feeding behavior.

Methods: In 22 families recruited from a regional children's heart center, 9 infants had hypoplastic left heart syndrome (high severity); 13 had lower severity defects. The primary caregiving parent, all but two who were mothers, were interviewed about parenting experience and about video-taped feeding interaction. Interviews were coded using directed content analysis to identify motivating forces. Ten Parent-Child Early Relationship Assessment scales were used to construct an adaptability variable, Parental Support, Attunement, and Warmth (warm tone of voice, positive affect, lack of withdrawn/depressed mood, quantity of visual contact, quantity of verbal contact, structuring and mediating the environment, sensitive responsiveness, flexibility, predictability, and lack of intrusiveness).
Results: Motivating forces were classed as Caring for Baby (supporting development, protecting); Relating (to baby, family); Parenting Identity; Self-Maintaining (promoting, protecting self); and Doing the Needed. Scored high or low, these classes and heart defect severity, in latent cluster analysis, revealed two clusters undifferentiated by severity (see Figure 1). Cluster 1 parents more often identified Parenting Identity and Baby Care motivating forces, whereas Cluster 2 parents more often identified Doing the Needed as a motivating force. Cluster 2 showed higher scores, on average, on Parenting Support, Attunement, and Warmth and less variability than Cluster 1.

Conclusions: Motivating forces, but not heart defect severity, sorted parents into clusters that may predict feeding support, attunement, and warmth.

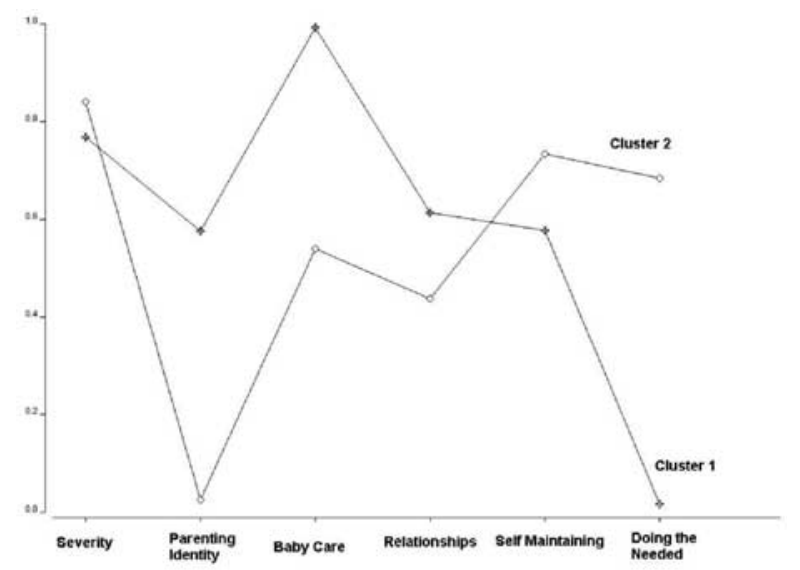

Figure 1. Latent clusters by severity of heart defect and parental

motivating forces.

7326

Emergency Visits by Young Children with Significant Heart Disease

Lee A Pyles, University of Minnesota Department of Pediatrics and Emergency Medicine, United States

Margaret Scheid, University of Minnesota Department of Pediatrics, United States

Objective: The purpose of this investigation is to describe the breadth of emergency visits of children with cardiac diseases less than 2 years of age, enrolled in a program to prepare for emergencies of children with heart disease.

Methods: Children treated for heart failure or arrhythmia and those who have undergone cardiac surgery had presenting complaint, results and charges from emergency visits tabulated from $9 / 26 / 02$ to $2 / 28 / 05$. Children were drawn from the 3 cardiac programs in a US metropolitan area. Visits not including new presentations.

Results: 170 children had 189 emergency visits in 1.72 years, average 0.65 visits per patient/year of study with 44 admissions (23\%) including 8 critical admissions. Cardiac diagnoses for the children included TOF 15\%, VSD 15\%, Aortic Coarctation 13\%, Transposition 12\%, SVT $6 \%$ and other congenital anomalies. The presenting complaints included the following emergencies: 19 cardiac (including 15 visits for arrhythmia, 1 cardiac arrest, 2 heart failure, 1 hypercyanotic spell), 60 respiratory (23 difficulty breathing, 18 cough, 7 wheezing, 6 hypoxia, 1 respiratory arrest, 1 apnea), 48 gastrointestinal, 39 infectious (30 fever and 7 otitis), neuro 10 , trauma 7 , and skin 6 . ED charges averaged $\$ 1205$ $+/-\$ 1260$ and varied with age of child $(\mathrm{p}<0.0001)$. 
Conclusions: Children with known heart disease rarely present with important cardiac emergencies other than SVT. Emergencies related to feeding issues were common. Hospital admission rate of $23 \%$ equals published literature but the ED visit rate of 0.65 visits/year is 3 times the usual rate for other Children with Special Health Care Needs.

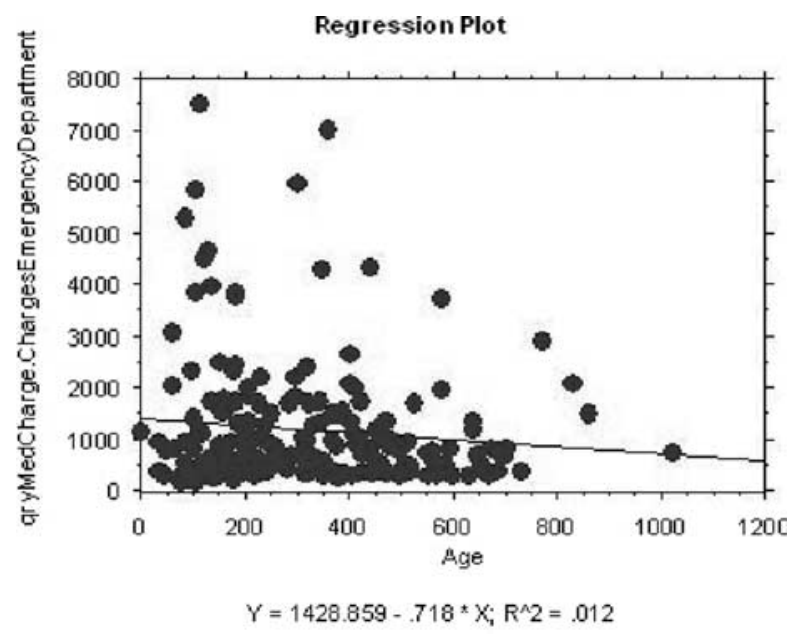

7327

Constrictive pericarditis in children

Swee Chye Quek, National University of Singapore, Singapore Kit Yee Chan, Singapore Baby and Child Clinic, Singapore William Yip, Singapore

Objective: Constrictive pericarditis $(\mathrm{CP})$ is rare, resulting from pericardial disease impeding diastolic filling of ventricles. We report case series outlining clinical manifestations, treatment and outcomes.

Patients: 6 patients $(\mathrm{M}=4, \mathrm{~F}=2)$ were diagnosed over 10 years. Mean age at presentation was $7.6( \pm 4.5)$ years, duration from onset to diagnosis was $1.6( \pm 2.2)$ years. Abdominal distension (ascites) was universal symptom; others included reduced effort tolerance, lethargy, loss of appetite. Pericardial/pleural effusions, raised JVP, edema, hepatomegaly were common signs. Echocardiography revealed dilation of atria, small ventricles, and jerkiness of the interventricular septum. There were marked respiratory swings on Doppler interrogation of mitral and tricuspid inflow velocities consistent with constriction. Significant pericardial effusion was present, but did not cause hemodynamic compromise. Additionally, IVC and hepatic veins were engorged. All patients had structurally normal hearts with no previous cardiovascular disease. Chest X-ray did not reveal calcification, but CT/MRI (heart) showed pericardial thickening. None was subjected to cardiac catheterization. A check for secondary causes revealed SLE in 1, the rest were "idiopathic" CP. Treatment was surgical(total pericardiectomy in 3, partial pericardiectomy in 2) and medical (for SLE) in 1.

Results: All patients survived, within follow-up period of 2.6 $( \pm 6.6)$ years. Outcome was good with resolution of clinical signs. Post-procedural echocardiography also improved, except for 1 (partial pericardiectomy) where there were residual signs. Conclusions: Although $\mathrm{CP}$ is uncommon, and its diagnosis frequently delayed, combination of ascites, pleural/pericardial effusions should raise possibility of $\mathrm{CP}$ in a child. Echocardiography is diagnostic. In absence of secondary causes, there is little role for medical treatment. Total pericardiectomy is definitive treatment and associated with good outcome.
7328

Cardiopulmonary Exercise Testing After Surgical Repair of Tetralogy of Fallot

$R$ Suresh Kumar, Dept of Pediatric Cardiology, ICVD, Madras

Medical Mission, Chennai, India, India

Roy Varghese, Dept of Pediatric Cardiology, ICVD, Madras Medical Mission, Chennai, India, India

G Madhusudan, Dept of Pediatric Cardiology, ICVD, Madras Medical

Mission, Chennai, India, India

Atul Prabhu, Dept of Pediatric Cardiology, ICVD, Madras Medical

Mission, Chennai, India, India

L Varatharajan, Dept of Pediatric Cardiology, ICVD, Madras Medical Mission, Chennai, India, India

Sreeja Pavithran, Dept of Pediatric Cardiology, ICVD, Madras Medical Mission, Chennai, India, India

John Valliatu, Dept of Pediatric Cardiology, ICVD, Madras Medical

Mission, Chennai, India, India

Objective: Cardiopulmonary exercise testing (CPT) is a powerful predictor for adverse events in congestive heart failure. The prevalence and significance of exercise intolerance in operated congenital heart disease merits investigation. This study was done to compare cardiopulmonary exercise parameters in patients after surgical repair of Tetralogy of Fallot (rTOF) with operated Atrial Septal Defect (ASD) and normal controls.

Method: 23 patients aged $>10$ years (Group 1, range 10-36 years, median 19.5 years) who had undergone surgical repair of TOF at a median age 10 years, underwent CPT using upright bicycle ergometry using MedGraphics BreezeSuite PFX UltimaTM till exhaustion. The results were compared with operated ASD patients (Group 2) and normal controls (Group3).

\begin{tabular}{lcccc}
\hline Parameter & $\begin{array}{c}\text { Group 1 } \\
(\mathbf{n}=23)\end{array}$ & $\begin{array}{c}\text { Group 2 } \\
(\mathbf{n}=21)\end{array}$ & $\begin{array}{c}\text { Group 3 } \\
(\mathbf{n}=20)\end{array}$ & $\begin{array}{c}\text { p } \\
\text { value }\end{array}$ \\
\hline $\begin{array}{l}\text { Peak VO2-Peak oxygen } \\
\text { consumption (ml/kg/min) }\end{array}$ & 23.1 & 28.2 & 30.3 & 0.005 \\
$\begin{array}{l}\text { VO2AT- Oxygen } \\
\text { consumption at anaerobic } \\
\text { threshold (ml/kg/min) }\end{array}$ & 11.9 & 13.8 & 14.7 & 0.18 \\
$\begin{array}{l}\text { VR or VE/VCO2 Slope - } \\
\text { Ventilatory response to } \\
\text { exercise }\end{array}$ & 34.9 & 27.5 & 27 & 0.15 \\
$\begin{array}{l}\text { MEP- Maximal exercise } \\
\text { performance (Watt/Kg) }\end{array}$ & 3.6 & 7 & 6.9 & 0.003 \\
$\begin{array}{l}\text { RER-Respiratory exchange } \\
\text { ratio }\end{array}$ & 1.6 & 1.26 & 1.2 & 0.75 \\
\hline
\end{tabular}

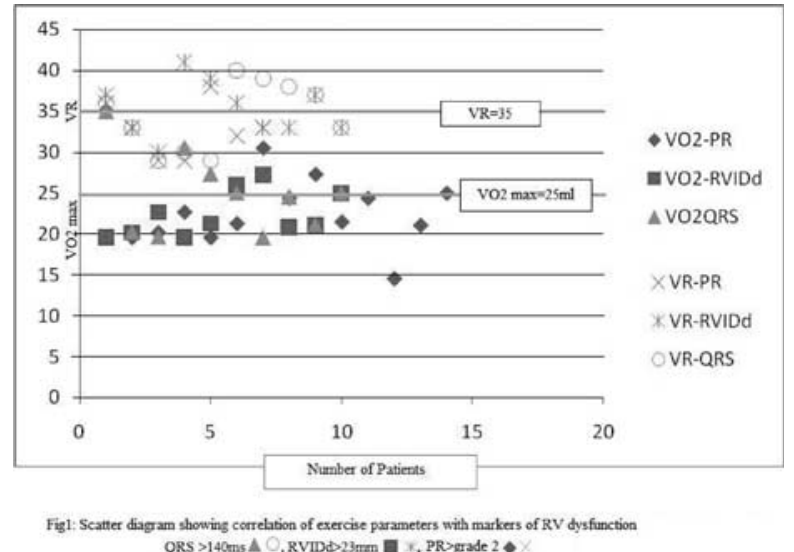

Results: Table 1 shows the group means of all exercise parameters. Though statistical significance was shown for only peakVO2 and 
MEP, other variables for rTOF also tended to have uniformly impaired values. Values crossing an arbitrary cut-off limit were almost confined to rTOF patients. rTOF patients with a combination of known markers of RV dysfunction (dilated RV, severe $\mathrm{PR}$ and prolonged QRS) were more likely to have impaired VO2max, VR and MEP (Figure 1).

Conclusion: rTOF patients frequently show impaired CPT parameters. This impairment is disease specific as operated ASD patients behaved like controls. RV dilatation, prolonged QRS duration and significant PR are more likely to be associated with impaired CPT parameters. The long term predictive value of these findings for adverse events needs prospective evaluation.

\section{2}

Congenital heart disease in 22q11 deletion syndrome: do variants in the folate genes have a modifier effect?

Gabriela M Repetto, Facultad de Medicina, Clinica AlemanaUniversidad del Desarrollo, Chile

Augusto San Martin, Facultad de Medicina, Clinica Alemana-

Universidad del Desarrollo, Chile

Alonso F Puga, Facultad de Medicina, Clinica Alemana-Universidad del Desarrollo, Chile

Juan Calderon, Facultad de Medicina, Clinica Alemana-Universidad del Desarrollo, Chile

Carmen P Astete, H Luis Calvo Mackenna, Chile

Mariana Aracena, H Luis Calvo Mackenna, Chile

Maria Luisa Guzman, Facultad de Medicina, Clinica Alemana-

Universidad del Desarrollo, Chile

Teresa Aravena, $H$ Sótero del Río, Chile

Microdeletion 22q11 syndrome (del22q11) has an estimated incidence of $1 / 4000$ and is a common known cause of congenital heart disease (CHD). Approximately $50-70 \%$ of patients have CHD. Most patients have a deletion of similar size and location; the reason for the incomplete penetrance of the cardiovascular anomalies is unknown. Low maternal folate levels and variants in folate metabolism genes have been associated with increased risk of non-syndromic CHD. We evaluated the association of 7 variants in 6 folate metabolism genes in 112 Chilean patients with del22q11. The patients, $50 \%$ of them with CHD, and their parents, were genotyped for variants C677T and A1298C in the MTHFR gene, A80G in SLC19A1, G1958A in MTHFD1, G742A in BHMT, A2756G in MTR and A66G in MTRR. We found marginal evidence of association of CHD with maternal allele $80 \mathrm{G}$ in the SLC19A1 gene: its allelic frequency was 0.72 in mothers of children with CHD and 0.44 in mothers of children without CHD (uncorrected p value 0.03; RR 1.35 (95\% CI $0.997-1.855)$. There were no statistically significant differences in allelic or genotypic frequencies for any of the other variants. SLC19A1 is involved in intestinal folate transport, the $80 \mathrm{G}$ allele decreases its efficiency and this may affect maternal folate absorption. The association found in this study suggests that maternal abnormalities in folate metabolism could modify the risk of cardiovascular abnormalities in patients del22q11.

\section{3}

\section{Congenital Heart Disease in Children in Fiji}

Kerrie A Richardson, Women's and Children's Hospital, Adelaide, Australia Malcolm E Richardson, Women's and Children's Hospital, Adelaide, Australia

Joseph Kado, Colonial War Memorial Hospital, Suva, Fiji

Objective: To describe the spectrum of congenital heart disease in a population of children attending a visiting paediatric cardiology clinic.
Methods: We reviewed the clinical records and echocardiograms of children attending a screening clinic that was part of an annual visiting cardiac surgical team.

Results: A total of 519 children were examined between 2002 and 2008. Ages ranged from 1 day to 18 years. Twenty-nine had normal hearts, 77 had rheumatic disease and 64 had postoperative studies. The remaining 349 had a variety of lesions. In decreasing order of frequency they included: ventricular septal defect $39 \%$, tetralogy of Fallot $16 \%$, duct $9 \%$, atrial septal defect $9 \%$, complex $8 \%$, pulmonary stenosis $2.6 \%$, congenital aortic valve disease $2.3 \%$, atrioventricular septal defect $2 \%$, coarctation $1.4 \%$ and transposition $1.4 \%$. An assortment of other lesions had a frequency of $1 \%$ or less. Aortic valve prolapse occurred in $38 \%$ and regurgitation occurred in $24 \%$ of all ventricular septal defects. Those with complex lesions tended to have pulmonary outflow obstruction or high pulmonary resistance protecting them from heart failure. Very few duct dependent lesions were seen.

Conclusion: The spectrum of lesions reflects the limited access to tertiary cardiac services. The most common complex lesions are those that allow medium term survival without surgical treatment. Aortic prolapse and regurgitation commonly complicate ventricular septal defects in these children.

\section{4}

Plastic Bronchitis after the Fontan Procedure - successful treatment with Tissue Plasminogen Activator

Malcolm E Richardson, Women's and Children's Hospital, Adelaide, Australia

James Martin, Women's and Children's Hospital, Adelaide, Australia

Carol Smith, Women's and Children's Hospital, Adelaide,

Australia

Michael Yung, Women's and Children's Hospital, Adelaide, Australia

Objective: To report the successful use of Tissue Plasminogen Activator (TPA) in a patient with respiratory failure from plastic bronchitis.

Methods: Case report.

Results: Approximately 18 months after modified Fontan surgery for complex heart disease that included obstructed anomalous right pulmonary venous drainage, an eight year old boy developed plastic bronchitis. He was treated with bronchodilators, physiotherapy and nightly nebulised DNAse. He remained well on this treatment over the next year but continued to produce bronchial casts on most days. During this period he required two brief admissions for worsening respiratory distress. These were managed successfully with intensification of bronchodilator treatment and physiotherapy. Sixteen months after the initial diagnosis he was admitted with rhinoviral infection, increased cast production and severe respiratory failure. He developed profound hypoxemia and continued to worsen despite all attempts at more conventional treatment including ventilation, nebulised DNAse, antibiotics, physiotherapy and repeated bronchoscopic removal of casts. After commencing nebulised TPA ( $5 \mathrm{mg}$ in $5 \mathrm{ml}$ four hourly) cast production ceased, air entry improved dramatically and systemic saturations rose from levels that were persistently below $50 \%$ back to previous baseline levels between 85 and 90\%. TPA treatment was successfully weaned as his infection settled. No adverse effects were noted. He was discharged 18 days later.

Conclusion: Nebulised TPA may be lifesaving in respiratory failure from plastic bronchitis. 
7335

Is the long-term outcome of the one \& one half physiology superior to the Fontan physiology in patients with hypoplastic right heart?

Koichi Sagawa, Fukuoka Children's Hospital, Japan

Shiro Ishikawa, Pediatric cardiology division, Fukuoka Children's

Hospital, Japan

Yuichi Ishikawa, Pediatric cardiology division, Fukuoka Children's

Hospital, Japan

Makoto Nakamura, Pediatric cardiology division, Fukuoka Children's

Hospital, Japan

Hiroya Ushinohama, Pediatric cardiology division, Fukuoka Children's

Hospital, Japan

Toshihide Nakano, Cardio-vasucular surgery division, Fukuoka

Children's Hospital, Japan

Hideaki Kado, Cardio-vasucular surgery division, Fukuoka Children's Hospital, Japan

Background: It is quite frequently to vacillate between the one \& one half repair and the Fontan-type repair in case of a choice of surgery in patients (pts) with hypoplastic right heart. To settle this issue, we addressed the comparison of the long-term outcome of the one $\&$ and one half physiology and the Fontan physiology in pts with hypoplastic right heart.

Subjects and methods: We reviewed twenty-one pts with hypoplastic right heart who had undergone functionally physiological corrections in our institute. Ten pts with one \& one half physiology and eleven age-matched pts with perfect Fontan physiology were enrolled. Both the groups had been treated with warfarin potassium, aspirin, and ACE-I/ARB/ $\beta$ blocker for cardiovascular protection. Results: Data were shown in the table.

Discussion: The one \& one half physiology is inferior to the Fontan physiology in the cardiac index and the plasma natriuretic peptide levels. Furthermore, it is suggested that the exercise tolerance is lower in the one \& one half physiology than in the Fontan physiology. On the other hand, the former is superior to the latter in the mean IVC pressure and the serum AST and $\gamma$ GTP levels. However, there was no significant difference of coagulability between the two physiologies under the treatment with warfarin potassium, aspirin, and ACEI/ $\mathrm{ARB} / \beta$ blocker. In conclusions, the one $\&$ one half physiology might be inferior to the Fontan physiology in the long-term outcome in pts with hypoplastic right heart.

\begin{tabular}{|c|c|c|c|}
\hline parametes & $\begin{array}{l}\text { one \& one half } \\
(n=10)\end{array}$ & $\begin{array}{l}\text { Fontan } \\
(\mathrm{n}=11)\end{array}$ & $\begin{array}{l}\mathrm{p} \\
\text { value }\end{array}$ \\
\hline age at examination (years) & 13.1 & 10.5 & 0.778 \\
\hline $\begin{array}{l}\text { postoperative period } \\
\text { (years) }\end{array}$ & 6.7 & 4.5 & 0.360 \\
\hline PT-INR & 1.64 & 1.45 & 0.130 \\
\hline Cardiac index $(\mathrm{L} / \mathrm{min} / \mathrm{kg})$ & 3.2 & 4.1 & $0.024 *$ \\
\hline $\begin{array}{l}\text { mean SVC pressure } \\
(\mathrm{mmHg})\end{array}$ & 12 & 10 & 0.073 \\
\hline $\begin{array}{l}\text { mean IVC pressure } \\
(\mathrm{mmHg})\end{array}$ & 6 & 10 & $0.004 *$ \\
\hline $\mathrm{SaO} 2(\%)$ & 96.6 & 95.2 & $0.017 *$ \\
\hline $\begin{array}{l}\text { exercise test endurance } \\
\text { time }(\% \mathrm{n})\end{array}$ & 72 & 86 & 0.068 \\
\hline peak HR (\% n) & 81 & 91 & 0.117 \\
\hline peak VO2 (\% n) & 73 & 90 & 0.117 \\
\hline hematocrit (\%) & 41.2 & 44.6 & $0.038^{*}$ \\
\hline paletelet $\left(\times 10^{\wedge} 3 / \mu \mathrm{l}\right)$ & 260 & 226 & 0.218 \\
\hline ANP (pg/ml) & 67 & 21 & $0.003 *$ \\
\hline $\mathrm{BNP}(\mathrm{pg} / \mathrm{ml})$ & 49 & 5 & $0.001 *$ \\
\hline
\end{tabular}

\begin{tabular}{|c|c|c|c|}
\hline parametes & $\begin{array}{l}\text { one \& one } \\
(\mathrm{n}=10)\end{array}$ & $\begin{array}{l}\text { half Fontan } \\
\quad(\mathrm{n}=11)\end{array}$ & $\begin{array}{l}\mathrm{p} \\
\text { value }\end{array}$ \\
\hline $\mathrm{TP}(\mathrm{g} / \mathrm{dl})$ & 7.1 & 7.2 & 0.751 \\
\hline albumin $(\mathrm{g} / \mathrm{dl})$ & 4.4 & 4.8 & 0.024 \\
\hline BUN (mg/dl) & 12 & 13 & 0.481 \\
\hline creatinine $(\mathrm{mg} / \mathrm{dl})$ & 0.5 & 0.5 & 0.725 \\
\hline AST $(I U / L)$ & 28 & 34 & $0.041 *$ \\
\hline $\operatorname{ALT}(\mathrm{IU} / \mathrm{L})$ & 18 & 24 & 0.091 \\
\hline$\gamma$-GTP (IU/L) & 20 & 48 & $0.001 *$ \\
\hline total bilirubin (mg/dl) & 0.9 & 0.9 & 0.307 \\
\hline $\begin{array}{l}\text { thrombin-antithrombin } \\
\text { complex (TAT: } \mathrm{ng} / \mathrm{ml} \text { ) }\end{array}$ & 2.0 & 5.4 & 0.529 \\
\hline activity of protein $\mathrm{C}(\%)$ & 61 & 51 & 0.418 \\
\hline antithrombin $(\%)$ & 91 & 101 & 0.275 \\
\hline $\begin{array}{r}\text { plasmin } \alpha 2 \text {-antiplasmin } \\
\text { complex }(\mathrm{PAP}: \mu \mathrm{g} / \mathrm{ml})\end{array}$ & 0.7 & 0.8 & 0.438 \\
\hline $\mathrm{d}$-dimer $(\mu \mathrm{g} / \mathrm{ml})$ & 0.4 & 0.3 & 0.360 \\
\hline thrombomodulin $(\mathrm{FU} / \mathrm{ml})$ & 2.0 & 2.4 & 0.168 \\
\hline
\end{tabular}

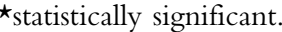

7336

The management for unbalanced ventricular heart in Down syndrome

Hirofumi Saiki, Hyogo Prefectural Kobe Children's Hospital, Department of Cardiology, Japan

Sachiko Kido, Hyogo Prefectural Kobe Children's Hospital, Department of Cardiology, Japan

Toshikatsu Tanaka, Hyogo Prefectural Kobe Children's Hospital, Department of Cardiology, Japan

Hideki Fujita, Hyogo Prefectural Kobe Children's Hospital,

Department of Cardiology, Japan

Kenta Tominaga, Hyogo Prefectural Kobe Children's Hospital, Department of Cardiology, Japan

Yoshihiro Oshima, Hyogo Prefectural Kobe Children's Hospital, Department of thoracic cardio-vascular surgery, Japan

Teruo Tei, Tei Pediatric Clinic, Japan

Teruo Tei, Tei Pediatric Clinic, Japan

Objective: Although the recent strategies for functional single ventricle have led to improved outcome, the establishment of Fontan circulation in Down syndrome is still challenging. The aim of this study is to elucidate the adequate strategies for unbalanced ventricle in Down syndrome.

Methods: 10 consecutive patients between 1997-2008 with Down syndrome and unbalanced ventricle were reviewed. The anatomical features were severe mitral stenosis in 1, unbalanced complete AVSD with abnormal mitral component and hypoplastic LV in 9. They also associated with variety of outlet anomalies. Because of clinical condition, 5 cases were decided on biventricular repair and 5 cases underwent TCPC with fenestration. There was no waiting case.

Results: The median age at operation, the mean follow up period after operation were $4.2(0.3-6.2) / 3.7(3.0-6.7)$ years, $6.6 \pm 3.2 /$ $4.5 \pm 1.8$ years in BVR and TCPC, respectively. The mortality was $20 \%$ (1 out of 5 ) in both, and there was no late death and take-down in either group. Excluding 2 fatal cases, 2 cases required re-operation for MVR and the other case underwent resuscitated cardiac arrest in BVR, while uneventful in TCPC. In contrast, pulmonary vasodilators including HOT after discharge were required in all TCPC cases, while only one in BVR. The frequency of out-patient and in-patient was 5.8/9.3 and $0.06 / 0.28$ per patient-year in BVR and TCPC, respectively. 
Conclusions: In spite of high morbidity, the patients with BVR have established higher QOL than the patients with TCPC. Patient selection for Fontan procedure in Down syndrome should be done more restrictively than non-Down patients.

\section{The present condition}

\begin{tabular}{|c|c|c|}
\hline & Biventricular repair & TCPC \\
\hline alive/death & $4($ re-ope: MVR 2)/ 1 & $4($ fenestrated 3$) / 1$ \\
\hline $\begin{array}{l}\text { BVR or TCPC (age) } \\
\text { / post-ope. period }\end{array}$ & $\begin{array}{c}3.6 \pm 2.3 \\
/ 6.6 \pm 3.2 \text { years }\end{array}$ & $\begin{array}{c}4.0 \pm 1.5 \\
/ 4.5 \pm 1.8 \text { years }\end{array}$ \\
\hline $\begin{array}{c}\text { meanPAp(mmHg) } \\
\text { / C.I.(Fick) }\end{array}$ & $\begin{array}{c}21 \pm 6 \\
13.4 \pm 0.08\end{array}$ & $\begin{array}{c}13 \pm 1 \\
/(2.8 \pm 0.25)\end{array}$ \\
\hline $\begin{array}{c}\text { Sat } 02 \text { (\%) } \\
\text { / home oxygen (case) }\end{array}$ & $\begin{array}{c}98 \pm 1 \\
/ 1 \text { ( tracheal stenosis) }\end{array}$ & $\begin{array}{c}90 \pm 4 \\
/ 4\end{array}$ \\
\hline $\begin{array}{l}\text { present medication } \\
\text { (case) }\end{array}$ & $\begin{array}{l}\text { ACE1 2, aspirin 2, } \\
\text { warfarin 2, diuretics 1, } \\
\text { digitalis } 1\end{array}$ & $\begin{array}{l}\text { beraprost } 3 \text {, ACE1 3, } \\
\text { aspirin } 3 \text {, warfarin } 3 \text {, } \\
\text { diuretics } 1\end{array}$ \\
\hline $\begin{array}{l}\text { out/in-patient after } \\
\text { operation ( average ) }\end{array}$ & $\begin{array}{l}5.8 / 0.06 \\
\text { /person-year }\end{array}$ & $\begin{array}{l}9.3 / 0.28 \\
\text { /person-year }\end{array}$ \\
\hline
\end{tabular}

\section{7}

Treatment of a large PAVF in a child after TCPC

Ivan Malcic, University Hospital Center Zagreb, Croatia, Croatia Andreas Eicken, German Heart Centre Munich, Germany

John Hess, German Heart Centre Munich, Germany

Christian Scheiber, German Heart Centre Munich, Germany

Hrvoje Kniewald, University Hospital Center Zagreb, Croatia, Croatia

Rudiger Lange, German Heart Centre Munich, Germany

Introduction: Late complications of RV by-pass procedures appear as arrhythmias, protein losing enteropathy, chronic heart failure and PAVF. Treatment of large PAVF is particularly difficult and may require complete resection of pulmonary segments affected by a fistula.

Case study: A girl P.T, born 08/2000 with a CHD, with basic diagnoses: Situs inversus abdominalis, left atrial isomerism, levocardia, functional single ventricle, single AV-valve, TGA, right aortic arch, infundibular and valvular PS, LVCS, azygos continuity, RVCS. Treatment: Ao-PA central shunt (Zagreb, 10/00), bidirectional Glenn (Zg, 04/01), TCPC with fenestration (Zg, 12/02). During the first year the child had no difficulties, then a deeper cyanosis started developing which warned of PAVF development. Selective angiocardiography established large PAVF in the medium and lower lobe of the right lung. Test-occlusion by transcatheter closing of pulmonary branches of the right lung in the medium and lower lobe and closing the fenestra with an Amplatzer plug increased saturation from $60 \%$ to $90 \%$ After the test, the Amplatzer plug was implanted, $12 \mathrm{~mm}$ on the fenestration and $10 \mathrm{~mm}$ on the fistulas (01/07 DHM). Nonetheless, deep cyanosis was developing due to new R-L shunt development on the intrapulmonary fistulas level (R-L shunt 59\%). Resection of the medium and lower lobe of the right lung was performed. 18 months after the surgery she is still saturated $>90 \%$.

Conclusion: Large pulmonary arteriovenous fistulas sometimes can not be successfully closed using interventional diagnostics procedure and should be treated with pulmectomy of the pulmonary lobes affected by the fistulas.

7339

Clinical Implications of B-type Natriuretic Peptide in Patients late after the atrium-pulmonary artery connection

Eriko Shimada, Tokyo Women's Medical University, Japan
Kei Inai, Tokyo Women's Medical University, Japan

Toshio Nakanishi, Tokyo Women's Medical University, Japan

Objectives: Fontan type operation is characterized by high central venous pressure and reduced preload for systemic ventricle. It is well known that B-type natriuretic peptide (BNP) has prognostic significance in chronic heart failure. However, it is not well established that clinical implications of BNP levels in the Fontan patients. The aim of this study is to elucidate the clinical implication of BNP in the patients after the Fontan type operation, especially in atrium-pulmonary artery connection (APC).

Methods and Results: BNP levels, in addition to clinical and hemodynamic variables, were evaluated in 112 patients late after Fontan operation. One hundred one patients underwent APC and 13 patients total cavopulmonary connection (TCPC). BNP levels of APC patients were higher than that of TCPC patients (105 vs. $13 \mathrm{pg} / \mathrm{ml}, \mathrm{p}<0.0001)$. In APC patients, BNP levels of patients in NYHA I were lower than that of patients in NYHA II and III $(p=0.03)$. The patients with arrhythmia had higher value of BNP than patients without arrhythmia $(168 \mathrm{vs} 103 \mathrm{pg} / \mathrm{ml}$, $\mathrm{p}=0.02)$. BNP levels were positively correlated with right atrial volume which obtained by cardiac catheterization $(\mathrm{p}<0.0001)$. Kaplan-Meier cardiac event free rate was significantly higher in patients with $\mathrm{BNP}>200 \mathrm{pg} / \mathrm{mL}$ compared with patients $<200 \mathrm{pg} / \mathrm{mL}(\mathrm{p}=0.001)$.

Conclusions: BNP may be derived from the right atrium, in APC patients, and therefore it may be increased in patients with dilated right atrium. BNP levels can be used to predict cardiac event in patients after APC.

\section{0}

Ventricular Function Following Ventricular Septal Defect (VSD) Closure

Savitri Shrivastava, Escorts Heart Institute and Research center,

New Delhi, India

Neeraj Awasthy, Escorts Heart Institute and Research center,

New Delhi, India

Muneesh Tomar, Escorts Heart Institute and Research center,

New Delhi, India

$S$ Radhakrishnan, Escorts Heart Institute and Research center,

New Delhi, India

Parvati Iyer, Escorts Heart Institute and Research center,

New Delhi, India

Sunil Kaushal, Escorts Heart Institute and Research center,

New Delhi, India

K S Iyer, Escorts Heart Institute and Research center, New Delhi, India

Background: This study investigated changes in left ventricular (LV) systolic function after VSD closure and attempts to elucidate the time period of recovery of LV function and factors affecting the same.

Methods: Echocardiograms were recorded before \& after operative repair of VSD over a period of 2 years (January 2007-November 2008). Systolic - ejection fraction and fractional shortening was used for follow up. Data was analyzed with respect to demographic features, VSD profile, degree of shunt (based on clinical and echo criteria), and associated lesions and Effect of surgery was analyzed with respect to duration and cross clamp time. Post operative echocardiogram was performed at 7th, 30,90 and 180 postoperative day with uniform institutional medication protocol. Statistical analysis was done using SPSS (version 17).

Results: 357 cases (32\% of the total operated cases) underwent VSD closure. Ejection fraction, \& fractional shortening decreased 
after VSD repair $(\mathrm{p}<0.05)$ in $101(28 \%)$. The VSDs were perimembranous, doubly committed, muscular and multiple present in $68 \%, 10 \%, 12 \%$ respectively. $72.2 \%$ had large, $11 \%$ moderate shunt. Ventricular function recovered at 90th postoperative day in $71 \%$ cases. Flattened septal motion persisted upto 6 months in 66\%. Those having persistent LV dysfunction $(\mathrm{n}=12)$ had prolonged bypass time $(\mathrm{p}<0.05)$, and associated shunt lesions. $(\mathrm{p}<0.05)$.

Conclusions: Ventricular dysfunctions after VSD closure recovers in most patients over 6 months. Additional studies are needed to define changes in after load, contractility as well as diastolic dysfunction and systolic mechanics.

7341

Predictors of Cardiac arrhythmias in early postoperative period after surgery for congenital heart disease

Savitri Shrivastava, Escorts Heart Institute and Research center,

New Delhi, India

Neeraj Awasthy, Escorts Heart Institute and Research center,

New Delhi, India

Kartikeya Bhargava, Escorts Heart Institute and Research center,

New Delhi, India

Munesh Tomar, Escorts Heart Institute and Research center, New Delhi, India

S Radhakrishnan, Escorts Heart Institute and Research center, New

Delhi, India

Parvati U Iyer, Escorts Heart Institute and Research center, New Delhi, India

K S Iyer, Escorts Heart Institute and Research center, New Delhi, India

Objective: To analyze the incidence and type of cardiac arrhythmias (CA) and find the predictors of their occurrence in early post-operative period after surgery for congenital heart diseases (CHD).

Methods: Occurrence of early postoperative CA was prospectively analyzed in 511 consecutive patients undergoing surgery for various CHD between November 2007 \& August 2008. Various risk factors including demographic (age, sex), operative [type of surgery, cardiopulmonary bypass time(CPBT), aortic crossclamp time] and postoperative metabolic parameters (electrolyte levels, blood gases, inotropic support) were assessed. Statistical analysis was done using the SPSS 17.0 software.

Results: CA occurred in 35\% $(\mathrm{n}=178 / 511)$ patients and were more common after Fontan surgery, complex CHD surgery and VSD closure. Most common types of CA were premature ventricular ectopics (VPC s), 45\%, junctional ectopic tachycardia (JET) 26\%, atrial tachyarrythmias (atrial tachycardia/flutter) $18 \%$, atrioventricular block (9\%), ventricular tachyarrhythmias $(\mathrm{VF} / \mathrm{VT}), 1.5 \%$. Predictors of CA on univariate analysis were lower age, late presenters, longer CPBT ( $>75$ minutes), aortic crossclamp time (>50 minutes), hypokalemia and hypercarbia .In the multivariate logistic regression analysis, aortic cross clamp time and hypercarbia were found to be independent predictors of occurrence of CA.

Conclusion: Longer aortic crossclamp time during surgery and hypercarbia in the post operative period were found to be independent predictors of occurrence of CA in early postoperative period after CHD surgery and should be avoided.

\section{2}

The postoperative left ventricular obstruction in aortic arch coarctation or interrution and venstricular septal defect in infants

Jinyoung Song, Bucheon general hospital, Korea
Sujin Kim, Bucheon general hospital, Korea

Eunyoung Choi, Bucheon general hospital, Korea

Soik Jang, Bucheon general hospital, Korea

Wooseup Shim, Bucheon general hospital, Korea

Changha Lee, Bucheon general hospital, Korea

Chul Lee, Bucheon general hospital, Korea

Heungguk Lim, Bucheon general hospital, Korea

Introduction: The aortic coarctation or interruption with ventricular septal defect are usually associated with various extents of left ventricular outflow tract (LVOT) obstructions and we encountered significant LVOTO lesions after the operations. So we followed those patients after the operation and evaluated the left ventricular outflow tract.

Method: Total 73 patients was operated and followed after the operation of aortic arch reconstruction and closure of ventricular septal defect or pulmonary arterial banding. All the patients were diagnosed and operated before 1 year old age. The preoperative evaluation was done by $2 \mathrm{D}$ echocardiogram and left ventricular outflow tract stenosis was defined as a pressure difference over $15 \mathrm{mmHg}$.

Results: There were no pressure differences through LVOT before the operation in all the patients. But the postoperative obstruictive lesions in LVOT were found in 13 patients. The pressure differences through LVOT were from $15 \mathrm{mmg}$ to $90 \mathrm{mmHg}(41.2 \pm 25.0 \mathrm{mmHg})$. The levels of the obstruction were valvar area in 2 patients and subvalvar area in 11 patients including one fibrotic membrane. The postoperative presence of obstruction was not related neither with the follow up period $(p=0.273)$ nor with the preoperative LVOT dimension by echocardiogram statistically $(\mathrm{p}=0.538)$. Although the statistical significance was not exist, the aortic interruption was related with postoperative LVOT obstruction more closely than the aortic coarctation $(\mathrm{p}=0.051)$.

Conclusion: The LVOT obstruction after the operation of the coarctation or interruption of aorta with ventricular septal defect in infants is possible with no predictable preoperative data so that the close observation should be mandatory especially in aortic interruption.

\section{3}

Marfan síndrome in 106 children. Prevalence of clinical manifestations, cardiovascular impact and survival

M Elena Soto, Instituto Nacional de Cardiologia Ignacio Chavez, Mexico Nilda Espinola-Zavaleta, Instituto Nacional de Cardiologia Ignacio Chavez, Mexico

Rafael Bojalil, Instituto Nacional de Cardiologia Ignacio Chavez, Mexico Luis M Amezcua- Guerra, Instituto Nacional de Cardiologia Ignacio Chavez, Mexico

Luis E Enriquez-Rodriguez, Instituto Nacional de Cardiologia Ignacio Chavez, Mexico

Emilia J Patino-Bahena, Instituto Nacional de Cardiologia Ignacio

Chavez, Mexico

Pedro A Reyes, Instituto Nacional de Cardiologia Ignacio Chavez, Mexico

The Marfan syndrome (MS) is a heritable autosomal dominant disorder of connective tissue, due to the fibrillin gene mutations, essential component of elastic fibers. Cardiovascular, skeletal and ocular anomalies are the most common clinical manifestations. There is extensive clinical overlap in this syndrome because its association with 50 types of mutations in TGFBR1 and TGFBR 2 on 15 chromosomes. Retrospective study of clinical evaluation, surgical treatment and survival of 106 children, from 1993-2008 years. 
Material and methods: 106 children with MS according to the Ghent Criteria were studied. Initial symptoms echocardiogram, medical and surgical treatment and survival were investigated.

Results: $52 \%$ female with mean age of $8.5 \pm 5$ years. Inicial manifestations: blurred vision 31\%, families with MS 12\%, heart murmur $8 \%$, dyspnea $7 \%$, feet malformations $6 \%$, thoracic pain $5 \%$, aneurysms $5 \%$, pre-syncope $4 \%$, aortic abnormalities $3 \%$, hernia $3 \%$, arrhythmia $1 \%$, scoliosis $1 \%$. Major musculoskeletal criteria $88 \%$, minor $76 \%$, major ocular criteria $70 \%$, minor $11 \%$, major cardiovascular criteria 59\%, minor $68 \%$, dural ectasia $53 \%$. Only minor criteria: respiratory $9 \%$, skin $69 \%$. The one year survival with cardiovascular complications and surgical treatment is of $80 \%$, which decreased to $50 \%$ in 5 years with medical treatment; when the patients go to surgery the survival increased to 20 years in $20 \%$ of cases. Only a $5 \%$ of patients without surgery reached 15 years survival.

Conclusions: The survival is not compromised by the musculoskeletal manifestations. There are precise surgical indications in cardiovascular complications, which performed in time, decreased the morbidity and mortality.

\section{4}

Prevalence and lethal arrhythmic risk of short QT interval in school health examination

Hiroshi Suzuki, Division of Pediatrics, Niigata university Graduate school of Medical and Dental Science, Japan

Fujito Numano, Division of Pediatrics, Niigata university Graduate school of Medical and Dental Science, Japan

Yoshinori Haniu, Division of Pediatrics, Niigata university Graduate school of Medical and Dental Science, Japan

Satoshi Hasegawa, Division of Pediatrics, Niigata university Graduate school of Medical and Dental Science, Japan

Naoki Boku, Division of Pediatrics, Niigata university Graduate school of Medical and Dental Science, Japan

Satoshi Hoshina, Division of Pediatrics, Niigata university Graduate school of Medical and Dental Science, Japan

Makoto Uchiyama, Division of Pediatrics, Niigata Graduate School of Medical and Dental Sciences., Japan

Background: Short QT (SQT) syndrome is a novel inherited arrhythmia characterized by a persistently SQT interval in the ECG. Some reports have claimed that a SQT interval in the ECG is associated with an increased risk of dying suddenly. However, little is known of the prevalence of SQT interval and its clinical significance in the general population. Especially, there is no report on this issue in young population. We sought to assess the prevalence and lethal arrhythmic risk of SQT interval in a young general population.

Methods \& Results: QT intervals were measured from the ECGs of 29,531 children (15,143 males and 15,144 females) who were in 1st grade (6-7 years old) or 7th grade (12-13 years old) in a school health examination in Niigata city, Japan. Bazett's method was used to correct the QT interval for heart rate (QTc). Cutoff values for SQT intervals were defined as $350 \mathrm{~ms}$ (1st screening) and $330 \mathrm{~ms}$ (further screening). The prevalence of QT interval $<350 \mathrm{~ms}$ was $0.38 \%$ (73 males and 38 females), and the prevalence of QTc $<330 \mathrm{~ms}$ was $0.017 \%$ (four males and one female). In addition, echocardiography, Holter ECG, treadmill test and face immersion test were performed in children with a QTc $<330 \mathrm{~ms}$. There were no abnormal findings but persist SQT interval among children with a QTc $<330 \mathrm{~ms}$.

Conclusion: SQT interval is rare in children, similarly to adults. Children with SQT interval had no abnormal finding but persist SQT interval, suggesting the risk for lethal arrhythmia may be low in children with SQT.

\section{5}

High $R$ '/ $R$ in V1-2 leads represents right ventricular pressure and volume overload in ASD

Motoki Takamuro, Department of Cardiology, Hokkaido Medical

Center for Child Health and Rehabilitation, Japan

Keiji Haseyama, Departement of Pediatrics, Sapporo Medical University, Japan

King-ya Hatakeyama, Department of Cardiology, Hokkaido Medical Center for Child Health and Rehabilitation, Japan

Norihisa Horita, Departement of Pediatrics, Sapporo Medical University, Japan

Naomi Abe, Department of Cardiology, Hokkaido Medical Center for Child Health and Rehabilitation, Japan

Masato Yokozawa, Department of Cardiology, Hokkaido Medical Center for Child Health and Rehabilitation, Japan

Background: In atrial septal defect (ASD), electrocardiography (ECG) shows incomplete right bundle branch block pattern with high R' voltage in lead V1.[Purpose] To evaluate relationship between R' voltage in right precordial leads and volume/pressure overload in ASD. [Methods] Retrospective study. In catheterization cases, $\mathrm{R}$ and $R^{\prime}$ voltage and R'/R in lead V1 to 3 were compared to Qp/Qs, mean pulmonary artery pressure, \%RVEDV, RVEDV/LVEDV, hANP and BNP. With $\mathrm{p}$ value $<0.05$ as significance.

Patients: 33 cases with ASD. Age at catheterization was 1t o 20 (Median 7) years old. Male 15, Female 18.

Results: Mean and standard deviation of each parameters were showed as follows; Qp/Qs $2.4 \pm 0.6, \mathrm{mPAP} 17 \pm 6.9 \mathrm{mmHg}$, \%RVEDV $162 \pm 33 \%$, hANP $43 \pm 38 \mathrm{ng} / \mathrm{ml}$, BNP $28 \pm 23 \mathrm{ng} /$ $\mathrm{ml}$. R'/R were $3.8 \pm 2.2$ in $\mathrm{V} 1,1.4 \pm 1.3$ in $\mathrm{V} 2,0.5 \pm 1.0$ in V3. In V3 lead, because over the half of cases have no R', excluded V3 data. In both of V1 and V2, R'/R was correlated to mPAP, \%RVEDV, RVEDV/LVEDV, hANP, BNP. However simple R' voltage has no correlation to all parameters.

Discussion: In ASD, although R' voltage in right precordial leads is not reflected to right heart overload, R'/R may predict pressure and/or volume overload of right ventricle. Correlation between $\mathrm{R} / \mathrm{R}^{\prime}$ and pressure overload is better in V1 than V2. Even in statistically not significant, Qp/Qs has better R2 and p-value in V2 rather than V1. When right heart overload in ASD is discussed with ECG, V2 lead should be added to V1 in point of view.

7346

The incidence of Aortic Stenosis and Subaortic Stenosis in patients with Coarctation of the Aorta in a Pediatric Cardiology Service

Carolina Talini, Brazil

Adriana C Bresolin, Universidade Estadual do Oeste do Paraná

(UNIOESTE), Brazil

Camille B Pompeu, Universidade Estadual do Oeste do Paraná, Brazil

Objective: Investigate the incidence and the diagnoses time of aortic valve stenosis (AVS) and subaortic stenosis (SUBAS) in combination with aortic coarctation (COA). Methods: A retrospective review of 36 patients with COA who were evaluated between 2003 and 2008 in a pediatric cardiology service.

Results: At the time the COA was diagnosed, median age was 25 months (range 6 days to 10 years 3 months) among these patients $61,1 \%$ were male. The median age, when the AVS was diagnosed, was 21 months (range 2 days to 11 years 3 months) and for SUBAS the age was 41 months (range 85 days to 8 years). Within the 13 patients who presented AVS, 5 of them were diagnosed with COA at the same moment, 4 others had AVS identified before COA and 4 after the first evaluation. For the 
SUBAS, 7 patients were diagnosed after the COA and just 1 at the same time. Twenty seven pediatric patients underwent COA surgery, $14,8 \%$ were considered neonates, $29,6 \%$ infants and $55,6 \%$ children. There was no death. Balloon angioplasty was the treatment in 2 patients with native COA, one of them had to undergo a surgery later. Three patients needed the angioplasty due to recoarctation.

Conclusion: In the patients with COA, the incidence of associated AVS was $36,1 \%$ and the SUBAS was $22,2 \%$, presenting itself higher than dades found in literature. The early diagnoses was made in 9 of the patients with AVS $(69,2 \%)$. Therefore the SUBAS diagnose was late.

\section{7}

Anomalous origin of the left coronary artery from pulmonary artery: Ten years experiences in diagnosis and surgical repair

Xiao Tan, Department of Pediatric Cardiology, Shanghai Children's

Medical Center, Shanghai Jiaotong University, Shanghai, China

Kun Sun, Department of Pediatric Cardiology, Shanghai Children's

Medical Center, China

Objective: To review our experience in ten years with the diagnosis of children with anomalous origin of the left coronary artery from pulmonary artery (ALCAPA) and to evaluate surgical results and short-middle term outcomes.

Methods: 35 cases of ALCAPA hospitalized between 1998 and 2008 in our center were enrolled. Electrocardiographic and echocardiographic features were compared between the infant group $(<1 \mathrm{Y}, \mathrm{n}=18)$ and the older children group $(>1 \mathrm{Y}$, $\mathrm{n}=17) .34$ patients were underwent surgical repair and shortmiddle term outcomes were evaluated.

Results: The diagnosis of ALCAPA was obtained in 27 patients through echocardiography with diagnostical rate of $77 \%$. The presence of abnormal Q wave in lead aVL (26/35), anterior lateral papillary echogenic $(28 / 35)$ and coronary collateral vessel signals $(25 / 35)$ were high in both groups $(\mathrm{P}>0.05)$. The presence of RCA dilation was lower in the infant group(7) than in the older children (16) group $(\mathrm{P}<0.05)$. Cardiac dysfunction was assessed in 16 infants but none in older children. Six collateral anastomosis sites were detected in 23 patients through angiography and the collateral flow pattern was age-dependent. Dual coronary repair was performed in 32 patients and LCA ligation in 2.LV function and mitral regurgitation improved in 22 patients during short-middle term follow-up.

Conclusion: Diagnosis can be established by combination of echocardiography and electrocardioagraphy. Abnormal Q wave in lead aVL, anterior lateral papillary echogenic and collateral vessel signals were the most valuable features to predict ALCAPA. Survival rate of surgical repair was satisfactory. Dual coronary repair was preferred with good short-middle term outcomes.

\section{8}

Platelet Vascular Endothelial Growth Factor Level Is Strongly Correlated with Coronary Artery Abnormalities in Kawasaki Syndrome

Kentaro Ueno, Department of Pediatrics, Kagoshima University Graduate School of Medical and Dental Sciences, Japan Yuichi Nomura, Department of Pediatrics, Kagoshima University Graduate School of Medical and Dental Sciences, Japan Kiminori Masuda, Department of Pediatrics, Kagoshima Medical Association Hospital, Japan

Yasuko Morita, Department of Pediatrics, Kagoshima Medical Association Hospital, Japan
Taisuke Eguchi, Department of Pediatrics, Kagoshima University Graduate School of Medical and Dental Sciences, Japan

Teruto Hashiguchi, Laboratory and Vascular Medicine, Kagoshima University Graduate School of Medical and Dental Sciences, Japan Ikuro Maruyama, Laboratory and Vascular Medicine, Kagoshima University Graduate School of Medical and Dental Sciences, Japan Yoshifumi Kawano, Department of Pediatrics, Kagoshima University Graduate School of Medical and Dental Sciences, Japan

Objective: Vascular endothelial growth factor (VEGF) is an endothelial cell specific mitogen that induces major angiogenesis and microvascular permeability. Since activated platelets abundantly release VEGF in the early stage of vascular inflammation, we evaluated the clinical relevance of VEGF from a single platelet (platelet VEGF) and ascertain any correlation with coronary artery abnormalities (CAA) in Kawasaki syndrome (KS).

Methods: Clinical features and results of blood examinations before IVIG treatment were compared $80 \mathrm{KS}$ patients (69 IVIG responders, 11 IVIG non-responders) and 26 febrile patients with infections. We evaluated serum VEGF corrected using hematocrit and platelet counts.

Results: KS patients had significantly higher levels of platelet VEGF $(\times 10-8 \mathrm{pg})(18.8 \pm 11.1)$ than did patients with infectious diseases $(8.1 \pm 3.0) \quad(\mathrm{p}<0.0001)$, although there was no significant difference in WBC or CRP. Platelet VEGF were higher in non-responders $(25.2 \pm 17.6)$ than those in responders $(17.8 \pm 8.1)$ but this difference was not statistically significant. Platelet VEGF was significantly higher in patients who showed higher maximum CAA z-score $(>3)(27.5 \pm 13.6)$ than in those who showed lower $z$-score $(<3)(16.1 \pm 6.9)(\mathrm{p}<0.0001)$, while there were no significant differences in serum VEGF levels $(983.5 \pm 601.8$, and 902.9 \pm 347.8 , respectively, $p=0.29)$. Platelet VEGF was significantly correlated with $\mathrm{z}$-score $(\mathrm{R}=$ 0.524, $\mathrm{p}<0.0001)$, while serum VEGF level was not $(\mathrm{R}=0.21)$. Conclusions: Platelet VEGF level before IVIG treatment was strongly correlated with $\mathrm{z}$-score and might be a useful predictor for CAA in KS patients.

\section{9}

What determines nutritional recovery in malnourished children undergoing correction of congenital heart defects? Balu Vaidyanathan, Amrita Institute of Medical Sciences, Elamakkara P.O, Kochi, Kerala, India, India

$R$ Reshma, Amrita Institute of Medical Sciences, Elamakkara P.O, Kochi, Kerala, India, India

A S Deepa, Amrita Institute of Medical Sciences, Elamakkara P.O, Kochi, Kerala, India, India

KR Sundaram, Amrita Institute of Medical Sciences, Elamakkara P.O, Kochi, Kerala, India, India

Krishna Kumar R, Amrita Institute of Medical Sciences, Elamakkara P.O, Kochi, Kerala, India, India

Objective: To identify determinants of malnutrition in a consecutively selected cohort of children undergoing corrective surgery or catheter intervention for congenital heart disease (CHD) in a center in the developing world and examine the impact of corrective intervention on nutritional recovery.

Methods: Patients with CHD admitted for corrective intervention were evaluated for nutritional status before and 2 years after the procedure. Anthropometric measurements were obtained at presentation and at 6-month intervals and Z-scores calculated. Malnutrition was defined as weight-for-age, height-for-age and weight/height $\mathrm{Z}$-score $<-2$. Determinants of malnutrition were entered into a multivariate logistic regression analysis model. 
Results: 476 consecutive children (mean age: $15.2+16.2$ months) undergoing corrective intervention were included. Weight Z-score $<-2$ was found in $59 \% ; 26.3 \%$ had height $\mathrm{Z}$-score $<-2$ at presentation. Predictors of malnutrition at presentation included congestive heart failure (CHF), age at correction, birth weight and parental height. On follow-up (425 patients; duration $20.63+13.1$ months), there was significant improvement in the Z-scores for weight $(-2.19+1.16$ vs. $-1.42+1.03 ; \mathrm{p}<0.001)$ and weight/height $(-2.09+1.3$ vs. $-1.15+1.25 ; \mathrm{p}<0.001)$. Height $\mathrm{Z}$-scores remained unchanged (Figure-1). Malnutrition persisted in 116 (27.3\%) on follow-up and was predicted by birth weight, nutritional status at presentation and parental height. Cardiac diagnosis, dietary intake and socio-economic factors did not impact malnutrition. Conclusions: Significant malnutrition is common in patients with $\mathrm{CHD}$ and is reversible once corrective intervention is performed. Persistent malnutrition after corrective intervention is predicted by nutritional status at presentation, birth weight and parental anthropometry.

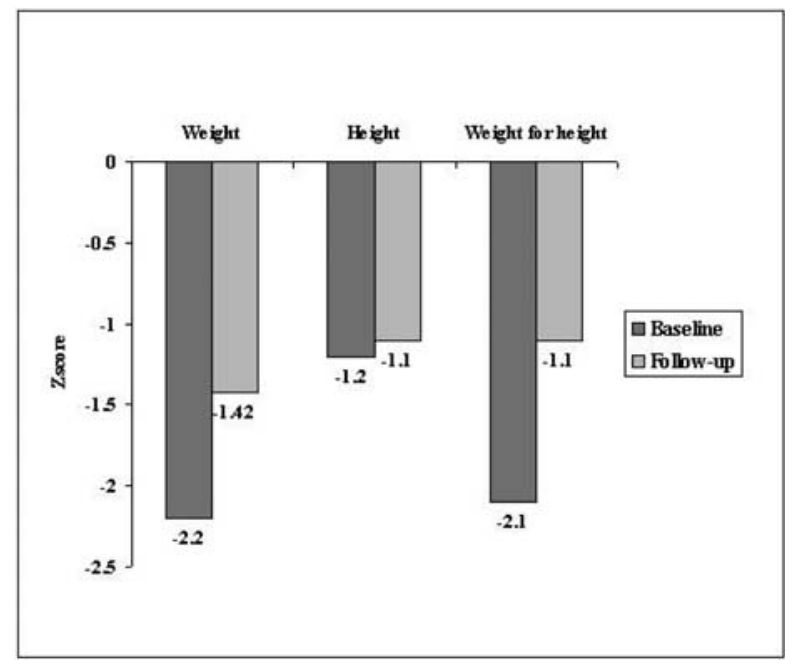

\section{0}

Incidence and etiological evaluation of murmurs in full term neonates

Hariganesh Rajgopal, Gandhi Medical College, Bhopal, India Yogesh Varma, Gandhi Medical College, Bhopal, India

Jyotsna Shrivastava, Gandhi Medical College, Bhopal, India

Rashmi Dwivedi, Gandhi Medical College, Bhopal, India

BalbirSingh Yadav, Gandhi Medical College, Bhopal, India

Objectives: To study the incidence, etiological basis and natural history of murmurs in full term healthy neonates

Methodology: The present study included 3348 neonates. It was conducted in the postnatal ward starting Nov 2007 till September 2008. Inclusion criteria: All full term neonates with a murmur detected on routine examination within the first week of life. Exclusion criteria: neonates ( $<36$ weeks), sick neonates with comorbid conditions.

Study design: Prospective/cohort study: All newborns were screened for the murmur by Residents at birth and later.A detailed history and anthropometric assessment was done. ECG, Chest X-ray, SPO2, color doppler by a neonatal probe of $7 \mathrm{MHz}$ on Acuson color doppler machine was done by a Cardiologist initially and repeated at 2,6 and 12 weeks. The cases were classified as Normal, physiological variants, structural heart diseases and assessed for single or multiple lesions, severity and progression.
Results: Out of 3348 newborn murmurs were detected in 46 (1.3\%). The incidence was $13.73 / 1000$. Among those with the murmur 20 (43\%) had structural heart diseases either alone or in combination, VSD (58\%), ASD (25\%), Pulmonary stenosis (12.5\%), TOF (4.1\%), Mitral atresia (4.1\%). Physiologial variants showed PFO (38\%), PDA (34\%), TR (26\%). X-ray chest had sensitivity of $45 \%$, specificity $90 \%$ and negative predictive value of $69 \%$. Only 3 with proven CHD had abnormal ECG. No statistical significance was noted between the day of appearance, location or the intensity of the murmur in relation to the structural cardiac lesion $(p>.05)$. Growth monitoring showed a statistically significant fall in weight among those with structural heart lesion $(\mathrm{p}<.01)$. Consanguinity did not affect the incidence of CHD.

Conclusion: Murmurs are a clue to underlying cardiac lesions. However, confirmation is based on various supportive investigations.

\section{1}

Role of Creatine Kinase(CK) and Creatine KinaseMuscle brain fraction(CKMB) in retrospective diagnosis of perinatal asphyxia and their correlation with cardiovascular dysfunction

Yogesh Varma, Gandhi Medical College, Bhopal, India

Rashmi Dwivedi, Gandhi Medical College, Bhopal, India

Sushant Shridhar, Gandhi Medical College, Bhopal, India

Gurmeet Singh, Gandhi Medical College, Bhopal, India

Objectives: Role of Creatine Kinase (CK) and Creatine KinaseMuscle brain fraction (CKMB) in retrospective diagnosis of perinatal asphyxia and their correlation with cardiovascular dysfunction.

Methods: Cases consisted of 40 asphyxiated full term neonates $(>2.0 \mathrm{~kg})$ [1 min Apgar score $<7$, requiring assisted ventilation with abnormal neurological examination within first $24 \mathrm{hrs}$ of birth], subgrouped for severity of asphyxia by levene staging for HIE. [Mild moderate and severe asphyxia as group I II and III respectively] Controls were unasphyxiated healthy neonates. Serum CK and CKMB were determined at $24 \mathrm{hrs}$ of birth.

Results: Mean $\mathrm{Ck}$ and $\mathrm{CKMB}$ were significantly higher in controls vs group 2 (826 vs 2949 and 26.2 vs 89.6 respectively, $\mathrm{P}<.001)$ and group 3 (826 vs 3475 and 26.2 vs 367.2 respectively $\mathrm{P}<.001)$ but not in controls vs group 1 (1107 vs $826 p=0.26$ and 35.8 vs $24.4 p=.06$ ). Overall CK was more sensitive than CKMB for retrospective diagnosis of asphyxia (72.5\% vs $47.5 \%$ ) but less specific (60\% vs $100 \%)$. Cardiovascular dysfunction on echocardiography was seen only in group 2 and group 3 cases $[15 \%$ and $71.4 \%$ respectively]. Presence of cardiovascular dysfunction significantly increased mean CKMB (355 vs $62 \mathrm{P}<.001$ ) but not mean CK levels (3108 vs 2276 $\mathrm{p}=0.25)$.

Conclusion: Both enzymes are unable to detect mild asphyxia and have low sensitivity for retrospective diagnosis of asphyxia though CKMB is highly specific. CKMB correlates well with both the severity of asphyxia and cardiovascular dysfunction and can be used as a predictor of cardiovascular dysfunction in HIE.

\section{2}

\section{Patent Foramen Ovale- Is there need for Follow-up?}

Juan Villafane, United States

A Patent Foramen Ovale (PFO) is found in over $75 \%$ of neonates and about $25-35 \%$ of normal adult hearts. A PFO may be associated with migraine headaches, stroke, myocardial infarction, 
orthodeoxia platypnea syndrome, decompression sickness and high-altitude pulmonary hypertension or edema. To date, there still exists controversy about which PFOs need closure. A much more common dilemma faced by the cardiologist is if there is a need for follow-up in all or certain patients with a PFO.

Methods: In September 2008, a survey was taken via PediHeartNet to find out the outpatient management of children diagnosed with a PFO.

Results: Out of 68 responses, we found that $40 \%$ of physicians do not schedule any follow-up for children with an isolated PFO. Only $6 \%$ of respondents would follow all children with any size PFO. About half of the physicians would recommend follow-up if unclear about the interatrial communication being a PFO or a small Atrial Septal Defect. The most chosen follow-up interval was one to two years. Of the group of "follow-up" responders, about $2 / 3$ of them would reevaluate children with a prominent right ventricle, atrial septal aneurysm, atypical atrial shunt or a PFO larger than 3 (to 7) mm. Almost $1 / 2$ of the "follow-up" responders stated that they wanted to confirm closure of the PFO as one of the reasons for a follow-up ehocardiogram.

Conclusions: A recent survey in PediHeartNet shows that $40 \%$ of cardiologists do not schedule any follow-up for children with isolated PFO. Only 6\% of respondents would follow all children.

\section{3}

An Overview of Heart Rhythm Disturbances in Children with Palpitations or Syncope

Lidija Vucajnk, General Hospital Celje, Slovenia

Objective: To asses the heart rhythm disturbances (HRD) in children with palpitations or syncope.

Methods: 504 children aged from 1 to 18 years with complains of palpitations or syncope were investigated by 24 hour ECGHolter monitoring. There were 235 boys (47\%) and 269 girls (53\%). Only children with normal echocardiography, somatic and neurological status were included.

Results: Among 504 children HRD were seen in 240 of them (48\%): in $49 \%$ of boys and in $46 \%$ of girls. Life threatening HRD was found in 1 child $(0,4 \%)$ : in 16 years old girl with LQT syndrome (QTc $560 \mathrm{~ms}$ ). Intermitent supraventricular tachycardia was found in 5 children $(2,1 \%)$; WPW in 4 children $(1,7 \%)$; permanent or intermitent AV block I with occasional Wenckenbach periodicity during the sleep in 14 children $(5,8 \%)$. Most often HRD were ectopic heart beats: in 123 children $(51,3 \%)$ supraventricular (SVE) and in $86(35,8 \%)$ ventricular (VE). In 6 children there was intermitent sinus coronarius rhythm (2,5\%). One child $(0,4 \%)$ had 6,5 seconds lasting sinus pause during tilt test. Most often HRD in girls were SVE $(45,2 \%)$ and $\operatorname{VE}(43,5 \%)$ and in boys SVE $(59,5 \%)$. HRD were most often seen in girls aged 15 and 16 years and in boys aged 16. During Holter monitoring only one child had altered consciousness (on tilt test) and $16 \%$ of children complained of palpitations.

Conclusion: HRD are seen in approximately half of the children with palpitations or syncope, but life threatenig or tachicardic HRD are rare.

\section{4}

\section{Our approach to alleviate cyanosis after the Fontan} procedure

Mamie Watanabe, Pediatric Cardiology, Kyushu Koseinenkin Hospital, Japan
Yoshie Ochiai, Cardiovascular Surgery, Kyushu Koseinenkin Hospital, Japan

Takuro Ohno, Pediatric Cardiology, Kyushu Koseinenkin Hospital, Japan

Tetuji Yuge, Pediatric Cardiology, Kyushu Koseinenkin Hospital, Japan Sayuri Kishimoto, Pediatric Cardiology, Kyushu Koseinenkin Hospital, Japan

Akira Sese, Cardiovascular Surgery, Kyushu Koseinenkin Hospital, Japan

Yutaka Imoto, Cardiovascular Surgery, Kyushu Koseinenkin Hospital, Japan

Kunitaka Joo, Pediatric Cardiology, Kyushu Koseinenkin Hospital, Japan

Objective: To improve functional status following the Fontan procedure, it is important to alleviate cyanosis. Factors contributing to cyanosis following the Fontan procedure are systemic venous-to-pulmonary venous collaterals, pulmonary arteriovenous malformations (PAVMs), or shunting through residual intra-atrial communication. The current study was undertaken to review our institutional experience to alleviate cyanosis after the Fontan procedure.

Patients and Method: Records of 93 patients who survived the Fontan procedure between February 1993 and June 2008 at our institution were reviewed. Our combined approach is to perform contrast echocardiography, pulmonary perfusion scan, multidetector-row computed tomography, and cardiac catheterization. Results: We performed coil embolization for systemic venous-topulmonary venous collaterals or PAVMs in 19 patients. After coil embolization, arterial oxygen saturation as measured using pulse oximetry (SpO2) was improved from 92.9\% to 95.6\%. For surgical reinterventions, repair of lateral tunnel baffle leak was performed in two patients. The $\mathrm{SpO} 2$ was dramatically improved from $87.0 \%$ to $94.0 \%$ after leak repair. Redirection of the hepatic venous flow was performed in two polysplenia patients to attain the balanced hepatic flow to both lungs. Immediately after surgery, $\mathrm{SpO} 2$ was improved from $81.0 \%$ to $93.5 \%$. One of the patients kept high $\mathrm{SpO} 2(96.0 \%)$ and showed regression of the PAVMs 5.7 years after redirection. Recent $\mathrm{SpO} 2$ was $94.8 \pm 3.7 \%$ in 93 patients during the follow-up period (8.5 \pm 4.3 years).

Conclusion: Interventions following the Fontan procedure to alleviate cyanosis can be safely performed. We should continue our efforts to lessen cyanosis and improve the quality of life for the patients.

\section{5}

Quality of life in children with mitral valve prolapse Bozena Werner, Department of Pediatric Cardiology and General Pediatrics, Medical University of Warsaw, Poland, Poland Izabela Janiec, Department of Pediatric Cardiology and General Pediatrics, Medical University of Warsaw, Poland, Poland

Jolanta Sieminska, Chair and Department of Experimental and Clinical Physiology, Medical University of Warsaw, Poland, Poland

Objective: The goal of the study was to assess the health related quality of life (HRQoL) in children with mitral valve prolapse (MVP).

Methods: The prospective study involved 67 patients of Department of Pediatric Cardiology with MVP, 47 girls and 20 boys, aged $8-18$ years (average 13,9 $\pm 2,61$ years). Control group consisted of 31 healthy children, matched according to age and sex. We used Polish version of Kidscreen-27 to access health related quality of life. Kidsceen-27 is an international selfreported questionnaire for children and consists of 27 items 
which are used to assess HRQoL across 5 dimensions: physical well-being (maximal number of points to achieve 25), psychological well-being ( $\max 35$ points), autonomy and parent relation (max 35 points), peers and social support (max 20 points), school environment ( $\max 20$ points).

Results: The results are presented in the table. The statistically significant difference was found only for one dimension of HRQoL - physical well-being (explores the level of child's physical activity, energy and fitness).

Conclusion: Children with mitral valve prolapse demonstrate similar quality of life compared to healthy children, except of physical well being which is significantly lower.

\begin{tabular}{lcccccc}
\hline & \multicolumn{2}{c}{$\begin{array}{c}\text { Children with MVP } \\
\text { range }\end{array}$} & \multicolumn{2}{c}{ Control group } & pverage \\
range & average & \\
\hline $\begin{array}{l}\text { physical well-being } \\
\text { psychological well- } \\
\text { being }\end{array}$ & $7-25$ & $16,84 \pm 0,52$ & $12-24$ & $19,84 \pm 0,52$ & 0,001 \\
$\begin{array}{c}\text { autonomy and parent } \\
\text { relation }\end{array}$ & $15-35$ & $27,09 \pm 0,57$ & $14-35$ & $28,97 \pm 0,77$ & 0,061 \\
$\begin{array}{c}\text { peers and social } \\
\text { support }\end{array}$ & $4-20$ & $14,22 \pm 0,50$ & $8-20$ & $14,81 \pm 0,66$ & 0,503 \\
& $\begin{array}{c}\text { school } \\
\text { environ- } \\
\text { ment }\end{array}$ & $5-20$ & 14,64 & $11-20$ & $15,35 \pm 0,45$ \\
& & $\pm 0,39$ & & $25,84 \pm 0,85$ & 0,414 \\
& & & & & \\
\hline
\end{tabular}

\section{6}

Arrhythmia in children with mitral valve prolapse Bozena Werner, Department of Pediatric Cardiology and General Pediatrics, Medical University of Warsaw, Poland, Poland Izabela Janiec, Department of Pediatric Cardiology and General Pediatrics, Medical University of Warsaw, Poland, Poland

Objective: The goal of the study was to assess the incidence of arrhythmias in children with mitral valve prolapse (MVP).

Methods: The study involved 67 children with MVP 47 girls, 20 boys, aged $8-18$ years (average 13,9 $\pm 2,61$ years). Control group consisted of 31 healthy children matched according to age and sex. In all children ECG, 24-hour Holter ECG monitoring and treadmill test according to Bruce protocol were performed.

Results: ECG showed arrhythmia in 7 (10,4\%) children with MVP. In 24-hour Holter ECG monitoring arrhythmia was detected in 19 $(28,3 \%)$ patients, including $8(11,9 \%)$ patients with ventricular and $11(16,4 \%)$ with supraventricular arrhytmia. Ventricular arrhythmia was classified according to Lown scale as I grade in 1 case, II grade in 3 patients and IV grade in 4 cases. Complex atrial arrhythmias including atrial tachycardia and atrial couplets were present in 4 $(5,9 \%)$ patients. $8(11,9 \%)$ children with MVP had positive treadmill test result due to arrhythmia: in 6 children because of arrhythmia induced by exercise (with presyncopal stage in one patient), in 2 because of arrhythmia exacerbation. Complete suppression of arrhythmia during treadmill test was observed in $6(8,9 \%)$ children and in one $(1,5 \%)$ child the effort had no influence on arrhythmia. Conclusions: 1/ In children with mitral valve prolapse arrhythmia is detected in about 30\% cases. 2/ Physical activity should be restricted only in patients, with positive result of treadmill test due to new rhythm disturbances or exacerbation of arrhythmia.

\section{7}

The assessment of blood pressure variability in children with vasovagal syncopes using ambulatory blood pressure monitoring

Barbara Wójcicka-Urbanska, Depatment of Pediatric Cardiology and General Pediatrics, Medical University of Warsaw, Poland
Piotr Wieniawski, Depatment of Pediatric Cardiology and General Pediatrics, Medical University of Warsaw, Poland

Jacek Skiendzielewski, Depatment of Pediatric Cardiology and General Pediatrics, Medical University of Warsaw, Poland

Tomasz Florianczyk, Depatment of Pediatric Cardiology and General Pediatrics, Medical University of Warsaw, Poland

Bozena Werner, Depatment of Pediatric Cardiology and General Pediatrics, Medical University of Warsaw, Poland

Objective: The aim of the study was to evaluate blood pressure variability (BPV) using ambulatory blood pressure monitoring (ABPM) in children with vasovagal syncopes (VVS). Methods: In 37 children, 27 girls, 10 boys, aged 9-18 years (mean 14,4 $\pm 2,2$ ) with syncope (23) or presyncope (14) VVS were diagnosed based on anamnesis and noninvasive evaluation of the circulatory system. In 12 children tilt test was performed. Standard deviations of the mean values of the following ABPM parameters were analyzed: systolic (SBP) and diastolic (DBP) blood pressure, diurnal (D-SBP) and nocturnal (N-SBP) systolic blood pressure, diurnal (D-DBP) and nocturnal (N-DBP) diastolic blood pressure, heart rate (HR), day (D-HR) and night (N-HR) heart rate and nocturnal dipp. Results were compared to control group consisted of 22 children matched according height and weight. Statistical analyses were obtained by using t-Student test and Fischer's exact test.

Results: There were no significant differences between study vs control group regarding heart rate: $\mathrm{HR}$ (/min.) 12,83 vs 13,35; D$\operatorname{HR}(/ \min$.) 12,19 vs 12,96 ; N-HR (/min.) 9,32 vs 9,52 . The results of other ABPM parameters are presented in the table. There were no significant differences between parameters of BPV in children with syncopes and presyncopes compared to control group.

Conclusions: PV did not differ significantly in children with VVS compared to control group. Potential parasympathetic hiperactivation in subjects with VVS seems to have no influence on BPV during everyday activity. Research is still in progress.

\begin{tabular}{lccccccc}
\hline & $\begin{array}{c}\text { SBP } \\
(\mathrm{mmHg})\end{array}$ & $\begin{array}{c}\text { DBP } \\
(\mathrm{mmHg})\end{array}$ & $\begin{array}{c}\text { D-SBP } \\
(\mathrm{mmHg})\end{array}$ & $\begin{array}{c}\text { N-SBP } \\
(\mathrm{mmHg})\end{array}$ & $\begin{array}{c}\text { D-DBP } \\
(\mathrm{mmHg})\end{array}$ & $\begin{array}{c}\text { N-DBP } \\
(\mathbf{m m H g})\end{array}$ & $\begin{array}{c}\text { dipp } \\
(\%)\end{array}$ \\
\hline $\begin{array}{c}\text { Study group } \\
(37)\end{array}$ & 10,31 & 10,1 & 9,74 & 6,97 & 9,6 & 6,55 & 12,34 \\
$\begin{array}{c}\text { Control } \\
\text { group }(22)\end{array}$ & 10,52 & 10,1 & 9,7 & 8,2 & 9,56 & 7,3 & 12,69 \\
$\mathrm{p}$ & $\mathrm{ns}$ & $\mathrm{ns}$ & $\mathrm{ns}$ & $\mathrm{ns}$ & $\mathrm{ns}$ & $\mathrm{ns}$ & $\mathrm{ns}$ \\
\hline
\end{tabular}

7358

Population-based Study of Pediatric Sudden Death in Taiwan

Mei-Hwan Wu, Department of Pediatrics, National Taiwan University Hospital, Taiwan

Hui-Chi Chen, Taiwan

Jou-Kou Wang, National Taiwan University Hospital, Taiwan

Hsin-Hui Chiu, Taiwan

Shin-Chung Huang, Taiwan

San-Kuei Huang, Taiwan

Background: The epidemiological data of pediatric sudden death (SD) are scarce and not yet described in Asians.

Methods: Patients who met the criteria listed for SD were identified from Taiwan National Health Insurance databases 2000-2006 that contained health care data from $>95 \%$ of the population.

Results: In this pediatric population (0 to18 years) of 5.44 millions, infant mortality, postneonatal infant mortality and under-five mortality was 6, 2.81 and 8 per 1000 live births, 
respectively. There were 1598 SDs (male/female rate ratio, 1.44). In the population 1-18 years, annual incidence of SD was 3.5 (95\% CI:3.3-3.7), ranging from $0.6(0.3-1.2$, aged 12 years) to 7.1 (6.0-8.5, aged 17 years) per 100,000. Male predominance was noted $(4.3(4.0-4.6)$ versus $3.0(2.7-3.3), p=0.0002)$. In infants, the incidence was $0.37 / 1,000$ live births (male/female rate ratio, 1.07). The proportionate mortality ratio by SD ranged $1.8-12 \%$ (median 9.3\%, 9.2 $\pm 2.4 \%$ ) and was lowest in the age group of 12 years. The proportionate mortality ratio by SD was $1.0 \%$ and $12.1 \%$ in the neonates or postneonatal infants.

Conclusions: The incidence of pediatric SD in Taiwan, an Asian country with neonatal mortality, postneonatal mortality and mortality of population 1-18 years comparable to the States, was within the reported range of previous Western reports as well as with a male predominance. The proportion of mortality accounted by SD was however relatively low, even in infants.

\section{9}

Incidence of Congenital Heart Disease in General Population of an Asian Country, Taiwan, from 2000 to 2006

Mei-Hwan Wu, National Taiwan University Hospital, Taiwan

Hui-Chi Chen, Taiwan

Jou-Kou Wang, National Taiwan University Hospital, Taiwan

Shin-Chung Huang, Taiwan

San-Kuei Huang, Taiwan

Objectives: We investigated the spectrum of congenital heart disease (CHD) in Taiwan population (23 millions).

Background: Epidemiological data of CHD are scarce in Asia and institutional studies showed racial-related differences.

Methods: Health care records of CHD patients were collected from National Insurance databases 2000-2006.

Results: CHD incidence per 1,000 live births was 29 (95\% CI 23-26): secundum atrial septal defect (ASDII, 14.7) was most common, followed by ventricular septal defect (VSD, 6.8), patent ductus arteriosus (PDA, 4.8), anomalies of pulmonary valve (3.4), tetralogy of Fallot (TOF, 0.76), endocardial cushion defect (ECD, 0.53 ), coarctation of aorta (CoA, 0.43), transposition of great arteries (TGA, 0.42), aortic stenosis (AS, 0.16), tricuspid atresia, Ebstein's anomaly and hypoplastic left heart syndrome (0.07). While female predominance was observed in all CHD, ASDII, VSD, ECD, anomalies of pulmonary valve and PDA, male was more in TGA, TOF and AS. Trend of increase was noted in all CHD, ASDII, PDA and CoA whereas decrease was in common truncus, common ventricle and anomalies of pulmonary valve. But, only $30 \%$ patients with ASDII, VSD, anomalies of pulmonary valve and PDA still needed CHD-specific medical care after age 3. Ratios of international incidences to ours revealed high for AS (2.43), tricuspid atresia (1.62) and hypoplastic left heart syndrome (4.10) but low for ASDII (0.34), PDA (0.77) and truncus (0.57).

Conclusion: In this Asian population, the incidence of CHD was at the high end of reported range due to more with simple lesions but fewer with left-sided obstructions and tricuspid atresia.

\section{2}

\section{Serial change of Matrix Metalloproteinases (MMPs) and} their inhibitors in Kawasaki Disease (KD)

Sin-Weon Yun, Department of Pediatrics, College of Medicine, Chung-Ang University, Korea

Mi Kyung Lee, Department of Laboratory Medicine, College of Medicine, Chung-Ang University, Korea

Jung-Ju Lee, Department of Pediatrics, College of Medicine, Chung-Ang University, Korea

Soo-Ahn Chae, Department of Pediatrics, College of Medicine, Chung-Ang University, Korea
In-Seok Lim, Department of Pediatrics, College of Medicine, Chung-Ang University, Korea

Dong-Keun Lee, Department of Pediatrics, College of Medicine, Chung-Ang University, Korea

Eung-Sang Choi, Department of Pediatrics, College of Medicine, Chung-Ang University, Korea

Byoung- Hoon Yun, Department of Pediatrics, College of Medicine, Chung-Ang University, Korea

Objective: Histopathological findings of coronary artery lesion (CAL) in KD indicate destruction of coronary artery wall with diffuse vasculitis. Matrix Metalloproteinases (MMPs) and their endogenous tissue inhibitors of MMPs (TIMPs) play central roles in this process. This study was performed to investigate the pathophysiologic role of MMPs and TIMPs in KD.

Methods: We compared 48 patients with typical KD and 15 control group. Serum MMP1, 2, 9 and TIMP1, 2 were measured by ELISA and compared accordong to clinical stages, responsiveness to IVIG and cardiac involvement.

Results: In acute stage MMP2, MMP9, TIMP1, TIMP2 were significantly increased in KD than febrile control $(\mathrm{P}<0.05)$. MMP1 and MMP1/TIMP1 are significantly increased into subacute phase, and significantly decreased into convalescent phase $(\mathrm{P}<0.05)$. MMP2 and MMP2/TIMP2 expressions are significantly lower in acute phase and significantly elevated into subacute and convalescent phase $(\mathrm{P}<0.05)$. Acute MMP9 levels are significantly decreased into subacute and convalescent phase $(\mathrm{P}<0.05)$. In acute phase, CAL group, MMP1, TIMP1 were lower than non CAL group, but, significantly increased in the subacute phase $(\mathrm{P}<0.05)$. In the CAL group, MMP1/TIMP1 ratio was persistently increased into convalescent phase $(\mathrm{P}<0.05)$.

MMPs and TIMPs has positive correlation with total WBC count and CRP and negative correlation with hemoglobin level and albumin level in the subacute phase $(\mathrm{P}<0.05)$.

Conclusions: These results suggest MMPs and MMP/TIMP might play an important role in the pathophysiology of KD and IVGG responsiveness and CAL. However further larger study will be needed.

\section{3}

Cardio-Facio-Cutaneous syndrome: First report of 2 cases with aortic stenosis

Keyhan Sayadpour Zanjani, Children's Medical Center, Teheran

University of Medical Sciences, Tehran, Iran, Iran

Ali Akbar Zeinaloo, Children's Medical Center, Teheran University of Medical Sciences, Tehran, Iran, Iran

Mohammad Yousef Aarabi Moghadam, Shaheed Rajayee CardioVascular Center, Tehran, Iran, Iran

Introduction: Cardio-Facio-Cutaneous (CFC) syndrome is a rare disease characterized by congenital heart diseases (CHD), facial abnormalities and cutaneous manifestations. Severe phenotypes of CFC syndrome have feeding abnormality and severe mental retardation in addition to classic organ involvements. Milder phenotypes have good mental and gastrointestinal functions, so their CHD are the main prognostic factor. We describe here two patients of CFC syndrome.

Case Presentations: The first patient was a 10-year-old boy with wide-spread biopsy-proven keratosis pilaris, flexion deformities of both knees requiring several surgeries and of fingers and toes, sparse scalp hair, nail dystrophy, and supra-orbital ridge hypoplasia. His CHD consisted of severe valvar aortic stenosis and closed patent ductus arteriosus. His parents refused any cardiac surgery or intervention. 
The second case was a 9-year-old girl with wide-spread biopsyproven keratosis pilaris, growth retardation, and sparse scalp hair. She had subaortic membranous stenosis leading to severe aortic regurgitation. She had two cardiac surgeries: subaortic membrane resection and Ross operation. None of the patients had neurologic, mental or gastrointestinal manifestations.

Discussion: Association of CFC syndrome with many congenital heart diseases has been reported but not yet with valvar or subvalvar aortic stenoses. Cutaneous manifestations of CFC syndrome are diverse, among them keratosis pilaris involving the skin of trunk and extremities is characteristic.

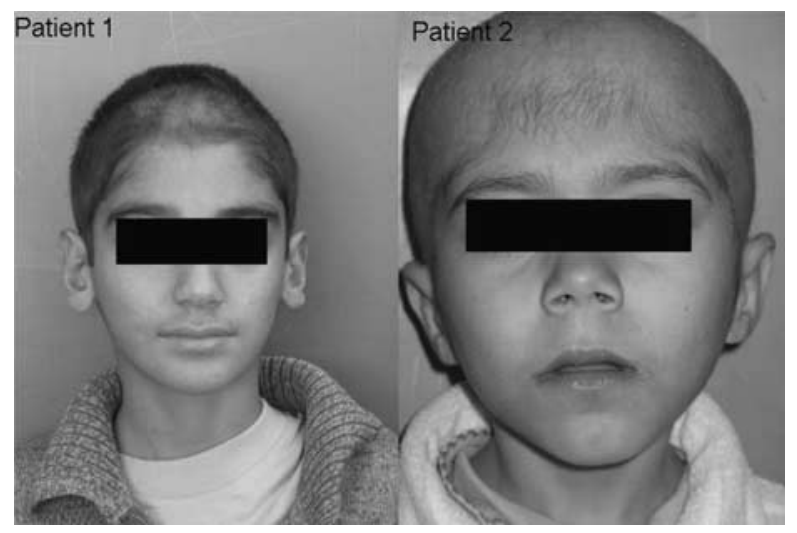

7364

Effects of chronic hypoxia on the expression of calmodulin and calcicum/calmodulin dependent protein kinase II and the calcium activity in myocardial cells in young rats

Pengjun Zhao, Shanghai Children s Medical Center, Shanghai Jiao Tong University, China

Kun Sun, Department of Pediatric Cardiology, Shanghai Children's Medical Center, China

Abstract Objective: Calcium plays an important role in the impairment of heart function and arrhythmia under the condition of chronic hypoxia. This study is aimed to investigate the expression of calmodulin $(\mathrm{CaM})$ and calcicum/calmodulin dependent protein kinase II (CaMKII) that regulate the calcium activity in myocardial cells through an animal model of chronic hypoxia .

Methods: The expression of mRNA and protein of CaM and CaMKII $\gamma /$ and CaMKII $\delta$ in myocardial cells were measured by RTPCR and Western Blot in normal rats and hypoxia rats one and three weeks after exposure. The cardiac cells from the control group and the 3-week hypoxia group were cultured. The intracellular calcium activity was detected using laser confocal equipment. The effect of CaMKII on the calcium activity was evaluated by the application of KN-62 (CaMKII specific inhibitor).

Results: The expression of CaM and CaMKII mRNA increased in hypoxia rats compared with that in control $(\mathrm{P}<0.01)$. The CaM and CaMKII $\delta \mathrm{mRNA}$ expression was different between 1 week and the 3 week hypoxia groups $(\mathrm{P}<0.01)$. The laser confocal demonstrated that the amplitude of calcium wave in hypoxic cells was not diferent from that in control, but the duration of calcium wave in hypoxic cells was longer than that in control $(\mathrm{P}<0.01)$. After KN-62 used,the amplitude of calcium wave decreased and the duration of calcium wave prolonged significantly.

Conclusions: The contents of CaM and CaMKII in myocardial cells increased under condition of chronic hypoxia as a compensation to keep calcium homeostasis in a certain time.

\section{5}

Risk factors predicting homograft stenosis after Ross operation- single center 12 year experience

Marek Kopala, Polish Mothers' Memorial Hospital, Lodz, Poland Jadwiga A Moll, Polish Mothers' Memorial Hospital, Lodz, Poland Katarzyna Mludzik, Polish Mothers' Memorial Hospital, Lodz, Poland

Maciej Moll, Polish Mothers' Memorial Hospital, Lodz, Poland

Jacek Moll, Polish Mothers' Memorial Hospital, Lodz, Poland

Objective: Since first Ross operation many investigators have been founding homograft-related factors influencing follow-up after surgery. The goal of this study was to establish echocardiographical and radiological factors identifying natural dynamic of pulmonary stenosis in mid and long-term follow-up and predicting postoperative outcome.

Methods: We followed up 86 patients (mean age, 9,6 $\pm 2,7$ years) who had Ross or Konno-Ross procedure by transthoracic echocardiography and 64-slice CT. There were 45 pts below 15 yrs of age. Follow-up was $6 \pm 3$ years and was $100 \%$ complete. Cryopreserved homografts were implanted to reconstruct RVOT during Ross operation. Up-to-size homografts were applied as a rule in children to avoid early replacement. Allograft stenosis was analyzed and risk factors were identified by univariate, multivariate, and survival analysis methods. Stenosis was defined as a mean ECHO gradient greater or equal to $20 \mathrm{~mm} \mathrm{Hg}$.

Results: There was no reoperation for homograft stenosis during available mid-term( 45 pts ) and late (30 pts) follow-up. Stenosisfree survival was $85 \pm 5 \%$ and $80 \pm 7 \%$ after $3-5$ and above 5 years, respectively. As independent predictor for stenosis transhomograft gradient greater than $10 \mathrm{~mm} \mathrm{Hg} 1$ year after procedure was revealed. Oversizing of homograft wasn't risk factor for stenosis. A cut-off value of $10 \mathrm{~mm} \mathrm{Hg}$ at 1 year of follow-up could predict occurrence of stenosis.

Conclusions: Homograft oversizing and age of recipient aren't important factors for pulmonary stenosis after Ross operation. Echocardiographic transhomograft gradient more than $10 \mathrm{~mm}$ $\mathrm{Hg}$ in early follow-up might predict occurance of stenosis.

\section{6}

Fate of pulmonary autograft (risk factors and clinical consequences) in patients who underwent Ross procedure- long-term follow-up

Marek P Kopala, Polish Mothers, Memorial Hospital, Lodz, Poland Jadwiga A Moll, Polish Mothers, Memorial Hospital, Lodz, Poland Katarzyna Mludzik, Polish Mothers, Memorial Hospital, Lodz, Poland

Maciej Moll, Polish Mothers, Memorial Hospital, Lodz, Poland Jacek J Moll, Polish Mothers, Memorial Hospital, Lodz, Poland

Objective: Dilatation of the pulmonary autograft root exposed to systemic pressure is still the crucial problem in the long-term follow-up after Ross operation. We assessed the prevalence, risk factors, and clinical consequences of late autograft dilatation.

Methods: History of 86 pts (mean age, 9,6 $\pm 2,7$ years) who underwent Ross or Konno-Ross surgery between 1995-2008 were reviewed. There were 45 pts below 15 years of age. Autograft annulus size, autograft sinus diameter and valve insufficiency (AI) were assessed using transthoracic echocardiography one week after procedure, 6 months and then annually after operation and were compared with normal valves values. V/s index (autograft annulus to sinus diameter), Z-score for autograft annulus and Z-score rate of change per year $(\mathrm{g} / \mathrm{y})$ was calculated. To verify these measurements multi-slice CT was completed in selected patients. 
Results: End-points of the study were freedom from autograft dilatation, from moderate or severe autograft regurgitation and from reoperation. Late autograft dilatation was identified in 27 (31\%) patients and regurgitation in $6(7 \%)$. Freedom from dilatation, from regurgitation and from reoperation (mechanical valve $-1 \mathrm{pt}$ ) was calculated. Autograft root diameters were compared to normal values (Z-score) referred to annulus, sinus of Valsalva and sinotubular junction. Older age was identified as predictive for autograft dilatation.

Conclusions: Autograft dilatation was revealed more frequently in patients who underwent Ross procedure above 7 yrs of age but it wasn't associated with increased autograft valve insufficiency. Higher Z-score in younger patients was a result of physiological discrepancy in diameters between pulmonary and aortic valves.

\section{7}

\section{Comparison of arrhythmia incidence after the} intracardiac lateral tunnel Fontan completion versus the extracardiac conduit

Vrej Sarkis, Heart Center, University Hospital of Cologne, Germany Markus Kruessell, Germany

Narayanswami Sreeram, Heart Center, University Hospital of Cologne, Germany

Konrad Brockmeier, Heart Center, University Hospital of Cologne, Germany

Mathias Emmel, Pediatric Cardiology, University of Cologne, Germany Gerardus Bennink, Germany

Background: Early and late arrhythmias are common following Fontan completion. The incidence of late arrhythmias has been shown to diminish with the use of an intracardiac lateral tunnel (ILT) compared to direct atriopulmonary connection. Little data are available for arrhythmia incidence following the use of an extracardiac conduit (ECC) for Fontan completion.

Patients and methods: The postoperative course of 51 consecutive survivors (surviving $>6$ months after Fontan completion) was analysed; 26 ILT Fontans (median age at Fontan completion 5.5 years; follow-up 58 to 120 months) versus 25 ECC Fontans (Fontan completion 3.4 years: follow-up 8 to 84 months).

Results: Early postoperative arrhythmias $(<30$ days)were observed in 14 patients (9 ILT-3 sinus node disease, 2 complete AV block, 4 IART versus $5 \mathrm{ECC}-2$ sinus node disease, 1 EAT, 1 AV block and 1 IART). Late arrhythmias (at $>6$ months post-Fontan) were seen in 15 patients (13 ILT -5 late AV block and 2 sinus node disease (all requiring a permanent pacemaker), 5 IART (3 successfully ablated at catheterization) and $1 \mathrm{VT}$ (requiring an ICD); versus 2 ECC (1 EAT (also observed in the early postoperative period) and 1 late AV block requiring pacing). There was 1 late death in the ILT subgroup, which was unrelated to arrhythmia.

Conclusions: The 2 groups differed substantially in age at Fontan completion and duration of follow-up. The preliminary data show that the incidence of early and late arrhythmia appears to be diminished following the routine practice of ECC Fontan completion.

\section{8}

Muscle sparing thoracotomy in pediatric age: a comparative study with standard posterolateral thoracotomy Nezihi Kucukarslan, Turkey

Ata Kirilmaz, Gata Haydarpasa Military Training Hospital Department of Cardiology, Turkey

Yucesin Arslan, Gata Haydarpasa Military Training Hospital Department of Cardiovascular Surgery, Turkey
Yavuz Sanisoglu, Gata Military Medical Faculty Department of

Bioistatistic, Turkey

Ertugrul Ozal, Gata Military Medical Faculty Department of

Cardiovascular Surgery, Turkey

Harun Tatar, Gata Military Medical Faculty Department of

Cardiovascular Surgery, Turkey

Objective: Alternative approaches to the standard posterolateral incision for thoracotomy have been developed to minimize its post operative pain and wound related side effects. Muscle-sparing (MS) thoracotomy has been a well-known substitution to the standard posterolateral thoracotomy for this purpose; however it has not been studied in the pediatric age group in detail.

Methods: We studied retrospectively the patients with thoracotomy for non-cardiac, thoracic surgical procedures. Group 1 included the patients with standard posterolateral thoracotomy for the surgery. The patients with MS thoracotomy composed Group 2. The related data were collected retrospectively. The early post operative morbidities (time requiring for regaining shoulder girdle movement, extubation, intensive care unit stay, and hospital stay) and late musculoskeletal anomalies (scoliosis, elevation of the shoulder, winged scapula, asymmetry of the nipples) were compared between groups.

Results: A total of 90 patients were included in the study. Group 1 constituted 50 patients with an average age of $4.24 \pm 2.91$ yearold. Group 2 included 40 patients with an average age of $4.20 \pm 2.92$ year-old. Comparison of the demographics and the baseline characteristics of the patients were not different between groups. In comparison of operative characteristics, there was lesser morbidity in group 2 as re-exploration for bleeding, wound infection, wound healing and fewer intensive care unit and hospital stay days. Late follow up revealed a significant increase in musculoskeletal deformities in group 1.

Conclusions: We conclude that muscle sparing incision should be preferred to the standard posterolateral thoracotomy in pediatric age.

\section{9}

Intraoperative transesophageal echocardiography in chidren weghing less than $5 \mathrm{~kg}$

Snehal Kulkarni, India

Swati Garekar, Wockhardt Pediatric and Congenital Heart Center, India Shreepal Jain, India

Pankaj Kasar, India

Suresh Joshi, India

We present our experience of intraoperative transesophageal echocardiography (TEE) in children weighing less than $5 \mathrm{~kg} .70$ infants weighing less than $5 \mathrm{~kg}$ underwent surgical repair during last one year. Intraoperative TEE was planned in 48 infants. Mean age of the patients was $3.1+/-2.6$ months and weight ranged from 2.28 to $5 \mathrm{~kg}$ (mean of $3.76 \mathrm{~kg}$ ). TEE examination was performed using pediatric TEE probe with Vivid I (GE) echocardiography equipment. Successful intraoperative TEE could be performed in 42 patients. TEE probe could not be inserted in 4 patients due to retrognathia. TEE probe had to be removed in 2 patients prior to imaging due pulmonary hypertensive crisis in patients with obstructed total anomalous pulmonary venous connection. There were no complications like eosophageal tear or bleeding. The mean airway pressure increased by $3 \mathrm{~mm} \mathrm{H}_{2} \mathrm{O}$ in 12 childrean and mean arterial pressure increased by $5 \mathrm{~mm} \mathrm{Hg}$ in 5 children after removing probe who were weighing less than $3 \mathrm{Kg}$. 4 children needed revision of surgical repair in 4 children due to residual defects. One patient needed closure of residual VSD, two needed revision of 
anastomosis in patients with repair of total anomalous pulmonary venous connection and one had anastomosis narrowing after arterial switch repair which needed revision.

We conclude that infants weighing less than $5 \mathrm{~kg}$ can safely undergo intraoperative TEE without major complications. Patients in this age group present with complex intracardiac defects which need early repair. Use of intraopeartive TEE will help to minimise the residual defects.

\section{0}

\section{Blalock-Taussig Shunt Performed By Using Biological} Graft

Ali Kutsal, Dr. Sami Ulus Pediatric Research and Training Hospital, Departments of Cardiovascular Surgery, Turkey

Murat Koc, Dr. Sami Ulus Pediatric Research and Training Hospital, Departments of Cardiovascular Surgery, Turkey

OMurat Disli, Dr. Sami Ulus Pediatric Research and Training

Hospital, Departments of Cardiovascular Surgery, Turkey

Rusen Melih Nebigil, Dr. Sami Ulus Pediatric Research and Training

Hospital, Departments of Cardiovascular Surgery, Turkey

Ali Bolat, Dr. Sami Ulus Pediatric Research and Training Hospital, Departments of Cardiovascular Surgery, Turkey

Sebahat Unlu, Dr. Sami Ulus Pediatric Research and Training

Hospital, Departments of Anesthesiology, Turkey

Burhan Ocal, Dr. Sami Ulus Pediatric Research and Training Hospital, Departments of Pediatric Cardiology, Turkey

Selmin Karademir, Dr. Sami Ulus Pediatric Research and Training

Hospital, Departments of Pediatric Cardiology, Turkey

Objective: The idea of improving systemic oxygen saturation in patients with cyanotic congenital heart disease and decreased pulmonary blood flow by creating a shunt between a systemic artery and the pulmonary artery was introduced clinically by Blalock and Taussig in 1945.

Indications for systemic pulmonary shunt are variable, the general ones are cyanotic complex defect, hypoplastic pulmonary artery, hypoplasia of pulmonary artery annulus, neonates with TOF and pulmonary atresia, tricuspid atresia. In such cases two stage corrections decrease porstoperative early mortality.

Methods: From June 2008 to December 2008, 3 patients underwent systemic-pulmonary shunt of the modified Blalock Taussig type, using a new type of biological graft (Shelhigh NOREACT $^{\mathbb{R}}$ tubular graft).

Result: No patient died at postoperative period, none of the cases were re-operated due to acute graft occlusion and/or thrombosis and/or kinking. No bleeding was observed during surgery or in the postoperative period, drainages were at acceptable limits. Echocardiography was performed on all patients and patent turbulent flows of shunts were seen.

Discussion: The biological graft proved to be effective for replacing inorganic grafts in modified Blalock-Taussig shunts, because of its excellent performance with no bleeding during surgery and in the postoperative period, easy technical management, technical reproducibility. We think that biological graft is a good alternative to PTFE, and it can replace PTFE grafts in the future.

\section{1}

\section{Outcome of Biventricular Outflow Tract Recontructions} with Rastelli Procedure

Ali Kutsal, Dr. Sami Ulus Pediatric Research and Training Hospital, Departments of Cardiovascular Surgery, Turkey

Murat Koc, Dr. Sami Ulus Pediatric Research and Training Hospital, Departments of Cardiovascular Surgery, Turkey
Rusen Melih Nebigil, Dr. Sami Ulus Pediatric Research and Training Hospital, Departments of Cardiovascular Surgery, Turkey Olcay Murat Disli, Dr. Sami Ulus Pediatric Research and Training Hospital, Departments of Cardiovascular Surgery, Turkey Suleyman Surer, Dr. Sami Ulus Pediatric Research and Training Hospital, Departments of Cardiovascular Surgery, Turkey Sebahat Unlu, Dr. Sami Ulus Pediatric Research and Training Hospital,Departments of Anesthesiology, Turkey

Burhan Ocal, Sami Ulus Pediatric Research and Training Hospital, Departments of Pediatric Cardiology, Turkey

Selmin Karademir, Sami Ulus Pediatric Research and Training

Hospital, Departments of Pediatric Cardiology, Turkey

The Rastelli operation was initially utilized for the repair of dtransposition of the great vessels with ventricular septal defect and pulmonary stenosis. It has subsequently been utilized for a variety of congenital heart defects characterized by two ventricles and overriding of the aorta with severe pulmonary stenosis (PS) or pulmonary atresia.

From July 2007 to December 2008, seven patients underwent correction of heart defects with rastelli procedure. Patients were aged from 4 month to 7 year, and four patients were female.

In all cases right ventriculotomy were made to repair the VSD with a Dacron patch by creating an intraventricular tunnel between the left ventricle and the aorta. The main pulmonary artery was divided and the proximal end was closed. A homograft conduit was implanted between the right ventricle and the main pulmonary artery. (Three of them were Labcor Stentless Valved Pulmonary Conduit and four of them were Contegra Pulmonary Bovine Conduit).

The Rastelli procedure can completely correct the right ventricular outflow tract stenosis, and right to left shunt, and avoid injuring the right coronary artery. It is a long and complex surgical procedure. It involves extensive and invasive monitoring of the patient before, during and after the operation. This includes catheters in a vein, an artery and the left atrium. Additionally, various medications will be used as necessary to alter the workload of the heart and blood vessels.

Satisfactory postoperative hemodynamics are dependent upon free, unobstructed egress of blood from both the left ventricle and the right ventricle.

\section{2}

Long-Term Results after Mitral Valve Repair in Children Cheul Lee, Department of Thoracic and Cardiovascular Surgery, Sejong General Hospital, Korea

Chang-Ha Lee, Department of Thoracic and Cardiovascular Surgery, Sejong General Hospital, Korea

Hong-Gook Lim, Department of Thoracic and Cardiovascular Surgery, Sejong General Hospital, Korea

Jae Gun Kwak, Department of Thoracic and Cardiovascular Surgery, Sejong General Hospital, Korea

Objective: We analyzed the long-term results of mitral valve repair in children.

Methods: We reviewed clinical records of 139 children $(<18$ years) who underwent mitral valve repair between 1988 and 2007. Patients with AVSD, single ventricle, or AV discordance were excluded. Median age was 2.3 years (2 months-17.6 years). Mitral regurgitation was predominant in 125 patients (90\%), and mitral stenosis was predominant in 14 patients $(10 \%)$. Associated cardiac lesions were present in 111 patients (80\%). Various surgical techniques were used according to the functional and pathologic findings of mitral valve. 
Results: There was no early death. Median follow-up was 8 years (2 months -20 years). Twenty six patients required 29 mitral reoperations, and 11 of these required mitral valve replacement. At 15 years, freedom from reoperation and mitral valve replacement was $77 \%$ and $90 \%$, respectively. Diagnosis of MS and valve status on discharge (MR grade $\geqslant 3$ or MS gradient $\geqslant 10 \mathrm{mmHg}$ ) were significant risk factors for reoperation. There were 3 late deaths, and overall survival was $97 \%$ at 15 years. Among 136 survivors, 111 patients (82\%) underwent echocardiography during follow-up. The degree of MR decreased significantly and only 5 patients showed MR grade $\geqslant 3$. The degree of MS decreased significantly and median MS gradient was $2.8 \mathrm{mmHg}(0-10 \mathrm{mmHg})$. All survivors remain in NYHA class I or II.

Conclusions: Mitral valve repair in children showed excellent survival, acceptable reoperation rate, and satisfactory valve function at long-term follow-up. Residual valve dysfunction was a significant risk factor for reoperation, but re-repair was successful in more than half of the patients who underwent reoperation.

\section{3}

The clinical results of one stage and staged total cavopulmonary anastomosis in pediatric populations over three years old in china

Shoujun Li, Surgical department, FUWAI Hospital, Peiking Union Medical College, China

Objective: To compare the outcome of one stage and staged total cavopulmonary connection(TCPC) in more than three-years-old patients.

Materials and Methods: Retrospectively analyzed consecutive 114 over-three-years-old patients with functional single ventricle (SV) enrolled in Fuwai Hospital. All the patients were divided into two groups: group $A(n=36)$ underwent staged TCPC ; group $B$ $(n=78)$ recieved one-stage TCPC. Group A were performed glenn procedure firstly because of high risk factor. Glenn procedure age were $5.9 \pm 2.8$ years, and the TCPC age were $8.3 \pm 3.1$ years. There was a interval of $26.0 \pm 32.0$ months between the two procedures. Mean pulmonary arterial pressure after Gleen procedure was $14.9 \pm 3.1 \mathrm{mmHg}$. 16 had fenestration. The TCPC age of were $9.4 \pm 5.6$ years. 35 of Group B had fenestration.

Results: The mortality of group A was $8.3 \%$ (3/36). One directly died of low cardiac output syndrome (LCOS), the other 2 died of LCOS-related complications. 5 (31.3\%) with drainage for more than 14 days in fenestration patients, $10(50 \%)$ in no fenestration patients. The mortality of Group B was 3.8\% (3/78). Two death were LCOS-related causes and 1 because of neurological complication. $10(28.6 \%)$ with drainage for more than 14 days in fenestration patients, $19(44.2 \%)$ in no fenestration patients. The Mortality of group A was higher than that of group B. Conclusion: Functional SV patients with high-risk factors can got better outcome by staged TCPC in more than three-years-old patients. Planned fenestration reduced the amount of drainage.

\section{4}

Tetralogy of Fallot Surgery-30 years experience from a single institution

Harald L Lindberg, Rikshospitalet, University of Oslo, Norway

Sigurd Birkeland, Rikshospitalet, University of Oslo, Norway

Kjell Saatvedt, Rikshospitalet, University of Oslo, Norway

Tom N Hoel, Rikshospitalet, University of Oslo, Norway

Egil Seem

Aim of study: To evaluate all patients with a diagnosis of Tetralogy of Fallot (TOF) and related congenital malformations (Double
Outlet Right Ventricle of TOF type, Pulmonary Atresia of TOF type, TOF with absent Pulmonary Valve, TOF with AV-canal) surgically treated in our Department during the period 1979 through 2007.

Methods: Patient records and the database of the department were evaluated, including earlier procedures performed in all patients. The official death registry of Norway was used for follow up. Results: A total of 420 patients were included in the study. There were 22 early and 23 late deaths. 399 patients have had a reparative procedure performed, with an EM of 11 (2,75\%). 151 cases has undergone a second repair, 47 have had a third operation performed, ten patients have been operated for a 4th time. Only three patients have been operated on with sternotomy for the 5th time. Mortality in this group was 15 early and 14 late. Our current policy is to do a primary repair at any age if the pulmonary valve is of adequate size.

Conclusion: Surgical treatment of the Tetralogy of Fallot and related congenital cardiac malformations has been performed for a long time with good results in our Department. In this cohort of patients almost $40 \%$ required additional procedures later on, in some cases as many as four additional surgeries. Reoperations carries a low risk in the second and third procedure, but may be more hazardous doing sternotomy for the fourth or fifth time.

\section{Patients repaired after 1978}

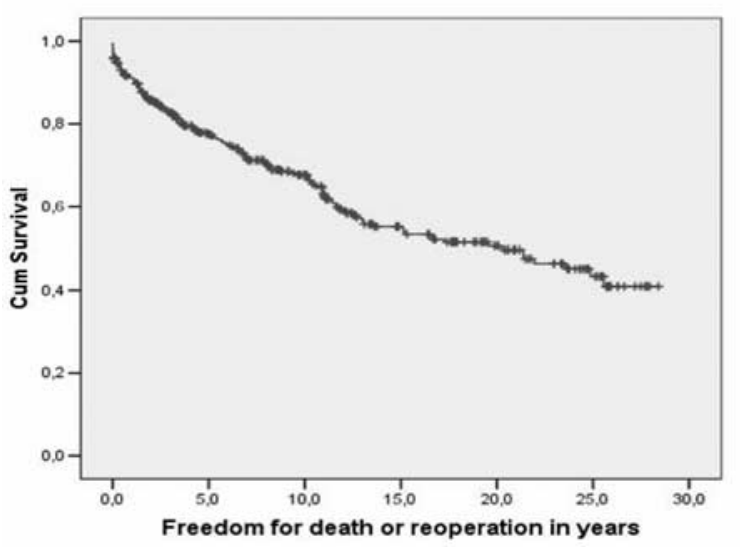

7375

A New Reconstructive Operation for the Tricuspid Valve of Ebstein's Anomaly

Jinfen Liu, Shanghai Children's Medical Center, Shanghai Jiaotong University School of Medicine, China

Zhiwei Xu, Shanghai Children's Medical Center, Shanghai Jiaotong University School of Medicine, China

Haibo Zhang, Shanghai Children's Medical Center, Shanghai Jiaotong University School of Medicine, China

Lisheng Qiu, Shanghai Children's Medical Center, Shanghai Jiaotong University School of Medicine, China

Yanan Lu, Shanghai Children's Medical Center, Shanghai Jiaotong University School of Medicine, China

Haifa Hong, Shanghai Children's Medical Center, Shanghai Jiaotong University School of Medicine, China

Objectives: We sought to describe a new technique for tricuspid valve repair in Ebstein's anomaly and to report early echocardiographic results, as well as early clinical outcomes.

Methods: From 2004 through 2008, 27 consecutive patients with Ebstein's anomaly aged 2 months to 17 years underwent operation for Ebstein anomaly with new method. Tricuspid 
incompetence was severe in 21 patients and moderate in 6 patients. The surgical strategies include palication of atrialized right ventricle to form the new tricuspid anulus in the normal position, mobilization of tricuspid valve completely which then be sutured in the new tricuspid anulus. For the patients with hypoplasia right ventricle, the Glenn procedure was also performed to decrease the right ventricle load.

Results: There was 1 (4\%) hospital death because of ventricular arrhythmia. Early postoperative echocardiograms have shown good result. After operation, Tricuspid Regurgitation was mild in 22 patients and moderate in 5 patients. Median aorta clamp time is 65 Mins and median ventilation time is $6.75 \mathrm{~h}$. Except one patient with ventricular arrhythmia, there was no other serious ayyhythmia occurred after operation.

Conclusions: The immediate outcomes of this new surgical techonology can have excellent results. From our point of view there has been an improvement in keeping the function the trcuspid valve in the operation for Ebatein's anomoly. The follow-up is needed for evaluate the long-term function of trucuspid valve.

\section{6}

25 Years Solid Collaboration between Project HOPE and Shanghai Children's Medical Center(SCMC) and Its Contribute to the Progress of Pediatric Cardiac Surgery in SCMC

Jinfen Liu, Shanghai Children's Medical Center, Shanghai Jiaotong University School of Medicine, China

Objectives: To introduce 25 years Solid Collaboration between Project HOPE and Shanghai Children's Medical Center (SCMC) and its contribute to the progress of pediatric cardiac surgery in SCMC.

Methods(History): Since 1985, Project HOPE has sent her cardiac team to Xinhua Hospital, Shanghai every year. Young doctors were sent to USA leading institutions for extended periods of training and clinical observation. Projest Hope also supported Shanghai government to build a new hospital-SCMC, which includes one heart center with nearly 250 beds.

Results: SCMC has became one of the biggest clinical medical centers for pediatric CHD in China. The Heart Center of SCMC has performed almost all procedures for complex CHD, including hypoplastic left heart syndrome (HLHS), TGA, pulmonary atresia with intact ventricular septum (PA/IVS), pulmonary atresia with VSD (PA/VSD), DORV, total anomalous pulmonary venous connection (TAPVC), etc. In 2007, the cardiovascular surgery team performed 2,850 procedures of CHD cases. This substantial number of cases probably is one of the largest quantities in CHD repair of the world.

Conclusions: This successful collaboration is a great example of medical exchange and support between developing and developed countries. It created a cascade reaction in the field of CHD surgery in China.

\section{8}

Feasibility of left ventricular retraining for arterial switch operation in "older age" in a developing country: first results

Pier F Lombardi, Clínica General del Norte, Barranquilla, Colombia Francisco De la Hoz, Clínica General del Norte, Barranquilla, Colombia

Arterial switch operation (ASO) is the procedure of choice for anatomic correction of transposition of the great arteries (TGA) with or without minor or major associated anomalies. In infants older than 21 days of age, left ventricular (LV) retraining followed shortly by ASO represents a good alternative to primary ASO for patients with TGA and intact ventricular septum (IVS). There are some instances, like social situations in developing countries, in which babies with TGA don't reach a cardiac center on time. Our short experience is represented by 2 patients who underwent rapid 2-stage ASO. LV geometry, LV mass index, mass/ volume ratio, LV diastolic posterior wall thickness/LV diastolic diameter ratio and the presence of a ductus arteriosus were considered on preoperative echocardiogram, as well as the LV mass.

Patient 1. Female. 2 months old. Diagnosis was TGA with IVS. A $4 \mathrm{~mm}$ MBTS together with a pulmonary artery banding (PAB) were made. ASO was performed after 6 days. Follow-up is 10 months. Good ventricular and valvar function was seen on Echocardiographic examination.

Patient 2. Female. 4 months old, underwent a $4 \mathrm{~mm}$ MBTS associated with a PAB. The pos operative course was complicated by sepsis. Eventually she had an ASO after 22 days. Follow-up is 4 months, with good ventricular and valvar performance.

A number of patients in our area cannot reach a primary cardiac center on time. In patients with TGA and IVS, when LV mass/volume ratio $<1.5$, we consider rapid 2-stage ASO, with encouraging results.

\section{9}

\section{Ross surgery in a pediatric patient without blood} transfusion

Pier F Lombardi, Clínica General del Norte, Barranquilla, Colombia Emre Belli, Marie Lannelongue Hospital, Paris-Sud University, Le Plessis-Robinson, France

Aortic valve replacement with patient's own pulmonary autograft is a good alternative for treatment of aortic valve disease in certain patients, especially if they're young. Patients of the Jehovah's Witness faith needing a Ross procedure still represent a dilemma for surgeons. We present a case of a young girl with aortic disease, who underwent a Ross procedure, without the use of any blood product.

A 9 years old girl presented to our institution with a diagnosis of double aortic lesion (severe insufficiency, moderate stenosis). She was of Jehovah's Witness faith. Ross surgery was recommended earlier, but she was refused for treatment in other centers. After discussion with her parents, who confirmed that she should not be given blood, she was scheduled for Ross aortic root replacement. The only strategy used for blood conservation was treatment with recombinant human erythropoietin. At operation the patient's own pulmonary valve was inverted onto the aortic root, and a $19 \mathrm{~mm}$ aortic homograft was implanted in the RVOT. No Fibrin glue or reinforced suture was used. Patient was sent home after 6 days in good conditions with a hemoglobin level of 9.4.

Ross procedure represents a challenging situation in a patient who refuses blood transfusion. This operation can be done safely. In our patient human erythropoietin was the only technique used for blood conservation. No cell-saving system or autotransfusion were used.

\section{0}

Tricuspid valve surgery in symptomatic low body weight neonates: a multidisciplinary approach

Giovanni Battista Luciani, Division of Cardiac Surgery, University of Verona, Verona, Italy, Italy

Luca Barozzi, Division of Cardiac Surgery, University of Verona, Verona, Italy, Italy 
Francesca Viscardi, Division of Cardiac Surgery, University of Verona, Verona, Italy, Italy

Mara Pilati, Division of Cardiology, University of Verona, Verona, Italy, Italy

Fabrizio De Rita, Division of Cardiac Surgery, University of Verona, Verona, Italy, Italy

Maria Antonia Prioli, Division of Cardiology, University of Verona, Verona, Italy, Italy

Giuseppe Faggian, Division of Cardiac Surgery, University of Verona, Verona, Italy, Italy

Alessandro Mazzucco, Division of Cardiac Surgery, University of Verona, Verona, Italy, Italy

Objectives: To define indications and outcome of surgical repair in premature or low birth weight $(\leqslant 2.5 \mathrm{~kg})$ neonates with symptomatic tricuspid valve (TV) disease.

Methods: Between 2004-2008, 6 neonates, with mean birth weight of $2.1 \pm 0.3 \mathrm{~kg}$ (range $1.7-2.5 \mathrm{~kg}$ ), were treated for symptomatic TV pathology. Severe TV stenosis was present in 3 (tumor in 2, mycetoma 1) and severe regurgitation in 3 (3 Ebstein/-like dysplasia), with marked RA dilatation in all. Five (83\%) had severe pulmonary hypertension and 3 ductal dependent pulmonary blood flow.

Results: Preoperative stabilization required vasoactive drugs in 5 patients, mechanical ventilation with PGE1 infusion in 3 (mean $37 \mathrm{dd}$ ), associated with iNO in 2 (mean $21 \mathrm{dd}$ ) and atrial septostomy in 1 . Surgery, deferred to mean age of $2.8 \pm 3.1$ mos (20 dd-6 mos) and weight of $2.9 \pm 1.5 \mathrm{~kg}(2.2-4.5 \mathrm{~kg})$, consisted in: mass excision $+\mathrm{TV}$ repair in 3 , TV repair $+\mathrm{RA}$ remodelling in 2 , stage 1 palliation followed by RA remodelling+Glenn shunt in 1 . There was 1 hospital death: 4 patients required postoperative iNO and 3 were discharged on sildenafil/bosentan therapy. During a 4-year follow-up (mean $2.1 \pm 1.4$ ), there was 1 late death with a $67 \%$ event-free survival. Follow-up echocardiography showed absent-trivial TV regurgitation in all late survivors, with normalized right heart dimensions.

Conclusions: Symptomatic tricuspid valve pathology in newborns is a highly lethal disease, due to association with low birth weight/ prematurity and pulmonary hypertension. Preoperative hemodynamic stabilization and elective surgery in early infancy is feasible in the majority of patients increasing the likelihood of durable repair.

\section{1}

\section{Reparative surgery of the pulmonary autograft:} experience with Ross reoperations

Giovanni Battista Luciani, Division of Cardiac Surgery, University of Verona, Verona, Italy, Italy

Luca Barozzi, Division of Cardiac Surgery, University of Verona, Verona, Italy, Italy

Francesca Viscardi, Division of Cardiac Surgery, University of Verona, Verona, Italy, Italy

Mara Pilati, Division of Cardiology, University of Verona, Verona, Italy, Italy

Maria Antonia Prioli, Division of Cardiology, University of Verona, Verona, Italy, Italy

Giovanni Puppini, Division of Radiology, University of Verona, Verona, Italy, Italy

Giuseppe Faggian, Division of Cardiac Surgery, University of Verona, Verona, Italy, Italy

Alessandro Mazzucco, Division of Cardiac Surgery, University of Verona, Verona, Italy, Italy

Objectives: Autograft valve and root pathology is the leading cause of Ross procedure failure. In order to define feasibility and outcome of autograft valve/root repair at reoperation, a 14-year clinical experience was analyzed.

Methods: One-hundred-twenty-five patients surviving an average of $7.0 \pm 1.9$ years (range 0.5-14) after the Ross procedure underwent cross-sectional clinical and echocardiographic examination. End-points of the study were freedom from autograft reoperation, from root/valve replacement, functional outcome after valve/root repair.

Results: Seventeen (14\%) patients underwent 21 cardiac procedures at an average of $6.0 \pm 3.1$ years (range $0.08-14.3$ years) after Ross operation. Eleven had undergone root replacement, 5 inclusion cylinder and 1 subcoronary grafting. Among these, 11 $(65 \%)$ required root and 6 valve reoperation. Surgery consisted in valve/root repair in 12 and AVR in 5, with no hospital mortality. Freedom from any cardiac reoperation was $78 \pm 5 \%$ at 14 years. During follow-up $4.9 \pm 1.9$ years (range $0.4-10.2$ years), 4/12 patients having autograft valve/root repair required AVR, while 8 present mild autograft valve inusfficiency or less. Actuarial freedom from autograft valve replacement was $86 \pm 4 \%$ at 14 years. Predictors of reoperation were older age at Ross $(p=0.04)$, use of root technique $(p=0.001)$ and length of follow-up $(p=0.02)$. Failure of valve/root repair was associated with isolated valve pathology $(p=0.001)$, severity of autograft insufficiency $(p=0.01)$ and earlier reoperation $(p=0.002)$.

Conclusions: Autograft reoperation with pulmonary valve repair is feasible in half of patients with Ross failure. Coexistent root pathology and absence of severe valve dysfunction are predictive of successful and durable repair.

\section{2}

Optimal size of the transannular patch for the reconstruction of a hypoplastic pulmonary root: preliminary results of the application of a new geometrical rule

Ignacio Lugones, Ricardo Gutiérrez Children's Hospital, Argentina María I Román, Ricardo Gutiérrez Children's Hospital, Argentina Andrés J Schlichter, Ricardo Gutiérrez Children's Hospital, Argentina

Objective: we present a prospective study designed to evaluate a new geometrical rule to determine the exact width of the transannular patch for each patient undergoing correction of right ventricular outflow tract obstruction.

Methods: the width of the transannular patch is obtained by subtracting the perimeter of the preoperative pulmonary annulus from the normal desired one. A table to be used in the operating room was designed, in which this value can easily be obtained. This table was tested from May to December 2008. Seven consecutive patients (mean age 13 months) underwent surgery for correction of tetralogy of Fallot with severely hypoplastic pulmonary annulus. Pre and postoperative echocardiograms were performed to assess pulmonary annulus diameter and transpulmonary gradients. Clinical and echocardiographic follow-up were also carried out.

Results: average percentage of deviation of postoperative pulmonary annulus diameters from their normal values was $5 \%$, indicating that each one of them was within $1 \mathrm{~mm}$ around the normal diameter. Average transpulmonary gradient was $21 \mathrm{mmHg}$, meaning mild residual pulmonary stenosis.

Conclusions: this new geometrical rule accurately determines the exact width of the transannular patch for the reconstruction of a hypoplastic pulmonary root, avoiding residual gradients and severe pulmonary regurgitation in order to prevent dilatation of the right ventricle in the late follow-up of these patients. 
7383

A Novel Hybrid Method Creating Porcine Model of Cyanotic Congenital Heart Defect with Decreased Pulmonary Blood Flow

Xiaodong Lv, Fuwai Hospital, China

Yaoqiang $\mathrm{Xu}$, China

Objective: To create a novel experimental model of cyanotic congenital heart defect (CHD) with a hybrid method.

Methods: An chronic model of CHD with decreased pulmonary blood flow and chronically developed cyanosis was surgically established in 8 piglets (1-2 months old) by creating an artificial atrial septal defect and surgical pulmonary artery banding (cyanosis group). Another 8 piglets underwent sham operations that did not include atrioseptestomy and pulmonary artery banding (control group). After a mean duration of 2 months following the hybrid procedure, these piglets underwent median sternotomy. Recorded Right ventricular and pulmonary artery pressures and hematocrit, $\mathrm{PaO} 2$ and $\mathrm{SaO} 2$ preoperatively and 2 months postoperatively. The blood gas samples were drawn from aortic, right atrium, pulmonary vein and pulmonary artery for calculating the Qp/Qs.

Results: At a mean duration of 2 months postoperatively, there were 6 long-term survivors in cyanosis group whose body weight increased to $20.6 \mathrm{~kg}$. The resting gradient across the pulmonary artery band was $53.7 \mathrm{mmHg}$. Qp/Qs reached 0.54:1. The arterial oxygen tension $(\mathrm{PaO} 2)$, arterial oxygen saturation $(\mathrm{SaO} 2)$, hematocrit value (HCT) and hemoglobin concentration $(\mathrm{Hb})$ were $52.9 \mathrm{mmHg}, 85.6 \%, 49.8 \%$ and $16.6 \mathrm{~g} / \mathrm{dl}$ in cyanosis group versus $118.0 \mathrm{mmHg}, 98.0 \%, 37.9 \%$ and $12.2 \mathrm{~g} / \mathrm{dl}$ in control group respectively (all $\mathrm{P}<0.001)$.

Conclusions: A porcine model of cyanotic congenital heart defect with decreased pulmonary blood flow was established by a hybrid method. Application of this experimental design may enhance our understanding and possibly influence the treatment of patients having cyanotic heart disease with decreased pulmonary blood flow.

\section{4}

The Possible and Extended Indication of Bilateral Pulmonary Banding for the Neonates with HLHS Compatible, Especially with Small for date

Yoshito Maehata, Division of Congenital Cardiovascular Surgery, Osaka City General Hospital, Osaka, Japan, Japan

Kyoichi Nishigaki, Division of Congenital Cardiovascular Surgery, Osaka City General Hospital, Osaka, Japan, Japan

Yoichi Kawahira, Division of Congenital Cardiovascular Surgery, Osaka City General Hospital, Osaka, Japan, Japan

Yosuke Murakami, Division of Congenital Cardiology, Osaka City General Hospital, Osaka, Japan, Japan

Eiji Ehara, Division of Congenital Cardiology, Osaka City General Hospital, Osaka, Japan, Japan

Tsugutoshi Suzuki, Division of Congenital Cardiology, Osaka City General Hospital, Osaka, Japan, Japan

Yuki Ozawa, Division of Congenital Cardiology, Osaka City General Hospital, Osaka, Japan, Japan

Objective: The aim of this study is to evaluate bilateral pulmonary banding $(\mathrm{BPAB})$ in neonates as a first palliative procedure for the patients with HLHS compatible, especially with small for date (SFD).

Patients \& methods: From April 2003 to October 2008, BPAB was performed in 20 patients at $3.3 \pm 2.9$ days after birth. Their body weight $(\mathrm{BW})$ at the procedure was $2.7 \pm 0.7 \mathrm{~kg}$ (range:0.9-3.6).
Diagnosis was HLHS $(n=8)$, CoA complex $(n=5)$, AA + VSD $(\mathrm{n}=4)$ and others. BPAB was performed by bold silk suture and with a circumference of $9.5 \pm 0.6 \mathrm{~mm}$ (range: $8-11$ ).

Results: $\mathrm{BPAB}$ decreased $\mathrm{SpO} 2$ from $87.7 \pm 3.8$ to $77.8 \pm 5.2 \%$ and improved BP from $43.8 \pm 12.4$ to $59.2 \pm 14.1 \mathrm{mmHg}$. Serum lactate was decreased from $102.0 \pm 94.9$ to $12.1 \pm 3.2$ $\mathrm{mg} / \mathrm{dl}(\mathrm{p}<0.05)$. One patient needed ASD creation under CPB. There were 2 operative deaths. The remaining 18 patients had no accident in hemodynamics immediately after BPAB. At 3 months $(90.9 \pm 29.3$ days) after the procedure, 16 patients $(80 \%)$ had received second one; Norwood type procedure $(n=11)$, EEDA \pm VSD closure $(n=3)$ and others. Their BW gained to $4.0 \pm 0.9 \mathrm{~kg}$ (range:2.6 to 5.2 ) at the time. As a sequential disease after $\mathrm{BPAB}$, there were left pulmonary artery stenosis in 7 patients (44\%) and left PA thrombosis in 1 patient. They needed additional PTA or AP shunt for the growth of the lung. Conclusion: BPAB would be effective as a choice of the first palliative procedure for HLHS compatible. Especially, the patients with SFD were considered to have possibility that they could be performed each first open cardiac surgery safety.

\section{5}

The Evolution of Paediatric Cardiac Surgery in a developing country; The Libyan experience

Hasan Ahmed Maghur, Tripoli Medical Center, Libya

Objective: Since 1992 Paediatric Cardiac Surgery (PCS) in Libya was performed in a sporadic manner mainly by visiting surgeons from Europe and was confined to older children. Since 1992, a program was started to establish the specialty of Paediatric Cardiac Surgery in Libya.

Method: A total of 1302 children, age 3 days to 16 years old, were operated upon over a two 6-year period. The first 6-year period was between 1992-1998 and the second period between 2002-2008.

Results: During the first period (92-98), a total of 505 operations were performed; 354 (70\%) were closed heart operations while $154(30 \%)$ were open heart operations. The majority 318 (63\%) were children older than 1 year and 190 (37\%) were infants. One neonate with Transposition of Great Arteries (TGA) underwent a successful Arterial Switch Operation (ASO) in 1996.

This trend changed during the second period (02-08). The total number of operations increased to 800 cases (60\% increase) with an increase in number of open heart procedures to 405 (51\%) with 395 (49\%) being closed heart cases. The number and percentage of infants increased to 438 (54\%) with 362 (46\%) older than 1 year. The number of ASO for TGA increased to 44 cases (4400\% increase).

Conclusion: This paper discusses the evolution of Paediatric Cardiac Surgery in Libya (a developing country) over these two periods and how newborns with TGA are now operated upon routinely.

\section{6}

Right ventricle remodeling surgery in congenital heart disease, using porcine Pulmonary Prosthesis

Miguel Angel Maluf, Universidade Federal de São Paulo, Brazil Celia Camelo Silva, Universidade Federal de São Paulo, Brazil Werther Brunow Carvalho, Universidade Federal de São Paulo, Brazil Antonio Carlos Carvalho, Universidade Federal de São Paulo, Brazil

The reconstruction of right ventricle outflow tract in congenital heart disease has deserved the interest of cardiac surgeons determined to alleviate anatomic obstruction and to return ventricular function. 
From June 1991-March 2008, 191 consecutive patients, aged 4 months to 35 years $(M=3.0)$ were operated. The patients were classified in 5 groups: G1 - Tetralogy of Fallot with pulmonary hypoplasia, $136(71.2 \%)$ cases; G2 - Pulmonary atresia with ventricular septal defect, 30 (15.7\%) cases; G3 - Arterial trunk, $10(5.2 \%)$ cases; G4 - Pulmonary atresia with intact ventricular septum, $07(3.6 \%)$ cases and G5 - Transposition of the great arteries with pulmonary stenosis, 08 (4.1\%) cases. Repair consisted of patch closure of the ventricular septal defect $(\mathrm{n}=184)$, tricuspid valve repair $(\mathrm{n}=15)$. Transannular pulmonary prosthesis, was performed in all cases. The Lecompte procedure was employed in seven cases and one and a half ventricle repair, in seven cases.

There were 15 hospital deaths (7.8\%) and six (3.4\%) late death. Fourteen $(8.2 \%)$ patients were lost during the follow-up. Fifteen $(8.8 \%)$ cases were reoperated for correction of residual lesions or prosthesis dysfunction. There was one $(6.6 \%)$ hospital death. One hundred and seventy $(89.0 \%)$ surviving patients were followed up from 3 to 204 months $(M=98.0)$. One hundred and forty one $(82.9 \%)$ patients, are free of reoperation.

Right ventricular remodeling constitutes a safe and standardized technique. The early reconstruction of the pulmonary valve and right ventricle outflow tract, could be preserving ventricular performance for a long period, while the prosthesis function is analyzed.

\section{7}

\section{Cavopulmonary anastomosis and variants} techniques in staging toward Fontan operation surgical results

Miguel Angel Maluf, Universidade Federal de São Paulo, Brazil Antonio Carlos Carvalho, Universidade Federal de São Paulo, Brazil

Celia Camelo Silva, Universidade Federal de São Paulo, Brazil Werther Brunow Carvalho, Universidade Federal de São Paulo, Brazil

Background: Due to large variability of the anatomy and associated lessons to the univentricular heart, there are many different techniques to perform the total cavopulmonary connections (TCPC). In this study we present three variations of TCPC procedure.

Patients: Between 1990 to 2008, 64 patients, age: 2 to 14 years $(\mathrm{M}=4.2)$, Female: $51 \%$, were operated. The cardiac malformation were: Tricuspid atresia, 25 (39.1\% cases, Common Ventricle, $19(29.6 \%)$ cases, Pulmonary atresia w/ intact ventricular septum, $15(23.5 \%)$ cases, Ebstein anomaly, 5 (7.8\%) cases. Three techniques were employed: G1- Intraatrial tunnel, 41 (64.1\%) cases; G2- One and a half ventricle operation, $16(25 \%)$ cases and G3- Kawashima operation, 7 (10.9\%) cases. Fifty two (81.2\%) patients had bidirectional cavopulmonary connection (BCPC). In the $\mathrm{G} 1$, the interaatrial tunnel was performed with a thin or tubular bovine pericardio and in the G2, the right ventricle outlet tract, was reconstructed with suine pulmonary prosthesis. Results: There were $4(6.2 \%)$ hospital death and 1 (1.5\%) late death. One $(1.5 \%)$ case was submitted to reoperation, for substitution of the pulmonary prosthesis. Fifty nine (92.1\%) surviving patients were followed up from 6 to 192 months and all are free of reoperation.

Conclusion: Early unloading of the functional univentricular heart by means of BCPC, allow a good condition to completion to TCPC. The mid-term results of one and a half ventricle repair, seem to be acceptable. These three procedures can be a good alternative to TCPC repair in suitable patients.

\section{8}

Lecompte procedure for correction of the transposition of the great arteries associated with ventricular septal defect and left ventricle outflow tract obstruction

Miguel Angel Maluf, Universidade Federal de São Paulo, Brazil

Celia Camelo Silva, Universidade Federal de São Paulo, Brazil

Werther Brunow Carvalho, Universidade Federal de São Paulo, Brazil

Antonio Carlos Carvalho, Universidade Federal de São Paulo, Brazil

Background: The Lecompte procedure, which is called $\mathrm{R}$ 'eparation l'etage ventricular- used for the anatomic repair of transposition of the great arteries associated with ventricular septal defect and pulmonary stenosis, to day is employed in an effort to avoid the limitations of Rastelli operation.

Methods: Between February 1994 and July 2007, eight patients with ages between 2 and 8 years (median: 2,9) with anomalies of Ventricular-Arterial connection, ventricular septal defect and pulmonary outflow tract obstruction were considered to fulfill the condition required for anatomic repair. After the intraventricular connection of the left ventricle to the aorta, to use the natural pulmonary orifice for the pulmonary outflow tract reconstruction. In six cases, were used Heterograft prosthesis (bicuspid $=4$ and tricuspid $=2$ ), one case, used Polytetrafluoroethylene prosthesis annd one case, used Homograft. The first patient of this serie was converted from Rastelli operation to Lecompte procedure, due to right ventricular-pulmonary artery prosthesis obstructio.

Results: There was one hospital death (12.5\%) and not late reoperations or death. seven patients whose follow-up time was 12 to 160 months (median 95.3) with clinical and echocardiographyc evaluation. No stenosis of the aortic flow tract was found. three patients have a trivial to moderate pulmonary insuficiency.

Conclusion: Lecompte procedure has the following advantages: 1Surgical indication for infants. 2- Low morbidity and mortality. 3- Free from reoperation over the long term. 4- Possibility of conversion of Rastelli procedure to Lecompte procedure.

\section{9}

Intraoperative echocardiographic approach to Off-Pump Pulmonary Valve Replacement

Stefano M Marianeschi, Pediatric Cardiac Surgery. Niguarda Hospital Milan, Italy

Emanuele Catena, Cardiac Anesthesia. Niguarda Hospital Milan Pascal A Berdat, Cardiovascular Center, Clinic Im Park, Zurich, Switzerland

Elena Ribera, Pediatric Cardiac Surgery. Niguarda Hospital. Milan, Italy

Gabriele Vignati, Pediatric Cardiology. Niguarda Hospital. Milan, Italy Lugi Mauri, Pediatric Cardiology. Niguarda Hospital. Milan, Italy Paola Austoni, Pediatric Cardiology. Niguarda Hospital. Milan, Italy Francesco Santoro, Pediatric Cardiac Surgery. Niguarda Hospital. Milan, Italy

Background: Pulmonary regurgitation is a frequent sequela after repair of Tetralogy of Fallot negatively affecting long-term prognosis and necessitating re-interventions.

The Shelhigh Injectable Stented Pulmonic Valve (SISPV) allows pulmonary valve replacement without cardiopulmonary bypass under direct control minimizing the impact of surgery on cardiac function.

Objective: The aim of the works is to describe our initial clinical experience with the intraoperative echocardiographic approach. Methods: Between April 2007 and December 2008, 5 symptomatic patients with severe pulmonary regurgitation and progressive right 
ventricular dysfunction after Tetralogy of Fallot repair received SISPV in our institution. All patients underwent trans-esophageal 2-D echocardiography during the surgical procedure.

Results: Valve insertion, delivery, and placement were successful in all patients but one that required the repositioning of the same valve in cardiopulmonary by pass. Early recovery was uneventful and all the patients were discharged home after a mean length of hospital stay of $6.3 \pm 2.4(2-12)$ days. Intraoperatively, transesophageal echocardiography was the unique tool to guide device positioning and verify surgical results. In two patients echo documented a valve displacement after delivery and guided the repositioning (one in cardiopulmonary by-pass). In the immediate postoperative course serial echocardiographic examinations documented right ventricle reverse remodeling and excluded later complications.

Conclusion: Trans esophageal echocardiography plays a pivotal role in the intraoperative management of patients with significant pulmonary regurgitation after repair of Tetralogy of Fallot underwent pulmonary valve replacement with injectable valves.

\section{1}

Pulse oximetry home monitoring in newborns with single ventricle physiology and a BT or Sano shunt as the only source of pulmonary blood flow

Mats Mellander, Department of Pediatric Cardiology, The Queen Silvia Childrens Hospital, Göteborg, Sweden, Sweden

Eva Strömvall-Larsson, Department of Pediatric Cardiology, The Queen Silvia Childrens Hospital, Göteborg, Sweden, Sweden Boris Nilsson, Department of Pedatric Cardiac Surgery, The Queen Silvia Childrens Hospital, Göteborg, Sweden, Sweden

We have experienced an increasing number of thrombotic shunt occlusions after Norwood surgery for HLHS and other hearts with single ventricle physiology. Our first thought was that this might be related to the introduction of the Sano modification in 2002. However none of 12 newborns with classical HLHS that had a Sano shunt 2002-2004 developed shunt occlusion compared to 4 of 9 operated 2005-2007.

Objective: To test if pulse oximetry home monitoring prevents death from shunt occlusion.

Methods: Newborns with single ventricle physiology and a BT or Sano shunt were included. Parents were taught to make one measurement daily while the baby was calm and if the saturation was $70 \%$ or less, confirmed on a repeat measurement, contact the local hospital.

Results: Eight of the 10 first patients on the programme had Norwood surgery and 7 had a Sano shunt. Four patients had saturations below 70\%. One had a distal Sano narrowing and therefore an early bidirectional Glenn (101 d). Another had a partial thrombotic shunt occlusion and the shunt was replaced the same day as the low saturation reading (59 d). Two patients had no apparent cause and saturations normalized without surgical intervention. One patient with good saturations died suddenly a few hours after a reading above $90 \%$. Autopsy showed thrombotic shunt occlusion. Conclusions: Home monitoring may have prevented 1-2 deaths from shunt occlusion in these first 10 patients on the home monitoring programme. Rapid development of thrombosis in one patient resulted in death in spite of home monitoring.

\section{2}

A retrospective analysis of the presentations and outcomes of pediatric patients treated for vascular ring malformations Brian Solomon, University of Connecticut School of Medicine, United States
Gary S Kopf, Yale-New Haven Hospital, United States

Dennis M Mello, Connecticut Children's Medical Center, United States

Introduction: Vascular ring malformations pose a significant, but surgically correctable, problem in pediatric cardiothoracic patients. Current studies fail to reach a consensus regarding the optimal means of diagnosis and surgical outcomes of these anomalies. Furthermore, these studies have massive variability in reported post-operative outcomes.

Methods: A retrospective review of 27 patients treated for vascular rings between 1999 and 2007 analyzed surgical outcomes with respect to age, weight, and type of vascular ring. Analysis provided an overview of presenting statistics of these malformations and correlated age of treatment, weight, and type of vascular ring with length of stay (LOS), length of post-operative ventilation (LOV), and surgical morbidity. Additionally, this study compared the rate of successful anomaly prediction by various diagnostic modalities.

\begin{tabular}{lccccc} 
Diagnostic Modalities Predictive of Vascular Ring Malformations \\
\hline Modality & $\begin{array}{l}\text { Number } \\
\text { Performed }\end{array}$ & $\begin{array}{c}\text { Number } \\
\text { Predictive } \\
\text { of Vascular } \\
\text { Ring }\end{array}$ & $\begin{array}{c}\text { Number } \\
\text { Predictive } \\
\text { of Specific } \\
\text { Type of } \\
\text { Vascular } \\
\text { Ring }\end{array}$ & $\begin{array}{c}\text { Percentage } \\
\text { Predive } \\
\text { of Vascular } \\
\text { Ring }\end{array}$ & $\begin{array}{c}\text { Percentage } \\
\text { Predictive } \\
\text { of Specific } \\
\text { Type of } \\
\text { Vascular } \\
\text { Ring }\end{array}$ \\
\hline CT Scan & 18 & 18 & 17 & $100 \%$ & $94.4 \%$ \\
MRI & 6 & 6 & 6 & $100 \%$ & $100 \%$ \\
Barium & 15 & 12 & 1 & $80 \%$ & $6.7 \%$ \\
$\begin{array}{l}\text { Esophagram } \\
\text { Echocardiography }\end{array}$ & 20 & 7 & 6 & $35 \%$ & $30 \%$ \\
$\begin{array}{l}\text { Bronchoscopy } \\
\text { Chest X-ray }\end{array}$ & 5 & 1 & 0 & $20 \%$ & $0 \%$ \\
\hline & 7 & 0 & 0 & 0 & $0 \%$ \\
\hline
\end{tabular}

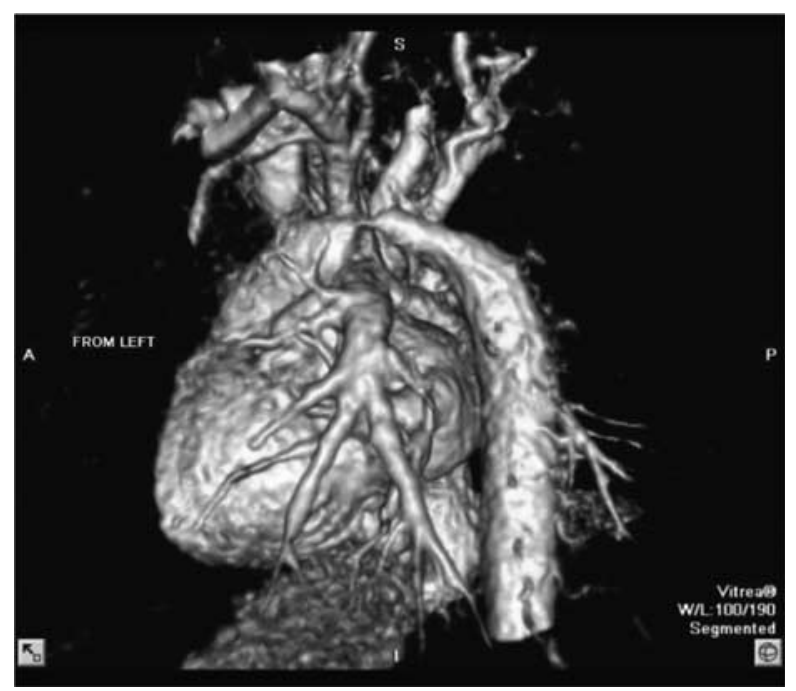

Results: Data indicated that age $<90$ days at time of treatment, weight $<6 \mathrm{~kg}$ at the time of treatment, and double aortic arch variant (DAA) were all factors predictive of increased LOV and LOS. CT and MRI were able to diagnose the specific type of malformation in $>94 \%$ of cases. Echocardiography and barium esophagraphy provided low sensitivity and specificity at detecting malformations. Finally, age of $<90$ days and DAA were predictive of increased post-operative tracheomalacia.

Conclusions: Age, weight, and specific malformation influence LOS, LOV, and cost of procedure. Specific symptoms were 
predictive of malformation type. DAA malformations have significantly increased rates of post-operative tracheomalacia. CT or MRI appear to be more effective means of diagnosis of vascular rings, while barium esophagraphy is a more appropriate screening modality.

7393

Total Anomalous Pulmonary Venous Connection: Outcome of surgical correction and long term follow up of 75 infants

Yasser Mohamed Menaissy, Faculty of Medicine.Cairo University, Egypt

Mohamed Rady Aboul Ezz, Faculty of Medicine. Cairo University, Egypt

Objective: We report a retrospective analysis of the demographic and clinical profiles of patients in order to assess the results and timing of operative repair for total anomalous pulmonary venous connection (TAPVC) and the long term follow up.

Methods: Between January 1998 and January 2008,75 patients underwent repair for total anomalous pulmonary venous connection. Ages ranged from 2 weeks to 1.6 years (mean 4 months). The mean weight was $5.4 \mathrm{~kg}$. The anomalous connection was supracardiac in $57(76 \%$ ) (including 3 cases in which only the left superior pulmonary vein connected to the vertical vein, 3 other veins connected to left atrium ), cardiac in $12(16 \%)$, infracardiac in $3(4 \%)$ and mixed in $3(4 \%)$ patient. Fifteen $(20 \%)$ patients had obstructed drainage and 24 patients (32\%) had moderate or severe pulmonary arterial hypertension. For supracardiac and infracardiac connections, transcardiac approach was used for anastomosis. In cardiac type, coronary sinus was unroofed and the resultant defect along with atrial septal defect was closed with a single patch.

Results: All the patients were operated upon using hypothermic CPB. There were 3(4\%) in-hospital deaths. Two patients died of pulmonary hypertensive crisis and one developed severe chest infection (died 3 weeks post-operatively). Follow-up ranged from 9 to 118 months (mean 68 months). There were no late deaths, but 2 patients developed venous obstruction and required balloon dilatation. Conclusions: Mortality rate has improved markedly in infants with TAPVC. Severe pulmonary arterial hypertension appears to be the most important predictor of operative mortality. Late referrals, severe malnutrition and delayed diagnosis or surgery possibly contribute to the mortality.

\section{4}

Emergency Arterial Switch Operation for Transposition of the Great Arteries as an alternative to ECMO

Jonathan G Mervis, Paediatric and congenital cardiac services Starship Children's Hospital, New Zealand

Kirsten Finucane, Starship Children's Hospital, New Zealand

John Beca, Paediatric Intensive Care Unit Starship Children's Hospital, New Zealand

Thomas Gentles, Paediatric and congenital cardiac services Starship

Children's Hospital, New Zealand

Background: A significant minority of newborns with Transposition of the Great Arteries (TGA) remain severely hypoxaemic despite Balloon Atrial Septostomy, prostaglandin and pulmonary vasodilators. We have adopted a strategy of urgent arterial switch operations (ASO) in these infants rather than ECMO.

Methods: Infants with TGA and an inability to achieve stable oxygen saturations $>65 \%$ and haemodynamic stability after intensive medical therapy were included in this study. Between 1996-2008 twenty-one (8\%) of 265 infants with TGA fitted this criteria. Pre-operative and operative management, and outcome were detailed for this subset. Results were compared to infants undergoing elective ASO and international data for cardiac infants treated with ECMO.

Results: There were two (9.5\%) early post operative deaths. Mean follow up was $3.4 \mathrm{yrs}$ with no late deaths. The mean ICU duration was 7.2 days and mean hospital stay 15 days. Two patients required post-operative ECMO. This compared to an overall early mortality for TGA of $2.3 \%$ and a survival of $38 \%$ in cardiac neonates treated with ECMO(international registry) over the same time period.

Conclusion: Emergency ASO rescues infants with TGA and severe hypoxaemia unresponsive to treatment and should be considered as an alternative to pre-operative ECMO.

\section{6}

Use of the "FIRST" score for patient selection during a humanitarian pediatric cardiac surgical mission to Mongolia

Kirk A Milhoan, Wilford Hall Medical Center, San Antonio Texas, United States

Ariuntsatsral Erdenebileg, Mongolia

Kimberly D Milhoan, UTHSCSA, San Antonio, Texas,

United States

Minette Son, UTHSCSA, San Antonio, Texas, United States

John P Kupferschmid, Texas Transplant Institue, San Antonio, Texas, United States

Background: The waiting list for children to receive cardiac surgery in developing countries is prohibitively long for humanitarian teams to meet all of the needs.

Objectives: We sought to develop a scoring system to identify those children for surgery that would best serve the short- and long-term goals of developing independent cardiac care in underserved countries.

Methods: Preselected children were screened by the pediatric cardiologist of the visiting team. The evaluation included: exam, echocardiogram, and assignment of a FIRST score (5-20 pts). F: Functional status (1-4 pts); I: Improvement after surgery (1-3pts); R: Risk of inoperability (1-4pts); S: Surgical risk (inversely awarded 1-4 pts); T: Teaching opportunities provided by the case (1-4pts). One point was given if an intangible reason for surgery existed. The score for an asymptomatic child with an ASD would be 8 .

Results: Seventy-three children were screened for ten surgical slots. Children with a higher FIRST score were given priority. The average FIRST score for the children chosen was 13.15 (11-15.5). Surgeries included repairs of six VSDs, three ASDs, and one PDA. All ten surgeries were successful. There were no major complications and none remained intubated $>24$ hours.

Conclusions: The "FIRST" score provides a system by which five separate considerations can be used to guide patient selection. The tool was successfully used to decrease patient selection bias, increase short-term success of the visiting team and improve the ability of international surgical teams to more effectively advance the proficiency of burgeoning programs in pediatric cardiac care.

\section{7}

Anatomical Repair for Pseudo-sequestration of the Lung: a Case Report

Yoshinori Miyahara, Department of Cardiovascular Surgery, Okayama University Graduate School of Medicine, Dentistry, and Pharmaceutical Sciences, Japan 
Shingo Kasahara, Department of Cardiovascular Surgery, Okayama University Graduate School of Medicine, Dentistry, and Pharmaceutical Sciences, Japan

Shunji Sano, Department of Cardiovascular Surgery, Okayama University Graduate School of Medicine, Dentistry, and Pharmaceutical Sciences, Japan

Unlike true sequestration, the patient with pseudo-sequestration has normal bronchial distribution and intact involved lung parenchyma, as termed anomalous systemic arterial supply to the normal basal segments of the lung. Therefore, in order to conserve optimal lung function, anatomical repair should be considered. However, pseudo-sequestration has been treated by surgical lobectomy or coil embolization.

Herein we present a case of 6-year-old boy with pseudosequestration in the left lower lung. We performed an anatomical anastomosis between the anomalous vessel and the pulmonary artery via a left thoracotomy. Six months after the operation, a lung perfusion scan showed a significant improvement in the affected lobe. Our case suggests clinical efficacy of the anatomical repair for this rare abnormality.

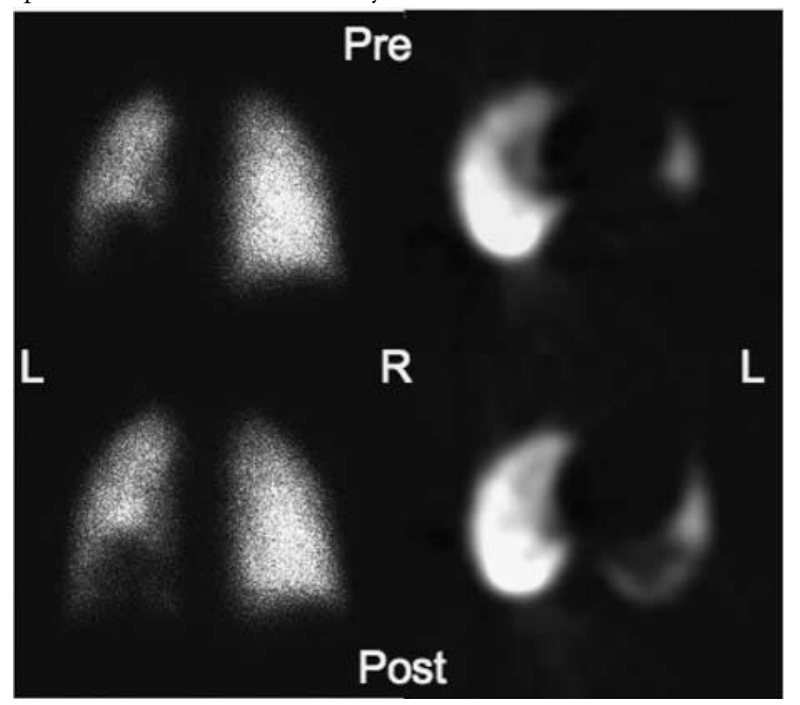

\section{8}

Successful Valve-Sparing Aortic Root Replacement in a 3-year-old child with Marfan Syndrome

Yoshinori Miyahara, Department of Cardiovascular Surgery, Okayama University Graduate School of Medicine, Dentistry, and Pharmaceutical Sciences, Japan

Masami Takagaki, Department of Cardiovascular Surgery, Okayama University Graduate School of Medicine, Dentistry, and Pharmaceutical Sciences, Japan

Shingo Kasahara, Department of Cardiovascular Surgery, Okayama University Graduate School of Medicine, Dentistry, and Pharmaceutical Sciences, Japan Shunji Sano, Department of Cardiovascular Surgery, Okayama University Graduate School of Medicine, Dentistry, and Pharmaceutical Sciences, Japan

Aortic root dilatation is rare in children, and often secondary to Marfan syndrome. Aortic root replacement for children under 5 -year-old is extremely rare. We experienced a case of 3-year-old boy $(92 \mathrm{~cm}, 12 \mathrm{~kg})$ with Marfan syndrome presenting dilated aortic root (the sinuses of Valsalva of $40 \mathrm{~mm}$ ). We electively performed a valve-sparing aortic root replacement using a $24 \mathrm{~mm}$ Gelweave Valsalva ${ }^{\mathrm{TM}}$ graft. Although the patient required mitral valve repair due to infective endocarditis postoperatively, the recovery from the second surgery was uneventful. The patient is currently doing well at home. This case is one of the youngest children of valve-sparing aortic root replacement in the literature.

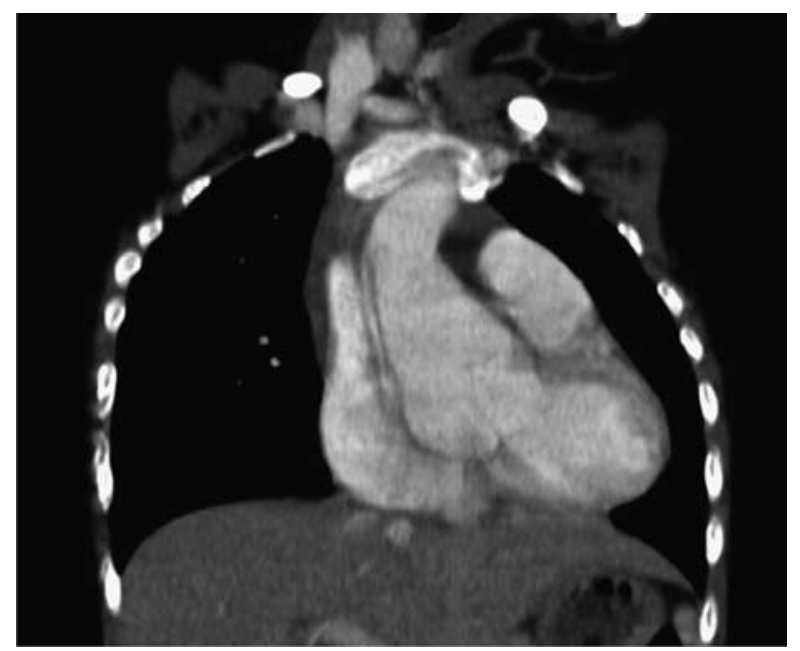

7399

Mechanical Mitral Valve Replacement in Children: single center experience

Murtada Mohamed, University of Khartoum, Sudan

Yassir Ali, University of Khartoum, Sudan

Abdalla Alamin, Ahmad Gasim Cardiac Center, Sudan

Hani Eltayeb, Ahmad Gasim Cardiac Center, Sudan

Abdelmoneim Abdellah, University of Khartoum, Sudan

Objectives: To study the outcome of mechanical valves in childrens with rheumatic mitral valve diseases in Ahmed Gasim Cardiac Center from January 2003 to December 2008.

Methodology: Retrospective descriptive hospital based study. The data was collected manually by the authors and analyzed by a computer programe (SPSS).

Results: Sixty four children underwent mitral valve replacement with mechanical valve, only one had a congenital mitral valve disease. $76.5 \%$ of the patients were between $12-15$ years with female predominance (57.8\%). Most of patients present with advanced disease and patients with NYHA III and IV constitute $64.1 \%$ of the study population. The duration of symptoms was 1-2years in $43.8 \%$. MR was the most common lesion (65.7\%), mitral stenosis $12.5 \%$ and combined mitral disease $17.2 \%$. Pulmonary artery pressure of $>50 \mathrm{mmHg}$ was detected in $56.3 \%$ but LVEF was $>40 \%$ in the majority (92.2\%). Mean cross clamp time was 63 minutes and mean bypass time 84 minutes. 25 patients received Sorin and 22 received St Jude mechanical valves. Three patient were explored for bleeding and another three had transient depression in LVF (4.7\%)with one in-hospital mortality. $70 \%$ of the population has no morbidities. Late mortality was $12.5 \%$.

Conclusion: Young patients present with advance rheumatic valve disease to our center. Valve repair was rarely feasible. Valve replacement with mechanical prosthesis prooved to have excellent outcome in short term follow up. However, long term results in developing countries yet to be determind.

\section{0}

Coronary arteries in long-term follow-up after arterial switch operation - morphometric study Maciej J Moll, Department of Cardiac Surgery, Polish Mother's Memorial Hospital-Research Institute, Poland 
Andrzej Sysa, Department of Cardiology, Polish Mother's Memorial Hospital, Research Institute, Lódz, Poland

Pawel Dryzek, Polish Mothers' Memorial Hospital, Lodz, Poland Katarzyna Mludzik, Department of Cardiology, Polish Mother's

Memorial Hospital, Research Institute, Lódz, Poland

Marek Kopala, Department of Cardiosurgery, Polish Mother's Memorial Hospital, Research Institute, Lódz, Poland

Tomasz Moszura, Department of Cardiology Polish Mother's Memorial Hospital-Research Institute, Lodz, Poland

Jadwiga A Moll, Department of Cardiology, Polish Mother's Memorial Hospital, Research Institute, Lódz, Poland

Jacek J Moll, Department of Cardiac Surgery, Polish Mother's Memorial Hospital-Research Institute, Poland

Background: Coronary transfer is the most crucial part of the arterial switch procedure. In $33 \%$ of all the patients with TGA atypical coronary pattern is found. Morphometric study of coronary arteries in patients 6 to 10 years after ASO was performed to assess the long-term outcome of the operation.

Material and method: Between 1992 and 2007496 children with TGA underwent arterial switch operation. In 136 of these patients (aged 6 to 10 years) selective coronary arteriography was performed. In each case coronary pattern was analyzed. Indexed diameter and cross-section area of LAD, Cx and RCA divided by BSA was measured.

Patients were divided into 3 groups:

1. TGA, IVS- $87 \mathrm{pts}$

2. TGA and VSD - 39pts

3. TGA with anomalies of the aortic arch -10 pts

Further, every group was divided into subgroup with typical (T) and anomalous (A)coronary anatomy.

The indexed diameter of LAD, Cx and RCA was compared between groups and subgroups.

Results: In group 1 there was a significant difference $(\mathrm{p}<0,001)$ in the indexed diameter of $\mathrm{Cx}$ between patients $\mathrm{T}$ and $\mathrm{A} . \mathrm{Cx} /$ BSA was higher in group A.

In group 2 there was a significant difference $(p<0,006)$ in the diameter of indexed RCA between patients $\mathrm{T}$ and $\mathrm{A}$. RCA/BSA was higher in group with $\mathrm{T}$.

Indexed diameter of RCA was significantly higher comparing groups 1 and 2 in patients with $\mathrm{T}$.

Conclusions: Size of the coronary arteries post ASO depends on coronary artery anatomy and the coexistence of VSD.

\section{1}

Reoperation for left atrioventricular valve regurgitation after repair of atrioventricular septal defect

Witold D Pietrzykowski, Department of Cardiac Surgery, Polish

Mother's Memorial Hospital-Research Institute, Poland

Jadwiga A Moll, Department of Cardiology, Polish Mother's Memorial Hospital-Research Institute, Poland

Maciej J Moll, Department of Cardiac Surgery, Polish Mother's

Memorial Hospital-Research Institute, Poland

Marek Kopala, Department of Cardiac Surgery, Polish Mother's

Memorial Hospital-Research Institute, Poland

Piotr Jarosik, Department of Cardiac Surgery, Polish Mother's Memorial Hospital-Research Institute, Poland

Jacek J Moll, Department of Cardiac Surgery, Polish Mother's Memorial Hospital-Research Institute, Poland

Objectives: Significant left atrioventricular valve regurgitation (LAVVR) after atrioventricular septal defect (AVSD) requires a reparative approach, finally valve replacement.
Aim of the study: Determine the outcomes of reoperations of LAVVR.

Methods: Between 1991-2008 we operated on 283 infants with complete form of AVSD (mean aged 4,3 months). Down's syndrome (DS) was diagnosed in 215 patients (76\%). Overall hospital mortality after AVSD repair was $8,8 \%$, the lowest in patients operated in second month of age $(4,6 \%)$.

Results: 13 patients (mean age 3,4 months) underwent first reoperation. There were 7 patients non-DS, 10,3\% of all non-DS and 6 DS patients, $2,8 \%$ of all DS $(p<0,05)$. Interval to reoperation was 1 week to 34 months (mean 10, median 5 months). In all patients partial clefts closures were performed (also Alfieri plasty in 1).

Second reoperation, i.e. valve replacement was required in 5 patients, 3 non-DS and 2 DS. Interval from first reoperation was 3 weeks to 6 months (mean 2,6, median 2 months). Significantly higher need for replacement was in non-DS $(4,4 \%)$ than in DS patients $(0,9 \%)(\mathrm{p}<0,05)$.

There was 1 hospital death after valve repair (patient underwent previously operation for oesophageal atresia). There were 4 hospital deaths after valve replacement, 3 in non-DS patients and 1 in DS patient. Definitive hospital mortality in reoperated patients was $5 / 13=38,5 \%$. Remaining patients had moderate or mild LAVVR and were clinically asymptomatic.

Conclusion: In this series non-DS patients had a significantly higher need for LAVVR reoperations and higher risk for complication after LAVV replacement.

\section{2}

No-patch technique for complete atrioventricular canal repair

Patrick O Myers, Division of Cardiovascular Surgery, Geneva

University Hospital and Medical School, Switzerland

Mustafa Cikirikcioglu, Division of Cardiovascular Surgery, Geneva

University Hospital and Medical School, Switzerland

Jorge Sierra, Division of Cardiovascular Surgery, Geneva University

Hospital and Medical School, Switzerland

Maurice Beghetti, Division of Pediatric Cardiology, Geneva University

Hospital and Medical School, Switzerland

Cécile Tissot, Division of Pediatric Cardiology, Geneva University

Hospital and Medical School, Switzerland

Yacine Aggoun, Division of Pediatric Cardiology, Geneva University

Hospital and Medical School, Switzerland

Afksendiyos Kalangos, Division of Cardiovascular Surgery, Geneva

University Hospital and Medical School, Switzerland

Background: Although the no-patch technique was the first described surgical treatment for complete atrioventricular canal (CAVC) by Lillihei in 1955, single or double patch techniques, which are more complex, are currently more widely used. We report our initial experience with this simple technique.

Methods: Eight consecutive patients underwent operation using the no-patch technique between April 2007 and August 2008. The CAVC was closed using pledgeted interrupted sutures placed on the right side of the VSD crest, passed through the bridging leaflet and to the facing part of the ostium primum defect, with closure of the left atrioventricular (AV) valve cleft and sliding plasty of the right AV septal leaflet.

Results: The median age was 11 months (4-58 months). The median weight was $5.2 \mathrm{~kg}$ (4.4-8.6). The mean VSD size was $8.4 \pm 1.8 \mathrm{~mm}$. Trisomy 21 was present in 7 patients. Cross-clamp and cardiopulmonary bypass were a mean of $52 \pm 10$ and $77 \pm 16$ minutes. Two patients required concomitant right $\mathrm{AV}$ valve annuloplasty. There were no early deaths and all patients 
were in sinus rhythm. On discharge echocardiogram, no patient presented a significant residual VSD or LVOT obstruction. One patient presented grade II left AV valve regurgitation and grade III right $\mathrm{AV}$ valve regurgitation; all others had no or trivial regurgitation. There were no late deaths or reoperations.

Conclusions: Surgical treatment of CAVC using the no-patch technique produces results comparable to single or double patch techniques, while avoiding the use of foreign material. These initial results are promising, but further studies are required.

\section{3}

Transatrial approach for operative repair of tetralogy of Fallot does not reduce but delays the need for secondary pulmonary valve replacement

Zsolt Nagy, Department of Thoracic, Cardiac and Vascular Surgery, University Hospital Tübingen, Germany

Gesa Wiegand, Germany

Renate Kaulitz, Department of Pediatric Cardiology, University

Hospital Tübingen, Germany

Michael Hofbeck, Germany

Gerhard Ziemer, Germany

Methods: Follow-up study of 101 patients operated with the diagnosis of uncomplicated tetralogy of Fallot (TOF) between 12.1994 and 12.2007. There were three perioperative deaths. From the 98 survivors $\mathrm{N}=55$ (56.1\%) were operated either via a classical transpulmonary/transanular, $\mathrm{N}=5(5.1 \%)$ via pure transventricular and $\mathrm{N}=38(38.8 \%)$ through a pure transatrial approach. Intraoperatively the pulmonary valve was either resected during the transanular repair, or progressively torn with Hegar dilators in all other cases. 6 patients were palliated preoperatively, either with a shunt $(\mathrm{N}=3)$ or a palliative balloon pulmonary valvuloplasty $(\mathrm{N}=3)$.

Results: The transatrially operated patients were older than those operated by a non-transatrial approach (11.82 vs 5.48 months $\mathrm{p}=0.037)$. Over an average follow-up of 79.94 months $(\mathrm{SD}=50.97) 18$ patients $(18.3 \%)$ required secondary pulmonary valve replacement (16 pulmonary homografts and 2 xenografts). The incidence of the PVR was not significantly different between the various approaches $11 / 55$ in the transanular vs $7 / 43$ in the non-transanular group $(\mathrm{p}=0.79)$, however, PVR was required earlier postoperatively in the transanular group than in the non-transanular patients (69.9 vs 126.8 months $\mathrm{p}=0.02$ ). Age alone (independent of the surgical approach) at the primary operation did not predict the need for PVR either: $1 / 11$ neonates vs $17 / 87$ nonneonates $(p=0.35)$ or $8 / 39$ vs $11 / 59$ $(\mathrm{p}=0.42)$ in young infants $(<6$ months old $)$ vs older patients.

Conclusion: Secondary pulmonary valve replacement is required in a significant proportion of surgically corrected TOF. Transatrial approach does not significantly reduce the incidence of the PVR, although in our series it significantly delays it.

\section{4}

Residual cleft: the only cause for reoperation on the left $\mathrm{AV}$ valve after surgical repair of common atrioventricular canal

Zsolt Nagy, Department of Thoracic Cardiac and Vascular Surgery,

University Hospital Tübingen, Germany

Gesa Wiegand, Germany

Renate Kaulitz, Department of Pediatric Cardiology, University

Hospital Tübingen, Germany

Michael Hofbeck, Germany

Gerhard Ziemer, Germany
Methods: Retrospective study of 80 consecutive patients operated upon with the diagnosis of CAVC between 1994 and 2006. 39 of them had complete and 41 had incomplete CAVC. For the complete form the single patch technique with autologous was preferentially used. Complete cleft closure was performed if no atypical valvular anomaly precluded it. Follow-up Doppler echocardiographic examinations performed at 1month, 3 months, one year, postoperatively as well as the latest examination (total mean FU 58.3 months for complete and 48.6months for the incomplete AV canals) were reviewed.

Results: Perioperative mortality was $1 / 39$ in the complete and $1 / 41$ in the incomplete forms. Reoperation rate for left AV valve insufficiency (LAVVI) was $3 / 39(7.6 \%)$ for complete and 1/41 $(2.4 \%)$ for incomplete forms respectively. In the survivors the pattern of the LAVVI was stable in 44, progressive in 19 , regressive in 5 and undulating in 9 patients. From the 4 reoperated patients due to LAVVI (all produced by incomplete cleft closure and successfully reoperated with additional cleft sutures) 3 showed a progressive pattern and one an undulating pattern.

Conclusions: The majority of the patients operated upon with CAVC did not progress to reoperation on LAVVI, showing a stable or regressive pattern of the LAVVI postoperatively. About $25 \%$ show a slight worsening over the time, this progression not being associated with any particular identifiable factor. An incomplete cleft closure, which was not perceived as such by the surgeon at the time of the repair, was the single cause of the 4 reoperations.

\section{5}

Use of Temporary Left Ventricular Mechanical Circulatory Support after Repair of Anomalous Left Coronary Artery from the Pulmonary Artery

Boris A Nasseri, Deutsche Herzzentrum Berlin, Germany

Vladimir Alexi-Meskishvili, Deutsche Herzzentrum Berlin, Germany

Boris Schmitt, Deutsche Herzzentrum Berlin, Germany

Sarah Eder, Deutsche Herzzentrum Berlin, Germany

Yu-Guo Weng, Deutsche Herzzentrum Berlin, Germany

Christof Stamm, Deutsche Herzzentrum Berlin, Germany

Felix Berger, Deutsche Herzzentrum Berlin, Germany

Roland Hetzer, Deutsche Herzzentrum Berlin, Germany

Background: Recovery of the left ventricular (LV) function after direct aortic re-implantation of the left coronary artery (LCA) for anomalous left coronary artery from pulmonary artery (ALCA$\mathrm{PA}$ ) is well described. In some patients with severe LVdysfunction temporary LV mechanical circulatory support (MCS) could be necessary. We report the mid-term results in 14 infants after ALCAPA-repair.

Methods: Fourteen infants (median age 4.2, range 3-12 months) underwent direct aortic re-implantation of the LCA. Seven patients had severe mitral regurgitation, and 7 had severe LVdysfunction. Six patients (LVAD-group) needed temporary MCS support after unsuccessful weaning from $\mathrm{CPB}$.

Results: All infants survived the operation, were alive at follow-up (median follow-up 10.4, range 1.4-17 years) and free of reoperation. Median preoperative LVEF (28 vs. 43\%) and LV-FS ( 9 vs. $22 \%)$ were significant lower $(\mathrm{p}=0.0119$ and $\mathrm{p}=0.0019)$, and LVEDD (47 vs. $32 \mathrm{~mm}$ ) and LVEDP (20 vs. $12 \mathrm{mmHg}$ ) were significant higher in the LVAD-group compared with the Non-LVAD-group $(p=0.0017$ and $p=0.0202)$. MCS support ranged from 4-12 days. Eleven patients had follow-up evaluation and all showed widely patent LCA anastomosis. LVEF had further improved compared to discharge with no significant different between groups $(p=0.3015)$. MRI evaluation in 
10 patients showed a perfusion deficits in $30 \%$, wall motion abnormalities in $50 \%$ and scar formation in $40 \%$ of the patients. Conclusion: Direct aortic re-implantation for ALCAPA even using temporary MCS can be performed with low mortality in infants. However, all patients need regular life-long evaluation after ALCAPA repair, especially after temporary MCS support due to myocardial damage.

7406

Aortic arch reconstruction using continuous low-flow cerebral perfusion without total circulatory arrest Mohammad Ali Navabi, Department of cardiac surgery, Tehran University of medical science, Iran

Hussein Rayatzadeh, Department of cardiac surgery, Tehran University of medical science, Iran

Objective: Aortic arch reconstruction in neonates routinely requires deep hypothermic circulatory arrest. The use of deep hypothermic circulatory arrest (DHCA) has played an important role in developing successful repair techniques for complex congenital heart disease. However, there have been a trend away from the use of DHCA because of the potential adverse neurological out come. We reviewed our experience with techniques of continuous low-flow cerebral perfusion (LFCP) avoiding total circulatory arrest.

Method: Since 2003, eight patients requiring aortic arch reconstruction were operated using continuous low-flow cerebral perfusion (LFCP). Intacardiac lesions when present were corrected during rewarming.

Results: Our initial limited experience with continuous low-flow cerebral perfusion (LFCP) is promising. We had no mortality and no neurological sequel in this small group.

Conclusion: Continuous low-flow cerebral perfusion (LFCP), avoids staged operation, when intracardiac lesions associates with aortic arch anomalies. Regional perfusion or continous low-flow cerebral perfusion is feasible and can be used with acceptable results. It may reduce potential complications following aortic arch reconstruction using circulatory arrest.

\section{8}

Polyethileneglycol: a novel tissue preservation towards improvement of bioprosthesis durability in children

Vinicius S Nina, University Hospital of The Federal University of Maranhão, São Luis-MA, Brazil, Brazil

Rachel V Haickel Nina, University Hospital of The Federal University of Maranhão, São Luis-MA, Brazil, Brazil

Vinicius G Mendes, University Hospital of The Federal University of Maranhão, São Luis-MA, Brazil, Brazil

Eduardo C Ferreira, University Hospital of The Federal University of Maranhão, São Luis-MA, Brazil, Brazil

Eduardo D Figueredo, University Center of Maranhão, UNICEUMA, São Luis-MA, Brazil, Brazil

José C Sampaio Filho, University Center of Maranhão, UNICEUMA, São Luis-MA, Brazil, Brazil

José Anselmo C Lopes, University Hospital of The Federal University of Maranhão, São Luis-MA, Brazil, Brazil

André Luis C Cantanhede, University Hospital of The Federal

University of Maranhão, São Luis-MA, Brazil, Brazil

Objective: To compare the acute inflammatory reaction and late calcific degeneration of bovine pericardium implants with two different tissue preservation.

Methods: Isolated segments of $1.0 \mathrm{~cm} 2$ of bovine pericardium were implanted subcutaneously in the paravertebral region of young rats which were divided into 4 groups consisting of 10 animals each: Group A and $\mathrm{A}^{1}$ (control - glutaraldehyde) and Group B and $\mathrm{B}^{1}$ (test: polyethyleneglycol - PEG). At the end of 1 and 8 weeks animals of groups $\mathrm{A}$ and $\mathrm{B}$ and $\mathrm{A}^{1}$ and $\mathrm{B}^{1}$ were sacrificed respectively, and the grafts removed for histological analysis with hematoxylin-eosin (for inflammation) and von Kossa (for calcification). For statistical analysis the Fisher's exact test was used.

Results: Some degree of inflammation was observed in all grafts with both tissue preservation; however, the collagen structure was preserved in $70 \%$ of the samples treated with PEG compared with $100 \%$ in the control group $(p=0.21)$. After 8 weeks calcification was observed in $30 \%$ of the grafts treated with PEG versus $90 \%$ of the segments treated with glutaraldehyde $(p=0.03)$.

Conclusion: The features of acute inflammatory reaction were similar with both tissue preservation; however, PEG seems to provide a greater resistance to calcification which ultimately could guarantee a long-term durability of a bioprosthetic material when compared to conventional preservation with glutaraldehyde.

\section{9}

Surgery for rheumatic valvulopathy in children: what we learned from the experience of a public hospital in a developing country

Vinicius S Nina, University Hospital of The Federal University of Maranhão, São Luis-MA, Brazil, Brazil

Rachel V Haickel Nina, University Hospital of The Federal University of Maranhão, São Luis-MA, Brazil, Brazil

Danila S Pacheco, University Hospital of the Federal University of Maranhão, São Luis-MA, Brazil, Brazil

Leonardo S Carneiro, University Hospital of the Federal University of Maranhão, São Luis-MA, Brazil, Brazil

carlos Eduardo E Machado, University Hospital of the Federal

University of Maranhão, São Luis-MA, Brazil, Brazil

Hilton $S$ Guimarães Jr, University Hospital of the Federal University of Maranhão, São Luis-MA, Brazil, Brazil

Diego Rafael B Leite, University Hospital of the Federal University of Maranhão, São Luis-MA, Brazil, Brazil

Karina S Luz, University Hospital of the Federal University of

Maranhão, São Luis-MA, Brazil, Brazil

Objective: To analyze the surgical outcomes of rheumatic valvular heart disease in children assisted in a public south-american hospital.

Methods: From January 2003 through June 2008 a retrospective study was performed with 50 children ( 33 female) with mean age of 13.4 years and mean weight of $35.9 \mathrm{Kg}$ who were submitted to surgical treatment of rheumatic valvular heart disease; of whom 37 had univalve (4 reoperations) and 13 multivalve disease. Operations were performed under cardiopulmonary bypass and consisted on insertion of a bioprosthesis in $72 \%$ (36), valvuloplasty in $22 \%$ (11), mechanical valve implant in $4 \%$ (2) and a combined procedure in $2 \%$ (1). Logistic regression model was applied to identify predictors of mortality.

Results: In-hospital mortality was 10\% (5 cases). There were no late deaths. The average bypass and cross-clamp times were 109.1 and 63.6 minutes respectively. The mean length of hospital stay was 15.7 days. The morbidity rate was $40 \%$. The major morbid events were: bleeding(8), hypotension (4) and arrhythmia (3). Multivariate analysis showed that preoperative heart failure, age under 11 years and weight under $28 \mathrm{Kg}$ were independent risk factors for mortality in this population $(\mathrm{p}<0.05)$.

Conclusion: Rheumatic heart disease in children is a multifaceted and challenging malady specially when requires an operation because other factors rather than the pathology itself may 
interfere in the outcome such as: demography (age/weight) and socio-economic status.

7410

Experience of utilization of pedicled autologous pericardium as a viable material for extracardiac total cavo-pulmonary connection: Long term results

Takako Nishino, Department of Cardiovascular Surgery,

Kinki University School of Medicine, Japan

Hitoshi Kitayama, Department of Cardiovascular Surgery,

Kinki University School of Medicine, Japan

Toshihiko Saga, Department of Cardiovascular Surgery, Kinki

University School of Medicine, Japan

Kousuke Fujii, Department of Cardiovascular Surgery, Kinki University

School of Medicine, Japan

Shintarou Yukami, Department of Cardiovascular Surgery, Kinki

University School of Medicine, Japan

Back ground: Although extracardiac total cavo-pulmonary connection (eTCPC) eliminates the potential problems which standard intracardiac TCPC has, prosthetic extracardiac conduit has the risk of obstruction because of peel formation and no potential for growth. We have used pedicled autologous pericardium as a viable material to create IVC-pulmonary circulation. At present study, we evaluate the long term results of the eTCPC using pedicled pericardium.

Patients: Between 1996 and 2008, 10 patients (1.5-11 years: the median 2.5 years, 7 univentricular heart (UVH), and 3 tricuspid atresia) underwent eTCPC using pedicled pericardium. Pedicled pericardium was used in 7 as extracardiac tube graft, and in 3 as extracardiac lateral tunnel, which made with pedicled pericardial flap and atrial wall. In 2 patients with an apicocaval juxtaposition, pericardial conduits were placed behinde the ventricle. A fenestration was created in 8 patients. All patients had early postoperative cardiac catheterization, and 8 patients underwent follow-up catheterization 2 to 9 years after surgery. The mean follow-up period was $10.0 \pm 1.7$ years $(8.2-12.8$ years).

Results: No patient showed conduit obstruction. Mid-term angiography showed that the IVC-PA conduits did not show remarkable increase in diameter but did grow in length with the passage of time.

Conclusions: Pedicled pericardium might have potential for growth. The IVC-PA conduits lengthened with time. Therefore, it should be expected avoiding re-operation as patients grew long term after eTCPC.

\section{1}

Surgical repair of truncus arteriosus communis with absent right pulmonary artery in a newborn

Matej Nosal, Childrens Heart Centre Slovak Republic, Slovak Republic Rudolf Poruban, Childrens Heart Centre Slovak Republic, Slovak Republic

Pavel Valentik, Childrens Heart Centre Slovak Republic, Slovak Republic

Ikenna Chima Omeje, Childrens Heart Centre Slovak Republic, Slovak Republic

Michal Sagat, Childrens Heart Centre Slovak Republic, Slovak Republic

Introduction: We present a HDV video of surgical repair of a type A 3 truncus arteriosus communis in a $3 \mathrm{~kg}$ newborn. The view is from patient's head.

Methods: A newborn was admitted to the Children's Heart Centre Slovakia with episodes of desaturation and hypercapnia.
On echo a truncus arteriosus communis type A3 with absent right pulmonary artery was diagnosed. On cathetrisation only the hilar part of the right pulmonary artery was found. The right pulmonary artery was connected to a right sided ductus, which had spontaneously closed. The patient was referred for complete repair. During the operation the conal defect was closed through a right ventriculotomy with a dacron patch by continuous suture. The left pulmonary artery was excised with a button of surrounding tissue from ascending aorta. The opening in the aorta was closed by direct suture. The hilar part of the right pulmonary artery was mobilised and anastomosed in an end to end fashion to the mobilised left pulmonary artery. The right ventriculotomy was connected to the inferior margin of the pulmonary bifurcation by an $11 \mathrm{~mm}$ pulmonary homograft. The foramen ovale was left patent.

Results: In the postoperative course the patient recieved sildenafil for episodes of pulmonary hypertension and was extubated on the 6 th postoperative day. The patient was discharged home on the 18th postoperative day with patent pulmonary arteries and a patent outflow tract from the right ventricle.

Conclusion: Good functional result can be achieved by complete surgical repair of truncus arteriosus type A 3 even in a neonate.

\section{2}

Ross-Konno operation for congenital aortic stenosis in a $2,9 \mathrm{~kg}$ infant

Matej Nosal, Childrens Heart Centre Slovak Republic, Slovak Republic Pavel Valentik, Childrens Heart Centre Slovak Republic,

Slovak Republic

Michal Sagat, Childrens Heart Centre Slovak Republic, Slovak Republic

Ikenna Chima Omeje, Childrens Heart Centre Slovak Republic, Slovak Republic

Rudolf Poruban, Childrens Heart Centre Slovak Republic, Slovak Republic

Introduction: We present a HDV video of a Ross-Konno procedure for congenital aortic stenosis in a $2,9 \mathrm{~kg}$ infant. The view is from patient's head.

Methods: A prenatally diagnosed newborn was admitted to our centre with signs of critical aortic stenosis. He underwent balloon valvuloplasty of aortic valve with a decrease in gradient from 100 to $45 \mathrm{~mm} \mathrm{Hg}$. After 1,5 months the patient was readmitted with failure to thrive and a rise in the gradient on aortic valve to $90 \mathrm{~mm} \mathrm{Hg}$, with 1st degree aortic insufficiency and 2nd degree mitral insufficiency. During the operation a thickened aortic valve with a $5,5 \mathrm{~mm}$ annulus was found. The valve was excised and the annulus was enlarged by conal incision. After excision, the pulmonary autograft was implanted onto the neoaortic annulus. The right ventricular outflow tract was reconstructed using an $11 \mathrm{~mm}$ pulmonary homograft. In the postoperative course the patient was extubated after 96 hours.

Results: The patient was discharged home on the 14th postoperative day with no gradient across the aortic valve with trace aortic insufficiency. The left ventricular function has improved. Between 10/2006-10/2008 we have used the Ross-Konno operation in 5 newborns with critical aortic stenosis. The median age was 11 days (2-53), median weight $3.3 \mathrm{~kg}(3.2-3.8)$ and median aortic annulus was $6 \mathrm{~mm}(4-10)$. At median follow up of 1.5 years all patients are alive in a good functional status.

Conclusion: Good functional result can be achieved by the RossKonno procedure even in very small babies. 
7413

Cor triatriatum sinister with persistent left superior vena cava and dilated coronary sinus: a novel surgical approach

Emmanouil Tsounias, University of Texas Health Science Center at Houston, United States

Monesha Gupta-Malhotra, University of Texas Health Science Center at Houston, United States

Georgios Hartas, University of Texas Health Science Center at Houston, United States

Felix $W$ Tsai, University of Texas Health Science Center at Houston, United States

Cor triatriatum is a congenital anomaly in which the left atrium is divided into two parts by a fold of tissue, a membrane or a fibromuscular band. Rarely, it is associated with a persistent left superior vena cava draining into a dilated coronary sinus, which can cause left ventricular inflow obstruction. Even after standard surgical resection of the cor triatriatum membrane, obstruction can persist. We report the case of a 21 year old female with cor triatriatum, diagnosed on routine physical exam. She underwent a novel approach, combining concomitant membrane resection with unroofing and patch enlargement of the coronary sinus, preserving the left superior vena cava. We present a review of this rare entity and the treatment options.

\section{4}

Perioperative Risk Factors in Reoperations for Adults with Congenital Heart Disease

Halkawt A Nuri, IRCCS Policlinico San Donato, Milan, Italy, Italy Alessandro Giamberti, IRCCS Policlinico San Donato, Milan, Italy, Italy

Massimo Chessa, IRCCS Policlinico San Donato, Milan, Italy, Italy Jaspal S Dua, IRCCS Policlinico San Donato, Milan, Italy, Italy Carlo De Vincentiis, IRCCS Policlinico San Donato, Milan, Italy, Italy

Raul Abella, IRCCS Policlinico San Donato, Milan, Italy, Italy Mario Carminati, IRCCS Policlinico San Donato, Milan, Italy, Italy Alessandro Frigiola, IRCCS Policlinico San Donato, Milan, Italy, Italy

Objectives: Congenital cardiac surgery in adults with congenital heart disease (ACHD) presents major difficulties in management and technique. Although, reoperations are more frequent than primary surgery in these patients, limited knowledge exists on perioperative risk factors associated with reoperations.

Methods: From January 2002 to December 2007, 164 ACHD (mean age $\pm \mathrm{SD}: 31 \pm 12.4$ years) underwent reoperation in our institute. All the pre, intra, and postoperative data were retrospectively analysed in order to identify perioperative risks factors for mortality and morbidity. The most frequent reoperations performed were: pulmonary valve implantation $n=43$, conduit replacement $n=17$, residual ASD closure $n=16$, various aortic valve procedures $n=15$, conversion to modified Fontan circuit $\mathrm{n}=13$ and Bentall operation $\mathrm{n}=12$.

Results: Early mortality was $3.6 \%(\mathrm{n}=5)$. Mean mechanical ventilation time was 26 hours; mean intensive care stay was 3.1 days; mean postoperative hospital stay was 8.2 days. Serious postoperative complications occurred in 24 patients (15.1\%). Cardiopulmonary by-pass time $(\mathrm{p}=0.001)$, correction after palliation $(p=0.004)$, preoperative hematocrit $(p=0.004)$, previous number of operations $(p=0.01)$ and the presence of preoperative congestive heart failure $(p=0.02)$ were significant perioperative risk factors for severe morbidity. No significant perioperative risk factors associated with mortality were found.
Conclusions: Reoperations in ACHD are mostly for right ventricular outflow tract lesions and can be associated with low risk if performed meticulously and in larger centres with high volume of reoperations. Important perioperative risk factors for morbidity remain high hematocrit (cyanosis), congestive cardiac failure, number of previous operations, long standing palliated heart and long cardiopulmonary by-pass time.

\section{6}

Outcomes after surgical treatment of children with partial anomalous pulmonary venous connection to the superior vena cava

Yoshie Ochiai, Kyusyu Koseinenkin Hospital, Japan

Yutaka Imoto, Kyusyu Koseinenkin Hospital, Japan

Masato Sakamoto, Kyusyu Koseinenkin Hospital, Japan

Akira Sese, Kyusyu Koseinenkin Hospital, Japan

Mamie Watanabe, Kyusyu Koseinenkin Hospital, Japan

Tetsuji Yuge, Kyusyu Koseinenkin Hospital, Japan

Takuro Ohno, Kyusyu Koseinenkin Hospital, Japan

Kunitaka Joo, Kyusyu Koseinenkin Hospital, Japan

Objective: Repair of anomalous pulmonary venous connection (PAPVC) to the superior vena cava (SVC) using an internal patch may be complicated by obstruction of the SVC or pulmonary veins (PV), or both, and sinus node dysfunction. Since 1996, we have adopted the Williams' method in which the SVC is divided and the proximal end is anastomosed to the right atrial appendage, and the distal SVC serves as a conduit for pulmonary drainage to the left atrium through the atrial septal defect (ASD). We retrospectively compared the results of the patch repair versus the Williams' method.

Methods: Between 1985 and 2007, 19 patients who underwent surgical treatment of PAPVC to the SVC were included. Mean age was 10.5 years (range, 1.5 to 41 ). In our early 7 patients, internal tunnel patch was used to close the ASD and reroute the anomalous PV into the left atrium, and another patch was used for enlargement of SVC and right atrial wall (double patch method). One patient was treated by only an internal tunnel patch, and 11 patients underwent the Williams' method.

Results: There were no early or late deaths. Postoperative obstruction of the SVC and the right superior PV was recognized in two patients who underwent the double patch method. One of these patients showed sick sinus syndrome. In contrast, 11 patients with the Williams' method did not show sinus node dysfunction or any obstruction of SVC or PV.

Conclusions: The Williams' method appears to be safe and effective procedure without any complication.

7417

Staged surgical repair for single ventricle with systemic outflow tract obstruction

Shinichiro Oda, Department of cardiovascular Surgery, Fukuoka Children's Hospital, Fukuoka, Japan

Toshihide Nakano, Department of cardiovascular Surgery, Fukuoka Children's Hospital, Fukuoka, Japan

Kazuhiro Hinokiyama, Department of cardiovascular Surgery, Fukuoka Children's Hospital, Fukuoka, Japan

Takahisa Sakurai, Department of cardiovascular Surgery, Fukuoka Children's Hospital, Fukuoka, Japan

Kunihiko Joo, Department of cardiovascular Surgery, Fukuoka Children's Hospital, Fukuoka, Japan

Hideaki Kado, Department of cardiovascular Surgery, Fukuoka Children's Hospital, Fukuoka, Japan 
Objectives: We review our experience with initial pulmonary artery banding (PAB) followed by a Damus-Kaye-Stansel procedure with bidirectional Glenn (BDG) in the subset of patients with functional single ventricles (SV) and systemic outflow tract obstructions (SOTO) to determine if this staged operative strategy influences the outcome of Fontan completion. Methods: Between January 1990 and October 2008, 27 patients with functional SV with SOTO were included. Our criteria for initial $\mathrm{PAB}$ in these patients are over $1 \mathrm{~mm}$ plus body weight in diameter of SOTO. Seventeen patients had concomitant aortic coarctation or interrupted aortic arch repair (63\%).

Results: The overall mortality was $11 \%$ (three of 27 patients) with a mean follow up period of $7.5 \pm 1.0$ years (range, 3 months to 18 years). Significant development of SOTO was recognized in 2 patients $(7 \%)$ after an initial $\mathrm{PAB}$. The other patient's average pressure gradient across SOTO before DKS + BDG procedure was $6.7 \pm 1.5 \mathrm{mmHg}$. Twenty-five patients $(25 / 27,93 \%)$ have undergone DKS+BDG procedure. Twenty patients (20/27, $74 \%$ ) have undergone the completion Fontan procedure and 4 patients are awaiting the next stage. Cardiac catheterization data (CVP $9.7 \pm 0.5 \mathrm{mmHg}$, EDP $4.2 \pm 0.6 \mathrm{mmHg}, \mathrm{SaO} 294.7 \pm 0.2 \%$ ) and cardiorespiratory response to exercise data (peak VO2: $92.5 \pm 7.4 \%$ of normal) after Fontan completion revealed sufficient results of this approach.

Conclusion: In patients with subpopulation of functional SV with SOTO, an initial PAB coupled with planned DKS+BDG procedure can be an acceptable operative strategy.

\section{8}

Long-term Results of Conotruncal Repair for Tetralogy of Fallot - 20 Years Tokyo Experiences

Toru Okamura, Department of Cardiovascular Surgery, Tokyo Women's Medical University, Japan

Takahiko Sakamoto, Department of Cardiovascular Surgery, Tokyo

Women's Medical University, Japan

Naruhito Watanabe, Department of Cardiovascular Surgery, Tokyo

Women's Medical University, Japan

Takuma Miyamoto, Department of Cardiovascular Surgery, Tokyo

Women's Medical University, Japan

Takeshi Konuma, Department of Cardiovascular Surgery, Tokyo

Women's Medical University, Japan

Masahide Komagamine, Department of Cardiovascular Surgery, Tokyo

Women's Medical University, Japan

Toshio Nakanishi, Department of Pediatric Cardiology, Tokyo Women's Medical University, Japan

Hiromi Kurosawa, Department of Cardiovascular Surgery, Tokyo

Women's Medical University, Japan

Objective: Conotruncal repair for tetralogy of Fallot (TOF) was introduced in 1985 to achieve low CVP, no heart murmur, and no conduction disturbance. The purpose of this study was to evaluate the long-term results of this procedure.

Patients and Methods: One hundred forty-three (143) TOF patients underwent conotruncal repair with hand-made monocuspid patch between October 1985 and January 1991. Patients with pulmonary atresia were excluded. The age of patients ranged from 2 months to 27 years (mean; 4.2 years).

Results: There was no early death. Two late deaths occurred over a mean follow-up period of 17.8 years (CHF, pneumonia). There were five reoperations (residual PAPVC repair (2), pacemaker implantation for preoperative II AV block (2), residual VSD (1)). No patient required reoperation for residual outflow reconstruction and new onset AV block. Cardiac catheterization in the midterm (1 month-5.7 years) revealed $\mathrm{RVp}$ of
$49.5 \pm 12.9 \mathrm{mmHg}, \quad \mathrm{CVP}$ of $9.0 \pm 2.8 \mathrm{mmHg}$, and CI of $3.8 \pm 0.9 / \mathrm{min} / \mathrm{m} 2$. In the long-term period, chest X-ray showed CTR of $51.8 \pm 4.4 \%$, and ECG revealed PQ interval of $0.17 \pm 0.03 \mathrm{msec}$ and QRS of $0.12 \pm 0.03 \mathrm{msec}$. All patients were in sinus rhythm, except for 4 patients of I AV block, and 3 of II AV block. Echocardiogram showed RV outflow pressure gradient of $16.7 \pm 9.0 \mathrm{mmHg}$, estimated $\mathrm{RVp}$ of $39.9 \pm$ $8.1 \mathrm{mmHg}$, moderate PR in 25 patients, and moderate TR in 2. All patients were in NYHA class I.

Conclusion: Conotruncal repair for TOF has provided excellent long-term outcomes with minimum incidence of morbidity. The further careful observation is mandatory.

\section{9}

Right Ventricular Outflow Tract Reconstruction using the Freestyle Porcine Aortic Root Stentless Bioprosthesis in adult and paediatric patients

Yishay Orr, Royal Perth Hospital, Australia

Andrew Bullock, Princess Margaret Hospital, Australia

David R Andrews, Princess Margaret Hospital and Royal Perth

Hospital, Australia

Objective: The optimal conduit for reconstruction of the right ventricular outflow tract (RVOT) in patients with congenital heart disease remains controversial. The Freestyle stentless aortic root bioprosthesis, which offers potential advantages including accessibility, extensive size range and anti-calcification treatment, has been used increasingly for this indication however the outcome for this strategy of RVOT reconstruction remains to be defined.

Methods: Outcomes for patients undergoing RVOT reconstruction using Freestyle stentless aortic root bioprostheses in Perth, Australia from December 2000 to September 2008 were retrospectively reviewed. Clinical data was acquired prospectively and all patients underwent routine post-operative follow-up by a cardiologist and cardiothoracic surgeon.

Results: 61 patients (mean age $23.6 \pm 2.3 \mathrm{yr}$; 59\% male) underwent 65 pulmonary valve replacements using Freestyle aortic root bioprotheses (mean size $24.2 \pm 0.5 \mathrm{~mm}$ ) for heterogenous indications (pulmonary regurgitation $(n=29)$, RV-PA conduit stenosis $(n=23)$, pulmonary stenosis $(n=6)$, pulmonary atresia $(\mathrm{n}=5)$, truncus arteriosus $(\mathrm{n}=1)$ and other $(\mathrm{n}=1))$. Operative mortality was $0 \%$ however overall mortality was $4.9 \%(n=3)$ during follow-up. No episodes of thromboembolism occurred and redo surgery was required in 4 patients (endocarditis $(n=2)$, conduit stenosis $(\mathrm{n}=2)$ ). Peak pulmonary valve gradient during short to medium term follow-up was $23.8 \pm 1.9 \mathrm{mmHg}$.

Conclusion: The Freestyle stentless aortic root bioprosthesis is an effective conduit for reconstructing the RVOT in a heterogenous patient cohort with diverse native or prosthetic pulmonary valve pathology. Longer follow-up is required to accurately determine the lifespan of this bioprosthesis in the pulmonary position, particularly in relation to valve and patient size.

\section{0}

Atrioventricular valve repair in Fontan candidates Yoshihiro Oshima, Department of Cardiovascular Surgery, Kobe Children's Hospital, Kobe, Japan, Japan

Ayako Maruo, Department of Cardiovascular Surgery, Kobe Children's Hospital, Kobe, Japan, Japan

Chikashi Shimazu, Department of Cardiovascular Surgery, Kobe Children's Hospital, Kobe, Japan

Tomonori Higuma, Department of Cardiovascular Surgery, Kobe Children's Hospital, Kobe, Japan 
Takeshi Inoue, Department of Cardiovascular Surgery, Kobe Children's Hospital, Kobe, Japan

Background: Significant atrioventricular valve (AVV) insufficiency has been associated with increased mortality and morbidity in Fontan candidates. The outcomes of patients undergoing AVV repair during Fontan palliation were reviewed to determine the optimal technique and timing of repair.

Methods: We retrospectively reviewed our experience with AVV repair in Fontan candidates from July 2004 to June 2008.

Results: Of the 15 first-time AVV repairs (8 Tricuspid valves, 7 common AV valves), 3 were performed in neonates, 7 at bidirectional Glenn, 1 at fenestrated Kawashima in 3-month-old infant, 2 after Glenn and 2 at Fontan. The median age at the first AVV repair was $11(0.1-131)$ months. Edge to edge repair was performed in 9 patients and semicircular annuloplasty in 6 . As additional procedures, Norwood (1), primary sutureless repair of TAPVC (1) and PA plasty with RV-PA shunt (1) were performed respectively in 3 neonates. Preoperatively, the median echocardiographic severity of AVV regurgitation was grade 3 (range: $2-4)$. At median follow up of 30 months (5-52 months) the severity of AVV regurgitation was reduced significantly to median grade 2 (range: $2-3 ; p<0.001$ ). Six patients required a reoperation for AVV regurgitation; 1 at Glenn, 3 pre-Fontan, 2 at Fontan. Thereafter, 3 of them required AVV replacement. There were no operative death and one late death arising from pulmonary venous obstruction. Eleven of 14 survivors have completed Fontan with no early and no late deaths.

Conclusion: Aggressive treatment of concomitant AVV insufficiency since neonatal period contributes to improved outcome.

\section{1}

Management of pulmonary artery sling associated with tracheal stenosis or intracardiac anomalies

Yoshihiro Oshima, Department of Cardiovascular Surgery, Kobe Children's Hospital, Kobe, Japan, Japan

Ayako Maruo, Department of Cardiovascular Surgery, Kobe Children's Hospital, Kobe, Japan, Japan

Chikashi Shimazu, Department of Cardiovascular Surgery, Kobe Children's Hospital, Kobe, Japan

Tomonori Higuma, Department of Cardiovascular Surgery, Kobe Children's Hospital, Kobe, Japan

Takeshi Inoue, Department of Cardiovascular Surgery, Kobe Children's Hospital, Kobe, Japan

Eiji Nishijima, Department of Pediatric Surgery, Kobe Children's Hospital, Kobe, Japan, Japan

Background: This study focused on the influence of concomitant anomalies and the individual surgical procedure.

Methods: Between 1984 and 2008, 32 patients underwent surgical repair of pulmonary artery sling (median, 7 month; range, 29 days to 9 years) in our institute. Twenty eight patients underwent left pulmonary artery reimplantation and tracheoplasty, whereas the other 4 had left pulmonary re-implantation only. For long segment tracheal stenosis, the early 4 patients were treated with a costal cartilage graft, and 19 patients with slide tracheoplasty. Five patients underwent tracheal resection with end-to-end anastomosis. Associated intracardiac anomalies were repaired concomitantly with left pulmonary artery reimplantation and tracheoplasty in 12 patients.

Results: There were 2 early deaths from low output syndrome and ventricular arrhythmia. Follow-up is complete in all patients (median, 5.1 years) with 3 late deaths from residual tracheal stenosis or pulmonary hypertension. Two survivors with a costal cartilage graft are free of respiratory problems. Of 16 survivors with slide tracheoplasty, 8 are fully active without tracheostomy and 8 with tracheostomy from tracheomalacia. Of 5 survivors with tracheal resection, 4 are doing well without tracheostomy. By echocardiography, the left pulmonary artery was patent in all assessed patients, but 2 patients had balloon arterioplasty.

Conclusion: Current strategy of left pulmonary re-implantation with simultaneous repair of tracheal stenosis and intracardiac anomalies results in a low operative mortality and satisfactory patency of left pulmonary artery. However, the management of younger infants with tracheoplasty for long segment stenosis involving carina or complex cardiac anomalies is still challenging.

\section{3}

\section{Longterm Results of Downsizing Valve Allografts in the Pulmonary Position}

Jun-Yen Pan, Cardiovascular Surgery, Kaohsiung Veterans General Hospital, Taiwan

Kai-Sheng Hsieh, Pediatrics, Kaohsiung Veterans General Hospital, Taiwan

Shu-Min Kuo, Cardiovascular Surgery, Kaohsiung Veterans General Hospital, Taiwan

Purpose: The purpose of this study was to examine the longterm results of downsizing cryopreserved homograft valved conduits used in the pulmonary position for surgical correction of congenital heart defects.

Materials and Methods: We made a bicuspid valved conduit by excising a complete cusp together with a longitudinal strip of the arterial wall of adult valved conduit. Between November 1994 and November 2008, 24 patients underwent surgical reconstruction for congenital heart defects using downsizing cryopreserved homograft valved conduit implantation in pulmonary position. There were 16 males and 8 females, and the median age was 3.5 years (range, 1.3-9.3 years). There were 17 aortic homografts and 7 pulmonary homografts. Diagnosis included VSD with pulmonary atresia $(\mathrm{n}=9)$, D-TGA with VSD and PS $(\mathrm{n}=2)$, corrected-TGA $(\mathrm{n}=6)$, and DORV $(\mathrm{n}=2)$.

Results: Three patients died early after operation (12.5\%). The mean follow-up period for 21 survival patients was $10.1+0.9$ years (range, 8.5-11.6 years). Six patients (29\%) underwent reoperation for graft failure including 5 aortic homografts and 1 pulmonary homograft. Average time to reoperation was 6.3 years (range, 5.3-8 years). There was no late death.

Conclusion: Downsizing cryopreserved homograft valved conduit function satisfactorily in the pulmonary position at longterm follow-up. The pulmonary homograft valved conduit appears to be more durable and less calcification than the aortic homograft valved conduit in the pulmonary position.

\section{4}

Early and late outcomes of surgery for low birth weight infants $(\mathrm{LBW}<2.5 \mathrm{~kg}$ ) with congenital heart disease

Ramesh C Parmar, The Children's Hospital at Westmead, Sydney. Australia

Bart Koene, Catharina Hospital, Eindhoven., The Netherlands Julian G Ayer, The Children's Hospital at Westmead, Sydney, Australia

Andrew D Cole, The Children's Hospital at Westmead, Sydney, Australia

Karen Walker, The Children's Hospital at Westmead, Sydney, Australia

Gary F Sholler, The Children's Hospital at Westmead, Sydney, Australia 
Nadia Badawi, The Children's Hospital at Westmead, Sydney, Australia

David S Winlaw, The Children's Hospital at Westmead, Sydney, Australia

Improvements in neonatal care and peri-procedural outcomes have reduced the weight at which cardiac surgery is routinely contemplated. As for normal birth weight infants (NBW, $2.5-4 \mathrm{~kg})$ quality of outcome measures should include assessments of both physical and neurodevelopmental status however these data are not widely available.

Methods: We reviewed our experience between October 2003 and December 2007 and categorised data according to the recent STS database report by Curzon et al. 2008. We examined the role of non technical human factors in a semi-structured interview with attending staff. Finally, we are following the LBW group to determine their cardiologic and neurodevelopmental status.

Results: Outcomes for the LBW group $(\mathrm{n}=41)$ vs. the NBW $(\mathrm{n}=260)$ were compared. 30 day mortality was $22 \%$ vs. $3 \%$ with weight at surgery being associated with a worse outcome. The most common diagnoses in the LBW group were pulmonary atresia and coarctation. Human factors identified included care and parental fatigue, conflict in perceptions of long term outcome, communication between subspecialty teams involved and experience of the surgeon. Outcomes were rarely influenced by an issue of diagnostic accuracy or technical performance. Developmental assessments suggest significant rates of neurodevelopmental impairment.

Conclusions: Low birth weight children continue to represent a significant challenge. Whilst operative mortality is satisfactory and consistent with published reports there is significant early morbidity and many long term problems. Tight integration of sub-specialty groups is required to improve outcomes, and routine neurodevelomental follow up should be mandatory in this group to facilitate early intervention.

7425

A 28 year experience with surgical repair of Tetralogy of Fallot: risk analysis for mortality or late reintervention Andrzej Pastuszko, Poland

Andrzej Kansy, Children's Memorial Health Institute, Warsaw, Poland Piotr Burczynski, Children's Memorial Health Institute, Warsaw, Poland Mariusz Birbach, Children's Memorial Health Institute, Warsaw, Poland

Wojciech Lipinski, Children's Memorial Health Institute, Warsaw, Poland

Malgorzata Mirkowicz-Malek, Children's Memorial Health Institute, Warsaw, Poland

Grazyna Brzezinska, Children's Memorial Health Institute, Warsaw, Poland

Bohdan Maruszewski, Children's Memorial Health Institute, Warsaw, Poland

Objective: Early results after repair of Tetralogy of Fallot have improved importantly during last decades. Data on long term outcomes are limited.

Methods: 773 patients underwent repair of TOF including TOF with pulmonary atresia between 1980 and 1999. Mean age at surgery was 73,6 months before 1990 and 57,5 after $(p<0,001)$. $70 \%$ of patients (540) underwent one-stage repair. Transventricular approach was used in 399 patients, 283 had transannular enlargement, 69 were repaired transatrially. 22 RV-PA conduits were interposed. IPPV and LOS times significantly decreased in later decade as well as number of complications. Mean hospital mortality was $10,6 \%$ before and 4,6\% after 1989. Hospital mortality did not differ between surgical techniques. Multivariate analysis showed that only surgical era and type of repair were risk factors for early death.

Results: Mean late follow-up was 18,6 years (8,9-28,2,SD 5,5) and was available in 660 patients $(95,5 \%)$. Late dysrhythmia occured in $23 \%$ of survivors, most frequently VEx, $11,5 \%$. There were 11 reoperations and 31 catheter interventions. Freedom from any reintervention after repair was 97,6\%, 95,8\% and $94,1 \% 5,10$, and 15 years respectively. The lowest risk of late reintervention was associated with transventricular approach $(3,19 \%)$. Late mortality was $1,2 \%$ (8 deaths). Cumulative overall survival is $88,1 \%$ independent on surgical technique, age at surgery and era at repair.

Conclusions: Early mortality after repair of TOF has significantly decreased over last decades and late survival is excellent. Continuous monitoring of late outcomes in recent population of young infants after primary repairs is mandatory.

\section{6}

Main Pulmonary Artery Dilatation after the Hybrid Procedure Exceeds Neo-aortic Dilatation after the Norwood/Sano Procedure: Is this a Cause for Concern? Peter Pastuszko, Rady Children's Hospital of San Diego, San Diego, $C A$, United States

Howaida G EL-Said, Rady Children's Hospital of San Diego,

San Diego, CA, United States

John W Moore, Rady Children's Hospital of San Diego, San Diego, $C A$, United States

Jolene M Kriett, Rady Children's Hospital of San Diego, San Diego, $C A$, United States

Dana Connolly, Rady Children's Hospital of San Diego, San Diego, $C A$, United States

John J Lamberti, Rady Children's Hospital of San Diego, San Diego, $C A$, United States

Objectives: The Hybrid procedure (Bilateral PA Bands + PDA stent) is an alternative repair technique in Hypoplastic Left Heart Syndrome (HLHS). We have noted marked dilatation of the main pulmonary artery (MPA) after the Hybrid operation and postulate that the Hybrid procedure predisposes to greater MPA dilatation than the Norwood/Sano procedure.

Methods: Between July 2007 and June 2008 six high-risk newborns underwent the Hybrid procedure. They were compared to twentyone matched controls (Norwood/Sano). Echocardiographic data were reviewed, including the pulmonary valve annulus (PV) and $z-$ score (PVz), the MPA and $z$-score (MPAz), and degree of pulmonary regurgitation (PR).

Results: Mean age at procedure for all patients $(\mathrm{N}=27)$ was 10 days \pm 8 and at follow-up was 4.5 months \pm 1 . Seventeen $(63 \%)$ were female; $10(37 \%)$ were male. In controls, there were significant increases in all diameters. Median increase in PV diameter was $3.2 \mathrm{~mm} \pm 0.48 \quad(\mathrm{p}=001), \quad \mathrm{PVz} 0.64 \pm 0.33 \quad(\mathrm{p}=0.009) . \quad$ MPA diameter increase was $2.6 \pm 0.46(p=0.001), \mathrm{MPAz} 0.71 \pm 0.29$ $(p=0.001)$. In the hybrid group, only the MPA diameter increased significantly, with a median increase of $4.7 \mathrm{~mm} \pm 1.43(\mathrm{p}=0.011)$. $\mathrm{MPAz}$ was $1.8 \pm 1.09(\mathrm{p}=0.069)$. The change in PR was not significant in either group. There was a significant difference in MPA dilatation between Hybrids and controls ( $\mathrm{R} 2=0.85$, $p=0.009)$, not in other parameters.

Conclusions: Dilatation of the MPA in short-term follow-up of Hybrids is concerning. It may be related to increased outflow impedance, altering timing and surgical strategy for stage II operation. 
7427

Phrenic Nerve Injury after Cardiac Surgeries for Congenital Heart Disease: A Review of 10 years experience Yi Chen Peng, China Medical University Hospital, Taichung, Taiwan, Taiwan

Jeng Sheng Chang, China Medical University Hospital, Taichung, Taiwan, Taiwan

Ping Chun Li, China Medical University Hospital, Taichung, Taiwan, Taiwan

Background: Phrenic nerve injury (PNI) is an annoying complication after cardiac surgery. Severe respiratory distress, prolonged ventilator use and hospital stay are common problems in the infantile patients. Early diaphragm plication vs. conservative therapy has been a debatable issue.

Methods: We retrospectively review the medical records of our pediatric patients having heart surgeries for congenital heart disease (CHD) in this tertiary referring hospital during Oct. 1999 through Oct. 2008. Diagnosis of PNI was confirmed by chest X-ray, ultrasound and fluoroscope studies. The CHD of these PNI patients include TAPVR, TOF, Asplenic syndrome, ECD and VSD. Their demographic data and details of operations and postoperative course are compared between these 2 groups of management.

Result: Thirteen of 545 patients (2.4\%) are found to be complicated with postoperative PNI. Eight patients suffered right side PNI, four left side and only one was bilateral PNI. The Age, ventilator days and hospitalization days in patients of conservative treatment are 8-days-to-5-years, $26.3 \pm 20.9$ days and $50.1 \pm 30.9$ days respectively, as well as 4-days-to-9-months, $71.6 \pm 14.1$ days and $80.0 \pm 26.8$ days respectively in patients receiving diaphragm plication therapy. Also, in all PNI patients older than 6 months of age extubation is always successfully in two weeks of operation whereas in those younger than 6months prolonged ventilator dependence is the rule.

Conclusion: We recommend early diaphragm plication on PNI patients younger than 6 months of age or suffering bilateral PNI.

\section{8}

Very Early Surgical Repair in Complete Atrioventricular Septal Defect

Daneil Perez, Paediatric Cardiovascular Unit, Santa Maria Clinic, Santiago, Chile

Stephan Haecker, Paediatric Cardiovascular Unit, Santa Maria Clinic, Santiago, Chile

Luis Cardendas, Paediatric Cardiovascular Unit, Santa Maria Clinic, Santiago, Chile

Gabreiela Enriquez, Paediatric Cardiovascular Unit, Santa Maria Clinic, Santiago, Chile

Pilar Soler, Paediatric Cardiovascular Unit, Santa Maria Clinic, Santiago, Chile

Karen Apablaza, Paediatric Cardiovascular Unit, Santa Maria Clinic, Santiago, Chile

Carola Carreño, Paediatric Cardiovascular Unit, Santa Maria Clinic, Santiago, Chile

Luis Leon, Paediatric Cardiovascular Unit, Santa Maria Clinic, Santiago, Chile

Few reports of early surgical repair of complete atrioventricular septal defect (AVSD) exist. EACTS database shows relatively late age and significant surgical mortality in the infant undergoing early AVSD repair. Our policy is to repair whenever CHF symptoms occur or elective surgery at 3 months. Between Jan 2006 and Dec 2008, 24 patients with Complete AVSD underwent definitive repair. Median age at surgery was 52
( \pm 42$)$ days and median weight was $3.6( \pm 1,0) \mathrm{Kg}, 22$ patients were $<2$ months, 6 patients were $<2.5 \mathrm{Kg}$. Four had unbalanced AVSD. All but one patient had Down's syndrome.

CPB time was 57 ( \pm 13$)$ min; Aortic crossclamp time was 33 (士7) $\mathrm{min}$.

Two pericardial patches were used. Mitral annuloplasty was often achieved with the Ventricular Septal Defect (VSD) patch (70\%); cleft closure was performed in $26 \%$. The Sternum was closed in $100 \%$. Median mechanical ventilation time was 24 hrs. ICU stay was a median $5.4( \pm 5)$ days. No surgical or late mortality occurred. Two patients with unbalanced AVSD were reoperated for residual VSD. All 24 pts are doing well on follow up (2y), $83 \%$ are on no medication. $96 \%$ have mild or trivial MI. One patient has moderate mitral incompetence and may need further surgery. In conclusion, early surgery with no mortality and limited morbidity can be achieved even in smaller patients with our simplified surgical technique. Shortened operating times and reduced needs of post operative support are part of our surgical strategy. Early repair reduces preop morbidity and allows earlier normal growth and psychomotor stimulation.

\begin{tabular}{lcc}
\hline & $\begin{array}{c}\text { EATCS } \\
(1997-2006)\end{array}$ & $\begin{array}{c}\text { Santa María } \\
\text { Clinic }\end{array}$ \\
\hline Age at surgery (months) & $4.9 \pm 2.3$ & $1.7 \pm 1.4$ \\
Weight (kg) & $4.8 \pm 1.1$ & $3.6 \pm 1$ \\
CPB (min) & $124.9 \pm 55$ & $57 \pm 13$ \\
Aortic cross clamp (min) & $79.6 \pm 32$ & $33 \pm 7$ \\
Mortality & $7.14 \%$ & $0 \%$ \\
LOS (days) & $15 \pm 17$ & $9 \pm 20$ \\
\hline
\end{tabular}

\section{9}

Neurohormonal profile predicts outcome following single ventricle palliation

Christian Pizarro, Alfred I duPont Hospital for Children, United States

Jacek Kolcz, Alfred I duPont Hospital for Children, United States Emilio Quezada, Alfred I duPont Hospital for Children, United States Carol Prospero, Alfred I duPont Hospital for Children, United States Robert Akins, Alfred I duPont Hospital for Children, United States

Objective: To asses the expression of fluid-regulating hormones, vasopressin, angiotensin II (AT II), atrial (ANP) and brain natriuretic peptide (BNP) and evaluate their influence on early outcome following single ventricle (SV) palliation.

Methods: Preoperative and postoperative plasma samples were prospectively collected among patients undergoing SV palliation $(\mathrm{BDG}=23$ and Fontan $=25)$. Analysis was performed to determine the potential influence of preoperative neurohormonal profile on clinical outcome(incidence of prolonged pleural effusions, lenght of mechanical ventilation $[\mathrm{MV}]$, ICU and hospital stay [LOS].

Results: The median duration of MV, ICU and LOS for the entire cohort were 6 hrs, 29 hrs and 11 days respectively. Median duration of CPB and myocardial ischemia were 73 and 37 minutes. There was no operative mortality. Significant differences across SV stages were observed for QP/QS $(p<0.001)$, PA pressure $(p<0.01)$, transpulmonary gradients $(\mathrm{p}<0.03)$ and SVC saturation $(p<0.0001)$. Preoperative BNP predicted likelihood of prolonged MV $(p<0.00001)$, and LOS $(p<0.05)$ in the Fontan cohort while preoperative AT II predicted duration of MV $(\mathrm{p}<0.000000)$, ICU $(p<0.00001)$ and LOS $(p<0.0003)$ among patients undergoing BDG. Perioperative expression of ANP and BNP did not correlate 
with the morphology of the SV, duration of cardiopulmonary bypass or myocardial ischemia.

Conclusion: Preoperative neurohormonal profile can predict early postoperative course following SV palliation. The influence of different hormones during the SV staging sequence suggests specific mechanisms possibly related to the underlying physiology, rather than operative factors.

\section{2}

Requirements for an optimized intraoperative myocardial protection in pediatrics

Claus J Preusse, University Bonn, Cardiac Surgery, Germany

Intraoperative myocardial protection in pediatrics is always still challenging. The morphologic but mainly metabolic differences to adult hearts demand for a sophisticated approach to this topic.

Besides aspects of anaesthesia and cardiopulmonary bypass application cardioplegia plays the most significant role. Concerning cardioplegia two parameters exist: Formulation of the solution applied and application mode.

Numerous experimental studies in 'immature' hearts have demonstrated that single dosing is superior to multidosing being mostly used worldwide. This discrepancy is related to one biochemical fact: Rate of intra-ischemic lactate production is much higher in 'immature' hearts than in adults. To minimize myocardial acidification either pediatric hearts must be cardioplegically reperfused at certain intervals (10-30 mins) or a highly buffered cardioplegic solution is applied. However, adding of a highly concentrated buffer to a cardioplegic solution is only possible if an intracellular type of solution (low $\mathrm{Na}$ ) is used.

The only cardioplegic solution world wide, that is highly buffered, is the histidine buffered Bretschneider HTK solution (CUSTODIOL ${ }^{\circledR}$ ), which allows safe x-clamping periods upto 3 hours in pediatrics including neonates after only single, initial application. The prerequisite for this longterm protection bases on an optimal initial equilibration of the extracellular space with HTK solution. Such an equilibration requires an initial perfusion time of about 4-6 mins.

Furthermore experimental data have proven that slightly increased magnesium and low calcium concentration in any solution improve protective power in pediatric hearts.

Physiological aspects and specific intraoperative clinical data in pediatrics using HTK solution are presented and discussed.

\section{3}

Outcomes of Contegra valved conduit in Paediatric Cardiac Surgery

Natasha Prior, Alder Hey Foundation NHS Trust, Liverpool, United Kingdom

Ramana RV Dhannapuneni, Alder Hey Foundation NHS Trust, Liverpool, United Kingdom

Andreas Hoschtitzky, Alder Hey Foundation NHS Trust, Liverpool, United Kingdom

Gordon Gladman, Alder Hey Foundation NHS Trust, Liverpool, United Kingdom

Ian Peart, Alder Hey Foundation NHS Trust, Liverpool, United Kingdom Annette Davis, Alder Hey Foundation NHS Trust, Liverpool, United Kingdom

Nelson Alphonso, Alder Hey Foundation NHS Trust, Liverpool, United Kingdom

Antonio F Corno, Alder Hey Foundation NHS Trust, Liverpool, United Kingdom
Objectives: Contegra, a glutaraldehyde fixed bovine jugular vein, has been developed for use in the surgical treatment of complex congenital heart defects. In this study we retrospectively evaluated our clinical experience with Contegra biological valved conduit implantations.

Methods: From January 1999 until December 2008196 Contegra valved conduit implantations were performed. In this retrospective study the outcomes in terms of early and late mortality, length of ICU and hospital stay, conduit failure requiring reoperation or cardiological interventional procedure were reviewed. Results: The mean patient age at time of surgery was $6.4 \pm 5.6$ years and the mean weight was $22.5 \pm 20.9 \mathrm{Kg}$. Surgical indication included right ventricular outflow tract reconstruction $(\mathrm{n}=130)$, replacement of a previously implanted conduit from the right ventricle to the pulmonary artery $(n=36)$, Ross procedure $(n=24)$ and aortic arch reconstruction $(n=6)$. The mean diameter of the implanted Contegra valved conduit was $18.1 \pm 3.5 \mathrm{~mm}$. There were $5(2.6 \%)$ early deaths and $1(0.5 \%)$ late death. The mean ICU stay was $4.3 \pm 6.9$ days and the mean post-operative hospital stay was $13.6 \pm 14.6$ days. The mean follow-up period was $784.3 \pm 823.7$ days, for a total of 1.8 year/ patient follow-up (range 1 month to 10 years). Nine (4.5\%) patients required cardiological interventional procedure within a mean interval of $2.5 \pm 1.4$ years, while 11 (5.6\%) patients required surgical revision following implantation of Contegra.

Conclusions: After 10 years of experience with Contegra we continue to use this biological valved conduit as a reliable alternative to homografts, without any significant negative impact in terms of outcome.

\section{4}

Outcome of AV valve function in 261 patients after AV canal repair; up to $\mathbf{1 0}$ years follow up

Howaida M O Al Qethamy, Head of Cardiac Surgery, Prince Sultan Cardiac Centre, Saudi Arabia

Mohamed E A Ibrahim, Research \& Delopment, Prince Sultan Cardiac Centre, Sudan

Yahya M Al Faraidi, Senior Consultat Ped. Cardiac Surgery, Prince

Sultan Cardiac Centre, Saudi Arabia

Hatim A Mohamed, Prince Sultan Cardiac Centre, Sudan

Reda M El Oakley, Consultant Adult Cardiac Surgery. Prince Sultan Cardiac Centre, United Kingdom

Introduction: Multiple studies presented early results of AV canal repair with relatively low risk of redo surgery. However little mid or long-term data have been published. In this study we present, the midterm results of 261 consecutive patients who had repair of AV canal between January 1999 to December 2008 in our institutions.

Methods and results: Retrospective review of 261 cases with mean age of $36+2.47$ months, male to female ratio $1: 2$; and complete AV canal with associated other cardiac anomalies in 39.8\%. 25\% had banding at a mean age of 2.3 months in preparation for stage two repairs that followed in an average of 1.8 years ( $0.6-4.3$ years range). The double patch technique with standard hypothermic cardiopulmonary bypass and cold blood cardioplegia were performed. The mean hospital stay was $17.52+1.44$ days (3-180 days range). The mean follow up time was $39+2.37$ months that $(1-113$ months range). The in hospital mortality was $5 \%$ with acturial survival using Kaplan Mayer of $95 \%+00$. The proportional hazards were investigated by cox-regression for gender, age, anomalies and AVC defect. The complete AV canal was the only significant ( $\mathrm{P}$ value $0.011, \operatorname{Exp}(\mathrm{B}) 0.016$ with $95 \%$ CI $0.001,0.391)$. Postoperatively, $84.3 \%$ of patients had zero to mild Mitral valve regurgitation, 
$13.4 \%$ had mild to moderate and $2.4 \%$ moderate to severe. Redo surgeries were done for $4.7 \%$ of the patients.

Conclusion: Double patch technique can offer satisfactory midterm results of AV canal repair.

\section{5}

\section{The treatment of aortic valve disease}

Andrea Quarti, Azienda Ospedaliera, ospedali Riuniti, Ancona, Italy Alessandro D'Alfonso, Azienda Ospedaliera, ospedali Riuniti, Ancona, Italy

Maria Grazia Bettuzzi, Azienda Ospedaliera, ospedali Riuniti, Ancona, Italy

Massimo Colaneri, Azienda Ospedaliera, ospedali Riuniti, Ancona, Italy

Alessandra Baldinelli, Azienda Ospedaliera, ospedali Riuniti, Ancona, Italy

Pier Luigi Colonna, Azienda Ospedaliera, ospedali Riuniti, Ancona, Italy

Roberto Ricciotti, Azienda Ospedaliera, ospedali Riuniti, Ancona, Italy Marco Pozzi, Azienda Ospedaliera, ospedali Riuniti, Ancona, Italy

Objective: Aortic valve repair techniques have evolved over the years. However the Ross operation has been considered to be the best option in small children despite some drawbacks. In the last two years we have adopted a more aggressive approach for valve repair with a consequent delay or avoidance of the Ross procedure in many cases.

Methods and Results: between 1993 and 2006 more than 200 patients have been operated on for aortic valve disease, using commissurotomy (29 patients $2 \mathrm{dd}-1$ yy) or Ross procedures (172 patients, 3 dd-59 yy). During the last 2 years the Ross procedure has been replaced by aortic valve repair in patients with normal or large annulus, using cusps extension (9 patients), annulus plication (2 patients), commissures resuspension (3 patients). During this period the Ross procedure associated to the Konno modification has been applied only to patients with hypoplastic aortic annulus or LVOTO.

Comment: New surgical options for children with aortic valve disease have changed our policy in the management of aortic valve. The Ross procedure is now our golden standard for neonates and children with an hypoplastic aortic annulus or as a fall back in cases of failed repair. In the others, aortic valve repair has become our first choice. In the last 2 years we widely applied cusps extension, cusps resuspension, annulus plication in this subset of aortic anatomy with good early and medium term results. A longer follow up will be necessary to have a better understanding of the validity of the aortic valve repair.

\section{6}

\section{Minimally invasive approaches in paediatric cardiac} surgery

Andrea Quarti, Azienda Ospedaliera Ospedali Riuniti, Ancona, Italy Alessandro D'Alfonso, Azienda Ospedaliera Ospedali Riuniti, Ancona, Italy

Maria Grazia Bettuzzi, Azienda Ospedaliera Ospedali Riuniti, Ancona, Italy

Massimo Colaneri, Azienda Ospedaliera Ospedali Riuniti, Ancona, Italy Alessandra Baldinelli, Azienda Ospedaliera Ospedali Riuniti, Ancona, Italy

Pier Luigi Colonna, Azienda Ospedaliera Ospedali Riuniti, Ancona, Italy

Roberto Ricciotti, Azienda Ospedaliera Ospedali Riuniti, Ancona, Italy Marco Pozzi, Azienda Ospedaliera Ospedali Riuniti, Ancona, Italy
Objective: Over the last years many minimally invasive approaches (MIA) have been described to treat different cardiac anomalies in the paediatric population. Simple pathologies can be safely treated with small incision reducing the psychological trauma without an increased surgical risk.

Methods: During the last 3 years 35 patient were treated with a MIA. A superior J ministernotomy was used in subaortic valvular stenosis in 5 patients (mean age 11,6 yy), an inferior J ministernotomy was used in 18 patients (mean age 5,7 yy) with type secundum ASD and a right antero-lateral minithoracotomy was adopted in 12 patients (mean age 16 yy) with a type secundum ASD.

Results: There were no death and only one patient with an ASD treated with a ministernotomy required an extension to a full sternotomy for difficulty in the aortic cannulation. No surgical related events were recorded.

Comment: The psychological impact of the scar in the young patients should not be underestimated. In the last 3 years we treated more than $90 \%$ of ASDs with a minimally invasive approach while in the last 2 years all patients presenting with an isolated subvalvular aortic stenosis were treated with a ministernotomy. The use of a wide spectrum of minimally invasive approaches allowed us to tailor the best approach for every single patient according to pathology, age, copathologies and to increase the number of patients that can benefit from MIA. Probably further use of different approaches will allowed us to widen even more the spectrum of pathologies and patients treateble with minimally invasive approach.

7437

\section{Repair of the atrio-ventricular valves}

Andrea Quarti, Azienda Ospedaliera, ospedali Riuniti, Ancona, Italy Alessandro D'Alfonso, Azienda Ospedaliera, ospedali Riuniti, Ancona, Italy

Maria Grazia Bettuzzi, Azienda Ospedaliera, ospedali Riuniti, Ancona, Italy

Massimo Colaneri, Azienda Ospedaliera, ospedali Riuniti, Ancona, Italy Alessandra Baldinelli, Azienda Ospedaliera, ospedali Riuniti, Ancona, Italy

Pier Luigi Colonna, Azienda Ospedaliera, ospedali Riuniti, Ancona, Italy Roberto Ricciotti, Azienda Ospedaliera, ospedali Riuniti, Ancona, Italy Marco Pozzi, Azienda Ospedaliera, ospedali Riuniti, Ancona, Italy

Background: Isolated atrioventricular valve regurgitation is uncommon in the paediatric population. Many techniques have been described to treat the wide spectrum of abnormalities causing valve regurgitation.

Methods: From April 2005 to September 2008, 14 patients, mean age 7,2 yy (range 2-18 yy) underwent tricuspid (group A: 8 patients) or mitral valve repair (group B: 6 patients). Among patients in group $\mathrm{A}$ in one case a Clover technique with annuloplasty ring insertion was adopted while the others were treated with annuloplasty ring insertion. In group B in 5 cases the edge to edge technique with annuloplasty ring was used for anterior or bileaflet prolapse and in one case a plasty of the commissure was used.

Results: There was no death in either groups and one patient in group B (treated with edge to edge repair) required a reoperation for mitral subvalvar stenosis. No patient had mitral or tricuspid regurgitation more than $1+$ at a mean follow-up of 16 months. None of the patients had any valve related event.

Conclusions: The repair of the atrioventricular valve is an uncommon procedure in the paediatric population. The edge to edge technique is a safe and useful technique for the anterior or bileaflet prolapse of 
the mitral valve and can be safely and successfully employed in the paediatric population, generally associated with an annuloplasty. The Clover technique ("extension" of the edge to edge to a tri-leaflet valve) can be useful in patients with tricuspid regurgitation. Early results are encouraging but a longer follow-up is needed to evaluate the long-term prognosis.

\section{8}

Early results of surgical correction of anomalous left coronary artery from pulmonary artery (ALCAPA) Samuel E Ramirez-Marroquin, "Ignacio Chávez" National Cardiology Institute of Mexico, Mexico

Jorge L Cervantes, "Ignacio Chávez" National Cardiology Institute of Mexico, Mexico

Pedro J Curi-Curi, "Ignacio Chávez” National Cardiology Institute of Mexico, Mexico

Juan Calderon-Colmenero, "Ignacio Chávez” National Cardiology Institute of Mexico

Jaime Garcia, "Ignacio Chávez" National Cardiology Institute of Mexico, Mexico

Emilia Patiño-Bahena, "Ignacio Chávez” National Cardiology

Institute of Mexico, Mexico

Jose A Garcia-Montes, "Ignacio Chávez” National Cardiology Institute of Mexico, Mexico

Alfonso Buendia, "Ignacio Chávez" National Cardiology Institute of Mexico, Mexico

Objective: To describe early and mid term results of surgical treatment of anomalous left coronary artery from pulmonary artery (ALCAPA).

Methods: We studied retrospectively 11 patients ( $82 \%$ female) with mean age of 4.9 years that underwent surgical correction of ALCAPA with coronary implantation technique in a 17 year period.

Results: Symptoms presented at a mean time of 12 months (range, 15 days to 6 years). Initial diagnosis of ALCAPA was made in 4 patients (33\%) and the rest of them were admitted as mitral regurgitation (5), dilated myocardiopathy (2), or restrictive myocardiopathy (1). Mitral regurgitation presented in $83 \%$ of the cases, and was severe in $58 \%$. Chest X-ray showed a congestion pattern in $66 \%$, and cardiomegalia in all patients. Myocardial perfusion gammagraphy was performed in 8 cases and showed ischemia in all of them except one. Echocardiography in 25\% of the patients had ejection fraction $\leqslant 30 \%$. All patients were operated with coronary implantation technique, 3 cases had a concomitant mitral valve repair and one patient recquired a mitral valve replacement. There was no operative mortality. During the first postoperative day $75 \%$ of the cases were extubated. Mean time of ICU was 4 days and mean time for hospital discharge was 9.9 days. Conclusion: Dual-coronary system can be established in patients with ALCAPA by the coronary implantation technique with excellent early results. A careful clinical and echocardiographic analysis is recommended for the diagnosis of ALCAPA in all patients with cardiac failure due to mitral regurgitation or dilated myocardiopathy.

\section{9}

Long term outcome after Fontan Procedure at the altitude of Mexico city

Samuel E Ramirez-Marroquin, "Ignacio Chávez” National Cardiology Institute of Mexico, Mexico

Jorge L Cervantes, "Ignacio Chávez" National Cardiology Institute of Mexico, Mexico
Pedro J Curi-Curi, "Ignacio Chávez" National Cardiology Institute of Mexico, Mexico

Jose A Garcia-Montes, "Ignacio Chávez" National Cardiology Institute of Mexico, Mexico

Emilia Patino-Bahena, "Ignacio Chávez" National Cardiology

Institute of Mexico, Mexico

Juan Calderon-Colmenero, "Ignacio Chávez" National Cardiology

Institute of Mexico

Objective: To evaluate the early and long-term outcome of those patients that have completed their staging protocol by means of a Fontan procedure at the altitude of Mexico city $(2420 \mathrm{~m}$ over the sea level) in a 17 year period.

Methods: We studied retrospectively 98 patients (51\% female) with mean age of 8 years (range, 1 to 22.7 years) that had univentricular heart physiology and underwent a Fontan procedure, 61 with an intracardiac lateral tunnel technique (41 fenestraded, 20 non fenestrated) and 37 with an extracardiac conduit (29 fenestrated, 8 non fenestrated). Review of their clinical, surgical, echocardiographic, angiographic and hemodynamic data was performed to identify risk factors for mortality. Follow up was complete in all survivors.

Results: The most common causes of early morbidity were pleural effusions (98\%) and ascitis (24\%). Early mortality was as high as $17 \%$ with intracardiac lateral tunnel technique but dropped to $5 \%$ with extracardiac fenestrated technique since 1998-1999. Pulmonary artery pressure $(\mathrm{p}<0.01)$, left atrial pressure $(p<0.01)$ and quantity of effusions $(p<0.05)$ were risk factors for early death. Mean follow up was 8.6 years. Overall survival at ten years was $73 \%$. Late reinterventions were necessary in $22(30 \%)$ of the survivors and 9 of them (12.6\%) had neurological disorders.

Conclusions: Our best results were with extracardiac fenestrated technique, comparable with those of larger series, but our morbidity (effusions and ascitis mostly) is higher, maybe due to Mexico city altitude. Better selection of patients help to reach a long-term survival with improvement in the quality of life.

\section{0}

Hypoplastic left heart syndrome - The influence of surgical strategy on outcomes

Mónica S Rebelo, Hospital Cruz Vermelha Portuguesa, Portugal Maria Ana Sampaio Nunes, Hospital Cruz Vermelha Portuguesa, Portugal

Ana R Fernandes, Hospital Cruz Vermelha Portuguesa, Portugal Manuel J Ferreira, Hospital Cruz Vermelha Portuguesa, Portugal Graziela Nero, Hospital Cruz Vermelha Portuguesa, Portugal Manuel Pedro Magalhães, Hospital Cruz Vermelha Portuguesa, Portugal

Hypoplastic left heart syndrome (HLHS) is the 4th most common lesion requiring neonatal open heart surgery and is the most common anomaly resulting in death from congenital heart disease within the first year of life.

Objectives: Compare the results of two different surgical techniques for aortic arch reconstruction (Group 1:autologous pericardium patch versus Group 2:homograft) in Norwood procedure.

Methods: This is a single institutional retrospective study performed in 43 paediatric patients submitted to Norwood procedure from 1998 to 2008.

Results: Median age of the studied population at the surgery was:13 days (1-90). More males. Post operative mortality (6 pts). Median follow-up was 3,4 years $(0.1-10)$. Ten patients are at $3 \mathrm{rd}$ 
surgical stage (Fontan). Group 1:22 pts; Group 2: 16 pts. 5 pts were excluded. There's no statistically difference between two groups considering clinical outcome and morphology of aorta. Conclusion: The incidence of aortic coarctation using both techniques is lower.

In studies the use of autologous pericardium patch was preferable considering economic aspects and the possibility of a future cardiac transplantation.

7441

Mid-term outcome of right ventricular outflow tract reconstruction using a handmade GoreTex tri-leaflet conduit

Oleksandr M Romaniuk, Ukrainian Children's Cardiac Center, Ukraine

Jaroslav Y Ivanov, Ukrainian Children's Cardiac Center, Ukraine Oleksandr D Bablyak, Ukrainian Children's Cardiac Center, Ukraine Oleksandr S Kondrachuk, Ukrainian Children's Cardiac Center, Ukraine

Illya M Yemets, Ukrainian Children's Cardiac Center, Ukraine

Objective: Since 2006 we started to implant handmade polytetrafluoroethylene (Gore-Tex) tri-leaflet conduits for right ventricular outflow tract reconstruction (RVOT).This report assesses the mid-term outcome of these prostheses.

Methods: From 2006 to 2008, 27 handmade whole GoreTex trileaflet conduits were implanted in 27 patients. The age at operation ranged from 15 days to 16 years (median: 8.9 months). The diagnoses included pulmonary atresia with ventricular septal defect in 9 patients, truncus arteriosus -5 , tetralogy of Fallot with absent pulmonary valve syndrome-1, aortic valve pathology (Ross procedure) -12 patients. The median conduit size was $16 \mathrm{~mm}$ (range: $12-24 \mathrm{~mm}$ ).

Results: There was 1 early death, not related to the conduit Follow-up was complete for all patients with a mean of $10,1 \pm 7,6$ months. Echocardiography was performed in all patients and MRI-in 7. None of the conduits required reoperation for conduit obstuction (mean peak pressure gradient by echocardiography $-16 \pm 8 \mathrm{~mm} \mathrm{Hg}$ ). Mean regurgitant fraction on conduit by MRI was $10 \pm 5.7 \%$. Valve leaflet motion was documented by echocardiography and MRI. All patients are NYHA class I.

Conclusions: Handmade tri-leaflet conduits is a reliable alternative to other methods of RVOT reconstruction in children and provide a good mid-term outcome. Longer follow-up is needed to determine how well whole GoreTex tri-leaflet conduits will function.

\section{2}

Anomalous Origin of One Pulmonary Artery from the Aorta (Hemitruncus): a Single Institution Experience

Mark Ruzmetov, Indiana University School of Medicine, Indianapolis, Indiana, United States

Mark D Rodefeld, Indiana University School of Medicine, Indianapolis, Indiana, United States

Mark W Turrentine, Indiana University School of Medicine, Indianapolis, Indiana, United States

John $W$ Brown, Indiana University School of Medicine, Indianapolis, Indiana, United States

Objective: Anomalous origin of one pulmonary artery from the aorta (hemitruncus) is a rare congenital lesion with a high mortality and morbidity if early diagnosis is not made and correction is not undertaken. In this study, we report a description of our approach and review of our 25-year experience in patients surgically treated for hemitruncus.

Methods: Between 1983 and 2008, fourteen patients (neonates $50 \% ; 7 / 14$ ) were operated on for hemitruncus. Median age at presentation was 26 days (range, 5 days to 8 months). Five patients (36\%) had associated cardiac lesions. All patients presented with congestive cardiac failure. Diagnosis was established by echocardiography in all patients. Eleven of the 14 had anomalous right pulmonary artery (RPA) from aorta, and 3 had left pulmonary artery from aorta.

Results: There was no operative or late mortality. Follow-up period ranged from 6 months to 20 years (median 15 years). Two patients required reoperations: for thrombosis of anastomosis when RPA was very small $(n=1)$ and for RPA stenosis $(n=1)$ 1 and 2 years after surgery, respectively. At 20 years, survival by Kaplan-Meier was $100 \%$, and freedom from reoperation, $86 \%$. All patients except 1 has normal hemodinamic function and were in NYHA class I.

Conclusions: Early repair of hemitruncus results in excellent hemodinamic and anatomic results. Survival is excellent, with a low incidence of reoperation or reintervention.

\section{3}

\section{Comparison of Contegra and Homograft Pulmonary} Conduits in Children Less Than Age 2

Andrew C Fiore, St.Louis University School of Medicine, St.Louis, MO, United States

Mark Ruzmetov, Indiana University School of Medicine, Indianapolis, Indiana, United States

Daniel Huynh, St.Louis University School of Medicine, St.Louis, MO, United States

Mark D Rodefeld, Indiana University School of Medicine, Indianapolis, Indiana, United States

Mark W Turrentine, Indiana University School of Medicine, Indianapolis, Indiana, United States

John W Brown, Indiana University School of Medicine, Indianapolis, Indiana, United States

Background: The optimal pulmonary valved conduit for infants and small children remains controversial. This report compares the initial insertion outcome of small caliber Contegra $(12-14 \mathrm{~mm})$ with pulmonary homografts $(10-15 \mathrm{~mm})$ in patients under age 2 .

Methods: From December 1998 to December 2008, 79 children (mean age $8.4+8.5 \mathrm{mo})$ received Contegra $(\mathrm{n}=48)$ or homograft $(\mathrm{n}=31)$ conduits. Mean $\mathrm{Z}$-score for Contegra was 2.2 (range, $-0.8-3.3$ ) and for homograft 2.1 (range, 0.8-4.2; $\mathrm{P}=0.2$ ). The two cohorts were similar with respect to age, BSA, conduit indication, bypass and cross clamp time. Graft dysfunction is defined as right ventricular outflow tract obstruction with peak echo-Doppler gradient $>40 \mathrm{~mm} \mathrm{Hg}$ and/or grade III/IV conduit valve regurgitation. Graft failure is defined as need for conduit replacement or need for catheter or surgical reintervention. Follow up was greater in homografts (Contegra, $4.0+$ 2.6 yrs vs. homograft, $5.3+2.9$ yrs; $\mathrm{P}=0.05$ ).

Results: These data demonstrate conduit dysfunction and the need for explantation were worse for small homografts albeit at longer follow-up interval. Small Contegra conduits trended to have less overall conduit failure. Distal stenosis and actuarial survival were similar in both cohorts. $\left({ }^{\star}\right.$ Values expressed as percent actuarial freedom from).

Conclusions: This study suggests the early performance of small Contegras may be more advantageous than homografts. A Contegra 
conduit is an appropriate first choice for conduit replacement in patients less than two years of age.

\begin{tabular}{llll}
\hline Actuarial Survival* & 81 & 87 & 0.57 \\
Conduit Dysfunction* & 85 & 24 & $<0.0001$ \\
Conduit Explantation* & 89 & 48 & 0.0001 \\
Conduit Failure* & 67 & 45 & 0.06 \\
Distal Conduit Stenosis* & 67 & 55 & 0.33 \\
\hline
\end{tabular}

\section{4}

Comparing Neurocognitive disturbances after off-pump and on-pump Coronary Artery Bypass Graft

Ali sadeghpour Tabaee MD, Arak university of medical science, Iran

Alireza Rostami MD, Arak university of medical science, Iran

Background: Neurocognitive complication after cardiac surgery with Cardiopulmonary bypass (CPB) is a common complication (in up to $53 \%$ of patients). In many patients this complication is only mild and transient, but in $5 \%$ of cases, it is severe.

Method: In this prospective study, from June 2002 to July 2004, in Rajaee Heart Center 186 patients were operated as on-pump or offpump Coronary artery bypass graft(CABG), they were evaluated for neurocognitive complications by mini-mental status test examination. Results: Average age of the patients was $56 \pm 6.2$ years, $62 \%$ were male and $38 \%$ were female. 121 patient were operated as on-pump and 65 patient were operated off-pump. Mini-mental status test examinations were normal in $50 \%$ of off-pump operations and in $43 \%$ of on-pump operations, very mild disturbance were seen in $48 \%$ of off-pump operations and in $54 \%$ of on-pump operations (pvalue $=0.192, \mathrm{NS})$, mild disturbance were seen in $2 \%$ of off-pump and in $3 \%$ of on-pump surgeries ( $p$-value $0.392, \mathrm{NS}$ ). Moderate or severe disturbances were not seen in any groups.

Conclusion: In our studies there was not significance difference in neurocognitive complication between off-pump and op-pump pump Coronary artery bypass graft (CABG) .

Key Words: Neurocognitive complication; Cardiopulmonary bypass, Coronary artery bypass graft.

\section{5}

Long term results and surgical outcome of conventional Fontan operation

Sadahiro Sai, Dept. of Cardiovascular Surgery, Miyagi children's hospital, Japan

Junetsu Akasaka, Dept. of Cardiovascular Surgery, Tohoku Univesity, Japan

Shunsuke Kawamoto, Dept. of Cardiovascular Surgery, Tohoku

Univesity, Japan

Yoshikatsu Saiki, Dept. of Cardiovascular Surgery, Tohoku Univesity, Japan

Osamu Adachi, Dept. of Cardiovascular Surgery, Tohoku Univesity, Japan Akinobu Konishi, Dept. of Cardiovascular Surgery, Miyagi children's hospital, Japan

Koichi Tabayashi, Dept. of Cardiovascular Surgery, Tohoku Univesity, Japan

Some patients who underwent conventional Fontan operation with atriopulmonary connection(APC), tend to present exercise intolerance, thromboembolic events and refractory arrhythmia with time.

Objective: We examined surgical outcome and late results in post Fontan patient with APC and efficacy of Total cavopulmonary connection (TCPC) conversion.
Method: Fifteen patients, ranged $2.5 \sim 12.6$ years old (mean 8.2) at Fontan, with tricuspid atresia $(\mathrm{n}=11)$, single ventricle and pulmonary atresia $(n=2$, each). Underwent APC between March 1978 and January 1995. Seven patients had conversion to TCPC. Interval period between initial Fontan and conversion was 12.1 year in average. There were six patients followed for more than 20 years.

Indication for conversion were pulmonary emboli $(n=2)$, Fontan pathway obstruction including conduit stenosis $(n=4)$ and atrial dysrhythmia $(n=1)$. All patients underwent the conversion to TCPC with an extracardiac conduit and the right atrial reduction plasty, while PV isolation was performed in two patients.

Results: There were 5 late deaths including two non cardiac related deaths due to post transfusion hepatitis. Actuarial survival rate and Freedom from reintervention were $66.8 \%$ and $51.9 \%$ respectively at 20 years.

Postconversion central venous pressure decreased significantly from 18.8 to $13.8 \mathrm{mmHg}$.

Exercise performance improved from grade 3.0 to 2.0 in NYHA functional class. Three of five patients with atrial dysrhythmia converted to sinus rhythm postoperatively in conversion cases.

Conclusion: Surgical outcome of Conversion to TCPC was satisfactory and it led clinical improvement by decreasing right atrial preload. In untreated post Fontan patients, the conversion should be undertaken prior to clinical signs of chronic rhythm disturbances or exercise impairment.

\section{6}

\section{Japanese multi-center clinical study of total anomalous} pulmonary venous connection

Kisaburo Sakamoto, Shizuoka Children's Hospital, shizuoka, Japan

Yuko Tosaka, Shizuoka Children's Hospital, shizuoka, Japan

Hidefumi Kishimoto, Japan

Toshihide Asou, Kanagawa Children's Medical Center, Japan

Arata Murakami, The University of Tokyo, Japan

Hiroshi Imoto, Kyushu Kouseinenkin Hospital, Japan

Objectives: It is said that operation results of total anomalous pulmonary venous connection (TAPVC) have been improved with the times, and some leading institutes had reported the excellent results of it. However there were not so many multicenter reports from any countries. In this report, we would like to talk about the exact results and consideration of TAPVC therapy in Japan, through multi-center data.

Methods: One hundred thirty-one patients with surgical repair of TAPVC from 1998 to 2007, who had no additional cardiac anomalies, were collected from 5 institutes among Japanese Congenital Heart Surgeon's Society group. We divided the whole into four groups (G-supra: 62 pts, G-cardiac: 21 pts, G-infra: 37 pts, G-mixed: 11 pts) and made some analysis retrospectively.

Results: Median day and body weight at TAPVC repair: G-supra 17 days $/ 3.4 \mathrm{~kg}, \mathrm{G}$-cardiac 26 days $/ 3.5 \mathrm{~kg}$, G-infra 8 days $/ 3.0 \mathrm{~kg}$, G-mixed 53 days $/ 3.3 \mathrm{~kg}$.

Postoperative survival rate(\%) at 1 year and 10 years: all groups 92.4/90.5, G-supra 91.9/91.9, G-cardiac 95.2/90.5, G-infra 94.6/90.5, G-mixed 81.8/81.8.

Freedom from postoperative pulmonary venous obstruction(\%) at 3 months, 1 year and 10 years: all group 82.3/77.2/ 69.0, G-supra 84.1/80.4/78.3, G-cardiac 85.2/73.9/73.9, Ginfra 81.1/78.4/53.4, G-mixed 70.0/60.0/60.0.

Conclusions: Postoperative results of TAPVC have acceptably improved in this decade and death after 1 year from TAPVC repair was rare, even in the groups of infracardiac and mixed type. 
However postoperative pulmonary venous obstruction have been also a major cause of early death yet.

7447

Impact of our novel surgical procedure for tetralogy of Fallot with an absent pulmonary valve using an in-situ mono cusp valve

Shigeru Sakurai, Department of cardiovascular surgery, Okayama university hospital, Japan

Shingo Kasahara, Department of cardiovascular surgery, Okayama university hospital, Japan

Youichi Inoue, Department of cardiovascular surgery, Okayama university hospital, Japan

Masami Takagaki, Department of cardiovascular surgery, Okayama university hospital, Japan

Sadahiko Arai, Department of cardiovascular surgery, Okayama

university hospital, Japan

Teiji Akagi, Department of cardiac care unit, Okayama university hospital, Japan

Shunji Sano, Department of cardiovascular surgery, Okayama university hospital, Japan

Background: In Japan, homografts and bovine jugular vein conduits were rare chances to use. The operative techniques for tetralogy of Fallot with absent pulmonary valve (TOF/APV) remains controversial as to whether the reconstruction of right ventricular outflow tract (RVOT) and remodeling of pulmonary artries.Morever,treatment of infants has been associated with increased mortality. With respect to the reconstruction of RVOT, some patients need re-operation in the future, and prevention of pulmonary regurgitation (PR) reduce the risk of late right ventricular dysfunction. We suggest a novel procedure for primary repair of TOF/APV.

Methods: Between January 1991 and December 2008, 8 consecutive patients including 6 infants underwent repair of TOF/APV with our new technique.Median age at repair was 9.5 months(range 1-55 months), and median weight was $5.6 \mathrm{~kg}$ (range 2.4-14.2 kg). Our surgical technique includes plication of dilatated pulmonary artery and RVOT reconstruction using autologous anterior pulmonary wall as an in-situ mono cusp valve.

Results: There was no early or late death during follow-up (median 54.5 months). One patient required re-operation due to bronchial compression. No patients had severe PR. The ratio of right and light ventricle end diastolic distance (RVEDD/ LVEDD) was reduced from median 0.73 to 0.60 . Morever, PR didn't deteriorate in 215 months follow-up, regardless of the somatic growth of the patient.

Conclusions: Based in our findins, we believe that our novel technique results in good outcomes and be feasible in infants or otherwise. Furtheremore, the in-situ mono cusp valve has a possibility to be avoid re-operation in long-term.

\section{8}

Outcome of Univentricular Heart palliation

Ana R Fernandes, Hospital Cruz Vermelha Portuguesa, Portugal Mónica Rebelo, Hospital Cruz Vermelha Portuguesa, Portugal Manuel J Ferreira, Hospital Cruz Vermelha Portuguesa, Portugal Maria Ana Sampaio Nunes, Hospital Cruz Vermelha Portuguesa, Portugal Graziela Nero, Hospital Cruz Vermelha Portuguesa, Portugal Manuel P Magalhães, Hospital Cruz Vermelha Portuguesa, Portugal

Objective: To evaluate the outcome of patients with $\mathrm{UH}$ submitted to the various stages of the Fontan procedure.
Methods: We retrospectively reviewed the registers patients (pts) with UH managed at our centre (1998-2008), and analysed their clinical outcome according to the type of systemic ventricle (G1: 2 ventricles, G2: left, G3: right and G4: indeterminate).

Results: 82 pts. The average present age is 4,3 years-old ( 2 months -12 years) and $57 \%$ are male. The first, second and third stages of the Fontan procedure were done at an average age of 36 days, 11,4 months and 4,4 years, respectively.

G1 (10 pts): $1 \mathrm{pt}$ in the first, 3 in the second and 4 in the last stage of the Fontan procedure; two patients was lost. Most patients are doing well, with minimum or no symptoms.

G2 (26 pts): 5 pts in the first, 9 in the second and 11 in the last stage of the Fontan procedure; one patient was lost. Most patients are also doing well.

G3 (45 pts): 15 pts in the first, 12 in the second and 18 in the last stage of the Fontan procedure; two patients was lost. Seven patients have mild to moderate limitation of activity and/or have mild to moderate cyanosis. Deaths (9) occurred in this group at or after the first stage.

G4 (1 pts): at the second stage and is clinically well.

Conclusion: The overall results of the palliation of patients with univentricular heart are good.Worse prognosis in patients with right type $\mathrm{UH}$.

\section{9}

Surgical aortic valvotomy for critical aortic stenosis in neonates and small infants

Shunji Sano, Okayama University Hospital, Japan

Shingo Kasahara, Okayama University Hospital, Japan

Ko Yoshizumi, Okayama University Hospital, Japan

Yasuhiro Kotani, Okayama University Hospital, Japan

Yasuhiro Fujii, Okayama University Hospital, Japan

Sadahiko Arai, Okayama University Hospital, Japan

Kozo Ishino, Showa Yokohama North Hospital, Japan

Masaaki Kawada, Tochigi Children's Hospital, Japan

Objective: Optimal management for critical aortic stenosis (AS) in neonates and early infants remains a challenge to cardiologists and cardiac surgeons. In our institution, surgical valvotomy is the first-line treatment for critical AS, although balloon valvuloplasty has become most popular treatment in elsewhere.

Methods: A retrospective review of neonates and small infants undergoing surgical valvotomy in single institution between 1993 and 2007 was carried out. Clinical and echocardiographic follow up data were analyzed. Twelve infants less than 6 months (mean age: 47 days) underwent surgical valvotomy for critical AS. Mean body weight at surgery was $4.2 \mathrm{~kg}$ (range: $2.6-6.5 \mathrm{~kg}$ ). Surgery included aortic commissurotomy and excision of myxomatous valve tissue. One patient who underwent balloon valvuloplasty at other institution was referred to us for severe aortic regurgitation (AR).

Results: There were no mortality with a mean follow up of 73 months. There were 4 reoperations including 2 re-valvotomy and 2 Ross in 3 patients. All 3 patients had unicusp or bicusp. One patient who underwent balloon valvuloplasty elsewhere after surgical valvotomy, had developed severe $\mathrm{AR}$ and required Ross procedure. Another patient required re-valvotomy at 15 months after and required Ross. Most recent echocardiogram showed less than Grade $1 \mathrm{AR}$ in 10 patients and Grade 2 in 2 . No patient has developed significant re-AS. Freedom from re-intervention at 4 years was $75 \%$. Freedom from AVR or Ross was $83 \%$.

Conclusions: Surgical valvotomy and excision of myxomatous tissue for critical AS results in a low early and late mortality, a low recurrence of significant $\mathrm{AR}$ and $\mathrm{AS}$. 
7450

Impact of Arch Reconstruction on Late results in Modified Norwood Procedure

Shunji Sano, Okayama University Hospital, Japan

Shingo Kasahara, Okayama University Hospital, Japan

Ko Yoshizumi, Okayama University Hospital, Japan

Yasuhiro Kotani, Okayama University Hospital, Japan

Yasuhiro Fujii, Okayama University Hospital, Japan

Sadahiko Arai, Okayama University Hospital, Japan

Kozo Ishino, Okayama University Hospital, Japan

Shin-ichi Otsuki, Okayama University Hospital, Japan

Objective: One of the technical points of Norwood Procedure is how to reconstruct the non-obstructed pathway from RV to descending aorta. We have reconstructed Neo-aorta without patch material in most of the patients of HLHS using cerebral perfusion since 1998, although we have modified our technique during 10 year period. We retrospectively reviewed late outcome of arch reconstruction.

Patients and Methods: Since 1998, we have performed 71 modified Norwood procedure using arch reconstruction without patch materials in 63 patients and with small pericardial patch in 9 patients. No homograft was used. During 10 year periods, 2 technical modifications in arch reconstruction were adopted. One was proximal arch plasty (Aortic arch and ascending aorta just opposite site of innominate artery were sutured inferiory to extend the width of aortic arch and shortened the neo-aortic suture line). Another was distal arch plasty (application of Brawn's modification).

Results: Proximal arch plasty was applied in 21 patients and distal arch plasty was applied in 40 patients. With a mean follow up of 64 months, re-CoA was found to have in 10 patients (16\%) Pressure gradient across the re-CoA was $5-23 \mathrm{mmHg}$ with a mean of $15 \mathrm{mmHg}$. Re-CoA was found in 1 patient out of 9 patch material group (11\%) and 9 patients with 62 non patch group (14\%) All reCoA was balloon dilated successfully except one who required surgical intervention.

Conclusion: Arch reconstruction was possible without patch material in most of the patients. Most of the re-CoA was repaired by balloon dilation.

7451

Arterial Switch Operation in Late Presenters with Transposition of Great Arteries and Intact Ventricular Septum: How Late is Late?

Anita Saxena, All India Institute of Medical Sciences, New Delhi, India, India

Akshay K Bisoi, All India Institute of Medical Sciences, New Delhi, India, India

Sandeep Chauhan, All India Institute of Medical Sciences, New Delhi, India, India

Srikrishna M Reddy, All India Institute of Medical Sciences, New

Delhi, India, India

Pranav Sharma, All India Institute of Medical Sciences, New Delhi, India, India

Rajnish Juneja, All India Institute of Medical Sciences, New Delhi, India, India

Shyam S Kothari, All India Institute of Medical Sciences, New Delhi, India, India

S Ramakrishnan, All India Institute of Medical Sciences, New Delhi, India, India

Objective: Patients with transposition of great arteries and intact ventricular septum (TGA IVS) who present late for surgery are considered high risk for primary arterial switch operation (ASO). However late presentation is not unusual in several parts of the world. This study reports our experience in patients with TGA IVS presenting beyond six weeks of life who underwent primary ASO.

Methods: Hospital records of patients who underwent ASO for TGA IVS beyond 6weeks of life over the last 4 years were reviewed for preoperative information including echocardiography. Outcomes were measured as in-hospital mortality and need for extra corporeal membrane oxygenator (ECMO) in postoperative period.

Results: Out of a total of 185 ASO procedures, 32 were performed at age $>6$ weeks for TGA IVS (17.3\%). The age ranged from 42 days to 9 years (median 60 days). Subvalvular LV outflow obstruction was present in 5 cases. 20 cases had been recently treated with antibiotics for sepsis and/or respiratory tract infection. Echo revealed a favorable geometry of LV in 22 cases and a borderline LV in 10 cases. In-hospital mortality was $21.8 \%$ (7/32), LV geometry was borderline in $4 / 7$ cases. The length of stay in ICU and hospital was 4 and 18 days respectively. ECMO support was required in 4 .

Conclusion: Our data shows that in select cases of TGA IVS presenting late, primary ASO can be performed successfully. Accurate assessment of LV by echo is crucial to make this decision. Some patients may require ECMO support.

\section{2}

Management of patients with bidirectional Glenn shunt and multiple arterio veneous malformation Prashant D Shah, Miot children cardiac care, chennai, India

K Sivakumar, Miot children cardiac care, chennai, India

Robert Coelho, Miot children cardiac care, chennai, India

Introduction: Patients with BDG and multiple pulmonary arterio venous malformation (PAVM)is a difficult group to deal with. management options are limited This is a small study of Fontan completion in this group of patients.

Material and methods: Study from March 2007 to December 2008 at MIOT Children cardiac care, Chennai, India.There are 4 patients age range from 2 years to 13 yrs, sex-m:f $=1$ :3.non pulsaltile glen was done in 2 pts, 1 patient had kawashima while other had pulsaltile bdg.

Average duration of previous surgery was 24 months.all patient presented with desaturation average saturation was $60 \%$ on room air. one patient was oxygen dependent 2 patients had bilateral PAVM while other 2 had right sided diffuse PAVM. All patients underwent catheterization with meanPVR was 1.4 woods $/ \mathrm{m} 2$. extra cardiac fontan procedure done with using 20 to $23 \mathrm{~mm}$ ptfe tube graft as a conduit and using beating heart cardio pulmonary bypass. Average CPB time was 102 minutes. post op ventilation was mean 8 hours, ICUstay -5 days, hospital stay 11 days. On discharge average saturation was $85 \%$. On follow up no patient had cath but 3 patients had improvement in symptoms and saturation, while 1 patient on 6 month follow up still saturating $70 \%$ and require intermittent oxygen.

Conclusion: Fontan completion is a good option for this high risk patients. It has shown improvement in symptoms and long term cath follow up require to show its effect on regression of PAVM.

\section{3}

Management of stented Patent Ductus arteriosus-our experience

Prashantkumar Dineshchandra Shah, Miot hospitals, chennai, India, India 
K Sivakumar, Miot hospitals, chennai, india, India

Robert Coelho, Miot hospitals, chennai,india, India

Introduction: In the era of intervention, cardiologists are now putting stents in each and every possible arteries. Recently PDA stent is becoming popular for neonates with patent ductus arteriosus (PDA) dependent Pulmonary blood flow, which prevent neonatal shunt procedure and gives time to grow child little older for surgery for staged or complete repair. Here we are sharing our surgical experience dealing with stented PDA.

Material \& Methods: 3 patients having PDA stent at neonatal age period diagnosis was Pulmonary atresia with Intact ventricular septum \& Right ventricle dependent coronary circulation- 2 patients, Tricuspid atresia,severe pulmonary stenosis with PDA-1 Age at time of surgery was 4 months 5 and 11 month accordingly. After Sternotomy we have dissected the PDA and encircled, after establishing $\mathrm{CPB}$, on beating heart PDA clamped by straight Vascular clamp and divided with tough scissor. Aortic end was sutured with continuous suture but sutures were not holding properly so it requires additional pledgeted sutures to control bleeding. Stent fragments, which were embedded into the pulmonary artey, had taken out and pulmonary end repaired with pericardial patch to avoid any narrowing. Because of previous patient experience we changed technique and repair Aortic end with interrupted felt pledgetted sutures, which was more secure and pulmonary end deal with the same way.

Conclusion: Stented PDA should deal very respectively, interrupted suture at aortic end gives a secure hemostasis and patching of pulmonary artery at PDA insertion site prevent origin stenosis of branch pulmonary artery.

\section{4}

Anomalous systemic venous connection causing cyanosis - surgical options

Prashantkumar Dineshchandra Shah, miot children cardiac care, India $R$ Satish, miot children cardiac care, India

$K$ Sivakumar, miot children cardiac care, India

Robert Coelho, miot children cardiac care, India

Introduction: Anomalous systemic venous connection is very rare and this anomaly causing cyanosis is rarer. All possible causes we have reviewed and described our experience.

Material and methods: Single institute study from March 2007- till date. SVC anomaly includes B/L SVC with large unroofed coronary sinus -2 patients (group-1)

Common atrium with LSVC to roof of LA -2 patients. (group-2)

Isolated RSVC to LA with PAPVC - 1 patient (group-3).

IVC to LA after ASD closure - 2 patients (group-4)

Surgery performed in all patients with roofing of coronary sinus with gortex patch in group1, baffling of LSVC to RA with autologous pericardial patch in group 2.Direct anastamosis of RSVC to RA in group3 without cardiopulmonary bypass.

Anastamosis of LSVC to RA with interposition of $18 \mathrm{~mm}$ PTFE tube graft -1 patient.

In group 4 Baffling of IVC to RA with pericardial patch after excising previous patch.

Conclusion: Various anomalies of SVC and IVC can be delt with appropriate surgical technique with good surgical outcome.

\section{5}

Palliative Arterial Switch for biventricular and univentricular hearts with Arterial Malposition

Rajesh Sharma, Escorts Heart Institute and Research Centre, India
Ashutosh Marwah, Escorts Heart Institute and Research Centre, New Delhi, India

Vishal K Singh, Escorts Heart Institute and Research Centre, New Delhi, India

Arun Ramaswamy, Escorts Heart Institute and Research Centre, New Delhi, India

Sunita Maheshwari, Narayana Hrudayalaya Institute of Medical

Sciences, Bengalooru, India

Sejal Shah, Narayana Hrudayalaya Institute of Medical Sciences, Bengalooru, India

Objective: To evaluate the role of Palliative Arterial Switch Cperation in the context of biventricular and univentricular hearts with malposed great arteries.

Methods and results: Seven children underwent arterial switch with pulmonary artery banding with partial/non addressal of their intracardiac communications at initial operation. Underlying diagnoses were: A). D-Transposition of the great arteries (TGA) with significant right ventricular hypoplasia and ventricular septal defect $(n=3) B)$. Double outlet of the right ventricle with complex ventricular septal anatomy and multiple ventricular septal defects(VSD) with complex atrioventricular valve anatomy $(\mathrm{n}=2)$, and $\mathrm{C})$. Univentricular hearts with $\mathrm{D}$ Transposition of the great arteries and subaortic obstruction $(n=2)$. One in each of the biventricular and univentricular groups had associated aortic arch obstruction that was tackled simultaneously at the time of the arterial switch. Ages ranged from 15 days to 18 months at time of initial repair (median75d). There were six early survivors. Two of the patients have gone on to receive second stage surgery in the form of a closure of VSD along with pulmonary artery reconstruction in GpA. One of the 2 patients in $\mathrm{Gp} \mathrm{C}$ has gone on to a second stage superior cavopulmonary shunt. Others are on follow up for appropriate timing for second stage repair.

Conclusion: Encouraging results with this alternative approach merit consideration of its use in complex situations associated with malposed great arterial relationships in either uni or biventricular subsets as an alternative to conventional surgical strategies of Stage One Norwood or Stansel type connections or high risk one- stage repairs, respectively.

\section{6}

Preoperative Autologous Donations of Blood in Children Undergoing Open Heart Surgery

Fumiaki Shikata, Department of Cardiovascular Surgery,

Ehime University, Ehime, Japan, Japan

Mitsugi Nagashima, Japan

Takashi Higaki, Department of Pediatric Cardiology, Ehime University, Ehime, Japan, Japan

Eiichi Yamamoto, Department of Pediatric Cardiology, Ehime

University, Ehime, Japan, Japan

Masaaki Ohta, Department of Pediatric Cardiology, Ehime University,

Ehime, Japan, Japan

Kikuko Murao, Department of Pediatric Cardiology, Ehime University, Ehime, Japan, Japan

Eiichi Ishii, Department of Pediatric Cardiology, Ehime University,

Ehime, Japan, Japan

Kanji Kawachi, Department of Cardiovascular Surgery, Ehime

University, Ehime, Japan, Japan

Objective: To investigate the effective method of preoperative autologous donations of blood in small children who are scheduled for open heart surgery.

Methods: Forty-five patients weighing less than $15 \mathrm{~kg}$ (age range, 10 months-5 years; weight range, $6.6-14.8 \mathrm{~kg}$ ) underwent 
preoperative autologous donation and cardiac surgery to treat a simple anomaly at Ehime University and Ehime Prefectural Central Hospital. Donations of $10 \mathrm{~mL} / \mathrm{kg}$ each were collected via the femoral vein under mild general anesthesia preoperatively. Twenty-six patients were given $400 \mathrm{IU} / \mathrm{kg}$ erythropoietin intracutaneously immediately after each donation (Group E). Sixteen patients underwent perioperative autologous donation without the use of erythropoietin (Group C). The perioperative hemoglobin and mean corpuscular hemoglobin concentrations, minimum hemoglobin level during $\mathrm{CPB}$, platelet count, total protein level and the total postoperative blood loss were analyzed.

Results: There was no serious complication related to autologous blood collection. Forty-two patients (93\%) underwent surgery without homologous blood transfusion. No significant differences in the hemoglobin levels were found between Group E and C before, during or after surgery. Postoperative recovery in hemoglobin levels of the patients who underwent surgery more than 22 days after the donation, was significantly better than the patients who underwent surgery less than 22 days (Figure 1).

Conclusions: Preoperative autologous donation can be performed more effectively even in children weighing less than $15 \mathrm{~kg}$ when the period between the donation and surgery is more than 22 days.

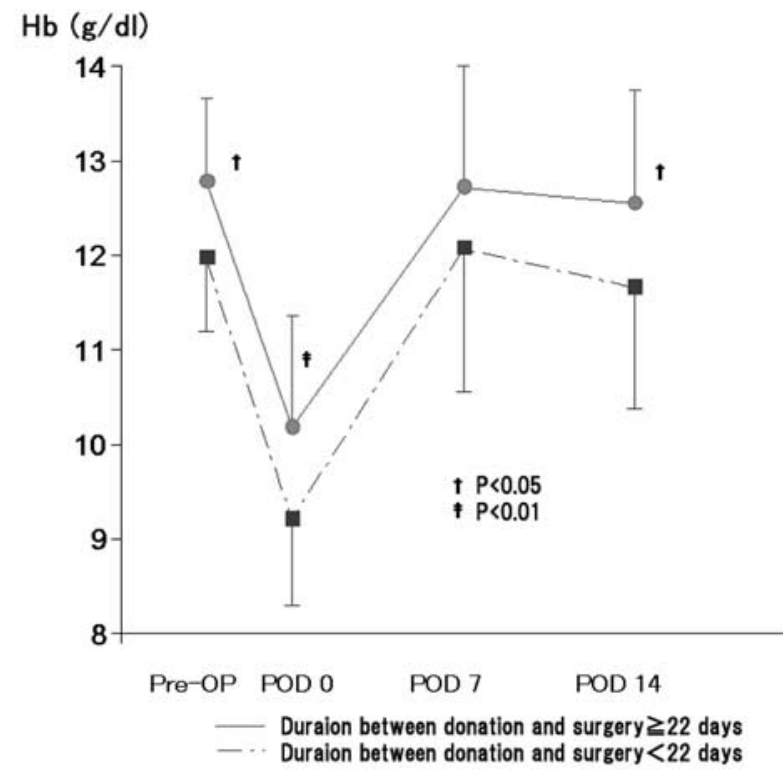

Figure 1. Perioperative Changes in Hemoglobin Levels

\section{7}

Surgical Repair of Ruptured Sinus of Valsalva Aneurysms: Early and Midterm Outcome

Man-shik Shim, Department of Thoracic and Cardiovascular Surgery, Sungkyunkwan University, School of Medicine, Samsung Medical Center, Seoul., Korea, Democratic People's Rep

Pyo Won Park, Department of Thoracic and Cardiovascular Surgery, Sungkyunkwan University, School of Medicine, Samsung Medical Center, Seoul., Korea, Democratic People's Rep

Young Tak Lee, Department of Thoracic and Cardiovascular Surgery, Sungkyunkwan University, School of Medicine, Samsung Medical Center, Seoul., Korea, Democratic People's Rep

Wook Sung Kim, Department of Thoracic and Cardiovascular Surgery, Sungkyunkwan University, School of Medicine, Samsung Medical Center, Seoul., Korea, Democratic People's Rep
Ki-ick Sung, Department of Thoracic and Cardiovascular Surgery, Sungkyunkwan University, School of Medicine, Samsung Medical Center, Seoul., Korea, Democratic People's Rep

Ji Hyuk Yang, Department of Thoracic and Cardiovascular Surgery, Sungkyunkwan University, School of Medicine, Samsung Medical Center, Seoul., Korea, Democratic People's Rep

Tae-Gook Jun, Department of Thoracic and Cardiovascular Surgery, Sungkyunkwan University, School of Medicine, Samsung Medical

Center, Seoul., Korea, Democratic People's Rep

Background: Ruptured sinus of Valsalva aneurysm is a rare lesion. We reviewed our experience to assess the outcome of surgical repair of ruptured sinus of Valsalva aneurysm and the development of aortic regurgitation(AR)after the surgery.

Method: Between January 1995 and December 2008, 39 patients was undergone curative surgical correction for ruptured sinus of Valsalva aneurysm. We retrospectively analyzed medical records and evaluate the treatment outcome. The median age was 33 (14-63) years. The mean follow up duration was $4.3(0.03-13.4)$ years.

Results: Primary closure alone was performed in 6 patients (single in 5 patients, double in 1 patient). Patch closure alone was performed in 9 patients (single in 5 patients, double in 4 patients). Combined patch and primary closure was performed in 19 patients. Aortic valve replacement (AVR) was performed in 5 patients. There was no hospital mortality. The median length of hospital stay was $7(5-58)$ days. Postoperative complications included arrhythmia in 3, systemic thromboembolism in 2, pericarditis/pericardial effusion in 2 , and subdural hemorrhage in 1 patients. 8 patients had AR more than moderate after the operation. The interval periods were between 4.4 and 60 months. In 7 patients, the cause of AR was prolapse of diseased cusp and the moderate AR developed within 33 months. 1 of 8 patients who had AR was performed AVR.

Conclusion: The early outcome of repair of ruptured sinus of Valsalva aneurysms was good. However, progressive AR may develop during the follow-up. Careful observation for $\mathrm{AR}$ is required.

\section{8}

Correcting mediastinum left displacement improves the imbalance in the pulmonary vascular bed and lung volume in children with pectus excavatum after the Nuss procedure

Shinya Shimoyama, Department of Pediatrics, Gunma University Graduate School of Medicine, Gunma, Japan/Maebashi Saiseikai Hospital, Japan, Japan

Tohru Kobayashi, Department of Pediatrics, Gunma University Graduate School of Medicine, Maebashi, Gunma, Japan, Japan Hiroyuki Mochizuki, Department of Pediatrics, Gunma University Graduate School of Medicine, Maebashi, Gunma, Japan, Japan Akito Hamajima, Department of Plastic and Reconstructive Surgery, Gunma Children's Medical Center, Shibukawa, Gunma, Japan, Japan

Tomio Kobayashi, Department of Cardiology, Gunma Children's Medical Center, Shibukawa, Gunma, Japan, Japan

Shinitu Hatakeyama, Department of Radiology, Gunma Children's

Medical Center, Shibukawa, Gunma, Japan, Japan

Hirokazu Arakawa, Department of Pediatrics, Gunma University Graduate School of Medicine, Maebashi, Gunma, Japan, Japan

Objectives: The imbalance of pulmonary perfusion and lung volume in patients with pectus excavatum (PE) after the Nuss procedure has not been objectively assessed. The study sought to assess the degree of impairment of the cardiovascular system in 
patients with PE and to investigate any changes caused by surgical correction.

Methods: Chest radiography, computed tomography (CT) and pulmonary perfusion scintigraphy were performed before surgery and at medium-term follow-up (2.7 \pm 0.3 years) in 34 patients operated on for PE. Chest radiography was used to calculate the vertebral index (VI) and the left displacement index (LDI), which is the ratio between the left border of the mediastinum and the left border of the thorax to the transverse thoracic dimension on posterioanterior chest radiography. Left-to-right count ratio for lung scintigraphy (Ls/Rs), and the left-to-right thorax volume ratio from $\mathrm{CT}(\mathrm{Lv} / \mathrm{Rv})$ were also measured.

Results: Preoperative left pulmonary perfusion and lung volume were significantly impaired compared with the right lung. After the Nuss procedure, Ls/Rs and Lv/Rv were significantly increased (Ls/ Rs; $0.81 \pm 0.09$ vs. $0.89 \pm 0.09, \mathrm{P}=0.001: \mathrm{Lv} / \mathrm{Rv} ; 0.68 \pm 0.08$ vs. $0.79 \pm 0.08, \mathrm{P}<0.001)$. LDI correlated with $\mathrm{Ls} / \mathrm{Rs}(\mathrm{R}=0.510$, $\mathrm{P}<0.001)$ and $\mathrm{Lv} / \mathrm{Rv}(\mathrm{R}=0.769, \mathrm{P}<0.001)$; there was no correlation for VI with Ls/Rs.

Conclusions: Our findings indicated that the imbalance of pulmonary perfusion and lung volume improves in PE patients after completion of the Nuss procedure. The alternations might be related to the improvement from the left shift of the mediastinum.

\section{9}

\section{Definitive Conal Enlargement for Recurrent Subaortic} Stenosis

Ashutosh S Singh, The Children's Hospital at Westmead, Sydney, Australia David S Winlaw, The Children's Hospital at Westmead, Sydney, Australia

Andrew D Cole, The Children's Hospital at Westmead,

Sydney, Australia

Richard B Chard, The Children's Hospital at Westmead, Sydney, Australia

Recurrence of subaortic stenosis (SAS) after initial resection occurs in up to $20 \%$ of cases. Options include re-resection, classical Konno aortoventriculoplasty with aortic valve replacement, valve sparing aortoseptal approach (Vouhe) or - as we suggest - valve sparing conal enlargement with patch augmentation of the sub-aortic region accessed via an infundibulotomy (popularised by Vouhe, initially described by Cooley). Repeated conservative approaches are associated with persisting gradients, heart block, ventricular septal aneurysm and VSD.

Patients: We performed conal enlargement in four children aged $2-19$ years. 3 had a canal defect and previously underwent transaortic resection and myectomy. 1 had Williams syndrome, initially treated for supra AS but subsequently developed muscular SAS.

A conal-enlargement type of resection and patch augmentation of the LVOT was successfully performed with patch augmentation of the subaortic area as described above. Complete relief of obstruction was obtained and no obstruction has recurred. The patient with Williams syndrome later required an aortic valve replacement but the subaortic area did not require further attention. Relief of obstruction has been sustained over a mean of 36 months. No heart block or low cardiac output was observed.

Discussion: The strength of this procedure is effective augmentation of the LVOT and amelioration of angulation which maintains the substrate for later obstruction. Conal enlargement is rarely described beyond the initial reports suggesting that this approach is underappreciated.

In our experience, comprehensive conal enlargement should be undertaken where conservative approaches had previously failed to gain an adequate long term relief of SAS.

\section{0}

Does the Kalangos biodegradable ring promote annular remodelling and preserve the growth potential following mitral valve repair for congenital mitral regurgitation? Sivakumar Sivalingam, National Heart Institute, Kuala Lumpur, Malaysia

Jeswant Dillon, National Heart Institute, Kuala Lumpur, Malaysia Mazeni Alwi, National Heart Institue, Kuala Lumpur, Malaysia Azhari Yakub, National Heart Institute, Kuala Lumpur, Malaysia Afksendiyos Kalangos, University Hospital of Geneva, Geneva, Switzerland

Objectives: The Kalangos biodegradable ring in experimental studies has been shown to promote annular remodelling and preserve annular growth potential. In this study we report the feasibility of this concept when implanted as concomitant annuloplasty for mitral valve repair in a pediatric population. Methods: Between January 2006 and December 2007, 20 patients with a median age of 3 years (6 months -13 years) underwent mitral valve repair with concomitant annuloplasty using the biodegradable ring. Regurgitation was due to annular dilatation in 3(15\%), prolapsed leaflets in $16(85 \%)$ and restrictive leaflet motion in $1(5 \%)$ patients. Pre-operative indexed mitral valve diameter and $\mathrm{Z}$-values were compared with those obtained at follow-up. The annular diameter in the immediate post-operative period and follow-up were compared to assess the growth potential of the annulus.

Results: There were no early deaths. The median follow-up period was 22.5 months. At follow-up regurgitation grades were none in 6 , mild in 13 and moderate in 1 patients. There were no significant changes in the mean gradient between the immediate post-operative period and follow-up $(4.47+/-1.41 \mathrm{mmHg}$ vs. $3.94+/-1.61 \mathrm{mmHg}$. p-0.179). Annuloplasty resulted in significant reduction of the indexed mitral valve diameter $40.28+/$ $-12.08 \mathrm{~mm} / \mathrm{m}^{2}$ to $18.70+/ 2.98 \mathrm{~mm} / \mathrm{m}^{2} \quad(\mathrm{p}<0.001)$ and $\mathrm{Z}$-value $0.80+/-1.01$ to $-0.74+/-0.98(\mathrm{p}<0001)$. There was a significant increase in the annular diameter by $7.9 \%$ between immediate post-operative period and follow-up $(17.41+/-2.9 \mathrm{~mm}$ to $18.70+/-2.9 \mathrm{~mm})(\mathrm{p}<0.0001)$.

Conclusion: Annuloplasty with Kalangos biodegradable ring has demonstrated to optimize the results of mitral valve repair in pediatric patients. It has further shown to promote annular remodelling and preserve the growth potential of the native annulus.

\section{1}

Primary arterial switch for late presenting simple D Transposition of the great arteries

Kini M Srinivas, Escorts Heart Institute and Research Centre, New Delhi., India

Bijender S Bali, Escorts Heart Institute and Research Centre,

New Delhi., India

Sabir Khan, Escorts Heart Institute and Research Centre, New Delhi., India

Shamsuddin Abul Kalam, Escorts Heart Institute and Research Centre, New Delhi., Bangladesh

Neeti Chandra, Escorts Heart Institute and Research Centre,

New Delhi., India

Vishal K Singh, Escorts Heart Institute and Research Centre,

New Delhi., India

Ashutosh Marwah, Escorts Heart Institute and Research Centre,

New Delhi., India

Rajesh Sharma, Escorts Heart Institute and Research Centre,

New Delhi., India 
Objective: Prospective evaluation of a protocol for primary arterial switch in late presenting simple TGA.

Methods: Sixteen patients with diagnosis of TGA with intact interventricular septum presenting since May 2007 with age ranging from 45 days to 185 days (median 120 days) had the following LV characteristics: Left ventricular posterior wall thickness $2.0 \mathrm{~mm}-3.0 \mathrm{~mm}$ (median $2 \mathrm{~mm}$ ) with a pancaked left ventricular cavity or a flat interventricular septum.7 had had a balloon septostomy elsewhere.All underwent a primary arterial switch.

Results: Weaning from cardiopulmonary bypass was achieved in all with initial reversal of heparin for hemostasis. Six patients could be managed without extracorporeal support while ten experienced high left atrial pressures and low systemic arterial pressure and had to be put on extracorporeal support. Time on ECMO ranged from 24-96 hours (median 72 hours). There were 2 deaths in the ECMO group(sepsis-1, pulmonary hemorrhage-1), none in the nonECMO group.Mean time to extubation was similar in both groups 12 days in the nonECMO and 10 days in the ECMO group.

Echo at hospital discharge showed a mean LVEF of 55\%.

Conclusion: Primary arterial switch with ECMO as standby can be offerred with reasonable safety to all simple transpositions presenting upto six months of age. Use of this modality in older patients is limited by the length of period on ECMO that may be required for left ventricular preparation.

\section{2}

Human Cardiac Tissue Signaling Associated With Regeneration-Related Events

Ann M Steele, All Children's Hospital, United States

Peter O Steele, All Children's Hospital, United States

William Schleif, All Children's Hospital, United States

Jasmine A Steele, All Children's Hospital, United States

Jeffrey P Jacobs, Univ. South Florida/All Children's Hospital/Cardiac Surgical Associates, United States

Wilfredo Chamizo, All Children's Hospital, United States

Vincent Molnar, All Children's Hospital, United States

Robert J Boucek, Seattle Children's Hospital, United States

Objective: The poorly characterized paracrine function of human heart is postulated to be a significant factor in myocardial responses to injury. Reported herein is a novel method that characterizes cytokine responses to injury associated with cell trafficking and proliferation.

Methods: Freshly discarded samples of human heart $(\mathrm{n}=15)$ were minced and explant was cultured in media (DMEM+20\% FCS). Media was sampled before and after addition of tissue and after 3, $7,14,28$ and 56 days in culture. The relative abundance of 120 cytokines was determined using monoclonal antibody array with chemi-luminescent detection (RayBio, USA). Based on cytokine-specific antibody binding on an arbitrary scale between 0 (negative control) to $100 \%$ (positive control), an increase to $>10 \%$ was defined as newly expressed.

Results: Stereotypic cellular events in culture were observed. By day 7 , phase bright cells, putative progenitor cells, were noted to bud from the tissue and loosely attach to the bottom of the flask. By day 14, a subpopulation of these cells formed aggregates or cardiospheres. Up to day 28, single cells and cardiospheres increased in number and underwent spontaneous differentiation. The number of newly expressed cytokines were: 14 in the first 3 days; 3 between day 4 and 7 preceding CPC out-trafficking; 4 between day 7-14 preceding cardiosphere formation; and 14 between day 14-28 with individual cell and cardiosphere differentiation.
Conclusions: Unique candidate cytokines were identified that were temporally associated with tissue trafficking, proliferation and differentiation of human cardiac tissue-derived progenitor cells.

\section{3}

Repair Of Coarctation Of The Aorta In Neonates And Infants

Senthil K Subbian, KK Women's and Children's Hospital, Singapore, Singapore

Sriram Shankar, KK Women's and Children's Hospital, Singapore, Singapore

Keng Y Wong, KK Women's and Children's Hospital, Singapore, Singapore

Teng H Tan, KK Women's and Children's Hospital, Singapore, Singapore

Objectives: To analyze the outcomes of repair of coarctation of aorta in neonates and infants.

Methods: Forty nine neonates and infants underwent coarctation repair between November 1998 and May 2008. 21 had simple coarctation (Group I), 9 had complex coarctations (Group II) and 19 had an associated VSD (Group III). Group I had a left thoracotomy and resection and end-to-end anastamosis. Group II had a left thoracotomy, resection and end-to-end anastamosis in 4, primary sternotomy and repair in 2 and a left thoracotomy followed by a sternotomy in the same anaesthesia to revise the anastamosis by joining the left subclavian and left common carotid artery in 3 . The patients with an associated VSD had a left thoracotomy for coarctation repair followed by a sternotomy for VSD closure with a median time interval of 2 days. The mean follow-up period was $8.8 \pm 4.5$ years.

Results: There was one in hospital death of a $1.2 \mathrm{~kg}$ premature neonate (Group III). 5 patients had a residual coarctation that was treated with balloon aortoplasty. Hoarseness of voice was observed in 3 patients.

Conclusions: Resection and end-to-end anastamosis for coarctation repair yields excellent outcomes. Augmenting the distal arch in complex coarctations by incorporating the lower ends of the left subclavian artery and the left common carotid artery is a useful technique. A shorter interval between the 2 stages of repair of coarctation and the associated VSD provides excellent results in addition to freedom from complications of circulatory arrest and a pulmonary artery band.

\section{4}

Clinical experience of ePTFE valved conduit in right ventricular outflow tract reconstruction

Si Chan Sung, Pusan National University Children's Hospital, Korea

Yun Hee Chang, St. Mary's Hospital, Catholic University, Korea Soo Kyung Park, Pusan National University Children's Hospital, Korea

Hyoung Doo Lee, Pusan National University Children's Hospital, Korea

Ji Ae Park, Pusan National University Children's Hospital, Korea Ji Eun Ban, Pusan National University, Korea

Objective: There has been no reasonably durable conduit for right ventricular outflow tract reconstruction (RVOTR) in small pediatric patients so far. We developed ePTFE valved conduit having thin bicuspid ePTFE membrane valve. The early results of this valved conduit were evaluated.

Methods: From April 2005 to August 2008, 11 patients underwent RVOTR using valved ePTFE conduit. Mean age at 
operation was $21.9 \pm 17.8$ months(7days -68.8 months) and mean body weight was $9.6 \pm 2.9 \mathrm{~kg}(2.98-13 \mathrm{~kg})$. The diagnosis was $\mathrm{TOF} / \mathrm{PA}$ in 9 patients, TGA/VSD/PA in 1 and TOF/APV in 1. The conduit size was $12 \mathrm{~mm}$ in 1 patient, $14 \mathrm{~mm}$ in $1,16 \mathrm{~mm}$ in $3,18 \mathrm{~mm}$ in 6. Mean follow-up duration was 16.5 months (4-43.7months). The conduit function was evaluated with transthoracic echocardiography.

Results: There was no operative mortality and late death. There were no conduit-related reoperations or postoperative complications including postoperative bleeding, thromboembolism, and thrombotic occlusion. Mean peak systolic pressure gradient between RV and distal MPA at discharge was $14.2 \pm 10.0 \mathrm{mmHg}$ $(0-29.2 \mathrm{mmHg})$. Conduit valve regurgitation was none in 7 patients, trivial in 3 , mild in 1 . Valve motion was good in 9 patients, not confirmed in 2 because of poor echo window. There were no significant aggravations of valve regurgitation in all patients during follow-up. Among the patients $(n=3)$ who followed up more than 20 months, small increases of pressure gradient between RV and distal MPA were observed (from $17.9 \mathrm{mmHg}$ [mean] to $27.1 \mathrm{mmHg}$ ).

Conclusions: ePTFE valved conduit has excellent function in terms of valve regurgitation and conduit pressure gradient without any conduit related complications. Further longer follow-up for this valved conduit function is mandatory.

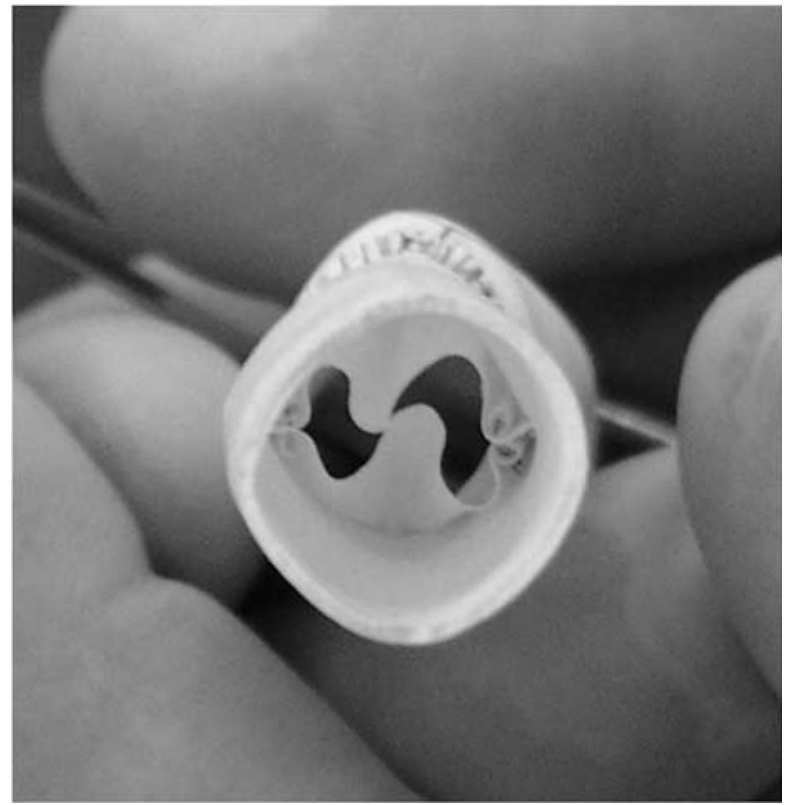

7465

Long-term survival after pediatric cardiac surgery during 10 years (1994-2003); Experience from one institution in Sweden

Jan T Sunnegardh, The Queen Silvia Children's Hospital, Sahlgrenska University Hospital, Göteborg, Sweden

Haikan Berggren, The Queen Silvia Children's Hospital, Sahlgrenska University Hospital, Göteborg, Sweden

Mats Synnergren, The Queen Silvia Children's Hospital, Sahlgrenska University Hospital, Göteborg, Sweden

Objective: To gather reliable and complete data for evaluation of long-term survival after pediatric cardiac surgery.

Methods: All 1652 patients (765 girls/887 boys) operated due to congenital heart defects in our institution before the age of 18 years from Jan 1st 1994 to Dec 31st 2003 were included. Patient files were cross-checked as of Dec 1st 2008, against the National Population Registry in Sweden, allowing for reliable and complete data on survival.

Results: Median age at surgery was 0.38 years (0-17.8 years). 1443 patients $(87 \%)$ had surgery for biventricular correction and 209 patients (13\%) had univentricular heart palliations. Median age of survivors at follow up was 12.4 years (5.0-31.6), and median follow up time after first surgery was 10.7 years (4.9-30.4). 133 deaths occurred (8,0\%). Median age at death was 0.5 years $(0-23.3)$ with median survival time after last surgery 0.01 years (0-13.5). 74 (5.1\%) deaths after surgery for biventricular correction corresponded to 59 deaths $(28.2 \%)$ after palliation for univentricular heart defects $(p<0.001)$. 30/54 $(55.5 \%)$ patients died after Norwood surgery for classic hypoplastic left heart syndrome compared to $29 / 155(18.7 \%)$ after surgery for other univentricular heart defects $(\mathrm{p}<0.001)$. Conclusions: Total survival was $92 \%$, with more than $50 \%$ of all deaths occurring later than 30 days after the last major surgical procedure, reflecting the need for long-term follow up. The total survival after surgery for biventricular correction was $94.9 \%$, after Norwood surgery in classic hypoplastic left heart syndrome $44.5 \%$ and in all other types of univentricular heart patients $81.3 \%$

\section{6}

A novel method of evaluating the critical Fontan candidates with markedly developed V-V shunts by balloon occlusion of the IVC

Shoji Suzuki, Department of Surgery, University of Yamanashi, Japan Shigeaki Kaga, Department of Surgery, University of Yamanashi, Japan Minako Hoshiai, Department of Pediatrics, University of Yamanashi, Japan

Toshie Kadono, Department of Pediatrics, University of Yamanashi, Japan Kohsuke Kise, Department of Pediatrics, University of Yamanashi, Japan Yoshihiro Honda, Department of Surgery, University of Yamanashi, Japan

Kisho Shimura, Department of Surgery, University of Yamanashi, Japan Masahiko Matsumoto, Department of Surgery, University of Yamanashi, Japan

Background: The markedly developed $\mathrm{V}-\mathrm{V}$ shunts via venous plexus after bidirectional Glenn operation (BDG) is a hurdle in preoperative assessment for a definitive Fontan operation, because it leads to underestimation of the pulmonary arterial pressure (PAP) due to the stealing flow. Moreover, it may occasionally cause a progressive desaturation which can not be treated by coil embolization.

Method: Two HLHS patients, 32 and 34 months old, weighing 11.5 and $10.2 \mathrm{~kg}$ respectively, were evaluated. They had undergone staged Norwood operations, and developed critical desaturation after BDG due to huge V-V shunts between the upper and the lower body, probably related to the small central PA behind the reconstructed neo-aorta. The IVC was occluded by a $15 \mathrm{~mm}$ balloon, and hemodynamics was observed for more than 5 minutes. Subsequently, an IVC venography was done.

Results: The arterial saturation significantly jumped up, by $6-12 \%$. The IVC pressure had increased by $4-5 \mathrm{mmHg}$, whereas the systemic blood pressure remained unchanged. Interestingly, the mean PAP declined by $2-3 \mathrm{mmHg}$ during the occlusion. The venography showed a reversed upstream of the shunt flow. We performed fenestrated TCPC based on these data, and got satisfactory results.

Comments and Conclusions: A decrease in the PAP suggests the effect of the declined atrial pressure or the presence of an 
unknown PA flow-mediated reaction. The IVC occlusion test should be contemplated to assess the possibility of Fontan circulation in critical candidates.

\section{7}

Modified surgical procedures in the first stage bilateral pulmonary artery banding strategy

Shin Takabayashi, Mie University Graduate School of Medicine, Japan Kazuto Yokoyama, Mie University Graduate School of Medicine, Japan Noriko Yodoya, Mie University Graduate School of Medicine, Japan Hiroyuki Ohashi, Mie University Graduate School of Medicine, Japan Yoshihide Mitani, Mie University Graduate School of Medicine, Japan Hideto Shimpo, Mie University Graduate School of Medicine, Japan

Objective: For hypoplastic left heart syndrome, the stage I bilateral pulmonary artery banding and the stage II Norwood and bilateral Glenn procedure is an useful option. Our modification of surgical procedures in this strategy are presented.

Methods: Nine hypoplastic left heart syndrome patients (2002-2008, AA/MA:6, AS/MS:2, 7-19d，2.6-3.5 kg) were performed the stage I bilateral pulmonary artery banding. Two patients had chromosome anomaly and one had closed ASD. The stage I was performed by $8.5-14 \mathrm{~mm}$ in circumference using ePTFE tape $(0.4 \mathrm{~mm}$ in thickness, $2 \mathrm{~mm}$ in width). After the stage I, prostaglandin E1 administration was continued in 7 . The 2nd stage operation was performed in $8 / 9(89 \%)$ patients $(3-9 \mathrm{~m}$, $2.9-5.2 \mathrm{~kg}$ ). In the stage II, we kept continuity between aortic arch and descending aorta using descending aortic cannulation. Distal pulmonary artery was closed vertically. Before five month old, pulmonary artery angioplasty was not required. Aortic arch angioplasty using glutaraldehyde treated own pericardium was performed in 2. Bilateral Glenn procedure was concomitantly performed in all. Fontan procedure was completed in 4 $(2 \mathrm{y} 4 \mathrm{~m}-4 \mathrm{y} 4 \mathrm{~m}, 10-14 \mathrm{~kg})$.

Results: 5/9 (56\%) patients are alive and well. There were no postoperative pulmonary artery distortion and unequal pulmonary artery growth (right/left pulmonary artery diameter: 0.95-1.15). There were no recoarctation or aneurysm of the aorta after aortic arch angioplasty. Chromosome anomaly, multiple non-cardiac anomary, or low cardiac function were associated in the deceased patients.

Conclusion: In this strategy, good Fontan circulation was obtained by our modified surgical procedures. This modification is an useful option for hypoplastic left heart syndrome patients.

\section{9}

Myocardial contractility after deep hypothermic circulatory arrest vs. mild hypothermic cardiac arrest in a neonatal piglet model

Theodor Tirilomis, Dept. for Thoracic, Cardiac, and Vascular Surgery, University Goettingen, Germany

Oliver J Liakopoulos, Dept. for Thoracic, Cardiac, and Vascular Surgery, University Goettingen, Germany

Lars Nolte, Dept. for Thoracic, Cardiac, and Vascular Surgery,

University Goettingen, Germany

Marc Bensch, Dept. for Thoracic, Cardiac, and Vascular Surgery,

University Goettingen, Germany

Katja Steinke, Dept. for Thoracic, Cardiac, and Vascular Surgery, University Goettingen, Germany

Jan D Schmitto, Dept. for Thoracic, Cardiac, and Vascular Surgery, University Goettingen, Germany

Friedrich A Schoendube, Dept. for Thoracic, Cardiac, and Vascular

Surgery, University Goettingen, Germany
Back ground: Perfusion and reperfusion during and after cardiopulmonary bypass may influence outcome of cardiac surgery. Aim of the study was to compare hemodynamics of neonates after cardiopulmonary bypass (CPB) with deep hypothermic circulatory arrest (DHCA) or mild hypothermic cardiac arrest in a piglet model.

Methods: Newborn piglets (younger then 7 days of age) were operated on cardiopulmonary bypass. Total CPB time was 180 minutes, including a periode of 90 minutes of cardioplegic cardiac arrest $\left(32^{\circ} \mathrm{C} ; \mathrm{MHCaA}\right.$ group; $\left.\mathrm{n}=10\right)$ or a periode of 90 minutes of deep hypothermic circulatory arrest $\left(18^{\circ} \mathrm{C}\right.$; DHCA group; $\mathrm{n}=8)$. Left ventricular $(\mathrm{LV})$ pressures were measured through a transapical Millar catheter. Regional myocardial contractility was determined as sonomicrometric measured myocardial wall thickeness (WT). After termination of $\mathrm{CPB}$ hemodynamics were examined for up to 6 hours. Animals did not receive any inotropic support postoperatively.

Results: In DHCA group three animals died during the obeservation period of 6 hours (vs. six animals in MHCaA group; n.s.). In both groups LV systolic pressures remained stable and although amplitude of WT decreased after CPB termination it remained constant thereafter. There were no significant differences between the two groups.

Conclusions: DHCA did not alter myocardial contractility and hemodynamics after surgery in a neonatal piglet model.

\section{0}

Results after repair of complex heart diseases with aortic arch obstruction using isolated cerebral perfusion

Tomas Tlaskal, Kardiocentrum, University Hospital Motol, Czech Republic

Pavel Vojtovic, Czech Republic

Vladimir Kucera, Czech Republic

Objective: To assess experience with the primary repair of complex heart diseases with aortic arch obstruction using isolated cerebral perfusion.

Methods: During 2002-2008 thirty-one patients, median age 6 days, with complex heart lesions and IAA (17 patients) or COA (14 patients) were primarily corrected. All patients had VSD, 10 patients had subvalvar AS, 7 PTA, 5 DORV and 4 TGA. The surgery was performed in low-flow mid-hypothermic bypass and isolated perfusion of the head. Aortic arch was reconstructed by direct anastomosis of descending and ascending aorta. In 11 patients the anastomosis was enlarged with a pericardial patch. The surgery required VSD closure in all patients, myectomy in 9 patients, conduit implantation in 8 patients and arterial switch operation in 8 patients. Duration of extracorporeal circulation was $218 \pm 55$, aortic cross-clamping $93 \pm 36$ and isolated cerebral perfusion $42 \pm 18$ minutes.

Results: There was no early death. Three (9.7\%) patients died late. One patient died from heart failure after Ross-Kono operation and two died from aspiration. Five (16.1\%) reoperations were required for stenosis of the ascending aorta (2 patients), valvar aortic stenosis with regurgitation, complex pulmonary stenosis, left bronchus compression and bleeding. Severe neurological dysorders were observed in 2 patients. During follow-up, all remaining patients are in good or very good clinical condition.

Conclusion: Complex congenital heart diseases with aortic arch obstruction can be corrected with low early mortality. Midterm results are very good but the need for reoperation is increased. Continuous cerebral perfusion decreases the risk of neurological complications. Supported by VZ 00064203-6804. 
7471

Long-term results after repair of anomalous origin of the left coronary artery from the pulmonary artery (ALCA) by reimplantation and Takeuchi operation

Tomas Tlaskal, Kardiocentrum, University Hospital Motol, Czech Republic

Bohumil Hucin, Czech Republic

Roman Gebauer, Kardiocentrum and Cardiovascular Research Center, University Hospital Motol, Prague, Czech Republic, Czech Republic Pavel Vojtovic, Czech Republic

Jiri Gilik, Czech Republic

Oleg Reich, Czech Republic

Objective: To assess long-term results after repair of ALCA performed by two surgical procedures in a single centre.

Methods: Between 1991-2008 repair of ALCA was performed in 33 patients at median age 0.8 years (range $0.1-10.0$ years). Seventeen (51.5\%) patients had grade 3-4 mitral insufficiency (MI) and 8 (24.2\%) had severe LV dysfunction. In 22 (66.6\%) patients reimplantation of the left coronary artery (LCA) and in $11(33.3 \%)$ Takeuchi intrapulmonary patch redirection was performed. Concommitant MV annuloplasty (2 patients) or replacement (2 patients) were necessary in 4 patients. Patients were followed in our institution and examined by ECHO.

Results: There was no early nor late death. Postoperatively, mechanical circulatory support was necessary in 3 patients. Patients were followed up for $7.8 \pm 4.2$ years (range 0.1-17.2 years). Nine reoperations were necessary in 6 patients (3 MV replacements in 2 patients, stenosis of LCA in 2, supravalvar PS in 2 , coronary fistula in 1 and severe bleeding in 1). During followup all patients remain in good clinical condition, 24 are in NYHA class I, 8 in class II. Moderate dysfunction of LV and MI persist in $8(24.2 \%)$ patients who had severe LV dysfunction prior to surgery. No difference between reimplantation and Takeuchi operation was observed.

Conclusion: Reimplantation and Takeuchi operation can be performed with good long-term results. There is no difference between these operations as far as the occurance of complications and need for reoperation. Long-term results depend mainly on the severity of LV dysfunction prior to surgery. Supported by VZ 00064203-6804

7472

Direct End-to-Side Anastmosis of Superior Vena Cavae in Patients with Bilateral Superior Vena Cavae for Biventricular Repair

Shinya Ugaki, Okayama University Hospital, Japan

Yasuhiro Fujii, Department of Cardiovascular Surgery, Okayama

University Hospital, Japan

Sadahiko Arai, Okayama Univeristy Hospital, Japan

Shingo Kasahara, Okayama University Hospital, Japan

Shunji Sano, Okayama University Hospital, Japan

Background: Anatomy of bilateral superior vena cavae (SVC) without innominate vein could make it more complex to perform complete repair. We reviewed our cases of direct endto-side anastmosis of SVCs to facilitate anatomical repair of SVC-right atrial connection for biventricular repair.

Methods: From July 2007 to December 2008, five patients received direct end-to side anastmosis of SVCs. Operative diagnosis are 2 partial atrioventricular septal defect with left isomerism, 1 complete atrioventricular septal defect with left isomerism, 1 complete atrioventricular septal defect without isomerism and 1 atrioventricular discordance and double outlet right ventricle with right isomerism. Mean age at the operation was $20 \pm 23$ months (4-58) and body weight was $7.8 \pm 3.4 \mathrm{~kg}$ (4.8-12.7). Preoperative measurement of distance between SVCs by cardiac catheterization was $32.9 \pm 2.1 \mathrm{~mm}$ (30-35.5). After completion of intracardial repair, the SVC to left atrium was diveded at SVC-atrial junction. It was end-to side anastmosed to SVC to right atrium utilizing side-clamp during cardiopulmonary bypass. Postoperative anticoagulant was continued for 8 weeks after surgery.

Results: No early and late death occurred during follow-up of $8.4 \pm 6.9$ months (1-17). All children had neither disturbed flow nor occlusion at the anastmosis site of SVCs by transthoracic UCG. One patient received postoperative cardiac catheterization, which demonstrated no pressure gradient between SVCs. Conclusions: Direct end-to-side anastmosis of SVCs seems to be more advantageous technique in complex anomalies with bilateral SVCs compared to intraatrial rerouting. This technique achieved excellent anatomical SVC-RA connection repair in biventricular repair.

\section{3}

Postoperative changes of coagulability and fibrinolytic function in Fontan circulation : Possibility of the conversion of anticoagulation therapy

Yoshimasa Uno, Department of Cardiac Surgery, The Jikei University School of Medicine, Japan

Kiyozo Morita, Department of Cardiac Surgery, The Jikei University School of Medicine, Japan

Kazuhiro Hashimoto, Department of Cardiac Surgery, The Jikei

University School of Medicine, Japan

Masahito Yamashiro, Department of Cardiac Surgery, The Jikei

University School of Medicine, Japan

Gen Shinohara, Department of Cardiac Surgery, The Jikei University

School of Medicine, Japan

Background: There is still no consensus concering the postoperative use and duration of Warfarin on Fontan patients. Recently, we evaluate changes of coagulability and fibrinolytic function after the surgery and then convert the anticoagulation therapy for patients with normal results.

Methods: We have measured plasma levels of TAT (Thrombin Antithrombin-3 complex) as the index of coagulability and PIC $(\alpha 2$-Plasmin Inhibitor-plasmin complex) as it of fibrinolytic function for 20 Extracardiac Fontan patients (mean age at operation 4.2 years) without complication. In all patients, intracardiac thrombus was also detected using primarily transthorasic echocardiography during the periods of this study. Mean follow-up was 18.7 months (6 to 60 months).

Results: There are no late death nor thromboembolism in those patients. Both levels of TAT and PIC remained in higher than normal within 6 months after surgery, even in taking Warfarin. Then they started declining gradually and almost normalized by 12 months. Confirming these results, we have converted anticoagulation therapy from Warfarin to antiplatlet agent for those cases. After its conversion, plasma levels of them have kept in lower and no patient showed thromboembolic event in echocardiography.

Conclusions: This study suggests that Fontan patients might need Warfarin as anticoagulation therapy for the first postoperative year, because of their activated status of coagulability. However, Warfarin could be replaced by antiplatlet agent for the patients who show normal results and no major complication since 12 months after the surgery. We are also reminded that farther evaluation and follow-up are important and necessary. 
7474

The impact of additional pulmonary blood flow on a bidirectional cavopulmonary shunt

Ymkje J van Slooten, University Medical Centre Groningen,

The Netherlands

Nynke J Elzenga, University Medical Centre Groningen,

The Netherlands

Tjalling $W$ Waterbolk, University Medical Centre Groningen,

The Netherlands

Rolf M Berger, University Medical Centre Groningen, The Netherlands Tjark Ebels, University Medical Centre Groningen, The Netherlands

Purpose: Additional pulmonary blood flow (APBF) with a bidirectional cavopulmonary shunt (BDCPS) is contentious. Disadvantages might include: venous congestion and ventricular volume overload. Advantages might include: better quality of life during a longer interval until total cavopulmonary connexion (TCPC). We investigated the impact of APBF in our patients with BDCPS.

Methods: From 1990 to 2006, 70 patients received a BDCPS, excluding patients with hypoplastic left heart syndrome. APBF consisted of a banded or stenotic pulmonary trunk or a BlalockTaussig shunt calibrated to an oxygen saturation (SO2) of approximately $85 \%$. The BDCPS-to-TCPC interval was dependent upon symptoms. The effects of APBF were investigated retrospectively. Variables were: interstage interval, age, body surface area, SO2, morbidity and TCPC details.

Results: Fifty-one patients (73\%) had APBF, of which 18 remain TCPC candidates $(35 \%), 5$ died $(10 \%), 1$ is TCPC contraindicated $(2 \%)$ and 27 received a TCPC (53\%) of which 2 patients died (7\%). Nineteen patients (27\%) had BDCPS only, of which 13 received a TCPC $(68 \%), 3$ remain TCPC candidates (16\%) and 3 died (16\%). Seventeen patients with APBF (60\%) vs. 4 without APBF (31\%) had an extracardiac conduit TCPC. Patients with APBF had a mean interstage interval of 3.3 years vs. 2.5 years in patients without APBF. With APBF, SO2 during the interstage interval remained higher and body surface area at TCPC was larger $(0.72$ vs. $0.65 \mathrm{~m} 2)$.

Conclusions: APBF may result in insertion of a larger extra cardiac conduit. Furthermore, APBF diminished haemodynamic symptoms after BDCPS and had no accountable negative impact.

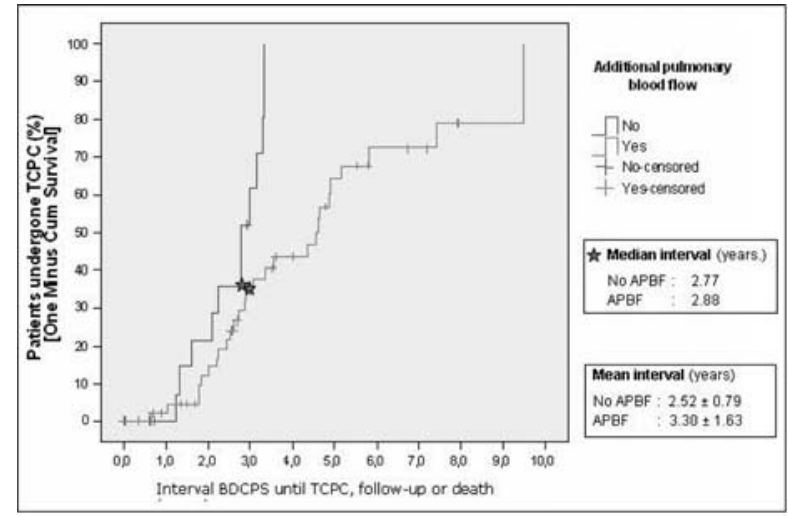

7475

Impact Of Antegrade Pulmonary Flow On The Outcome Of Bidirectonal Cavopulmonary Shunt

Roy Varghese, Institute of Cardiovascular Diseases, Madras Medical Mission, Chennai, India

$R$ Kumar Suresh, Institute of Cardiovascular Diseases, Madras Medical Mission, Chennai, India
Suraj Kumar Varma, Institute of Cardiovascular Diseases, Madras Medical Mission, Chennai, India

John Valliath, Institute of Cardiovascular Diseases, Madras Medical Mission, Chennai, India

Introduction: Preservation of antegrade flow has been considered to be of advantage in bi-directional cavopulmonary shunts (BCPS). This report studied impact of antegrade pulmonary blood flow on the outcome following the procedure.

Material and methods: During the period January 2003 through September 2008, 83 patients underwent the BCPS. Mean age was 48 months and mean weight $12 \mathrm{kgs}$. 28 were performed in infancy. Per operatively, antegrade pulmonary blood flow was preserved in 28 patients (group I) and interrupted in 62 patients (Group II). 7 patients from group I were taken back to the operating room and the flow was interrupted due to raised superior vena caval pressures. Mean pre operative $\mathrm{SpO} 2$ in group I and II were $68.8 \%$ and $70.8 \%$ respectively. Postoperative superior caval pressures, duration of ventilation, ICU stay, hospital stay, oxygen saturations at regular intervals and morbidity were assessed (Table 1). Patients were followed up at intervals of three months, one and two years.

Results: There was no hospital mortality. There was no statistically significant difference in the immediate and intermediate term outcome between the two groups. There were two cases of diffuse A-V malformations - one died and the other had resolution following Fontan completion.

Conclusion: Preservation of antegrade pulmonary blood flow does not seem to confer any additional benefit on immediate or intermediate term outcome following BCPS. The decision to preserve or interrupt the antegrade flow requires to be individualized.

\begin{tabular}{|c|c|c|c|}
\hline Variable & Group I & Group II & P-value \\
\hline Post-op CVP & $13.7+3.6$ & $12.8+3.4$ & 0.33 \\
\hline Pre discharge $\mathrm{SpO} 2$ & $84.6+6.4$ & $82+6.4$ & 0.16 \\
\hline ICU Stay (days) & $2.5+1.3$ & $3.6+3.6$ & 0.174 \\
\hline $\begin{array}{l}\text { Duration of ventilation } \\
\text { (hrs) }\end{array}$ & $11.4+16.3$ & $24.6+44.8$ & 0.21 \\
\hline Hospital Stay (days) & $12.8+6.7$ & $14.8+6.7$ & 0.23 \\
\hline $\mathrm{SpO} 2$ at 3 months & $84.3+5.5$ & $83.5+7.4$ & 0.65 \\
\hline $\mathrm{SpO} 2$ at 1 year & $82+3.6$ & $81+6.6$ & 0.73 \\
\hline $\mathrm{SpO} 2$ at 2 years & $81+2.6$ & $81.5+3.8$ & 0.77 \\
\hline
\end{tabular}

7476

Early and Intermediate Experience With Extracardiac Fontan Operation

Roy Varghese, Institute of Cardio-Vascular Diseases, Madras Medical Mission, Chennai, India

E Kumar Suresh, Institute of Cardio-Vascular Diseases, Madras Medical Mission, Chennai, India

G Madhusudan, Institute of Cardio-Vascular Diseases, Madras Medical Mission, Chennai, India

Atul Prabhu, Institute of Cardio-Vascular Diseases, Madras Medical Mission, Chennai, India

John Valliath, Institute of Cardio-Vascular Diseases, Madras Medical Mission, Chennai, India

Introduction: There are conflicting reports regarding the two current techniques of Fontan completion. This report evaluates the outcome in 33 consecutive cases of Extracardiac Fontan operation in a single centre. 
Patients and Methods: During 1999-2008, 33 patients (M:F = 19:14; Median age $=9.5$ years, range 5 to 29 years) underwent extracardiac Fontan procedure for univentricular physiology. The diagnostic categories are shown in diagram 1.28 patients had prior superior cavopulmonary anastomosis. The median preoperative $\mathrm{SPO} 2$ was $77+8.2 \%$. The mean PA pressure was $8+3.2 \mathrm{~mm}$ of $\mathrm{Hg}$. The operation consisted of connecting the intra-pericardial IVC to the PA using a conduit (Gore-tex tube-31, median size$22 \mathrm{~mm}$ ) with a fenestration to the RA performed on the beating heart under normothermic perfusion.

Results: The immediate post-operative outcome is shown in table 1. All patients received warfarin for the first year, and subsequently warfarin and/or aspirin. On follow up $(\mathrm{n}=30$, median duration 14 months, range $=4-108$ months), all patients were in functional class 1 or 2 . There was no mortality. SPO2 at last follow up was $92+7.6 \%$. All patients, including 5 with $>60$ months follow-up, in preoperative sinus rhythm continued to be so. Three patients had asymptomatic SVT. There were no "Fontan failures", thromboembolic episodes, or protein-losing enteropathy. No patient required fenestration closure or conduit revision.

Conclusion: Extracardiac Fontan operation can be performed in univentricular hearts with excellent functional outcome in the short and intermediate term.

\begin{tabular}{lc}
\hline Mortality & Nil \\
\hline Mechanical ventilation (hours) & Median -8 (Range $=4-36)$ \\
ICU stay (days) & Median $-11($ Range $=2-28)$ \\
Chest tube drainage (days) & Median $-12($ range $=3-30)$ \\
Pleurodesis & $17(51.5 \%)$ \\
Transient low cardiac output & $4(12 \%)$ \\
Renal Failure & $2(6 \%)$ \\
Transient elevation of liver enzymes & $4(12 \%)$ \\
Arrhythmias & $8(24 \%)$ \\
\hline
\end{tabular}

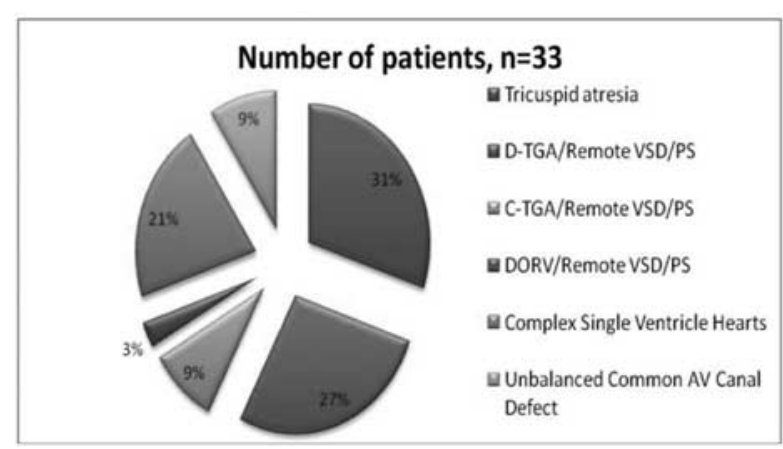

7477

The switch back Ross operation: Report of two cases Walter VA Vicente, Hospital das Clinicas of Ribeirão Preto, Brazil Cesar A Ferreira, Hospital das Clinicas of Ribeirão Preto, Brazil Jyrson G Klamt, Hospital das Clinicas of Ribeirão Preto, Brazil Paulo H Manso, Hospital das Clinicas of Ribeirão Preto, Brazil Oswaldo C Almeida Filho, Hospital das Clinicas of Ribeirão Preto, Brazil Ana Paula C Carlotti, Hospital das Clinicas of Ribeirão Preto, Brazil Lidiane R Arantes, Hospital das Clinicas of Ribeirão Preto, Brazil Jorge Haddad, Hospital das Clinicas of Ribeirão Preto, Brazil

Objective: The switch back Ross operation (SBR) is a challenging option for the treatment of neoaortic valve dysfunction late after the Jatene operation. So far, only one case was reported in the literature. We herein report two additional cases.

Methods: The first pt. (8yo boy) presented to our Institution in 1997, with TGA + LV outlet obstruction. The Jatene operation + membranous septum aneurysm resection + ASD patch closure was performed. One month later, moderate neoaortic valve regurgitation with preserved LV function was noticed (echo). He remained asymptomatic until 2005 when, at age $16 y$, he became dyspneic on moderate physical exertion. A $49 \mathrm{~mm}$ neoaortic root aneurysm + severe neoaortic valve regurgitation $+\mathrm{LV}$ dilatation $(65 \mathrm{~mm}$ diastolic) were diagnosed. SBR was performed on 15.02.2006. The second pt. had been operated on (Jatene operation + transpulmonary VSD closure) at age $1 \mathrm{mo}$. He did well initially, but 5 y later he was diagnosed with sinus of Valvalsa aneurysm + neoaortic valve regurgitation + severe pulmonary anastomotic stricture + subaortic membrane. A SBR was carried out on 24.04.2007.

Results: By-pass and aortic X-clamp times were: Pt 1-300 min and $202 \mathrm{~min}, \mathrm{Pt} 2-235 \mathrm{~min}$ and $164 \mathrm{~min}$. Both pts. did well postoperatively and were put on follow-up.

Conclusions: Notwithstanding its complexity, the switch back Ross operation deserves consideration for the difficult and rarely reported conditions of neoaortic dilatation and valve dysfunction in childhood.

\section{8}

On by-pass transternal tracheal reconstruction after the Jatene operation, in low birth weight a baby

Walter VA Vicente, Hospital das Clinicas of Ribeirão Preto, Brazil Cesar A Ferreira, Hospital das Clinicas of Ribeirão Preto, Brazil Paulo H Manso, Hospital das Clinicas of Ribeirão Preto, Brazil Ana Paula P Carlotti, Hospital das Clinicas of Ribeirão Preto, Brazil Lidiane R Arantes, Hospital das Clinicas of Ribeirão Preto, Brazil Mauro C Jurca, Hospital das Clinicas of Ribeirão Preto, Brazil Jorge Haddad, Hospital das Clinicas of Ribeirão Preto, Brazil Jyrson Klamt, Hospital das Clinicas of Ribeirão Preto, Brazil

Objective: Tracheal stenosis is rarely reported following the Jatene operation. We herein present such a case in a low birth weight baby, with right aortic arch and a malpositioned descending aorta located in front of the spine.

Methods: A $2500 \mathrm{~g}$ neonate with TGA underwent a straightforward Jatene operation with the LeCompte maneuver on 05.2006. Post-operative was straightforward until respiratory discomfort early after extubation required re-intubation. Bronchoscopy demonstrated supracarinal vascular compression. Trachea vascular decompression was attempted through a left sided thoracotomy on p.o. day 63 . Intraoperatively, a $1.5 \mathrm{~cm}$ supracarinal malatia segment was found, compressed by a vascular ring formed by the right aortic arch in continuation with a malposicioned descending aorta sitting right in front of the spine, and the left pulmonary artery. Two weeks later, at age $3 \mathrm{~m} 9 \mathrm{~d}$, four ring tracheal resection followed by end to end anastomosis was performed transternally, on cardiopulmonary-pass.

Results: After prolongued hospitalization due to tracheal anastomotic granulomae and difficulty to thrive, the baby has been faring well on ambulatory follow up.

Conclusions: Tracheal compression may be elicited by the Jatene operation in the presence of right aortic arch and malpositioned descending aorta. Transternal tracheoplasty on by-pass may be required post-operatively.

\section{9}

Mid-term evaluation of prosthetic valves in the pulmonary position

Hunaid A Vohra, Wessex Cardiothoracic Centre, Southampton, United Kingdom 
Gedrius Baliulis, Wessex Cardiothoracic Centre, Southampton, United Kingdom

Gruschen Veldtman, Wessex Cardiothoracic Centre, Southampton, United Kingdom

Kevin Roman, Wessex Cardiothoracic Centre, Southampton, United Kingdom

Joseph J Vettukattil, Wessex Cardiothoracic Centre, Southampton, United Kingdom

James Gnanapragasam, Wessex Cardiothoracic Centre, Southampton, United Kingdom

Tony Salmon, Wessex Cardiothoracic Centre, Southampton, United Kingdom

Marcus P Haw, Wessex Cardiothoracic Centre, Southampton, United Kingdom

Aim: To examine the mid-term clinical outcome of pulmonary valve replacement (PVR) with prosthetic valves.

Methods: We reviewed 24 consecutive patients who underwent PVR with prosthetic valves between September 1999 and October 2008. The median age was 21.5 years (range 6-70 years; 4 children). The primary diagnosis was Tetralogy of Fallot in 12 patients $(50 \%)$. Valve pathology was regurgitation in 17 patients (70.8\%). Cardiac surgery had been previously performed in 23 patients $(95.8 \%)$. PVR was performed with bioprosthetic valves in 18 patients and mechanical valves in 6 patients (median size $25 \mathrm{~mm}$; range 21-32 mm). The median follow-up was 36 months (range $1.2-108$ ) and the cumulative follow-up was 74.7 patient-years.

Results: There were no early valve-related deaths. Hospital mortality was $4.1 \%(\mathrm{n}=1)$ and no patient required early rereplacement of prosthesis. Two patients required permanent pacemaker insertion. During follow-up, there was no late death, re-operation for structural valve degeneration or valve thrombosis. Only 1 patient required repeat operation for endocarditis at 37 months follow-up. The actuarial survival at 5 years was $95.6 \pm 4.2 \%$. Overall freedom from re-operation after PVR at 5 years was $90 \pm 9.5 \%$. At last follow-up, 21 patients (91.3\%) were NYHA class I versus 13 patients (54.1\%) preoperatively $(p<0.05)$. In the 22 alive patients who did not undergo redo-PVR, there was no regurgitation and the peak PV gradient was $20.8 \pm 11.9 \mathrm{mmHg}$. Twenty one patients $(91.3 \%)$ had good right ventricular function compared to 18 patients (75\%) pre-operatively.

Conclusions: PVR with prosthetic valves can be performed with good mid-term survival, functional status and haemodynamics.

\section{0}

Long-term follow-up after primary complete repair of truncus arteriosus with homograft: a $\mathbf{4 0}$ year experience Hunaid A Vohra, Wessex Cardiothoracic Centre, Southampton, United Kingdom

Alicia XF Chia, Wessex Cardiothoracic Centre, Southampton, United Kingdom

Gruschen Veldtman, Wessex Cardiothoracic Centre, Southampton, United Kingdom

Kevin Roman, Wessex Cardiothoracic Centre, Southampton, United Kingdom

Joseph J Vettukattil, Wessex Cardiothoracic Centre, Southampton, United Kingdom

James Gnanapragasam, Wessex Cardiothoracic Centre, Southampton, United Kingdom

Tony Salmon, Wessex Cardiothoracic Centre, Southampton, United Kingdom

Marcus P Haw, Wessex Cardiothoracic Centre, Southampton, United Kingdom
Background: To determine the long-term performance of homograft and truncal valve after complete repair of truncus arteriosus communis (TAC).

Methods: From January 1964 to June 2008, 32 patients (median age 14 days; range 5 days-2.5 years) underwent primary homograft repair of TAC. Twenty four $(75 \%)$ were neonates. The homograft used in RVOT was aortic in 24 patients and pulmonary in 8 patients (median diameter $16 \mathrm{~mm}$; range $8-24 \mathrm{~mm}$ ). The median follow-up was 24.5 years (range $5.6-43.5$ years).

Results: There were 3 hospital deaths and 1 late death. The actuarial survival at 30 years was $83.1 \pm 6.6 \%$. Of the 28 survivors, 25 reoperations were performed in 19 patients $(76 \%)$. The median time to homograft re-operation was 12.1 years (range 1.0-26.1 years). Overall freedom from homograft re-operation after 10, 20 and 30 years was $68.4 \pm 8.7 \%, 37.4 \pm 9.5 \%$ and $26.7 \pm 9.3 \%$, respectively. Twelve patients retained the original homografts at a median followup of 16.4 years (range $0-30.2$ years). Six underwent a truncal valve replacement (TVR) with mechanical prosthesis at a median of 10.5 years (range 3.4-22 years). Freedom from TVR at 10 and 30 years was $93.1 \pm 4.7 \%$ and $81.8 \pm 8.9 \%$, respectively. In the 22 alive patients who did not undergo TVR, the peak truncal valve gradient was $8.9 \pm 8.3 \mathrm{mmHg}$ at a median follow-up of 24.5 years (range $=5.6-32.9$ years). At last follow-up, 27 patients (96.4\%) had good LV function and 24 patients (85.7\%) were NYHA I. Conclusions: After complete primary repair of TAC, homografts can be expected to last $>12$ years before requiring rereplacement. Long-term TV haemodynamics are good.

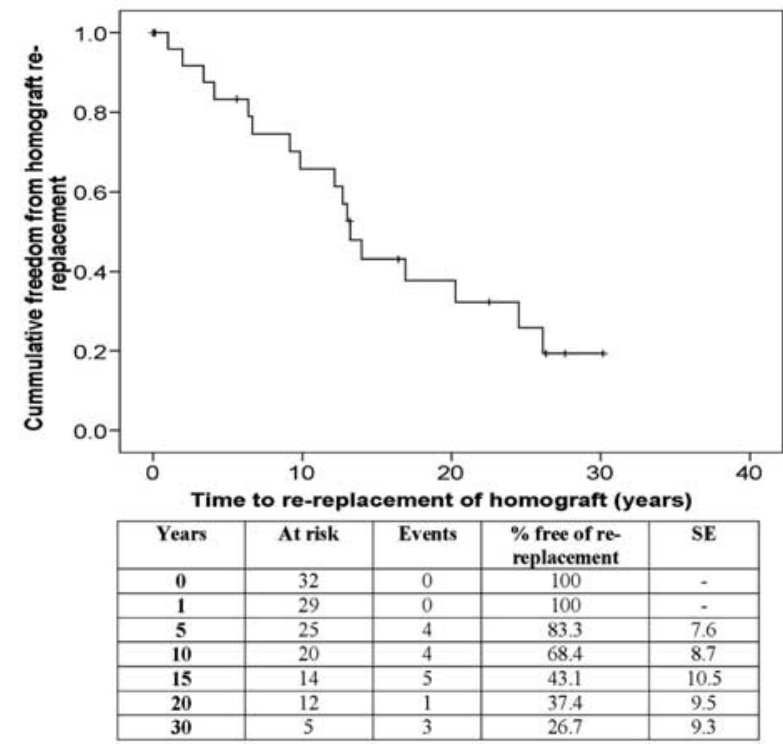

7481

Prevention of adhesions In Redo Paeiatrci Cardiac Surgey Using a Synthetic Hydrogel

Nihal A Weerasena, Leeds general Infirmary. Leeds, United Kingdom Pace Napoleone, Bologna Unvi.Hosp, Italy

A Valori, Osp.Regnia Margherita.Torino, Italy

G Crupi, Osp.Riuniti.Bergamo, Italy

C Marcelletti, Osp.Civico Palermo, Italy

F Santo, Osp. Niguarda Milano, Italy

$P$ Vouche, Hosp. Necker Paris, France

G Gargiulo, Bologna Univ Hosp, Italy

Objectives: Adhesions make resternotomy hazardous, increasing surgical time and potential complications. A synthetic hydrogel 
reduced pericardial adhesions in a limited series of reoperations. To expand this preliminary data, a prospective, open-label, observational study was conducted to evaluate efficacy, safety and ease-of-use.

Methods: Seven European paediatric cardiac surgery centers recruited patients requiring primary sternotomy for staged repair of congenital heart defects. Patients with unplanned re-operations, or re-operations in $<3$ or $>8$ months were excluded. A bodyweight-adjusted dose of synthetic hydrogel (Coseal ${ }^{\circledR}$, Baxter, USA) was sprayed onto the heart surface at the end of surgery. Adhesions were evaluated at first re-operation. Primary outcome included the presence, location and severity of adhesions, secondary outcome safety, duration of surgery and ease of use.

Results: Seventy-nine consecutive patients (male $=45$, median age $=37$ days) were recruited between 2005-2007. All underwent major surgical procedures and received hydrogel (mean volu$\mathrm{me}=2.37 \mathrm{~mL}$, range $=1-4 \mathrm{~mL}$ ). Thirty-six patients were re-operated in the defined time. Mean \pm SD duration of the first surgery was $3.65 \pm 1.83$ hours (range $=1-10)$, and $3.45 \pm 1.79$ hours (range $=1-10)$ for the second. Total adhesions $(\mathrm{N}=252)$, measured at seven different sites, were categorized as either filmy $(n=213$; $84.5 \%)$, dense $(n=29 ; 11.5 \%)$, cohesive $(n=9 ; 3.6 \%)$ or undetermined $(n=1 ; 0.4 \%)$. Ease of hydrogel application was assessed as $9.9 \pm 6.8$ (range $=0-26)$, based on a $0-100$ (very easy-very difficult) scale. Seventeen serious adverse events occurred; three were confirmed as product-related and resolved without sequela.

Conclusions: In this study the majority of adhesions were filmy and easily dissected. Use of a synthetic hydrogel may reduce severity of cardiac adhesions.

Table 1 Description of population of re-opearated children at least 3 months after the first surgery: Re-operation - Assessment of adhesion (Primary criteria)

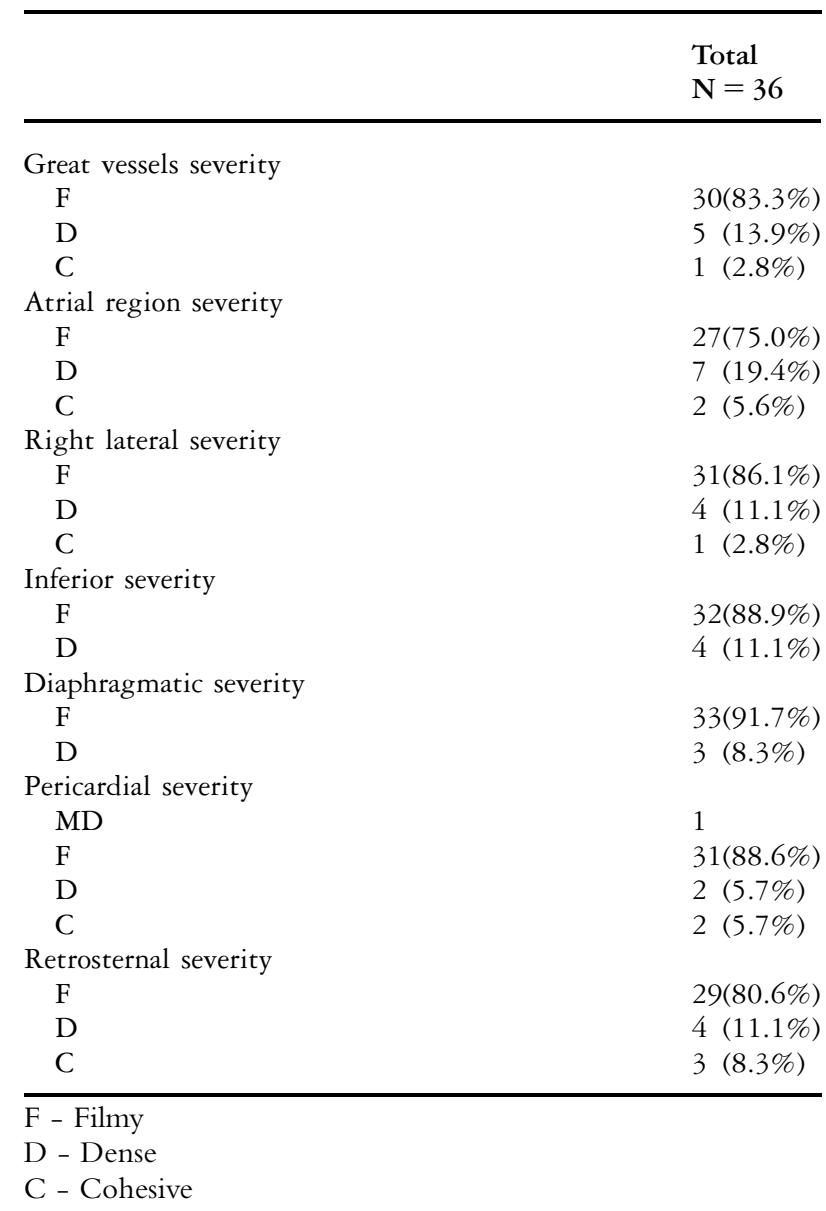

\section{2}

Anomalous Origin of the Coronary Artery from the Pulmonary Artery

Shye-Jao Wu, MacKay Memorial Hospital, Taiwan

Ming-Ren Chen, MacKay Memorial Hospital, Taiwan

Haw-Kwei Hwang, MacKay Memorial Hospital, Taiwan

Chang-Hsien Yu, MacKay Memorial Hospital, Taiwan

Shan-Miao Lin, MacKay Memorial Hospital, Taiwan

Objectives: The anomalous origin of the coronary artery from the pulmonary artery is a rare disease entity. We report our surgical results to restore two-coronary system for our patients.

Methods: Two male babies, 1-month-old and 2-month-old, who suffered from anomalous origin of the left coronary artery from the pulmonary artery, presented with intermittent shortness of breath and tachypnea with cyanosis respectively. The third male baby, 4-month-old, who suffered from anomalous origin of the right coronary artery from the pulmonary artery, presented with shortness of breath leading to cardiogenic shock. LV dilatation was found in all the patients. Regarding mitral regurgitation, moderate degree for the first patient, severe degree for the second, and mild-to-moderate degree for the last. The Takeuchi procedure to re-route the left coronary artery to the ascending aorta through the intra-pulmonary artery baffle was done for the first two patients. Mitral valvuloplasty was done for the second patient. Re-implantation of the right coronary artery was performed for the last patient.

Results: All the patients survived the operation and were discharged from the hospital. Peritoneal dialysis was used for the first two patients in the immediately postoperative period. During the follow up (7-83 months), all the patients regained normal LV function and have good physical activity. As for the mitral regurgitation, trivial degree for the first patient, moderate degree for the second and none for the last.

Conclusions: Restoration of two-coronary system is the surgical goal for patients with anomalous origin of the coronary artery from the pulmonary artery.

\section{3}

Strategy for Bi-ventricular repair of Transposition of the Great Arteries with Ventricular Septal Defect and Pulmonary Stenosis -Nikaidoh's or Rastelli's and Modified REV procedure?

Zhi wei Xu, Shanghai Children's Medical Center,Medical College, Shanghai Jiao-Tong University, China

Qin Yan, Shanghai Children's Medical Center,Medical College, Shanghai Jiao-Tong University, China

Jin fen Liu, Shanghai Children's Medical Center,Medical College,

Shanghai Jiao-Tong University, China

Xin wei Du, Shanghai Children's Medical Center,Medical College, Shanghai Jiao-Tong University, China

Zhen jing Shi, Shanghai Children's Medical Center,Medical College, Shanghai Jiao-Tong University, China

De ming Zhu, Shanghai Children's Medical Center,Medical College, Shanghai Jiao-Tong University, China

Objective: To study the early results of the management of D-TGA/ VSD/PS by Nikaidoh's and Rastelli's modified REV procedure. Method From Jan.2000 to June 2008, 44 patients had underwent Nikaidoh's or Rastelli's and REV procedure for the management of D-TGA/VSD/PS. In Nikaidoh group there were 20 patients, with a median age of 15 months and reconstruction of RVOT by autograft of pericardium patch. In Rastelli group, the reconstruction of RVOT was made by homogrft on 15 patients who is with a 
median age of 4 years old. There were 9 patients in REV group. Results There was one death result in severe heart failure in Nikaidoh group, no death in Rastelli group. One patient died in REV group, because of residual LVOTO and RVOTO, and arrhythmias. The postoperative Echo examination showed no residual RVOTO or LVOTO in Nikaidoh group, but there were $20-27 \%$ cases with RVOTO or LVOTO in the other groups $(\mathrm{p}<0.05)$, and pulmonary insufficiency (PI) were frequently in Nikaidoh group (87.5\% patients) and M.Lecompte group (60\%) compared with Rastelli group $(\mathrm{p}<0.05)$. There was no significance difference in the heart function all of groups, but younger surgical age in Nikaidoh group. No death at follow -up. Conclusions The three procedures are all valuable surgical option for management of D-TGA/VSD/PS. Nikaidoh procedure is suitable for younger age and more anatomically correct alignment between the ventricle and great artery. It would be further required to study for the outcome of long-term follow-up in three procedures.

Supported Foundation: Shanghai Science Technology Committee (044119627).

\section{4}

Surgical strategy in D-TGA and Taussig-Bing anomaly with aortic arch abnormally: single-stage repair

Zhi wei Xu, Shanghai Children's Medical Center, Medical College, Shanghai Jiao tong University, China

Qin Yan, Shanghai Children's Medical Center, Medical College, Shanghai Jiao tong University, China

Jin fen Liu, Shanghai Children's Medical Center, Medical College, Shanghai Jiao tong University, China

Zhen ying Shi, Shanghai Children's Medical Center, Medical College, Shanghai Jiao tong University, China

De ming Zhu, Shanghai Children's Medical Center, Medical College, Shanghai Jiao tong University, China

Objective: To evaluate perioperative and early-mid term results after single-stage arterial switch operation associated with aortic arch repair.

Method: From Jan. 2000 to Dec. 2007, 333 consecutive patients underwent arterial switch operation(ASO) for Transposition of Great Artery (D-TGA) or Taussig-Bing (T-B) anomaly; 23 (6.90\%) patients of them had associated aortic arch repair, which were 12 patients with TGA and 11 T-B anomaly. Aortic arch anatomy showed: localized aortic coarctation $(\mathrm{n}=15)$ and coarctation with hypoplastic aortic arch $(n=3)$ and IAA $(n=5)$. VSD was present in $21(86.9 \%)$ patients. The complex coronary artery anatomy $(\mathrm{n}=5)$ were common findings. The repair of arch was accomplished under deep hypothermic circulatory arrest or low flow, employing a wide pericardial patch to reconstruction of arch in most patients. Aortic arch reconstruction was achieved either by resection and extended anastomoses $(\mathrm{n}=6)$.

Results: Hospital mortality was $13.04 \%(\mathrm{n}=3)$. The high risk factors of death were unsuitable reconstructed arch, complex coronary anatomy or severe pulmonary hypertension. There were no late deaths. There were $95 \%$ freedom of reintervention and re-operation for re-obstruction of arch and 95\% freedom of reintervention and for Suprapulmonary stenosis at 5years of Follow-up. Two patients had mild-moderate aortic regurgitation at 5 years of follow-up.

Conclusion: Single-stage repair for D-TGA and T-B anomaly with aortic arch abnormally is suitable for infant, and operative survivors is favorable. Elimination of the tension at the two aortic end-to-side anastomoses would be necessary. Pericardial patch augmentation of hypoplastic aortic arch is a reliable technique for arch reconstruction.

\section{5}

Influencing factors of in the early outcome of one stage or two stages Fontan operation

Qin Yan, Shanghai Children's Medical Center, Medical College,

Shanghai Jiao tong University, China

Zhi wei Xu, Shanghai Children's Medical Center, Medical College, Shanghai Jiao tong University, China

Jin fen Liu, Shanghai Children's Medical Center, Medical College,

Shanghai Jiao tong University, China

Zhen ying Shi, Shanghai Children's Medical Center, Medical College, Shanghai Jiao tong University, China

De ming Zhu, Shanghai Children's Medical Center, Medical College, Shanghai Jiao tong University, China

Zhao kang Su, Shanghai Children's Medical Center, Medical College, Shanghai Jiao tong University, China

Wen xiang Ding, Shanghai Children's Medical Center, Medical

College, Shanghai Jiao tong University, China

Objective: To review a single-center experience with the Fontan operation and to determine risk factors influencing early outcome of one stage or two stages Fontan.

Methods: Records of 254 consecutive patients who had a Fontan operation at one Center between Jan.1986 and Dec.2007 were reviewed. The perioperative variables affecting early mortality were analysed by univariate and multivariate.

Results: The early mortality after one stage Fontan operation decreased significantly from $22.8 \%$ in the first 16 years of the experience to $4.8 \%$ in the last 8 years. The risk factors of the early mortality were PAI $(\mathrm{p}<0.01)$, aortic cross-clamping (ACC) time $\geqslant 80$ minutes $(p=0.04)$, Heterotaxy syndrome (HS) and morphologically Single Ventricle (MSV) $(p=0.02)$. In a multivariate model, the following variables were associated with the factors of the early mortality after Fontan: HS $(\mathrm{p}<0.03), \quad$ MSV $(\mathrm{p}<0.01), \quad$ CAVV with regurgitation $(\mathrm{p}=0.02)$, TAPVC with supra-cardiac or mixed type $(\mathrm{p}<0.05)$, hypoplastic pulmonary branch $(\mathrm{p}<0.05)$, major systemic or pulmonary artery and venous collaterals $(p=0.03)$, longer ACC time $(p=0.001)$ and the absence of a baffle fenestration $(p<0.01)$. The factor affecting higher early mortality and MOFin the two stage Fontan was longer durational time $(>3$ years $)$ between Glenn and Fontan $(p<0.001)$ and collaterals $(\mathrm{p}<0.01)$.

Conclusion: The influencing factors of the early mortality were morphologically and homodynamic state perioperative Fontan and surgical techniques. Declined mortality in the late experience is related in part to procedural modification. It were important to occlude collaterals for decreasing mortality after Fontan operation, especially two stage.

Supported Foundation: Fontan Prize of EACTS.

\section{6}

Strategy for Reconstruction of right ventricular outflow tract in primary palliative management of PA/VSD: conduit or transannular patch?

Qin Yan, Shanghai Children's Medical Center, Medical College, Shanghai Jiao tong University, China

Zhi wei Xu, Shanghai Children's Medical Center, Medical College, Shanghai Jiao tong University, China

Jin fen Liu, Shanghai Children's Medical Center, Medical College, Shanghai Jiao tong University, China

Zhong qun Zhu, Shanghai Children's Medical Center, Medical College, Shanghai Jiao tong University, China

Zhen jing Shi, Shanghai Children's Medical Center, Medical College, Shanghai Jiao tong University, China 
De ming Zhu, Shanghai Children's Medical Center, Medical College, Shanghai Jiao tong University, China

Zhao kang Su, Shanghai Children's Medical Center, Medical College, Shanghai Jiao tong University, China

Wen xiang Ding, Shanghai Children's Medical Center, Medical College, Shanghai Jiao tong University, China

Objective: The study was to compare conduit or transannular patch of the re-constructed right ventricular outflow tract (RVOT) in terms of early-term clinical consequences, and the follow-up outcomes.

Methods: Between July 2001 and June 2008, 96 consecutive patients with PA/VSD underwent reconstruction of RVOT in primary palliative procedure including non-valved Gore-Tax conduit $(\mathrm{CONT}, \mathrm{n}=63$ ) and non-valved transannular pericardial patch (TAP, $n=33$ ). The patient profile is shown in table-1. Unifocalization of pulmonary artery were done in 3 patients with PA/VSD and MAPCAs in the same time.

Results: The surgical survival was significant higher in CONT group $(93 \%)$ than in TAP $(79 \%, p<0.05)$. Post -op Echocardiography showed that PI or TR was visible in TAP group than in CONT. There was no significantly difference in the average mechanical ventilation time and CICU stay between two groups. The interval of staged repair PA/VSD in CONT versus TAP group was $(16 \pm 7)$ month versus $(23 \pm 14)$ month, $\mathrm{p}<0.05$. The complications after staged repair PA/VSD did not differ between both patients. There was no late death and re-operation and normal heart function in each group during follow-up. Conclusion: Gore - Tax conduit reconstruct RVOT in primary palliative procedure is a better surgical option for the treatment of patients with Pulmonary Atresia with VSD in lower hospital mortality and reasonable heart function.

\begin{tabular}{lcc}
\hline & TAP $(\mathrm{n}=33)$ & Conduit $(\mathrm{n}=\mathbf{6 3})$ \\
\hline Age & 49 month & 66 month \\
Weight & $8.6 \mathrm{~kg}$ & $9.6 \mathrm{~kg}$ \\
MAPACs & 1 & 5 \\
PDA & 31 & 53 \\
PAI $<100 \mathrm{~mm} 2 / \mathrm{m} 2$ & 30 & 59 \\
BTS & 1 & 1 \\
Hospital Death & 7 & 5 \\
II staged repair & 12 & 42 \\
\hline
\end{tabular}

\section{7}

Diagnosis and Treatment of Atrioventricular Septal Defect in Children: Results of 239 cases from Shanghai Children's Medical Center

Kaihua Yang, Shanghai Children's Medical Center, China

Kun Sun, Shanghai Children's Medical Center, China

LanPing Wu, Shanghai Children's Medical Center, China

Objective: To evaluate the diagnosis and surgical outcomes of atrioventricular septal defect (AVSD) in Children.

Methods: Two hundred and thirty nine consecutive patients accepted surgery for biventricular correction of AVSD from 2003 to 2007 were enrolled. There were 121 males and 118 females, the age ranged between 1 month to 14 years. Angiogram data, records of surgery, echocardiogram data of pre-surgery assessment, 2 days post surgery and short-term follow up and outpatient data were collected.

Results: The number of patients needing pre-surgery angiogram was decreased in the past five years. The diagnosis of 31 patients
$(13 \%)$ was altered after surgery. Early mortality rate was $2.1 \%$ and 5 infants died after surgery. Six patients had reoperation during the short-term follow-up, and 3 of them had severe left atrioventricular valve regurgitation. Most patients presented inferior left atrioventricular valve (LAVV) function in shortterm follow up (from 3 months to 50 months after surgery, the average follow up time was 13.5 months), while infants had valve repair besides cleft suture presented subsequently worse LAVV function postoperatively.

Conclusions: Pre-operative echocardiogram is recommended for patients with AVSD. Early death and reoperation rate following surgical repair is acceptable, but left atrioventricular valve regurgitation remained significant among these patients. The outcome of valve repair in patients with AVSD is not satisfactory and better procedure of the valve repair is needed.

\section{8}

Influences of Pulmonary Valve Replacement on RV Volume and Function in Repaired TOF

Byung Won Yoo, Division of Pediatric Cardiology, Yonsei University, College of Medicine, Korea

Young Jin Kim, Department of Radiology, Yonsei University, College of Medicine, Korea

Nam Kyun Kim, Division of Pediatric Cardiology, Yonsei University, College of Medicine, Korea

Jae Young Choi, Division of Pediatric Cardiology, Yonsei University, College of Medicine, Korea

Han Ki Park, Department of Cardiovascular Surgery, Yonsei University, College of Medicine, Korea

Young Hwan Park, Department of Cardiovascular Surgery, Yonsei University, College of Medicine, Korea

Jun Hee Sul, Division of Pediatric Cardiology, Yonsei University, College of Medicine, Korea

Objectives: In patients with corrected tetralogy of Fallot (TOF), it has been reported that beneficial effects of pulmonary valve replacement (PVR). The aim of our study was to evaluate the effects of PVR and to analyze whether a threshold exists above which the RV does not improve further after surgery.

Methods: Twenty-one patients who underwent PVR after TOF repair, were studied by cardiac magnetic imaging (CMR) in Yonsei cardiovascular center from Jan 2004 to Aug 2008. The pre- and post-operative (preop, postop) CMR parameters (RV EDV, ESV, EF) were compared and we analyze whether a threshold can be identified for normalization of RV volumes after surgery in each preop parameters.

Results: The RV volume indexes were significantly decreased after PVR(RVEDVi:167.5 $\pm 33.6 \mathrm{ml} / \mathrm{BSA}, 109.8 \pm 15.5 \mathrm{ml} /$ BSA, $\mathrm{P}<0.001$; RVESVi: $95.9 \pm 28.7 \mathrm{ml} / \mathrm{BSA}, 55.3 \pm 14.5 \mathrm{ml} /$ BSA, $\mathrm{P}<0.001)$, and RVEF was improved (44.0 $\pm 9.7 \%$, $50.1 \pm 8.2 \%, \quad \mathrm{P}=0.01)$. Postop RVEDVi was significantly correlate with preop RVEDVi ( $\mathrm{r}=0.66, \mathrm{P}=0.001)$, RVES$\operatorname{Vi}(\mathrm{r}=0.55, \mathrm{P}=0.01)$ and inversely with $\operatorname{RVEF}(\mathrm{r}=-0.52$, $\mathrm{P}=0.017$ ). Postop RVESVi was correlated with preop RVES$\operatorname{Vi}(\mathrm{r}=0.52, \mathrm{P}=0.015)$ and inversely with RVEF ( $\mathrm{r}=-0.57$, $\mathrm{P}=0.007)$, but was not with preop RVEDVi. For normalization of RVEDVi, sensitivity and specificity were $71 \%$ and $93 \%$ with a preop RVEDVi cutoff value of $180 \mathrm{Ml} / \mathrm{m}^{2}$, and $71 \%$ and $79 \%$ with a preop RVESVi cutoff value of $100 \mathrm{Ml} / \mathrm{m}^{2}$. For normalization of RVESVi, these were $50 \%$ and $91 \%$ with a preop RVEDVi $180 \mathrm{Ml} / \mathrm{m}^{2}$, and $80 \%$ and $91 \%$ with a preop RVESVi $90 \mathrm{Ml} / \mathrm{m}^{2}$. Conclusion: All patients who underwent PVR showed significant $\mathrm{RV}$ volume reduction, but RV volume did not reach the normal value in some patients. We suggest that RV remodeling after 
PVR is insufficient in patients who had severe RV dilatation (RVEDVi $>180 \mathrm{Ml} / \mathrm{m}^{2}$, RVESVi $>90 \mathrm{Ml} / \mathrm{m}^{2}$ ) and poor $\mathrm{RV}$ function $(\mathrm{RVEF}<40 \%)$ before PVR.

\section{9}

\section{Surgical management of multiple ventricular septal} defects

Naoki Yoshimura, First Department of Surgery, University of Toyama, Graduate School of Medicine, Japan

Hironori Matsuhisa, First Department of Surgery, University of Toyama, Graduate School of Medicine, Japan

Shingo Otaka, First Department of Surgery, University of Toyama, Graduate School of Medicine, Japan

Masaya Aoki, First Department of Surgery, University of Toyama, Graduate School of Medicine, Japan

Kazuhiro Watanabe, Department of Pediatrics, University of Toyama, Graduate School of Medicine, Japan

Keiichi Hirono, Department of Pediatrics, University of Toyama, Graduate School of Medicine, Japan

Fukiko Ichida, Department of Pediatrics, University of Toyama, Graduate School of Medicine, Japan

Takuro Misaki, First Department of Surgery, University of Toyama, Graduate School of Medicine, Japan

Objective: Recently, the felt sandwich technique is widely used to close muscular ventricular septal defect (VSD). We evaluated the early and midterm results of our strategy (a combination of sandwich technique and direct closures) and assessed the role of the sandwich technique in the treatment of multiple VSDs.

Methods: Thirty-six consecutive patients underwent an operation for multiple VSDs and associated cardiac malformations. Median age and body weight at operation were 6.5 months and $5.9 \mathrm{~kg}$, respectively.

Results: One-hundred-and-twenty-eight VSDs were closed in 36 patients. There were no ealry and late deaths. No reoperation was required. There were no heart block and no significant residual shunts in the latest follow-up study. Two patients with Swiss cheese septum had postoperative congestive heart failure. Three muscular VSDs were closed by the sandwich technique in these 2 patients, whereas 1 or less VSD was closed by the sandwich technique in the other 34 patients. Eight of 11 patients (72.7\%) who underwent the sandwich procedure showed septal dysfunction, whereas 6 of other 25 patients (24.0\%) showed septal dysfunction $(\mathrm{p}<0.05)$.

Conclusions: The outcome of the surgical repair of multiple VSDs was satisfactory. Although the sandwich technique is simple and effective, the use of numerous felt patches disturbed the movement of the interventricular septum. To avoid postoperative cardiac dysfunction, an effort should be made to close the muscular VSD directly. Large apical VSDs especially those locate just underneath the moderator band are considered suitable for the sandwich technique.

\section{0}

Surgical Treatment of left atrial appendage herniation through partial pericardial defect and left atrial appendage aneurysm

Jae-Hyeon Yu, Departmet of Cardiac Surgery, Chungnam National University Hospital, Daejeon, Korea

Yong-Ho Kim, Departmet of Cardiac Surgery, Chungnam National University Hospital, Daejeon, Korea

Suk-Ki Lee, Departmet of Cardiac Surgery, Chungnam National University Hospital, Daejeon, Korea
Hong-Ryang Gil, Departmet of pediatric cardiology, Chungnam National University Hospital, Daejeon, Korea

Shin-Kwang Kang, Departmet of Cardiac Surgery, Chungnam National University Hospital, Daejeon, Korea

Myung-Hun Na, Departmet of Cardiac Surgery, Chungnam National University Hospital, Daejeon, Korea

Seung-Pyung Lim, Departmet of Cardiac Surgery, Chungnam National University Hospital, Daejeon, Korea

Young Lee, Departmet of Cardiac Surgery, Chungnam National University Hospital, Daejeon, Korea

Objective: Distinguishing between LAA herniation through a pericardial defect and LAA aneurysm may be difficult because of the similarities of the presentations and radiographs but surgical techniques different. We report two cases-a LAA herniation and a LAA aneurysm-that were successfully treated with surgical intervention.

Method E Results: Case 1: A 14-year-old female presented with symptoms of exertional dyspnea and chest pain. Chest radiograph and chest CT showed abnormal bulging of left superior cardiac border and partial pericardial defect with LAA herniation. Using Left thoracotomy, the pericardial defect, measuring $5 \times 6 \mathrm{~cm}$ was closed to prevent subsequent herniation with bovine pericardium. The patient recovered without complications and has remained asymptomatic since the operation. Case 2: A 13-year-old male presented with abnormal bulging of left cardiac border on chest radiograph. Chest MDCT showed that LAA was enlarged and pericardial defect was suspected. Echocardiography showed enlarged LA with some evidence of thrombi inside. After diagnosed as a pericardial defect, left thoracotomy was made. On operation, there was a bulging of pericardium without defect. After opening pericardium, the LAA aneurysm was found, $5 \times 6 \mathrm{~cm}$ with a neck of diameter $2.0 \mathrm{~cm}$. The aneurysm excised using Endo GIA staplers without cardiopulmonary bypass. On resection, there is no thrombus inside.

Conclusion: Although distinguishing between Congenital LAA aneurysm and LAA herniations through partial pericardial defect may be demanding, early surgical intervention is preferable to eliminate symptoms and to prevent potential fatal complications.

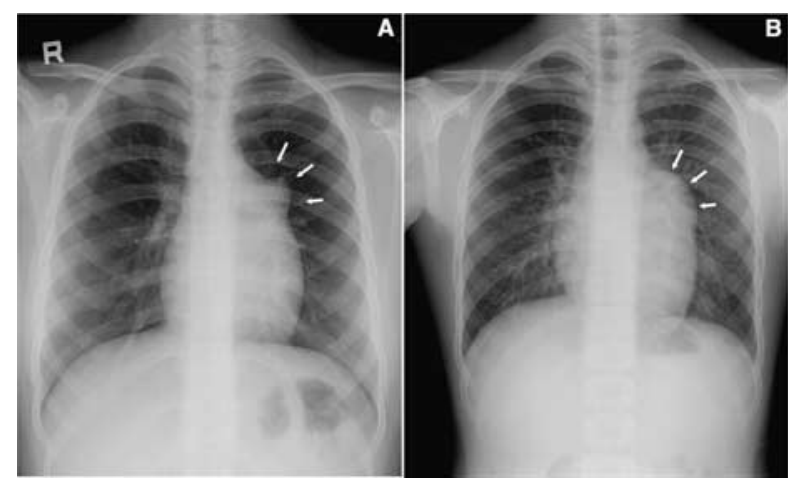

7491

Staged and "One-Stop" Hybrid Approach for Treatment of Tetralogy of Fallot with Aortopulmonary Collateral Arteries Zhe Zheng, Fu Wai Hospital, China Shoujun Li, Fu Wai Hospital, China De Wang, Fu Wai Hospital, China Shengshou Hu, Fu Wai Hospital, China

Objective: To compare the early results of one-stop hybrid procedure with conventional staged procedure of for TOF with APCAs. 
Methods: From January 2003 to July 2008, fifty-one patients were diagnosis of TOF and redundant APCAs and were performed with combined therapy of APCAs occlusion and complete surgical repair. From January 2003 to May 2007, 24 patients were defined as Staged hybrid group (A). APCAs were occluded in catheter laboratory before (group A1, 11 cases) or after (group A2, 13 cases) surgical correction. From June 2007 to July 2008, 36 patients were included as one-stop hybrid group (B). Occlusion of APCAs and surgical correction were concomitantly performed in one-stop hybrid operation room. The data of these two groups were compared and analyzed.

Results: Compare with group A2, group A1 and group B had shorter cardiopulmonary bypass duration and aortic clamping duration. Group B had shorter tracheal intubation duration, ICU stay, hospital stay and less hospitalization cost than group A1 and A2. After use of the one-stop hybrid procedure in June 2007, the morbidity and mortality of TOF with APCAs decreased in our center.

Conclusion: one-stop hybrid procedure is a safe and effective approach for TOF with redundant APCAs.

\section{2}

Focus on 2007: A clinical audit of the Red Cross War Memorial Children's Hospital Paediatric Cardiac Surgery Program

Liesl J Zuhlke, Western Cape Pediatric Cardiac Services, Red Cross and Tygerberg Hospitals, Cape Town, South Africa

Rik De Decker, Western Cape Pediatric Cardiac Services, Red Cross and Tygerberg Hospitals, Cape Town, South Africa

John Lawrenson, Western Cape Pediatric Cardiac Services, Red Cross and Tygerberg Hospitals, Cape Town

John Hewitson, Western Cape Pediatric Cardiac Services, Red Cross and Tygerberg Hospitals, Cape Town, South Africa

Andre Brooks, Western Cape Pediatric Cardiac Services, Red Cross and Tygerberg Hospitals, Cape Town, South Africa

Introduction: Paediatric Cardiac Surgery has a long and rich history in South Africa with the first bypass operation performed by Prof Chris Barnard in a child at Red Cross Childrens Hospital in 1958. Our hospital remains the largest public service unit with 250 cases per year. We undertook a clinical audit of all patients operated upon in 2007 to critically review deficiencies within the service and define ways of managing our reduced resources in a more efficient fashion.

Results: A total of 252 surgical procedures were reviewed. Demographic parameters reviewed included age, age at diagnosis and at surgery, mortality, days spent in ICU and total days spent in hospital. Surgical parameters included time of surgery, bypass times, reoperations and re-intervention rates. The total mortality was $7.5 \%$ with in hospital mortality rates at $5.8 \% .43 \%$ of patients resided within the Cape Metropole while 22\% resided in provinces other than the Western Cape. $64 \%$ of patients were diagnosed within the first year of life, most as neonates. Conclusion: In this audit we sought to focus on 2007 and critically appraise as many aspects of our surgical service as possible. We hope to now institute clinical audit as a regular part of our program in particular to aid us in managing our limited resources in a more efficient way and hopefully in so doing improving our service delivery.

\section{2}

Acute Chagas Disease by Oral Transmision in Children. Cases Reported in Urban Population: Caracas, Venezuela Cielo Rojas, Hospital Universitario de Caracas, Venezuela Amando Martín, Hospital Universitario de Caracas, Venezuela
Iván A Machado, Hospital Universitario de Caracas, Venezuela Luis Villarroel, Hospital Universitario de Caracas, Venezuela Iván J Mendoza, Instituto de Medicina Tropical - Universidad Central de Venezuela, Venezuela

Juan A Marques, Instituto de Medicina Tropical - Universidad Central de Venezuela, Venezuela

Belkisolé Noya, Instituto de Medicina Tropical - Universidad Central de Venezuela, Venezuela

Raiza Ruiz, Instituto de Medicina Tropical-Universidad Central de Venezuela, Venezuela

In our country, Chagas Disease vectorial transmisión (insects) has been extensively studied. However, oral transmission has been described since 1921. Tripanosoma cruzi can resist gastric $\mathrm{pH}$, penetrate the mucosae and induce immune response.

On December 2007, we had an acute outbreak of Acute Chagas Disease, transmitted by oral route. 250 persons were affected in Caracas, 11 hospitalized in our Department of Pediatrics: $73 \%$ males, $27 \%$ females. The more evident symptoms were: fever 9/11pts (81\%), bipalpebral edema 6/11pts (54\%), chest pain 5/11pts (45\%), effort dysnea 1/11pts (9\%). 90\% of the patients had clinical cardiovascular evaluation, echocardiogram and Holter ECG. Acute Myocarditis was evident in 1/11 (9\%), pericardial effusion 9/11 (18\%), supraventricular arrhythmias 5/11 (45\%).

Diagnosis was made by a positive ELISA test in $81 \%$ of patients, and in $18 \%$ by parasitologic tests. Acute Phase Reactants, CK, CKMB and transaminases determination were made on admisión and as follow-up. Patients were dismissed only after normalization of C- Reactive protein of their erithrocyte sedimentation rate. $45 \%$ of the patients were treated with nifurtimox, 54\% with beznidazole. Secondary effects detected: epigastralgia 3/11(27\%), maculo-papular rash 2/11(18\%), visual allucinations $1 / 11(9 \%)$, thrombocytopenia $1 / 11(9 \%)$.

Acute Chagas Disease by oral transmisión is a reality. It has been proved by experimental, clinical and epidemiological data. Its true incidence is probably underestimated, specially in urban population. It is important to inform properly to the Medical Community and all the population about this transmission mechanism. Appropiate strategies about food manipulation, Access to specific treatment and promote research programs are necessary.

\section{3}

Acute myocarditis or idiopathic dilative cardiomyopathy? A six-month-follow up of NT-proBNP and left ventricular diameters in children presenting with left ventricular dilatation and dysfunction

Farnaz Darbandi-Mesri, Department of Pediatric Cardiology, Medical University of Vienna, Austria

Doris Luckner, Department of Pediatric Cardiology, Medical University of Vienna, Austria

Ulrike Salzer-Muhar, Department of Pediatric Cardiology, Medical University of Vienna, Austria

Objectives: To evaluate plasma N-terminal pro-B-type natriuretic peptide (NT-proBNP) levels and echocardiographic left ventricular (LV) parameters at initial presentation and after 6 months in children with heart failure, left ventricular dilatation/ dysfunction due to acute myocarditis or idiopathic dilative cardiomyopathy (DCM).

Methods: Retrospective study performed in a tertiary pediatric cardiology centre from 2004 to 2006. Ten children with acute myocarditis $(n=6)$ or idiopathic DCM $(n=4)$ were included. 
NT-proBNP values (in pg/ml)and left ventricular (LV) measurements were retrieved from medical records.

Values of enddiastolic/endsystolic LV diameters (LVEDD, LVES) were expressed as percentages of mean normal reference values. A p-value $<0.05$ was considered significant.

Results: Median NT-proBNP level was $20241 \mathrm{pg} / \mathrm{ml}$ initially and $522.95 \mathrm{pg} / \mathrm{ml}$ after 6 months $(\mathrm{p}<0.05)$. Patients with acute myocarditis had higher initial NT-proBNP levels $(29813.7 \pm 3113.8)$ than children with DCM $(7813.8 \pm 2192.3)$. This difference was noticeable, but not statistically significant.In patients with myocarditis, plasma NT-proBNP levels dropped after initiation of therapy and were normal or mildly elevated in all after 6 months.

Mean LVEDD was $167.6 \%$ of the mean normal reference value initially and decreased to $149.6 \%$ after 6 months, when $8 / 10$ patients still had enlarged LVEDD. Mean LVES was 243.3\% of normal initially and decreased to $197.3 \%(\mathrm{p}<0.05)$

Conclusion: Children with myocarditis had higher NT-proBNP levels at presentation than children with DCM and showed a rapid decrease after initiation of treatment. Despite clinical improvement and normal/mildly elevated NT-proBNP values, marked left ventricular dilatation was still found in two thirds after 6 months.

\section{4}

Left ventricular non-compaction (LVNC): clinical presentation, laboratory findings, and outcome Valeria Acevedo, Clinica Alemana-Hospital Roberto Del Rio, Chile Patricia Alvarez, Clinica Alemana-Hospital Roberto Del Rio, Chile Rodrigo Neghme, Congenital Heart Institute, Orlando - FL., United States

Marcela Alburquenque, Hospital Roberto Del Rio, Chile

Katty Quezada, Hospital Roberto Del Rio, Chile

Sonia Gonzalez, Hospital Exequiel Gonzalez, Chile

Rodrigo Soto, Clinica Alemana, Chile

Carols Fernandez, Hospital Roberto Del Rio, Chile

We report 36 patients $(\mathrm{Pt}), 21$ male, mean age 5.8 years with LVNC. EKG and echocardiography were available in all, Holter in 29, MRI in 27, biopsy in 6 and autopsy in 1. Follow-up ranges from 1 to 216 months.

Isolated LVNC was seen in $29(80 \%), 6(17 \%)$ had an associated VSD. Family history was present in $6(17 \%)$. At initial presentation, CHF was seen in $19(52 \%)$, dilated cardiomyopathy in $20(55 \%)$, SVT in $5(14 \%)$, VT in $2(5 \%)$, syncope in $2(5 \%)$.

The diagnosis was made by echocardiography in all with abnormal trabeculations seen at the apex in $36(100 \%)$, lateral wall in 35 (97\%), and apical septum in 17 (47\%). Restrictive flow pattern was seen in 14 (39\%) and mitral regurgitation (MR) in 31 (86\%). MRI correlated with echo findings in all. EKG and Holter showed left ventricular hypertrophy in 14 (39\%), biventricular hypertrophy in $8(22 \%)$, WPW in $8(22 \%)$, SVT in $5(14 \%)$, isolated premature ventricular contractions in 9 $(25 \%)$, VT in $2(6 \%)$. Six $(17 \%)$ died, all infants, because of refractory CHF. Treatment helped 30/36 survivors who are now in NYHA I or II.

Conclusion: CHF and arrhythmias were frequent, but thromboembolism was not seen. WPW (22\%) and MR (86\%) were findings not previously reported with such frequency in children. Mortality in infants from intractable CHF was significant (17\%). Anticongestive and arrhythmia therapy seems indicated along with early consideration for transplantation, particularly in infancy, if improvement is not promptly seen.

\section{5}

Serum cardiovascular growth factors during doxorubicin chemotherapy in children with leukemia

Steven E Lipshultz, University of Miami Miller School of Medicine, United States

Rebecca E Scully, University of Miami Miller School of Medicine, United States

Holly M Smith, Vanderbilt University School of Medicine, United States

Brent Anderson, Vanderbilt University School of Medicine, United States

Tracie L Miller, University of Miami Miller School of Medicine, United States

Stuart R Lipsitz, Brigham and Women's Hospital, United States Stephen E Sallan, Dana-Farber Cancer Institute, United States Douglas B Sawyer, Vanderbilt University School of Medicine, United States

Objective: To better understand individual susceptibility to doxorubicin (DOX) chemotherapy-related cardiotoxicity in children undergoing treatment for leukemia, we examined serum levels of neuregulin-1beta (NRG), cardiotrophin-1 (CT-1), vascular endothelial growth factor (VEGF), and interleukin-6 (IL-6), each of which alter myocardial responses to DOX in animals.

Methods: Serum collected during treatment from 90 DOX-treated children $\left(30 \mathrm{mg} / \mathrm{m}^{2}\right.$ every 3 weeks for 10 cycles) at baseline, midand late-treatment was analyzed for NRG, CT-1, IL-6 and VEGF levels. Serum cardiac troponin $\mathrm{T}(\mathrm{c} \operatorname{TnT})$ and high-sensitivity Creactive protein levels (hsCRP) were assessed previously.

Results: CT-1 and NRG levels decreased significantly with time; mean CT-1 was $800 \mathrm{pg} / \mathrm{ml}$ at baseline, $205 \mathrm{pg} / \mathrm{ml}$ mid-treatment and $19.1 \mathrm{pg} / \mathrm{ml}$ at late-treatment $(\mathrm{p}<.001)$. Mean NRG was $17.9 \mathrm{pg} / \mathrm{ml}$ at baseline, $13.5 \mathrm{pg} / \mathrm{ml}$ mid-treatment, and $7.7 \mathrm{pg} / \mathrm{ml}$ late $(\mathrm{p}<.001)$. VEGF was stable between baseline $(145 \mathrm{pg} / \mathrm{ml})$ and mid-treatment $(177 \mathrm{pg} / \mathrm{ml})$, but increased at late-treatment $(292 \mathrm{pg} /$ $\mathrm{ml})$ compared to mid-treatment $(\mathrm{p}=0.0035)$. Mean IL-6 decreased from baseline $(32.3 \mathrm{pg} / \mathrm{ml})$ to mid-treatment $(9.2 \mathrm{pg} / \mathrm{ml}$; $\mathrm{p}<0.001)$, but not between baseline and late-treatment $(21.5 \mathrm{pg} /$ $\mathrm{ml} ; \mathrm{p}=0.13)$. IL-6 correlated with hsCRP $(\mathrm{r}=.405, \mathrm{p}<.001)$ and was higher when $c \operatorname{TnT}$ was elevated $(\mathrm{cTnT} \geqslant 0.01 \mathrm{ng} / \mathrm{ml}$; median IL-6: 17.6 vs. $4.1 \mathrm{pg} / \mathrm{ml}, \mathrm{p}=0.017)$.

Conclusions: CT-1 and NRG decline with DOX-treatment promoting increased cardiac apoptosis, left ventricular remodeling, and decreased left ventricular function and mass. IL-6 and VEGF changes suggest DOX-related inflammation and angiogenesis, especially in the setting of myocardial injury.

\section{6}

Functional Status is Impaired and Correlated with Clinical Status in Pediatric Cardiomyopathy

Lynn A Sleeper, New England Research Institutes, United States

Jeffery A Towbin, Baylor College of Medicine, United States

Steven D Colan, Harvard Medical School, United States

Daphne T Hsu, Children's Hospital at Montefiore, United States

James D Wilkinson, University of Miami Miller School of Medicine, United States

Tracie L Miller, University of Miami Miller School of Medicine, United States

Steven E Lipshultz, University of Miami Miller School of Medicine, United States

Background: Little systematic information exists on the functional status of children with cardiomyopathy (CM) and its association 
with socioeconomic status (SES), growth, cardiac status, and risk of death/transplant.

Methods: The NHLBI Pediatric CM Registry measured parentreported functional status using the Child Health Questionnaire and Functional Status IIR (FSIIR) in 249 children with cardiomyopathy.

Results: Subjects (mean age $7.9 \pm 5.9 \mathrm{yr}$ ) had lower functional status vs. historical controls (CHQ Physical summary score $43.2 \pm 13.1$ vs. $53.0 \pm 8.8 ; \quad$ Psychosocial $47.9 \pm 10.7$ vs. $51.2 \pm 9.1$ ), but $74 \%$ and $91 \%$ were in normal range, respectively. Poorer physical and psychosocial functioning was associated with impaired growth and parental non-completion of high school. In patients with dilated cardiomyopathy, younger age at $\mathrm{dx}$ $(\mathrm{P}=.004)$ and lower $\mathrm{LV}$ mass $\mathrm{z}$-score $(\mathrm{R}=-0.32$; slope -2.6 ; $\mathrm{P}=.01)$ independently correlated with better physical function. In patients with hypertrophic cardiomyopathy, better physical function was independently correlated with parental education $(\mathrm{P}<.001)$, lower $\mathrm{LV}$ posterior wall thickness $(\mathrm{R}=-0.29$; $\mathrm{P}<.001)$ and closer to normal $\mathrm{FS}(\mathrm{R}=-0.26 ; \mathrm{P}=0.04)$. Clinical status was also associated with emotional impact of CM on parents. Across all pts, lower CHQ Physical summary score was a risk factor for death/transplant [hazard ratio 0.75 per 5 -point increase in score, $\mathrm{p}=.013$; hazard ratio 4.41 for score below vs. within normal range (logrank test $\mathrm{p}=.034)$ ].

Conclusions: Functional status in children with CM is impaired relative to healthy children and is associated with SES, concurrent LV structure and function, and growth. Physical functioning is associated with risk of death and transplant.

\section{8 \\ LVAD as a Bridge to Recovery in Young Children with Cardiomyopathy: A Longitudinal Echocardiographic Study}

Chelsea L Knudson, University of Arizona College of Medicine, United States

Hannah Zimmerman, University of Arizona Department of Surgery, United States

Jack G Copeland, University of Arizona Department of Surgery Section of Cardiothoracic Surgery, United States

Daniela Lax, University of Arizona Department of Pediatrics Section of Pediatric Cardiology, United States

Diane Covington, University of Arizona Marshall Foundation Artificial Heart Lab, United States

Richard G Smith, University Medical Center, United States

Brian J Blair, University of Arizona Department of Pediatrics Section of Pediatric Cardiology, United States

Brent J Barber, University of Arizona Department of Pediatrics Section of Pediatric Cardiology, United States

Objective: Echocardiographic follow-up of young children who were successfully weaned from left ventricular assist device (LVAD).

Methods: Children with dilated cardiomyopathy failing maximal inotropic support were placed on LVADs. They were followed longitudinally with multiple echo studies.

Results: A total of 26 children were placed on mechanical circulatory support, 14 were transplanted and 12 (46\%) demonstrated left ventricular (LV) recovery. Eight (67\%) of the 12 were weaned off mechanical support and discharged home, 1 was weaned and subsequently transplanted, 2 died in the hospital after weaning, and 1 had LV recovery, but died prior to device explantation.

Of the 12 patients, $9(75 \%)$ were female, the median age was 12 months (1-72 months), and the mean weight $8.5 \mathrm{~kg}$ (mean BSA $=0.42 \mathrm{~m} 2$ ). Devices implanted included: MEDOS, Berlin, and Jostra, with a mean duration of support of 16.5 days (2-70 days).

The following echo parameters were followed:

Pre LVAD: LV diameter end diastole (LVED) Z score $=+5.3-14.6 \quad($ mean $=+10.5) ; \quad$ Fractional Shortening $(\mathrm{FS})=8-13 \%($ mean $9 \%) ; 90 \%$ had mild-severe mitral regurgitation (MR), and $70 \%$ had mild-moderate tricuspid regurgitation (TR).

Post LVAD: (1-57 months [mean 22, median 17] post LVAD wean): LVED Z score significantly improved to +1-8.4; (mean $+5 ; \mathrm{p}=0.0001) ; \mathrm{FS}$ improved (mean 33\%); and MR (trace 66\%) and TR (trace 55\%) also improved.

Conclusions: We report the successful weaning of children $\leqslant 6$ years of age from LVAD and the echocardiographic demonstration of reverse remodeling with improvements in LV dimension, function and $\mathrm{AV}$ valve regurgitation.

\section{9}

Parents experiences of having an asymptomatic child diagnosed with hypertrophic cardiomyopathy through family-screening

Ewa-Lena Bratt, Sahlgrenska Academy, Institute of Clinical Sciences, Department of Pediatrics, University of Gothenburg, Sweden Ingegerd Östman-Smith, Sahlgrenska Academy, Institute of Clinical Sciences, Department of Pediatrics, University of Gothenburg, Sweden Asa B Axelsson, Sahlgrenska Academy, Institute of Health and Care Sciences, University of Gothenburg, Sweden

Background: Hypertrophic Cardiomyopathy (HCM) is an inherited cardiac disease with a prevalence of 1:500. Children with a parent diagnosed with HCM have a 50\% risk of inheriting the mutation. HCM is the commonest medical cause of sudden death in the age 8-16 years and may have implications for the psychosocial well-being of the child, as well as for the family. A HCM-diagnosis may cause fear and anxiety, because of the risk of complications, and due to lifestyle-modifications the patient is advised to make. These restrictions in sports-participation may bring profound changes to the affected individual's lifestyle and impacts the whole family.

Aim: To describe parents' experiences of how the diagnosis affects everyday family-life.

Method: 12 parents with asymptomatic children diagnosed with HCM through family-screening, were interviewed 12-24 months after diagnosis. Analysis was conducted with qualitative content-analysis.

Results: The analysis resulted in two themes and six sub-themes. The parents struggled for normalization of life as the diagnosis was followed by lifestyle-modifications which they too had to adapt to.

1. A drastic change of life:

- Death as a reality

- Loss of freedom of choice

- Faith in the future

2. A strive for normalization of life:

- To feel secure

- To protect without circumscribing

- To adapt to a new life

Conclusion: Diagnosis of asymptomatic children affects the parents who strive to protect their child without circumscribing their life too much, and to achieve a new state of normality. The main changes perceived were ascribed to lifestyle modifications and the awareness of the vulnerability of life. 


\section{0}

Selective or non-selective high-dose betablockade evaluation of exercise capacity in children and adolescents with hypertrophic cardiomyopathy Ewa-Lena Bratt, Sahlgrenska Academy, Institute of Clinical Sciences, Department of pediatrics, University of Gothenburg, Sweden Ingegerd Östman-Smith, Sahlgrenska Academy, Institute of Clinical Sciences, Department of pediatrics, University of Gothenburg, Sweden Asa B Axelsson, Sahlgrenska Academy, Institute of Health and Care Sciences, University of Gothenburg, Sweden

Background: Hypertrophic cardiomyopathy (HCM) is a common medical cause of sudden death during exercise. High-dose therapy with metoprolol or propanolol reduces risk of sudden death. There is a perception that exercise ability will be drastically curtailed, with non-selective beta-blockade being worse, but on the other hand beta-blockade improves diastolic function in HCM. This study evaluates the exercise capacity in HCMpatients with metoprolol- or propranolol-therapy compared to HCM-patients without treatment.

Methods: $20 \mathrm{HCM}$ patients have >one year follow-up, 12 in treatment-group (6 metoprolol and 6 propanolol), median age 15 years (range 9-25), and 8 randomised controls, median age 14.5 years (range 7-24), with no therapy but same life-style modifications. Before study entry, and after one year, they underwent incremental bicycle exercise tests with a ramp protocol starting at $1 \mathrm{~W} / \mathrm{kg}$, with $10 \mathrm{~W}$ increments each minute. We monitored 12 lead ECG, blood pressure and respiratory rate every minute.

Results: Maximum exercise capacity in $\mathrm{W} / \mathrm{kg}$ in metoprolol-group before treatment, median $2.85 \mathrm{~W} / \mathrm{kg}$ (range 2.2-3.3) was not different from controls, median $2.6 \mathrm{~W} / \mathrm{kg}(2.1-3.3 ; \mathrm{p}=0.51)$. After one year of metoprolol-therapy the median exercise capacity was $2.55 \mathrm{~W} / \mathrm{kg}$ (range 2.1-3.0) and no different from the controls, median $2.4 \mathrm{~W} / \mathrm{kg}(1.4-2.9 ; \mathrm{p}=0.51)$. Maximum exercise capacity in propranolol-group before treatment was $2.65 \mathrm{~W} / \mathrm{kg}(2.1-3.8)$, no different from controls $(p=0.79)$. After one year of propanololtherapy the exercise capacity was $2.35 \mathrm{~W} / \mathrm{kg}$ (1.6-2.8), still no different from controls $(p=0.95)$.

Conclusion: Neither selective nor non-selective beta-blockade causes significant reduction in exercise capacity in HCMpatients, probably because improvement in diastolic filling increases the stroke volume to compensate.

\section{1}

A single center experience of intracardiac thrombosis in children with Dilated Cardiomyopathy

Soo Han Choi, Department of Pediatrics, Sungkyunkwan University School of Medicine, Samsung Medical Center, Seoul, Korea, Korea Soo In Jeong, Department of Pediatrics, Sungkyunkwan University School of Medicine, Samsung Medical Center, Seoul, Korea, Korea Ji-Hyuk Yang, Department of Thoraxic surgery, Sungkyunkwan University School of Medicine, Samsung Medical Center, Seoul, Korea, Korea

I-Seok Kang, Department of Pediatrics, Sungkyunkwan University School of Medicine, Samsung Medical Center, Seoul, Korea, Korea Tae-Gook Jun, Department of Thoraxic surgery, Sungkyunkwan University School of Medicine, Samsung Medical Center, Seoul, Korea, Korea

Heung-Jae Lee, Department of Pediatrics, Sungkyunkwan University School of Medicine, Samsung Medical Center, Seoul, Korea, Korea June Huh, Korea

Introduction: Intracardiac thrombosis in dilated cardiomyopathy (DCMP) could occur with subsequent serious morbidity. This study was aimed to assess risk factors and outcome of intracardiac thrombosis in children with DCMP.

Patients and Method: Retrospective review of clinical records was done in 83 children with DCMP who was diagnosed from January 1995 to December 2008.

Results: Intracardiac thrombi were detected in five patients $(6.0 \%)$ (LV (3) and LA (1), RV (1) respectively). The etiology of DCMP was idiopathic (2), VSD with pulmonary hypertension (1), repaired VSD with complete atrioventricular block (1), and after chemotherapy for AML (1). Average duration from diagnosis to detection of thrombi was 9.4 months $(0 \sim 23 \mathrm{mo})$. One showed LA thrombus in the pouch of interatrial septum at the time of diagnosis. The other 4 patients had thrombi in ventricles when rapid deterioration of ventricular function occurred. All showed poor ejection fraction of left ventricle $(15.5 \sim 27 \%)$. Cerebral infarction of left MCA territory was noted in one with LV thrombus after CRT implantation. One showed LV thrombus after volume reduction operation of LV. RV thrombus was found in unrepaired VSD with pulmonary hypertension. All were treated with heparinization $(15 \sim 20 \mathrm{U} / \mathrm{kg} / \mathrm{hr}$, average treatment duration 7.8 days). LV thrombectomy was performed in a patient with postoperative volume reduction operation. Three showed complete absorption without further embolic complications after anticoagulation. Two patients died of respiratory infection and pump failure.

Conclusion: Careful evaluation and aggressive anticoagulation are necessary for preventing intracardiac thrombus in children with poor ventricular function.

8012

Aorto-arteritis in the setting of dilated cardiomyopathy Swati Garekar, Wockhardt Pediatric and Congenital Heart Center, India

Snehal Kulkarni, India

Zakiya Khan, India

Akhbar Haque, India

Dilated cardiomyopathy (DCM) is a relatively common diagnosis encountered in the pediatric cardiac outpatient clinic (PCOPD). We present a case where a presumed viral myocarditis leading to DCM revealed itself to be aorto-arteritis (AA). The purpose of this submission is to highlight AA as an important differential in all cases of dilated cardiomyopathy.

An 8 yr old girl presented to our PCOPD with a dyspnea on exertion. 2 months prior she had been diagnosed with DCM of unknown etiology. At that time, her echocardiogram showed a severely dilated and poorly contractile left ventricle. This was confirmed in our clinic. She was placed on routine decongestive and cardiosupportive medications. Subsequently she was seen in 2 other PCOPDs in the country where intravenous immunoglobulins were given after endomyocardial biopsy. At her next visit with us, she was hypertensive and had weight loss. A CT scan revealed isolated coarctation of the lower thoracic aorta. The pulse wave Doppler of the abdominal aorta showed depressed waveforms. Mantoux test was positive; anti Kochs therapy was initiated. Balloon angioplasty was successfully performed. 6 months post angioplasty, her hypertension is under control but her DCM has not improved. A diagnosis of AA related primary DCM was made and the patient is currently being comanaged by a rheumatologist.

Non specific inflammation on endomyocardial biopsy is not specific for viral myocarditis. AA should be considered in the differential diagnosis of every child presenting with DCM in a third world country. 
8013

Clinical features of left ventricular noncompaction in infants and children

Sayaka Watanabe, University of Toyama, Japan

Kazuyoshi Saito, University of Toyama, Japan

Ibuki Keijirou, University of Toyama, Japan

Kazuhiro Watanabe, University of Toyama, Japan

Keiichi Hirono, University of Toyama, Japan

Keiichiro Uese, University of Toyama, Japan

Fukiko Ichida, University of Toyama, Japan

Toshio Miyawaki, University of Toyama, Japan

Background: INVM is characterized by a left ventricle with prominent trabecular meshwork, which has been thought to be due to an arrest of myocardial morphogenesis during fetal life. However, increasing number of juvenile or adult cases, with longer clinical course have been also reported recently.

Methods: We analyzed the clinical features and anatomical properties of infantile INVM ( $<2$ years: 43 cases) in comparison with juvenile (2-15 years: 45 cases). We developed a new echocardiographic criteria "noncompaction score" to estimate the severity of noncompacted myocardium add to N/C ratio.

Results: Although most of patients in infantile type had clinically signs or symptoms of heart failure such as dyspnea at initial presentation (63\%), a greater number of asymptomatic patients were identified because of such as abnormalities on screening ECG in juvenile type (60\%). The incidence of WPW syndrome was higher $(16.4 \%)$, in contrast, the incidence of LBBB and VT was rarer than those reported among adults. In echocardiographic findings, the maximum N/C ratio was observed in the apex in both types. Noncompaction score and N/C score were not significant difference in both groups. Therefore LVEF at initial presentation in infantile type was lower than juvenile type significantly $(45.8 \pm 4.0$ vs. $61.6 \pm 2.0: \mathrm{p}<0.001)$. Although the survival analysis showed poor prognosis in infantile type, the significant risk factor was below 50\% LVEF $(\mathrm{p}=0.008$, $\mathrm{HR}=18.8)$, and heart failure at diagnosis $(\mathrm{p}=0.008, \mathrm{HR}=13.4)$. Conclusions: INVM in both type showed poor prognosis correlated with depressed LVEF and heart failure at diagnosis.

\section{4}

Mitochondrial Disorders: Characteristics and Outcome from the Pediatric Cardiomyopathy Registry

Gerald F Cox, Genzyme, United States

Steven D Colan, Harvard Medical School, United States

Lynn A Sleeper, New England Research Institute, United States

Jeffrey A Towbin, Baylor College of Medicine, United States

Paul Kantor, Hospital for Sick Children, Canada

James D Wilkinson, University of Miami Miller School of Medicine, United States

Steven E Lipshultz, University of Miami Miller School of Medicine, United States

Background: Little systematic, large-sample information exists on the characteristics and long-term outcome of children with mitochondrial disorders and cardiomyopathy (CM).

Methods: The NHLBI Pediatric Cardiomyopathy (CM) Registry has collected clinical and long-term outcome data on over 3,500 children meeting strict echocardiographic criteria for CM since 1990. Ninety-nine cases of mitochondrial disease have been indentified.

Results: At time of CM diagnosis, 49\% had dilated CM (DCM), $26 \%$ had hypertrophic CM (HCM), 22\% had mixed/other type of $\mathrm{CM}(\mathrm{M} / \mathrm{OCM})$, and 2 patients had restrictive CM. Barth syndrome was the etiology in $27 \%$, respiratory chain defects in $17 \%$, and Kearns-Sayre, MELAS, and Senger syndromes in 10\%, with the reminder unspecified. In $40 \%$, mitochondrial disease was diagnosed $>30 \mathrm{~d}$ after CM presentation. Outcome differed by type of CM $(p=0.022)$ with 2 -yr mortality being highest in patients with HCM (49\%). Two-yr mortality for patients with M/OCM was $36 \%$ and with DCM was $17 \%$. Age $<1 \mathrm{yr}$ at diagnosis of CM was a significant risk factor for death in patients with HCM.

Conclusions: Approximately 3\% of pediatric CM can be attributed to mitochondrial disease, which is likely under diagnosed. Notably, $46 \%$ of these patients had unspecified mitochondrial disease and $27 \%$ had Barth syndrome. Mitochondrial disorders were associated with all functional types of CM. Patients presenting with HCM, particularly infants, had the poorest survival ( $51 \%$ at 2 years). These first attempts to characterize the patient population may help to better understand the underlying disease pathophysiology leading to earlier and more specific diagnosis.

\section{5}

Effects of Prednisolone Treatment on Cardiac Functions in Patients with Duchenne Type Muscular Dystrophy Osman Kucukosmanoglu, Department of Pediatric Cardiology, Faculty of Medicine, Cukurova University, Adana Turkey, Turkey

Tulin Dede, Turkey

Nazan Ozbarlas, Cukurova University, Turkey

Duchenne muscular dystrophy is an inherited myopathy characterized by progressive skeletal muscle weakness leading to loss of ambulation, respiratory and cardiac failure.

Currently, there is no cure for Duchenne muscular dystrophy. Although corticosteroids are clinically effective pharmacologic therapy for skeletal muscle function, there is limited data documenting the impact on cardiac functions.

Objective: This study aimed to detect effects of prednisolone on cardiac functions in patients with Duchenne muscular dystrophy. Patients and methods: This study consisted of 26 boys with Duchenne muscular dystrophy and 19 healthy boys in the control group. All patients have taken prednisolone at least six months. Patients were examined regularly 3-6 months interval. To evaluate cardiac functions all patients underwent ECG, Holter and echocardiography study. Echocardiography studies performed to the control group. Left atrial and ventricular dimensions, left ventricular ejection fraction and fractional shortening were measured by two-dimensional echocardiography. By Doppler echocardiography mitral inflow velocities, Mitral E/Mitral A ratio, and time intervals were measured. Tissue Doppler velocities of septal and lateral sites of the mitral annulus were measured by tissue Doppler echocardiography.

Results: The results compared with the normal values and control group. There was no significant impairment at systolic and diastolic functions in the patient group during whole follow-up. Conclusion: Although, normal cardiac functions were probably due to young age, steroid therapy may have some protective effects on cardiac functions. The steroid treatment seems to be preventive for development of ventricular dysfunction but the long term studies necessitate at this topic.

\section{6}

Extracorporeal Membrane Oxygenation for children with acute fulminant myocarditis in Singapore

Anna Mani, K K Women and Children's Hospital, Singapore Sriram Shankar, KK Women and Children's Hospital, Singapore Keng Yean Wong, K K Women and Children's Hospital, Singapore 
Objective: The purpose of this study was to evaluate outcome of patients requiring extracorporeal membrane oxygenation (ECMO) for acute myocarditis in our institution.

Methods: The hospital records of all patients requiring ECMO for myocarditis in KK Women and Children's Hospital were reviewed.

Results: Eight patients with myocarditis were put on ECMO for cardiopulmonary support from January 2002 to October 2008. Patient's age ranged from 3 to 12 years (median 6 years). Duration on ECMO ranged from 89-502 hours. 5 patients were discharged well (62.5\%). One of these patients (Patient 4) developed dilated cardiomyopathy and died after 18 months post- ECMO survival.

Patient 7 had severe mitral regurgitation after weaning off ECMO and underwent mitral valve repair successfully. Patient 8 had no cardiac contractility for the initial 2 weeks but regained good cardiac function after 21 days of support, was weaned off ECMO and discharged home well. Complications include left hemiparesis in Patient 7 and left hemothorax in Patient 2 and Patient 8. Two patients who collapsed and required cardiac massage prior to ECMO did not survive.

Conclusion: Recovery of cardiac function and good outcome can be anticipated in patients with acute myocarditis requiring ECMO. Initiation of ECMO before cardiovascular collapse increases likelihood of survival.

\section{7}

Utility of Tissue Doppler in Diagnosis of Myocardial Compromise in Duchenne Disease

Juan P Miranda, Hospital Roberto Del Rio, Chile

Patricia Alvarez, Hospital Roberto Del Rio, Chile

Valeria Acevedo, Hospital Roberto Del Rio, Chile

Karin Kleinsteuber, Hospital Roberto Del Rio, Chile

Maria Avaria, Hospital Roberto Del Rio, Chile

Rodrigo Neghme, Congenital Heart Institute, Orlando-fl, Chile

Daniel Aguirre, Hospital Roberto Del Rio, Chile

Duchenne Muscular Dystrophy (DMD) is caused by the absence of dystrophin in cardiac and skeletal muscle. Myocardial involvement has been traditionally diagnosed by conventional echocardiography. Our objective was to evaluate the role of Tissue Doppler (TD) in the preclinical diagnosis of myocardial involvement in DMD.

We report 15 patients, mean age 11 years (4-17 years) with DMD. All patients were studied with echocardiography with TD. Eight children had decreased functional capacity, 5 palpitations, 5 weight loss, and 4 presented with dyspnea. EKG was abnormal in $14 / 15$ (93\%). Holter showed ventricular extrasystoles in 3 patients. Conventional echocardiography showed decreased LV function in 4/15 patients (27\%). LV enlargement was seen in 3 patients. LV shortening fraction was decreased in 4 patients, who had a mean SF of $23 \%$ (17-28\%). Conventional Doppler showed an E/A relation $<1$ and enlargement of the left atrium in 1 patient, trivial mitral insufficiency in 3 and mild pulmonary hypertension in 2 patients. $11 / 15$ patients $(73 \%)$ had abnormal TD analysis in the lateral portion of the mitral ring: $\mathrm{e}^{\prime} 7$ to $21 \mathrm{~cm} / \mathrm{s}$ (8 patients under the normal limit for age); a' 4 to $13 \mathrm{~cm} / \mathrm{s}$ ( 3 patients over the normal range) and s' 5 to $13 \mathrm{~cm} / \mathrm{s}$ (4 patients under the normal range).

Conclusion: TD evaluation is more sensitive (73 vs. $27 \%$, $\mathrm{p}<0.05)$ than conventional echocardiography in detecting early ventricular dysfunction in patients with DMD allowing early therapeutic intervention. TD should be part of the routine evaluation in DMD patients.
8018

QT Dispersion Predicts Cardiac Events after Hematopoietic Stem Cell Transplantation in Children Noriko Motoki, Department of Pediatrics, Shinshu university of medicine, Japan

Takashi Shimizu, Department of Pediatrics, Shinshu university of medicine, Japan

Yukako Itoh, Department of Pediatrics, Shinshu university of medicine, Japan

Youhei Akazawa, Department of Pediatrics, Shinshu university of medicine, Japan

Kazuo Sakashita, Department of Pediatrics, Shinshu university of medicine, Japan

Kenichi Koike, Department of Pediatrics, Shinshu university of medicine, Japan

Background: In hematopoietic stem cell transplantation (SCT) of leukemia, the heart failure is one of the serious complications, but has difficulty with a prediction. QT dispersion (QTD) has been studied as a predictive factor of heart failure and fatal arrhythmia in adults. The purpose of this study is to examine whether QTD predicts cardiac events, such as heart failure and arrhythmia, after SCT in children.

Methods: Fourteen patients (mean age: 6.6 years; 6 men; 8 acute lymphoblastic leukemia, 6 acute myeloblastic leukemia) who received SCT were studied retrospectively. Twelve lead electrocardiograms and echocardiography were performed for all patients before conditioning therapy. QTD, corrected QT dispersion $(\mathrm{QTCD})$, echocardiographic functional parameters and cumulative anthracycrine dose (ADR) were evaluated. Patients were divided into two groups, patients with cardiac event group $(\mathrm{CE}+)$ and without cardiac event group (CE-) after SCT. We compared these values between 2 groups.

Results: All patients were treated with cyclophosphamide and performed total body irradiation in the conditioning therapy. Six patients experienced cardiac events (arrhythmia in 2, heart failure in 4). There were significant differences as QTD (CE+ vs. CE-; 48.3 vs. $27.8 \mathrm{~ms}, \mathrm{p}=0.003)$ QTcD (60.7 vs. $37.5 \mathrm{~ms}, \mathrm{p}=0.003)$ and ADR (297.6 vs. $166.8 \mathrm{mg} / \mathrm{m} 2, \mathrm{p}=0.002)$. Echocardiographic parameters did not show significant difference between 2 groups. Conclusion: QTD could be used as a noninvasive predictor of cardiac events after SCT in children. Despite the absence of echocardiographic abnormalities, patients with a very high QTD were needed care of cardiac events associated with SCT.

8019

Endomyocardial biopsy in children on cardiac extracorporeal support

Nitha Naqvi, Great Ormond Street Hospital For Sick Children, London, United Kingdom

Sachin Khambadkone, Great Ormond Street Hospital For Sick Children, London, United Kingdom

Phillip Rees, Great Ormond Street Hospital For Sick Children, London, United Kingdom

Michael Burch, Great Ormond Street Hospital For Sick Children, London, United Kingdom

Michael Ashworth, Great Ormond Street Hospital For Sick Children, London, United Kingdom

Robert Yates, Great Ormond Street Hospital For Sick Children, London, United Kingdom

Kate Brown, Great Ormond Street Hospital For Sick Children, London, United Kingdom

Graham Derrick, Great Ormond Street Hospital For Sick Children, London, United Kingdom 
Objectives: To investigate whether endomyocardial biopsy in children on ECMO, performed at the time of decompressing balloon atrial septostomy (BAS), is safe and useful for management and prognosis.

Methods: Transluminal ventricular biopsy was performed in a cohort of children on cardiac ECMO support at the time of BAS. Echocardiographically guided biopsies were taken, using modified biopsy forceps and sheath. The left ventricle (LV) was sampled by trans-septal approach. Right ventricular (RV) biopsies were taken when a safe approach to LV sampling was not technically possible.

Results: Median age was 9.5 years (range 5 months- 14.4 yrs) and median weight $22 \mathrm{~kg}$ (range $7-52 \mathrm{~kg}$ ). Seventeen patients underwent attempted biopsy (12 LV, 2 RV, 3 unsuccessful) with minimal addition to procedure time (10 minutes) and no biopsy related complications.

Histology revealed recoverable disease in 7 patients $(5$ acute myocarditis, 1 acute rejection [previous heart transplant], 1 antiphospholipid syndrome). Probably unrecoverable disease was seen in 4 patients (cardiomyopathy). Non-specific findings were seen in 3 patients, of which myocarditis was subsequently proved in surgical biopsy (1 patient) and post-mortem biopsy (1 patient).

Results of biopsy helped stratify management strategy into bridge to recovery or bridge to transplantation/withdrawal.

Conclusions: Endomyocardial biopsy is feasible and safe in children on ECMO cardiac support, with minimal addition to theatre time and resources, and can provide useful information when histological changes are diagnostic. However, care must be taken with the interpretation of non-diagnostic results, since misleading false negatives may be due to patchy myocardial involvement.

\section{0}

Important role of SHP2 mutants in morphological changes in cardiaomyocyte hypertrophy

Jun Narita, Osaka university oggraduate school of medicine, Japan Shigetoyo Kogaki, Osaka university oggraduate school of medicine, Japan

Toshiki Utikawa, Osaka university oggraduate school of medicine, Japan Hiroaki Ichimori, Osaka university oggraduate school of medicine, Japan Hidekazu Ishida, Osaka university oggraduate school of medicine, Japan Yoko Okada, Osaka university oggraduate school of medicine, Japan Keiichi Ozono, Osaka university oggraduate school of medicine, Japan

Background: Hypertrophic cardiomyopathy (HCM) is the frequent cardiac phenotype in Noonan syndrome (NS) and LEOPARD syndrome (LS). We have recently demonstrated a possible correlation between phenotype of cardiac hypertrophy and phosphatase activities of SHP2, a product of the PTPN11 gene in NS and LS. The aim of the current study is to investigate whether SHP2 mutants could induce different types of cardiac myocyte hypertrophy in vitro.

Methods: Patients $(\mathrm{n}=8)$ with HCM in NS or LS were screened for mutations in the PTPN11 gene. The six mutations were isolated and cloned into a pAd DEST/V5 vector. Neonatal rat cardiomyocyte were isolated and infected. We performed the quantitative morphometry (surface area) in each cardiomyocyte and investigate the signal transduction pathways responsible for the cardiac hypertrophy.

Results: WT(wild type)-SHP2 expressed cardiomyocyte showed significant increase in length and surface area compared to control (LacZ-expressed, p < 0.01). LS mutant (Y279C, Q510E) caused more remarkable increase in surface area of cardiomyocyte than WT $(\mathrm{p}<0.01)$. Among several signaling, only pStat3 after
LIF stimulation could be activated in Y279C and Q510E, whereas significantly not activated in WT and D61N.

Summary: All SHP2 mutants induced hypertrophic changes in cardiomyocyte in vitro. LS mutants with severe HOCM induced more significant changes in surface area of cardiomyocyte. Low phosphatase activity of SHP2 in cardiomyocyte may affect the severity of cell hypertrophy via unknown pathway other than LIF-Stat pathway. We need further study to elucidate a key role of SHP2 in cardiomyocytes.

\section{1}

Long-term mechanical circulatory support in children Evgenij Potapov, Deutsches Herzzentrum Berlin, Germany Christof Stamm, Deutsches Herzzentrum Berlin, Germany Yuguo Weng, Deutsches Herzzentrum Berlin, Germany Boris Nasseri, Deutsches Herzzentrum Berlin, Germany Michael Hübler, Deutsches Herzzentrum Berlin, Germany Oliver Miera, Deutsches Herzzentrum Berlin, Germany Felix Berger, Deutsches Herzzentrum Berlin, Germany Roland Hetzer, Deutsches Herzzentrum Berlin, Germany

Background: Ventricular assist devices (VAD) are increasingly being used in children with end-stage heart failure requiring or medium- and long-term support. We analyzed the development of our clinical practice with pediatric VAD over 19 years.

Methods: Since 1990, 102 VAD have been implanted in 99 patients ( $<18$ yrs) at our institution (Berlin Heart Excor $\mathrm{n}=100$, $\mathrm{BH}$ Incor $\mathrm{n}=1$ and Novacor $\mathrm{n}=1$ ). This cohort was divided in two groups: Period I - devices implanted between 1990 and $2002(\mathrm{n}=46)$ and Period II - devices implanted since 2002 $(\mathrm{n}=56)$, and the outcome of both groups was compared.

Results: There were no significant differences in the preoperative patient data except for time of support (Period I, median 10 days (range $0-591)$ vs. Period II, 37 (1-420 days), $p<0.001)$. In period I more patients were supported with a biventricular VAD, $64 \%$, vs. $26 \%, \mathrm{p}<0.001)$. In the later period more children were extubated while on the VAD ( $38 \%$ vs. $62 \%, p=0.02)$. Hospital discharge following either weaning from the system or heart transplantation was achieved in $49 \%$ during period I and in $70 \%$ during period II $(\mathrm{p}=0.035)$, and an increasing proportion of those was $<1$ yr old ( $8 \%$ vs. $44 \%, p=0.088$ ). Moreover, the discharge rate of patients with postcardiotomy heart failure improved (17\% vs. $80 \% \mathrm{p}=0.028)$.

Conclusion: Earlier implantation, device modifications, and improvements in the anticoagulation monitoring regime have led to a significant increase in the survival and discharge rate of children on VADs, especially those under 1 year of age.

\section{2}

A Case of Wolff-Parkinson-White Syndrome Associated with Atypical Dilated Cardiomyopathy

Masahiro Ohtsuki, Juntendo University School of Medicine, Japan Takeshi Furukawa, Juntendo University School of Medicine, Japan Hideo Fukunaga, Juntendo University School of Medicine, Japan Tomoyuki Sato, Juntendo University School of Medicine, Japan Katsumi Akimoto, Juntendo University School of Medicine, Japan Ken Takahashi, Juntendo University School of Medicine, Japan Masahiko Kishiro, Juntendo University School of Medicine, Japan Toshiaki Shimizu, Juntendo University School of Medicine

Wolff-Parkinson-White (WPW) syndrome is a common preexcitation syndrome. In this paper, we report a case of a 6 yearold boy with WPW syndrome who developed atypical dilated 
cardiomyopathy. He presented to the hospital with a cardiac murmur. Electrocardiogram revealed type B WPW syndrome, and ultrasound showed mitral regurgitation, thickening of the chorda tendinea, ventricular aneurysm with partial thinning of the interventricular septum and paradoxic motion, reduced ejection fraction, and cardiac enlargement. In addition, delayed enhanced magnetic resonance imaging detected cardiomyopathy of the intraventricular septum in the same area, and it is currently under observation as an atypical dilated cardiomyopathy. Reports of cardiac aneurysm with dilated cardiomyopathy in WPW syndrome are rare. Furthermore, persistent elevation of antistreptolysin $\mathrm{O}$ titer and thickening of the chorda tendinea in the present case can suggest possible association with rheumatic fever. It is unclear whether cardiac aneurysm developed by chance in WPW syndrome causing its progression to cardiac enlargement or whether it has been complicated by other diseases such as rheumatic fever, but this may be a new category of cardiomyopathy. Continued follow-up and accumulation of cases are necessary.

\section{3}

Impact of Highly Active Anti-Retroviral Therapy on Paediatric Human Immuno-deficiency Virus Associated Left Ventricular Dysfunction within the Johannesburg Teaching Hospital Complex

Lungile Pepeta, Nelson Mandela Academic Hospital, Walter Sisulu University, South Africa

Antoinette M Cilliers, University of Witwatersrand, South Africa

Willy Hendson, University of Witwatersrand, South Africa

Deliwe Ngwezi, University of Witwatersrand, South Africa

Firoza Motara, University of Witwatersrand, South Africa

Paul E Adams, University of Witwatersrand, South Africa

Hopewell N Ntsinjana, University of Witwatersrand, South Africa

Objective: To analyze the outcome of paediatric patients with left ventricular (LV) dysfunction placed on Highly Active AntiRetroviral Therapy (HAART).

Method: Retrospective review of records of HIV positive children with LV dysfunction managed at Johannesburg Teaching Hospital Complex. Baseline characteristics were documented. The following variables: fractional shortening (FS), CD4 percentage, viral load and nutritional status were compared before and after commencement HAART.

Results: Thirty four patients with LV dysfunction had their records reviewed.

Eighteen patients with LV dysfunction (group 1) received HAART, and 16 (group 2) were HAART naive (pre HAART era). The mean age of group 1 patients at initial visit was 75 months, with a M:F ratio of 9:9. Seventeen patients showed improved LV function, from a mean FS of $18 \%$ to $32.8 \%$, ( $\mathrm{p}$-value $<0.0001)$. There was an increase in CD4 percentage from a median of $12 \%$ to $30.5 \%$ ( $p$ value $<0.0001$ ). There was viral load suppression from an initial median viral copies of 24900 to $<25$ copies ( $p$ value $<0.0001$ ). There was weight gain following HAART from a median z-score of -1.70 pre-HAART to -1.32 during HAART ( $\mathrm{p}$ value $=0.0083$ ). There was no statistically significant difference between group 1 and group 2 regarding FS and nutritional $z$-scores at the initial visit.

Conclusion: The findings are in keeping with other reports that have shown improvement in left ventricular function in patients with HIV associated cardiomyopathy treated with HAART. The recovery of myocardial function is associated with improvement of their initially poor immunological and nutritional status.

\section{4}

Use of cardiac markers as monitoring of doxorubixin induced cardiotoxicity in children with cancer

Yupada Pongprot, Department of Pediatrics, Chiang Mai University, Thailand, 50200, Thailand

Rekwan Sittiwangkul, Department of Pediatrics, Chiang Mai

University, Thailand, 50200, Thailand

Pattra Thanarattanakorn, Department of Pediatrics, Chiang Mai

University, Thailand, 50200, Thailand

Pimlak Charoenkwan, Department of Pediatrics, Chiang Mai

University, Thailand, 50200, Thailand

Suchaya Silvilairat, Department of Pediatrics, Chiang Mai University, Thailand, 50200, Thailand

Background: Anthracyclines chemotherapy are associated with increased risk of arrhymias, pericarditis and ventricular dysfunction. Echocardiography has been used as a screening test in children with cancer after anthracycline therapy.

Objective: To evaluate NT-proBNP, cardiac troponin $\mathrm{T}$ and CKMB comparing with echocardiographically cardiac function in childhood cancer treated with doxorubicin.

Method: We performed prospective study of systolic, diastolic function and tissue Doppler imaging by echocardiogram in children with cancer who received accumulative dose of doxorubicin between $50-350 \mathrm{mg} / \mathrm{m} 2$. Blood level for NTproBNP, cardiac troponin T and CK-MB were analyzed within 6 hours of cardiac study.

Result: Of 22 patients 8 (36\%) had abnormally high mean NTproBNP level of $389 \pm 71 \mathrm{pg} / \mathrm{ml}$ compared with $74.91 \pm 60 \mathrm{pg} /$ $\mathrm{ml}$ in normal level group. As the drug dose increased, NTproBNP level increased correlated significantly with decreased ejection fraction (EF) $(\mathrm{r}=-0.36, \mathrm{P}=0.05)$ and decreased deceleration time (DT) $(\mathrm{r}=-0.37, \mathrm{P}<0.05)$. In this study, tissue Doppler imaging could not early detect systolic and diastolic dysfunction. CKMB and cardiac troponin $\mathrm{T}$ were abnormal in only 3 and 2 patients respectively.

Conclusion: NT-proBNP is a useful cardiac marker for monitoring of anthracycline cardiotoxicity due to its good correlation with systolic and diastolic function. CKMB and cardiac troponin $\mathrm{T}$ were suggested to be insensitive.

\section{5}

Cardiac status in hypocalcemic infants

Shakuntala S Prabhu, B.J. Wadia Hospital For Children, India Sumitra Venkatesh, India

Nandkumar Kene, India

Aim: To study the cardiac manifestations of hypocalcemia in infants and note the effect of treatment.

Method: 30 infants (1 month to 1 year) with hypocalcemia diagnosed by biochemistry along with 15 age and sex-matched healthy controls were included. Clinical manifestations, X-ray chest, ECG and Echocardiographic data were assessed.

Results: The presenting symptoms were convulsions in 23 infants, failure to thrive in 11, respiratory distress in 3 (of which 2 had congestive heart failure) and no symptoms in 2. Cardiothoracic ratio was abnormal in 8 and 11 had ECG abnormalities (Sinus tachycardia in 2, prolonged PR interval in 2, abnormal QRS axis for age in 2 and T- wave abnormalities in 4). The Echocardiographic/Color doppler evaluation revealed abnormal findings in $13 / 30$ patients ( $\mathrm{p}$ value $<0.05$ ). Mild to moderate tricuspid regurgitation in 4 , hypercontractile heart in 7 , left ventricular dilatation in 3 and LV dysfunction in 2 . The 2 patients with LV dysfunction needed IV calcium infusion along with other 
inotropes and showed marked improvement in ejection fraction and fractional shortening on therapy. The other patients were treated with intravenous or oral calcium and Vitamin D supplementation. Normalization of cardiac status was noted after one month in all with said abnormalities.

Conclusion: Cardiac manifestations are sub-clinical in hypocalcemic infants. X-ray \& ECG changes may be seen but 2 DEchocardiographic and colour Doppler evaluation is necessary to note the cardiac status (especially Left ventricular function). Hypocalcemia must be ruled out in all infants with cardiomyopathy.

\section{7}

Dobutamine stress echocardiography and tissue/flow myocardial performence indexes for early detection of the anthracycline-induced cardiotoxicity in asymptomatic children

Sedef Tunaoglu, Gazi University, Medical Faculty, Department of Pediatric Cardiology, Ankara, Turkey

Ayse Yildirim, Gazi University, Medical Faculty, Department of

Pediatric Cardiology, Ankara, Turkey

Güçlü Pinarli, Gazi University, Medical Faculty, Department of

Pediatric Oncology, Ankara, Turkey

Rana Olguntürk, Gazi University, Medical Faculty, Department of

Cardiology, Ankara, Turkey

Deniz Oguz, Gazi University, Medical Faculty, Department of

Pediatric Cardiology, Ankara, Turkey

Serdar Kula, Gazi University, Medical Faculty, Department of Pediatric Cardiology, Ankara, Turkey

Aynur Oguz, Gazi University, Medical Faculty, Department of

Pediatric Oncology, Ankara, Turkey

Ceyla Karadeniz, Gazi University, Medical Faculty, Department of

Pediatric Oncology, Ankara, Turkey

Objective: Assessment of systolic and diastolic functions and tissue Doppler indices by stress echocardiography for the early determination of late anthracycline-induced cardiotoxicity in asymptomatic children.

Method: Dobutamine stress echocardiography was performed on 20 anthracycline-treated asymptomatic patients $(14 \mathrm{M}, 6 \mathrm{~F}$; mean age $18.4 \pm 3.2$ years) and age-matched healthy control group (14 F, 4 E; mean age: $19.2 \pm 4.0$ years).

Echocardiographic examination was performed : at rest, 5, 10, 15 and $20 \mu \mathrm{g} / \mathrm{kg} / \mathrm{min}$ dobutamine infusions with 5 minutes interval. Tissue Doppler measurements were recorded at annular and mid segment of right ventricle, interventricular septum and left ventricle. Results: At rest patients' isovolemic contraction and isovolemic relaxation times were longer than controls; by dobutamine infusions patients' percentage of left ventricular posterior wall thickeness values were decreased and left ventricular systolic wall stress, LVDd, LVSd, mitral accelaration and deceleration time were increased, Although ejection fractions and fractional shortening measurements were in normal limits during dobutamine infusions; right ventricule, interventricular septum and left ventricular tissue MPI and LV flow MPI values were higher than controls during test. Patients LV tissue MPI was increased much more than LV flow MPI by stress echocardiography Cumulative anthracycline doses had positive correlation between LV tissue MPI and showed negative correlation with percentage of LV posterior wall thickening by stress echocardiography.

Conclusion: Tissue myocardial performance indexes are more sensitive measurements for the determination of left ventricular disfunction and stress echocardiography is sensitive technique for find out of the late cardiac toxicity in anthracycline-treated asymptomatic patients.
8029

Are Cardiac Biomarkers Useful in Assessing Cardiac Structure and Function in Children Exposed to the Human Immunodeficiency Virus In Utero?

James D Wilkinson, University of Miami, United States

William T Shearer, Baylor College of Medicine, United States

Louis I Bezold, University of Kentucky, United States

Bruce Thompson, Clinical Trials and Survey Corporation, United States Irene Cheng, Clinical Trials and Survey Corporation, United States Kenneth C Rich, Univeristy of Illinois- Chicago, United States Steven E Lipshultz, University of Miami, United States

Background: Increased left ventricular (LV) mass and decreased fractional shortening are associated with mortality in Human Immunodeficiency Virus (HIV) seropositive infants and children. We hypothesized that cardiac biomarkers would be associated with abnormal cardiac structure and function in HIV-seronegative children born to HIV-seropositive mothers.

Methods: We analyzed serial paired observations of cardiac biomarkers and echocardiograms in 273 HIV-seronegative children (ages $0-58$ months), exposed to HIV in utero. were log-transformed. Linear associations between 425 serial paired biomarker values $[\mathrm{N}$-terminal probrain natriuretic peptide (NTproBNP), high sensitivity C-reactive protein (HsCRP) and cardiac troponin $\mathrm{T}(\mathrm{cTnT})$ levels] and $\mathrm{ECHO}$ parameters z-scores were analyzed using generalized estimating equations (GEE) and were also dichotomized and the Cochran-MantelHaenszel test was used to assess associations between them.

Results: In the linear analyses, elevated NT-proBNP levels were associated with increased LV mass $(p=0.053)$ and there was a trend toward a significant association between elevated $c \operatorname{TnT}$ levels at birth and decreased LV fractional shortening $(p=0.068)$. In the categorical analyses, elevated cTnT levels $(>0.01 \mathrm{ng} / \mathrm{mL})$ were significantly associated with a cardiac intraventricular septal wall thickness z-score $>2(\mathrm{OR}=9.16$; 95\% CI 1.24-67.41; $\mathrm{p}=0.007)$. Conclusions: Our findings indicate significant or borderline significant associations between abnormal cardiac biomarker levels (NT-proBNP and cTnT) and increased LV mass and cardiac septal wall thickness and decreased LV function (fractional shortening). Cardiac biomarkers may be a resource-efficient modality in monitoring the cardiac status of children exposed to HIV in utero and also identify those children who require a more complete cardiac evaluation.

\section{0}

Antiretroviral Therapy and HIV-Associated Left Ventricular Diastolic Dysfunction in Uninfected but ART-Exposed Infants Born to HIV-Infected Women: The Prospective NHLBI CHAART-1 Study

Sharon E O'Brien, Boston Medical Center, United States

Benjamin W Eidem, Mayo Clinic, United States

Steven C Colan, Harvard University, United States

William T Shearer, Baylor College of Medicine, United States

James D Wilkinson, University of Miami, United States

Bruce Thompson, Clinical Trials and Survey Corporation, United States Steven E Lipshultz, University of Miami, United States

Background: Antiretroviral therapy (ART) has greatly reduced vertical transmission of HIV. However, the long-term effects on left ventricular diastolic function in children after in utero exposure to ART and HIV have not been evaluated.

Methods: We compared Doppler indices of myocardial function from echocardiograms taken at birth through 48 months of age from 148 ART-exposed children enrolled in the Cardiac Status of 
Highly Active Antiretroviral Therapy (CHAART-1) study to those from 130 controls. Differences in diastolic measures between the two cohorts were analyzed using Generalized Estimating Equations models.

Results: Within the CHAART-1 cohort mitral valve (MV) early and late atrial velocities were below normal at all ages (p?0.001), MV $\mathrm{E}: \mathrm{A}$ was above normal at 1,12 and 24 -months of age $(\mathrm{p}=0.01$, $\mathrm{p}<0.0001, \mathrm{p}=0.0008)$ indicative of impaired compliance. Peak myocardial early diastolic velocity was below normal at all ages $(p=0.01)$ consistent with impaired relaxation. The systolic ejection time was below normal at 1 month of age $(p=0.001)$. The myocardial performance index was above normal at all ages $(\mathrm{p}<0.0001)$ indicative of impaired systolic and diastolic function. Conclusion: HIV-uninfected children with fetal exposure to ART and HIV have statistically significant echocardiographic evidence of impaired left ventricular function during the first four years of life. We speculate that fetal ART and HIV exposure may impact myocardial growth and subsequently affect myocardial contractility and relaxation. This effect may be modulated by age and gender. Follow-up of left ventricular function in this group appears warranted.

\section{1}

Study on longitudinal myocardial function of left ventricle in children with dilated cardiomyopathy using velocity vector imaging

Shu-wen Zhong, Department of Pediatric Cardiology, Shanghai Children's Medical Center, Shanghai Jiaotong University, Shanghai, China Yu-qi Zhang, Department of Pediatric Cardiology, Shanghai Children's Medical Center, Shanghai Jiaotong University, Shanghai, China Kun Sun, Department of Pediatric Cardiology, Shanghai Children's Medical Center, China

Li-jun Chen, Department of Pediatric Cardiology, Shanghai Children's Medical Center, Shanghai Jiaotong University, Shanghai, China

Objective: To evaluate the accuracy of velocity vector imaging (VVI) in assessing the longitudinal myocardial function of left ventricle in children with dilated cardiomyopathy (DCM).

Methods: The peak systolic velocity (VS), systolic peak strain $(\varepsilon)$, systolic peak strain rate (SRS) and the time to peak systolic longitudinal velocity (TV) were measured in 25 children with DCM and 25 normal children in apical four chamber view using VVI. Left ventricular $\mathrm{dp} / \mathrm{dtmax}$ ( $\mathrm{LV} \mathrm{dp} / \mathrm{dtmax}$ ) were estimated using the spectrum of mitral regurgitant by continuous doppler. Left ventricular end-diastolic dimension (LVDD) and left ventricular end-systolic dimension (LVDS) were measured in short axis view of left ventricular with $M$ mode echocardiography and ejection fraction (EF) was calculated. The correlation among different values was analyzed with linear regression.

Results: The EF of the normal children was $65.8 \pm 4.2 \%$ and the SRS of all segments was $-1.26 \sim-1.43 / \mathrm{s}$; The EF of DCM children was $41.2 \pm 14.5 \%$ and the SRS of all segments was $-0.31 \sim-0.51 / \mathrm{s}$; the absolute values of SRS in each segment in $\mathrm{dcm}$ group were lower than those of the normal children $(\mathrm{P}<0.05)$; the $\mathrm{LV} \mathrm{dp} / \mathrm{dtmax}$ of DCM patients was $521.8 \pm 283.4 \mathrm{mmHg} / \mathrm{s}$, SRS of DCM coerelated well with $\mathrm{LV} \mathrm{dp} / \mathrm{dtmax}(\mathrm{P}<0.05, \mathrm{r}=0.86)$.

Conclusions: The longitudinal myocardial function of left ventricle in children with dilated cardiomyopathy could be accurately estimated by VVI.

8032

Assessment of longitudinal myocardial function in children with dilated cardiomypathy by velocity vector imaging Shu-wen Zhong, Department of Pediatric Cardiology, Shanghai Children's Medical Center, Shanghai Jiaotong University, Shanghai, China
Yu-qi Zhang, Department of Pediatric Cardiology, Shanghai Children's Medical Center, Shanghai Jiaotong University, Shanghai, China Kun Sun, Department of Pediatric Cardiology, Shanghai Children's Medical Center, China

Li-jun Chen, Department of Pediatric Cardiology, Shanghai Children's Medical Center, Shanghai Jiaotong University, Shanghai, China

Objective: To assess the left ventricular longitudinal systolic function in children with dilated cardiomyopathy by syno Velocity Vector Imaging.

\begin{tabular}{|c|c|c|c|c|c|c|c|c|}
\hline $\begin{array}{l}\text { Seg- } \\
\text { mental } \\
\text { EF }(\%)\end{array}$ & $\begin{array}{c}\text { Base } \\
\text { of } \\
\text { sepum }\end{array}$ & $\begin{array}{l}\text { Middle } \\
\text { of } \\
\text { septum }\end{array}$ & $\begin{array}{l}\text { Apex of } \\
\text { septum }\end{array}$ & $\begin{array}{c}\text { Base of } \\
\text { LV }\end{array}$ & $\begin{array}{l}\text { Middle of } \\
\text { LV }\end{array}$ & $\begin{array}{c}\text { Apex of } \\
\text { LV }\end{array}$ & $\begin{array}{c}\mathrm{V} \max \\
(\mathrm{ml})\end{array}$ & $\begin{array}{c}V_{\min } \\
(\mathrm{ml})\end{array}$ \\
\hline DCM & $\begin{array}{r}26.4 \pm \\
10.8\end{array}$ & $\begin{array}{r}32.4 \pm \\
12.1\end{array}$ & $\begin{array}{r}39.9 \pm \\
12.7\end{array}$ & $\begin{array}{r}27.3 \pm \\
13.0\end{array}$ & $\begin{array}{r}26.9 \pm \\
12.6\end{array}$ & $\begin{array}{r}26.9 \pm \\
11.8\end{array}$ & $\begin{array}{c}107.4 \pm \\
80.0\end{array}$ & $\begin{array}{r}66.0 \pm \\
26.6\end{array}$ \\
\hline $\begin{array}{l}\text { Nor- } \\
\text { mal }\end{array}$ & $\begin{array}{c}47.7 \pm \\
7.3\end{array}$ & $\begin{array}{r}49.3 \pm \\
6.3\end{array}$ & $\begin{array}{c}46.0 \pm \\
8.5\end{array}$ & $\begin{array}{c}43.6 \pm \\
6.2\end{array}$ & $\begin{array}{c}47.4 \pm \\
7.8\end{array}$ & $\begin{array}{r}48.4 \pm \\
11.5\end{array}$ & $\begin{array}{r}81.9 \pm \\
67.8\end{array}$ & $\begin{array}{r}38.3 \pm \\
19.8\end{array}$ \\
\hline
\end{tabular}

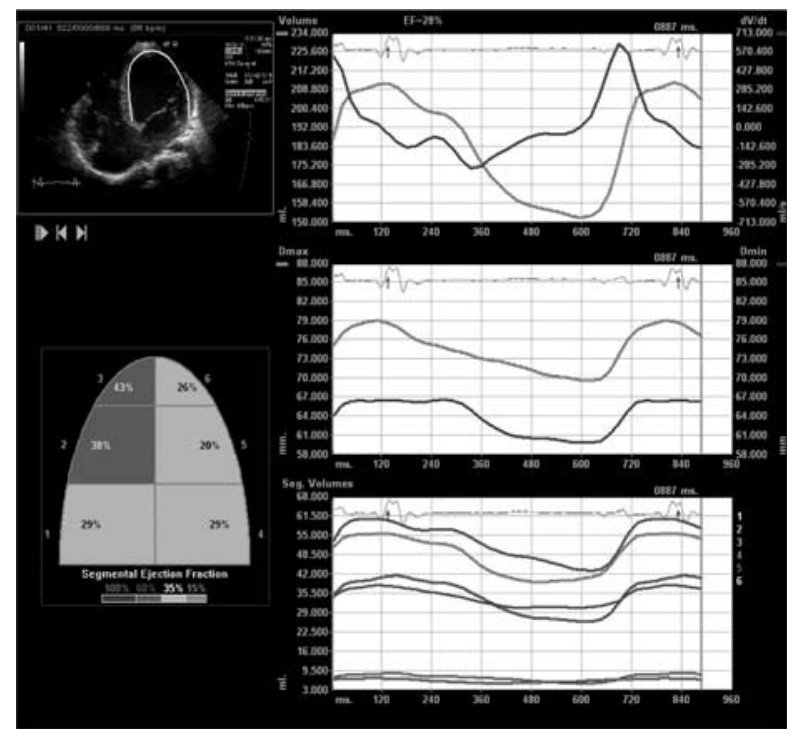

Method: LV images (apical four-chamber view, high frame capture) frome 23 DCM children and 20 normal children were stored and analyzed. Segmental ejection fractions were determined at basal, middle and apical segments of LV free wall and ventricular septum by velocity vector imaging. LV volume was also automatically obtained throughout the cardiac cycle. These parameters of the two groups were compared.

Result: All the parameters of the DCM were significantly lower than the those of the normal group $(\mathrm{P}<0.001)$.

Conclusion: VVI provide a useful and noninvasive method for evaluating regional cardiac function.

8033

Post surgical recoartation in patients with age less than 1 year

Jose M Velasco, Pediatric Heart Institute, University Hospital 12 de Octubre, Madrid, Spain

Stefano M Marianeschi, Pediatric Cardiac Surgery, Niguarda Hospital, Milan, Italy

Lorenzo Boni, Pediatric Heart Institute, University Hospital 12 de Octubre, Madrid, Spain

Enrique Garcia, Pediatric Heart Institute, University Hospital 12 de Octubre, Madrid, Spain 
Alberto Mendoza, Pediatric Heart Institute, University Hospital 12 de Octubre, Madrid, Spain

Maria Dolores Herrera, Spain

Lorenzo Galletti, Pediatric Cardiac Surgery, Ospedali Riuniti di

Bergamo, Bergamo, Italy

Juan V Comas, Pediatric Heart Institute, University Hospital 12 de

Octubre, Madrid, Spain

Introduction: Recurrent stenosis after repair of aortic coartation is not an uncommon clinical occurrence. This study values the results and the follow-up of post surgical recoartation treatment, either with balloon valvuloplasty and surgical aortic arch reconstruction, in patients initially operated under 1 year of age. Materials and Methods: Between February 1998 and December 2008 we followed 173 infants previously operated, under one year of age, of aortic coartacion repair (simple/complex). The recoartation rate was $24.2 \%$ (42 patients); as first treatment, 37 patients underwent balloon angioplasty, which was initially effective in 32 (group A) and 5 patients directly underwent reparative surgery (group B), including 5 additional patients, coming from the initial angioplasty group. Echocardiographic, hemodynamic, angiographic, MRN data were review.

Results: A successful balloon angioplasty was achieved in 32/37 patients $(86.4 \% ; 6$ of these 37 patients underwent subsequent surgery. In group A (effective angioplasty), peak gradient decreased from $38 \pm 16$ to $9 \pm 6 \mathrm{mmHg}(\mathrm{p}<0.001)$. In group B (surgical aortoplasty), peak gradient decreased from $56 \pm 20$ to $15 \pm 17 \mathrm{mmHg}(\mathrm{p}<0.001)$. During 10 years of follow-up, the restenosis rate was $1 / 10(10 \%)$ in group $\mathrm{B}$ and $6 / 32(18.7 \%)$ in group A; two of these 6 patients underwent a new successful angioplasty and four underwent surgical repair.

Conclusion: Balloon angioplasty can be safely performed in infants with good term results. The risk of restenosis is low, and can be successfully managed with repeat angioplasty. The need for further surgical intervention in those with transverse arch hypoplasia remains high.

\section{4}

Five Year Experience of Delivering Prenatally-Diagnosed Infants with Hypoplastic Left Heart Syndrome with Intact Atrial Septum in the Cardiac Catheterization Suite

Shyam K Sathanandam, Hope Children Hospital, Oak Lawn, IL, United States

Andrew Van Bergen, Hope Children Hospital, Oak Lawn, IL, United States

David A Roberson, Hope Children Hospital, Oak Lawn, IL, United States

Dhaval Patel, Hope Children Hospital, Oak Lawn, IL, United States

Tarek S Husayni, Hope Children Hospital, Oak Lawn, IL, United States

Bettina F Cuneo, Hope Children Hospital, Oak Lawn, IL, United States

Michel N Ilbawi, Hope Children Hospital, Oak Lawn, IL, United States

Alexander J Javois, Hope Children Hospital, Oak Lawn, IL, United States

Objective: The objective was to review our five year experience of utilizing the cardiac catheterization suite for cesarean section (C-section) delivery and immediate catheter intervention to create adequate interatrial communication (IAC) for infants with prenatal diagnosis of hypoplastic left heart syndrome (HLHS) with intact atrial septum (IAS) or severely restrictive IAC (SR-IAC).
Methods: The period of study was January 2003-December 2008. Group 1 included 117 infants with HLHS with adequate IAC. Group 2 included 7 infants with prenatal diagnosis of HLHS with IAS or SR-IAC delivered in the catheterization suite with immediate transcatheter intervention. Group 3 included 7 undiagnosed infants with SR-IAC who either underwent delayed intervention or no intervention before stage 1 palliation. Results: Incidence of HLHS with IAS or SR-IAC during the study period was 14 of $131(10.7 \%)$. Overall survival to stage 2 was $68 \%$ (Group 1), 43\% (Group 2) and $0 \%$ (Group 3). Of the 7 infants in Group 2, two have completed stage 3 palliation. In Group 3, four of seven infants were discharged after Norwood, but had interstage deaths. Statistical analysis revealed that survival differences between Groups 1 and 2 were due to chance. Survival benefits were observed both clinically and statistically between Groups 2 and 3.

Conclusion: Using a multidisciplinary approach, time-saving measures can be instituted by delivering prenatally-diagnosed infants with HLHS with IAS via C-section in the cardiac catheterization suite where left atrial decompression can be performed quickly and safely. Our five year institutional experience proves that immediate intervention may improve survival.

\section{6}

Alternate uses of PDA occluders to close cardiac and extra cardiac defects

Anil Radha Sivadasan, Department of Pediatric Cardiology, Apollo

Health City, Hyderabad, India

Girish Basavaraj Navasundi, Department of Pediatric Cardiology,

Apollo Health City, Hyderabad, India

Kanhaiya Himatlal Jeswani, Department of Pediatric Cardiology,

Apollo Health City, Hyderabad, India

Deepa Mohan Sharma, Department of Pediatric Cardiology, Apollo

Health City, Hyderabad, India

Meena Trehan, Department of Pediatric Cardiology, Apollo Health

City, Hyderabad, India

$S$ G Sunil, Department of Pediatric Cardiology, Apollo Health City, Hyderabad, India

Biswajit Bandopadhya, Rabindranath Tagore International Institute of Cardiac Sciences, Kolkata, India

Ashutosh Raghuvanshi, Rabindranath Tagore International Institute of Cardiac Sciences, Kolkata, India

We describe the use of PDA occluder to close intra and extra cardiac defects different from its intended use. These were used for closure of aorto pulmonary window (1), Blalock Taussig shunt(1), coronary AV fistula (3), rupture of sinus of valsalva aneurysm-(2), Muscular VSD(3) and MAPCA(1). We used either Amplatzer (ADO)or a Lifetech occluder (LDO).

1. A 3 year old boy with $6 \mathrm{~mm}$ distal APW was closed using a 8-6 mm LDO. 2. A $6 \mathrm{~mm}$ modified BT shunt which was unligated after intracardiac repair in a 28 year old woman was blocked with a 10-8 ADO. 3. Three patients underwent closure of coronary AV fistula, two had fistula from RCA and were blocked with 6-4 ADO and 8-6 LDO. The third had a fistula from the LMCA and was occluded with 6-4 mm ADO. A 5 year old boy had a RSOV aneurysm into left ventricle and this was from the venous end after transeptal puncture of the interatrial septum. The defect was closed with 10-8 ADO with no residual flow. Three patients with muscular VSD underwent closure. One had a $5 \mathrm{~mm}$ anterior muscular VSD with mild PAH and was closed with 10-8 ADO. The other two patients had posterior muscular VSD which were closed with 8-6 LDO device. 
A 7 year old boy with VSD-PA after unifocalization and intracardiac repair with conduit, developed recurrent pleural effusions. one large MAPCA draining directly to RPA and was closed with 10-8 ADO.

Conclusions: Transcatheter closure of cardiac and extra cardiac defects can be safely and effectively accomplished with PDA occluders and offers good alternative to surgical treatment.

\section{7}

Serial Troponin as an Early Indicator for Need to Re-intervene After the Hybrid Procedure

Howaida G EL-Said, Rady Children's Hospital of San Diego, San Diego, CA, United States

John W Moore, Rady Children's Hospital of San Diego, San Diego, $C A$, United States

Peter Pastuszko, Rady Children's Hospital of San Diego, San Diego, $C A$, United States

John J Lamberti, Rady Children's Hospital of San Diego, San Diego, $C A$, United States

Objectives: The Hybrid procedure (Bilateral PA Bands + PDA stent) is an alternative treatment in HLHS. The cornerstone of this procedure is flow in the transverse arch. We report our experience with serial measurements of Troponin to evaluate the adequacy of transverse arch flow after the Hybrid procedure.

Methods: Patients who underwent the Hybrid procedure at our institution were retrospectively reviewed. Troponin levels were drawn shortly after the procedure, then every 8 hours for 48 hours, and subsequently every 24 hours for 3 days. Troponin levels were drawn thereafter if transverse arch inadequacy was suspected.

Results: From July 2007 through November 2008, 11 patients underwent the Hybrid procedure (7 HLHS, 2 IAA, 1 Heterotaxy, 1 aortic atresia/VSD). There were no operative mortalities. One patient died at 60 days post-Hybrid. Two patients underwent a Norwood/Glenn repair. Two had a biventricular repair. Two had a Sano procedure due to inadequate transverse arch flow, and four are awaiting repair. Troponin levels were followed in 7 cases. Median Troponin level at $1 \mathrm{hr}$ was $1.02 \mathrm{ng} / \mathrm{ml}$, at $8 \mathrm{hrs} 2.5$, at $16 \mathrm{hrs} 1.37$ and at $24 \mathrm{hrs}$ 1.27 , and 0.06 at discharge. The two patients requiring intervention had a $20 \%$ increase in Troponin level compared to levels at discharge which were equal to the post-operative nadir. Troponin level did not correlate with the measured gradient in the transverse arch.

Conclusions: Troponin level elevations occur after the Hybrid procedure but return to normal within one week. Late increase in Troponin may assist in the decision to re-intervene.

\section{8}

Catheter dilatation of critical stenosis of the pulmonary valve in neonates

Utku Arman Orun, Dr. Sami Ulus Pediatric Research and Training Hospital, Departments of Pediatric Cardiology, Turkey

Senem Ozgur, Dr. Sami Ulus Pediatric Research and Training

Hospital, Departments of Pediatric Cardiology, Turkey

Burhan Ocal, Dr. Sami Ulus Pediatric Research and Training Hospital, Departments of Pediatric Cardiology, Turkey

Filiz Senocak, Dr. Sami Ulus Pediatric Research and Training

Hospital, Departments of Pediatric Cardiology, Turkey

Selmin Karademir, Dr. Sami Ulus Pediatric Research and Training

Hospital, Departments of Pediatric Cardiology, Turkey

Murat Koc, Dr. Sami Ulus Pediatric Research and Training Hospital, Departments of Cardiovascular Surgery, Turkey
Ali Kutsal, Dr. Sami Ulus Pediatric Research and Training Hospital, Departments of Cardiovascular Surgery, Turkey

Critical pulmonary stenosis can be a potential lethal condition in neonates. It is defined as a solemn obstruction in pulmonary artery with right to left shunt at interatrial level. To establish the adequate pulmonary blood flow is necessary for survival. Management strateggies may vary. Patients clinical status and the development stage of right ventricle are the most important criterias of choosing the treatment modality. Percutaneus baloon valvuloplasty is one of these management strategies and it is widely performed all over the world. We report a case of newborn with critical pumonary stenosis within seven days after birth. We performed a different mechanical perforation and baloon valvuloplasty tecnique. We used 7 F High Flow Cordistm $\left(\mathrm{DUCOR}{ }^{\mathrm{R}}\right.$ ) cathether to perforate pulmonary valve. Afterwards baloon valvuloplasty was succesfully done, the gradient has decreased to $40 \mathrm{mmHg}$ from $100 \mathrm{mmHg}$ at the pulmonary valve level. Improvement of clinical and laboratory paramaters established within 24 hour after the treatment. The patient was discharged on the third day. Patient follow up still goes in our department. Our modified mechanichal technique can be a good alternative for other approaches.

\section{9}

Percutaneous balloon valvotomy in pulmonary atresia with intact ventricular septum in a neonate

Utku Arman Orun, Dr. Sami Ulus Pediatric Research and Traning Hospital Departmant of Pediatric Cardiology, Turkey

Senem Ozgur, Dr. Sami Ulus Pediatric Research and Traning Hospital Departmant of Pediatric Cardiology, Turkey

Burhan Ocal, Dr. Sami Ulus Pediatric Research and Traning Hospital Departmant of Pediatric Cardiology, Turkey

Filiz Senocak, Dr. Sami Ulus Pediatric Research and Traning Hospital Departmant of Pediatric Cardiology, Turkey

Selmin Karademir, Dr. Sami Ulus Pediatric Research and Traning

Hospital Departmant of Pediatric Cardiology, Turkey

Vehbi Dogan, Dr. Sami Ulus Pediatric Research and Traning Hospital Departmant of Pediatric Cardiology, Turkey

Murat Koc, Dr. Sami Ulus Pediatric Research and Traning Hospital Departmant of Cardivascular Surgery, Turkey

Ali Kutsal, Sami Ulus Pediatric Research and Traning Hospital, Turkey

Intact ventricular septum with pulmoner atresia or critical pulmonary stenosis is a rare anomaly of the Congenital Heart Diseases (CHD). It is seen aproximately in 3\% of all CHDs. Management strateggies may vary. Patients clinical status, the development stage of right ventricle, tricuspit area, right ventricle end diastolic diameter and right ventricle dependent coronary arterial circulation are the most important criteria of choosing the treatment modality. Transient transcatheteric approaches and urgent surgical procedures play an important role as the life-saving procedures in patients that have a chance of biventricular operation and ductus dependent circulation in the newborn period. We described in here pulmonary atresia with intact ventriculary septum with newborn was perforeted with transient transcatheteric approaches. Firstly $4 \mathrm{~F}$ right coronary catheter was placed at the level of the pulmonary atrethic valve. Then atrethic valve was perforated by gentle end of 0.014 guidewire. When 0.014 guidewire placed on pulmonary arter, 0.021 wire was pushed forward to pulmonary arter, then advanced to the patent ductus arteriosus. Snare wire was pushed forward to descending aorta within 4 F multipurpose catheter. Guide wire was caught by snare catheher which was fixed at descending aorta. 
Therefore support was provided to balon angioplasty procedure. Finally baloon angioplasty was performed with $6 \times 3 \mathrm{~mm}$ baloon. By the opaque injection passage from pulmonary valve was demonstrated but tricuspit failure was still keeping on. Spo2 obtained by pulse oximeter was $89 \%$. Our modified mechanichal technique can be a good alternative for other approaches.

\section{0}

Transcatheter closure of Atrial Septal Defects in children less than 10 years: Immediate and intermediate follow up Anil Radha Sivadasan, Department of Pediatric Cardiology, Apollo Health City, Hyderabad, India

Girish Basavaraj Navasundi, Department of Pediatric Cardiology, Apollo Health City, Hyderabad, India

Kanhaiya Himatlal Jeswani, Department of Pediatric Cardiology, Apollo Health City, Hyderabad, India

Deepa Mohan Sharma, Department of Pediatric Cardiology, Apollo

Health City, Hyderabad, India

Meena Trehan, Department of Pediatric Cardiology, Apollo Health City, Hyderabad, India

$S$ G Sunil, Department of Pediatric Cardiology, Apollo Health City, Hyderabad, India

Background: This is a retrospective study of patients who underwent ASD device closure using either Amplatzer (ASO) or Lifetech ASD occluder (LSO) from July 2004-October 2008. Results: 33 patients underwent percutaneous closure who were less than 10 years (range 11 months- 10 years, $18<5$ years). The procedure was done under conscious sedation in 10 with TTE and the rest under general anesthesia and TEE.The defect size ranged from $10 \mathrm{~mm}-24 \mathrm{~mm}$ (median: $16 \mathrm{~mm}$ ). The device sizes ranged from $10-32 \mathrm{~mm}$. The device chosen was the maximum diameter of the defect on echocardiogram (TEE when this was done). Over sizing was done in 4 patients with floppy rims (by $2-4 \mathrm{~mm}$ ). In 4 patients (age $2-6$ years, ASD $>15 \mathrm{MM}$ ), the initial device was replaced by a smaller device due to repeated failure of deployment. Balloon assisted closure was done in 18 patients (Electively in 12 with deficient or floppy rims and on failure of initial attempt in the rest). The mean fluoroscopic time was 12 minutes (range 4-40.2 minutes) and the procedural time was 57 minutes ( 25 to 170 minutes). Follow up echocardiography showed complete closure immediately, at 24 hours, and 1 year in $86 \%, 92 \%$ and $96 \%$ for ASO and $92 \%, 94 \%$ and $94 \%$ for LSO respectively.

Complications: SVT-2, transient sinus node dysfunction-1, Device embolization-1.

Conclusions: The early and intermediate results of transcatheter closure of atrial septal defects shows that it is feasible and safe in children less than 10 years. Balloon sizing was not done in any of the patients. Balloon assisted closure simplifies the procedure especially in patients with floppy rims.

\section{1}

Transcatheter closure of atrial septal defects (ASD) guided by transthoracic echocardiography in children and adults Celal Akdeniz, Dr. Siyami Ersek Hospital for Cardiology and Cardiovascular Surgery, Turkey

Ahmet Celebi, Turkey

Cenap Zeybek, Dr. Siyami Ersek Hospital for Cardiology and Cardiovascular Surgery, Turkey

Abdullah Erdem, Dr. Siyami Ersek Hospital for Cardiology and Cardiovascular Surgery, Turkey

Turkay Saritas, Dr. Siyami Ersek Hospital for Cardiology and Cardiovascular Surgery, Turkey
Ender Odemis, Dr. Siyami Ersek Hospital for Cardiology and Cardiovascular Surgery, Turkey

Halil Demir, Dr. Siyami Ersek Hospital for Cardiology and Cardiovascular Surgery, Turkey

Yalim Yalcin, Dr. Siyami Ersek Hospital for Cardiology and Cardiovascular Surgery, Turkey

Objectives: We used to perform transesophageal echocardiography (TEE) under general anesthesia in all patients underwent transcatheter ASD closure during the initial experience. After getting more experience in 24 patients by TEE, we started to use transthoracic echocardiography (TTE) for guidance in all patients if it is possible. We aimed to evaluate the safety and efficacy of transcatheter ASD closure guided by TTE.

Material and method: We analyzed the data of 161 patients with ASD who underwent transcatheter closure after we started to use TTE guidance. Balloon sizing for device selection was performed in all patients. Amplatzer, Cardiofix and Cardia septal occluders were used

Results: 128 ASDs were closed under TTE guidance. Implantation was successful in all and showed complete occlusion without major complication. TEE guidance is performed in 33 patients because of poor transthoracic acoustic windows (16), multiple ASDs (7) and very large defects $>25 \mathrm{~mm}$ (10). One unsuccessful attempt and two device migrations have observed in TEE group. Defect diameters were similar $(18.1 \pm 6.6 \mathrm{~mm}$ vs $16.1 \pm 5.5 \mathrm{~mm})$ but the age distribution were slightly higher in TEE group $(21.1 \pm 17.5$ vs $14.2 \pm 13.0$ years; $p=0.047)$. Duration of intervention was significantly shorter in TTE group $(66.4 \pm 27.3$ vs $79.3 \pm 26.6$ minutes, p: 0.02). Fluoroscopy times were similar.

Conclusion: TTE, as a noninvasive guiding imaging tool, provides accurate definition of the defect anatomy in most of the patients including adults for transcatheter ASD closure. It is possible to avoid the complications of general anesthesia and endotracheal intubation and also save time by using TTE.

\section{2}

Lung Perfusion Studies after Transcatheter Closure of Patent Ductus Arteriosus (PDA): Analysis of Three Different Devices

Tugcin Polat, Dr. Siyami Ersek Hospital for Cardiology, Thoracic and Cardiovascular Surgery, Turkey

Cenap Zeybek, Dr. Siyami Ersek Hospital for Cardiology, Thoracic and Cardiovascular Surgery, Turkey

Ahmet Celebi, Turkey

Yalim Yalcin, Dr. Siyami Ersek Hospital for Cardiology, Thoracic and Cardiovascular Surgery, Turkey

Elnur Imanov, Dr. Siyami Ersek Hospital for Cardiology, Thoracic and Cardiovascular Surgery, Turkey

Turkay Saritas, Dr. Siyami Ersek Hospital for Cardiology, Thoracic and Cardiovascular Surgery, Turkey

Ender Odemis, Dr. Siyami Ersek Hospital for Cardiology, Thoracic and Cardiovascular Surgery, Turkey

Celal Akdeniz, Dr. Siyami Ersek Hospital for Cardiology, Thoracic and Cardiovascular Surgery, Turkey

Objective: Impaired left lung perfusion has been described after coil occlusion of PDA. This study was conducted to evaluate lung perfusion following PDA closure with three different devices including Amplatzer Duct Occluder (ADO).

Method: Lung perfusion scans were performed in 130 of 360 children after PDA closure, including 41 patients with large PDAs $(\geqslant 4 \mathrm{~mm}$ ) using ADO device (Group 1), 49 patients with moderate PDAs (2.5-4 mm) using Cook Detachable coils (Group 
2) and 40 patients with small PDA's $(\leqslant 2.5 \mathrm{~mm})$ using Gianturco coils (Group 3). Variables were examined to predict the risks for reduced left lung perfusion.

Results: Decreased left lung perfusion, defined as $<40 \%$ of total pulmonary blood flow, was found in 15/41 (\%37) in Group 1, $10 / 49(\% 20)$ in Group 2 and $3 / 40(\% 7)$ in Group 3 $(\mathrm{X} 2=10.195, \mathrm{p}=0.006)$. In Group 1, multivariate logistic regression analysis revealed that tubular and short ducts were significant risk factors for abnormal left lung perfusion $(p=0.032)$. In Group 2, decreased perfusion was significantly correlated with multiple coils usage $(\mathrm{p}=0.043)$ and number of loops deployed at the pulmonary side $(\mathrm{p}=0.013) .1 .5$ loops deployed at the pulmonary side in two of three patients with abnormal perfusion was the only finding in Group 3 .

Conclusion: Abnormal left lung perfusions were found to be more frequently in patients with moderate to large PDAs after transcatheter closure. It seems to be related to ductal morphology (short and tubular shape) for ADO, multiple coils usage and leaving more loops at the pulmonary side for coils.

\section{3}

Intrasept occluder (Cardia) in transcatheter closure of secundum atrial septal defects (ASD): Early results

Abdullah Erdem, Dr. Siyami Ersek Hospital for Cardiology and Cardiovascular Surgery, Turkey

Ahmet Celebi, Turkey

Celal Akdeniz, Dr. Siyami Ersek Hospital for Cardiology and Cardiovascular Surgery, Turkey

Turkay Saritas, Dr. Siyami Ersek Hospital for Cardiology and Cardiovascular Surgery, Turkey

Cenap Zeybek, Dr. Siyami Ersek Hospital for Cardiology and Cardiovascular Surgery, Turkey

Elnur Imanov, Dr. Siyami Ersek Hospital for Cardiology and Cardiovascular Surgery, Turkey

Fadli Demir, Dr. Siyami Ersek Hospital for Cardiology and Cardiovascular Surgery, Turkey

Nurdan Erol, Dr. Siyami Ersek Hospital for Cardiology and

Cardiovascular Surgery, Turkey

Objectives: The Cardia septal occluder (Intrasept, CSO) is a new transcatheter ASD occlusion device. We sought to analyze safety, efficacy, and follow-up results of percutaneous closure of ASD using this new device.

Methods: Between April 2007 and December 2007, we attempted transcatheter closure of ASD with CSO device under the guidance of transthoracic (8) or transesophageal echocardiography (2) at our institution. Indications of ASD closure are significant left to right shunt and suitable defect rims showed by detailed transthoracic echocardiographic examination.

Results: Device implantation was successful in all ten patients $(\% 100)$. The median age was $7.5(4.5-24)$ years and median weight was $30(16-64 \mathrm{~kg}) \mathrm{kg}$. The median Qp/Qs ratio was 1.7 (range, 1.5 to 2.4), and the median ASD size as assessed by transthoracic echocardiography was $15.3 \mathrm{~mm}$ (range, 9.7 to $15 \mathrm{~mm}$ ). The median device size used was $20 \mathrm{~mm}$ (14 to $24 \mathrm{~mm}$ ). Total occlusion rate was $60 \%(6 / 10)$ immediately after completion of the procedure, rising to $90 \%(9 / 10)$ the day after. Displacement of aortic side wing from right side to left causing residual shunt was the only complication encountered. Small residual shunt persisted in this patient but no intervention was needed during follow up.

Conclusions: Transcatheter closure of ASD with this new device is an effective and safe procedure in medium sized ASD's. Our results should be confirmed in larger number of patients.

\section{4}

Combined Percutaneous Transcatheter Interventions in the Same Session for Patients with Multiple Congenital Cardiovascular Defects

Ahmet Celebi, Turkey

Turkay Saritas, Dr. Siyami Ersek Hospital for Cardiology and Cardiovascular Surgery, Turkey

Ender Odemis, Dr. Siyami Ersek Hospital for Cardiology and

Cardiovascular Surgery, Turkey

Celal Akdeniz, Dr. Siyami Ersek Hospital for Cardiology and Cardiovascular Surgery, Turkey

Abdullah Erdem, Dr. Siyami Ersek Hospital for Cardiology and Cardiovascular Surgery, Turkey

Fadli Demir, Dr. Siyami Ersek Hospital for Cardiology and

Cardiovascular Surgery, Turkey

Nurdan Erol, Dr. Siyami Ersek Hospital for Cardiology and Cardiovascular Surgery, Turkey

Yalim Yalcin, Dr. Siyami Ersek Hospital for Cardiology and

Cardiovascular Surgery, Turkey

Objective: To investigate the methods of combined percutaneous transcatheter interventions for congenital heart disease with multiple defects and to evaluate its efficacy in children.

Methods: 17 cases (10 boys, 7 girls, ages 3 days -16 years, body weight $3.4-35 \mathrm{~kg}$ ) that underwent multiple transcatheter interventions for congenital heart disease with multiple defects were retrospectively analyzed and presented. The sequence of the interventions was planned according to the nature and localization of the defects.

Result: Additional PDA closure (6) was performed following VSD closure (2), ASD closure (1), coarctation angioplasty (2), pulmonary balloon valvuloplasty (1); coarctation angioplasty (5) was performed following aortic valvuloplasty (3), VSD closure (1) and balloon atrial septostomy (1) for transposition of great arteries. Ductal stent implantation was the combined procedure in 2 patients after pulmonary valve perforation and/or pulmonary balloon valvuloplasty with hypoplastic right ventricles. Other combined procedures were ASD closure after pulmonary balloon valvuloplasty (1); pulmonary balloon valvuloplasty after aortic balloon valvuloplasty (1); coil embolization of a pulmonary lobar sequestration after coarctation angioplasty (1); and palliative pulmonary balloon valvuloplasty after recanalization and angioplasty of the systemic-pulmonary shunt in tetralogy of Fallot (1). There was no mortality or major morbidity.

Conclusion: For the treatment of combined congenital cardiovascular defects, multiple transcatheter interventions in the same session are feasible, safe and effective with satisfactory good results. Second intervention may be performed as complementary or substantive to the first procedure.

\section{5}

Innovative approach for Balloon Pulmonary Valvuloplasty in Critical Valvar Pulmonary Stenosis

Supratim Sen, Rabindranath Tagore International Institute of Cardiac Sciences, India

Biswajit Bandyopadhyay, Rabindranath Tagore International Institute of Cardiac Sciences, India

Dhritabrata Das, Rabindranath Tagore International Institute of Cardiac Sciences, India

Mily Ray, Rabindranath Tagore International Institute of Cardiac

Sciences, India

Amitabha Chattopadhyay, Rabindranath Tagore International Institute of Cardiac Sciences, India

PS Bhattacharyya, Rabindranath Tagore International Institute of Cardiac Sciences, India 
A 2 day old neonate weighing $2.1 \mathrm{~kg}$ presented with respiratory distress and a systolic murmur. He was diagnosed with critical valvar pulmonary stenosis, intact ventricular septum with moderate RV dysfunction and mild LV dysfunction. There was right to left shunting across a stretched PFO and severe TR (gradient $74 \mathrm{~mm} \mathrm{Hg}$ ). The pulmonary valve annulus was $6 \mathrm{~mm}$ with a restrictive opening and an underestimated gradient of $45 \mathrm{mmHg}$.

During cardiac catheterization, the pulmonary valve could not be crossed anterogradely from the RV. Aortic angiogram in lateral view showed a closed ductus. Prostaglandin E1 infusion was started $(0.1 \mathrm{mcg} / \mathrm{kg} / \mathrm{min})$ and after 5 minutes, the ductus opened up. PDA was then crossed from the aortic end with a $4 \mathrm{~F} \mathrm{JR}$ catheter. The pulmonary valve was crossed retrogradely with a $0.014 \times 190$ PTCA wire which was stationed in the RV. The valve was dilated with a $7 \times 2 \mathrm{~mm}$ mini Tyshak balloon with full disappearance of the waist. Post-balloon angiogram showed good opening of the pulmonary valve and RV-PA pressure gradient was reduced to $13 \mathrm{~mm} \mathrm{Hg}$. Prostaglandin infusion and ventilation were tapered off within 24 hours and the baby was discharged 3 days later.

Balloon pulmonary valvuloplasty is the recommended treatment for congenital pulmonary valve stenosis. In this case, as the procedure was performed on day 2 of life, only functional closure of the ductus had occurred. Thus, prostaglandin infusion effected reestablishment of ductal patency and allowed a retrograde approach through the stenosed pulmonary valve.

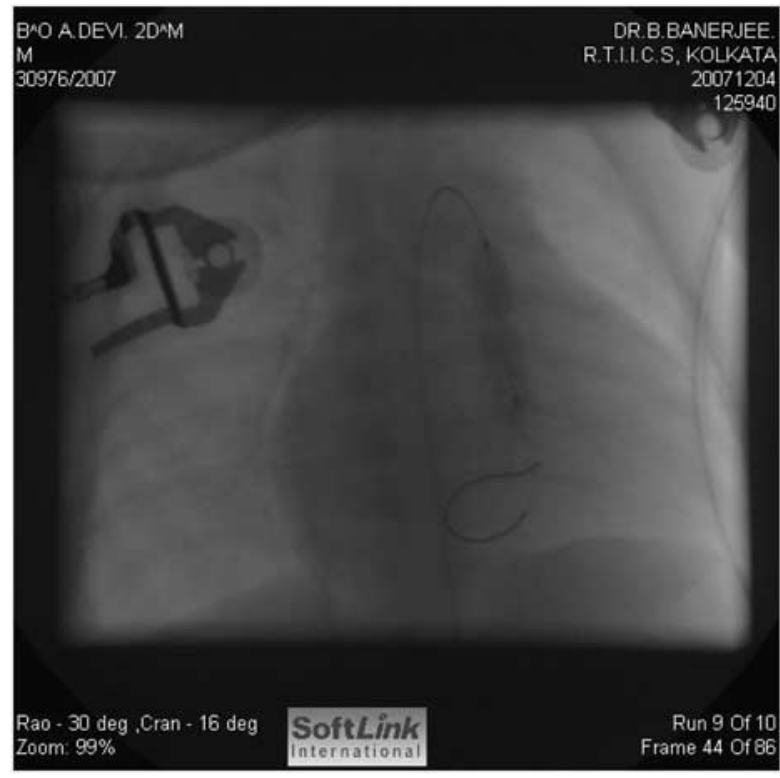

\section{6}

Clinical Profile and Management Options of Ruptured Sinus of Valsalva

Supratim Sen, Rabindranath Tagore International Institute of Cardiac Sciences, Kolkata, India

Biswajit Bandyopadhyay, Rabindranath Tagore International Institute of Cardiac Sciences, Kolkata, India

Amitabha Chattopadhyay, Rabindranath Tagore International Institute of Cardiac Sciences, Kolkata, India

Mily Ray, Rabindranath Tagore International Institute of Cardiac Sciences, Kolkata, India

The first successful surgical repair of Rupture of aneurysm of the sinus of Valsalva (RSOV) was in 1957. It is now accepted that RSOVs are amenable to transcatheter device closure. We analyzed demographics and lesion characteristics of RSOV, and outcomes of surgical repair and device closures. Isolated RSOVs were closed by transcatheter device closure. Patients with RSOV associated with other lesions underwent surgical repair.

Twenty-one patients presented to our institute with RSOV between January 2004 and January 2008. The mean age of presentation was 29.9 years. Aortic regurgitation and VSD were the commonest associated lesions. Eight patients with isolated RSOVs underwent device closure. The average hospital stay was 2.9 days as compared to 8.2 days for surgical repair. There were no mortalities and no significant short term complications in both the patients with surgical and device closures.

We conclude that transcatheter device closure is a viable and safe treatment option for isolated RSOVs, whereas surgical repair is better suited for those patients with associated cardiac malformations.

\begin{tabular}{lcccc}
\hline Origin & \multicolumn{4}{c}{ RSOV Lesion Characteristics: Exit Site } \\
\cline { 2 - 5 } & RA & RV & RVOT & Total \\
\hline Right coronary sinus & 2 & 3 & 11 & 16 \\
Non-coronary sinus & 4 & 0 & 1 & 5 \\
Left coronary sinus & 0 & 0 & 0 & 0 \\
Total & 6 & 3 & 12 & 21 \\
\hline
\end{tabular}

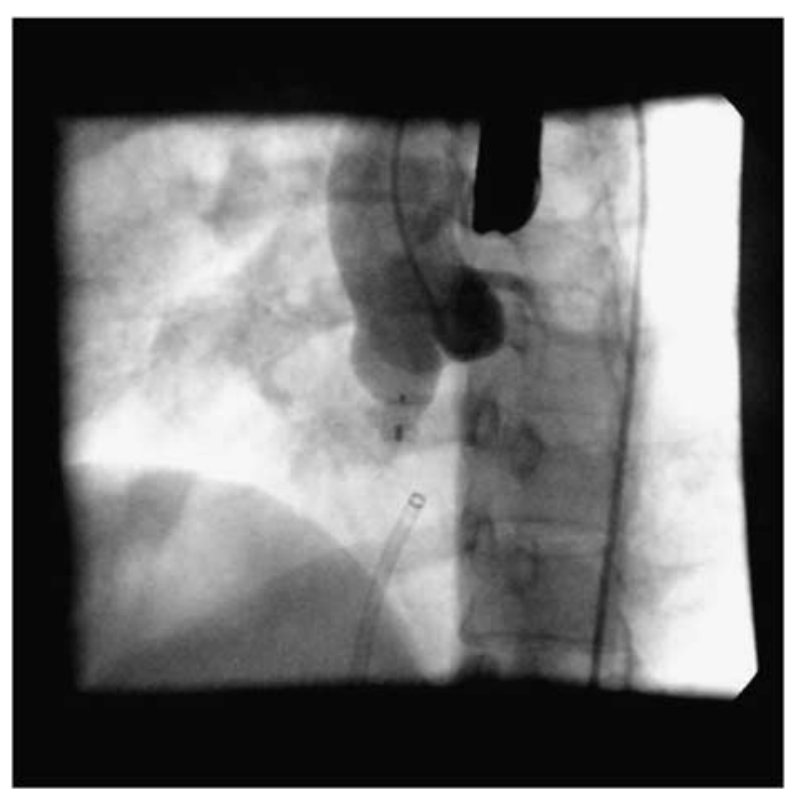

Aortic injection after device placement

\section{7}

Safety and efficacy of transcatheter closure of atrial septal defects under transthoracic echocardiographic guidance in cardiac catheterization laboratory

Martin NL Wong, Sarawak General Hospital, Malaysia

Boonhock Choo, Sarawak General Hospital, Malaysia

Kuihian Sim, Sarawak General Hospital, Malaysia

Objectives: Conventionally, transcatheter device closure of atrial septal defects (ASD) are performed under the guidance of either transoesophageal echocardiography (TEE, which requires general anaesthesia) or intracardiac echocardiographic (which is costly) in the cardiac catheterization laboratory. We aimed to assess the safety and efficacy of using transthoracic echocardiography to guide this procedure. 
Methods: 18 patients (median age $32.5 \pm 1.7$ years) with haemodynamic significant and suitable ASD underwent transcatheter ASD closure using Amplatzer Septal Occluder. Appropriate device size was selected prior to the procedure based on TEE assessment performed earlier at outpatient echocardiography laboratory. Implantation of the device was guided solely by fluoroscopy and transthoracic echocardiography in the cardiac catheterization laboratory.

Results: The median ASD diameter of the cohort was $20 \mathrm{~mm}$ (range 11 to $26 \mathrm{~mm}$ ) and the corresponding size of devices implanted ranged from 16 to $36 \mathrm{~mm}$ (median $28 \mathrm{~mm}$ ). Procedure was successful in 17 patients without any complication. Only in 1 case, the device could not be deployed in proper position and was retrieved. The median procedural and fluoroscopy times required were short at $28.0 \pm 11.7 \mathrm{mins}$ and $4.8 \pm 2.8$ mins respectively. At 6 months follow up, complete closure and regression of right heart dilatation were documented in all the 17 cases.

Conclusions: Transcatheter closure of ASD under transthoracic echocardiography guidance is safe, fast and feasible.

\section{8}

Catheter Balloon De-Banding Of The Pulmonary Artery: Feasibility And Safety (a Hybrid technique)

Howaida G El-Said, University of California San Diego, United States Rabih K Hamzeh, University of California San Diego, United States John J Lamberti, University of California San Diego, United States John W Moore, University of California San Diego, United States

Objective: Non-surgical adjustment of Pulmonary artery band $(\mathrm{PAB})$ diameter is an attractive method for increasing pulmonary blood flow (PBF). This study assessed the feasibility/safety of band loosening/debanding by balloon dilatation.

Methods: All pts who underwent PAB balloon dilatation (PABB) between 10/2006 \& 4/2008 were reviewed. At surgery, PAB diameter was fixed with hemoclips in a manner designed to permit progressive dislodgement of the clips in a controlled manner. A balloon inflated at the PAB site resulted in its gradual loosening until desired clinical outcome is achieved.

Results: Eight PABB were performed in 6pts (2pts had 2 dilations). Mean age at catheterization 6 months (range 3-14) (mean weight $5 \mathrm{~kg}$ ). Median time from $\mathrm{PAB}$ placement 4.5 months (range 1.8-15). Diagnosis at PAB: CoA/hypoplastic transverse arch with multiple apical muscular VSDs $(n=4)$ and functional single ventricle with un-protected $\operatorname{PBF}(\mathrm{n}=2)$. PABB required a single balloon in 7 (serial dilations in 5) and double balloon in 1. Balloons ranged from $4-12 \mathrm{~mm}$; inflated to $8-24$ atms. The procedure was successful in all patients. Saturation increased from a mean of 76 to $89 \%(\mathrm{p}=0.001)$. Gradient decreased from a mean of 67 to $36 \mathrm{mmHg} \quad(p=0.001)$. Angiographically measured band site diameter increased from a median of 3.8 to $6.7 \mathrm{~mm}(\mathrm{p}=0.002)$. One patient had a reperfusion injury, requiring mechanical ventilation for 5 days. There were no other complications.

Conclusion: $\mathrm{PABB}$ is a safe and effective method for increasing $\mathrm{PBF}$ in a staged/controlled manner. PABB may eliminate the need for surgical removal in some cases.

\section{9}

Experience in atrioseptostomy using radiofrequency perforation and stenting of the atrial septum Fernando S Ballesteros, Gregorio Marñón Hospital. Madrid., Spain Jose L Zunzunegui, Gregorio Marñón Hospital. Madrid., Spain
Teresa Alvarez, Gregorio Marnón Hospital. Madrid., Spain Constancio Medrano, Gregorio Marñón Hospital. Madrid., Spain Manuela Camino, Gregorio Marñón Hospital. Madrid., Spain Enrique Maroto, Gregorio Marñón Hospital. Madrid., Spain Miriam Centeno, Gregorio Marnón Hospital. Madrid., Spain Ruben Greco, Gregorio Marñón Hospital. Madrid., Spain

Objectives: The possibility of creating an interatrial communication in cardiopathies with high pressure of the left atrium facilitates medical management and improves the postoperative prognosis. The aim of this report is to describe our experience in these procedures using Nykanen radiofrequency catheter and stenting. Methods: A retrospective study reviewing four patients' records who had undergone the procedure from May 2006-Oct 2008. The indication was mitral stenosis in three cases and ventricle dysfunction in one. The mean age was 3, 5 months ( 3 days old- 4, 5 months); mean weight was $3,6 \mathrm{Kg}(2,5-4,5 \mathrm{Kg})$. The procedure was performed with transesophageal echocardiographic guidance with an intracardiac probe (10F Acunav). An average of 4, 5 pulses at 8 watts were delivered depending on septum thickness. The stents implanted were $5 \times 18 \mathrm{~mm}$ Herculink stents (2), $4 \times 12 \mathrm{~mm}$ Herculink stent, and 2, $5 \times 12 \mathrm{~mm}$ Micro-Driver stent.

Results: Success was obtained in all four cases with a decrease in the left atrium pressure; postprocedure echocardiograms showed no pericardial effusions and no interference with atrioventricular valve function or venous inflow to the atrium.In three the stent was removed in later surgery (mean time to explantation of 10 days), and the orifice diameter was conserved with no evidence of thrombus. One patient died 1,5 months after the procedure in multiorganic failure.

Conclusions: In our experience radiofrequency atrioseptostomy stenting of the atrial septum is safe and effective. This permits control of the orifice size, and can be performed beyond the neonatal period.

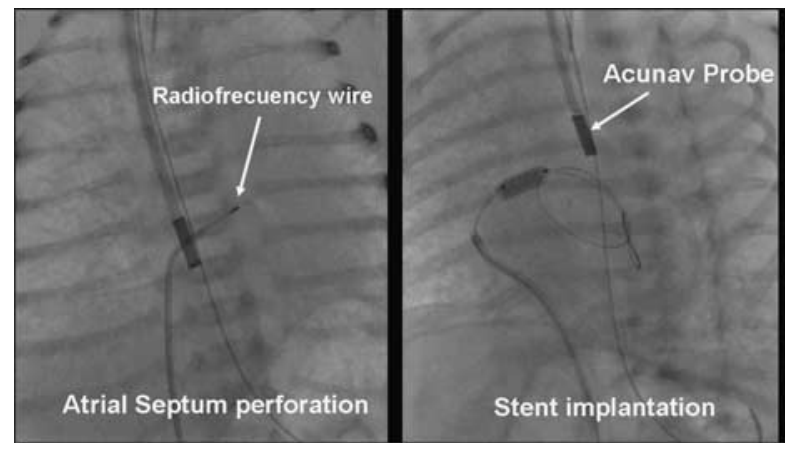

\section{0}

Scottish experience of percutaneous VSD closure, 2002-2007

Elena Grant, Royal Hospital for Sick Children, Glasgow,

United Kingdom

Stuart Lilley, Royal Hospital for Sick Children, Glasgow,

United Kingdom

Neil Wilson, John Radcliffe Infirmary, Oxford, United Kingdom

Brodie Knight, Royal Hospital for Sick Children, Glasgow,

United Kingdom

Objectives: To review our experience of percutaneous transcatheter closure of VSD using Amplatzer membranous (AMembVSDO) or muscular (AMuscVSDO) VSD Occluders.

Background: Since the advent of specifically designed devices, transcatheter closure of ventricular septal defects (VSD) is an attractive alternative to surgical closure in selected patients. 
Methods: Between 10/2002 and 10/2007, transcatheter VSD closure was attempted in 34 patients with either AMembVSDO (27) or AMuscVSDO (7). Median age was 8 years (range 0.6-34) and median weight was $25.4 \mathrm{~kg}$ (range $8.1-77.0$ ). The mean length of device follow-up was 35 months (range 8-59).

Results: Devices were successfully placed in all but three patients who are excluded from all further analysis. Mean fluoroscopy time of 41 min (range 15-107) and mean total procedure time of 181 min (range 100-330) include time spent on other unrelated interventions $(\mathrm{n}=3)$. Median device size was $8 \mathrm{~mm}$ (range 4-18). Complete closure rate was $61.3 \%$ within 1 week, $80.6 \%$ within 2 months and $87.1 \%$ at last follow-up. For AMembVSDO the respective rates were $64 \%, 88 \%$ and $92 \%$ and for AMuscVSDO $57.1 \%, 57.1 \%$ and $71.4 \%$. No deaths or long term morbidity could be attributed to device implantation. Complications included: transient intra-procedural complete heart block $(\mathrm{n}=1)$ and $\operatorname{SVT}(\mathrm{n}=1)$, transient $\operatorname{RBBB}(\mathrm{n}=3)$, new trivial aortic regurgitation $(n=2)$, new or increased trivial tricuspid regurgitation $(n=4)$, femoral artery pseudoaneurysm $(\mathrm{n}=1)$, brachial plexopathy $(\mathrm{n}=1)$ and pyrexia of unknown origin $(\mathrm{n}=1)$.

Conclusion: Transcatheter VSD closure using AMembVSDO and AMuscVSDO appears to be a safe and effective alternative to open heart surgery.

\section{1}

Is there a genetic predisposition to the occurrence of atrioventricular block after atrial septal percutaneous closure?

Sylvia Abadir, Sainte Justine Hospital, Canada

Shiwei Yang, Sainte Justine Hospital, Canada

Georgia Sarquella-Brugada, Sainte Justine Hospital, Canada

Kathleen Riopel, Sainte Justine Hospital, Canada

Roxanne Gendron, Sainte Justine Hospital, Canada

Nagib Dahdah, Sainte Justine Hospital, Canada

Gregor Andelfinger, Sainte Justine Hospital, Canada

Background: Atrioventricular block (AVB) was reported following atrial septal defect (ASD) occlusion by Amplatzer septal occluder (ASO). The main hypothesis is the impingement of ASO on the conduction system. However, in knock out mice, hypoplasia of the conduction system was described in association with NKX 2.5, Id 2 and GATA4 mutations. We hypothesized that mutations of these genes predispose to AVB following ASO in humans.

Methods: Patients with new onset AVB or worsened AV conduction following ASO were compared to controls matched for previously identified risk factors (device size, size/height ratio). Clinical evaluation, three-generation family history, echocardiograms, electrocardiograms and genetic sequencing of candidate genes were performed.

Results: Twenty eight patients $(6.4 \pm 4.2$ yo, $23.2 \pm 14.9 \mathrm{~kg}$, ASO size $25.4 \pm 5.2 \mathrm{~mm}$ ) and 28 controls were enrolled. Second or third degree AVB occurred in 15 patients, first degree AVB (AVB-1) in 13. AVB resolved in 24/28 patients (median 8 days, $0.5-240)$. AVB-1 remains in $4,4.17 \pm 2.35 \mathrm{y}$ later. A positive family history of cardiac malformation $(n=17 / 55)$ did not correlate with AVB after ASO $(\mathrm{OR}=1.6$, CI $0.5-5, \mathrm{p}=0.3)$. Gene sequencing identified a mutation affecting the NKX 2.5 homeodomain (Arg189Leu) in a patient with preexisting borderline PR evolving towards AVB-1/2. No coding mutations were detected in GATA4 or ID2.

Conclusion: In this series, mutations in NKX2.5, GATA4 or ID2 were rare events, and mechanical stress of the ASO on the conduction system remains a plausible cause for AVB. However, this study provides the first proof-of-principle that NKX2.5 homeodomain mutations can predispose to worsening of AVB after ASO.

\section{2}

Covered stents for moderate-severe native aortic coarctation

Sylvia Abadir, Sainte Justine Hospital, Canada

Stéphane Noble, Montreal Heart Institute, Canada

Georgia Sarquella-Brugada, Sainte Justine Hospital, Canada

Reda Ibrahim, Montreal Heart Institute, Canada

Nagib Dahdah, Sainte Justine Hospital, Canada

Joaquim Miro, Sainte Justine hospital, Canada

Background: Covered stents (CS) have been suggested as an alternative to bare stents to decrease aortic complications in moderate-severe native CoA.

Methods: We retrospectively reviewed our 18 patients $(26.7 \pm 14.6$ yo; 12 to 58$)$ who underwent CS implantation since 2003 (Cheatham-Platinium stent, 8 zig, length 22 to $45 \mathrm{~mm})$.

Results: Preimplantation systolic blood pressure was $147.9 \pm 17.5$ $\mathrm{mmHg}$, with mean pressure gradient of $63.3 \mathrm{mmHg}$ (41 to 103). $11 / 16$ hypertensive patients were under medication, $8 / 11$ had at least 2 medications. Significant collaterals were present in $15 / 18$ patients. All attempts were successful. Lesion diameter/aortic diameter at diaphragm increased from $20.7 \% \pm 11.0 \%$ to $90.2 \% \pm 15.8 \%$. Invasive gradient (under general anesthesia) decreased from $33.4 \pm 11.1$ to $1.4 \pm 2.8 \mathrm{mmHg}$. One major complication occurred (mild cerebrovascular ischemic event in a 46.9 yo). Sub-clavian artery was partially jailed in $4 / 18$, with none losing radial pulse. 4 patients had almost atretic lesion $(<1 \mathrm{~mm})$, one needing radiofrequency perforation for acquired interruption.

During follow up (mean 15.8 mo, 1 to 49.5), 13 patients have already undergone non invasive imaging, with one demonstrating a small $(6 \mathrm{~mm})$ aneurysm. No stent fracture was observed. Three of our 8 teenager patients underwent further dilatation to accommodate aortic growth. Normal blood pressure was obtained in 8/16 previously hypertensive patients. Only one patient has blood pressure gradient $>20 \mathrm{mmHg}$, due to aortic arch hypoplasia.

Conclusion: CS implantation is safe in moderate-severe native $\mathrm{CoA}$, and provides excellent transcoarctation gradient relief. These results compare favorably to our previously reported experience with balloon dilatation and bare stents implantation in native coarctation.

\section{3}

Outcomes of transcatheter closure of patent ductus arteriosus at Chris Hani Baragwanath Hospital, South Africa: review of 15 years experience

Paul E Adams, Chris Hani Baragwanath Hospital, The University of the Witwatersrand, South Africa

Matthew F Chersich, Reproductive Health and HIV Research Unit, The University of the Witwatersrand, South Africa

Lungile Pepeta, Chris Hani Baragwanath Hospital, The University of the Witwatersrand, South Africa

Hopewell N Ntsinjana, Chris Hani Baragwanath Hospital, The University of the Witwatersrand, South Africa

Firosa Motara, Chris Hani Baragwanath Hospital, The University of the Witwatersrand, South Africa

Antoinette M Cilliers, Chris Hani Baragwanath Hospital, The

University of the Witwatersrand, South Africa 
Objectives: Review outcomes of transcatheter closure of patent ductus arteriosus (PDA), role of PDA shape and changes in practice over time.

Methods: Retrospective analysis of patient files and clinic database was performed on children who had transcatheter PDA closure at Chris Hani Baragwanath Hospital between 01/01/1993 and 30/06/2008.

Results: Over 15 years, 1254 PDAs were diagnosed, of which 293 required closure (167 had surgery and 139 transcatheter closure). Median age at transcatheter closure was 1.8 years (IQR $=1-4.5-$ years); $66.2 \%$ were female (92/139). Mean PDA diameter was $3.2 \mathrm{~mm}(\mathrm{sd}=1.6 \mathrm{~mm})$, with an average $2: 1$ shunt. Transcatheter closure was performed using COOK Flipper coils $(n=93 ; 18$ required multiple coils) or Amplatzer devices ( $n=46: 37$ with ADO1, 8 AVP and 1 ADO2). Twenty children required repeat procedures. Early occlusion rates for coils were 52\% (39/75); late occlusion occurred in 90.6\% (68/75). Amplatzer devices, available since 2003, are now overwhelmingly used. Amplatzer early occlusion rates were $94.3 \% \quad(33 / 35)$ with $100 \%$ late occlusion. Outcome was related to PDA shape; $88 \%$ of type A and $\mathrm{E}$ closed successfully, 67\% type B, 28\% type C and $0 \%$ of type $\mathrm{D} ; \mathrm{P}<0.001$. Surgical closure reduced over time: from 94 (1993-1997), to 40 (1998-2002) and 32 (2003-2008).

Conclusion: Transcatheter PDA occlusion is safe and effective in our setting, with outcomes similar to reports elsewhere. Shape and size of PDAs are important determinants of device selection and procedure outcome. Transcatheter occlusion helps minimise surgical waiting lists. Overall findings support more widespread use of transcatheter closure in similar settings.

\section{4}

Morphology of the patent ductus arteriosus; A predictor of the outcome of Stenting in Duct-Dependent Pulmonary Circulation; An Experience in Children Hospital, Cairo University

Hala M Agha, Children Hospital, Cairo University, Egypt

Hala S Hamza, Children Hospital, Cairo University, Egypt

N Sreeram, Cologne university, Germany

K Brockmeier, Cologne university, Germany

y Hussein, Children Hospital, Cairo University, Egypt

A Esmat, Children Hospital, Cairo University, Egypt

Objectives: To present an institutional experience with stent placement in patent ductus arteriosus (PDA) in the neonates and infants with duct-dependent pulmonary circulation and to assess the outcome of the procedure.

Background: PDA has been proposed as an alternative to surgical shunt on account of postoperative complications of surgical shunting. Methods: Twelve patients with duct-dependent pulmonary circulation who were sent to the cardiac catheterization laboratory for percutaneous ductal stenting from April 2008 to November 2008 constitute the study population. Six patients did not qualify because of complex tortuous ductus arteriosus or branch pulmonary artery stenosis. Atrial septostomy by balloon dilatation was performed as required.

Results: The mean age of the patients at the time of stent implantation was $34 \pm 16$ days. The mean body weight was $4 \pm 1 \mathrm{Kg}$. Ductal stenting was successful in 6 patients; four of them had straight horizontal duct, while the other two patients had a vertical duct. The mean ductal diameter was $3.6 \pm 0.2 \mathrm{~mm}$. Balloon-expandable coronary stents of $4 \mathrm{~mm}$ in diameter and $5 \mathrm{~mm}$ longer than the PDA length were used. The duration of hospital stay was $5.83 \pm 6.4$ days. The mean duration of follow-up was 4.75 months and mean oxygen saturation at last follow-up was $85.2 \pm 15.5 \%$. The cumulative mortality was $2 / 6$ (33.3\%).

Conclusions: Stenting the PDA in selected patients is effective for short-term palliation in infants with duct-dependant pulmonary circulation. Morphology of the PDA predicts the outcome of this procedure. Stent should be longer than the ductus length to avoid its constriction.

\section{6}

Acute Myocardial Ischaemia following Transcatheter Occlusion of Coronary Artery Fistula in an Infant: A Case Report

Norliza Ali, Paediatric Institute, Kuala Lumpur Hospital, Malaysia Liang Choo Hung, Paediatric Institute, Kuala Lumpur Hospital, Malaysia

Hasri Samion, National Heart Institute, Kuala Lumpur, Malaysia

Introduction: Congenital coronary artery fistula is a rare anomaly defined as a direct communication between the coronary artery and any surrounding cardiac chamber or vascular structure. Transcatheter closure of the fistula has been advocated as an effective alternative to surgical repair. It has been associated with uncommon complications, such as transient ischaemic electrocardiographic changes, arrhythmias, device embolization, myocardial infarction, and rarely death.

Case Report: We described here a case of congenital right coronary artery - right ventricle fistula which was diagnosed postnatally. The patient developed overt heart failure soon after birth which was difficult to manage medically. He underwent successful transcatheter occlusion of the fistula with vascular plug at the age of 50 days old and was put on aspirin soon after the procedure. He developed symptoms of myocardial ischaemia nine days after the procedure with electrocardiography changes, raised cardiac enzyme, and well organised thrombus in the right coronary artery with poor myocardial contractility on echocardiogram. He responded to antithrombolytic therapy with ECG resolution followed by resolution of the thrombus. At follow up, he remained well with normal ECG and good myocardial contractility.

Conclusion: Congenital coronary artery fistula is a rare anomaly which can be successfully managed with transcatheter intervention. However, the patient needs to be closely followed up for possible complications post procedure.

\section{8}

Early Result Of Stage I and II Hybrid Approach For Hypoplastic Left Heart Syndrome In Saudi Arabia Mashail A Alobaidan, Prince Sultan Cardiac Center, Saudi Arabia Jassim Abdulhameed, Prince Sultan Cardiac Center, Saudi Arabia Abdulroof Alsaedi, Prince Sultan Cardiac Center, Saudi Arabia

Background: Till recently hypo plastic left heart syndrome (HLHS) is considered the most congenital heart disease with very bad prognosis and outcome, the results remain suboptimal for the traditional staged repair of these patients.

Utilizing surgical and transcatheter techniques, a hybrid strategy was developed to achieve the Fontan circulation.

Method \& Result: Total of 13 newborns underwent hybrid stage I for hypoplastic left heart syndrome (HLHS) between May 2005 and Feb 2007, mean age is 11 days (6-24 D), mean weight is $3.1 \mathrm{~kg}(2.3-3.7 \mathrm{~kg})$, male : female ratio is $1.6: 1$, median hospital stay is 10 days $(7-51 \mathrm{D})$, median ventilatory support is 4 days (3-12 D), all except one need inotropic support for few days. Mortality is $23 \%$. 
7 patients achieved stage II, mean age is 7.9 mo (7-12 mo), mean weight is $6.7 \mathrm{~kg}(5.6-8.7 \mathrm{~kg})$, median ventilatory support is 11 days (4-17 days), median hospital stay is 23 days (8-30 days). One patient Complicated with LPA occlusion needing LPA stent implantation. One death (14.3\%). 6 patients are pending the stage III.

Conclusion: Though small number and short experience, the result of hybrid approach in our center is good and comparable to other center and it can achieve acceptable short term outcomes in patient with HLHS .Therefore we recommend doing it and with improving learning curve we believe it will be considered as a replacement or an alternative to Norwood operation.

8059

Experience of Transcathetr Completion of Fontan In Prince Sultan Cardiac Center (Saudi Arabia) Mashail A Alobaidan, Prince Sultan Cardiac Center, Saudi Arabia Jassim Abdulhameed, Prince Sultan Cardiac Center, Saudi Arabia Atif Alsahari, Prince Sultan Cardiac Center, Saudi Arabia

Introduction: Fontan surgery and its modifications are wellrecognized palliation for univentricular types of heart lesions. Until recently the surgical route was the only reasonable approach, but nowadays palliation can be achieved by combined surgical and transcatheter approaches, which offer good immediate and early results for the high-risk patient.

Method: 7 patients underwent stage I (hemifontan) which is purely surgical, $3(48.2 \%)$ of them underwent stage II transcatheter completion of Fontan using uncovered stent and ASD II device closure of the Fenestration, 2 of them (66.6\%) has adjunctive closure of pulmonary forward flow using Amplatzer muscular VSD device closure.

Result: Mean age 6.8 year, mean weight is $20 \mathrm{~kg}$, median hospital saty is 2 days, median follow up 1 year, no immediate or early complication, rhythm is sinus, no reintervention.

Conclusion: We conclude that Trans catheter completion of Fontan approach in selected patient is an alternative approach.

\section{0}

Single Center Experience of PDA Stent In Saudi Arabia Mashail A Alobaidan, Prince Sultan Cardiac Center, Saudi Arabia Jassim Abdulhameed, Prince Sultan Cardiac Center, Saudi Arabia Atif Alsahari, Prince Sultan Cardiac Center, Saudi Arabia Tarek Moamenah, Saudi Arabia

Background: Transcatheter PDA stenting is considered now as amodality to improve the pulmonary blood flow, as an alternative to Surgical aortopulmonary shunt in a selected patients who are suitable for the procedure.

Method: Between Jan 2005 to Oct 2008, 51 patients underwent PDA stenting in PSCC after full assessment by echocardiogram and angiogram 30 (59\%) patient have PA \& VSD 19 of them with 2 ventricles and the remaining 11 with single ventricle morphology, 13 (25\%) patients have TOF, 8 patients (16\%) have PA \& IVS, 5 patients have Laser wire/RF perforation of the pulmonary valve same time. Median age is 8 days, median weieght is $3.2 \mathrm{~kg}$, procedure is prograde or retrograde.

Result: Mean ventilatory support is 2 days, median hospital stay is 5 days, median saturation is $79 \%$, median follow up is 18 mo (1-46mo), median floroscopy time is 17.6 minutes $(7.7-43 \mathrm{~min})$, $6(11.7 \%)$ of them has NEC first few days post stenting, $4(7.8 \%)$ has stent migration, 4 (17.8\%) lost follow up, 2 (3.9\%) deaths, Conclusion: We conclude that PDA stenting is a safe and alternative procedure to Surgical aortopulomnary shunt.
8062

Hybrid Procedure for Hypoplastic Left Heart Syndrome: Adjustable x Fixed PA Bands

Renato S Assad, Hospital Samaritano Sao Paulo - Brazil, Brazil Maria Fernanda S Jardim, Hospital Samaritano Sao Paulo - Brazil, Brazil

Simone F Fontes-Pedra, Hospital Samaritano Sao Paulo - Brazil, Brazil

Marina M Zamith, Hospital Samaritano Sao Paulo - Brazil, Brazil Leonardo A Miana, Hospital Samaritano Sao Paulo - Brazil, Brazil Petronio G Thomaz, Hospital Samaritano Sao Paulo - Brazil, Brazil Ana C Aliman, Hospital Samaritano Sao Paulo - Brazil, Brazil Carlos A Pedra, Hospital Samaritano Sao Paulo - Brazil, Brazil

Objective: We present our initial clinical experience with hybrid procedure for HLHS, comparing the use of fixed $\mathrm{x}$ adjustable PA bands.

Methods: Between September 2004 and December 2008, we have performed Hybrid Stage I palliation for HLHS using adjustable $\mathrm{PA}$ bands (APAB group) in 3 patients weighing between $1.8-2.8 \mathrm{~kg}$ and traditional bands (TPAB group) in 3 patients weighing between $2.0-3.3 \mathrm{~kg}$. Implantation of the APAB was straightforward from the technical standpoint. Fine adjustments of the pulmonary blood flow could be easily achieved percutaneously.

Results: The first APAB case demised during to interstage 1-2 (65 days old) due to atrioseptostomy complications. The second APAB case successfully completed the Fontan circulation at 2 years of age, and the third patient has reached the second stage (intraoperative death due to hemorrhagic complications). Regarding TPAB group, the first patient died at six months of life due to pulmonary infection and the two other cases underwent the second stage operation (intra and postoperative deaths). The graph shows the $\mathrm{O} 2$ sat behavior of both groups during interstage 1-2.

Conclusions: Customization of the pulmonary blood flow seemed to result in a more precise balance between the pulmonary and systemic circulations during the inter-stage period. The use of adjustable PA bands in the hybrid procedure for HLHS may favor a more stable condition of the patients during interstage 1-2, according to the rapid somatic growth and underlying clinical needs of the patient.

\section{3}

Fetal Atrial Septostomy for Hypoplastic left heart Syndrome: Postnatal Outcome and late Pulmonary Vascular Morphological Assessment

Renato S Assad, Hospital Samaritano Sao Paulo, Brazil, Brazil Carlos A Pedra, Hospital Samaritano Sao Paulo, Brazil, Brazil Fábio Peralta, Hospital Samaritano Sao Paulo, Brazil, Brazil Simone F Fontes-Pedra, Hospital Samaritano Sao Paulo, Brazil, Brazil Maria Fernanda S Jardim, Hospital Samaritano Sao Paulo, Brazil, Brazil

Marina M Zamith, Hospital Samaritano Sao Paulo, Brazil, Brazil Ana C Aliman, Hospital Samaritano Sao Paulo, Brazil, Brazil Vera D Aiello, Laboratory of Pathology, Heart Institute University of São Paulo Medical School, Brazil

Objective: The association of hypoplastic left heart syndrome (HLHS) and restrictive atrial septum (RAS) carries a bad prognosis, related to profound cyanosis after birth. Relief of pulmonary hypertension in the fetal life may attenuate secondary pulmonary abnormalities. We report the outcome of a fetus with HLHS and highly RAS, submitted to intrauterine 
atrioseptostomy. Pulmonary structural changes were evaluated 6 months after birth.

Methods: Fetal atrioseptostomy was carried out at 33 weeks' gestation. Atrial septum was dilated with a balloon catheter, relieving left atrial hypertension. The fetus was delivered at term, with stable hemodynamics and respiratory function. Hybrid procedure was carried out on the 5th day of life, with PDA stent and adjustable pulmonary artery bands. Patient developed a restrictive atrial communication at 3 weeks of life, requiring radio frequency septal access to implant a stent across the atrial septum. At 6 months, a comprehensive stage 2 procedure was performed.

Results: Patient did not survive stage 2 procedure due to hemorrhagic complications. Lung biopsy revealed signs of persistent edema of the interlobular septa, besides focal thickenning of the alveolar walls. There was thickening of the vein wall, with duplication of the elastic lamina, and lymphatic dilation as well. The arterial bed showed only mild hypertrophy of the medial layer. Conclusions: Fetal atrioseptostomy allowed a relief of pulmonary venous hypertension, and probably alter secondary pulmonary vascular changes, since good respiratory function and stable hemodynamics were evident postnatally. The ideal timing of the procedure to completely reverse associated pulmonary abnormalities remains unclear.

\section{4}

Does experience make a difference in percutaneous closure of atrial septal defects?

Mehnaz Atiq, Aga Khan University Hospital, Pakistan

Sohail A Khan, Aga Khan University Hospital, Pakistan

Fateh A Tipu, Aga Khan University Hospital, Pakistan

Muhammed Hamid, Aga Khan University Hospital, Pakistan

Mansoor Khan, Aga Khan University Hospital, Pakistan

Muneer Amanullah, Aga Khan University Hospital, Pakistan

Percutaneous closure of atrial septal defects (ASD) is a safe alternative to surgery. We undertook a study to evaluate how the learning curve and experience has affected percutaneous ASD closure in our centre.

Methods: Ninety two patients underwent attempted percutaneous closure of ASDs over last 5 years. There were 38 males. Patients were divided into 2 groups "A" (initial) (\$2 $\$ tfrac $\{1\}\{2\} \$$ years) and "B" (subsequent ) $(\$ 2 \backslash$ tfrac $\{1\}\{2\} \$$ yrs) representing early and late experience of the operator. There were 35 patients in group A and 57 patients in group B.

Results: The mean age in group A was $29 \pm 14$ (range $8-56$ yrs) and $17 \pm 11$ yrs (range $0.5-54$ yrs) in group B. The mean size of the ASD on transesophageal echocardiogram was $20 \pm 8 \mathrm{~mm}$ in group $\mathrm{A}$ and $22 \pm 12 \mathrm{~mm}$ group $\mathrm{B}(\mathrm{p}=0.03)$. Mean $\mathrm{QP} / \mathrm{QS}$ in group $\mathrm{A}$ was $2.1 \pm 0.8$ and $2.7 \pm 1.8$ in group $\mathrm{B}$. Balloon sizing was done in all patients in group A and 24 patients in group B. Ratio of device size to TEE size of defect was $1.45 \pm 0.2$ in group $A$ and $1.2 \pm 0.08$ $(p=0.04)$. There were failure of device placement in 2 patients in Group A and 3 in group B. Device embolization occurred in 2 patients in group B and none in group A. Atrial fibrillation occurred in 3 patients in Group A and 1 in Group B.

Conclusion: Our study showed closure of larger ASDs, younger patient age and use of relatively smaller device to defect ratio with experience. Embolizations were seen only in group B.

\section{6}

Challenges of management of infantile critical aortic and pulmonary stenosis

Vijayalakshmi Balekundri, Sri Jayadeva Institute of Cardiology,

Bangalore, Karnataka, India
Chitra Narasimhan, Sri Jayadeva Institute of Cardiology, Bangalore, Karnataka, India

Anurakti Srivastava, Sri Jayadeva Institute of Cardiology, Bangalore, Karnataka, India

Usha M Kodandaram Sastry, Sri Jayadeva Institute of Cardiology,

Bangalore, Karnataka, India

Transcatheter balloon valvuloplasty in critical infantile aortic and pulmonary stenosis is difficult. Mortality with AS is as high as $25 \%$. Hence many centers prefer surgery.

Aim: to evaluate the success of balloon valvuloplasty in infants with coronary hardware.

Material \& Methods: Out of 175 cases of ABV, 11 were infants $(6.3 \%)$. Out of 365 of PBV, 44 were infants $(12 \%)$. Aortic valve was successfully crossed in all with Shinobi $0.014 \times 180$ guide wire with hydrophilic coating and radio opaque tip. Pulmonary valves were crossed with high torque floppy $0.014 \times 190$ guide wire, with loop in RA. Dilatation done with 1.5, 2.5, $4 \mathrm{~mm}$ PTCA balloons and $6 \mathrm{~mm}$ Tyshak balloons.

Results: ABV was successful in all cases. Youngest, 14 hours old hydrops foetalis neonate on ventilator. Echo showed pin point critical AS with EF-30\%, gradient $36 \mathrm{~mm} \mathrm{Hg}$, post ABV with $4 \mathrm{~mm}$ PTCA balloon, EF improved to $60 \%$, after 48 hours neonate was off ventilator. $1.8 \mathrm{kgs}, 2$ days old, was youngest case of PS. 8 cases of TOF with severe hypoxemia with mean $\mathrm{SO} 2-48+8 \%$. After PBV, SO2 improved to $85+5 \%$. PV peak Grdt. $-100+2$ came down to $24+6 \mathrm{~mm} \mathrm{Hg}$. Successful in all cases with only one mortality (1.8\%). Loss of femoral pulse in 2 cases.

Conclusions: BV though riskiest and demanding is literally life saving in infants. Usage of better hardware and PTCA balloons have significantly reduced the morbidity and mortality in infants.

\section{8}

Transcatheter closure of shunt anomalies: experience in a Bangladeshi center Fatema N N Begum, Bangladesh Army, Bangladesh

Background: Congenital heart disease occurrence is 25/1000 live birth in Bangladesh which is very high compared to many countries. About $48 \%$ of CHD is contributed by VSD, ASD and PDA. We have started closure of PDA in our country back in 1999, ASD in 2001 and VSD in 2004.

Objective: Objective of this study is to show the outcome of these procedures in a developing country like Bangladesh where resources are limited.

Methods: This is a retrospective study carried out in pediatric cardiology unit of Combined Military Hospital, Dhaka from January 1999 to November 2008. All the patients who were taken into the cath lab with an intention of device closure are included in the study.

Results: Among a total of 582 cases, 180 were attempted for ASD device closure where 169 (93.89\%) cases were successful. VSD device closure was attempted in 32 cases and 30 (93.75\%) were successful. PDA coil/device closure was attempted in 370 cases and 363 of them were successful (98.1\%). In ASD and VSD cases there was one case of embolization for each.

5 cases of PDA coil embolization was experienced which were later removed and a bigger device was implanted.

All patients were discharged 24 hours after procedure excepting VSD cases in which discharge followed 72 hours after procedure. Conclusion: Device Closure of shunt anomalies is an excellent modality of treatment for closing ASD, VSD and PDA. Outcome of these interventions are excellent in out center and we never encountered any complications during follow up of these cases. 
8069

Symptomatic tachy- and bradyarhythmias after transcatheter closure of interatrial communications with Amplatzer devices

Jacek Bialkowski, Silesian Center fort Heart Diseases, Poland Malgorzata Szkutnik, Poland

Jacek Kusa, Poland

Introduction: Transcatheter closure of interatrial communications (ASD) with Amplatzer occluders (ASO) became standard treatment in many centers. We analyzed the incidence of cardiac arrhythmias after such treatment.

Methods: The group of 759 patients (pts) after transcatheter closure of ASD with ASO were retrospectively analyzed. Only pts with a new and symptomatic arrhythmias were included to the study. All pts, who had arrhythmias prior to ASD closure, were excluded. Results: New tachy- and bradyarhtymias after implantation of ASO were observed in $11 \mathrm{pts}(1,5 \%)$. There were 9 pts (mean age 36,7 y) with atrial tachyarhythmias (AF in 8 and SVT in $1 \mathrm{pt}$ ), which appeared between 1 day and 3 months after implantation. Seven pts were treated initially by pharmacotherapy; in 2 of them sinus rhythm returned just after cardioversion. In another 2 cardioversion was performed as initial therapy. In non but one recurrence of tachycardia was observed, however 7 of them had prolonged (till one year) pharmacotherapy. In 2 pts aged 15 and 16 years complete a-v block appeared 4,3 and 1,5 year after ASO implantation respectively. In the first one intermittent II degree a-v block (Mobitz II) was observed before ASD closure. DDDR pacemaker was implanted in both pts.

Conclusions: Transcatheter closure of ASD with ASO is associated with the risk of new atrial tachyarythmias (usually early after the procedure and in older patients). The risk of complete heart block is low, but it can appear in late follow-up. Thence close long term follow-up of all patients is obligatory.

\section{0}

Patent ductus artieriosus (PDA) in lowland and highland patients

Jacek Bialkowski, Silesian Center for Heart Diseases, Zabrze, Poland Carlos Zabal, Instituto Nacional de Cardiologia, Mexico City, Mexico Ramon Bermudez-Canete, Hospital Ramon y Cajal, Madrid, Spain Ramon Flores, Hospital CNM 20 de Noviembre ISSSTE, Mexico City, Mexico

Hernan Diaz, Hospital UNICAR, Guatemala City, Guatemala Ramiro Menacho, Hospital Obrero CNS, La Paz, Bolivia Jaqueline Kreutzer, Children Hospital, Pittsbourgh, United States

Objective: To compare the physiopatomorphology of PDAs in 1367 patients (pts) living in lowland (at altitude 0-700 m) Group L and highland 1500-4200 $\mathrm{m}-$ Group H.

Method: The hemodynamic data of 670 pts from Group H(age $9,5+/-12,8$ y) and 697 from Group $\mathrm{H}$ (age 8,2+/-19,7 y) were analyzed. No preliminary selection of the pts with PDA were made.

Results: The diameter of the PDA was smaller and the mean pulmonary artery pressure was lower in Group L compared to Group H: $2,3+/-1,2$ vs 4,1 +/-1,2 mm $(\mathrm{p}<0,001)$ and 17,9 $+/-5,9$ vs $25,5+/-12,3 \mathrm{mmHg} \quad(\mathrm{p}<0,001)$. PDA types (according Kirchenko) seen in Group L and Group $\mathrm{H}$ were respectively present as follows: type A $57,2 \%$ vs $81,2 \%$ ( $\mathrm{p}<0,001$, type B $4,5 \%$ vs $2,9 \%$, type C $4,9 \%$ vs $2,6 \%$, type D $7,9 \%$ vs $3,2 \% \quad(p<0,001)$ and type $\mathrm{E} \quad 25,5 \%$ vs $10,2 \%$ $(p<0,001)$. According to morphology of PDA $68,1 \%$ of pts in Group L were treated with coils, 25,1\% with Amplatzer Duct
Occluders (ADO), 5,5\% with Rashkind or Starflex umbrellas. In Group H 92,5. \% pts were treated with ADO and 7,5\% received coils. In both Groups in two pts Amplatzer Muscular VSD Occluder was implanted.

Conclusions: PDAs in patients living at high altitude have a different anatomical and hemodynamic features compared to those living at lowland. This finding has important implication for further strategy of trans-catheter closure at different sites.

\section{1}

Cryo-balloon Angioplasty of Pulmonary Vein Stenosis: Successful Therapy or "Freezer Burn"

Michael A Bingler, The Children's Hospital Denver, United States

Jeffrey R Darst, Children's Hospital Boston, United States

David T Balzer, St. Louis Children's Hospital, United States

Thomas E Fagan, The Children's Hospital Denver, United States

Objective: Current therapy for most forms of pulmonary vein stenosis (PVS) is less than satisfactory due to recurrent, progressive re-stenosis and neointimal proliferation. We sought to determine the safety and effectiveness of cryo-balloon angioplasty (CbA) for PVS in pediatric patients.

Methods: We reviewed the catheterization database, hospital records, imaging studies and pathologic specimens in all patients who had pulmonary vein (PV) CbA using Boston Scientific PolarCath TM peripheral dilation system.

Results: Ten patients (13 PVs) had CbA from 8/06-12/08. Median age 18 months (4 mo-18.5 yrs), weight $8.9 \mathrm{~kg}$ (3.8-47.7). Five had additional structural heart lesions (2 repaired TAPVR). Three PVs had previous surgical and 4 previous transcatheter therapy. Median balloon size/stenosis diameter and balloon size/normal vessel diameter ratios were 2.69 (1.82-3.33) and $1.2(0.87-2.07)$. Results are summarized below. Six patients (11 PVs) had follow up cath 6 months $( \pm 4.1)$ later. Improved PVS diameter was maintained in only 2 PVs. Two veins had restenosis but maintained diameter greater than before initial $\mathrm{CbA}$. All vessels had repeat intervention with acute relief of PVS. Stroke occurred within 24 hours of $\mathrm{CbA}$ in one patient. One patient underwent lung transplantation after repeat non-CbA and PV stent. Two patients died; one of progressive PVS 2.5 months after initial $\mathrm{CbA}$ and one of unknown cause 3 months after second CbA.

Conclusion: Cryo-balloon angioplasty of pulmonary vein stenosis is safe and results in acute relief of stenosis. However, cryoballoon angioplasty appears minimally effective as sole therapy in maintaining long term relief of pulmonary vein stenosis.

\begin{tabular}{lccccc}
\hline & $\begin{array}{c}\text { Stenosis } \\
\text { Diameter } \\
(\mathrm{mm})\end{array}$ & $\begin{array}{c}\text { Mean } \\
\text { Gradient } \\
(\mathrm{mmHg})\end{array}$ & $\begin{array}{c}\text { Stenosis: } \\
\text { Normal } \\
\text { Vessel Ratio }\end{array}$ & $\begin{array}{c}\text { Stenosis } \\
\text { Diameter at } \\
\text { Follow Up } \\
(\mathrm{mm})\end{array}$ & $\begin{array}{c}\text { Mean } \\
\text { Gradient at } \\
\text { Follow Up } \\
(\mathrm{mmHg})\end{array}$ \\
\hline $\mathrm{N}$ & 13 & 13 & 13 & 11 & 11 \\
Pre & $2.4( \pm 0.6)$ & $12.3( \pm 6.9)$ & $0.55( \pm 1.9)$ & & \\
Post & $4.1( \pm 1.1)$ & $3.6( \pm 2.9)$ & $0.98( \pm 3.6)$ & $5.0( \pm 2.1)$ & $2.55( \pm 2.2)$ \\
$\begin{array}{l}\text { F/U } \\
\text { P value }\end{array}$ & $<0.001$ & 0.001 & $<0.001$ & $\begin{array}{c}2.67( \pm 1.2) \\
0.003\end{array}$ & $\begin{array}{c}13.1( \pm 10.0) \\
0.017\end{array}$ \\
\hline
\end{tabular}

8072

Transapical left ventricular access for difficult to reach interventional targets in the left heart

Derize E Boshoff, UZ Leuven, Belgium

Stephan Brown, Bloemfontein, South Africa

Filip Rega, UZ Leuven, Belgium 
Werner Budts, UZ Leuven, Belgium

Hein Heidbuchel, UZ Leuven, Belgium

Marc Gewillig, UZ Leuven, Belgium

Objective: Interventional targets may be "excluded" due to vascular problems or complex surgery. This study reviews our experience using transapical ventricular puncture.

Patients: Patient 1 (74y, 2 sternotomies), patient 2 (66y, 5 sternotomies) and patient 5 (69 y, 3 sternotomies) had paravalvular mitral valve leaks. Patient 3 (6.3y, 2 sternotomies) extracardiac Fontan, residual leak after 2 surgical attempts of patch closure of regurgitant atrioventricular valve. Patient 4 (10mo) had failure of standard ablation of the posteroseptal region of the mitral valve with persistent life-threatening ventricular tachycardia.

Methods: general anesthesia. Entry: LV apex percutaneous 3; mini-thoracotomy 2 (1 conversion). 1-3 6Fr sheaths were placed, followed by the procedure as required. Direct surgical closure of the puncture site was done in 4 patients and in patient 3 , a percutaneous vascular occlusion device was used.

Results: Easy and immediate access was obtained in all patients. The paravalvular leaks were crossed within seconds and completely closed with 1-3 Amplatzer occluders. In patient 3 the valve was crossed using a Brokenbrough needle and a $12 \mathrm{~mm}$ Amplatzer device was placed in the patch leak. Patient 4 was successfully ablated using a $7 \mathrm{Fr}$ irrigated catheter endo- and epicardially. Complications were in the percutaneous puncture group only: coronary artery puncture 1 , hemothorax 1 , both treated percutaneously.

Conclusion: Direct left ventricular puncture offers a very useful alternative access site in selected patients to reach "inaccessible" targets for certain percutaneous interventions in patients where standard approaches may be impossible or difficult.

8073

Hybrid Approach As Bridge To Biventricular Repair In A Neonate With Critical Aortic Stenosis And Borderline Left Ventricle

Derize E Boshoff, UZ Leuven, Belgium

Stephan Brown, Bloemfontein, South Africa

Benedicte Eyskens, UZ Leuven, Belgium

Ruth Heying, UZ Leuven, Belgium

Marc Gewillig, Dept. Paed. Cardiol., UZ - KU Leuven ; B-3000

Leuven, Belgium, Belgium

Introduction: Critical neonatal aortic stenosis remains a difficult condition to manage. Small left ventricular size, poor left ventricular function and the presence of endocardial fibroelastosis are all associated with higher morbidity and mortality. Methods: A 4,2 kg newborn of mother with poorly controlled insulin-dependant diabetes mellitus presented with poor circulation and critical aortic stenosis. Initial $6 \mathrm{~mm}$ balloon dilatation was successful, but the infant tolerated biventricular circulation poorly: small left ventricular volume and significant pulmonary hypertension; a single ventricle hybrid approach was decided upon. This strategy would buy some time and allow the left ventricle potentially to grow.

Report: At day 25 : Rashkind and duct stented 9/19 mm, followed by $4 \mathrm{~mm}$ Goretex bands on branch pulmonary arteries. At 8 months, test occlusion of the ductus and interatrial septum indicated that biventricular repair was possible. The bands were progressively dilated to $8 \mathrm{~mm}$ and the ductus closed with a $4 / 6$ Amplatzer ductal occluder and $8 \mathrm{~mm}$ Amplatzer vascular occlusion device. At 3.2 years he is doing well with a biventricular circulation and small residual interatrial shunt.
Conclusion: Hybrid procedure may be considered in neonates with borderline left heart as a bridge to possible biventricular repair. Biventricular repair may be achieved by percutaneous intervention avoiding further surgery.

\section{4}

Coarctation of the aorta treated with the new Advanta V12 LD stent. Acute results

Elchanan Bruckheimer, Schneider Children's Medical Center, Israel

E Birk, Schneider Children's Medical Center, Israel

Sergio LN Braga, Instituto Dante Pazzanese de Cardiologia, Brazil T Dagan, Schneider Children's Medical Center, Israel

Cesar A Esteves, Instituto Dante Pazzanese de Cardiologia, Brazil

Carlos AC Pedra, Instituto Dante Pazzanese de Cardiologia, Brazil

Although CoA stenting has become popular, some safety and technical issues still remain including risks of aneurysm formation, aortic rupture and vascular injury. We report the early clinical results of CoA stenting using the new ePTFE covered Advanta V12 LD stent (Atrium) in 2 centers. This stainless steel open-cell design stent comes premounted on 12, 14 and $16 \mathrm{~mm}$ balloons and requires a 9-11Fr delivery system. Two layers of a thin ePTFE membrane cover the stent in its inner and outer portions. From 09 to 12/2008, 13 patients (median age and weight: 14.5 years and $44.5 \mathrm{kgs}$, respectively) with either native CoA (10) or ReCoa (3) underwent the procedure under general anesthesia. Balloons of a diameter sufficient to anchor the stent in the coarctation site were selected using the smallest available delivery system. Secondary dilation with larger diameter balloons was performed until the pressure gradient was less than $20 \mathrm{mmHg}$ and the stent was apposed to the isthmus wall. Coarctation diameter increased from $5.2 \pm 2.9$ to $14.4 \pm 2.9 \mathrm{~mm}[\mathrm{p}<0.01]$. Peak pressure gradient decreased from $29.4 \pm 13.2$ to $2.2 \pm 3.4 \mathrm{mmHg}[\mathrm{p}<0.001]$. The stent achieved the desired diameter in all cases. There were no complications. At shortterm median follow-up of 0.8 months all patients are alive and well with no evidence of recoarctation. These initial results show that the use of the new covered Advanta V12LD stent through a low profile delivery system is feasible, safe and effective in the immediate treatment of CoA. Long term follow up is required.

\section{5}

Cardiac catheterisation in infants and children on Extra Corporeal Membrane Oxygenation (ECMO) support Abdul Rasheed, Glenfield Hospital, Leicester, United Kingdom Mohammad D Khan, Glenfeld Hospital, United Kingdom Frances A Bu'Lock, Glenfield Hospital, Leicester, United Kingdom Abdul K Duke, Glenfield Hospital, Leicester, United Kingdom Magdi Tofeig, Glenfield Hospital, United Kingdom Richard K Firmin, Glenfield Hospital, Leicester, United Kingdom Giles J Peek, Glenfield Hospital, Leicester, United Kingdom

Cardiac catheterisation on a patient on ECMO support is a challenging procedure. However timely catheterisation is important for interventional procedures such as decompressing the left atrium or for critical anatomic/physiological information, especially when ECMO weaning is difficult. We reviewed our experience of catheterising infants and children receiving ECMO support.

Patient and Methods: Retrospective case note review of all paediatric patients undergoing cardiac catheterisation while on ECMO support, between August 2004 and May 2008.

Results: Please see the attached table 
Outcome: Significant abnormality was identified in 6 patients who underwent diagnostic cardiac catheterisation and all of them had subsequent cardiac surgery and came off ECMO. However two patients died subsequently. All patients who underwent radiofrequency ablation made full recovery of their ventricular function. Among the patients with cardiomyopathy who required creating or enlarging inter-atrial communication, one patient required cardiac transplant, the second patient required Berlin heart support for a month and was successfully weaned off and the third had successful surgical repair of abnormal coronary artery anatomy.

Complications from cardiac catheterisation were rare. One patient had excessive bleeding from the vascular access site and another patient had transient desaturation

Conclusion: Both diagnostic and interventional cardiac catheterisation of patients during ECMO support is possible and safe. It is mandatory and should be considered early in patients who fail to wean from ECMO for cardiac support particularly following surgery. Early atrial septostomy for left heart venting is essential to prevent lung consolidation and facilitate recovery of the poorly functioning left ventricle.

\begin{tabular}{|c|c|c|c|}
\hline $\begin{array}{c}\text { Total number of } \\
\text { patients - } 13\end{array}$ & $\begin{array}{l}\text { Life threatening } \\
\text { arrhythmia - } 3\end{array}$ & $1-4$ years -4 & $\begin{array}{l}\text { Radiofrequency } \\
\text { ablation for } \\
\text { arrhythmia-3 }\end{array}$ \\
\hline $\begin{array}{l}\text { Total number of } \\
\text { procedures - } 14\end{array}$ & $\begin{array}{l}\text { Respiratory } \\
\text { distress/Sepsis }\end{array}$ & $\begin{array}{l}\text { Diagnostic } \\
\text { catheterisation - } 7\end{array}$ & $\begin{array}{l}\text { Stenting of branch } \\
\text { pulmonary } \\
\text { arteries-1 }\end{array}$ \\
\hline $\begin{array}{l}\text { Congenital heart } \\
\text { disease }-7\end{array}$ & Newborn -4 & \multicolumn{2}{|l|}{$\begin{array}{l}\text { Interventional } \\
\text { procedures - } 7\end{array}$} \\
\hline $\begin{array}{l}\text { Dilated } \\
\text { Cardiomyopathy-2 }\end{array}$ & $\begin{array}{l}\text { Infant }(1-12 \\
\text { months })-5\end{array}$ & \multicolumn{2}{|c|}{$\begin{array}{l}\text { Creating/enlarging inter atrial } \\
\text { communication - } 3\end{array}$} \\
\hline
\end{tabular}

\section{6}

Percutaneous closure of multiple ASD: results and long-term follow-up

Gianfranco Butera, Policlinico San Donato IRCCS, Italy Enrico Romagnoli, Policlinico San Donato IRCCS, Italy Massimo Chessa, Policlinico San Donato IRCCS, Italy Luciane Piazza, Policlinico San Donato IRCCS, Italy Angelo Micheletti, Policlinico San Donato IRCCS, Italy Diana Negura, Policlinico San Donato IRCCS, Italy Carmelo Arcidiacono, Policlinico San Donato IRCCS, Italy Mario Carminati, Policlinico San Donato IRCCS, Italy

Aim: To assess feasibility of percutaneous treatment of multiple ASDs.

Methods: Between 1998 and 2007, 165 out of 1280 consecutive patients undergoing ASD percutaneous closure at our institution showed multiple defects. According to the septum anatomy and the clinical characteristics multiple ASDs were classified in four categories: double atrial septal defects (d-ASD), multi-fenestrated atrial septal defects (f-ASD), multi-fenestrated defects with no signs of right heart overload (f-PFO), and complex cases (c-ASD). End-points were: 1) immediate procedural success; 2) long term safety and efficacy.

Results: In this study, up to $81 \%$ of multiple ASDs was suitable for percutaneous closure. Accurate ASD morphology assessment and appropriate device selection were key elements to obtain procedural success. Multiple device implantations were required in $47 \%$ of cases, especially in patients with d-ASD and c-ASD. Complication rate, residual shunt and long term outcome were comparable among the four different categories. In particular, at long term follow-up ( $6 \pm 1.8$ years) no patient required further surgical or percutaneous treatment and complete closure was confirmed in $98 \%$ of cases.
Conclusions: Percutaneous closure of multiple ASDs is feasible and associated with a good outcome. A thorough identification and analysis of morphological aspects are mandatory in order to select the appropriate device and the optimal strategy. The implantation of multiple devices does not seem to be associated to an increased risk of complications at long term follow up.

\section{7}

Long-term follow-up of percutaneous closure of secudnum atrial septal defect

Gianfranco Butera, Policlinico San Donato IRCCS, Italy

Tommaso Infusino, Policlinico San Donato IRCCS, Italy

Paolo Ferrero, Policlinico San Donato IRCCS, Italy

Mario Carminati, Policlinico San Donato IRCCS, Italy

Alessandro Frigiola, Policlinico San Donato IRCCS, Italy

Sara Foresti, Policlinico San Donato IRCCS, Italy

Massimo Chessa, Policlinico San Donato IRCCS, Italy

Riccardo Cappato, Policlinico San Donato IRCCS, Italy

Objectives: To study long-term results of percutaneous closure of secundum ASD.

Methods: Between January 2000 and January 2004, 333 (111 males) patients underwent percutaneous closure of secundum ASD. All patients were included in the present study and followed up. Median age at implantation was 30 years (2-81). Comorbidities were present in 84 subjects (26\%). Median NYHA class was I (range I-IV). Mean ASD diameter was $21 \pm 6 \mathrm{~mm}$. Qp/Qs ratio and sistolic pulmonary pressures were $2 \pm 0.5$ and $16 \pm 4 \mathrm{mmHg}$, respectively.

Results: Closure was performed by using an Amplatzer device in 289 pts $(86 \%)$ and a StarFlex device in $44(14 \%)$. Procedure and fluoroscopic times were $68 \pm 28$ and $12 \pm 9$ minutes, respectively. Complex ASD (Multiple ASD, ASD+atrial septal aneurysm) were treated in 48 pts $(15 \%)$.

Procedure was successfully performed in 317 pts (95\%). Significant early complications (within 1 month) occurred in pts $11(3.3 \%)$ (device embolization needing cardiac surgery, cardiac tamponade, intraoperative TIA, device thrombosis). Patients were followed up for $6+/-1$ years. Complete closure was shown in all patients. We reported no case of late device embolization, thrombus formation, endocarditis. NYHA class improved significantly (NYHA I pre-procedure: $60 \%$ versus $85 \%$ post-procedure; $\mathrm{p}=0.001)$. Arrhythmias improved after closure (pre-procedure: $16 \%$ versus post-procedure: $10 \% ; p=0.04$ ). Late reintervention was needed in 5 pts $(1.5 \%)$ : occurrence of aortoright atrial fistula (1 pt), development of significant mitral insufficiency $(1 \mathrm{pt})$, vascular surgery due to the occurrence of femoral artero-venous fistula (3 pts).

Conclusions: Percutaneous ASD closure is safe and effective.Early results are stable during long-term follow-up.

\author{
8078 \\ Percutaneous closure of residual post-surgical ventricular \\ septal defects \\ Gianfranco Butera, Policlinico San Donato IRCCS, Italy \\ Jaspal Dua, Policlinico San Donato IRCCS, Italy \\ Massimo Chessa, Policlinico San Donato IRCCS, Italy \\ Luciane Piazza, Policlinico San Donato IRCCS, Italy \\ Diana Negura, Policlinico San Donato IRCCS, Italy \\ Vlasta Fesslova, Policlinico San Donato IRCCS, Italy \\ Alessandro Giamberti, Policlinico San Donato IRCCS, Italy \\ Mario Carminati, Policlinico San Donato IRCCS, Italy
}


Background: Residual VSD may occur after surgery. Redo operation have higher rate of morbidity and mortality.

Patients and methods: Between January 00 and November 08, 162 pts underwent percutaneous VSD closure. Nineteen pts had a residual postsurgical VSD. They were operated on a median of 8 years before the percutaneous procedure. All pts had echocardiographic signs of left ventricle volume overload. The Amplatzer muscular VSD occluder (mVSD) and the eccentric VSD occluder (pmVSD) were used.

Results: Median age at procedure was 18 years (range 1,5-64 yrs). Median weight was $55 \mathrm{kgs}$ (range 9-80 kgs). Median QP/QS was 1,8/1 (range 1,5-2.5). Mean fluoroscopy and procedure times were $41+/-31$ and $135+/-91 \mathrm{~min}$, respectively. The median size of the VSD was $8 \mathrm{~mm}$ (range $6-18 \mathrm{~mm}$ ). In 12 pts a mVSD device was used, while in 7 cases a pmVSD device was implanted. In one subject two procedures and devices were needed. No death occurred. Two pts required immediate external cardioversion due to ventricular tachycardia.

A mild to trivial intraprotesic residual shunt was showed in three pts. All subjects but one were discharged home 48 hours after the procedure. Median follow-up was 18 months (range 2-48 mts). Complete occlusion was shown in all subjects. No late complications occurred. One subject experienced recurrent VSD due to patch dehiscence and finally she was sent to surgical repair. Conclusion: In the current era, percutaneous closure of residual post surgical VSD can be achieved safely and successfully.

\section{9}

Transcatheter closure of congenital ventricular septal defects (VSD) with various devices and coils; early and mid-term results

Ahmet Celebi, Turkey

Abdullah Erdem, Dr Siyami Ersek Hospital for Cardiology and Cardiovascular Surgery, Turkey

Cenap Zeybek, Dr Siyami Ersek Hospital for Cardiology and Cardiovascular Surgery, Turkey

Turkay Saritas, Dr Siyami Ersek Hospital for Cardiology and Cardiovascular Surgery, Turkey

Celal Akdeniz, Dr Siyami Ersek Hospital for Cardiology and

Cardiovascular Surgery, Turkey

Ender Odemis, Dr Siyami Ersek Hospital for Cardiology and

Cardiovascular Surgery, Turkey

Halil Demir, Dr Siyami Ersek Hospital for Cardiology and Cardiovascular Surgery, Turkey

Yalim Yalcin, Dr Siyami Ersek Hospital for Cardiology and

Cardiovascular Surgery, Turkey

Objectives: We sought the safety, efficacy, and follow-up results of transcatheter closure of VSD using different types of devices and coils.

Methods: 47 patients underwent to transcatheter closure of VSD during the last two years. In perimembranous VSDs, left side of the device was inserted either to left ventricular side of the septum or into the aneurysm to avoid AV block.

Results: The median age was $6(0,42-50)$ years. The median Qp/Qs ratio was $1.74(1.2-4.0)$, and the median VSD diameter was $9 \mathrm{~mm}$ $(3.5-20 \mathrm{~mm})$. Implantation was successful in $44(93.6 \%)$ patients with 31 perimembranous, 13 muscular VSDs. 15 Amplatzer muscular and 6 membranous VSD occluders, 9 Nitt-occlud coil (PFM), 7 Cardiofix muscular VSD occluders, 6 Amplatzer duct occluders and one detachable coil (Cook) were used. Complete occlusion rate was $50 \%$ at completion of the procedure, rising to $66 \%$ the day after and $72,7 \%$ at sixth month during the follow-up. No major complication was encountered except transient complete AV block and hemolysis in one patient. Patients that the device implanted into the aneurysm have high residual shunts the day after the procedure, comparing to patients that the left disc of the device inserted to the left ventricular side $(7 / 11$ vs $3 / 20$, respectively, $p=0,006)$.

Conclusions: Transcatheter closure of VSD with different types of devices is an effective and safe procedure. Device insertion into aneurysm is an alternative in perimembranous VSD thought to have decreased possibility of AV block but tend to have increased residual shunt in early period.

\section{0}

A new technique for transcatheter closure of moderate to large PDA using Cook detachable coils: Deployment of all loops in the aortic ampulla

Ahmet Celebi, Turkey

Yalim Yalcin, Dr Siyami Ersek Hospital for Cardiology and

Cardiovascular Surgery, Turkey

Tugcin Bora Polat, Dr Siyami Ersek Hospital for Cardiology and Cardiovascular Surgery, Turkey

Abdullah Erdem, Dr Siyami Ersek Hospital for Cardiology and

Cardiovascular Surgery, Turkey

Ender Odemis, Dr Siyami Ersek Hospital for Cardiology and

Cardiovascular Surgery, Turkey

Cenap Zeybek, Dr Siyami Ersek Hospital for Cardiology and

Cardiovascular Surgery, Turkey

Turkay Saritas, Dr Siyami Ersek Hospital for Cardiology and

Cardiovascular Surgery, Turkey

Elnur Imanov, Dr Siyami Ersek Hospital for Cardiology and

Cardiovascular Surgery, Turkey

Objective: Deploying at least one loop at the pulmonary artery (PA) is recommended to prevent coil migration to the aorta. With increasing experience, we have used a new technique as leaving all loops in the aortic ampulla using transvenous route to close moderate to large PDAs with detachable coils, and compared to standard approach.

Method: 83 of 144 patients who underwent PDA closure with detachable coils, have a minimum PDA diameter of 3- $4.0 \mathrm{~mm}$, were included into the study. Patients were divided into two groups according to leaving coil loop at the PA side (Group I) or not (Group II).

Results: Age, weight and minimum PDA diameter were similar in Groups. However, complete occlusion was higher at catheter laboratory $(28 / 40$ vs $19 / 43, \mathrm{p}=0.018)$ and so additional coil requirement $(1 / 40$ vs $10 / 43, \mathrm{p}=0.003)$ was lower in Group I. The prevalence of residual shunt with time tended to be lower in new technique $(\mathrm{P}=.032)$. Thus, three patients in Group I and one patient in Group II required reintervention at one year. Coil migrations to the PA were encountered $1 / 40$ in Group I and $3 / 43$ in Group II, but not to the aorta. A relative decrease of the left lung perfusion was observed in patients in whom initial coil was deployed with leaving loop at the PA side $(\mathrm{p}=0.018)$.

Conclusions: Coil occlusion of PDAs with leaving all loops in the aortic ampulla is feasible and safe. It is superior in immediate and overall complete occlusion rate with lower complications.

\section{1}

Transcatheter closure of distal aortopulmonary window with Amplatzer duct occluder devices Anpon Bhagyavathy, MIOT Hospital, Chennai, India Kothandam Sivakumar, MIOT Hospital, Chennai, India Ramyashri Chandrasekaran, MIOT Hospital, Chennai, India Radhakrishnan Satish, MIOT Hospital, Chennai, India 
Arunkumar Govindarajan, MIOT Hospital, Chennai, India

Robert Coelho, MIOT Hospital, Chennai, India

Objective: Distal aortopulmonary window (APW) are located away from aortic and pulmonary valves and may be amenable for catheter closures. We present our experience in transcatheter distal APW closure.

Methods: Patients with distal APW and good margin away from semilunar valves are selected on echocardiogram. Carefully chosen patients were taken for closure in catheterization laboratory after informed consent. After ascending aortogram and hemodynamic assessment under sedation with ketamine, APW is cannulated from pulmonary side with an internal mammary catheter and guidewire tip parked in right innominate artery. Through a long sheath from venous access, Amplatzer duct occluder device $2 \mathrm{~mm}$ more than the size of APW is deployed under angiographic control.

Results: 4 patients aged 8 months to 15 years had device closure of distal APW. The body weight ranged from $4.6-30 \mathrm{~kg}$. Two of these patients were operated in past through left thoracotomy for diagnosis of patent ductus arteriosus, but continued to have continuous murmurs and left to right shunt through APW. The pulmonary artery hypertension was mild in two and moderate in rest. The shunt ratio was $2.9+/-1.1$. The device used were $8 \times 6$ in one, $12 \times 10$ in two and $14 \times 12$ in one patient. Echocardiogram at 24 hours showed complete closure with no procedural complications. On a follow up ranging from 6 months to 5 years, there is no residual shunt, no pulmonary artery flow disturbance.

Conclusions: Transcatheter closure of distal APW is a safe and less morbid alternative to surgery in patients appropriately selected on echocardiogram.

\section{2}

Initial palliation in symptomatic patients with complex Tetralogy of Fallot

Zdenka Reinhardt, Birmingham Children's Hospital-NHS Trust, UK, United Kingdom

Oliver Stumper, Birmingham Children's Hospital-NHS Trust, United Kingdom

Paul Miler, Birmingham Children's Hospital-NHS Trust,

United Kingdom

Vinay Bhole, Birmingham Children's Hospital-NHS Trust, United Kingdom

John Stickley, Birmingham Children's Hospital-NHS Trust, United Kingdom

Douglas Wall, Birmingham Children's Hospital-NHS Trust, United Kingdom

John GC Wright, Birmingham Children's Hospital-NHS Trust, United Kingdom

Joseph V De Giovanni, Birmingham Children's Hospital-NHS Trust, United Kingdom

Objective: To evaluate the effectiveness of RVOT stenting in symptomatic neonates and infants with complex TOF physiology. Method: Retrospective data analysis of RVOT stent implantation as a primary procedure between 2005 and 2008.

Results: Eight patients underwent primary RVOT stenting over the 3.7 year period. Four had 2 stents implanted during the first procedure due to instability or inadequate cover of the entire length of the infundibulum. A further 2 required repeat stenting subsequently.

Indications for stenting were severe cyanosis and spells $(n=6)$, TOF with cAVSD $(n=2)$. Four patients suffered from an associated genetic syndrome.
Patient's median age at stenting was 66 days (23-373), median weight $3.8 \mathrm{~kg}(1.75-8 \mathrm{~kg})$. Pulmonary vasculature was hypoplastic in all with a mean pulmonary valve diameter of $4.5 \mathrm{~mm}$, RPA diameter of $3.2 \mathrm{~mm}$ and LPA of $3 \mathrm{~mm}$.

Stents implanted were premounted Liberte coronary balloon expandable stents (Boston Scientific) in all $(n=8)$, peripheral vascular Jomed (Abbott) in 1 and Genesis (Cordis) in 1.

RVOT stenting improved systemic oxygen saturations from a median of $75 \%(40-82)$ to $90 \%(81-97)$ [p $<0.05]$.

There were two major complications: 1 perforation and tamponade requiring emergency RVOT patch surgery, 1 transient pulmonary oedema. There were no deaths. Three patients required reintervention, 2 catheter, 1 surgery. One patient had subsequent complete repair, with the others awaiting further management. Conclusion: Stenting of the RVOT is a feasible and effective palliation and offers a promising new treatment option in the initial management of patients with complex Tetralogy of Fallot.

8083

Transcatheter Closure of Ventricular Septal Defect: an Early Experience

Mulyadi M Djer, University of Indonesia, Jakarta, Indonesia

Sukman T Putra, University of Indonesia, Jakarta, Indonesia

Hasri Samion, Institut Jantung Negara, Kuala Lumpur, Malaysia

Background: Percutaneous transcatheter closure ventricular septal defect (VSD) is recently more popular because of shorter hospitalization, eliminated the need for thoracotomy, and better cosmetic results without scar.

Objective: To evaluate the efficacy and safety of transcatheter closure of VSD.

Methods: A case series of patients who underwent transcatheter closure of VSD at Dr. Cipto Mangunkusumo Hospital, Jakarta, Indonesia.

Results: In our division since February 2002, transcatheter closure of VSD has been done in 14 patients. The VSD consisted of 12 perimembranous VSD that was closed using membranous VSD occluder and the remaining 2 was muscular VSD that was closed using Amplatzer muscular VSD occluder and Amplatzer septal occluder, respectively. Nine of patients were females and 5 males. The age ranged from 3 to 26 years (median 8 years) and the median of body weight was 23.5 (range 14-68) kg. All patients were discharged at following day after procedure. Patients were followedup periodically by clinical and echocardiography to monitor the presence of residual shunt or complication. During procedure and follow-up there was no significant complication occurred and no patient had complete AV block.

Conclusion: Based on our experience we conclude that percutaneous transcatheter closure of VSD is effective and save. Keyword: Ventricular septal defect, Amplatzer occluder.

\section{4}

Acute and late obstruction of a modified BlalockTaussig (BT) shunt: Multi centre experience of different catheter-based methods of treatment

Tomasz Moszura, Department of Cardiology Polish Mother's Memorial Hospital-Research Institute, Lodz, Poland

Maria Zubrzycka, Catheterization Laboratory, Children's Memorial Health Institute, Warsaw, Poland

Pawel Dryzek, Department of Cardiology Polish Mother's Memorial

Hospital-Research Institute, Lodz, Poland

Krzysztof Michalak, Department of Cardiology Polish Mother's

Memorial Hospital-Research Institute, Lodz, Poland 
Bozena Rewers, Catheterization Laboratory, Children's Memorial Health Institute, Warsaw, Poland

Jacek Moll, Department of Cardiosurgery Polish Mother's Memorial Hospital-Research Institute, Lodz, Poland

Andrzej Sysa, Department of Cardiology Polish Mother's Memorial Hospital-Research Institute, Lodz, Poland

Obstruction of modified Blalock-Taussig shunt is serious complication.

The purpose of this study is a retrospective evaluation of the effectiveness of various transcatheter recanalisation methods for the treatment of occlusion or critical stenosis of BT shunts in 24 pts, aged from 2 weeks to 8 years.

All 24pts were accepted for BT due to complicated anatomy of CHD (no cardiac flow to pulmonary arteries in 15/24) and/or pulmonary arteries hipoplasia.

In $12 \mathrm{pts}$ the occlusion was detected in the postoperative period (1-14days), in $10 / 24 \mathrm{pts}$ it occurred during the long-term follow-up.

Methods of BT recanalisation: local rtpa infusion via catheter in BT was performed in all 24pts for short period of time (appr $10 \mathrm{~min}$ ) followed by balloon angioplasty of BT in 22pts and additionally stents implantation to BT in 3pts. Due to severe pulmonary arteries hypoplasia and poor clinical result of BT recanalisation additionally in $1 \mathrm{pt}$ stent was implanted to the pulmonary artery, in $1 \mathrm{pt}$ to RVOT.

The emergency transcatheter BT recanalization was successful in 22/24pts (92\%), with arterial saturation increase (average: $31 \%$; SD $-16 \% ; p<0,001)$. Unrestricted blood flow in the recanalized BT was achieved in 19/24pts. In 2pts new BT shunt was performed due to suboptimal clinical result of recanalisation.

No procedure related complications were observed.

Conclusions:

1) Emergency transcatheter recanalization of occluded BlalockTaussig shunts is safe and effective, so it is an alternative to surgical treatment.

2) The presence of fixed lesions with neointimal hypertrophy significantly reduces the effectiveness of isolated balloon angioplasty and indicates endovascular stent implantation
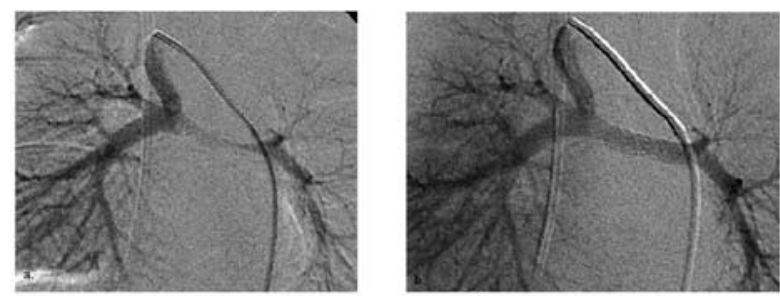

Figure 1. Patient after B-T recanalisation and LPA hypeplasio

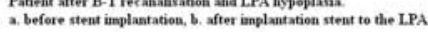

\section{5}

Interventional treatment of aortic coarctation in newborns and neonates-one centre results

Pawel Dryzek, Department of Cardiology, Polish Mother's Memorial Hospital, Research Institute, Poland

Tomasz Moszura, Department of Cardiology, Polish Mother's Memorial Hospital, Research Institute, Poland

Beata Politowska, Department of Cardiology, Polish Mother's Memorial Hospital, Research Institute, Poland

Katarzyna Ostrowska, Department of Cardiology, Polish Mother's

Memorial Hospital, Research Institute, Poland

Jadwiga Moll, Department of Cardiology, Polish Mother's Memorial

Hospital, Research Institute, Poland
Jacek Moll, Department of Cardiosurgery, Polish Mother's Memorial Hospital, Research Institute, Poland

Andrzej Sysa, Department of Cardiology, Polish Mother's Memorial Hospital, Research Institute, Poland

Aortic coarctation occurs in $5-8 \%$ of newborns with congenital heart disease. Surgical treatment is an obligatory standard of clinical management in children with primary coarctation under 6 month of age.

The aim of this study is to present our experience in interventional cardiology procedures for primary and secondary aortic coarctation.

39 pts aged from 6 days to 12 months required balloon angioplasty while in 6 pts stent implantation was done.

Primary coarctation of aorta appeared in 16 pts ( 9 newborns) aged from 6 to 75 days( mean 23).

Intervention was performed as an emergency management in pts not qualified for surgical techniques due to severe condition or additional pathology (critical aortic valve stenosis, multi-organ insufficiency, pulmonary hypertension or severe infection).

Recurrent or residual coarctation was present in 23 pts, aged from 1.5 to 12 months (mean 152 days), coexisting usually with other complex heart defects (TGA + VSD + CoA, TaussigBing + CoA, VSD + CoA, IAA + VSD).

In all pts interventional procedures were used efficiently. The mean pressure gradient decreased from 35.7 to $11.4 \mathrm{mmHg}$ in pts with primary CoA and from 37.8 to $8.6 \mathrm{mmHg}$ in pts with restenosis (evaluated by cardiac catheterization).

4 newborns with additional critical aortic valve stenosis were undergone balloon valvuloplasty simultaneously with good effectiveness.

Stent implantation was successfully done in 4 pts with primary tubular stenosis of aortic isthmus and concomitant hipoplastic aortic arch and in 2 pts due to recoarctation.

Conclusions: Emergency interventional cardiology methods in children with primary CoA under 6 month of age are worth to consider.

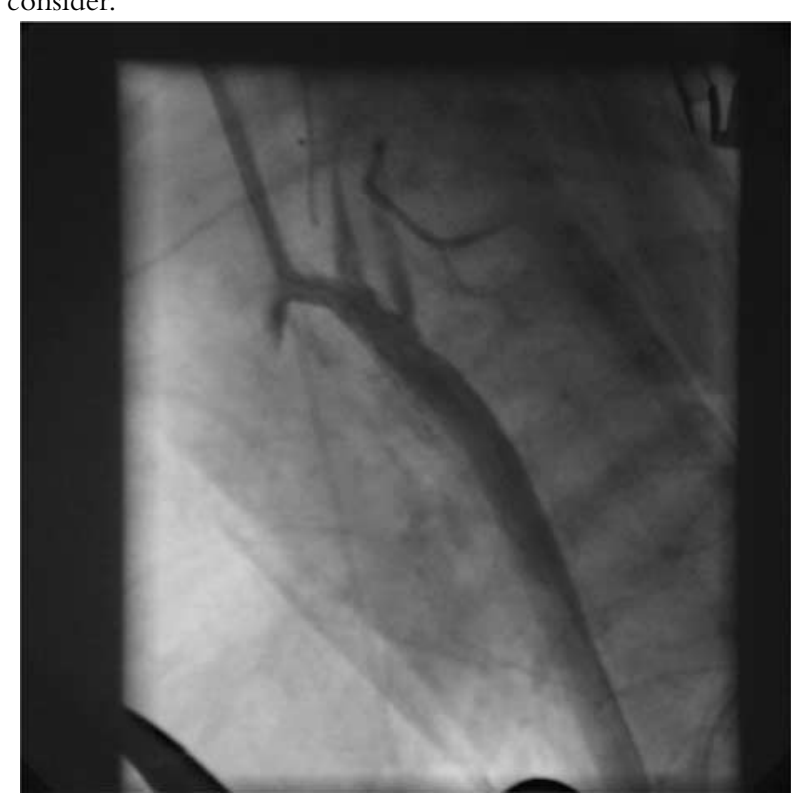

8086

Percutaneous management of residual shunts after initial patent foramen ovale (PFO) device closure

Jaspal S Dua, IRCCS Policlinico San Donato, Milan, Italy, Italy 
Massimo Chessa, IRCCS Policlinico San Donato, Milan, Italy, Italy Alessandro Giamberti, IRCCS Policlinico San Donato, Milan, Italy, Italy

Halkawt Nuri, IRCCS Policlinico San Donato, Milan, Italy, Italy Luciane Piazza, IRCCS Policlinico San Donato, Milan, Italy, Italy Gianfranco Butera, IRCCS Policlinico San Donato, Milan, Italy, Italy Claudio Bussadori, IRCCS Policlinico San Donato, Milan, Italy, Italy Mario Carminati, IRCCS Policlinico San Donato, Milan, Italy, Italy

Objective: Percutaneous closure of the patent foramen ovale (PFO) is well established, though residual shunts or iatrogenic atrial septal defects increase the risk of recurrent events by upto fourfold. Treatment options include medical therapy, surgery or possible percutaneous implantation of second device. The best therapeutic approach is not clear; it is also not clear if a second device implantation is feasible, what are the risks and long-term follow-up?

We report four cases with percutaneous management of significant residual atrial shunting.

Methods: Between 01/2000-12/2006, 1100 patients underwent percutaneous PFO closure. A significant proportion had history of paradoxical embolism or migraine. The patients were followed-up at 1,6,12 months (Transcranial Doppler at 6 months). Amongst these, four had a significant residual shunt and/or a second neurological event and underwent cardiac catheterisation.

Results: All four had significant residual shunts and underwent an Amplatzer device implantation (See Table) under fluoroscopy and echocardiographic guidance. They were discharged on Aspirin $300 \mathrm{mgs}$ for 6-months. None had any residual shunt postprocedure and are doing well on follow-up at 3 yrs.

Conclusion: All patients had the second device closure at least 6-months after the first device, by which, the endothelisation of the initial device was likely to be complete. Technically, the second device implantation was no more challenging than the first procedure. In this small series, the second 'interval' device implantation has proved to be safe and effective in eliminating a significant residual or iatrogenic shunt and the associated neurological risks. A larger series and a longer follow-up are mandatory.

\begin{tabular}{|c|c|c|c|c|}
\hline $\begin{array}{l}\text { Age } \\
\text { (yrs) }\end{array}$ & Diagnosis & $\begin{array}{l}\text { First Device } \\
\text { Implanted }\end{array}$ & $\begin{array}{c}\text { Repeat } \\
\text { Neurological } \\
\text { Event }\end{array}$ & $\begin{array}{c}\text { Second } \\
\text { Intervention } \\
\text { At }\end{array}$ \\
\hline 67 & Stroke, PFO & $\begin{array}{l}\text { Amplatzer PFO } \\
\text { Occluder } 25 \mathrm{~mm}\end{array}$ & No & 12 months \\
\hline 22 & $\begin{array}{l}\text { Stroke, } \\
\text { WPW, } \\
\text { PFO }\end{array}$ & $\begin{array}{l}\text { Amplatzer PFO } \\
\text { Occluder } 25 \mathrm{~mm}\end{array}$ & No & 12 months \\
\hline 39 & Stroke, PFO & Starflex Device $28 \mathrm{~mm}$ & Yes: TIA & 11 months \\
\hline 57 & TIA, PFO & $\begin{array}{l}\text { Amplatzer Cribriform } \\
\text { Occluder } 25 \mathrm{~mm}\end{array}$ & Yes: TIA & 10 months \\
\hline
\end{tabular}

\begin{tabular}{|c|c|c|c|c|}
\hline $\begin{array}{l}\text { Age } \\
\text { (yrs) }\end{array}$ & $\begin{array}{l}\text { Residual Shunt Pre- } \\
\text { Second Intervention }\end{array}$ & Etiology & $\begin{array}{l}\text { Second Device } \\
\text { Implanted }\end{array}$ & $\begin{array}{l}\text { Residual Shunt } \\
\text { at Follow-Up }\end{array}$ \\
\hline 67 & Moderate & $\begin{array}{r}\text { Probable } \\
\text { erosion }\end{array}$ & $\begin{array}{c}\text { Amplatzer Septal } \\
\text { Occluder } 6 \mathrm{~mm}\end{array}$ & None \\
\hline 22 & Moderate & Iatrogenic ASD & $\begin{array}{l}\text { Amplatzer Septal } \\
\text { Occluder } 4 \mathrm{~mm}\end{array}$ & None \\
\hline 39 & Moderate & $\begin{array}{l}\text { Shunt through } \\
\text { PFO tunnel }\end{array}$ & $\begin{array}{l}\text { Amplatzer PFO } \\
\text { Occluder } 18 \mathrm{~mm}\end{array}$ & None \\
\hline 57 & Large & $\begin{array}{c}\text { Shunt through } \\
\text { PFO }\end{array}$ & $\begin{array}{l}\text { Amplatzer PFO } \\
\text { Occluder } 18 \mathrm{~mm}\end{array}$ & None \\
\hline
\end{tabular}

\section{7}

Spectrum of Management in Coronary Artery Fistula: Single Centre Experience

Jaspal S Dua, IRCCS Policlinico San Donato, Milan, Italy, Italy Massimo Chessa, IRCCS Policlinico San Donato, Milan, Italy, Italy Diana Negura, IRCCS Policlinico San Donato, Milan, Italy, Italy Luciane Piazza, IRCCS Policlinico San Donato, Milan, Italy, Italy Angelo Micheletti, IRCCS Policlinico San Donato, Milan, Italy, Italy Halkawt Nuri, IRCCS Policlinico San Donato, Milan, Italy, Italy Gianfranco Butera, IRCCS Policlinico San Donato, Milan, Italy, Italy Mario Carminati, IRCCS Policlinico San Donato, Milan, Italy, Italy

Objective: Congenital coronary artery fistulae (CAF) are rare. Two-thirds of CAF arise from the right coronary artery (RCA). About $90 \%$ of CAF drain into the right side. We report the variation in clinical characteristics and management of CAF. Methods: Retrospective review of database identified 18 patients with CAF between January 2001 and December 2008 (8 yrs).

Results: Median age was 15.7 years (11days -50 yrs). Mean followup was $42+/-30.4$ months.

Origin sites were RCA $(\mathrm{n}=9 ; 50 \%)$, left main $(\mathrm{n}=8 ; 45 \%)$ and LAD $(n=1 ; 5 \%)$. Common drainage sites were the right ventricle $(n=7) \&$ right atrium $(n=7)$.

Therapeutic strategies were based on symptoms and shunt size. Three patients $(16 \%)$ with small shunts had conservative management. 6 patients $(33 \%)$ had surgical ligation. 9 patients $(50 \%)$ were considered for transcatheter occlusion. One patient has been referred for surgery because of the extreme tortuosity of CAF. One patient had a diagnostic angiogram and was then lost to followup. 4 patients had coil occlusion with various different coils, 1 had Amplatzer PDA device, 1 had Amplatzer mVSD device and 1 had Amplatzer Vascular Plug. All had complete occlusion. The only complication was AV dissociation in one patient. There was no death. All patients are doing well at follow-up.

Conclusion: CAF have variable clinical presentation and management depending upon the size, type and manifestations. Our results are comparable with those in the literature (similar efficacy and morbidity but less mortality). Most of the series in literature are small; an international registry will provide further thoughts for optimal management.

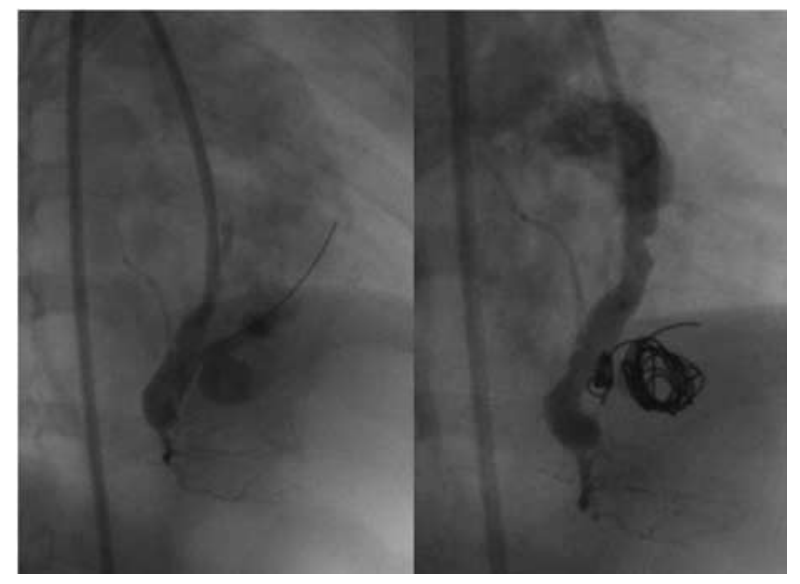

\section{8}

Long Term Single Centre experience with the Helex Septal Occluder

Lucas J Eastaugh, The Royal Children's Hospital, Melbourne, Australia

James L Wilkinson, The Royal Children's Hospital, Melbourne, Australia 
Geoffrey K Lane, The Royal Children's Hospital, Melbourne, Australia

Introduction: The Helex(TM) Septal Occluder is a purposed designed device for transcatheter atrial septal defect (ASD) closure. We report our institutions experience with this device. Methods: All patients who underwent implantation of a Helex(TM) Septal Occluder were reviewed. Procedures were performed using general anaesthesia with transoesophageal echocardiography guidance. Procedural success was defined as accurate placement of a device. Clinical review with transthoracic echocardiography was performed at $24 \mathrm{hrs}, 1 \mathrm{mth}, 6 \mathrm{mths}$ and $1 \mathrm{yr}$ following deployment and fluoroscopic screening was performed at 6 mths.

Results: 49 patients underwent device implantation between May 2001 and Dec 2008. Two patients had device closure of Fontan fenestrations, two had closure of patent foramen ovale and the remaining 45 procedures were for secundum ASD closure. The average ASD size was $11.9 \mathrm{~mm}$ (range $5-22 \mathrm{~mm}$ ). Age range at implantation was $1-18$ yrs with a weight range of $9.6-76.8 \mathrm{~kg}$ and the median follow up was 2.05 years ( 1 mth-7.58 years). In 15 patients a residual leak was noted immediately post-procedure with only 1 patient having a residual haemodynamically significant shunt at 1 year due to device malposition. There were two device embolization's and both were retrieved percutaneously. There has been 1 device frame fracture identified. Five patients experienced migrainous headaches post-procedure which in 2 patients persisted beyond 6 months. No patients developed any pathological rhythm disturbances or complaints of palpitations.

Conclusions: The Helex(TM) device has proven to be an effective device with the added benefit of easy retrievability. The occurrence of frame fracture has not been of clinical concern.

\section{9}

Initial study for transcatheter closure of PDA with Amplatzer Duct Occluder II

Sonia A El Saiedi, Cairo University Children Hospital, Egypt

Amal M El Sisi, Cairo University Children Hospital, Egypt

Rasha I Ammar, Cairo University Children Hospital, Egypt

The occlusion of certain anatomical types of PDA, especially in infants and young children, remain a challenge. The search for the perfect device is pursued by different companies to cover all the shapes and types of PDAs.

Aim of the study is to report the initial experience with transcatheter closure of PDA with ADOII (AGA medical).

Methods: Ten cases with different shapes of PDA were included, their age ranged from 0.08 to $5 \mathrm{yrs}$ and wt ranges from $3 \mathrm{Kg}$ to $20 \mathrm{Kg}$.

The device used is a modified ADOII made of fabric free nitinol wire mesh. The device configuration is a central waist with two retention discs. The two discs are designed to be deployed on the arterial and venous sides of the defect. Device is available in two lengths.

Results: ADO II can be used to close ducts beyond the neonatal period if less than $4.5 \mathrm{~cm}$ in diameter. The device can be used from both the arterial and the venous side. Occlusion is not attained immediately due to absence of fabric in this device but it is complete within one month. This work demonstrates how to choose the suitable length of the device.

Conclusion: The ADO II is a promising addition to our choices for PDA closure.

Further studies will be needed to define clearly when to use the new device.

\section{1}

Transcatheter Closure of Large Coronary Artery Fistula Using Shape-memory Alloy Occluder

Yuan Feng, Department of Cardiology, West China Hospital of Sichuan University, China

Purpose: To evaluate the feasibility and effectiveness of transcatheter closure of large coronary artery fistula with shape-memory alloy occluder.

Method: Nine patients (aged $20 \pm 9.9$ years, 2 males) with large coronary artery fistula were enrolled for percutaneous transcatheter closure using shape-memory alloy occluders (Cardi-O-Fix Occluder System, Starway Medical Technology Inc, Beijing, China), including 4 patients with right coronary artery to right ventricle fistula, 2 with left anterior descending coronary artery to right ventricle, 1 with left circumflex coronary artery to right ventricle, 1 with left circumflex coronary artery to right atrium and 1 with right coronary artery to left ventricle. The narrowest diameter of fistulas was $7 \pm 3.9 \mathrm{~mm}(3.3-15 \mathrm{~mm})$ while the widest diameter $23 \pm 8.8 \mathrm{~mm}(13-38 \mathrm{~mm})$ by coronary angiogram.

Result: The procedure was successful in 7 patients, of whom 6 patients received patent ductus arteriosus occluder (sized $10-20 \mathrm{~mm}$ ) while 1 received a $16 \mathrm{~mm}$ muscular ventricular septal defect occluder plus 10 coils (sized 5-10 mm). Angiogram immediately after the procedure demonstrated a complete occlusion in 6 patients and a small residual shunt in 1 patient which was not detected by echocardiography at 4 days after the procedure. The retrograde passing of delivery sheath was failed in the other 2 patients who were subsequently corrected by surgical technique. All the 9 patients survived during the follow-up of 1-6 months without notable symptoms.

Conclusion: Transcatheter closure of large coronary artery fistula with shape-memory alloy occluder is an effective, safe and reliable method.

Keywords: coronary artery fistula, transcatheter closure, shapememory alloy occluder.

\section{2}

Outcome of pulmonary artery angioplasty in young children

Alain Fraisse, Timone Children's Hospital, Marseille

Andreea Dragulescu, Timone Children's Hospital, Marseille, France

Pascal Amedro, Arnaud de Villeneuve Hospital, Montpellier, France

Dominique Metras, Timone Children's Hospital, Marseille

Bernard Kreitmann, Timone Children's Hospital, Marseille, France

Serge Camilleri, Timone Children's Hospital, Marseille

Objective: To clarify the outcome of unilateral pulmonary artery (PA) angioplasty in young children.

Patients and Results: Since February 1998, 10 patients (7 boys, 3 girls) $<15 \mathrm{Kg}$ underwent 22 unilateral PA angioplasty at a median age and weight of $1.9(0.7-3.6)$ years and 10.2 (7.9-15) $\mathrm{Kg}$, respectively. In 8 cases, the PA stenosis developed after an initial surgical reconstruction of the PA, during or after repair of associated cardiac disease: tetralogy of Fallot $(n=8), D-$ transposition of the great arteries $(n=1)$ and right ventricular outflow obstruction $(n=1)$. The interventions were successful in 95\% (18 of 20) of the cases. The narrowest mean diameter of the stenotic PA increased from $2.7 \pm 1.2 \mathrm{~mm}$ to $8.9 \pm 3 \mathrm{~mm}$ and the mean pressure gradient dropped from $15.8 \pm 10.8 \mathrm{mmHg}$ to $4.5 \pm 5.5 \mathrm{mmHg}$. Ten stents were implanted in 8 patients: 8 in the left, 2 in the right PA. Lung scan showed a mean increase in perfusion to the affected lung from $15 \%(+11 \%)$ to $30 \%(+10 \%)$, $\mathrm{p}=0.001$. Twelve PA redilations were performed in 6 patients. 
Minor complications were transient pulmonary edema $(\mathrm{n}=1)$ and transient hemoptysis $(n=1)$. One stent that migrated in left PA distally was redilated there, whereas another stent was implanted in the stenosis. All patients are asymptomatic after a mean follow-up of $5.8(+0.9$ to 9.9$)$ years, with a mean lung perfusion of $32 \%$ $(+6.5 \%)$.

Conclusion: PAA is efficient in small children with a significant gain in lung perfusion that is maintained during follow-up. Stent placement is often necessary and can be redilated to accommodate somatic growth.

\section{3}

Stenting of patent ductus arteriosus (PDA) as a palliative procedure for older children and adults with congenital cyanotic heart disease

Edwin Francis, Amrita Institute of Medical Sciences and Research

Center, India

Kappanayil Mahesh, Amrita Institute of Medical Sciences and Research Center, India

Balu Vaidyanathan, Amrita Institute of Medical Sciences and Research Center, India

Raman Krishna Kumar, Amrita Institute of Medical Sciences and Research Center, India

Objective: To describe the indications, technique and results (early and short-term follow-up) of palliative PDA stenting in selected patients ( 2 years and older) with congenital cyanotic heart disease with reduced pulmonary blood flow who were not candidates for definitive surgery in the immediate future.

Methods: Hospital records of patients (2 years and older) undergoing PDA stenting between Jan 2007-Dec 2008 were reviewed. The access (femoral vein or artery, axillary/radial artery) and approach was dictated by the anatomy of the PDA. Coronary or peripheral vascular balloon expandable stents were used. A coronary guiding catheter $(5-7 \mathrm{~F})$ or a long sheath (4-7 F) was used to access the PDA.

Results: Nine patients (median age 14 years; range 2-18 years; median weight $20 \mathrm{Kg}$; range $7-57 \mathrm{Kg}$ ) were included (3 with single ventricle physiology and 6 with TOF and pulmonary atresia). Indication for intervention was hypoxia (mean saturation $71 \pm 4 \%$, hemoglobin $20 \pm 1.9 \mathrm{gm} / \mathrm{dl}$ ) with no immediate prospect of definitive surgical correction because of unsuitable anatomy $(n=8$, disconnected pulmonary arteries in 4, hypoplastic pulmonary artery in 4) or economic considerations $(\mathrm{n}=1)$. Stents of $4-8 \mathrm{~mm}$ diameter and $16-38 \mathrm{~mm}$ length were used. Successful stenting was accomplished in all (fluoroscopy time: $22 \pm 8$ minutes) with no complications and the saturations improved to $88 \pm 1.5 \%$. The most recent oxygen saturation on follow-up (1-22 months) was $84 \pm 3 \%$. One patient underwent corrective repair.

Conclusion: PDA stenting can provide effective palliation in selected older patients with cyanotic congenital heart disease.
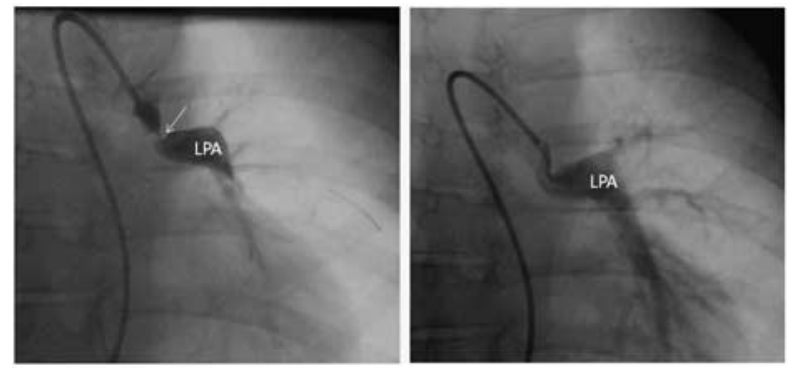

Frames of angiography before and after ductal stenting for a patient with TOF pulmonary atresia and disconnected pulmonary arteries The arrow points to the site of narrowing in the PDA. LPA: left pulmonary artery
8094

Balloon dilatation or stenting is an effective interim palliation of critical coarctation in infants

Edwin Francis, Amrita Institute of Medical Sciences and Research Center, India

Gayatri Satheesh, Amrita Institute of Medical Sciences and Research Center, India

Balu Vaidyanathan, Amrita Institute of Medical Sciences and Research Center, India

Bhava RJ Kannan, Vadamulayan Hospital, Madurai, Tamil Nadu, India Raman Krishna Kumar, Amrita Institute of Medical Sciences and Research Center, India

Objective: To describe the immediate and follow up outcome of critically ill infants with coarctation who underwent balloon dilation or stenting as a rescue procedure.

Methods: We retrospectively analyzed data of all critically ill newborns and infants who presented to us with severe coarctation and left ventricular dysfunction from July 2003-July 2008 and who had underwent transcatheter intervention.

Results: There were total of 10 patients (weight $2.9 \pm 0.4 \mathrm{Kg}$; median age 4 weeks range 1-12 weeks); additional cardiac lesions were present in 7. Stenting of the coarctation segment was done in 5 patients. Procedural success was achieved in all patients with no mortality (systolic gradient reduced from $51 \pm 12 \mathrm{~mm} \mathrm{Hg}$ to $13 \pm 4 \mathrm{~mm} \mathrm{Hg}$ ). Complications included cardiopulmonary arrest requiring brief CPR $(n=1)$, sepsis $(n=1)$ and temporary loss of lower limb pulse $(n=2)$. Left ventricular function improved in all patients. Average ICU stay was $3 \pm 2$ days and hospital stay was $6 \pm 2$ days. One patient was lost to follow up. Others have been seen at $18 \pm 12$ weeks. 3 patients ( 2 with stents) underwent elective coarctation repair. Two patients underwent VSD closure and coarctation repair and one (large posterior muscular VSD) underwent PA banding. One patient is awaiting surgical repair. Two patients (1 with stent) underwent re-dilatation for re-stenosis and one patient (Shone complex) is on medical follow up.

Conclusion: Balloon dilatation with or without stenting can be safely undertaken with good immediate relief of symptoms in sick newborns with severe coarctation and left ventricular dysfunction as a bridge to definitive surgery.
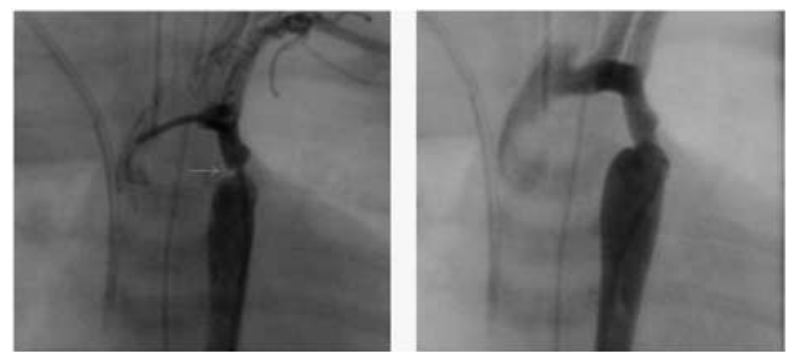

Pre and post angiographic frames of a newborn coarctation stenting. Arrow indicates the site of narrowing.

\section{5}

Transcatheter occlusion of large patent ductus arteriosus in small infants ( $5 \mathrm{Kg}$ or under) using first generation duct occluders

Edwin Francis, Amrita Institute of Medical Sciences and Research Center, India

Shehan Perera, The Royal Children's Hopsital, Australia

Kappanayil Mahesh, Amrita Institute of Medical Sciences and Research Center, India

Balu Vaidyanathan, Amrita Institute of Medical Sciences and Research Center, India 
Kavasseri S Remadevi, Amrita Institute of Medical Sciences and Research Center, India

Raman Krishna Kumar, Amrita Institute of Medical Sciences and Research Center, India

Objective: To describe our institutional experience with device closure of large patent ductus arteriosus (PDA) in symptomatic infants weighing $\leqslant 5 \mathrm{~kg}$ using the first generation duct occluders. Methods: Case selection was based on echocardiographic anatomy of the PDA, anticipated device size and patient weight. Salient features of the technique included extrusion of device in the aorta close to the duct ampulla and partial deployment of the aortic retention disc within the duct ampulla (figure).

Results: During the study period (January 2004-December 2008), of 631 patients undergoing transcatheter closure, 112 weighed $\leqslant 5 \mathrm{~kg}$. 38 symptomatic infants (age: 1-9 months; weight $3.9 \pm 0.8 \mathrm{~kg}$ ) with relatively large PDA (size $-4.3 \pm 0.9 \mathrm{~mm}$ ) underwent closure using the Amplatzer or Lifetech nitinol duct occluders and remaining underwent coil occlusion. Successful closure was achieved in 36 patients (device sizes $6-4$ to $12-10$, fluoro time of $8.4 \pm 3.9$ minutes). Arterial access was avoided in 12 patients. Seven had transient lower limb pulse loss, one of whom required thrombolysis. Device dislodged into adjacent aorta in one requiring surgical retrieval. Significant issues on follow up (18 \pm 10.6 months) included left pulmonary artery stenosis $(n=2)$, and descending aortic gradients $(n=2$, see figure). Complete flow elimation was accomplshed in all. Conclusion: In selected infants $\leqslant 5 \mathrm{~kg}$ transcatheter device closure of PDA is feasible. However, careful follow-up is mandated by development of LPA stenosis and aortic narrowing from the retention disc in a small fraction of patients.

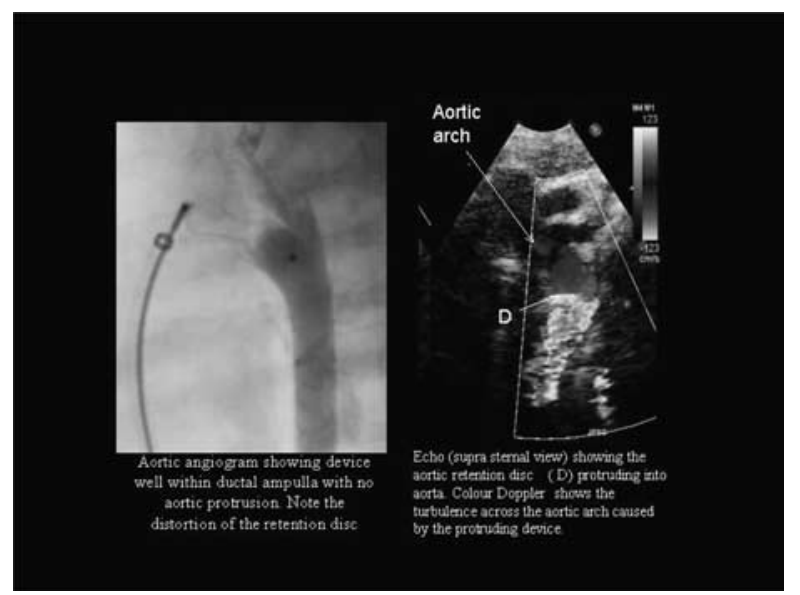

8096

Outcome of large left ventricular tumors with functional HLH (hypoplastic left heart) after management with Hybrid approach and subsequent conversion to Glenn shunt Grace A Freire, Saint Louis University School of Medicine and Cardinal Glennon Children's Medical Center, United States

Saadeh B Jureidini, Saint Louis University School of Medicine and Cardinal Glennon Children's Medical Center, United States

Andrew C Fiore, Saint Louis University School of Medicine and Cardinal Glennon Children's Medical Center, United States Kenneth O Schowengerdt, Saint Louis University School of Medicine and Cardinal Glennon Children's Medical Center, United States

Purpose: To describe single-center results utilizing hybrid approach for treatment of neonates with large ventricular tumors and univentricular physiology.
Outcomes of neonates with large cardiac tumors resulting in functional HLH are discouraging. We report on the outcome of 2 surviving infants after innovative use of hybrid approach and transition to Glenn shunt.

Methods: 2 patients were diagnosed prenatally with large, obstructive left ventricular tumors and echocardiography findings of ductal dependency. At birth, ductal patency was maintained with prostaglandin infusion until 1 week of age when a hybrid procedure was performed consisting of placement of bilateral pulmonary bands and ductal stent. Balloon atrial septostomy followed one week later. Initially, the patient suspected to have a rhabdomyoma demonstrated decrease in tumor size, but at about 5 months of age the tumor size increased. The second patient underwent cardiac biopsy that confirmed suspicion of a fibroma; tumor size did not change in this patient. At 5 and 6 months of age, Glenn shunt palliation was accomplished with good results. Results: The 2 patients are thriving at 15 and 16 months of age. The tumors have demonstrated no further growth. We plan future biventricular repair for these patients with possible resection of the tumors.

Conclusion: Initial hybrid procedure and subsequent Glenn shunt palliation can be safely performed in neonates with large spaceoccupying left ventricular tumors. Conversion to univentricular physiology by this approach is an alternative to the Norwood procedure and may permit safer tumor resection, conversion to biventricular repair, or transplantation in the future.

\section{7}

Effect on Right Ventricular Size of Transcatheter Closure of Atrial Septal Defect

Yun-Ching Fu, Taichung Veterans General Hospital, Taiwan

Chi-Lin Ho, Taiwan

Ming-Chih Lin, Taichung Veterans General Hospital, Taiwan

Sheng-Ling Jan, Taichung Veterans General Hospital, Taiwan

Background: Atrial septal defect (ASD) causes right ventricular volume overloading. The purpose aimed to investigate the effect on right ventricular size of transcatheter closure of ASD.

Methods: Between January 2005 and December 2006, 94 patients (age range 2.8 to 61.8 years, mean: 18.2 ) with secundum type ASD underwent successful closure with an Amplatzer septal occluder were divided into 3 groups according to Qp/Qs $(<1.5$, $\mathrm{n}=22 ; \geqq 1.5$ and $<2, \mathrm{n}=27 ; \geqq 2, \mathrm{n}=45$ ). Right ventricular body dimension in end diastolic (RVd) and systolic (RVs) phases were recorded by $\mathrm{M}$ mode in parasternal short axis view before, the following day, 1 month and 6 months after the closure of ASD. The data of RVd and RVs were corrected by BSA for each patient.

Results: In the small shunt group $(\mathrm{Qp} / \mathrm{Q} s<1.5)$, right ventricular size significantly reduced 1 month after closure of ASD (RVd/BSA: $1.81 \pm 1.33$ vs $1.40 \pm 0.96$; RVs/BSA: $1.75 \pm 1.01$ vs $1.46 \pm 0.67)$. In median $(1.5 \mathrm{QP} / \mathrm{Qs}<2)$ group, right ventricular size also significantly reduced one month later $(\mathrm{RVd} / \mathrm{BSA}: 1.90 \pm 1.41$ vs $1.40 \pm 0.85 ; \mathrm{RV} / \mathrm{BSA}: 1.84 \pm 1.29$ vs $1.43 \pm 0.83)$. In the large shunt group $(\mathrm{Qp} / \mathrm{Qs}>1.5)$ right ventricular size significantly reduced on the following day $(\mathrm{RVd} /$ BSA: $2.44 \pm 1.29$ vs $1.95 \pm 1.14$; RVs/BSA: $2.2 \pm 1.24$ vs $1.92 \pm 1.12)$.

Conclusion: The right ventricular size was significantly reduced on the following day and continued to shrink significantly till 6 months after the device closure in the large shunt group (Qp/ $\mathrm{Qs} \geq 2)$. In groups of small and median shunt $(1<\mathrm{Qp} / \mathrm{Q} s<2)$, $\mathrm{RV}$ size change seems to occur within one month after ASD closure. 


\section{8}

Clinical Outcomes of a Surgical Closure and a Device closure of Atrial Septal Defects in Adult Patients More Than 40 Years of Age

Yasuhiro Fujii, Okayama University Hospital, Japan

Teiji Akagi, Okayama University Hospital, Japan

Manabu Taniguchi, Okayama University Hospital, Japan

Tomoko Tomii, Okayama University Hospital, Japan

Shinichi Otsuki, Okayama University Hospital, Japan

Sadahiko Arai, Okayama Univeristy Hospital, Japan

Shingo Kasahara, Okayama University Hospital, Japan

Shunji Sano, Okayama University Hospital, Japan

Objective: To describe the outcomes of a surgical closure and a device closure of ASDs using an Amplatzer Septal Occluder in patients more than 40 years of age.

Methods: Between Mar 1991 and Feb 2008, 21 surgical closures of ASDs (S group) and 44 device closures of ASDs using the Amplatzer Septal Occluder (ASO group) were performed in patients more than 40 years of age. These outcomes were retrospectively reviewed and compared.

Results: There was no mortality and no major complication in either group. In the $\mathrm{S}$ group, the hospital stay was significantly longer than in the ASO group $(\mathrm{P}<0.001)$. In compared to the ASO group using univariate analysis, the $\mathrm{S}$ group tended to have a significantly higher total incidence of postoperative atrial arrhythmias ( $\mathrm{S}$ vs. $\mathrm{ASD}=8 / 21$ vs. $7 / 44, \mathrm{P}=.047$ ), however, there was no significance in multivariate analysis $(\mathrm{P}=0.348)$. A higher incidence of a new onset of atrial arrhythmia was observed in the $\mathrm{S}$ group $(2 / 14$ vs. $0 / 33, \mathrm{P}=0.074)$, however, both of the new-onset arrhythmias were temporary in the early postoperative period. The existence of atrial arrhythmia in the mid-to-long term period was similar in the both groups $(5 / 21$ vs. $7 / 44, \mathrm{P}=0.752$ ).

Conclusions: Both a surgical closure and device closure of ASD showed satisfactory outcomes for patients more than 40 years of age. Although early postoperative atrial arrhythmia is a concern in the $\mathrm{S}$ group, there was no difference in the incidence of atrial arrhythmia in the mid-to-long term period.

\begin{tabular}{llll}
\hline & Surgery $(\mathbf{n}=21)$ & ASO $(\mathbf{n}=44)$ & P \\
\hline Age (years) & $52.5+/-8.1$ & $58.8+/-11.9$ & 0.014 \\
ASD diameter (mm) & $25.9+/-9.5$ & $18.8+/-6.7$ & 0.001 \\
Hospital Stay (days) & $14.7+/-7.0$ & $3.0+/-1.6$ & $<0.001$ \\
Follow up (months) & $55.9+/-52.2$ & $19.4+/-9.0$ & 0.005 \\
Total incidence of atrial & $38 \%(8 / 21)$ & $16 \%(7 / 44)$ & 0.047 \\
$\quad$ arrhythmia (AA) & $24 \%(5 / 21)$ & $16 \%(7 / 44)$ & 0.752 \\
AA in the Mid-to-long term & $14 \%(2 / 14)$ & $0 \%(0 / 33)$ & 0.074 \\
New onset of AA & $14 \%(1 / 7)$ & $36 \%(4 / 11)$ & 0.444 \\
Resolution rate of & & & \\
$\quad$ preoperative AA & & & \\
\hline
\end{tabular}

\section{9}

Histological Changes at Sites of Pulmonary Venous Stenting in a Normal Porcine Model

Takeshi Furukawa, Department of Pediatrics, Juntendo University School of Medicine, Japan

Masahiko Kishiro, Department of Pediatrics, Juntendo University School of Medicine, Japan

Hideo Fukunaga, Department of Pediatrics, Juntendo Nerima Hospital, Japan

Masahiro Ohtsuki, Department of Pediatrics, Juntendo University School of Medicine, Japan
Ken Takahashi, Department of Pediatrics, Juntendo University School of Medicine, Japan

Katsumi Akimoto, Department of Pediatrics, Juntendo University School of Medicine, Japan

Toshiaki Shimizu, Department of Pediatrics, Juntendo University

School of Medicine, Japan

Shiori Kawasaki, Department of Cardiovascular Surgery, Juntendo University School of Medicine, Japan

Objective: The outcome of stent implantation by catheter intervention for pulmonary vein stenosis (PVS) remains poor in children. Meanwhile, use of drug eluting stents (DES) in PVS has never been reported, and its effectiveness remains unknown. Methods: In this study, 3 bare-metal stents (BMS) and sirolimuseluting stents (SES) each were implanted in one-month-old pigs which were autopsied 8 weeks later to compare restenosis.

Results: The extent of neointimal thickness by injury score was significantly less in the SES groups than in the BMS group (injury score 1: SES $0.226 \pm 0.031 \mathrm{~mm}$ versus BMS $0.351 \pm 0.033 \mathrm{~mm}$ $(\mathrm{P}<0.01)$; injury score 2 : SES $0.609 \pm 0.208 \mathrm{~mm}$ versus BMS $1.232 \pm 0.244 \mathrm{~mm}(\mathrm{P}<0.01))$. Neointimal thickness for injury score 3 did not differ significantly. Pathological findings with BMS showed a confluence of abscessed inflammatory cells around the stent wires as well as granuloma formation around it. Granuloma formation was not seen with SES.

Conclusions: Restenosis was significantly reduced in the SES group, suggesting the effectiveness of DES in PVS treatment. Due to the small number of cases in the present study and the considerable spread in injury scores and balloon to vein ratios, future studies with more cases are needed.

\section{0}

Stent implantation in the residual pulmonary artery stenosis in 5 children with congenital heart disease Wei Gao, Department of Cardiology, Shanghai Children's Medical Center, School of Medicine, Shanghai, Jiao Tong University, China

Zhi-qing Yu, Department of Cardiology, Shanghai Children's Medical Center, School of Medicine, Shanghai, Jiao Tong University, China Fen Li, Department of Cardiology, Shanghai Children's Medical Center, School of Medicine, Shanghai Jiao Tong University, China Mei-rong Huang, Department of Cardiology, Shanghai Children's Medical Center, School of Medicine, Shanghai, Jiao Tong University,, China

Yun Li, Department of Cardiology, Shanghai Children's Medical Center, School of Medicine, Shanghai, Jiao Tong University, China Jian-ping Yang, Department of Cardiology, Shanghai Children's Medical Center, School of Medicine, Shanghai, Jiao Tong University, China

Yu-min Zhong, Department of Cardiology, Shanghai Children's Medical Center, School of Medicine, Shanghai, Jiao Tong University, China

Yu-qi Zhang, Department of Cardiology, Shanghai Children's Medical Center, School of Medicine, Shanghai, Jiao Tong University, China

Objective: Balloon expandable stent placement in branch pulmonary arteries is limited by fragile hemodynamics during wire/sheath positioning and technical difficulties. Recent advances in delivery techniques and stent design have allowed the delivery of large stents, diliatable to adult size, through small sheaths. We report our short and mid-term results of branch pulmonary arteries stents implated in children.

Methods: Stent implantation in the residual pulmonary artery stenosis with congenital heart disease was performed in 5 cases 
from 2005 to 2007 . The age was 7 to 15 years. Stent delivery occurred through an 10 12F Mullins sheath or Amplatzer ASD delivery system on a BIB balloon (NuMed, Hopkinto, NY, USA) or a Z-Med balloon (B. Braun Medical Inc., Bethelehem, PA). Follow up with echocardiography and clinical examination was done.

Results: The CP stent (NuMed, Hopkinto, NY, USA) and Palmaz stent (Cordis J \& J Interventional Sistems) were placed in the cath lab in 8 vessels. Mean minimum diameter of left and right branch pulmonary arteries increased from $5.5 \pm 1.3 \mathrm{~mm}, 8.7 \pm 0.5 \mathrm{~mm}$ to $12.2 \pm 1.8 \mathrm{~mm}$ and $16.0 \pm 2.6 \mathrm{~mm}$. Mean pressure gradient decreased from $40 \pm 14.50 \mathrm{mmHg}, \quad 41.7 \pm 11.8 \mathrm{mmHg}$ to $2.75 \pm 0.83 \mathrm{mmHg}$ and $8.3 \pm 2.6 \mathrm{mmHg}$. Major complications included stent migration to main pulmonary artery in one patient. Conclusion: Branch pulmonary arteries can be stented safely and effectively in small patients. Long term follow up is necessary to determine if these stents can be dilated to adult size.

Keywords: pulmonary artery stenosis ; interventional therapeutic; stents

8101

Initial experience with the Solysafe ${ }^{\circledR}$ device for percutaneous closure of secundum atrial septal defect in pediatric patients

Francisco J Garay, Hospital Clínico. Universidad Católica de Chile, Santiago, Chile

Simon B Duque, Hospital de Niños Dr. Roberto Gilbert. Guayaquil, Ecuador

Isabel Freire, Hospital de Niños Dr. Roberto Gilbert. Guayaquil, Ecuador

Alex Alcantara, Hospital Dr. Sótero de Río. Santiago., Chile

Gonzalo Urcelay, Hospital Clínico. Universidad Católica de Chile. Santiago, Chile

Felipe Heusser, Hospital Clínico. Universidad Católica de Chile. Santiago, Chile

Solysafe is a new self-centering device with helicoidal structure made of Phynox and two polyester patches. Coaxial control catheters are screwed to each end, so patch deployment is independently controlled. Implantation is through a $10 \mathrm{Fr}$ short sheath in femoral vein. The device is advanced over a 0.018 " guide-wire through the ASD and deployed by manipulating the control catheters. Patches are locked with a clicking mechanism. The device is released by unscrewing the control catheters. The procedure can be reversed.

We report the initial experience with Solysafe for ASD closure in pediatric patients.

ASD closure procedures using Solysafe from May to October 2008 were reviewed. Procedures were performed under fluoroscopic and echocardiographic guidance. Device size was selected according to balloon stretched diameter.

Solysafe device was used in 7 patients ( 3 female), median age 7 years (6-14), median weight $22.8 \mathrm{~kg}(19.5-58)$ and median stretched diameter $17 \mathrm{~mm}$ (9-18). Six devices were implanted. In one patient needing a Solysafe 25 device, a Solysafe 20 device (largest available at that time) was deployed but prolapsed through the defect and was uneventfully removed. Intraprocedure echocardiography demonstrated complete closure in 4 patients and small residual shunt in 2. A very low device profile was remarkable. No intraprocedure complications occurred. Median follow-up of 6 months (2-8) demonstrated 100\% closure rate and no complications.

The new Solysafe device is safe and effective for ASD closure in pediatric patients. The implanted device profile is remarkable.
10 Fr sheath needed for implantation limits its use in small children.

8102

Coa Stenting - a single operator experience

Tiow H Goh, Monash Medical Centre, Melbourne, Australia

COA management is a life long process beginning with surgical repair. With growth and time, 1. recoarctation, 2. hypoplastic arch segments (transverse arch (TAh) between IA and LCA, distal arch (DAh) between LCA and LSCA, isthmus (AIh) between LSCA and Desc.AO (DAO), 3. arch distortion (AD) and 4. Proximal hypertension can develop, necessitating further surgical or interventional management.

Aim: To review efficacy of single operator interventional COA stents (yr2000-2008) to address the above issues.

Methods and observation: Of 100 pts followed up from 1-30 yrs serially with 2D, Bp, exercise tests and $\mathrm{mri} / \mathrm{cta}, 13$ pts. (12 yr-30 yr,wt. 43-121 kgm 64.9) had "recurrence" and 5pts with native COA underwent J\&J/CP stenting.

\begin{tabular}{llllll}
\hline \multicolumn{2}{l}{ ANATOMY OF ARCH } & & & & \\
COA type & PATIENTS & AD & TAh & DAh & Alh \\
NATIVE & 5 & 0 & $1^{*}$ & $1^{*}$ & $4+1^{*}$ \\
SUBCLFLAP & 7 & 4 & 3 & 6 & 1 \\
END-END & 6 & 2 & 2 & $4 *$ & $4 *$ \\
$*$ same pts & & & & \\
STENT PLACEMENT SITE (DILATED 16-20MM) \\
COA TYPE & TAh & DAh+AIh & AIh & \\
NATIVE & $1 *$ & $2+1^{*}$ & $2+1^{*}$ & \\
SUBCLFLAP & 0 & 6 & 1 & \\
END-END & 2 & 4 & 0 & & \\
\hline
\end{tabular}

Results: 1. Gradients $>30 \mathrm{mmhg}$ reduced to below $5 \mathrm{mmhg}$. 2 . Aortic arch diameter increased to $15-20 \mathrm{~mm}$ at stented site. 2 . Hypertension improved in most cases. 3 Better exercise endurance 4. Arch distortion improved 3pts.

Complications: 1 . Stent malposition in 3 pts,-parked safely 2 .aortic intimal tear 1pt-resolved. 3. Prolonged catheter 1pt. 4. RFA haematoma 5pts. 5. "jailed "LSCA 5pts, LCA 1pt. NO MAJOR COMPLICATIONS.

Conclusion: 1. segmental aortic arch stenting can be safely undertaken. 2. arch anatomy and diameter are improved 3. hypertension and exercise tolerance are improved. 4. surgery is minimised. 5. long term effects of coa stents however remain unknown.

\section{3}

Ductal stenting for duct-dependent pulmonary circulation: a multi-center experience

Hala Hamza, University Children's Hospital, Cairo, Egypt

Hala Agha, University Children's Hospital, Cairo, Egypt

Amira Essmat, University Children's Hospital, Cairo, Egypt

Yasser Hussain, University Children's Hospital, Cairo, Egypt

Vikas Kohli, Indraprastha Apollo Hospital, New Delhi, India

Mathias Emmel, Pediatric Cardiology, University of Cologne, Germany

Markus Kruessell, Germany

Narayanswami Sreeram, Heart Center, University Hospital of Cologne, Germany

Background and Aims: Ductal stenting in neonates and infants with pulmonary atresia-variants potentially avoids the need for a surgical shunt. We report a multi-center experience with this approach. Patients and methods: 31 consecutive infants (17 female) from 3 centers, catheterised on an intention to treat are presented. 
The demographic data were as follows: age 3 to 120 days; weight 2.2 to $4.0 \mathrm{~kg}$. The ductal morphology was horizontal and straight $(n=22)$, tortuous $(n=5)$ and vertical $(n=4)$. Stenting was performed as an adjunct to RF perforation of the pulmonary valve in 16 patients. Prostaglandin E was discontinued 6 hours prior to the procedure in 23 infants.

Results: In 6 patients, a stent was not implanted for the following reasons (failure to enter the duct $n=4$ ); tortuous duct with concomitant stenosis of a branch PA $(n=2)$. In the remainder, stents ranging from 3.5 to $4.5 \mathrm{~mm}$ in diameter of varying lengths were implanted (retrogradely in 14, anterogradely in 11; 2 stents in series in 2 patients). The procedure was uncomplicated in all; 1 infant in whom the initial stent did not cover the entire duct required a second stent at an interval of 3 days. Following stent implantation, the systemic saturation at rest ranged from 75 to $88 \%$. The duration of hospital stay ranged from 1 to 66 days. Patients were discharged from hospital on aspirin $5 \mathrm{mg} / \mathrm{kg} /$ day. Conclusions: Despite a learning curve, ductal stenting is relatively straightforward and provides adequate medium-term palliation. It is of particular importance in centers with limited surgical backup.

\section{5}

Successful new strategy - stent implantation for pulmonary venous drainage route - of single ventricle with total anomalous pulmonary venous connection

Takashi Higaki, Department of Pediatric Cardiology, Stroke E Cardiovascular Center Ehime University Hospital, Japan

Eiichi Yamamoto, Department of Pediatric Cardiology, Stroke \& Cardiovascular Center Ehime University Hospital, Japan

Takeshi Nakano, Department of Pediatrics, Ehime University Graduate School of Medicine, Japan

Masaaki Ohta, Department of Pediatrics, Ehime University Graduate School of Medicine, Japan

Kikuko Murao, Department of Pediatrics, Ehime University Graduate School of Medicine, Japan

Mitsugi Nagashima, Department of Cardiovascular Surgery, Stroke \& Cardiovascular Center Ehime University Hospital, Japan

Fumiaki Shikata, Department of Cardiovascular Surgery, Stroke E Cardiovascular Center Ehime University Hospital, Japan

Eiichi Ishii, Department of Pediatrics, Ehime University Graduate

School of Medicine, Japan

Operative mortality rate of the palliative repair of Asplenia and single ventricle with total anomalous pulmonary venous connection (TAPVC) during neonate is extremity high. Although the surgical results is improving in the modern surgical era, It will be still extremely challenging in these patients. Therefore, alternative method to treat TAPVC in the neonate period has been expected.

Catheter intervention is one of the most important treatments in complex heart disease. Stent implantation for pulmonary venous drainage route early after birth could be effective and less invasive than surgery to prevent pulmonary venous occlusion maintaining stable hemodynamics in early infantile and neonatal period.

In addition, this procedure may delay invasive open cardiac surgery. We succeeded in three neonatal cases for stent implantation to vertical vein (VV) or ductus venosus (DV) for Asplenia syndrome with supracardiac or infracardiac type of TAPVC. One of them was diagnosed during pregnancy by the fetal echocardiogram. We scheduled her delivery and performed stent implantation. For better result, it is expected to make an intrauterine diagnosis by the fetal echocardiogram, premediated delivery and subsequent stent implantation, especially with infracardiac type TAPVC.

Although it is considered that the appropriate size of the stent was $8 \mathrm{~mm}$ for these neonates in our study, an appropriate size of the stent for the longer patency should be analyzed in the future.

In conclusion, stent implantation for pulmonary venous drainage route early after birth is effective and less invasive therapy in neonates with complex congenital heart disease.

\section{7}

Analysis of Ventricular Dopppler Tissue Imaging in Patients Underwent Transcatheter Closure and Surgical Repair of Perimembranous Ventricular Septal Defect Xue-cun Liang, Pediatric Heart Center, Children's Hospital of Fudan University, Shanghai, China

Guo-ying Huang, Pediatric Heart Center, Children's Hospital of Fudan University, Shanghai, China

Fang Liu, Pediatric Heart Center, Children's Hospital of Fudan University, Shanghai, China

Feng Sheng, Pediatric Heart Center, Children's Hospital of Fudan

University, Shanghai, China

Objective: Aim to investigate and compare the ventricular function of perimembranous ventricular septal defect(VSD) patients after transcatheter closure and surgical repair by Doppler tissue imaging (DTI).

Methods: Seventy-seven age-matched consecutive patients underwent perimembranous VSD closure: 35 patients were treated with transcatheter occluder at age of $5.4 \pm 3$ years and 42 patients were treated surgically at age of $4.8 \pm 2.6$ years. Ventricular function was assessed using DTI and conventional echocardiography at $0.8 \pm 1$ year (from 1 month to 30 months) after VSD closure.Results:There was a significantly higher mitral annular myocardial systolic velocity in patients with occluder closure $(9.03 \pm 2.07 \mathrm{~cm} / \mathrm{s}$ vs $7.16 \pm 1.02 \mathrm{~cm} / \mathrm{s}, \mathrm{p}<0.001)$ than those after surgical repair, although the end-diastolic volume and ejection fraction of left ventricle(LV) measured by conventional echocardiography and the LV myocardial performance index (MPI), mitral annular isovolumic contraction acceleration (IVA), early (e) to late (a) diastolic mitral annular velocity ratio calculated by DTI were all similar $(\mathrm{p}=\mathrm{NS})$. More interestingly, all right ventricular(RV) parameters excepting tricuspid annular $\mathrm{e} / \mathrm{a}$ ration $(\mathrm{p}=\mathrm{NS})$ measured by DTI were significantly better in occluder patients: the RVMPI was lower $(0.32 \pm 0.07$ vs $0.39 \pm 0.08, \mathrm{p}=0.019)$; the tricuspid annular IVA $(2.46 \pm$ $0.94 \mathrm{~m} / \mathrm{s} 2$ vs $1.60 \pm 0.33 \mathrm{~m} / \mathrm{s} 2, \quad \mathrm{p}=0.003)$ and myocardial systolic velocity $(12.69 \pm 2.56 \mathrm{~cm} / \mathrm{s}$ vs $8.98 \pm 1.59 \mathrm{~cm} / \mathrm{s}$, $\mathrm{p}<0.001)$ were both higher. In both cohorts of the patients, the size of the VSD was no significant difference $(5.92 \pm 1.7 \mathrm{~mm}$ vs $6.92 \pm 2.58 \mathrm{~mm}, \mathrm{p}=\mathrm{NS}$ ).

Conclusion: In our small sample study, the transcatheter closure of perimembranous VSD is promising technique that can be used in suitable cases, which is probably helpful to reserve the ventricular function, especially the RV function. Further and large-sample studies are required.

\section{8}

Leaflet entrapment causing acute myocardial ischemia after balloon aortic valvuloplasty

Hye Sun Hyun, Department of Pediatrics, Samsung Medical Center, SungKyunKwan University School of Medicine, Seoul, Korea

Soo In Jeong, Department of Pediatrics, Samsung Medical Center, SungKyunKwan University School of Medicine, Seoul, Korea, Korea 
June Huh, Department of Pediatrics, Samsung Medical Center, SungKyunKwan University School of Medicine, Seoul, Korea, Korea Ji-Hyuk Yang, Department of Thoracic Surgery, Samsung Medical Center, SungKyunKwan University School of Medicine, Seoul, Korea, Korea

Tae-Gook Jun, Department of Thoracic Surgery, Samsung Medical Center, SungKyunKwan University School of Medicine, Seoul, Korea, Korea

Heung-Jae Lee, Department of Pediatrics, Samsung Medical Center, SungKyunKwan University School of Medicine, Seoul, Korea, Korea I-Seok Kang, Department of Pediatrics, Samsung Medical Center, SungKyunKwan University School of Medicine, Seoul, Korea, Korea

Objective: We report a case of acute aortic insufficiency by $\mathrm{AV}$ leaflet entrapment after balloon valvuloplasty (BVP) of aortic valve $(\mathrm{AV})$.

Method: A 4-year-old girl underwent surgical aortic valvuloplasty with leaflet extension for congenital aortic steno-insufficiency (AsR). After operation she suffered from dizziness and decreased activity. Postoperative echocardigram showed mild aortic stenosis (AS) of peak pressure gradient (PG) $42 \mathrm{mmHg}$ and minimal AR. Five months after operation, she collapsed after complaining chest pain at playground. Neurologic and cardiac evaluation showed no significant finding but mild AS of above PG. We tried BVP of the AV because we thought her symptom was related with AS despite mild PG. The PG was $40 \mathrm{mmHg}$ at catheterization. The AV annulus was $15-16 \mathrm{~mm}$ and the sinotubular junction was narrow on angiogram. Initially we tried BVP with $12 \mathrm{~mm}$ balloon, but there was no change of PG. During retrial with $14 \mathrm{~mm}$ balloon, sudden bradycardia and hypotension developed, which recovered soon after medication. However, in a few minutes bradycardia reappeared which led to cardiac arrest. Angiogram during resuscitation showed open, fixed, thick leaflet causing aortic regurgitation and narrowing left coronary sinus. Under ECMO support she underwent emergency surgery. On operation field, there was no vessel or leaflet tearing, and the left coronary sinus was small.

Conclusion: Aortic cusp entrapment is a very rare complication of BVP that results acute aortic regurgitation and possible coronary insufficiency. In a case of aortic stenosis with small coronary sinus and sinotubular junction, this complication should be concerned and BVP should be done carefully.

\section{9}

\section{Successful tricuspid and mitral isthmus ablation in an} infant with right and left atrial flutter

Hiromi Iguchi, Nihon University School of Medicine, Japan

Naokata Sumitomo, Nihon University School of Medicine, Japan Takahiro Nakamura, Nihon University School of Medicine, Japan Tomonori Matsumura, Nihon University School of Medicine, Japan Hiroshi Kanamaru, Nihon University School of Medicine, Japan Mamoru Ayusawa, Nihon University School of Medicine, Japan Hideo Mugishima, Nihon University School of Medicine, Japan Shozaburo Doi, Tokyo Medical and Dental University, Japan

We report successful ablation of an infant with typical and atypical left atrial flutter (AFL) without any heart disease. A 3 year-old boy was admitted to our hospital for recurrence of AFL treatment. Echocardiogram showed no cardiac anomaly, however chest X-ray showed mild cardiomegaly. Electrocardiogram showed junctional rhythm with heart rate 49 beat per minute. Electrophysiological study showed prolonged sinus node recovery time $(4592 \mathrm{~ms})$. AFL with the cycle length of $270 \mathrm{~ms}$ was spontaneously induced during $0.02 \mu \mathrm{g} / \mathrm{kg} / \mathrm{min}$ of isoproterenol infusion, AFL. (AFL1) Propagation mapping of AFL1 showed counter clockwise rotation around the tricuspid valve by $3 \mathrm{D}$ electroanatomical mapping (CARTO). (Fig) AFL1 was successfully terminated by linear ablation from tricuspid valve to inferior vena cava (tricuspid isthmus). However, AFL2 was recurred in the next day of the first ablation. AFL2 with cycle length of $270 \mathrm{~ms}$ was induced spontaneously or by rapid atrial pacing at pacing cycle length of $230 \mathrm{~ms}$ from high right atrium during $0.02 \mu \mathrm{g} / \mathrm{kg} / \mathrm{min}$ of isoproterenol infusion. Propagation mapping of the left atrium showed clockwise rotation around the mitral valve. Linear ablation from left lower pulmonary vein to mitral valve annulus (mitral isthmus) was successfully terminated AFL2. (Fig) Radiofrequency catheter ablation in the children with common AFL was also widely performed. However, only a few report of atypical AFL after surgical corrected congenital heart disease in children is reported. This is a first report of infant with right and left AFL without any heart disease.

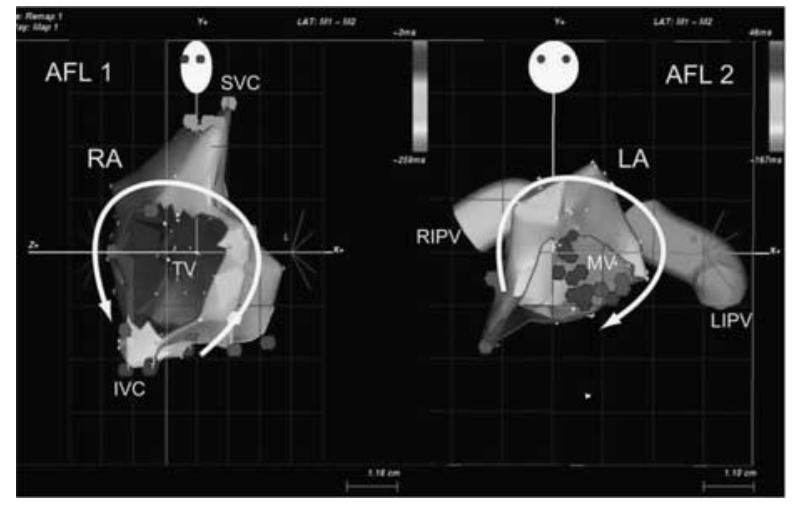

8110

Pericardiocentesis using the left lateral approach in children and infants

Frank F Ing, Texas Children's Hospital, United States

John Breinholt, Texas Children's Hospital, United States

Richard O'Brian, Texas Children's Hospital, United States Christopher Petit, Texas Children's Hospital, United States Nelson David, Texas Children's Hospital, United States

Matthewson James, St. Joseph Hospital and Medical Center, United States

Objectives: Pericardiocentesis has traditionally utilized the subxiphoid approach originally described by Marfan over 100 years ago. However, the left lateral (LL) approach using echocardiographic guidance offers an equally effective but shorter, more direct and perhaps safer route, especially for those pericardial effusions (PCE) that are loculated and/or posterolaterally located. We report our experience with this approach.

Methods: The records of all pts who underwent pericardiocentesis using the LL approach from $1 / 07$ to $12 / 08$ at Texas Children's Hospital were reviewed. Procedural data included location of entry, depth of needle required to enter the pericardium, amount and type of fluid removed, and complications.

Results: 18pts (20procedures) underwent this approach. Median age was $8.8 \mathrm{yr}$ (range: $1 \mathrm{mo}-17.25 \mathrm{yr}$ ). Presumed etiology was related to blood dycrasias (7), post viral (4), post-op (2), and others (5). Mean effusion was $7.8 \pm 4.2 \mathrm{~mm}$ inferiorly and $18.5 \pm 6.7 \mathrm{~mm}$ posterolaterally. Needle entry was all in the $\mathrm{LL}(16)$ or peri-apical region (2). Depth of needle entry ranged 5-20 mm. Amount of fluid drained averaged 209 $\pm 126 \mathrm{cc}$. Serous fluid was noted in all but 3 (serosanguious). Pericardial drains were left in place in 3. Minor complications included a small anterior pneumothorax (1) and inadvertent entrance into 
pleural space (2) without further sequelae. No major complications occurred.

Conclusion: The LL approach for pericardiocentesis is safe \& effective when there is a significant posterolateral PCE. Entrance from skin to pericardium is no more than $2 \mathrm{~cm}$ thereby minimizing errors related to angle and distance of needle penetration. Rare minor complications include pneumothorax and entrance into pleural space. This approach should be considered in the majority of pts with PCE.

\section{1}

Effects of right ventricular outflow tract of Tetralogy of Fallot on pulmonary valve development

Masahiro Kamada, Department of Pediatric Cardiology, Hiroshima City Hospital, Japan

Naomi Nakagawa, Department of Pediatric Cardiology, Hiroshima City Hospital

Hisako Kiguchi, Department of Pediatric Cardiology, Hiroshima City Hospital

Objects: We investigated the effects of the right ventricular outflow tract (RVOT) of Tetralogy of Fallot (ToF) on pulmonary valve (PV) growth following balloon valvuloplasty of the pulmonary valve (BPV).

Background: In ToF patients, BPV promotes pulmonary artery and PV growth, though a transannular patch may be required.

Methods: Clinical and catheterization data from 26 infant patients who underwent preoperative BPV during the past 9 years were retrospectively studied.

Results: The PV annulus grew significantly from -4.3 SD before BPV to -1.9 SD before ToF radical surgery, and then to $-2.0 \mathrm{~S}$ $\mathrm{D}$ in all patients except one. No significant increase in RVOT Zscore was accompanied by decreased RVOT size in 11 patients. There was no significant relationship between the RVOT at the time of BPV and PV annulus prior to total correction, between the RVOT and PV annulus prior to total correction, or between the RVOT diameter before BPV and increase in PV Z-score. In one patient, there was a significant relationship between increase in RVOT-Z score and increase in PV Z-score. In 3 patients complicated with a cyanotic spell, RVOT diameter rapidly decreased and the balloon size/PV annulus ratio (\%) during BPV was less than $120 \%$. Conclusion: PV annulus size just prior to ToF total repair is determined by $\mathrm{PV}$ annulus size at the first examination plus PV growth, which is dependent on RVOT growth after BPV. A balloon catheter greater than $120 \%$ of the PV annulus should be used during BPV to accelerate RVOT growth after the procedure.

\section{2}

Transcatheter closure of ruptured sinus of Valsalva aneurysm using the Amplatzer duct occluder: Immediate and intermediate-term results

Prafulla G Kerkar, King Edward VII Memorial Hospital, Parel. Mumbai 400 012, India

Prasanna Y Nyayadhish, King Edward VII Memorial Hospital, Parel, Mumbai 400 012, India

Charan P Lanjewar, King Edward VII Memorial Hospital, Parel, Mumbai 400 012, India

Nidheesh Mishra, King Edward VII Memorial Hospital, Parel, Mumbai 400 012, India

Milind S Phadke, King Edward VII Memorial Hospital, Parel, Mumbai 400 012, India

Gaurav S Verma, King Edward VII Memorial Hospital, Parel, Mumbai 400 012, India
Amit B Sharma, King Edward VII Memorial Hospital, Parel, Mumbai 400 012, India

Isaac Mammen, King Edward VII Memorial Hospital, Parel, Mumbai 400 012, India

Objective: To assess the immediate and intermediate-term results of transcatheter closure (TCC) of congenital ruptured sinus of Valsalva aneurysm (RSVA).

Methods: Between July 2004 and January 2009, TCC of RSVA was attempted in 17 patients (8F/9M, aged $17-47$ years). Most $(10 / 17)$ were in NYHA Class III or IV. Three had previous surgeries for CAD, VSD and RSVA. None had associated defects requiring surgery. Echocardiography revealed RSVA from right coronary sinus to RA in 3 and $\mathrm{RV}$ outflow in 4, while noncoronary sinus ruptured into RA in 9 and RV inflow in 1. The echo/angio estimated diameter of the defect at its aortic end was $4-11 \mathrm{~mm}$. After establishing an arterio-venous wire loop, a 2-4 mm larger Amplatzer duct occluder (ADO) was deployed by venous approach, ensuring closure at aortic end and nonencroachment on aortic valve/coronaries by online TEE/ angiography.

Results: The ADO deployment failed in 2 \{large defect size $(11 \mathrm{~mm})$ in one and significant procedure-related AR (PRAR) in other\}. Of 15 successful deployments, 10 had complete closure at 24 hours. Five had residual shunts (4 small, 1 moderate with selfabating hemolysis). Trace PRAR occurred in 4 . On a median follow-up of 26 months, 12 patients were in Class I and 3 in Class II. Residual shunts disappeared in 2 and were small in 3; PRAR vanished in 3 of 4 . There was no AR progression, recurrence or infective endocarditis.

Conclusions: Thus, TCC of RSVA using ADO is a safe and effective alternative to surgery. However, a longer follow-up is warranted.

\section{3}

Unusual thrombus formation complicating transdermal closure of interatrial communication

Maria G Kiaffas, Onassis Cardiac Surgery Center, Greece

Eugenia Bei, Onassis Cardiac Surgery Center, Greece

Andrew Chatzis, Onassis Cardiac Surgery Center, Greece

Spyridon Rammos, Onassis Cardiac Surgery Center, Greece

Objective: Presentation of unsual thrombogenic complication during transdermal atrial septal defect (ASD) closure.

Methods: Patients' data undergoing transdermal closure of ASD or patent foramen ovale (PFO) were reviewed, with emphasis on thrombus formation during such procedures imaged by transesophageal echocardiography (TEE).

Results: Two patients were identified with thrombi during transdermal ASD closure; a 13 year old status post surgical correction of common atrioventricular canal and a residual ASD, and a 28 year old with a PFO and history of strokes. In both, during catheterization and immediately after balloon sizing of their defects, TEE revealed a large echogenic structure in the left atrium associated with the guiding wire and perceived as a thrombus. Routine heparin anticoagulation and therapeutic clotting time (ACT) was documented before inflation of the sizing balloon. Both pts were immediately managed with anticoagulation and thrombolysis. Resolution of the thrombus was achieved in the first case in a few hours, with subsequent removal of the guiding wire and closure of his defect at a later date. In the other case despite thrombolytic efforts the thrombus increased in size and was surgically removed followed with simultaneous surgical closure of the ASD. No evidence of 
systemic embolization was identified in either pt after clot removal.

Conclusions: Thrombi during transdermal ASD closure are not rare, but are usually associated with the device's surface. Thrombi formed on guiding-wires used during the procedure, are also an existing risk. TEE is extremely helpful in their imaging and in preventing systemic embolism with their prompt identification.

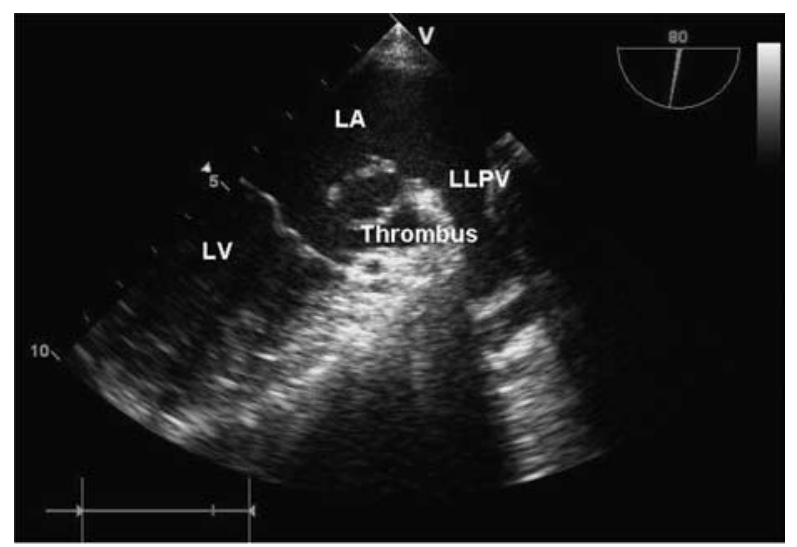

\section{4}

Patent foramen ovale; the only culprit for systemic embolization?

Maria G Kiaffas, Onassis Cardiac Surgery Center, Greece Sotiria Apostolopoulou, Onassis Cardiac Surgery Center, Greece Spyridon Rammos, Onassis Cardiac Surgery Center, Greece

Objective: Presentation of possible systemic embolism sources found incidentally during transdermal patent foramen ovale (PFO) closure.

Methods: Patients' data with neurological symptoms due to suspected systemic emboli secondary to the presence of a PFO, who underwent transdermal closure of their defect were reviewed. Emphasis was given in incidental anatomic anomalies identified by transesophageal echocardiograpy (TEE) or angiography during the procedure.

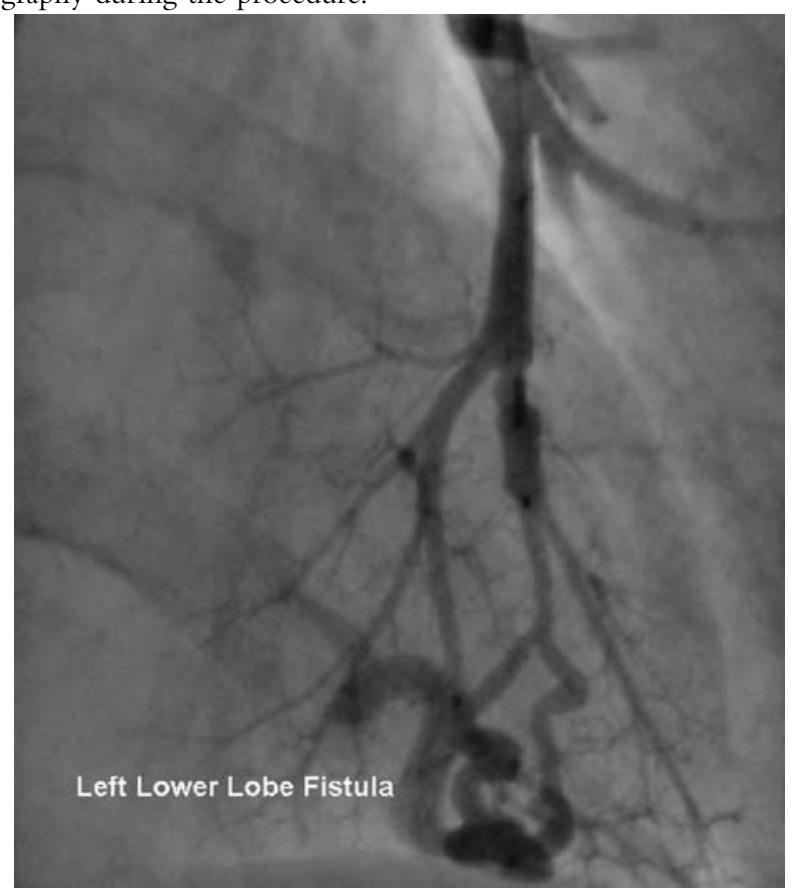

Results: Incidental identification of possible thrombotic sources other than a PFO were found in two patients with neurological symptoms and an echocardiographically diagnosed PFO with right-to-left shunt. Case \#1, a 44 year old woman with history of ischemic strokes, during catheterization for PFO closure and routine pulmonary angiography was found to have a large pulmonary arteriovenous fistula in her left lower lobe. This was embolized successfully with an Amplatzer plug following her PFO closure. Case \#2, a 20 year old man with history of migraines and syncope attributed to transient ischemic attacks, during PFO closure was found to have by TEE myocardial non-compaction involving both the LV and RV; this was confirmed by ventriculography of both ventricles during catheterization. The PFO was closed and myocardial biopsy results are pending.

Conclusions: The presence of a PFO with right-to-left shunting in view of neurological symptoms attributed to transient central nervous ischemia might not always be the culprit for systemic embolization. Thorough TEE examination and angiography might reveal other less common sources of systemic embolism; both imagers and interventionalists should be alert for the identification of such uncommon lesions.

\section{7}

Thrombolytic therapy for femoral artery thrombosis after left cardiac catheterization in children

Shihua Zhao, Cardiovascular Institute and Fuwai Hospital, Peking Union Medical College and Chinese Academy of Medical Sciences, China

Chaowu Yan, Cardiovascular Institute and Fuwai Hospital, Peking Union Medical College and Chinese Academy of Medical Sciences, China

Shiliang Jiang, Cardiovascular Institute and Fuwai Hospital, Peking Union Medical College and Chinese Academy of Medical Sciences, China

Zhongying Xu, Cardiovascular Institute and Fuwai Hospital,

Peking Union Medical College and Chinese Academy of Medical Sciences, China

Lianjun Huang, Cardiovascular Institute and Fuwai Hospital, Peking Union Medical College and Chinese Academy of Medical Sciences, China

Jian Ling, Cardiovascular Institute and Fuwai Hospital, Peking Union Medical College and Chinese Academy of Medical Sciences, China Hong Zheng, Cardiovascular Institute and Fuwai Hospital, Peking Union Medical College and Chinese Academy of Medical Sciences, China

Yun Wang, Cardiovascular Institute and Fuwai Hospital, Peking Union Medical College and Chinese Academy of Medical Sciences, China

Objective: The study was conducted to investigate the feasibility and effectiveness of thrombolytic therapy for femoral artery thrombosis after left cardiac catheterization in children. Methods Thrombolytic therapy with urokinase was carried out in children with femoral artery thrombosis after left cardiac catheterization. Patients were given a bolus injection of heparin $(100 \mathrm{u} / \mathrm{Kg})$. $30000-100000 \mathrm{u}$ boluses of urokinase (UK) were injected intravenously, and then a continuous infusion of 10000$50000 \mathrm{u} / \mathrm{h}$ was administered. Transcatheter thrombolysis was performed once previous procedures failed. Results Eight patients (age range 8 months to 7 years [ $3.1 \pm 2.3$ years], body weight $7 \mathrm{~kg}$ to $20 \mathrm{~kg}$ [13.1 $\pm 4.2 \mathrm{~kg}]$ ) presented lower limbs ischemia after left cardiac catheterizations was performed. Seven patients accepted thrombolytic therapy with UK. In 5 patients, peripheral intravenous thrombolysis was successful with restoration 
of a normal pulse. In the other 3 cases, peripheral intravenous thrombolysis failed, followed by successful transcatheter thrombolysis. The average duration of therapy was $7.25 \pm 5.31 \mathrm{~h}[1-17 \mathrm{~h}]$. The average dose of heparin and UK were $1600 \pm 723 \mathrm{u}$ [800-3000 u] and $268571 \pm 177240 \mathrm{u}$ [50000-500000u], respectively. There were no statistically significant differences in partial thromboplastin time before and during UK therapy (40.6 \pm 22.3 to $49.9 \pm 39.2$ seconds). However, the prothrombin time was significantly longer $(12.7 \pm 2.58$ to $48.1 \pm 18.6$ seconds, $\mathrm{P}<0.05)$. Patency of the target vessel was evaluated in all the patients for 2 weeks and no occlusion recurred. Conclusion Thrombolytic therapy with UK is a safe and useful modality in children with femoral artery thrombosis after left cardiac catheterization.

\section{8}

Occlusion of Fontan fenestrations using the Amplatzer septal occluder Amira AAH Al-Hay, Chest Hospital, Kuwait

Lulu Abu Shaban, Chest Diseases Hospital, Kuwait

Mostafa Al Qbandi, Chest Diseases Hospital, Kuwait

Muath Al Anbaei, Chest Diseases Hospital, Kuwait

Objective: To assess the efficacy and safety of the Amplatzer septal device for occlusion of Fontan fenestrations percutaneously. Background: Fenestration improves postoperative outcomes in children undergoing Fontan repair. Many of these fenestrations close spontaneously; ultimately persistent fenestrations cause desaturation and a potential cause of paradoxical embolism.

Methods: This is a retrospective review of 26 consecutive patients who underwent transcatheter closure of Fontan fenestration in a tertiary cardiology centre in Kuwait. The median age was 9.2 years (range, 1.5 to 18.3). After assessment of hemodynamic suitability for fenestration closure, an appropriate balloon wedge catheter sizing of fenestration was obtained. Occlusion was accomplished using a 4-7 mm single Amplatzer septal device in 12 patients and $8-13 \mathrm{~mm}$ devices in the other thirteen patients while two devices were deployed in one patient. The median fluoroscopic and procedure times were 22 and 143.5 minutes.

Results: There was one immediate complication. A $100 \%$ occlusion rate of the fenestration was achieved in both types of Fontan circulation. Mean $\pm \mathrm{SD}$ arterial oxygen saturation increased from $84.7 \pm 4.7 \%$ to $95.2 \pm 1.7 \%$ (paired $t$ test $\mathrm{p}<0.001) 10$ minutes after deployment of the occluder, while the mean Fontan circuit pressure increased but within the acceptable range $(12.9 \pm 3.2$ vs. $14.6 \pm 2.6 \mathrm{mmHg}, \mathrm{p}<0.001)$. No complications or device failures were seen during follow up. Conclusion: The Amplatzer septal occluder device is safe, and effective to close Fontan fenestration in the lateral tunnel and extracardiac conduit types. However, outpatients follow up with serial trasthoracic echocardiographic monitoring for systemic venous congestion or thrombosis is warranted.

\section{9}

Bone Marrow-Derived Hematopoietic and Non-Hematopoietic Stem Cells contribute to distinct cell lineages in pulmonary vascular diseases in chronically hypoxic mice Taichi Kato, Mie University Graduate School of Medicine, Japan Yoshihide Mitani, Mie University Graduate School of Medicine, Japan Noriko Yoyoda, Mie University Graduate School of Medicine, Japan Junko Maruyama, Mie University Graduate School of Medicine, Japan Masahiro Masuya, Mie University Graduate School of Medicine, Japan Hiroyuki Ohashi, Mie University Graduate School of Medicine, Japan
Kazuo Maruyama, Mie University Graduate School of Medicine, Japan Yoshihiro Komada, Mie University Graduate School of Medicine, Japan

Background: Bone marrow (BM) stem cells differentiate into various cells under physiological and pathological conditions. However, whether BM-derived cells incorporated into pulmonary vascular diseases (PVD) are of hematopoietic stem cell (HSC) origin or of non-HSC origin, is unknown. We tested the hypothesis that different BM stem cell fractions differentiate into discrete cell lineages in PVD.

Methods \& Results: Lethally irradiated C57BL6 mice, transplanted with whole BM cells $(2 \mathrm{x} 106)$ (TNC study, $\mathrm{n}=25)$ or 6,500 cells from c-kit+, Sca-1+, Lin- HSC-rich fraction (KSL study, $\mathrm{N}=12$ ) from littermates expressing eGFP, were kept in hypobaric hypoxia $(380 \mathrm{mmHg})$ or in the ambient air, for 21 days. In TNC study, compared with controls, percentages of $\mathrm{CD} 31+\mathrm{eGFP}+\mathrm{BM}$-derived endothelial cells/ whole CD31+ cells $(4.0 \pm 0.5$ vs $1.7 \pm 0.4)$ and of MOMA2+eGFP+BMderived macrophages/ whole MOMA2 + cells $(38.4 \pm 2.6$ vs $20.0 \pm 5.0)$ were higher in hypoxic mice $(\mathrm{p}<.05)$. However, $\alpha$ actin + eGFP+BM-derived smooth muscle cells were not found in either group investigated. In KSL study, CD31+ eGFP+ endothelial cells were rarely seen both in hypoxic and normoxic mice. In contrast, MOMA2+eGFP+ cells were more frequently seen in hypoxic mice.

Conclusions: BM-derived macrophages and endothelial cells, which were increased in PVD, mainly originate from an HSCrich fraction and a non-HSC-rich fraction, respectively. These findings suggest differential roles of BM-derived HSC and nonHSC in the development of pulmonary hypertension.

\section{0}

Estimation of Pulmonary Vascular Resistance:

Correlation between Echocardiography and Catheterization Data In Patients With Congenital Heart Disease Dheeraj Bhatt, Post Graduate Institute of Medical Education \& Research - chandigarh India, India

Rajiv Mahajan, Post Graduate Institute of Medical Education \& Research - chandigarh India, India

Manojkumar Rohit, Post Graduate Institute of Medical Education \& Research - chandigarh India, India

The Doppler-derived ratio of TRV/TVIRVOT has been previously used to measure pulmonary vascular resistance (PVR). The applicability of this ratio in measuring PVR in patients with congenital heart disease has not been adequately evaluated.

Aim: To measure PVR in patients with congenital heart disease using Doppler echocardiography and compare with invasively derived PVR.

Method: Twenty patients with post-tricuspid shunt lesions who were planned to undergo cardiac catheterization as a part of their management were enrolled for the study. The formula, PVR $(\mathrm{WU})=10 \mathrm{x}$ TRV/TVIRVOT was used to calculate PVR noninvasively. Peak tricuspid regurgitation velocity (TRV) and the time velocity integral of the right ventricular outflow tract (TVIRVOT) were measured using continuous wave and pulsedwave Doppler respectively. PVR was also determined by cardiac catheterization. The peak systolic velocity of the lateral wall of tricuspid annulus ( $\mathrm{tSm}$ ) was also measured by tissue Doppler and correlated with invasively determined PVR.

Results: There was a significant correlation between the ratio TRV/TVI RVOT and PVR CATH $r=0.635(p=0.003)$. This correlation was better in the subgroup with lower PVR $r=0.817$ 
(PVR $<6 \mathrm{WU})$. However, there was a significant difference between the mean PVR obtained by echocardiography and catheterization, mean PVR 1.8 WU vs 6.9 WU respectively $(p<0.0001)$. Bland-Altman analysis showed very wide limits of agreement which was clinically unacceptable. There was no correlation between $\mathrm{tSm}$ and invasively determined PVR.

Conclusion: Although there is positive correlation between the TRV/TVIRVOT and invasively determined PVR, this ratio cannot be reliably used to estimate the PVR in post-tricuspid shunts.

8121

Pulmonary Veno-Occlusive Disease in a Child with Prothrombine Gene Mutation

Makbule Eren, Turkey

Birsen Ucar, Professor in Eskisehir Osmangazi University Faculty of Medicine Department of Pediatrics, Section of Cardiology, Turkey Baris Urhan, Assistant in Eskisehir Osmangazi University, Faculty of Medicine, Department of Pediatrics, Turkey

Ragip Özkan, Professor in Eskisehir Osmangazi University Faculty of Medicine Department of Radiology, Turkey

Mustafa Acikalin, Associate Professor in Eskisehir Osmangazi

University, Faculty of Medicine, Department of Pathology

Zubeyir Kilic, Turkey

Pulmonary veno-occlusive disease (PVOD) is one of the rare causes of pulmonary hypertension. Although various factors have been claimed to be a risk factor, the role of thrombotic diathesis in the pathogenesis needs to be clarified. To date increased platelet adhesiveness and some risk factors for hypercoagulability, such as oral contraceptive use were found to be associated with PVOD. Here we reported a child with PVOD and prothrombine gene mutation which is the first case of PVOD with prothrombine gene mutation.

A 13-year-old girl presented with a history of dyspnea and cyanosis. Physical examination revealed cyanosis, tachypnea, ortophnea, bilaterally decreased pulmonary sounds and a loud second heart sound. Chest radiography and echocardiography findings were consistent with pulmonary hypertension and pulmonary edema. Thorax computed tomography showed bilateral ground glass appearance, inter-lobular septal thickening and increased pulmonary arterial diameter suggesting PVOD. Right heart catheterization was performed and increased pulmonary arterial but normal wedge pressure was observed. Lung biopsy confirmed PVOD; intimal fibrosis, increased wall thickness, and luminal occlusion in the small interlobular veins. Other laboratory evaluations revealed heterozygous prothrombine gene mutation with normal serological results for rheumatologic, infectious and hereditary thrombophilias. Thrombocyte aggregation and secretion tests were normal. Despite of treatment with morphine, furosemid and iloprost inhalation pulmonary edema progressed. So iloprost was discontinued, warfarin and corticosteroid treatment were started. But she worsened and died at 33thday of her admission.

In conclusion prothrombine gene mutation may be a risk factor for PVOD. But its association should be verified with further reports.

8123

Single-Center Experience in Large Cohort of Patients with Pulmonary Arterial Hypertension Related to Congenital Heart Disease over 15 years: Effect of New Therapies Sotiria C Apostolopoulou, Onassis Cardiac Surgery Center, Athens, Greece, Greece
Alexandros Tsoutsinos, Onassis Cardiac Surgery Center, Athens, Greece, Greece

Maria Kiaffas, Onassis Cardiac Surgery Center, Athens, Greece, Greece Cleo V Laskari, Onassis Cardiac Surgery Center, Athens, Greece, Greece Spyridon Rammos, Onassis Cardiac Surgery Center, Athens, Greece, Greece

Objective: This study examines the epidemiology, 15-year F/U and effect of emerging therapies on a large cohort of patients with pulmonary arterial hypertension (PAH) related to congenital heart disease (CHD).

Methods: Clinical, echocardiographic, exercise and hemodynamic data were recorded on all patients diagnosed with PAH related to CHD between 1993 and 2008.

Results: Sixty patients aged $21 \pm 14(0.1-51)$ years $(24(40 \%)$ $<18$ yrs) at diagnosis were studied; $44(73 \%)$ had unrestrictive mixing and the rest non-mixing lesions, mainly repaired CHD. Seven (12\%) WHO Class II patients received no therapy, 45 $(75 \%)$ in Class II-III monotherapy (34 bosentan, 1 discontinuation after repair, 6 sildenafil, 5 sitaxsentan) with clinical and exercise improvement. Due to deterioration, sildenafil was added in $6(10 \%)$ patients after $3 \pm 1$ years of bosentan monotherapy and epoprostenol in $2(3 \%)$ patients after 1 year combination therapy. Over $6 \pm 4(1-15) \quad \mathrm{F} / \mathrm{U}$ years, 49 (82\%) patients remained stable on no or monotherapy, 4 (7\%) deteriorated after 1-5 years monotherapy but stabilized on combination therapy and 7 (12\%) died (3 right heart failure, 4 suddenly). The unrestrictive mixers had $2(4 \%)$ and the non-mixers $5(33 \%)$ deaths respectively.

Conclusions: Long-term improvement and stabilization is possible in $\mathrm{PAH}$ related to $\mathrm{CHD}$ with monotherapy or combination therapy in case of deterioration overtime. Death may be sudden or due to right heart failure, while survival is better in unrestrictive mixers. Larger long-term registry studies are needed to evaluate survival and clinical condition and improve medical management of these patients in the current treatment era.

\section{4}

Impact on clinical parameters using new farmacological therapies in patients with pulmonary arterial hypertension due to congenital heart disease

Paola Argiento, Chair of Cardiology, Second University of Naples, Monaldi Hospital, Naples, Italy, Italy

Michele D'Alto, Chair of Cardiology, Second University of Naples, Monaldi Hospital, Naples, Italy, Italy

Emanuele Romeo, Chair of Cardiology, Second University of Naples, Monaldi Hospital, Naples, Italy, Italy

Giuliano D'Alterio, Chair of Cardiology, Second University of Naples, Monaldi Hospital, Naples, Italy, Italy

Berardo Sarubbi, Chair of Cardiology, Second University of Naples, Monaldi Hospital, Naples, Italy, Italy

Giuseppe Santoro, Chair of Cardiology, Second University of Naples, Monaldi Hospital, Naples, Italy, Italy

Maria Giovanna Russo, Italy

Raffaele Calabro', Chair of Cardiology, Second University of Naples, Monaldi Hospital, Naples, Italy

Objective: To evaluate the impact of new farmacological options in adults with PAH CHD-related.

Methods: We enrolled between January 2001 - November 2008 all the patients with PAH CHD-related.

Results: The patients were 65 (27 M, 38 F, mean age $38.4 \pm 13.5)$. 22 had ventricular septal defect, 17 atrio-ventricular canal, 10 atrial septal defect, 7 single ventricle, 5 atresia of pulmonary valve 
with ventricular septal defect, 4 persistent ductus arteriosus. At the rest, the NYHA class was, III 47 for patients and IV for 18 patients. The $\mathrm{O} 2$ saturation at rest was $80.4 \pm 10.5 \%$. The mean pulmonary pression was $76.4 \pm 21.6 \mathrm{mmHg}$; the mean pulmonary artery resistances was $8.7 \pm 4.8$ Wood Unit $/ \mathrm{m} 2$; the mean $\mathrm{QP} / \mathrm{QS}$ was $0.9 \pm 0.5$. Then, 30 patients are under therapy with oral bosentan $(125 \mathrm{mg} \times 2 /$ day or $62.5 \mathrm{mg} \times 2 /$ day $), 7$ with oral sildenafil $(20 \mathrm{mg} \times 3 /$ day), 5 patients with oral sitaxentan $(100 \mathrm{mg} /$ day), 17 with oral bosentan and oral sildenafil, 2 with oral sitaxentan and oral sildenafil, 4 with oral bosentan, oral sildenafil and epoprostenol ev $(9.5 \mathrm{ng} / \mathrm{kg} / \mathrm{min})$. At the follow-up (mean 3,1 $\pm 2,2 \mathrm{yrs}$ ), the NYHA class was improved for 50/65 (77\%) patients, the actual NYHA class is I for 7 patients, II for 31 patients, III for 21 patients and IV for 6 patients.

Conclusion: A complex therapeutical management is required for patient with PAH CHD-related. In our experience the new farmacological treatments were safe and well tolerated in adults patients with PAH CHD-related; we observed an unexpected and significatevely improvement of NYHA class.

\section{5}

Fetal and neonatal ventricular load markers in experimental congenital diaphragmatic hernia Maria J Baptista, Pediatric Cardiology, H. S. João, Porto; Life and Health Sciences Research Institute, Minho University, Portugal Cristina Nogueira-Silva, Life and Health Sciences Research Institute, Minho University, Portugal

José C Areias, Pediatric Cardiology, H. S. João, Porto, Portugal Jorge M Correia-Pinto, Life and Health Sciences Research Institute, Minho University, Portugal

Background: In congenital diaphragmatic hernia $(\mathrm{CDH})$, vascular pulmonary underdevelopment could induce severe pulmonary hypertension; however, its significance in perinatal ventricular load conditions is largely unknown. Ventricular pressure overload induces increased myocardium gene expression of B-type natriuretic peptide (BNP), components of the renin-angiotensinogen and endothelin (ET)-1 system. Our aim was to evaluate perinatal myocardium expression of these genes in a nitrofeninduced $\mathrm{CDH}$ rat model.

Methods: In the nitrofen-induced $\mathrm{CDH}$ rat model, fetuses from dated pregnant Sprague-Dawley rats at 15.5, 17.5, 19.5 and 21.5 days postcoitum as well as newborn pups were assigned to 3 experimental groups: control, nitrofen (exposed to nitrofen, without $\mathrm{CDH}$ ), and $\mathrm{CDH}$ (exposed to nitrofen, with $\mathrm{CDH}$ ). Myocardial samples collected from the right and left ventricle were processed for quantification of mRNA of BNP, angiotensinogen, and ET-1.

Results: The perinatal expression of BNP, angiotensinogen, and ET-1 mRNA in the right and left ventricle of the control group revealed daily changes. During gestation, the expression of BNP and angiotensinogen mRNA underwent significant oscillation compared with control in both nitrofen-exposed fetuses, although there is no significant differences between the nitrofen and $\mathrm{CDH}$ groups. After birth, occurs a significant increasing expression of all studied genes only in the right ventricle of CDH pups.

Conclusions: The pre-natal myocardial quantification of BNP, angiotensinogen, and ET-1 mRNA demonstrated that $\mathrm{CDH}$ is only associated with significant molecular alterations in the right ventricle and after birth, suggesting that vascular alterations does not have significant pre-natal impact in fetal hemodynamic.

\section{6}

Cardiac function in pulmonary hypertension: the usefulness of biochemical markers and echo in management of $\mathrm{CDH}$ infants

Maria J Baptista, Pediatric Cardiology, H. S. João, Porto; Life and Health Sciences Research Institute, Minho University, Portugal Gustavo Rocha, Neonatology Service, Hospital S. João, Porto, Portugal Fátima Clemente, Neonatology Service, Hospital S. João, Porto, Portugal Luis Azevedo, Bioestatistica e Informática Médica, Faculdade de Medicina, Porto, Portugal

José C Areias, Pediatric Cardiology, H. S. João, Porto, Portugal Jorge Correia-Pinto, Pediatric Surgery Dpt, H S João, Porto; Life and Health Sciences Research Institute, Minho University, Portugal

Background: The severity of pulmonary hypertension $(\mathrm{PH})$ in congenital diaphragmatic hernia $(\mathrm{CDH})$ seems to be determinant of clinical outcome. The increased right ventricle (RV) afterload could interfere in heart function contributing for the poor outcome in most affected newborns. Our aim was to evaluate the clinical usefulness of biochemical and echocardiographic markers of ventricular overload in management of $\mathrm{CDH}$ infants.

Methods: 28 newborns, $\mathrm{CDH}(\mathrm{n}=18)$ and age-matched controls $(\mathrm{n}=10)$ were enrolled in a prospective study. We assessed $\mathrm{N}$ terminal-pro-B type natriuretic peptide (NT-proBNP) plasmatic levels, echo parameters of $\mathrm{PH}$ and biventricular function at $24 \mathrm{~h}$ after delivery as well as survival outcome.

Results: In comparison to control group, $\mathrm{CDH}$ infants presented higher estimated mean pulmonary pressure and NT-proBNP. Regarding echocardiographic parameters of RV function, $\mathrm{CDH}$ infants presented higher RV Tei index and lower peak pulmonary velocity as well as E/A wave tricuspid ratio, suggesting impairment in RV function. Concerning left ventricle (LV), $\mathrm{CDH}$ infants had a significant increase in LV ejection fraction and a decreased E/A wave mitral ratio, evocative of $\mathrm{LV}$ function changes. We found significant correlations between NT-proBNP and estimated pulmonary mean pressure, RV Tei index and tricuspid E/A ratio. Additionally, we found that $\mathrm{CDH}$ infants with NT-proBNP higher than $11.500 \mathrm{pg} / \mathrm{ml}$ experienced a worse prognosis.

Conclusions: We demonstrated that PH is associated with NTproBNP elevation and heart function adaptation in $\mathrm{CDH}$ infants. We believe that echocardiographic study and early elevations in NT-proBNP levels could alert for a subset of $\mathrm{CDH}$ infants with worse prognosis.

\section{7}

Prevalence and prognostic value of acute pulmonary vasodilator response in children and adults with pulmonary arterial hypertension

J Menno Douwes, Center for Congenital Heart Diseases, Dept of Pediatric Cardiology, University Medical Center Groningen,

The Netherlands

Rosa Laura E van Loon, Center for Congenital Heart Diseases, Dept of Pediatric Cardiology, University Medical Center Groningen

Elke S Hoendermis, Center for Congenital Heart Diseases, Dept of Cardiology, University Medical Center Groningen

Melle Talsma, Center for Congenital Heart Diseases, Dept of Pediatric Cardiology, University Medical Center Groningen

Rolf MF Berger, Center for Congenital Heart Diseases, Dept of

Pediatric Cardiology, University Medical Center Groningen

Objective: To investigate the prevalence and prognostic value of acute pulmonary vasodilator response in children and adults with pulmonary arterial hypertension (PAH). 
Methods: Sixty-six PAH patients (38 children, 28 adults; 20 with and 46 without post-tricuspid shunt) underwent acute pulmonary vasodilator challenge. Responders were identified according to three currently used criteria:

1) Rich-criteria, 1992: decrease in mean pulmonary arterial pressure (mPAP) and pulmonary vascular resistance (PVR) of $>20 \%$;

2) Barst-criteria, 1986: decrease in mPAP of $>20 \%$, unchanged or increased cardiac index and decreased or unchanged pulmonary to systemic vascular resistance ratio (PVR/SVR);

3) Sitbon-criteria, 2005: decrease in $\mathrm{mPAP}$ of $>10 \mathrm{mmHg}$ reaching a mPAP of $\leqslant 40 \mathrm{mmHg}$ and an increased or unchanged cardiac output.

We evaluated the prevalence of acute response in all patients, separately in children versus adults and separately in patients with versus without post-tricuspid shunt. Next, we analyzed whether responders had better survival.

Results: The prevalence of acute pulmonary vasodilator response in all groups depended on the criteria used (Figure). "Sitbonresponders" tended to have better survival than non-responders (5-year survival $100 \%$ vs $77.4 \%$ respectively, $p=0.29$ ), whereas "Rich- and Barst-responders" showed no difference in survival compared to non-responders. Lower PVR/SVR and mPAP/ mSAP ratio both at baseline and after vasodilator challenge predicted improved survival.

Conclusions: The prevalence of acute pulmonary vasodilator response is highly dependent on the criteria used, appears to be higher in children, but absent in patients with a post-tricuspid shunt. Only responders according to the strict Sitbon-criteria showed improved survival. PVR/SVR and $\mathrm{mPAP} / \mathrm{mSAP}$ ratio appear good predictors of survival in PAH.

\section{Prevalence of acute pulmonary vasodilator response}

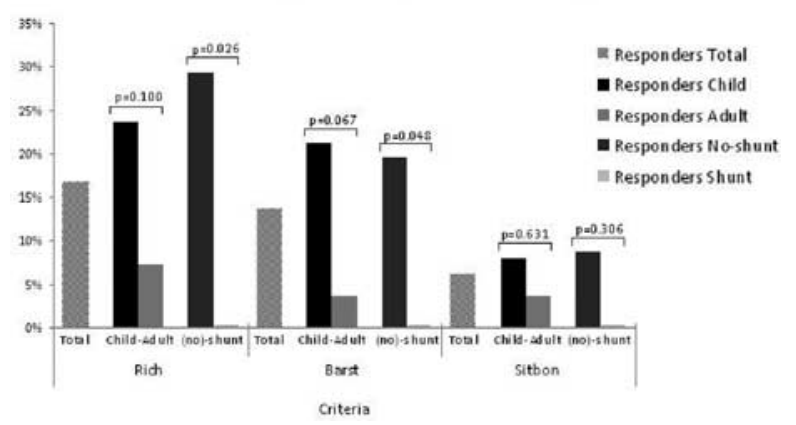

8128

The Impact of Current Therapies on Survival in Children with Idiopathic Pulmonary Arterial Hypertension

Tara Bharucha, Australia

Michelle L Rose, The Royal Children's Hopsital, Melbourne, Australia Suzanna Vidmar, Australia

Ingrid P King, Australia

Daniel J Penny, The Royal Children's Hopsital Melbourne E Murdoch Children's Research Institute, Australia

Peta Alexander, The Royal Children's Hospital, Melbourne, Australia Robert G Weintraub, The Royal Children's Hopsital, Australia

Objective: Childhood idiopathic pulmonary arterial hypertension (IPAH) is a devastating and rapidly progressive disease. Combination medical therapies are frequently employed, despite limited data about efficacy. We reviewed outcomes for all children aged 0-18 years with IPAH, referred to a single statewide cardiology service since 1998 (current patients).
Methods: Standard statistical methods were used to assess survival. Freedom from initiation of intravenous epoprostenol was calculated for those started on first-line oral therapies. Historic controls comprised children with IPAH diagnosed between 1980-1997.

Results: The mean (SD) age at diagnosis for 15 current IPAH patients was 7.3 (4.2) years compared to 6.9 (4.2) years for 13 historic controls $(p=0.83)$. The two groups were matched for haemodynamic parameters at right heart catheterisation. A PDE5 inhibitor was administered to 12 of $15(80 \%)$ current patients, an endothelin receptor antagonist in 14 (93.3\%) patients, epoprostenol in $9(66.7 \%)$ and a calcium channel blocker in 2 $(13.3 \%)$ patients. Eight current patients have been treated with a combination of $3 \mathrm{PAH}$ specific therapies, 6 patients with two therapies, and one patient is receiving monotherapy. Survival free from lung transplantation for current patients was $100 \%$ at 1 year after diagnosis and $56.4 \%$ at 5 years, compared to $50 \%$ at 1 year and $33.3 \%$ at 5 years for historic controls $(p=0.06)$. Survival free from epoprostenol initiation for current patients was only $22.4 \%$ at 5 years.

Conclusions: Combination therapies are associated with modestly improved survival in children with IPAH. The probability of epoprostenol utilisation within 5 years of diagnosis remains high.

\section{9}

A descriptive analysis of pulmonary arterial hypertension in children from the East of Scotland 1983 to 2008

Janet E Burns, Royal Hospital for Sick Children, Edinburgh, United Kingdom

Muhammed Walayat, Royal Hospital for Sick Children, Edinburgh, United Kingdom

Phil Booth, Aberdeen Maternity Hospital, United Kingdom

Iain Laing, Simpson Memorial Maternity Pavilion, Edinburgh, United Kingdom

In the 1980s, paediatic pulmonary arterial hypertension (PAH) was rare but commonest in patients with late diagnosis of structural heart faults, often with associated Down syndrome, and neonates with persistent fetal circulation. Occasional children were seen with primary pulmonary hypertension/idiopathic pulmonary arterial hypertension (IPAH), sometimes in a familial context. Those who responded to calcium channel blockers $(\mathrm{CaCBs})$ tended to survive and those who did not died within a few months or years of diagnosis. Patients with pulmonary hypertension associated with a structural heart fault with the potential for a large left to right shunt were likely to die at surgical repair if they had not shown evidence for significant left heart volume loading and associated heart failure.

\begin{tabular}{llll}
\hline & 1980-89 & 1990-99 & 2000-2008 \\
\hline $\begin{array}{l}\text { Downs with } \\
\text { inoperable disease }\end{array}$ & $15(10)$ & $8(4)$ & $4(20)$ \\
PFC/PPHN & $12(8)$ & $11 / 4$ & $3 / 2$ \\
IPAH & $5(2)$ & $3(3)$ & $2(2)$ \\
$\begin{array}{l}\text { Post operative } \\
\text { PAH }\end{array}$ & & \\
& & $\begin{array}{c}\text { PAH with other } \\
\text { problems }\end{array}$ \\
\hline
\end{tabular}

In the 21st century, screening of Down syndrome infants for structural heart faults with earlier surgical repair and better neonatal care have almost eliminated the formerly most common 
causes of PAH. Their places have been taken by infants with congenital diaphragmatic hernia, occasional post repair persistent $\mathrm{PAH}$ in survivors and patients with a wide variety of other systemic problems including bone marrow disorders. Most diagnosed patients are treated with a variety of agents and $\mathrm{CaCBs}$ are uncommonly used.

In this review of paediatric patients from the East of Scotland, the prevalence of different types of $\mathrm{PAH}$ is compared and the unexpectedly favourable outcome in some patients discussed This may have relevance to the practice of routine presciption of anti IPAH treatment for all patients.

\section{0}

Inhaled iloprost In Control Of Postoperative Inhaled iloprost In Control Of Postoperative Pulmonary Hypertension After Pediatric Cardiac Surgery: A One Year, Single Center, Open Label Pilot Trial

Xiaoman Cai, Department of cardiovascular surgery, Shanghai Children's Medical Center, Medical college of Shanghai Jiaotong University, China

Zhuoming Xu, Department of cardiovascular surgery, Shanghai Children's Medical Center, Medical college of Shanghai Jiaotong University, China

Jinfen Liu, Department of cardiovascular surgery, Shanghai Children's Medical Center, Medical college of Shanghai Jiaotong University, China Zhenying Shi, Department of cardiovascular surgery, Shanghai Children's Medical Center, Medical college of Shanghai Jiaotong University, China

Limin Zhu, Department of cardiovascular surgery, Shanghai Children's Medical Center, Medical college of Shanghai Jiaotong University, China Xinrong Liu, Department of cardiovascular surgery, Shanghai Children's Medical Center, Medical college of Shanghai Jiaotong University, China Zhaokang Su, Department of cardiovascular surgery, Shanghai Children's Medical Center, Medical college of Shanghai Jiaotong University, China

Objectives: Pulmonary hypertension is a major postoperative complication in children with congenital heart disease (CHD) and has significant impact on morbidity and mortality in the postoperative period. The aerosolized administration of the prostacyclin analogue iloprost is a alternative with pulmonaryselective vasodilation and virtually no systemic side-effects. The aim was to evaluate the efficacy of inhaled iloprost for treatment of $\mathrm{PH}$ after CHD repair in children.

Methods: During January 2008-December 2008, 46 pts with mean age of $13.7 \pm 28.8$ month(range $0.8-132$ months) and significant postoperative $\mathrm{PH}(\mathrm{Pp} / \mathrm{Ps} \geqslant 0.5$ or $\mathrm{mPAP} \geqslant 30 \mathrm{mmHg})$ after repair of CHD (Table 1). PH was evaluated through PA line and/or echocardiography. Inhaled iloprost was administered via nebulized aerosol at a dose of $50 \mathrm{ng} / \mathrm{kg} / \mathrm{min}$ lasting $10 \mathrm{~min}$ q2h to q6h.

Result: All patients were weaned off ventilators successfully in CICU and no drug related complications happened. The duration of mechanical ventilation was $53.8 \pm 36.1 \mathrm{hr}$ and CICU stay was $6.1 \pm 3.6$ days. The reintubation rate was $7.2 \%$ (3 pts) for pulmonary infection. One patient dead (hospital mortality was $2.4 \%$ ) due to LCOS. Inhaled iloprost induced a statistically reduction $\mathrm{mPAP}$ at the end of the inhalation period in comparison to baseline $(\mathrm{P}<0.05)$. The $\mathrm{Pp} / \mathrm{Ps}$ ratio decreased over this period $(\mathrm{P}<0.05)$. There were no change in $\mathrm{HR}$ and ABP during inhalation (Table 2).

Conclusion: Inhaled iloprost appears to be a selective pulmonary vasodilator and may be effective in the treatment of postoperative $\mathrm{PH}$ after Paediatric Cardiac Surgery.
Table 1 Information for all patients' demographic and operative characteristics

\begin{tabular}{ll}
\hline Age (month) & $13.72 \pm 28.81$ \\
Weight (kg) & $11.32 \pm 18.61$ \\
Gender & \\
Male & $32(69.57 \%)$ \\
Female & $14(30.43 \%)$ \\
Diagnosis & \\
D-TGA/VSD/PH & $11(23.91 \%)$ \\
DORV/VSD/PH & $10(21.74 \%)$ \\
COA/VSD/PH & $8(69.57 \%)$ \\
IAA/VSD/PH & $6(17.39 \%)$ \\
PTA/VSD/PH & $6(17.39 \%)$ \\
VSD/PH & $5(10.87 \%)$ \\
Prognosis & \\
survival & $45(97.82 \%)$ \\
death & $1(2.17 \%)$ \\
\hline
\end{tabular}

Table 2 Pulmonary and systemic hemodynamics pre- and postiloprost inhalation

\begin{tabular}{llll}
\hline & $\mathrm{T}_{\mathbf{0}}$ & $\mathrm{T}_{1}$ & $\mathrm{~T}_{2}$ \\
\hline MAP (mmHg) & $68.81 \pm 10.51$ & $56.68 \pm 12.33$ & $61.72 \pm 11.40$ \\
MPAP (mmHg) & $42.42 \pm 12.26$ & $23.65 \pm 6.31^{*}$ & $20.70 \pm 4.72^{*}$ \\
Pp/Ps & $0.62 \pm 0.12$ & $0.42 \pm 0.10^{*}$ & $0.33 \pm 0.11^{*}$ \\
HR & $108.23 \pm 21.32$ & $115.01 \pm 13.61$ & $106.32 \pm 8.50$ \\
CVP & $11.82 \pm 4.53$ & $9.10 \pm 3.07$ & $9.15 \pm 3.02$ \\
\hline
\end{tabular}

$\mathrm{T}_{0}=$ before inhalation-baseline; $\mathrm{T}_{1}=$ end of the inhalation period; $\mathrm{T}_{2}=30 \mathrm{~min}$ after the end of the inhalation period.

$\mathrm{MAP}=$ mean arterial pressure; $\mathrm{MPAP}=$ mean pulmonary arterial pressure; $\mathrm{Pp} / \mathrm{Ps}=\mathrm{a}$ ratio of pulmonary to systemic systolic pressure; $\mathrm{HR}=$ heart rate $\mathrm{CVP}=$ central venous pressure

$\star \mathrm{P}<0.05$ in comparison to baseline.

\section{1}

Ventricular septal defect closure with double patch technique in paediatric patients with increased pulmonary vascular resistance

Jorge L Cervantes, "Ignacio Chávez" National Cardiology Institute of México, Mexico

Pedro J Curi-Curi, "Ignacio Chávez" National Cardiology Institute of México, Mexico

Samuel E Ramirez-Marroquin, "Ignacio Chávez" National Cardiology Institute of México, Mexico

Jose A Garcia, "Ignacio Chávez" National Cardiology Institute of México, Mexico

Juan Calderon-Colmenero, "Ignacio Chávez” National Cardiology Institute of México, Mexico

Objetive: To describe our experience in surgical closure of ventricular septal defects (VSD) with double patch technique in paediatric patients with increased pulmonary vascular resistance (PVR).

Methods: We studied retrospectively 24 patients (63\% female), with a mean age of 7 years (range, 1 to 18 years) and VSD associated with increased PVR $(10.6 \pm 5.6 \mathrm{U}$ Wood) that were operated with double patch VSD closure at a 4 year period. We describe the early and mid term outcome with emphasis in morbidity and mortality.

Results: The defects were classified as simple (VSD as an isolated lesion) in 18 cases (75\%), or complex (VSD associated with other mayor cardiac lesions) in 6 cases $(25 \%)$. All VSD were closed with 
a bovine pericardial patch with a mean restrictive fenestration of $5.3 \mathrm{~mm}$ (range, 4.5 to $8 \mathrm{~mm}$ ). Another small patch was placed at the left side of the first one in order to allow a unidirectional right to left shunt. Only one of these patients died in the early postoperative period because of pulmonary hypertension. Early morbidity causes were: pulmonary hypertension ( 8 cases: $36 \%)$, right ventricular failure (5 cases: $23 \%$ ) and pneumonia (3 cases: $14 \%)$. There have been no deaths in the mid term follow-up and only one case (4.5\%) developed severe mitral and aortic regurgitation. Conclusions: Placement of a unidirectional flap valve VSD patch in children with pulmonary hypertension and increased PVR has reasonable morbidity and mortality, and reduces the need for ECMO or nitric oxide in these high risk group of patients.

\section{3}

The Effect of Perioperative inhaled Iloprost Treatment on Congenital Heart Disease with Pulmonary Arterial Hypertension

Deok Young Choi, Department of Pediatrics, Gil medical Center, Gachon University of Medicine and Science, Korea

Mi Jin Jung, Department of Pediatrics, Gil medical Center, Gachon University of Medicine and Science, Korea

Chang Hyu Choi, Department of Cardiovascular Surgery, Gil medical Center, Gachon University of Medicine and Science, Korea

Objectives: Congenital heart disease(CHD) with pulmonary arterial hypertension (PAH) has somewhat high risk of postoperative morbidity and mortality. Inhaled iloprost has been approved to be effective in the treatment of $\mathrm{PAH}$. We assessed the efficacy and safety of inhaled iloprost treatment in cases of CHD with PAH before and after operation.

Methods: Total 20 patients who had VSD or ASD with PAH showing bidirectional shunt or right ventricular pressure(RVP) estimated more than $60 \%$ of systemic pressure in the preoperative echocardiogram were enrolled. 10 patients (Group I) were treated with inhaled iloprost before (mean 7.4 days) and after (mean 5.8 days) operation. 10 patients (Group II) were not given. We investigated subcutaneous oxygen saturation ( $\mathrm{SaO} 2), \mathrm{RVP}$ in the echocardiogram, and postoperative clinical course like duration of ventilator application, inotropic support, ICU stay, and chest tube drainage.

Results: Mean age $(13.6 \pm 16.5$ vs $10.3 \pm 12.7, \mathrm{P}=0.3)$ and weight $(26.1 \pm 22.3$ vs $20.8 \pm 16.2, \mathrm{P}=0.44)$ in both groups were not different statistically. No perioperative mortality was encountered. There were increased $\mathrm{SaO} 2 \quad(92.4 \pm 2.9 \%$ to $96.8 \pm 2.4 \%, \quad \mathrm{P}<0.005)$ and decreased estimated RVP $(84.6 \pm 18.1 \mathrm{mmHg}$ to $74.9 \pm 14.2 \mathrm{mmHg}, \quad \mathrm{P}=0.13)$ with preoperative use of inhaled iloprost in Group I. In the postoperative clinical course, Group I showed shorter duration in days compared to Group II, statistically insignificant though : mechanical ventilation $(1.5 \pm 1.1$ vs $2.2 \pm 1.5, \quad \mathrm{P}=0.14)$, inotropic support $(3.4 \pm 1.9$ vs $4.3 \pm 1.4, \mathrm{P}=0.13)$, ICU stay $(3.5 \pm 1.9$ vs $4.8 \pm 1.6, \mathrm{P}=0.07)$ and chest tube drainage $(4.0 \pm 0.9$ vs $5.2 \pm 1.9, \mathrm{P}=0.057)$.

Conclusion: Our study represented that perioperative medical intervention with inhaled iloprost was effective to reduce pulmonary arterial pressure and to improve postoperative clinical course in congenital heart diseases with pulmonary hypertension.

\section{4}

The role of Rho A/Rho-kinase pathway on the pathogenesis of pulmonary hypertension secondary to left ventricular dysfunction

Zen-Kong Dai, Dept. of Pediatrics, Kaohsiung Medical University, Taiwan
Ming-Shin Tan, Dept. of Pediatrics, Kaohsiung Medical University, Taiwan

Jong-Hau Hsu, Dept. of Pediatrics, Kaohsiung Medical University, Taiwan

Jiunn-Ren Wu, Dept. of Pediatrics, Kaohsiung Medical University, Taiwan Bin-Nan Wu, Dept. of Pediatrics, Kaohsiung Medical University, Taiwan Chaw-Chi Chiu, Dept. of Surgery, Kaohsiung Medical University, Taiwan Shah-Hwa Chou, Dept. of Surgery, Kaohsiung Medical University, Taiwan

Rho A/Rho Kinase (ROCK) signaling plays a key role in the pathogenesis of experimental pulmonary hypertension $(\mathrm{PH})$, such as hypoxic $\mathrm{PH}$, and monocrotaline (MCT)-induced $\mathrm{PH}$. In this study, we test whether Rho A/Rho Kinase signaling was involved in the pathogenesis of $\mathrm{PH}$ in aortic banded rats. Four weeks after ascending aortic banding, compared with the shamoperated rats, the aortic banded Wistar rats developed $\mathrm{PH}$ $(28 \pm 3($ Mean $\pm \mathrm{SE}) \mathrm{mmHg}, \mathrm{n}=8$; and $13 \pm 1 \mathrm{mmHg}, \mathrm{n}=8$, $\mathrm{p}<0.01)$ with medial hypertrophy in pulmonary arteriole. Compared with in sham-operated rats, there were increases in ratio of membrane RhoA/cytosolic RhoA (1.0 \pm 0.08 , $1.5 \pm 0.16, \mathrm{p}<0.05)$ and in ROCK II protein $(1.0 \pm 0.3$; $2.1 \pm 0.2, p<0.01)$, respectively, in lungs of banded rats, In extralobar pulmonary arteries, we could detect cleaved (constitutive active) ROCK $\mathrm{I}$, as well as increase in ratio of membrane RhoA/cytosolic Rho A $(1.0 \pm 0.2 ; 2.4 \pm 0.3, \mathrm{p}<0.01)$ and in ROCK II protein $(1.0 \pm 0.3 ; 1.9 \pm 0.2, \mathrm{p}<0.01)$ in banded rats, respectively. In addition, there were increased phosphorylated ERM (ezrin, radixin, and moesin) at T567 (ezrin), T5648 (radixin), and T558 (moesin) in lungs of banded rats. Interestingly, the Rho kinase inhibitor, Fasudil $(10 \mathrm{mg} / \mathrm{kg} / \mathrm{iv}$, within $5 \mathrm{~min}$ ) could significantly decrease the mean pulmonary arterial pressure $(\mathrm{P}<0.01)$, without any change of systemic arterial pressure detected. However, these results suggest that the activation of Rho A/ROCK signaling is involved in the development of PH secondary to left ventricular dysfunction.

\section{8}

Hypoventilation easily leads to severe pulmonary hypertension in Down syndrome patients with congenital heart disease

Yuji Hamamichi, Osaka Medical Center and Research Institute for Maternal and Child Health, Japan

Syu Maekawa, Osaka Medical Center and Research Institute for Maternal and Child Health, Japan

Akane Kadota, Osaka Medical Center and Research Institute for Maternal and Child Health, Japan

Yukiko Kawazu, Department of Pediatric Cardiology, Osaka Medical Center for Maternal and Child Health, Osaka, Japan

Noboru Inamura, Osaka Medical Center and Research Institute for

Maternal and Child Health, Japan

Futoshi Kayatani, Department of Pediatric Cardiology, Osaka Medical Center for Maternal and Child Health, Osaka, Japan, Japan

Hiroaki Kawata, Japan

Hidefumi Kishimoto, Japan

Congenital heart disease in patients with Down syndrome commonly has potential for early development of pulmonary hypertension. Moreover hypoventilation due to upper airway obstruction often causes severe pulmonary hypertension. We investigated how much hypoventilation had effect on pulmonary hypertension of cardiovascular anomalies. We reviewed the medical records of 65 Down syndrome patients who had cardiac catheterization between 1993 and 2008. Cardiac catheterization was performed under only intravenous sedation in 56 patients 
and under intubation in 9 patients. Study age was between 7 days and 11 months (median 5 months). They had cardiac malformations, left to right shunt such as ventricular septal defect, atrioventricular septal defect, patent ductus arteriosus and atrial septal defect. We defined mean pulmonary artery pressure $55 \mathrm{mmHg}$ or over as severe pulmonary hypertension, and arterial PCO2 $46 \mathrm{mmHg}$ or over as hypoventilation. Contrary to our expectation there was no severe pulmonary hypertension in patients with intubation. In patients with only sedation severe pulmonary hypertension was significantly related to hypoventilation and high left atrial pressure by univariate analysis. Study age and cardiovascular malformation types showed no significant relationship to severe pulmonary hypertension. After multiple logistic regression model, severe pulmonary hypertension was independently associated with an odds ratio of $15.6(95 \% \mathrm{CI}$, 2.7-89.1:p $=0.035) \quad$ for hypoventilation, $5.3 \quad(95 \% \mathrm{CI}$, $1.1-25.4: \mathrm{p}=0.002)$ for high pressure of left atrium $(\geqq 8 \mathrm{mmHg})$. Under sedation pulmonary hypertension may be emphasized. Conversely under intubation, pulmonary hypertension may be underestimated. These results show that hypoventilation can easily bring about severe pulmonary hypertension in Down syndrome patients with left to right cardiovascular anomalies.

\section{9}

Combination therapy of bosentan, beraprost and HOT improve pulmonary artery hypertension related to congenital heart disease in contraindications for right heart bypass surgery

Keijirou Ibuki, Department of Pediatrics, Faculty of Medicine, University of Toyama, Japan

Keiichi Hirono, Department of Pediatrics, Faculty of Medicine, University of Toyama, Japan

Kazu Saito, Department of Pediatrics, Faculty of Medicine, University of Toyama, Japan

Kazuhiro Watanabe, Department of Pediatrics, Faculty of Medicine, University of Toyama, Japan

Sayaka Watanabe, Department of Pediatrics, Faculty of Medicine, University of Toyama, Japan

Naoki Yoshimura, 1st department of surgery, Faculty of Medicine, University of Toyama, Japan

Fukiko Ichida, Department of Pediatrics, Faculty of Medicine, University of Toyama, Japan

Toshio Miyawaki, Department of Pediatrics, Faculty of Medicine,

University of Toyama, Japan

Background: Bosentan, an oral dual endothelin (ET-A/ET-B) receptor antagonist has been shown to be effective in patients with idiopathic $\mathrm{PAH}$ and $\mathrm{PAH}$ related to connective tissue disease, improving long term quality of life. However, there are few reports so far on the effect of bosentan for increased pulmonary vascular resistance (PVR) in patients with single ventricle (SV) physiology.

Objective: We examine successful combination therapy of bosentan, beraprost and HOT in five patients with congenital heart disease (CHD) who could not undergo right heart bypass surgery because of PAH initially.

Methods: All enrolled patients had PAH in World Health Organization (WHO) functional class III related to CHD with or without prior surgical repair with fixed elevated pulmonary vascular resistance.

Results: Combination therapy successfully reduced PAP and PVR in all cases. Mean PAP and PVR at baseline and after bosentan therapy, respectively, were; mean main PA pressure $(\mathrm{mmHg})$ : $27.0 \pm 9.4$ to $19.4 \pm 14.5$ (p<0.05), PVRI (unit/m2): $5.5 \pm 2.4$ to $1.9 \pm 0.8(\mathrm{p}<0.05)$, PVR/SVR: $0.36 \pm 0.19$ to $0.06 \pm 0.02$ $(\mathrm{p}<0.05)$. Each patient had improved clinical symptoms and modified New York Heart Association class, from III to II. Bidirectional Glenn was performed in 4 cases, and 3 cases underwent successful Fontan operations.

Conclusions: Combined therapy with bosentan, beraprost and HOT may widen the surgical options and improve outcome in Fontan candidates. Larger studies with long-term bosentan are needed to assess the optimal doses and possible therapeutic role of bosentan in this population.

\section{0}

Gene Expression of Endothelin-1 and Endothelin Receptor A on Monocrotaline-Induced Pulmonary Hypertension in Rats after Bosentan Treatment Young Mi Hong, Department of Pediatrics, School of Medicine, Ewha Womans University Hospital, Korea

Kyoung Ah Lim, Deparment of Pediatrics, Phochun Cha University, Korea

Hae Soon Kim, Department of Pediatrics, School of Medicine, Ewha Womans University, Korea

Kwan Chang Kim, Thoracic and Cardiovascular Surgery,

School of Medicine, Ewha Womans University, Korea

Min-Sun Cho, Department of Pathology, School of Medicine, Ewha Womans University, Korea

Endothelin (ET)-1, a potent endothelium-derived vasoconstrictor peptide, has several properties suggestive of its potential pathophysiological role in pulmonary hypertension. Bosentan is an endothelial receptor antagonist and efficacious in treating pulmonary hypertension. The objectives of this study were to evaluate the effect of bosentan on monocrotaline (MCT)induced pulmonary hypertension in rats and to correlate it with the gene expression of ET-1 and ET A receptors.

Sprague-Dawley rats were divided into three groups: control group, MCT group $(60 \mathrm{mg} / \mathrm{kg}$ ), bosentan group (MCT $60 \mathrm{mg}$ / $\mathrm{kg}$ plus $6.25 \mathrm{mg} /$ day bosentan orally). The mean body weights of the rats in the MCT and bosentan groups were significantly lower than the control group on day 7, 14, and 28 . Administration of bosentan significantly inhibited the progression of right ventricular hypertrophy on day 28. Quantitative analysis of peripheral pulmonary arteries revealed that the increase in medial wall thickness after MCT injection was significantly attenuated in the bosentan group on day 28. In addition, an increase in the number of intra-acinar muscular arteries after MCT injection was reduced by bosentan on day 14 . The expressions of ET-1 (4.19 \pm 1.84 vs $1 \pm 0.20)$ and ET receptor A $(3.35 \pm 1.26$ vs $1 \pm 0.34)$ gene were significantly increased in MCT group on day 5 . Serum ET-1 concentrations in the MCT group were higher than the control group on day 28 .

In conclusion, ET-1 expression correlates with the progression of cardiopulmonary alterations in rats with MCT-induced pulmonary hypertension. Administration of bosentan inhibited pulmonary vascular hypertrophy and right ventricular hypertrophy during development of MCT-induced pulmonary hypertension in rats.

\section{1}

Gene Expression of Nitric Oxide Synthase and Matrix Metalloproteinase-2 in Monocrotaline-Induced Pulmonary Hypertension in Rats

Young Mi Hong, Department of Pediatric, School of Medicine, Ewha Womans Univeristy, Korea 
Hee Sun Koo, Korea

Kyoung Ah Lim, Deparment of Pediatrics, Pochun Cha University, Korea

Nitric oxide (NO) is a major endothelium dependent vasomediator and growth inhibitor. NO is catalysed by NO synthase (NOS), and whether the expression of NOS is altered in association with the ventricular hypertrophy is not known. NO can also produce peroxynitrite, which activates matrix metalloproteinases (MMPs). The purposes of this study were to determine the gene expression of endothelial NOS and MMP2 in the lung in a rat model of pulmonary hypertension. SpragueDawley rats were treated with monocrotaline (MCT) $(60 \mathrm{mg} / \mathrm{kg})$ to produce pulmonary hypertension. The mean body weight significantly decreased in the MCT group compared with the control group. The $\mathrm{RV} /(\mathrm{LV}+$ septum $)$ ratio significantly increased in the MCT group compared with the control group on days $14 \& 28$. The expression of eNOS mRNA significantly increased in the MCT group compared with the control group $(3.51 \pm 1.18$ vs $1 \pm 0.37)$ on day 28 . Serum NO level did not show significant change in either group. Serum MMP-2 level $(254.21 \pm 36.27 \mathrm{ng} / \mathrm{mL}$ vs $198.37 \pm 10.57 \mathrm{ng} / \mathrm{mL})$ and MMP-2 gene expression $(4.55 \pm 1.98$ vs $1 \pm 0.34)$ significantly increased in the MCT-treated rats compared with the control group on 28 day. These data suggest that elevated eNOS expression may be responsible for the activation of MMP-2. The causal relationship between eNOS and MMP-2 and their role in pulmonary hypertension require further investigations.

\section{3}

Outcome of surgical repair of patients with left-to-right (LR) shunt lesion and severe pulmonary arterial hypertension (PAH)

Soo In Jeong, Department of Pediatrics, Samsung Medical Center, SungKyunKwan University School of Medicine, Korea

Cha Gon Lee, Department of Pediatrics, Samsung Medical Center, SungKyunKwan University School of Medicine, Korea

Soo Han Choi, Department of Pediatrics, Samsung Medical Center, SungKyunKwan University School of Medicine, Korea June Huh, Department of Pediatrics, Samsung Medical Center, Sungkyunkwan University School of Medicine, Korea

Heung-Jae Lee, Department of Pediatrics, Samsung Medical Center, SungKyunKwan University School of Medicine, Korea

Ji-Hyuk Yang, Department of Thoracic Surgery, Samsung Medical Center, SungKyunKwan University School of Medicine, Korea Tae-Gook Jun, Department of Thoracic Surgery, Samsung Medical Center, SungKyunKwan University School of Medicine, Korea I-Seok Kang, Department of Pediatrics, Samsung Medical Center, Sungkyunkwan University School of Medicine, Korea

Objective: To report outcome of surgical closure of LR shunt lesions associated with severe $\mathrm{PAH}$.

We reviewed medical records of 21 patients who underwent surgical closure of LR shunt lesions associated with Rp above 8 Wood unit (Wu) after 1 year of age.

Results: Median age of operation was 26 years (range: 1-58). Shunt lesions were ASD $(n=11)$, VSD $(n=4)$, VSD with PDA $(n=4)$ and PDA $(n=2)$. NYHA class was III in 10 patients and II in 11 patients. Median Rp was $10.8 \mathrm{Wu}(8.3 \sim 29.7)$. Median $\mathrm{Rp} / \mathrm{Rs}$ was 0.32 (0.15-0.72). Median PAP was $58 \mathrm{mmHg}$ (40-91). There were response to pulmonary vasodilator in 4 patients, partial response in 2 patients and no response in 15 patients. After test occlusion of shunt with or without pulmonary vasodilator, six of 8 non-responders showed decrease in pressure.
Complete closure was done in 5 patients, and partial closure $(n=12)$ or ASD creation $(n=4)$ in others. During postoperive follow-up of median 27months (4-117), estimated RVSP was decreased in 20 patients and was normalized in 9 patients. NYHA class was improved in 20 patients and unchanged in 1 patient whose RVSP didn't decrease. Postoperatively we used pulmonary vasodilators in 16 patients and discontinued it in 6 patients during follow up. Immediate postoperative PAP was correlated with that of test occlusion. There was no mortality or significant morbidity.

Conclusion: Surgical repair of LR shunt with PAH can be performed safely in selected subjects. With recent advances in intensive care and pulmonary vasodilator therapy, guideline of surgical repair of LR shunt with PAH should be reconsidered.

\section{4}

Effect of hydrogen sulfide on the apoptosis of pulmonary artery smooth muscle cell in rats with pulmonary hypertension induced by high pulmonary blood flow Hongfang Jin, Department of Pediatrics, Peking University First Hospital,Beijing, China

Wei Li, Department of Pediatrics, Peking University First Hospital, Beijing, China

Xiaohui Li, Department of Pediatrics, Peking University First Hospital, Beijing, China

Junbao Du, Department of Pediatrics, Peking University First Hospital, Beijing, China

Chaoshu Tang, Key Lab. Molecular Cardiovascular Diseases, Ministry of Education, Peking University, Beijing,

Objective: To investigate whether induction of apoptosis of pulmonary artery smooth muscle cells (PASMCs) was involved in the mechanism responsible for the protective role of hydrogen sulfide in the development of pulmonary hypertension induced by high pulmonary blood flow.

Methods: In the present study, we used a rat model of high pulmonary blood flow induced pulmonary hypertension established by an abdominal aorta-inferior vena cava shunt operation. DLPropargylglycine (PPG), an inhibitor of endogenous hydrogen sulfide production, was administrated intraperitoneally at a dose of $37.5 \mathrm{mg} / \mathrm{kg}$ per day for 4 week since the shunting operation. On the other side, sodium hydrosulfide (NaHS), a hydrogen sulfide donor, was administrated intraperitoneally at a dose of $56 \mu \mathrm{mol} / \mathrm{kg}$ per day for 11 weeks since the shuntiong operation.

Results: After 4 week shunting operation, the apoptosis of PASMCs, expression of Fas and caspase- 3 were significantly decreased $(\mathrm{P}<0.01)$, but expression of $\mathrm{Bcl}-2$ significantly increased $(\mathrm{P}<0.01)$. PPG administration further promoted the apoptosis of PASMCs, downregulated the expression of Fas and caspase-3 (P<0.01), and increased the expression of Bcl-2 $(\mathrm{P}<0.01)$; After 11 weeks shunting operation, the apoptosis of PASMCs, expression of Fas and Caspase- 3 were significantly decreased $(\mathrm{P}<0.01)$, but expression of $\mathrm{Bcl}-2$ significantly increased $(\mathrm{P}<0.01)$. NaHS administration significantly increased the apoptosis of PASMCs, upregulated the expression of Fas and caspase-3, and inhibited the expression of Bcl-2.

Conclusion: Hydrogen sulfide might exert a protective role in the development of pulmonary hypertension induced by high pulmonary blood flow by inducing the apoptosis of PASMCs.

8147

Reversible Course of Pulmonary Arterial Hypertension

Alisa Limsuwan, Division of Pediatric Cardiology, Ramathibodi

Hospital, Mahidol University, Thailand

Samart Pakakasama, Department of Pediatrics, Ramathibodi Hospital, Mahidol University, Thailand 
Suradej Hongeng, Department of Pediatrics, Ramathibodi Hospital, Mahidol University, Thailand

Pulmonary arterial hypertension $(\mathrm{PAH})$ is considered to be a rare but serious complication of bone marrow transplantation (BMT). Majority of the series reports demonstrated a potential fatal outcome and the treatments have been believed to require an indefinite duration of therapy.

Objective: To describe cases of reversible PAH related to BMT in two consecutive patients.

Methods and Result: Two patients with PAH after BMT were worked up for the common secondary causes PAH with negative result. Therefore BMT was considered to be an uncommon cause of PAH. The first patient is a 20 year-old male. He was diagnosed with relapse acute lymphoblastic leukemia underwent successful BMT complicated with PAH 10 months after transplantation in 2003. He was in functional class IV and initially treated with combination of aerosolized iloprost and oral sildenafil. His functional class gradually improved over the past few years while his medication was gradually titrated down and be able to switch to oral amlodipine. His pulmonary arterial pressure was currently normalized with the recent treatment. The second patient is an 18 year-old female known diagnosis of chronic myeloid leukemia underwent BMT in 2004 and developed PAH 4 month after BMT. She was in functional class II and initially treated with sildenafil and beraprost. With improvement of her symptoms and normal exercise test, her medication was discontinued after 4 months of therapy. Cardiac catheterization showed normal pulmonary artery pressure.

Conclusion: BMT was considered to be an uncommon cause of $\mathrm{PAH}$ and amenable to reverse may occasionally be achievable.

\section{8}

Outcome of children with pulmonary arterial hypertension in the era of antiproliferative medication Rosa Laura E van Loon, Department of Pediatric Cardiology, University Medical Center Groningen, Groningen, The Netherlands Marc T R Roofthooft, Department of Pediatric Cardiology, University Medical Center Groningen, Groningen, The Netherlands

Lennie van Osch-Gevers, Department of Pediatric Cardiology, Erasmus Medical Center Rotterdam, Rotterdam, The Netherlands Tammo Delhaas, Department of Pediatric Cardiology, University Hospital Maastricht, Maastricht, The Netherlands

A Derk-Jan ten Harkel, Center for Congenital Anomalies of the Heart, Amsterdam/Leiden, The Netherlands

Jan L M Strengers, Department of Pediatric Cardiology, University

Medical Center Utrecht, Utrecht, The Netherlands

Hans L Hillege, Department of Cardiology and Epidemiology,

University Medical Center Groningen, Groningen, The Netherlands

Rolf M F Berger, Department of Pediatric Cardiology, University

Medical Center Groningen, Groningen, The Netherlands

Objectives: To investigate the outcome of children with pulmonary arterial hypertension $(\mathrm{PAH})$ treated in the era of antiproliferative medication (prostanoids, endothelin-receptorantagonists and 5-phosphodiesterase-inhibitors).

Methods: Fifty-two children with idiopathic $(\mathrm{n}=29)$ and systemic-to-pulmonary shunt associated PAH $(n=23)$ underwent baseline and follow-up assessments at a national referral center for pediatric PAH. Treatment was initiated depending on functional class (WHOclass) and acute pulmonary vasoreactivity responder status.

Observed survival was compared to expected survival using the NIH registry formula for PAH survival (D'Alonzo,1991).
Potential baseline predictors for survival and effect of antiproliferative medication on clinical and laboratory follow-up assessments were evaluated.

Results: Median age was 6.1 years (range 0.04-17.4), WHOclass I/II/III/IV $(\mathrm{n}=1 / 13 / 26 / 12)$, median follow-up 3.2 years (range $0.02-14.2)$.

Treatment (mono- and combination-) included calcium channel blockers $(n=9)$, epoprostenol $(n=10)$, bosentan $(n=34)$ and sildenafil $(\mathrm{n}=11)$.

Observed survival in the current era of antiproliferative medication was improved compared to expected survival (Figure).

The following baseline variables were associated with decreased survival: higher WHOclass $(\mathrm{p}=0.02$, Hazard Ratio2.2, 95\% Confidence Interval $1.1-4.5)$, respiratory rate $(p=0.02)$, pulmonary-to-systemic arterial pressure ratio $(p=0.04)$; and lower blood pressure $(p=0.02)$, cardiac index $(p=0.04)$ and systemic venous saturation $(p=0.05)$.

After start of antiproliferative medication, 6-minute walk distance initially increased, but eventually decreased after longer follow-up. No changes were seen in transcutaneous oxygen saturation, body mass index or serum markers.

Conclusions: Current survival of pediatric PAH appeared improved compared to survival before the introduction of antiproliferative medication. After start of antiproliferative mediacation functional capacity improved during short-term, but declined during long-term follow-up.

\section{Survival during and before era of antiproliferative medication}

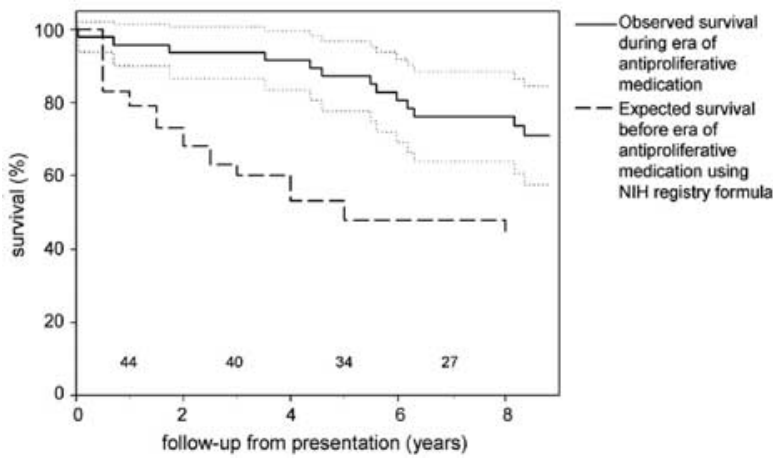

8149

Efficacy of combination of Pulmonary Artery Banding and oral Sildenafil in children with early Eisenmenger VSD: 3 year Follow up

Viresh Mahajan, Batra Hospital, New Delhi, India

Anil Bhan, Batra Hospital, New Delhi, India

Rajesh Sharma, Batra Hospital, New Delhi, India

Pradipta Acharya, Batra Hospital, New Delhi, India

Objective: Large VSD presenting late with right to left shunt systemic PA pressures and unfavorable cardiac catheterization data are considered inoperable even in absence of clubbing and visible cyanosis. The rationale of treatment was based on the fact that if left untreated they progress to develop cyanosis and clubbing over next few years.

Method: In this prospective study PA banding \& sildenafil was used in patients with criteria a. Systemic PA Pressures b. Basal right to left shunt (sat<90\%) c. No clubbing d. PVR $>6$ wood units on $100 \%$ oxygen +40 ppmNO. Total of 15 patients had mean age -8 yrs (range $2-14 \mathrm{yrs}$ ), weight of $25 \mathrm{~kg}$ (range $8-45 \mathrm{~kg}$ ). Results: PA Band was well tolerated in 14/15. The PA/Ao pressure in OR was $0.80-0.85$. Sildenafil was started and Follow up was done with Echo+Cath upto 3 yr. 5/15 have undergone 
catheterization \& PA/Ao pressure varied between 0.45 to 0.6. 4 patients (26.6\%) have undergone Debanding \& VSD Closure with smooth course and PA/Ao was $40-50 \% .9$ patients are 6 mo to $1 \mathrm{yr}$ post PA band \& band gradient is 45 to $50 \mathrm{~mm} \mathrm{Hg}$. 1 patient did not tolerate the PA Band had to be debanded in OR. There was no mortality in the study.

Conclusion: PA Band followed by oral sildenafil therapy is safe and efficacious. This can possibly change the natural history of Early Eisenmenger VSD's.

\section{0}

Use of ductus arteriosus in management of pulmonary artery hypertension

Anna Mani, K K Women and Children's Hospital Singapore, Singapore

Sriram Shankar, K K Women and Children's Hospital, Singapore

Vijay M Hanjoora, K K Women and Children's Hospital, Singapore Keng Yean Wong, K K Women and Children's Hospital, Singapore

Severe pulmonary hypertension is a significant determinant of mortality following repair of total anomalous pulmonary venous drainage (TAPVD). Several strategies are used in its management. We describe the use of the ductus arteriosus in managing the postoperative pulmonary hypertension in these patients. Between 1996 and 2006, 28 patients underwent repair of TAPVD, 3 patients were managed by leaving the ductus arteriosus open to decompress the pulmonary artery. There were no deaths in this series. Decompression of the pulmonary artery by leaving the ductus arteriosus open is a useful maneuver in the early postoperative period in patients with persistent suprasystemic pulmonary artery pressure resulting in right ventricular decompensation.

8151

Pulmonary and systemic hypertension in the setting of Moya-Moya disease due to disseminated fibromuscular dysplasia

Takeshi Nakano, Department of Pediatric Cardiology, Ehime central prefectural Hospital, Ehime, Japan, Japan

Shin Ono, Department of Pediatric Cardiology, Ehime central prefectural Hospital, Ehime, Japan, Japan

Toshiyuki Chisaka, Department of Pediatric Cardiology, Ehime central prefectural Hospital, Ehime, Japan, Japan

Takashi Higaki, Department of Pediatrics, Ehime University Graduate School of Medicine, Japan

Eiichi Yamamoto, Department of Pediatrics, Ehime University Graduate School of Medicine, Japan

Masaaki Ohta, Department of Pediatrics, Ehime University Graduate School of Medicine, Japan

Kikuko Murao, Department of Pediatrics, Ehime University Graduate School of Medicine, Japan

Eiichi Ishii, Department of Pediatrics, Ehime University Graduate

School of Medicine, Japan

\section{A case report}

The patient, a girl aged 13 years, had been diagnosed acquired cytomegalovirus encephalitis at the age of a month and followed up as epilepsy since then. She was diagnosed Moya-moya disease due to cataplexy of lower extremity at the age of 10 years and underwent surgical treatment, but she had felt general fatigue and shortness of breath on exertion since these time.

At the age of 12 years, she fell unconscious and was diagnosed plumonary hypertension. ECG pattern was marked right ventricle hypertrophy. Pulmonary perfusion scintigraphy showed multifocal perfusion defects.

Cardiac catheterization revealed systolic pressure of aorta/ pulmonary artery of $160 / 100 \mathrm{mmHg}$ and pulmonary vascular resistance was $17 \mathrm{Um} 2$. Selective pulmonary angiography demonstrated multiple stenosis of peripheral pulmonary artery, and intimal thickening was observed by the intravascular ultrasound.

Segmental stenosis at the proximal potion of the celiac trunk and narrowing of the coronary artery, supramesenteriac artery and abdominal aorta extending to the femoral arteries were noted. No findings of segmental stenosis of the renal arteries and serum renin level was not elevated. Systemic blood pressure at rest is fluctuating at the range of 120 to $160 \mathrm{mmHg}$ because the elasticity of the systemic arteries is decreased.

She is currently receiving warfarin, beraprost, bosentan and O2 inhalation, her symptoms almost disappear. Brain natriuretic peptide level and six-minutes walk test are improved.

We estimate the stenotic lesions are progressive, therefore we are considering to perform the balloon angioplasty for pulmonary stenosis if the symptoms worsen in future.

\section{2}

Oral Sildenafil for Persistent Pulmonary Hypertension Early After Congenital Cardiac Surgery

Shintaro Nemoto, Department of Cardiovascular Surgery, Osaka

Medical College Hospital, Japan

Tomoyasu Sasaki, Department of Cardiovascular Surgery, Osaka

Medical College Hospital, Japan

Hideki Ozawa, Department of Cardiovascular Surgery, Osaka Medical College Hospital, Japan

Mari Kakita, Department of Cardiovascular Surgery, Osaka Medical College Hospital, Japan

Takahiro Katsumata, Department of Cardiovascular Surgery, Osaka

Medical College Hospital, Japan

Kanta Kishi, Department of Pediatrics, Osaka Medical College

Hospital, Japan

Kenichi Okumura, Department of Pediatrics, Osaka Medical College

Hospital, Japan

Yasuhiko Mori, Department of Pediatrics, Osaka Medical College

Hospital, Japan

Background: Sildenafil is widely known as a strong pulmonary vasodilator by increasing the intracellular cGMP concentration through inhibition of phosphodiesterase 5 . The benefit of oral sildenfil treatment for persistent pulmonary hypertension $(\mathrm{PH})$ early after congenital cardiac surgery was assessed.

Methods: After admission to the intensive care unit, sildenafil was administered at a starting dose of $0.5 \mathrm{mg} / \mathrm{kg}$. With careful monitoring hemodynamics, the dose of sildenafil was increased stepwise by $0.5 \mathrm{mg} / \mathrm{kg}$ every 4 to 6 hours up to a maximum dose of $2.0 \mathrm{mg} / \mathrm{kg}$. After successful weaning from ventilator and other vasodilators, sildenafil was gradually discontinued during the next 5 to 7 days. Medical records of 90 cases were retrospectively reviewed. Results: Age distribution; $<1$ month: 25, 1 to less than 6 months: 29, 6 to less than 12 months: 17,1 to 3 years: 8,4 to 9 years: 9 , $>10$ years: 2. Operations; VSD closure: 16 , arterial switch: 30 , truncus repair: 10, complete AVSD repair: 10, TAPVD repair: 6, ASD+VSD closure: 2, other open heart: 16. Concomitant inhaled nitrous oxide treatment: 64. Changes in pulmonary arterial pressure (monitored in 26 cases); decreased: 20, unchanged: 5, elevated: 1 . Bosentan was added in 2 cases with persistent symptoms due to $\mathrm{PH}$ despite sildenafil treatment. Modest oxygen desaturation after sildenafil administration: 7. No 'rebound' pulmonary hypertension after sildenafil discontinuation and no significant adverse events during sildenafil treatment. Conclusions: Our results suggest that oral sildenafil treatment is a safe and effective alternate for persistent $\mathrm{PH}$ after congenital heart surgery in children. 
8153

Aggressive pulmonary vasodilator therapy, together with coil embolization for the associated systemic venous collaterals, against pulmonary vascular disease in high-risk Fontan candidates after Glenn procedure: a mid-term result

Hiroyuki Ohashi, Mie University Graduate School of Medicine, Japan Yoshihide Mitani, Mie University Graduate School of Medicine, Japan Noriko Yodoya, Mie University Graduate School of Medicine, Japan Hirofumi Sawada, Mie University Graduate School of Medicine, Japan Hidetoshi Hayakawa, Mie University Graduate School of Medicine, Japan

Shin Takabayashi, Mie University Graduate School of Medicine, Japan Hideto Shimpo, Mie University Graduate School of Medicine, Japan Yoshihiro Komada, Mie University Graduate School of Medicine, Japan

Background: Pulmonary vascular disease, frequently associated with systemic venous collaterals in patients after Glenn procedure, is a grave hazard for the successful Fontan completion in such patients. Recently, some pulmonary vasodilators were demonstrated to improve exercise capacity and pulmonary hemodynamics in patients with pulmonary arterial hypertension. We therefore hypothesized that aggressive pulmonary vasodilator therapy, together with coil embolization for the associated systemic venous collaterals, may improve pulmonary hemodynamics in high-risk Fontan candidates with pulmonary vascular disease after Glenn procedure. Methods and results: 7 patients (mean age: $20 \mathrm{~m} \pm 3 \mathrm{~m}$ ) (hypoplastic left heart syndrome 3 , isomerism heart 2 , single ventricle +21 trisomy 1 , pulmonary atresia with intact ventricular septum 1) after Glenn procedure, associated with mean pulmonary artery pressure $\geqslant 15 \mathrm{mmHg}$ or significant systemic venous collaterals, were enrolled in the present study and observed for a mean interval of $20 \mathrm{~m}$. Home oxygen therapy and beraprost administration was performed in all the 7 patients. Sildenafil was administered in 3 patients, while coil embolization for systemic venous collaterals was performed in 5 patients. In the follow-up, mean pulmonary artery pressure decreased from $17 \pm 1$ to $12 \pm 1 \mathrm{mmHg}(\mathrm{p}<.05)$, PA index increased from $164 \pm 23$ to $175 \pm 22$, and $\mathrm{SpO} 2$ increased from $77 \pm 2$ to $83 \pm 1 \%(\mathrm{p}<.01)$. Five out of seven patients completed Fontan procedure, while the remaining two patients are waiting. Conclusions: The present mid-term results suggest that aggressive pulmonary vasodilator therapy, together with coil embolization for the associated systemic venous collaterals, may be an effective therapy for pulmonary vascular disease in high-risk Fontan candidates after Glenn procedure.

\section{4}

Pretreatment with phosphodiesterase inhibitors (Sildenafil/Milrinone) prevents pulmonary hypertensive crisis in children undergoing cardiac surgery Usha Pratap, Department of Pediatric Cardiology,Deenanath Mangeshkar Hospital,Erandawana, Pune 411004, India Ranjit $R$ Jagtap, Department of Cardiac Surgery, Deenanath Mangeshkar Hospital,Erandawana, Pune 411004, India Ashutosh A Hardikar, Department of Cardiac Surgery, Deenanath Mangeshkar Hospital, Erandawana, Pune 411004, India Nilesh M Juvekar, Department of Cardiac Intensive Care, Deenanath Mangeshkar Hospital, Erandawana, Pune 411004, India Vinayak N Desurkar, Department of Cardiac Intensive Care, Deenanath Mangeshkar Hospital, Erandawana, Pune 411004, India

Introduction: An acute pulmonary hypertensive crisis is a dreaded complication in the immediate post operative period. We assessed whether pretreatment with sildenafil and or milrinone before cardiac surgery would have any impact on the number and severity of postoperative hypertensive crisis.

Aims and objectives: To assess the number and severity of pulmonary hypertensive crisis in children undergoing repair of CHD with pulmonary hypertension who were pretreated with chronic phosphodiesterase inhibitor therapy

Preoperative: PDE group 40 consecutive children undergoing surgery for CHD with PA pressures more than $50 \%$ systemic, were included in the study group. Patients were given sildenafil in a dose of $0.5 \mathrm{mg} / \mathrm{kg} /$ dose 6 hourly prior to surgery. Children who required surgery soon after diagnosis received IV milrinone $(0.5 \mathrm{mcg} / \mathrm{kg} / \mathrm{min}$, no loading) with or without sildenafil.

Control group: 33 historical age, weight, diagnosis matched controls who underwent repair before the study period. They did not receive phosphodiesterase inhibitors prior to surgery

The primary end points were 1) Number of crisis 2) Episodes of increased PA pressures to $75 \%$ systemic or more not requiring intervention 3) 3. Death presumed to be due to crisis.

Results: Patients in the PDE group received either received sildenafil $(n=39)$ or milrinone $(n=1)$ or $\operatorname{both}(n=10)$. The duration therapy was 29 days (SD 52.6) for sildenafil and 1.5 days(SD 0.9) for milrinone.

Other data are included in the table

Conclusions: Pretreatment with phosphodiesterase inhibitors increases the odds of not having a postoperative pulmonary hypertensive crisis $(\mathrm{NNT}=4)$,increases the odds of tolerating high PA pressures without a crisis $(\mathrm{NNT}=3)$, and the odds of survival $(\mathrm{OR}=3.3)$.

\begin{tabular}{|c|c|c|c|c|}
\hline & $\begin{array}{l}\text { Controls } \\
\mathbf{N}=33\end{array}$ & $\begin{array}{c}\text { PDE group } \\
\mathrm{N}=40\end{array}$ & & \\
\hline $\begin{array}{l}\text { Age (years) } \\
\text { median (SD) }\end{array}$ & $0.83(3.42)$ & $0.5(2.92)$ & & \\
\hline $\begin{array}{l}\text { Weight (kg) } \\
\text { median (SD) }\end{array}$ & $5.2(4.6)$ & $4.75(7.5)$ & & \\
\hline Disease profile & $\begin{array}{r}\text { TAPVC } 10, \\
\text { Shunts } 23\end{array}$ & $\begin{array}{r}\text { TAPVC } 15, \\
\text { Shunts } 25\end{array}$ & & \\
\hline $\begin{array}{l}\text { Ventilation hrs } \\
\text { mean, (SD, } \\
95 \% \mathrm{CI})\end{array}$ & $\begin{array}{l}100(133.76 \\
52.6 \text { to } 147)\end{array}$ & $\begin{array}{l}38.5(55.14 \\
20.34 \text { to } 56.3)\end{array}$ & $\begin{array}{c}\mathrm{p}=0.0095 \\
\text { (highly significant) }\end{array}$ & \\
\hline $\begin{array}{l}\text { Inotrope Score } \\
\text { (SD) }\end{array}$ & $1782.9(2070)$ & 1130.1 (926) & $\begin{array}{l}\mathrm{p}=0.76 \text { (not } \\
\text { significant) }\end{array}$ & \\
\hline Survival $\%$ & 84.85 & 95 & $\begin{array}{c}\mathrm{OR}=3.3(95 \% \\
\mathrm{CI} 0.6 \text { to } 18.7)\end{array}$ & \\
\hline $\begin{array}{l}\text { Avoiding PA } \\
\text { crisis \% }\end{array}$ & 39.9 & 72.5 & $\begin{array}{l}\text { OR- } 4.05(95 \% \\
\text { CI } 1.5 \text { to } 10.8)\end{array}$ & $\mathrm{NNT}=4$ \\
\hline $\begin{array}{l}\text { Tolerating high } \\
\text { PA, no crisis } \%\end{array}$ & 4.7 & 47 & $\begin{array}{c}\mathrm{OR}=9.5(95 \% \\
\mathrm{CI} 1.1 \text { to } 81)\end{array}$ & $\mathrm{NNT}=3$ \\
\hline
\end{tabular}

8155

Use of bosentan hydrate, an endothelin-1 dual receptor blocker, for treatment of impaired pulmonary circulation after congenital cardiac surgery

Tomoyasu Sasaki, Department of Cardiovascular Surgery, Osaka Medical College Hospital, Japan

Shintaro Nemoto, Department of Cardiovascular Surgery, Osaka

Medical College Hospital, Japan

Hideki Ozawa, Department of Cardiovascular Surgery, Osaka Medical College Hospital, Japan

Mari Kakita, Department of Cardiovascular Surgery, Osaka Medical College Hospital, Japan

Takahiro Katsumata, Department of Cardiovascular Surgery, Osaka Medical College Hospital, Japan

Kanta Kishi, Department of Pediatric, Osaka Medical College Hospital, Japan 
Kenichi Okumura, Department of Pediatric, Osaka Medical College Hospital, Japan

Yasuhiko Mori, Department of Pediatric, Osaka Medical College

Hospital, Japan

Background: Severe pulmonary excess flow and marginal growth of the pulmonary artery often cause impairment of pulmonary circulation early after congenital cardiac surgery. The resultant elevated vascular resistance may lead to pulmonary hypertension $(\mathrm{PH})$, which is a major cause of postoperative morbidity. Endothelin-1 (ET-1) plays a key role in this process and bosentan hydrate (BOS), an ET-1 dual receptor blocker, may therefore be a logical treatment for impaired pulmonary circulation. We review our initial experience with BOS treatment in ten pediatric patients.

Patients and Methods: Age distribution; neonate: 1, 1-3 months: 3, 4-6 months: 5, 6-12 months: 1 . Down syndrome: 5. Operation performed; AVSD repair: 3 , VSD closure: 2, TAPVD repair: 1 , PAPVD repair: 1, DORV repair: 1, Truncus repair: 1, bidirectional cavopulmonary shunt: 1 . BOS was administered orally or through a nasogastric tube at a dose of $0.75 \mathrm{mg} / \mathrm{kg}$ twice a day.

Results: All patients were treated with oral sildenafil citrate (SIL) prior to BOS treatment. Three patients suffered from $\mathrm{PH}$-related symptoms despite SIL treatment. After adding BOS, all patients were weaned from SIL uneventfully without the symptoms. BOS was discontinued shortly after hospital discharge, except for three patients who had persistent PH. Only one patient had a robust increase in liver transaminase, which was normalized after discontinuation of BOS. There were no other adverse events during BOS treatment.

Conclusions: Our early experience suggests that ET-1 blockade may be an effective alternative approach for impaired pulmonary circulation that are refractive to existing therapies in the early postoperative period after congenital cardiac surgery.

\section{6}

Paediatric Pulmonary Arterial Hypertension: Effects of Long-Term Treatment with Bosentan

Shahin Moledina, Great Ormond Street Hospital, London, UK, United Kingdom

Alison A Hislop, Great Ormond Street Hospital, London, UK, United Kingdom

Ingram Schulze-Neick, Great Ormond Street Hospital, London, UK, United Kingdom

Sheila G Haworth, Great Ormond Street Hospital, London, UK, United Kingdom

Objectives: To investigate the long term effects of treatment with bosentan in childhood pulmonary arterial hypertension $(\mathrm{PAH})$ Methods: This retrospective case note study included 103 children treated with bosentan, with or without a prostanoid or sildenafil, between January 2002 and April 2008. 42 had Idiopathic PAH (IPAH) and 61 had Associated PAH (APAH), all with congenital heart disease. Haemodynamic data were recorded at baseline $(\mathrm{n}=79)$ and $20+/-8$ months after treatment initiation ( $\mathrm{n}=17)$. 6-minute walk distance, WHO functional class, somatic growth and Quality of Life (SF10) were assessed at baseline and at regular intervals for up to 5 years. Survival estimates were determined for all 103 patients.

Results: The median duration of treatment was 36 months (range 6-77 months) with no untoward side effects. At the end of study only $21 \%$ with IPAH, remained on bosentan monotherapy, compared with $41 \%$ with APAH. Kaplan-Meier survival estimates at 1, 2 and 3 years were $96 \%, 89 \%$ and $83 \%$ respectively. Exercise tolerance improved. The six-minute walk distance increased from a baseline of $258+/-127$ meters (mean $+/$ $-\mathrm{SD}, \mathrm{n}=50)$ to $312 \mathrm{~m}$ at 6 months $(\mathrm{p}<0.01, \mathrm{n}=36)$, an increase maintained at 1,2 and 3 years $(\mathrm{p}<0.01$ for all; $\mathrm{n}=33$, $\mathrm{n}=27, \mathrm{n}=19$ respectively). Repeat cardiac catheterisation showed no deterioration in either pulmonary vascular resistance index (21v17units.m2 at baseline) or pulmonary artery pressure (56v52 $\mathrm{mmHg}$ at baseline).

Conclusion: Bosentan is safe and effective as a monotherapy or given in combination, as appropriate, in children with $\mathrm{PAH}$.

\section{7}

Twenty three years old man who was treated as PPH for 11 years finally revealed as multiple peripheral PS: A case report Yasuhiro Tsuji, Division of Pediatrics and Perinatology, Tottori University, Japan

Hiroaki Funata, Division of Pediatrics and Perinatology, Tottori University, Japan

Yuichirou Hashida, Division of Pediatrics and Perinatology, Tottori

University, Japan

Youichi Mino, Division of Pediatrics and Perinatology, Tottori University, Japan

Hiroki Kuranobu, Division of Pediatrics and Perinatology, Tottori University, Japan

Susumu Kanzaki, Division of Pediatrics and Perinatology, Tottori

University, Japan

Primary pulmonary hypertension (PPH) is progressive, and no effective cure is currently available. Patients with $\mathrm{PPH}$ usually die within several years after onset. We report a 23 years old man who was treated as PPH for 11 years finally revealed as multiple peripheral pulmonary stenosis (PS).

Case report: This male patient was referred to our hospital when he was 12 years old because of right ventricular hypertrophy. His right ventricular hypertrophy was noticed by the electrocardiogram examination routinely performed during the first-year of junior high school in our prefecture. The cardiac catheterization showed high pulmonary artery pressure [93/75 (82) $\mathrm{mm} \mathrm{Hg}$, and the imaging view of the pulmonary artery showed proximal artery dilation and progressive narrowing suitable for $\mathrm{PPH}$. We diagnosed him as PPH, and treated using belaprost sodium, ACE-I, and aspirin. Though 11 years passed since his PPH was diagnosed, he did not show rapid aggravation of either his physical condition (NYHA I-II) or pulmonary pressure. Therefore, we referred him to the cardiovascular division of another hospital that specially deals with adult PPH patients. Cardiac catheter examination conducted in the hospital showed that pulmonary artery pressure was high [96/ $33(56) \mathrm{mm} \mathrm{Hg}$. Cardiovascular angiography revealed he had multiple pulmonary artery stenosis in peripheral area. He was finally diagnosed not as PPH but as multiple peripheral PS.

Our case suggested that we should seek another cause of $\mathrm{PH}$ whenever patients with $\mathrm{PPH}$ whose symptoms do not progress rapidly.

\section{8}

Relationship of VEGF/receptors level with hemodynamic parameters in cyanotic congenital heart disease with diminished pulmonary flow

Sedef Tunaoglu, Gazi University, Medical Faculty, Department of Pediatric Cardiology, Turkey

Ilknur Aslan, Gazi University, Medical Faculty, Department of Pediatrics, Turkey 
Velit Halit, Gazi University, Medical Faculty, Department of Cardiovascular surgery, Turkey

Serdar Kula, Gazi University, Medical Faculty, Department of Pediatric Cardiology, Turkey

Rana Olguntürk, Gazi University, Medical Faculty, Department of Pediatric Cardiology, Turkey

Tetralogy of Fallot (TF) is most frequently seen cyanotic congenital heart disease with diminished pulmonary flow causes hypoxia and elevated serum VEGF levels in patients were reported in cyanotic congenital heart disease. Despite of these potent stimulators, pulmonary vascular development is not sufficient in some patients with TF. This study was planned to investigated the relationship of serum VEGF levels and VEGF receptors of pulmonary vascular bed in patients with TF.

Method: Twenty-four patients who were undergoing open-heart surgery were included the study. Patients were divided into two groups: Group 1 (Cyanotic group : 13 patients with TF; 4F, 9M; mean age: $29 \pm 13.8$ months); Group 2 (Acyanotic group: 11 patients with ASD, VSD, PDA; 8F, 3M; mean age: $48 \pm 30.7$ months). Lung biopsies were taken during operation from patients in cyanotic group for VEGF receptors.

Results: VEGF levels were negatively correlated with arterial oxygen saturation in Group 1, since there was no correlation in Group 2. Arterial VEGF levels were significantly elevated in Group 2 compared with Group1 (222,9 $\pm 219,9$ vs $59,5 \pm 54,8 \mathrm{pg} / \mathrm{ml})$. There was no significant difference in pulmonary artery VEGF levels between groups (Group 2: $128,5 \pm 161,9$, Group 1: $61,5 \pm 57,2 \mathrm{pg} / \mathrm{ml}$ ) VEGF tissue staining were find negative in 11 of 13 patients in Group 1. $\mathrm{KDR} / \mathrm{Flk}-1$ receptors were find positive in 11 patients of group 1. Flt-1 receptors were find positive in 6 patients.

Conclusion: Further studies are required on VEGF/receptor relationship, gene expression and production in patients with diminished pulmonary flow.

\section{9}

Evaluation of acute hemodymamic effect of adenosine infusion in patients with chronic thromboembolism pulmonary hypertension

Guang-yi Wang, Department of cardiology, Chinese PLA general hospital, China

Hai-jun Hou, China

Zhi-feng Wang, China

Objective: To determine the effect of acute hemodynamic after adenosine infusion in patients with Chronic thromboembolic pulmonary hypertension (CTEPH).

Method: Right heart catheterization was performed in 25 patients (mean age 51 years, New York Heart Association class I-III) at the time of diagnosis of CTEPH. Mean pulmonary arterial pressure (mPAP), systolic pulmonary arterial pressure (sPAP), cardiac output (CO), pulmonary vascular resistance (PVR), systemic arterial pressure (MAP), oxygen saturation were obtained before and after infused adenosine (Beginning initial dose from $50 \mathrm{ug} / \mathrm{min} / \mathrm{kg}$ to maximal dose for $300 \mathrm{ug}$ increase by degreed).

Results: Parameters of pulmonary arterial hemodynamics were observed following the infusion of adenosine (before to after infusion): the mean dosage of infusion adenosine was $275 \mathrm{Ug} / \mathrm{kg} /$ min. PVR: $1012+/-305.6$ to $834.3+/-295.3$ dyn.s.cm(-5), $\mathrm{p}<0.05$; mPAP: $59.2+/-16.9$ to $53.3+/-16.2 \mathrm{~mm} \mathrm{Hg}$, $\mathrm{p}<0.05$, sPAP $87.1+/-23.3$ to $79.1+/-16.6 \mathrm{mmHg}$ CO: $3.79+/-1.26$ to $4.56+/-1.251 / \mathrm{min}, \mathrm{p}<0.05$. SO2: 92.2 $+/-3.9 \%$ to $96.2+/ 3.4 \%, \mathrm{P}>0.05$. Systemic artery pressure slightly decreased but did not display statistical difference: $128.4+$ / -14.7 to $113.6+/-11.1 \mathrm{mmHg}, \mathrm{p}>0.05$.

Conclusions: Hemodynamic changes after infusion of adenosine suggest a advantage of a reversible component of vasoconstriction to pulmonary arterial hypertension in patients with CTEPH.

\section{0}

Evaluation of acute hemodymamic effect of inhaled iloprost in pulmonary artery hypertension with right heart catheterization

Guang-yi Wang, Department of cardiology, Chinese PLA general hospital, China

Hai-jun Hou, China

Zhi-feng Wang, China

Objective: In this study, we evaluated acute hemodynamic effects of inhaled iloprost in patients with CTEPH.

Method: Right heart catheterization was performed in 15 patients (mean age 53 years, New York Heart Association class II-III) at the time of diagnosis of CTEPH. Pulmonary vascular resistance (PVR), mean pulmonary arterial pressure (mPAP), cardiac output (CO), mean systemic arterial pressure (MAP) were obtained before and $20 \mathrm{~min}$ after inhaling $20 \mathrm{ug}$ iloprost. Subsequently, all patients were evaluated for pulmonary endarterectomy. Three patients were eligible for surgery.

Results: Significant changes in pulmonary hemodynamics were observed following the inhalation of iloprost (before to after inhalation): PVR: $986+/-405.3$ to $707.3+/-315.3$ dyn.s.cm $(-5), \mathrm{p}<0.05$; mPAP: $55.1+/-13.9$ to $45.3+/-12.2 \mathrm{~mm}$ $\mathrm{Hg}, \mathrm{p}<0.05$, CO: $4.89+/-1.16$ to $5.47+/-1.12 \mathrm{l} / \mathrm{min}$, $\mathrm{p}<0.05$. Systemic artery pressure slightly decreased but did not display statistical difference: $123.4+/-12.8$ to $116.6+/$ $-11.5 \mathrm{mmHg}, \mathrm{p}>0.05$.

Conclusions: Hemodynamic changes after inhalation of iloprost suggest a significant advantage of a reversible component of vasoconstriction to pulmonary arterial hypertension in patients with CTEPH.

\section{1}

Severe Pulmonary Hypertension in an Infant Girl with Incontinentia Pigmenti

Kenji Yasuda, Department of Pediatrics, Shimane University Faculty of Medicine, Japan

Noriaki Minami, Department of Pediatrics, Shimane University Faculty of Medicine, Japan

Yoko Yoshikawa, Department of Pediatrics, Shimane University Faculty of Medicine, Japan

Takeshi Taketani, Japan

Seiji Fukuda, Department of Pediatrics, Shimane University Faculty of Medicine, Japan

Seiji Yamaguchi, Department of Pediatrics, Shimane University Faculty of Medicine, Japan

Introduction: Incontinentia pigmenti (IP) is a rare X-linked dominant genodermatosis that presents multiple disorders of the ectodermal tissues. IP is caused by mutations in the NF-kappaB essential modulator (NEMO) gene. There are a few reports of cardiovascular involvement in IP; however, fatal pulmonary hypertension (PH) in IP has seldom been reported and little is known about its etiology. We report an infant girl with IP accompanied by severe $\mathrm{PH}$, and her histo-pathological findings of pulmonary artery (PA).

Case Presentation: The patient was clinically diagnosed as IP during her neonatal period based on the specific skin lesion, 
neonatal seizure and the family history of IP. At the age of 2 months, systolic heart murmur was pointed out, and severe $\mathrm{PH}$ was detected by echocardiography and cardiac catheterization. Although oxygen inhalation, oral Bosentan and predonisolone treatment were initiated for the $\mathrm{PH}$ and hypereosinophilia, severe $\mathrm{PH}$ persisted and she died of $\mathrm{PH}$ crisis at the age of 5 months. Deletion of the NEMO gene was identified, and she was genetically diagnosed as IP. The postmortem histo-pathological findings of PA demonstrated grade IV of Heath-Edwards' grading system. Neither apparent structural cardiac abnormalities, nor infiltration of eosinophil into lung was detected.

Discussion and Conclusion: The histo-pathological findings confirmed her diagnosis of pulmonary arterial hypertension (PAH), suggesting that diffuse microangiopathy might be involved in IP. The modulation of NF-kappaB signaling or hypereosinophilia may be important factor for the development of PAH in IP. Further examination is necessary to determine the mechanism responsible for $\mathrm{PAH}$ in IP.

\section{2}

Sildenafil Improves Oxygen Saturation in Hypoxemic Patients with Complex Congenital Heart Diseases and Diminished Pulmonary Vascular Bed in the Early and Mid-Term Follow-Up Period

Noriko Yodoya, Mie University Graduate School of Medicine, Japan Yoshihide Mitani, Mie University Graduate School of Medicine, Japan Hiroyuki Ohashi, Mie University Graduate School of Medicine, Japan Hidetoshi Hayakawa, Mie University Graduate School of Medicine, Japan

Shin Takabayashi, Mie University Graduate School of Medicine, Japan Hideto Shimpo, Mie University Graduate School of Medicine, Japan Yoshihiro Komada, Mie University Graduate School of Medicine, Japan

Background: Sildenafil, a selective pulmonary vasodilator by a blockade of phosphodiesterase type 5, improves exercise capacity and pulmonary hemodynamics in patients with pulmonary arterial hypertension. We tested the hypothesis that sildenafil improves oxygen saturation in hypoxemic patients with complex congenital heart diseases (CHD), diminished pulmonary vascular bed and right to left (RL) shunting in the early and mid-term follow-up period.

Methods and results: Nine patients with complex CHD and decreased pulmonary blood flow, 4 patients with systemic venous collaterals after bidirectional Glenn procedure, 2 patients after fenestrated Fontan procedure, and 3 patients with Fallot's tetralogy with severely hypoplastic unifocalized pulmonary arteries, were investigated at the starting age of 29 (mean) $\pm 18 \mathrm{~m}$ (SD) and after an interval $(13 \mathrm{~m} \pm 4 \mathrm{~m})$ under chronic sildenafil administration $(1.5-2.0 \mathrm{mg} / \mathrm{kg} /$ day $)$. Before chronic therapy, acute sildenafil loading $(0.5-0.7 \mathrm{mg} / \mathrm{kg} /$ dose $)$ increased $\mathrm{SpO} 2$ from $80.3 \pm 4.0$ at the baseline to $84.7 \pm 4.0 \%$ at $30-60 \mathrm{~min}$ after the loading ( $\mathrm{p}<.0001$, paired $\mathrm{T}$ test). In the mid-term follow-up period after chronic treatment, acute loading similarly increased $\mathrm{SpO} 2$ from $80.7 \pm 5.4$ to $84.8 \pm 4.1 \%(\mathrm{p}=.0004)$. Similarly, a significant increase in $\mathrm{SpO} 2$ in response to sildenafil was confirmed at these time points in subgroups of patients: a group of pre- and post-Fontan patients, and a Fallot's group.

Conclusion: Sildenafil administration had continued acute effects on disturbed pulmonary circulation in patients with complex CHD, low pulmonary blood flow, and RL shunting in the mid-term follow-up, as evidenced by a reactive increase in $\mathrm{SpO}$. The present findings suggest that chronic sildenafil administration could be one of adjunctive treatment options in such patients.

\section{3}

Intravenous Transplantation of Syngenic Bone Marrowderived Mononuclear Cells Improves Pulmonary Artery hypertension by Paracrine Effect through VEGF-VEGF Receptor System

Homare Yoshida, Department of Cardiovascular Surgery, IHBS,

The University of Tokushima Graduate school, Japan

Takashi Kitaichi, Department of Cardiovascular Surgery, IHBS,

The University of Tokushima Graduate school, Japan

Masahisa Urata, Department of Cardiovascular Surgery, IHBS,

The University of Tokushima Graduate school, Japan

Hirotsugu Kurobe, Department of Cardiovascular Surgery, IHBS,

The University of Tokushima Graduate school, Japan

Tamotsu Kanbara, Department of Cardiovascular Surgery, IHBS,

The University of Tokushima Graduate school, Japan

Tatsuo Motoki, Department of Cardiovascular Surgery, IHBS,

The University of Tokushima Graduate school, Japan

Tetsuya Kitagawa, Department of Cardiovascular Surgery, IHBS,

The University of Tokushima Graduate school, Japan

Objective: We investigated the effects and possible mechanism of intravenous transplantation of syngenic bone marrow-derived mononuclear cells (BMMNCs) on pulmonary artery hypertension $(\mathrm{PAH})$ induced by monocrotaline (MCT).

Methods: MCT of $80 \mathrm{mg} / \mathrm{kg}$-weight was administrated to C57BL/ 6 mice, and it induced PAH 4 weeks later. BMMNCs harvested from syngenic donor mice were injected intravenously to those mice 4 weeks after MCT-administration. Right ventricle/septum-left ventricle weight ratio, the number of small pulmonary artery, and medial thickness of PA were observed. Western immnoblotting of the lung tissue was performed to observe vascular endothelial growth factor (VEGF) expression at one week after BMMNCs transplantation. VEGFR-2 inhibitor was administrated to the PAH mice simultaneously with BMMNCs transplantation.

Results: $\mathrm{RV} / \mathrm{S}+\mathrm{LV}$ weight ratio increased, the number of $\mathrm{PA}$ decreased, and medial thickness increased significantly 28 days after MCT-injection compared to those of vehicle-injected mice, respectively $(0.296 \pm 0.04$ vs $0.203 \pm 0.02 ; 5.1 \pm 1.7$ vs $20.9 \pm 4.8$; $21.5 \% \pm 5.2 \%$ vs $7.5 \% \pm 2.1 \%, \mathrm{p}<0.01)$. These indices of MCTinjected mice improved significantly 28 days after BMMNCs transplantation compared to those mice of 8 weeks after MCT/ PBS-injection, respectively $(0.22 \pm 0.02$ vs $0.31 \pm 0.02 ; 17.1 \pm 2.6$ vs $8.2 \pm 1.7 ; 7.7 \% \pm 2.2 \%$ vs $20 \% \pm 2.1 \%, p<0.01)$. However, BMMNCs were not incorporated into the lung during one week after the transplantation. Upregulation of VEGF was observed significantly in the lung tissue one week after transplantation. Moreover improvement of PAH was inhibited by administration of VEGFR-2 inhibitor.

Conclusions: These results indicate that intravenous transplantation of syngenic BMMNCs improves MCT-induced PAH through VEGF-VEGFR system. Intravenous transplantation of syngenic BMMNCs has the potential as a new therapeutic option for $\mathrm{PAH}$.

\section{4}

The changes of pulmonary arterial pressure after tonsillectomy and sdonoidectomy in childhood Gyoung-bin Yun, Department of Pediatrics, Chungnam National University Hospital, Korea

Sang-yun Lee, Department of Pediatrics, Chungnam National University Hospital, Korea

Yong-min Kim, Department of Otorrhinolaryngology, Chungnam National University Hospital, Korea 
Hong-ryang Kil, Department of Pediatrics, Chungnam National University Hospital, Korea

Introduction: Pulmonary Hypertension is characterized by normal pulmonary artery wedge pressure $(<15 \mathrm{mmHg})$, mean pulmonary arterial pressure (mPAP) $>25 \mathrm{mmHg}$ at rest, andmPAP $>30 \mathrm{mmHg}$ during exercise. If severe upper airways obstruction occurs, there will be symptoms such as severe alveolar hypoventilation, hypoxemia and the hypercarbia, secondary pulmonary arterial hypertension, right ventricular hypertrophy, and potent excretion of pulmonary vasoconstrictors. The characteristic clinical signs of upper air way obstruction in childhood are noisy breathing such as snoring or stridor, chest wall recession, restless sleep with frequent arousal, excessive daytime somnolence, nocturnal enuresis, behavioral changes and morning headache, systemic hypertension and failure to thrive. The aim of this study is to evaluate the changes of PAP after tonsillectomy and adenoidectomy and its contributing factors.

Methology: 13 patients were selected who were diagnosed as tonsilar and adenoidal hypertrophy at Chungnam National University Hospital. Each patient underwent echocardiogram at 1st, 2nd, 3rd and 30th day after operation. We checked mPAP and tricuspid valve E/A ratio measured in room air and $\mathrm{O} 2$ supply (5 L/min via mask) state. The proBNP checked before operation. Statistical analysis was made by chai square test.

Result: The mean age of patient were $7.69 \pm 3.22$ year (4-12). mPAP is markedly reduced after surgery $(\mathrm{P}<0.05)$. Tricuspid Valve $\mathrm{E} / \mathrm{A}$ ratio was not reduced after surgery. O2 supplmentation reduced $\mathrm{mPAP}$ before surgery, but did not reduce $\mathrm{MPAP}$ after surgery. E/A ratio didn't influenced by $\mathrm{O} 2$ supplmentation.

Conclusion: The hypertrophy of tonsil and adenoid produced the pulmonary arterial hypertension. The pulmonary hypertension by airway obstruction is improved by tonsillectomy and adenoidectomy.

\section{6 \\ Characteristics and Outcomes of Children with Pulmon- ary Arterial Hypertension Referred to a Single Statewide Service \\ Michelle L Rose, The Royal Children's Hopsital, Melbourne, Australia Tara Bharucha, Australia \\ Suzanna I Vidmar, Australia \\ Daniel J Penny, The Royal Children's Hopsital Melbourne E Murdoch Children's Research Institute, Australia \\ Ingrid P King, Australia \\ Robert G Weintraub, The Royal Children's Hopsital, Australia}

Objective: Childhood pulmonary hypertension is associated with varied aetiologies and outcomes. Existing therapies are largely based on studies of adult patients with idiopathic pulmonary arterial hypertension (IPAH). We performed a retrospective review of demographic characteristics and outcomes of all patients referred for ongoing long-term management of $\mathrm{PAH}$ to a single, statewide paediatric service.

Methods: Records of 90 patients with PAH who were managed treated between July 1998 and July 2008 were reviewed. Survival analysis was undertaken in patients who fulfilled the following criteria: age $>3$ months of age at referral, $\mathrm{PAH}$ persisting for at least 6 months after surgery for congenital heart disease (CHD), treatment with a PAH-specific therapy for $>14$ days, and a mean PA pressure $>25 \mathrm{mmHg}$ or a TR velocity $>3.5 \mathrm{~m} / \mathrm{s}$. Patients were grouped into one of 4 principal diagnostic categories: IPAH, CHD, chronic lung disease (CLD) and repaired diaphragmatic hernia.

Results: IPAH was the principal diagnosis in 17 of 90 patients, CLD in 14 , CHD in 43 , and repaired diaphragmatic hernia in 8 . The accompanying Table shows freedom from death or lung transplantation at specified time intervals after diagnosis for each group. Conclusions: The majority of children referred for management of PAG have associated heart or lung disease. The period of greatest risk for children with CLD and repaired diaphragmatic hernia is $<12$ months after diagnosis, whereas the risk for children with IPAH is ongoing.

\begin{tabular}{lccc}
\hline & \multicolumn{3}{c}{ Probability of survival } \\
Group & 1 year & 3 years & 5 years \\
\hline IPAH & 1.0 & 0.85 & 0.56 \\
CHD & 0.8 & 0.67 & 0.67 \\
CLD & 0.79 & 0.79 & 0.79 \\
Diaphraghmatic & 0.45 & 0.45 & 0.45 \\
hernia & & & \\
\hline
\end{tabular}

\section{7}

Impact: When Healthcare Leaders and Providers Communicate Poorly

Maryanne W Kessel, Children's Hospital of Wisconsin, United States Kathy Mussatto, Children's Hospital of Wisconsin, United States Mary Beth Petersen, Children's Hospital of Wisconsin, United States Lee Anne Eddy, Children's Hospital of Wisconsin, United States Maureen Otto, Children's Hospital of Wisconsin

Background: Communication among healthcare providers directly affects patient care. In 2005, 69\% of The Joint Commission sentinel events reported communication as root cause. $73 \%$ of those resulted in patient deaths. In an American Association of Critical Care Nurses sponsored study, 84\% surveyed physicians reported coworkers taking shortcuts dangerous to patients. Impact: Nearly three in four medical errors are related to miscommunication. Yet, $<10 \%$ were willing to confront colleagues about concerns.

Methods: Evaluated case reviews for: diagnostic, medication and surgical errors, and deaths. Communication errors were not defined. Goal: Identify communication as a "risk factor" in patient care. Determine Believable, Useable, and Practical communication tools: 1) Immediate communication ("right data to right person at right time") via SBARR (Situation, Background, Assessment, Recommendation, Response). 2) Change culture through Crucial Conversations skills.

SBARR education introduced hospital-wide. Crucial Conversation taught to Herma Heart Center leadership via outside consultant. Initial class: 20 early adopters and skeptics attended Mastery Course. Phase two: 30 interested staff and faculty. Phase three: 20 staff attended "exposure" course.

Results: 70 team members engaged in a significant time commitment and exposure to culture change. Anecdotal, qualitative observations (similar to social sciences) describe behavioral changes. Team reported an increased success in "safe" communications with challenging patient care interactions.

Conclusion: To improve patient care, cardiac leadership implemented new communication tools. The challenge remains to create and sustain effective, safe communication. Barriers include lack of tools for quantitative measurement of improvement tied to patient outcomes.

\section{8}

Paediatric Cardiac Surgery For Palestinian Children

Mahmoud Nashashibi, Makassed Hospital, Jerusalem, Israel 
Steve Sosebee, Palestine Children's Relief Fund, United States

Vivian Bader, Makassed Hospital, Jerusalem, Israel

Guido Van Nooten, University of Ghent, Belgium

Giancarlo Crupi, Bergamo, Italy

Babulal Sethia, Royal Brompton Hospital, United Kingdom

Alan Kerr, ex- Green lane Hospital, Auckland, New Zealand

Paediatric cardiac services in the Occupied Territories of Israel (Pop $3.8 \mathrm{M}$ ) are inadequate due to the tragic political situation and resultant socioeconomic deprivation. At least 500 children need surgery annually.

The Palestine Children's Relief Fund, a secular, apolitical US NGO has sponsored and coordinated a program at the Makassed Hospital in East Jerusalem to serve West Bank and Gaza. Objectives are provision of a near-continuous service by international teams, provision of facilities and equipment and recruitment and training of staff with the goal an autonomous service. Groups from Belgium, Italy, France, England, New Zealand and USA have made regular visits. Facilities provided include a dedicated fully equipped OR, a 7 bed ICU and 2 echo machines.

Between Sept 2003 and Dec 2008764 operations have been performed (2003 27, 2004 111, 2005 144, 2006 102, 2007 192, 2008 188). Overall mortality is $3.9 \%$. Over the same period European donations have allowed referral of some children to Israeli hospitals. This has allowed progression at Makassed hospital towards younger and more complex cases without significant increase in mortality. Many operations are now done with the sole support of Palestinian staff.

Differences in philosophy and protocols between visiting groups have been minimized by annual meetings and discussions. Ongoing problems include patient and staff access, follow-up care and staff recruitment and training.

This model of cardiac care for children in a combat zone has proved effective and may be unique.

\section{9}

Pediatric and Congenital Heart Surgery in Less Developed Country: tales of two countries

Hafil B Abdulgani, Faculty of Medicine. Universiti Teknologi MARA, Malaysia

Yahya Awang, Faculty of Medicine, Universiti Teknologi Mara, Malaysia Khalid Yusoff, Fakulti of Medicine, Universiti Teknologi MARA,

Malaysia

Hamed Oemar, Malaysia

In 1980s a comprehensive, organized, and modern cardiovascular care, in less developed countries, was initiated by the Establishment; i.e. National Heart Center (Indonesia) and National Heart Institute (Malaysia). Although surgery of congenital heart disease (CHD) were scarcely performed, previously, the center/institute did focus on managing coronary heart disease. This task was in response to public health surveys that coronary heart disease became the number one cause of death in the region. At the other end, advanced management of congenital heart disease [(CHD) albeit constant in prevalence] were slow to gain momentum. Lack of expertise, as well as limited funding, were considered to be primary reasons. Nevertheless, despite more than two decades of progress, expert-shortages continued to alter the end-result of medical/surgical care of infant/children with CHD. Unattractive financial rewards were also felt to have a negative impact on the recruitment process of young medical graduates to excel as pediatric cardiologist and/or pediatric cardiac surgeon. Alternative solution to provide a sustainable care to those infants and children with CHD, to a more developed neighboring countries, were short-termed and unpopular. Such measure challenged the existence of highly specialized, centralized health-care system. This paper is an appeal, to the World Society for Pediatric and Congenital Heart Surgery, for reformed service development and quality management policies for lessdeveloped countries in the current global financial turmoils.

\section{0}

Written Patient Information in the 21st Century

Janet E Burns, Royal Hospital for Sick Children, Edinburgh,

United Kingdom

Sheila Wurr, Royal Hospital for Sick Children, Edinburgh, United Kingdom

Muhammed Walayat, Royal Hospital for Sick Children, Edinburgh, United Kingdom

Since 1999, some families in Scotland have been routinely sent an information letter prior to admission for an elective cardiac catheter procedure in their child. The information is explicit in describing potential complications including local vascular injury, brain damage and death.

The pilot was sent to 20 parents whose children had recently had a catheter procedure. They commented on the accuracy and helpfulness of the information, what should be included or excluded, and whether they thought it an appropriate letter to receive. 18 parents responded. None objected to the letter or felt it would have adversely affected consent. None felt that it gave additional information but all indicated it was helpful to have a written communication.

In 10 years it has been sent to 427 families, enclosing 2 copies so that one may be signed and returned to confirm that the parent wishes to proceed with the procedure. It is made clear that the child's name will be added to the waiting list for the procedure once the form has been returned. 42 have needed further chasing before returning it and none have declined catheterisation. Some minor changes have been made.

One colleague now uses the standard letter but others find it too explicit and plan a simpler letter.

Conclusion: Many clinicians still use verbal communication only to give risk/benefit information relating to procedures. It is however possible to devise a simple computer generated letter to complement this and ensure that potential complications are acknowledged by families prior to admission.

\section{1}

Operation Open Heart - a 22 year experience

Andrew D Cochrane, Monash Medical Centre, Melbourne, Australia Annette Baldwin, Sydney Adventist Hospital, Australia Ian Nicholson, Westmead Children's Hospital, Sydney, Australia Graham Nunn, Mater Children's Hospital, Brisbane, Australia

Operation Open Heart has been conducted by the Sydney Adventist Hospital for 22 years, since the first trip to Tonga in 1986. There have been 71 cardiac teams in total which have regularly visited 12 countries in the Pacific, Asia and Rwanda. The major recipients of this support have been Fiji (17 visits) and Papua New Guinea (16 visits). The teams have performed cardiac surgery on 1,635 children and adults, with a predominance of paediatric congenital surgery over adult valve surgery. The early mortality is low at $2 \%$. The teams generally include a cardiologist, cardiac surgeon, anaesthetist, intensivist, perfusionist, physiotherapist, ICU and ward nursing staff, and a biomedical technician. The financial value of this contribution were these operations to have been done in Australia would be AUD $\$ 25$ million. 
All members of the teams are volunteers and contribute financially as well as medically, by paying for their own airfares. The teams attempt to bring all of their equipment and disposable items in order to minimize the impact on the recipient hospital. The program is supported by AusAid, by donations/discounts from surgical equipment companies, and by reduced air freight charges.

In addition to the service component, the capacity building in the recipient country is equally important. This may involve training nursing staff in the operating room, ICU or ward, by working with local cardiac surgeons and anaesthetists, training local surgeons to do simple procedures such as PDA ligation and shunts, teaching medical students, and by upgrading local infrastructure.

8172

Missions of Hope: The New Face of Pediatric Cardiology Sonia A ElSaiedi, Cairo university Children Hospital, Egypt Fadia M Mahmoud, Cairo University Children Hospital, Egypt Mohamed R Abu El ezz, Cairo University Children Hospital, Egypt Wael M Abdel Aal, Cairo University, Egypt

Hala M Agha, Cairo University Children Hospital, Egypt

For so many years, thousands of children living outside the metropolis and other large population towns in Egypt have been unable to be seen by cardiologists for diagnosis and help, due to the logistics and costs involved in travelling from these remote regions to tertiary health care centers. Over the past decade, steps have been taken to change the situation by means of "Missions" going to these outlying towns and villages. The concept being that doctors going to the patients rather than patients coming to the doctors.

During these missions large numbers of patients are clinically examined over a short period of time where qualified decisions are taken on the spot as to whether continuing medical treatment or adjustment of medications versus referral for intervention or surgery.

The goal is putting the patients on the right track and this can only be achieved by planning a proper schedule of management with follow up examination dates or intervention dates.

The system continued to gain momentum and success by the collaboration of paediatric cardiologists, echo-cardiographers, cardiac surgeons and charity social workers all working as one team.

These missions had made a great impact on the morbidity and mortality of CHD in our community. New boundaries are set, Links across the country are established, communications improved.

All the challenges, the preparations and the outcome will be presented.

\section{3}

How to start and sustain a successful pediatric cardiac intensive care program: A combined clinical and administrative strategy

Li Juan Fu, Heart Center of Shanghai Children's Medical Center, China Lei Zheng, Heart Center of Shanghai Children's Medical Center, China

Zhi Wei Xu, Thoracic \& Cardiovascular Surgery of Shanghai Children's Medical Center, China

Yong Mei Guan, Thoracic \& Cardiovascular Surgery of Shanghai Children's Medical Center, China

Objective: To describe key factors of advanced pediatric cardiac intensive care unit. To introduce concepts of teamwork, common perspective and scorecard, which measure excellence in such a new program.

Methods: Review of current clinical research data in concepts of teamwork, common perspective and scorecard. Compare clinical research data and administrative business between pediatric cardiac intensive care unit in China and USA.

Results: Clinical concepts are almost same in patients' treatment in two countries. Principles are useful as basic philosophical strategies at the bedside. There is still difference on administrative principles, concepts, strategies, human resource and applications, which make different results.

Conclusions: Starting a pediatric cardiac intensive care program is vital to start and sustain excellence, as well as a difficult endeavor. Monitoring excellence in such a program warrants application of a scorecard system. Well administrative principles and effective application ensure the clinical strategy which is a combined clinical and administrative approach.

Key Words: pediatric cardiac intensive care unit, administrative principles, operational strategies.

\section{5}

Tracking Cardiovascular Morbidity: Utility of a

Hand-Held Device to Create a Cardiovascular

Complications Database

Derek G Human, Division of Cardiology, Department of Pediatrics, British Columbia Children's Hospital and The University of British Columbia, Canada

Julie Ng, Division of Cardiology, Department of Pediatrics, British Columbia Children's Hospital and The University of British Columbia, Canada

James E Potts, Division of Cardiology, Department of Pediatrics, British Columbia Children's Hospital and The University of British Columbia, Canada

Background: In this era of declining cardiovascular mortality, other measures of Quality Assurance (QA) become important. We were concerned that the Discharge Abstract Database (DAD) provided through Hospital Records was neither timely nor complete.

Objectives: To analyze incongruent information, determine sources of error, and provide a true incidence of complications. Methods: Five cardiologists used Palm OS based software (Smartlist to Go) to enter all procedures (cardiac catheterization (CC), closed-heart operation $(\mathrm{CHO})$ or open-heart operation $(\mathrm{OHO})$ ) and associated complications (chosen from pre-defined pick lists) noted during their on-call duties. Following a brief trial period in 2005, all data captured over 2 years were compared.

Results: DAD identified 152 cases not in the Cardiovascular Complications Database (CCD): $53 \%$ were out of scope of CCD, $37 \%$ missed CC, $4 \%$ CHO and 6\% OHO. Detailed comparison of $55 \mathrm{OHO}$ cases for all potential complications revealed 46 positive in DAD and CCD, 324 negative in both, 75 positive in DAD, but not in CCD and 50 positive in CCD and not in DAD. This gave a $25 \%$ discrepancy between the two systems, accounted for by the use of non-specific coding terms in DAD and a low threshold for recording events. Using the corrected data from CCD, control charts with quarterly rates for all complications were derived: Those related to CC were $1 / 10$ cases, CHO 3/10 cases and OHO 5.5/10 cases.

Conclusions: Significant anomalies were found between the CCD and $\mathrm{DAD}$; creation of reliable $\mathrm{QA}$ data requires rigorous protocols congruent between physician and administrative perspectives.

\section{6}

Development and Validation of Revised Risk Adjustment for Congenital Heart Surgery Method

Kathy J Jenkins, Children's Hospital Boston, United States 
Jeffrey P Jacobs, University of South Florida, United States Marshall Jacobs, Drexel University College of Medicine, United States Emile Bacha, Children's Hospital Boston, United States Bohdan Maruszewski, Children's Memorial Health Institute, Poland James H Moller, University of Minnesota, United States Sean O'Brien, Duke University Medical Center, United States Kimberlee Gauvreau, Children's Hospital Boston, United States

Background: The Risk Adjustment for Congenital Heart Surgery (RACHS-1) method has been used extensively for comparative analyses of risk of death for children undergoing congenital heart surgery. Ongoing risk reduction, emergence of standardized nomenclature and new procedures necessitate reconsideration of the original methodology.

Methods: A panel of experts used clinical judgement, and then examined mortality rates from multiple data sources (Society of Thoracic Surgeons, European Association for Cardiothoracic Surgery), Pediatric Cardiac Care Consortium, Pediatric Health Information System, and Kids Inpatient Database) to group procedures, using International Pediatric and Congenital Cardiac Codes, into candidate risk groups (1 to 5 ) with similar withingroup risk of in-hospital death.

Results: Using input from 30 pediatric cardiologists and cardiac surgeons, procedure-based risks of death for 180 procedures were considered. Procedural mortality rates were calculated using data from nearly 100,000 cases. Median mortality rates for preliminary risk categories are: $1-0.4 \%, 2-1.7 \%, 3-4.1 \%, 4-6.4 \%, 5-20.7 \%$. Final risk category development and decisions about incorporation of original RACHS-1 clinical variables (age, prematurity, noncardiac anomaly) or other clinical factors will be determined, based on statistical significance, contribution to ROC area, and consistency of findings, at the next meeting of the panel in January 2009. The final model will be validated in subsequent databases.

Conclusion: Similar to RACHS-1, this updated version of the Risk Adjustment for Congenital Heart Surgery method should be useful to adjust for current, baseline risk of death after congenital heart surgery, using various clinical data sources.

\section{7}

Establishment of a new tertiary-care pediatric cardiology and cardiac surgery center: initial experience of case-mix and factors of quality of care

Laszlo Kiraly, Sheikh Khalifa Medical City/Cleveland Clinic, United Arab Emirates

Csaba Tamas, Sheikh Khalifa Medical City/Cleveland Clinic, United Arab Emirates

Stephen Shipton, Sheikh Khalifa Medical City/Cleveland Clinic, United Arab Emirates

Johannes Du Plessis, Sheikh Khalifa Medical City/Cleveland Clinic, United Arab Emirates

Haitham Talo, Sheikh Khalifa Medical City/Cleveland Clinic, United Arab Emirates

Hansjorg Mossinger, Sheikh Khalifa Medical City/Cleveland Clinic, United Arab Emirates

Kerstin Mutze, Sheikh Khalifa Medical City/Cleveland Clinic, United Arab Emirates

Edgar Szekely, Sheikh Khalifa Medical City/Cleveland Clinic, United Arab Emirates

Objective: While establishing a new cardiovascular program in a multicultural environment, many challenges are faced. The chief test is living up to the high patient expectations in order to meet the contemporary excellent surgical results achieved worldwide.
We present our initial experience of a new tertiary-care pediatric cardiology/cardiac surgery center in UAE.

Methods: Our multidisciplinary team drew up a detailed continuum of care plan and performance criteria were employed to monitor care. Having established the necessary OR/ICU environments, full range of pediatric cardiac services was offered including neonatal open-heart surgery. Continuous auditing and outcome review allowed adjustments in organizational and professional care to achieve excellent results.

Results: Since the start of the program in April 2007 to date, 295 pediatric cardiac operations and 189 catheter-interventions were performed. The surgical patient population comprised of 117(39.6\%) neonates, infants(1-6 months of age): $85(29 \%)$, >6months: 93(31.4\%), including $10 \mathrm{GUCH}$ cases. Aristotle Basic Complexity Score Levels were: I (simplest):18(16.3\%), II (simple):63(21.4\%), III (complex):52(17.6\%), IV (most complex):132(44.7\%). Introduction of integrated pediatric cardiac ICU/HDU resulted in marked reduction of LOS (mean:13.1 \pm 5.04 to $7.12 \pm 3.1$ days, $\mathrm{p}=.001$ ).

Conclusions: The rapidly growing population of the UAE requested the foundation of a national pediatric cardiac center. Our program has quickly won recognition indicated by a steadily growing number of referrals from all over the country. Neonates and infants ( $<6$ months of age) requiring urgent/emergent intervention have been overrepresented since the start. Continuous review and audit permits a developing model of care. Initial experience demonstrates that our program is capable of becoming a national center of excellence.

\section{8}

Comparison of Vascular Complication between Conventional Treatment and Bone Marrow Transplantation in Children with B-Thalassemia Disease

Alisa Limsuwan, Division of Pediatric Cardiology, Department of Pediatrics, Ramathibodi Hospital, Mahidol University, Thailand Darin Tubtom, Division of Pediatric Cardiology, Department of Pediatrics, Ramathibodi Hospital, Mahidol University, Thailand Samart Pakakasama, Department of Pediatrics, Ramathibodi Hospital, Mahidol University, Thailand

Ampiwan Jaunsumrit, Department of Pediatrics, Ramathibodi Hospital, Mahidol University, Thailand

B-thalassemia patients may be predisposed to premature atherosclerosis due to vascular dysfunction with increased arterial stiffness and endothelial deregulation. This assumption has been observed largely in adult with $\beta$-thalassemia major. Whether atherosclerosis changes occur early in childhood of $B$-thalassemia disease (BTD) patients is needed to be defined in order to prevent this cardiovascular complication in the young. Moreover, it remains uncertain if curative treatment with bone marrow transplantation (BMT) actually improves this vascular alteration. Methods: Thirty-seven $B$-thalassemia children, with an average age of $10.1 \pm 2.7$ years, classified into: group 1 comprises 25 children with BTD currently managed with conventionally medical treatment and group 2 is BTD children who underwent BMT $(n=12)$. Twenty nine age-matched healthy children were studied simultaneously and served as controls. The carotid stiffness index and intima-media thickness (IMT) was measured. Result: Conventionally treated BTD children (group 1) had a greater arterial stiffness index compare with the controls $(4.57 \pm 1.78$ VS $2.87 \pm 1.07, \mathrm{p}<0.001)$. While the carotid IMT was significantly greater in both groups of BTD children than controls (group 1: $0.45 \pm 0.03 \mathrm{~mm}$ VS $0.34 \pm 0.04 \mathrm{~mm}$, $\mathrm{p}<0.001$ and group 2: $0.43 \pm 0.03$ VS $0.34 \pm 0.04, \mathrm{p}<0.001)$. 
Conclusion: Carotid IMT and arterial stiffness is increased in conventionally treated B-thalassemia children, suggesting an early atherosclerotic change in these children. While BTD children underwent BMT had an increased carotid IMT but normal arterial stiffness. Whether BMT treatment alters the vascular complication is needed to be uncovered through future long term cohort study.

\section{9}

\section{Outpatients' cardiac screening in a tertiary referral} hospital in Eritrea

Ornella Milanesi, Department of Paediatrics, University of Padova, Italy Tsegereda Gebrehiwot, Orota Paediatric Hospital of Asmara, Eritrea Paola Cogo, Department of Paediatrics, University of Padova, Italy Elena Reffo, Department of Pediatrics, University of Padua, Italy Nicola Maschietto, Department of Pediatrics, University of Padua, Italy Raffaele Bonato, Department of Pharmacology and Anaesthesiology, University of Padua,, Italy

Giovanni Stellin, Department of Cardiac, Thoracic and Vascular Science, University of Padua, Italy

Andreas Urban, Hammer Forum e V, Director of the Project, Germany

A humanitarian collaboration between the Paediatric Cardiac and Cardiac Surgical Units of our University and the Paediatric Cardiac Unit of the Orota Hospital in Asmara has been established since 2004. In the last 3 years, an electronic database of the evaluated patients has been created. This paper aims at describing the typology of patient referring to the cardiac outpatients' clinic in Asmara and outlining guidelines for the optimization of the economical resources. A total of 561 children were screened, in 3 weeks, one per year. Simple cardiac lesions (ASD, VSD, PDA, PS, AoS, simple ToF) were present in 199 of them $(35,2 \%)$, chronic rheumatic disease (CRD) in $97(17,2 \%)$, complex CHD (single ventricle, asplenia syndrome, common arterial trunk) in $45(8 \%)$, other medical lesions (cardiomyopathies, MV prolapse, arrhythmia) in $37(6,6 \%)$. One hundred forty-nine cases $(24,7 \%)$ had a normal heart, $44(7,8 \%)$ were followed postoperatively. Among the 199 simple lesions, 81 (PDA, AoS or PS) (41\%) could be treated also by interventional cardiology, at an estimated lower cost than surgery. Moreover, in most of the 29 ASD patients a percutaneous closure was feasible, at a cost comparable to surgery. In conclusion, in a very low income country such Eritrea, patients with complex CHD are a minority of those referring to the tertiary hospital, surviving the first months of life. Among simple lesions, completely curable, at least $40 \%$ can be treated in the catheterization laboratory. Rheumatic disease is a sanitary emergency in Eritrea and its prophylaxis deserves resources allocation.

\section{0 \\ An Evaluation of Pediatric Cardiology Outpatient Clinics in A tertiary Level Hospital In Kuwait}

Esmail M Redha, Ministry Of Health, Mubarak Al-Kabeer Hospital, Kuwait

Objective: To examine an outpatient department (OPD) in a hospital setting where patients are health care customers who draw conclusions about service quality, which are influenced by their service experiences.

Method: We measured the level of satisfaction of patients for the health service quality in a pediatric OPD clinics in a tertiary level hospital over a period of 6 weeks. 245 parents, either father or mother, received self-administered questionnaire of 14 questions. At least one interviewer was present ready for any clarification.
Results: Mean age of the patients was $1.9 \pm 0.4$ years, ratio of male: female patients are about 1:1. The mother accompanied patient for most of clinic visits (77.1\%). Medical team (doctors, nurses, secretaries and pharmacists) attitude towards patients was the most important related factor with their evaluation of pediatric OPD reaching $p$-value $<0.0001$. Other factors, including provision of information about the visit, illness \& treatment had significant impact on patients' evaluation of health service. Protection of privacy had similar significance on the outcome of patients' satisfaction. Car parking near the hospital and pediatric OPD and information on medication and their effects had great influence on results (p-values of 0.013 and 0.022 respectively). Unexpectedly, availability of the treatment had no effect on the overall evaluation $(p=0.180)$.

Conclusion: Patient's satisfaction with a health service is the main goal of product design in the field of quality of health service. The provision of better health service quality in pediatric OPD is an area where much improvement can be made with minimal effort.

\section{1}

International Quality Improvement Collaborative for Congenital Heart Surgery in the Developing World Melyssa S Sueiro, Children's Hospital Boston, United States Jan T Christenson, University Hospital of Geneva, Switzerland William M Novick, University of Tennessee, United States Aldo $R$ Castaneda, Unidad de Cirugia Cardiovascular de Guatemala, Guatemala

Jean A Connor, United States

Kimberlee Gauvreau, Children's Hospital Boston, United States

Ravi Agarwal, Frontier Lifeline Hospital, India

Frank Molloy, Internacional Children's Herat Foundation, United States

Juan Velez, Clinica Medellin, Colombia

Iftikhar Ahmed, Armed Forces Institute of Cardiology/National

Institute for Heart Diseases, Pakistan

Sunil Gunness, Cardiac Centre, Mauritius

Zhaohui Lu, Shanghai Children's Medical Center, China

Ivan Vides, Cardiovascular Unit (UNICAR) Hospital in Guatemala, Guatemala

Vitaly Dedovich, Childrens Cardiac Centre, Hospital No 1, Belarus

Kathy J Jenkins, Children's Hospital Boston, United States

Background: Partnerships between established and developing programs have accelerated availability of congenital heart surgery globally. The need for benchmarking data and the opportunity for collaborative quality improvement was identified at the Global Forum for Humanitarian Medicine in Cardiology and Cardiac Surgery, Geneva 2007. Seven international sites participated in the pilot phase. Representatives traveled to Boston in June 2008 to attend the first International Quality Improvement Collaborative for Congenital Heart Surgery meeting. Consensus was established on clinical, procedural and outcome data to be collected.

Methods: Sites submit data using a web-based data tool to a central data repository. Diagnostic and procedural information are entered using International Pediatric Congenital Cardiac Codes, including occurrence of in-hospital or 30-day death, and surgical site or blood stream infection. Institutions will receive biannual reports with individual and comparative data, including riskadjusted mortality rates using the RACHS-1 method.

Results: Data collected $(\mathrm{n}=410$ cases thus far) from September 2008 to March 2009 will be included in the first report. Initial findings will be evaluated at the next meeting in Geneva, June 
2009. Sites will also work together to develop and implement improvement strategies, based on the initial results, and new institutions will be invited to participate.

Conclusion: A collaborative approach to quality improvement devoted to developing programs should accelerate the spread of best practices to children with congenital heart disease globally.

\section{2}

The Spectrum of Congenital Heart Disease in Uganda: Natural and Unnatural History

Stephanie Lacey, University of Florida/Wolfson Children's Hospital, United States

Peter Lwabi, Uganda Heart Institute/Mulago Hospital, Uganda

Keith Kocis, University of North Carolina, United States

Craig Sable, Children's National Medical Center, United States

Background: Limited access to treatment and genetic and environmental factors impact the spectrum of pediatric heart disease in sub-Saharan Africa

Objective: Characterize children with heart disease in Uganda.

Methods: Seven medical missions were carried out to Kampala, Uganda (Mulago Hospital) over a 5 year period to diagnose and facilitate treatment of children with heart defects and build a sustainable cardiac surgery program. Diagnoses (echocardiography), age, pulse oximetry, and outcomes were tracked during the 3 most recent missions.

Results: 309 patients were evaluated. Diagnoses included ASD $(\mathrm{n}=33)$, VSD $(\mathrm{n}=46)$, PDA $(\mathrm{n}=21)$, pulmonary stenosis $(n=17)$, TOF $(n=58)$, subaortic stenosis $(n=5)$, atrioventricular septal defect $(n=8)$, truncus arteriosus $(n=11)$, double outlet right ventricle $(\mathrm{n}=11)$, TGA $(\mathrm{n}=10)$, pulmonary atresia/ $\operatorname{VSD}(n=5)$, tricuspid atresia $(n=6)$, hypoplastic left heart $(\mathrm{n}=3)$, TAPVR $(\mathrm{n}=3)$, rheumatic disease $(\mathrm{n}=5)$, and cardiomyopathy $(\mathrm{n}=5) .33$ patients had no significant disease. Mean age and pulse oximetry (unrepaired) of the most common defects were: ASD (11 years, 96\%), VSD (3.5 years, 95\%), and TOF (6.4 years, 82\%). 92 patients underwent surgery or catheterization in North America $(n=45)$, Europe $(n=3)$, India $(n=12)$, South Africa $(n=2)$, and Uganda $(n=30 ; 17$ open cases). The most common repairs were ASD $(n=22)$, VSD $(\mathrm{n}=16)$, TOF $(\mathrm{n}=25)$, PDA $(\mathrm{n}=12)$, and pulmonary stenosis $(n=7)$. There were 3 post-operative deaths (none in Uganda). 12 children who did not undergo repair died.

Conclusions: There is a preponderance of TOF among Ugandan children referred for cardiac evaluation; other conotruncal anomalies are also highly represented. Effective intervention can be provided for a significant subset of patients.

\section{3}

Morbidity and psychomotor development after neonatal cardiac surgery

Cornelia Wörner, Heart Center Leipzig, Department of Pediatric

Cardiology, Germany

Nadine Wolf, Heart Center Leipzig, Department of Pediatric

Cardiology, Germany

Jan Janousek, Heart Center Leipzig, Germany

Martin Kostelka, Heart Center Leipzig, Department of Pediatric Cardiac Surgery, Germany

Introduction: Given the low surgical mortality after contemporary neonatal cardiac surgery (Heart Center Leipzig, 1998-2006, $\mathrm{N}=439,30$ days mortality $=5,2 \%)$ this study focused on morbidity, psychomotor development, social aspects, and quality of life.
Methods: Pilot study. Structured questionnaires were separately sent to parents and pediatricians of 59 consecutive neonates (d-transposition of great arteries $=15$, coarctation of the aorta $=14$, hypoplastic left heart syndrome $=10$, tetralogy of Fallot $=5$, pulmonary atresia with ventricular septal defect $=4$, and other $=11)$ after corrective $(\mathrm{N}=42)$ or palliative $(\mathrm{N}=17)$ cardiac surgery operated over a period of 1 year (median age at surgery $=6$ days, median Aristotle complexity score $=9.0$ ) . Postoperative data were retrieved from medical records.

Results: The median hospital stay was 28 days (8-169), including median of $12 \mathrm{~d}$ on ICU and 4.1 ventilation days. Secondary chest closure was performed in 16/59 (27.1\%), postoperative ECMO was necessary in $2 / 59$ (3.4\%) and non-obligatory surgical/catheter re-interventions (those given by staged surgical strategy excluded) in $23 / 59(38,9 \%)$ of patients. One early $(1.7 \%)$ and 4 late $(6.8 \%)$ death occured. Completed questionnaires could be obtained from 31/64 survivors at a median age of 2.3 years. More than $80 \%$ of children had an overall normal development, $65 \%$ parents stated a normal exercise tolerance, $86 \%$ normal speech comprehension, $48 \%$ normal active speech, $17,4 \%$ mild behavioural disorders, and $21,7 \%$ concentration difficulties.

Conclusion: Despite high complexity and postoperattive morbidity neonatal cardiac surgery carries normal psychomotor development in the majority and acceptable extent of negative social impact on the children's families.

\section{4}

Serial Measurements of Exercise Performance in Pediatric Heart Transplant Recipients

Joanne P Yeung, Division of Cardiology, Department of Pediatrics, British Columbia Children's Hospital and The University of British Columbia, Canada

James E Potts, Division of Cardiology, Department of Pediatrics, British Columbia Children's Hospital and The University of British Columbia, Canada

George GS Sandor, Division of Cardiology, Department of Pediatrics, British Columbia Children's Hospital and The University of British Columbia, Canada

Derek G Human, Division of Cardiology, Department of Pediatrics, British Columbia Children's Hospital and The University of British Columbia, Canada

Introduction: Heart transplantation has become an increasingly acceptable therapeutic option for pediatric patients with end-stage heart disease. With advances in surgical technique, immunosuppressive therapy and follow-up care, long-term survival and health-related outcomes need to be assessed. We report the results of serial exercise testing performed over a 5-year period using stress echocardiography in a cohort of pediatric heart transplant patients (HTP) that we follow.

Methods: HTP $(\mathrm{n}=7)$ exercised on a semi-recumbent cycle ergometer with the workload increased in 20-40 watt increments every 3 minutes until volitional fatigue. EchocardiographyDoppler measurements, heart rate and blood pressure were taken during each stage. Results were compared with healthy controls $(\mathrm{CON}, \mathrm{n}=12)$. Median values over the 5 -year period are reported.

Results: Patient demographics including age, height, and weight were similar between HTP and CON. The resting left ventricular end-diastolic (3.6 vs $4.4 \mathrm{~cm}, \mathrm{p}<0.0001)$ and endsystolic (2.1 vs $2.7 \mathrm{~cm}, \mathrm{p}<0.0001)$ dimensions and the aortic cross-sectional area $\left(2.5\right.$ vs $\left.3.7 \mathrm{~cm}^{2}\right)$ were smaller in HTP. The stroke volume index ( 36 vs $\left.49 \mathrm{~mL} / \mathrm{m}^{2}, \mathrm{p}<0.003\right)$, cardiac index 
(5.55 vs $\left.9.03 \mathrm{~L} / \mathrm{min} / \mathrm{m}^{2}, \mathrm{p}<0.0001\right)$, and heart rate (169 vs $185 \mathrm{bpm}, \mathrm{p}>0.05)$ were lower in HTP at peak exercise. Although measures of contractility were similar, ejection force $\left(25.5\right.$ vs $\left.55.3 \mathrm{cmg} / \mathrm{s}^{2}, \mathrm{p}<0.009\right)$ and work $(984 \mathrm{vs} 1219 \mathrm{~J} / \mathrm{kg}$, $\mathrm{p}<0.008)$ were lower in HTP at peak exercise.

Conclusions: Exercise tolerance is reduced in heart transplant patients. Both central and peripheral limitations may be responsible. Over time, hemodynamics and ventricular function have remained relatively constant in our cohort.

\section{5}

Outcomes of pediatric heart transplantation for congenital versus acquired heart disease

Stacey Pollock-BarZiv, Hospital for Sick Children, Canada

Cedric Manlhiot, Hospital for Sick Children, Canada

Brian W McCrindle, Canada

Anne I Dipchand, Hospital for Sick Children, Canada

Background: The objectives of this study were to ascertain perioperative and post-heart transplant ( $\mathrm{HTx}$ ) outcomes for congenital vs acquired heart disease.

Methods: Review of HTx patients (1989-Dec 2007). Data collected included demographics, pre-HTx medical characteristics (surgical history, HLA senzitization, days wait-listed), and perioperative and post-HTx outcomes including ICU time, ECMO use and survival.

Results: 181 patients underwent HTx, of whom 118 had CHD (43 hypoplastic left heart syndrome; and 63 acquired heart disease (AHD)(30 male, 48\%; 2 LV fibromas, and 61 CM: 11 restrictive CM, 48 dilated CM; 2 Anthracycline CM). Of the CHD patients, $64(54 \%)$ had prior cardiac surgeries: failed univentricular palliation $(n=35)$; failed biventricular repair $(n=28)$. Mean follow-up time of the groups was similar: 3.8 yrs (CHD) vs 3.5 yrs (AHD). Kaplan Meier survival outcomes were better for AHD vs CHD at 1 yr post-Htx: $88 \%$ vs $80 \% ; 3$ yrs: $86 \%$ vs $74.5 \%$; 5 yrs: $73 \%$ vs $68.5 \%$.

CHD patients were more likely to be HLA sensitized (13\% vs $6 \%, \mathrm{p}<0.01)$, be younger at HTx (4.8 yrs vs 7.3 yrs, $\mathrm{p}<0.05)$, have longer mean days wait-listed (105 vs $64, \mathrm{p}<0.01)$, use ECMO post-HTx (17 vs $5, \mathrm{p}<0.01)$, and have greater mortality $(37$ vs $13, \mathrm{p}<0.05)$ than AHD patients.

Conclusions: Patients with CHD have higher mortality after HTx, compared to those with AHD. The increased risk of allosensitization and prior cardiac surgeries likely contributes to the increased risk of death in patients with CHD.

\section{8}

Cardiorespiratory Function of Pediatric Heart Transplantation Recipients in Early Postoperative Period

Hsin-Hui Chiu, Taiwan

Ssu-Yuan Chen, National Taiwan University Hospital and National Taiwan University College of Medicine, Taiwan

Shoei-Shen Wang, National Taiwan University Hospital and National Taiwan University College of Medicine, Taiwan

Ching Lan, National Taiwan University Hospital and National Taiwan University College of Medicine, Taiwan

Nai-Kuan Chou, National Taiwan University Hospital and National Taiwan University College of Medicine, Taiwan

Jin-Shin Lai, National Taiwan University Hospital and National Taiwan University College of Medicine, Taiwan

Mei-Hwan Wu, National Taiwan University Hospital and National Taiwan University College of Medicine, Taiwan
Objective: In adults after successful heart transplant, the cardiorespiratory function is still suboptimal. Such data in pediatric recipients are still scarce.

Methods: From April 2007 to November 2008, six children (five male and one female), four with dilated cardiomyopathy and two with complex heart disease, underwent orthotropic heart transplantation $(\mathrm{OHT})$ at the age of $14 \pm 3.1$ years. Grade exercise testing with breath-by-breath gas analysis was performed using an incremental cycling at $31.5 \pm 7.3$ days after surgery.

Results: Pediatric recipients had significant impairment in workload (58 \pm 31 watts), oxygen consumption (18.4 \pm $3.5 \mathrm{~mL} / \mathrm{Kg} / \mathrm{min})$, and oxygen pulse $(7.6 \pm 2.4 \mathrm{~mL} /$ beat $)$ at peak exercise one month after OHT. Typical heart rate responses of a denervated heart with the manifestation of higher resting heart rate $(96 \pm 10$ beats $/ \mathrm{min})$, lower peak heart rate $(119 \pm 22$ beats/min) during exercise, and continuous heart rate increase toward 1 to 2 minutes after cessation of exercise were also demonstrated.

Conclusions: Pediatric OHT recipients have a low cardiopulmonary endurance during the early postoperative period. Such early cardiorespiratory response to exercise was also observed in adult recipients. Therefore, an early multidisciplinary cardiac rehabilitation program given in adults should also be incorporated into the postoperative follow-up of pediatric recipients to enhance the physical capacity and improve the quality of life.

\section{9}

Factors Affecting Waitlist Times In Pediatric Patients

Anne I Dipchand, Hospital for Sick Children, Canada

Cedric Manlhiot, Hospital for Sick Children, Canada

Paul F Kantor, Hospital for Sick Children, Canada

Seema Mital, Hospital for Sick Children, Canada

Brian W McCrindle, Canada

Purpose: To profile the waitlist time and mortality in pediatric patients, and to assess risk factors for longer waiting times and for waitlist mortality.

Institutional review of patients listed for HTx (1990-2008). Competing risk analysis to model survival to HTx, death on waitlist, delisting, or improvement. Associated factors sought in multivariable parametric survival models.

308 listings in 280 patients ( 2 in 18; 3 in 5 patients). Median age at listing was $1.6 y(0-17.9 y)$. Competing risk analysis: 1 month after listing 47\% remained listed (transplanted at 1 month: 33\%; deceased/delisted at 1 month: 15\%); 5 months later 11\% remained listed (transplanted at 6 months: 62\%; deceased/delisted at 6 months: 19\%). Younger patients had the highest waitlist mortality: $\mathrm{n}=72$ age $0-1$ month $(54 \%$ transplanted at 6 months; $28 \%$ deceased/delisted at 6 months), $\mathrm{n}=63$ age 1-12 months $(67 \% ; 20 \%), \mathrm{n}=55$ age $1-5$ years $(53 \% ; 25 \%)$ and $\mathrm{n}=128$ age $>5$ years $(69 \% ; 10 \%)$. Diagnosis was associated with differing waitlist mortality: congenital heart disease, $\mathrm{n}=179$ (53\%; 22\%); cardiomyopathy, $\mathrm{n}=107$ (73\%; 15\%); allograft vasculopathy, $\mathrm{n}=12(58 \% ; 8 \%)$; primary graft failure, $\mathrm{n}=10(70 \% ; 30 \%)$. Blood group: $\mathrm{O}, \mathrm{n}=149$ (52\%;19\%); $\mathrm{A}, \mathrm{n}=105$ (77\%; 14\%); $\mathrm{B}$, $\mathrm{n}=41(63 \% ; 27 \%) ; \mathrm{AB}, \mathrm{n}=13(46 \% ; 38 \%) .38$ were on $\mathrm{a}$ $\operatorname{VAD}(24 \mathrm{ECMO}, 14$ Berlin), waitlist mortality of $38 \%$ on ECMO; $14 \%$ on Berlin Heart.

Blood groups $\mathrm{O} / \mathrm{B}$, younger patients and patients with congenital heart disease waited the longest. Higher status patients and those on mechanical support had the shortest wait times but the highest mortality. Berlin heart reduced mortality by more than half. These need to be considered when listing for HTx. 


\section{0}

24-hours ambulatory blood pressure monitoring and heart rate variability in pediatric heart transplant recipients

Ugo Giordano, Cardiorespiratory and Sports Med. Unit - Bambino Gesù Children's Hospital - Rome, Italy

Giorgia Grutter, Cardiology and Cardiac Surgery Dept - Bambino Gesù Children's Hospital - Rome, Italy

Roberta Meta, Cardiorespiratory and Sports Med. Unit - Bambino Gesù Children's Hospital - Rome, Italy

Francesca Calò Carducci, Cardiology and Cardiac Surgery

Dept - Bambino Gesù Children's Hospital - Rome, Italy

Armando Calzolari, Cardiorespiratory and Sports Med.

Unit - Bambino Gesù Children's Hospital - Rome, Italy

Francesco Parisi, Cardiology and Cardiac Surgery Dept - Bambino

Gesù Children's Hospital - Rome, Italy

Hypertension is a significant complication after orthotopic heart transplant (OHT); as a sign of diminished parasympathetic activity also reduced heart rate variability (HRV) is depressed. Aim of the study was to investigate correlations between Blood Pressure (BP) and HRV during 24 hours BP/ECG simultaneous recording (Cardio(x)plore device-Meditech). Cardiovascular risk as a degree of HRV depression was considered.Twenty OHT patients (P) (7 F, 13 M-18,7 $\pm 5,2$ yrs) were evaluated. Mean systolic and diastolic BP (SBP, DBP) (24 hours, daytime and nighttime) and total spectral power of HRV were examined; patients were also considered for cardiovascular risk (low, medium or high, respectively SDNNA $>100$, SDNNA between 50 and 100 , SDNNA $<50$ ). Six $\mathrm{P}$ were treated for hypertension and $3 / 20$ were affected by hypertension before transplantation. Hypertensive $\mathrm{P}$ showed higher cyclosporine blood levels while no significant correlations were found between BP values and HRV. When considered for SDNNA, 7/20 (35\%) were at high risk, 11/20 at medium risk $(55 \%)$ and only 5 patients $(10 \%)$ were at low risk. ABPM showed 4 P (20\%) with mean BP values higher than 95th during the day, $7 \mathrm{P}(35 \%)$ had reduced nocturnal fall in SBP and $2 \mathrm{P}$ had a nocturnal rise in BP. Abnormalities of BP and evidence of a depressed HRV were frequent in our population. The well-tolerated simultaneous 24-hours BP/ECG recording was helpful to obtain BP and HRV profiles during the same daily-activities. Antihypertensive treatment was effective in terms of class of drug choice, time and dose of administration.

\section{2}

Cardiac transplantation in patients under 16 year of age - single center experience

Katarzyna Januszewska, Klinikum Grosshadern, Ludwig Maximilians University, Munich, Germany

Edward Malec, Department of Cardiac Surgery, Klinikum Grosshadern, Ludwig Maximilians University, Munich, Germany

Julia Birnbaum, Department of Pediatric Cardiology, Klinikum

Grosshadern, Ludwig Maximilians University, Munich, Germany Alexandra Fuchs, Department of Pediatric Cardiology, Klinikum Grosshadern, Ludwig Maximilians University, Munich, Germany Robert Dalla-Poza, Department of Pediatric Cardiology, Klinikum Grosshadern, Ludwig Maximilians University, Munich, Germany Christoph Schmitz, Department of Cardiac Surgery, Klinikum Grosshadern, Ludwig Maximilians University, Munich, Germany Ralf Sodian, Department of Cardiac Surgery, Klinikum Grosshadern, Ludwig Maximilians University, Munich, Germany Heinrich Netz, Department of Pediatric Cardiology, Klinikum Grosshadern, Ludwig Maximilians University, Munich, Germany
Objective: Cardiac transplantation is nowadays an established last option for treatment of complex congenital hearts defects and cardiomyopathy in end-stage heart failure. The aim of the study was to present single center experience with heart transplantation in patients under 16 year of age.

Methods: Between March 1992 and September 2008, 72 patients aged from 5 days to 15.9 years (mean, 6.6 $+/-5.8$ years) underwent orthotopic heart transplantation in our center. The preoperative diagnosis was cardiomiopathy in $49(68.1 \%)$ and congenital heart defect in $23(31.9 \%)$ children. $16(22.2 \%)$ patients had history of previous cardiac surgery. 9 children required ventricular assisted device as a bridge to cardiac transplantation. Demographic, clinical perioperative and midterm data were retrospectively analyzed.

Results: The mean follow-up was $6.6+/-4.6$ years (range, 3 months to 16.8 years). The mean waiting for transplant time was $65.5+/-95.7$ days. Early (30-days) and late postoperative mortality was $5.6 \%(\mathrm{~N}=4)$ and $10.3 \%(\mathrm{~N}=7)$, respectively. Actuarial 1, 5 and 10 year survival was $89 \%, 89 \%$ and $87.5 \%$. The mean ventilatory support time was $6.1+/-7.3$ days, and the mean hospital stay time was $45.4+/-20.4$ days. The diagnosis of congenital heart defect had significantly higher incidence among non-survivals in comparison with survivals $(p=0.036)$. Conclusions: Heart transplantation in patients below 16 year of age has very good midterm results. The diagnosis of congenital heart defect is a risk factor for death after transplantation in this group of age.

\section{3}

Cardiac and non cardiac operations after pediatric heart transplantation

Marcelo B Jatene, Heart Institute - University of São Paulo - São Paulo - SP, Brazil

Estela Azeka, Heart Institute - University of São Paulo - São PauloSP, Brazil

Rafael Tineli, Heart Institute - University of São Paulo - São Paulo SP, Brazil

Arlindo Riso, Heart Institute - University of São Paulo - São Paulo SP, Brazil

Carla Tanamati, Heart Institute - University of São Paulo - São Paulo - SP, Brazil

Anderson Dietrich, Heart Institute - University of São Paulo -

São Paulo - SP, Brazil

Antonio Augusto Lopes, Heart Institute - University of São Paulo São Paulo - SP, Brazil

Miguel Barbero Marcial, Heart Institute - University of São Paulo São Paulo - SP, Brazil

Objective: The most prevalent complications after pediatric heart transplantation (PTx) are infecction, rejection, coronary disease and linphatic tumors. Cardiac and non cardiac operations prevalence after PTx are unknown. The objective of this study is to present our institution experience with these operations.

Methods: Between 1992 e 2008, 71 children (2,87 \pm 2 2,2years) were transplanted, being myocardiopathies $(76 \%)$ the most commom indication. Actuarial survival was $89 \%$, 73\% e 57\%, respectively with 1,5 e 10 years post PTx. Cardiac operations after PTx were retransplantation, myocardial revascularization, coronary stent implantation, ascending aorta aneurysm correction, ECMO and PM implantation and bleeding review. Non cardiac operations were colecistectomy, lung biopsies, linphonode biopsies, lung tumors excision, traqueostomy, chest drainage, diafragmatic plicature, gastrostomy and abdominal tumor excision. 
Results: Sixteen children (22,5\%) were submitted to 31 interventions $(2,0 \pm 1,1$ /paciente). The most prevalent cardiac operations were bleeding review in 4 , retransplantation in 3 ; ECMO support in 3 and coronary stent implantation in 2 . The non cardiac operations were amigdalectomy and lung biopsy in 3, pericardial drainage and colecistectomy in 2 . The mortality was $7.1 \%$, being retransplantation the major cause. Complications related to immunossupressive therapy(coronary disease and linphoproliferative diseases) were $26.6 \%$ of interventions. Conclusion: Cardiac and non cardiac operations after PTx showed low morbidity and mortality, being the complications directly related to immunossupressive therapy the most prevalent.

\section{4}

\section{Transcoronary Bone Marrow-Derived Mononuclear} Progenitor Cells

Alisa Limsuwan, Division of Pediatric Cardiology, Ramathibodi

Hospital, Mahidol University, Thailand

Suradej Hongeng, Department of Pediatrics, Ramathibodi Hospital, Mahidol University, Thailand

Pongsak Khowsathit, Division of Pediatric Cardiology, Ramathibodi Hospital, Mahidol University, Thailand

Pavit Pienvichit, Department of Internal Medicine, Ramathibodi

Hospital, Mahidol University, Thailand

Rattanaporn Pornkul, Department of Radiology, Ramathibodi Hospital, Mahidol University, Thailand

Suvipaporn Siripornpitak, Department of Radiology, Ramathibodi Hospital, Mahidol University, Thailand

Somchai Sangkitporn, Clinical Reserch Center, Ministry of Public Health, Thailand

Recent advance in stem cell therapy to restore the cardiac function has a great promise for patients with congestive heart failure after myocardial infarction in adult population.

Objective: Whether pediatric patients would be feasible and benefit from this bone marrow-derived progenitor cells treatment modality is needed to clarify.

Method: After standard therapy for congestive heart failure for years, we treated 3 consecutive children ( 2 girls) with dilated cardiomyopathy (age 10, 18 and 13 years) by intracoronary transplantation of autologous bone marrow mononuclear cells. The progenitor cell suspension was injected via a transcoronary catheter into dominant coronary without any complication.

Result: Three month after stem cell therapy, the patients' cardiac function assessed by both cardiac magnetic resonance (MRI) and echocardiogram has been improved compare to the baseline. The MRI showed left ventricular ejection fraction of 35,12 , and $31 \%$ versus the baseline of 19,5 , and $16 \%$. The patient's functional class (NYHC) and well being was also improved.

Conclusion: This is the first pediatric experience of successful transcoronary injection of the bone marrow-derived progenitor cell for children with impaired cardiac function. The initial result seems to be effective under clinical condition. The procedure is considered safe and feasible in pediatric population.

\section{5}

Five years after ABO Incompatibility Pediatric Heart Transplantation - Cruising the barrier succesfully Gerardo Naiman, Hospital Nacional de Pediatria, Argentina Horacio Vogelfang, Hospital Nacional de Pediatria, Argentina Luis Quiroga, Hospital Nacional de Pediatria, Argentina Alejandra Villa, Hospital Nacional de Pediatria, Argentina Marita De La Riba, Hospital Nacional de Pediatria, Argentina
Estela Carmona, Hospital Nacional de Pediatria, Argentina Gustavo Burlli, Hospital Nacional de Pediatria, Argentina Ana Del Pozo, Hospital Nacional de Pediatria, Argentina

Materials: A Female patient, 16 months old, $7.8 \mathrm{Kg}$ weight, blood group B (-), was admitted at the cardiovascular ICU in December 2003, with a dilated cardiomiopathy (post-viral). After 25 days on waiting list, she was supported with assisted mechanical ventilation, i.v. inotropic drugs and peritoneal dialysis, she underwent transplantation with an $\mathrm{AB}(+)$ organ. The female donor was 17 months old and weighted $12 \mathrm{Kg}$.

Methods: West Protocol for ABO incompatible donors was slightly modified according to our patient who had high levels isohaemagglutinin. Standard surgery technique was used, with cava-cava anastomosis. By-pass 28 C. Aprotinin and hemofiltration.

During cardiopulmonary by-pass, three times the total blood was exchanged, oxigenator were changed too. Plasma AB and red cells B (+) were used for replacement. Aprotinin and hemofiltration was used.

Results: The immediate course was uneventful. Dopamine and Milrinone was used. Extubated four days later. Gal, azathioprine, cyclosporine and steroids were used. She was discharged 19 days after transplantation. Haemagglutinins titers were 1 to $1 / 4$.

Follow up: 5 years follow up show hemodinamically good condition, without steroid requirements. Haemaglutinins titers remain similar. NYHA: Class 1. DDLV, SDLV were normal. Cath: normal coronary arteries, and no rejection signs in biopsy. Now she received Mycophenolate and low doses cyclosporine. Conclusion: Hearts donors is a problem worldwide.

This patient's evolution supports the idea that $\mathrm{ABO}$ incompatible heart transplantation may be performed safely in some children beyond the West protocol age.

This technique thus contributes to reduce mortality among infants on the heart waiting list.

\section{6}

Heart Transplantation for Systemic Ventricular Failure following Atrial switch Operation for TGA

Amit Pawale, Freeman Hospital, Newcastle Upon tyne, NE7 7DN, United Kingdom

Milind Chaudhary, United Kingdom

Gareth Parry, United Kingdom

Neil Wrightson, United Kingdom

Leslie Hamilton, United Kingdom

Massimo Griselli, United Kingdom

John H Dark, United Kingdom

Asif Hasan, Freeman Hospital, Newcastle Upon Tyne,UK, United Kingdom

Background: Systemic ventricular failure is a well documented long term consequence of atrial switch procedures (ASP). Heart transplantation remains the only available treatment.

Objective: To evaluate outcome of heart transplantation for systemic ventricular failure late after atrial switch procedure.

Materials and methods: Retrospective analysis of patients undergoing orthotopic heart transplantation following previous atrial switch procedure. During the period 1985-2008 we performed orthotopic heart transplants in 824 patients. 13 patients had a primary diagnosis of Transposition of the Great Arteries (TGA) of whom 8 had prior Mustard and 5 had prior Senning procedures.

Results: In these 13 patients there were 3 deaths within the first 30 days after transplant (donor organ failure, stroke, and acute 
rejection), 2 deaths later in the first year (acute rejection, lymphoma) and one further death at nearly seven years posttransplant (graft coronary artery disease). Actuarial survival (compared with other adult recipients) was $76.0 \%(86.8 \%)$ at 30 days, $56.5 \%(80.7 \%)$ at one year and $56.5 \%(71.8 \%)$ at 5 years. Conclusion: While heart transplantation for failed atrial switch carries a relatively high early post-operative mortality, mid to long term results are good.However, technical challenges remain and there is still room for improvement.

\section{7}

Transplant coronary artery disease contributes to late mortality in patients requiring low dose intropic support for acute rejection

Christina M Phelps, The Children's Hospital of Denver, United States Cecile Tissot, The Children's Hospital of Denver, United States

Shelley D Miyamoto, The Children's Hospital of Denver, United States

Acute graft rejection in pediatric heart transplant patients is a cause of significant mortality. A retrospective review of the heart transplant database at our institution from 6/96 until 2/07 was conducted with IRB approval. All patients who required inotropic support during an episode of acute graft rejection were identified and included for analysis. The incidence of transplant coronary artery disease (TCAD) before and after hemodynamically significant rejection was examined.

Fifty-three patients had 70 rejection episodes requiring intravenous inotropic support. Low dose support, defined as $\leqslant 2$ inotropes [milrinone $(\leqslant 0.5 \mathrm{mcg} / \mathrm{kg} / \mathrm{min})$, dopamine or dobutamine at $<10 \mathrm{mcg} / \mathrm{kg} / \mathrm{min}$ ] was required in 32 patients. Survival to hospital discharge was $41 \%$ in the high dose group (HDG) compared to $94 \%$ in the low dose group (LDG). There was no difference in graft age or survival to discharge for those with and without TCAD at presentation with acute rejection. At 6 months, no additional patients in the HDG died compared to continued graft loss ( $47 \%$ graft survival) in the LDG.

Of the 9 patients in the LDG who died suddenly at home, 8 had documented TCAD. New TCAD developed in 5, while 2 had stable TCAD and 1 had progression of TCAD since the rejection episode. In contrast, only one patient in the HDG developed new TCAD or had progression of disease following treatment for rejection. Patients with TCAD have a high late mortality risk following an episode of hemodynamically significant rejection even if only low dose inotropes are required.

\section{8}

Neurodevelopmental Outcomes in Pediatric Cardiac Transplantation

Luis G Quinonez, University of Alberta, Stollery Children's Hopital, Canada

Ari R Joffe, University of Alberta, Stollery Children's Hopital, Canada Charlene MT Robertson, University of Alberta, Stollery Children's

Hopital, Canada

Gwen Y Alton, University of Alberta, Stollery Children's Hopital, Canada

James Y Coe, University of Alberta, Stollery Children's Hopital, Canada

David B Ross, University of Alberta, Stollery Children's Hopital, Canada

Ivan M Rebeyka, University of Alberta, Stollery Children's Hopital, Canada

Objective: To determine neurodevelopmental outcomes and their predictors in children after cardiac transplantation.
Methods: Children <6yrs having cardiac transplant between 2000-2008 had demographic, preoperative recipient and donor, operative, and postoperative variables recorded. The association of variables with outcomes $>6$ mos post-transplant were determined by univariate and multiple regression analyses.

Results: Thirty-seven children (25 [65\%] male) had transplantation at age 268 [IQR 82-820] days, with 4 (11\%) deaths. Twenty-nine $(88 \%)$ of survivors had neuro-developmental assessment $19 \pm 8 \mathrm{mos}$ after transplantation (age $32 \pm 13 \mathrm{mos}$ ). Between the congenital heart disease (CHD)(15) and non$\mathrm{CHD}(14)$ groups there was no difference in pre-transplant days ventilated, highest creatinine, lactate, inotrope score, time to transplant, urgency of transplant, age or size at transplant, panel reactive antibodies, ischemic time, or donor weight $(p>0.05)$. Patients with CHD had longer cardiopulmonary-bypass times, more hypothermic circulatory-arrest, and higher post-operative inotrope scores, lactate, and days of ventilation $(\mathrm{p}<0.05)$. Thirteen $(45 \%)$ had disability: cerebral palsy (3), visual impairment (3), sensorineural hearing loss (2), epilepsy (1), isolated mental delay (6) [total mental delay 11 (38\%)]. The Adaptive Behaviour Assessment SystemGeneral Adaptive Composite was $74 \pm 20$ (normative mean $100 \pm 15$ ) and Motor Score was $6.5 \pm 4.3$ (normative mean $10 \pm 3$ ). On multivariate logistic regression only year of transplant predicted death (OR 0.28; 95\% CI. 09-0.91); and CHD predicted disability (OR 14.3; 95\% CI 1.4-143.7).

Conclusion: A significant proportion of children after cardiac transplantation have disability and below-average developmental scores. Patients with CHD are more likely to have disability. These data should help to appropriately counsel parents prior to proceeding with transplantation.

\section{9}

Early Experience in Mechanical Cardiac Support as Bridge to Transplant in Pediatrics

Horacio Vogelfang, Hospital de Pediatria "J.P. Garrahan" -Buenos

Aires-Argentina, Argentina

Gerardo Naiman, Hospital de Pediatria "J.P. Garrahan" -Buenos

Aires-Argentina, Argentina

Alejandra Villa, Hospital de Pediatria "J.P. Garrahan" -Buenos

Aires-Argentina, Argentina

Alberto Charroqui, Hospital de Pediatria "J.P. Garrahan" -Buenos

Aires-Argentina, Argentina

Guillermo Moreno, Hospital de Pediatria "J.P. Garrahan" -Buenos

Aires-Argentina, Argentina

Luis Quiroga, Hospital de Pediatria "J.P. Garrahan" -Buenos

Aires-Argentina, Argentina

Estela Carmona, Hospital de Pediatria "J.P. Garrahan" -Buenos

Aires-Argentina, Argentina

Maria de Carmen de la Riba, Hospital de Pediatria "J.P. Garrahan"Buenos Aires-Argentina, Argentina

Objetive: To describe the experience using a pulsatile electropneumatic assist device (Excor Berlin Heart ${ }^{\mathbb{R}}$ ) as bridge to transplantation.

Methods: Since march 2006 till agoust 2008, 6 children had been supported. Age range: 31 to 126 months. Weight range: 10 to $31 \mathrm{~kg}$. Results: Case I: 71 month, male, $19 \mathrm{~kg}$. Dilated Cardiomyophaty with end stage cardiac dysfunction; no response to inotropes infusion; mechanical respiratory support. After biventricular device implant early improvement of systemic functions. Extubation 48 hs. after. Assistance: 14 days. Transplantated.

Case II: 31 month, female, $11 \mathrm{~kg}$. Restrictive Cardiomyophaty and Systemic Pulmonary Hypertension. Severe heart failure. Biventricular Assistance. Extubation 72 hs after. Pulmonary 
Hypertension levels after 45 days: 50\%. Assistance: 264 days. Transplantated.

Case III: 56 month, female, $11 \mathrm{~kg}$. Dilated Cardiomyophaty. Severe left ventricle dysfunction. Univentricular assistance. Extubated 10 days after. Assistance:147 days. Endochraneal bleeding.

Case IV: 126 month, female, $31 \mathrm{~kg}$. Dilated Cardiomyophaty. Severe left ventricle dysfunction. Univentricular assistance. Extubated 24 hs after. Assistance:70 days. Endochraneal bleeding.

Case V: 46 month, female, $10 \mathrm{~kg}$. Dilated Cardiomyophaty. Severe heart function deterioration; mechanical respiratory support. Univentricular assistance. Extubated $24 \mathrm{hs}$ after. Assistance: 246 days. Transplantated.

Case VI: 48 month, male, $18 \mathrm{~kg}$. Dilated Cardiomyophaty. Severe left ventricle dysfunction. Univentricular assistance. Extubated 3 days after. Assistance: 96 days. Transplantated. Conclusions: Pulsatile electropneumatic assist device system seems to be a satisfactory device for bridging to transplantation infants and children in desperate heart failure condition. Decision for implantation in those early stage experience will largely depends on each institutional criteria.

\section{0}

Role of Immunosuppression Regimen in Post-Transplant Lymphoproliferative Disorder in Pediatric Heart Transplant Patients

Jeffrey D Dayton, Morgan Stanley Children's Hospital of New YorkPresbyterian, Columbia University Medical Center, United States Marc E Richmond, Morgan Stanley Children's Hospital of New YorkPresbyterian, Columbia University Medical Center, United States Robert G Weintraub, The Royal Children's Hopsital, Australia Anne T Shipp, The Royal Children's Hopsital, Australia Manuela Orjuela, Morgan Stanley Children's Hospital of New YorkPresbyterian, Columbia University Medical Center, United States Linda J Addonizio, Morgan Stanley Children's Hospital of New YorkPresbyterian, Columbia University Medical Center, United States

Objective: To examine the impact of type of immunosuppressive therapy on risk of post-transplant lymphoproliferative disorder (PTLD) in pediatric heart transplant recipients.

Methods: All heart transplant patients followed by two children's hospitals (1984-2008) were reviewed. Patients surviving longer than 2 months post primary transplant were included $(n=324)$. Mann-Whitney, Chi-square, and Cox hazard modeling were used as appropriate.

Results: Indications for transplant included cardiomyopathy (59\%) and congenital heart disease (39\%). PTLD developed in 39 patients $(12 \%)$ at a median time of 2.8 years post transplant (range $0.2-12$ years). Median age at transplant for the study population was 10.2 years and was significantly younger in patients who developed PTLD (3.7 vs 10.8 years, $p=0.002$ ). Patients with and without PTLD did not differ by sex. Except for 3 patients, all patients were initially treated with cyclosporine post transplant; $31 \%$ were changed to tacrolimus at a median of 149 days post transplant (range 5 days-15.7 years). PTLD-free survival was decreased in patients receiving tacrolimus as compared to those receiving cyclosporine only (Figure 1), with a hazard ratio of 3.6 (95\% CI 1.9-6.9). The frequency of rejection did not differ between patients with and without PTLD (0.56 vs 0.6 episodes per patient year, $\mathrm{p}=0.89)$. Overall survival was worse in patients with PTLD with a hazard ratio of 2.5 (95\% CI 1.4-4.3).

Conclusions: Pediatric heart transplant patients treated with tacrolimus have a higher risk of PTLD than those treated with cyclosporine alone.

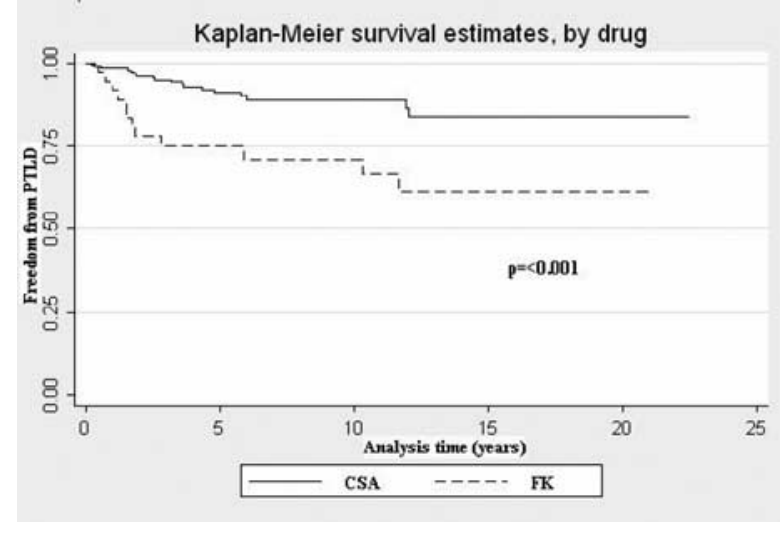

8201

How Often Do Electrocardiograms and Holter Monitors Identify Unsuspected Arrhythmias in Adults With Congenital Heart Disease?

Fred H Rodriguez, Texas Children's Hospital, United States

Douglas S Moodie, Ochsner for Children, United States

Marc Neeland, Ochsner for Children, United States

Christopher S Snyder, Ochsner for Children, United States

Background: Adults with congenital heart disease (ACHD) are at risk of developing arrhythmias. The purpose of this study is to assess the incidence of arrhythmias encountered on ECG and Holter monitors in ACHD patients.

Methods: A review of ACHD patients from 7-04-12-07. Data collection included: ECGs, and 24-hour Holter monitor results. Inclusion criteria: age $\geqslant 18$ years, congenital heart disease, ECG and 24-hour Holter monitor.

Results: We identified 333 patients with 500 visits. Inclusion criteria was met by 140 patients. ECGs revealed 109 normal and 31 abnormal (22\%), with most common abnormalities consisting of ectopy (7\%), supraventricular tachycardia (6\%), ST abnormalities (4\%), junctional rhythm (3\%), WPW (2\%), pacemaker issues (2\%), prolonged QT interval (2\%. Holter monitors revealed 43 (31\%) normal and 97 (69\%) abnormal, including ectopy (66\%), ventricular couplets (11\%), supraventricular tachycardia (10\%), ventricular tachycardia (6\%), high grade atrioventricular block (5\%), junctional rhythms (4\%), pacemaker problems (3\%), and WPW (1\%). In ACHD with normal ECGs, $69 \%$ had abnormal Holters. In ACHD with abnormal ECGs, $71 \%$ had abnormal Holters. In 48 patients with multiple visits, 33 (69\%) had significant changes on Holter monitors.

Conclusions: Abnormal ECGs and/or Holter monitors are present in a significant percentage of ACHD patients. In ACHD patients with a normal ECG, a majprity have abnormal Holters. We strongly recommend that a Holter be performed on all ACHD patients regardless of ECG results. In addition, repeat Holter monitoring can identify significant changes.

8202

Mapping of familial ventricular septal defects and aneurysms to chromosome $\mathbf{1 0}$

Dorothy J Radford, The Prince Charles Hospital, Brisbane, Australia Mugur I Nicolae, The Prince Charles Hospital, Australia Jonathan Ginns, Australia

Gregor Andelfinger, Sainte Justine Hospital, Montreal, Canada Maryse Thibeault, Sainte Justine Hospital, Montreal, Canada Roxanne Gendron, Sainte Justine Hospital, Montreal, Canada Geraldine Asselin, Montreal Heart Institute, Canada 
Marie-Pierre Dube, Montreal Heart Institute, Canada

Background: Although ventricular septal defects (VSD) are the most common congenital heart disease, familial clustering has been described only in rare instances. We have ascertained an extended family with VSDs and septal aneurysms requiring surgical intervention.

Methods: Detailed family history, physical exam, ECG, echocardiography and chart review was performed. Blood was sampled for genetic studies. Informed consent was obtained from all participants. Genotyping was performed using the Illumina Linkage 12 panel. MERLIN was used for statistical analysis, with standard parameters for a rare autosomal dominant trait (penetrance set to 0.90 , phenocopy set to 0.01 and disease allele frequency set to 0.001 ).

Results: 18 family members in three generations could be ascertained, out of whom 10 are affected (2 atrial septal defect, 3 septal aneurysm, 4 VSD, 1 Tetralogy of Fallot). Parametric multipoint LOD scores reach significance on chromosome 10p15.3-10p15.2 (max. 3.13). The LOD score support interval is in a gene-poor region which overlaps with numerous known cytogenetic anomalies causative in septal defects, but not the Di George syndrome 2 region on 10p.

Conclusion: The successful mapping of a rare familial form of VSDs/septal aneurysms provides evidence for further genetic heterogeneity of Mendelian cardiovascular traits. Fine-mapping, haplotype construction and resequencing will provide a unique opportunity for the pathogenesis of septal defects and shed light on candidate genes of heart development.

8203

Surgery for Congenital Heart Disaeses in Adults in Developing Country: Ahmed Gasim Cardiac Center Abdelmoneim Abdellah, Sudan

Murtada Mohamed, University of Khartoum, Sudan

Hani Eltayeb, Ahmed Gasim Cardiac Center, Sudan

Yassir Ali, University of Khartoum, Sudan

Abdalla Elsheikh, Ahmed Gasim Cardiac Center, Sudan

Objectives: To review the pattern of adult congenital heart disease needed surgical intervention in Ahmed Gasim Cardiac Center in a ten years duration.

Methodology: This study is retrospective descriptive hospital based from Jan 1998 to Dec 2008. The data is collected manually by the authers and analyzed by a computr program (SPSS).

Results: A total of 101 patients were operated on during the study period. $54.5 \%$ were females. The patient's ages range from 18 to 58 years with a mean of 27.9. 17 patients underwent closed cardiac procedure. the majority of open cardiac procedure is for atrial septal defects (37 patients) followed by Fallot' tetralogy (17 patients). 10 patients had subaortic membrane, two of them required replacement of the aortic valve as well as membrane excision. No intraoperative mortality in this group. Immediate postoperative complications include excessive bleeding which needed reopening, prolonged inotrpic support, and comlpete heart block. Late postoperative complications noted were infective endocarditis and severe pulmonary regurgitation with right ventricular failure. Both groups of complications were noted mainly in patients with Fallot's tetralogy repair. There were three late mortalities all in Fallot's tetralogy group.

Conclusions: Arount 10\% of the total cardiac operations in our center are adult congenital heart disease. The presentation of the patients is very late, yet there is an excellent early and late surgical outcome.

\section{4}

Outcome of pregnancy in complex congenital heart disease: case report

Khalid S Alnajashi, Prince Sultan cardiac Center, Saudi Arabia Saleh S Alghamdi, Prince Sultan cardiac Center, Saudi Arabia Mohammed A Awadh, Riyadh Millitary Hospital, Saudi Arabia Faisal M Al Attawi, Prince Sultan cardiac Center, Saudi Arabia

Objectives: This case series report outcomes of pregnancy in patient with complex congenital heart disease.

Background: Increasing numbers of women with repaired/ palliated complex CHD reaching reproductive age might have inappropriate pregnancy counseling.

Methods: Four patients referred to the adult CHD service. Case 1, 22 years old lady with tricuspid atresia, pulmonary atresia underwent modified Fontan; advice not to get pregnant.

Case 2, 20 years old lady had multiple ventricular septal defect underwent pulmonary artery banding and Glenn operation, high pulmonary artery pressure preclude completion of Fontan, advice against pregnancy.

Case 3,26 years old lady with repaired truncous arteriosus, Case 4, 24 years old lady, had transposition of great artery underwent Mustard procedure, both of them never been taught about pregnancy risk.

Result: Case 1, she delivered term baby, her echocardiography study showed good ventricular function; no arrhythmias postpartum.

Case 2 , she delivered prematurely at 28 week of gestation, no postpartum arrhythmia, her baby remained in the neonatal unit.

Case 3 , she delivered at term by cesarean section for obstetric indication, echocardiography study showed mild RV to PA conduit stenosis with good biventricular function, and no arrhythmias post partum.

Case 4 , at 35 weeks she underwent cesarean section because of patient request, echocardiography study showed no systemic or pulmonary veins baffle obstruction, no AVVR, good biventricular function, normal sinus rhythm in 24 hour holter.

Conclusion: Appropriate pregnancy counseling for women with complex CHD need to be delivered to the patient some of them might take the risk of having a baby.

\section{5}

Early results with the use of biodegradable ring for atrioventricular valve repair

Fotios A Mitropoulos, Department of Pediatric and Congenital Cardiac surgery, Onassis Cardiac Surgery Center, Athens, Greece, Greece Meletios A Kanakis, Department of Pediatric and Congenital Cardiac surgery, Onassis Cardiac Surgery Center, Athens, Greece, Greece Constantinos Contrafouris, Department of Pediatric and Congenital Cardiac surgery, Onassis Cardiac Surgery Center, Athens, Greece, Greece Nicholaos M Giannopoulos, Department of Pediatric and Congenital Cardiac surgery, Onassis Cardiac Surgery Center, Athens, Greece, Greece Andreas Chatzis, Department of Pediatric and Congenital Cardiac surgery, Onassis Cardiac Surgery Center, Athens, Greece, Greece Michael Mylonakis, Department of Pediatric and Congenital Cardiac surgery, Onassis Cardiac Surgery Center, Athens, Greece, Greece Prodromos Azariades, Department of Pediatric and Congenital Cardiac surgery, Onassis Cardiac Surgery Center, Athens, Greece, Greece

Objective: We describe our early results with the use of biodegradable ring for atrioventricular (AV) valve repair in patients with congenital heart diseases.

Patients-Methods: 25 patients (aged from 11 months to 58 years old, 9 males and 16 females) underwent cardiac surgery for 
congenital heart diseases during the period of March 2006December 2008.

6 patients underwent mitral valve annuloplasty, 18 tricuspid valve annuloplasty and 1 combined. All patients underwent A-V valve annuloplasty using the biodegradable ring Kalangos (Bioring SA, Lonay, Switzerland). The sizes of the ring for the mitral and tricuspid position were $16-26 \mathrm{~mm}$ and $26-34 \mathrm{~mm}$ respectively. Mean follow-up was 16.2 months.

Results: Success of implantation was $100 \%$. There were no perioperative or postoperative complications. Mean operative time for ring placement was $7 \mathrm{~min}$. Mean hospital stay was 8 days. Latest transthoracic echocardiography during the follow-up showed trace mitral valve regurgitation (MVR) in 5 and mild in 1 ; trace tricuspid valve regurgitation (TVR) in 10, mild in 8 and mild to moderate in 1 patient. Furthermore, there was a significant decrease of the left ventricular dimensions in patients with MVR and significant decrease of the right heart chambers size in patients with TVR. All patients are currently in excellent clinical status (NYHA I: 22 and NYHA I-II: 3) without symptoms.

Conclusion: The use of biodegradable ring for AV valve annuloplasty is a safe, quick and reliable technique with excellent short term results. Further follow-up is needed in order to evaluate the long term results.

\section{6}

Age at repair affects outcome of secundum atrial septal defects: benchmark for trans-catheter therapy

Elisabetta Zorzi, Division of Cardiology, University of Verona, Verona, Italy, Italy

Mara Pilati, Division of Cardiology, University of Verona, Verona, Italy, Italy

Francesca Viscardi, Division of Cardiac Surgery, University of Verona, Verona, Italy, Italy

Maria Antonia Prioli, Division of Cardiology, University of Verona, Verona, Italy, Italy

Luca Barozzi, Division of Cardiac Surgery, University of Verona, Verona, Italy, Italy

Corrado Vassanelli, Division of Cardiology, University of Verona, Verona, Italy, Italy

Alessandro Mazzucco, Division of Cardiac Surgery, University of Verona, Verona, Italy, Italy

Giovanni Battista Luciani, Division of Cardiac Surgery, University of Verona, Verona, Italy, Italy

Objective: To define the very-long term history of surgically repaired secundum atrial septal defect (ASD) in the trans-catheter therapy era.

Methods: All consecutive patients who underwent repair of isolated ASD between 1970 and 2007 were assessed directly or by telephone interview. Hospital records and death certificates were obtained if interim hospitalization or death had occurred.

Results: Two-hundred-twenty-two patients (152 female, 68\%), had repair at a mean age of $22 \pm 19$ years (range $1-70$ years) with no hospital mortality. During follow-up (mean $14 \pm 8$, range $1-36$ years) 14 deaths occurred, 4 due to cardiac cause. Actuarial 30 -year survival and freedom from cardiac death were $86 \pm 4 \%$ and $96 \pm 2 \%$, respectively. Symptomatic SVT were present in 38 patients (17\%) and 9 patients (4\%) required pacing. At follow-up 196 patients $(88 \%)$ were in NYHA class I, $19(8 \%)$ in II, 7 (3\%) III and $2(1 \%)$ in IV. Thirty-three patients (15\%) were on cardiac medication, 14 on warfarin. Multivariate analysis showed older age at operation $(\mathrm{p}=0.01)$ as independent predictor of major cardiovascular events.
Conclusions: Very-long term outcome after ASD repair shows excellent survival and low morbidity, which represent the current gold standard for trans-catheter therapy. Considering the association between older age at repair and late morbidity, choice between surgical and trans-catheter approach must not delay repair beyond early childhood.

\section{8}

Factors influencing review frequency of adults with congenital cardiac disorders

Janet E Burns, Edinburgh Royal Infirmary, United Kingdom Peter Bloomfield, Edinburgh Royal Infirmary, United Kingdom Hamish Walker, Glasgow Western Infirmary, United Kingdom

Although national guidelines in the UK recommend that all patients with significant adult congenital heart disorders (ACHD) are followed within a specialised adult congenital or grown up congenital heart $(\mathrm{GUCH})$ service, there has been no evidence base to support particular intervals for review nor the investigations that should be done at each visit.

Patients followed at the adult congenital service based in Edinburgh, either within Edinburgh or at outreach clinics serviced from Edinburgh, are seen less frequently than in larger centres. The main reason is lack of resource, particularly clinic room and technical support availability and lack of consultant time.

Despite this, there have been no emergency admissions provoked by the review interval and only 2 patients in the past 5 years have had an unexpected deterioration in their condition, requiring intervention within 3 months of their latest attendance.

The recruitment of DGH cardiologists who were prepared to develop an interest in ACHD has permitted an increase in the number of clinic slots from 300 to 480 with minimal loss of quality as all provide 12 lead ECG and TTE availability but not necessarily exercise testing at the same visit.

This is still insufficient to permit the frequency of review desired by the consultants in charge. Plans to accommodate this in future include written information to patients indicatign when earlier review should be sought, nurse directed telephone consultations to confirm earlier review is not required and interim attendance for investigations outwith regular outpatient attendances.

\begin{tabular}{lcccccc}
\hline & $\begin{array}{c}\text { total visits } \\
\text { (patients): } \\
\text { deaths }\end{array}$ & Tetralogy & TGA & $\begin{array}{c}\text { Eisen- } \\
\text { mengers }\end{array}$ & $\begin{array}{c}\text { Single } \\
\text { ventricle }\end{array}$ & Coarctation \\
& & & & & & \\
\hline 2004 & $514(370): 1$ & $56(48): 0$ & $57(39): 0$ & $16(14): 0$ & $22(20)$ & $57(53): 0$ \\
2005 & $440(367): 3$ & $51(44): 0$ & $59(42): 0$ & $14(12): 1$ & $25(19)$ & $45(45): 0$ \\
2006 & $479(393): 6$ & $61(53): 1$ & $54(39): 1$ & $17(12): 0$ & $36(23)$ & $58(47): 0$ \\
2007 & $416(358): 6$ & $52(45): 0$ & $54(47): 1$ & $16(12): 1$ & $32(21)$ & $66(55): 1$ \\
2008 & $381(359): 1$ & $63(57): 0$ & $39(37): 0$ & $12(10): 0$ & $31(25)$ & $56(54): 0$ \\
Total & $2230(1300): 17$ & $308(187): 1$ & $263(75): 2$ & $75(27): 2$ & $160(48)$ & $262(119): 1$ \\
\hline
\end{tabular}

\section{9}

Cardiopulmonary Stress Echocardiography: A Useful Tool in Assessment of Tetralogy of Fallot with Transannular Patch and Obligate Pulmonary Valve Insufficiency Devyani Chowdhury, Penn State Hershey Children's Hospital, United States

Christina Tofani, Penn State College of Medicine, United States Jennifer Ting, Penn State Hershey Medical Center, United States Steven Cyran, Penn State Hershey Children's Hospital, United States William Davidson, Penn State Hershey Medical Center, United States 
Objective: The objective of this study was to evaluate cardiopulmonary stress echocardiograms (CPSE) and RV function (Tei index) in patients with Transannular Patch repair of Tetralogy (TOF/TAP). TOF/TAP creates obligate pulmonary insufficiency (PI) causing RV dilation and failure.

Methods: Retrospective study. 218 patients underwent TOF/TAP repair at Penn State Hershey Medical Center (2/74-7/08). 47 underwent pulmonary valve replacement (PVR) $(29 \pm 18 \mathrm{yr})$. Group A: pre-PVR, Group B: post-PVR, Group C: TOF/TAP/ PI not yet needing PVR; $\mathrm{n}=171$.

Results: Exercise time was improved in Group B (12 Vs 9 in Group A) ( $<$.001); VO2max was not different. O2 pulse, ml/ beat, was higher in Group B (12 vs. 9 in Group A, p <.001). RV augmentation during exercise (measured on a scale of 0 to $-2,0$ normal, -2 severe dysfunction) was better in Group B ( -0.9 vs. Group A $-1.7, \mathrm{p}<.0001)$; as was RV Tei index $(0.2$ versus Group A $0.4, \mathrm{p}<0.0001)$.

The exercise duration of 161 patients in Group C was higher $(11 \mathrm{~min})$ than in Group A $(9 \mathrm{~min})(\mathrm{p}<.0001)$. VO2max $((\mathrm{ml} /$ $\mathrm{kg} / \mathrm{min})$ was 28 in Group C vs. 22 in $\mathrm{A}(\mathrm{p}=.007)$. RV augmentation $(-.07 \mathrm{Vs}-2.0$ in Group $\mathrm{A}, \mathrm{p}<.0001)$ and Tei index $(0.2$ versus 0.3 , Group A, $\mathrm{p}<.0001)$ were both superior in Group C.

Conclusions: CPSE demonstrated that in patients with TOF/ TAP/PI O2 pulse and both resting and exercise RV systolic function improves after PVR with preserved exercise capacity. CPSE offers a means to risk stratify TOF/TAP/PI for PVR and demonstrate physiologic benefits of surgery.

\section{0}

Histopathology of Ascending Aorta and the Risk Factors Related to Histopathological Conditions and Aortic Dilatation in Patients with Tetralogy of Fallot

Ujjwal Kumar Chowdhury, All India Institute of Medical Sciences New Delhi-110029, INDIA, India

Objectives: To evaluate the aortic wall histopathology and the risk factors related to histopathology and aortic dilatation in patients undergoing intracardiac repair of tetralogy of Fallot (TOF).

Patients and Methods: Operatively excised full thickness aortic wall tissue from 118 consecutive patients undergoing intracardiac repair of TOF aged 6 months to 47 years (mean, 110.6 \pm 94.8 ; median 70 months) were studied by light microscopy. The receiver operating characteristic (ROC) curve analysis was done to quantify the diagnostic accuracy of loss of lamellar counts and multiple logistic regression model.

Results: Thirty (25.4\%) aortic tissue specimens were indicated as histologically normal and were used as normal controls. The incidence of elastic fragmentation, increased ground substance, medionecrosis, smooth muscle disarray, and fibrosis was $74.6 \%$, $49.1 \%, 38.9 \%, 26.2 \%$ and $52.5 \%$ respectively. A lamellar count of $<60$ was associated with a sensitivity of $80 \%$ and a specificity of $87.67 \%$. Area under the ROC curve indicated that $93.37 \%$ $(\mathrm{SE} \pm 0.039)$ of the time the value of lamellar count was lower for the abnormal histopathology group compared with normal $(p<0.001)$. The risk of aortic dilatation was 16.27 times higher in patients with histopathologically abnormal aorta.

Conclusions: The great majority of aortic media of the ascending aorta in cyanotic TOF indicates significant loss of lamellar units, and pre-existing intrinsic aortopathy. The changes are present since infancy and are more pronounced in older patients subjected to long-standing cyanosis and volume overload and may account for or may coexist with the higher incidence of aortic dilatation encountered in these patients.
8211

Criterions for Selection of Patients for, and Results of a Novel Technique of Transfer of Anomalous Left Coronary Artery from the Pulmonary Artery using Autogenous Aortic and Pulmonary Arterial Flaps

Ujjwal Kumar Chowdhury, All India Institute of Medical Sciences, India

Background: Direct re-implantation of the anomalous left coronary artery (LCA) into aorta is the preferred surgical option for a dual coronary arterial system in patients with anomalous LCA from the pulmonary artery (PA). However, it can be applied only to patients with anomalous LCA arising from the right posterior pulmonary sinus. We report a new technique of LCA re-implantation to the aorta using combined autogenous aortic and PA flaps in situations when a direct connection was not possible.

Patients and methods: A cohort of four patients aged, 3 months, 6 months, 18 months, and 27 years with anomalous LCA from the left posterior pulmonary sinus underwent this technique of coronary artery transfer due to lack of coronary arterial length, diminished vessel elasticity, and extensive collaterals around the pulmonary sinuses.

Results: There was no early or late death. Postoperatively, all patients are in class-I with good function at a median follow-up of 74 ( 9-96) months. Postoperative coronary angiogram in one patient showed good LCA flow without any distortion.

Conclusions: The benefits of this new "trapdoor" technique are: i) excellent operative exposure, ii) use of autogenous, viable tissue capable of growth, iii) avoidance of injury to aortic and pulmonary valvular apparatus and right ventricular outflow tract obstruction, iv) elimination of use of pericardium for augmentation of the neo-aortic tube, v) achievement of the anastomosis with correct angling and length, and vi) possibility of implantation in all patients including adults regardless of the distance from the aorta or coronary artery configuration.

8212

Late onset severe pulmonary hypertension after successful atrial or atrial switch procedure for transposed great arteries

Rachael L Cordina, Royal Prince Alfred Hospital, Australia David S Celermajer, Royal Prince Alfred Hospital, Australia Shamus O'Meagher, Royal Prince Alfred Hospital, Australia

Common complications after surgery for transposition of the great arteries (TGA) include systemic ventricular dysfunction and arrhythmia after atrial baffle $(A B)$ repair and outflow tract stenosis or regurgitation after the arterial switch (AS). Severe pulmonary hypertension (PHT) is a rarely reported problem after $\mathrm{AB}$ and has not been noted after AS. We report three cases of PHT from our Adult Congenital Heart Clinic, two after $\mathrm{AB}$ and one after AS. We currently follow 78 patients with repaired TGA; 70 had atrial and 8 had arterial switch procedures. Two patients with $A B$ have developed severe pulmonary hypertension. One presented at age 27 and pulmonary artery pressure was $105 / 65 \mathrm{mmHg}$ at cardiac catheterization. She had simple TGA with Mustard repair at age 5 months. Two years after the diagnosis of PHT, she remains functionally impaired on Bosentan and Sildenafil. Another patient presented at age 34 years with suprasystemic pulmonary hypertension late after repair. She was born with complex TGA and had had AB and VSD closure at 8 months. Despite Bosentan, Sildenafil and inotropic support, she died four months after the diagnosis had been made, awaiting heart/lung transplantation. 
Finally, a 16-year-old boy presented with suprasystemic pulmonary pressures after an arterial switch procedure in the neonatal period, for simple TGA. He's currently on Bosentan therapy with good therapeutic response.

$4 \%$ of our adult CHD patients with TGA have developed late severe pulmonary hypertension. As novel therapies are available for this complication, regular surveillance and early treatment for this condition should be considered.

8213

Re-cardiac surgery in grown-up congenital heart disease Kazuyuki Daitoku, Hirosaki University School of Medicine, Japan Sanae Yamauchi, Hirosaki University School of Medicine, Japan Yoshiaki Saito, Hirosaki University School of Medicine, Japan Kozo Fukui, Hirosaki University School of Medicine, Japan Yasuyuki Suzuki, Hirosaki University School of Medicine, Japan Ikuo Fukuda, Hirosaki University School of Medicine, Japan

The number of grown-up patients with congenital heart disease $(\mathrm{GUCH})$ is constantly increasing. Re-cardiac surgery in the same group is also increasing. We evaluated the problem of $\mathrm{GUCH}$ who underwent re-cardiac surgery. Between February 2002 and December 2008, 13 patients were performed re-cardiac surgery in Hirosaki University Hospital. There were 7 male and 6 female, and mean age were $37.6 \pm 11.2$ years old. Diagnosis of congenital heart disease were as follows; Atrial septal defect (ASD) 2, ASD + mitral regurgitation 1 , ASD+ventricular septal defect (VSD) 1, VSD 4, Tetralogy of Fallot (TOF) 1, Congenital aortic valve stenosis 1, Coarctation of the aorta $(\mathrm{CoA})+\mathrm{VSD} 1$, Patent ductus arteriosus (PDA) 1, Double outlet right ventricle (DORV) 1. The reasons for re-operation were divided by related disease and acquired heart disease. Five cases were required repair of residual defects after correction. Three cases were needed replacement of valve failure after valve plasty. Two cases (TOF and $\mathrm{CoA}$ ) were presenting for expected reoperations after correction in childhood. Two cases were required another surgery because of developing Stanfotd type A of acute aortic dissection. The case of DORV was presenting for functional repair after palliative surgery. Although all cases were successfully repaired and uneventfully discharged, residual ASD or VSD might be avoided at the first surgery. First corrective surgery with subclavian flap method was failed in CoA case. It is important to perform corrective surgery for simple cardiac anomaly for the first time and necessary to manage and follow up all patients' life in GUCH.

\section{4}

Echocardiographic assessment of ventricular septal defect in the adulthood. A retrospective study of 136 cases

Nilda Espinola-Zavaleta, Instituto Nacional de Cardiología "Ignacio

Chavez", Mexico

María Elena Soto, Instituto Nacional de Cardiología "Ignacio Chavez", Mexico

Christian Buelna-Cano, Instituto Nacional de Cardiología "Ignacio Chavez", Mexico

Emilia J Patiño-Bahena, Instituto Nacional de Cardiología "Ignacio Chavez", Mexico

Introduction: The ventricular septal defect (VSD) in the adult patients is rare. The evolution of the VSD depends on its location and size.

Objective: Describe the experience of adult patients with VSD. Methods: 136 patients: 77 females (56.6\%), with mean age of $34 \pm 11.6$ years. All patients had clinical history and transthoracic echocardiography. Sixteen patients $(12 \%)$ went to surgery.
Results: The $80 \%$ had symptoms such as: dyspnea (57\%), palpitations (17\%), chest pain (12\%) and exertional syncope $(12 \%) .113$ patients $(82 \%)$ had isolated VSD and the rest in association with other shunts (ASD-13, PDA-12). The VSD was primembranous in 93 cases (68\%), muscular in 25 (18\%) and 18 of infundibular type (13\%). A small and medium VSD (2 a $15 \mathrm{~mm}$ was found in 108 patients and a large VSD $(>$ de $15 \mathrm{~mm})$ in patients. Seventy eight patients (57\%) had pulmonary hypertension. In the analysis of the mean life between patients without surgery and with normal pulmonary pressure versus non operated patients but with pulmonary hypertension was of 18 and 8 years, respectively $(\mathrm{p}=0.02)$.

Conclusion: The VSD is a congenital heart disease that in the adulthood is symptomatic in the majority of cases. The mean life is decreased when there are a perimembranous type and a large size of VSD and when it is associated to moderate and severe pulmonary hypertension.

\section{5}

Right ventricular function in patients with pulmonary hypertension. An echocardiographic study

Nilda Espinola-Zavaleta, Instituto Nacional de Cardiología "Ignacio Chavez", Mexico

Emilia J Patino-Bahena, Instituto Nacional de Cardiología "Ignacio Chavez", Mexico

Background: Right ventricular (RV) function is a key determinant of survival in pulmonary hypertension (PHT). The aim of this study was to assess the right ventricular function by echocardiography in patients with PHT.

Methods: We studied a cohort of 44 consecutive patients with idiopathic or shunt-associated pulmonary hypertension by two dimensional color Doppler flow echocardiography for measurement the parameters of right ventricular function.

Results: The mean age was $34.4 \pm 11.3$ years and $84 \%$ were female. The mean FS RV areas was $25.4 \pm 8.6 \%$, mean TAPSE was $10.2 \pm 3 \mathrm{~cm}$, mean diastolic EI was $1.81 \pm 0.4$, mean systolic EI was $1.78 \pm 0.6$, mean $\mathrm{RV}$ free wall thickness was $7.9 \mathrm{~mm} \pm 2.1$, mean $\mathrm{RV} / \mathrm{LV}$ ratio was $2.0 \pm 0.8$, mean tricuspid ratio was $1.4 \pm 0.6$, mean RIMP was $0.84 \pm 0.3$, mean VC was $5.5 \pm 2.3$, mean PAP was $110 \mathrm{mmHg} \pm 33$ and LVEF was $66.4 \% \pm 8.4$. The $\mathrm{RV} / \mathrm{LV}$ ratio $(\mathrm{r}=62 \%, \mathrm{p} \leqslant 0.000)$, systolic EI $(r=64 \%, p \leqslant 0.000)$, and diastolic EI $(r=64 \%, p \leqslant 0.000)$ was directly associated with PHT and the FSRV is inversely associated with $\mathrm{PHT}(\mathrm{r}=32 \%, \mathrm{p} \leqslant 0.03)$.

Conclusions: Echocardiography is a non invasive technique that allows quantification of right ventricular function and detection of RV dysfunction in PHT. A direct relationship between a RV/ LV ratio, systolic and diastolic EI and PHT was found and the relationship between FSRV and PHT was inverse.

\section{6}

Post-operative arrhythmias in adult patients with congenital heart disease

Hideo Fukunaga, Department of Pediatrics, Juntendo University

Nerima Hospital, Japan

Katsumi Akimoto, Department of Pediatrics and Adolescent Medicine, Juntendo University School of Medicine, Japan

Masahiko Kishiro, Department of Pediatrics and Adolescent Medicine, Juntendo University School of Medicine, Japan

Toshiaki Shimizu, Department of Pediatrics and Adolescent Medicine, Juntendo University School of Medicine, Japan

Shiori Kawasaki, Department of Cardiovascular Surgery, Juntendo University School of Medicine, Japan 
Shinichi Niijima, Department of Pediatrics, Juntendo University Nerima Hospital, Japan

Objective: In the long-term postoperative course of the congenital heart disease (CHD), arrhythmia is one of the most important prognostic factor. The complication of arrhythmia in adult congenital heart disease (ACHD) was examined.

Methods: For 42 adult patients with CHD (VSD12, TOF11, TGA8, TAPVR4, AVSD1, CoA2, ASR2, PVS1, PDA1) who were received radical operation, we examined incidence of lateonset arrhythmia (LOA) retrospectively.

Results: 10 patients (23.8\%, average 25.0-year-old) had LOA; eight patients $(80 \%)$ had supraventricular arrhythmia (Af6, PSVT1, APCs1). In TOF, one had APC, VPC and PSVT, one had VT. In TGA, five patients $(62.5 \%)$ complicated supraventricular arrhythmia; Af in four cases, sick sinus syndrome in one case. All of them received intraatrial switch operations. In VSD, three patients $(25 \%)$ complicated arrhythmia; Af in two cases, VPCs in one case. In these cases, residual shunt or atrioventricular valve regurgitation caused atrial overload.

Conclusions: In this study, we speculate the risk factors for LOS are as follows, atrial pressure overload, intraatrial switch operations, residual ventricular shunt, recurrent surgical infiltration and long incision line. In our hospital, right ventricle incision line was $1 \mathrm{~cm}$ or less at present and this minimization was efficacious for the arrhythmia prevention. In the future, the number of ACHD patients will increase inevitably, therefore medical care system following though there life period is needed by cooperation among pediatrician, physician and surgeons.

\section{7}

Do adolescents with congenital heart disease know their medical condition?

Elisabeth Getz, Rikshospitalet University Hospital, Norway

Mona Knutsen, Rikshospitalet University Hospital, Norway

Per Morten Fredriksen, Rikshospitalet University Hospital, Norway

Thomas Möller, Vestfold Hospital Trust, Norway

Christian Neukamm, Rikshospitalet University Hospital, Norway

Henrik Brun, Norway

Erik Thaulow, Norway

Objective: Seventy-two adolescents with congenital heart disease (CHD), born 1990-94, attended a special designed program before transfer to the GUCH service.

Methods: The patients' knowledge about their disease was examined by fill-in-forms. A comprehensive medical examination was performed.

Results: Thirty-four had coarctation of the aorta (CoA), 22 had tetralogy of Fallot (TOF) and 16 had total cavopulmonary connection (TCPC). Only 38\% of the CoA patients knew the name of their defect. However, $75 \%$ reported to be able to explain the mechanisms of the defect. Ninety per cent of CoA patients felt healthy and 79\% were attending sports. Of the TOF patients, 74\% knew the name of their defect, but only $63 \%$ had knowledge about the hemodynamic impact. $79 \%$ of the TOF patients felt healthy, but only $42 \%$ attended sports. $69 \%$ of the TCPC patients knew the name of their defect and only $50 \%$ said they were able to explain the hemodynamic effect. Of the TCPC patients $56 \%$ felt healthy and $69 \%$ participated in sports. The program included a medical examination that led to 1 patient with CoA and 2 TOF patients having surgery. In addition, $1 \mathrm{CoA}$ patient was stented.

Conclusions: A comprehensive medical and educational follow-up of adolescents with CHD prior to transfer to an adult department seems warranted.

\section{8}

Surgery for GUCH (grown-up congenital heart disease) in a tertiary hospital of a developing country

Rachel V Haickel Nina, University Hospital of the Federal University of Maranhão, São-Luis-MA, Brazil, Brazil

Vinicius S Nina, University Hospital of the Federal University of Maranhão, São-Luis-MA, Brazil, Brazil

José A Figueiredo Neto, University Hospital of the Federal University of Maranhão, São-Luis-MA, Brazil, Brazil

Isabela B Silva, University Hospital of the Federal University of

Maranhão, São-Luis-MA, Brazil, Brazil

Alexandre A Andrade, University Hospital of the Federal University of Maranhão, São-Luis-MA, Brazil, Brazil

Rafael M Gonçalves, University Hospital of the Federal University of Maranhão, São-Luis-MA, Brazil, Brazil

Sarah B Ramos, University Hospital of the Federal University of

Maranhão, São-Luis-MA, Brazil, Brazil

José Daniel A Costa, University Hospital of the Federal University of Maranhão, São-Luis-MA, Brazil, Brazil

Objective: To analyze the surgical outcomes of GUCH (grown-up congenital heart disease in a tertiary south-american public hospital.

Method: From January 2003 through June 2008 a retrospective study was performed on 63 patients (41 female) with mean age of 31.4 years (15-72 years) who were undergone to surgical correction of GUCH. All patients had a preoperative echocardiogram and angiogram for evaluation of the ventricular function, pulmonary artery pressure (PAP) and to rule out other cardiac lesions. Operations were performed under cardiopulmonary bypass through a mid-sternotomy. Logistic regression model was applied to identify predictors of adverse outcome.

Results: Predominant pathologies were ASD in 41 cases $(65,2 \%)$, VSD in 12 cases (19\%) followed by combined lesions in 10 cases $(15,8 \%)$. The mean systolic PAP was $40.3 \mathrm{mmHg}$ (20$100 \mathrm{mmHg}$ ). Twenty-two $(34,9 \%)$ patients had PAP $>35 \mathrm{mmHg}$. In-hospital mortality was $1.5 \%$ (1 case). There were no late deaths. The morbidity rate was $31.7 \%$ (20 cases). The major morbid events were: arrhythmias, low $\mathrm{CO}$ and transient neurologic impairment. Multivariate analysis showed that preoperative PAP greater than $43 \mathrm{mmHg}$ was a predictor of postoperative cardiac and neurologic dysfunction $(\mathrm{p}<0.05)$. Conclusion: Late presentation for surgery makes GUCH a very common finding in developing countries. Although it imposes a demanding clinical assessment, it can be surgically treated with low mortality and acceptable morbidity.

\section{9}

Surgical correction of patent ductus arteriosus in adults: is there a concern ?

Rachel V Haickel Nina, University Hospital of the Federal University of Maranhão, São Luis-MA, Brazil, Brazil

Vinicius S Nina, University Hospital of the Federal University of

Maranhão, São Luis-MA, Brazil, Brazil

José A Figueiredo Neto, University Hospital of the Federal University of Maranhão, São Luis-MA, Brazil

Ana Carolina $M$ de Castro, University Hospital of the Federal

University of Maranhão, São Luis-MA, Brazil, Brazil

Camila B Nogueira, University Hospital of the Federal University of Maranhão, São Luis-MA, Brazil, Brazil

Caroline S Ramos, University Hospital of the Federal University of Maranhão, São Luis-MA, Brazil, Brazil

Leiliana M Coelho, University Hospital of the Federal University of Maranhão, São Luis-MA, Brazil, Brazil 
Silmara M Rodrigues, University Hospital of the Federal University of Maranhão, São Luis-MA, Brazil, Brazil

Objective: The aim of this study was to analyze the outcomes of the surgical correction of Patent Ductus Arteriosus (PDA) in the adult population.

Methods: From January 2003 through June 2008, 22 patients (17 female) with mean age of 23.09 years underwent surgical correction of PDA. All patients had a preoperative angiogram to measure the pulmonary artery pressure (PAP). The surgical approach consisted on a left thoracotomy in the fourth intercostal space followed either by division and suture of the ductus in 15 or by a purse-string suture only in 7 (calcified ductus).

Results: Major preoperative symptoms were exertional dyspnea (22), chest pain (6) and palpitations (5). Mean systolic PAP was $44.8 \mathrm{mmHg}(20-110 \mathrm{mmHg})$. The average of mechanical ventilation time was 3.1 hours. There was no mortality and the mean length of hospital stay was 5.8 days. The complication rate was $50 \%$ - 11 cases of which: hypertension (4), bleeding (3), arrhythmia (2), pericarditis (1) and chest pain (1). There were no specific complications related to either techniques used to close the PDA.

Conclusion: Surgical correction of PDA in adults is sometimes technically demanding due to the occurrence of calcification, friable tissues and pulmonary hypertension. However, it can be safely performed with low mortality and acceptable morbidity.

\section{0}

\section{Pregnancy Outcome in Complex Congenital Heart} Disease

Rafael Hirsch, Rabin Medical Center, Israel

Alexander Dadashev, Rabin Medical Center, Israel

Leonard Blieden, Rabin Medical Center, Israel

Pregnancy is an important issue pertaining to patients (pts) with complex congenital heart disease. We reviewed our database for pregnancies in pts with transposition (complete and congenitally corrected)(TGA and CTGA), Fontan physiology, Eisenmenger syndrome and unrepaired cyanotic heart disease.

There were 36 pts with 56 pregnancies (no.): CTGA - 11 pts (24), TGA -10 pts (atrial switch -8 pts, arterial switch -2 pts) (13), Fontan -5 pts (6), unoperated Fallot -2 pts (3), Eisenmenger -8 pts (12).

Five CTGA pts had additional anomalies or previous operations. TGA post atrial switch pts included two with bio-prosthetic tricuspid valve. All but one pregnancy in this group were uneventful. There was no deterioration of myocardial function or worsening tricuspid regurgitation.

Two Fontan pts received medication for early heart failure. One pt had termination of pregnancy due to fetal hypoplastic left heart. One pt had severe post partum bleeding, accelerated protein losing enteropathy and is now on chronic hemodialysis. The two pts with unoperated TOF had three uneventful pregnancies and deliveries.

Two pts with ASD and Eisenmenger syndrome had 5 children before the diagnosis was made. 2 pts died in the immediate post partum period after successful delivery of their first baby. One pt with 2 children died at age 22 years.

Conclusion: Pregnancy can be successful and safe in pts with a systemic right ventricle and unoperated cyanotic anomalies. Pregnancy in Eisenmenger syndrome remains dangerous and contraindicated. Pts with Fontan physiology may have serious complications.
8221

Percutaneous Closure of Patent Arterial Ducts in Adults; Procedural and Clinical Results

Rafael Hirsch, Rabin Medical Center and Sackler School of Medicine, Tel Aviv University, Israel

Alex Dadashev, Rabin Medical Center and Sackler School of Medicine, Tel Aviv University, Israel

Elena Gleimer, Rabin Medical Center and Sackler School of Medicine, Tel Aviv University, Israel

Abid Assali, Rabin Medical Center and Sackler School of Medicine, Tel Aviv University, Israel

Ran Kornowski, Rabin Medical Center and Sackler School of Medicine, Tel Aviv University, Israel

Leonard Blieden, Rabin Medical Center and Sackler School of Medicine, Tel Aviv University, Israel

Background: Clinically important patent arterial ducts (PDA) requiring intervention are rare in adults.

Aim: To examine the procedural and clinical outcome of PDA closure in an adult congenital heart unit.

Results: In 14 years, thirty three pts ( 24 female) had PDA closure; mean age at catheterization was 39.6 $\pm \mathrm{SE} 2.7$ years (range 18-72). 23 pts had a continuous murmur. FC was II or worse in 21 pts. LV diastolic diameter was $56 \pm$ SE $1.3 \mathrm{~mm}$ and left atrial area $24 \pm \mathrm{SE} 1.1 \mathrm{~cm} 2$. LV systolic function was impaired in 6 and diastolic function in 7. Duct diameter by echo was $4.8 \pm \mathrm{SE} \quad 0.3 \mathrm{~mm}$ and by angiography $3.8 \pm \mathrm{SE} \quad 0.2 \mathrm{~mm}$. $\mathrm{Qp} / \mathrm{Qs}=1.6 \pm \mathrm{SE}$ 0.07. LVEDP was $17 \pm \mathrm{SE} 1.2 \mathrm{mmHg}$. Mean pulmonary artery pressure was $22 \pm \mathrm{SE} 2 \mathrm{mmHg}$ and cardiac index $3.2 \pm \mathrm{SE} 0.1 \mathrm{l} / \mathrm{min} \times$ msq. Devices used (no): Rashkind umbrella (3), Gianturco coils (8), Amplatzer PDA occluders (19) and NitOcclud (3). Procedural success and complications: One patient with Amplatzer device and 2 with coils had mild residual leaks. One coil embolized to the pulmonary artery and was successfully retrieved. There were no late complications. Clinical outcome: LV diastolic diameter decreased to $52 \pm$ SE $2.6 \mathrm{~mm}$ $(p=0.001)$ and left atrial area to $21 \pm$ SE $1.4 \mathrm{~cm} 2(p=0.003)$. $13 / 21$ pts with pre-catheterization reduced NYHA FC improved by one FC. One patient died 3 years post procedure from worsening heart failure despite initial improvement.

Conclusions: Clinically significant PDAs in adults of all ages can be treated successfully and safely with percutaneous devices with a significant clinical benefit.

\section{2}

Plasma fibrinogen level is associated with endothelial function and hemodynamics in the working skeletal muscle in patients after the Fontan operation

Kei Inai, Department of Pediatric Cardiology, Tokyo Women's Medical University, Japan

Eriko Shimada, Department of Pediatric Cardiology, Tokyo Women's Medical University, Japan

Toshio Nakanishi, Department of Pediatric Cardiology, Tokyo Women's Medical University, Japan

Objectives: We have demonstrated that patients after the Fontan operation have endothelial dysfunction and abnormal skeletal muscle hemodynamics, which were related to exercise intolerance. Fibrinogen is an independent cardiovascular risk factor causing inflammatory activation and endothelial dysfunction. The aim of this study is to investigate whether the fibrinogen level is associated with endothelial dysfunction and skeletal muscle hemodynamics in patients after the Fontan operation. 
Methods: We studied 30 patients after the Fontan operation and an 10 age-matched control group. All subjects underwent cardiopulmonary exercise test with near-infrared spectroscopy monitoring on the vastus lateralis muscle. They also underwent endothelial function study with high-resolution ultrasound. Plasma fibrinogen level was measured using immunonephelometry.

Results: In patients without aortic desaturation, endotheliumdependent vasodilation (FMD) was diminished, but endothelium-independent vasodilation (NTG) was similar compared with controls. In patients with desaturation, both of FMD and NTG was diminished. In patients without aortic desaturation, fibrinogen level was high compared with patients with desaturation. Fibrinogen level was negatively correlated with FMD, but not with NTG. Increased fibrinogen level was associated with an attenuated postexercise resaturation of the working skeletal muscle. In multivariate analysis, an independent association was established between fibrinogen level and FMD.

Conclusion: The increased plasma fibrinogen level is associated, at least in patients without aortic desaturation, with endothelial dysfunction and abnormal skeletal muscle hemodynamics. This implies that increased fibrinogen level has a possible contribution to the pathophysiology of endothelial dysfunction and exercise intolerance in patients after the Fontan operation.

8223

Pulmonary valve replacement and ventricular tachycardia following tetralogy of Fallot repair. Intraoperative right ventricle outflow tract cryoablation or postoperative internal cardioverter defibrillator?

Jakub Kadlec, Oxford Heart Centre, Cardiothoracic Surgery, United Kingdom

Nihal A Weerasena, Yorkshire Heart Centre, Cardiothoracic Surgery, United Kingdom

Kevin G Watterson, Yorkshire Heart Centre, Cardiothoracic Surgery, United Kingdom

John D Thomson, Yorkshire Heart Centre, Congenital Cardiology, United Kingdom

Kate M English, Yorkshire Heart Centre, Congenital Cardiology, United Kingdom

Rafael R Guerrero, Yorkshire Heart Centre, Cardiothoracic Surgery,

United Kingdom

Michael E Blackburn, Yorkshire Heart Centre, Congenital Cardiology, United Kingdom

Objectives: This study aims to define prevalence of ventricular tachycardia and review indications for intraoperative RVOT cryoablation and postoperative ICD implantation in patients indicated for PVR following TOF repair in our unit.

Methods: Retrospective data were collected from clinical notes of 34 survivors of PVR following TOF repair between January 2003 and November 2008, one died patient was excluded.

Results: PVR was performed at median age of 24.4 years (range 15.7-50.1) and 21.0 years (10.8-44.3) after TOF repair.

Preoperatively, $22(64.7 \%)$ patients reported palpitations, including $12(35.3 \%)$ with preoperative VT (nine had history of documented VT on ECG, one newly recorded nonsustained VT on 24 hours' tape and two newly inducible VT on EPS).

Three (8.8\%) patients underwent intraoperative RVOT cryoablation (due to VT on preoperative EPS in two, recurrent VF during resternotomy and preoperative QRS $>180 \mathrm{~ms}$ in one).

Ten $(29.4 \%)$ patients received ICD at median of 0.4 years (0.1-4.2) postoperatively. All RVOT cryoablated patients were palpitations free, one had negative and one is awaiting EPS.
Conclusion: This study shows that prevalence of VT among patients after TOF repair indicated for PVR is as high as 35.3\%. These patients were primarily indicated for postoperative ICD implantation. Recently, we decided to indicate all patients with VT prior to PVR for intraoperative RVOT cryoablation followed by postoperative EPS. Only patients with positive postoperative EPS will be referred for ICD. This can decrease patients' morbidity and spare financial resources. Our initial limited results are promising and are part of current prospective study.

\section{4}

Medical and healthcare problems in adults with isolated discrete subaortic stenosis operated on in childhood Anna I Kaneva-Nencheva, National Heart Hospital, Sofia, Bulgaria Vladimir B Pilossoff, National Heart Hospital, Sofia, Bulgaria Mila I Markova, National Heart Hospital, Sofia, Bulgaria Margarita R Tzonzarova, National Heart Hospital, Sofia, Bulgaria

The aim of the study is to evaluate the medical and healthcare problems of the ACHD with operated on isolated discrete subaortic stenosis (IDSS)in childhood.

Thirty seven patients were selected, mean age 25.6 \pm 5.4 years, followed up for $13.3 \pm 5.1$ years after surgery. The postoperative result was assessed as unsatisfactory in cases with peak gradient (PG) over $50 \mathrm{~mm} \mathrm{Hg}$ and moderate-severe aortic regurgitation (AR). Social functioning was compared to the official statistical data. Healthcare was assessed on the basis of frequency of the medical check-up.

PG increased from $15 \pm 13 \mathrm{~mm} \mathrm{Hg}$ immediately after the operation to $33 \pm 27 \mathrm{~mm} \mathrm{Hg}$ at last control $(\mathrm{p}=0.0001)$. Twelve pts $(35.5 \%)$ had unsatisfactory postoperative result due to restenosis (90\%), AR (25\%). ReOp was performed in 7 (18.9\%). The probability for satisfactory postoperative result decreased from $86 \%$ to $79 \%, 73 \%$ and $52 \%$ at the age of $18,25,30$ and 35 years. When compared to the official data, there is higher unemployment rate $((p=0.005)$ despite the higher educational level $(p=0.015)$. The period with no specialized medical check-up for the study group is $5.8 \pm 4.5$ years, significantly longer when compared to patients below 18 years $(\mathrm{p}=0.0001)$.

There are significant medical problems in at least $1 / 3$ of the ACHD patients operated on for IDSS in childhood that have to be assessed and treated in a specialized medical center. The quality of life of the patients is good in the setting of deteriorated social functioning.

8225

The effect of circulating hepatocyte growth factor in protein-losing enteropathy after Fontan operation

Gi Beom Kim, Seoul National University Children's Hospital, Korea Eun Jung Bae, Seoul National University Children's Hospital, Korea Chung Il Noh, Seoul National University Children's Hospital, Korea Jung Yun Choi, Seoul National University Children's Hospital, Korea Yong Soo Yun, Seoul National University Children's Hospital, Korea Woong Han Kim, Seoul National University Children's Hospital, Korea

Jeong Ryul Lee, Seoul National University Children's Hospital, Korea Yong Jin Kim, Seoul National University Children's Hospital, Korea

Objective: Protein-losing enteropathy (PLE) is devastating sequela of Fontan operation, of which pathophysiology is poorly understood and treatment is incomplete. Hepatocyte growth factor (HGF) is known as potent lymphangiogenetic factor. The purpose of this study was to evaluate the potential role of HGF in the development of PLE after Fontan operation. 
Methods: Twenty-nine patients who underwent Fontan operation were enrolled in this study. Among them, 10 patients were under the treatment of PLE and 19 patients showed no evidence of PLE. We reviewed medical records and measured serum HGF, vascular endothelial growth factor (VEGF), heparin, albumin, random stool $\alpha-1$ antitrypsin concentration and other factors. We also checked echocardiogram to evaluate hemodynamic status including ventricular function and cardiac index.

Results: Mean age was $15.7 \pm 8.7$ in the patients and $17.0 \pm 6.6$ year-old in the controls. Serum albumin level was significantly lower in the patients than the controls $(2.82 \pm 0.96$ versus $4.28 \pm 0.36 \mathrm{~g} / \mathrm{dL}, \mathrm{p}<0.001)$ and stool $\alpha-1$ antitrypsin concentration was significantly higher in the patients than the controls $(116.4 \pm 108.3$ versus $5.1 \pm 3.2 \mathrm{mg} / \mathrm{dL}, \mathrm{p}<0.001)$. Serum HGF was significantly higher in the patients than the controls $(0.61 \pm 0.27$ versus $0.42 \pm 0.12 \mathrm{ng} / \mathrm{mL}, \mathrm{p}=0.031)$. There was no significant difference of serum VEGF and heparin between two groups. Elevated serum HGF showed significant correlation with decreased serum albumin $(\mathrm{r}=-0.474, \mathrm{p}=0.011)$ and borderline significance in the correlation with increased stool $\alpha-1$ antitrypsin concentration $(r=0.365, p=0.067)$.

Conclusions: Increased circulating HGF may play a role in the development of PLE after Fontan operation. Further studies about the role of HGF in the PLE pathogenesis are needed.

\section{6}

Emergency Admissions In Adult Congenital Heart Disease Patients: Predictors Of Increased Length Of Stay And Patterns Of Resource Utilization

Angela $S$ Koh, Singapore

Betty $T$ Yap, Singapore

Ju Le Tan, National Heart Centre, Singapore, Singapore

Background: In the area of acute emergency care, the needs of adult congenital heart disease patients (ACHD) are unique and burden on hospital resources are likely substantial. We aim to understand the predictors of increased hospital length of stay (LOS) and impact on hospital resources.

Methods: We evaluated 600 ACHD patients in our adult congenital database. Patients who required emergency admissions between January 2007 and December 2007 were studied from hospital records. Results: Sixty-eight ACHD patients (11\%) required emergency admissions, culminating in a total of 108 admissions. Mean age was 37.6 years (SD 18.0), with a female preponderance (56\%). Most were either schooling or working (62\%) and were single (62\%). Atrial septal defect (24\%), ventricular septal defect (19\%) and Tetralogy of Fallot (13\%) formed the majority of diagnoses, mirroring proportions in our cohort. A third of the admissions were for cardiac reasons including arrhythmia, heart failure, endocarditis, and thromboembolism; remaining two-thirds were for non-cardiac reasons. Mean hospital LOS was 3.1 days (SD 3.7). Those who were older ( $\mathrm{p}=0.02$, 95\%CI $0.02-0.108)$ and neither employed nor schooling $(\mathrm{p}=0.021, \quad 95 \% \mathrm{CI}$ 0.255-3.133) had longer LOS. Thromboembolism accounted for longer LOS $(p=0.047,95 \%$ CI $0.035-6.141) .33 \%$ of the admissions that required interdisciplinary referrals had increased LOS ( $p<0.001,95 \%$ CI 1.415-4.257), with utilization of noncardiac investigations $(p=0.002)$. Increased LOS was not associated with adverse clinical outcome $(\mathrm{p}=0.68)$.

Conclusion: Older age groups, unemployment and thromboembolic complications were associated with increased LOS. Noncardiac conditions required interdisciplinary resources and contributed to increased LOS. Understanding their diverse acute needs will improve care and reduce LOS.
8227

Right ventricular global and regional function in two different models of RV overload

Ewa Kowalik, Institute of Cardiology, Poland

Miroslaw Kowalski, Institute of Cardiology, Poland

Mariusz Kusmierczyk, Institute of Cardiology, Poland

Jacek Rózanski, Poland

Piotr Hoffman, Poland

Objective: Aim of the study was to compare long-term effects of right ventricular (RV) overloads on global function and regional deformation indices in patients with tetralogy of Fallot (TOF, pressure and/or volume overload model) and in patients with atrial septal defect (ASD, volume overload model). On standard echo examination both groups were not different in terms of RV end-diastolic diameter.

Methods: 50 adults $(24 \mathrm{~F} / 26 \mathrm{M})$ aged $18-52$ yrs after total repair of TOF and 40 patients $(30 \mathrm{~F} / 10 \mathrm{M})$ with ASD with significant leftto-right shunt were examined. Patients were characterized by tricuspid annular plane systolic excursion (TAPSE), fractional area change (FAC) and myocardial performance index (MPI). Regional longitudinal systolic and diastolic Strain Rate (SR) and maximal strain (S) were analyzed off-line using GE Echopac 2D Strain. All values were averaged from three consecutive heart cycles.

Results: We found no difference in FAC and MPI between ASD and TOF patients (FAC 0,26 vs 0,29 ; MPI 0,39 vs 0,41 ). TAPSE was significantly increased in patients with ASD $(32,8 \mathrm{~mm}$ vs $20,7 \mathrm{~mm} ; \mathrm{p}<0,05)$. Maximal strain values were significantly lower in subjects with TOF for all analyzed RV segments $(-20,92 \%$ vs $-27,37 \%$ for basal; $-20,14 \%$ vs $-31,81 \%$ for mid; $-18,42 \%$ vs $-33,05 \%$ for apical segments, respectively).

Conclusions: Comparing to ASD, TOF patients demonstrate impaired regional RV function. Among the RV global function indices only TAPSE distinguishes between patients with RV volume and pressure/volume overload.

\section{8}

Long-term outcome of congenitally corrected transposition of the great arteries in adults - one center experience

Ewa Kowalik, Institute of Cardiology, Poland

Miroslaw Kowalski, Institute of Cardiology, Poland

Piotr Hoffman, Poland

Objective: Congenitally corrected transposition of the great arteries (ccTGA) is a rare anomaly which can lead to reduced survival in adult age. We aimed to determine what potential problems those patients may face and what is their prognosis in longer time perspective.

Methods: Clinical records since 1995 of all patients with ccTGA were retrospectively reviewed and analyzed for clinical status, systemic right ventricular (RV) dysfunction, atrioventricular regurgitation, heartblock, arrhythmias, surgical procedures and mortality.

Results: Data were obtained from 30 patients. The mean length of follow-up was 7 years. Out of 30, 4 patients died $(13,3 \%)$ at a mean age of 49,5 years. The longest survival was 77 years! The most common anomaly associated with ccTGA was pulmonary stenosis $(33,3 \%)$ and VSD (30\%). Surgery was performed in 13 patients (43.3\%). Twelve patients have had permanent pacemakers implanted because of complete heart block which developed either spontaneously or as a consequence of surgery. Endocarditis was 
observed in one patient. By univariate analysis, atrial fibrillation was a risk factor for increased mortality $(p=0,01)$. Risk factors for developing heart failure included age $(p=0,03)$, atrial fibrillation $(p=0,002)$, tricuspid valve replacement $(p=0,0008)$, RV enddiastolic diameter $(\mathrm{p}=0,01)$.

Conclusions: ccTGA is not a benign lesion in adults although long survivals can be observed. Large group of ccTGA patients show tendency to have complete heart block. Atrial fibrillation is associated with heart failure and increased mortality.

\section{9}

Right Ventricle Function in Patients after Ross Operation: Short-, Mid- and Long-Term Echocardiographic

Follow-ups

Andrii Kurkevych, Ukrainian Children's Cardiac Center, Kyiv,

Ukraine, Ukraine

Oleksandr Romanyuk, Ukrainian Children's Cardiac Center, Kyiv,

Ukraine, Ukraine

Valentyna Khanenova, Ukrainian Children's Cardiac Center, Kyiv,

Ukraine, Ukraine

Ihor Lebid', Crimea State Medical University, Simferopol, Ukraine

Nadia Rudenko, Ukrainian Children's Cardiac Center, Kyiv, Ukraine, Ukraine

Illya Yemets, Ukrainian Children's Cardiac Center, Kyiv, Ukraine, Ukraine

Objective: To assess the right ventricle (RV) function by echocardiography in early, middle and late period after Ross operation (RO).

Material and Methods: We studied 52 patients after RO: group 1 $(\mathrm{n}=19$, aged 10,8 $\pm 6,4)$, group $2(\mathrm{n}=19$, aged 13,9 $\pm 5,2)$, and group $3(\mathrm{n}=13$, aged $19,1 \pm 5,7)$, with mean follow-up time: $5,8 \pm 2,6 ; 31,1 \pm 11,9 ;$ and $82,3 \pm 23,0$ months, respectively; plus 15 healthy individuals (control group, aged 15,3 $\pm 7,9$ ). RV function was assessed quantitatively by: 1) ejection fraction (RVEF) and indexed RV end-diastolic volume (RVEDVi), 2) RV myocardial tissue velocities (systolic Sa, early-diastolic Ea, and late-diastolic Aa) and Tei Index, obtained by Tissue Doppler Imaging (TDI) method.

Results: The longer period after $\mathrm{RO}$, the more RV dilation occurs (RVEDVi from $84,2 \pm 15,9 \mathrm{ml} / \mathrm{m}^{2}$ in group 1 to $93,5 \pm 18,1 \mathrm{ml} / \mathrm{m} 2$ in group 3$)$, with a significant increase compared to control group $\left(74,1 \pm 20,3 \mathrm{ml} / \mathrm{m}^{2}\right)$ during mid- and long-term follow-ups ( $\mathrm{p}=0.003$ and $\mathrm{p}=0.014$, respectively). Compared to RVEF in control group $(58,6 \pm 9,3 \%)$, RVEF was significantly lower in groups $1-3(44,5 \pm 8,6 \%, p=0.0001$; $45,9 \pm 9,8 \%, p=0.0005$; and $46,5 \pm 11,4 \%, p=0.005$, respectively), but without tendency to decrease with time. Same pattern was observed with TDI parameters. The global RV function, assessed by Tei Index, was significantly decreased early after RO $(0,54 \pm 0,08$ vs. $0,41 \pm 0,04$ in control group, $\mathrm{p}<0.001)$, with progressive decrease in long-term period $(0,64 \pm 0,11, \mathrm{p}<0.001)$.

Conclusions: By quantitative echocardiographic assessment (RVEF, RVEDVi and TDI velocities), we identified slight early postoperative RV systolic and diastolic dysfunction with no negative progression with time. Tei index could be the most sensitive marker for RV function monitoring after RO.

\section{0}

Renal vascular function in aorta coarctation

Benedetta Leonardi, Department of Cardiology, Bambino Gesu' Hospital, Rome, Italy
Tom Gentles, Department of Cardiology, Green Lane Hospital, Auckland, New Zealand

Mauro Colajacomo, Department of Radiology, Bambino Gesu' Hospital, Rome, Italy

Lidia Monti, Department of Radiology, Bambino Gesu' Hospital, Rome, Italy

Andrea Magistrelli, Department of Radiology, Bambino Gesu'

Hospital, Rome, Italy

Fabrizio Gimigliano, Department of Cardiology, Bambino Gesu' Hospital, Rome, Italy

Ugo Giordano, Cardiorespiratory and Sports Medicine Unit-Dept of

Pediatrics, Bambino Gesu' Hospital, Rome, Italy

Stephen Pruett Sanders, Department of Cardiology, Bambino Gesu' Hospital, Rome, Italy

Background: Despite operative repair, subjects with coarctation of the aorta have a high risk of abnormal systemic vascular characteristics and late hypertension. The effect of these pathological changes on the left ventricle has been well described, but their impact on other organs less well defined. Objective: Our aim was to assess renal vascular function in patients late after coarctation repair.

Methods: 16 patients, age $21 \pm 7$ years, underwent a renal artery duplex ultrasound. The resistance index (RI) was measured (max systolic velocity-diastolic velocity/systolic velocity). Results were correlated with MRI parameters including LV size and mass.

Results: RI was abnormal in 2 patients and borderline abnormal in another (19\%). They had long standing arch obstruction ( $>10$ years) that had been repaired in the last 1 to 3 years. Two of the three required treatment with antihypertensive agents and had abnormal with night time hypertension on 24 hour monitor and excessive BP response to exercise. An abnormal exercise BP response was present in 2/2 (100\%) of those with an abnormal RI who had exercise tests, compared to 4 of $10(40 \%)$ of those with a normal RI $(p=0.12)$. Those with an abnormal RI tended to have greater LV mass $(82 \mathrm{vs} .64 \mathrm{~g} / \mathrm{m} 2$, $\mathrm{p}=0.10$ ), and a larger LV (94 vs. $80 \mathrm{ml} / \mathrm{m} 2, \mathrm{p}=0.17$ ) assessed by MRI.

Conclusions: There is a risk of renal vascular dysfunction late after coarctation repair. This may be particularly so when repair is delayed with persistent hypertension, and/or an abnormal blood pressure response to exercise.

\section{1}

Electrocardiographic Characteristics In Predicting

Hemodynamically Significant Secundum ASD

Bee Chian Lim, National Heart Centre, Singapore

Daniel Chong, National Heart Centre, Singapore

Mahendra Achyut Kare, National Heart Centre, Singapore

Fei Qiong Huang, China

Lisha Na, China

Lay Kian Goh, National Heart Centre, Singapore

Lay Wai Khin, National Heart Centre, Singapore

Ju Le Tan, National Heart Centre, Singapore

Objective: To determine the correlation between various electrocardiographic characteristics and presence of hemodynamically significant secundum ASD.

Method: Retrospective study looking at pre-closure electrocardiograms of 84 consecutive echocardiographically proven secundum ASD patients who attended our clinic from 2005 till 2008. ECG features of Right Bundle Branch Block (RBBB), right axis deviation (RAD) and prolongation of $\mathrm{PR}$ interval were 
assessed. Pre-closure echocardiograms were evaluated for ASD size, right ventricular and right atrial dilatation.

Results: Of all the 84 consecutive echocardiographically proven secundum ASD patients (mean age of $40.2 \pm 15.9$ years, 65\% female), electrocardiographic features of incomplete RBBB, complete RBBB and RAD were observed in $69 \%, 11 \%$ and $58 \%$ respectively (Table 1 ). RBBB appeared to be the only statistically significant electrocardiographic characteristic in predicting hemodynamically significant secundum ASD ( $\mathrm{P}$ value $<0.0001)$, with sensitivity, specificity, positive predictive value and negative predictive value of $90 \%, 63 \%, 91 \%$ and $59 \%$ respectively. Both RAD and PR interval prolongation showed high positive predictive value, but poor sensitivity and specificity in predicting hemodynamically significant ASD (Table 2,3).

Conclusions: The presence of $\mathrm{RBBB}$ pattern in our study population predicted $91 \%$ of all the moderate to large secundum ASD defects with RV dilatation, who required closure of ASD. However, the absence of RBBB could not be utilized to exclude the presence of significant ASD and RV dilatation.

Table 1 Prevalence of ECG abnormalities in echocardiographically proven secundum ASD patients.

\begin{tabular}{lll}
\hline & & Frequency \\
\hline Condution abnormality & Normal & $20 \%$ \\
& RBBB incomplete & $69 \%$ \\
& RBBB complete & $11 \%$ \\
Frontal axis & Normal & $31 \%$ \\
& RAD & $58 \%$ \\
PR interval & LAD & $11 \%$ \\
& Normal & $78 \%$ \\
& Prolonged & $22 \%$ \\
\hline
\end{tabular}

- $\mathrm{RBBB}$ incomplete $=\mathrm{QRS}<120$ milliseconds, $\mathrm{RBBB}$ complete $=\mathrm{QRS}>120$ milliseconds, $\mathrm{RAD}=$ Right Axis Deviation, $\mathrm{LAD}=$ Left Axis Deviation, prolonged PR interval > 200 milliseconds.

Table 2 Sensitivity, Specificity, Positive Predictive Value and Negative Predictive Value for Different ECG Criteria in predicting hemodynamically significant secundum ASD

\begin{tabular}{lccccc}
\hline \multicolumn{5}{c}{ ASD moderate to large } \\
\cline { 3 - 6 } & & Sensitivity & Specificity & PPV & NPV \\
\hline \multirow{2}{*}{ RBBB } & present & 90 & 63 & 91 & 59 \\
& absent & 10 & 37 & 41 & 9 \\
RAD & present & 59 & 57 & 86 & 24 \\
\multirow{2}{*}{ PR prolonged } & absent & 41 & 43 & 76 & 14 \\
& present & 26 & 93 & 94 & 22 \\
& absent & 74 & 7 & 78 & 6 \\
\hline
\end{tabular}

\begin{tabular}{lcccccc}
\hline \multicolumn{5}{c}{ RV dilatation } \\
\cline { 3 - 6 } & & Sensitivity & Specificity & PPV & NPV \\
\hline \multirow{2}{*}{ RBBB } & present & 85 & 40 & 87 & 36 \\
& absent & 15 & 60 & 64 & 13 \\
RAD & present & 51 & 44 & 83 & 15 \\
\multirow{2}{*}{ PR prolonged } & absent & 49 & 56 & 85 & 17 \\
& present & 19 & 90 & 90 & 19 \\
& absent & 81 & 10 & 81 & 10 \\
\hline
\end{tabular}

Table 3 Correlations between Electrocardiogram and Echocardiogram feautures of hemodynamically significant secundum ASD

\begin{tabular}{lcclllll}
\hline & \multicolumn{2}{c}{$\begin{array}{l}\text { Moderate to large } \\
\text { ASD size }\end{array}$} & \multicolumn{2}{l}{$\begin{array}{l}\text { RV } \\
\text { dilatation }\end{array}$} & $\begin{array}{l}\text { RA } \\
\text { dilatation }\end{array}$ \\
\cline { 2 - 8 } ECG features & $\%$ & P value & $\%$ & P value & $\%$ & P value \\
\hline RBBB present & 91 & $<0.0001$ & 87 & 0.068 & 90 & 0.006 \\
RBBB absent & 41 & $<0.0001$ & 64 & 0.068 & 40 & 0.006 \\
RAD present & 86 & 0.279 & 83 & 0.805 & 86 & 0.513 \\
RAD absent & 77 & 0.279 & 85 & 0.805 & 77 & 0.513 \\
PR prolonged & 94 & 0.13 & 90 & 0.49 & 100 & 0.097 \\
PR normal & 78 & 0.13 & 81 & 0.49 & 75 & 0.097 \\
\hline
\end{tabular}

8233

Development of a Specialist Adult Congenital Heart Unit at the Prince Charles Hospital Brisbane Queensland Australia-Trialling a Unique Model of Transition Care Theresa Malpas, The Prince Charles Hospital Brisbane Queensland Australia, Australia

Jillian Kaisar, The Prince Charles Hospital Brisbane Queensland Australia, Australia

Background: Since 1968 this hospital has successfully treated the paediatric and ever-increasing adult population with congenital heart conditions. In 2005 the Adult Congenital Heart Unit was established. In 2008 paediatric cardiology relocated to Mater Hospital, South Brisbane. A Transition policy, model of care and evaluation process has commenced. Current psychological research within the unit includes state-wide psycho-social profiling of the adult congenital heart population with Interpretive Phenomenological Analysis of experiences of young Australian adults with congenital heart conditions including parental perspectives.

Aims: Our unit aims to provide specialist inpatient and outpatient services to adults; seamless transition from paediatric to adult services; and multidisciplinary response to psycho-social problems. Research and psychological screening aims to inform Transition related interventions and increase awareness of psycho-social issues for Australians living with congenital heart conditions.

Method: The Director, Staff Specialist, Fellow in medicine, Psychologist and Nurse Coordinator facilitate the Transition Clinic, training of medical and nursing staff, and management of adult congenital heart patients. Patients receive ongoing access to progressive surgery, the heart transplant unit, specialist cardiology interventions and diagnostic services located at the hospital. Outcomes: Increased clinic attendance rates, treatment compliance and patient satisfaction.

Decreased inpatient occurrence and decreased length of hospital stays. Improved quality of life for patients. Better management of adults through continuous co-ordinated specialist care. Teaching, training and research within nursing, medical and allied health disciplines, maintaining best-practice standards.

8234

A Computed Tomography Study Of Coronary Arteries In Adult Mustard Patients: Anatomy and Clinical Implications

Simona A Marcora, Bambino Gesù Pediatric Hospital, Pediatric Cardiology Department, Rome, Italy 
Salvatore Giannico, Bambino Gesù Pediatric Hospital, Pediatric Cardiology Department, Rome, Italy Alberto Bellelli, Fatebenefratelli Isola Tiberina Hospital, Radiology Department, Rome, Italy

Marco Pierleoni, Fatebenefratelli Isola Tiberina Hospital, Radiology Department, Rome, Italy

Stephen P Sanders, Bambino Gesù Pediatric Hospital, Pediatric Cardiology Department, Rome, Italy

Paolo Di Renzi, Fatebenefratelli Isola Tiberina Hospital, Radiology

Department, Rome, Italy

Objective: Little is known about coronaries in adult patients (pts) after Mustard operation for TGA. We want to determine coronary anatomy in adult Mustard pts using computed tomography (CT).

Methods: A retrospective ECG gating 64-multislice CT (64MSCT) examination was performed for ventricular function evaluation in 26 Mustard pts ( $22 \pm 3$ years) not suitable for cardiac magnetic resonance because of a pacemaker.Two observers independently described origin, epicardial course and size of the major coronary vessels.

Results: 64-MSCT identified origin and course of the coronary arteries in all pts. The coronary origins were usual for TGA in 17 pts $(65 \%)$ and unusual in 9 pts. The left coronary system was hypoplastic in most pts: the left main coronary artery (LMCA) absent or short in $17 / 26$ pts and the left circumflex coronary artery (LCx) hypoplastic in $17 / 26$ pts (diameter range $0.7-1.2 \mathrm{~mm}$ ). An inter-arterial course of the LMCA reported to be associated to sudden death and a course of a LCx very close to a caval baffle stent that could complicate an interventional procedure were observed in 2 pts. The diagnoses were totally concordant between the 2 operators.

Conclusions: 64 MSCT is an excellent non-invasive technique for studying coronary anatomy in adult pts after Mustard and can show potentially dangerous coronary courses. Interestingly the left coronary system was hypoplastic in the majority of our Mustard pts and could cause ischemic injury to the left ventricle (LV) such as fibrosis and myocardial dysfunction, with important implications for late LV retraining or biventricular resynchronization.

\section{5}

Structural Abnormalities of the pulmonary trunk in Tetralogy of Fallot: A morphological study

Elisabeth Bédard, Royal Brompton Hospital, United Kingdom

Karen P McCarthy, Cardiac Morphology Unit, National Heart \& Lung

Institute, Imperial College London, United Kingdom

Konstantinos Dimopoulos, United Kingdom

Georgios Giannakoulas, United Kingdom

Michael A Gatzoulis, United Kingdom

Siew Yen Ho, United Kingdom

Objective: Little is known about pulmonary trunk (PT) histology in patients with tetralogy of Fallot (TOF), especially in the era of palliative surgery and total repair during childhood. We hypothesized that intrinsic histological abnormalities of the PT are present from birth and may be affected by palliative surgery and/or total repair.

Methods and Results: We studied 39 formalin-fixed necropsy heart specimens with TOF and compared them with 17 normal control hearts. Sections of the PT were studied by light microscopy using various stains; histologic findings were graded according to severity. Amongst the TOF group (1 foetus, 11 infants, 14 children, 13 adults), 11 patients had undergone palliative and 10 reparative surgery at median age of 8 (range
$2.5-18)$ years. Grade $\geqslant 2$ PT histological changes were present in $59 \%$ (medionecrosis), 36\% (fibrosis), 56\% (cyst-like formation) and $56 \%$ (abnormal elastic tissue configuration, Figure 1) of TOF patients. Total histology grading scores were higher in TOF hearts (median 6; range 1-9) compared with controls (median 1; range $0-6)$. Histological abnormalities were present amongst infants (median score 3.5, range 1-9), after palliative surgery (median score 5, range 2-9) or repair (median score 7.5, range 4-9).

Conclusions: Marked intrinsic histological abnormalities in the PT of hearts with TOF exist compared to controls. These changes were present from infancy and amongst patients who had undergone palliative or reparative surgery, albeit the latter was performed late. Our data suggest structural abnormalities of the PT, which may affect overload i.e. pulmonary regurgitation, and thus outcome.

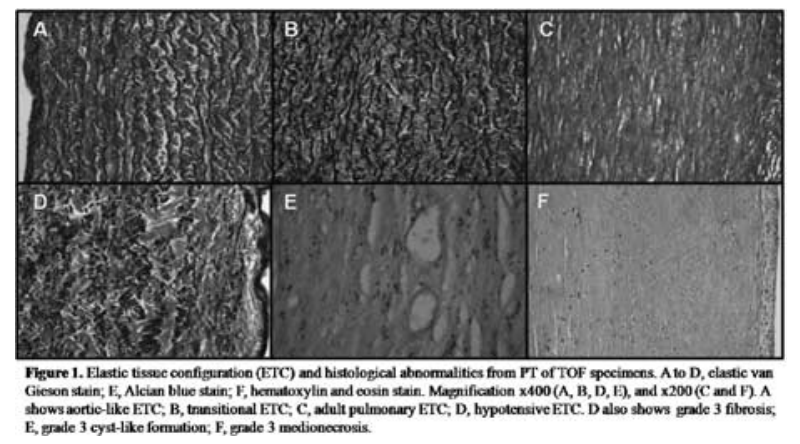

8236

Is Paediatric Intensive Care the most appropriate area of care for post operative adult congenital heart patients? Caroline McPherson, Paediatric Intensive Care Unit, Freeman Hospital, Newcastle Upon Tyne, UK, United Kingdom Lynne Scott, Paediatric Intensive Care Unit, Freeman Hospital, Newcastle Upon Tyne, UK, United Kingdom

Objective: To identify the nursing issues surrounding this expanding group of patients and to examine how we can plan our future service needs.

Background: Currently adults who are undergoing complex corrective congenital surgery are admitted post operatively to the Paediatric Intensive Care Unit (PICU). However, with the number of adult congenital patients now greater than those in childhood, it has forced us to ask the question....

Is the PICU the most appropriate area of care for this expanding group of patients?

Results: We examined our current practice and problems encountered by the adult congenital patient being cared for in the PICU. A series of issues were identified which impact on care delivered and include:

1) The experience and understanding of both nursing and medical staff.

2) The previous levels of training and the subsequent training needs.

3) The ethical implications.

4) The opportunity cost in the PICU of using PICU beds for adults.

5) The psychological implications for the patient and their families prior to their PICU admission.

6) Practical nursing issues.

7) The current national practices.

Conclusion: Clearly this is a highly complex group of patients with specific needs and requirements. As a team of motivated, 
innovative and dynamic practitioners we must learn from the issues we identified. We need to reflect on and develop our current practice to continue to provide the best nursing, medical, psychological and emotional care for both our patients and their families in the most appropriate clinical area.

8237

Aortic Valve Replacement with Stentless Bioprosthesis in the Young

Guido Michielon, Italy

Fiore Iorio, Cardiac Surgery Department Ospedale Pediatrico Bambino Gesù, Roma, Italy

Gianluca Oricchio, Cardiac Surgery Department Ospedale Pediatrico

Bambino Gesù, Roma, Italy

Duccio Di Carlo, Cardiac Surgery Department Ospedale Pediatrico

Bambino Gesù, Roma, Italy

Gianluca Brancaccio, Cardiac Surgery Department Ospedale Pediatrico Bambino Gesù, Roma, Italy

Antonio Amodeo, Cardiac Surgery Department Ospedale Pediatrico

Bambino Gesù, Roma, Kenya

Giorgia Grutter, Cardiac Surgery Department Ospedale Pediatrico

Bambino Gesù, Roma, Italy

Roberto M Di Donato, Cardiac Surgery Department Ospedale

Pediatrico Bambino Gesù, Roma, Italy

Objective: Ross operation is a double root procedure widely used in children with aortic valve (AoV) disease. Freedom from autograft and allograft reoperations and long-term durability in patients with bicuspid AoV are still under debate. We report the first preliminary experience with stentless aortic bioprostheses in selected children and adolescents with AoV disease.

Methods: 25 patients (mean age $16.8+/-8$ years) were enrolled in this prospective study between 2003 and 2008. Serial echocardiographic evaluations were available in all. Mean follow-up was 24.2 months.

Results: Indication for AoV replacement were severe AI in 9 $(36 \%)$, AS in $6(24 \%)$ and combined AS/AI in $10(40 \%)$. Seventeen patients $(68 \%)$ had undergone previous cardiac surgery, including AoV plasty in $8(32 \%)$. A bioprosthesis was preferred for pregnancy desire (7), contraindication to anticoagulation (8), and patient's preference (10). Surgical anatomy included unicuspid AoV (2), bicuspid AoV (14), tricuspid AoV (8) and failed mechanical prosthesis (1). Subcoronary implantation was preferred in 21 (84\%). Associated procedures were performed in 9 (36\%). Kaplan Meier survival is $95.2+/-4.6 \%$ at 64 months. Freedom from thromboembolism, prosthetic endocarditis, structural valve degeneration and reoperation is $100 \%$ at 64 months. Freedom from AI is $95.2+/-4.6 \%$ at 64 months. Mean transprosthetic gradient within 6 months from surgery is $10.6+/-4.7 \mathrm{mmHg}$. Full recovery of left ventricular function was observed in all. No patient is currently on anticoagulants and one patient delivered full-term without complications.

Conclusions: AoV replacement with stentless bioprosthesis is safe and may represent a valid alternative to the Ross operation in young selected patients.

\section{8}

Beating heart replacement of the pulmonary valve using stented bioprosthesis in patients with surgical corrected Tetralogy of Fallot

Fotios A Mitropoulos, Department of Pediatric and Congenital heart surgery, Onassis Cardiac Surgery Center, Athens, Greece, Greece
Meletios A Kanakis, Department of Pediatric and Congenital heart surgery, Onassis Cardiac Surgery Center, Athens, Greece, Greece Nicholaos M Giannopoulos, Department of Pediatric and Congenital heart surgery, Onassis Cardiac Surgery Center, Athens, Greece, Greece

Constantinos Contrafouris, Department of Pediatric and Congenital heart surgery, Onassis Cardiac Surgery Center, Athens, Greece, Greece Andreas Chatzis, Department of Pediatric and Congenital heart surgery, Onassis Cardiac Surgery Center, Athens, Greece, Greece Michael Mylonakis, Department of Pediatric and Congenital heart surgery, Onassis Cardiac Surgery Center, Athens, Greece, Greece Prodromos Azariades, Department of Pediatric and Congenital heart surgery, Onassis Cardiac Surgery Center, Athens, Greece, Greece

Objective: We present our early results of pulmonary valve PV replacement with the beating heart technique using oversized bioprosthetic valves in patients with surgical corrected Tetralogy of Fallot (SCTOF).

Patients-Methods: From September 2005 to December 2008, 35 patients (aged from 9 years to 56 years, old 24 males and 11 females) with SCTOF underwent PV replacement after a period ranged from 4 to 35 years from the anatomic correction. Our choice of prosthesis was the stented bioprosthetic Aortic Magna (Edwards, Lifesciences) valve. Thirty two valves were size $27 \mathrm{~mm}$, two $25 \mathrm{~mm}$ and one $23 \mathrm{~mm}$. The operation was performed with the beating heart technique under normothermic extracorporeal circulation. Concomitant surgical procedures included tricuspid valve annuloplasty $(n=22)$, resection of aneurysmal outflow tract patches $(\mathrm{n}=18)$, augmentation of stenotic pulmonary arteries $(\mathrm{n}=8)$ and right ventricular remodeling $(\mathrm{n}=9)$.

Results: There was one perioperative death $(2.8 \%)$ and one patient $(2.8 \%)$ developed atrial flutter. Mean hospital stay was 9 days. Cardiac echocardiography during the latest follow-up (from 6 months to 38 months after the operation) revealed bioprosthesis without significant stenosis or regurgitation $(n=34)$. Significant decrease of the right heart dimensions was also observed $(\mathrm{n}=34) .32$ patients are in NYHA I and 2 patients are in NYHA I-II, up to day.

Conclusions: The use of the new bioprosthetic valves using the beating heart technique provide excellent immediate and shortterm outcome. Further follow-up is necessary to evaluate the durability of this valve in the pulmonary position.

\section{9}

The Effect of Percutaneous Atrial Septal Defect Closure on Echocardiographic Parameters of the Left and Right Heart

Oliver J Monfredi, Manchester Royal Infirmary, Oxford Road, Manchester, UK, United Kingdom

Matthew Luckie, Manchester Royal Infirmary, Oxford Road, Manchester, UK, United Kingdom

Petra Jenkins, Manchester Royal Infirmary, Oxford Road, Manchester, UK, United Kingdom

Helen Buckley, Manchester Royal Infirmary, Oxford Road, Manchester, UK, United Kingdom

Bernard Clarke, Manchester Royal Infirmary, Oxford Road, Manchester, UK, United Kingdom

Vaikom S Mahadevan, Manchester Royal Infirmary, Oxford Road, Manchester, UK, United Kingdom

Percutaneous device closure is an established treatment for secundum atrial septal defects (ASDs). 26 consecutive patients undergoing percutaneous ASD closure underwent prospective echocardiography at baseline, 24 hours post-, and 6-8 weeks 
post-closure. Echo parameters were analysed by a single blinded operator.

Mean age of the study population was 46 years $(+/-17.8)$. $69 \%$ were female. Mean device size was $27 \mathrm{~mm}$ (range 11-40 mm). Right atrial (RA) area fell by $4.2 \mathrm{~cm}^{2}$ over 8 weeks $(\mathrm{p}<0.05)$, whilst mean right ventricular $(\mathrm{RV})$ diameter fell by $0.9 \mathrm{~cm}(\mathrm{p}<0.05)$. RV diastolic and systolic areas also fell by statistically significant amounts.

Fractional area change (FRAC) of the RV decreased by a mean of $6.6 \%$ on day 1 post-ASD closure, and by a total of $11.7 \%$ at 8 weeks $(p<0.05)$. Mean RV tricuspid annular plane systolic excursion (TAPSE) decreased by $0.24 \mathrm{~cm}$ on day 1 , and by $0.49 \mathrm{~cm}$ at 8 weeks $(\mathrm{p}<0.05)$. Mean septal mitral annular E/E' ratio increased from 12.4 to 16.4 on day 1 , and to 20.3 at 8 weeks $(\mathrm{p}<0.05)$. Mean lateral mitral annular E/E' ratio showed similar statistically significant increases.

The study confirms that RV remodeling occurs following ASD closure. It demonstrates for the first time that certain echocardiographic effects are evident as early as the first post-procedural day. These effects are presumably related to decreases in RV volume loading consequent on ASD closure, and continue to at least 8 weeks post-closure. Further correlation studies are required to elucidate whether certain pre-closure parameters can accurately predict favourable RV remodeling following ASD device closure.

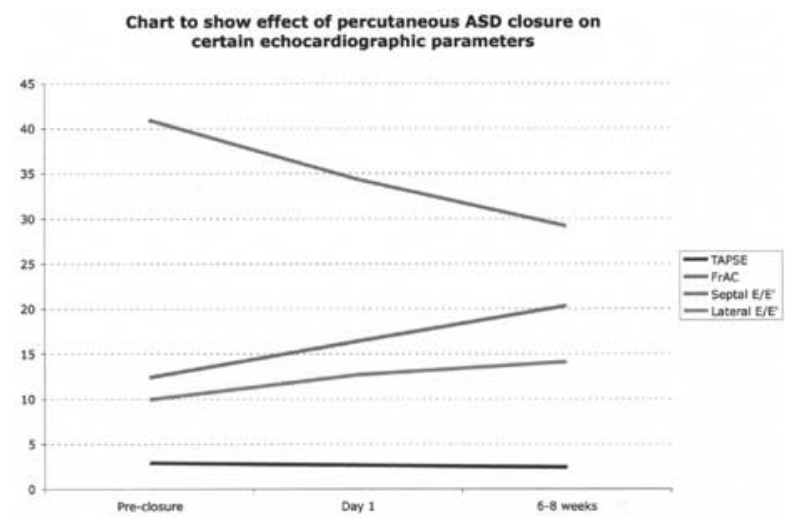

8240

Atrial Septal Defect with Tricuspid Regurgitation. Surgical Results

Orlando Moreno, Hospital Universitario de Caracas, Venezuela Iván A Machado, Hospital Universitario de Caracas, Venezuela Fernando Alvarado, Hospital Universitario de Caracas, Venezuela Chadi Nasser, Hospital Universitario de Caracas, Venezuela Xiomara Regoli, Hospital Universitario de Caracas, Venezuela Pedro Graziano, Hospital Universitario de Caracas, Venezuela Luane Piamo, Hospital Universitario de Caracas, Venezuela Fernando Bosch, Hospital Universitario de Caracas, Venezuela

Atrial Septal Defect (ASD), can be found up to 30\% among adults with Congenital Heart Disease. Generates a progressive chronic volume overload of the Right Ventricle (RV), specially in large defects. Over time dilates the Tricuspid Annullus, generates ventricular disfunction, heart failure, arrhythmias and intracavitary thrombosis, more evident after the fourth decade of life. Pulmonary Artery Hipertension generates chronic RV pressure overload. We believe that many patients with indication of ASD closure also need Tricuspid repair with annuloplasty (TA).

Methods: Prospective and longitudinal study with mid-term follow up. From August 2006-October 2008, we performed 18 ASD closures with TA using MC3 Edwards or Duran Rings, with Transesophageal Ecocardiographic during surgery (TTEE), clinical and transthoracic echocardiogram follow-up (FU).

Results: Mean age at surgery: 39,38 years (range 23-56), more frequent in females: 16 cases (88\%). ASD types: Ostium Secundum 8 cases (44\%), Sinus Venosus 6 cases (34\%), Ostium Primum 2 cases (11\%), residual post surgical 2 cases (11\%). Preoperative TR: severe ( 9 cases, $50 \%$ ), moderate (9 cases, $50 \%$ ). In all cases, we performed ASD closure with bovine pericardial patch. Durán Ring, 5 cases (27-33 mm); MC3 Ring 13 cases (28-34 mm). TTEE revealed TR improvement in all cases. Short and mid-term follow up TR: none, 6 cases; trivial, 3 cases; mild 7 cases, moderate in 2 cases.

Conclusions: In patient that fill criteria por ASD closure and TR, we consider useful TA. It improves post-surgical recovery. During mid-term follow-up improves ventricular performance.

\section{1}

The clinical outcome in adult patients with Fontan physiology

Makoto Nakamura, fukuoka children's hospital, Japan

Shiro Ishikawa, fukuoka children's hospital, Japan

Yuichi Ishikawa, fukuoka children's hospital, Japan

Hiroya Ushinohama, fukuoka children's hospital, Japan

Koichi Sagawa, Fukuoka Children's Hospital, Japan

Naoki Fusazaki, fukuoka children's hospital, Japan

Hideaki Kado, fukuoka children's hospital, Japan

Objective: We have experienced 894 patients (pts) with functionally univentricular hearts except hypoplastic left heart syndrome from September 1980 through October 2008. The overall rate of Fontan procedure is $56 \%$ in our institution. This study evaluated the clinical outcome in adult pts $(20$ year-old and more) with Fontan physiology.

Methods: Eighty-one adult pts with Fontan physiology were enrolled. We analyzed post-operative morbidity, hemodynamics, and medications retrospectively.

Table The characteristics of adult patients with Fontan physiology $(\mathrm{n}=81)$

Gender

Age at Fontan ope.

Present age

Diagnosis

male/female $42 / 39$

Diagnosis

Fontan procedure

(4y-24y)

meadin; 23y3m (20y-37y)

SRV or SLV: 26, Heterotaxy: 24,

TA: 20, S-I heart: 5, PA IVS: 4, others: 2

APC: 7, TCPC(LT): 39, TCPC(EC): 33 ,

TCPC(IC): 2, others: 2

Clinical manifestations Death: 2, dysrhythmia: 10, DM: 1,

PLE: 1, Hemoptysis: 1, DCM: 1,

Brain infarction: 1, CHF: 1

Drop out: 11

Medication after

Warfarin: 67, aspirin: 49, ACE-I: 35;

Fontan ope.

$\overline{\mathrm{SRV}}$; single right ventricle, SLV; single left ventricle, TA; tricuspid artresia, S-I heart; superior inferiror heart, PA IVS; pulmonary atresia intact ventricular septum, APC; atripulmonary connection, TCPC; total cavopulmonary connection, LT; lateral tunnel, EC; extracrdiac conduit, IC; intracardiac conduit, DM; diabetes mellitus, DCM; dilated Cardiomyopathy, CHF; congestive heart failure, ACE-I; angitensin converting enzyme inhibitor, ARB; angiotensin II receptor blockade

Results: The table shows a summary of the characteristics of the pts enrolled. The median age at Fontan operation was 10 years and the median age at the examination was 23 years. Twenty-six pts had diagnosed as single right or left ventricle heart, 24 as heterotaxy and 20 as tricuspid atresia. Two late-deaths were 
observed and 11 drop-outs. The New York Heart Association functional classification I was in 68 pts whose medications were warfarin potassium in 66 pts $(66 / 68=97 \%)$, aspirin in 49 pts $(72 \%)$, angiotensin converting enzyme inhibitors in 35 pts (51\%), carvedilol in 24 pts (35\%), angiotensin II receptor blockers in 15 pts $(22 \%)$ and others in 37 pts (54\%). Ten pts had been treated for dysrhythmias, in which 3 pts had undergone catheter ablation, 2 pts concomitant surgical therapy at fontan operation and 2 pts pacemaker implantation.

Conclusions: In our institute, warfarin potassium, aspirin and ACE-I/ARB/carvedilol are basic medications for cardiovascular protection in patients with Fontan physiology. The present data show the encouraging survival rate in adult pts with Fontan physiology, but that the morbidity of dysrhythmias remains suboptimal.

\section{2}

Fate of Coronary Cusp Prolapse and Aortic Incompetence in non-operated Subpulmonary Ventricular Septal Defect after 28 years Follow-up in a Chinese Population

Yin Ming Ng, Department of Paediatrics, Queen Elizabeth Hospital, Kowloon, Hong Kong, Hong Kong

Shuk Han Lee, Department of Paediatrics, Queen Elizabeth Hospital, Kowloon, Hong Kong, Hong Kong

Dora Mei Ling Wong, Department of Paediatrics, Queen Elizabeth

Hospital, Kowloon, Hong Kong, Hong Kong

Louisa Poon, Department of Paediatrics, Queen Elizabeth Hospital, Kowloon, Hong Kong, Hong Kong

Objectives: The subpulmonary defects (SVSD) were followed longitudinal and the progress of Coronary Cusp Prolapse (CCP) and Aortic Incompetence (AI) were assessed regularly since birth. Method: Patients diagnosed SVSD between 1972 and 1982 were followed prospectively, would only be referred for surgery with indications such as previous infective endocarditis, cardiomegaly, heart failure and patients' choice. 45 patients diagnosed to have SVSD were regularly followed. Standard views of trans-thoracic echo were performed regularly to assess AI and CCP. At least one cardiac catheterization would be performed. The severity of $\mathrm{CCP}$ and $\mathrm{AI}$ was graded using the following system: $0=$ none, $1=$ mild, $2=$ moderate and $3=$ severe.

Results: There were 30 Doubly Commited VSD (DCVSD) (66.7\%)and 15 Outlet Muscular (OMVSD) (33.3\%). The mean age was 26 years (median 27 year, range 20 years to 36 years) and the mean follow up duration is 28 years. There were 31 males and 14 females. Nine (7 DCVSD, 2 OMVSD) were closed by patch closure. The three patients with large defects were all operated early at age of 12-13 years. For the 6 with small defects, 2 were operated because of patient's choice, the others were operated because of indications mentioned above. Those who are not operated only have small subpulmonary VSD and mostly grade $1 \mathrm{AI}$ and/ or CCP with minimal progression over 26-35 years.

Conclusion: This series of 45 Chinese patients shows that the small subpulmonary VSD are usually static and will not show much progression and can be followed and treated conservatively.

\section{3}

Heart failure therapy in systemic right ventricular systolic dysfunction

Mugur I Nicolae, The Prince Charles Hospital, Australia

Jonathan Ginns, Australia

Theresa Malpas, The Prince Charles Hospital, Australia
Dorothy Radford, Australia

Richard Slaughter, Australia

Introduction: Systemic right ventricular dysfunction is a common occurrence in patients with Transposition of Great Arteries, with an incidence of $32-48 \%$ at $15-18$ years follow-up, and this only tends to worsen with age.

A few small studies have been inconclusive in demonstrating any benefit from medical therapy in this situation, despite some improvement in the peak oxygen consumption, and increased exercise capacity.

Our study is a prospective, non-randomised case series of patients with TGA, commenced on medical therapy and followed with MRI at 12 months time.

Methods: Patients with complete or congenitally corrected TGA were invited to participate in this study after written informed consent. 13 patients were included, of which 11 were commenced on medical therapy (two patients declined medication and they were included in the control group). A cardiac MRI was performed at the commencement of the study and then repeated after 12 months of follow-up. In the treatment group, six patients were on a combination of ACEI/A2 blocker and beta blockers (Carvedilol in three patients, Bisoprolol in one patient, and Atenolol in two patients). Three patients were on a single ACEI only, and one patient was on angiotensin II receptor blocker alone. Only one patient was on beta blocker alone (Metoprolol).

Statistical analysis was performed using the Wilcoxon test.

Conclusions: Medical therapy in patients with systemic right ventricular dysfunction in TGA may results in an improvement of the right ventricular ejection fraction, mainly by increasing the indexed stroke volume of the systemic right ventricle.

\begin{tabular}{lll}
\hline & Control group & Treatment group \\
\hline RVEDVi at baseline & $149.5 \mathrm{ml} / \mathrm{m}^{2}$ & $157.36 \mathrm{ml} / \mathrm{m}^{2}$ \\
RVEDVi end of study & $156 \mathrm{ml} / \mathrm{m}^{2}$ & $158.45 \mathrm{ml} / \mathrm{m}^{2}$ \\
RVESVi at baseline & $92 \mathrm{ml} / \mathrm{m}^{2}$ & $100.81 \mathrm{ml} / \mathrm{m}^{2}$ \\
RVESVi end of study & $97 \mathrm{ml} / \mathrm{m}^{2}$ & $99.09 \mathrm{ml} / \mathrm{m}^{2}$ \\
SVi at baseline & $58.5 \mathrm{ml} / \mathrm{m}^{2}$ & $57.45 \mathrm{ml} / \mathrm{m}^{2}$ \\
SVi end of study & $59 \mathrm{ml} / \mathrm{m}^{2}$ & $59.36 \mathrm{ml} / \mathrm{m}^{2}$ \\
RVEF at baseline & 0.415 & 0.369 \\
RVEF end of study & 0.38 & 0.384 \\
\hline
\end{tabular}

\begin{tabular}{llll}
\hline & Baseline & 12 months follow-up & P value \\
\hline RVEDVi & $157.36 \mathrm{ml} / \mathrm{m}^{2}$ & $158.45 \mathrm{ml} / \mathrm{m}^{2}$ & 0.91 \\
RVESVi & $100.81 \mathrm{ml} / \mathrm{m}^{2}$ & $99.09 \mathrm{ml} / \mathrm{m}^{2}$ & 0.6 \\
Svi & $57.45 \mathrm{ml} / \mathrm{m}^{2}$ & $59.36 \mathrm{ml} / \mathrm{m}^{2}$ & 0.58 \\
RVEF & 0.369 & 0.384 & 0.18 \\
\hline
\end{tabular}

8244

Examination of cardiac arrhythmias in occurring late after repair of tetralogy of Fallot

Masahiro Ohtsuki, Juntendo University School of Medicine, Japan Katsumi Akimoto, Juntendo University School of Medicine, Japan Masahiko Kishiro, Juntendo University School of Medicine, Japan Ken Takahashi, Juntendo University School of Medicine, Japan Keiko Sato, Juntendo University School of Medicine, Japan Masao Ohtaka, Juntendo University School of Medicine, Japan Kanako Tahara, Juntendo University School of Medicine, Japan 
Toshiaki Shimizu, Juntendo University School of Medicine

Background: Arrhythmias occurring late after repair of tetralogy of Fallot (TOF) are common. Due to the risk of sudden cardiac death, attention is focused towards ventricular tachycardias (VT).

Aim: The aim of this study is the examination of cardiac arrhythmias such as VT in occurring late after repair of TOF. Results: 133 patients, $77 \mathrm{men}$ and 56 women, age 1 to 38 years (the average of 20.4 years), were included. 3 patients had arrhythmias. One was atrial flutter (AF). The others were VT. Case 1 was 27 years old man. The radical repair was performed at 3 years old. AF appeared in 10 years after repar. Patch closure and the cryoablation were performed for residual ventricular septal defect and AF at 20 years old. Case 2 was 27 years old man. The radical repair was performed at 4 years old. VT appeared in 15 years after repair. He was observed for Atenolol. Case 3 was 9 years old boy. The radical repair was performed at 2 years old. VT appeared in 6 years after repair. VT disappeared after the catheter ablation.

Conclusions: High right ventricular pressure (RVp) was found in 3 patients. No other factors were found in 3 patients. However, all patients with high RVp did not have any arrhythmias. The difference of patients with arrhythmias and without arrhythmias are unknown. We will study the causes of arrhythmias, such as $\mathrm{RVp}$, pulmonary regarditation, operation style and so on.

\section{5}

The characterisitics of the blood gas analysis in adult congenital heart disease patients

Yoko Okada, Department of Pediatrics, National Cardiovascular Center, Japan

Hideo Ohuchi, Department of Pediatrics, National Cardiovascular Center, Japan

Background: The blood gas analysis data in adult patients with congenital heart disease vary with the disease-specific characteristics. For example, Fontan patients show hypocapnea and Hypoxemia.

Objectives: To elucidate the mechanisms of the respiratorycirculatory relations in adult congenital heart disease patients.

Method: In 129 Tetralogy of Fallot (TF) patients, 153 Fontan patients, and 36 cyanotic patients (Cy), the data of blood gas, serum norepinephrine (NE) concentration, serum BNP concentration, caridac output, pulmonary capillary wedge pressure (PCWP), central venous pressure, ventilatory capacity (VC) and FEV were examined and compared.

Results: In Fontan patients hypoxemia and hypocapnea and low cardiac output were pointed out compared to TF and Cy patients (pCO2; Fontan $35.0 \mathrm{mmHg}$, TF $39.6 \mathrm{mmHg}$, Cy $38.2 \mathrm{mmHg}$, pO2:Fontan $71.5 \mathrm{mmHg}$, TF $92.5 \mathrm{mmHg}$, Сy $45.3 \mathrm{mmHg}$, CI:Fontan 2.48, TF 2.92, Cy $2.64 \mathrm{~L} / \mathrm{min} / \mathrm{M} 2$ ). The NE concentration in Fontan patients was higher than TF patients (Fontan 309.9ng/ml, TF $219.5 \mathrm{ng} / \mathrm{ml}$ ). VC and FEV didn't differ in these 3 groups statistically. In TF and Cy patients the $\mathrm{pCO} 2$ and $\mathrm{pO} 2$ showed negative correlation, while in Fontan patients there were no relation. In 3 groups no relation was found between $\mathrm{pCO} 2$ and $\mathrm{NE}$ concentration. In further analysis, the hypocapnea in Fontan patients was related to Age, BSA, Cardiac index, VC.

Conclusions: The characterisitics of resipiratory-circulatory state in Fontan patients are low cardiac output, hypocapnea, hypoxemia, elevated NE concentration, lower VC, however, the hypocapnea is not related to $\mathrm{pO}_{2}$, the norepinephrine concentration, and PCWP, which have been considerd as resipiratory driving factors in adult heart failure patients. In Fontan patients, there might be more complexed mechanisms of respiratory-circulatory relation.

\section{6}

Distribution of types of atrial septal defects in adults

Bilgehan S Oz, GATA Cardiovascular Surgery, Turkey

Mehmet Yokusoglu, GATA Cardiology, Turkey

Erkan Kaya, GATA Cardiovascular Surgery, Turkey

Mehmet Uzun, GATA Cardiology, Turkey

Harun Tatar, GATA Cardiovascular Surgery, Turkey

Objective: Atrial septal defect is the most common congenital heart disease in adulthood. In this study we present morphological and clinical features of our operated 591 adult patients with atrial septal defect.

Methods: Between september 1991 and november 2008, 591 patients were operated for atrial septal defect in our hospital. There were 514 men and 77 women and mean age during operation was $20 \pm 2$ years old (age ranges between 18 and 61 years old). Diagnosis was achieved by transesophageal echocardiography and then cardiac catheterisation was performed in all patients before surgery. The presence and type of atrial septal defects and accompanying cardiac abnormalities recorded. In this retrospective study categorical variables were analyzed by Chi-square test and continuous variables were analyzed by Kruskal-Wallis test. Mann-Whitney U test was used in intergroup comparisions.

Results: 473 of patients (80\%) were secundum type atrial septal defect, 79 patients (13\%) were sinus venosus type atrial septal defect and 39 patients (7\%) were primum type atrial septal defect. Main clinical sign in ostium primum type atrial septal defect patients was palpitation (63\%). Most of the secundum type atrial septal defect patients were asymptomatic (93\%). There were no significant differences among the types regard to sex and ages. Conclusion: Patients with atrial septal defect can easily diagnose and operate in developed regions in childhood but types oand clinical signs of atrial septal defects must be kept in mind of surgeons who work in undeveloped countries.

\section{7}

Survival and prognostic factors in the absence of atrioventricular connection

Emilia J Patino-Bahena, Instituto Nacional de Cardiología "Ignacio Chavez", Mexico

Nilda Espinola-Zavaleta, Instituto Nacional de Cardiología "Ignacio Chavez", Mexico

Luis Muñoz-Castellanos, Instituto Nacional de Cardiología "Ignacio Chavez", Mexico

María Elena Soto, Instituto Nacional de Cardiología "Ignacio Chavez", Mexico

Alfonso Buendia, Instituto Nacional de Cardiología "Ignacio Chavez", Mexico

Carlos Zabal, Instituto Nacional de Cardiología "Ignacio Chavez", Mexico

Juan Calderon-Colmenero, Instituto Nacional de Cardiología "Ignacio Chavez", Mexico

Fause Attie, Instituto Nacional de Cardiología "Ignacio Chavez", Mexico

Introduction: The absence of right atrioventricular connection is the third most common cyanotic congenital heart disease. The absence of atrioventricular connection can be right or left. The therapeutic management varies according to the anatomy. 
Objectives: Determine the clinical and morphologic characteristics that allow the survival of patients with absence of atrioventricular connection and highlight the importance of echocardiographic technique.

Methods: Twenty four patients were studied. All had a clinical history, echocardiogram and hemodynamic study.

Results: The NYHA functional class of the group was I/II in the $87.5 \%$. The electrocardiogram showed sinus rhythm in the $92 \%$ and dilatation of left ventricle, the chest X-ray cardiomegaly II in $83 \%$. The echocardiogram showed situs solitus and absence of right atrioventricular connection in the $92 \%$ of cases. The most frequent ventriculoarterial connection was concordant (71\%). All patients had atrial septal defect, 21 ventricular septal defect, 21 decreased pulmonary flow. The ejection fraction of the main ventricle (EFMV) of the group was of $55 \pm 10 \%$. The factors that diminished the survival of these patients were: small atrial septal defect and EFMV less than $40 \%$.

Conclusions: The presence of a wide atrial sepal defect and normal EFMV were the factors that allow the survival of these patients to the adulthood.

The echocardiography gives to the clinicians and surgeons very important information.

\section{8}

Evaluation of exercise capacity long term after surgical correction of d-transposition of great arteries (d-TGA) by Senning atrial switch

Artur Z Pietrucha, Coronary Disease Department, Medical School of Jagiellonian University, John Paul II Hospital, Cracow, Poland, Poland Beata J Pietrucha, Children Cardiology Department, University Children Hospital Jagiellonian University, Cracow, Poland

Nader El-Massri, Coronary Disease Department, Medical School of Jagiellonian University, John Paul II Hospital, Cracow, Poland, Poland Danuta Mroczek-Czernecka, Coronary Disease Department, Medical School of Jagiellonian University, John Paul II Hospital, Cracow, Poland, Poland

Andrzej Gackowski, Coronary Disease Department, Medical School of Jagiellonian University, John Paul II Hospital, Cracow, Poland, Poland Ewa Konduracka, Coronary Disease Department, Medical School of Jagiellonian University, John Paul II Hospital, Cracow, Poland, Poland Andrzej Rudzinski, Children Cardiology Department, University Children Hospital Jagiellonian University, Cracow, Poland

Wieslawa Piwowarska, Coronary Disease Department, Medical School of Jagiellonian University, John Paul II Hospital, Cracow, Poland, Poland

Aim of the study: evaluation exercise capacity in young people, long term after surgical correction of d-TGA by Senning atrial switch.

We observed 21 pts (18 men, 3 women) at age 21-25 after Senning procedures, performed at 3-7 months of life.

All pts underwent standard and 24-hour ECG, echocardiography examination, cardiac MRI and cardiopulmonary exercise treadmill test (ETT). Left (LV) and right ventricle (RV) function, ETT duration and workload, max oxygen uptake (VO2max) and anaerobic threshold (AT) were evaluated. VO2max, ETT work were also expressed as \% of predicted values. Serum level of NT-proBNP was measured in all pts.

Results: LV function was not reduced in all pts (LV diastolic diameter $46 \pm 4,2 \mathrm{~mm})$ LV EF $68 \pm 14,7 \%)$. RV dilatation $(38,2 \pm 12,3 \mathrm{~mm}$ with moderately reduced ejection fraction (RV EF $47 \pm 13,9 \%$ ) were observed in pts after TGA correction. Cardiac MRI discovered significant RV dilatation with hypertorophy of RV walls with magnified trabeculation. Exercise was well tolerated by all pts. - they achieved about $100 \%$ of predicted workload $(207,6 \pm 65,3 \mathrm{~W} ; 12 \pm 2,5 \mathrm{METS})$. Value of VO2max was decreased in all pts. [24,3 $\pm 9,1 \mathrm{ml} / \mathrm{kg} / \mathrm{min}]$ - about $42,8 \%$ of predicted values. The level of AT was decreased in comparison to predicted values $(0,97 \pm 0,121 / \mathrm{min} ; 26,8 \pm 9,6 \% \mathrm{VO} 2 \mathrm{max})$. Serum level of NTproBNP was moderately increased $(328 \pm 127,8 \mathrm{pg} / \mathrm{ml})$.

Conclusions:

- Young people after surgical treatment of d-transposition of great arteries by Senning atrial switch revealed good exercise toleration.

- In spite of good exercise toleration, mild to moderate reduction of exercise capacity and function of circulatory system was observed in these patients.

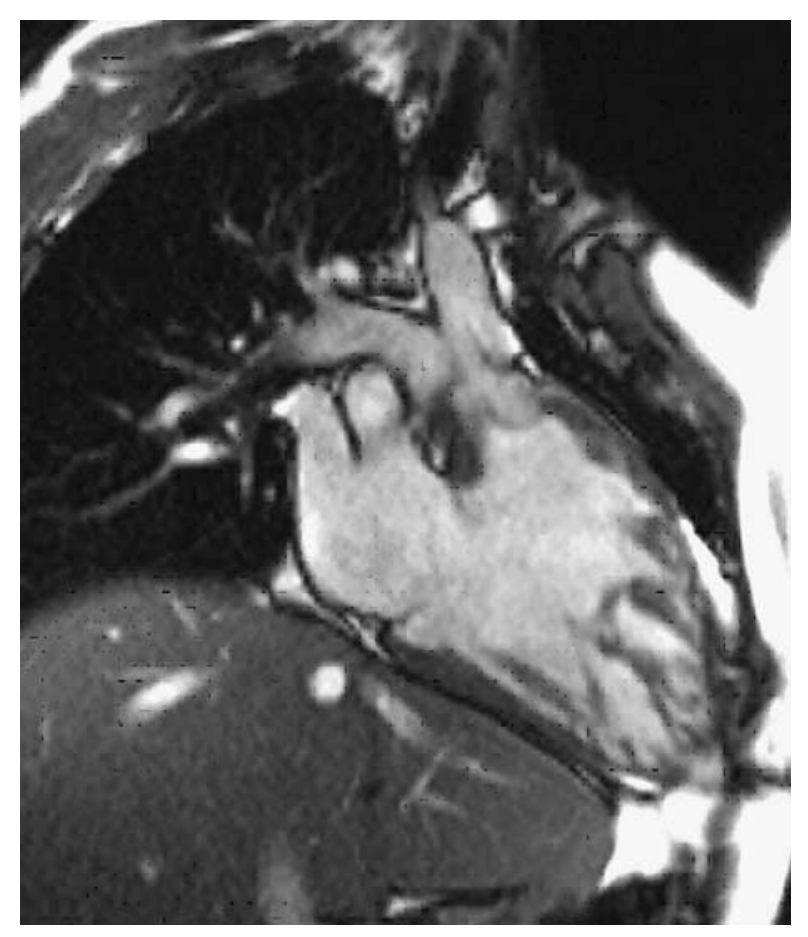

8249

Exercise capacity in young people long term after surgical treatment of tetralogy of Fallot

Artur Z Pietrucha, Coronary Disease Department, Medical School of Jagiellonian University, John Paul II Hospital, Cracow, Poland

Beata J Pietrucha, Children Cardiology Department, University Children Hospital Jagiellonian University, Cracow, Poland

Nader El-Massri, Coronary Disease Department, Medical School of Jagiellonian University, John Paul II Hospital, Cracow

Andrzej Gackowski, Coronary Disease Department, Medical School of Jagiellonian University, John Paul II Hospital, Cracow

Danuta Mroczek-Czernecka, Coronary Disease Department, Medical School of Jagiellonian University, John Paul II Hospital, Cracow Anton Chrustowicz, Coronary Disease Department, Medical School of Jagiellonian University, John Paul II Hospital, Cracow Andrzej Rudzinski, Children Cardiology Department, University Children Hospital Jagiellonian University, Cracow, Poland Wieslawa Piwowarka, Coronary Disease Department, Medical School of Jagiellonian University, John Paul II Hospital, Cracow

The aim of study was evaluation of physical efficiency and function of circulatory system in young people, long term after surgical treatment of tetralogy of Fallot (TOF). 
We observed 22 pts. (7 woman and 15 man) aged 19-23 yrs (mean age 21,2) after correction of TOF. TOF was corrected at the age 10 months and 8th years of life.

All pts underwent echocardiography examination and cardiopulmonary exercise treadmill test (ETT). Left and right ventricle function, pulmonary valve function, ETT duration and workload, and max oxygen uptake (VO2max) and anaerobic threshold (AT) were evaluated. VO2max, ETT work were also expressed as \% of predicted values

Results: (see table.)

Left ventricle function was not reduced. Right ventricle dilatation with moderately reduced ejection fraction and significant pulmonary valve insufficiency was observed in all pts.

Exercise was well tolerated by all pts. - they achieved about $90 \%$ of predicted workload.

Value of VO2max was decreased in all pts. AT was decreased in comparison to predicted values. These results indicate mild to moderate reduction of function of circulatory system.

Conclusions: Young people after surgical treatment of tetralogy of Fallot revealed good exercise toleration in spite of mild to moderate reduction of right ventricle function and pulmonary insufficiency.

\begin{tabular}{lc}
\hline VO2max [ml/kg/min] & 24,7(50,5\%) \\
\hline AT [1/min] & 0,91 \\
AT [\% VO2max] & 28 \\
Work [W] & $167(88 \%)$ \\
METS & 11 \\
RV [mm] & 46 \\
RV EF [\%] & $50,1 \%$ \\
LV [mm] & $48 / 32$ \\
LV EF [\%] & 65 \\
\hline
\end{tabular}

\section{0}

Surgical Outcome of Major Open Heart Surgery in Adults with Congenital Heart Disease

$R$ Suresh Kumar, Dept of Pediatric Cardiology, ICVD, Madras

Medical Mission, Chennai, India, India

Roy Varghese, Dept of Pediatric Cardiology, ICVD, Madras Medical

Mission, Chennai, India, India

G Ganesh Kumar, Dept of Pediatric Cardiology, ICVD, Madras

Medical Mission, Chennai, India, India

Suraj K Varma, Dept of Pediatric Cardiology, ICVD, Madras Medical Mission, Chennai, India, India

J Vimala, Dept of Pediatric Cardiology, ICVD, Madras Medical

Mission, Chennai, India, India

G Selvakumar, Dept of Pediatric Cardiology, ICVD, Madras Medical Mission, Chennai, India, India

Udaycharan Murmu, Dept of Pediatric Cardiology, ICVD, Madras

Medical Mission, Chennai, India, India

John Valliatu, Dept of Pediatric Cardiology, ICVD, Madras Medical

Mission, Chennai, India, India

Objective: There is scanty data on the surgical outcome of complex congenital heart disease in adults. This is a retrospective study of Adult congenital heart disease (ACHD) patients in an Indian tertiary care centre with focus on surgical outcome of complex ACHD.

Method: Case records of 746 consecutive patients $\geqslant 21 \mathrm{yrs}$ who attended a Pediatric Cardiology/ACHD unit during the period June 2003 through August 2008 were reviewed retrospectively. 153 patients underwent open heart surgery. Atrial Septal Defect (Ostium secundum or Sinus venosus) closure was considered "simple" (Group A) while all the other open heart surgeries were considered "Major" (Group B). The results of intracardiac repair (ICR) for Tetralogy of Fallot were compared with those of 25 children who had the same surgery.

Results: There were 102 patients in Group A and 51 in Group B. Diagram I and Table I show diagnostic categories, demographic profile and outcome variables in the group.

Outcome of ICR was comparable in adults $(n=23)$ and children $(n=25)$, but for one mortality $(4 \%)$ and higher incidence of major complications (22\% vs $7.1 \%)$.

At a median follow up of 24 months, there was no late mortality.

All patients were physically active and in NYHA class I or II. Two patients in Group A and one patient in Group B had major arrhythmias (AF/SVT).

Conclusion: In the current era, major open heart surgery can be performed in adults with low mortality and morbidity, even with the different disease profile in emerging economies.

\begin{tabular}{lccc}
\hline Variable & Group A (n=102) & Group B (n=51) & p value \\
\hline Age (yrs) & $33.4 \pm 9.1$ & $29.1 \pm 6.6$ & 0.004 \\
& $(\mathrm{M}: \mathrm{F}=37: 65)$ & $(\mathrm{M}: \mathrm{F}=29: 22)$ & \\
Weight (kg) & $56.8 \pm 12.9$ & $52.7 \pm 12.5$ & 0.075 \\
Bypass time (min) & $67.8 \pm 26.4$ & $127.2 \pm 56.5$ & $<0.001$ \\
Aortic x clamp time (min) & $36.1 \pm 21.5$ & $79.9 \pm 48.1$ & $<0.001$ \\
ICU stay (days) & $1.9 \pm 1.2$ & $4.1 \pm 2.9$ & $<0.001$ \\
Ventilation $>24 \mathrm{hrs}$ & $1(1 \%)$ & $13(27.6 \%)$ & $<0.001$ \\
Complications & $6(5.8 \%)$ & $11(21.5 \%)$ & 0.004 \\
Mortality & $\mathrm{Nil}$ & $2(4 \%)$ & 0.044 \\
\hline
\end{tabular}

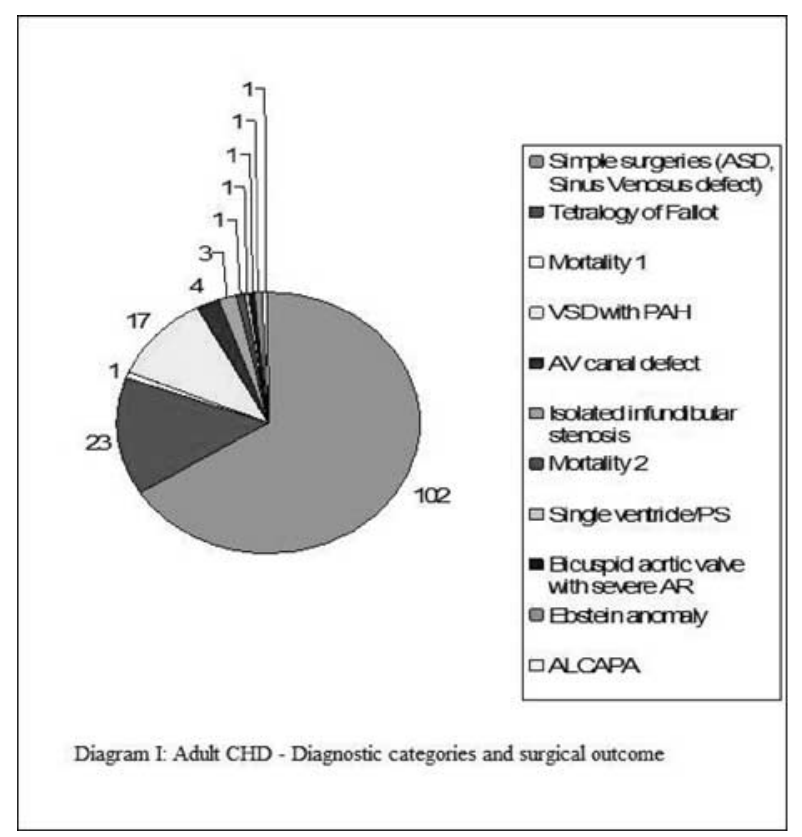

8251

Sildenafil Therapy for Pulmonary Hypertension Prior to Trial Closure of ASD with Severe Pulmonary Hypertension

Nomee A Radzi, Institut Jantung Negara, Kuala Lumpur, Malaysia Geetha Kandavello, Institut Jantung Negara, Kuala Lumpur, Malaysia Mazeni Alwi, Institut Jantung Negara, Kuala Lumpur, Malaysia Hasri Samion, Institut Jantung Negara, Kuala Lumpur, Malaysia Haifa Latiff, Institut Jantung Negara, Kuala Lumpur, Malaysia

Objective: To assess the symptoms and ability of patients with Atrial Septal Defect (ASD) and severe pulmonary hypertension (PHT) to undergo closure after oral Sildenafil. 
Method: Patients more than 16 years of age with ASD and severe PHT (mean PA:Ao ratio $>0.5$ and pulmonary vascular resistance $(\mathrm{PVR})>3$ woods units) were enrolled from January 2005 to April 2007. All were given oral Sildenafil (100-150 mg daily) for at least 6 months. The NYHA functional class, 6 minute walk, cardiopulmonary test and cardiac catheterization were done at baseline and at 6 months follow-up. Patients with $\operatorname{PVR}<8$ woods unit were subjected to closure of the ASD either by trial closure with device (initial part of study) or surgical closure with a fenestrated patch. Patients were followed up with echocardiogram, exercise test and repeat cardiac catheterization.

Results: 12 patients with mean age of 31.6 years were enrolled. The mean PA/Ao pressure was 0.56 and PVR 9 woods units. 5 patients underwent closure of the ASD (mean PA: Ao ratio 0.56, PVR 7.3). 4 out of 5 are currently well at a mean of 27 months post procedure with a mean estimated PA pressure of $24 \mathrm{mmHg}$. One patient died secondary to accelerated pulmonary vascular disease a year following closure. All patients had improvement in symptoms, 6 minute walk test and cardiopulmonary test.

Conclusion: Oral Sildenafil improved functional class status in patients with severe PHT. It had shown potential use as treatment prior to closure of ASD.

\section{2}

Evidence-based developments of the New Zealand Adult Congenital Heart Disease Service: a 5-year Action Research Summary

Annette F Rief, Auckland City Hospital, New Zealand

Developed from critical science or critical theory, action research is based on Kurt Lewins' psychological theories, who, in 1946, describes his method as a way of generating knowledge about a social system while simultaneously trying to change it, placing importance and meaning of critical thinking, analysis and reflection in every day life. When applied to the workplace, the action research methodology is based on strategic planning, effective decision making, creative problem solving and situational leadership in combination with research and development activities as well as organisational team building. This makes action research the ideal tool to introduce a new service to an existing health care organisation.

In case of the development of the New Zealand wide, Auckland based, Adult Congenital Heart Disease (ACHD) service action research was used as a quick and sharp evaluation tool, which proved ideal for assessing the initial service needs as well as the subsequent progress of the service development. A total of eight action research cycles provided ongoing evaluation of the service development process over the past 5 years. The action research cycles were used to undertake an initial needs analysis, establish teaching outcome measures and service development outcome measures using implementation and evaluation phases. A range of methods were used to examine the development progress, including semi-structured interviews, field notes and audits utilising qualitative and quantitative research methods.

\section{3}

Evaluation of 53 cases of pulmonary regurgitation following Tetralogy Of Fallot repair, in Rajaei heart center, from 2003 to 2007

Alireza Rostami MD, Arak university of medical science, Iran

Gholamreza Omrani, Iran

Bahador Baharestani, Iran

Mohamad ali Yusefnia, Iran

Nader Givtaj, Iran

Ali sadeghpour Tabaee MD, Arak university of medical science, Iran
Background: Pulmonary regurgitation (PR) is the most important residual lesion remaining after repairing tetralogy of Fallot. Through a thorough review of the data statistics of patients undergoing pulmonary valve replacement following total correction for tetralogy of fallot, and analyzing these data, the following study was performed and presented below.

Material and Methods: Database search for medical records of patients undergoing pulmonary valve replacement following total correction for tetralogy of fallot, from 2003 to 2007, that are 53 cases are gathered, analysed, and presented here.

Results and data synthesis: Age $(22.21+/-6.98$ years old), duration between the 2 operations (138.47 +/ -79.99 months), Right ventricular ejection fraction (mildly decreased in $18.6 \%$ of cases, moderately decreased in $67.9 \%$ of cases, and severely decreased in $12.2 \%$ of cases), aneurysm in outflow tract of right ventricle (20.8\%), tricuspid regurgitation (56.6\%), tricuspid stenosis (in 1 case). The valve used for pulmonary valve replacement (biologic 86.6\%), (metallic 11.2\%), (Homograft $1.9 \%$ ). Pulmonary artery pressure (lower than $25 \mathrm{mmhg}$ in 34 cases (64.2\%), between $25 \mathrm{mmhg}$ to $50 \mathrm{mmhg}$ in 7 cases $(13.2 \%)$, between $50 \mathrm{mmhg}$ and $75 \mathrm{mmhg}$ in 1 case(1.9\%), and more than 75 mmhg in 1 case.

Conclusions: Although right ventricular volume load due to severe pulmonary regurgitation after repaired tetralogy of Fallot can be tolerated for years, there is now evidence that the compensatory mechanisms of the right ventricular myocardium ultimately fail and that if the volume load is not eliminated or reduced the dysfunction might be irreversible.

\section{4}

Inflammatory Markers Are Elevated in Eisenmenger Syndrome

$S$ Ramakrishnan, All India Institute of Medical Sciences,

New Delhi, India

Amit Pandharkar, All India Institute of Medical Sciences,

New Delhi, India

Shyam S Kothari, All India Institute of Medical Sciences,

New Delhi, India

Anita Saxena, All India Institute of Medical Sciences, New Delhi, India

Balram Bhargava, All India Institute of Medical Sciences,

New Delhi, India

Rajnish Juneja, All India Institute of Medical Sciences,

New Delhi, India

Ganesan Karthikeyan, All India Institute of Medical Sciences, New Delhi, India

$R$ Lakshmy, All India Institute of Medical Sciences, New Delhi, India

Purpose: Inflammation is considered to be one of the important factors in the progression Eisenmenger syndrome (ES). Markers of systemic inflammation are not yet studied in Eisenmenger syndrome.

Methods: Twenty-two consecutive symptomatic patients of ES with a mean age of $24 \pm 10.6$ years, were enrolled in the study. Six $(27 \%)$ patients had atrial septal defect, 8 patients (36\%) had ventricular septal defect and 8 patients (36\%) had patent ductus arteriosus. Thirteen (59\%) patients were in WHO functional class II and nine (41\%) patients were in WHO class III. Inflammatory markers including hs-CRP (high sensitivity C-reactive protein), interleukin-2, interleukin-6 and interferon $\gamma$ were assayed with commercially available kits using ELISA and the values were compared with 6 healthy controls.

Results: There is significant elevation in hs-CRP and interferon $\gamma$ levels as compared to controls. The results are summarized in Table 1. 
Conclusion: Inflammatory markers including hs-CRP and interferon $\gamma$ are significantly elevated in Eisenmenger syndrome. The causes, consequences and prognostic significance of such elevations need further evaluation.

\begin{tabular}{lccc}
\hline Biomarker & $\begin{array}{c}\text { Eisenmenger } \\
\text { Syndrome } \mathbf{n}=22\end{array}$ & $\begin{array}{c}\text { Control } \\
\mathbf{n}=6\end{array}$ & $\begin{array}{c}\mathbf{P} \\
\text { Value }\end{array}$ \\
\hline hs CRP $(\mathrm{mg} / \mathrm{L})$ & $3.5+2.8$ & $0.6+0.4$ & 0.04 \\
Interferon $-\gamma(\mathrm{pg} / \mathrm{ml})$ & $21.7+12.70$ & $8.7+3.94$ & 0.02 \\
Interleukin $-6(\mathrm{pg} / \mathrm{ml})$ & $12.07+19.59$ & $2+1.22$ & 0.22 \\
Interleukin $-2(\mathrm{pg} / \mathrm{ml})$ & $19.41+13.56$ & $15+12.24$ & 0.52 \\
\hline
\end{tabular}

\section{5}

Cardiac Resynchronization Therapy for CHF after the total repair of TOF in a adult patient

Yoshiaki Satio, Department of Thoracic and Cardiovascular Surgery, Hirosaki University School of Medicine, Japan

Kazuyuki Daitoku, Department of Thoracic and Cardiovascular

Surgery, Hirosaki University School of Medicine, Japan

Sanae Yamauchi, Department of Thoracic and Cardiovascular Surgery,

Hirosaki University School of Medicine, Japan

Yasuyuki Suzuki, Department of Thoracic and Cardiovascular Surgery, Hirosaki University School of Medicine, Japan

Ikuo Fukuda, Department of Thoracic and Cardiovascular Surgery,

Hirosaki University School of Medicine, Japan

Tetralogy of Fallot (TOF) in adult patients are rare and only few cases have been reported its surgical outcomes. We report a case of 65-year-old female TOF patient with a history of transient loss of consciousness presented with cyanosis and shortness of breath. Her oxygen saturation was $82 \%$ in room air. Subsequent investigations revealed remarkable right ventricular hypertension of 99/edp10 $\mathrm{mmHg}$, obstruction to right ventricular outflow with PA pressure of $15 / 9$ (11) $\mathrm{mmHg}$, and underling NYHAIII chronic heart failure $(\mathrm{CHF})$ with complete $\mathrm{A}-\mathrm{V}$ block. The patient was preoperatively treated with medications and inserted a percutaneous pacemaker for bradyarythmia. We performed ventricular septal defect closure, pulmonary valve replacement associated with right ventricular outflow tract repair, tricuspid valve repair and pacemaker implantation. Although her recovery from the surgery was uneventful, she revealed symptomatic CHF one and a half years after the surgery. Cardiac catheterization showed significant left ventricular enlargement and diffuse poor wall motion of the heart muscle. We managed the CHF providing with cardiac resynchronization therapy (CRT), which is an adjunctive treatment currently indicated for CHF patients. Left ventricular (LV) hypokinesis with an ejection fraction (EF) of $15.3 \%$ on admission improved to normal LV function with an EF of 58.1\% 2 years after starting CRT. She remains asymptomatic and has lived without oxygen therapy. In conclusion, concrete surgical repair for TOF in adult patients is recommended. Although the exact origin of the $\mathrm{CHF}$ is still unknown, CRT was efficient for managing postoperative cardiac dysfunction.

\section{6}

Prevalence of Adult Patients with Congenital Heart Disease in Japan

Yumi Shiina, Chiba Cardiovascular Center Department of Adult Congenital Heart Disease and Pediatrics, Japan

Tomohiko Toyoda, Chiba Cardiovascular Center Department of Adult Congenital Heart Disease and Pediatrics, Japan
Yasutaka Kawasoe, Chiba Cardiovascular Center Department of Adult Congenital Heart Disease and Pediatrics, Japan

Shigeru Tateno, Chiba Cardiovascular Center Department of Adult Congenital Heart Disease and Pediatrics, Japan

Takeaki Shirai, Chiba Cardiovascular Center Department of Adult Congenital Heart Disease and Pediatrics, Japan

Yuko Wakisaka, Chiba Cardiovascular Center Department of Adult Congenital Heart Disease and Pediatrics, Japan

Kozo Matsuo, Chiba Cardiovascular Center Department of Adult Congenital Heart Disease and Pediatrics, Japan

Koichiro Niwa, Chiba Cardiovascular Center Department of Adult

Congenital Heart Disease and Pediatrics, Japan

Background: Today most patients with congenital heart disease (CHD) can survive into adulthood. But data regarding the number of adults with CHD in Japan are scarce. Our study aims to define the number of adults with CHD.

Material and Methods: The expected numbers of infants with major CHDs since 1947 were calculated from birth and mortality rates. We estimated the numbers of CHD survivors from data on natural history and long-term mortality rate. The number of death from 1968 to 1997 was analyzed using individual death certificates held by Japanese Government.

Results: In 1967, 140,495 patients with CHD including 50,704 adults were assumed to be alive. From 1968 to 1997, 548,360 patients with CHD were born and 82,919 died. Except for the patients with spontaneous closure of VSD, 523,682 patients were estimated to be alive in 1997 with 217,084 children (42\%) and 306,598 adults $(58 \%)$. In 2007, 442,773 adults are alive. From 1997 to 2007, about 13,000 adults/year have been increasing. Conclusions: The prevalence in adults with CHD has been explosively increasing from 1967 to 2007 . Total 442,773 adults with CHD are alive in 2007 with annual increase of 13,000. These data are useful for further establishment of the special facilities and resources for adult CHD patients not only in Japan but also in other Asian countries.

8257

Assessment of cardiac function using tissue Doppler echocardiography during pregnancy in adult congenital heart disease patients

Mikiko Shimizu, Toyko Women's Medical University, Japan Yoshio Matsuda, Toyko Women's Medical University, Japan Tokuko Shinohara, Toyko Women's Medical University, Japan Toshio Nakanishi, Toyko Women's Medical University, Japan

Objective: The number of adult congenital heart disease patients (ACHD) is increasing, and the management of pregnancy in ACHD is still challenging. There is no established data regarding cardiac function during pregnancy in ACHD. This study was to evaluate cardiac function during pregnancy using tissue Doppler echocardiography (TDI). TDI indices have been shown to be less load dependent than conventional method, thus sought to be suitable for evaluation during pregnancy.

Method: Sixteen ACHD patients with NYHA class I/II were recruited. Three patients were with systemic RV. Age matched control were also enrolled $(n=14)$. Three serial echocardiographies were performed at 11-17, 21-25, and 31-34 weeks of gestation. Conventional indices; EF, FS, in-flow pattern, and Tei index were measured as well as TDI indices including isovolumic acceleration (IVA), systolic wall velocity (SW), E and A velocities. Mode of delivery, gestational age and birth weight were also recorded.

Results: All the TDI indices stayed unchanged in both groups throughout pregnancy (repeated measure of ANOVA). Two out 
of three with systemic RV showed significant deterioration of the $\mathrm{RV}$ function (decrease IVA and increased $\mathrm{E} / \mathrm{Ea}$ and Tei), and required medical intervention. The birth weight of the baby from systemic RV was $17 \pm 3.5 \%$ tile, where systemic LV was $51.4 \pm 39.5 \%$ tile. Those who showed significant deterioration of LV IVA and/or FS had smaller babies (28 $\pm 22 \%$ tile) than who stayed unchanged $(71 \pm 41 \%$ tile).

Conclusion: TDI was useful tool to evaluate cardiac function of pregnant ACHD patients. Careful management is warranted when deterioration of LV IVA, FS and E/Ea are observed.

\section{8}

Fontan surgery and pregnancy: long-term outcomes and management

Tokuko Shinohara, Tokyo Women's Medical University, Japan

Tetsuko Ishii, Tokyo Women's Medical University, Japan

Aki Ikeda, Tokyo Women's Medical University, Japan

Shuhei Fujita, Tokyo Women's Medical University, Japan

Daiji Takeuchi, Tokyo Women's Medical University, Japan

Kazuhiro Takahashi, Tokyo Women's Medical University, Japan

Yoshiki Mori, Tokyo Women's Medical University, Japan

Toshio Nakanishi, Tokyo Women's Medical University, Japan

Aim: To obtain the long-term outcomes after pregnancy in the patients with Fontan circulation [objective] Five women aged 25-42 years at the time of the study, who had Fontan (APC:3, Bjork:1, TCPC:1) operation in 1976-1995 and successful pregnancy in 1997-2007 at one institution.

Methods: The cardiac history of each patient before/after Fontan operation and pregnancy, with the major events such as heart failure, arrhythmia, symptomatic thrombosis, TCPC conversion was clarified in a style of the chronological table retrospectively utilizing medical record.

Results: Follow-up time after pregnancy was $2-12$ years $(m=6)$. The time between Fontan operation and pregnancy was 13 years at average (7-16 years). Three in five $(60 \%)$ had major events in $2-10$ years after pregnancy. One had TCPC conversion with a pacemaker implantation (PMI) following significant atrial thrombosis, pulmonary infarction and cerebral vascular event 6 years after pregnancy. Difficult atrial brady-tachyarrhythmia was occurred in one who required EPS/ ablation and PMI in 10 years later. The deep vein thrombosis was seen and treated in the same patient. One had atrial flutter with heart failure 2 years after pregnancy, which was 16 years from Fontan operation. Four patients were in NYHA class I, and one in II.

Conclusions: The successful pregnancy did not affect apparently the outcomes of Fontan surgery in long term in our institution. The timing of pregnancy in a chronological management of female Fontan patients was important in addition to the essential condition of highly selected good Fontan status.

\section{9}

Review of surgical correction of Scimitar Syndrome at TPCH, Brisbane, Australia

Sunil Kumar Swain, The Prince Charles Hospital, Brisbane, Australia Andrew Clarke, The Prince Charles Hospital, Brisbane, Australia Homayoun Jalali, The Prince Charles Hospital, Brisbane, Australia Robert Tam, The Prince Charles Hospital, Brisbane, Australia Peter Tesar, The Prince Charles Hospital, Brisbane, Australia Peter Pohlner, The Prince Charles Hospital, Brisbane, Australia

Background: Scimitar Syndrome is a rare complex anomaly where one or all right sided pulmonary veins drains to the IVC, hepatic veins or azygous vein. It is rare with an incidence of $0.5 \%$ of all congenital heart disease. Despite various surgical procedures, the reported surgical outcome are very disappointing. We reviewed seven cases of Scimitar syndrome, operated at Prince Charles hospital between 1994 to 2007.

Materials and Methods: Between Dec-1994 to June-2007, seven patients having a scimitar syndrome underwent corrective surgery at our hospital. There were 3 females and 4 males. The youngest was 3 yrs old and the oldest was 74 yrs old. Their follow up ranges from $1 \mathrm{yr}$ to $14 \mathrm{yrs}$. All patients underwent baffle correction of scimitar vein.

Results: All patients undergoing baffle correction of a scimitar vein to the LA were extubated the day following the operation. Immediate post operative period was satisfactory. There was no thirty day mortality. One adult patient had a non coronary cusp aortic leaflet perforation and underwent aortic valve replacement three weeks after surgery. Two patients developed baffle obstruction over a two year period at subsequent follow up. Of the remaining five patients, four are well. One elderly patient died eight years post operatively from a non cardiac cause.

Conclusion: Though rare and technically demanding, correction of scimitar syndrome at all ages is feasible with minimal risk to survival. However disappointing functional results remain in a significant number.

\section{0}

Aortic Compliance Determination in Post-Repaired Tetralogy of Fallot Patients Using Magnetic Resonance Imaging

Thu-Thao Le, National Heart Centre, Singapore

Ru-San Tan, National Heart Centre, Singapore

Ada X Ng, King's College of London, United Kingdom

Sann $\mathrm{Nu}$ Wai, National Heart Centre, Singapore

Myo Myint Aung, Nanyang Technological University, Singapore

Ju-Le Tan, National Heart Centre, Singapore

Background \& Aim: Intrinsic histological changes in the aorta, such as fibrosis, elastic fragmentation, and disruption of elastic lamellae, may alter the aortic elastic properties and lead to progressive aortic root dilatation in post-repaired tetralogy of Fallot (TOF) patients. We aim to compare the aortic compliance, using magnetic resonance imaging (MRI), between post-repaired TOF patients and normal controls.

Methods: We retrospectively studied 31 post-repaired TOF patients (22 \pm 8 years, 23 men) and 24 normal controls (22 5 years, $12 \mathrm{men}$ ) who underwent MRI scans (1.5T Siemens). Left ventricular (LV) mass, LV and RV end-diastolic (EDV) and end-systolic (ESV) volumes were determined from short-axis cine MR images using standard methodology and indexed to body surface area. Ejection fraction $(E F)$ was calculated. Minimum (Amin) and maximum (Amax) aortic diameters were obtained from velocity-encoded flow mapping of the ascending aorta (ARGUS, Siemens). Aortic compliance was calculated as (Amax-Amin)/(Amin $\times$ pulse pressure), where pulse pressure is the difference between systolic and diastolic blood pressure.

Results: Indexed LVEDV, RVEDV, RVESV, LVEF and RVEF were significantly difference between the 2 groups (Table). Compared with the normal controls, aortic compliance was significantly lower in post-repaired TOF patients.

Conclusions: Decreased aortic compliance indicates increased aortic stiffness in post-repaired TOF patients in comparison to normal controls. This non-invasive assessment agrees with the findings of intrinsic histological abnormalities in TOF and may have potential implications for clinical management. 
Table. MR-derived LV and RV functional parameters, and aortic compliance in post-repaired TOF patients and normal controls.

\begin{tabular}{|c|c|c|c|}
\hline Parameters & $\begin{array}{l}\text { Post-repaired } \\
\text { TOF }(\mathrm{n}=31)\end{array}$ & $\begin{array}{l}\text { Normal } \\
\text { Controls } \\
(n=24)\end{array}$ & $\mathrm{p}$ value \\
\hline Indexed LVEDV $\left(\mathrm{ml} / \mathrm{m}^{2}\right)$ & $77.9 \pm 23.9$ & $92.3 \pm 13.2$ & 0.011 \\
\hline Indexed LVESV $\left(\mathrm{ml} / \mathrm{m}^{2}\right)$ & $35.1 \pm 14.3$ & $36.9 \pm 10.5$ & ns \\
\hline Indexed LVM $\left(\mathrm{g} / \mathrm{m}^{2}\right)$ & $50.9 \pm 16.0$ & $50.8 \pm 8.1$ & ns \\
\hline $\operatorname{LVEF}(\%)$ & $55.7 \pm 7.1$ & $60.5 \pm 6.7$ & 0.015 \\
\hline Indexed RVEDV $\left(\mathrm{ml} / \mathrm{m}^{2}\right)$ & $171.8 \pm 41.6$ & $88.9 \pm 15.3$ & $<0.001$ \\
\hline Indexed RVESV $\left(\mathrm{ml} / \mathrm{m}^{2}\right)$ & $99.4 \pm 32.3$ & $36.7 \pm 13.0$ & $<0.001$ \\
\hline $\operatorname{RVEF}(\%)$ & $42.9 \pm 7.3$ & $59.6 \pm 8.0$ & $<0.001$ \\
\hline $\begin{array}{c}\text { Aortic compliance } \\
(0.001 / \mathrm{mmHg})\end{array}$ & $5.64 \pm 4.19$ & $11.82 \pm 6.02$ & $<0.001$ \\
\hline
\end{tabular}

\section{1}

Erectile Dysfunction in Young Men with Congenital Heart Disease

David Tanous, Australia

Adrienne H Kovacs, Toronto Congenital Cardiac Centre for Adults, Peter Munk Cardiac Centre, University Health Network/Toronto General Hospital, Canada

Matthias Greutmann, Toronto Congenital Cardiac Centre for Adults, Peter Munk Cardiac Centre, University Health Network/Toronto General Hospital, Canada

Olga H Balint, Toronto Congenital Cardiac Centre for Adults, Peter Munk Cardiac Centre, University Health Network/Toronto General Hospital, Canada

Nicholas Collins, Toronto Congenital Cardiac Centre for Adults, Peter Munk Cardiac Centre, University Health Network/Toronto General Hospital, Canada

Miguel Llano, Murray Koffler Urologic Wellness Centre, Mount Sinai Hospital, Toronto, Canada

Candice K Silversides, Toronto Congenital Cardiac Centre for Adults, Peter Munk Cardiac Centre, University Health Network/Toronto General Hospital, Canada

Erwin Oechslin, Toronto Congenital Cardiac Centre for Adults, Peter Munk Cardiac Centre, University Health Network/Toronto General Hospital, Canada

Background: Men with acquired heart disease are at risk for erectile dysfunction (ED); however, the extent to which this is an issue in young men with congenital heart disease (CHD) is unknown. The goal of this study was to determine the prevalence and risk factors of ED in men with CHD.

Methods: Males attending an adult CHD clinic completed the International Index of Erectile Function, International Prostate Symptom Score; Short Form-12; and Satisfaction with Life Survey. Univariate and independent risk factors were determined for the presence of ED.

Results: Of 128 respondents, 102 (mean age $36 \pm 13$ years) were in stable relationships and/or sexually active and therefore, suitable for analysis. Twenty-eight percent reported some degree of ED. The predictors of ED were age $(p<0.01)$ and hypertension $(p<0.05)$, yet functional class $(p=0.53)$, defect complexity $(p=0.46)$, pulmonary hypertension $(p=0.53)$ or ventricular dysfunction $(p=0.86)$ were not. Patients with ED had poorer perceived health $(p<0.01)$ and lower life satisfaction $(p<0.05)$. Using a multivariate logistic regression model, age was the only independent predictor of ED (OR 1.04/year, 95\% CI 1.01 to $1.08, \mathrm{p}=0.01$, or OR $4.2,95 \%$ CI 1.4 to 12.8 , $\mathrm{p}=0.01$, for age $>50$ years in a repeat analysis).
Conclusions: Erectile dysfunction was present in $28 \%$ of the men and correlated with poorer quality of life measures. Age and hypertension, but not disease complexity or functional class were the predictors of $\mathrm{ED}$, suggesting that $\mathrm{CHD}$ per se does not contribute to the presence of ED, rather that it relates to underlying vascular disease.

\section{2}

Improvement in Left Ventricular Function after Pulmonary Valve Replacement in Patients with Tetralogy of Fallot David Tanous, Australia

Jasmine Grewal, Toronto Congenital Cardiac Centre for Adults, Peter Munk Cardiac Centre, University Health Network/Toronto General Hospital, Canada

Laura Dos Subira, Toronto Congenital Cardiac Centre for Adults, Peter Munk Cardiac Centre, University Health Network/Toronto General Hospital, Canada

Christopher Calderone, Division of Cardiovascular Surgery, Hospital for Sick Children, Toronto, Canada

Erwin Oechslin, Toronto Congenital Cardiac Centre for Adults, Peter Munk Cardiac Centre, University Health Network/Toronto General Hospital, Canada

Candice K Silversides, Toronto Congenital Cardiac Centre for Adults, Peter Munk Cardiac Centre, University Health Network/Toronto General Hospital, Canada

Background: Left ventricular (LV) dysfunction can occur in patients with repaired tetralogy of Fallot (rTOF), due partly to adverse ventricular-ventricular interactions. The purpose of this study was to determine whether pulmonary valve implantation (PVR) and subsequent right ventricular (RV) remodelling, was associated with improvement in LV function.

Methods: Pre- and post-operative echocardiograms of consecutive patients with rTOF undergoing PVR between 2003 and 2008 at a single centre were reviewed. Exclusion criteria for patients included: a) inadequate echocardiographic images, b) not in sinus rhythm at either examination, c) predominant pulmonary stenosis, d) undergoing concomitant tricuspid, aortic or mitral valve surgery. Global $\mathrm{RV}$ and LV function was measured using the myocardial performance index (MPI) and LV volumes using the area-length method. Comparisons between pre- and post-operative data were made using Wilcoxon signed-rank test.

Results: Twenty-four patients (mean age $32 \pm 10$ years) were included. Mean age at intra-cardiac repair (transannular patch in $75 \%$ ) was $6 \pm 3$ years. The RV end-diastolic diameter decreased from $52 \pm 7 \mathrm{~mm}$ preoperatively to $43 \pm 9 \mathrm{~mm}$ post-operatively $(\mathrm{p}<0.001)$. Global RV (RV MPI $0.28 \pm 0.11$ vs. $0.23 \pm 0.12, p=0.03$ ) and LV (LV MPI $0.49 \pm 0.17$ vs. $0.42 \pm 0.15, p=0.04)$ function improved after surgery. The LV end-diastolic volume increased (101 \pm 39 vs. $114 \pm 35 \mathrm{~mL}, \mathrm{p}=0.03)$, but LV end-systolic volume was unchanged $(47 \pm 22$ vs. $48 \pm 17 \mathrm{~mL}, \mathrm{p}=0.47)$ with a resultant increase in $\mathrm{LV}$ ejection fraction ( $54 \pm 6 \%$ vs. $58 \pm 6 \%, p=0.02)$.

Conclusions: In patients with rTOF, global LV function shows improvement after PVR. Improvements in ventricular-ventricular interactions, as a result of reduction in RV size, are a possible explanation for this finding.

\section{6}

Gender evaluates clinical course in adult patients with congenital heart disease

Olga Trojnarska, Cardiac Departmant University of Medical Sciences Poznan, Poland

Agnieszka Bartczak, Poland

Stefan Grajek, Poland 
Introduction: Clinical course and patients' $(\mathrm{P})$ prognosis with ischaemic disease and heart failure varies between genders.

The aim of this study: Evaluation if gender affects clinical course of adults with congenital heart defects (CHD) in long-term observation.

Material and method: From 1995 to 2006 (mean 5,53 $\pm 2,5$ years) we followed-up $1653 \mathrm{P}$ (860 female) aged 18-74 years $(33,4 \pm 10,2)$. Following clinical events were analysed: supraventricular arrhythmia (SVA): paroxysmal supraventricular tachycardia or/and atrial fibrillation/flutter, ventricular arrhythmia (VA): $\geqslant 30 / \mathrm{h}$, conduction and rhythm disturbances (CRD): block AVI-III, nodal rhythm, HR $<40 / \mathrm{min}$, pauses $\geqslant 2 \mathrm{~ms}$, NYHA $\geqslant$ III, death.

P represented 21 different CHD - simple: ASD, VSD, PDA, BAV, pulmonary stenosis, pulmonary venous connection anomaly, mitral insufficiency, Wiliams, Marfan, Ebstein, Eisenmenger syndromes, and complex: coarctation of aorta, Fallot tetralogy, ASDI, CAVC, subvalvular AS, univentricular heart, CCTGA, DTGA, DORV, pulmonary atresia. Kaplan-Meier curves were estimated, log-rank tests were calculated to compare curves for genders.

Results: SVA occurred among $10,7 \% \mathrm{P}$, after five years $-14,5 \% \mathrm{P}$. VA appeared in $6,3 \% \mathrm{P}$, after five years $-11,2 \% \mathrm{P}$. CRD occurred among $10,8 \% \mathrm{P}$, after five years $-11,2 \%$. NYHA $\geqslant$ III was observed among $2,6 \% \mathrm{P}$, after five years $-3,7 \% \mathrm{P}$, death among $2,3 \%$ P. Survival rate after five years- $96,8 \%$. Incidence of VA, SVA, NYHA $\geqslant I I I$, death did not differ between genders $(p=0,54),(p=0,32),(p=0,13),(p=0,45)$ respectively. Higher probability of CRD was observed among men $(p=0,005)$, especially with complex defects $(p=0,0002)$.

Conclusions: 1. Probability of conduction and rhythm disturbances is higher among men. 2. Incidence of SVA, VA, $\mathrm{NYHA} \geqslant \mathrm{III}$, death does not differ between genders.

\section{7}

Clinical problems during pregnancy and childbirth in patients with congenital heart diseases

Olga Trojnarska, Cardiology Department University of Medical Sciences Poland, Poland

Joanna Jarek, Cardiology Department University of Medical Sciences

Poland, Poland

Karolina Plaskota, Cardiology Department University of Medical

Sciences Poland, Poland

Agnieszka Bartczak, Poland

Stefan Grajek, Poland

Pregnancy and childbirth in patients $(\mathrm{P})$ with congenital heart defects (CHD) leads to cardiologic complications (CC). The aim of the study is to evaluate frequency of $\mathrm{CC}$ in pregnants with CHD.

Material and methods: from 2003-2008 we followed-up course of 232 pregnancies in 194 females aged $19-43$ years $(27,2 \pm 5,1)$ in tertiary center. $134 \mathrm{P}$ underwent surgery. $102 \mathrm{P}$ presented shunts defects (ASD/operated ASD, VSD/operated VSD, operated CAVC), 37P- dominant left ventricular leasions (operated aortic coarctation, operated/unoperated aortic stenosis and/or insufficiency, Marfan), 38P- dominant right ventricle anomalies and single ventricle (operated ToF, operated DORV, Ebstein, TGA-Senning, CCTGA, Eisenmenger, postFontan operation, operated truncus arteriosus), 7P- mechanical valve prosthesis, $48 \mathrm{P}$ - defects without hemodynamic significance (operated PDA, $\mathrm{BAV}$, operated pulmonary venous connection anomaly). CC in pregnancy: deterioration of cardiac function (HF) - increase NYHA class of al least one functional class, supraventricular arrhythmia (SVA): paroxysmal supraventricular tachycardia or/ and atrial fibrillation/flutter, ventricular arrhythmia (VA) $\geqslant 30 / \mathrm{h}$. Comparing multiple proportion determined differences in frequency of CC between analysed subgroups.

Results: Death was not observed. HF was found in 28P $(12,1 \%)$, VA in $15 \mathrm{P}(6,5 \%)$, SVA in $20 \mathrm{P}(8,6 \%)$. HF occurred significantly more often in $\mathrm{P}$ with right heart defects and single ventricle $(p<0,01)$ in comparison to every other group of P. Analysis of frequency occurrence of VA and SVA did not reveal any significant differences between subgroups.

Conclusions: 1. Pregnancy in $\mathrm{P}$ with $\mathrm{CHD}$ followed-up by specialized cardiologists and obstetricians is safe. 2. The most frequent complication is deterioration of HF observed especially in $\mathrm{P}$ with right heart defects and single ventricle.

\section{8}

Right Ventricular Strain Parameters Correlate with RV Ejection Fraction and Exercise Performance in Adults with Congenital Heart Disease Undergoing MRI

Uyen T Truong, Children's National Medical Center, United States Karen S Kuehl, Children's National Medical Center, United States Craig A Sable, Children's National Medical Center, United States Xiaokui Li, Oregon Health Science University, United States Russell Cross, Children's National Medical Center, United States Lowell Frank, Children's National Medical Center, United States David J Sahn, Oregon Health Science University, United States

Background: Assessment of right ventricular (RV) function plays a critical role in managing adults with congenital heart disease (CHD). We have previously validated vector velocity imaging (VVI) for determining ventricular mechanics on gradient cine MRI images compared to SPAMM tagging. We used VVI in this study to derive information on RV and left ventricular (LV) function and mechanics from cine MRI in patients with CHD. Methods: Adults with tetralogy of Fallot (ToF), and d- and 1transposition who underwent cardiac MRI were included. VVI parameters (strain, strain rate, and radial velocity) were obtained from short and long axis cines of the LV and RV. VVI parameters were compared by linear regression to RV end-diastolic volume, MRI ejection fraction (EF), and VO2 Max on exercise stress test. Results: VVI data were available for the LV in 26 and for the RV in 30 patients. MRI RV EF correlated with RV short axis radial strain $(\mathrm{r}=0.57, \mathrm{p}=0.005$, figure) and strain rate $(\mathrm{r}=0.57$, $\mathrm{p}=0.004)$. VO2 max correlated with $\mathrm{RV}$ radial velocity $(\mathrm{r}=0.76, \mathrm{p}<0.001)$ and strain rate $(\mathrm{r}=0.45, \mathrm{p}=0.08) . \mathrm{LV}$ long axis circumferential strain, radial displacement, and radial strain rate correlated with both MRI LV and RV EF in ToF. Conclusions: VVI analysis of gradient cine MRI images is a promising technique in assessing RV function and ventricularventricular interaction in adults with $\mathrm{CHD}$ affecting the right ventricle. RVEF on MRI could be predictive of both RV and LV strain mechanics.
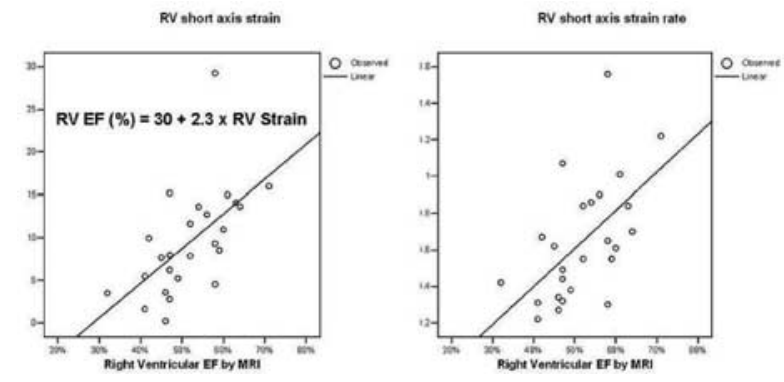
8269

Percutaneous Closure of Patent Foramen Ovale in Young stroke Patients with 36 months follow-up

Guang-yi Wang, Department of cardiology, Chinese PLA general hospital, China

Zhi-feng Wang, China

Hai-jun Hou, China

Yue Li, China

Objective: Rencently several years, clinical investigation of percutaneous PFO device trial showed the recurrent thromboembolic event is improved by the percutaneous PFO closure. The PFO Amplatzer occluder device (PFOAOD) for percutaneous closure can now be utilized. We conducted a prospective study collecting data regarding the success of percutneous PFO closure and the incidence of recurrent thromboembolic events in patients with paradoxical embolism.

Methods: 30 patients between the ages of 30 and 60 had undergone percutaneous PFO closure. All patients previously suffered from embolic strokes and were placed on anticoagulant therapy. 10 patients had suffered from additional embolic events. 10 patients presented with atrial septal aneurysms and 10 with symptoms of migraine. Follow-up: Medication was aspirin $100 \mathrm{mg}$ with plavix $75 \mathrm{mg}$ and prophylactic antibiotic therapy for 6 months post PFO closure. Patients discontinued prior wafarin therapy unless there was a concomitant illness requiring continued therapy (e.g DVT). The 60-day embolic event free success rate is $90 \%$. One patient had a transient event related to complicated procedure placement. All patients after discharge have been event free currently. Baseline and 36 months follow-up transesophageal echocardiogram with bubble contrast injection under valsalva maneuver will be reported. One patient had recurring symptoms of migraine.

Conclusion: Transcatheter closure of PFO can be accomplished safety and successfully. Preliminary closure results of PFO showed a decreased rate of recurrent embolic events. A large scale randomized control trial may be warranted to confirm these results.

8270

Transcatheter Closure of the Geriatric Secundum Atrial Septal Defects Using Amplatzer Device

Zhi-feng Wang, China

Guang-yi Wang, Department of cardiology, Chinese PLA general hospital, China

Hai-jun Hou, China

Objective: The purpose of this study was to evaluate the feasibility and safety of transcatheter closure of the geriatric secundum atrial septal defects (TCGASDs) using Amplatzer occluder device. This paper, however, is to report on our clinical experience with this device.

Materia and Methods: Between August in 1998 to October in 2007, the geriatric secundum atrial septal defects for 93 patients was diagnosed At a average age of 69.2 $\pm 6.1 \mathrm{yr}$ (range, $60 \sim 74 \mathrm{yr}$ ) and average weight of $63.6 \pm 14.6 \mathrm{Kg}$ (range $60 \sim 76 \mathrm{Kg}$ ). The average ASD diameter measured by transthoracic echocardiography (TTE) was $20.9 \pm 14.7 \mathrm{~mm}$ (range, $16 \sim 36 \mathrm{~mm}$ ) and transesophageal echocardiography (TEE) was $21.6 \pm 14.9 \mathrm{~mm}$ (range, $17 \sim 38 \mathrm{~mm}$ ), and the average ASD balloon stretched diameter was $24.2 \pm 12.8 \mathrm{~mm}$ (range, $19 \sim 38 \mathrm{~mm}$ ). All patients had right atrial and ventricular volume overload with a $\mathrm{QP} / \mathrm{QS}$ of $3.4 \pm 1.3(1.6 \sim 6.2)$ A F8 12 catheter was used for delivery of the device in all patients.
Results: The device was placed correctly in all patients. There was immediate and complete closure in 63/93 patients. There was one episode of device embolization in right ventricle after procedure for 36 hours. The death due to pericardial tamponade and the sinus bradycardia and hemourine caused by bladder tumor after released device was in one case respectively. From 24 hour to 1 year after procedure, there was complete closure of the ASD in all closured patients.

Conclusion: TCGASDs using Amplatzer occluder device was an more efficient and relatively safety nonsurgical method. The successful rate of technique was for $98 \%$ in this group.

\section{1}

Do patients over 40 years of age benefit from closure of atrial septal defects: a comparison of cardiac remodeling assessed by $2 \mathrm{D}$-echocardiography and plasma NTproBNP levels following ASD closure

Martin NL Wong, Sarawak General Hospital, Malaysia

Prashant Joshi, Sarawak General Hospital, Malaysia

Kimbee Lau, Sarawak General Hospital, Malaysia

Kuihian Sim, Sarawak General Hospital, Malaysia

Objectives: Previous studies suggested patients over 40 years old do not benefit as much from atrial septal defects (ASD) closure compared to younger patients. We aimed to evaluate and compare the magnitude of cardiac remodeling following ASD among them.

Methods: 20 patients (median age 27.6 years, range 6.5 to 64.6 years) with isolated ASD underwent ASD closure (6 transcatheter device closure, 14 surgical closure) and were studied. They were divided into two age groups: $<40$ years $(n=15)$ and $\geqslant 40$ years $(\mathrm{n}=5)$. Plasma NT-proBNP levels and the following echocardiographic parameters were measured before, at 2 months and at 6 months after ASD closure: right atrial (RA) and left atrial (LA) sizes, right ventricular $(\mathrm{RV})$ and left ventricular $(\mathrm{LV})$ volume, $\mathrm{LV}$ ejection fractions (LVEF) and LV eccentric index.

Results: RA size and RV volume decreased whereas LV volume, LVEF and LV eccentric indices increased significantly following ASD closure with similar magnitude of changes seen between the two age groups. The plasma NT-proBNP levels also decreased significantly (loge NT-proBNP values from $4.95 \pm 1.28 \mathrm{pg} / \mathrm{ml}$ to $4.50 \pm 1.01 \mathrm{pg} / \mathrm{ml} ; \mathrm{p}=0.02)$. Similar trend of reduction was seen in both age groups and at 6 months, there was no difference in the plasma NT-proBNP levels between them (loge NT-proBNP values $4.34 \pm 0.93 \mathrm{pg} / \mathrm{ml}$ versus $\left.4.94 \pm 1.19 \mathrm{pg} / \mathrm{ml} ; \mathrm{p}={ }^{`} 0.27\right)$.

\begin{tabular}{|c|c|c|c|c|c|c|c|}
\hline \multirow[b]{2}{*}{ Paremeter } & \multicolumn{2}{|c|}{$<40$ years } & \multicolumn{4}{|c|}{$\geqslant 40$ years } & \multirow{2}{*}{$\begin{array}{c}\begin{array}{c}\text { Between } \\
\text { age } \\
\text { groups }\end{array} \\
\text { p value }\end{array}$} \\
\hline & Before & After & $\mathrm{p}$ value & Before & After & $\mathrm{p}$ value & \\
\hline $\begin{array}{l}\text { RA size } \\
(\mathrm{cm} 2 / \mathrm{m} 2)\end{array}$ & $\begin{array}{c}17.0 \pm \\
3.4\end{array}$ & $\begin{array}{r}9.5 \pm \\
1.9\end{array}$ & 0.043 & $\begin{array}{c}20.0 \pm \\
9.7\end{array}$ & $\begin{array}{c}12.4 \pm \\
4.7\end{array}$ & $<0.001$ & 0.931 \\
\hline $\begin{array}{l}\text { LA size } \\
\qquad(\mathrm{cm} 2 / \mathrm{m} 2)\end{array}$ & $\begin{array}{c}10.2 \pm \\
3.1\end{array}$ & $\begin{array}{c}10.2 \pm \\
2.0\end{array}$ & NS & $\begin{array}{r}12.6 \pm \\
3.4\end{array}$ & $\begin{array}{c}10.8 \pm \\
1.9\end{array}$ & NS & 0.777 \\
\hline $\begin{array}{l}\text { RV volume } \\
(\mathrm{ml} / \mathrm{m} 2)\end{array}$ & $\begin{array}{r}112.0 \pm \\
31.2\end{array}$ & $\begin{array}{r}53.2 \pm \\
18.5\end{array}$ & 0.005 & $\begin{array}{c}109.9 \pm \\
31.9\end{array}$ & $\begin{array}{r}57.2 \pm \\
19.0\end{array}$ & $<0.001$ & 0.487 \\
\hline $\begin{array}{l}\text { LV volume } \\
(\mathrm{ml} / \mathrm{m} 2)\end{array}$ & $\begin{array}{r}26.7 \pm \\
8.4\end{array}$ & $\begin{array}{r}48.9 \pm \\
10.7\end{array}$ & 0.012 & $\begin{array}{r}29.6 \pm \\
12.2\end{array}$ & $\begin{array}{r}53.4 \pm \\
21.6\end{array}$ & $<0.001$ & 0.306 \\
\hline LVEF (\%) & $45 \pm 8$ & $64 \pm 9$ & 0.020 & $44 \pm 12$ & $63 \pm 10$ & $<0.001$ & 0.927 \\
\hline $\begin{array}{l}\text { LV eccentric } \\
\text { index }\end{array}$ & $\begin{array}{r}0.72 \pm \\
0.09\end{array}$ & $\begin{array}{r}0.94 \pm \\
0.10\end{array}$ & 0.023 & $\begin{array}{c}0.77 \pm \\
0.11\end{array}$ & $\begin{array}{r}1.02 \pm \\
0.07\end{array}$ & $<0.001$ & 0.788 \\
\hline
\end{tabular}

Conclusions: Patients above 40 years of age manifested similar degree of cardiac remodeling following ASD closure compared to younger patients. 


\section{3}

Transvenous CRT in complex congenital heart diseases dextrocardia with transposition of the great arteries after Mustard operation

Peter A Zartner, German Paediatric Heart Center, Sankt Augustin, Germany

Walter Wiebe, German Paediatric Heart Center, Sankt Augustin, Germany

Martin BE Schneider, German Paediatric Heart Center, Sankt

Augustin, Germany

Cardiac resynchronization therapy revealed first promising results in patients with a congenital heart disease and a systemic right ventricle.

Contrast enhanced magnet resonance imaging showed accessibility of the coronary sinus in an 18 year old male patient with mirror dextrocardia, d-transposition of the great arteries and ventricular septal defect (VSD) after Mustard operation and VSD patch closure. Entering the CS from the inferior vena cava, a well-developed vein draining from the apex and the right ventricular anterior wall could be identified. From the left subclavian vein an atrial and a left ventricular lead (Medtronic, 3830-59) were placed, followed by a CS-lead (Biotronik, Corox) (Figure 1), which initially caused diaphragmal contractions. Retraction could solve this problem with persistent good sensing (atrial lead $4.5 \mathrm{mV}$, sub pulmonic lead $13.4 \mathrm{mV}$ ) and pacing thresholds (atrial $0.4 \mathrm{~V} / 0.4 \mathrm{~ms}$, sub pulmonic $0.4 \mathrm{~V} / 0.4 \mathrm{~ms}$, sub aortic CS $1.1 \mathrm{~V} / 0.4 \mathrm{~ms})$. All system parameters were stable at 3 months follow-up. Bicycle test showed mild improvement in peak performance from $1.9 \mathrm{~W} / \mathrm{kg}$ to $2.5 \mathrm{~W} / \mathrm{kg}$. Pro-brain natriuretic peptide decreased from $649 \mathrm{ng} / 1$ to $239 \mathrm{ng} / 1$ [standard normal: $<64 \mathrm{ng} / \mathrm{l}]$.

In the view of an increasing number of adult patients after atrial switch operation (Mustard/Senning) presenting with cardiac failure and interventricular dyssynchrony, the least invasive transvenous approach to CRT is feasible also if additional anomalies are present and should be considered in the preprocedural evaluation.

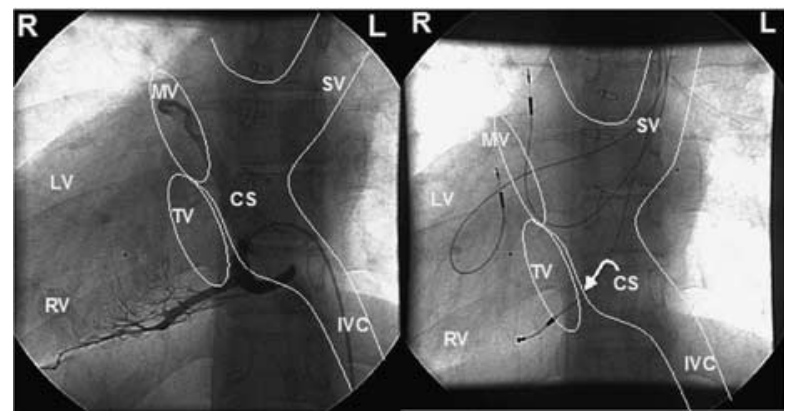

8274

Congenital unilateral absent pulmonary artery - clinical feature, diagnosis, treatment

Maria Zyla-Frycz, Silesian Centre for Heart Diseases Zabrze, Poland Jacek Kusa, Poland

Jan Frycz, Cardiology Dept. Municipal Hospital, Gliwice, Poland Malgorzata Szkutnik, Poland

Jacek Bialkowski, Silesian Centre for Heart Diseases Zabrze, Poland

Congenital unilateral absence of pulmonary artery (UAPA) is a rare malformation- $0,39 \%$ of congenital heart diseases, may involve right or left branch. Absent left pulmonary artery is more often associated with congenital heart diseases while absent right pulmonary artery usually is present as an isolated form. Early diagnosis of UAPA, especially of the isolated form is very important because of later consequences.

Aim: Having our own material as well as publicated date we present symptoms, clinical date and diagnostic methods useful in making diagnosis of UAPA as well as therapeutic procedures.

Material and methods: We analyzed the group or 6 patients (3 children and 3 adults). X-ray, ECG, ECO were done in all cases, in some of them we performed angioTK, MRI and hemodynamic investigation.

Results: UAPA was recognized in all cases, isolated absence of RPA in one case, absence of LPA was in 5 cases and in 4 of them was associated with congenital heart diseases. There was diminished pulmonary marking on X-ray in all patients as well as ECO examination revealed possibility of UAPA. In 2 cases the diagnosis was established during catheterization and in other cases angioCT or MRI was done. In one patient three interventional procedures were performed.

Conclusions:

1. The clinical date of UAPA are not specific. In cases with associated congenital diseases the most characteristic are symptoms caused by this malformation.

2. The early diagnosis is done on the basic of X-ray and ECO. MRI, angioCT and hemodynamic investigation allow to establish final diagnosis.

\section{5}

Management of symptomatic secundum atrial septal defects in children aged less than three years - Is catheter closure safe below three years of age?

Robert Coelho, MIOT Hospital, Chennai, India, India

Shrinivas Chakravarthi, MIOT Hospital, Chennai, India, India Ramyashri Chandrasekaran, MIOT Hospital, Chennai, India, India Anpon Bhagyavathy, MIOT Hospital, Chennai, India, India Kothandam Sivakumar, MIOT Hospital, Chennai, India, India Radhakrishnan Satish, MIOT Hospital, Chennai, India, India Arunkumar Govindarajan, MIOT Hospital, Chennai, India, India Hariprakash Suriyanarayanapillai, MIOT Hospital, Chennai, India, India

Objectives: Some secundum atrial septal defects (ASD) warrant closure below 3 years due to heart failure or retarded growth. In the asymptomatic group, echo measure of ASD progressively increases with age. If ASD device closure is safe under 3 years, smaller devices can be utilized and device surface exposed in the atria is reduced.

Methods: ASD closed below 3 years in our institution were analyzed. Surgery was offered only if the margins were totally absent. Patients with even deficient margins had attempted device closure after informed consent. Device size was equal to the largest ASD echo measurement; no balloon sizing was done. Surgery on cardiopulmonary bypass was done through sternotomy or thoracotomy. Results: Among 21 patients aged $2.1+/-0.7$ years, weighing $10.1+/-2.4 \mathrm{~kg}$ with ASD measuring $16.6+/-4.2 \mathrm{~mm}, 14$ weighed below tenth centile, 2 had heart failure, rest were asymptomatic. 16 patients had attempted device closure with device size of $17+/-4.6 \mathrm{~mm}$. In one patient weighing $8 \mathrm{~kg}$ with $16 \mathrm{~mm}$ ASD, catheter injury to right pulmonary vein caused hemothorax, needing surgery. Device closure was uncomplicated and successful in others. 5 patients with absent margins electively operated also had uncomplicated outcome. The mean duration of hospital and intensive stay was anticipatedly longer after surgery. There were no procedural deaths. 
Conclusions: Three fourths of ASD needing closure will be amenable anatomically for device closure. In patients below 3 years, device closure is safe and feasible. Due procedural care should be exercised to avoid procedural complications.

\section{6}

Atrioventricular septal defect with common arterial trunk: failure of septation at both atrioventricular and ventriculoarterial junctionos

Iki Adachi, Cardiac Morphology Unit, National Heart \& Lung Institute, Imperial College London, United Kingdom

Siew Yen Ho, Cardiac Morphology Unit, National Heart \& Lung Institute, Imperial College London, United Kingdom

Margot M Bartelings, Department of Anatomy \& Embryology, Leiden University Medical Center, The Netherlands

Karen P McCarthy, Cardiac Morphology Unit, National Heart \& Lung Institute, Imperial College London, United Kingdom

Anna Seale, Department of Paediatric Cardiology, Royal Brompton Hospital, United Kingdom

Hideki Uemura, Department of Cardio-Thoracic Surgery, Royal

Brompton Hospital, United Kingdom

Background: Coexistence of abnormalities in both atrioventricular and ventriculoarterial junctions occasionally represents a formidable challenge to the surgeon. Association of atrioventricular septal defect (AVSD) with common arterial trunk is such an example. To date, only two reports have described successful operative outcome. This paucity of success might reflect the anatomical complexity that could prevent favourable result.

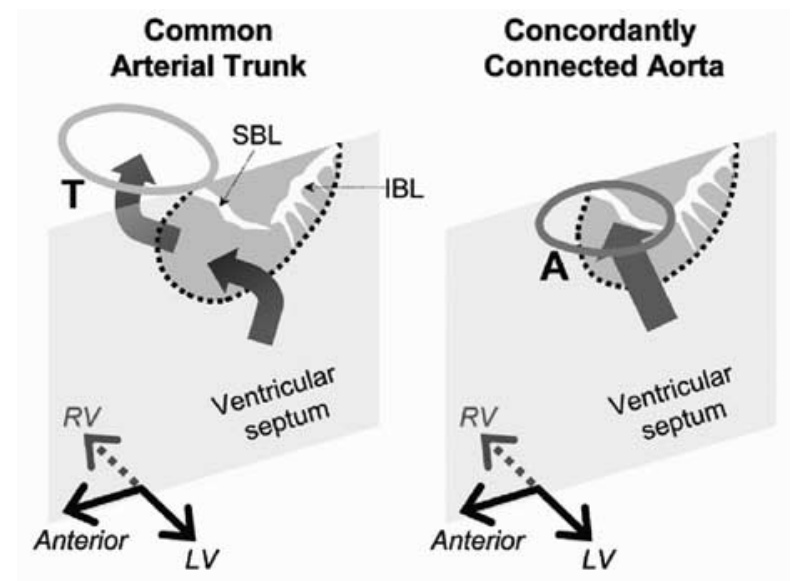

Methods: We reviewed 6 specimens with AVSD and common arterial trunk, focusing on how to establish a non-obstructed connection between the left ventricle (LV) and the truncal valve. Results: In all cases, the common trunk arose exclusively from the right ventricle $(\mathrm{RV})$, and the only exit from the LV was the ventricular component of AVSD (Fig). In particular, preferential route was limited to a space below the superior bridging leaflet (SBL) that did not have any tendinous cords inserting onto the ventricular crest in contrast to the inferior bridging leaflets (IBL) that were always tethered to the crest with many short cords. Accordingly, the size of potential LV outflow depended on the shape of the antero-superior margin of the ventricular crest below the SBL. The potential outflow was narrower than the truncal valvar area in all hearts but one having extensive anterosuperior excavation of the ventricular crest, suggesting the necessity of septal enlargement had anatomical repair been attempted during life.
Conclusions: Owing to the unique ventriculoarterial connection, the surgeon, considering anatomical repair, needs to pay attention to the antero-superior margin of the ventricular scoop, which determines the adequacy of LV outflow size.

\section{7}

Pentalogy of Cantrell: a 40 years experience at a single institution in México

Norma A Balderrábano, Hospital Infantil de México "Federico Gómez", Mexico

Erika G Sandoval, Hospital Infantil de México "Federico Gómez”, Mexico Alfredo Vizcaíno, Hospital Infantil de México "Federico Gómez", Mexico

Objective: To present 20 cases of Pentalogy of Cantrell seen in 40 years in a single institution.

Methods: We review the clinical records, image studies and pathology pieces of all cases with Pentalogy of Cantrell from 1968 to 2008. Patients were classified as: Group I (with Ectopia Cordis) and Group II (without Ectopia Cordis).

Results: Twenty patients with Pentalogy of Cantrell were seen. Age at diagnosis was 2,47 months. Eleven in group I (55\%). The congenital heart defects were: Group I: single ventricle: $4(36 \%)$, double outlet of right ventricle: $3(27 \%)$, ventricular septal defect: 2 (18\%), aortic coarctation:1 (9\%), atrial septal defect: 1 (9\%). Group II: double outlet of right ventricle: 3 (33\%), double inlet of left ventricle: $2(22 \%)$, ventricular septal defect: $2(22 \%)$, tetralogy of Fallot: 1 (11\%), hypoplasic left ventricular syndrome: $1(11 \%)$. Surgery was performed in twelve $(60 \%), 5$ in group I, 7 in II. Cardiac surgery was performed in $10(50 \%) ; 5$ in group I $(45 \%), 5$ in II $(55 \%)$. Sixteen patients died $(80 \%), 11$ in group I $(100 \%)$ and 5 in group II $(55 \%)(\mathrm{p}=<0.05)$. Seven of 12 patients with surgery died (58\%); $5 / 5(100 \%)$ of group I and $2 / 7(29 \%)$ of group II $(\mathrm{p}=<0.05)$. Four patients are alive and asymptomatic. Conclusions: There are approximately 185 cases of Pentalogy of Cantrell reported around the world. This is the largest group of cases reported to date. Pentalogy of Cantrell has elevated mortality especially if Ectopia Cordis is associated.

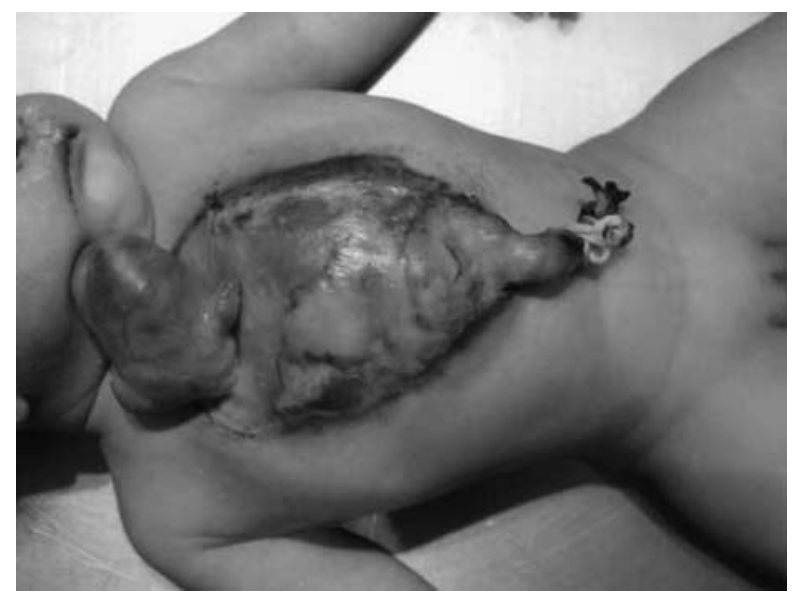

\section{8}

Systemic and Pulmonary Venous Connections in Heterotaxy

Louise Calder, Starship Children's Hospital, Pediatric and Congenital Cardiology Department, New Zealand

Objective: The expected types of connections in asplenia or polysplenia are well known. This study was undertaken to ascertain the number of exceptions. 
Methods: The connections were assessed in 38 specimens with asplenia and 13 with polysplenia.

Results: There was right atrial appendage isomerism in $95 \%$ of cases with asplenia and left isomerism in $77 \%$ with polysplenia. A unique patient with asplenia had right-sided juxtaposition of the atrial appendages. The atrial septum did not show lateralization in $84 \%$ with asplenia and $92 \%$ with polysplenia. Bilateral superior vena cavae were found in 63 with asplenia and in only 23\% with polysplenia. The coronary sinus was absent in $95 \%$ with asplenia but in only $50 \%$ with polysplenia. In polysplenia the suprarenal portion of the IVC was absent in $54 \%$ and pulmonary veins connected to ipsilateral atria in $62 \%$. There was total anomalous pulmonary venous connection in $74 \%$ with asplenia. Only 4 patients had normal pulmonary venous connections. In the others with connection to the atria, the pulmonary veins united into a common vessel which was often obstructed at the junction with the atrium. Eleven different patterns of systemic venous connections were seen in the asplenia cases plus additional variations with pulmonary venous connections to different sites. Ten patterns of systemic and pulmonary venous connections were seen in the polysplenia cases.

Conclusion: Detailed analysis of these connections is required before surgical treatment could be undertaken.

8279

A case of HLHS with congenital biliary atresia; an autopsy findings

Ai Kasuga, Hokkaido Medical Center for Child Health and

Rehabilitation, Japan

Motoki Takamuro, Hokkaido Medical Center for Child Health and

Rehabilitation, Japan

Manabu Watanabe, Hokkaido Medical Center for Child Health and Rehabilitation, Japan

Sachiko Kimura, Hokkaido Medical Center for Child Health and

Rehabilitation, Japan

Akihiro Nui, Hokkaido Medical Center for Child Health and

Rehabilitation, Japan

Keiji Haseyama, Sapporo Medical University

King-ya Hatakeyama, Hokkaido Medical Center for Child Health and Rehabilitation

Masato Yokozawa, Hokkaido Medical Center for Child Health and

Rehabilitation

Background: For hypoplastic left heart syndrome (HLHS), bilateral pulmonary artery banding (b-PAB) as initial intervention followed by Norwood and Glenn procedure over the neonatal period is recently promising result.

Case: A case of HLHS, bilateral superior vena cava, interruption of inferior vena cava (IVC) with azygos connection was diagnosed during fetal period. The baby weighed $2564 \mathrm{~g}$ was born 37 weeks at gestational age by elective cesarian section. Two days after birth b-PAB was done on continuous infusion of prostagrandin E1. Over the early neonatal period, jaundice persisted. Direct bilirubin and transaminase gradually increased. Imaging studies showed normal gall bladder and bile duct in size and appearance, so could not confirmed congenital bilirary atresia (BA). We concluded Norwood procedure followed by laparotomy and repair of BA if defined until 60 days old. However her parents decided conservative treatment and familial touching during her life. On 120 days after birth she was died being held by her mother.

Autopsy: Polysplenia. Foramen ovale was almost closed. Clinically undiagnosed partial anomalous pulmonary venous return (right upper pulmonary vein to SVC) was only cardiac return from lung. Pulmonary hypertension because of pulmonary congestion was more severe in left lung with thickened endothelium and lymphangiectasia. Not macroscopic, but microscopic examination revealed congenital BA (III, c2, beta).

Discussions: For peritoneal dialysis immediately after Norwood procedure, laparotomy was not done. Ethical issue for severe extracardiac complication with HLHS will be discussed.

\section{0}

Retroaortic innominate vein- incidence, association with congenital heart defects, clinical and embyological significance

Snehal M Kulkarni, Wockhardt Hospitals, India

Shreepal Jain, India

Pankaj Kasar, India

Swati Garekar, Wockhardt Pediatric and Congenital Heart Center, India

We analysed 2200 echocardiographies performed during last 18 months. Out of 1970 abnormal echocardiographies, 10 patients were found to have retroaortic innominate vein $(0.55 \%)$. It was diagnosed using suprasternal short axis view. Out of these 10 patients, 8 were diagnosed to have tetralogy of Fallot (TOF) and two had univentricular heart with severe pulmonary stenosis. It was not seen in normal echocardiograms. out of these 10 patients, seven patients had right aortic arch and two had pulmonary atresia. There was a high incidence of retroaortic innominate vein in patients with TOF. We had 108 patients with TOF out of which 8 had its presence (8.5\%). In 8 patients its presence was confirmed during surgery. No other imaging modality was used for confirmation. Though its presence in isolation has no clinical significance, its presence needs to screened carefully especially in patients with right ventricular outflow tract obstructions and right aortic arch. It can be mistaken for left superior vena cava or right pulmonary artery. If undiagnose, its presence may cause technical difficulty during pacemaker insertion, superior vana caval cannulation during surgery and exposure of pulmonary artery during Glenn anstomosis. Its presence has a embyological significane.Reduced shortening of aortic arch as seen in right aortic arch, compresses and prevents development of superior transverse venous plexus. Abnormal development of pulmonary arteries in pulmonary stenosis or pulmonary atresia encourages sparing of inferior transverse venous plexus leading to formation of anomalos retroaortic course of innominate vein.

\section{1}

Atrioventricular septal defect with only ventricular component: morphologic features

Karen P McCarthy, Cardiac Morphology Unit, National Heart \& Lung Institute, Imperial College London, United Kingdom

Iki Adachi, Cardiac Morphology Unit, National Heart \& Lung

Institute, Imperial College London, United Kingdom

Hideki Uemura, Department of Cardio-Thoracic Surgery, Royal

Brompton Hospital, United Kingdom

Siew Yen Ho, Cardiac Morphology Unit, National Heart \& Lung

Institute, Imperial College London, United Kingdom

Background: Atrioventricular septal defect with only ventricular component of septal deficiency is the least common of various forms of this malformation. We reviewed its morphology in heart specimens and compared our findings with the other forms for a better understanding of its surgical morphology.

Methods: We examined 78 cardiac specimens with atrioventricular septal defect; 56 (72\%) had common atrioventricular valvar 
orifice with both atrial and ventricular components (so-called "complete" form), while 22 (28\%) had separate valvar orifices (so-called "partial" or "incomplete" form) with 17 having only atrial component (so-called "ostium primum" form) and 5 having only ventricular component.

Results: Amongst hearts with atrioventricular septal defect, the hearts with only ventricular component of the defect had the mildest deformity of the ventricular mass characterised by less inlet-outlet disproportion, smaller "gap" between anterior and posterior parts of the atrioventricular junction and the least extensive septal deficiency. However, these hearts still possessed the characteristic common atrioventricular junction and had fiveleaflet configuration of the atrioventricular valve with similar proportions of mural leaflets in both valvar orifices as in other forms. Furthermore, owing to the unique relationship of the bridging leaflets to the septum, the leaflets were always 'upwardly' displaced as opposed to 'downwardly' displaced leaflets in "ostium primum" form.

Conclusions: Our observations suggest this entity might represent the mildest end of the whole spectrum of hearts with atrioventricular septal defect. Since 'upwardly' displaced leaflets are not modifiable and could be aggravated further following surgery, they might play a role in late valvar dysfunction.

\section{2}

Coronary anatomy of the right ventricular inferior wall in Ebstein malformation: relevance to "vertical" plication

Karen P McCarthy, Cardiac Morphology Unit, National Heart \& Lung Institute, Imperial College London, United Kingdom

Iki Adachi, Cardiac Morphology Unit, National Heart \& Lung

Institute, Imperial College London, United Kingdom

Hideki Uemura, Department of Cardio-Thoracic Surgery, Royal

Brompton Hospital, United Kingdom

Siew Yen Ho, Cardiac Morphology Unit, National Heart \& Lung

Institute, Imperial College London, United Kingdom

Background: "Vertical" plication of the atrialised chamber in Ebstein malformation has been the subject of debate. A major argument against the procedure is a risk of coronary arterial injury; yet the coronary anatomy in the malformation remains incompletely investigated.

Methods: We examined 17 autopsy hearts with the malformation. Special attention was paid to the course of the posterior descending artery (PDA) on the RV inferior wall relative to the interventricular septum.

Results: The right coronary artery was dominant in 11, while the remaining 6 had left dominance. In 6 hearts with right dominance, the right coronary artery gave rise to PDA before reaching the crux of the heart. This "early take-off" caused the entire PDA to be deviated rightward. Additionally, 2 hearts with right dominant pattern showed rightward deviation of PDA course after an initial short segment along the septum. In 2 hearts with left dominance, the left circumflex artery ran beyond the crux and then gave rise to PDA, again resulting in rightward deviation of PDA. These deviations allowed the PDA to run on the atrialised part of the RV inferior wall where endocardial stitches would be placed during "vertical" plication.

Conclusion: PDA was frequently $(10 / 17,59 \%)$ deviated toward right side relative to the interventricular septum in Ebstein malformation. Together with the thinness of the atrialised ventricular wall, care should be taken to the branch even with superficial endothelial stitches.

\section{Course of PDA on the RV inferior wall}

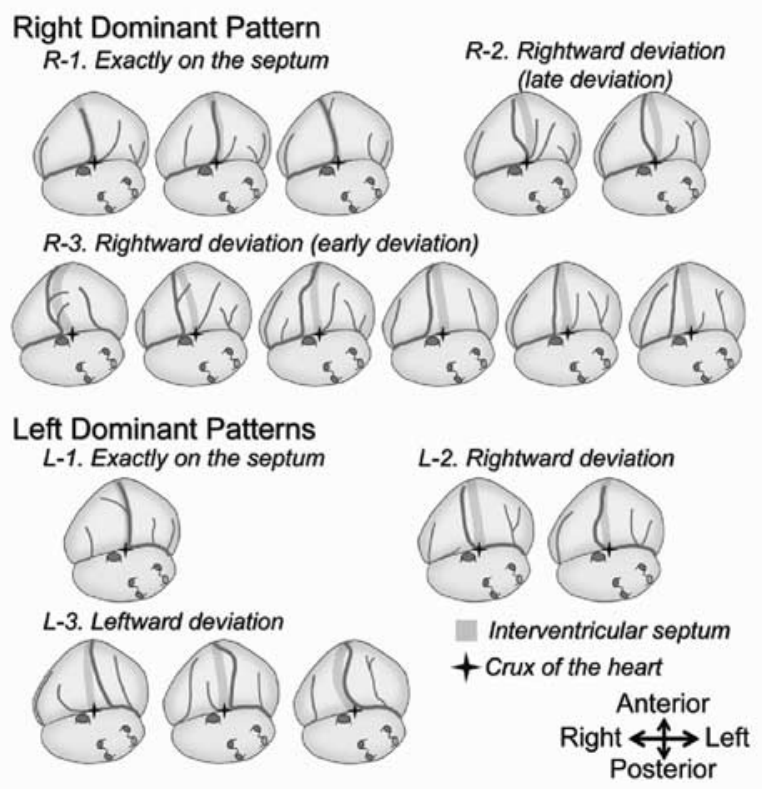

8283

Morphology of the right sinus venosus valve in fetuses between 10 and 23 week of gestation Cezary S Niszczota, Department of Anatomy, Center of Biostructure Research, Medical University of Warsaw, Poland, Poland Adam Kolesnik, Department of Anatomy, Center of Biostructure Research, Medical University of Warsaw, Poland, Poland

Right sinus venosus valve (RSVV) directs blood flow from inferior vena cava (IVC) to foramen ovale. Description of its anatomy related to fetal age can be important for the proper assessment of the heart on fetal autopsy. The study aimed to determine morphology of normally developed RSVV in the 1st and the 2nd trimester fetuses. Twenty normal fetal hearts aged from 10 to 23 weeks were submerged in water and dissected carefully to visualize the RSVV (figure). Nikon SMZ 1500 microscope, Nikon DS-Fi1 camera and NIS-Elements 3.00 software were used for observation and documentation. RSVV was present in all fetuses. Its inferior attachment was found

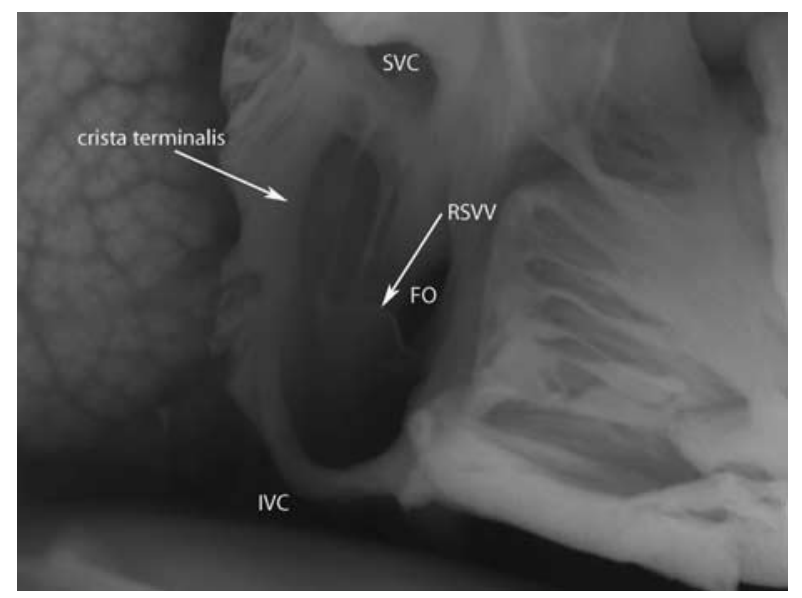

between ostium of coronary sinus (CS) and the septal leaflet of tricuspid valve. In fetuses aged 10-15 weeks it covered the ostium 
of CS completely. The superior attachment was located in the region of sagittal bundle in fetuses younger than 20 weeks and in precaval segment of crista terminalis in older specimens. Starting from 16th week of gestation the division of RSVV into the valve of IVC and valve of CS was observed. In the fetuses aged 20 weeks and older the division of RSVV was distinct. The place of division was located in the region of Todaro's tendon. Gradual regression of the RSVV was observed since 12th week of development.

Conclusions: RSVV is constantly present in small fetal hearts. Its involution and division into IVC and CS valves can be observed. Further studies can help to determine the norm useful for pathologists.

\section{4}

Relationship between the coronary arteries and the aortic arches. Study done in sharks

João C Saba, Federal University of Sao Paulo/unifesp, Brazil

Celia Maria C Silva, Federal University of Sao Paulo/unifesp, Brazil Antonio Carlos C Carvalho, Federal University of Sao Paulo/unifesp, Brazil

Carlos Eduardo Bernini Kapins, Escola Paulista de Medicina - Unifesp - SP, Brazil

Jose Carlos Prates, Brazil

Taking into account that the origin the coronary arteries from the pulmonary artery we made a hypothesis that the coronary arteries could be the last pair of aortic arches connected with the Pulmonary trunk (6th aortic arch, throughout a ductus, which we called as coronary ductus. These observations was taken from the knowledge of the existence of a shark that have seven branchias and also seven branchial arches (heptranchias perlo. We decided to study the coronary system of the sharks and its relations with the aorta and the branchial arches. The connections between the coronary arteries to the aorta in the embryological life still not yet completely understood. Our study was carried out with injections of contrast media into the aorta and coronary arteries of 16 shark's head sectioned at the inferior level of the last

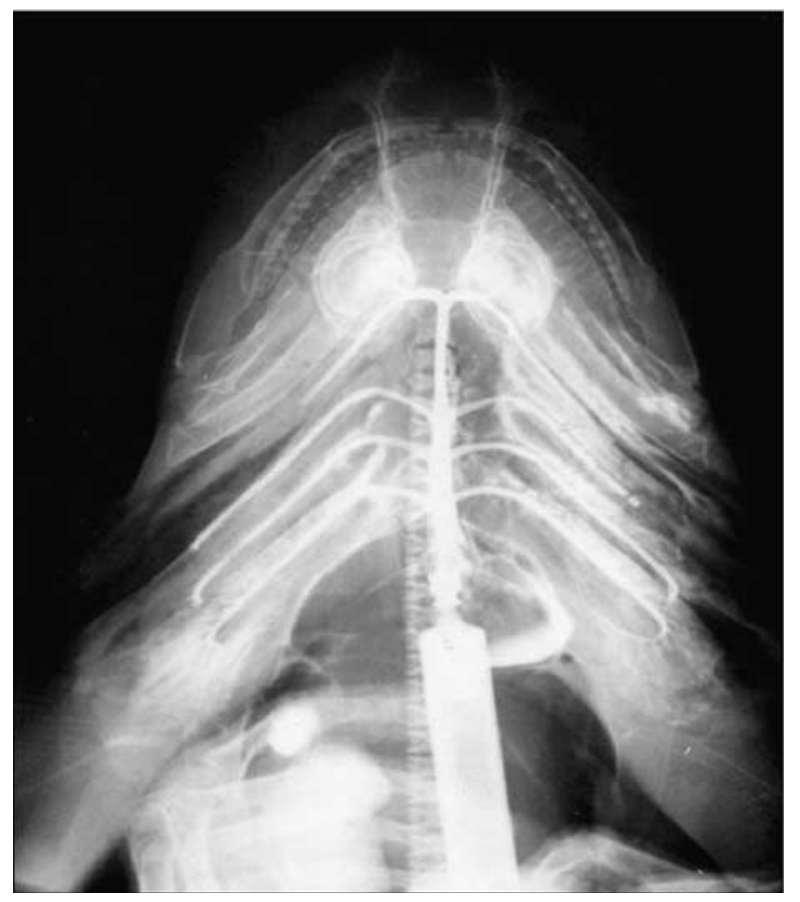

branchia,all of them with five branchias. We concluded that there was a clear relation with the coronary arteries and the branchial arches, and we do not find connections between the coronary arteries and the ascending aorta maybe because in that vessel only run venous blood. We were not able to prove that the coronary arteries were the last pair of the aortic arches because we never found connections of these arteries with the descending aorta. We still having doubts why when the coronary arteries arise from the pulmonary trunk, in generally they arise beyond the valve sinus, going into the opposite of the embryological theory of the connection of the coronary vessels with the aortics sinus.

\section{5}

Myocardial infarction caused by anomalous origin of the right coronary artery (RCA) from the left coronary sinus Dalibor Saric, University Hospital Center Zagreb, Croatia, Croatia Branko Marinovic, University Hospital Center Zagreb, Croatia, Croatia Ivan Malcic, University Hospital Center Zagreb, Croatia, Croatia Christian Scheiber, German Heart Center Munich, Germany Andreas Eicken, German Heart Center Munich, Germany Hrvoje Kniewald, University Hospital Center Zagreb, Croatia, Croatia

Anomalous origin of the RCA from the left coronary sinus with an interarterial course is a rare but clinically significant anomaly. It is found in only about $0.03-0.9 \%$ of patients undergoing coronary angiography. Manifestations vary from asymptomatic patients to those who present with angina pectoris, myocardial infarction, arrhythmias, and sudden death.

We describe the case of a 16 years old girl with this anomaly who presented with a complaint of precordial pain while she was resting. The patient's initial physical examination showed no abnormalities but electrocardiogram showed signs of inferior myocardial infarction. Her cardiac troponins were very elevated. She was treated conservatively and a few days later she was referred to our clinic for further evaluation. According her medical history coronary artery anomaly was suspected. ECHO showed normal origin and course left CA but we were not able to identify right CA origin. The function of both ventricle were normal. Stress myocardial perfusion scan with technetium $99 \mathrm{~m}$ sestamibi revealed a small irreversible inferior defect. Exercise stress test was within normal limits. Coronary angiography documented the anomalous origin of the RCA from the left sinus of Valsava. MSCT coronary angiography confirmed anomalous origin of the right coronary artery from the left coronary sinus with course of the proximal part RCA between the aorta and pulmonary trunk.

Our patient successfully underwent surgery (unroofing procedure).

8287

Multiplex, quantitative, real-time PCR is a sensitive, inexpensive and rapid assay to detect DiGeorge Syndrome (DGS1) and can be adapted to newborn screening Joshua M Larson, Medical College of Wisconsin, United States Aoy Tomita-Mitchell, Medical College of Wisconsin, United States Sujana Ghanta, University of Louisville, United States John Routes, Medical College of Wisconsin, United States Ulrich Broeckel, Medical College of Wisconsin, United States William Grossman, Medical College of Wisconsin, United States James S Tweddell, Medical College of Wisconsin, United States Michael E Mitchell, Medical College of Wisconsin, United States

Objective: DiGeorge Syndrome (DGS1), also known as 22q11.2 deletion syndrome, affects 1:4000 live births. The gold standard for detection of DGS1 is fluorescence in situ hybridization 
(FISH), with a probe at the TUPLE1 gene. However, FISH has a high false negative rate (15\%), is costly, and time-consuming. The goal of this study was to determine if multiplexed quantitative real-time PCR (MQPCR), a rapid and inexpensive assay, could be used to determine haploinsufficiency in DGS1.

Methods: A blinded study was performed on 384 patients following congenital heart surgery. 14 were FISH-positive for DGS1, and seven additional patients were diagnosed clinically. In the remaining 363, 68 were FISH-negative. Using real-time PCR, the cycle threshold $(\mathrm{Ct})$ value was used to calculate the relative gene copy number $(\mathrm{rGCN})$ in the region of the TBX1 gene.

Results: 363 patients were identified as normal, mean rGCN value 1.00 (95\%CI 0.71-1.3), indicating two copies, and 21 patients were identified as DGS1, mean rGCN value 0.51 (95\%CI 0.33-0.69), indicating a deletion ( $\mathrm{p}<0.0001)$. All 14 (FISH-positive) DGS1 patients test were successfully identified. Six of seven clinically diagnosed DGS1 patients were found to carry a deletion (one did not), all confirmed via microarray (Affymetrix 6.0). One patient not previously identified as DGS1 was detected and confirmed via microarray. The sensitivity (21/ $21)$ and specificity $(363 / 363)$ of the assay was $100 \%$.

Conclusion: In summary, the MQPCR assay is rapid ( $<4 \mathrm{hrs}$ ), inexpensive $(<\$ 1)$, sensitive, and specific for DGS1. The assay is readily adaptable to high-throughput and ideal for populationbased newborn screening.

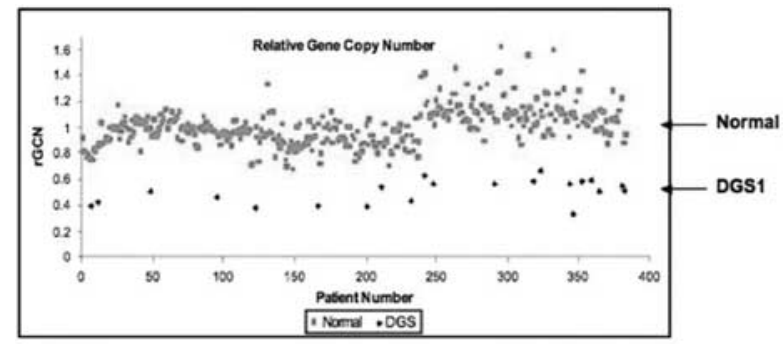

\section{9}

High-resolution genome-wide copy number analysis diagnoses 28 out of 150 patients with idiopathic syndromic congenital heart defects

Jeroen Breckpot, Center of Human Genetics, University Hospitals

Leuven, Belgium, Belgium

Bernard Thienpont, Center of Human Genetics, University Hospitals Leuven, Belgium, Belgium

Hilde Peeters, Center of Human Genetics, University Hospitals Leuven, Belgium, Belgium

Joris $R$ Vermeesch, Center of Human Genetics, University Hospitals

Leuven, Belgium, Belgium

Marc Gewillig, Paediatric Cardiology Unit, University hospitals

Leuven, Belgium, Belgium

Koenraad Devriendt, Center of Human Genetics, University Hospitals Leuven, Belgium, Belgium

Array Comparative Genomic Hybridization (aCGH) is a screening technique for genome-wide detection of chromosomal imbalances, surpassing the limited resolution of conventional karyotyping. We investigate the usefulness of aCGH as a diagnostic tool in 150 selected individuals with syndromic congenital heart defects (CHDs). A subgroup of 29 patients is moreover analyzed by higher resolution aCGH to assess its value. We also systematically assess which phenotypic characteristics are significantly associated with a positive diagnostic outcome to provide a handle for selecting patients.
Methods: 150 patients with an idiopathic CHD and additional features suggesting a chromosomal defect were selected. aCGH was performed on an in-house made microarray of 1 Megabase resolution. In 29 patients, a higher resolution analysis was performed on Agilent 244K arrays (resolution: 30 kilobase).

Results: Chromosomal imbalances not previously described as polymorphisms were detected in 46/150 patients (30\%). 26 of these $(17 \%)$ carry imbalances which overlap with well-described disease-related regions or comprise genes known for causing CHD when mutated, and therefore are considered to be causal. Higher resolution aCGH revealed the presence of 2 causal de novo imbalances in a subgroup of 29 subjects.

Conclusion: This study demonstrates the ability of aCGH to provide an etiological diagnosis in a large proportion of patients with syndromic CHD. Besides their usefulness in genetic counselling, identified chromosomal aberrations may aid in medical follow-up. Higher resolution aCGH contributes to the etiological diagnosis to a lesser extent but highlights the need for a handle to evaluate the causality of copy number variants in syndromic CHD.

\section{0}

Chronically elevated left ventricular afterload in fetal sheep prevents gestational increase in isovolumic acceleration

Paul A Brooks, The Royal Children's Hopsital, Murdoch Children's

Research Institute, University of Melbourne, Australia

Andrew T Hattam, Murdoch Children's Research Institute, Department of Paediatrics University of Melbourne, Australia Michael MH Cheung, The Royal Children's Hopsital, Murdoch Children's Research Institute, University of Melbourne, Australia Daniel J Penny, The Royal Children's Hopsital, Murdoch Children's Research Institute, University of Melbourne, Australia Joseph J Smolich, Murdoch Children's Research Institute, Department of Paediatrics University of Melbourne

Aims: Fetal ventricular contractility is generally assessed by echocardiographic indices whose acute load dependence is well recognized. Color tissue Doppler (cTDI) isovolumic acceleration (IVA), an index of contractility relatively insensitive to acute changes in loading conditions, may permit more accurate evaluation of fetal systolic function. However, the impact of chronic loading changes on cTDI indices in the fetus is unknown. This study assessed the effect of chronically elevated left ventricular (LV) afterload on systolic cTDI indices in fetal sheep.

Methods: In 17 twin ovine pregnancies, one fetus had a surgical nonconstrictive ascending aortic band placed at a median 91 days gestation (range 90-98 days, term $=147$ days), whilst the other served as an un-operated control. Echocardiograms of both fetuses were performed under maternal sedation at $\sim 2$ weekly intervals until near term. Left and right ventricular (RV) IVA and peak systolic (S') velocities were obtained at their respective atrioventricular valve annulus. Ventricular outputs and peak ascending aortic gradient were obtained with standard Doppler techniques.

Results: Gestation related increases in LV and RV outputs did not differ in banded and control groups despite a moderate peak ascending aortic gradient in near-term banded fetuses $(23.9 \pm 11.5 \mathrm{mmHg})$. LV and RV S' and RV IVA showed no differences between banded and control groups. However, the gestation related increase in LV IVA present in control fetuses was absent in banded animals $(\mathrm{p}=0.004)$.

Conclusions: These results suggest a significant impact of chronically elevated afterload on IVA in the fetus, the mechanism of which requires clarification with invasive studies. 


\section{1}

Biventricular diastolic function is unaffected by chronically elevated left ventricular afterload in fetal sheep

Paul A Brooks, The Royal Children's Hopsital, Murdoch Children's

Research Institute, University of Melbourne, Australia

Andrew T Hattam, Murdoch Children's Research Institute, Department

of Paediatrics University of Melbourne, Australia

Michael MH Cheung, The Royal Children's Hopsital, Murdoch

Children's Research Institute, University of Melbourne, Australia

Daniel J Penny, The Royal Children's Hopsital, Murdoch Children's

Research Institute, University of Melbourne, Australia

Joseph J Smolich, Murdoch Children's Research Institute, Department of

Paediatrics University of Melbourne

Aims: In contrast to the mature heart, fetal ventricular diastolic dysfunction is a late and ominous finding in conditions accompanied by long-standing elevated ventricular afterload. Little is known, however, about longitudinal changes in fetal diastolic function occurring in this setting. The aim of this study was to assess biventricular diastolic properties using echocardiography in a fetal sheep model of chronically elevated left ventricular (LV) afterload.

Methods: In 17 twin ovine pregnancies, one fetus had a surgical non-constrictive ascending aortic band placed at mid-gestation (range 90-98 days, term $=147$ days), whilst the other served as an un-operated control. Echocardiograms of both fetuses were performed under maternal sedation at $\sim 2$ weekly intervals until near term, with biventricular assessment of color tissue Doppler (cTDI) early relaxation (E') and late filling (A') myocardial velocities at their respective atrioventricular valve annulus, along with pulsed wave Doppler early (E) and late (A) inflow velocities and deceleration time.

Results: Although a moderate peak ascending aortic gradient was present in the banded fetuses near term $(23.9 \pm 11.5 \mathrm{mmHg})$, neither cTDI myocardial velocities nor pulsed wave Doppler $E$ and A wave velocities or their ratio (E/A) were significantly different for either ventricle between groups. However, mitral inflow deceleration time in the banded fetuses tended to be shorter with advancing gestation after adjustment for heart rate $(p=0.06)$.

Conclusion: Isolated moderate and chronically increased LV afterload in fetal sheep produces no significant change in biventricular diastolic function. The contribution of chronically elevated afterload to fetal diastolic dysfunction requires further investigation.

\section{2}

Acute Effects of Coarctation of the Aorta on Cardiac Sensory Neuronal Activity in Piglets

Robert P Chen, IWK Health Centre, Canada

Introduction: Persistent systemic hypertension after surgical repair of coarctation of the aorta (CoA) is not well understood. We have shown that congenital heart disease, such as patent ductus arteriosus, and cardiovascular surgery of the right ventricular outflow tract alters the ability of nodose ganglioncardiac sensory neurites to transduce mechanical and chemical stimuli in piglet models. These cardiac afferent signals modify circulatory behaviour. Therefore, we believe persisting systemic hypertension may be the result of modifying cardiac afferent neuronal behaviour.

Objective: We hypothesised that the development of acute CoA will alter the ability of neonatal cardiac sensory neurites to transduce the physiologic milieu of the heart as reflected in the activity of nodose ganglion cardiac afferent neurons.

Method: Nine 8 weeks old piglets $(13 \pm 0.98 \mathrm{~kg})$ underwent surgery to expose the nodose ganglion in the neck and to place a ligature around the thoracic descending aorta near the aortic isthmus. Spontaneous activity from nodose ganglion cardiac afferent neurons was measured in situ and extracellular recording techniques. The ligature was tightened to produce a pressure gradient simulating an acute coarctation. Neuronal activity of cardiac sensory neurites associated with nodose ganglion afferent neurons was measured before and after creating a gradient across the aortic ligature, and then after release of the ligature.

Results: See table.

Conclusion: Sensory cardiac neuronal activity tends to fall $(p=0.21)$ during acute $\mathrm{CoA}$ with a $78 \mathrm{mmHg}$ gradient without a significant change in heart rate or diastolic descending aortic pressure. Neuronal activity remains depressed after removal of the CoA.

\begin{tabular}{lccc} 
Table (mean \pm standard error) & $\begin{array}{c}\text { Before } \\
\text { CoA }\end{array}$ & $\begin{array}{c}\text { With } \\
\text { CoA }\end{array}$ & $\begin{array}{c}\text { After } \\
\text { CoA }\end{array}$ \\
\hline Heart Rate (beats/min) & $167 \pm 12$ & $170 \pm 16$ & $150 \pm 18$ \\
Systolic LVP (mmHg) & $95 \pm 8$ & $138 \pm 15$ & $89 \pm 9$ \\
DsAo BP - Systolic (mmHg) & $94 \pm 8$ & $61 \pm 12$ & $83 \pm 9$ \\
DsAo BP - Diastolic (mmHg) & $59 \pm 8$ & $51 \pm 10$ & $53 \pm 9$ \\
CoA Gradient (mmHg) & $2 \pm 2$ & $78 \pm 21$ & $6 \pm 3$ \\
Neuronal Activity (impulses/ & $249 \pm 162$ & $29 \pm 10$ & $115 \pm 59$ \\
min) & & & \\
\hline
\end{tabular}

\section{3}

The Preoperative Risk Attenuates the Number of Circulating Endothelial Progenitor Cells during Cardiopulmonary Bypass - Implications for Cardiac Cell Therapy Yeong-Hoon Choi, Dept. of Cardiothoracic Surgery, Heart Center of the University of Cologne, Germany

Christof Stamm, Dept. of Cardiothoracic Surgery, German Heart Center Berlin, Germany

Klaus Neef, Dept. of Cardiothoracic Surgery, Heart Center of the University of Cologne, Germany

Thorsten Wittwer, Dept. of Cardiothoracic Surgery, Heart Center of the University of Cologne, Germany

Henning Bovenschulte, Dept. of Radiology, University of Cologne, Germany

Oliver J Liakopoulos, Dept. of Cardiothoracic Surgery, Heart Center of the University of Cologne, Germany

Thorsten Wahlers, Dept. of Cardiothoracic Surgery, Heart Center of the University of Cologne, Germany

Objectives: The number of circulating endothelial progenitor-cells (EPC) depends on cytokine release and is also associated with cardiovascular risk factors. During cardiopulmonary bypass (CPB) the endothelium is the first organ to be affected by mechanical and immunological stimuli. We hypothesized that the magnitude of EPC mobilization by CPB correlates with the preoperative risk.

Methods: EPCs were quantified in blood-samples from 20 patients who underwent $\mathrm{CABG}$ by magnetic-bead-isolation (MACS) and FACS. For determination of endothelial injury, circulating endothelial cells (CEC) were quantified in the same manner. Patients were divided into two groups based on the EuroSCORE-system: low-risk (LR) and high-risk (HR). 10 healthy volunteers served as controls. Samples were obtained preoperatively, $1 \mathrm{~h}$ and $24 \mathrm{~h}$ postoperatively. Plasma cytokine- and growthfactors-levels were measured in parallel.

Results: All CPB-patients showed a significantly reduced basal number of EPCs compared to controls (LR:3.26 $\pm 0.3 / \mathrm{ml}$, HR:2.67 $\pm 0.39 / \mathrm{ml}, \quad$ control:5.6 $\pm 0.52 / \mathrm{ml}, \quad \mathrm{p}<0.01 \quad$ (control/ LR); $<0.001$ (control/HR)). CPB-induced EPC release which 
peaked at $1 \mathrm{~h} \quad$ (preop:2.97 $\pm 0.26 / \mathrm{ml} ; \quad 1 \mathrm{~h}: 48.17 \pm 4.34 / \mathrm{ml}$; 24h:5.67 $\pm 0.87 / \mathrm{ml}, \mathrm{p}<0.001$ (preop/1h); $\mathrm{p}<0.001$ (preop./ 24h). EPC-count correlated with the duration of CPB. However, EPC-release was significantly attenuated in HR-patients $(28.53 \pm 2.85 / \mathrm{ml}, \quad 67.8 \pm 3.84 / \mathrm{ml}$ (HR/LR@1h, p<0.0001) and inversely correlated with the preoperative EuroSCORE. GCSF-, SCF- and VEGF-levels increased throughout the observation period and correlated with the EPC-count. Endothelial injury, determined by CEC-count, von-Willebrand-factor and solublethrombomodulin, was similar in both groups.

Conclusions: Cardiovascular risk factors directly influence the mobilization of EPCs after stimulation by CPB. This could be secondary to impaired mobilization or the result of increased EPC-turnover, and may have implications for cell therapy strategies in cardiac surgical patients.

\section{5}

Morphological and Microstructural Changes of Rat Mitral Valve Chordae Tendinae During Postnatal Maturation

Michael G Dickinson, Childrens Hospital Los Angeles, Department of Cardiothoracic Surgery, University of Southern California, Los Angeles., The Netherlands

Ivan Vesely, Childrens Hospital Los Angeles, Department of Cardiothoracic Surgery, University of Southern California, Los Angeles., United States

Background: Mitral valve chordae tendinae are an essential component for proper functioning of the human heart. The microstructural make-up of the chordae is responsible for their tensile properties and gradually changes with age. However, little is known about the maturation of chordae and their microstructure.

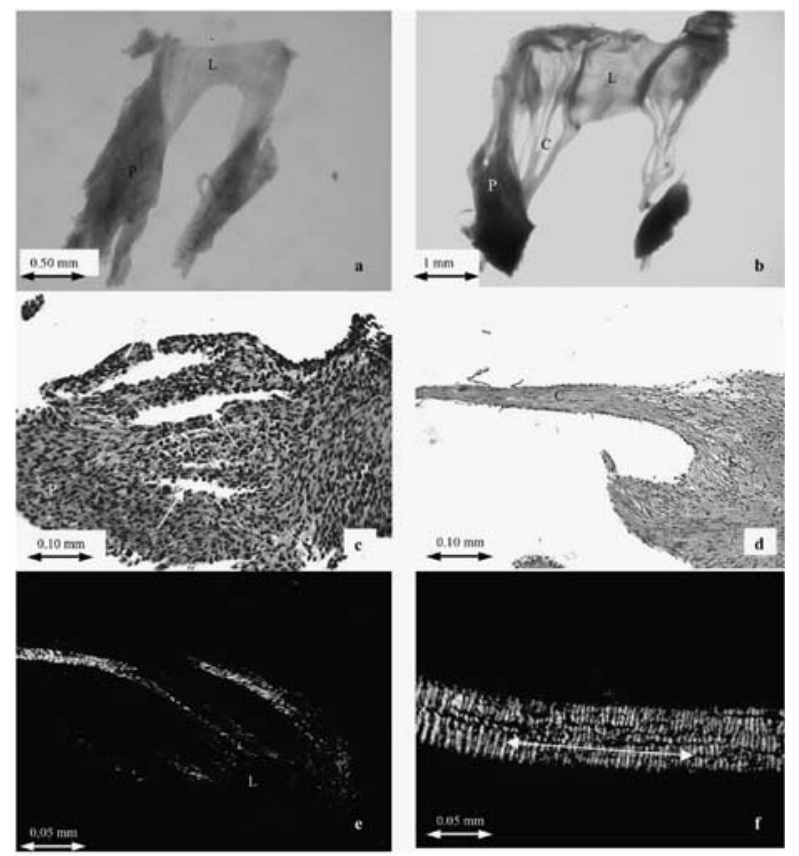
Figure 1. Morphological and microstructural differences between rat mitral valve chordac one day after binh and
adult. The mitral valve was cut in half after valve extraction. (a): Day 1 after birth shows no chordac between the leaflet and papillary musele (b): Adult mitral valve with numerous chordac, all inserting into the free edge of the leaflet.

Trichrome staining of mitral valve chordac. (c): Day I after birth, connective tissue of the leaflet is uniformly amanged throughout the tissue, consisting of collagen and glycosaminoglycans. Gaps surrounded by collagen (rofi) are visible (arrows). (d): Adult ching

Polarized light imagery of picroserius red-stained mitral valve chordac. Fibril crimp period is illustrated by the presence of dark extinction bands. Crimp periods were measured along the direction of the arrows as shown in $(0)$ (e): Day I after birth collagen has not completely formed, crimp is not seen. At adult age (f) chordae are uniformly aligned and show a constant crimp period. L: leanlet; C: chordae, P; papillary muscle
Objective and Methods: To examine the maturation of the mitral valve chordae tendinae, we studied the structural changes in chordae tendinae in rats at 1, 3, 7, 15,30 days of postnatal life and of adult. The differences in microstructure of the chordae of each age group were observed using light microscopy. Collagen fibril crimp period was measured using polarized light microscopy.

Results: At day 1 after birth the chordae have yet to develop and the lateral sides of the mitral valve leaflets were completely attached to the papillary muscles. Chordae developed through the formation of gaps in the leaflet tissue. From day 7 on, numerous chordae were seen. No organized collagen fibrils were seen at day 1 after birth. As the chordae matured, crimped collagen fibrils were formed and started to align in a longitudinally packed core with increasing density. Collagen crimp period increased significantly with age $(\mathrm{P}<0,001)$. No differences were found between average crimp period and chordae diameter.

Conclusion: Rat chordae tendinae have yet to develop 1 day after birth. Morphological development and microstructural maturation of the chordae are not completed until adulthood. A better understanding of normal chordal maturation may help improve methods for the development of tissue-engineered chordae.

\section{7}

Role of maternal hypercholesterolemia and hyperglycemia in the onset of cardiac disease in childhood and adults Adriana C Gittenberger-de Groot, Department of Anatomy and Embryology, Leiden University Medical Center, The Netherlands Fanneke E Alkemade, The Netherlands Pauline AM Roest, The Netherlands

Ko Willems van Dijk, The Netherlands

Robert E Poelmann, Department of Anatomy and Embryology, Leiden University Medical Center, The Netherlands

Ulf Eriksson, Sweden

Marco C DeRuiter, The Netherlands

Background: The fetal origins hypothesis postulated by Barker in the last century suggests that an adverse intrauterine environment induces changes that persist into adulthood. This intrauterine programming is not achieved by alterations in DNA sequence but by epigenetic mechanisms. We have investigated the role of epigenetics in hypercholesterolemia (HC), as well as in hyperglycemia (HG) models.

Methods: For the HC study we generated genetically identical heterozygous ApoE-deficient and LdLr-deficient progeny from mothers with a wild-type or a null-background. For the HG study we studied the effect of high glucose levels on neural crest cell contribution and applied antioxidant treatment to possibly reduce cardiac malformation damage in a chicken model. Endglycation product, VEGF and Smad expression were studied in a diabetic rat model with genetic susceptibility for cardiac malformations.

Results: In HC we showed that the maternal background was essential for development of profound atherosclerosis in the offspring. The combined information from both the ApoE and Ldlr-deficient offspring showed that $\mathrm{HC}$ was not the primary initiator. The inflammatory status of the ApoE-deficient mother seems to be very important. In the latter altered histone methylation was found in endothelial and smooth muscle cells of the affected offspring. In HG we showed that oxidative stress was involved in the development of congenital heart disease with predominance for outflow tract anomalies.

Conclusion: The studied models underscore the effect of in utero priming of the offspring on childhood and adult disease. 
Epigenetic factors such as altered methylation guide us towards further mechanistic research.

\section{8}

Mutation analysis of CRELD1 gene in children with atrioventricular septal defect

Ying Guo, Shanghai Children's Medical Center, China Kun Sun, Shanghai Children's Medical Center, China Jie Shen, Shanghai Children's Hospital, China

Objective: Atrioventricular septal defect (AVSD) is one form of major congenital cardiovascular malformations and CRELD1 is the first identified AVSD candidate gene. We hypothesize that CRELD1 was involved into the process of extracellular matrix production/deposition and valvuloseptal formation as a cell adhesion molecule. Mutations of the CRELDs might result in its functional changes and then affect the process of heart development.

Method: The genomic DNA of 66 children with AVSD were analyzed for the variation in the CRELD1 gene by sequencing. Western-blot and FQ RT-PCR was applied to examine the expression of CRELD1, Tenascin-C and Aggrecan of mutational gene.

Results: We found one missense mutation C857G (P286R) and one new polymorphisms T1136C (M379T). The P286R missense mutation of CRELD1 make the gene gain function. Compared with the un-load, the Aggrecan mRNA expression was down-regulation in wild-type and mutant-type ( $\mathrm{t}$ value was 140.27 vs $26.36, \mathrm{P}<0.01)$. However, the down-regulation was more significant in mutant-type than in wild-type $(\mathrm{t}=-25.69$, $\mathrm{P}=0.002)$. There was no significant difference of the Tenascin$\mathrm{C}$ expression between wild-type and the un-load $(\mathrm{t}=1.167$, $\mathrm{P}>0.05)$, but the Tenascin-C expression was up-regulation in mutant-type $(\mathrm{t}=6.66, \mathrm{P}=0.022)$.

Conclusion: Low prevalence of CRELD1 mutations and the normal phenotype with mutational gene indicated that AVSD is either a complex trait, influenced by both genetic and environmental susceptibility factors, or a monogenic trait caused by a few AVSD genes. The P286R mutation of CRELD1 downregulates the expression of Aggrecan and upregulates the expression of Tenascin-C, both of which are crucial extracellular matrix in the formation of the atrioventricular septal.

\section{9}

Higher preoperative serum lactate and sub-clinical infection predict poor outcome in open heart surgeries of congenital heart disorders: 1H NMR study

Nirmal Gupta, Sanjay Gandhi Postgraduate Institute of Medical

Sciences, India

Partima Tripathi, Sanjay Gandhi Postgraduate Institute of Medical Sciences, India

Raja Roy, Sanjay Gandhi Postgraduate Institute of Medical Sciences, India

C L Khetrapal, Sanjay Gandhi Postgraduate Institute of Medical Sciences, India

Serial postoperative serum lactate (PoSL) measurements are used to assess the clinical progress in critically ill children following corrective surgery. Elevated PoSL levels after cardiac surgery are also associated with hypoperfusion and tissue hypoxia. To the best of our knowledge there are no reports of Preoperative Serum Lactate (PrSL) estimations in patients undergoing openheart surgeries. The use of $1 \mathrm{H}-\mathrm{NMR}$ spectroscopy is demonstrated to examine the role of pre-and-post operative metabolic changes in such patients. Mixed venous and arterial blood samples were drawn in fifty-two patients with congenital heart diseases; before, at the termination of surgery and thereafter at 24 hour intervals till the patient expired or for 5 days. The concentrations of the metabolites were determined using $1 \mathrm{H}-$ NMR technique. Eight non-survivors had significantly raised PrSL levels [median (range) 5.3(1.9-6.4) $\mathrm{mM}$ ] vs. survivors [median (range) $2.8(0.6-11.0) \mathrm{mM}] \quad(\mathrm{p}<0.02)$. Two of the non-survivors also had significant amounts of bacterial metabolites (Acetate, Pyruvate, Succinate and Formate) in their preoperative samples. Routine preoperative tests and preclinical assessments in all the patients did not reveal any infection or cardio-renal dysfunction. Forty-four survivors, with low PrSL levels and absence of metabolites indicative of infection had their PoSL levels returned to baseline after an initial rise. In all the eight non-survivors PoSL levels remained significantly higher [median(range) $7.3(5.3-8.6) \mathrm{mM}$ ] compared to the forty-four survivors [median (range) $3.9(1.1-10.2) \mathrm{mM}](\mathrm{p}<0.01)$. This study underlines the significance of PrSL and bacterialmetabolite concentrations in controlling sub-clinical infection or cardio-renal dysfunction to achieve better postoperative outcomes.

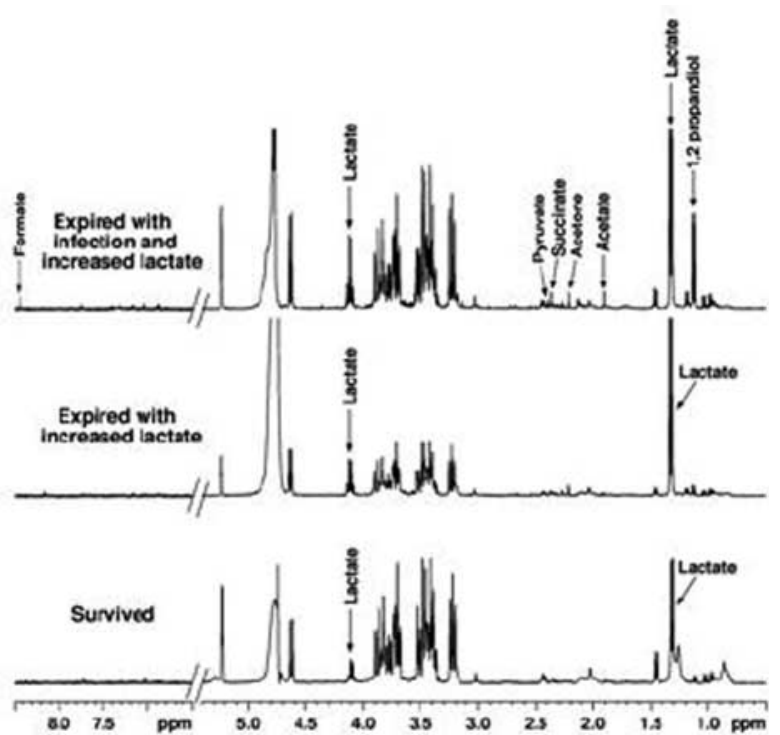

8300

Arterial haemodynamics and blood gas status are largely preserved in fetal sheep with chronically increased left ventricular afterload

Andrew T Hattam, Heart Research Group, Murdoch Children's

Research Institute, Australia

Magdy Sourial, The Royal Children's Hopsital, Australia

Paul A Brooks, Heart Research Group, Murdoch Children's Research Institute, Australia

Daniel J Penny, The Royal Children's Hopsital, Melbourne, Australia Joseph J Smolich, Heart Research Group, Murdoch Children's Research Institute, Australia

Objective: Although recent studies from our laboratory suggest that chronically increased left ventricular (LV) afterload in the fetus is associated with LV remodelling, the effect of such remodelling on haemodynamics and arterial blood gas status is unknown. We addressed this question in near-term fetal sheep with chronically increased LV afterload imposed at midgestation. 
Methods: In twelve twin pregnancies, one fetus underwent non-constrictive ascending aortic banding under anaesthesia at $92 \pm 3$ days gestation (term $=147$ days), with the other fetus serving as a control. After near-term $(140 \pm 1$ days $)$ anaesthesia of the ewe, both fetuses underwent echocardiography followed by insertion of axillary arterial (Ax), pulmonary arterial (PA) and left atrial (LA) fluid-filled catheters, and in a subgroup $(\mathrm{n}=6)$, an apical LV micromanometer catheter.

Results: An ascending aortic pressure gradient of $26 \pm 13 \mathrm{mmHg}$ was present on echocardiography in banded fetuses, without evidence of cardiac failure. Mean Ax $(48 \pm 6$ vs $52 \pm 7 \mathrm{mmHg})$ and PA pressures $(49 \pm 6$ vs $53 \pm 7 \mathrm{mmHg}$ ) were similar in control and banded fetuses. However, in banded fetuses, mean LA pressure $(6 \pm 2$ vs $4 \pm 2 \mathrm{mmHg}, \mathrm{p}<0.05)$ and LV enddiastolic pressure $(11 \pm 3$ vs $8 \pm 2 \mathrm{mmHg}, \mathrm{p}<0.05)$ were lower, while the maximal rate of rise of $\mathrm{LV}$ pressure was higher $(1401 \pm 279$ vs $1950 \pm 480 \mathrm{mmHg} / \mathrm{s}, \mathrm{p}<0.05)$. Apart from a lower $\mathrm{pH}(7.273 \pm 0.052$ vs $7.215 \pm 0.093 ; \mathrm{p}<0.05)$ and higher PCO2 $(51.3 \pm 5.5$ vs $57.5 \pm 7.8 \mathrm{mmHg} ; \mathrm{p}<0.05)$ in banded fetuses, Ax blood gas variables were not different between groups.

Conclusion: Arterial blood pressures are maintained and blood gas status is largely unimpaired in fetal sheep with chronically increased LV afterload.

\section{1}

Chronically increased afterload alters left ventricular growth patterns in fetal sheep

Andrew T Hattam, Heart Research Group, Murdoch Children's

Research Institute, Australia

Paul A Brooks, Heart Research Group, Murdoch Children's Research Institute, Australia

Daniel J Penny, The Royal Children's Hopsital, Melbourne, Australia Joseph J Smolich, Heart Research Group, Murdoch Children's Research Institute, Australia

Objective: Although the mid-gestation fetus with aortic stenosis and normal left ventricular (LV) chamber geometry frequently evolves into hypoplastic left heart syndrome in late-gestation, little is known about the effects of a chronic increase in LV afterload on fetal LV growth patterns. We addressed this question in fetal lambs with chronically increased LV afterload induced by aortic banding at mid-gestation.

Methods: Surgery was performed in 17 anaesthetised twinpregnancy ewes (gestation $=92 \pm 3$ days; term $=147$ days), with one fetus undergoing non-constrictive ascending aortic banding, and the second serving as the un-operated control. Serial fetal echocardiography studies were performed under maternal sedation at fortnightly intervals until near-term (140 \pm 1 days) to measure LV wall and chamber dimensions. Changes in LV growth patterns were assessed by comparing the slopes of the relationship between echocardiography parameters and gestation in banded and control fetuses.

Results: With aortic banding, peak ascending aortic pressure gradient rose from $8 \pm 4 \mathrm{mmHg}$ at mid-gestation to $26 \pm$ $13 \mathrm{mmHg}$ near-term, with no evidence of cardiac failure. This elevated LV afterload was accompanied by an increase in the slope of the LV posterior-wall thickness relationship $(\mathrm{p}<0.01)$, a decrease in the slope of the LV diastolic short-axis chamber diameter relationship $(p<0.001)$, and an unchanged LV longaxis relationship. Furthermore, the slope of the LV short-to-long axis ratio relationship was decreased in banded fetuses $(\mathrm{p}<0.05)$. Conclusion: Chronically elevated LV afterload in fetal sheep results in substantial changes in LV growth patterns, manifest not only via alterations in LV wall thickness and cavity dimensions, but also in LV chamber geometry.

\section{2}

Factors related to adverse drug reaction of digoxin in patients with impaired renal function

Ching-Shan Huang, Central Taiwan University of Science and Technology, Taiwan

Jen-Wen Chiang, Central Taiwan University of Science and Technology, Taiwan

The transport of digoxin was observed to be mediated by the multidrug resistance protein 1 (MDR1) gene product, P-glycoprotein and probably by organic anion transporting polypeptide 2 (OATP2). A total of 94 Taiwanese with serum creatinine values $=1.5 \mathrm{mg} / \mathrm{dl}$ who received low dose digoxin treatment $(0.125 \mathrm{mg}$ per day) were enrolled in this study. The record of coadministered P-glycoprotein inhibitors was reviewed for every patient. The most important single nucleotide polymorphisms of the MDR1 (C1236T, G2677T and C3435T) and OATP2 (G388A and $\mathrm{T} 521 \mathrm{C}$ ) genes were determined by the methods of polymerase chain reaction-restriction fragment length polymorphism. The 94 study subjects were divided into case group $[\mathrm{N}=20$ with adverse drug reaction (ADR), a trough serum digoxin $=2.5 \mathrm{ng} / \mathrm{mL}$ was observed within a one-year period subsequent to treatment] and control group $(\mathrm{N}=74$ without $\mathrm{ADR})$. The results demonstrated that there was neither difference of MDR1 $1236 \mathrm{C}>\mathrm{T}, 2677 \mathrm{G}>$ A,T and C3435C $>$ T haplotypes nor difference of OATP2 $388 \mathrm{G}$ $>\mathrm{A}$ and $521 \mathrm{~T}>\mathrm{C}$ haplotypes between the case and the control groups $(\mathrm{P}=0.532 \sim 0.991)$. However, significant difference of the frequency for co-administration with $\mathrm{P}$-glycoprotein inhibitors was observed $(\mathrm{P}=0.004)$. In conclusion, for the patients with impaired renal function, digoxin ADR may be caused by P-glycoprotein inhibitor co-administration rather than by MDR1 and OATP2 polymorphisms.

\section{3}

The endothelin system in pulmonary arteries of patients with failed Fontan circulation

Hidekazu Ishida, Department of Pediatrics, Osaka University Graduate School of Medicine, Osaka, Japan, Japan

Shigetoyo Kogaki, Department of Pediatrics, Osaka University Graduate School of Medicine, Osaka, Japan, Japan

Hiroaki Ichimori, Department of Pediatrics, Osaka University Graduate School of Medicine, Osaka, Japan, Japan

Jun Narita, Department of Pediatrics, Osaka University Graduate School of Medicine, Osaka, Japan, Japan

Takayoshi Ueno, Department of Cardiovascular Surgery, Osaka University Graduate School of Medicine, Osaka, Japan, Japan

Hajime Ichikawa, Department of Cardiovascular Surgery, Osaka University Graduate School of Medicine, Osaka, JAPAN, Japan Yoshiki Sawa, Department of Cardiovascular Surgery, Osaka University Graduate School of Medicine, Osaka, JAPAN, Japan

Keiichi Ozono, Department of Pediatrics, Osaka University Graduate School of Medicine, Osaka, JAPAN, Japan

Objectives: An outcome of the Fontan procedure has been improved recently, however not a few patients have failure of the Fontan circulation because of high pulmonary vascular resistance. Endothelin-1 (ET-1) is one of the most important vasoconstrictive substances, but there are only a few studies focused on ET-1 and especially on its receptors after the Fontan 
procedure. The aim of this study is to clarify the expression levels of ET-1 and endothelin receptor type A (ETAR) and type $\mathrm{B}$ (ETBR) in the pulmonary arteries of patients with failed Fontan circulation.

Methods: We retrospectively analyzed ten autopsy lung tissues from the patients died after the Fontan procedure (group F), and compared to four normal lung tissues without cardiovascular disease (group N). We evaluated expression levels of ET-1, ETAR and ETBR in their pulmonary arteries using immunohistochemistry and quantitative real-time PCR.

Results: The distal pulmonary arteries in group $\mathrm{F}$ displayed intimal and medial hypertrophy with increased wall thickness $(p<0.05$ vs Group N). The expressions of ET-1, ETAR and ETBR in group $F$ was significantly increased, and mRNA expression of ET-1 was also increased ( $p<0.05$ vs Group N). Conclusion: The pulmonary arteries of patients with failed Fontan circulation showed significant medial hypertrophy, and the expressions of ET-1, ETAR and ETBR were significantly increased. High ET-1 and its receptors expression in the small pulmonary arteries might deteriorate Fontan circulation by elevating pulmonary artery pressure and promoting vascular remodeling with medial hypertrophy.

\section{4}

Effect of Hydrogen Sulfide on the Proliferation of the Aortic Smooth Muscle Cells of Normal Rats and Spontaneous Hypertensive Rats

Hongfang Jin, Department of Pediatrics, Peking University First

Hospital, Beijing, China

Hui Yan, Department of Pediatrics, Peking University First Hospital, Beijing, China

Junbao Du, Department of Pediatrics, Peking University First Hospital, Beijing, China

Chaoshu Tang, Key Laboratory of Molecular Cardiovascular Diseases, Beijing, China

Objective: Hydrogen sulfide, a well-known toxic gas with the smell of rotten eggs, was proved to be generated in vivo in human and animal organisms and participated in many pathophysiological processes. Hypertension is one of the most common diseases harmed seriously, while the mechanisms are far from clear. Proliferation of aortic smooth muscle cells (ASMCs) is a pivot component of the development of hypertension. To investigate the effect of hydrogen sulfide on the proliferation of ASMCs of normal rats and spontaneous hypertensive rats (SHRs).

Methods: ASMCs of normal rats and spontaneous hypertensive rats were cultured and separated into experimental group and control group, respectively. The experimental group was added into NaHS (hydrogen sulfide donor) or PPG (the inhibitor of hydrogen sulfide synthesis) of different consistencies, while the control group was added into DMSO of equal volume. PCNA and p-ERK in ASMCs were detected by Western blot at each time point.

Results: NaHS had no effect on the proliferation of ASMCs of normal rats and the expression of p-ERK. PPG advanced the proliferation of ASMCs of normal rats and the expression of p-ERK obviously. NaHS inhibited the proliferation of ASMCs of SHR and the expression of p-ERK obviously. PPG advanced the proliferation of ASMCs of SHR and the expression of p-ERK obviously.

Conclusion: Hydrogen sulfide could regulate the proliferation of ASMCs of normal rats and SHR through inhibiting the expression of $\mathrm{p}$-ERK.

\section{5}

Phenotypic predictors of chromosome 22q11.2

microdeletion : A prospective study of 150 children

$<2$ years with conotruncal malformations

Mahesh Kappanayil, Amrita Institute of Medical Sciences and Research Center, Kochi, Kerala, India, India

Alka Anil Kumar, Amrita Institute of Medical Sciences and Research Center, Kochi, Kerala, India, India

Sheela Nampoothiri, Amrita Institute of Medical Sciences and Research Center, Kochi, Kerala, India, India

MV Thampi, Amrita Institute of Medical Sciences and Research Center, Kochi, Kerala, India, India

KR Sundaram, Amrita Institute of Medical Sciences and Research Center, Kochi, Kerala, India, India

Krishna Kumar, Amrita Institute of Medical Sciences and Research

Center, Kochi, Kerala, India, India

Background: 22q11.2 microdeletion in patients with conotruncal malformations significantly impacts patient management and counseling. Marked phenotypic variability makes clinical prediction difficult. Diagnostic cytogenetic testing has limited availability in most developing nations and may take several days. Objectives: To identify frequency of 22q11 microdeletion and the most specific phenotypic markers for its clinical prediction in a large cohort of Indian children $\leqslant 2$ years with conotruncal malformations.

Methods: Prospective, hospital-based, observational study conducted at a tertiary care hospital in India. Conotruncal anomalies were identified through echocardiography. Independent phenotypic evaluation was done by pediatric geneticist (blinded to cardiac diagnosis). Cytogenetic diagnosis was established through fluorescent in-situ hybridization (FISH). Genotype-phenotype correlations were studied using multivariate logistic regression analysis.

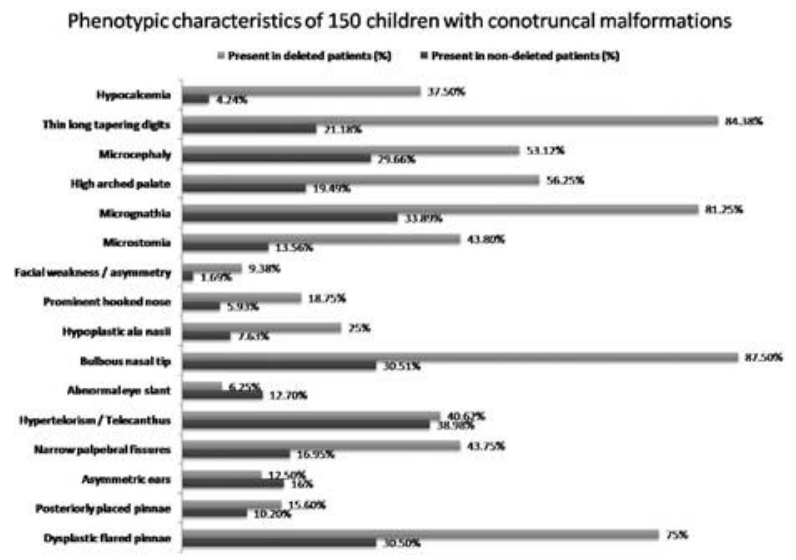

Results: FISH was positive for microdeletion $22 \mathrm{q} 11.2$ in 32 of 150 patients (21.3\%). Frequency in individual lesions wastruncus arteriosus $(55.6 \%)$, interrupted aortic arch $(50 \%)$, TOF/ PA (30\%), conoventricular VSD (21.1\%), DORV (18.8\%), simple TOF $(12.9 \%)$ and TOF/absent pulmonary valve $(0 \%)$. Among the 24 phenotypic variables studied, bulbous nasal tip (OR-16.7, $\mathrm{p}<0.001$ ), dysplastic flared pinnae (OR-9, p 0.003), microstomia (OR-10.7, p 0.002), thin long fingers (OR-3.4, p 0.066), and hypocalcemia (OR-25, p 0.001) emerged as predictors of 22q11 microdeletion on multivariate analysis. Discriminant analysis provided a computational scoring model to clinically predict 22q11.2 deletion with correct classification in $75.3 \%$ cases. 
Conclusions: This study identifies specific phenotypic guidelines for bed-side prediction of 22q11 microdeletion and may assist in better management and counseling for conotruncal malformations when cytogenetic diagnosis is awaited or unavailable.

\section{6}

Experience of three cases with genetically proven Loeys-Dietz Syndrome

Sung Hoon Kim, Korean Society of Pediatric Cardiology and Cardiac Surgery, Korea

Joon Huh, Korean Society of Pediatric Cardiology and Cardiac Surgery, Samsung Medical Center, Korea

I-Seok Kang, Korean Society of Pediatric Cardiology and Cardiac Surgery, Samsung Medical Center, Korea

Heung Jae Lee, Korean Society of Pediatric Cardiology and Cardiac Surgery, Samsung Medical Center, Korea

Chang-Suk Ki, Department of Laboratory Medicine and Genetics,Samsung Medical Center, Korea

Loeys-Dietz syndrome is described as an aortic-aneurysm syndrome with widespread systemic involvement which is caused by heterozygous mutations in the genes encoding transforming growth factor $B$ receptors 1 and 2 ( TGFBR1 and TGFBR2, respectively). This disease is characterized by arterial tortuosity and aneurysms, hypertelorism, and bifid uvula or cleft palate and lack of ocular findings of Marfan syndrome and often present in childhood with significant cardiovascular problems. Marfan clinic in Samsung medical center is composed of many departments such as pediatrics, internal medicine, cardiovascular surgery, orthopedics, ophthalmology, radiology, laboratory medicine and genetics and coordinator and we describe 3 patients with Loeys-Dietz syndrome who was diagnosed by genetic test at various ages.

\begin{tabular}{cccc}
\hline & & & \\
patient 1 & mutation ; & nucleotide : & amino acid : \\
& TGFBR1Exon6 & c.1124C $>\mathrm{T}$ & Thr375Arg \\
patient 2 & mutation; & nucleotide : & amino acid \\
& TGFBR2Exon4 & c.1130A $>$ G & :His378Arg \\
patient 3 & mutation ; & nucleotide : c.915_ & ? \\
& TGFBR2Exon4 & 916delCCinsTCATG & \\
\hline
\end{tabular}

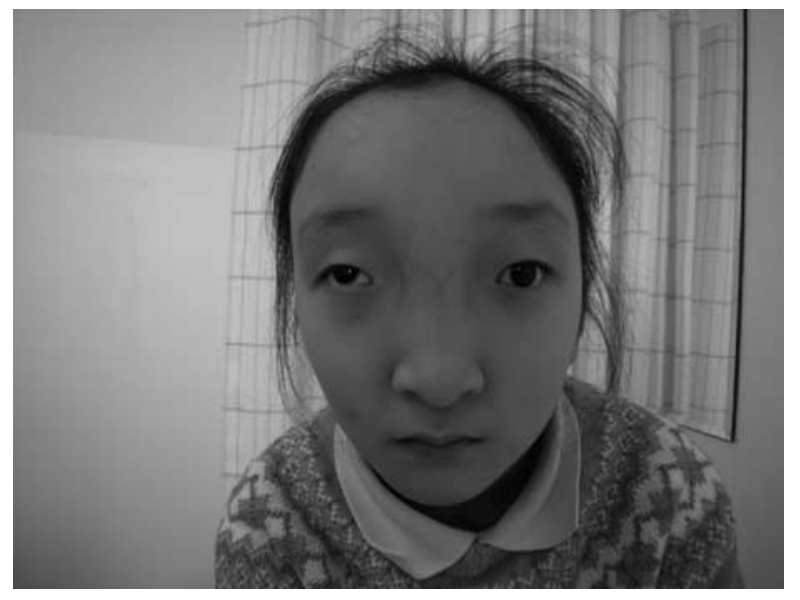

8307

Pulsed doppler echocardiographic assessment in monocrotaline induced pulmonary hypertensive rats Kanta Kishi, Japan

Denan Jin, Department of pharmacology, Osaka medical college, Japan

Shinji Takai, Department of pharmacology, Osaka medical college, Japan
Kenichi Okumura, Department of pediatrics, Osaka medical college, Japan

Yasuhiko Mori, Department of pediatrics, Osaka medical college, Japan Hiroshi Tamai, Department of pediatrics, Osaka medical college, Japan Mizuo Miyazaki, Department of pharmacology, Osaka medical college, Japan

We investigated whether pulsed Doppler echocardiography can assess the progression of pulmonary hypertension $(\mathrm{PH})$ and the right ventricle $(\mathrm{RV})$ function in rats.

Six-week-old S-D rats were injected with monocrotaline (MCT $60 \mathrm{mg} / \mathrm{kg}$ ) or vehicle and underwent serial echocardiography. The ratio of pulmonary acceleration time to ejection time (AcT/ET) and RV Tei index were measured by pulsed Doppler echocardiography. Furthermore, gross anatomic evaluation of heart and measurements of pulmonary arterial systolic pressure (PASP) were performed.

PASP were significantly elevated in MCT group two weeks after injection and more elevated four weeks after injection. The ratio of RV weight to left ventricle plus septum weight were not significantly different between two groups two weeks after injection but were significantly increased in MCT group four weeks after injection. Similarly, AcT/ET was significantly less in the MCT group two weeks after injection (MCT: $0.30+/$ -0.01 vs control: $0.39+/-0.01 \mathrm{p}<0.001)$ and became more less four weeks after injection (MCT: $0.21+/-0.01$ vs control: $0.39+/-0.01 \mathrm{p}<0.001)$. RV Tei index was not significantly different between two groups two weeks after injection but significantly greater in the MCT group four weeks after injection (MCT: $0.70+/-0.06$ vs control: $0.34+/-0.02 \mathrm{p}<0.001$ ). Furthermore, AcT/ET correlated inversely with PASP $(\mathrm{r}=-0.86 \mathrm{p}<0.001)$ and RV Tei index correlated linearly with the ratio $\mathrm{RV}$ weight to left ventricle plus septum weight $(\mathrm{r}=0.79 \mathrm{p}<0.001)$.

We concluded that AcT/ET and RV Tei index can assess the progression of $\mathrm{PH}$ and $\mathrm{RV}$ dysfunction due to RV hypertrophy.

\section{9}

Intercalated disk damage and cardiac pump function reduction in rats treated with zidovudine and preventive effect of vitamin $C$

Ornella Milanesi, Department of Paediatrics, University of Padova, Italy Anna S Belloni, Department of Human Anatomy and Physiology, University of Padua, Italy

Lucia Petrelli, Department of Human Anatomy and Physiology, University of Padua

Ezia Ruga, Department of Pediatrics, University of Padua, Italy Silvia Ricato, Department of Pediatrics, University of Padua, Italy Muarizio Cavalli, Department of Pharmacology and Anaesthesiology, University of Padua, Italy

Gabriella Cargnelli, Department of Pharmacology and Anaesthesiology, University of Padua, Italy

Sergio Bova, Department of Pharmacology and Anaesthesiology, University of Padua, Italy

Zidovudine (AZT; 3'-azido-3'-deoxythymidine), highly active antiretroviral drug, has been hypothesized to induce myocardial toxicity during long term treatment. This experimental study aims at evaluating by echocardiography, electron microscopy (EM) and immunocytochemistry (ICC) the cardio-toxicity of chronic administration of AZT in rats and the possible preventing effect of vitamin C. Six adult Wistar Kyoto rats received AZT $(1 \mathrm{mg} / \mathrm{ml})$ in the drinking water for 8 months, six vitamin $\mathrm{C}$ $(10 \mathrm{~g} / \mathrm{kg}$ of food) and AZT, six vitamin $\mathrm{C}$ alone, and six served as 
controls. A 2D-derived m-mode echocardiographic evaluation of the LV Fractional Shortening (FS) was performed at the beginning and before the sacrifice. LV fragments from each rat were processed for EM and light and EM and ICC for visualization of connexin-43, a protein expressed in the in cardiac intercalated discs. Cardiac FS was significantly reduced, compared to untreated animals, by $19.3 \%(\mathrm{p}<0.05)$ and by $10.1 \%(\mathrm{p}<0.05)$ in rats treated with AZT and AZT + vitamin C, respectively. However, no statistical difference was detected between these two groups ( $p>0.05$ ). Vitamin $\mathrm{C}$ by itself did not modify FS $(p>0.05)$. EM showed striking alteration of intercalated discs, totally reverted by the administration of vitamin C. Light microscopy ICC evidenced connexin-43 immunopositivity. EM demonstrated that anti-connexin-43 immunogold was exclusively located in the zones of the intercalated discs. In conclusion, chronic AZT therapy causes a reduction of LV SF and damage of intercalated disk in rats. Concomitant assumption of vitamin $\mathrm{C}$ reverse the morphologic damage but restores only partially the functional impairment.

\section{0}

Acute hemodynamic changes after experimental Fontan circulation in a swine model without the use of bypass

Fotios A Mitropoulos, Department of Pediatric and Congenital Heart Surgery, Onassis Cardiac Surgery Centre, Athens, Greece, Greece

Meletios A Kanakis, Center for Experimental Surgery Biomedical Research Foundation Academy of Athens, Athens, Greece, Greece Dimitrios Angouras, Department of Cardiac Surgery, Attikon Hospital, University of Athens, Greece

Constantine Dimitriou, Center for Experimental Surgery

Biomedical Research Foundation Academy of Athens, Athens,

Greece, Greece

Michael Katsimpoulas, Center for Experimental Surgery Biomedical Research Foundation Academy of Athens, Athens, Greece, Greece Chris Rokkas, Department of Cardiac Surgery, Attikon Hospital, University of Athens, Greece, Greece

Panayiotis E Karayannacos, Center for Experimental Surgery Biomedical Research Foundation Academy of Athens, Athens, Greece, Greece Constantine E Anagnostopoulos, Department of Cardiac Surgery, Attikon Hospital, University of Athens

Objective: Our study uses a swine model of total cavopulmonary connection (TCPC) to test the hemodynamic performance before and after the establishment of (TCPC) without the use of cardiopulmonary bypass (CPB) or other means of temporary bypass.

Methods: 8 anesthetized animals (mean weight $43 \mathrm{~kg}$, mean age 4.5 months) underwent (TCPC). This was established by the use of an appropriate size Y-shaped conduit connecting and draining superior and inferior caval veins (end to end anastomosis) to the pulmonary trunk (end to side anastomosis). Decompression of the right ventricle was achieved with the use of a roller pump. Before and after the establishment of TCPC, hemodynamic parameters were recorded.

Results: All animals needed volume loading during the performance of the end to end anastomosis between the graft and the great veins. One animal needed inotropic support.

All animals demonstrated hemodynamic stability after the institution of the Fontan circulation. When the TCPC was established, a statistically significant decrease was observed in the mean arterial pressure $(\mathrm{P}<0.001)$, cardiac output $(\mathrm{P}<0.0001)$, mean pulmonary artery pressure $(\mathrm{P}<0.001)$ and heart rate $(\mathrm{P}<0.01)$. A statistically significant increase in the inferior vena caval pressure $(\mathrm{P}<0.01)$ and in the pulmonary vascular resistance $(\mathrm{P}<0.05)$ was also observed. Changes in left atrial pressure and in systemic vascular resistance were not statistically significant.

Conclusions: The establishment of a beating heart Fontan circulation without the use of $\mathrm{CPB}$ or other means of temporary bypass is feasible and our results are in concordance with the pattern of the Fontan paradox.

\begin{tabular}{|c|c|c|c|}
\hline Parameter & $\begin{array}{l}\text { Baseline (mean } \\
\text { value } \pm \mathrm{SE})\end{array}$ & $\begin{array}{c}\text { Fontan (mean } \\
\text { value } \pm \mathrm{SE})\end{array}$ & p-value \\
\hline Mean Aortic Pressure $(\mathrm{mmHg})$ & $44.35 \pm 2.36$ & $28.57 \pm 1.53$ & $<0.001$ \\
\hline $\begin{array}{l}\text { Mean Inferior Vena Caval } \\
\text { pressure }(\mathrm{mmHg})\end{array}$ & $13.8 \pm 1.77$ & $25.85 \pm 2.00$ & $<0.01$ \\
\hline $\begin{array}{l}\text { Mean Left Atrial pressure } \\
(\mathrm{mmHg})\end{array}$ & $6.37 \pm 0.92$ & $4.15 \pm 0.53$ & Not Significant \\
\hline $\begin{array}{l}\text { Mean Pulmonary Artery } \\
\text { Pressure }(\mathrm{mmHg})\end{array}$ & $18.38 \pm 0.60$ & $11.03 \pm 1.10$ & $<0.001$ \\
\hline $\begin{array}{l}\text { Pulmonary Vascular Resistance } \\
\text { (Wood's units) }\end{array}$ & $3.49 \pm 0.21$ & $5.97 \pm 0.73$ & $<0.05$ \\
\hline Cardiac Output (lt/min) & $3.48 \pm 0.27$ & $1.17 \pm 0.14$ & $<0.0001$ \\
\hline $\begin{array}{l}\text { Systemic Vascular Resistance } \\
\text { (Wood's units) }\end{array}$ & $11.11 \pm 0.86$ & $15.86 \pm 2.19$ & Not Significant \\
\hline Heart Rate (per min) & $89.45 \pm 4.05$ & $70.70 \pm 3.33$ & $<0.01$ \\
\hline
\end{tabular}

8311

Creatine Kinase Increase was Identified in Cardiac Tissue in Enhanced Maturation by Perinatal Glucoocrticoid Therapy on Fetal and Neonate Rats

Masanori Mizuno, Department of Pediatrics, St. Marianna University School of Medicine, Japan

Kentaro Asoh, Department of Pediatrics, St. Marianna University

School of Medicine, Japan

Yuko Takeba, Department of Pharmacology, St. Marianna University School of Medicine, Japan

Yachiyo Kurihara, Department of Pediatrics, St. Marianna University School of Medicine, Japan

Kenjiro Gotoh, Department of Pediatrics, St. Marianna University School of Medicine, Japan

Masaki Arima, Department of Pediatrics, St. Marianna University

School of Medicine, Japan

Yoshimitsu Tsuzuki, Department of Pediatrics, St. Marianna

University School of Medicine, Japan

Yasushi Koitabashi, Department of Pediatrics, St. Marianna University School of Medicine, Japan

Objective: Maturation of heart is characterized by increase of Creatine Kinase. We have reported that cardiac-function related proteins increased with prenatal glucocorticoid administration in the fetal rat models. The aim of the present study was to demonstrate the effect of prenatal glucocorticoid administration on the creatine kinase level in fetal and neonatal cardiac tissues.

Methods: Dexamethasone (DEX) was administrated to pregnant rat for 2 days on day 19 and 20 of gestation to simulate preternal DEX theraphy. Mitochondria was extracted from the fetal and neonatal cardiac tissues, then the expression of creatine kinase in mitochondria was analyzed using western blotting and immunohistochemistry. Uptake of JC-1 (5,5',6,6'-tetrachloro-1,1',3,3'-tetraethyl-benzimidazolocarbocyanine iodide) to mitochondria was measured using fluorometry, as a parameter of its function.

Results: Expression of creatine kinase in 21-day fetus and 1-day neonates of DEX groups were significantly increased compared to the control. The uptake of JC-1 to mitochondria was also significantly increased in fetal and neonatal DEX group. 
Conclusions: These results will suggest that perinatal glucocorticoid administration increases cardiac contraction-related proteins and may contribute to the maturation of the cardiac tissue and also to the mitochondrial activation in the fetal rats.

\section{2}

Computer modelling of fetal blood flow distribution in hypoplastic left heart syndrome: effect of pulmonary trunk/ductal enlargement and aortic coarctation Jonathan P Mynard, Heart Research Group, Murdoch Children's Research Institute, Australia

Andrew T Hattam, Heart Research Group, Murdoch Children's Research Institute, Australia

Malcolm R Davidson, Department of Chemical and Biomolecular Engineering, University of Melbourne, Australia

Daniel J Penny, Heart Research Group, Murdoch Children's Research Institute, Australia

Joesph J Smolich, Heart Research Group, Murdoch Children's Research Institute, Australia

Objective: Recently, we developed a computer model of the fetal circulation which produced blood flow distributions seen in vivo. The aim of this study was to use this model to investigate flow patterns in hypoplastic left heart syndrome (HLHS), a condition characterised by reduced left ventricular output and ascending aortic (AA) diameter, and which is often associated with pulmonary trunk (PT) and ductus arteriosus (DA) enlargement, and aortic isthmus (AI) coarctation. Particular attention was directed to changes in upper body (UB, including cerebral) flow.

Methods: The computer model consisted of one-dimensional representations of left/right ventricles (LV/RV) and large arteries, and a lumped-parameter model of downstream circulations. A spectrum of HLHS severity was simulated by reducing LV output and AA diameter, from normal to aortic valve atresia (AVA, 10\% of normal AA diameter and zero LV output). With AVA, PT/DA diameters were then progressively increased (to $80 \%$ above normal). Subsequently, AI diameter was reduced to simulate coarctation.

Results: With increasing HLHS severity, combined ventricular output and UB flow fell progressively to $17 \%$ and $26 \%$ below normal respectively for AVA, despite RV output increasing by $29 \%$ and the appearance of retrograde AI flow. PT/DA enlargement increased RV output further (by 54\%), thereby ameliorating UB flow reduction to $13 \%$. However, superimposition of AI coarctation progressively reduced UB flow towards zero after AI diameter had been reduced below $40 \%$ of normal.

Conclusions: Computer modelling of the fetal circulation suggests that PT/DA enlargement and aortic coarctation have a major impact on UB flow in HLHS.

\section{3}

Multiple circulatory fates of fetal pulmonary waves determined using linear transmission line theory Jonathan P Mynard, Heart Research Group, Murdoch Children's Research Institute, Australia

Daniel J Penny, Heart Research Group, Murdoch Children's Research Institute, Australia

Joseph J Smolich, Heart Research Group, Murdoch Children's Research Institute, Australia

Objective: Recent studies in lambs using wave intensity (WI) analysis suggest that a characteristically low fetal lung blood flow is related to an extremely large backward-running compression wave (BCW) present in the left (LPA) and right pulmonary arteries (RPA), which is partially transmitted via the main pulmonary artery (MPA) into the pulmonary trunk $(\mathrm{PT})$ as a pressure-increasing, flow-reducing wave, and into the ductus arteriosus (DA) as a pressure- and flowincreasing wave. The aim of this study was to more fully elucidate the circulatory fates of the fetal pulmonary $\mathrm{BCW}$ by assessing transmission and reflection properties of the LPA/RPA/MPA and MPA/PT/DA junctions.

Methods: Pressure, flow and WI reflection and transmission coefficients at the LPA/RPA/MPA and MPA/PT/DA junctions were calculated using linear transmission line theory. Vessel diameter and wave speed values required for these calculations were obtained from fetal lamb studies.

Results: LPA and RPA BCW were partially transmitted into the MPA (pressure/flow/WI coefficients: 0.5/0.92/0.24 and 0.58/ $0.92 / 0.32$ respectively). The resulting MPA BCW was partially transmitted into the PT (coefficients: 0.52/1.16/0.25) and, with lesser flow and WI consequences, into the DA (coefficients: 0.52/ $0.33 / 0.15)$. LPA and RPA BCW were also partially re-reflected at the LPA/RPA/MPA junction as pressure-reducing waves. However, the re-reflected wave in the LPA was largely cancelled by partial transmission of the RPA BCW into the LPA, and vice versa. Conclusion: Fetal pulmonary BCW have multiple circulatory fates, but the most haemodynamically important are transmission into the PT and DA, causing flow reduction and augmentation respectively, with pressure augmentation in both vessels.

\section{4}

Therapeutic effects of ghrelin in a neonatal model of pulmonary hypertension

Ana L Neves, Hospital S. João, Portugal

Daniel M Gonçalves, Faculdade de Medicina do Porto, Portugal

Mário S Santos, Faculdade de Medicina do Porto, Portugal

Maria J Baptista, Hospital S. João, Portugal

Tiago H Coelho, Hospital S. João, Portugal

Jorge C Pinto, Hospital S. João, Portugal

José C Areias, Hospital S. João, Portugal

Adelino L Moreira, Faculdade de Medicina do Porto, Portugal

Introduction: Pulmonary hypertension $(\mathrm{PH})$ is a severe disease with a poor prognosis. Different forms of PH are characterized by vascular remodeling, resulting in increased vascular resistance and subsequent right heart failure. Significant progress in understanding the pathophysiology of $\mathrm{PH}$ has resulted in shift in therapeutic targets. Ghrelin (GHR) is a vasoactive peptide with several cardiovascular effects. We have recently described an infantile model of $\mathrm{PH}$ induced by monocrotaline (MCT).

\begin{tabular}{lcccc}
\hline & Ctrl & Ctrl + GHR & MCT & MCT + GHR \\
\hline $\begin{array}{l}\text { Body Weight } \\
\text { (BW), g }\end{array}$ & $46 \pm 1$ & $61 \pm 2 \mathrm{a}$ & $42 \pm 2$ & $56 \pm 4 \mathrm{a}, \mathrm{c}$ \\
$\mathrm{RV} / \mathrm{LV}, \mathrm{g} / \mathrm{g}$ & $0.29 \pm 0,02$ & $0.29 \pm 0,04$ & $0.49 \pm 0,05 \mathrm{a}$ & $0.50 \pm 0,03 \mathrm{a}, \mathrm{b}$ \\
$\mathrm{Lung} / \mathrm{BW}, \mathrm{g} / \mathrm{g}$ & $8.70 \pm 0.46$ & $8.23 \pm 0.11$ & $12.14 \pm 0.79 \mathrm{a}$ & $11.03 \pm 1.12 \mathrm{a}, \mathrm{b}$ \\
$\mathrm{RVPmax}, \mathrm{mmHg}$ & $18.3 \pm 1.9$ & $19.5 \pm 3$ & $41.9 \pm 1.5 \mathrm{a}$ & $36.7 \pm 4.6 \mathrm{a}, \mathrm{b}, \mathrm{c}$ \\
$\mathrm{RV} \mathrm{dP/dtmax}$ & $657 \pm 157$ & $712 \pm 56$ & $988 \pm 95 \mathrm{a}$ & $1288 \pm 162 \mathrm{a}, \mathrm{b}$ \\
$\mathrm{mmHg} / \mathrm{s}$ & & & & \\
$\mathrm{RV} \mathrm{dP/dtmin,}$ & $-440 \pm 60$ & $-473 \pm 43$ & $-765 \pm 141 \mathrm{a}$ & $-823 \pm 135 \mathrm{a}, \mathrm{b}$ \\
$\mathrm{mmHg} / \mathrm{s}$ & & & & \\
$\mathrm{RV}-\mathrm{Tau}, \mathrm{ms}$ & $20 \pm 1$ & $18 \pm 3$ & $32 \pm 3 \mathrm{a}$ & $26 \pm 8 \mathrm{c}$ \\
$\mathrm{LVPmax}, \mathrm{mmHg}$ & $58.7 \pm 2.8$ & $42.2 \pm 6.9$ & $39.9 \pm 3.5 \mathrm{a}$ & $52.6 \pm 1.8 \mathrm{c}$ \\
\hline
\end{tabular}

Objective: The aim of this work was to establish a neonatal model of pulmonary hypertension induced by MCT and to evaluate hemodynamic and morphometric effects of GHR administration. 
Methods: Wistar rats 3-day-old randomly received MCT (30 mg/ $\mathrm{Kg}$, subcutaneous) or equal volume of vehicle (Group Ctrl). At D7, animals treated with MCT randomly received GHR $(100 \mu \mathrm{g} / \mathrm{kg}$, subcutaneous, bid) (Group MCT-GHR) or vehicle (Group MCT) for 2 weeks. Between D21-23, animals were instrumented to evaluate the following right ventricle (RV) and left ventricle (LV) hemodynamic parameters: pressure at peak systole (Pmax), peak rates pressure rise ( $\mathrm{dP} / \mathrm{dtmax})$, pressure fall (dP/dtmin) and time constant $\tau$. At the end, heart and lungs were excised and weighted.

Results: Presented in the table as means \pm SE. $\mathrm{p}<0.05$ : a vs. Ctrl; b vs. Ctrl-GHR; c vs. MCT.

Conclusions: Administration of GHR induced an increase in body weight and attenuated hemodynamic alterations induced by MCT. GHR may present as a future candidate for therapeutic use in $\mathrm{PH}$ in neonatal period.

\section{5}

Do Bolus Doses of UFH Contribute to Increased Bleeding Risks in Children?

Fiona Newall, The Royal Children's Hopsital, Australia

V Ignjatovic, The University of Melbourne, Murdoch Children's

Research Institute and The Royal Children's Hospital, Australia

L Johnston, The University of Melbourne and Queens University Belfast

Geoff Lane, Australia

P Monagle, The University of Melbourne; Murdoch Children's Research Institute; The Royal Children's Hospital, Australia

Clinical reports suggest UFH-related major bleeding rates of up to $24 \%$ in children. Current recommendations for UFH therapy recommend a $75-100 \mathrm{IU} / \mathrm{kg}$ bolus followed by an agedependent continuous infusion rate. This recommendation is based on a study that first measured UFH response 6 hrs post commencement; at that time, 39\% of patients achieved a minimum level APTT $(>55 \mathrm{sec})$. We evaluated laboratory response to a single UFH bolus dose of $75-100 \mathrm{u} / \mathrm{kg}$ in children $<16$ years of age $(n=64)$ to determine the independent impact of UFH bolus on anticoagulation levels in children. Venous blood was collected from a dedicated IV at 15, 30, 45 and 120 mins post UFH dose. APTT and anti-Xa assays were performed. The upper limit of the APTT assay was modified to measure time to clot formation up to 999 secs. APTT and anti$\mathrm{Xa}$ results for samples collected at 15 and 120 mins post bolus are presented in the table.

$67 \%$ of all APTT assays performed at 15, 30 and 45 mins post bolus were $>999$ secs. Current bolus recommendations give very high anticoagulant effect out to 120 mins post bolus dose, without concurrent continuous infusion. Despite very high APTT and anti-Xa levels, there were no major bleeding events. Lack of target APTT range achievement in previous paediatric studies may reflect the need for modified continuous.

\author{
8316 \\ Correlation of UFH Measures of Effect with Protamine \\ Titration in Children \\ Geoff Lane, Australia \\ Fiona Newall, The Royal Children's Hopsital, Australia \\ V Ignjatovic, The University of Melbourne, Murdoch Children's \\ Research Institute and The Royal Children's Hospital, Australia \\ L Johnston, The University of Melbourne and Queens University \\ Belfast \\ R Summerhayes, Australia
}

P Monagle, The University of Melbourne; Murdoch Children's Research Institute; The Royal Children's Hospital, Australia

Therapeutic ranges for UFH therapy are based on measures of UFH effect corresponding to a UFH concentration of 0.2 to $0.4 \mathrm{IU} / \mathrm{mL}$ by protamine titration (Pr.T.). In adults, this corresponds to an anti-Xa assay of $0.35-0.7 \mathrm{IU} / \mathrm{mL}$. Such a reference range has never been validated in children. This study aimed to determine the strength of correlation between Pr.T., APTT, anti-Xa and TCT (6 NIH units) in plasma collected from children given a $75-100 \mathrm{IU} / \mathrm{kg}$ bolus of UFH. Blood samples were collected from children aged $<16$ years $(n=64)$ at 15,30 , 45 and 120 minutes post UFH. Agreement between assays was determined using Lin's Correlation Coefficient. Correlation coefficients between Pr.T. and APTT, anti-Xa and TCT were $0.49,0.49$ and 0.47 , respectively across the whole population. 47 anti-Xa assays were within 0.35 to $0.7 \mathrm{IU} / \mathrm{mL}$. Table 1 describes the agreement between anti-Xa assays of 0.35 to $0.7 \mathrm{IU} / \mathrm{mL}$ with therapeutic range determined by Pr.T.

Thus for the majority of time points (34/47) for which the anti $\mathrm{Xa}$ assay was therapeutic, the gold standard Pr.T. was supratherapeutic. APTT and TCT were similarly lacking agreement with Pr.T. Given that, in adults, the rationale for using antiXa, APTT or TCT as a monitoring test for UFH is based on the correlation with Pr.T., this lack of correlation in children suggests that these assays are not ideal for monitoring UFH in paediatrics. Further clinical outcome studies are required to determine optimal methods of monitoring UFH in children.

\section{8}

Association between Pulmonary Hypertension in Secundum Atrial Septal Defect and Gene Mutation Sri Endah Rahayuningsih, Faculty of Medicine Padjadjaran University Bandung Indonesia, Indonesia

Bambang Madiyono, Faculty of Medicine Indonesia University Jakarta Indonesia, Indonesia

Atrial septal defect (ASD) is a congenital heart disease where there is a defect in the atrial septum which cause a shunt from left to right atrium. Most ASD cases resolves spontaneously, while the rest who don't will have an increased blood flow to the lung and develop into pulmonary hypertension. ASD is a multifactorial disease, including genetic factor, where there is a mutation in NKX.5, GATA4, TBX5 and MYH6 gene.

Objective: The aim of this study was to find an association between pulmonary hypertension in secundum ASD and gene mutation.

Methods: The subjects of this study were secundum ASD patients who fulfilled the inclusion criteria. A genetic epidemiology study was carried out to detect a mutation in NKX.5, GATA4, TBX5 and MYH6 gene using DNA isolation sequencing test. The diagnosis of ASD and pulmonary hypertension was made based on echocardiography.

Results: This study showed that seven children with ASD had a mutation in NKX2.5, GATA4, and MYH6 gene. There was no mutation in TBX5 gene. Five of the children had pulmonary hypertension and it occurs in secundum ASD with diameter between 14-32 mm. There was an association between pulmonary hypertension in secundum ASD and mutation in NKX2.5, GATA4, and MYH6 gene (P=0.037).

Conclusion: ASD with gene mutation more often has pulmonary hypertension;. Besides diameter, gene mutation has a role in the development of pulmonary hypertension in ASD. 


\section{9}

Bradycardia and atrial Fibrillation in utero and Short QT syndrome A mutation-specific disease

Georgia Sarquella-Brugada, Centre de Genètica Cardiovascular, Universitat de Girona, Spain

Oscar Campuzano, Centre de Genètica Cardiovascular, Universitat de Girona, Spain

Begoña Benito, Centre de Genètica Cardiovascular, Universitat de Girona, Spain

José Santos de Soto, Hospital Virgen del Rocio, Sevilla, Spain

Josefina Grueso, Hospital Virgen del Rocío, Sevilla, Spain

Josep Brugada, Hospital Clínic de Barcelona, Spain

Ramon Brugada, Centre de Genètica Cardiovascular, Universitat de Girona, Spain

Atrial fibrillation in the structurally normal heart is extremely rare in the fetus. In 2005 we described a new genetic disease characterized by bradycardia, atrial fibrillation in utero and short QT syndrome. Here we report the clinical and genetic investigation of two unrelated individuals with this characteristic phenotype.

Methods: Genetic, electrophysiological, echocardiogram and electrocardiographic analysis has been performed. Electrical cardioversion has been attempted in both.

Results: In patient \#1 pregnancy was interrupted at 38 weeks due to severe bradycardia and irregular rhythm, suggesting AF. Electrophysiological study revealed irregular atrial electrograms with a normal HV interval, confirming diagnosis of AF with slow ventricular response. QTc was $280 \mathrm{msec}$. The patient underwent one attempt at electrical cardioversion, which did not terminate AF. Patient \# 2 was delivered at week 37. After her birth her ecg showed irregular heart rhythm with bradycardia. Electrocardiograms showed paroxysmal atrial fibrillation and QTc of $290 \mathrm{msec}$. Intracardiac electrogram revealed atrial fibrillation. Genetic analyses revealed a single base $G$ to A substitution at nucleotide 421 in KCNQ1. At follow-up, both patients are developing well and the heart is growing normally though they both have conduction block with ventricular escape rhythm.

Conclusions: The presence of bradycardia and atrial fibrillation inutero and short QT syndrome is a characteristic phenotype of a gain of function mutation in KCNQ1. Despite of the severity of the clinical picture, the babies are developing well. The identification of a same mutation in two unrelated patients suggests that this is a hot spot for the disease.

\section{0}

Adverse effects of remote ischemic preconditioning in the immature heart

Michael R Schmidt, Aarhus University Hospital, Skejby, Denmark, Denmark

Nicolaj B Støttrup, Aarhus University Hospital, Skejby, Denmark, Denmark

Marie M Michelsen, Aarhus University Hospital, Skejby, Denmark, Denmark

Torsten T Nielsen, Aarhus University Hospital, Skejby, Denmark, Denmark

Hans Erik Bøtker, Aarhus University Hospital, Skejby, Denmark, Denmark

Raj K Kharbanda, John Radcliffe Hospital, Oxford, UK, United Kingdom

Andrew N Redington, Hospital for Sick Children, Toronto, Canada

Background: Remote ischemic preconditioning (rIPC) reduces myocardial injury in patients undergoing cardiac surgery.
Dialyzed plasma from rabbits undergoing rIPC protects isolated rabbit hearts against ischemia-reperfusion (IR)-injury. However, newborn hearts are known to be resistant to local preconditioning. We investigated whether protection against IR-injury is achieved in the newborn heart by 1) in-vivo rIPC and 2) dialysate from adult rabbits undergoing rIPC.

Methods: 21 newborn (1-4 days old) rabbits randomized into three groups: control $(n=7)$, in-vivo $\operatorname{rIPC}(n=7)$ and adult dialysate group $(n=7)$. Remote preconditioning in adult and newborn rabbits was induced by four 5-minute cycles of lower limb ischemia. Plasma from the remotely preconditioned adult rabbits was dialyzed through a $15 \mathrm{kDa}$ membrane. All isolated hearts underwent 55 minutes of stabilization while perfused with either standard Krebs-Henseleit buffer (control and in-vivo rIPC) or buffer with added dialysate, followed by 40 minutes of global no-flow ischemia and 120 minutes of reperfusion. Left ventricular (LV) function was assessed throughout using a balloon-tipped catheter and extent of infarction measured by tetrazolium staining.

Results: Baseline LV function (LV developed pressure (LVDP)) was similar in all groups. However, at 120 minutes of reperfusion, the reduction in LVDP was less in controls (50.1 $\pm 22.9 \%$ ) compared to in-vivo $(72.3 \pm 13.2 \%, \mathrm{p}=0.03)$ and dialysate $(82.4 \pm 13.7 \%, \mathrm{p}=0.01)$ groups. Infarct size (\% of $\mathrm{LV})$ was smaller in controls $(58.3 \pm 6.5 \%)$ than in $\operatorname{rIPC}(70.0 \pm 8.1 \%$, $\mathrm{p}=0.01)$ and dialysate $(73.4 \pm 8.4 \%, \mathrm{p}=0.008)$ groups.

Conclusion: Remote ischemic preconditioning worsens IR injury in the immature heart, either when applied in-vivo, or induced by dialysate from remotely preconditioned adult rabbits.

8322

The potent vasoregulatory mediator urotensin-II is produced in the fetus and demonstrates a delayed increase after birth

Catherine M Simpson, Murdoch Childrens Research Institute, Melbourne, Australia, Australia

Joseph J Smolich, Murdoch Childrens Research Institute, Melbourne, Australia, Australia

Andrew T Hattam, Murdoch Childrens Research Institute, Melbourne, Australia, Australia

Lara S Shekerdemian, The Royal Children's Hopsital, Melbourne, Australia

Daniel J Penny, The Royal Children's Hopsital, Melbourne, Australia

Objective: The mediators involved in perinatal regulation of vascular function are incompletely understood, although enothelin-1 (ET-1) is known to be important. Another potent vasoactive agent, urotensin-II (U-II), has been implicated in regulation of vascular tone, but its potential roles in the fetus or in circulatory adjustments at birth are unknown. The aims of this study were to determine if U-II was produced by the fetus and to compare changes in circulating U-II and ET-1 levels at birth.

Methods: Eighteen anaesthetised fetal lambs were instrumented with aortic and pulmonary arterial (PA) catheters for blood sampling and pressure measurement. In seven fetuses blood was simultaneously sampled from the ewe's carotid artery. In a subgroup of eight lambs, PA blood samples were taken $0.5,1$ and $4 \mathrm{hr}$ after delivery by caesarean-section. Plasma U-II and ET-1 concentrations were measured by radioimmunoassay.

Results: In fetuses, PA and aortic U-II levels were near-identical $(1.29 \pm 0.4$ vs. $1.28 \pm 0.35 \mathrm{pmol} / \mathrm{L})$, and were higher than in ewes $(0.72 \pm 0.3 \mathrm{pmol} / \mathrm{L}, \mathrm{p}<0.02)$. After birth, in conjunction with a reduced PA $(\mathrm{p}<0.006)$ and unchanged aortic blood pressure $(\mathrm{p}>0.3)$, ET-1 levels at all postnatal time points were greater than 
in the fetus $(\mathrm{p}<0.001)$. By contrast, U-II levels were unchanged from the fetus at 0.5 and $1 \mathrm{hr}$, but were greater by $4 \mathrm{hr}(\mathrm{p}=0.01)$. Conclusions: These findings suggest that the fetus produces U-II. However, the delayed elevation of circulating U-II levels following delivery implies that, unlike ET-1, this agent may not play a major role in the immediate haemodynamic changes at birth.

\section{3}

Urotensin-II receptor blockade lowers pulmonary vascular resistance in a perinatal model of meconium aspiration syndrome

Catherine M Simpson, Murdoch Childrens Research Institute, Melbourne, Australia, Australia

Joseph J Smolich, Murdoch Childrens Research Institute, Melbourne, Australia, Australia

Lara S Shekerdemian, The Royal Children's Hopsital, Melbourne, Australia

Daniel J Penny, The Royal Children's Hopsital, Melbourne, Australia

Objectives: Endothelin-1 (ET-1) contributes to the pathogenesis of meconium aspiration syndrome (MAS), however, failure of ET-receptor antagonists to completely reverse associated haemodynamic abnormalities suggests a role for other vasoconstrictors. The potent vasoactive peptide urotensin-II (U-II), has been implicated in the pathophysiology of pulmonary hypertension, but its contribution to MAS is unknown. This study has determined the role of U-II in a perinatal model of MAS via measurement of circulating U-II and ET-1 levels, and U-IIreceptor blockade studies.

Methods: After instrumentation with aortic, pulmonary trunk and left atrial catheters and pulmonary trunk/left pulmonary artery flow probes, 19 anaesthetized fetal lambs were randomised to the following groups: 1) control $(n=5), 2)$ control plus specific $U$ II-receptor blockade with palosuran $(10 \mathrm{mg} / \mathrm{kg} / \mathrm{hr}$ infusion; $\mathrm{n}=5), 3)$ tracheal instillation of $20 \%$ human meconium $(3 \mathrm{~mL} /$ $\mathrm{kg} ; \mathrm{n}=5)$, 4) meconium instillation plus palosuran $(\mathrm{n}=4)$. Haemodynamics and plasma U-II/ET-1 levels were measured for 6 hours after delivery during mechanical ventilation.

Results: After birth in control lambs, U-II levels increased $(\mathrm{p}<0.05)$, pulmonary vascular resistance (PVR) fell $(\mathrm{p}=0.01)$, and this fall was prevented by palosuran. By contrast, meconium animals displayed an exaggerated increase in U-II levels $(\mathrm{p}<0.05$ vs control) with an elevation in PVR $(p<0.005)$ which palosuran reduced by $>50 \%(\mathrm{p}<0.001)$. ET-1 levels rose in meconium $(p<0.025)$ and meconium-palosuran $(p<0.05)$ groups, but were not different between these groups.

Conclusion: In a perinatal model of MAS, raised U-II levels in combination with a reduction in PVR by U-II-receptor blockade, suggest that U-II plays a major role in the pathophysiology of this condition.

\section{4}

Immature hearts respond metabolically different to remote ischemic preconditioning than mature hearts: A tracer study

Nicolaj B Støttrup, Department of Cardiology, Aarhus University Hospital Skejby, Denmark

Michael R Schmidt, Department of Cardiology, Aarhus University Hospital Skejby, Denmark

Marie M Michelsen, Department of Cardiology, Aarhus University Hospital Skejby, Denmark

Keld Sorensen, Department of Cardiology, Aarhus University Hospital Skejby, Denmark
Torsten T Nielsen, Department of Cardiology, Aarhus University Hospital Skejby, Denmark

Hans Erik Bøtker, Department of Cardiology, Aarhus University Hospital Skejby, Denmark

Raj K Kharbanda, John Radcliffe Hospital, Oxford, United Kingdom Andrew N Redington, The Hospital for Sick Children, Toronto, Canada

Background: Carbohydrates constitute the dominant energy substrate in the immature and hypoxic mature heart. The effects of remote ischemic preconditioning (rIPC) on myocardial metabolism during ischemia and reperfusion are not fully understood. Dialyzed plasma from mature rabbits undergoing rIPC protects isolated mature rabbit hearts in a Langendorffmodel.

Objectives: To characterize differences between adult and neonatal response to different rIPC stimuli on glucose metabolism.

Methods: 21 newborn (1-4 days old) and 24 adult rabbits $(3 \mathrm{~kg}$.) were randomized into 3 groups: control, in-vivo rIPC and adult dialysate group. Hearts were mounted in a Langendorff-model and perfused for $55 \mathrm{~min}$ stabilization, $40 \mathrm{~min}$ global ischemia and $120 \mathrm{~min}$ reperfusion. Plasma from the remotely preconditioned adult rabbits was dialyzed, added to the buffer and administrated to the dialysate groups. Glycolytic flux was traced using $5-3 \mathrm{H}-$ labled glucose.

Results: Baseline exogenous glycolytic flux was 2.6 times higher in immature $(3.77 \pm 0.41 \mu \mathrm{mol} \times \min -1 \times \mathrm{g}$ dry wt-1) compared to mature hearts $(1.43 \pm 0.17 \mu \mathrm{mol} \times$ min- $1 \times \mathrm{g}$ dry wt-1; $\mathrm{P}=0.0001)$. During early reperfusion, mature hearts decreased glycolytic flux in $\operatorname{rIPC}(5.35 \pm 0.77 ; \mathrm{P}=0.01)$ and dialysate groups $(6.18 \pm 1.3 ; \mathrm{P}=0.05)$ compared to controls $(11.067 \pm 1.37 \mu \mathrm{mol} \times$ min-1 $\times$ g dry wt-1). No significant changes were observed in immature hearts. Lactate release during early reperfusion was reduced in mature rIPC and dialysate $(48.78$ and $53.80 \%, \mathrm{p}<0.05)$ when compared to controls. No differences were observed in the immature groups.

Conclusion: rIPC modulates adult glucose metabolism by inhibition of glycolytic flux during ischemia and early reperfusion. Immature hearts are incapable of this regulation.

\section{5}

Collaboratively charting the gene-to-phenotype network of human congenital heart defects

Bernard Thienpont, Center for Human Genetics, University of Leuven, Belgium

Jeroen Breckpot, Center for Human Genetics, University of Leuven, Belgium

Roland Barriot, Department of Electrotechnical engineering ESAT$S C D$, University of Leuven, France

Steven Van Vooren, Department of Electrotechnical engineering ESATSCD, University of Leuven, Belgium

Leon-Charles Tranchevent, Department of Electrotechnical engineering ESAT-SCD, University of Leuven, Belgium

Marc Gewillig, Department of Pediatric Cardiology, University Hospital Leuven, Belgium

Koenraad Devriendt, Center for Human Genetics, University of

Leuven, Belgium

Yves Moreau, Department of Electrotechnical engineering ESAT-SCD, University of Leuven, Belgium

The genetics of congenital heart defects is complex, due to a huge etiological heterogeneity. Using a collaborative Wiki, we develop a knowledge base and gene prioritization portal aimed at mapping genes involved in congenital heart defects (CHDs) and untangling their relations with corresponding human 
phenotypes. This portal, CHDWiki accessible at http://www. esat.kuleuven.be/ $\sim$ bioiuser/chdwiki, is not only an evolving community repository of current knowledge on the genetic basis of congenital heart defects, but also a collaborative environment for the study of candidate genes potentially implicated in CHDs, in particular by integrating recent strategies for the statistical prioritization of candidate genes. It thus serves the broad community that is facing CHDs, ranging from the pediatric cardiologist and clinical geneticist to the basic investigator of cardiogenesis. We will demonstrate the usefulness of this portal as a clinical guide for the genetic diagnosis of CHD using illustrative clinical cases.

\section{6}

Spatiotemporal expression of histone acetyltransferases p300 and CBP during mouse cardiogenesis

Jie Tian, Department of Cardiology, Children's Hospital of Chongqing Medical University, China

Guozhen Chen, Department of Cardiology, Children's Hospital of Chongqing Medical University, China

Jing Zhu, Department of Molecular Biology, Pediatric Institute of

Chongqing Medical University, China

Gang Wu, Department of Cardiology, Children's Hospital of

Chongqing Medical University, China

Huichao Sun, Department of Cardiology, Children's Hospital of

Chongqing Medical University, China

Xupei Huang, Department of Biomedical Science, Charlie E. Schmidt

College of Biomedical Science, Florida Atlantic University, United States

Objectives: The aim of this study was to analyze the protein distribution and mRNA expression levels of histone acetyltransferases p300 and CBP in mouse heart during embryonic development and in the adult heart.

Methods: A total of 39 normal mouse embryonic hearts ranging in age from E7.5 to E18 as well as postnatal day 1 and adult hearts were used to analyze p300 and CBP protein distribution by immunohistochemistry. In addition, p300 and CBP mRNA levels were determined by RT-PCR using E10.5 E18 embryonic hearts as well as postnatal 1 day and adult hearts.

Results: Immunohistochemical staining revealed that p300 was highly expressed in nearly every region of the hearts during different stages of embryonic development as well as in postnatal day 1 and adult hearts. No CBP expression was evident in E7.5 heart. However, CBP protein was detectable at the subseuqnt stages, but at a relatively low level. Examination of p300 and CBP mRNA levels showed a peak expression at stage E10.5 heart, which was gradually decreased during the subsequent embryonic development stages and throughout the postnatal to the adult stages.

Conclusion: Our results demonstrate a dynamic expression pattern of p300 and CBP during mouse heart development, indicating that they may play an important role during cardiogenesis.

\section{7}

Cytokine and neutrophil number postoperative increase as probable pneumonia predictors

Alexey V Trepakov, Filatov;s Children Hospital Moscow, Russia Lidiy K Romanova, Institute of Human Morphology of Russian Academy of Medical Sciences Moscow, Russia

Lidiy F Makarova, Institute of Human Morphology of Russian Academy of Medical Sciences Moscow, Russia

Rimma S Bugrilova, Institute of Human Morphology of Russian Academy of Medical Sciences Moscow, Russia
Introduction: Surgery in infants is often associated with pulmonary inflammation. Sometimes, the blood level of proinflammatory factors IL-6 and IL- 8 is increased. The number of neutrophils is raised as well. A qualitative evaluation of the factors, cellular composition analysis of nonbronchoscopic trachebronchial suction (NTL) combined with clinical findings can help early diagnose pneumonia. The objective of the study was to reveal the blood level of proinflammatory cytokines, as well as examine the NTL cellular composition and cytokine level in infants before and after surgery.

Methods: We studied 24 infants. 15 patients underwent CPB surgery, 9 patients were operated without CPB. In 7 cases a diagnosis of pneumonia was made between 3 and 5 days postoperatively.

Results: The blood cytokine concentration in operated infants pre- and postoperatively is presented in the study. A significant increase in proinflammatory factors after surgery can be observed. We examined the NTL of 9 infants who underwent surgery and who did not develop pneumonia. We noticed that number of neutrophils increased significantly in all patients after CPB surgery, sometimes reaching $80 \%$. We consider it as a sign of pulmonary inflammation. The number of nonviable alveolar macrophages before and after surgery exceeded 50\%. It indicates a decrease in cellular pulmonary protection.

Conclusion: Thus, an increase in the blood level of proinflammatory cytokines was observed in infants who underwent $s$ urgery. At sometimes, the NTL relative number of neutrophils was increased. An early detection of the mentioned factors appears to be a diagnostic marker of the pulmonary inflammation onset.

\section{8}

Identification of molecular target proteins related to cardiac function in fetal rat tissues after antenatal glucocorticoid administration using a proteomics approach

Yoshimitsu Tsuzuki, Department of Pediatrics, St. Marianna University School of Medicine, Japan

Kentaro Asoh, Department of Pediatrics, St. Marianna University

School of Medicine, Japan

Yuko Takeba, Department of Pharmacology, St. Marianna University School of Medicine, Japan

Yachiyo Kurihara, Department of Pediatrics, St. Marianna University School of Medicine, Japan

Kenzirou Gotoh, Department of Pediatrics, St. Marianna University School of Medicine, Japan

Masaki Arima, Department of Pediatrics, St. Marianna University

School of Medicine, Japan

Masanori Mizuno, Department of Pediatrics, St. Marianna University School of Medicine, Japan

Yasushi Koitabashi, Department of Pediatrics, St. Marianna University School of Medicine, Japan

Objective: Antenatal glucocorticoid therapy has been shown to improve acute conditions including infant respiratory distress syndrome and reduce mortality, although little known is about the effects on cardiac function-related factors in the fetus or neonate. We investigated whether cardiac function-related factors are altered in cardiac tissues of fetuses and neonates born to pregnant rats administered glucocorticoid.

Methods: Dexamethasone $1 \mathrm{mg} / \mathrm{kg}$ s.c. or vehicle was administered for 2 days to pregnant rats on days 17 and 18 or 19 and 20 of gestation, and cardiac tissues of 19- and 21-day fetuses and $1-, 3-$, and 5-day neonates were analyzed using a proteomic 
technique with liquid chromatography-mass spectrometry/mass spectrometry.

Results: The five proteins alpha-enolase, creatine kinase-M type, beta-tubulin, troponin T, and ATP synthase beta-chain were identified.

In Western blot analysis, the glycolytic enzyme enolase was increased significantly in the 19-day and 21-day fetal groups after antenatal dexamethasone administration compared with the respective control groups. Levels of cAMP as a metabolic product of ATP were significantly increased in the 19-day and 21-day fetal groups after antenatal dexamethasone. Immunohistologically, the cross-sectional area of ventricular myocardium was significantly increased in the 21-day fetal and 1-day neonatal dexamethasone groups. The increase in heart size might have resulted from increased alpha-enolase levels in cardiac tissues with antenatal glucocorticoid administration.

Conclusions: These results suggest that antenatal glucocorticoid therapy may accelerate cardiac development of the preterm heart.

\section{9}

\section{Vascular changes after bone-marrow transplantation in} children

Maila I Turanlahti, Hospital for Children and Adolescents, Department of Pediatric Cardiology, Helsinki, Finland, Finland

Mervi Taskinen, Hospital for Children and Adolescents, Department of Hematology-Oncology and Stem Cell Transplantation, Helsinki, Finland, Finland

Ulla Pihkala, Hospital for Children and Adolescents, Department of Hematology-Oncology and Stem Cell Transplantation, Helsinki, Finland, Finland

Eero Jokinen, Hospital for Children and Adolescents, Department of Pediatric Cardiology, Helsinki, Finland, Finland

Objective: To study arterial stiffness and endothelial function in children after bone-marrow transplantation. Increased risk for metabolic syndrome has been observed as late effect of bonemarrow transplantation.

Methods: Noninvasive vascular ultrasonic measurements were performed in 25 children 2.7-11.6 years after bone-marrow transplantation, and in 22 controls (age range 6.4-15.5 years). Mechanical changes of the common carotid artery and flowmediated dilatation of brachial artery were measured. Blood glucose, serum insulin and plasma lipid measurements were examined after an overnight fast.

\begin{tabular}{lcc}
\hline & Study PATIENTS & Controls \\
\hline Number (F/M) & $25(12 / 13)$ & $22(12 / 10)$ \\
Age, yrs (range) & $11.0(6.4-14.9)$ & $11.3(6.7-15.5)$ \\
Weight, kg (range) & $35.4(18.5-70.0)$ & $43.1(22.3-71.8)$ \\
Total cholesterol, mmol/l & $4.5 \pm 0.8$ & $4.4 \pm 0.7$ \\
HDL cholesterol, mmol/l & $1.6 \pm 0.4$ & $1.7 \pm 0.3$ \\
Triglycerides, mmol/l & $1.1 \pm 0.7$ & $0.7 \pm 0.3$ \\
fB-Gluc, mmol/l & $4.9 \pm 0.5$ & $4.8 \pm 0.4$ \\
fB-insulin, mmol/l & $14.2 \pm 30.3$ & $5.8 \pm 3.8$ \\
\hline
\end{tabular}

Results: The distensibility of carotid artery was significanty lower in the patients $\left(0.48 \pm 0.19\right.$ versus $0.64 \pm 0.28 \mathrm{mmHg}^{-1} \times 10^{-2}$, $\mathrm{p}=0.024)$. The distensibility decreased with the time past BMT $(p=0.009)$ but was dependent of the age only in the control group $(p=0.034)$. The compliance of carotid artery was significantly decreased in the patients $(0.10 \pm 0.04$ versus
$0.13 \pm 0.05, \quad \mathrm{~mm} 2 \mathrm{xmmHg}-1, \mathrm{p}=0.041)$. The incremental elastic modulus ( $\mathrm{E}$ inc) was higher in BMT patients $(2.05 \pm 0.7$ versus $1.6 \pm 0.6 \mathrm{~mm} / \mathrm{Hg} \times 103, \mathrm{p}=0.019)$ and was dependent of the time after BMT $(\mathrm{p}=0.036)$.

There was no difference in the flow-mediated dilatation between the two groups. Serum triglycerides were elevated in the patient group compared to controls $(1.1 \pm 0.7$ versus $0.7 \pm 0.3 \mathrm{mmol} / 1, \mathrm{p}=0.026$ )

Conclusions: Early mechanical changes of arterial wall were found after bone-marrow transplantation. The ultrasound measurements may offer a noninvasive method to find early alterations of the vascular bed and to optimize a preventive treatment of atherosclerosis.

8330

Assessment of the Right Ventricle Mass Acquisition in an Experimental Model of Intermittent Pulmonary Trunk Banding

Acrisio S Valente, Heart Institute University of Sao Paulo Medical School, Brazil, Brazil

Renato S Assad, Heart Institute University of Sao Paulo Medical School, Brazil, Brazil

Maria Cristina D Abduch, Heart Institute University of Sao Paulo Medical School, Brazil, Brazil

Gustavo J Silva, Heart Institute University of Sao Paulo Medical School, Brazil, Brazil

Petronio G Thomaz, Heart Institute University of Sao Paulo Medical School, Brazil, Brazil

Leonardo A Miana, Heart Institute University of Sao Paulo Medical School, Brazil, Brazil

Jose A Krieger, Heart Institute University of Sao Paulo Medical School, Brazil, Brazil

Noedir A Stolf, Heart Institute University of Sao Paulo Medical School, Brazil, Brazil

Objective: To evaluate the mass acquisition of the right ventricle (RV) of young goats, submitted to 24, 48, 72 and 96 hours of intermittent pressure load.

Methods: Four groups of seven young goats were submitted to 12-hour period of RV systolic overload, alternated with a resting period of 12 hours. The groups were divided in 24, 48, 72 and 96-hour training periods. Echocardiographic and hemodynamic evaluations were performed every day. After completing the training program of each group, the animals were killed for water content and cardiac masses evaluation. Another seven animals were used as a control group.

Results: Important RV dilation was observed at 24-hour period, with significant increase in the RV wall thickness starting at the 48 -hour group $(\mathrm{p}<0.05)$. There was a significant increase in the RV volume to mass ratio at 24 -hour training period $(p=0.003)$, with a trend for recovery throughout the protocol. The RV mass of the 96-hour trained group $(1.76 \pm 0.52 \mathrm{~g} / \mathrm{Kg})$ was significantly greater than the other groups $(p<0.0001)$, with no significant myocardial water content changes in that group. The daily mean increase in RV mass was $21.57 \% \pm 26.78 \%$, with $104.7 \%$ increase in the 96-hour trained group as compared to the control group. The rate of RV mass acquisition for the overall study period was $0.084 \pm 0.035 \mathrm{~g} / \mathrm{h}$. RV wall stress peaked rapidly at 48 -hour period, followed by progressive decrease throughout the protocol.

Conclusions: Intermittent banding of the pulmonary trunk has permitted a significant RV mass acquisition only in the 96-hour trained group. 
8331

Balloon pulmonary valvuloplasty for neonates and nursing infants with early-stage tetralogy of Fallot Hisako Kiguchi, Department of Pediatric Cardiology, Hiroshima City Hospital

Masahiro Kamada, Department of Pediatric Cardiology, Hiroshima City Hospital, Japan

Naomi Nakagawa, Department of Pediatric Cardiology, Hiroshima City Hospital

Purpose: To evaluate balloon pulmonary valvuloplasty (BPV) effectiveness for neonatal and nursing infants with tetralogy of Fallot (ToF). Subjects and Methods: Tirty-five neonatal to early nursing infants with Tof undergoing BPV were examined for changes in oxygen saturation, pulmonary valve (PV) diameter, pulmonary vascular tree diameter [Nakata's PA index (PAI)], and pulmonary arterial valve regurgitation (PR), as well as effects on a radical operation. Results: At the BPV, ages ranged from 5-95days (median 27), body weights from $2.1-5.6 \mathrm{~kg}$ (3.4), PV diameters from 3.5-6.6 mm (5.5), and PAI from 74-306 (136). A balloon equivalent to $80-157 \%$ (121\%) of PV diameter was used. $\beta$ blocker administration was given after surgery. Mild pulmonary edema was observed in 1 case and right ventricle outflow tract dissection in $1 . \mathrm{SpO} 2$ was significantly elevated from preoperative $86.6 \pm 7.7 \%$ to postoperative $94.6 \pm 4.6 \%$. However, it declined to $85.8 \pm 7.8 \%$ before the radical operation. Pulmonary valve area (PVA) increased from $69.3 \pm 13.8 \%$ (normal) before BPV to $87.0 \pm 20.4 \%$ before the radical operation. PAI increased in all cases from mean 163 to 339 . A radical operation was completed in 31 subjects, of whom 18 required a transannular patch repair. An m-BT shunt operation was performed in 2 with repeated cyanotic fits and 1 with pulmonary coarctation while waiting for the radical operation. One with Down's syndrome died from pneumonia while waiting for the radical operation.

Conclusion: BPV has limitations for avoiding transannular patch repair, as $\mathrm{SpO} 2$ was elevated and the pulmonary vascular tree and annulus increased following the procedure.

\section{2}

Clinical Course after Transcatheter Closure of Multiple Atrial Septal Defects Using Single Amplatzer Septal Occluder

Nam Kyun Kim, Division of Pediatric Cardiology, Severance Cardiovascular Center, Yonsei University Health System, Korea Jae Young Choi, Division of Pediatric Cardiology, Severance Cardiovascular Center, Yonsei University Health System, Korea Su Jin Park, Division of Pediatric Cardiology, Severance Cardiovascular Center, Yonsei University Health System, Korea

Byung Won Yoo, Division of Pediatric Cardiology, Severance Cardiovascular Center, Yonsei University Health System, Korea Jun Hee Sul, Division of Pediatric Cardiology, Severance Cardiovascular Center, Yonsei University Health System, Korea

Objectives: We investigated the outcome of transcatheter closure using a single device in patients with multiple ASDs.

Methods: From May 2003 to November 2008, 429 patients underwent transcatheter closure of ASD in our institute. Among them, 65 patients had multiple ASDs, and transcatheter closure was performed using a single device in all patients. We reviewed the outcome of the procedure, complications and serial echocardiographic findings.

Results: The presence of multiple defects was diagnosed by echocardiography before the procedure in 50 patients $(77 \%)$, and by transesophageal or intracardiac echocardiography at the time of procedure in 15 patients (23\%). Largest defect size was $16.9 \pm 6.0 \mathrm{~mm} \quad(7 \mathrm{~mm}-32 \mathrm{~mm})$, smallest defect size was $5.8 \pm 3.0 \mathrm{~mm}(2 \mathrm{~mm}-13 \mathrm{~mm})$ and total defect size was $23.0 \pm 7.6 \mathrm{~mm}(10 \mathrm{~mm}-37 \mathrm{~mm})$. Distance between the margins of the defects was ranged from $2 \mathrm{~mm}$ to $19 \mathrm{~mm}$. The average balloon occlusive diameter and device size were $22.9 \pm 7.6 \mathrm{~mm}$ and $23.1 \pm 7.7 \mathrm{~mm}$. Follow-up echocardiography performed after one day of the procedure revealed 51 patients (78.5\%) with complete closure or trace residual shunt flow, $87.7 \%$ after 1 month, 91.5\% after 3month, 94.8\% after 6month and 100\% after 1year. All patients had no major complications.

Conclusion: Transcatheter closure of ASD with a single ASO is an effective and feasible therapeutic option in patients with multiple defects. Distance between the defects and device size larger than total defect may have an important role in complete closure of the multiple ASDs with single device. Nevertheless, remaining defects, even in distant locations, can be closed spontaneously or further reduced in size after closure of the largest defect.

\section{3}

Midterm Outcomes in Patients with Atrial Septal Defect and Pulmonary Hypertension Treated with Single Atrial Septal Occluder

Nam Kyun Kim, Division of Pediatric Cardiology, Severance Cardiovascular Center, Yonsei University Health System, Korea Jae Young Choi, Division of Pediatric Cardiology, Severance Cardiovascular Center, Yonsei University Health System, Korea Su Jin Park, Division of Pediatric Cardiology, Severance Cardiovascular Center, Yonsei University Health System, Korea

Byung Won Yoo, Division of Pediatric Cardiology, Severance Cardiovascular Center, Yonsei University Health System, Korea Jun Hee Sul, Division of Pediatric Cardiology, Severance Cardiovascular Center, Yonsei University Health System, Korea

Objectives: We assessed clinical course of patients with pulmonary artery hypertension (PAH) after transcatheter closure of secundum atrial septal defect (ASD).

Methods: From May 2003 to December 2007, 359 patients underwent transcatheter closure of ASD. Among them, 43 patients $(12 \%)$ had PAH, defined as peak systolic pulmonary artery pressure (PAPsyst) above $40 \mathrm{mmHg}$. PAH was classified as mild (PAPsyst $=40-49 \mathrm{mmHg}$ ), moderate (PAPsyst $=50-59 \mathrm{mmHg}$ ), severe (PAPsyst $>60 \mathrm{mmHg}$ ) according to pulmonary artery pressure measured by cardiac catheterization. Estimated right ventricular systolic pressures were measured by Doppler echocardiography before the procedure, during and follow-up visits.

Results: In mild PAH group ( $\mathrm{n}=25), 18$ patients $(72 \%)$ showed normalization, immediately after the closure of ASD. Remaining 7 patients showed normalization during follow-up. In moderate PAH group ( $n=10), 8$ patients $(80 \%)$ showed normal PAPsyst after closure of ASD and 2 patients became normal within 1 month. In severe PAH group $(n=8)$, only 1 patient $(12.5 \%)$ showed immediate normalization after transcatheter closure. Six patients (75\%) had remaining $\mathrm{PAH}$ after the procedure but showed normalization during follow-up. One patient (12.5\%) with severe PAH was treated with oral pulmonary artery vasodilator (Bosentan) and mild PAH remained at last fallow-up. Major complications related to ASD occlusion or PAH were not found.

Conclusion: Transcatheter ASD closure is safe and effective, even in patients with PAH. PAH regresses after the closure of defect in most patients and shows further improvement as time goes by. Further study in larger population is warranted, to determine long-term outcome. 
8334

Catheter Intervention After Norwood Stage-I Palliation Sung-Hae Kim, Department of Cardiology, Shizuoka Children's Hospital, Japan

Wataru Soda, Department of Cardiology, Shizuoka Children's

Hospital, Japan

Norie Mitsushita, Department of Cardiology, Shizuoka Children's Hospital, Japan

Masaki Nii, Department of Cardiology, Shizuoka Children's Hospital, Japan

Yasuhiko Tanaka, Department of Cardiology, Shizuoka Children's Hospital, Japan

Yasuo Ono, Department of Cardiology, Shizuoka Children's Hospital, Japan

Kisaburo Sakamoto, Department of Cardiovacsular Surgery, Shizuoka Children's Hospital, Japan

Background: Our institution mainly perform Norwood stage-I palliation for hypoplastic left heart variants and severe AS with VSD and/or single ventricle physiology and the survival improved over $85 \%$ in this decade. However, strict pulmonary blood flow control is indispensable for early to mid-term postoperative mortality and morbidity. After the palliation, we perform interventions to increase pulmonary forward flow as well as other techniques, as a bridge to next stage elective surgery.

Study Population: Fifty-eight consecutive patients who underwent Norwood procedure since 1999 until 2008. HLHS and variants $(\mathrm{n}=30)$, and non-HLHS $(\mathrm{n}=23)$ are included.

Results: Hospital death within 30 days after Norwood was 8/58, and among the 50 survivors, 20 patients (40\%) were underwent intervention subsequently. The median age of Norwood, intervention, and the next stage surgery such as Glenn or Rastelli were 6days, 5-months, 6-months old, respectively. Among the 20 interventions, 11 included dilatation of BT or RV-PA shunt by a balloon or a stent, and 8/11 included releasing of hemoclips primarily loaded for flow restriction. Coil occlusion for aortopulmonary collaterals was performed in 17/20 cases. High-pressure balloon angioplasty was successfully performed for coarctation of neo-Aorta in $7 / 20$ cases. $15 / 20$ cases recieved multiple interventions simultaneously. No procedural complications occurred, and 18/20 underwent next stage surgery with favorable postoperative course. Conclusions: Shunt dilatation including "declipping" procedure and occlusion of aortopulmonary collaterals were safe and effective in the aspect of pulmonary blood flow control, from restriction to promotion. Simultaneous multi-interventional procedures can be performed with minimal invasion in hemodynamically unstable infants.

\section{5}

Significance of Vascular plug in various abnormal vascular communications

Nageswara Rao Koneti, CARE Hospitals, The Institute of Medical

Sciences, Hyderabad, India, India

Raghava Raju Penumatsa, India

Vasudevan Kanchi, CARE Hospitals, The Institute of Medical Sciences, Hyderabad, India, India

TNC Padmanabham, Krishna Institute of Medical Sciences, Hyderabad, India, India

Objective and Methods: We studied the usage and efficacy of vascular plug in closing various vascular communications from 2003 to 2008. Total 16 vascular plugs were deployed during the study period in 11 patients ( 7 males and 4 females). The mean age was $12.6 \pm 16.7$ (range 1-60 years). The vascular abnormality includes systemic collateral to pulmonary circulation in 4 patients, coronary arterial-venous fistulae (CAVF) in 3, pulmonary arterial- venous (PAVF) fistula in 2, patent ductus arteriosus in 1 and residual BT shunt in 1.

Results: Multiple plugs were deployed in $1 \mathrm{PAVF} \& 1$ systemic collateral and in the rest one plug was deployed in each. The ten minutes angiogram revealed persistence of shunt in all systemic collaterals whereas no significant residual shunt was seen in the rest. There was significant improvement of the saturation noted in both the cases of PAVF. There were no procedural related complications. There was no significant murmur noted in systemic collateral closure at 6 weeks follow up.

Conclusions: We conclude that vascular plug is useful in closing vascular communications of wide spectrum. Immediate closure was achieved in low flow (PAVF) or smaller (CAVF) communications whereas delayed closure is seen in high flow systemic collaterals.

8336

Efficacy and safety of new Amplatzer Duct Occluder device (ADO II) in closing Patent Ductus Arteriosus (PDA) in young children

Nageswara Rao Koneti, Care Hospital, The Institute of Medical Sciences, India

Suman Vyas Yandamury, Care Hospital, The Institute of Medical Sciences, India

Vasudevan Kanchi, CareHospital, The Institute of Medical Sciences, India

Sitaram Dosapati, Care Hospital, The Institute of Medical Sciences, India

Kashif Azam Syed, India

Objective: We report the efficacy of new Amplatzer Duct Occluder (ADO II) in young children with hemodynamically significant small to moderate Patent Ductus Arteriosus (PDA) from October to December 2008.

Methods: A total of 13 children diagnosed to have PDA (diameter $<5 \mathrm{~mm}$ ) were included in the study. The average age of the children was $18 \pm 15.06$ months (4 to 48 months). The body weight was $7.9 \pm 2.31 \mathrm{~kg}$ ( 3.5 to $11 \mathrm{~kg}$ ). There were 11 females and 2 males. All children were thoroughly evaluated clinically and echocardiographically. The procedure was done under general anesthesia after obtaining informed consent. Descending thoracic aortic angiogram was done in lateral view in all cases and RAO view in selected cases. PDA diameter, length and ampulla were measured angiographically and correlated with echocardiogram. The device size was selected 1.5 to $2 \mathrm{~mm}$ higher than the narrowest PDA diameter whereas the length was chosen $4 \mathrm{~mm}$ and $6 \mathrm{~mm}$ for the duct length lesser and greater than $4 \mathrm{~mm}$ respectively. The descending aortic and the pulmonary artery angiogram were done in all cases before final deployment.

Results: The procedure was successful in all children. There were no procedure related complications. There was mild residual leak in one child at $24 \mathrm{hrs}$ which disappeared at $72 \mathrm{hrs}$. We conclude that ADO II is safe and effective in closing small to moderate $\mathrm{PDA}$ in young children using $4 \mathrm{f}$ and $5 \mathrm{f}$ delivery systems.

\section{7}

Transcatheter closure of coronary arterial-venous fistulae (CAVF) and management of complications

Nageswara Rao Koneti, CARE Hospital, The Institute of Medical

Sciences, India

Raghava Raju Penumatsa, India 
Vasudevan Kanchi, CARE Hospital, The Institute of

Medical Sciences, India

suman Vyas Yendamary, India

Srinivas Kumar Arramraju, India

We report transcatheter closure of coronary arterial-venous fistulae (CAVF) in 10 patients (7 males and 3 females) from March 2002 to October 2008. The mean age was $14.5 \pm 12.2$ years. Right coronary artery (RCA) fistulae were seen in 8 patients whereas left coronary artery (LCA) fistulae in 2. Of these, 5 patients had fistula emptying into right atrium, 2 each in right ventricle (RV) \& pulmonary artery (PA) and in superior vena cava in 1 . The CAVF was closed with multiple coils in 7 patients and vascular plug in 3 patients. There was residual leak in 2 patients in-spite of multiple coils who had multiple exit points into PA. One child developed significant ST-T changes during procedure due to retrograde propagation of thrombus which was managed with intracoronary Urokinase, in whom, the CAVF was communicating from LCA to RV apex. One child had transient ST-T changes immediately after the procedure which was managed with intravenous heparin. All patients were discharged with anti-platelet medication and advised regular follow-up. The mean follow-up period was $3.1 \pm 1.99$ years. During the follow-up, apart from routine ECG and echocardiogram, tread mill test was done in 6 patients. One patient with poor compliance presented with inferior wall myocardial infarction which was treated with intravenous Urokinase. The check angiogram after six weeks showed dilated RCA with normal patency of coronary arteries. We conclude that transcatheter closure of CAVF is feasible however needs meticulous assessment, careful observation and close long term follow-up with oral anti-platelet medication.

\section{9}

Reopening totally occluded segments of cavopulmonary connections in patients with single ventricle physiology Markus Kruessell, Germany

Narayanswami Sreeram, Heart Center, University Hospital of Cologne, Germany

Mathias Emmel, Pediatric Cardiology, University of Cologne, Germany Gerardus Bennink, Germany

Background: Early recognition of occlusion of any segment of a cavopulmonary connection (CPC) is mandatory, and efforts at immediate recanalization should be undertaken to optimise Fontan physiology.

Patients and methods: Six consecutive patients (age range 3 months to 5 years) with clinical suspicion of occlusion of a CPC segment are presented. All had undergone a Norwood type of palliation, followed by a bidirectional Glenn shunt at between 3 and 5 months. The oldest patient had undegone Fontan completion using an extracardiac conduit. The interval from surgery to clinical presentation ranged between 1 day and 3 months. At emergency cardiac catheterization, total thrombotic occlusion was seen in the following segments: left pulmonary artery $(n=5)$, superior vena cava $(n=3)$, inferior vena cava (within the extracardiac conduit, $\mathrm{n}=1$ ). All occluded segments could be crossed with a 4F endhole catheter and guidewire combination. Serial balloon dilation and stent implantation $(n=11$ stents, dilated to between $6 \mathrm{~mm}$ and $15 \mathrm{~mm}$, depending on the size of the native vessel) were successfully undertaken.

Results: All occluded segments were reopened completely. Two patients died during further follow-up ( 2 to 10 days later), both due to multiple recurrent thrombotic obstructions, despite subsequent tPA therapy (and redilation/re-recanalization in one). The remaining patients are alive and well, and 2 of the survivors have had Fontan completion.

Conclusions: Early and aggresssive therapy can result in salvage of a significant proportion of acutely occluded CPC segments.

\author{
8340 \\ Vascular complications of pediatric cardiac \\ catheterization and effects on morbidity \\ Nezihi Kucukarslan, Turkey \\ Bilgehan $\mathrm{S} O z$, Gata Military Medical Faculty Department of \\ Cardiovascular Surgery, Turkey \\ Ertugrul Ozal, Gata Military Medical Faculty Department of \\ Cardiovascular Surgery, Turkey \\ Vedat Yildirim, Gata Military Medical Faculty Department of \\ Cardiovascular Surgery, Turkey \\ Vedat Okutan, Gata Military Medical Faculty Department of Pediatric \\ Cardiology, Turkey \\ Harun Tatar, Gata Military Medical Faculty Department of \\ Cardiovascular Surgery, Turkey
}

Objective: In this study we observed the vascular complications following the cardiac catheterization used both for diagnosis and treatment in pediatric patients. We also examined the characteristics, types and treatment methods of these complications and the effects of application on morbidity.

Method: This retrospective study included the records of 556 patients (ages 0-14) who had cardiac catheterization between January 1996 and May 2004 in pediatric cardiology clinic. Vascular complications were hematoma, embolus, pseudoaneurysm, A-V fistula, hemorrhage, infection and mortality. Surgical endications were determined by physical examination and arterial venous doppler ultrasonography.

Results: Vascular complications developed in 49 patients (8.81\%). Thirty of them (61.22\%) had surgical intervention. Hematoma in 18 patients $(3.23 \%)$, embolus in 12 patients $(2.15 \%)$, pseudoaneurysm in 2 patients $(0.35 \%)$, A-V fistula in 2 patients $(0.35 \%)$, hemorrhage in 8 patients $(1.43 \%)$, deep venous trombosis in 2 patients $(0.35 \%)$, infection in 4 patients $(0.71 \%)$ were found. Vascular complications extended the hospitalization period $5+2$ days.

Conclusion: The small body sizes increase the morbidity rate, causing trouble in finding vessels to apply the catheter. A complete sedation and application of catheterization and vascular surgery by experienced hands decreases the vascular complications following cardiac catheterizations. During the treatment of vascular complication in pediatric ages the medical treatment must be discussed while keeping surgical intervention in mind.

\section{2}

Outcome After Balloon Aortic Valvuloplasty of Critical Aortic Stenosis in Neonates

Yulia L Kuzmenko, GU Ukrainian Children's Cardiac Center, Ukraine

Andriy V Maksymenko, GU Ukrainian Children's Cardiac Center, Ukraine

Arkadiy A Dovgaliuk, GU Ukrainian Children's Cardiac

Center, Ukraine

Olexandr M Romaniuk, GU Ukrainian Children's Cardiac

Center, Ukraine

Objective: We present a review of our experience primary interventional treatment and the surgical treatment of residual aortic valve disease after BAV of critical aortic stenosis in neonates. 
Methods and results: Between 1996-2008 balloon dilatation of critical aortic stenosis were performed in 53 neonates with biventricular circulation. Median age at the BAV procedure was 13,5 days (range 1 to 30 days), weight was $3,38 \mathrm{~kg}$ (range 2,2 to $4,45 \mathrm{~kg}$ ), ratio balloon/aortic annulus $=0,9$, ejaction fraction $<55 \%$ (mean 40,4+11,9\%; p < 0.001) All patients presented with variang degrees of congestive failure as evidenced by tachypnoea, cardiomegaly and hepatomegaly. Prostaglandin infusion was initiated 38 patients (71\%), 21 patients (40\%) required assisted ventilation, 35(66\%) needed an inotropic infusion or for hospital transfer. The mean Doppler gradient was reduced from $52,8+23,8$ to $21,5+11,5 \quad(p<0.001)$. During a median follow-up was 3,7 years ranging up to 12,1 years, there were 14 reintervention on the LV outflow tract in 11 (21\%) and 5 deaths (9\%). Hospital mortality 2\% (1 patient), 3 patient had died during one year after initial BAV, 1 patient $-2,5$ years after initial BAV. The mean time interval between the initial dilation and reintervention was $45,5+27,7$ monts (range $2-77 \mathrm{~m}$, $\mathrm{p}<0.001) ; 3$ patients required balloon procedure, 6 a Ross operation and 2 a open valvulotomy.

Conclusion: Transcatheter BAV offers effective immediate reduction of gradient in critical aortic stenosis with biventricular circulation resulting in the relief of heart failure and the need for continued ventilation and inotropic support.

\section{4 \\ Biologic Response to HELEX ${ }^{\mathrm{TM}}$ Septal Occluder Implantation in the Canine Heart \\ Geoffrey K Lane, The Cleveland Clininc Foundation, United States Nicholas P Macri, W.L. Gore \& Associates Inc, United States John F Rhodes, The Cleveland Clininc Foundation, United States C Igor Mesia, The Cleveland Clininc Foundation, United States Lourdes $R$ Prieto, The Cleveland Clininc Foundation, United States Warren J Cutright, W.L. Gore \& Associates Inc, United States Evan M Zahn, Miami Children's Hospital, United States Larry A Latson, The Cleveland Clininc Foundation, United States}

The HELEX ${ }^{\mathrm{TM}}$ Septal Occluder device is a purpose designed device for atrial septal defect closure. The purpose of this study was to evaluate the biologic response of this device in the canine heart.

Thirty-four animals had percutaneous implantation of a HELEX $^{\mathrm{TM}}$ device. Animals were sacrificed at intervals from 2 days to 2 years post implantation (PI). Gross and histological assessment of the extent and type of tissue coverage was performed. Complete fibrous connective tissue coverage was seen in 2 of 5 devices examined 14 days PI. Fibrous connective tissue completely covered the left atrial component in 13/14 devices implanted $>30$ days. One device at 6 months PI had complete coverage of the right atrial side with extensive coverage of the left atrial side. Endothelial-like cell coverage ranged from multifocal to complete in 10/13 devices implanted $>30$ days. One device examined by scanning electron microscopy and immunohistochemistry confirmed that the endothelial-like cells seen with light microscopy were true endothelial cells. The lungs, brain and kidneys of each dog implanted longer than 30 days were examined for thrombo-embolism and no infarction was seen.

The biologic response following implantation of the HE$\mathrm{LEX}^{\mathrm{TM}}$ device is progressive with an initial fibrous connective tissue coverage followed by endothealisation. There was complete coverage of the left atrial side of the device with fibrous connective tissue by 30 days post implantation in almost all animals. These data provide evidence of the biocompatibility of the HELEX ${ }^{\mathrm{TM}}$ Septal Occluder.

\section{5}

Transcatheter Intervention in Patients with Pulmonary Atresia Intact Ventricular Septum or Critical Pulmonic Stenosis

Hyoung-Doo Lee, Pusan National University Children's Hospital, Korea

Ji-Ae Park, Pusan National University Children's Hospital, Korea Min-Jung Cho, Pusan National University Children's Hospital, Korea

Objective: To evaluate the result of transcatheter intervention in Korean baby with these anomalies in the PDA stent era.

Method: Since Jan. 2004, 24 neonates (11 males, 13 females) were diagnosed as PA IVS or critical PS. We tried transcatheter staged balloon pulmonary valvuloplasty in 21 patients, and successful in 17 cases $(80.9 \%)$, among them PDA stenting was required in 8 patients. Right ventricular outflow tract reconstruction with transannular patch and central shunt was done for the failed 4 patients. For the another 3 neonates, who had markedly hypoplastic right ventricle or fistulous communication between right ventricle and coronary artery, we made systemic to pulmonary shunt and interatrial communication. We reviewed change of tricuspid z-value and longevity of ductal patency in PDA stent group and complications, and compared tricuspid zvalue between balloon only group and PDA stent group.

Results: Tricuspid showed growth of tricuspid valve with time in stent group. Follow up duration was 2 months $\sim 3.5$ years $(1.84 \pm 1.04)$. The stents were occluded in 2 patients, which were patent at least 2.5 and 1.2 years after implantation. The other stents are patent in median 1.9 years follow up.

Conclusion: Transcatheter intervention in patients with pulmonary atresia intact interventricular septum or critical pulmonic stenosis is useful therapeutic method in neonates. PDA stenting can prevent from additional surgical procedure to increase pulmonary blood flow. The longevity of shunt through the ductal stent was good in Korean infant.

\section{6}

Transcatheter Closure of Large Pulmonary Arteriovenous Malformations Using Amplatzer Vascular Plug/Duct Occluder: Report of a Family with Hereditary Hemorrhagic Telangiectasia

Hyoung-Doo Lee, Pusan National University Children's Hospital, Korea

Min-Jung Cho, Pusan National University Children's Hospital, Korea Ji-Ae Park, Pusan National University Children's Hospital, Korea

Background: Although transcatheter embolization with coils has been considered the classic method for interventional occlusions of pulmonary arteriovenous fistulas (PAVFs), it is not ideal in fistulas with large feeding arteries because of the risk for device embolization. We report two experiences with uses of Amplatzer Vascular Plugs/Duct Occluders for transcatheter closure of multiple PAVFs in a family with hereditary hemorrhagic telangiectasia (HHT)

Methods and results: Case 1. A 8-year-old female was evaluated for recurrent epistaxis and diagnosed as HHT, a fibrovascular disorder characterized by mucocutaneous telangiectases, arteriovenous malformations of various organs, and inheritance. Radiologic studies revealed two large PAVFs in the posterobasal and mediobasal segment of the left lower lung. Percutaneous transcatheter embolization was then performed using the Amplatzer Duct Occluder $(10 \times 12 \mathrm{~mm})$ for the fistula having feeding vessel of $8.2 \mathrm{~mm}$ in diameter and Gianturco coil $(4 \times 3 \mathrm{~mm})$ for the smaller fistula $(2.9 \mathrm{~mm})$. After the procedure, 
percutaneous oxygen saturation at rest rose to $>95 \%$ on room air which was about $71 \%$ before.

Case 2. Her father, having mucocuteneous telangiectases on the tongue and in the nasal mucosa, was also confirmed the diagnosis of bilateral multiple PAVFs. Selective angiography of pulmonary arteries showed 4 PAVFs of right upper (feeding vessels of $2.8 \mathrm{~mm})$, right lower $(3.5 \mathrm{~mm})$, left upper $(3.2 \mathrm{~mm})$ and left lower $(4.2 \mathrm{~mm})$ lung. In all 4 PAVFs, percutaneous closure with Amplatzer Vascular Plugs were performed $(4 \times 7 \mathrm{~mm}$ for right upper lesion, $6 \times 7 \mathrm{~mm}$ for right lower and left upper lesions, and $8 \times 7 \mathrm{~mm}$ for left lower lesion).

Conclusions: Amplatzer Duct Occluders/Vascular Plugs are easy and safe devices to occlude large PAVFs.

\section{8}

Cheatham-Platinum Stent Implantation for Pulmonary Artery Stenosis Associated with Congenital Heart Disease in Children and Adolescents: Immediate and Midterm Results

Fen Li, Department of Cardiology, Shanghai Children's Medical Center Affiliated to Shanghai Jiaotong University School of Medicine, China Aiqing Zhou, Department of Cardiology, Shanghai Children's Medical Center Affiliated to Shanghai Jiaotong University School of Medicine, China Wei Gao, Department of Cardiology, Shanghai Children's Medical Center Affiliated to Shanghai Jiaotong University School of Medicine, China

Zhiqing Yu, Department of Cardiology, Shanghai Children's Medical Center Affiliated to Shanghai Jiaotong University School of Medicine, China Kun Sun, Department of Cardiology, Shanghai Children's Medical Center Affiliated to Shanghai Jiaotong University School of Medicine, China

Meirong Huang, Department of Cardiology, Shanghai Children's Medical Center Affiliated to Shanghai Jiaotong University School of Medicine, China

Yun Li, Department of Cardiology, Shanghai Children's Medical Center Affiliated to Shanghai Jiaotong University School of Medicine, China Wu Zhao, Department of Cardiology, Shanghai Children's Medical Center Affiliated to Shanghai Jiaotong University School of Medicine, China

Objective: Over the past 10 years, application of NuMED Cheatham-Platinum (CP) stent in pediatric pulmonary artery stenosis has been relatively uncommon. The aim of this study was to evaluate the immediate and midterm results of $\mathrm{CP}$ stent implantation for treatment of pulmonary artery stenosis associated with congenital heart disease (CHD) in children and adolescents.

Methods: From August 2005 to May 2007, 4 consecutive patients ( 2 boys and 2 girls) with pulmonary artery stenosis associated with CHD underwent stent implantation. Follow-up was carried out by transthoracic echocardiography.

Results: We performed totally 5 stent placement procedures and implanted $7 \mathrm{CP}$ stents (8-zig, 22-39 $\mathrm{mm}$ in length) in these patients. All stents but one were immediately successfully placed in the target lesions without displacement during the procedures. After procedure, the systolic pressure gradient across the stenosis decreased from $36.67 \pm 20.08$ to $3.67 \pm 3.20 \mathrm{~mm} \mathrm{Hg}$ $(\mathrm{P}=0.005)$ and the narrowest diameter of the stenotic segment increased by $112 \%$, from $6.97 \pm 2.22$ to $13.40 \pm 4.40 \mathrm{~mm}$ $(\mathrm{P}=0.013)$. During a median follow-up of 20 months (range, 13-34 months), the results have been stable without complications except 2 stents which developed intrastent restenosis 6 months later. Conclusions: Our experience suggests that the CP stent implantation is safe and feasible for the treatment of pulmonary artery stenosis in children and adolescents with CHD. The immediate and midterm results are encouraging, but long-term results remain to be further evaluated and demand many more cases to be studied.

\section{9}

Repair of Ruptured Sinus of Valsalva Aneurysm without VSD:Comparisons between Transcatheter Closure and Surgery

Ming-Tai Lin, Department of Pediatrics, National Taiwan University Hospital, Taipei, Taiwan, Taiwan

Shuenn-Nan Chiu, Department of Pediatrics, National Taiwan

University Hospital, Taipei, Taiwan, Taiwan

Chun-An Chen, Department of Pediatrics, National Taiwan University Hospital, Taipei, Taiwan, Taiwan

En-Ting Wu, Department of Pediatrics, National Taiwan University Hospital, Taipei, Taiwan, Taiwan

Jou-Kou Wang, National Taiwan University Hospital, Taiwan Mei-Hwan Wu, Department of Pediatrics, National Taiwan University Hospital, Taipei, Taiwan, Taiwan

Shu-Hsun Chu, Department of Surgery, National Taiwan University Hospital, Taipei, Taiwan, Taiwan

Chi-Ren Hung, Department of Surgery, National Taiwan University Hospital, Taipei, Taiwan, Taiwan

Background: Percutaneous transcatheter closure of ruptured sinus of valsalva aneurysm (RSVA) has been an alternative approach to surgery. However, there is still no objective comparison between these two strategies.

Method: From 1968 to 2007, 42 patients (27 males, 15 females) age from 18 years to 55 years (median 34 years) were identified to have RSVA without VSD in this institution. Of them, 6 patients received transcatheter closure ("CATH group", one with Gianturco coil, five with Amplatzer ductal occluders) and 36 received surgical repair ("SURGERY group", 7 with infective endocarditis). Echocardiographic and/or operative findings revealed the rupture of right coronary sinus into right ventricle, right atrium and left ventricle in 21, 6 and 1 cases, respectively; while non-coronary sinus ruptured into RA in 9 cases and into $\mathrm{RV}$ in 3 cases. The demographic characteristics, hemodynamics and outcome including residual shunt and aortic regurgitation of the CATH (6 patients) and SURGERY (36-7 $=29$ patients) groups were compared.

Results: There is no significant difference in age, sex, origin, size and opening of aneurysm, ratio of Qp/Qs and percentage of aortic regurgitation before repair between the CATH and SURGERY groups. After intervention, percentage of patients possessing AR and residual shunt (immediate and 3 years later) was very low and similar in both groups $(\mathrm{p}>0.05$, details shown in table).

Conclusions: The short term efficacy of transcatheter closure is similar to that of conventional surgical repair in the repair of ruptured sinus of valsalva aneurysm. Long term follow-up is mandatory.

\begin{tabular}{lcccccccc} 
Group & No. & $\begin{array}{c}\text { Age }(\mathrm{yr}, \\
\text { median) }\end{array}$ & $\begin{array}{c}\text { Size } \\
(\mathrm{mm})\end{array}$ & $\begin{array}{c}\text { AR } \\
\text { before immediate } \\
\text { repair }\end{array}$ & $\begin{array}{c}\text { AR 3 } 3 \\
\text { years } \\
\text { after }\end{array}$ & $\begin{array}{c}\text { Residual Residual } \\
\text { shunt } \\
\text { immediate } \begin{array}{c}3 \text { years } \\
\text { later }\end{array}\end{array}$ \\
\hline Cath & 6 & 29.5 & $\begin{array}{r}8(7- \\
8.5)\end{array}$ & $1 / 6$ & $0 / 6$ & $0 / 6$ & $1 / 6$ & $0 / 6$ \\
Surgery & 29 & 30 & $\begin{array}{r}10(7- \\
12.6)\end{array}$ & $6 / 29$ & $2 / 29$ & $2 / 29$ & $1 / 29$ & $1 / 36$ \\
\hline
\end{tabular}




\section{0}

Perventricular Device Closure of Muscular Ventricular Septal Defects in 3 Chinese Young Children

Fang Liu, Cardiac Center, Children's Hospital of Fudan University, China

Guo ying Huang, Cardiac Center, Children's Hospital of Fudan University, China

Bing Jia, Cardiac Center, Children's Hospital of Fudan University, China Lin Wu, Cardiac Center, Children's Hospital of Fudan University, China Feng Sheng, Cardiac Center, Children's Hospital of Fudan University, China Xiao jing Ma, Cardiac Center, Children's Hospital of Fudan University, China

Xue cun Liang, Cardiac Center, Children's Hospital of Fudan University, China

Three successful cases of intraoperative perventricular device closure of muscular ventricular septal defects (VSDs) on the beating heart in China were reported.

Patients and Methods: Between October 2006 to March 2007, 3 patients with large muscular VSDs were admitted to our center, with age from 6 months to 3 years 8 months, body weight from 5.4 to $14 \mathrm{~kg}$. The size of muscular VSD was $14 \mathrm{~mm}, 13 \mathrm{~mm}$ and $11 \mathrm{~mm}$ respectively, with multiple small shunts around in case 1 and 2 , previous pulmonary artery (PA) banding in case 1 , and aortic coarctation (CoA) in case 2. Via a sternotomy, the right ventricle free wall was punctured and an Amplatzer muscular VSD occluder device was deployed under transesophageal echocardiography guidance and monitoring. PA debanding and CoA repairing were performed at the same procedure in case 1 and 2. No cardiopulmonary bypass was needed in all cases.

Results: $16 \mathrm{~mm}, 14 \mathrm{~mm}$ and $12 \mathrm{~mm}$ muscular VSD occluders were successfully deployed respectively with mild residual shunt immediately. No complication intra- or post-operation. At 22 to 28 months follow-up, all patients were asymptomatic, echocardiography showed tiny residual shunt in 2 cases, and no shunt in one case. Cardiac size recovered to normal and no arrhythmia was found in both ECG and 24 hours Holter in all 3 cases.

Conclusion: Perventricular device closure can be applied to close muscular VSDs successfully and safely, especially suitable for young infants and patients with previous PA banding or associated with other defects.

Key Words: perventricular device closure, muscular ventricular septal defects.

\section{3}

Percutaneous Pulmonary Valve Implantation is Superior to Bare Metal Stenting in Improving Biventricular Efficiency in Patients with Right Ventricular Outflow Tract Obstruction - Utilization of a Catheter/Magnetic Resonance Hybrid Lab

Philipp Lurz, UCL Institute of Child Health and Great Ormond Street Hospital for Sick Children, United Kingdom

Johannes Nordmeyer, UCL Institute of Child Health and Great

Ormond Street Hospital for Sick Children, Germany

Vivek Muthurangu, UCL Institute of Child Health and Great Ormond Street Hospital for Sick Children, United Kingdom

Sachin Khambadkone, UCL Institute of Child Health and Great

Ormond Street Hospital for Sick Children, India

Graham Derrick, UCL Institute of Child Health and Great Ormond Street Hospital for Sick Children

Silvia Schievano, UCL Institute of Child Health and Great Ormond Street Hospital for Sick Children, Italy

Philipp Bonhoeffer, UCL Institute of Child Health and Great Ormond Street Hospital for Sick Children, Germany
Andrew M Taylor, UCL Institute of Child Health and Great Ormond Street Hospital for Sick Children, Germany

Background: Treatment of right ventricular outflow tract obstruction (RVOTO) is possible with bare metal stenting (BMS), though this causes pulmonary regurgitation (PR). In this study, we assessed the acute physiological effects of BMS vs. percutaneous pulmonary valve implantation (PPVI) using an $\mathrm{X}$ ray/magnetic resonance (MR) hybrid lab.

Methods: 14 children (median age 12.9) with RVOTO (echo gradient $>50 \mathrm{mmHg}$ ) were included. Patients were initially placed in the MR scanner and the ventricular volumes and great vessel blood flow assessed under general anaesthetic (GA). Patients were then transferred into the bi-plane catheter lab (under the same GA, on a sliding table) and a BMS inserted. Catheters and wires were removed and patients were transferred back to MR for repeat assessment. This whole process was repeated for a PPVI, which was placed into the BMS

Results: BMS significantly reduced the right ventricle (RV) to systemic pressure ratio $(0.75 \pm 0.17$ vs. $0.41 \pm 0.14 \% ; p<0.001)$ with no further change post-PPVI $(0.42 \pm 0.11 ; \mathrm{p}=1.0)$. However, BMS resulted in free PR $(21.3 \pm 10.7$ vs. $41.4 \pm 7.5 \% ; \mathrm{p}<0.001)$, which was nearly abolished following PPVI $(3.6 \pm 5.6 \% ; \mathrm{p}<0.001)$. Effective RV stroke volume (RV stroke volume - pulmonary regurgitant volume) post-BMS remained unchanged $(33.8 \pm 7.3$ vs. $32.6 \pm 8.7 \mathrm{ml} / \mathrm{m} 2 ; \mathrm{p}=1.0)$, but was increased after re-valvulation with PPVI $(41.0 \pm 8.0 \mathrm{ml} /$ $\mathrm{m} 2 ; \mathrm{p}=0.004)$.

These improvements post-PPVI were accompanied by a significant heart rate reduction (post-BMS $75.5 \pm 17.7$ vs. postPPVI $69.0 \pm 16.9 \mathrm{bpm} ; \mathrm{p}=0.006)$ at maintained cardiac output $\left(2.5 \pm 0.5\right.$ vs. $2.4 \pm 0.5$ vs. $\left.2.7 \pm 0.5 \mathrm{ml} / \mathrm{min}^{\star} \star_{\mathrm{m}} ; \mathrm{p}=0.14\right)$.

Conclusion: Using an X-ray/MR hybrid lab, we have demonstrated that PPVI is superior to BMS in improving biventricular efficiency in patients with RVOTO.

\section{4}

Early and Intermediate Outcomes After Transcatheter Valvotomy in Newborns With Pulmonary Atresia and Intact Ventricular Septum

Andrey V Maksymenko, Ukrainian Children's Cardiac Center, Ukraine Yulia L Kuzmenko, Ukrainian Children's Cardiac Center, Ukraine Arkadiy A Dovgaliuk, Ukrainian Children's Cardiac Center, Ukraine Elena P Boyko, Ukrainian Children's Cardiac Center, Ukraine Ilya N Yemets, Ukrainian Children's Cardiac Center, Ukraine

This study analyzes outcomes and predictive factors in cases of percutaneous mechanical perforation and balloon dilation of the atretic valve in cases of Pulmonary atresia with intact interventricular septum (PA-IVS).

Methods and Results: Between September 2003 and November 2008, 20 of 26 neonates with PA-IVS underwent attempted percutaneous balloon pulmonary valvoplasty. In 18 it was successful. According to Z-scores of TV and right ventricle 5 patiens (group1) had no significant hypoplasia of right heart structures (Z-scoreTV $>\ll-2 »), 9$ patients (group2) had moderate hypoplasia $(\mathrm{Z}-$ score $\mathrm{TV}=\ll-2 »-\ll-4 »)$ and 4 patients (group3) had severe hypoplasia (Z-Score TV $<\ll-4 »)$. In all cases of severe hypoplasia balloon atrial septostomy was performed in addition to valvuloplasty procedure. There was no early and late deaths. Six patients were free of reintervention $(n=4$ from group1, $\mathrm{n}=2$ from group2). Modified Blalock-Taussig shunt $(\mathrm{n}=2$ from group $2, \mathrm{n}=4$ from group 3$)$ or right ventricular outflow tract reconstruction ( $\mathrm{n}=1$ from group2) were done 
between 1 and 9 days after valve dilation. During follow-up of 1 to 48 months 7 additional procedures in 5 patients performed. Twelve patients (from group1 and group2) are planned for biventricular repair, 4 a $1 \frac{1}{2}$-ventricle circulation (from group 2 and group 3), and 2 patients from group3 waiting for staged univentricular correction.

Conclusions: Percutaneous balloon valvotomy is an effective treatment strategy for cases of PA-IVS with well formed right ventricle and absence of right ventricular dependent coronary circulation. There is a high rate of reinterventions in patients with moderate and severe right heart structures hypoplasia.

8356

Pulmonary artery stenting without long introducer sheath (LIS). One centre experience

Tomasz Moszura, Department of Cardiology Polish Mother's Memorial Hospital, Research Institute, Poland

Pawel Dryzek, Department of Cardiology Polish Mother's Memorial Hospital, Research Institute, Poland

Krzysztof Michalak, Department of Cardiology Polish Mother's

Memorial Hospital, Research Institute, Poland

Jacek Moll, Department of Cardiosurgery Polish Mother's Memorial

Hospital, Research Institute, Poland

Jadwiga Moll, Department of Cardiology Polish Mother's Memorial

Hospital, Research Institute, Poland

Andrzej Sysa, Department of Cardiology Polish Mother's Memorial

Hospital, Research Institute, Poland

Pulmonary artery stenting without a long introducer sheath has special significance particularly in children with low body weight. Using only a short sheath often permits implanting a stent of appropriate parameters and improves access to peripherally located stenoses.

The aim of this paper is to present the results of balloonexpandable stent implantation into pulmonary arteries without LIS in 50 patients. The subjects were divided into two groups. Group 1 comprised 28 patients (mean age-3,5 years) with a single-ventricle heart after bi-directional Glenn procedure, in whose case using only a short sheath usually allows angiographic control of the stent's position before expansion. Group 2 consisted of 22 patients (mean age 9,3 years) with a twoventricle heart, as stenting using the femoral approach does not permit such control.

In our data, no statistically relevant differences between the two groups in the number of complications and incorrect stent position following the implantation were noted (3\% vs $4 \%$ ). Good treatment results, with a decrease in gradient, an increase in saturation and a widening of the stenosis were obtained in majority of cases ( $\mathrm{p}<0,01$ dependent $\mathrm{t}$-test) in both groups $(97 \%$ vs $96 \%)$.

Conclusions: The obtained results suggest that pulmonary artery stenting with a short introducer sheath (SIS) can be successfully performed in children if proper procedures are observed. In the case of single-ventricle hearts after a Glenn procedure, SIS might be the method of choice, as except its standard advantages, it also permits angiographic control of the stent's position directly before expansion.

\section{7}

Occlusion of PDA and BT shunts with the pfm duct occluder device

Jorge M Moreira, Pediatric Cardiology, Hospital S Joao, Porto, Portugal Maria J Baptista, Pediatric Cardiology, Hospital S Joao, Porto, Portugal
Eduardo D Silva, Cardiac Catheterization Laboratory, Hospital S Joao, Portugal

Joao C Silva, Pediatric Cardiology, Hospital S Joao, Porto, Portugal

Background: Transcatheter coil therapy is currently the accepted method to close small to moderate patent ductus arteriosus (PDA), and is also used for redundant modified Blalock Taussig $(\mathrm{mBT})$ shunt occlusion. The aim of this study is to report our experience with the Duct-occlud pfm device in those clinical conditions.

Methods: Between November 2006 and December 2008, 45 patients with a median age of 7 years (range 0,9-19) and a median weigth of $25 \mathrm{~kg}$ (range 7,8-74) underwent attempted closure of their aortopulmonary connection. Forty three had a PDA and two had a mBT shunt. The device implantation was transvenously in PDA and transaortically in BT shunt.

Results: All the patients had a pfm coil implanted with no subsequent embolization, vascular obstructions or hemolisys. All PDA configurations, according to Krichenko classification, were found, except B type (A 18/43, C 7/43, D 8/43, E 12/43); pulmonary end size ranged from 0.5 to $3.2 \mathrm{~mm}$ (mean 1.4) and aortic end from 1 to $12 \mathrm{~mm}$ (mean 5,9). Smaller devices $(4 \times 4$ and $5 \times 4$ ) were implanted in $32 \%$ of patients. Immediate postprocedure minimal residual shunt was frequent $(47 \%)$, but in 24 hours echoDoppler control it was rare $(9 \%)$. The only patient with a moderate residual shunt had a second device implanted ten months latter. Both $\mathrm{mBT}$ shunts were completly occluded the day after the procedure.

Conclusions: The pfm DuctOcclud device is an effective and safe method for closure of as redundant modified BT shunts, as well as, the small-to-moderate PDA, whatever its morphology.

\section{8}

Covered Stent, A New Concept in the Management of Complex COA in Children and Young Patients. How to Avoid Repetitive surgery?

Hojjat Mortezaeian Langroudi, Rajaie Heart Center (RHC), Iran Paridokht Nokhostin Davari, Rajaie Heart Center (RHC), Iran

Akbar Shahmohammadi, Rajaie Heart Center (RHC), Iran

Mohmood Meraji, Rajaie Heart Center (RHC), Iran

Mohammad Yossef Aarabi, Rajaie Heart Center (RHC), Iran

Gholamreza Omrani, Iran

Avisa Tabib, Rajaie Heart Center (RHC), Iran

Yasaman Khalili, Rajaie Heart Center (RHC), Iran

Background: We implanted covered CP stents as a rescue treatment in patients with $\mathrm{CoA}$ aneurysms in patients with previous history of surgical or trans catheter COA repair, patients with COA\&PDA and systemic $\mathrm{PH}$, patients with dissection of aorta while catheterization and in patients at risk of developing complications such as complex CoA anatomy near-aortic arch interruption. This study sought to evaluate the use of covered stents in the treatment of complex COA specially in children and young patients.

Methods: Fifteen covered CP stents were implanted in 13 patients, 6 patients had had previous history of surgery COA repair or balloon angioplasty, 3 patients had complex or near-atretic CoA, 3 patients with COA \& Large PDA with systemic PH, 1 patient with dissection while catheterization. Cheatham-platinum stents covered with expanded polytetrafluoroethylene were used in all patients (NuMED Inc). The maximum balloon diameter was chosen based on either the transverse or the distal arch diameter, whichever was greater, and on occasions 1 to $2 \mathrm{~mm}$ greater. The stent was crimped onto a BIB balloon (NuMED Inc). Rapid right ventricular pacing was used during stent deployment in all 
cases. Heparin at a dose of $75-100 \mathrm{IU} / \mathrm{kg}$ was given. Heparin infusion or subcutaneous low-molecular-weight heparin was administered for a total of $24 \mathrm{~h}$. Antibiotics were given at the beginning of the procedure and continued for $24 \mathrm{~h}$. Aspirin was administered to all patients after the procedure at a dose of 3 to $5 \mathrm{mg} / \mathrm{kg}$ and continued for 6 months with clopidogrel $1 \mathrm{mg} / \mathrm{kg}$ for at least 1 month.

Results: The mean patient age and weight were $14 \pm 6$ years (range 7 to 24 years), and $32 \pm 9 \mathrm{~kg}$ (range 19 to $64 \mathrm{~kg}$ ), respectively. The systolic gradient across the CoA decreased from a mean $( \pm \mathrm{SD}$ ) of $32 \pm 15 \mathrm{~mm} \mathrm{Hg}$ before to a mean of $8 \pm 4 \mathrm{~mm}$ $\mathrm{Hg}$ after the procedure $(\mathrm{p}<0.001)$, and the diameter of the CoA increased from $4 \pm 3.5 \mathrm{~mm}$ to $16 \pm 2 \mathrm{~mm}(\mathrm{p}<0.001)$ A successful outcome was defined as a peak systolic pressure gradient after stent implantation of $<20 \mathrm{~mm} \mathrm{Hg}$. The follow-up period was up to 24 months (mean, 12 months). All stents were patent and in good position on Echocardiography, angiogram or computed tomography.

Conclusions: Covered CP stents may be used as the therapy of choice in patients with complex CoA. In the light of our experience and published data, we believe that covered CP stents are very useful tools for treating various congenital malformations. In particular, they would seem to be indicated in the following clinical settings: (I) subatretic native aortic coarctation (II) severe aortic coarctation or recoarctation (III) native aortic coarctation or recoartation with aneurysm (IV) aortic coarctation and patent ductus arteriosus (V) collateral vessels creating left to right shunting and (VI) bail-out in cases of complications in the catheterization laboratory. There are, however, few reports on the use of covered stents in congenital heart diseases, a setting in which their role remains to be clearly defined.

\section{9}

The efficacy of percutaneous transluminal angioplasty (PTA) in patients with coarctation of the aorta (CoA) Naomi Nakagawa, Hiroshima City Hospital, Department of Pediatric Cardiology, Japan

Hisako Kiguchi, Hiroshima City Hospital, Department of Pediatric Cardiology, Japan

Masahiro Kamada, Hiroshima City Hospital, Department of Pediatric Cardiology, Japan

Kunikazu Hisamochi, Hiroshima City Hospital, Department of Cardiac Surgery, Japan

Osamu Ooba, Hiroshima City Hospital, Department of Cardiac Surgery, Japan

Objective: We evaluated the efficacy of percutaneous transluminal angioplasty (PTA) in patients with coarctation of the aorta $(\mathrm{CoA})$.

Materials and Methods: We performed 32 PTAs in 28 patients with CoA (age range, 17 days to 16 years old; median, 8 months) and divided them into native $\operatorname{CoA}(n=20)$ and post-surgical CoA $(\mathrm{n}=12)$ groups.

Results: There were no significant differences in body weight and sex between the groups, while native CoA had a lower median age ( 2.1 vs 6.1 years). The median period from surgery to PTA in post-surgical CoA was 43 months (3-194). Peak systolic gradient across the coarcted segment decreased from $36.2 \pm 10.7$ to $10.2 \pm 1.6$ and $35.8 \pm 4.7$ to $7.3 \pm 7.3 \mathrm{mmHg}$ (both, $\mathrm{p}<0.001$ ) in native $\mathrm{CoA}$ and post-surgical $\mathrm{CoA}$, respectively. Five in native CoA, each under 2 months old, developed re-coarctation after initial decline in the trans-coarctation gradient. Those underwent a re-PTA and re-coarctation was resolved in 4 . There were no failures in post-surgical $\mathrm{CoA}$ and 3 in native $\mathrm{CoA}, 2$ of whom needed surgery for low cardiac function and 1 for a large residual gradient. Follow-up angiography and/or computed tomography in 16 cases revealed 2 aneurysms in native CoA. There were 3 complications, including obliteration of the femoral artery in the neonatal period in 2 and erroneous dilation of ductus arteriosus in 1 .

Conclusion: PTA is an adequate alternative for both native and post-surgical CoA cases. However, caution is needed for restenosis and femoral artery obliteration in infants, as well as aneurysm formation, especially in native CoA cases.

\section{0}

Follow up after interventional procedures; is it time for consensus?

Nitha Naqvi, Royal Brompton and Harefield Hospital NHS Trust, United Kingdom

Alan G Magee, Royal Brompton and Harefield Hospital NHS Trust, United Kingdom

Objective: To investigate variations in current UK practice for follow up management of children undergoing common interventional cardiology procedures.

Method Design and setting: A standardised questionnaire was e mailed to all UK paediatric interventional cardiology consultants in all the 13 recognised units.

Main outcome measures: Follow up care for device closure of ASD, PDA and VSD were studied, regarding timings and frequency of follow up appointments, time to discharge, use of aspirin and endocarditis prophylaxis.

Results: All thirteen centres responded with 28 questionnaires returned. $88 \%$ of all interventional consultants responded. There was enormous variation in practice. After ASD and VSD closures 1st follow up appointment time varied from 1 week to 12 weeks. After PDA closure follow up varied from none to annual visits until 16 years of age. Discharge from follow up after ASD closure varied from 1 year post-procedure to lifelong follow up. All respondents agreed to lifelong follow up post VSD device closure. Aspirin duration following ASD closure varied from 6 weeks to 1 year, and 3 months to lifelong after VSD closure. There was no agreement on endocarditis prophylaxis.

Conclusion: Due to the lack of evidence based practice there is wide variation in follow up management leading to large differences in resource and cost implications. There is frequent variation between individuals in the same units as well as between units. There is a need for consensus guideline.

\section{1}

Hybrid therapy for hypoplastic left heart syndrome with intact atrial septum

Naoki Ohno, Department of Pediatrics, Okayama University Graduate School of Medicine, Dentistry and Pharmaceutical Sciences, Japan Shinichi Ohtsuki, Department of Pediatrics, Okayama University Graduate School of Medicine, Dentistry and Pharmaceutical Sciences, Japan Yoshio Okamoto, Department of Pediatrics, Okayama University Graduate School of Medicine, Dentistry and Pharmaceutical Sciences, Japan

Maiko Kondo, Department of Pediatrics, Okayama University Graduate School of Medicine, Dentistry and Pharmaceutical Sciences, Japan

Yoshihiko Kurita, Department of Pediatrics, Okayama University Graduate School of Medicine, Dentistry and Pharmaceutical Sciences, Japan Takahiro Eitoku, Department of Pediatrics, Okayama University Graduate School of Medicine, Dentistry and Pharmaceutical Sciences, Japan 
Tsuneo Morishima, Department of Pediatrics, Okayama University Graduate School of Medicine, Dentistry and Pharmaceutical Sciences, Japan

Shunji Sano, Department of Cardiovascular Surgery, Okayama University Graduate School of Medicine, Dentistry and Pharmaceutical Sciences, Japan

Objective: In cases of hypoplastic left heart syndrome (HLHS) with intact atial septum, we discuss hybrid therapy and the postoperative course.

Methods: The subjects were two patients with HLHS who underwent hybrid therapy in our hospital. Hybrid therapy: Immediately after birth, needle puncture of the interatrial septum via the right auricular appendage was carried out with the heart beating, static balloon atrioseptostomy (BAS) and bilateral pulmonary artery banding (PAB) was performed.

Results: Case 1: Stenting for the interatrial septum was performed due to restenosis. A decrease in pulmonary artery pressure (PAP) was indicated by pressure gradient in $\mathrm{PAB}$ of $65 \mathrm{mmHg}$, and Norwood procedure was performed at the age of one month. Bidirectional Glenn procedure was performed at the age of one year and currently waiting for total cavopulmonary connection. Case 2: BAS was performed again twice due to restenosis. But there was a gradual decrease in PAP with pressure gradient in $\mathrm{PAB}$ of $30 \mathrm{mmHg}$. Norwood procedure was performed at the age of 3 months due to unstable hemodynamics. However, postoperative lung biopsy revealed notable hypoplasia of pulmonary small arteries. We considered that there was not surgical indication. The patient was treated with Sildenafil and Bosentan but died from $\mathrm{PH}$ crisis .

Conclusions: Careful consideration must be given to the indication and timing of the Norwood procedure and also indication for further surgery based on changes in the pressure gradient in $\mathrm{PAB}$ as an index of PAP after interartial communication has been sufficiently established.

\section{2}

Early and midterm results of the patients with PDA and VSD closed using Amplatzer duct occluder Nazan Ozbarlas, Department of Pediatric Cardiology, Faculty of Medicine, Cukurova University, Adana Turkey, Turkey Osman Kucukosmanoglu, Department of Pediatric Cardiology, Faculty of Medicine, Cukurova University, Adana Turkey, Turkey Sevcan Erdem, Department of Pediatric Cardiology, Faculty of Medicine, Cukurova University, Adana Turkey, Turkey Alev Kiziltas, Department of Pediatric Cardiology, Faculty of Medicine, Cukurova University, Adana Turkey, Turkey

Abdi Bozkurt, Department of Cardiology, Faculty of Medicine, Cukurova University, Adana Turkey, Turkey

In this report we review initial and 4-year results following transcatheter occlusion of patent ductus artriozus (PDA) and ventricular septal defect (VSD) using Amplatzer duct occluder (ADO) and ADO II.

Between November 2004 and 2008, 66 patients, ages ranged 7 months to 66 years, underwent transcatheter closure using the ADO and ADO II. Two muscular VSDs were closed with ADO.

The mean pulmonary artery pressure was $25,8 \mathrm{mmHg}$ (median $24 \mathrm{mmHg}, 12$ to $60 \mathrm{mmHg}$ ) and 24 of them were greater then $25 \mathrm{mmHg}$. The mean PDA diameter (at the pulmonary end) was $3,3 \mathrm{~mm}$ (range 1,6 to $7,5 \mathrm{~mm}$ ), the PDA length was $7,0 \mathrm{~mm}$ (range 3 to $16 \mathrm{~mm}$ ) and the mean ampulla diameter (at the aortic end) was $10,5 \mathrm{~mm}$ (range 5,3 to $23 \mathrm{~mm}$ ). For closure of PDA, 52 $\mathrm{ADO}$ and 14 ADO II were used. Two patient's muscular VSD were closed with ADO. Occlusion of femoral artery was developed only one patient and there was not another complication in early period. Complete closure was observed in $67 / 68(98,5 \%)$ patients. None of the patients developed increased velocity across the left pulmonary artery and descending aorta. The mean follow up period was 7,9 months (median 2,5 months, 1 to 42 months). No early or late evidence of device failure, recanalization, endocarditis, thromboembolism, or hemolysis were observed.

Our results provide that transcatheter closure using ADO can safely and effectively in treatment of PDA and it can be use for closure of some of muscular VSD.

\section{3}

Effectiveness of Stent therapy of Coarctation of Aorta in Reducing systemic blood Pressure

Najma Patel, National Institute of Cardiovascular Diseases, Pakistan Kalimuddin Aziz, National Institute of Cardiovascular Diseases

Shakeel Qureshi, St.Thomas Hospital. London, United Kingdom, United Kingdom

Objective: To assess the effectiveness of Stent therapy (ST) for Coarctation of Aorta $(\mathrm{CoA})$ in reducing Systemic Blood Pressure (BP)

Method: Follow up (f/u) data after ST was analyzed. 37 patients were studied for ST. 30 with native and 7 with recurrent CoA. 32 had ST, 5 were not suitable. 26/32 were hypertensive. On Aortograms, discrete CoA was found in 15, long segment in 13 while 4 had near atretic Co A.

BIB Balloon (B) and CP stent were used. Dilated to the B size $2 \mathrm{~mm}$ smaller than the diameter of Arch or equal to the diameter of descending aorta. 2 with near atresia had hypoplastic proximal segment, so were dilated up to the diameter of proximal segment. Result: Mean gradient across CoA decreased from $55.2 \mathrm{mmHg}$ (SD \pm 19.2 , range $21-90)$ to $12.3 \mathrm{mmHg}$ (SD \pm 12.47 , range 2-30) $\mathrm{p}<0.000$. Diameter at Coarctation site increased from mean $3.78 \mathrm{~mm}$ (SD \pm 1.47 , range $1.2-6.4)$ to $15.9 \mathrm{~mm}$ $(\mathrm{SD} \pm 1.78$, range 12.8-19) $\mathrm{p}<0.000$. All patients had four limbs BP recording preprocedure and then in $\mathrm{f} / \mathrm{u}$ period. Predilatation mean SBP was $172 \mathrm{mmHg}$ (SD \pm 24 , range 120-190) and mean DBP was $98.8 \mathrm{mmHg}$ ( \pm SD11.3, range 80-120). At mean $\mathrm{f} / \mathrm{u}$ of 30.6 months, mean SBP was $138 \mathrm{mmHg}, \quad(\mathrm{SD} \pm 14.7120-165) \mathrm{p}<0.002$, and mean DBP $84.1 \mathrm{mmHg}(\mathrm{SD} 7.7$, range 80-100) $\mathrm{p}<0.000$. Predilatation 6 were not taking any antihypertensive drugs, 12 were taking two and 14 were taking 3 drugs, Postdilatation 9 were not taking any medication, 10 were taking only one, 10 were taking 2 , only 3 were taking 3 drugs.

Conclusion: We conclude that ST provides long term reduction in systemic hypertension.

\section{4}

\section{Asd Closure By Amplatzer Septal Occluder}

Najma Patel, National Institute of Cardiovascular Diseases, Pakistan Kalim uddin Aziz, National Institute of Cardiovascular Diseases, Pakistan

Habiba Tasneem, National Institute of Cardiovascular Diseases, Pakistan

Shakeel Qureshi, Evelena Children's Hospital, United Kingdom Zahid Amin, Cardiac Catheterization and interventions, University of Nebraska/Creighton University Children's Hospital of Omaha, United States James Wilkinson, Department of Cardiology, Royal Children's Hospital, Melbourn, Australia 
Closure of large atrial septal defects(ASD)continue to pose problems for percutaneus closure.We report our learning experience of 106 procedures in patients age 12.8years ( \pm 8.99), using Amplatzer Septal occluder (ASO).

Methods: ASO was used in all cases. General anaestesia used with transoesophageal echocardiography. Sizing Balloon used to size all ASD initially but lately to size only those with variable diameters in various echo views.

Results: ASD size was $24.23 \mathrm{~mm}( \pm 6.11)$ either with sizing balloon or colour Doppler. Device size was $27.86 \mathrm{~mm}$ ( \pm 7.44 ). Two had multiple ASD, one had aneurysm of IAS with multiple ASD, each closed with one device. Two devices were used in 2 and 1 deployment was unsuccesful. Did not attempt device closure in 5.3 had IVC and superior rims deficient, 2 due to ASD too large for 5 and 6 years old. Used Balloon assisted (BAT) in 8 and catheter assisted technique (CAT) in 2 with small inferior or superior rims. Deployed part of LA disk in RUPVorLUPV with deployement of RA disk in RA and then pulled the LA disk for large ASD. One patient had PDA, closed by coil,3 had pulmonary valvoplasty for pulmonary stenosis simultaneously.

Major complication was pericardial effusion in 1, developed after 3 days required pericardiocentesis. Minor complications were transient AV block type II in 1, SVT in 1 complete heart block in 1.

Conclusions: We conclude that large ASD continue to be a challenge in selection, deployment and procedure time. Complication were seen in large ASD. For us cost issue makes use of more than 1 devices prohibitive, and a greater stress in sizing and device selection.

\section{5}

A multicenter experience with perventricular closure of mucular VSDs in South America

Carlos Pedra, Dante Pazzanese Instituto de Cardiologia, Brazil Simone RF Fontes-Pedra, Instituto Dante Pazzanese de Cardiologia, Brazil

Paulo Chaccur, Instituto Dante Pazzanese de Cardiologia, Brazil Marcelo Jatene, Hospital do Coracao, Brazil

Justo Santiago, Hospital Universitario de Merida, Venezuela Isac Tueti, Hospital Cardiologico Infantil, Venezuela Guillermo Villoria, Hospital Cardiologico Infantil, Venezuela Igor Donis, Hospital Cardiologico Infantil, Venezuela

Perventricular closure of muscular VSDs has become an attractive treatment modality for infants. However, its reproducibility worldwide remains to be seen. We report a multicenter experience in South America. From 07/2007 to 08/2008, 7 non-consecutive patients (median age and weight: 9 months and $6 \mathrm{kgs}$, respectively) underwent the procedure in the $\mathrm{OR}$ under TEE guidance using Amplatzer devices. All pts but one were in $\mathrm{CHF}$ and had PAH. One patient was status post PA banding and 2 also had CoA, which were all repaired at the same session. Six pts had single defects $(5$ midmuscular, 1 apical) measuring $10.5 \pm 3.4 \mathrm{~mm}$ and 1 patient had multiple apical defects that required 2 devices. Eight devices were all implanted successfully (median size: $12 \mathrm{~mm}$ ), with 2 having to be fixed in the RV wall with a surgical suture. One patient each developed $\mathrm{R}$ and LBBB. In 1 patient with a $14 \mathrm{~mm}$ defect, the inferior portion of a $16 \mathrm{~mm}$ device prolapsed through the inferior rim of the defect towards the LV after 10 days, requiring surgical removal with patch closure of the mid-muscular VSD. After a median follow-up of 9 months, the patient with 2 devices remained with a $1 \mathrm{~mm}$ residual shunt with normal LV size. Perventricular closure of muscular VSDs seems to be reproducible, feasible, relatively safe and effective in our hands. Larger defects may need device oversizing $>3 \mathrm{~mm}$ or suture fixation for adequate stabilization. More patients and longer follow up are needed.

\section{6}

Preliminary experience with fetal cardiac interventions in Brazil

Simone RF Fontes-Pedra, Hospital do Coracao, Brazil

Carlos Pedra, Dante Pazzanese Instituto de Cardiologia, Brazil

Fabio Peralta, Hospital do Coracao, Brazil

Marina Zamith, Hospital Samaritano, Brazil

Fernanda S Jardim, Hospital Samaritano, Brazil

Vera Aiello, Instituto do Coracao, Brazil

Cristina Alliman, Hospital Samaritano, Brazil

Renato Assad, Hospital Samaritano, Brazil

Because few centers in the world perform fetal cardiac interventions, its reproducibility worldwide is unknown. We report a preliminary experience from Brazil. From 07/07 to 08/ 08, 6 fetuses underwent interventions for HLHS and restrictive/ absent ASD ( 3 cases), critical AS and impending HLHS ( 2 cases) and PA and IVS with small RV (1 case) between 27-32 weeks of gestation. Procedures were performed under maternal sedation/ regional blockade and fetal anesthesia. Cardiac access was achieved with Chiba needles through the maternal abdominal/ uterine walls and fetal chest wall under echocardiographic guidance. Coronary artery balloons and wires were used for dilation. New ASDs were successfully created in the 3 HLHS cases and the AV was successfully dilated in the 2 AS cases. The PV was perforated and crossed in the PA case but could not be dilated because the RV was punctured too close to the PV. Complications included successfully drained tamponade (3 cases), bradicardia (2), thrombus formation in the LA (1) and fetal demise the following day (1 case of HLHS, transplacentary?). One HLHS neonate died after a hybrid procedure due to white lungs and the other after stage II operation due to pulmonary vein obstruction. One AS fetus was born premature (32 weeks) and died and the other required a Norwood operation due to a small LV. The PA patient underwent successful neonatal RF perforation/dilation. Fetal interventions were feasible in our hands despite a high rate of complications. Although they were immediately successful, they may not alter the natural history of some defects.

\section{7}

Occlusion of Fontan fenestration in children with univentricular heart. Hemodynamic results and risk factors for unfavourable response

Jaana Pihkala, Hospital for Children and Adolescents, University of Helsinki, Finland

Merit Järvelä, University of Helsinki, Finland

Eero Jokinen, Hospital for Children and Adolescents, University of

Helsinki, Finland

Talvikki Boldt, Hospital for Children and Adolescents, University of Helsinki, Finland

Ilkka Mattila, Hospital for Children and Adolescents, University of

Helsinki, Finland

Anneli Eerola, University Hospital of Tampere, Finland

Background: Fenestrating Fontan circulation improves outcome but is associated with cyanosis and thromboembolic risk.

Objective: To review operative history and clinical and catheterization data on patients undergoing fenestration test occlusion, and to define risk factors for unfavourable response to test occlusion.

Methods: Fifty-two patients underwent hemodynamic evaluation and fenestration test occlusion in median 2.3 years after Fontan operation. Test occlusion failure was defined as $>4 \mathrm{mmHg}$ 
increase in central venous pressure (CVP) or $>10 \%$ reduction in central venous oxygen saturation (CVSat).

Results: Thirty-two patients passed test occlusion (group P) and fenestration was closed. Twenty patients failed it (group F) and fenestration was left patent. Groups didn't differ in preoperative hemodynamics, age at Fontan, or time interval between Fontan and catheterization. Failure was more common in patients with pulmonary artery stenoses in preoperative angiography $(p=0.02)$, in patients having undergone repair for aortic arch $(p=0.03)$, and in those with dominant right ventricular $(\mathrm{RV})$ morphology $(\mathrm{p}<0.05)$. Clinical and catheterization data are shown on Table. No complications occurred during catheterization.

Conclusions: Fenestration closure is safe with improvement in oxygen saturation in patients with favourable response to test occlusion. During test occlusion, change in CVSat reflects hemodynamic response more significantly than change in CVP. Systemic ventricular morphology of RV type and history of pulmonary artery stenoses and repair for aortic arch predispose patients to test occlusion failure.

Table. Clinical and catheterization data.

\begin{tabular}{|c|c|c|}
\hline & Group P & Group F \\
\hline Age (years) & $5.2(3.1-10.6)$ & $5.3(3.4-19.5)$ \\
\hline $\begin{array}{l}\text { Systemic ventricular } \\
\text { morphology } \mathrm{RV} / \mathrm{LV}\end{array}$ & $12 / 20$ & $13 / 7$ \\
\hline $\begin{array}{l}\text { Pulse oximetry admission/ } \\
\text { discharge }(\%)\end{array}$ & $\begin{array}{l}90(74-95) / 95 \\
\quad(90-99) * \S\end{array}$ & $\begin{array}{c}90(80-94) / 90 \\
(83-94) *\end{array}$ \\
\hline $\begin{array}{l}\text { Hemoglobin concentration } \\
(\mathrm{g} / \mathrm{l})\end{array}$ & $149(133-190)$ & $153(127-190)$ \\
\hline $\begin{array}{l}\text { Mean pulmonary artery } \\
\text { pressure }(\mathrm{mmHg})\end{array}$ & $11(8-17) \#$ & $13(10-16) \#$ \\
\hline $\begin{array}{l}\text { CVP before/during occlusion } \\
(\mathrm{mmHg})\end{array}$ & $\begin{array}{c}12(8-17) / 13 \\
(8-19) d x\end{array}$ & $\begin{array}{c}13(10-16) / 15 \\
\quad(10-18) d\end{array}$ \\
\hline $\begin{array}{l}\text { CVSat before/during occlusion } \\
(\%)\end{array}$ & $\begin{array}{l}62(43-75) / 66 \\
\quad(56-92) * \S\end{array}$ & $\begin{array}{c}64(47-75) / 47 \\
\quad(29-61) * \S\end{array}$ \\
\hline $\begin{array}{l}\text { Aortic saturation before/ } \\
\text { during occlusion }(\%)\end{array}$ & $\begin{array}{c}90(68-99) / 96 \\
(90-99) \S\end{array}$ & $\begin{array}{c}89(83-95) / 95 \\
\quad(84-98) \S\end{array}$ \\
\hline
\end{tabular}

$\star_{\mathrm{p}}<0.001, \# \mathrm{p}<0.05$ between groups

$\S \mathrm{p}<0.001,0 \mathrm{p}<0.01$ within groups

\section{8}

Covered Stent Implantation in Kawasaki Disease with Giant Coronary Aneurysm

Worakan Promphan, Queen Sirikit National Institute of Child Health, Thailand

Worawut Tassanawiwat, Sappasittiprasong Hospital, Thailand

Napa Siriwiwattanakul, Rajvithi Hospital, Thailand

Tawatchai Kirawittaya, Queen Sirikit National Institute of Child Health, Thailand

Chaisit Sangtaweesin, Queen Sirikit National Institute of Child

Health, Thailand

Thanarat Layangool, Queen Sirikit National Institute of Child Health, Thailand

We reported an 8 year-old-girl who has been implanted a $3 \times 15 \mathrm{~mm}$ covered stent in the mid right coronary artery (RCA) aneurysm. She has been diagnosed as a Kawasaki disease in 2001 without immunoglobulin therapy. Early echocardiographic follow up showed multiple aneurysms at proximal left main coronary artery (LMCA), left anterior descending (LAD) and RCA with the diameters of $5.4,8.5$, and $5 \mathrm{~mm}$ respectively. During follow up, though aneurysms have not regressed, she has been doing well with an intact left ventricular systolic function. She has been commencing with low dose aspirin and oral warfarin during follow up. Last coronary angiography, March 2008 , showed a significant stenosis $(>90 \%)$ of proximal and distal part of a huge mid RCA aneurysm. There was no scar or infarction on cardiac MRI study.

Covered stent has been implanted in April 2008 with a favorable outcome. The mid RCA aneurysm has been obliterated and excellent distal RCA flow has been achieved. She has been anticoagulated with oral warfarin (INR $>2$ ) and low dose aspirin after the treatment. However, significant in-stent restenosis has demonstrated 6 months later requiring another $3 \times 23 \mathrm{~mm}$ drug eluting stent placement over the previous one.

Though coronary intervention using covered stent has been feasible, in-stent restenosis has occurred quite early after the treatment.

\section{9}

Efficacy and Safety of Low-molecular-weight Heparin in the Treatment of Children after Transcatheter Closure of Atrial Septal Defects

Jun Qin, Cardiovascular Disease Research Center, Xinqiao Hospital, Third Military Medical University, China

Objective: To observe the efficacy and safety of low-molecularweight heparin (LMWH) in the treatment of children after transcatheter closure of atrial septal defects (ASD).

Methods: 48 patients were randomized into unfractionated heparin (UFH) group or LMWH group. $20 \mathrm{mg}$ LMWH (enoxaparin sodium) was administered twice through subcutaneous injection in abdominal wall after occlusion. But in the control group UFH $(10 \mathrm{IU} / \mathrm{Kg} / \mathrm{h})$ was intravenously given for 24 hours after occlusion. Aspirin therapy, which began right after the occlusion of ASD, lasted for 6 months in all the patients. Activated partial thromboplastin time (APTT) were determined before the occlusion of ASD, at $6 \mathrm{~h}, 24 \mathrm{~h}$ and $48 \mathrm{~h}$ after device implantation. Transthoracic echocardiograph (TTE) were performed 1, 3, and 6 months after the occlusion of ASD.

Results: The follow-up has been performed on 43 patients (LMWH 22, UFH 21). Neither thrombus on ASD occluders nor severe hemorrhage complications were found during the followup period. Before occlusion no significant difference in APTT was noted in the two groups. But after occlusion APTT of the UFH group was significantly prolonged at $6 \mathrm{~h}$ and $24 \mathrm{~h}$ but returned to normal range at $48 \mathrm{~h}$. However, such significant changes were not found in the LMWH group. Inter-group comparison revealed that APTT was more significantly prolonged in UFH group at $6 \mathrm{~h}$ and $24 \mathrm{~h}$ but no significant inter-group differences were found at $48 \mathrm{~h}$.

Conclusions: Enoxaparin sodium is safe, effective and convenient in the treatment of children after transcatheter closure of ASD. Key words: Low-molecular-weight Heparin Children Atrial septal defect Occlusion

\section{0 \\ PDA Angiographic Morphology in Cyanotic Congenital Heart Diseases \\ Nomee A Radzi, Institut Jantung Negara, Kuala Lumpur, Malaysia \\ Mazeni Alwi, Institut Jantung Negara, Kuala Lumpur, Malaysia \\ Hasri Samion, Institut Jantung Negara, Kuala Lumpur, Malaysia \\ Haifa Latiff, Institut Jantung Negara, Malaysia \\ Geetha Kandavello, Institut Jantung Negara, Malaysia}

Objective: To examine the morphology of the patent ductus arteriosus (PDA) in cyanotic congenital heart diseases. 
Method: A retrospective review of angiographic records of patients with duct-dependant heart lesions over a period of 2002-2008 was done. The types of PDA were classified according to the origin of the PDA from the aorta: Type I: distal to the subclavian artery (SCA), Type II: between the SCA and the carotid artery, Type III: distal to the carotid artery and Type IV: from the innominate or SCA. Tortuosity was expressed as 'simple' if only one curve is present or 'complex' when there is more than one.

Results: 237 records were examined. Type I was the commonest, $149 / 237$ (63\%), followed by type II, 50/237 (22\%), type III 28/ 237, (11\%) and type IV, 10/237 (4\%). The duct in Type I was straight in 30\%, simple 33\% and complex 37\%; Type II straight in $12 \%$, simple $64 \%$, complex $24 \%$; Type III straight in $39 \%$, simple $43 \%$, complex $18 \%$ and Type IV simple and complex divided equally. The PDA insertion was into the MPA 70\%, LPA 26\% and RPA 4\%. Branch PA stenosis occurred in 40\%. Patients with PAIVS and tricuspid atresia tend to have Type I morphology whereas those of TOF/PA had the more distal type.

Conclusion: This study showed that the PDA in cyanotic heart diseases tends to arise more proximal and is often long and tortuous. Stenosis occurs when the PDA inserts into the branch PAs which is not uncommon especially in TOF/PA.

\section{1}

Mid to Long Term Outcome of Transcatheter Closure of Ventricular septal Defects

Nomee A Radzi, Institut Jantung Negara, Kuala Lumpur, Malaysia

Hasri Samion, Institut Jantung Negara, Kuala Lumpur, Malaysia

Mazeni Alwi, Institut Jantung Negara, Kuala Lumpur, Malaysia

Haifa Latiff, Institut Jantung Negara, Kuala Lumpur, Malaysia

Geetha Kandavello, Institut Jantung Negara, Kuala Lumpur, Malaysia

Objective: To establish the medium to long term outcome of transcatheter closure of subaortic ventricular septal defect (VSD) using the AMVOD as an alternative to surgical closure.

Methods: Retrospectively collected data from 2004 to 2006 of 76 patients who underwent transcatheter closure of their VSD were analysed. 64 had perimembranous, 10 had doubly-committed subarterial and 2 had muscular type VSD. 53 had no aortic regurgitation (AR), 19 trivial and 4 mild. The patients were followed up for a minimum of two years focusing on cardiac rhythm and status of the aortic valve.

Results: The median age was 8.9 years months and the median weight $26 \mathrm{~kg}$. The mean VSD size was $9 \mathrm{~mm}$ and the mean device size $10 \mathrm{~mm}(4-18 \mathrm{~mm}) .69$ (91\%) patients showed no worsening of AR. Only $7(9 \%)$ patients had worsening of AR, 3 from nil to trivial, 3 from trivial to mild and one progressed to severe AR requiring aortic valve replacement. 8 patients had transient arrhythmia. 4 developed new RBBB whereby one of them reverted back to sinus rhythm after a year. 2 other patients developed LBBB at 6 and 12 months post procedure - one progressed to complete heart block requiring PPM insertion and the other developed LV dysfunction for which we are considering cardiac resynchronisation therapy. Another patient was found to have $\mathrm{CHB}$ at 10 months post procedure and had a PPM inserted. Conclusion: Transcatheter closure offers an attractive alternative to surgical closure however caution must be taken in view of emerging arrhythmias.

\section{2}

Transcatheter Closure of Secundum Atrial Septal Defects with Amplatzer Septal Occluder : Experience at Dr Soetomo Hospital Surabaya Indonesia
Mahrus A Rahman, Division of Paediatric Cardiology Department of Paediatric Dr Soetomo Hospital Surabaya, Indonesia Muljadi M Djer, Division of Paediatric Cardiology Department of Paediatric Dr Cipto Mangunkusumo Hospital Jakarta, Indonesia Ahmad Lefi, Indonesia

Background: Secundum atrial septal defect (ASD2) causes right heart volume overload and produces symptoms after third decade of life. Treatment until now has been open heart surgery. Many devices were introduced to close ASD2 transcatheter.

Objectives: To review our experience with transcatheter closure of ASD2 using Amplatzer septal occluder.

Methods: Between November 2004 and November 2008, there were 17 patients, median age 27 years (6-52 years). Diagnosis was established by transthoracic (TTE) and transesophaeal echocardiography (TEE). Implantation was performed under local and general anesthesia through femoral vein with the guidance of TTE, TEE and fluoroscopy. Blood pressure was monitored through femoral artery. The device size chosen by balloon-stretched diameter or without balloon sizing.

Results: Implantation was successfull in all 17 patients.

Mean ASD2 diameter by TTE and TEE $21+6.9 \mathrm{~mm}$, mean stretched diameter was $25+4.0 \mathrm{~mm}$. Mean pulmonary flow: systemic flow was $2.45+1$. Mean procedure time was $107.8+$ 42.7 minutes. Mean fluoroscopy time was $24.8+11.9$ minutes. The complications were transient arrhythmia (premature atrial contraction) in one pasien, and transient migraine in 2 pasien. Conclusions: Transcatheter closure of ASD2 with Amplatzer septal occluder is a safe and effective alternative to surgical closure.

\section{3}

Transcatheter Closure of Coronary Artery Fistulae Mahrus A Rahman, Division of Cardiology Department of Paediatric Dr Soetomo Hospital Surabaya, Indonesia

Alit Utamayasa, Division of Paediatric Cardiology Department of Paediatric Dr Soetomo Hospital Surabaya, Indonesia

Teddy Ontoseno, Division of Cardiology Department of Paediatric Dr Soetomo Hospital Surabaya, Indonesia

Background: Transcatheter closure of Coronary Artery Fistulae (CAF) has been advocated with many kind of device as a minimally invasive alternative to surgery.

Objectives: We report a case of coronary artery fistulae after transcatheter closure with single Gianturco coil.

Methods: Coronary artery fistula was diagnosed by transthoracal echocardiography and confirmed with coronary angiography. Anterograde approached using multipurpose endhole catheter \#4F. Heparin and prophylactic antibiotic were given before procedure and 2 other doses antibiotic were given after procedure. One $8 \mathrm{~mm} \times 5 \mathrm{~cm}$ Gianturco coil was placed at the mouth of the CAF. Repeted coronary angiography after procedure was made.

Results: Single coronary artery fistula from right coronary artery draining into right atrium. The smallest diameter was $2 \mathrm{~mm}$ and the largest diameter was $8 \mathrm{~mm}$. Flow ratio was 2. Postdeployment angiogram demonstrated small residual flow. Follow-up echocardiogram 24 hour after procedure showed no residual shunt. Early complication was not found.

Conclusions: Transcatheter closure of small CAF with Gianturco coil is an acceptable alternative to surgery.

\section{4}

First Percutanous device closure to coronary artery fistula in 18 month old Libyan boy

Salem M Rahouma, Benghazi Cardiac Center, Libya 
Naima I Ghobaa, benghazi children's hospital, Medical University Benghazi Libya, Libya

Rasmia H Faituri, university department of child health Benghazi medical University, Libya

18 month old libyan boy (fig1) was diagnosed with a large coronary artery fistula that originated from the left coronary artery and emptied into the right atrium via coronary sinus . The patient was referred with heart murmur noted in neonatal routine check where echocardiogram carried out at benghazi's children's hospital confirm large coronary artery fistula (Fig 2) catheterization at age of 6 month confirm echocardiogram studies (fig3).

Aims/objectives: The early diagnosis and referral for intervention procedure is the gold standard of pediatric cardiology where the services needed in conjunction with surgical cover to the eastern region of Libya.,

Methods and materials: Benghazi cardiac center serves nearly half country of popultion over 6 , million (fig4) skilled pediatric echocardiography, catheterization lab diagnostic and intervention procedures in house facilities placement a 8/6-mm Amplatzer PDA device. Discussion/Results: we discuss the use of transcatheter methods including vascular plugs or PDAl/VSD occluder devices for coronary artery fistulae,

Our retrograde approach is simple and need to establish arteriovenous loops or to insert additional devices, we compare our results with previous reports. To our knowledge, this report the first case in north africa that describes the closure of a coronary fistula in an child at age of 18 month by use of an Amplatzer ductal occluder (fig5). Conclusion: We conclude the safety, the feasibility, and the standard approach to deployment to be established.as we provide the only single cardiac center experience which serve whole country for interventional in structure/congenital heart disease in the eastern region of Libya.

\section{5}

Nitinol vascular plug for embolisation of large

shunts- Can it be improved?

Premsekar Rajasekaran, Frontier Lifeline Hospital (Dr. K.M.Cherian

Heart Foundation), India

Anuradha Sridhar, India

Subramaniam Raghavan, India

Yogesh Sathe, India

Shanthi Chidambaram, India

Kotturathu M Cherian, India

Objective: Nitinol vascular plugs are designed for occluding large shunts. We share our single institutional experience in use of this device in various shunt lesions.

Method: A total of 7 implantations were identified in six patients aged 11 months to 14 years between July 2006 and December 2008. 2 plugs, $10 \mathrm{~mm}$ and $12 \mathrm{~mm}$ respectively were used to occlude large coronary cameral fistula to right atrium in two children aged 3 and 10 years. 2 plugs, $10 \& 12 \mathrm{~mm}$ were implanted in an 11 month infant with PAVM of the right lower lobe of lung. A $16 \mathrm{~mm}$ plug was implanted in a 2 year old post TAPVC repair child with an unligated ascending vein. 2 plugs of $6 \mathrm{~mm}$ size were used in two patients aged 8 and 14 years occluding BT shunt and aortopulmonary collateral respectively. Results: All 7 implantations achieved the desired end result. It was noted consistently that the immediate post-procedure angiography revealed significant shunt through the body of the plug. This was more so in the case of shunts of arterial origin. None of the 6 patients have been subjected to repeat angiography to document complete shunt abolition.
Conclusion: The vascular plug owing to its design lends itself well to closing moderate to large shunt lesions. However, the lack of occlusive fabric inside the Nitinol wire mesh delays thrombosis and shunt abolition. This leads us to wonder whether incorporating a polyester patch as in PDA, VSD, and ASD occluders would enhance the function of the vascular plug.

8376

Experience with the Amplatzer Vascular Plug in the Paediatric Catheterization Laboratory

Katja R Reineker, Dep. of Paediatric Cardiology, UK S-H, Campus

Kiel, Germany, Germany

Hans-Heiner Kramer, Dep. of Paediatric Cardiology, UK S-H, Campus Kiel, Germany, Germany

Gunther Fischer, Dep. of Paediatric Cardiology, UK S-H, Campus

Kiel, Germany, Germany

Objective: The Amplatzer ${ }^{\mathbb{R}}$ Vascular Plug (AVP) is a retrievable, selfexpanding cylindrical nitinol wire mesh device designed for embolisation of arterial and venous vascular malformations.

Patients and Methods: 13 patients (age: 12 days-15 years) with underlying complex congenital heart disease $(\mathrm{n}=8)$ or an isolated malformation $(\mathrm{n}=5)$ underwent embolisation with a total of 18 AVP. Devices of 4 to $12 \mathrm{~mm}$, which had at least twice the diameter of the malformation at its narrowest segment were deployed through a 5 to $7 \mathrm{~F}$ guide catheter. Once, 2 AVP were positioned in series. During routine catheterization, 8 patients with single ventricle palliation had occlusion of aortopulmonary $(n=6)$ and venovenous $(n=4)$ collaterals or suture dehiscence of the Fontan tunnel $(n=2)$. One patient each underwent embolisation of a coronary artery fistula, sinus valsalva aneurysm, A. brachialis aneurysm, V. verticalis, and the ductus venosus Arantii.

Results: Complete occlusion was confirmed in all patients by angiography or echocardiography within the first 24 hours after device placement. There were no device embolisations or other procedure related complications.

Conclusion: The AVP is an easy to use and effective device for embolisation in a wide variety of clinical situations and contributes to reduce procedure and fluoroscopy times. In most cases, placement of a single device results in effective occlusion of the target malformation.

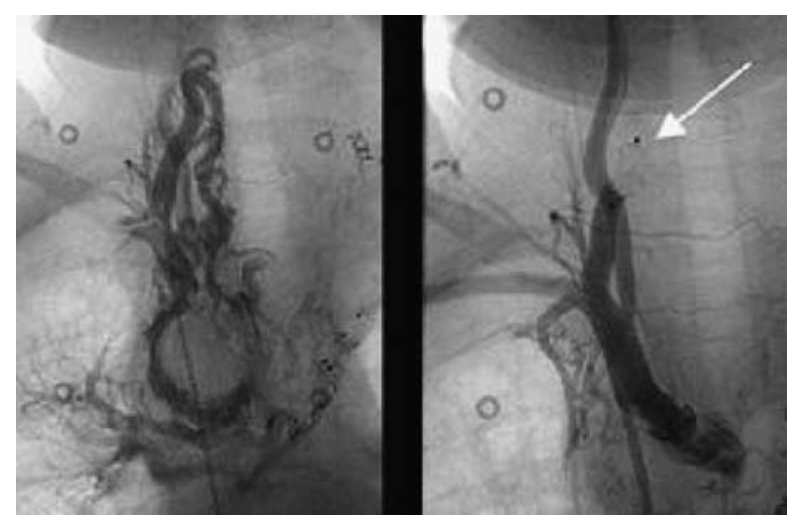

8377

Catheter interventions in the management of Hypoplastic Left Heart Syndrome

Zdenka Reinhardt, Birmingham Children's Hospital, UK, United Kingdom

Vinay K Bhole, Birmingham Children's Hospital, UK, United Kingdom John Stickley, Birmingham Children's Hospital, UK, United Kingdom 
Joseph V De Giovanni, Birmingham Children's Hospital, UK, United Kingdom

Rami Dhillon, Birmingham Children's Hospital, UK,

United Kingdom

Paul Miller, Birmingham Children's Hospital, UK, United Kingdom

Aashish Chikermane, Birmingham Children's Hospital, UK,

United Kingdom

Oliver Stumper, Birmingham Children's Hospital, UK,

United Kingdom

Aim: To define the contribution of catheter interventions in the management of patients with HLHS.

Methods: Retrospective case note review of all 505 patients with HLHS, undergoing Norwood palliation over a 15 year period (1993-2008).

Results: 505 patients with HLHS were treated. A total of 268 catheter interventions were performed in 168 patients. Two patients underwent initial hybrid Stage I procedure.

Pre-Norwood Stage 1 interventions: 5 patients underwent emergency balloon atrial septostomy (1\%).

Pre-Cavopulmonary Shunt (93 interventions in 85/336 patients -25\%): 69 pts underwent balloon angioplasty of re-coarctation (20.5\%), and 10 had stenting of a RV-PA conduit (1 bail-out). Six patients had other procedures (balloon/stent of veins or atrial septum).

Pre-Fontan Completion (106 interventions in 74/285 patients -26\%): 26 LPA angioplasty, 28 LPA stenting, 22 occlusion of veno-venous collaterals and 20 coarctation angioplasties were performed. A further 10 other procedures were performed (balloon/stent of veins or atrial septum, EPS).

Post Fontan Completion (60 interventions in 43/280 patients15\%): 16 Fontan fenestration closures, 14 stenting of Fontan fenestration and 10 LPA balloons, 16 LPA stents, and 4 others. Coarctation balloon angioplasty was effective in all with freedom from reintervention in $59 / 73$ pts $(81 \%)$. LPA angioplasty was of only limited benefit (freedom from reintervention in 14/36 (39\%), making LPA stenting the preferred technique. There were 1/44 (2.3\%) late occlusion after LPA stent.

Conclusion: Interventional cardiac catheterization constitutes an integral part in the management of patients with HLHS.

\section{8}

Balloon pulmonary valvotomy for palliation of patients with cyanotic congenital heart diseases and pulmonary stenosis

Kavasseri S Remadevi, Amrita Institute of Medical Sciences and

Research Center, India

Edwin Francis, Amrita Institute of Medical Sciences and Research

Center, India

Balu Vaidyanathan, Amrita Institute of Medical Sciences and Research Center, India

Bhava RJ Kannan, Vadamulayan Hospital, Madurai, Tamil Nadu,

India

Raman Krishna Kumar, Amrita Institute of Medical Sciences and

Research Center, India

Background: While corrective repair and definitive palliation is the ideal goal in cyanotic congenital heart diseases with reduced pulmonary blood flow (CHDPS), it is often not easily accomplished because unsuitable anatomy. Additionally, in the developing world, there are severe economic and logistic difficulties. Balloon pulmonary valvotomy (BPV) can potentially be used as an inexpensive and less invasive alternative to aortopulmonary shunts in selected patients.
Objectives: To describe case selection, immediate and short-term follow-up results of palliative BPV in a diverse group of patients with CHDPS.

Materials and methods: Case records of consecutive patients with CHDPS who underwent BPV (June 2004-Dec 2008) were reviewed. BPV was offered for all patients with: significant hypoxia (saturation $<80 \%$ ), predominantly valvar PS, anatomy not suitable for surgery (corrective or definitive palliation) or anticipated delays in surgery.

Results: The demographics of 73 patients included are indicated in table. The median balloon-annulus ratio was $1.2(0.6-2)$ and fluoroscopy time was 20 (3-72) minutes. The systemic saturation increased significantly (from $72 \pm 8.6 \%$ to $88 \pm 6 \%$ ) in 68 patients and remained unchanged in 5 . The pulmonary valve could be crossed in all but one. There were no instances of pulmonary hypertension or edema. Major complications included a fatal cyanotic spell $(\mathrm{n}=1)$ and nonfatal cardiac perforation $(\mathrm{n}=1)$. During follow up (median 12 range: $1-56)$ months), 23 have undergone corrective and 6 underwent palliative operations.

Conclusion: Balloon pulmonary valvotomy offers satisfactory interim palliation in selected patients with diverse congenital cyanotic heart disease and pulmonary stenosis.

\begin{tabular}{|c|c|}
\hline Variable & Details \\
\hline Age & 1 year (10 days -37 years) \\
\hline Weight & $8(1.9-55) \mathrm{Kg}$ \\
\hline $\begin{array}{l}\text { Oxygen } \\
\text { Saturation }\end{array}$ & $72 \pm 8.6 \%$ \\
\hline Hemoglobin & $16.3(9.05-26.4) \mathrm{g} / 100 \mathrm{ml}$ \\
\hline Diagnosis & $\begin{array}{l}\text { Tetralogy of Fallot ( } \mathrm{n}=61 \text {; included } 5 \text { with } \\
\text { hypoplastic branch pulmonary arteries, } 4 \text { with } \\
\text { disconnected pulmonary arteries, } 4 \text { with coronary } \\
\text { crossing right ventricular outflow tract, } 2 \text { with } \mathrm{AV} \\
\text { canal) Double outlet right ventricle }(5) \text {, Corrected } \\
\text { transposition (2), Single ventricle palliation } \\
\text { candidates (4) }\end{array}$ \\
\hline $\begin{array}{c}\text { Indication } \\
\text { for BPV }\end{array}$ & $\begin{array}{l}\text { Interim palliation (young infants, syndromic } \\
\text { patients, anatomic issues precluding immediate } \\
\text { correction 42), anticipated delay due to financial } \\
\text { constraints (8), Preoperative stabilization for } \\
\text { severe hypoxia (3), Unsuitable for corrective } \\
\text { surgery or definitive palliation (20) }\end{array}$ \\
\hline
\end{tabular}
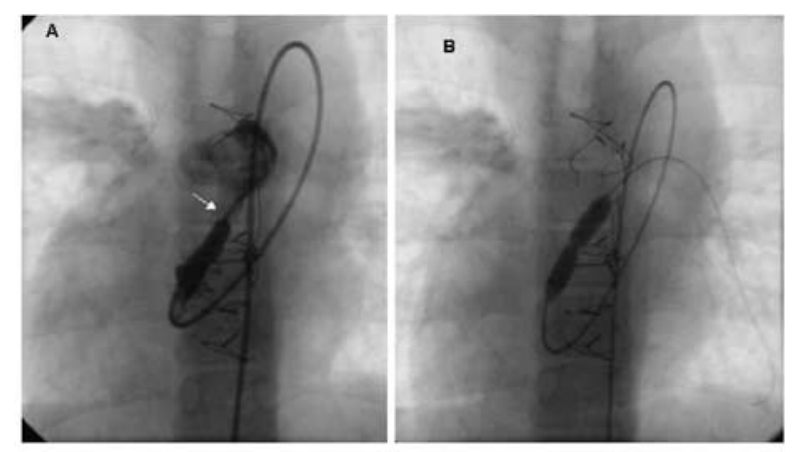

Balloon pulmonary valvotorry in a 11 year old with corrected transpostion, ventricular septal defect and pulmonary stenosis. A. This frame shows valvar stenosis with a doming valve and a discrete jet (arrow) with a small annulus. B:Balloon dilatation with a $8 \mathrm{~mm}$ balloon forming a waist which cisappeared with further inflation. The valve was crossed in a retrograde fashion (via femoral artery and venticle). 
8379

Catheter interventions (CI) after arterial switch operation (ASO) for transpostion of the great arteries (TGA) - the importance of major aortopulmonary collaterals (MAPCAs)

Frank T Riede, Heart Center, University of Leipzig, Germany Martin Kostelka, Heart Center, University of Leipzig, Germany Vit Razek, Heart Center, University of Leipzig, Germany

Jorg Hambsch, Heart Center, University of Leipzig, Germany Michael Weidenbach, Heart Center, University of Leipzig, Germany

Introduction: Perioperative mortality after ASO has decreased significantly over the last years. However, there is an acceptable but still significant rate of CI and reoperations after ASO.

Aim of the study: Analysis of type and frequency of CI after ASO with special focus on the role of MAPCAs on which only few data exist.

Methods: Retrospective single center study. All patients after ASO from $08 / 1998$ to $12 / 2008$ were included.

Patients: 155 patients underwent ASO from 08/1998 to 12/2008. Median age at operation was 9 days. Median follow up was 219 days (3 days -10 years). Overall mortality was $0,65 \%$.

Results: 29 patients $(18,8 \%)$ underwent CI. Freedom from CI was $81 \%$ after 1 year and 64\% after 5 and 10 years. Early CI: 11 patients had 12 CI (MAPCAs $n=6(50 \%)$, SVC stenosis $n=3$, coarctation $\mathrm{n}=2$ and LPA stenosis $\mathrm{n}=1$ ). Late CI: 18 patients underwent $25 \mathrm{CI}$, during which 28 lesions were treated: supravalvular $(\mathrm{n}=12)$ /valvular $(\mathrm{n}=3)$ pulmonary stenosis, $($ re- $)$ coarctation/aortic arch stenosis $n=7$ and MAPCA $n=6(21 \%)$. The indication for early MAPCA closure were severe left ventricular failure, pulmonary hyperperfusion/edema and failure to wean form ventilatory or inotropic support in 4 and radiologic signs of pulmonary hyperfusion in 2 of 6 patients. Patients with late MAPCA closure were asymptomatic.

Conclusion: Especially in the early postoperative period, timely recognition and treatment of MAPCAs contributes to reduced postoperative morbidity and mortality in patients after ASO.

\author{
8380 \\ Challenging closure of huge hypertensive patent ductus \\ arteriosus using Amplatzer septal occluder: PSU \\ experience \\ Supaporn Roymanee, Prince of Songkla University, Had Yai, Thailand, \\ Thailand \\ Saranwan Phetphisal, Prince of Songkla University, Had Yai, Thailand, \\ Thailand \\ Nakharin Tonklang, Prince of Songkla University, Had Yai, Thailand, \\ Thailand \\ Saranyou Suwan-uksorn, Thailand \\ Treechada Visartpong, Prince of Songkla University, Had Yai, \\ Thailand, Thailand \\ Sririchai Chewathanakornkul, Prince of Songkla University, Had Yai, \\ Thailand, Thailand \\ Tawai Ngernsritakul, Thailand
}

Objective: To report a challenging huge hypertensive patent ductus arteriosus (PDA) closed by transcatheter technique.

Method and Result: A 38-year-old cachetic female with NYHA FC III dyspnea underwent transcatheter closure of hypertensive PDA. Right and left heart catheterization had taken only under room air and oxygen inhalation (selective pulmonary vasodilators are unavailable). The aortogram revealed huge aneurysmatic and calcified PDA $24 \mathrm{~mm}$ with ampulla diameter of $40 \mathrm{~mm}$ (aorta only $20 \mathrm{~mm}$ ). Both pulmonary arteries (PA) also had become aneurysmatic with its size nearly $60 \mathrm{~mm}$. There was systemic pulmonary artery pressure (PAP) and no any change of PAP before and after oxygen inhalation. However, Qp:Qs was increased from 1.5 to 2.8 , pulmonary vascular resistance was decreased from 9.2 to $2.6, \mathrm{Rp}$ :Rs was decreased from 0.67 to 0.23 , and aortic saturation was increased from $95 \%$ to $99 \%$. Intention to trial occlusion using Amplatzer septal occluder (ASO) $26 \mathrm{~mm}$, we expected that $40 \mathrm{~mm}$-left atrial disc would fit at aortic ampulla, however device passed through the huge PDA and huge PA aneurysm. Subsequently, ASO $38 \mathrm{~mm}$ was successfully placed. After last 30 minutes, the PAP was 116/ $40 \mathrm{mmHg}$ (mean 74) while systemic pressure was $122 / 64 \mathrm{mmHg}$ (mean 88) and no aortic desaturation. The device was implanted without obstruction to aorta or PA branches. There was mild residual central shunt and transient microscopic hematuria which both disappeared completely 48 hours later.

Conclusion: According to new innovation of device technology and technique leading to successful and safety closure of huge hypertensive ductus arteriosus.

\section{1}

Use of Amplatzer Vascular Plug for Complex Congenital Interventions

$S$ Ramakrishnan, All India Institute of Medical Sciences, New Delhi, India

Shyam S Kothari, All India Institute of Medical Sciences,

New Delhi, India

Rajnish Juneja, All India Institute of Medical Sciences,

New Delhi, India

Anita Saxena, All India Institute of Medical Sciences, New Delhi, India

Background: Amplatzer vascular plug (AVP) is an underutilized item in the armamentarium of interventional cardiologists.

Methods: We describe 9 cases of unusual interventions using the AVP. The patient age ranged from 2-20 years. The diagnosis included coronary aterio-venous fistula (3 cases), baffle leak following Fontan operation (1 case), Coil embolization of AP collaterals (2 plugs), pulmonary artery aneurysm (1 case) and post operative Blalock-Taussig shunt (through arterial approach 1 case and through Glenn circuit 1 case). In 3 of the cases, delivery of duct/septal occluder failed due to inability to adequately advance the delivery sheath or the occluder.

Results: The size of AVP used ranged from $4 \mathrm{~mm}$ to $12 \mathrm{~mm}$.In all the cases, the delivery of AVP was relatively unproblematic as it could be advanced over a $5 \mathrm{~F}$ or $6 \mathrm{~F}$ right coronary guiding catheter. The procedure was successful in all the cases. Complete occlusion of shunt could be achieved with AVP alone, despite concerns over less thrombogenicity.

Conclusion: In selected cases, Amplatzer vascular plug offers an alternative innovative solution for the interventionists. The better profile and manoeuvrability was useful in all the cases.

\section{2}

Coarctation of the aorta in adolescents and adults- everyone shall receive a covered stent Masood Sadiq, Punjab Institute of Cardiology, Pakistan Asif Rehman, Punjab Institute of Cardiology, Pakistan Shakeel Qureshi, Evelena Children's Hospital, United Kingdom

Background: Covered stents has decreased the risk of complications in coarctation of the aorta $(\mathrm{CoA})$ and are now being used as primary treatment in selected patients. 
Objective: To evaluate the use of covered Cheatham-Platinum (CP) stents in the management of native CoA.

Patients \& Methods: 35 covered CP stents were implanted in 31 pts with native CoA from Jan $02-\operatorname{Dec} 08$. This represents $66 \%$ of all patients having coarctation stenting ( $>85 \%$ in last 2 years). Indications were severe and near atretic lesion, long segment CoA, transverse arch coarctation, Turner syndrome, associated PDA and rescue treatment in aortic dissection. This patient with bare stent related dissection had 4 covered stents in total.

Results: Mean age and weight were $24( \pm 13.7)$ years and 56 $( \pm 13) \mathrm{kg}$. The procedure was successful in $30 / 31 \mathrm{pts}$. One pt with complete interruption needed surgery. The systolic gradient across the CoA decreased from $52( \pm 28) \mathrm{mmHg}$ to $5( \pm 3.5) \mathrm{mmHg}$ $(p<0.0001) \&$ diameter of the CoA increased from $5.2( \pm 1.9) \mathrm{mm}$ to $18( \pm 4.5) \mathrm{mm}(\mathrm{p}<0.0001)$. There was one death 3 days post procedure due to intracranial hemorrhage. Follow up period was up to 82 (mean 34) months. All stents were patent and in good position on CT performed 3-6 months later. In 33\% of pts antihypertensive medication was either decreased or stopped.

Conclusion: Covered stents may be used safely and effectively as therapy of choice in adolescents/adults with severe and complex CoA. They are argued as preferred form of treatment for all adolescents and adults.

\section{3}

Follow up after Balloon Aortic Valvuloplasty from the 1990s - a single centre 10 year cohort

Ricardo A Samson, University of Arizona, United States

Tim Jellyman, Waitakere Hospital, New Zealand

Clare O'Donnell, Starship Hospital and Greenlane Clinical Services, New Zealand

Tom L Gentles, Starship Hospital and Greenlane Clinical Services, New Zealand

Nigel J Wilson, Starship Hospital and Greenlane Clinical Services, New Zealand

Objective: To review long term outcomes from percutaneous Balloon Aortic Valvuloplasty (BAV).

Methods: Single centre, retrospective audit of all BAVs for aortic stenosis from 1991 to 2000. Valve survival was defined by freedom from repeat BAV or aortic valve surgery.

Results: 30 children aged 1 month to 18 years underwent BAV. 18 had immediate valve gradient reduction $>50 \%$. Balloon-annulus ratio (BAR) did not correlate with gradient reduction or development of aortic regurgitation (AR). Acute severe AR occurred in 1 patient. Long-term complications: femoral artery occlusion 1, brachial plexus palsy 1 .

Overall survival $8-18$ years was $100 \%$. Median survival of the native aortic valve was 5.9 years. 9 required surgery for significant progresive AR, 3 within 2 years of BAV, 6 after $4.2-9.5$ years.

$4 / 30$ patients developed re-stenosis and underwent repeat BAV 3.7-7.6 years after initial BAV. 3 of these 4 required surgery within 6 months ( 1 for inadequate stenosis relief, 2 for significant AR). 6 others underwent surgery for persistent or recurrent stenosis; $1-5.6$ years after BAV.

Overall 20 of 30 required subsequent aortic valve surgery or repeat BAV. Freedom from re-intervention at 5 years was $53 \%$ and 10 years was $27 \%$.

Conclusions: BAV is a palliative procedure for aortic stenosis delaying requirement for further procedures over 5 years in the majority of cases. Aortic regurgitation can be progressive late following BAV and in this series was as frequent as recurrent aortic stenosis.

\section{4}

Transcatheter Arterial Duct Closure In Symptomatic Infants Younger Than 12 Months

Giuseppe Santoro, Cardiology, Monaldi Hospital, Naples, Italy

Chiara Marrone, Italy

Gianpiero Gaio, Italy

Maria Teresa Palladino, Italy

Carola Iacono, Italy

Raffaella Esposito, Italy

Maria Giovann Russo, Italy

Raffaele Calabro, Cardiology, II University of Naples, Naples, Italy, Italy

Background: Transcatheter arterial duct (AD) closure still remains technically challenging in very young children. This study evaluated the feasibility and mid-term results of this approach in symptomatic infants.

Methods: 48 of 339 patients (14.1\%) submitted to percutaneous AD closure at our Institution were symptomatic infants younger than 12 months (age $7.7+2.9$ mos, range $2.0-12.9$; weight $7.6+2.3$, range 3.6-13). Clinical indications for treatment were failure to thrive and/or recurrent respiratory infections. All pts were on anticongestive pharmacological therapy. Based on ductal and local anatomy, $\mathrm{AD}$ closure was attempted using a detachable-coil (Cook, Bloomington, Indiana, USA) approach in 18 cases (Group I) or the Amplatzer Duct Occluder device (AGA Medical Corporation, Golden Valley, Minnesota, USA) in 30 cases (Group II).

Results: Mean AD diameter was 2.5 $+1.0 \mathrm{~mm}$ (range 1.5-4.5). Mean Qp/Qs was $2.1+1.2$ (range 1.0-5.9) without any significant difference between groups. Overall feasibility of the procedure was $93.8 \%$ (45/48 patients), without any difference between groups $(94.4 \%$ vs $93.3 \%, p=N S)$. Fluoroscopy and procedural time were $16.6+14.2$ and $88.3+43.2 \mathrm{~min}$, respectively and were significantly higher in Group I $(22.5+5.5$ and $115.8+23.7 \mathrm{~min})$ than in Group II $(6.2+2.0$ and $61.0+40.3 \mathrm{~min})(\mathrm{p}<0.001)$. Occlusion rate at discharge was $62.8 \%$, rising to $93.4 \%$ at last follow-up control, without any significant difference between groups ( 23.5 vs $46.4 \%$ at 24 h; 5.9 vs $7.1 \%$ at last $\mathrm{F}-\mathrm{U}, \mathrm{p}=\mathrm{NS}$ for both comparisons).

Conclusions: Percutaneous closure of symptomatic ADs might be considered feasible, effective and safe in young infants, without differences between controlled-release coil approach and ADO device option.

8387

Transcatheter Closure of Rupture of Sinus of Valsalva Aneurysm to left ventricle using Life Tech PDA occluder device

Anil Radha Sivadasan, Department of Pediatric Cardiology, Apollo Health City, Hyderabad, India

Girish Basavaraj Navasundi, Department of Pediatric Cardiology, Apollo Health City, Hyderabad, India

Kanhaiya Himatlal Jeswani, Department of Pediatric Cardiology, Apollo Health City, Hyderabad, India

Deepa Mohan Sharma, Department of Pediatric Cardiology, Apollo

Health City, Hyderabad, India

Meena Trehan, Department of Pediatric Cardiology, Apollo Health

City, Hyderabad, India

S G Sunil, Department of Pediatric Cardiology, Apollo Health City, Hyderabad, India

Introduction: We describe the first case of transcatheter closure of RSOV draining to the left ventricle using a Amplatzer PDA occluder (ADO) deployed from the venous side. 
Case: A 5 year old boy presented with failure to thrive and congestive heart failure. 2D echocardiogram showed a RSOV from left sinus draining into the LV. In addition he had a Coronary AV fistula from LMCA draining into right atrium and $2.5 \mathrm{~mm}$ PDA. The RSOV had a proximal narrow neck of $6 \mathrm{~mm}$ and a distal opening of $12 \mathrm{~mm}$ into the LV. The procedure was done under general anesthesia and initially the coronary AV fistula and the PDA were closed using two 6-4 ADOs. Through a $8 \mathrm{~F}$ venous access a trans septal puncture was done and a $8 \mathrm{~F}$ Mullins sheath was tracked into the left atrium.Using a balloon tipped catheter a 0.014 PTCA wire was passed retrograde through the RSOV into the left ventricle and was snared into the Mullin sheath. This wire was exchanged for a 0.032 terumo wire to from a arterio-venous loop passing through the aneurysm. The $8 \mathrm{~F}$ Mullins was advanced over this wire into the aorta and a 10-8 ADO was deployed into the proximal opening. Post release showed no residual flow. On follow up at 6 months, there is no residual flow and had gained $2 \mathrm{~kg}$.

Conclusion: We report this case as transcatheter closure of RSOV to the left ventricle has not been previously described. This patient is unique as he had three left to right shunts and were closed by transcatheter technique.

\author{
8388 \\ Percutaneous device closure of PDA in children less than \\ $10 \mathrm{~kg}$ without arterial access: Immediate and intermediate \\ follow up \\ Anil Radha Sivadasan, Department of Pediatric Cardiology, Apollo \\ Health City, Hyderabad, India \\ Girish Basavaraj Navasundi, Department of Pediatric Cardiology, \\ Apollo Health City, Hyderabad, India \\ Kanhaiya Himatlal Jeswani, Department of Pediatric Cardiology, \\ Apollo Health City, Hyderabad, India \\ Deepa Mohan Sharma, Department of Pediatric Cardiology, Apollo \\ Health City, Hyderabad, India \\ Meena Trehan, Department of Pediatric Cardiology, Apollo Health \\ City, Hyderabad, India \\ $S$ G Sunil, Department of Pediatric Cardiology, Apollo Health City, \\ Hyderabad, India
}

Arterial access is often obtained during PDA device closure and this would require heparinization and carries a small risk of femoral arterial occlusion. This is a retrospective study of the patients who underwent PDA device closure with only venous access from July 2004-October 2008 using either Amplatzer (ADO) or Lifetech occluder (LDO).

Method: The duct size and the adequacy of the ductal ampulla for device closure was decided on transthoracic echocardiogram.Duct angiogram was done in lateral and $\mathrm{RAO}$ views through the Mullins sheath. The position of the device is checked by $2 \mathrm{D}$ or 3D echocardiogram to see for residual flow, LPA and RPA flow turbulence and for aortic obstruction. The suprasternal view which profiles the long axis of the descending aorta was used to study the relative position of the retention skirt of the device in relation to isthmus and descending aorta.

Results: During this period 42 patients underwent successful device closure of PDA of which 23 were children less than $10 \mathrm{kgs}$. Age ranged from 5 months to 3 years (Wt:4.2-10 kgs), duct size ranged from $3.5 \mathrm{~mm}$ to $12 \mathrm{~mm}$ (mean $5+/-2 \mathrm{~mm}$ ). The device size ranged from 6-4 to 16-14 mm (ADO:13,LDO: 10). Arterial access was obtained only in three patients. (Inadvertent arterial puncture-1, difficulty to retrieve the device after initial deployment-2)

Conclusion: Device closure of ducts without arterial access is feasible and safe in selected patients less than $10 \mathrm{~kg}$. 2D and 3D echocardiogram gives sufficient information regarding the device position and its relation to structures like LPA/RPA and aorta to permit assessment of safe deployment.

\section{9}

Safety and feasibility of transcatheter closure of large patent ductus arteriosus in patients weighing less than $6 \mathrm{~kg}$ Kothandam Sivakumar, MIOT Hospital, Chennai, India, India Ramyashri Chandrasekaran, MIOT Hospital, Chennai, India, India Anpon Bhagyavathy, MIOT Hospital, Chennai, India, India Hariprakash Suriyanarayanapillai, MIOT Hospital, Chennai, India, India

Radhakrishnan Satish, MIOT Hospital, Chennai, India, India Arunkumar Govindarajan, MIOT Hospital, Chennai, India, India Robert Coelho, MIOT Hospital, Chennai, India, India

Objectives: Large patent duct arteriosus (PDA) in children weighing under $6 \mathrm{~kg}$ challenge the interventionist due to need for a large delivery system, sheath kink and encroachment of aorta or pulmonary artery (PA) by the occluder. Many institutions prefer surgery. We report our approach in closure of large PDA associated with severe pulmonary arterial hypertension in babies weighing under $6 \mathrm{~kg}$.

Methods: This is a retrospective analysis of all consecutive patients weighing under $6 \mathrm{~kg}$ with large PDA associated with PA systolic pressures more than half of systemic pressures since 2003. Preterm ducts and PDA associated with coarctation were excluded. After explaining the pros and cons of transcatheter approach, patients were taken for transcatheter closure. Ducts with a roomy ampulla was given a trial closure with multiple coils aided with bioptome, other ducts were closed with occluders.

Results: 38 patients aged 1-21 months (median 6months) and weighing $2.6-6 \mathrm{~kg}$ (median $4.7 \mathrm{~kg}$ ) were diagnosed to have large PDA (mean diameter $6.4+/-2.1 \mathrm{~mm}$ ). 3 parents opted for surgery; rest were taken for transcatheter closure. 36 of 38 procedures were successful, four with multiple coils and rest with occluders. Two failures due to sheath kink and aortic protrusion proceeded to surgery. Four patients needed occluder upsizing; four needed blood transfusions. There were no deaths. On a mean follow up of $37+/-14$ months, there was no residual flows, aortic or pulmonary artery encroachment by devices. Conclusions: Transcatheter closure of large ducts with pulmonary hypertension in infants weighing under $6 \mathrm{~kg}$ is safe and effective on midterm followup.

\section{0}

'Temporary stent' for correcting unbalanced pulmonary blood flow

Wataru Soda, Shizuoka children's Hospital pediatric cardiology, Japan Sung-Hae Kim, Shizuoka children's Hospital pediatric cardiology, Japan Norie Mitsushita, Shizuoka children's Hospital pediatric cardiology, Japan

Masaki Nii, Shizuoka children's Hospital pediatric cardiology, Japan Yashiko Tanaka, Shizuoka children's Hospital pediatric cardiology, Japan Yasuo Ono, Shizuoka children's Hospital pediatric cardiology, Japan Kisaburo Sakamoto, Shizuoka children's Hospital cardiovascular surgery, Japan

Backgroud: In general, congenital arterial lesions such as pulmonary coarctation, non-confluency, and major aortopulmonary collaterals associated with pulmonary atresia are prone to be remainig pulmonary branch stenosis and subsequent hypoplasia of unilateral pulmonary blood flow, after the first palliative SP shunt procedure To overcome such problem, we introduced 
"temporary stent" which include setnt implantation via the SP shunt to persistent unilateral stenotic pulmonary artery and perform next stage open heart surgery with removal of the stent and plasty of the vessel within three months.

Aims: To evaluate the indication and effect of temporary stent to correct unbalanced pulmonary blood flow.

Subjects: Four cases of pulmonary atresia including 3 infants (cTGA/PA/VSD, DORV/PA, PA/VSD/MAPCA (Allagile syndrome)), and one eight-year-old child (PA/VSD/non-confluent PA/bilateral PDA).

Methods: Relatively small stents (two Medtronic Drivers, one Genesis on Slalom, one Express LD) was deployed using 0.014", or 0.018 " supportive wire through BT shunt or RV-PA conduit. Results: All the stents were precisely placed, and removed easily in the next stage operations ( 3 Rastelli, and one bidirectional Glenn).

Discussion: In the condition that the stent was temporarily placed within 3 months until completely endothelialized, the lowest profiled assembly was applied via SP shunt of small children. Long-term evaluation is still warranted.

8391

A Novel Technique for Stenting Pulmonary Artery and Conduit Bifurcation Stenosis

Oliver Stumper, The Heart Unit, Birmingham Children's HospitalNHS Trust, UK, United Kingdom

Zdenka Reinhardt, The Heart Unit, Birmingham Children's HospitalNHS Trust, United Kingdom

Vinay K Bhole, The Heart Unit, Birmingham Children's Hospital-

NHS Trust, United Kingdom

Sara Thorne, University Hospital Birmingham, United Kingdom

Aim: The stenting of proximal pulmonary artery or conduit bifurcation stenosis remains challenging. We sought to place a stent across such lesions which was mounted onto two balloon angioplasty catheters placed within the respective distal branch pulmonary arteries.

Methods: Over a 4 year period four patients were identified with significant branch pulmonary artery stenosis just proximal to a bifurcation, which could not be improved by single and double balloon angioplasty. The insertion of a standard stent was judged to be unsatisfactory, due to potential jailing of a branch PA, or having to severely oversize the balloon. A large bore Mullins sheath was placed just proximal to the stenosis, two guidewires were placed within the branch PAs. A stent with minimal shortening characteristics (EV3 in 3, Genesis in 1) was mounted onto two balloon angioplasty catheters in tandem and then delivered.

Results: Mullins sheath sizes were $16 \mathrm{~F}$ in one, $14 \mathrm{~F}$ in two and $12 \mathrm{~F}$ in one. In one patient an $18 \mathrm{~mm}$ angioplasty and a $5 \mathrm{~F}$ Judkins were used for mounting the stent. The procedures were uneventful in all. Conduit gradients were reduced in 3 from mean 55-15 mmHg. One patient with branch PA stenosis had marked improvement in morphology and perfusion.

Conclusion: The described technique is an effective and safe technique in treatment of selected proximal bifurcation stenoses.

\section{2}

A Three Dimensional Simulation System for Determining the Optimal Size of Amplatzer Septal Occluder before the Procedure of Transcatheter Occlusion of Atrial Septal Defect: A pilot feasibility study

Kun Sun, Department of Pediatric Cardiology, Shanghai Children's Medical Center, China
Wenjing Hong, Department of Pediatric Cardiology, Shanghai Children's Medical Center, Shanghai Jiaotong University, China Jian Guo Yu, School of computer science, Shanghai Fudan University, China

Nan Zhao, School of computer science, Shanghai Fudan University, China LanPing Wu, Department of Pediatric Cardiology, Shanghai Children's Medical Center, Shanghai Jiaotong University, China

Objective: To evaluate the feasibility of our self-designed three dimensional simulation system (3DSS) for determining the optimal size of Amplatzer septal occluder before the procedure of transcatheter occlusion of atrial septal defect (ASD).
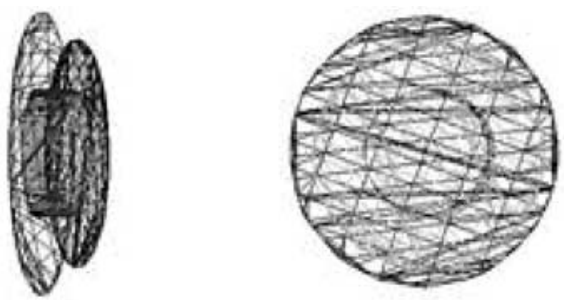

Simulated octluder sown froth different ditctions and angles
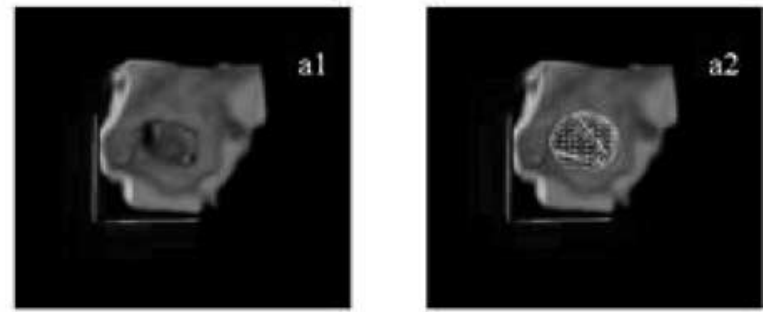

The simulated devise was "deployed" into ASD

Methods: 3D volume rendering and 3D visualization methods were used for the development of 3DSS. 30 children (9 male and 21 femal, aged $6.37 \pm 3.49$ years) with ASD who have been successfully underwent transcatheter occlusion were recruited in this study. 3D modeling method was used for simulating the shape and the size of each occlusion device. The simulated devise was "deployed" into ASD, which was reconstructed from 3DE data set of each patient before ASO occlusion, with the axes of the devise at the center of the decussation of the major and minor axis of ASD. The size of right disk and left disk of the simulated device and the distance between the inferior margin of device and mitral and tricuspid valve of simulation occluder were measured using 3DSS, and the results were compared with that measured from the $3 \mathrm{D}$ imaging of the same patient one day after successfully underwent transcatheter closure of ASD using Tomtec 3DE system.

Results: No significant difference between simulation occluder and ASO in size and the distance between the inferior margin of device and mitral and tricuspid valve measured by 3DSS and by the Tomtec 3DE system one day after successful occlusion $(\mathrm{P}>0.05)$.

Conclusions: The 3DSS could be a usefull noninvasive method for ASO selection before the procedure of transcatheter occlusion of ASD.

\section{3}

Neonatal Arterial Duct Stenting in palliating Pulmonary atresia and VSD

Sreekanthan Sundararaghavan, Manipal Hospital, India

Ravi Narayan, Manipal Hospital, India 
Anand Shenoy, Manipal Hospital, India

Anil Bhatt, Manipal Hospital, India

Stenting of the ductus arteriosus in pulmonary atresia and VSD is considered to be challenging due to its tortuous and vertical course. We describe the early experiences in this unique subset of patients. Methods: A retrospective review of all patients diagnosed with pulmonary atresia and VSD in the year 2008 was undertaken. All patients underwent a detailed clinical examination and echocardiogram to determine ductal dependency. A total of 5 patients underwent cardiac catheterization with intent to stent the ductus arteriosus.

Results: All 5 patients (mean age 32 days and mean weight $3.2 \mathrm{Kgs}$ ) had successful deployment of bare metal stents in the ductus arteriosus by two primary operators. The mean narrowest ductal diameter was $2.3 \mathrm{~mm}$ and mean length was $12.2 \mathrm{~mm}$. The mean preprocedure saturation was $70 \%$ which increased to $80 \%$ post implantation. 1 patient died while on ventilator due to sepsis and pneumonia. On follow up (mean 110 days), 3 of 4 patients have oxygen saturations around $80 \%$ and showed adequate physical growth. 1 patient with overhanging stent at the MPA, has branch PA gradient of $32 \mathrm{~mm} \mathrm{Hg}$. One patient with LPA origin stenosis became progressively desaturated and awaits surgical shunt to LPA. Conclusions:

1. It is feasible to perform ductal stenting in the challenging group of pulmonary atresia and VSD with low procedural mortality, however, the major complication rate may be higher.

2. The estimated stent length is at best an approximation and operator dependent.

3. Branch pulmonary artery stenosis worsens after stent implantation necessitating further surgical intervention.

\section{4}

Interventions in pulmonary hypoplasia in uni- and biventricular hearts

Tomasz Moszura, Department of Cardiology Polish Mother's Memorial Hospital-Research Institute, Lodz, Poland

Anna Mazurek-Kula, Department of Cardiology Polish Mother's

Memorial Hospital-Research Institute, Lodz, Poland

Pawel Dryzek, Department of Cardiology Polish Mother's Memorial

Hospital-Research Institute, Lodz, Poland

Jacek Moll, Department of Cardiosurgery Polish Mother's Memorial

Hospital-Research Institute, Lodz, Poland

Jadwiga Moll, Department of Cardiology Polish Mother's Memorial

Hospital-Research Institute, Lodz, Poland

Andrzej Sysa, Department of Cardiology Polish Mother's Memorial

Hospital-Research Institute, Lodz, Poland

Background: Correct development of pulmonary arteries is one of the most important factors influencing outcome of surgical treatment in children with congenital heart defects.

Material: Between 1998 and 2008, we performed 50 interventions (39 patients; age 25 days- 6 years) with pulmonary stenosis or hypoplasia.

Group I 17 patients with pulmonary hypoplasia and presence of MAPCA-candidates for biventricular repair (ToF, DORV, ToF+PA).

Group II 22 patients with HLHS and postoperative pulmonary stenosis.

Methods and results: In group I 12/17 patients were qualified for biventricular repair. Two patients after stent implantation into RVOT required next stent implantation. 3 patients are in observation. One patient (Alagille syndrome and multiperipheral pulmonary stenosis) was qualified to B-T shunt.
In group II. balloon angioplasty was effective in 6 patients with pulmonary stenosis and ineffective in 15 children with pulmonary artery hypoplasia. In these patients, primary or delayed stent implantation resulted in increase of vessel diameter from $/$ mean (SD) $/ 4,56(1,17) \mathrm{mm}$ to $9(1,73) \mathrm{mm}(\mathrm{p}<0,001)$. This was associated with reduction of pulmonary arterial pressure from/mean (SD)/ $15,56(6,85) \mathrm{mmHg}$ to $13(5,35) \mathrm{mmHg}(\mathrm{p}=0,0054)$.

Conclusions:

1. Redilation of previously implanted stents allows for adaptation of diameter of pulmonary artery to keep pace with the growth of the patient.

2. Interventional closure of MAPCAs, reduces the risk of pulmonary hypertension and improves flow by primary pulmonary arteries.

3. Stent implantation into RVOT in patient with ToF and hypoplastic pulmonary arteries increases pulmonary blood flow by physiological way and leads to improvement of pulmonary arteries development.

\section{5}

Transcatheter closure of ruptured sinus Valsalva aneurysms with Amplatzer occluders

Malgorzata Szkutnik, Poland

Jacek Kusa, Poland

Jacek Bialkowski, Silesian Center for Heart Diseases, Poland

Maria Zyla-Frycz, Silesian Center for Heart Diseases, Poland

Introduction: Ruptured Sinus Valsalva Aneurysm (RSVA) is a rare, usually surgically treated pathology. Own experience in transcatheter closure of RSVA is presented.

Material and methods: From 5 patients (pts) with RSVA two had right- and one noncoronary sinus opened to the right atrium (RA). One of them, previously treated surgically (with recanalization), had 2 RSVA orifices. Another pt had right RSVA opened to RVOT and the fifth one (after previous surgical correction of Fallot Tetralogy) left RSVA opened to the pulmonary trunc. In the last case RVSA was very close to the origin of the left coronary artery. Mean age of pts was 33,2 (18-51) y, QP/QS ratio 1,84 (1,3-2,2). In all pts attempt of transcatheter closure was undertaken with application of Amplatzer devices (ADO or ASO).

Results: Six procedures were performed in 5 pts. The procedures were technically successful in all pts. There were 7 devices implanted: 6 ADO (in 1 pt ineffective - retrived) and 1 ASO. No early nor late embolization of the device occured. In one pt (with left RSVA) after deployment of ADO ST depression in ECG appeared, device was withdrawn and the procedure abandoned.In the pt with RSVA opened to RVOT during the procedure and 2 following days premature ventricular extrasystoles were observed. In follow-up mean 1,1 (from 0,6 to 1,5 )y in all treated pts complete closure of RSVA was confirmed in TTE.

Conclusion: Percutaneous closure of Ruptured Sinus Valsalva Aneurysm is feasible, although technically demanding procedure, which can replace surgical treatment.

\section{1}

Experiences with stent implantations into the stenosed segments of aortopulmonary collaterals in non surgically treated patients with pulmonary atresia, ventricular septal defect and multiple aortopulmonary collaterals in Slovak Republic

Peter Olejnik, Department of pediatric cardiology, Children's University Hospital Bratislava and Children's Cardiac Center, Slovak Republic Peter Tittel, Slovak Republic 
Zuzana Venczelova, Slovak Republic

Jozef Masura, Slovak Republic

Objectives: Presentation of four stents implantation in three patients with non-surgically treated pulmonary atresia, ventricular septal defect and multiple aortopulmonary collaterals (PA, VSD, MAPCAs), who underwent stent implantations into the stenosed MAPCAs.

This congenital cardiopathy has a severe prognosis. We have 21 non-surgically treated and 15 surgically treated patients with PA, VSD, MAPCAs in our institution. These patients could survive due to pulmonary blood perfusion through the aortopulmonary collateralls. As time passed, the stenoses of MAPCAs formed, resulting in progression of hypoxemia and cyanosis. The level of NYHA impaired and neurological complications were likely to be present as well. Stent implantations into the stenosed segments of MAPCAs are only possible non-surgical effective palliative intervention for these patients after precise isotropic high resolution multislice CT-angiography delineation of MAPCAs.

Methods: The comparison of blood oxygenation parameters, neurological complications and level of NYHA before and after stent implantations into the stenosed segments of MAPCAs in three adult patients with non-surgically treated PA, VSD, MAPCAs.

Results: All patients with non-surgically treated PA, VSD, MAPCAs after J\&J Palmaz Genesis $6 \mathrm{~mm}$ premounted stent implantations showed encouraging results in improvement of blood oxygenation parameters, neurological complications and level of NYHA. High resolution multislice CT-angiography in all cases confirmed proper site of implanted stents and improvement in blood perfusion of pulmonary segments supplied by dilated collateral.

Conclusions: Stent implantations into the stenosed segments of MAPCAs seem to be only effective non-surgical paliative intervention, which can improve quality of life in non-surgically treated patients with PA, VSD, MAPCAs.

8402

The Cassification and its key point of transcatheter closure of perimembranous ventricular septal defect using asymmetric membranous VSD device

Guang-yi Wang, Department of cardiology, Chinese PLA general

hospital, China

Zhi-feng Wang, China

Hai-jun Hou, China

Yue Li, China

Objective: To evaluate the Cassification and its key point of transcatheter closure of perimembranous ventricular septal defect (PVSD) using asymmetric membranous VSD Amplatzer occluder device (AAOD).

Methods: Between June in 2002 to October in 2007 PVSD for 128 patients was diagnosed At a average age of $10.6 \pm 5.9 \mathrm{yr}$ (range, $2 \sim 48 \mathrm{yr}$ ) and average weight of $44.3 \pm 10.2 \mathrm{Kg}$ (range $9 \sim 72 \mathrm{Kg}$ ). The configuration of PVSD was classified into the 7 types according to its angiographic form. A F6 $\sim 9$ catheter was used for delivery of the device in all patients.

Results: The configuration of PVSD was classified into the tube shape, funne shape, window shape, aneurysm shape, cauliflower shape, subseptal shape, irregular shape, aortic valve prolapse by its angiographic form. The different configuration of PVSD have different particularity of transcatheter closure of PVSD using this device.
Conclusion: Transcatheter closure of PVSDs using asymmetric perimembranous VSD Amplatzer occluder device was an excellently efficient, feasible nonsurgical method. The successful rate of technique was for $97.6 \%$ in this group.

Key words: Transcatheter closure; Perimembranous Ventricular Septal Defects; Asymmetric membranous Amplatzer occluder device.

\section{3}

Transcatheter closure of moderate-to-large patent ductus arteriosus in infants using Amplatzer duct occluder Jou-Kou Wang, National Taiwan University Hospital, Taiwan Mei-Hwan Wu, National Taiwan University Hospital, Taiwan Ming-Tai Lin, National Taiwan University Hospital, Taiwan Sheunn-Nan Chiu, National Taiwan University Hospital, Taiwan

Objective: There are difficulties in transcatheter closure of patent ductus arteriosus (PDA) in infants. We present the results of transcatheter closure of PDA in infants using Amplatzer duct occluder (ADO).

Methods: During a 7.5-year period, there were 46 infants undergoing PDA closure of using ADO. During this period of time, 5 infants with PDA were sent to surgery because of a too large ductus or a body weight $<3.5 \mathrm{~kg}$. The indication of using an $\mathrm{ADO}$ was a ductus diameter $\geqslant 2.5 \mathrm{~mm}$. Their age ranged from 2 to 12 months with a mean body weight of $6.3 \pm 1.6 \mathrm{~kg}$. Of them, 39 infants were symptomatic. Device diameter selected was $1-3 \mathrm{~mm}$ larger than ductal diameter.

Results: The mean systolic pulmonary artery pressure was $40.9 \pm 18.2 \mathrm{mmHg}$. The mean $\mathrm{Qp} / \mathrm{Qs}$ ratio was $3.1 \pm 1.2$. The mean ductus diameter was $3.3 \pm 0.8 \mathrm{~mm}$. ADO was successfully deployed in 45 patients. Failure occurred in an infant in whom a $6 \mathrm{Fr}$ sheath kinked. The mean diameter of device used was $5.4 \pm 1.1 \mathrm{~mm}$.No severe complications occurred. At the 1-month echocardiographic follow-up, a small residual shunt was present in 4 out of 45 patients and it disappeared in all 4 patients at 3-month follow-up. Mild left pulmonary artery stenosis $(\operatorname{Vmax}<2.5 \mathrm{M} / \mathrm{sec}$ ) was documented in 7 patients. One developed left ventricular outflow tract obstruction 2.3 year after the procedure.

Conclusions: Transcatheter closure of PDA in infants using ADO is a safe and effective method.

\section{4}

Transcatheter treatment of coronary artery fistula using a variety of devices

Jou-Kou Wang, National Taiwan University Hospital, Taiwan Mei-Hwan Wu, National Taiwan University Hospital, Taiwan Ming-Tai Ling, National Taiwan University Hospital, Taiwan Shuenn-Nan Chiu, National Taiwan University Hospital, Taiwan

Background: We report the results of transcatheter treatment of coronary artery fistula using different devices.

Methods: During an 11.5-year period, 19 patients underwent attempted transcatheter closure of coronary artery fistula. There were 7 females and 12 males with ages ranging from 2.5 to 72 years (median 12 years). Two had undergone surgery with significant residual shunts. Heart failure was present in 4 patients and angina in 5 . The fistula originated from right coronary artery in 9 , from left coronary artery in 8 and from both coronary arteries in 2.

Results: The procedure was successful in 17 patients, and failed in 2. Coils (Gianturo coils \& micro coils) were use in 12 patients, 
Amplatzer duct occluder in 3, Amplatzer vascular plug in 2, \& a stent graft was used in 1 . Of the 2 failure cases, 1 was operated later on \& the other is awaiting a second attempt. Distal embolization of devices (one coil \& one Amplatzer vascular plug) occurred in 2 patients. The devices were retrieved percutaneously. One developed hemolysis which was managed by placing more coils in the fistula. Two had transient ST-T wave changes on the ECG following the procedure. No residual shunt was detected in any patient. Most patients had improvement in symptoms.

Conclusions: Transcatheter treatment of coronary artery fistula using a variety of devices provides good results which are comparable to those of surgery.

\section{5}

Follow-up results of transcatheter valvotomy in patients with pulmonary atresia and intact ventricular septum Jou-Kou Wang, National Taiwan University Hospital, Taiwan Mei-Hwan Wu, National Taiwan University Hospital, Taipei, Taiwan, Taiwan

Ming-Tai Lin, National Taiwan University Hospital, Taipei, Taiwan, Taiwan

Chung-I Chang, National Taiwan university Hospital, Taipei, Taiwan, Taiwan

Yi-Sharn Chen, National Taiwan University Hospital, Taipei, Taiwan, Taiwan

Objective: To investigate the follow-up results of transcatheter valvotomy in patients with pulmonary atresia and intact ventricular septum (PA-IVS)

Methods: Between 1995 and 2008, 44 neonates diagnosed as PAIVS with a tricuspid valve $\mathrm{Z}$ score $>-3.5$ underwent attempted transcatheter valvotomy. A radiofrequency guide wire (PA120) was used in 38 and coronary guide wire in 6 for perforation of the atretic pulmonary valve. A subsequent balloon valvuloplasty was performed following perforation of the atretic valve.

Results: Transcatheter pulmonary valvotomy was successful in 39 patients \& failed in 5 . Three of 5 failure cases were complicated with pericardial effusion. Of the 39 patients, the mean right ventricular pressure decreased from $116 \pm 23$ to $56 \pm 14 \mathrm{mmHg}$. following valvuloplasty $(\mathrm{p}<0.01)$. Of the 39 patients, 9 required a right ventricular outflow tract (RVOT) patch with or without a shunt because of persisted cyanosis with significant RVOT obstruction and 2 required a shunt. Three required ligation of the ductus. Acute renal failure occurred in 2 requiring peritoneal dialysis. There were 4 mortalities. (2 sepsis \& 2 heart failure). During follow-up, 5 patients underwent balloon dilation for recurrent RVOT gradient. Two required a RVOT patch due to significant stenosis following balloon dilation. Two underwent balloon valvuloplasty for tricuspid valve stenosis. Transcatheter closure of atrial communications was performed in 3. After a mean follow-up period of 4.2 years, 35 surviving patients achieved biventricular circulation with a mean gradient of $18 \pm 12 \mathrm{mmHg}$ across RVOT.

Conclusions: Transcatheter valvotomy can be a definitive treatment in selected patients with PA-IVS.

\section{6}

Transcatheter Closure of Patent Ductus Ateriosus In High Altitude Areas of Qinghai Province - An Analysis of 1000 Clinical Cases

Liming Wang, Section of Cardiac Surgery, Qinghai Provincial Hospital of Cardiovascular Diseases, China
Purpose: The incidence of patent ductus ateriosus (PDA) in Qinghai province, situated in Qinghai-Tibetan Plateau, is estimated as high as $6 \%-7 \%$. Due to the harshness of natural condition (hypoxic, extremely cold and dry) in the high altitude areas (altitude: 2,226 $\mathrm{m} \sim 3,270 \mathrm{~m}$; atmospheric pressure: $77.33 \mathrm{Kpa}$ ), PDA patients suffer more with earlier complications such as pulmonary arterial hypertension and cardiac dysfunction. Transcatheter closure technique has been increasingly used in the treatment of congenital heart diseases due to its high success rate and minimal invasiveness.

Methods: This retrospective study analyzed the 1000 clinical cases (aged from 2 to 70 years, 463 males) who received transcatheter closure of PDA (Cardi-O-Fix PDA Occluder System, Starway Medical Technology Inc, Beijing, China) in Qinghai Provincial Hospital of Cardiovascular Diseases during the period from 2001 to 2006.

Results: Before the procedure, all patients were diagnosed by clinical and echocardiographic assessment as PDA with the narrowest portion of $3 \sim 26 \mathrm{~mm}$ in diameter, of whom 260 patients showed various degrees of pulmonary arterial hypertension. The procedure was successful in $98 \%$ of patients. During the follow up of 2 months to 5 years, 10 patients were found to have residual shunt while nobody suffered from either occluder dislodgement or reopening of PDA.

Conclusion: Transcatheter closure of PDA is feasible in patients coming from high altitude areas which results in good safety and effectiveness.

Key words: high altitude areas, PDA, transcatheter closure.

\section{7}

The Study on middle and long-term results of percutaneous mitral balloon valvuloplasty Zhi-feng Wang, China

Guang-yi Wang, Department of cardiology, Chinese PLA general hospital, China

Objective: This study reports the clinical 10 years follow - up results of patients who underwent percutaneous balloon mitral valvuloplasty (PBMV) in order to identify mid and long-term results, the incidence of restenosis and the determining factors of results and restenosis.

Methods: From January 1996 to June 2006, 83 patients were registry, including 12 male (14.46\%) and 71 female (85.54\%), with age of $20-68$ (mean age of $41.56 \pm 10.68$ ). The data including preprocedure, postprocedure 2 days and 5-10 years follow-up (mean year of $3.1 \pm 2.4$ ) clinical and echocardiography results were studied retrospectively by SPSS software.

Results: After PBMV, mitral valve area (MVA) increased from $1.05 \pm 0.23 \mathrm{~cm} 2$ before PBMV to $1.98 \pm 0.27 \mathrm{~cm} 2(\mathrm{P}<0.01)$. There were $95.18 \%$ patients who had at least one class improvement in cardiac function NYHA just after PBMV. At 5-10 years follow-up, survival rate was 98.8\%, 81.93\% patients were still in class I or II, MVA decreased to $1.69 \pm 0.27 \mathrm{~cm} 2$ gradually, restenosis rate was $31.33 \%$. Atrial fibrillation, cardiac function and Wilkins' score before PBMV were correlative factors with MVA and cardiac function during follow-up $(\mathrm{P}<0.05)$.

Conclusion: PBMV can achieve excellent and sustained mid and long-term results, especially sinus rhythm, Wilkins' score $\leqslant 8$, without poor cardiac function may have the best long-term results.

[Key Words] percutaneous balloon mitral valvuloplasty; mitral stenosis; echocardiography 


\section{8}

Effect of difrerent ocluders in transcatheter closure of congenital ventricular septal defect in children

Qijian Yi, Heart Center, Children's Hospital, Chongqing Medical University, China

Objective: To investigate the methods of selecting different shape occluders and to evaluate effect in transcatheter closures of congenital ventricular seplal defect (VSD) in children.

Methods: Transcatheter closures were performed in 150 childrens with congenital VSD during May, 2004 to July, 2007, under the guidance of transthoracic echocardiography (TTE). There were 4 patients with intracfistal VSD, 143 patients with perimembranous VSD and 3 patients below aortic valve VSD.

Left ventriculography and TTE were performed repeatedly after the procedure to assess the effect of occlusion. TTE, X-ray and electrocardiography (ECG) were scheduled 24 hours, 1, 3, 6, 12 months after closed in follow-up.

Results: The occluders were deployed successfully in 126 patients. The successful rate of operation was $84 \%$. Thin waist shape occluders were deployed in 7 patients; equal side shape occluders were deployed in 114 patients; eccentric shape occhders were deployed in 12 patients. There were 2 patients with residual shunt, that disappeared in third month of follow-up. There was no cardiac arrhythmia after closure.

Conclusion: Transcatheter closure of VSD is an effective and safety treatment in children with VSD. It is very important in selecting different shape occluders according to anatomy and pathologic characteristics. In general, equal side shape occluder is suitable for a large number of VSD patients. In some conditions, the other shape occluder may be considered for selection.

Key words: Ventricular septal defect, transcatheter closure, occluder, curative effect.

\section{9 \\ Morphometric Assessment of Patent Arterial Duct Before and After Device Closure \\ William CL Yip, National University of Singapore, Singapore \\ Wenxia Wu, Gleneagles Medical Centre, Singapore \\ Ting Fei Ho, National University of Singapore, Singapore \\ Kit Yee Chan, Gleneagles Medical Centre, Singapore \\ Swee Chye Quek, National University of Singapore, Singapore}

Objective: Transcatheter closure of patent arterial duct (PAD) has become the treatment of choice for patients after the neonatal period. This paper aims to assess the morphometric changes of PAD before and after device closure.

Methods: 80 patients with PAD were divided into Group A $(\mathrm{n}=30)$ with closure by Cook's detachable coils (CDC) and Group B $(\mathrm{n}=50)$ with closure by Amplatzer duct occluders (ADO). PAD size (S), PAD angle before (A1) and after (A2) closure, and narrowest diameter of the descending aorta adjacent to the PAD before (D1) and after (D2) closure were measured from the descending aortogram at the lateral projection. Derived indices included: Angle Change $(\mathrm{AC}=\mathrm{A} 1-\mathrm{A} 2)$, Relative Angle Change $[\mathrm{RAC}=(\mathrm{A} 1-\mathrm{A} 2) / \mathrm{A} 1]$, Diameter Change $(\mathrm{DC}=\mathrm{D} 1-\mathrm{D} 2)$ and Relative Diameter Change $[\mathrm{RDC}=(\mathrm{D} 1-\mathrm{D} 2) / \mathrm{D} 1]$. All measurements were in $\mathrm{mm}$ and degree, respectively.

Results: The age [5.8(4.3) vs. 12.5(16.5)yr, $\mathrm{p}<0.001]$ was younger and PDA S $[2.9(1.5)$ vs. $4.4(1.5)]$ smaller in Group A patients compared to Group B patients. However, A1 [130.8(9.9) vs. 134.6(9.7)] and $A 2[110(10.7)$ vs. 112.6(10.4)] were not different. D1 was marginally larger $[13.4(5.0)$ vs. $12.8(3.0)]$ due to larger patients in Group B compared to Group A. Significant
AC in Group A [21.9(9.6)] and in Group B [21.9(9.6)] and RAC in Group A [15.8(6.1)\%] and in Group B [16.2(6.9)\%], p < 0.01, were noted. There was also larger DC $[2.5(1.5)$ vs. $1.4(1.1)$, $\mathrm{p}<0.003$ ] and RDC [20.2(13.5)\% vs. 11.6(9.2)\%, $\mathrm{p}<0.001]$ after ADO closure compared to CDC closure.

Conclusions: Significant morphometric changes were noted in patients with PAD after device closure. This was associated with

\section{0}

Bivalirudin (Angiomax ${ }^{\circledR}$ ) As a Procedural Anticoagulant in the Pediatric Population Undergoing Intravascular Procedures for Congenital Heart Disease

Rolando Zamora, Methodist Children's Heart Institute. San Antonio, Texas, United States

Thomas J Forbes, Children's Hospital of Michigan, Detroit Michigan, United States

Jeremy Ringewald, Medical University of South Carolina, United States Robert Vincent, Emory University, United States

Athar Qureshi, The Cleveland Clinic, United States

Jonathan Rome, Children's Hospital of Philadelphia, United States Ziyad M Hijazi, Rush University Medical Center, Chicago IL, For the Angiomax Pediatric Study Investigators, United States

Objective: To determine bivalirudin's safety, plasma concentration $(\mathrm{PK})$, pharmacodynamics (PD), and dosing guidelines when used as a procedural anticoagulant in pediatric percutaneous intravascular procedures using the dose for adults undergoing PCI ( $0.75 \mathrm{mg} / \mathrm{kg}$ bolus, followed by a $1.75 \mathrm{mg} / \mathrm{kg} / \mathrm{hr}$ infusion)

Methods: This is a prospective, open-label, single-arm, multicenter, non-randomized trial. 110 patients were enrolled, 50\% received a device (ASD, PDA, stent, coil), 50\% had balloon angioplasty \& valvuloplasty or diagnostic procedures. There were 11 neonates, 33 infants/toddlers, 32 young children, and 34 older children. End-points were: bleeding (major \& minor), and thrombotic events.

Results: Drug concentration (PK) was higher in older children. The half-life was consistent with adult times. Drug clearance and volume of distribution increased with weight and age. PD: ACT levels are comparable post-dose between neonates and older children/adults. Weight based $(\mathrm{mg} / \mathrm{Kg})$ dosing provides a lower drug concentration in neonates compared to older children/ adults. End-points : Thrombosis and major bleeding events at 30 days were $8.2 \%$ and $1.8 \%$ respectively. All but one of the thrombosis events were sheath clots. Two major bleeds, both hematomas $>2.5 \mathrm{~cm}$, two patients treated for decreased pulses (1.8\%).

Conclusion: 1) Bivalirudin provided safe anticoagulation in all age groups. Major bleeding occurred in $1.8 \%$ of patient population. For the older age ( $>2$ yrs) categories, PK appears to be consistent with adult data. For neonates, weight-based dose results in lower PK, but anticoagulation did occur at that dose. Although end point definitions were sensitive in this study, they are comparable to published events using heparin.

\section{1}

Feasibility and Efficacy of Stent Redilatation in Aortic Coarctation

Keyhan Sayadpour Zanjani, Children's Medical Center, Tehran University of Medical Sciences, Iran

Titus Sabi, German Heart Institute Berlin, Berlin, Germany, Germany Stanislav Ovroutski, German Heart Institute Berlin, Berlin, Germany Bjoern Peters, German Heart Institute Berlin, Berlin, Germany Oliver Miera, German Heart Institute Berlin, Berlin, Germany 
Nikole Nagdyman, German Heart Institute Berlin, Berlin, Germany Felix Berger, German Heart Institute Berlin, Berlin, Germany Peter Ewert, German Heart Institute Berlin, Berlin, Germany

Objectives: We evaluated the efficacy and safety of stent redilation in patients with aortic coarctation (COA).

Methods and Results: In our retrospective study of 28 stent redilation procedures on 22 patients, the interval between implantation and redilation ranged from 2.5 month to 11 years (median 18 months). Ten patients had native COAs, one had repaired interrupted aortic arch. The remaining patients had residual COAs after surgery. Stent redilation was successful in 26 procedures. Pressure gradients decreased from a mean of $26 \pm 13.8 \mathrm{~mm} \mathrm{Hg}$ to $7.9 \pm 6.52 \mathrm{~mm} \mathrm{Hg}(\mathrm{P}<0.001)$. The mean diameter of the stents increased from $9.19 \pm 3.29 \mathrm{~mm}$ to $12.1 \pm 3.68 \mathrm{~mm}(\mathrm{P}<0.001)$. In a patient with Williams-Beuren syndrome, massive neointimal proliferation made stent redilation impossible. One other patient developed aortic aneurysm after redilation and was operated after 3 years.

Conclusions: Stent redilation for aortic COA is a safe and successful procedure.

\section{2}

Biodegradable Magnesium Stents in Emergency Interventions in Critically Ill Babies. First Clinical Experiences

Peter A Zartner, German Paediatric Heart Center, Sankt Augustin, Germany

Matthas Sigler, University and Heart Center, Goettingen, Germany

Robert Cesnjevar, Cardiothoracic Surgery, University of Erlangen,

Germany

Boulos Asfour, German Paediatric Heart Center, Sankt Augustin, Germany

Martin BE Schneider, German Paediatric Heart Center, Sankt

Augustin, Germany

With good early results and superior to balloon dilation only, stents have found its place in interventions in children with a congenital heart disease, but solutions are needed to cover early infancy and the neonatal period.

Under compassionate care regulations we implanted absorbable metals stents (AMS, Biotronik, Berlin, Germany) in three patients (bodyweight $1,7 \mathrm{~kg}, 7 \mathrm{~kg}, 2 \mathrm{~kg}$ ) with severe pulmonary vessel obstructions and critical clinical conditions. The AMS was made of an alloy of over $90 \%$ of magnesium and some rare earth elements. Projected degradation time was 4 to 8 weeks after implantation; intravascular stability of the stent was 1 to 3 weeks. All stents and the implantation procedures were well tolerated and follow-up was not noticeably influenced by the stent degradation. All three children recovered and could be dismissed home from the hospital.
After 4 weeks all stents had lost its stability and gave room to further procedures. One patient died 5 months after implantation due to pneumonia not related to the stent. Autopsy and histopathology revealed a patent vessel at the stented area, with the stent dissolved completely.

Biodegradable metal stents offer new strategies in the therapy of congenital heart diseases especially for newborns and small infants. Depending on the implantation site stent diameters of $6 \mathrm{~mm}$ and larger are needed. A prolonged degradation time may help to avoid early reintervention. To evaluate the possible domain of biodegradable metal stents in children with congenital heart diseases is an important challenge to come.

\section{3}

Stenting and Cutting Balloon in Stenosed Shunts and Pumonary Arteries in Patients After Norwood-Sano Operation

Jose Luis Zunzunegui, Gregorio Marañon Hospital, Spain

Fernando Ballesteros, Gregorio Marañon Hospital, Spain

Teresa Alvarez, Gregorio Marañon Hospital, Spain

Constancio Medrano, Gregorio Marañon Hospital, Spain

Ruben Greco, Gregorio Marañon Hospital, Spain

Erique Maroto, Gregorio Marañon Hospital, Spain

Esther Panadero, Gregorio Marañon Hospital, Spain

Mirian Centeno, Gregori Marañon Hospital, Spain

Introduction: Currently in the majority of the centers the first intervention for hypoplastic left ventricle syndrome is done with the insertion of a conduit from de RV to the pulmonary arteries (Sano procedure). The proximal and distal anastomosis stenosis are one of the complication in the follow up, it could produce relevant cyanosis and growth diminution of pulmonary branches previously Glenn operation.

Methods: 13 patients with Sano procedure; age 14 days- 5 months; weight $2.900-5.700$ grs. The interventional indications; 9 children with hypoxemia (satO2<70\%), 4 in ECMO for refractary cyanosis and right ventricle disfunction. 15 premounted stents (diameter 4-5 mm) were implanted; 10 in the conduit (5 in proximal position, 5 in the distal anastomosis); 5 in the pulmonary arteries (4 in the left, 1 in the right branch). 4 Cutting Ballon angioplasties in the left pulmonary artery origen.

Results: Diameter increase of the stenosis $>50 \%$ and improvement of satO2 $>70 \%(10-20 \%)$. Hypoxemia reappearance in one infant one month later for stent restenosis resolved by stent redilatation. One among the ECMO patients could be withdrawn of the bypass.Glenn procedure was performed in all the survivors patients Conclusion: Succesful relief of shunt and branches stenosis was achieved in all the patients with this approach. It is safe a feasible even in the early postoperative period and allow to delay the cavopulmonar anastomosis after 3 months of age with less morbimortality. 
https://doi.org/10.1017/S1047951109991946 Published online by Cambridge University Press 
Aarabi Moghadam, Mohammad Yousef; 7363 Aarabi, Mohammad Yossef; 8358 Abadir, Sylvia; 3061, 4061, 8051, 8052 Abdel Aal, Wael M; 8172

Abdellah, Abdelmoneim; 7399, 8203

Abduch, Maria C; 7395, 8330

Abdul Aziz, Yang Rafidah; 6057

Abdul Hamed, Jassim; 7156

Abdul Latiff, Haifa; 4031, 6057, 6082

Abdul-Khaliq, Hashim; 3121

Abdulgani, Hafil B; 8169

Abdulhameed, Jassim; 8058, 8059, 8060

Abe, Masanori; 7116, 7153

Abe, Naomi; 7345

Abe, Osamu; 7260

Abella, Raul; 4090, 4106, 7414

Abello, Mauricio S; 7031

Aboul Ezz, Mohamed Rady; 7393

Abreu, Georgina V; 7005

Abtahi, Saeed; 6062

Abu El ezz, Mohamed R; 4026, 6058, 8172

Aburawi, Elhadi H; 4026, 6058

Acar, Philippe; 7092, 7110

Acevedo, Valeria; 4064, 6054, 7035, 8004, 8017

Acharya, Anil; 7251

Acharya, Pradipta; 8149

Acikalin, Mustafa; 8121

Adachi, Iki; 3094, 8276, 8281, 8282

Adachi, Osamu; 7445

Adameczek, Agnieszka; 6206

Adams, Paul; 7292, 8023, 8053

Addonizio, Linda J; 8200

Advani, Najib; 7258

Affara, Nada; 4051

Agarwal, Aarti; 6002

Agarwal, Neeraj; 7297

Agarwal, Ravi; 6164, 7154, 8181

Agarwal, Surendra Kumar; 6006

Agematsu, Kohta; 2063, 3119

Aggoun, Yacine; 7402

Aggrawal, Pooja; 7295

Agha, Hala; 8103, 8054, 8172

Agrawal, Amit; 6167

Aguerrevere, Brígida; 7136

Aguirre, Daniel; 4064, 6054, 8017

Ahluwalia, Jasmina; 7051

Ahmad, Zaheer; 2009, 6059, 6069, 6087, 7064

Ahmadi, Ali Reza; 6074

Ahmed, Iftikhar; 8181

Aiello, Vera; 8366

Aiello, Vera D; 8063

Ajami, Gholamhossein; 6062, 6137, 7073

Ajithakumari, K; 7279

Akagi, Teiji; 7208, 7242, 7243, 7447, 8098

Akasaka, Junetsu; 7445

Akazawa, Youhei; 8018

Akdeniz, Celal; 8041, 8042, 8043, 8044, 8079

Akimoto, Katsumi; 7316, 8022, 8099, 8216, 8244

Akins, Robert; 7429

Aksit, Mehmet Arif; 7050

Aksjonova, Iryna O; 7168

Aktas, Ilknur; 7317

Al Attawi, Faisal M; 8204

Al Faraidi, Yahya M; 7155, 7434

Al Ghamdi, Saleh S; 7007

Al Qethamy, Howaida M O; 7155

Al Saeedi, Abdulraoof AH; 7156
Al-Khatib, Yasser; 7204, 7221

Al-Naami, Ghassan; 6115

AlAbdulkarim, Nawal A; 6014, 6015

Alamin, Abdalla; 7399

Alburquenque, Marcela; 4064, 6054, 8004

Alcaide, Paola; 6199

Alcantara, Alex; 8101

Alehan, Dursun; 6060

Alenius Dahlqvist, Jenny; 7004

Alexander, Peta; 8128

Alexi-Meskishvili, Vladimir; 3121, 7405

Alghamdi, Saleh S; 8204

Ali, Norliza; 8056

Ali, Yassir; 7399, 8203

Aliman, Ana C; 8062, 8063

Alkemade, Fanneke E; 8297

Allen, Peter; 6208

Alliman, Cristina; 8366, 8057

Almeida, Claudia; 4059

Almeida Filho, Oswaldo C; 7477

Alnajashi, Khalid S; 8204

Alobaidan, Mashail A; 8058, 8059, 8060

Alphonso, Nelson; 6025, 6211, 7189, 7195,7433

Alsaeedi, Abdul Raoof AH; 7157, 8058

Alsahari, Atif; 8059, 8060

Altamirano, Luis A; 6061

Alton, Gwen Y; 8198

Aluquin, Vincent PR; 7048

Alvarado, Fernando; 8240

Alvares, Silvia; 7066

Alvarez, Patricia; 4064, 6054, 7035, 8004, 8017

Alvarez, Teresa; 8049, 8413

Alwi, Mazeni; 4031, 6057, 6082, 7460, 8251, 8370, 8371

Amanullah, Muneer; 3116, 8064

Amedro, Pascal; 4062, 7092, 7110, 8092

Amezcua-Guerra, Luis M; 7343

Amin, Zahid; 8364

Ammar, Rasha I; 8089

Amodeo, Antonio; 8237

Amoozegar, Hamid; 6062, 6137

An, Qi; 3017

Anagnostopoulos, Constantine E; 8310

Al Anbaei, Muath; 8118

Andalaft, Rogério B; 7005, 7006

Andelfinger, Gregor; 4052, 8051, 8202

Anderson, Brent; 8005

Anderson, Robert H; 6059

Anderson, Vicki; 3050, 6220

Ando, Makoto; 3053, 7113, 7314

Ando, Tatsuya; 7067

Andou, Hisashi; 6089

Andrade, Alexandre A; 8218

Andrews, David R; 7419

Andropoulos, Dean B; 3028, 6003, 6004

Ang, Hak Lee; 7259

Angouras, Dimitrios; 8310

Anis, Aslam H; 3117

Aniteye, Ernest; 7197, 7198

Annerén, Goran; 7124

Aoki, Masaya; 7159, 7489

Aoki, Mitsuru; 7309

Aotsuka, Hiroyuki; 7309

Apablaza, Karen; 7428

Apitz, Christian; 7068

Apostolopoulou, Sotiria; 8114, 8123

Aracena, Mariana; 7332
Aragaki, Yoshio; 7127

Arai, Sadahiko; 7208, 7242, 7244, 7447, 7449, 7450, 7472, 8098

Arakawa, Hirokazu; 7231, 7278, 7458

Arantes, Lidiane R; 7477, 7478

Aravena, Teresa; 7332

Arcidiacono, Carmelo; 4090, 6021, 8076

Ardoin, Kipp; 7002

Areias, José C; 6036, 8125, 8126, 8314

Argiento, Paola; 4038, 8124

Arheart, Kristopher L; 3049

Arheden, Hakan; 4024, 6113

Arima, Masaki; 8311, 8328

Arizmendi Tapia, Felipe; 6063

Arnold, Philip D; 6211

Arora, Ramesh; 4057

Arramraju, Srinivas Kumar; 8337

Arslan, Yucesin; 7368

Arzt, Wolfgang; 3081

Asenjo, Manuela; 6179, 6180, 6181, 6194, 6198, 6199

Asfour, Boulos; 3083, 3086, 7160, 8412

Ashworth, Michael; 8019

Aslan, Ilknur; 8158

Asoh, Kentaro; 8311, 8328

Asou, Toshihide; 7446

Assad, Renato S; 7395, 8062, 8063, 8330

Assali, Abid; 8221

Asselin, Geraldine; 8202

Astete, Carmen P; 7332

Atiq, Mehnaz; 3116, 8064

Atiyah, Merna M; 7007

Attie, Fause; 8247

Aung, Myo Myint; 8260

Austoni, Paola; 7389

Avaria, Maria; 8017

Avila Rosales, Lucrecia; 6063, 6138

Awadh, Mohammed A; 8204

Awang, Yahya; 8169

Awasthy, Neeraj; 7340, 7341

Axelsson, Åsa B; 8009, 8010

Aydemir, Numan A; 7166, 7167

Ayer, Julian G; 7424

Ayusawa, Mamoru; 6085, 7028, 7260, 8109

Azancot, Annabelle; 4072

Azariades, Prodromos; 8205, 8238

Azeka, Estela; 8193

Azevedo, Luis; 8126

Azevedo, Ruben; 4083

Aziz, Kalimuddin; 7261, 8363, 8364

Bablyak, Oleksandr; 6064, 7161, 7168, 7441

Bacha, Emile; 8176

Badawi, Nadia; 3114, 7424

Bader, Vivian; 8168

Badle, Saket; 2009

Bae, Eun Jung; 7023, 7280, 8225

Baffa, Gina; 3020

Baharestani, Bahador; 8253

Bahl, Vinay K; 6109

Bailey, Leonard L; 7141

Bailliard, F; 6081

Balci, Oya; 7293

Balderrábano, Norma A; 8277

Baldinelli, Alessandra; 6143, 6144, 7070,

7192, 7435, 7436, 7437

Baldwin, Annette; 8171

Balekundri, Vijayalakshmi; 8066

Bali, Bijender S; 7461 
Balint, Olga H; 8261

Baliulis, Gedrius; 7479

Ballesteros, Fernando; 8049, 8413

Balzer, David T; 8071

Ban, Ji Eun; 7464

Banaszak, Pawel; 7042

Bandyopadhyay, Biswajit; 8036, 8045, 8046

Baptista, Maria J; 8125, 8126, 8314, 8357

Barber, Brent J; 8008

Barbero Marcial, Miguel; 7235, 8193

Barbosa, Fabiane B; 6086

Baris, Safa; 6041

Barnett, Bryanne E W; 6225

Barozzi, Luca; 7380, 7381, 8206

Barrea, Catherine; 6016

Barreta, Jorge; 7133

Barriot, Roland; 8325

Barron, David J; 2064, 3021

Bartczak, Agnieszka; 8266, 8267

Bartelds, Beatrijs; 4051, 4102

Bartelings, Margot M; 8276

Barth, Mary J; 7227

Bartkowska-Sniatkowska, Alicja; 7012

Bartlett, Harry; 6061

Bass, John L; 6107

Bassin, Levi; 7162

Bassler, Nicole; 4084

Baszko, Artur; 7011, 7012

Batinica, Maja; 7255

Battin, Malcolm; 7045

Bauer, Ulrike; 6224

Bautista, Edgar; 4037

Bax, Jeroen J; 6119

Baysal, Kemal; 7254, 8122

Bebbington, Michael; 3128

Beca, John; 2100, 3007, 7394

Bédard, Elisabeth; 8235

Beerbaum, Philipp; 7128

Beghetti, Maurice; 7402

Begum, Fatema N N; 8068

Bei, Eugenia; 8113

Belina, Drazen; 7091

Bellelli, Alberto; 8234

Belli, Emre; 7379

Bellinghausen, Winfried; 4103

Belloni, Anna S; 8309

Benito, Begoña; 8319

Benjacholamas, Vichai; 4059, 7164, 7367, 8339

Bensch, Marc; 7469

Benson, Lee; 4059, 4089

Berdat, Pascal A; 7389

Berger, Felix; 3121, 7405, 8021, 8411

Berger, Rolf MF; 4051, 4102, 7474, 8127, 8148

Berger, Wouter R; 7008

Berggren, Håkan; 7465

Berlage, Thomas; 6123

Bermudez-Canete, Ramon; 8070

Bernard, Pierre; 6016

Bernini Kapins, Carlos Eduardo; 8284

Bettuzzi, Maria G; 6143, 6144, 7070, 7192, 7435, 7436, 7437

Beyene, Joseph; 3012

Bezold, Louis I; 8029

Bhagyavathy, Anpon; 6068, 6169, 8081, 8275,8389

Bhamra, Jagjit; 3071

Bhan, Anil; 8149

Bhargava, Balram; 8254

Bhargava, Kartikeya; 7341

Bharucha, Tara; 8128, 8166

Bhatt, Anil; 8393

Bhatt, Dheeraj; 8120
Bhattacharyya, PS; 8045

Bhojani, Kaushik; 7101

Bhole, Vinay; 4060, 4071, 4109, 8082, 8377,8391

Bhoyar, Abhay; 7072

Bialkowski, Jacek; 7042, 8069, 8070, 8274, 8395

Bian, Jun; 6147, 6188

Bichara, Grace C V L; 7209, 7210, 7211

Bieganowska, Katarzyna; 7009, 7010, 7029

Biernacka-Zielinska, Malgorzata; 6112

Biffanti, Roberta; 3120

Bigdelian, Hamid; 7165

Bigras, Jean Luc; 4052

Bilal, Mehmet S; 7166, 7167

Bingler, Michael A; 8071

Biondi-Zoccai, Giuseppe; 4106

Birbach, Mariusz; 7009, 7174, 7425

Birk, E; 8074

Birkeland, Sigurd; 7374

Birnbaum, Julia; 3019, 8192

Bisoi, Akshay K; 7451

Bjorkhem, Gudrun; 7124

Blackburn, Michael E; 8223

Blair, Brian J; 8008

Blanc, Julie; 6066

Blaschczok, Hedwig-Christine; 3086

Blaser, Susan; 6067

Blieden, Leonard; 8220, 8221

Blom, Nico A; 6119

Bloomfield, Peter; 8208

Bo, Ping; 3122

Bobkowski, Waldemar; 7011, 7012, 7058, 7059

Boccuzzo, Giovanna; 3120, 7130, 7131

Bocsi, Jozsef; 4103

Bodor, Gábor; 7219

Bøe, Morten L; 3071

Boethig, Dietmar; 7213

Boettler, Petra; 7128

Bohuta, Lyubomyr Y; 7168, 7169

Bojalil, Rafael; 7343

Boku, Naoki; 7344

Bolat, Ali; 7370

Boldt, Talvikki; 8367

Bollen, Casper; 7098

Bolster, Edwin; 6218

Bonato, Raffaele; 8179

Bonello, Beatrice; 7060

Bonhoeffer, Philipp; 4028, 4085, 4111, 8353

Boni, Lorenzo; 8033

Bonnemains, Laurent; 6066

Bonnet, Damien; 6066

Booth, Phil; 8129

Boric, Igor; 7255

Boruta, Peter; 6120

Borzoee, Mohammad; 6062, 6137, 7073, 8055

Bosch, Fernando; 8240

Boshoff, Derize; 4110, 6022, 8072, 8073

Bøtker, Hans Erik; 8320, 8324

Bottega, Natalie; 4113, 4116

Boucek, Mark M; 3022

Boucek, Robert J; 7462

Bouchardy, Judith; 4116

Bouchier-Hayes, David; 3031, 7246

Boudjemline, Younes; 6066

Bourlon, François; 7110

Bouvagnet, Patrice; 4080

Bova, Sergio; 8309

Bovenschulte, Henning; 8293

Bowles, Neil E; 4070

Boyko, Elena P; 8354

Bozikov, Jadranka; 7255
Bozkurt, Abdi; 8362

Braby, Jeanne; 3008

Bradley, Timothy; 3012, 6067, 6201

Braga, Sergio LN; 8074

Brancaccio, Gianluca; 8237

Brankovic, Jelena; 3124, 6021

Bratt, Ewa-Lena; 8009, 8010

Bravo-valenzuela, Nathalie J M; 7074

Brawn, William J; 2064, 3021

Breckpot, Jeroen; 8289, 8325

Breinholt, John; 8110

Bresolin, Adriana C; 7346

Breymann, Thomas; 7213

Briggs, Kate; 4032

Brizard, Christian; 6215, 7146

Broberg, Craig S; 4037

Brockmeier, K; 7367, 8054

Broeckel, Ulrich; 8287

Brooks, Andre; 7170, 7492

Brooks, Paul A; 8290, 8291, 8300, 8301

Broomfield, Alex; 4073

Brown, John W; 3085, 7140, 7171, 7172, 7442, 7443

Brown, Karen; 6007

Brown, Kate; 8019

Brown, Katherine; 4067

Brown, Morgan L; 3123, 7173

Brown, Roger; 7325

Brown, Stephan; 6022, 8072, 8073

Brozik, Henryka; 6112

Bruckheimer, Elchanan; 8074

Brugada, Josep; 8319

Brugada, Ramon; 8319

Brun, Henrik; 4035, 8217

Bryant, Randall; 7026

Brzezinska, Grazyna; 7425

Brzezinska-Paszke, Monika; 7009, 7010, 7029

Brzezinska-Rajszys, Grazyna; 7115

Bu'Lock, Frances A; 6093, 6100, 8075

Buckley, Helen; 8239

Budhiwardana, Novik; 7216

Budts, Werner; 8072

Buelna-Cano, Christian; 8214

Buendia, Alfonso; 7438, 8247

Bugrilova, Rimma S; 8327

Buljevic, Bruno; 7021

Bullock, Andrew; 7419

Burch, Michael; 8019

Burczynski, Piotr; 7174, 7425

Burkhart, Harold M; 3087

Burlli, Gustavo; 8195

Burns, Janet E; 8129, 8170, 8208

Bush, Anneke C; 7075

Bush, David M; 7075, 7076

Busro, Pribadi W; 7216

Bussadori, Claudio; 8086

Butera, Gianfranco; 2010, 4090, 4106, 6021,

8076, 8077, 8078, 8086, 8087

Butler, Tanya L; 4017

Butt, W W; 6171

Byrne, John S; 3031, 7246

Cai, Sally; 6133

Cai, Xiaoman; 6174, 8130

Calabrò, Raffaele; 4030, 4038, 8124, 8384

Caldarone, Christopher A; 7248

Calder, Louise; 3095, 8278

Caldera, Kalhara; 6012

Calderon, Juan; 7332

Calderon-Colmenero, Juan; 7177, 7190,

7191, 7438, 7439, 8131, 8247

Calderone, Christopher; 8262

Calò Carducci, Francesca; 8190

Calzolari, Armando; 8190 
Camilleri, Serge; 8092

Camino, Manuela; 8049

Campuzano, Oscar; 4052, 8319

Canavan, Colin; 3031, 7246

Canessa, Roberto; 6212

Cannon, Bryan; 7026

Cantanhede, André Luis C; 7408

Capderou, Andre; 6066, 7134

Capelli, Claudio; 4028, 4111

Capelli, Horacio; 7024, 7043, 7121, 7133

Cappato, Riccardo; 8077

Caputo, Massimo; 7147

Carcioppolo, Victor; 6208

Cardendas, Luis; 7428

Cargnelli, Gabriella; 8309

Carlotti, Ana Paula P; 7477, 7478

Carlsson, Marcus; 4024, 4026, 6113

Carminati, Mario; 1127, 2010, 4090, 4106, 6021, 7414, 8076, 8077, 8078, 8086, 8087

Carmo, Carmen; 7066

Carmona, Estela; 8195, 8199

Carneiro, Leonardo S; 7409

Carrel, Thierry; 7311

Carreño, Carola; 7428

Carrozza, Marianna; 4030

Carvalho, Antonio C C; 6086, 7386, 7387. 7388,8284

Carvalho, Marisa; 7066

Carvalho, Werther Brunow; 7386, 7387, 7388

Casagrande, Ivan S J; 3056

Casey, Frank; 3126, 6033, 6034, 6219, 6227

Castaneda, Aldo R; 8181

Castejón, José M; 7136

Catena, Emanuele; 7389

Cavalcanti, Elaine; 7234

Cavalli, Muarizio; 8309

Çelebi, Ahmet; 7166, 7167, 8041, 8042, $8043,8044,8079,8080$

Celermajer, David S; 8212

Cender, Laurie; 3117, 6178

Centeno, Miriam; 8049, 8413

Cervantes, Jorge L; 7177, 7190, 7191, 7438, 7439,8131

Cervantes, Laura; 6194, 6199

Cesnjevar, Robert; 8412

Cetta, Frank; 3123

Cevik, Ayhan; 7054, 7318

Cevik, Berna Saylan; 6055, 7319

Chaccur, Paulo; 8365

Chae, Soo-Ahn; 7362

Chaker, Joao Saba; 6086

Chakrabarti, Shanta; 4033

Chakravarthi, Shrinivas; 6068, 8275

Chamizo, Wilfredo; 7462

Chan, Kak C; 3022

Chan, Kit Yee; 7327, 8409

Chan, Pui Ding; 6057

Chandra, Neeti; 7461

Chandra, Subash V; 7181

Chandrasekaran, Ramyashri; 6068, 6170, $8081,8275,8389$

Chang, Chung-I; 6070, 6222, 7178, 7179, 7180,8405

Chang, Jeng-Sheng; 6141, 7187, 7427

Chang, Jia-Kan; 7077, 7078

Chang, Yun Hee; 7464

Chanthong, Prakul; 6135

Chard, Richard B; 3072, 7036, 7162, 7459

Charlton, James; 6069

Charoenkwan, Pimlak; 8024

Charroqui, Alberto; 8199

Chattopadhyay, Amitabha; 8045, 8046

Chaturvedi, Rajiv; 4059, 4089

Chatzis, Andreas; 8113, 8205, 8238
Chaudhari, M; 7111, 7112, 8196

Chaudhuri, Maitri; 7181

Chauhan, Sandeep; 7451

Chen, Bin-Jin; 6127

Chen, Chun-An; 6070, 6222, 8349

Chen, Fong-Lin; 7079, 7080, 7081

Chen, Gang; 6210

Chen, Guo-Zhen; 4025, 4027, 4063, 6071, 6127, 6131, 8326

Chen, Hui-Chi; 7358, 7359

Chen, Huiwen; 7182

Chen, Li; 3048

Chen, Li-jun; 8031, 8032

Chen, Ming-Ren; 7482

Chen, Ri; 3029

Chen, Robert P; 8292

Chen, Rui; 4070

Chen, Shu-bao; 3088, 6072, 7082, 7274

Chen, Ssu-Yuan; 8188

Chen, Sun; 6072

Chen, Xingpeng; 2104, 3018, 7138

Chen, Yih-Sharang; 6070, 6222, 7178, 7179, 8405

Chen, Yuan; 4082

Chen, Zhang-gen; 6080, 6096, 6098, 7237

Cheng, Irene; 4068, 8029

Chenu, Caroline; 7183

Cherian, K M; 2047

Cherian, Kotturathu M; 6164, 7154, 8375

Chersich, Matthew F; 8053

Chessa, Massimo; 1127, 2010, 4090, 4106, 6021, 7414, 8076, 8077, 8078, 8086, 8087

Chetaille, Phillipe; 4052

Cheung, Michael; 6130, 6171, 7008, 8290, 8291

Cheung, Yiu-Fai; 1128, 6090, 7083, 7084

Chewathanakornkul, Sririchai; 8380

Chia, Alicia XF; 7480

Chiang, Jen-Wen; 8302

Chidambaram, Shanthi; 6164, 8375

Chikermane, Ashish; 8377

Chisaka, Toshiyuki; 8151

Chiu, Chaw-Chi; 8134

Chiu, Hsin-Hui; 7358, 8188

Chiu, Ing-Sh; 6070, 6222, 7178, 7179, 7180

Chiu, Sheunn-Nan; 8349, 8403, 8404

Cho, Hwa Jin; 7013

Cho, Mi-Young; 7185

Cho, Min-Jung; 6160, 8345, 8346

Cho, Min-Sun; 8140

Cho, SeongHo; 7186

Cho, Young-Kuk; 7013, 7085, 7086, 7286

Choi, Chang Hyu; 8133

Choi, Deok Young; 8133

Choi, Eun Young; 3082

Choi, Eung-Sang; 7362

Choi, Eunyoung; 7342

Choi, HyungSoon; 7022

Choi, Jae Young; 7120, 7488, 8332, 8333

Choi, Jung-Yun; 6051, 7023, 7280, 8225

Choi, Kyung-Sook; 6051

Choi, Soo Han; 8011, 8143

Choi, Woo-Yeon; 7013, 7085, 7086, 7286

Choi, Yeong-Hoon; 8293

Chong, Daniel; 8231

Choo, Boonhock; 6124, 6142, 8047

Choo, Jonathan TL; 7264

Choo, Kok Kuan; 4031

Chou, Nai-Kuan; 8188

Chou, Shah-Hwa; 8134

Chow, Pak-cheong; 1128

Chow, Sharon; 6178

Chowdhury, Devyani; 7087, 8209

Chowdhury, Ujjwal Kumar; 8210, 8211
Christenson, Jan T; 8181

Christov, Georgi v; 7018

Chrustowicz, Anton; 8249

Chu, Chen; 3127, 7088

Chu, Shu-Hsun; 8349

Chuang, Tzu-Yao; 7187

Chueng, David T; 3056

Cikirikcioglu, Mustafa; 7402

Cilliers, Antionette; 7292, 7307, 8023, 8053

Clarke, Andrew; 8259

Clarke, Bernard; 8239

Clarke, David; 6232

Clarke, David M; 6231

Claudia, Carvajal; 6212

Clemente, Fátima; 8126

Cleuziou, Julie; 2103, 7188

Cobos, Susana; 6179, 6180, 6181, 6194, 6198, 6199

Cochrane, Andrew; 3050, 4081, 6042, 6220, 7146, 8171

Coe, James Y; 8198

Coelho, Leiliana M; 8219

Coelho, Robert; 6169, 6170, 7452, 7453,

7454, 8081, 8275, 8389

Coelho, Tiago H; 8314

Cogo, Paola; 8179

Cohen, Laureen; 7060

Colajacomo, Mauro; 8230

Colak, Omer; 7050

Colan, Steven C; 8030

Colan, Steven D; 4068, 4069, 8006, 8014

Colaneri, Massimo; 6143, 6144, 7070, 7192, $7435,7436,7437$

Cole, Andrew; 3027, 3072, 3114, 7162, 7424, 7459

Coleman, Lee; 2100

Collins, Kathryn K; 3025

Collins, Nicholas; 8261

Colman, Jack M; 4114

Colonna, Pier Luigi; 7192, 7435, 7436, 7437

Cols, John G; 7248

Comas, Juan V; 8033

Connell, Vanessa; 7008

Conner, William; 6189

Connolly, Dana; 7426

Connolly, Heidi M; 3123

Connor, Jean A; 4093, 8181

Contrafouris, Constantinos; 8205, 8238

Cooper, Stephen G; 3072, 7321

Copeland, Jack G; 8008

Cordina, Rachael L; 8212

Cordova, Julio; 4037

Corno, Antonio F; 6211, 7189, 7195, 7433

Corpus, Rohan J; 4021

Correia-Pinto, Jorge; 8125, 8126

Costa, José Daniel A; 8218

Costa, José M; 3056

Costello, Claire; 6183, 6184

Cosulich, Michael T; 6078

Cousineau, Jocelyne; 7271

Coutinho, Ralph B; 6086

Covington, Diane; 8008

Cowan, Brett; 4066

Cox, Gerald F; 8014

Craig, Brian; 3126, 6033, 6219, 6227

Crawford, Jackie; 3024, 4012

Crepaz, Roberto; 3124

Cristovão, Salvador A B; 7209, 7210, 7211

Crone, Leonie; 4020

Crosby, Marsha C; 3117

Cross, Russell; 8268

Crossett, Marcus; 4023

Crupi, G; 7481

Crupi, Giancarlo; 8168 
Cui, Vivian; 4086, 6134

Cullen-Dean, Geraldine; 6189

Cullinan, Paul; 3098

Cuneo, Bettina F; 6134, 8034

Curi-Curi, Pedro J; 7177, 7190, 7191, 7438, 7439, 8131

Curtis, Stephanie; 4033

Cutright, Warren J; 8344

Cyran, Steven; 7087, 8209

Czyz, Krzysztof; 7011, 7012, 7058, 7059

D’Alfonso, Alessandro; 6143, 6144, 7192 7435, 7436, 7437

D'Alterio, Giuliano; 4038, 8124

D'Alto, Michele; 4038, 8124

d'Udekem, Yves; 6171, 6215, 7146

Dadashev, Alexander; 8220, 8221

Dähnert, Ingo; 3046

Dagan, T; 8074

Dahdah, Nagib; 3061, 4061, 7271, 8051, 8052

Dai, Zen-Kong; 8134

Daitoku, Kazuyuki; 8213, 8255

Dalla-Poza, Robert; 8192

Dancea, Adrian; 4061, 6176

Dangel, Joanna; 6013, 6017, 6019, 6023 $6031,6048,6049,7003$

Dangel, Maria M; 6017, 6018, 6019

Daniel, Jane; 6183, 6184

Danieli, Joseph; 7193

Daniels, Jane; 7072

Danielson, Gordon K; 3123

Darbandi-Mesri, Farnaz; 8003

Dark, John H; 8196

Darst, Jeffrey R; 8071

Das, Dhritabrata; 8045

Datta, Panchali; 7324

Daubeney, Piers EF; 3098, 4104

Davari, Paridokht Nokhostin; 8358

David, Nelson; 8110

Davidson, Malcolm R; 8312

Davidson, Simon; 3098

Davidson, William; 8209

Davis, Andrew; 3057, 4032, 7001, 7008

Davis, Annette; 7433

Davis, Christopher; 7052

Dayton, Jeffrey D; 8200

de Castro, Ana Carolina M; 8219

de Catte, Luc; 6022

De Decker, Rik; 7122, 7492

De Decker, Zandre; 7122

De la Hoz, Francisco; 7378

de la Riba, Maria de Carmen; 8199

De La Riba, Marita; 8195

De Luca, Francesco; 3124

de Paula, Ana Carolina; 7281, 7282

de Pont, Judith; 4020

De Rita, Fabrizio; 7380

de Roos, Albert; 6119

De Souza, Astrid M; 3113, 7047, 7267, 7268

De Souza, Eustace S; 3113, 7047

De Vincentiis, Carlo; 7414

de Windt, Leon J; 4102

Dean, Mathew; 7087

Dean, Suzanne; 6042, 6229

Dearani, Joseph A; 3087, 3123, 7173

Deavers, Julie A; 3008

Dede, Tulin; 8015

Dedovich, Vitaly; 8181

Deepa, A S; 7349

DeGiovanni, Joseph; 4060, 4071, 4109, 8082, 8377

Del Pozo, Ana; 8195

Delhaas, Tammo; 8148

Delvin, Edgar; 7271
Demir, Cansun; 6038

Demir, Fadli; 8043, 8044

Demir, Halil; 8041, 8079

Denjean, André; 4072

Denmark, Lloyd; 3024

Derby, Christopher D; 3020

Dereddy, Narendra; 4036

Derrick, Graham; 8019, 8353

DeRuiter, Marco C; 8297

Desurkar, Vinayak N; 8154

Devananda, N S; 7181

Devriendt, Koenraad; 8289, 8325

Dezfouli, Shala; 4084

Dhandayuthadani, Giri; 7147

Dhannapuneni, Ramana RV; 7195, 7433

Dhillon, Rami; 4060, 8377

Di Carlo, Duccio; 8237

Di Donato, Roberto M; 8237

Di Renzi, Paolo; 8234

Diamant, Ulla-Britt; 3112, 7014

Diaz, Hernan; 8070

Dicke, Frank; 7019

Dickinson, Michael G; 8295

Dietrich, Anderson; 7235, 8193

Dilber, Danijel; 7055

Dillon, Jeswant; 7460

Dilworth, Kelly; 3116

Dimitriou, Constantine; 8310

Dimopoulos, Konstantinos; 8235

Ding, Wen xiang; 7485, 7486

Dipchand, Anne I; 8185, 8189

Disli, Olcay Murat; 7145, 7370, 7371

Djer, Mulyadi M; 8083, 8372

Djukic, Milan M; 7089

Docherty-Skippen, Susan M; 6190

Dodd, Patricia M; 3056

Dogan, Vehbi; 6053, 8039

Doherty, Nicola; 6219

Doi, Shozaburo; 8109

Don-Wauchope, Andrew; 6189

Donis, Igor; 8365

Dorner, Sanja; 7055, 7090

Dortan, Maria; 7026

Dos Subira, Laura; 8262

Dosapati, Sitaram; 8336

Doublin, Nancy A; 7202

Douwes, J Menno; 8127

Dovgaliuk, Arkadiy A; 8342, 8354

Dragulescu, Andreea; 4062, 7060, 7092, 8092

Driscoll, David J; 3123

Dryzek, Pawel; 7400, 8084, 8085, 8356, 8394

Du, Junbao; 3048, 7015, 8144, 8304

Du Plessis, Jean H; 6075

Du Plessis, Johannes; 8177

du Sart, Desiree; 3057

Du, Xin wei; 7483

Du, Zhong-Dong; 7015

Dua, Jaspal S; 1127, 2010, 7414, 8078, 8086, 8087

Dube, Marie-Pierre; 8202

Dubowy, Karl O; 7128

Dudley, Joann; 7150

Duke, Abdul K; 6093, 8075

Duncan, Walter J; 7267, 7268

Dupont, Emmanuel; 4104

Duque, Simon B; 8101

Durham, Lucian A; 3087

Durongpisitkul, Kritvikrom; 4058

DuSart, Desiree; 7001

Dwivedi, Rashmi; 7350, 7351

Eastaugh, Lucas J; 4032, 7001, 8088

Ebata, Ryota; 7303, 7309
Ebels, Tjark; 7474

Ebishima, Hironori; 7196

Ebrahimi, Parisa; 6137

Echigo, Shigeyuki; 6103

Eddy, Carey-Anne; 3024, 4012

Eddy, Lee Anne; 8167

Eder, Sarah; 7405

Edgar, Rosemary A; 4071

Edwin, Frank; 7197, 7198

Eerola, Anneli; 7093, 8367

Egan, Jonathan R; 4017

Eguchi, Taisuke; 7291, 7348

Ehara, Eiji; 7384

Eicken, Andreas; 7337, 8285

Eidem, Benjamin W; 8030

Eitoku, Takahiro; 8361

Ejike, Janeth C; 7141

El Oakley, Reida M; 7157, 7434

El Saiedi, Sonia A; 8089

El Sisi, Amal M; 8089

el Zein, Chawki F; 3054, 7431

El-Massri, Nader; 8248, 8249

EL-Said, Howaida G; 7052, 7426, 8037, 8048

El-Segaier, Milad; 4080, 4108, 7238

Elamin, Hani Eltayeb; 7199

Ellims, A; 6130

ElSaiedi, Sonia A; 8172

Elsheikh, Abdalla; 8203

Elshershari, Huda; 7094

Eltayeb, Hani; 7399, 8203

elZein, Chawki F; 7227

Elzenga, Nynke J; 7474

Emmel, Mathias; 3058, 7367, 8103, 8339

Emmertsen, Kristian; 6226

Engel, Mark; 7253, 7305, 7306

English, Kate M; 8223

Enriquez, Gabreiela; 7428

Enriquez-Rodriguez, Luis E; 7343

Entsua-Mensah, Kow; 7197, 7198

Erdem, Abdullah; 8041, 8043, 8044, 8079, 8080

Erdem, Sevcan; 6038, 8362

Erdenebileg, Ariuntsatsral; 7075, 7396

Erdmenger, Julio; 7190, 7191

Eremija, Lazo S; 6214

Eren, Abdulkadir; 6159

Eren, Makbule; 8121

Erenberg, Francine; 7204, 7221

Erez, Eldad; 7202

Ergün, Mehmet Ali; 7317

Erhuma, Salem M; 7270

Eriksson, Ulf; 8297

Erol, Nurdan; 8043, 8044

Errami, Ab; 4080

Errico, Gabriella; 3124

Escudero, Maria; 6224

Eshelman, Jennifer C; 6162

Esmat, A; 8054

Espinola-Zavaleta, Nilda; 7343, 8214, 8215,8247

Esposito, Raffaella; 8384

Essmat, Amira; 8103

Esteves, Cesar A; 8074

Eum, Gwang-Hyun; 7013

Evruke, Cuneyt; 6038

Ewer, Andrew; 7072

Ewert, Peter; 3121, 8411

Eyskens, Benedicte; 4110, 6022, 8073

Ezwae, Salma M; 7270

Faella, Horacio; 7133

Fagan, Thomas E; 8071

Faggian, Giuseppe; 7380, 7381 
Faituri, Rasmia H; 8374

Fakri, Dicky; 7216

Falasco, Gianclaudio; 7130

Farouk, Ahmed; 7203, 7204, 7221

Fedderly, Raymond; 7325

Feituri, Rasmia H; 7270

Feldman, Brian M; 3012

Felix, Donald E; 6005

Feng, Chuan; 4082

Feng, Yuan; 8091

Fenton, Kathleen N; 7205

Ferek-Petric, Bozidar; 7021

Fernandes, Ana R; 7440, 7448

Fernandez, Ana Isabel; 6199

Fernandez, Carols; 8004

Ferreira, Cesar A; 7477, 7478

Ferreira, Eduardo C; 7408

Ferreira, Manuel J; 7440, 7448

Ferreiro, Carlos R; 7234

Ferrer, Soledad; 6194

Ferrero, Paolo; 8077

Fesslova, Vlasta; 3124, 6021, 8078

Festa, Marino; 3027, 4020

Figueira, José A; 7136

Figueiredo Neto, José A; 8218, 8219

Figueredo, Eduardo D; 7408

Fijten, Stephanie; 3027

Fink, Christo; 3083

Finucane, Kirsten; 7102, 7144, 7206, 7394

Fiore, Andrew C; 3085, 6177, 7443, 8096

Firmin, Richard K; 6093, 6100, 8075

Fischer, Gunther; 8376

Fischer, Krisztián; 7219

Fitts, James; 7141

Fleishman, Craig E; 6005

Flint, Julia; 7206

Floh, Alejandro A; 6146

Flores, Ramon; 8070

Florianczyk, Tomasz; 7357

Fogel, A; 6077

Fogel, Mark A; 4007, 6078

Foker, John E; 6107

Fong, Nai Chung; 7097

Fontes-Pedra, Simone; 7234 8062, 8063, 8365,8366

Forbes, Thomas; 4107, 8410

Forbess, Joseph M; 7150, 7207, 7215

Foreman, Celeste; 6204

Foresti, Sara; 1127, 8077

Fouilloux, Virginie; 4062, 7060, 7092, 7183

Fournier, Anne; 3061, 7271

Fragata, Claudia S; 7005, 7006

Fraisse, Alain; 4062, 7060, 7092, 7110, 8092

Francis, Edwin; 8093, 8094, 8095, 8378

Francis, Richard JB; 3051

Franich Ray, Candice; 3050, 6220

Frank, Lowell; 8268

Franklin, Rodney; 3075, 3098

Franks, Cherie; 4115

Fraser, Charles D; 3028, 6004

Frazer, Jeffrey R; 4087

Fredriksen, Per Morten; 4035, 8217

Freire, Grace A; 6177, 8096

Freire, Isabel; 8101

Freund, Jan Erik; 7098

Freund, Matthias; 7098

Frid, Christina; 7124

Friedberg, Mark K; 3045, 3115

Friedman, Lawrence B; 7287

Frigiola, Alessandro; 1127, 4106, 6021, 7414, 8077

Frimpong-Boateng, Kwabena; 7197, 7198

Frndova, Helena; 6135

Froemke, Cecily; 7099
Frycz, Jan; 8274

Fu, Li Juan; 6147, 8173

Fu, Lin-Shien; 7284

Fu, Yun-Ching; 6155, 7284, 8097

Fuchs, Alexandra; 8192

Fujii, Kousuke; 7410

Fujii, Takanari; 7100

Fujii, Yasuhiro; 7196, 7208, 7242, 7449, 7450, 7472, 8098

Fujimoto, Yasufumi; 7224

Fujimoto, Yoshifumi; 7226

Fujita, Hideki; 1126, 7336

Fujita, Shuhei; 8258

Fujiwara, Masako; 7067

Fujiwara, Tadashi; 7309

Fukazawa, Ryuji; 7116, 7153

Fukuda, Ikuo; 8213, 8255

Fukuda, Kazuyoshi; 7220

Fukuda, Seiji; 8161

Fukuhara, Hitoo; 7032, 7038, 7039

Fukuhara, Junji; 6085, 7028, 7260

Fukui, Kozo; 8213

Fukunaga, Hideo; 8022, 8099, 8216

Fuller, Corey; 7141

Funata, Hiroaki; 7127, 8157

Furlanetto, Beatriz H S; 7209, 7210, 7211

Furlanetto, Gláucio; 3056, 7209, 7210, 7211

Furmston, Alexandra; 7072

Furukawa, Takeshi; 8022, 8099

Fusazaki, Naoki; 7114, 8241

Gackowski, Andrzej; 8248, 8249

Gaio, Gianpiero; 4030, 8384

Galletti, Lorenzo; 8033

Gan, Huili; 3122, 7212

Gandy, Kimberly L; 3008

Ganesh Kumar, G; 8250

Ganeshalingham, Anusha; 7144

Ganeshkumar, G; 6163

Gao, Wei; 8100, 8348

Garay, Francisco J; 8101

Garcia, Enrique; 8033

Garcia, Jaime; 7438

Garcia, Jose A; 8131

Garcia-Montes, Jose A; 7438, 7439

Garekar, Swati; 6154, 7101, 7239, 7369, 8012, 8280

Gargiulo, G; 7481

Gatland, Rachel; 3091

Gatzoulis, Michael A; 8235

Gauvreau, Kimberlee; 4093, 8176, 8181

Gayathri, S; 3097

Gaynor, J William; 3080

Gayretli, Gökçe Zeynep; 7054

Ge, Shuping; 7079

Gebauer, Roman; 3023, 7016, 7471

Gebauer, Roman A; 3023

Gebrehiwot, Tsegereda; 8179

Gedlu, Etsegenet; 3075

Geiger, Ralf; 4034

Geldenhuys, Agnetha; 7170

Gellatly, Marcelee; 6183, 6184

Gendron, Roxanne; 4052, 8051, 8202

Gentles, Thomas; 3015, 4066, 7102, 7206, 7394, 8230, 8383

George, Kristen; 3047

Getz, Elisabeth; 6101, 8217

Geva, Judith; 6197

Gewillig, Marc; 4110, 6022, 8072, 8073, 8289,8325

Gewitz, Michael H; 4036

Ghanayem, Nancy; 6197

Ghanayem, Nancy S; 3008

Ghanta, Sujana; 8287
Ghathi, Musa A; 7270

Ghez, Olivier; 7183

Ghobaa, Naima I; 7270, 8374

Giacomuzzi, Carmen; 6208

Giamberti, Alessandro; 1127, 2010, 4106 , 7414, 8078, 8086

Giannakoulas, Georgios; 8235

Giannico, Salvatore; 8234

Giannopoulos, Nicholaos M; 8205, 8238

Gibson, Richard; 7136

Giese, Rachel A; 3051

Gil, Hong-Ryang; 7490

Gilík, Jirí; 6046, 7471

Gill, Andy; 6075

Gillespie, Matthew J; 4007, 6077, 6078

Gillett, Christopher; 7147

Gimigliano, Fabrizio; 8230

Ginns, Jonathan; 8202, 8243

Ginther, Richard M; 7207, 7215

Giordano, Ugo; 8190, 8230

Girotra, Sameer; 7233

Gittenberger-de Groot, Adriana C; 6107. 8297

Gitter, Roland; 7091

Giuffre, Michael; 7019

Givtaj, Nader; 8253

Gladman, Gordon; 6025, 6026, 7189, 7433

Gleimer, Elena; 8221

Glezlova, Anna; 6120

Gnanapragasam, James; 7479, 7480

Goerler, Heidi; 7213

Goh, Lay Kian; 8231

Goh, Tiow H; 4032, 7103, 8102

Gokalp, Selman; 6041, 7151

Golden, Alex; 7204, 7221

Gonçalves, Daniel M; 8314

Gonçalves, Rafael M; 8218

Gonzalez, Rodrigo; 7035

Gonzalez, Sonia; 8004

Gordon, Lavinia; 3057

Gorenflo, Matthias; 4110

Gorney, Ronald M; 7207, 7215

Gotoh, Kenjiro; 8311, 8328

Govindarajan, Arunkumar; 6068, 6169, 6170, $8081,8275,8389$

Gowda, Srinath T; 4107

Goyal, Puneet; 6002, 6006

Gracii, Vernon M; 6158

Grady, Anita M; 7104

Grajek, Stefan; 7011, 7012, 8266, 8267

Grant, Brian; 6067, 6227

Grant, Elena; 8050

Graziano, Pedro; 8240

Greco, Ruben; 8049, 8413

Greeley, William J; 2102

Greil, Sabine; 3019

Greutmann, Matthias; 4114, 8261

Greve, Gottfried; 3071

Grewal, Jasmine; 8262

Grigg, L; 6130

Griselli, Massimo; 8196

Grosse-Wortmann, Lars; 3032, 3045,

3115,6067

Grossman, William; 8287

Gruber, Peter; 3080

Grünig, Ekkehard; 4035

Gruenwald, Colleen; 6204

Grueso, Josefina; 8319

Grutter, Giorgia; 8190, 8237, 6186

$\mathrm{Gu}$, Xiaorong; 6186

Guan, Yong Mei; 6186, 8173

Guerguerian, Anne-Marie; 6204

Guerrero, Rafael R; 8223

Guhathakurta, Soma; 6164, 7154 
Gui, Yonghao; 3127, 4101, 7088

Guimarães Jr, Hilton S; 7409

Guimond, Jeanette M; 6187

Guirgis, Maurice; 4072

Guler Eroglu, Ayse; 6041, 7151

Guleserian, Kristine J; 7207, 7215

Gunal, Nazlihan; 7254, 8122

Gunn, Julia K; 2100, 3007

Gunness, Sunil; 8181

Guo, Ying; 8298

Gupta, Nirmal; 8299

Gupta, Reetesh; 7233

Gupta-Malhotra, Monesha; 7413

Gut, Krystyna; 7034

Gutierrez, Gerardo; 4037

Guzik, Przemyslaw; 7058, 7059

Guzman, Maria Luisa; 7332

Haas, Felix; 7098

Habib, Ricardo G; 7005, 7006

Haddad, Jorge; 7477, 7478

Hadibrata, Hariadi; 7216

Haecker, Stephan; 7428

Haenzka, Marie -Christin; 4103

Hager, Alfred; 7128

Haickel Nina, Rachel V; 7408, 7409, 8218, 8219

Halit, Velit; 8158

Hall, Kathleen; 4115

Halliday, Rober; 3114

Hamada, Hiromichi; 7299

Hamada, Satoshi; 7217

Hamajima, Akito; 7458

Hamamichi, Yuji; 6029, 8138

Hambsch, Jörg; 4103, 8379

Hamela-Olkowska, Anita; 6023, 6048, 7003

Hamer, Marion E; 6221

Hamid, Muhammed; 8064

Hamilton, Carole; 6207

Hamilton, JRL; 7112

Hamilton, Leslie; 8196

Hamilton, Mark; 4033

Hamilton, Robert; 7026

Hamza, Hala; 8054, 8103

Hamzeh, Rabih K; 8048

Han, Ui-Jeong; 7086

Haniu, Hisanori; 7105

Haniu, Yoshinori; 7344

Hanjoora, Vijay M; 8150

Hanke, Thorsten; 2103

Hanséus, Katarina; 7004

Happonen, J-M; 7017

Haq, Anwar U; 3116

Haque, Akhbar; 8012

Hara, Masanori; 7217

Harada, Yorikazu; 7218

Harahap, Eva M; 7216

Hardikar, Ashutosh A; 8154

Harris, Catharine; 7094

Harris, Kevin C; 3117

Harris, Matthew A; 4007, 6077, 6078

Harrison, Tondi M; 7106, 7325

Hartas, Georgios; 7413

Hartyánszky, István; 7219

Hasan, Asif; 3116, 7112, 8196

Hasegawa, Satoshi; 7105, 7344

Haseyama, Keiji; 7345, 8279

Hashida, Yuichiro; 7127, 8157

Hashiguchi, Teruto; 7348

Hashimoto, Kazuhiro; 7473

Hassan, Amina; 7156

Hata, Akira; 7299

Hatakeyama, King-ya; 7345, 8279

Hatakeyama, Shinitu; 7458
Hattabaugh, Kelly; 7099

Hattam, Andrew T; 8290, 8291, 8300, 8301, 8312,8322

Haugen, Bjørn Olav; 4022

Haun, Christoph; 3083, 3086, 7160

Haw, Marcus; 2009, 6059, 7479, 7480

Hawkins, Lois; 6178

Haworth, Sheila G; 8156

Al-Hay, Amira AAH; 8118

Hay, Margaret; 6228, 6231, 6232

Hayabuchi, Yasunobu; 7250

Hayakawa, Hidetoshi; 3090, 8153, 8162

Hayashi, Kitami; 7299

Hayashi, Tamaki; 7220

Haynes, S R; 7112

Hazeki, Daisuke; 7291

He, Chunlan; 6210

He, Pingping; 6186, 6188

He, Yan; 6150

Heidbuchel, Hein; 8072

Heinle, Jeffrey S; 3028, 6003, 6004

Heinonen, Sarah; 7141

Heitz, François; 7110

Helden, Liz; 6189, 6190

Helmig, Sarah; 7002

Henderson, Mark; 7203

Hendson, Willy; 8023

Hennein, Hani A; 7203, 7204, 7221

Henriques, Sandra S; 7209, 7210, 7211

Henson, Janine; 7099

Herceg-Cavrak, Vesna; 7255

Hernandez, Ignacio; 6054

Hernandez, Sergio; 7205

Herrera, Maria; 6054

Herrera, Maria Dolores; 8033

Hess, John; 7337

Hetzer, Roland; 3121, 7405, 8021

Heusser, Felipe; 8101

Hewitson, John; 7222, 7492

Heying, Ruth; 4110, 8073

Hickey, Michael J; 4084

Hickey, Patricia A; 4093

Hidvégi, Erzsébet; 7107

Hiew, Chee Chin; 3026

Higaki, Takashi; 6156, 7273, 7456, 8105, 8151

Higashi, Kouji; 7303

Higuma, Tomonori; 7230, 7420, 7421

Hiippala, Anita; 7017

Hijazi, Ziyad M; 8410

Hill, Rebecca; 6211

Hill, Samantha J; 6007, 7223

Hillege, Hans L; 8148

Hinchliffe, Mary; 7267, 7268

Hinokiyama, Kazuhiro; 3055, 7417

Hiramatsu, Takeshi; 3119

Hirono, Keiichi; 6223, 7108, 7159, 7296, 7489, 8013, 8139

Hirose, Akiko; 6024, 7027

Hirose, Keiichi; 7224, 7226

Hirsch, Rafael; 8220, 8221

Hirth, Asle; 3071

Hisamochi, Kunikazu; 8359

Hislop, Alison A; 8156

Hjortdal, Vibeke; 4080, 6226

Ho, Chi-Lin; 8097

Ho, Siew Yen; 3094, 8235, 8276, 8281, 8282

Ho, Ting Fei; 7049, 8409

Hochbergs, Peter; 4108

Hoel, Tom N; 7374

Hoendermis, Elke S; 8127

Hörer, Jürgen; 2103, 7188

Hörnsten, Rolf; 7004

Hofbeck, Michael; 7068, 7403, 7404
Hoffman, Piotr; 8227, 8228

Hogan, Kimberly A; 6005

Holland, Andrew; 3114

Holmstrøm, Henrik; 4035

Holtby, Helen; 3032, 6010, 6133

Honda, Takafumi; 7299

Honda, Yoshihiro; 7466

Hong, WJ; 6090

Hong, Haifa; 7375

Hong, Wen-Jing; 6127, 7083, 7084, 8392

Hong, Young Mi; 8140, 8141

Hongeng, Suradej; 8147, 8194

Hope, Sarah A; 4023

Horita, Norihisa; 7345

Horlick, Erick M; 4059

Hornung, Tim S; 7045, 7144

Horton, Stephen B; 6215

Horvath, Elizabeth; 7313

Hoschtitzky, Andreas; 7195, 7433

Hoshiai, Minako; 7466

Hoshina, Satoshi; 7344

Hou, Hai-jun; 7302, 8159, 8160, 8269, 8270 , 8402

Houde, Christine; 4061

Houghton, Kristin M; 7267, 7268

Hrachovy, Richard A; 6004

Hraska, Viktor; 3083, 3086, 7160

Hsieh, Kai-Sheng; 7077, 7078, 7301, 7423

Hsu, Daphne T; 8006

Hsu, Jong-Hau; 8134

Hsu, Pei-Chen; 7225

$\mathrm{Hu}$, Shengshou; 7148, 7491

Hu, Xiufen; 7082

Hua, Yu-Chuan; 6222

Huang, Ching-Shan; 8302

Huang, Fei Qiong; 8231

Huang, Feiqiong; 6150

Huang, Guo-ying; 3088, 6080, 6096, 6097. $6098,7082,7274,7285,8107,8350$

Huang, Jing; 4036

Huang, Lianjun; 8117

Huang, Mei-rong; 3088, 6071, 7274, 8100, 8348

Huang, Mi-Shu; 6155

Huang, Min; 3088, 7274

Huang, Rimao; 3029

Huang, Rong; 7207

Huang, San-Kuei; 7358, 7359

Huang, Shiang-Suo; 7079, 7080

Huang, Shin-Chung; 7358, 7359

Huang, Xupei; 8326

Huang, Yu-Chih; 6141

Huberth, János; 7107

Hubinont, Corinne; 6016

Hucin, Bohumil; 7471

Hübler, Michael; 8021

Hughes, Marina; 4067, 6008, 6081

Hugues, Nicolas; 7110

Huh, Joon; 8306

Huh, June; 6196, 7056, 8011, 8108, 8143

Huhta, James C; 6036

Human, Derek G; 3117, 7267, 7268, 8175, 8184

Human, Paul; 7170

Humpl, Tilman; 6204

Hung, Chi-Ren; 8349

Hung, Liang Choo; 7259, 8056

Hunt, Rod; 2100, 3007

Hunter, Jill V; 3028, 6003

Hurh, June; 7240

Husayni, Tarek; 4086, 8034

Hussain, Ijaz; 6082

Hussain, Yasser; 8054, 8103

Huynh, Daniel; 3085, 7443 
Hwang, Betau; 6084, 7225

Hwang, Da-Cheng; 7077

Hwang, Haw-Kwei; 7482

Hwang, Joo Min; 6196

Hyun, Hye Sun; 7056, 8108

Iacono, Carola; 8384

Ibrahim, Jill; 6162

Ibrahim, Mohamed E A; 7155, 7434

Ibrahim, Reda; 8052

Ibuki, Keijirou; 7108, 7296, 8139

Ichida, Fukiko; 4070, 6223, 7108, 7159,

7296, 7489, 8013, 8139

Ichihara, Yuki; 7232

Ichikawa, Hajime; 8303

Ichimori, Hiroaki; 8020, 8303

Ida, Hiroyuki; 7067

Ide, Yujiro; 7224, 7226

Iemura, Motofumi; 6024, 7027, 7275

Ignjatovic, V; 8315, 8316

Iguchi, Hiromi; 8109

Ikeda, Aki; 8258

Ikegami, Ei; 7153

Ikeyama, Yukiko; 3090

Ilbawi, Michel; 6134, 7227, 8034

Ilhan, Mustafa; 7318

Illyés, Miklós; 7107

Ilyin, Vladimir N; 7228, 7229

Imagawa, Hiroshi; 6156

Imai, Yasuharu; 2063, 3119, 7232

Imanov, Elnur; 8042, 8043, 8080

Imoto, Hiroshi; 7446

Imoto, Yutaka; 7354, 7416

Inage, Akio; 6088, 6115, 6117

Inai, Kei; 7339, 8222

Inamura, Noboru; 6029, 8138

Inder, Terrie; 2100, 3007

Infusino, Tommaso; 8077

Ing, Frank F; 4087, 8110

Inoue, Nao; 6050, 6116

Inoue, Takeshi; 7230, 7420, 7421

Inoue, Youichi; 7447

Iorio, Fiore; 8237

Iribarren, José R; 7136

Irving, Claire; 7111, 7112

Iserin, Franck; 4072

Ishibashi, Naoko; 7113

Ishibashi, Nobuyuki; 3030, 6213

Ishida, Hidekazu; 8020, 8303

Ishihara, Kazuaki; 3119, 7232

Ishii, Eiichi; 7273, 7456, 8105, 8151

Ishii, Masahiro; 6089, 7276

Ishii, Tetsuko; 8258

Ishii, Yoichiro; 7231

Ishikawa, Shiro; 6104, 7114, 7335, 8241

Ishikawa, Yuichi; 6104, 7114, 7335, 8241

Ishino, Kozo; 7449, 7450

Itam, Amin; 6057

Ithuralde, Alejandro; 7031

Ithuralde, Mariano; 7031

Itoh, Yukako; 8018

Ivanov, Jaroslav Y; 7441

Iwasa, Shizuko; 7232

Iwasaki, Tatsuo; 6151, 6166, 6172

Iwata, Yusuke; 3030

Iyer, Krishna S; 7233, 7340, 7341

Iyer, Parvati; 7233, 7340, 7341

Jackson, Shaun; 4084

Jacobs, Jeffrey P; 7462, 8176

Jacobs, Marshall; 8176

Jacobsen, Elisabeth B; 6226

Järvelä, Merit; 8367

Jagiellowicz, Dorota; 7115
Jagtap, Ranjit R; 8154

Jain, Shreepal; 7101, 7369, 8280

Jákli, Györgyi; 7107

Jalali, Homayoun; 8259

James, Matthewson; 8110

James, Paul; 3057, 7001

Jan, Sheng-Ling; 6155, 7284, 8097

Jang, So Ick; 3082, 7342

Janiec, Izabela; 7355, 7356

Janousek, Jan; 3023, 3075, 4103, 6121, $6123,7016,8183$

Januszewska, Katarzyna; 3019, 8192

Jardim, Fernanda S; 8366

Jardim, Maria Fernanda S; 8062, 8063

Jarek, Joanna; 8267

Jarosik, Piotr; 7401

Jarranganth, M; 7181

Jarvis, Jonathan C; 7189

Jarvis, Steve; 6204

Jatene, Ieda B; 7234

Jatene, Marcelo; 7234, 7235, 8193, 8365

Jaunsumrit, Ampiwan; 8178

Javois, Alexander J; 4086, 8034

Jehlicka, Petr; 7277

Jellyman, Tim; 8383

Jen, Wen-Yen; 7078

Jenkins, Kathy; 4003, 4093, 8176, 8181

Jenkins, Petra; 8239

Jensen, Steen M; 3112, 7014

Jeong, Soo In; 7056, 8011, 8108, 8143

Jeswani, Kanhaiya Himatlal; 8036, 8040, 8387, 8388

Jhang, Won-Kyoung; 7236

Ji, Gang; 6174, 6188

Jia, Bing; 6080, 6096, 6097, 6098, 6210, 7285,8350

Jiang, Qiu; 4101

Jiang, Shiliang; 8117

Jin, Denan; 8307

Jin, Dong Kyu; 7056

Jin, Hongfang; 8144, 8304

Jögi, Peeter; 4108, 7238

Joffe, Ari R; 8198

Johansson, Jens; 7238

Johansson, Sune B; 7238

Johnsen, Søren P; 6226

Johnson, Robert A; 6211

Johnston, L; 8315, 8316

Jokinen, Eero; 3011, 7093, 8329, 8367

Jonas, Richard A; 3030, 3051, 3052, 6213

Jones, Bryn; 6171

Jones, Caroline B; 6025, 6026

Jones, Timothy; 2064, 3021

Jonzon, Anders; 7124

Joo, Kunihiko; 3055, 7417

Joo, Kunitaka; 7354, 7416

Jordan, Brigid; 3050, 6220

Joshi, Prashant; 6142, 8271

Joshi, Suresh; 6154, 7101, 7239, 7369

Ju, Da-Hye; 6027, 6028

Juan, Chien-Chang; 6084

Jun, Tae-Gook; 6196, 7186, 7240, 7457, $8011,8108,8143$

Juneja, Rajnish; 6109, 7297, 7451, 8254, 8381

Jung, Mi Jin; 8133

Jurca, Mauro C; 7478

Jureidini, Saadeh B; 6177, 8096

Justo, Robert; 6061, 6183, 6184

Jutras, Luc; 4113, 6007

Juvekar, Nilesh M; 8154

Jystad, Marte A; 6224

Kádár, Krisztina; 7219

Kadlec, Jakub; 8223
Kadner, Alexander; 7311

Kado, Hideaki; 3055, 6104, 7335, 7417, 8241

Kado, Joseph; 6108, 7333

Kadono, Toshie; 7466

Kadota, Akane; 6029, 8138

Kaga, Shigeaki; 7466

Kaisar, Jillian; 6193, 8233

Kajimura, Ichige; 6050, 6116, 7218

Kakadekar, Ashok; 6218, 7019

Kakita, Mari; 7241, 8152, 8155

Kalam, Shamsuddin Abul; 7461

Kalangos, Afksendiyos; 7402, 7460

Kallio, Katariina H; 3011

Kamada, Masahiro; 8111, 8331, 8359

Kamberi, Suleman; 7008

Kamisago, Mitsuhiro; 7116, 7153

Kammache, Issam; 7092

Kanakis, Meletios A; 8205, 8238, 8310

Kanamaru, Hiroshi; 6085, 7028, 7260, 8109

Kanbara, Tamotsu; 7250, 8163

Kanchi, Vasudevan; 7057, 8335, 8336, 8337

Kandavello, Geetha; 4031, 6057, 6082, 8251, 8370,8371

Kanegane, Hirokazu; 7296

Kaneva-Nencheva, Anna I; 7018, 8224

Kang, I-Seok; 6196, 7056, 7240, 8011, 8108 , 8143,8306

Kang, Shin-Kwang; 7490

Kannan, Bhava RJ; 8094, 8378

Kannivelu, Arivalagan; 4060, 4071

Kansy, Andrzej; 2101, 7174, 7425

Kantoch, Michal J; 3059, 7019

Kantor, Paul; 3045, 3047, 3115, 8014, 8189

Kantorova, Andrea; 3083

Kanzaki, Susumu; 7127, 8157

Kapins, Carlos E B; 6086, 7210

Kaplanska, Anna; 6013, 6017

Kappanayil, Mahesh; 3096, 8146, 8305

Karaci, Ali R; 7166

Karademir, Selmin; 6053, 7145, 7315, 7370,

$7371,8038,8039$

Karadeniz, Ceyla; 8027

Karagkiozaki, Varvara; 6087

Karasawa, Kensuke; 6085

Karavannacos, Panayiotis E; 8310

Kare, Mahendra Achyut; 8231

Karimova, Ann; 6153

Karlsson, Marcus; 7004

Karthikeyan, Ganesan; 8254

Kasahara, Shingo; 6172, 7196, 7208, 7242, 7243, 7244, 7397, 7398, 7447, 7449, 7450, 7472, 8098

Kasar, Pankaj; 6154, 7101, 7239, 7369, 8280

Kashiwagi, Hiroko; 7312

Kasparian, Nadine A; 6225

Kasuga, Ai; 8279

Kaszuba, Agnieszka; 7009, 7010

Kato, Taichi; 4039, 8119

Katsimpoulas, Michael; 8310

Katsube, Yasuhiro; 7116, 7153

Katsumata, Takahiro; 7241, 8152, 8155

Katta, Umadevi S; 4036

Katz, Uriel; 7193

Kaufholz, Denise; 6200

Kaufman, Jon; 6162

Kaulitz, Renate; 7403, 7404

Kaushal, Sunil; 7340

Kaushal, Sunil K; 7233

Kawabata, Takuya; 7243, 7244

Kawachi, Kanji; 6156, 7456

Kawada, Masaaki; 7449

Kawahira, Yoichi; 7384

Kawalec, Wanda; 7009, 7029, 7115, 7174

Kawamoto, Shunsuke; 7445 
Kawamura, Naohisa; 6105

Kawano, Yoshifumi; 7291, 7348

Kawasaki, Shiori; 8099, 8216

Kawasoe, Yasutaka; 6230, 7037, 8256

Kawata, Hiroaki; 8138

Kawazu, Yukiko; 6029, 8138

Kaya, Erkan; 8246

Kayatani, Futoshi; 6029, 8138

Keatley-Clarke, Anne; 6224

Keenan, Niamh M; 3031, 7246

Keijirou, Ibuki; 8013

Kellas, Heather; 6191

Kemmotsu, Yasushi; 7296

Kempfert, Jörg; 7185

Kene, Nandkumar; 8025

Kenny, Damien; 4033, 7147

Kepski, Roman; 7010

Kerkar, Prafulla G; 8112

Kerkovits, Lorand; 7313

Kermeen, Fiona D; 4115

Kerr, Alan; 8168

Kerr, Steven; 6211

Keshavjee, Shaf; 6204

Kessel, Maryanne W; 8167

Khairy, Paul; 3061

Khalili, Yasaman; 8358

Khambadkone, Sachin; 4085, 8019, 8353

Khan, Asim; 3076, 3116, 7247

Khan, Khalid; 7072

Khan, Mansoor; 3116, 8064

Khan, Mohammad D; 8075

Khan, Sabir; 7461

Khan, Sohail A; 8064

Khan, Zakiya; 6154, 7101, 7239, 8012

Khanenova, Valentyna; 8229

Kharbanda, Raj K; 8320, 8324

Khetrapal, C L; 8299

Khin, Lay Wai; 8231

Khongphatthanayothin, Apichai; 7020, 7164, 7300

Khoo, Nee Scze; 4065, 4066, 6088, 6117

Khoshhal, Saad; 6087

Khositseth, Anant; 7119

Khowsathit, Pongsak; 7123, 8194

Ki, Chang-Suk; 8306

Kiaffas, Maria; 6030, 8113, 8114, 8123

Kido, Sachiko; 1126, 7336

Kiess, Marla; 7047

Kiguchi, Hisako; 8111, 8331, 8359

Kikuchi, Toru; 7105

Kil, Hong-ryang; 8164

Kilic, Zubeyir; 7050, 8121

Kim, Ahm; 6027

Kim, Chong A; 7281, 7282

Kim, Gi Beom; 7023, 7280, 8225

Kim, Hae Soon; 8140

Kim, Kwan Chang; 8140

Kim, Mi-kyung; 7062

Kim, Nam Kyun; 7120, 7488, 8332, 8333

Kim, Siho; 7248, 7249

Kim, Soo Jin; 3082, 7053

Kim, Sujin; 7342

Kim, Sung Hoon; 8306

Kim, Sung-Hae; 8334, 8390

Kim, Wook Sung; 7186, 7457

Kim, Woong Han; 8225

Kim, Yong Jin; 8225

Kim, Yong-Ho; 7490

Kim, Yong-min; 8164

Kim, YongBum; 7022

Kim, Young Jin; 7488

Kim, Young-Hwue; 7236

Kim, Young-Nam; 7086

Kim, YoungHwue; 7022
Kim, Yuria; 7120

Kimura, Sachiko; 8279

Kimura, Sumito; 6089

King, Ingrid P; 8128, 8166

King, Terry D; 7002

Kiraly, Laszlo; 8177

Kirawittaya, Tawatchai; 8368

Kirilmaz, Ata; 7368

Kirk, Edwin P E; 6225

Kise, Kohsuke; 7466

Kishi, Kanta; 6105, 7100, 8152, 8155, 8307

Kishimoto, Hidefumi; 7446, 8138

Kishimoto, Sayuri; 7354

Kishimoto, Shintaro; 6024, 7027, 7275

Kishiro, Masahiko; 7316, 8022, 8099, 8216, 8244

Kitagawa, Tetsuya; 7250, 8163

Kitahara, Junichiro; 7159

Kitaichi, Takashi; 7250, 8163

Kitamura, Noriko; 6035

Kitano, Masataka; 6103

Kitaoka, Chika; 7310

Kitaoka, Chisato; 7310

Kitayama, Hitoshi; 7410

Kitchiner, Denise; 6026

Kiziltas, Alev; 8362

Klamt, Jyrson; 7477, 7478

Kleinsteuber, Karin; 8017

Klemencz, Hajnalka; 7107

Klenk, Dore; 3020

Kniewald, Hrvoje; 7021, 7055, 7090, 7091, 7337, 8285

Knight, Brodie; 8050

Knowles, Michael R; 3051

Knox, Patty; 6178

Knudson, Chelsea L; 8008

Knutsen, Mona; 6101, 8217

Ko, Jae-Kon; 7022, 7236

Kobayashi, Tohru; 7231, 7278, 7458

Kobayashi, Tomio; 7231, 7278, 7458

Koc, Murat; 6053, 7145, 7370, 7371, 8038 , 8039

Koca, Bulent; 6041, 7151

Kocis, Keith; 8182

Kodandaram Sastry, Usha M; 8066

Koene, Bart; 7424

Kogaki, Shigetoyo; 8020, 8303

Koh, Angela S; 8226

Koh, Carline; 6090

Kohli, Vikas; 8103

Koike, Kenichi; 8018

Koirala, Bhagawan; 7251

Koirala, Ramesh Raj; 7251

Koitabashi, Yasushi; 8311, 8328

Kojucharov, Ivailo R; 7018

Kolcz, Jacek; 3020, 7252, 7429

Kolesnik, Adam; 6031, 6037, 8283

Komada, Yoshihiro; 3090, 4039, 8119, 8153, 8162

Komagamine, Masahide; 7418

Kondo, Maiko; 8361

Kondrachuk, Oleksandr; 6064, 6091, 6092 , 7441

Konduracka, Ewa; 8248

Koneti, Nageswara Rao; 7057, 8335, 8336, 8337

Konishi, Akinobu; 7445

Konishi, Kyoko; 7273

Konuma, Takeshi; 2063, 7418

Koo, Hee Sun; 8141

Kook, Hyun; 7013

Kopala, Marek; 3118, 7365, 7400, 7401, 7366

Kopf, Gary S; 7392

Kordon, Zbigniew; 3019, 6017
Kornerup Hansen, Axel; 7322

Kornouhov, Oleg J; 7229

Kornowski, Ran; 8221

Kostelka, Martin; 3023, 3046, 3075, 7185 , 8183, 8379

Kotani, Yasuhiro; 7196, 7208, 7242, 7449, 7450

Koteda, yusuke; 7275

Kotei, David; 7197, 7198

Kothandam, Sivakumar; 8132

Kothari, Shyam S; 6109, 7297, 7451, 8254 , 8381

Kovacs, Adrienne H; 8261

Kowalik, Ewa; 8227, 8228

Kowalski, Miroslaw; 8227, 8228

Koyama, Nobuya; 7217

Kozlik-Feldmann, Rainer; 3019

Kozlowski, Michal; 7174

Kraan, Roeland; 7306

Kramer, Hans-Heiner; 8376

Krauze, Tomasz; 7058, 7059

Kreitmann, Bernard; 4062, 7060, 7092, 7183, 8092

Kreutzer, Jaqueline; 8070

Krieger, Jose A; 8330

Kriett, Jolene M; 7426

Krishna R, Kumar; 3097

Krishnan, Ladish; 7279

Krjukov, Vladislav A; 7229

Kroft, Lucia JM; 6119

Krolikowski, Mary; 7325

Kruessell, Markus; 3058, 7367, 8103, 8339

Kubin, Joanna; 2102

Kubus, Peter; 3023, 7016

Kucera, Vladimir; 7470

Kucukarslan, Nezihi; 7368, 8340

Kucukosmanoglu, Osman; 6038, 8015, 8362

Kudo, yoshiyuki; 7275

Kudumula, Vikram; 6093, 6100

Kuehl, Karen S; 8268

Kuehne, Titus; 7128

Kula, Serdar; 7054, 7293, 7317, 7318, 8027,8158

Kuladhipati, Indra; 6109

Kulkarni, Snehal; 6154, 7101, 7239, 7369, 8012,8280

Kumar, Alka Anil; 8305

Kumar, Bobby; 7048, 7087

Kumar, Girish; 7233

Kumar, Niranjan; 7239

Kumar R, Krishna; 3096, 7279, 7349, 8093, 8094, 8095, 8305, 8378

Kuo, Shu-Min; 7079, 7080, 7081, 7423

Kupferschmid, John P; 7396

Kuptanon, Teeradej; 7119

Kuran, Joanna; 6013, 6017

Kuranobu, Hiroki; 7127, 8157

Kurihara, Yachiyo; 8311, 8328

Kurita, Yoshihiko; 8361

Kurkevych, Andrii; 8229

Kurobe, Hirotsugu; 7250, 8163

Kurosaki, Ken-ichi; 6103, 7030

Kurosawa, Hiromi; 2063, 3119, 7100, 7232, 7418

Kusa, Jacek; 7042, 8069, 8274, 8395

Kusmierczyk, Mariusz; 8227

Kutarski, Andrzej; 7009

Kutsal, Ali; 6053, 7145, 7370, 7371, 8038, 8039

Kuybulu, Ayca Esra; 7315

Kuzmenko, Yulia L; 8342, 8354

Kwak, Jae Gun; 7372

Kwon, Bo Sang; 7023, 7280 
L'Herault, Johanne; 6146

La Rotta, Gustavo; 6010

La-orkhun, Vidhavas; 7300

Laakso, Toby; 4021

Labombarda, Fabien; 6066

Lacey, Stephanie; 8182

Lacour-Gayet, Francois; 3083

Ladusans, Edmund J; 6211, 7195

Lafuente, Maria V; 7024, 7121

Lago, Paola; 7131

Lai, Jin-Shin; 8188

Laing, Iain; 8129

Lakshmy, R; 8254

Lal, Puroshattam; 4057

Lambert, Virginie; 7134

Lamberti, Anna; 4072

Lamberti, John J; 7052, 7137, 7149, 7426, 8037, 8048

Lan, Ching; 8188

Lane, Geoffrey K; 4032, 8088, 8315, 8316, 8344

Lange, Rüdiger; 2103, 7188, 7337

Lanjewar, Charan P; 8112

Larsen, Lars Allan; 4080

Larsen, Signe H; 6226

Larson, Joshua M; 8287

Laskari, Cleo; 6030, 8123

Latiff, Haifa; 8251, 8370, 8371

Latson, Larry; 4107, 8344

Latus, Heiner; 7068

Lau, K C; 7036

Lau, Kimbee; 8271

Laura, Juan P; 7133

Lavoie, Josee; 6176

Law, Ian; 7026

Lawrenson, John; 7122, 7222, 7306, 7492

Lax, Daniela; 8008

Layangool, Thanarat; 8368

Lazarov, Stojan D; 7018

Le, Thu-Thao; 8260

Lê, Trong Phi; 4058

Leal, Gabriela N; 7281, 7282

Lean, Fiona; 3091

Leanage, Ranjit; 6093, 6100

Leatherbury, Linda; 3051, 3052

Lebid', Igor G; 7168, 8229

Lee, Bing-Tsun; 6141

Lee, Cha Gon; 8143

Lee, Chang-Ha; 3082, 7342, 7372

Lee, Cheul; 3082, 7372

Lee, Chul; 7342

Lee, Dong-Keun; 7362

Lee, Heung-Jae; 6196, 7056, 7240, 8011, 8108, 8143, 8306

Lee, Hyoung-Doo; 6160, 8345, 8346, 7464

Lee, Jeong Ryul; 8225

Lee, Jung-Ju; 7362

Lee, Kang-wook; 7053, 7062

Lee, Kyong-Jin; 4059, 4089, 6146

Lee, Kyong-Soon; 6146

Lee, Mi Kyung; 7362

Lee, O-kyung; 7062

Lee, Pi-Chang; 6084, 7225

Lee, Pil Ryang; 6027

Lee, Sang-yun; 8164

Lee, Shuk Han; 8242

Lee, Suk-Ki; 7490

Lee, Young; 7490

Lee, Young seok; 7249

Lee, Young Tak; 7186, 7457

Lee, Young-Jin; 7085

Lee, Yu Kyung; 6196

Lefi, Ahmad; 8372

Legge, Lea; 6178
Lei, Qian; 6175

Leigh, Magaret W; 3051

Leite, Diego Rafael B; 7409

Lema, Guillermo; 6212

Lennon, Diana; 3015, 6191

Leon, Luis; 7428

Leonard, Steven R; 7207, 7215

Leonardi, Benedetta; 8230

Leone, Cláudio; 7281, 7282

Lertsapcharoen, Pornthep; 7020, 7164, 7300

Lesch, Wiebke; 6224

Leshko, Jenny; 6036

Leske, Jane; 6197

Leung, Mande T; 7061

Levitsky, Sidney; 3056

Li, Bei; 3029

Li, Chi Keung; 7097

Li, Fen; 8100, 8348

Li, Hongyin; 2104, 7138

Li, Jia; 6133

Li, Jun; 6147

Li, Lihuan; 6175

Li, Ping-Chun; 7187, 7427

Li, Shoujun; 7148, 7373, 7491

Li, Shun-Hwa; 3008

Li, Wei; 8144

Li, Xiaohui; 8144

Li, Xiaokui; 8268

Li, Yan; 7148

Li, Yi-Ching; 7079, 7080

Li, Yue; 8269, 8402

Li, Yun; 8100, 8348

Liakopoulos, Oliver; 7469, 8293

Liang, Xue-cun; 1128, 6080, 6097, 8107, 8350

Lianza, Alessandro C; 7281, 7282

Liao, Chung-Pin; 7080

Liao, Shih-Cheng; 6222

Lidov, Hart GW; 3030

Lilley, Stuart; 8050

Lillie, Christian; 3083

Lim, Bee Chian; 8231

Lim, Heungguk; 7342

Lim, Hong-Gook; 3082, 7372

Lim, In-Seok; 6025, 6026, 7362

Lim, Joyce SE; 6025, 6026

Lim, Kyoung Ah; 8140, 8141

Lim, Seung-Pyung; 7490

Lim, Vivien YJ; 7049

Lim, Zek; 6059, 6069, 6087

Lima, Cesar A; 7005, 7006

Lima, Danielle D; 7005, 7006

Limsuwan, Alisa; 7123, 8147, 8178, 8194

Lin, Chu-Chuan; 7077

Lin, Lin; 6011

Lin, Ming-Chih; 6155, 7284, 8097

Lin, Ming-Tai; 8349, 8403, 8405

Lin, Shan-Miao; 7482

Lin, Shiou-Jeng; 6141

Linask, Kersti; 6036

Lindberg, Harald L; 7374

Ling, Jian; 8117

Ling, Ming-Tai; 8404

Lipinski, Wojciech; 7425

Lipshultz, Steven E; 3049, 4068, 4069, 8005, 8006, 8014, 8029, 8030

Lipsitz, Stuart R; 4069, 8005

Litwin, Mieczyslaw; 7115

Liu, Fang; 6098, 7041, 7274, 7285, 8107, 8350

Liu, Jinfen; 6174, 7375, 7376, 7483, 7484, $7485,7486,8130$

Liu, Liping; 6188
Liu, Po-Yen; 7077

Liu, Xiao-qin; 3089, 3092, 6080, 6097

Liu, Xinrong; 8130

Liu, Ye; 6210

Liu, Yi-qing; 6132

Liu, Yinglong; 7148

Liuba, Petru; 6058, 7322

Llano, Miguel; 8261

Lo, Cecilia W; 3051, 3052

Loberman, Dan; 7193

Loeckinger, Alex; 4034

Loeff, Marcus; 3019

Lombardi, Pier F; 7378, 7379

Long, Sandy; 3091

van Loon, Rosa Laura E; 4051, 8148

Lopes, Antonio Augusto; 7235, 8193

Lopes, José Anselmo C; 7408

Lopes, Lilian M; 6032, 7209, 7210, 7211

Losay, Jean; 7134

Loureiro, Marilia; 7066

Love, Donald; 3024, 4012

Løvstakken, Lasse; 4022

Lowe, Boris S; 4113

Lu, Ping; 6122, 7138

Lu, Yanan; 7375

Lu, Ying; 7041, 7285

Lu, Zhaohui; 8181

Lucas, Victor; 7026

Luciani, Giovanni Battista; 7380, 7381, 8206

Luckie, Matthew; 8239

Luckner, Doris; 8003

Luey, Brenda; 6191

Lugones, Ignacio; 7382

Luize, Christian M; 7005, 7006

Lun, Kin-Shing; 7083, 7084

Lundell, Bo; 7124

Lundin, Anders; 4108

Luo, Wanjun; 3029

Lupi-Herrera, Eulo; 4037

Lupoglazoff, Jean Marc; 4072

Lurz, Philipp; 4085, 4111, 8353

Lusson, Jean-René; 7110

Luz, Karina S; 7409

Lv, Tewei; 4082

Lv, Xiaodong; 7383

Lwabi, Peter; 8182

Lyon, Joy E; 4091, 6192

Ma, Jae-Sook; 7013, 7085, 7086, 7286

Ma, Xiao-jing; 3088, 6080, 6096, 6097, 6098, 7274,8350

Macbeth-Curtis, Nicole; 6201

Macciocca, Ivan; 3057, 7008

MacCormick, Judith D; 7206

Machado, carlos Eduardo E; 7409

Machado, Iván A; 7136, 8002, 8240

Machado, Ricardo; 7066

Mackie, Andrew; 4065, 6088, 6115, 6117

Macri, Nicholas P; 8344

Madalena, Celia; 7066

Madhusudan, G; 6163, 7328, 7476

Madiyono, Bambang; 8318

Maehata, Yoshito; 7384

Maekawa, Syu; 6029, 8138

Maeno, Yasuki; 6024, 7027

Magalhães, Manuel P; 7440, 7448

Magee, Alan G; 8360

Maghur, Hasan Ahmed; 7385

Magioli Bravo-valenzuela, Nathalie Jeanne; 6032

Magistrelli, Andrea; 8230

Magnier, Suzel; 4072

Mahadevan, Vaikom S; 8239

Mahajan, Rajiv; 7295, 8120 
Mahajan, Viresh; 8149

Mahesh, Kappanayil; 8093, 8095

Maheshwari, Sunita; 7455

Mahmoud, Fadia M; 8172

Mahmud, Elsayed; 7033

Mahnke, Christopher B; 7149

Mak, Wai Yau; 7097

Makarova, Lidiy F; 8327

Maksymenko, Andrey V; 6099, 8342, 8354

Malcic, Ivan; 7021, 7055, 7090, 7091, 7337, 8285

Malec, Edward; 3019, 8192

Malek, Malgorzata; 7174

Malhotra, Arun; 6110

Malm, Torsten; 7238

Malpas, Theresa; 6193, 8233, 8243

Maluf, Miguel Angel; 7386, 7387, 7388

Mammen, Isaac; 8112

Mani, Anna; 7152, 8016, 8150

Manlhiot, Cedric; 3032, 4059, 6010, 6135, 8185,8189

Manso, Paulo H; 7477, 7478

Marath, Aubyn; 6208, 7099

Marcelletti, C; 7481

Marcora, Simona A; 823

Marek, Jan; 6046, 6081

Marelli, Ariane J; 4113, 4116

Marianeschi, Stefano M; 7389, 8033

Marinovic, Branko; 8285

Markova, Mila I; 8224

Maroto, Enrique; 8049

Maroto, Erique; 8413

Marques, Juan A; 8002

Marrone, Chiara; 4030, 8384

Martín, Amando; 8002

Martin, Edward C; 6107

Martin, Gerard R; 3052

Martin, James; 7334

Martin, Robin; 4033, 7147

Martinez, Raquel; 6179, 6180, 6181, 6194, 6198, 6199

Martinez, Susana; 6180, 6181

Martinez, Teresa; 6194

Martucci, Giuseppe; 4113, 4116

Maruo, Ayako; 7230, 7420, 7421

Maruszewski, Bohdan; 2101, 7174, 7425 8176

Maruszewski, Przemyslaw; 2101

Maruyama, Ikuro; 7348

Maruyama, Junko; 4039, 8119

Maruyama, Kazuo; 4039, 8119

Marwah, Ashutosh; 7455, 7461

Maschietto, Nicola; 8179

Masini, Annaclaudia; 3124

Mason, Jennifer; 4114

Masuda, Kiminori; 7291, 7348

Masuhara, Hiroshi; 7217

Masura, Jozef; 6120, 8401

Masuya, Masahiro; 4039, 8119

Matejka, Tomas; 3023

Mathew, Rajamma; 4036

Mathias, Leigh; 6036

Mathioudakis, Diana; 7195

Mathur, Sujeev; 4071, 6093, 6100

Matsuda, Osamu; 7273

Matsuda, Yoshio; 8257

Matsuhisa, Hironori; 7159, 7489

Matsui, Mie; 6223

Matsuishi, Toyojiro; 6024, 7027, 7275

Matsumoto, Masahiko; 7466

Matsumura, Masaharu; 7028, 7260

Matsumura, Tomonori; 8109

Matsuo, Kozo; 7037, 8256

Matsuzaki, Tachiyo; 6223
Matthiassen, Wendy; 7306

Mattila, Ilkka; 8367

Mauri, Lugi; 7389

Mayer, Otto; 7277

Mayosi, Bongani; 7253, 7305, 7306

Mazurek-Kula, Anna; 8394

Mazzucco, Alessandro; 7380, 7381, 8206

Mc Laughlin, M; 6195

McBrien, Angela; 3126, 6033

McCanta, Anthony C; 3025

McCarthy, Karen P; 3094, 8235, 8276, 8281, 8282

McCrindle, Brian; 4059, 6135, 8185, 8189

McCrossan, Brian A; 6034, 6227

McCusker, Chris G; 6219

Mcewan, Angus; 6008

McGuinness, Jonathan; 3031, 7246

McKenzie, Emmitt D; 3028, 6003, 6004

McKirkle, J M; 6195

McLoughlin, Darren; 3031, 7246

McNeil, Keith; 4115

McPherson, Caroline; 8236

Meagher, Simon; 6043

Medeiros, Marcus V L; 7209

Medeiros, Patricia M V P; 7209, 7210, 7211

Medrano, Constancio; 8049, 8413

Mehta, Chetan; 4073, 4109

Meira, Zilda MA; 3016, 7288

Mekraz, Ibrahim A; 7270

Melendez Ramirez, G; 6063

Meli, Sara; 3021

Mellander, Mats; 7391

Mello, Dennis M; 7392

Menacho, Ramiro; 8070

Menahem, Samuel; 3050, 4032, 6012, 6042, $6043,6220,6228,6229,6231,6232$

Menaissy, Yasser Mohamed; 7393

Mendes, Vinicius G; 7408

Mendez, Armando; 7287

Mendoza, Alberto; 2102, 8033

Mendoza, Iván J; 8002

Meng, Chung-Chang L; 7225

Meng, XiangChun; 4063

Meraji, Mohmood; 8358

Merino, Waldo; 6212

Mervis, Jonathan; 7102

Mervis, Jonathan G; 7394

Mesia, C Igor; 8344

Messiah, Sarah E; 3049

Meta, Roberta; 8190

Metras, Dominique; 4062, 7060, 7092, 7183, 8092

Meyns, Bart; 4110

Mhatre, Amit; 6154

Miana, Leonardo A; 8062

Michael, Helen C; 6025, 6026

Michalak, Krzysztof; 3118, 8084, 8356

Micheletti, Angelo; 1127, 2010, 4090, 6021, 8076, 8087

Michelsen, Marie M; 8320, 8324

Michielon, Guido; 8237

Middleton, Lee; 7072

Miera, Oliver; 8021, 8411

Milanesi, Ornella; 3120, 7131, 8179, 8309

Milas, Vesna; 7090

Milhoan, Kimberly D; 7396

Milhoan, Kirk A; 7075, 7076, 7396

Miller, Paul; 4060, 4109, 8082, 8377

Miller, Tracie L; 3049, 4069, 7287, 8005, 8006

Milovanovic, Vladimir B; 6214

Minami, Noriaki; 8161

Minette, Mary; 7099
Mino, Yoichi; 7127, 8157

Mir, Thomas S; 7128

Miranda, Juan P; 8017

Mirdehghan, Abolghasem; 7165

Mirkowicz-Malek, Malgorzata; 7425

Miro, Joaquim; 4061, 8052

Misaki, Takuro; 7159, 7489

Mishali, David; 7193

Mishra, Nidheesh; 8112

Miszczak-Knecht, Maria; 7009, 7010, 7029

Mital, Seema; 8189

Mitani, Yoshihide; 3090, 4039, 6216, 7467

8119, 8153, 8162

Mitchell, Michael E; 3008, 8287

Mitropoulos, Fotios A; 8205, 8238, 8310

Mitsui, Hideya; 7244

Mitsushita, Norie; 8334, 8390

Mittal, B R; 7051

Miura, Takashi; 7232

Mivahara, Yoshinori; 7242, 7243, 7397, 7398

Miyamoto, Shelley D; 8197

Miyamoto, Takuma; 7418

Miyawaki, Toshio; 7108, 7296, 8013, 8139

Miyazaki, Aya; 7030

Miyazaki, Mizuo; 8307

Mizrahi, Eli M; 6004

Mizuno, Masanori; 8311, 8328

Mizuno, Yoshiko; 6230

Mludzik, Katarzyna; 3118, 7365, 7366, 7400

Moamenah, Tarek; 8060

Mochizuki, Hiroyuki; 7458

Möller, Thomas; 4035, 6101, 8217

Mohamed, Hatim; 7155, 7434

Mohamed, Murtada; 7399, 8203

Mohaupt, Saffloer; 4051

Mohr, Friedrich Wilhelm FW; 7185

Moledina, Shahin; 8156

Moll, Jacek; 3118, 7365, 7366, 7400, 7401 $8084,8085,8356,8394$

Moll, Jadwiga; 3118, 7365, 7366, 7400, 7401, $8085,8356,8394$

Moll, Maciej; 7365, 7366, 7400, 7401

Moller, James H; 8176

Molloy, Bernadette; 6219

Molloy, Frank; 8181

Molnar, Vincent; 7462

Moltedo, Jose M; 7031

Monagle, P; 8315, 8316

Monfredi, Oliver J; 8239

Monges, Soledad; 7024

Moniotte, Stéphane; 6016

Monti, Lidia; 8230

Montiel Galvan, Olga; 6138

Montigny, Martine; 7271

Moodie, Douglas S; 8201

Moon, Ju Ryoung; 6196

Moore, John; 7052, 7149, 7426, 8037, 8048

Moreau, Yves; 8325

Moreira, Adelino L; 8314

Moreira, Dalmo R; 7005, 7006

Moreira, Jorge M; 8357

Morelli, Carmela; 4030

Moreno, Guillermo; 8199

Moreno, Orlando; 8240

Moreno-Cabral, Carlos E; 7137, 7149

Morgan, Gareth J; 4033, 4089, 6227

Mori, Katsuhiko; 7314

Mori, Yasuhiko; 6105, 8152, 8155, 8307

Mori, Yoshiki; 7100, 8258

Morikawa, Akihiro; 7278

Morimatsu, Hiroshi; 6166

Morishima, Tsuneo; 8361

Morita, Kiyoshi; 6151, 6166, 6172

Morita, Kiyozo; 7067, 7473 
Morita, Yasuko; 7291, 7348

Morrison, Katherine M; 6189, 6190

Mortezaeian Langroudi, Hojjat; 8358

Mos, Krista; 3027

Mossinger, Hansjorg; 8177

Moszura, Tomasz; 7400, 8084, 8085, 8356, 8394

Mota, Cleonice CC; 3016, 7288

Mota, Conceição; 7066

Motara, Firosa; 8053

Motara, Firoza; 7292, 7307, 8023

Motoki, Noriko; 8018

Motoki, Tatsuo; 7250, 8163

Mouratian, Mariela; 7133

Mozol, Krzysztof; 7174

Mroczek-Czernecka, Danuta; 8248, 8249

Mugishima, Hideo; 6085, 7028, 8109

Mulla, Neda F; 7141

Munkhammar, Peter; 4024, 6113

Muñoz-Castellanos, Luis; 8247

Munro, Hamish M; 6005

Murakami, Arata; 7446

Murakami, Yasuo; 7113, 7314

Murakami, Yoshitaka; 7273

Murakami, Yosuke; 7384

Murao, Kikuko; 7456, 8105, 8151

Murmu, Udaycharan; 6163, 8250

Mussatto, Kathleen A; 6197, 8167

Muta, Hiromi; 7276

Muthurangu, Vivek; 4067, 4085, 8353

Muto, Ayako; 7299

Mutze, Kerstin; 8177

Muusfeldt Birck, Malene; 7322

Myers, Kim A; 7061

Myers, Patrick O; 7402

Mylonakis, Michael; 8205, 8238

Mynard, Jonathan P; 4016, 8312, 8313

Na, Lisha; 8231

$\mathrm{Na}$, Myung-Hun; 7490

Nadig, M; 6110

Nagasawa, Kelly K; 7137

Nagashima, Mitsugi; 6156, 7273, 7456, 8105

Nagatani, Koji; 7273

Nagdyman, Nicole; 8411

Nagesh, CM; 6110

Nagy, Zsolt; 7403, 7404

Naiman, Gerardo; 8195, 8199

Naito, Yuji; 3084, 3119

Nakada, Masayuki; 6035

Nakagawa, Naomi; 8111, 8331, 8359

Nakahata, Yayoi; 6089

Nakajima, Hiromichi; 7309

Nakamura, Makoto; 6104, 7114, 7335, 8241

Nakamura, Takahiro; 6085, 7260, 8109

Nakamura, Toshimi; 7310

Nakamura, Tsuneyuki; 7310

Nakamura, Yoshihide; 7032, 7038, 7039

Nakamura, Yoshikazu; 7260

Nakanishi, Toshio; 2063, 3119, 7100, 7232,

7339, 7418, 8222, 8257, 8258

Nakano, Takeshi; 8105, 8151

Nakano, Toshihide; 3055, 6104, 7335, 7417

Nakano, Yusuke; 6050, 6116

Nakata, Tomohiro; 7224, 7226

Nakazawa, Makoto; 7067

Nakhleh, Nader J; 3051

Nampoothiri, Sheela; 3096, 8305

Nandurkar, Harshal; 4084

Nantarakchaikul, Palinee; 7119

Napoleone, Pace; 7481

Naqvi, Nitha; 3098, 6102, 8019, 8360

Narasimhan, Chitra; 8066

Narayan, Ravi; 8393
Narayanan, Arul; 4073

Narita, Jun; 8020, 8303

Narula, Nidhi; 7295

Nashashibi, Mahmoud; 8168

Nasis, Arthur; 4023

Nassar, S; 7044

Nasser, Chadi; 8240

Nasseri, Boris; 3121, 7405, 8021

Navabi, Mohammad Ali; 7406

Navarini-Meury, Susanne; 7311

Navasundi, Girish Basavaraj; 8036, 8040, 8387,8388

Nawa, Nobutoshi; 7312

Neas, Katherine; 3024

Nebigil, Rusen Melih; 7145, 7370, 7371

Nederhoff, Marcel; 4102

Neef, Klaus; 8293

Neeland, Marc; 8201

Neghme, Rodrigo; 4064, 7035, 8004, 8017

Negishi, Yoshie; 7316

Negura, Diana; 2010, 4090, 8076, 8078, 8087

Nehgme, Rodgrigo; 6054

Nelson, David P; 3028, 6003, 6004

Nemeth, Agnes; 7313

Nemoto, Shintaro; 7241, 8152, 8155

Neri, Daniella; 7287

Nero, Graziela; 7440, 7448

Netz, Heinrich; 8192

Neukamm, Christian; 8217

Neves, Ana L; 6036, 8314

Newall, Fiona; 8315, 8316

Newcombe, Jennifier B; 7141

Ng, Ada X; 8260

Ng, Julie; 8175

$\mathrm{Ng}$, Lawrence; 3012

$\mathrm{Ng}$, Yin Ming; 8242

Ngernsritakul, Tawai; 8380

Nguyen, Nguyen Vu; 6134

Ngwezi, Deliwe; 7307, 8023

Nicholson, Ian; 3072, 7036, 7162, 8171

Nicholson, Ross W; 3015

Nicolae, Mugur I; 8202, 8243

Nicolas, Aeschlimann; 6212

Nielsen, Torsten T; 8320, 8324

Niemirska, Anna; 7115

Niewiadomska-Jarosik, Katarzyna; 6112

Nii, Masaki; 6035, 6103, 8334, 8390

Niijima, Shinichi; 8216

Niksch, Alisa; 6134

Nilsson, Boris; 7391

Nina, Vinicius S; 7408, 7409, 8218, 8219

Ning, Shou Bao; 7285

Nishigaki, Kyoichi; 7384

Nishiguchi, Kosuke; 7314

Nishijima, Eiji; 7421

Nishimura, Rick A; 7173

Nishino, Hiroshi; 6024, 7027, 7275

Nishino, Takako; 7410

Nishiyama, Mitsunori; 7113

Nisida, Hitoshi; 7039

Niszczota, Cezary S; 6037, 8283

Niwa, Koichiro; 6230, 7037, 8256

Noble, Stéphane; 8052

Nock, Hermine; 6224

Nogueira, Camila B; 8219

Nogueira-Silva, Cristina; 8125

Noh, Chung Il; 7023, 7280, 8225

Nolte, Lars; 7469

Nomura, Yuichi; 7291, 7348

Noonan, Patrick M; 4109

Nordmeyer, Johannes; 4085, 4111, 8353

Nordqvist, Peter; 6224

Norman, Bronwyn; 4094

Norman, Paul; 6075
Northam, Elisabeth; 3050, 6220

Nosal, Matej; 7411, 7412

Novak, Milivoj; 7021

Novick, William; 7205, 8181

Nowak, Cheryl; 6197

Noya, Belkisolé; 8002

Ntsinjana, Hopewell; 7292

Ntsinjana, Hopewell N; 8023, 8053

Nui, Akihiro; 8279

Numan, Mohammed T; 7033

Numano, Fujito; 7105, 7344

Nunn, Graham; 8171

Nuri, Halkawt; 1127, 7414, 8086, 8087

Nyayadhish, Prasanna Y; 8112

Nyrnes, Siri Ann; 4022

O'Brian, Richard; 8110

O'Brien, Kathy; 4115

O'Brien, Sean; 8176

O'Brien, Sharon E; 8030

O'Byrne, Michael L; 6158

O’Donnell, Clare; 3015, 8383

O'Leary, Patrick W; 3087, 7173

O’Meagher, Shamus; 8212

O'Sullivan J J; 7112

Ocal, Burhan; 6053, 7370, 7371, 8038 , 8039

Occleshaw, Christopher; 4066

Ochi, Masami; 7153

Ochiai, Yoshie; 7354, 7416

Ochoa, Manuel; 6063

Oda, Hisayuki; 6104

Oda, Shin-ichiro; 3055

Oda, Shinichiro; 7417

Odemis, Ender; 8041, 8042, 8044, 8079, 8080

Odermarsky, Michal; 7322

Oechslin, Erwin; 8261, 8262

Oemar, Hamed; 8169

Örmeci, Ahmet Rifat; 7315

Östman-Smith, Ingegerd; 8009, 8010

Özkan, Ragip; 8121

Ofori-Amanfo, George; 6158

Ogawa, Shunichi; 7116, 7153

Ogier de Baulny, Hélène; 4072

Oguz, Aynur; 8027

Oguz, Deniz; 6159, 7054, 7293, 7315, 7317, 7318, 8027

Ohashi, Hiroyuki; 3090, 4039, 6216, 7467,

$8119,8153,8162$

Ohno, Naoki; 8361

Ohno, Takuro; 7354, 7416

Ohta, Masaaki; 6156, 7456, 8105, 8151

Ohtaka, Masao; 7316, 8244

Ohtsuki, Masahiro; 7316, 8022, 8099, 8244

Ohtsuki, Shinichi; 8361

Ohuchi, Hideo; 7030, 8245

Okada, Tomoo; 6085

Okada, Yoko; 8020, 8245

Okamoto, Yoshio; 8361

Okamura, Toru; 3030, 6213, 7232, 7418

Okazaki, Mieko; 6044

Oko-Lagan, Jolanta; 7034

Okumura, Kenichi; 6105, 8152, 8155, 8307

Okutan, Vedat; 8340

Olaly-Nyakundi, Elizabeth; 6158

Olejnik, Peter; 6120, 8401

Olgar, Seref; 7315

Olguntürk, Rana; 7054, 7317, 7318, 8027, 8158

Olgunturk, Rana F; 6159, 7293

Oliveira, Patricia M; 7234

Olmos, Mario C C; 7211

Omeje, Ikenna Chima; 7411, 7412 
Ommen, Steve R; 7173

Omrani, Gholamreza; 8253, 8358

Ono, Masamichi; 7213

Ono, Shin; 8151

Ono, Yasuo; 6035, 7320, 8334, 8390

Onouchi, Yoshihiro; 7299

Ontoseno, Teddy; 8373

Ooba, Osamu; 8359

Oowada, Natsuko; 6089

Oprea, Valéria; 7219

Orav, John; 4069, 7287

Oricchio, Gianluca; 8237

Orjuela, Manuela; 8200

Orr, Yishay; 7419

Orun, Utku Arman; 6053, 7145, 8038, 8039

Osaki, Masaki; 6035, 7224, 7226, 7320

Oshima, Yoshihiro; 6223, 7230, 7336, 7420, 7421

Osman, Hanif; 3026

Osmancik, Pavel; 4103

Ostrowska, Katarzyna; 8085

Ostrowsky, Jacob; 7203

Ota, Noritaka; 7223

Otaka, Shingo; 7489

Otani, Tstsuya; 7278

Otsuki, Shin-ichi; 7208, 7450, 8098

Otto, Maureen; 8167

Ovroutski, Stanislav; 8411

Oyamada, Jun; 6044

Oz, Bilgehan S; 8246, 8340

Ozaki, Noriyasu; 7032, 7038, 7039

Ozal, Ertugrul; 7368, 8340

Ozawa, Hideki; 7241, 8152, 8155

Ozawa, Tsukasa; 7217

Ozawa, Yuki; 7384

Ozbarlas, Nazan; 6038, 8015, 8362

Ozgunen, Fatma Tuncay; 6038

Ozgur, Senem; 8038, 8039

Ozkutlu, Suheyla; 6060

Ozono, Keiichi; 8020, 8303

Oztunc, Funda; 6041, 7151

Pacheco, Danila S; 7409

Padalino, Massimo A; 3120, 7130, 7131, 7132

Padmanabham, TNC; 8335

Paik, Kyung Hoon; 7056

Pakakasama, Samart; 8147, 8178

Palladino, Maria Teresa; 4030, 8384

Palominos, Gilberto; 4064, 7035

Pan, Guangyu; 2104, 6122

Pan, Jun-Yen; 7423

Pan, Silin; 3017

Panadero, Esther; 8413

Pandharkar, Amit; 8254

Pandya, Khyai; 7297

Papierz, Justyna; 6018

Parera, Shehan; 8343

Parisi, Francesco; 8190

Park, Han Ki; 7488

Park, In-Sam; 7113, 7314

Park, In-Sook; 6027, 6028, 7022, 7236

Park, Jeanny; 7104

Park, Jeong-Jun; 7236

Park, Ji-Ae; 6160, 7464, 8345, 8346

Park, Mi Kyung; 7053

Park, Pyo Won; 7186, 7240, 7457

Park, Sin-ae; 7053, 7062

Park, Soo Kyung; 7464

Park, Su Jin; 8332, 8333

Park, Sun-Ju; 7085

Park, Young Hwan; 7488

Parmar, Ramesh C; 7321, 7424

Parry, Andrew J; 7147
Parry, Gareth; 8196

Parry, Tameeka; 6061

Pascoe, Elaine; 6075

Passarelli, Maria L; 7074

Passerino, Carlos H; 3056

Pastuszko, Andrzej; 7425

Pastuszko, Anna; 2102

Pastuszko, Peter; 2102, 7052, 7426, 8037

Patankar, Nikhil S; 7294

Patel, Chetan; 6110

Patel, Dhaval; 4086, 8034

Patel, Najma; 4088, 7261, 8363, 8364

Paterson, Ian; 4065

Patil, Sachin; 6154, 7239

Patiño-Bahena, Emilia; 7438, 7439, 7343, $8214,8215,8247$

Patterson, Michael WH; 7047

Pau, Kiew Kong; 3026

Paul, Jean Francois; 6066

Pavithran, Sreeja; 7328

Pavlovic, Mladen; 7311

Pawale, Amit; 8196

Pawelec-Wojtalik, Malgorzata; 7011, 7012

Peart, Ian; 7433

Pedra, Carlos; 7234, 8062, 8063, 8074 8365, 8366,

Pedro, Becker; 6212

Peek, Giles J; 6093, 6100, 8075

Peersen, Kari; 4035

Peeters, Hilde; 8289

Pegas, Fabiano M; 7006

Pemberton, Victoria; 6197

Peng, Yi Chen; 7427

Penha, Juliano; 7235

Penner, Marie; 6178

Penny, Daniel J; 4016, 4081, 8128, 8166, $8290,8291,8300,8301,8312,8313$, 8322, 8323

Penumatsa, Raghava Raju; 8335, 8337

Pepe, Salvatore; 4081

Pepeta, Lungile; 7292, 8023, 8053

Peralta, Fábio; 8063, 8366

Perçin, Ferda; 7317

Perdeus, Piotr; 6106

Perera, Shehan; 8095

Perez, Daneil; 7428

Perryman, Richard; 3022

Persson, Kenneth; 7322

Pesonen, Erkki; 4024, 4026, 4108, 6058, 7322

Peter, Karlheinz; 4083, 4084, 6215

Peters, Bjoern; 8411

Peters, Brigitte; 7128

Petersen, Mary Beth; 8167

Petit, Christopher; 8110

Petrelli, Lucia; 8309

Pettersen, Eirik; 4035

Pfammatter, Jean-Pierre; 7311

Phadke, Milind S; 8112

Phelan, Dean; 3057, 7001

Phelps, Christina M; 6162, 8197

Phetphisal, Saranwan; 2008, 8380

Phornphutkul, Charlie; 7298

Photiadis, Joachim; 3083

Piamo, Luane; 8240

Piazza, Luciane; 2010, 4090, 8076, 8078, 8086, 8087

Pickardt, Thomas; 6224

Pienvichit, Pavit; 8194

Pierleoni, Marco; 8234

Pietrucha, Artur Z; 8248, 8249

Pietrucha, Beata J; 7034, 8248, 8249

Pietrzak, Radoslaw; 6106, 7323

Pietrzykowski, Witold D; 7401
Pihkala, Jaana; 7093, 8367

Pihkala, Ulla; 8329

Pilan, Maria L; 7043

Pilati, Mara; 7380, 7381, 8206

Pilossoff, Vladimir B; 8224

Pinarli, Güçlü; 8027

Pinero, Gloria; 6194

Pinto, Jorge C; 8314

Pinto, Manuel; 4064

Pippa, Simpson M; 3008

Pircher, Iris; 4034

Pires, M M; 7044

Pirzadeh, Afsaneh; 2102

Piskorski, Jaroslaw; 7058, 7059

Pitkönen, Olli; 7017

Piwowarska, Wieslawa; 8248, 8249

Pizarro, Christian; 3020, 7429

Plaskota, Karolina; 8267

Plymale, Jennifer M; 7104

Podolec, Piotr; 7252

Poelmann, Robert E; 8297

Pohlner, Peter; 8259

Polat, Tugcin; 8042, 8080

Polimenakos, Anastasios; 3054, 7227, 7430,

7431

Politowska, Beata; 8085

Pollock-BarZiv, Stacey; 8185

Pompeu, Camille B; 7346

Pongprot, Yupada; 7298, 8024

Poon, Louisa; 8242

Poonkodi, Priya; 6168

Porisch, Mary E; 7076

Pornkul, Rattanaporn; 8194

Poruban, Rudolf; 7411, 7412

Potapov, Evgenij; 8021

Potts, James E; 3113, 3117, 7047, 7061, 7267, $7268,8175,8184$

Potts, Mary T; 3113, 7047, 7061

Pozzi, Marco; 6143, 6144, 7070, 7192, 7435 , 7436, 7437

Prabhu, Atul; 6163, 7328, 7476

Prabhu, Shakuntala S; 7294, 7324, 8025

Pradhan, Siddhartha; 7251

Prandstraller, Daniela; 3124

Pratap, Usha; 8154

Prates, Jose Carlos; 8284

Preisman, Sergey; 7193

Preusse, Claus J; 7432

Preutthipan, Aroonwan; 7119

Pridham, Karen A; 7325

Prieto, Lourdes; 4107, 8344

Prioli, Maria Antonia; 7380, 7381, 8206

Prior, Natasha; 7195, 7433

Prodan, Zsolt; 7188

Promphan, Worakan; 8368

Prospero, Carol; 7429

Puga, Alonso F; 7332

Puga, Francisco J; 3087

Pulido, Tomas; 4037

Puppini, Giovanni; 7381

Putra, Sukman T; 8083

Pyles, Lee A; 6107, 7326

Al Qbandi, Mostafa; 8118

Al Qethamy, Howaida M O; 7434

Qian, Juan; 6114

Qin, Jun; 8369

Qiu, Lisheng; 6129, 7375

Quarti, Andrea; 6143, 6144, 7192, 7435, 7436, 7437

Quek, Swee Chye; 7327, 8409

Quezada, Emilio; 7429

Quezada, Katty; 4064, 6054, 7035, 8004

Quinonez, Luis G; 8198 
Quiroga, Luis; 8195, 8199

Qureshi, Athar; 8410

Qureshi, Shakeel; 8363, 8364, 8382

Racek, Jaroslav; 7277

Radford, Dorothy; 4115, 8202, 8243

Radtke, Wolfgang A; 3020

Radzi, Nomee A; 8251, 8370, 8371

Raghavan, Subramaniam; 6164, 8375

Raghuvanshi, Ashutosh; 8036

Rahayuningsih, Sri Endah; 8318

Rahman, Mahrus A; 8372, 8373

Rahmat, Budi; 7216

Rahouma, Salem M; 8374

Raitakari, Olli T; 3011

Raj, Benedict; 3072

Rajasekaran, Premsekar; 6164, 8375

Rajbanshi, Bijoy Gopal; 7251

Rajgopal, Hariganesh; 7350

Ramaciotti, Claudio; 7150

Ramakrishnan, S; 6109, 7451, 8254, 8381

Ramani, Meera; 7047

Ramaswamy, Arun; 7455

Ramirez-Marroquin, Samuel E; 7177, 7190, 7191, 7438, 7439, 8131

Rammos, Spyridon; 6030, 8113, 8114, 8123

Ramos, Caroline S; 8219

Ramos, Eloane G; 7289

Ramos, Sarah B; 8218

Ramsay, James M; 6075

Rasheed, Abdul; 8075

Rastan, Ardawan; 7185

Ravelo, Luis; 7136

Ray, Mily; 8045, 8046

Rayatzadeh, Hussein; 7406

Razack, Saleem; 6176

Razek, Vit: 3023, 6121, 6123, 8379

Razo, Antonio; 7177

Razzouk, Anees J; 7141

Re, Jennifer M; 6042, 6229

Reade, Erin; 2102

Rebelo, Mónica; 7440, 7448

Rebeyka, Ivan; 6115, 8198

Reddy, D Venu; 7149

Reddy, Pramod; 7057

Reddy, Srikrishna M; 7451

Reddy, Venu D; 7137

Redha, Esmail M; 8180

Redhyan, Abdulrahman E; 7157

Redington, Andrew; 3045, 3115, 6010, 8320, 8324

Redmond, J Mark; 3031, 7246

Rees, Mark; 4012

Rees, Phillip; 8019

Reffo, Elena; 8179

Rega, Felip; 4110

Rega, Filip; 8072

Regoli, Xiomara; 8240

Rehman, Asif; 8382

Reich, Oleg; 7471

Reichart, Bruno; 3019

Reineker, Katja R; 8376

Reinhardt, Zdenka; 2064, 3021, 4060, 4109 , $8082,8377,8391$

Rekawek, Joanna; 7009, 7010, 7029

Relaño, Cristina; 6179, 6180, 6181, 6194, 6198, 6199

Remadevi, Kavasseri S; 8095, 8378

Rempel, Gwen; 6178

Ren, Yunyun; 3127, 7088

Repetto, Gabriela M; 7332

Reshma, R; 7349

Rewers, Bozena; 8084

Reyes, Pedro A; 7343
Rhodes, John F; 8344

Ribera, Elena; 7389

Ricato, Silvia; 8309

Ricciotti, Roberto; 6143, 6144, 7070, 7192, 7435, 7436, 7437

Rich, Kenneth C; 4068, 8029

Richardson, Kerrie A; 6108, 7333

Richardson, Malcolm E; 6108, 7333, 7334

Richmond, Marc E; 8200

Richter, Andrea; 4052

Riede, Frank T; 3046, 8379

Rief, Annette F; 8252

Riley, Robyn; 6061

Ringewald, Jeremy; 8410

Riopel, Kathleen; 4052, 8051

Riso, Arlindo; 7235, 8193

Riza, Ali; 7167

Roberson, David A; 4086, 6134, 8034

Robertson, Charlene MT; 8198

Rocha, Fátima DM; 3016, 7288

Rocha, Gustavo; 8126

Roche, Lucy; 3045, 3115

Rodefeld, Mark D; 3085, 7140, 7171, 7442, 7443

Rodés-Cabau, Josep; 4061

Rodrigo, Gonzalez; 6212

Rodrigues, Silmara M; 8219

Rodriguez Hernandez, Lidia; 6063

Rodríguez Saldaña, Humberto; 6063

Rodriguez, Fred H; 8201

Roebiono, Poppy S; 7216

Roest, Arno AW; 6119

Roest, Pauline AM; 8297

Rohit, Manojkumar; 7051, 7295, 8120

Rojas, Cielo; 8002

Rokitska, Nadiya V; 6091, 6092

Rokkas, Chris; 8310

Rolland, Audrey; 7134

Romagnoli, Enrico; 8076

Roman, Kevin; 6059, 6069, 7479, 7480

Román, María I; 7382

Romaniuk, Oleksandr M; 7168, 7441, 8342

Romanova, Lidiy K; 8327

Romanyuk, Oleksandr; 8229

Rome, Jonathan; 4003, 4007, 8410

Romeo, Emanuele; 4038, 8124

Roodt, Sandra; 4092

Roofthooft, Marc T R; 8148

Rooney, Nichola; 6219

Rose, Michelle L; 8128, 8166

Rosenfeldt, Franklin L; 4081

Rosenthal, David; 6205

Ross, David; 6115, 8198

Rossi, María G; 7121

Rostami MD, Alireza; 7444, 8253

Roten, Lisa; 7202

Roth, Stephen J; 6205

Rotstein, Amichay; 6042, 6043

Rouault, Francis; 7110

Routes, John; 8287

Rowlinson, Giselle V; 4104

Roy, Raja; 8299

Roymanee, Supaporn; 2008, 8380

Rózanski, Jacek; 8227

Rudenko, Nadia; 8229

Rudzinski, Andrzej; 7034, 8248, 8249

Ruga, Ezia; 8309

Ruiz, Raiza; 8002

Russo, Maria Giovanna; 4030, 4038, 8124, 8384

Ruturi, Josiah AM; 6158

Ruzmetov, Mark; 3085, 7140, 7171, 7172, 7442, 7443

Rychik, Jack; 3080, 3125, 3128, 6045
Rydberg, Annika; 3112, 7004, 7014

Ryugo, Masahiro; 6156

Saadullah, Tareen; 7261

Saatvedt, Kjell; 7374

Saba, João C; 8284

Sabi, Titus; 8411

Sable, Craig; 8182, 8268

Sack, Ulrich; 4103

sadeghpour Tabaee MD, Ali; 7444, 8253

Sadiq, Masood; 3076, 7247, 8382

Saga, Toshihiko; 7410

Sagat, Michal; 7411, 7412

Sagawa, Koichi; 7335, 8241

Sagawa, Kouichi; 6104, 7114

Sahn, David J; 8268

Sai, Sadahiro; 7445

Saikawa, Yutaka; 7310

Saiki, Hirofumi; 1126, 7336

Saiki, Yoshikatsu; 7445

Saito, Kazu; 8139

Saito, Kazuyoshi; 7108, 7296, 8013

Saito, Ryota; 7067

Saito, Yoshiaki; 8213

Saji, Tsutomu; 7217, 7296

Sakaguchi, Heima; 7030

Sakamoto, Kisaburo; 7224, 7226, 7446, 8334,8390

Sakamoto, Kisaburou; 6035, 7320

Sakamoto, Masato; 7416

Sakamoto, Takahiko; 7218, 7418

Sakashita, Kazuo; 8018

Sakijan, Abdul Samad; 6082

Sakurai, Shigeru; 7447

Sakurai, Takahisa; 3055, 7417

Sakurai, Yoshihiko; 7220

Salgado, Gladys; 7043

Saliba, Zakhia; 4106

Salihoglu, Ece; 7166, 7167

Sallan, Stephen E; 8005

Sallan, Steven E; 4069

Salmon, Anthony P; 2009, 7479, 7480

Saltik, Levent; 7151

Salvadori, Sabrina; 7131

Salvi, Prasanna; 6154, 7239

Salzer-Muhar, Ulrike; 8003

Samion, Hasri; 3026, 4031, 6057, 6082, 8056, $8083,8251,8370,8371$

Samoukovic, Gordan; 7223

Sampaio Filho, José C; 7408

Sampaio Nunes, Maria Ana; 7440, 7448

Samson, Ricardo A; 8383

San Martin, Augusto; 7332

Sanatani, Shubhayan; 3059, 7019, 7267, 7268

Sanders, Stephen P; 8230, 8234

Sandor, George GS; 3113, 7047, 7061, 7267, 7268,8184

Sandoval, Erika G; 8277

Sandoval, Julio; 4037

Sands, Andrew; 3126, 6033, 6034, 6219, 6227

Sangkitporn, Somchai; 8194

Sangtaweesin, Chaisit; 8368

Sanisoglu, Yavuz; 7368

Sanli, Cihat; 6159, 7054, 7317, 7318

Sano, Shunji; 6172, 7127, 7196, 7208, 7242, $7243,7244,7397,7398,7447,7449,7450$ $7472,8098,8361$

Sano, Tetsuya; 7312

Santiago, Justo; 8365

Santo, F; 7481

Santoro, Francesco; 7389

Santoro, Giuseppe; 4030, 4038, 8124, 8384 
Santos de Soto, José; 8319

Santos, Luis; 4037

Santos, Mário S; 8314

Saraste, Antti; 7322

Saric, Dalibor; 7021, 7055, 7091, 8285

Sarikouch, Samir; 7128

Saritas, Turkay; 8041, 8042, 8043, 8044 8079,8080

Sarkis, Vrej; 7367

Sarquella-Brugada, Georgia; 4052, 8051, 8052,8319

Sarquella-BRugada, Georgia; 3061, 4061

Sarubbi, Berardo; 4038, 8124

Sasaki, Jennifer; 7149

Sasaki, Kaori; 7299

Sasaki, Tomoyasu; 7241, 8152, 8155

Sasoh, Masaki; 2063, 3119

Sathanandam Shyam K; 6134, 8034

Sathe, Yogesh; 8375

Satheesh, Gayatri; 8094

Satio, Yoshiaki; 8255

Satish, Gayathri; 3096

Satish, Radhakrishnan; 6068, 6169, 6170,

$7233,7340,7341,7454,8081,8275,8389$

Sato, Hitoshi; 7310

Sato, Junichiro; 7314

Sato, Keiko; 7316, 8244

Sato, Tomoyuki; 8022

Satomi, Gengi; 6050, 7218

Sawa, Yoshiki; 8303

Sawada, Hirofumi; 3090, 8153

Sawin, Kathleen; 6197

Sawyer, Douglas B; 8005

Saxena, Anita; 6109, 6110, 7297, 7451, 8254, 8381

Schaefer, Natasha; 7287

Schaff, Hartzell V; 3087, 7173

Schaffer, Michael S; 3025

Schears, Gregory J; 2102

Scheiber, Christian; 7337, 8285

Scheid, Margaret; 7326

Schell, David; 4020

Schiebold, Daniela; 4083, 6207

Schievano, Silvia; 4028, 4085, 4111, 8353

Schindler, Ehrenfried; 7160

Schleif, William; 7462

Schlichter, Andrés J; 7382

Schmid, Eckhard; 6207

Schmidt, Michael R; 8320, 8324

Schmitt, Boris; 7405

Schmitto, Jan D; 7469

Schmitz, Christoph; 8192

Schneider, Martin BE; 3060, 3086, 7040, $7160,8273,8412$

Schneider, Peter; 3046

Schneider, Rayfel; 3012

Schoeman, Mardelle; 7122

Schoendube, Friedrich A; 7469

Schoenwaelder, Simone; 4083

Scholl, Frank G; 3022

Schomisch, Steve; 7203

Schoof, Stephan; 7068

Schouten, Jan; 4080

Schowengerdt, Kenneth O; 6177, 8096

Schreiber, Christian; 7188

Schulze-Neick, Ingram; 8156

Schutte, Deborah; 7202

Schutte, Glynis; 7122

Schwienbacher, Martin; 4034

Sciegata, Alberto M; 7024

Scott, Gwendolyn B; 7287

Scott, Lynne; 8236

Scully, Rebecca E; 4069, 8005

Seale, Anna; 8276
Seale, Helen; 4115

Seed, Michael T; 6067

Seem, Egil; 7374

Segal, Evgen V; 7169

Sehic, Igor N; 6214

Seino, Yoshiki; 7312

Seitz, Michael; 3072

Sekelyk, Roman; 7161

Seki, Mitsuru; 7278

Selvakumar G; 6163, 8250

Sen, Supratim; 8045, 8046

Seneviratne, Sujith; 4023

Senocak, Filiz; 6053, 8038, 8039

Seo, Dong-Man; 6028, 7236

Serdari, Mohd Shafullah; 3026

Sereboe, Lawrence; 7197, 7198

Sermer, Mathew; 4114

Serwacki, Michael; 7076

Sese, Akira; 7354, 7416

Seshadri, Shivaprakash; 7203

Seth, Sandeep; 6109

Sethia, Babulal; 8168

Severs, Nicholas J; 4104

Sezgin, Melike Evim; 7050

Shaban, Lulu Abu; 8118

Shaboodien, Gasna; 7253

Shah, Prashant D; 7452

Shah, Prashantkumar Dineshchandra; 7453 , 7454

Shah, Sejal; 7455

Shahani, Jagdish M; 6009

Shahmohammadi, Akbar; 8358

Shahriari, Ali P; 7171

Shakya, Samjhana; 7251

Shaleve, Smadar; 6228

Shan, Lishen; 4070

Shaner, Marie; 7087

Shankar, Sriram; 7152, 7264, 7463, 8016, 8150

Sharma, Amit B; 8112

Sharma, Deepa Mohan; 8036, 8040, 8387, 8388

Sharma, Jyotindra; 7251

Sharma, Pranav; 7451

Sharma, Rajesh; 3116, 6167, 6168, 7455, 7461, 8149

Sharpe, Joan A; 6075

Shearer, William T; 4068, 8029, 8030

Shekerdemian, Lara S; 2100, 3007, 6171, 8322,8323

Shelling, Andrew; 3024, 4012

Shen, Jie; 6217, 8298

Shen, Rong; 6132

Shen, Xiangdong; 7148

Sheng, Feng; 7041, 8107, 8350

Shenoy, Anand; 8393

Sherwood, Megan; 3114

Shi, Live; 3127

Shi, Zhen jing; 7483, 7484, 7485, 7486

Shi, Zhenying; 8130

Shiina, Yumi; 7037, 8256

Shikata, Fumiaki; 6156, 7456, 8105

Shim, Man-shik; 7457

Shim, Wooseup; 3082, 7342

Shima, Midori; 7220

Shimada, Eriko; 7339, 8222

Shimada, Shunsuke; 6044

Shimazu, Chikashi; 7230, 7420, 7421

Shimizu, Kazuyoshi; 6151, 6166, 6172

Shimizu, Mikiko; 8257

Shimizu, Takashi; 8018

Shimizu, Toshiaki; 7316, 8022, 8099, 8216, 8244

Shimoyama, Shinya; 7458
Shimpo, Hideto; 4039, 6216, 7467, 8153, 8162

Shimura, Kisho; 7466

Shina, Yumi; 6230

Shinohara, Gen; 7473

Shinohara, Tokuko; 8257, 8258

Shipp, Anne T; 8200

Shipton, Stephen; 8177

Shirai, Lance K; 7137, 7149

Shirai, Takeaki; 6230, 7037, 8256

Shiraishi, Isao; 6111

Shockley RN, BSN, CCRN, Heather M; 6200

Sholler, Gary; 3072, 3114, 6225, 7036, 7162, 7424

Shridhar, Sushant; 7351

Shrivastava, Jyotsna; 7350

Shrivastava, Savitri; 7233, 7340, 7341

Shylaja, S; 7279

Siala, Konrad; 7277

Siegel, Rolland; 3056

Sieminska, Jolanta; 7355

Sierra, Jorge; 7402

Sieverding, Ludger; 7068

Sievers, Hans H; 2103

Sigler, Matthas; 8412

Siles, Ana; 7271

Silva, Celia M C; 6086, 7386, 7387, 7388,8284

Silva, Eduardo D; 8357

Silva, Isabela B; 8218

Silva, Joao C; 8357

Silva, M E; 7044

Silva, M L; 7044

Silva, Ransika De; 2009

Silverbach, Michael; 7099

Silverman, Earl D; 3012

Silverman, Lewis B; 4069

Silversides, Candice K; 4114, 8261, 8262

Silvilairat, Suchaya; 8024

Silviliarat, Suchaya; 7298

Sim, James Y; 7137, 7149

Sim, Kuihian; 6124, 6142, 8047, 8271

Simell, Olli G; 3011

Simmer, Karen; 6075

Simmons, Qasim; 6208

Simpson, Catherine M; 8322, 8323

Sinclair, Brian G; 3113

Singh, Ashutosh S; 7459

Singh, Gurmeet; 7351

Singh, Surjeet; 7051

Singh, Surjit; 7295

Singh, Vishal K; 6167, 6168, 7455, 7461

Sinimol, TM; 3097

Sinzobahamvya, Nicodeme; 3086, 7160

Sipahi, Tansu; 7315

Sirachainan, Nongnuch; 3093

Siripornpitak, Suvipaporn; 8194

Siriwiwattanakul, Napa; 8368

Sittiwangkul, Rekwan; 7298, 8024

Siu, Samuel C; 4114

Sivadasan, Anil Radha; 8036, 8040, 8387, 8388

Sivakumar, K; 7452, 7453, 7454

Sivakumar, Kothandam; 6068, 6169, 6170, $8081,8275,8389$

Sivalingam, Sivakumar; 3026, 7460

Sivanandam, Shanthi; 6107

Sivarajan, Venkatesan B; 6135

Sivarajen, Ben; 6204

Sivaratnam, D; 6130

Siwik, Ernest; 7204, 7221

Siwinska, Aldona; 7011, 7012, 7058, 7059

Sjöberg, Pia S; 7238 
Skalski, Janusz; 7252

Skerritt, Claire; 7147

Skiendzielewski, Jacek; 7357

Skinner, Jonathan R; 3024, 4012

Skogvoll, Eirik; 4022

Slater, Nancy R; 6201

Slaughter, Richard; 6061, 8243

Sleeper, Lynn A; 8006, 8014

Sloman, Felicity M; 4094

van Slooten, Ymkje J; 7474

Slorach, Cameron; 3012

Smallhorn, Jeffrey; 4065, 6088, 6115, 6117

Smit-van Oosten, Annemiek; 4102

Smith, Carol; 7334

Smith, Elisabeth C; 6202, 6203

Smith, Holly M; 8005

Smith, J H; 7112

Smith, Richard G; 8008

Smolewska, Elzbieta; 6112

Smolich, Joseph; 4016, 6215, 8290, 8291, 8300, 8301, 8312, 8313, 8322, 8323

Snider, Scott; 6208

Snyder, Christopher S; 7002, 7026, 7031, 8201

Soda, Wataru; 8334, 8390

Sodian, Ralf; 8192

Soler, Pilar; 7428

Solomon, Brian; 7392

Somanathan, Arjuna; 7146

Somarriba, Gabriel; 7287

Son, Minette; 7396

Song, Houyan; 4101

Song, Jin Young; 3082, 7342

Soni, Reeni; 7019

Soongswang, Jarupim; 4058

Sørensen, Karina Meden; 4080

Sørensen, Keld; 8324

Sosebee, Steve; 8168

Soto, M Elena; 7343, 8214, 8247

Soto, Rodrigo; 8004

Soule, Mauricio; 7190, 7191

Sourial, Magdy; 8300

Soylemezoglu, Oguz; 6159

Speggiorin, Simone; 7130, 7131, 7132

Spencer, Mary; 6178

Spiegelstein, Dan; 7193

Spigelblat, Linda; 7271

Sporing, Eileen M; 4093

Spray, Thomas; 3080

Sreeja, P; 6163

Sreeram, N; 3058, 7367, 8103, 8054, 8288, 8339

Sridhar, Anuradha; 6164, 8375

Srinivas, Kini M; 7461

Srivastava, Anurakti; 8066

St George-Hyslop, Cecilia; 6204

Stables, Simon; 3024

Stajevic, Mila; 6214

Stamm, Christof; 3121, 7405, 8021, 8293

Stanczyk, Jerzy A; 6112

Stark, Ann R; 3028, 6003, 6004

Stattin, Eva-Lena; 3112, 7014

Staveski, Sandra L; 4092, 6205

Stayer, Stephen A; 3028, 6003

Steele, Ann M; 7462

Steele, Jasmine A; 7462

Steele, Peter O; 7462

Stein, Joerg I; 4034

Steinberger, Julia; 6107

Steinke, Katja; 7469

Stellin, Giovanni; 3120, 7130, 7131, 7132, 8179

Stensbjerg, Jesper; 4080

Stephens, Derek; 3115
Stephensen, Sigurdur S; 6113

Stewart, Moira; 6219

Stickley, John; 3021, 8082, 8377

Stierle, Ulrich; 2103

Stirling, John; 3015

Stockton, Emma F; 6008

Stos, Bertrand; 6066, 7134

Støttrup, Nicolaj B; 8320, 8324

Stozicky, Frantisek; 7277

Straub, Andreas; 4083, 4084, 6207, 6215

Strengers, Jan; 7098

Strengers, Jan L M; 8148

Strömvall-Larsson, Eva; 7004, 7391

Strugnell, Wendy; 6061

Stumper, Oliver; 2064, 3021, 4060, 4109 $8082,8377,8391$

Su, Tonglin; 7036

Su, Zhao kang; 6174, 7485, 7486, 8130

Subbian, Senthil K; 7463

Subramanian, Chellappan; 7189

Succi, Fabiana P; 7234

Suda, Kenji; 6024, 7027, 7275

Sueiro, Melyssa S; 8181

Suemori, Tomohiko; 6151, 6166, 6172

Sugimura, Tetsu; 7275

Sul, Jun Hee; 7120, 7488, 8332, 8333

Sulik, Agata; 7034

Sumitomo, Naokata; 6085, 7028, 7260, 8109

Summerhayes, R; 8316

Sun, Ai-Min; 4027

Sun, Huichao; 8326

Sun, Jing; 6114

Sun, Jinghui; 7082

Sun, Kun; 4025, 4027, 4063, 6052, 6071, 6072, 6114, 6127, 6129, 6131, 6132, 7347, 7364, 7487, 8031, 8032, 8298, 8348, 8392

Sundaram, KR; 3097, 7349, 8305

Sundararaghavan, Sreekanthan; 8393

Sung, Kiick; 7186, 7457

Sung, Si Chan; 7464

Sunil, S G; 8036, 8040, 8387, 8388

Sunnegardh, Jan; 7124, 7465

Surer, Suleyman; 7371

Suresh, R Kumar; 6163, 7328, 7475, 7476, 8250

Suri, Deepti; 7051

Suriyanarayanapillai, Hariprakash; 6169, 6170, 8275, 8389

Surmacz, Rafal; 7059

Susheel, TK; 6213

Sutherland, Valerie; 7111

Suwan-uksorn, Saranyou; 8380

Suzuki, Hiroshi; 7105, 7344

Suzuki, Satoshi; 6151, 6166, 6172

Suzuki, Shoji; 7466

Suzuki, Takahiro; 7231

Suzuki, Tsugutoshi; 7384

Suzuki, Yasuyuki; 8213, 8255

Swain, Sunil Kumar; 8259

Swanevelder, Justiaan; 6093, 6100

Swanson, Veronica; 7099

Swast, Anita; 3125

Swisher, Matthew W; 3052

Syed, Kashif Azam; 8336

Synnergren, Mats; 7465

Sysa, Andrzej; 3118, 7400, 8084, 8085, 8356, 8394

Szabolcs, Judit; 7313

Szamosi, Tamas; 7313

Szatmári, András; 7219

Szekely, Edgar; 8177

Szkutnik, Malgorzata; 7042, 8069, 8274 8395

Szumowski, Lukasz; 7010, 7029
Szwast, Anita; 3080

Szymkiewicz-Dangel, Joanna; 6023, 6031, 7003

Tabayashi, Koichi; 7445

Tabib, Avisa; 8358

Tachi, Maiko; 7224, 7226

Taffe, John; 6042

Tagawa, Tetsuzo; 7312

Tahara, Kanako; 8244

Takabayashi, Shin; 6216, 7467, 8153, 8162

Takada, Yoshinobu; 7312

Takagaki, Masami; 7196, 7208, 7244, 7398, 7447

Takahashi, Kazuhiro; 8258

Takahashi, Ken; 6115, 7316, 8022, 8099 , 8244

Takahashi, Yukihiro; 3053, 7113, 7314

Takai, Shinji; 8307

Takamuro, Motoki; 7345, 8279

Takeba, Yuko; 8311, 8328

Takeda, Tomohiro; 7278

Takei, Kouta; 6050, 6116

Takens, Janny; 4102

Taketani, Takeshi; 8161

Takeuchi, Daiji; 8258

Takigiku, Kiyohiro; 6050, 6116, 7218

Takkenberg, Johanna J M; 2103

Talini, Carolina; 7346

Talo, Haitham; 8177

Talpur, Saeed; 7261

Talsma, Melle; 8127

Tam, Robert; 8259

Tam, Vincent KH; 7202

Tamai, Hiroshi; 6105, 8307

Tamas, Csaba; 8177

Tamatey, Martin; 7197, 7198

Tammo, Raad; 6092

Tamura, Masamichi; 6044

Tan, Ju-Le; 8226, 8231, 8260

Tan, Ming-Shin; 8134

Tan, Ru-San; 8260

Tan, Teng Hong; 7152, 7264, 7463

Tan, Xiao; 6114, 7347

Tanaka, Toshikatsu; 1126, 7336

Tanaka, Yashiko; 8390

Tanaka, Yasuhiko; 7320, 8334

Tanamati, Carla; 7235, 8193

Tang, Chaoshu; 7015, 8144, 8304

Tang, Yue; 6175

Taniguchi, Akira; 7312

Taniguchi, Kazuo; 7028

Taniguchi, Manabu; 8098

Tanous, David; 4114, 8261, 8262

Tao, Qilin; 6210

Tarayan, Marat V; 7228, 7468

Tarnok, Attila; 4103

Taskinen, Mervi; 8329

Tasneem, Habiba; 7261, 8364

Tassanawiwat, Worawut; 8368

Tatar, Harun; 7368, 8246, 8340

Tatebe, Shunsuke; 7309

Tateno, Shigeru; 6230, 7037, 8256

Taylor, Andrew; 4028, 4067, 4085, 4111, 6008, 6081, 8353

Taylor, Katherine; 3032, 6010

Taylor, Teressa; 7227

Tazawa, Seiichi; 6050, 6116

Tchervenkov, Christo I; 6007, 7223

Tei, Teruo; 7336

ten Harkel, A Derk-Jan; 8148

Terai, Masaru; 7299

Teramoto, Chikao; 7217

Tesar, Peter; 8259 
Tettey, Mark; 7197, 7198

Tewari, Prabhat; 6006

Tham, Edythe; 6088

Tham, Edythe B; 6117

Thampi, MV; 8305

Thanarattanakorn, Pattra; 8024

Thaulow, Erik; 4035, 6101, 8217

Thawerani, Hasina; 7261

Therrien, Judith; 4113, 4116

Thibeault, Maryse; 8202

Thienpont, Bernard; 8289, 8325

Thiruchelvam, Timothy R; 6171

Thomas, Timothy H; 7150

Thomaz, Petronio G; 8062

Thompson, Bruce; 4068, 8029, 8030

Thompson, Richard; 6115

Thomson, John D; 8223

Thorne, Sara; 8391

Tian, Jie; 4082, 8326

Tian, Xin; 3052

Tian, Zhiyun; 3080, 3125, 3128, 6045

Timala, Rabindra Bhakta; 7251

Timmins, Jo-Anne M; 4071

Tineli, Rafael; 8193

Ting, Jennifer; 8209

Tinker, Kathy; 6177

Tipu, Fateh A; 8064

Tirilomis, Theodor; 7469

Tissot, Cécile; 7402, 8197

Tittel, Peter; 8401

Tiveron, Marcos G; 7235

Tlaskal, Tomas; 7470, 7471

Tláskal, Tomáš; 6047

Tobota, Zdzislaw; 2101

Toda, Yuichiro; 6151, 6166, 6172

Tofani, Christina; 8209

Tofeig, Magdi; 6100, 8075

Tomar, Munesh; 7340, 7341

Tomek, Viktor; 3023, 6046, 6047

Tometzki, Andrew; 4033

Tomii, Tomoko; 8098

Tominaga, Kenta; 1126, 7336

Tomita-Mitchell, Aoy; 8287

Tomkiewicz-Pajak, Lidia; 7252

Tomyn-Drabik, Malgorzata; 8415

Tonklang, Nakharin; 2008, 8380

Torp, Hans; 4022

Tosaka, Yuko; 7224, 7226, 7446

Toussaint-Goetz, Nicole; 3060, 3086

Towbin, Jeffrey A; 4070, 8006, 8014

Toyoda, Tomohiko; 6230, 7037, 8256

Toyohara, Keiko; 7032, 7038, 7039

Toyono, Manatomo; 6044

Toyot, Sheena; 6069

Tranchevent, Leon-Charles; 8325

Trehan, Meena; 8036, 8040, 8387, 8388

Trenholme, Adrian A; 3015, 6191

Trepakov, Alexey V; 8327

Tripathi, Partima; 8299

Troendle, David; 7150

Trojnarska, Olga; 8266, 8267

Trotter, Andrew; 6061

Truong, Uyen T; 8268

Truszkowski, Krzysztof K; 6112

Tsai, Felix W; 7413

Tsai, Tsung-Po; 7081

Tsakistos, Andreas; 4092

Tsang, Victor; 4067

Tseng, Wen-Yih; 6070

Tsounias, Emmanouil; 7413

Tsoutsinos, Alexandros; 8123

Tsubata, Shinichi; 4070

Tsuchiya, Keiji; 7278

Tsuji, Yasuhiro; 7127, 8157
Tsuzuki, Yoshimitsu; 8311, 8328

Tubtom, Darin; 8178

Tueti, Isac; 8365

Tulzer, Gerald; 3081

Tunaoglu, Sedef; 6159, 7054, 7293, 7317, $7318,8027,8158$

Turanlahti, Maila I; 8329

Turner, Daniel; 4107

Turner, Mark; 4033

Turrentine, Mark W; 3085, 7140, 7171, 7172, 7442,7443

Tweddell, James S; 3008, 8287

Tyrrell, Pascal N; 3012

Tzonzarova, Margarita R; 7018, 8224

Ucar, Birsen; 7050, 8121

Uchiyama, Makoto; 7105, 7344

Udink ten Cate, Floris; 3058

Uebing, Anselm; 7068

Uemura, Hideki; 3094, 8276, 8281, 8282

Ueno, Kentaro; 7291, 7348

Ueno, Takayoshi; 8303

Uese, Keiichiro; 8013

Ugaki, Shinya; 7472

Ugljen, Ranko; 7021

Umezu, Kentaro; 7218

Unlu, Sebahat; 7370, 7371

Uno, Yoshimasa; 7473

Unver, Cigdem; 6053

Urashima, Takashi; 7067

Urata, Masahisa; 7250, 8163

Urban, Andreas; 8179

Urcelay, Gonzalo; 8101

Urhan, Baris; 8121

Ushinohama, Hiroya; 6104, 7028, 7114, 7335,8241

Utamayasa, Alit: 8373

Utikawa, Toshiki; 8020

Uzun, Mehmet; 8246

Vaidyanathan, Balu; 3097, 7349, 8093, 8094, 8095,8378

Vaidyanathan, Karthik; 7154

Valdes-Cruz, Lilliam; 3022

Valentik, Pavel; 7411, 7412

Valitova, Asija A; 7229

Valliath, John; 7475, 7476

Valliatu, John; 7328, 8250

Valori, A; 7481

van Albada, Mirjam E; 4051

Van Arsdell, Glen S; 7248

Van Bergen, Andrew; 6134, 8034

van den Boom, Jutta; 7045

van den Brink, Olivier W; 4081

van der Hulst, Annelies E; 6119

van Gilst, Wiek H; 4102

van Loon, Rosa Laura E; 8127

Van Nooten, Guido; 8168

van Osch-Gevers, Lennie; 8148

Van Vooren, Steven; 8325

Varatharajan, L; 7328

Varghese, Roy; 6163, 7328, 7475, 7476, 8250

Varma, Amit; 6167, 6168

Varma, Suraj K; 7475, 8250

Varma, Yogesh; 7350, 7351

Varvarovska, Jana; 7277

Vassanelli, Corrado; 8206

Vasudevan, Sanjeev; 7279

Vaughan, Anne; 4012

Velasco, Jose M; 8033

Veldtman, Gruschen; 2009, 7479, 7480

Velez, Juan; 8181

Venczelova, Zuzana; 6120, 8401
Venkatesh, Sumitra; 7294, 7324, 8025

Ventriglia, Flavia; 3124

Verebély, Tibor; 7219

Verma, Gaurav S; 8112

Vermeesch, Joris R; 8289

Vesely, Ivan; 8295

Veshti, Altin; 7130

Vettukattil, Joseph; 6059, 6069, 6087, 7064, 7479,7480

Vezmar, Marko; 4059

Vicente, Walter V A; 7477, 7478

Vida, Vladimiro L; 3120, 7130, 7131, 7132

Vides, Ivan; 8181

Vidmar, Suzanna; 8128, 8166

Vignati, Gabriele; 7389

Vijarnsorn, Chodchanok; 4058

Viljevac, Mladen; 7091

Villa, Alejandra; 8195, 8199

Villafane, Juan; 7352

Villafañe Molina, Solana; 7043

Villalba, Claudia N; 7121, 7133

Villarroel, Luis; 8002

Villoria, Guillermo; 8365

Vimala, J; 8250

Vincent, Robert; 8410

Visartpong, Treechada; 8380

Viscardi, Francesca; 7380, 7381, 8206

Vithessonthi, Kanyalak; 7300

Vizcaíno, Alfredo; 8277

Vlk, Radek; 6047

Vogelfang, Horacio; 8195, 8199

Vogt, Manfred; 7188

Vohra, Hunaid; 2009, 6059, 7479, 7480

Vojtovic, Pavel; 7470, 7471

von Scanzoni, Claudia; 7136

Vouche, P; 7481

Vucajnk, Lidija; 7353

Wada, Keiko; 7312

Wada, Naoki; 3053

Wagener, Frank ADTG; 4051

Wagner, Jasenka; 7090

Wagner, Robert; 6121, 6123

Wagner, Thomas; 6207

Wahlers, Thorsten; 8293

Wai, Sann Nu; 8260

Wakisaka, Yuko; 6230, 7037, 8256

Walayat, Muhammed; 8129, 8170

Walczak, Franciszek; 7010, 7029

Wald, Rachel M; 4114

Walker, Hamish; 8208

Walker, Karen; 3114, 7424

Walker, Kathie; 7306

Wall, Douglas; 8082

Wall, Douglas A; 2064, 3021

Walther, Thomas; 7185

Wang, Bing-Yao; 6084

Wang, Cheng; 7015

Wang, Chin Fu; 7301

Wang, De; 7491

Wang, Guang-yi; 4112, 7302, 8159, 8160, $8269,8270,8402,8407$

Wang, Jou-Kou; 6070, 6222, 7178, 7179,

7180, 7358, 7359, 8349, 8403, 8404, 8405

Wang, Li; 3048, 7015

Wang, Lianyi; 2104, 6122, 7138

Wang, Liming; 8406

Wang, Qi Feng; 6231, 6232

Wang, Qian; 4027

Wang, Rong; 6011, 6175

Wang, Rong-fa; 3088, 7274

Wang, Shoei-Shen; 8188

Wang, Xiaozhoub; 3017

Wang, Xu; 6150 
Wang, Yuexiang; 4101

Wang, Yuhong; 6175

Wang, Yun; 8117

Wang, Zhi-feng; 4112, 7302, 8159, 8160, 8269, 8270, 8402, 8407

Wanitkun, Suthep; 3093

Waragai, Tadashi; 7113

Warnes, Carole A; 3123

Warrier, KRR; 3097

Watanabe, Kazuhiro; 6223, 7108, 7159, 7489, 8013, 8139

Watanabe, Ken; 7039

Watanabe, Mamie; 7354, 7416

Watanabe, Manabu; 8279

Watanabe, Naruhito; 7418

Watanabe, Sayaka; 4070, 7108, 8013, 8139

Watanabe, Yoshinori; 7217

Waterbolk, Tjalling W; 7474

Watterson, Kevin G; 8223

Webb, Rachel H; 3015, 3091, 6191

Weerasena, Nihal A; 7481, 8223

Weidenbach, Michael; 3075, 6121, 6123, 8379

Weinberg, Paul M; 6077, 6078

Weintraub, Robert G; 8128, 8166, 8200

Weir, Kelly; 6183, 6184

Welke, Karl; 6208, 7099

Wen, Chih-Yun; 7080

Wendel, Hans Peter; 6207, 6215

Wendel, Hans-Peter; 4083

Weng, Yu-Guo; 3121, 7405

Weng, Yuguo; 8021

Weng, Zen-Chung; 7081

Weng, Zeng-Chung; 7225

Werner, Bozena; 6106, 6206, 7323, 7355, 7356, 7357

Westein, Erik; 4084

Westenberg, Jos JM; 6119

Westhoff-Bleck, Mechthild; 7213

Weyand, Katrin; 3086

Whistance, Robert; 2009

Whitehead, Kevin K; 4007, 6077, 6078

Wiebe, Walter; 8273

Wiegand, Gesa; 7403, 7404

Wieniawski, Piotr; 7357

Wiggins, Mark; 4020

Wijnberg, Hans; 4051

Wiklund, Urban; 7004

Wild, Florentine; 6121, 6123

Wilkinson, James D; 4068, 8006, 8014, 8029 , 8030

Wilkinson, James L; 4032, 8088, 8364

Will, Joachim; 7040

Willems van Dijk, Ko; 8297

Wilson, Judith; 3047

Wilson, Neil; 8050

Wilson, Nigel J; 3015, 3091, 6191, 7102, 8383

Winbo, Annika; 3112, 7014

Winlaw, David; 3027, 3072, 3114, 4017, $6225,7036,7162,7321,7424,7459$

Wittwer, Thorsten; 8293

Wlasienko, Pawel; 6013, 6017, 6018, 6019, 6048, 6049

Wörner, Cornelia; 3046, 3075, 8183

Wójcicka-Urbanska, Barbara; 7357

Wojtalik, Mariusz; 7011

Wolf, Nadine; 8183

Wolk, John H; 4036

Woloszyn, Marcela I; 7133

Won, Hye-Sung; 6027, 6028

Wong, SJ; 6090

Wong, Davina; 3098

Wong, Derek TH; 3047
Wong, Dora Mei Ling; 8242

Wong, Ken-Pen; 7077

Wong, Keng Y; 7463

Wong, Keng Yean; 7152, 7264, 7463, 8016 , 8150

Wong, Martin NL; 6124, 6142, 8047, 8271

Wongwandee, Ratthapon; 7123

Woodward, Dan; 6208

Wren, Christopher; 7112

Wright, Gail E; 6205

Wright, John G C; 4060, 4109, 7072, 8082

Wrightson, Neil; 8196

Wu, Bin-Nan; 8134

Wu, En-Ting; 8349

Wu, Gang; 8326

Wu, Jiunn-Ren; 8134

Wu, Lan-Ping; 4025, 4027, 6114, 6127, 6129, 7487, 8392

Wu, Lin; 6098, 7041, 7285, 8350

Wu, Mei-Hwan; 6070, 6222, 7178, 7179, 7180, 7358, 7359, 8188, 8349, 8403, 8404,8405

Wu, Qin; 3017

Wu, Qingyu; 2104, 3018, 6122, 7138

Wu, Shye-Jao; 7482

Wu, Wenxia; 7049, 8409

Wurr, Sheila; 8170

Xi, Jicheng; 3018

Xiao, Wenjing; 6175

Xie, Li-Jian; 3088

Xie, Yume; 7082

Xing, Quansheng; 3017

Xing, Yanlin; 4070

$\mathrm{Xu}$, Yaoqiang; 7383

Xu, Zhi wei; 7483, 7484, 7485, 7486

Xu, Zhi Wei; 8173

Xu, Zhiwei; 7182, 7375

Xu, Zhongying; 8117

$\mathrm{Xu}$, Zhou ming; 6147, 6174, 8130

Xu, Zhuoming; 6174, 8130

Xue, Hai-Hong; 6127

Xue, Hui; 7138

Xue, Qinhua; 6175

Yadav, BalbirSingh; 7350

Yagihara, Toshikatsu; 6223, 7030

Yakub, Azhari; 7460

Yalçin, Yalim; 7166, 7167, 8041, 8042, 8044, 8079, 8080

Yalynska, Tetyana; 6064, 6091, 6092, 6099

Yamada, Osamu; 7030

Yamaguchi, Masahiro; 7230

Yamaguchi, Seiji; 8161

Yamamoto, Eiichi; 6156, 7273, 7456, 8105, 815

Yamamoto, Masaki; 7030

Yamashiro, Masahito; 7473

Yamauchi, Sanae; 8213, 8255

Yan, Chaowu; 8117

Yan, Fuxia; 6011, 6175

Yan, Hui; 8304

Yan, Jun; 7148

Yan, Qin; 7483, 7484, 7485, 7486

Yan, Xiangang; 6210

Yandamury, Suman Vyas; 8336

Yañez Sanchez, JM; 6063

Yang, Ge; 4082

Yang, Ji-Hyuk; 6196, 7186, 7240, 7457, 8011, 8108, 8143

Yang, Jian-ping; 8100

Yang, Kaihua; 6129, 7487
Yang, Shiwei; 8051

Yano, Kiyotaka; 2063

Yao, Li-Ping; 4063, 6114, 6127

Yap, Betty T; 8226

Yasuda, Kenji; 8161

Yasukawa, Kumi; 7303

Yasukochi, Satoshi; 6050, 6116, 7218

Yates, Robert; 8019

Yazaki, Satoshi; 6103

Ye, Ming; 62107285

Yemets, Illya N; 6064, 6091, 6099, 7161 , 8229,8354

Yemets, Illya M; 7168, 7169, 7441

Yendamary, suman Vyas; 8337

Yershova, Yevgeniya B; 6092, 6099

Yeung, Joanne P; 8184

Yi, Qijian; 8408

Yildirim, Ayse; 8027

Yildirim, Selman Vefa; 8398, 8399 8400

Yildirim, Vedat; 8340

Yildiz, Yahya; 7166, 7167

Yip, William; 7049, 7327, 8409

Yodoya, Noriko; 6216, 7467, 8153, 8162

Yokoyama, Kazuto; 6216, 7467

Yokozawa, Masato; 7345, 8279

Yokusoglu, Mehmet; 8246

Yoo, Byung Won; 7120, 7488, 8332, 8333

Yoo, Shi-Joon; 6067

Yoshida, Homare; 7250, 8163

Yoshida, Yoko; 7038, 7039

Yoshikawa, Tadahiro; 7113, 7314

Yoshikawa, Yoko; 8161

Yoshikawa, Yoshiro; 7220

Yoshimoto, Jun; 7032, 7038, 7039

Yoshimura, Naoki; 6223, 7108, 7159, 7489,8139

Yoshizumi, Ko; 7449, 7450

Young, Alistair; 4066

Young-Borkowski, Lisa; 6197

Yoyoda, Noriko; 8119

Yu, Chang-Hsien; 7482

Yu, Chen-Yan; 3088, 7274

Yu, Hsi-Yu; 6070

Yu, Jae-Hyeon; 7490

Yu, Jian-Guo; 6127, 8392

Yu, Jung-Min; 7081

Yu, Zhi-qing; 8100

Yu, Zhiqing; 8348

Yuan, Yue; 7082

Yuge, Tetsuji; 7354, 7416

Yukami, Shintarou; 7410

Yun, Byoung- Hoon; 7362

Yun, Gyoung-bin; 8164

Yun, Sin-Weon; 6051, 7362

Yun, Tae-Jin; 7236

Yun, Yong Soo; 7023, 7280, 8225

Yung, Michael; 7334

Yung, Tak-Cheung; 7083, 7084

Yurt, Ayse; 6041

Yusefnia, Mohamad ali; 8253

Yusoff, Khalid; 8169

Yusoff, Yazmin; 3026

Zabal, Carlos; 7191, 8070, 8247

Zadinello, M; 7044

Zahka, Kenneth; 7204, 7221

Zahn, Evan M; 8344

Zamith, Marina; 8062, 8063, 8366

Zamora, Rolando; 8410

Zanjani, Keyhan Sayadpour; 7363, 8411

Zare, khobiar; 6062

Zartner, Peter A; 3060, 7040, 8273, 8412 
Zavalkoff, Samara; 6176

Zeinaloo, Ali Akbar; 7363

Zeng, Irene; 3091, 4066

Zentner, D; 6130

Zeybek, Cenap; 8041, 8042, 8043, 8079,8080

Zhang, Haibo; 7375

Zhang, Hargen; 6133

Zhang, Jian-qun; 3122

Zhang, Lifeng; 4101, 7041

Zhang, Po; 7082

Zhang, Qingyou; 7015

Zhang, Ting; 7223

Zhang, Yajuan; 6150

Zhang, Yanbo; 6150

Zhang, Yu-qi; 4025, 6131, 6132, 8031,

8032, 8100

Zhang, Yu-Qi; 4027, 6071

Zhang, Yuqi; 6114

Zhang, YuQi; 4063, 6052

Zhang, Zewei; 3017

Zhang, Zhi-fang; 6132
Zhang, Zhifang; 6114

Zhang, Zhong-de; 8317

Zhao, Lili; 4082

Zhao, Nan; 8392

Zhao, Pengjun; 7364

Zhao, Shihua; 8117

Zhao, Wu; 8348

Zheng, Hong; 8117

Zheng, Lei; 8173

Zheng, Zhe; 7148, 7491

Zhong, Shu-wen; 4025, 6131, 8031, 8032

Zhong, Yu-min; 8100

Zhong, Yu-Min; 4027

Zhou, Aiqing; 8348

Zhou, Qi-wen; 3122

Zhou, Zhi-bin; 4112

Zhu,; 6126

Zhu, De ming; 7483, 7484, 7485, 7486

Zhu, De Ming; 6217

Zhu, Jing; 4082, 8326

Zhu, Limin; 6174, 6188, 8130, 8165

Zhu, Min; 4027
Zhu, Rui; 3018

Zhu, Shanliang; 7082

Zhu, Zhong qun; 7486

Ziemer, Gerhard; 4083, 6207, 6215, 7117

7118, 7403, 7404

Zilla, Peter; 7222

Zimmerman, Hannah; 8008

Ziolkowska, Lidia; 8116

Zorzi, Elisabetta; 8206, 8207

Zubrzycka, Maria; 8084, 8115, 8116 ,

8414, 8415

Zühlke, Liesl J; 7253, 7305

Zuhlke, Liesl; 7122, 7170

Zuhlke, Liesl J; 7306, 7492

Zunzunegui, Jose L; 8049

Zunzunegui, Jose Luis; 8413

Zurakowski, David; 3030, 6213

Zyla-Frycz, Maria; 8274, 8395

Šaric, Dalibor; 7090

Škovránek, Jan; 6046, 6047

Špálová, Ivana; 6047 